\title{
CALENDAR YEAR 1993 GROUNDWATER QUALITY REPORT FOR THE UPPER EAST FORK POPLAR CREEK HYDROGEOLOGIC REGIME, Y-12 PLANT, OAK RIDGE, TENNESSEE
}

\author{
1993 Groundwater Quality Data and Calculated Rate \\ of Contaminant Migration
}

February 1994

Prepared by

HSW ENVIRONMENTAL CONSULTANTS, INC.

Under Purchase Order 70Y-EAQ10C

for

Environmental Management Department

Health, Safety, Environment, and Accountability Organization

Oak Ridge Y-12 Plant

Oak Ridge, Tennessee 37831

Managed by

MARTIN MARIETTA ENERGY SYSTEMS, INC.

for the U.S. Department of Energy

Under Contract No. DE-AC05-84OR21400 


\section{DISCLAIMER}

This report was prepared as an account of work sponsored by an agency of the United States Government. Neither the United States Government nor any agency thereof, nor any of their employees, makes any warranty, express or implied, or assumes any legal liability or responsibility for the accuracy, completeness, or usefulness of any information, apparatus, product, or process disclosed, or represents that its use would not infringe privately owned rights. Reference herein to any specific commercial product, process, or service by trade name, trademark, manufacturer, or otherwise does not necessarily constitute or imply its endorsement, recommendation, or favoring by the United States Government or any agency thereof. The views and opinions of authors expressed herein do not necessarily state or reflect those of the United States Government or any agency thereof. 


\section{TABLE OF CONTENTS}

Section $\quad \underline{\text { Page }}$

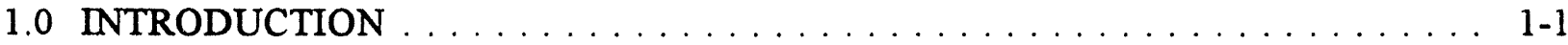

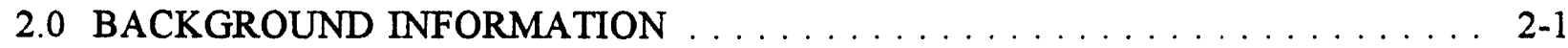

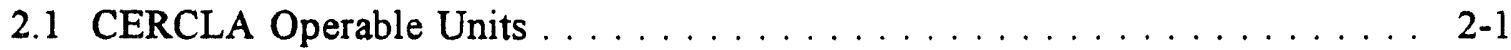

2.1.1 East Fork Poplar Creek OU $01 \ldots \ldots$. . . . . . . . . . . . . . 2

2.1.2 East Fork Poplar Creek OU $02 \ldots \ldots \ldots$ 2-4

2.1.3 East Fork Poplar Creek OU $03 \ldots \ldots$. . . . . . . . . . . . 2-4

2.1.3.1 Y-12 Salvage Yard ... . . . . . . . . . . . 2-5

2.1.3.2 S-2 Site . . . . . . . . . . . . . . . . . . . . 2-6

2.1.3.3 Waste Coolant Processing Area . . . . . . . . . . . 2-6

2.2 Y-12 Study Areas . . . . . . . . . . . . . . . . . . . 2-7

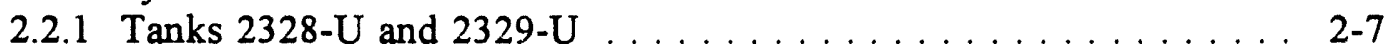

2.2.2 Interim Drum Yard . . . . . . . . . . . . . . . . 2-7

2.2.3 Beta-4 Security Pits . . . . . . . . . . . . . . . . . 2-8

2.3 New Hope Pond . . . . . . . . . . . . . . . . . . . . . . . . 2-8

2.4 Underground Storage Tanks . . . . . . . . . . . . . . . . . . . . . . . 2- 2-9

2.4.1 $9754-2$ Fuel Facility . . . . . . . . . . . . . . . . . . 2-10

2.4.2 Garage Underground Tanks . . . . . . . . . . . . . . . . 2-10

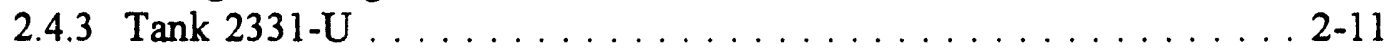

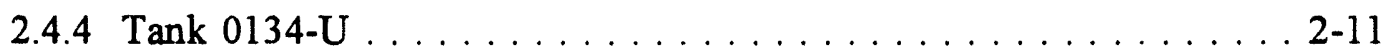

2.4 .5 Rust Garage Area . . . . . . . . . . . . . . . . . . . . . 2-12

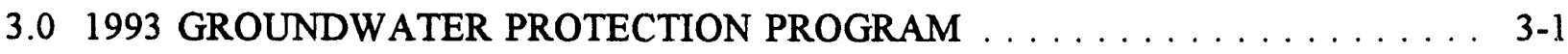

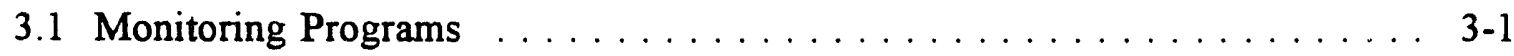

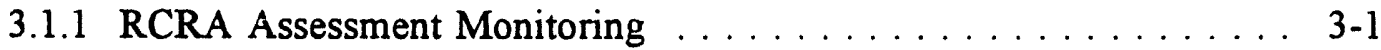

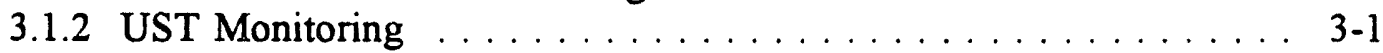

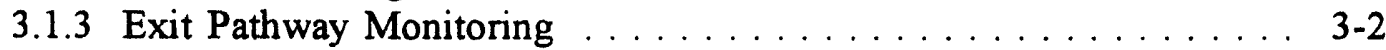

3.1 .4 Grid Well Monitoring . . . . . . . . . . . . . . . . 3-2

3.2 Sampling and Analysis Program . . . . . . . . . . . . . . . . . 3-2

3.2.1 Sampling Locations . . . . . . . . . . . . . . . . . . . 3-3

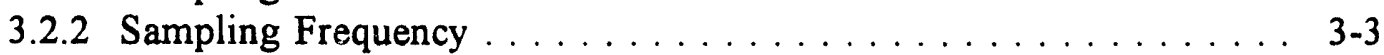

3.2.3 Sample Collection ...................... 3-3

3.2.4 Quality Assurance/Quality Control Sampling ... . . . . . . . . 3-4

3.3 Analytical Parameters . . . . . . . . . . . . . . . . . . . . . 3-4

3.4 Data Management . . . . . . . . . . . . . . . . . . . . . 3-5

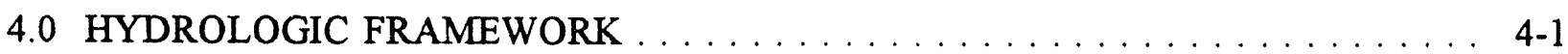

4.1 Geology . . . . . . . . . . . . . . . . . . . . . . . . 4-2

4.2 Groundwater System . . . . . . . . . . . . . . . . . . . . . 4-4

4.2.1 Stormflow Zone . . . . . . . . . . . . . . . . . . . . . . . 4-5

4.2 .2 Vadose Zone . . . . . . . . . . . . . . . . . . . 4-5

4.2.3 Groundwater Zone . . . . . . . . . . . . . . . . . . . . 4-6

4.2.3.1 Water-Table Interval . . . . . . . . . . . . . . 4-6

4.2.3.2 Shallow Interval $\ldots \ldots \ldots \ldots \ldots \ldots \ldots$ 4-7 
TABLE. OF CONTENTS

$\underline{\text { Section }}$

4.2.3.3 Intermediate Interval . . . . . . . . . . . . . . . . . 4-8

4.2.3.4 Deep Interval . . . . . . . . . . . . . . . . . . . . 4-8

4.2 .4 Aquiclude . . . . . . . . . . . . . . . . . . . . . . . . . . . 4 4-9

4.2.5 Groundwater Flow Directions . . . . . . . . . . . . . . . 4-10

4.2.6 Contaminant Transport . . . . . . . . . . . . . . . . . 4-11

4.2.6.1 Quantitative Estimates . . . . . . . . . . . . . . . 4-11

4.2.6.2 Qualitative Estimates . . . . . . . . . . . . . . . 4-13

4.3 Surface-Water System . . . . . . . . . . . . . . . . . . . . . 4-13

5.0 REFERENCES . . . . . . . . . . . . . . . . . . . . . . . $5-1$

Appendices
A Figures
B Tables
C Monitoring-Well Construction Details
D Sampling Sequence
E Groundwater Quality Data
F Field Duplicate Data
$G$ Ion-Charge Balance Calculations
$\mathrm{H}$ Trace Metal Data Summary
I QA/QC Data Summaries
J Validated Results of Volatile Organic Compound Analyses
K Radiochemical Data Summary
L Annual Average Nitrate (as N) Concentrations
M Groundwater Elevations 


\section{LIST OF FIGURES}

Figure

Page

$1 \quad$ Regional Location of the $\mathrm{Y}-12$ Plant $\ldots \ldots \ldots \ldots \ldots \ldots$

2 Hydrogeologic Regimes at the $\mathrm{Y}-12$ Plant $\ldots \ldots \ldots \ldots \ldots$ A-2

3 Waste-Management Sites, CERCLA Operable Units, and USTs Located in the Upper East Fork Poplar Creek Hydrogeologic Regime . . . . . . . . . . . . A A-3

4 Location of Monitoring Wells Sampled During $1993 \ldots \ldots \ldots \ldots \ldots \ldots$

5 Topography and Bedrock Geology in the Upper East Fork Poplar Creek Hydrogeologic Regime . . . . . . . . . . . . . . . . . . . . . A-5

6 Schematic Profile of Hydrostratigraphic Units in the Upper East Fork Poplar Creek Hydrogeologic Regime . . . . . . . . . . . . . . . . . . . . . A-6

7 Groundwater Elevations in the Water-Table Interval $\ldots \ldots \ldots \ldots \ldots \ldots$ A-7

8 Groundwater Elevations in the Shallow and Intermediate Bedrock Intervals . . . A-8 


\section{LIST OF TABLES}

Table

1 Waste-Management Sites, CERCLA Operable Units, and Underground

Storage Tanks Included in the 1993 Groundwater Protection Program . . . . . . . . B-1

2 Monitoring Wells Sampled During $1993 \ldots \ldots \ldots \ldots \ldots \ldots \ldots$. . . . . . .

3 General Construction Information for Monitoring Wells Sampled During 1993 B-8 


\section{ACRONYMS}

$\begin{array}{ll}\text { ARAR } & \text { Appropriate Relevant Applicable Requirement } \\ \text { BCV } & \text { Bear Creek Valley } \\ \text { CERCLA } & \text { Comprehensive Environmental Response, Compensation, and Liability Act } \\ \text { CY } & \text { Calendar Year } \\ \text { DOE } & \text { U.S. Department of Energy } \\ \text { EMD } & \text { Environmental Monitoring Database } \\ \text { EPA } & \text { U.S. Environmental Protection Agency } \\ \text { FFA } & \text { Federal Facility Agreement } \\ \text { GWPP } & \text { Groundwater Protection Program } \\ \text { ORNL } & \text { Oak Ridge National Laboratory } \\ \text { ORR } & \text { Oak Ridge Reservation } \\ \text { OU } & \text { Operable Unit } \\ \text { PCB } & \text { Polychlorinated biphenyl } \\ \text { PCPA } & \text { Post-Closure Permit Application } \\ \text { QA/QC } & \text { Quality Assurance/Quality Control } \\ \text { RCRA } & \text { Resource Conservation and Recovery Act } \\ \text { RIFS } & \text { Remedial Investigation/Feasibility Study } \\ \text { SWMU } & \text { Solid Waste Management Unit } \\ \text { TDEC } & \text { Tennessee Department of Environment and Conservation } \\ \text { TSD } & \text { Treatment, Storage, or Disposal (Unit) } \\ \text { UEFPC } & \text { Upper East Fork Poplar Creek } \\ \text { UST } & \text { Underground Storage Tank } \\ \text { VOC } & \text { Volatile Organic Compound } \\ \text { WCPF } & \text { Waste Coolant Processing Facility } \\ \text { WMCBF } & \text { Waste Machine Coolant Biodegradation Facility } \\ & \end{array}$




\subsection{INTRODUCTION}

This report contains groundwater quality data obtained during the 1993 calendar year (CY) at several waste management facilities and petroleum fuel underground storage tank (UST) sites associated with the U.S. Department of Energy (DOE) Y-12 Plant located on the DOE Oak Ridge Reservation (ORR) southeast of Oak Ridge, Tennessee (Figure 1). These sites are within the Upper East Fork Poplar Creek Hydrogeologic Regime (East Fork Regime), which is one of three regimes defined for the purposes of groundwater and surface-water quality monitoring at the Y-12 Plant. The Environmental Management Department of the Y-12 Plant Health, Safety, Environment, and Accountability Organization manages the groundwater monitoring activities in each regime as part of the Y-12 Plant Groundwater Protection Program (GWPP).

The annual groundwater report for the East Fork Regime is completed in two-parts; Part 1 (this report) containing the groundwater quality data and Part 2 containing a detailed evaluation of the data. The primary purpose of this report is to serve as a reference for the groundwater data obtained each year under the lead of the Y-12 Plant GWPP. However, because it contains information needed to comply with the Resource Conservation and Recovery Act (RCRA) interim status assessment $\mathrm{mc}$ toring reporting requirements, this report is submitted to the Tennessee Department of He's $\Omega$ and Environment (TDEC) by the RCRA reporting deadline (March 1 of the following CY). Part 2 of the annual groundwater report, to be issued mid-year, will contain a regime-wide evaluation of groundwater quality, present the findings and status of ongoing hydrogeologic studies, describe changes in monitoring priorities, and present planned modifications to the groundwater sampling and analysis activities. 


\subsection{BACKGROUND INFORMATION}

The Y-12 Plant is located in Bear Creek Valley (BCV), which is flanked on the north by Pine Ridge and on the south by Chestnut Ridge (directions in this report are in reference to the Y-12 Plant grid system). The East Fork Regime encompasses the Y-12 Plant complex, extending west from New Hope Pond to a topographic and hydrologic divide located near the west end of the plant that separates the East Fork Regime from the Bear Creek Hydrogeologic Regime (Bear Creek Regime) (Figure 2).

Groundwater quality monitoring in the East fork Regime during CY 1993 was performed at:

- sites scheduled for investigation under the Comprehensive Environmental Response, Compensation, and Liability Act (CERCLA);

- a RCRA treatment, storage, and disposal (TSD) facility (New Hope Pond); and

- petroleum fuel underground storage tanks (USTs).

A brief description of each of the sites, including waste inventories and disposal/operational histories, and a discussion of the regulatory status and groundwater monitoring history of each site are provided in the following sections.

\subsection{CERCLA Operable Units}

In 1984, the U.S. Environmental Protection Agency (EPA) and the TDEC determined that several of the waste-management sites at the Y-12 Plant were hazardous waste TSD units subject to regulation under RCRA. One of these TSD units, New Hope Pond, is located in the East Fork Regime and was granted RCRA interim status in 1986. Between 1987 and 1989, several other waste-management sites in the regime were identified as solid waste management units (SWMUs) subject to regulation under section 3004(u) of RCRA (Welch 1987; Welch et al. 1987; Wiggins and Welch 1988a and 1988b; and Murphy 1989). A RCRA Facility Investigation was planned for each of these sites to determine if contaminant releases to the environment had occurred. 
In November 1989, the ORR was added to the National Priority List and in January 1992, the DOE, the EPA, and the TDEC negotiated a Federal Facility Agreement (FFA) regarding environmental restoration at the ORR under CERCLA. This agreement changed the regulatory status several of the RCRA regulated SWMUs in the East Fork Regime to CERCLA Operable Units (OUs). As such, the DOE appealed the applicability of RCRA permitting, post-closure care, corrective action, and groundwater monitoring requirements to CERCLA OUs.

In April 1993, the DOE, TDEC, and Martin Marietta Energy Systems, Inc. signed an Agreed Order regarding a RCRA post-closure permit for the S-3 Site (a RCRA TSD unit/CERCLA OU located in the Bear Creek Regime), thereby resolving the appeal and formally agreeing to proceed with CERCLA as a lead regulatory program with RCRA as an appropriate, relevant, and applicable requirement (ARAR). Under this agreement, RCRA will be applied as an ARAR to the extent that post-closure maintenance and care of former TSD facilities will be conducted in compliance with the terms of RCRA post-closure permits. Groundwater monitoring is to be integrated with the CERCLA remedial investigation/feasibility study (RI/FS) programs with RCRA corrective action deferred to CERCLA. Groundwater monitoring data reporting will comply with RCRA post-closure permit conditions as well as RI/FS requirements.

Two types of OUs were established under the FFA; source control OUs and integrator

OUs. Source control OUs are individual or groups of waste sites for which a RI/FS will be performed, including any interim actions and/or remedial actions. Integrator OUs encompass groundwater and surface water, and are considered separate from source control OUs that contribute to the contamination of these media. Integrator OUs were established because available data showed that contaminants from multiple sources had intermingled in the groundwater system and migrated through a common exit-pathway (U.S. Department of Energy 1992). One integrator OU and two source control OUs established under the FFA lie within the East Fork Regime (Table 1).

\subsubsection{East Fork Poplar Creek OU 01}

East Fork Poplar Creek OU 01 is the integrator OU established by the FFA and includes groundwater in the regime and areas within the Y-12 Plant where elemental mercury was routinely handled during the extraction process for lithium isotopes at the Y-12 Plant between 
1955 and 1962 (U.S. Department of Energy 1992). The integrator OU was established because monitoring results obtained since the mid-1980s indicated that groundwater contamination in the regime resulted from releases from several separate sources, possibly including the mercury process spill areas.

Since 1990, efforts to characterize the extent of the groundwater contamination have been performed in accordance with the technical approach contained in the Comprehensive Groundwater Monitoring Plan for the Department of Energy Y-12 Plant (Comprehensive Monitoring Plan; Geraghty \& Miller, Inc. 1990). This plan defined the following objectives for an integrated groundwater contamination assessment program for the East Fork Regime:

- determine the horizontal and vertical extent of groundwater contamination in the regime through phased installation of monitoring wells along a pre-defined grid pattern;

- install additional monitoring wells in the Maynardville Limestone - the primary avenue for contaminant migration in groundwater;

- define and coordinate studies to determine the hydrogeologic characteristics of the flow system in the East Fork Regime;

- perform all sampling activities in accordance with RCRA assessment monitoring protocols (i.e., quarterly sampling frequency) to ensure continued regulatory compliance; and

- report all the monitoring results in a single regime-wide groundwater quality assessment report.

Implementation of the grid-network approach to groundwater contamination assessment in the East Fork Regime began in 1991. The grid network proposed in the Comprehensive Monitoring Plan incorporated many of the existing monitoring wells located at New Hope Pond and several SWMUs and USTs, but also included new monitoring wells proposed for installation in areas of the Y-12 Plant that lacked monitoring coverage. Most of the wells originally included in the grid network, and new wells added to the network between 1991 and 1993, have been incorporated into the ongoing quarterly sampling program.

The RI for East Fork Poplar Creek OU 01 is not scheduled to begin until the late-1990s. Nevertheless, continued implementation of the technical approach established in the Comprehensive Monitoring Plan is expected to provide a substantial portion of the groundwater 
quality data needed to effectively characterize the extent of contamination for the purposes of the RI. However, some objectives of the technical approach, such as following RCRA assessment monitoring protocols, are less applicable because of changing regulatory developments. Additionally, future monitoring priorities may be modified to support particular aspects of the RIs for the source control OUs in the regime.

\subsubsection{East Fork Poplar Creek OU 02}

The 1992 FFA designated the Abandoned Nitric Acid Pipeline as East Fork Poplar Creek OU 02 (Table 1). The pipeline is located in the northwestern portion of the East Fork Regime within the Y-12 Plant compound (Figure 3). Used between 1951 and 1983, the pipeline originally transported effluent, derived from operations in Buildings 9212 and 9206, to the S-3 Ponds. Subsequently, the pipeline was connected to the uranium recovery process lines from the H-1 Foundry (Building 9998). During its operation, an estimated 5,500 gallons per day of effluent, which generally consisted of nitric acid with uranium in solution, was pumped through the pipeline to the S-3 Ponds (Geraghty \& Miller, Inc. 1989a).

Available construction drawings indicate that the pipeline was constiucted of 1.5 to 3 -inch diameter No. 347 stainless-steel pipe. Some sections of the pipeline may have been constructed of 6-inch diameter stainless steel. The pipeline was buried up to 14 ieet $(\mathrm{ft})$ below ground level and sections that passed over water or sewer lines were encasec in concrete. According to drawings generated during the construction of the Y-12 Plant Perimeter Intrusion Detection and Alarm System, sections of the pipeline may have been abandoned and rerouted during its operational period (Geraghty \& Miller, Inc. 1989a).

\subsubsection{East Fork Poplar Creek OU 03}

East Fork Poplar Creek OU 03 is a source control OU comprised of several former RCRA SWMUs within the Y-12 Salvage Yard, the S-2 Site, and the Waste Coolant Processing Facility (Table 1). A RI work plan for this OU was reviewed by the EPA and TDEC and is currently being finalized (U.S. Department of Energy 1993a). Field work for the RI is scheduled to begin in the Spring of 1994. 


\subsubsection{Y-12 Salvage Yand}

The Y-12 Salvage Yard is in the northwestern portion of the East Fork Regime (Figure 3). Waste-management sites in the Y-12 Salvage Yard include the Scrap Metal Storage Area, the Oil/Solvent Drum Storage Area, the Oil Storage Tanks, the Drum Deheader, and three concrete sumps designated as tanks 2063-U, 2328-U, and 2329-U (Figure 3).

Th Salvage Yard Scrap Metal Storage Area has been used from 1950 to the present for the storage of scrap metal, some of which contains low levels of depleted or enriched uranium. Some mino contamination of surficial soils at the site has been reported (Welch et al. 1987).

Before being closed, the Salvage Yard Oil/Solvent Drum Storage Area consisted of two storage areas: the east drum storage area and the west drum storage area. Each area was closed as described in respective closure plans (Welch 1986; Lind and Welch 1989). The combined total storage capacity of both areas was approximately 175,000 gallons. Waste oils containing chlorinated organics, uranium and/or beryllium, chlorinated organic solvents, and non-chlorinated flammable solvents were stored in drums, and leaking drums and spills have been documented on the site. Although a dike is present around the downslope side of the site, the gravel/soil base of the dike may have permitted infiltration of spilled material into the subsurface (Welch et al. 1987).

Operation of the Salvage Yard Oil Storage Tanks began in 1978 when a 6,000 gallon tank was installed to store polychlorinated biphenyls (PCB)-contaminated oil. A 5,000 gallon tank was added to the site in 1980. Both tanks were surrounded by an earthen dike and were emptied in 1986 (Welch 1986). Spills and leaks have occurred, but were contained within the diked area (Welch et al. 1987).

The Salvage Yard Drum Deheader operated from 1959 to 1989 , and was used to cut off the tops and crush empty drums collected from various locations throughout the Y-12 Plant. The deheader is in a small shed with a concrete floor. Before deheading, the drum contents were emptied into other drums contained within a small sump (Tank 2063-U). Tank 2063-U was about $4 \mathrm{ft}$ long, $2 \mathrm{ft}$ wide, $2 \mathrm{ft}$ deep, and had a total capacity of about 130 gallons. Spills and overflows that occurred when the liquids were poured into the storage drums were contained in Tank 2063-U, and transferred through a connecting drain pipe to other sumps designated Tanks 
2328-U and 2329-U. Liquids still present when the drums were deheaded flowed into a floor drain connected to Tanks 2328-U and 2329-U (Stone 1989a).

A leak test was performed for Tank 2063-U in March 1989 and leak rate of 0.46 gallons per hour ( $\mathrm{gal} / \mathrm{hr}$ ) was determined. This rate exceeded the maximum allowable rate established under the TDEC UST regulations and the tank was subsequently excavated. Soils near the tank contained elevated concentrations of cadmium, lead, and mercury, and detectable levels of volatile organic compounds (VOCs) and PCBs (Stone 1989a).

\subsubsection{S-2 Site}

The S-2 Site is in the southwestern portion of the East Fork Regime on the northern flank of Chestnut Ridge (Figure 3). The site was an unlined, earthen reservoir used from 1945 to 1951 for percolation, evaporation, or neutralization of an unknown quantity of liquid wastes. Waste materials reportedly included nitrates of copper, nickel, and chromium; diethyl ether and pentaethers; nitric, hydrochloric, and sulfuric acids; sulfates; dibutyl carbinol and tributyl phosphates; aluminum nitrate; hydrogen fluoride; cadmium; natural and enriched uranium; and cyanide compounds (Kimbrough 1986). The site was closed in 1951, the remaining liquids were neutralized, and the reservoir was filled with soil and seeded with grass (Haase 1987).

\subsubsection{Waste Coolant Processing Area}

The Waste Coolant Processing Area, located in the west-central portion of the Y-12 Plant (Figure 3), is an area used to treat waste coolants collected from various shops within the plant complex. The site originally consisted of the Waste Machine Coolant Biodegradation Facility (WMCBF), an unloading/storage area, and a treatment basin/effluent drain field (Murphy 1989). Operation of the WMCBF ceased in 1985 when it was replaced with a new treatment unit, the Waste Coolant Processing Facility (WCPF) (Murphy 1989). The original unloading/storage area was retained as part of the WCPF, but the WMCBF and the treatment basin/effluent drain field were closed in accordance with a TDEC-approved closure plan (Stone and McMahon 1988). The TDEC certified final closure of the WMCBF on November 23, 1988. 


\subsection{Y-12 Study Areas}

Several sites that were not included in the source control OUs established for the East Fork Regime were designated in the FFA as Y-12 Study Areas and represent lower priority units that will be investigated under preliminary assessments/site investigations (U.S. Department of Energy 1992). New OUs or additions to established OUs may be necessary if results of these preliminary investigations indicate that a complete RI/FS is warranted.

\subsubsection{Tanks 2328-U and 2329-U}

As noted in Section 2.1.3.1, Tanks 2328-U and $22^{\circ} \mathrm{U}$ are located in the Y-12 Plant Salvage Yard and were used in conjunction with operation of the Salvage Yard Drum Deheader and Tank 2063-U. Located adjacent to each other, Tanks 2328-U and 2329-U were separated by a rubber baffle. Each tank was about $8 \mathrm{ft}$ long, $4 \mathrm{ft}$ wide, $4 \mathrm{ft}$ deep, and had a total capacity of about 950 gallons. The tops of the tanks were open at the ground surface. Consequently, rainwater and surface runoff would occasionally collect in the tanks. The baffle between the tanks helped to separate the oils and solvents from the rainwater and runoff. Once separated, the water was released through a drainpipe to the Y-12 Plant stormwater drainage system. The remaining oils and solvents were periodically pumped out, as necessary, and treated or disposed in the Bear Creek Burial Grounds Waste-Management Area (Stone 1989a).

Leak tests were performed on Tanks 2328-U and 2329-U in March 1989 and showed a respective leak rate of $0.14 \mathrm{gal} / \mathrm{hr}$ for each tank. This rate exceeded TDEC standards and the two tanks were subsequently excavated along with Tank 2063-U. Soils near the tanks contained elevated concentrations of cadmium, lead, and mercury, and detectable levels of VOCs and PCBs (Stone 1989a).

\subsubsection{Interim Drum Yard}

The Interim Drum Yard is located in the southwestern portion of the East Fork Regime (Figure 3). The site is currently a graveled, covered, and diked outdoor storage area that formerly was used to store drums containing various hazardous, mixed, and non-hazardous wastes 
including sludge containing chromium, mercury-contaminated wastes, chlorinated and nonchlorinated organics, and plating solutions. Materials contaminated with PCBs are not currently stored at the site but have been in the past. The site presently contains approximately 700 drums of waste, all in over packs. The southern portion of the site has been closed in accordance with a TDEC-approved closure plan (Willoughby et al. 1988).

\subsubsection{Beta-4 Security Pits}

The Beta-4 Security Pits are near the northwestern boundary of the East Fork Regime (Figure 3). The site was used from 1968 to 1972 for classified disposals of uranium and uranium alloys, scrap metal containing depleted and enriched uranium, organic compounds, acids, and miscellaneous debris (Welch et al. 1987).

\subsection{New Hope Pond}

New Hope Pond is a closed RCRA TSD unit located at the east end of the Y-12 Plant (Figure 3). Constructed in 1963, New Hope Pond regulated the flow and quality of water in Upper East Fork Poplar Creek (UEFPC) before exiting the Y-12 Plant, and provided a contingency for control of accidental spills of oils or other substances (PEER Consultants 1989). While in operation, discharge from the pond averaged approximately 8 million gallons per day (mgd). Water-quality sampling was performed in accordance with the conditions set forth in a National Pollutant Discharge Elimination System permit (Permit No. TN002968). Operation of New Hope Pond ceased on November 8, 1988.

In 1973, sediment removed from New Hope Pond was placed in the Chestnut Ridge Sediment Disposal Basin (Sediment Disposal Basin). Additionally, sediments from the inlet diversion ditch were periodically removed and disposed in the Sediment Disposal Basin. These sediments contained PCBs, mercury, and uranium, but Extraction Procedure Toxicity analyses indicated that they did not exhibit the characteristics of a hazardous waste (Saunders 1983; Kimbrough and McMahon 1988a and 1988b). Approximately 25,000 cubic yards of sediment remained in New Hope Pond when it was closed in 1988. Closure of New Hope Pond began in 1988 and was certified by the TDEC in December 1990. Closure involved stabilizing sediments 
left in the pond with coarse aggregate, and covering the pond with a multi-layer, low-permeability cap (Martin Marietta Energy Systems, Inc. 1988).

A RCRA post-closure permit application (PCPA) for the site was initially submitted to the TDEC in 1989 (Lee Wan \& Associates, Inc. 1989). Pertinent aspects of the PCPA are currently being revised to reflect integrated RCRA/CERCLA requirements contained in the Agreed Order signed in April 1993. Additional changes to the PCPA for New Hope Pond also may be necessary pending the outcome of ongoing negotiations over modifications to the post-closure permit issued for the S-3 Site by the TDEC in April 1993.

Lake Reality was constructed immediately downstream of New Hope Pond (Figure 3) and began operation on November 8, 1988. Water in UEFPC enters Lake Reality from an extension of the New Hope Pond inlet diversion ditch and exits through a weir in the west berm. The total surface area of Lake Reality is about 2.5 acres, and the average water depth is approximately $7 \mathrm{ft}$. During normal operations, Lake Reality contains approximately 6 million gallons of water (Lee Wan \& Associates 1989).

In October 1992, the Y-12 Plant GWPP Manager was informed that a pump had been installed in a 6-ft diameter, 20-ft deep dewatering sump installed near Lake Reality in 1990 (Turner 1992). Groundwater removal from the sump (the sump pump is automatically controlled via a pressure switch) relieved the hydrostatic pressure that tended to float the flexible liner on the bottom of Lake Reality (Turner 1992). Groundwater enters the sump from regolith and weathered bedrock, as well as from a french drain constructed beneath the concrete-lined diversion channel located on the southern and eastern boundary of New Hope Pond (Turner 1992). The french drain was reportedly constructed to capture water from one or more springs that had been intersected by the excavation for the diversion channel (Turner 1992). Except for brief periods (less than 1 hour), pumping ceased between September 1991 and October 1993, after which the sump pump was returned to automatic control. Operation of the sump pump may affect groundwater levels in nearby monitoring wells (Turner 1992).

\subsection{Underground Storage Tanks}

Several investigations of product releases from petroleum USTs in the East Fork Regime were initiated in 1989. The objectives of the investigations were to detect and recover any free 
product and determine the extent of petroleum hydrocarbons in soil and groundwater. These investigations have been performed in accordance with requirements specified in Rules of the Tennessee Department of Environment and Conservation, Division of Underground Storage Tanks, Chapter 1200-1-15, Underground Storage Tanks.

\subsubsection{4-2 Fuel Facility}

The Building 9754-2 Fuel Facility, located in the eastern portion of the East Fork Regime (Figure 3), was used from 1978 to 1989 to dispense gasoline and diesel fuel. The site had two USTs: a 20,000-gallon unleaded gasoline tank (Tank 0439-U) and a 10,000-gallon diesel tank (Tank 0440-U). Both tanks were in an unlined, gravel-filled pit about $12 \mathrm{ft}$ deep. The top of the tank pit was open to precipitation, and water that entered the pit was drained to a nearby collection basin through a 12-inch diameter concrete pipe (Welch 1989a).

In June 1989, free product was observed in the collection basin connected to the tank pit. A sump was subsequently excavated adjacent to the tank pit to facilitate free product recovery and removal of contaminated groundwater. Free product and contaminated water were regularly pumped from the sump for several weeks (Stone 1989b). A recovery well was then installed downgradient of the tank pit, but no free product was observed in the well (Stone 1989c).

Gasoline and diesel fuel spills and leaks at the site have been documented. Spills typically occurred when the tanks were overfilled. Gasoline leaks from the fill pipe and the discharge line for Tank 0439-U were confirmed during leak testing and subsequent tank excavation. During excavation of Tank 0440-U, it was noted that leaks from an associated discharge line had occurred (Welch 1989a). Both tanks were excavated in September 1989. Two additional monitoring wells were installed in September 1990 as part of an ongoing UST site investigation (Eaton and Ingram 1991).

\subsubsection{Garage Underground Tanks}

The Garage Underground Tanks were located about $100 \mathrm{ft}$ north of the old Building 9754 Fuel Facility (Figure 3), which went into service in 1944. The site originally contained a 10,000-gallon diesel fuel tank and a 10,000-gallon leaded gasoline tank. A 20,000-gallon 
unleaded gasoline tank was installed at the site in 1975. The tanks gravity-fed three 1,000-gallon tanks at dispenser islands in front of former Building 9754. Individual diesel fuel and gasoline dispensers were installed west of Building 9754 for emergency purposes. Product releases from overfilling the gravity-feed system have occurred.

The three large USTs at the site were converted to waste oil storage in 1978. These tanks were subsequently removed in October 1989 and the tank site is currently undergoing RCRA closure. Also in 1978, the three 1,000-gallon dispenser tanks were emptied and the emergency dispensers were removed. The dispenser tanks are being closed under UST regulations in conjunction with closure of the Building 9754-2 Fuel Facility (Stone 1989b).

\subsubsection{Tank 2331-U}

Tank 2331-U was a 560-gallon steel tank used to store gasoline. The tank and its associated piping were installed in 1973 to serve gasoline-powered equipment in Building 9201-1 (Figure 3). A leak test performed in September 1988 indicated that the tank was leaking. The tank was subsequently emptied and service was discontinued until its removal in December 1988. Soils near the tank pit reportedly contain detectable levels of toluene and xylenes (Welch 1989b). In August 1989, a monitoring well was installed downgradient of the tank pit to facilitate detection and recovery of free product. Although petroleum odors in the well were noted, no free product was observed (Stone 1989d).

\subsubsection{Tank 0134-U}

Tank 0134-U was a steel tank with a capacity of 117 gallons that was used to store gasoline for emergency electrical power generation at Building 9204-2 (Figure 3). The tank was installed in the mid-1960s and was removed from service in 1982. When the tank was excavated in August 1989, several holes were observed in the sides of the tank. Follow-up investigations at the site indicated that VOCs were present in soils and groundwater in the area (Stone 1989e). A monitoring well was subsequently installed within the tank pit to facilitate free product detection and recovery. No free product was observed, but petroleum odors in the well were noted (Stone 1989f). 


\subsubsection{Rust Garage Area}

The Rust Garage Area is located in the northwestern portion of the East Fork Regime about $650 \mathrm{ft}$ east of the S-3 Site (Figure 3). Building 9720-15, which houses the Rust Garage, was used as a vehicle and equipment maintenance shop and is currently used as a paint shop. Industrial products containing hazardous constituents that are stored on the site include lubricating oil, gasoline, diesel fuel, hydraulic fluid, anti-freeze, battery acid, and mineral spirits. A bulk-oil storage platform and an elevated gasoline tank are located south of the garage, and a wash pad is located on the east side of the building.

Building 9754-1 at the Rust Garage Area was originally a fueling station for DOE fleet vehicles. Four petroleum fuel USTs with associated piping were located at the site: a 12,000-gallon gasoline tank (Tank 1222-U), an 8,000-gallon gasoline tank (Tank 2082-U), a 12,000-gallon diesel fuel tank (Tank 1219-U), and a 1,000-gallon gasoline tank (Tank 2068-U). Tank 2068-U was connected to tanks $1222-U$ and $2082-U$ via a transfer line encased in concrete. All four tanks at the site were excavated in December 1989.

Gasoline and diesel fuel releases associated with operation of the USTs have been reported. Because of their proximity and similar operational history, product releases have been evaluated under a single investigation for the site, which started in September 1987. Temporary piezometers were installed during early-1988 to facilitate detection of the leaks, and free product was observed in at least one of these piezometers (Geraghty \& Miller, Inc. 1988). Monitoring wells were installed at the site in 1990 as part of a UST site investigation (Eaton and Van Ryn 1991). 


\subsection{GROUNDWATER PROTECTION PROGRAM}

The following sections describe the groundwater sampling activities performed during CY 1993 under the lead of the Y-12 Plant GWPP. Included are brief descriptions of the monitoring programs for which the sampling was performed, as well as details regarding sampling locations and frequencies, sample collection procedures, analytical parameters, and data management.

\subsection{Monitoring Programs}

Groundwater sampling activities during CY 1993 addressed the purposes of four monitoring programs: (1) RCRA Assessment Monitoring, (2) UST Monitoring, (3) Exit-Pathway Monitoring, and (4) Grid Well Monitoring. Each of these monitoring programs is implemented within the context of the technical approach contained in the Comprehensive Monitoring Plan. However, in light of the increase in regulatory requirements, an increasing number of wells are sampled to meet multiple programmatic goals.

\subsubsection{RCRA Assessment Monitoring}

Quarterly RCRA Assessment Monitoring was continued during 1993, but included only 12 monitoring wells located at New Hope Pond (Table 2). Assessment monitoring at the site is will be discontinued when a RCRA post-closure permit (which is expected to require RCRA compliance monitoring) is issued for the site by the TDEC.

\subsubsection{UST Monitoring}

Groundwater monitoring at petroleum USTs in the East Fork Regime was continued during 1993 and involved 20 wells, most located near the Building 9754-2 Fuel Facility and the Rust Garage Area (Table 2). Monitoring objectives have been achieved for the purposes of the TDEC UST regulations, and additional monitoring of most of these wells as part of the Y-12 Plant GWPP will be discontinued in 1995; sampling of selected wells at the Rust Garage Area will be continued. 


\subsubsection{Exit Pathway Monitoring}

In June 1990, the DOE revised Order 5400.1 regarding environmental protection standards for DOF facilities. This order required monitoring of groundwater that is, or could be, affected by operations at DOE facilities to demonstrate compliance with DOE requirements and applicable Federal, State, and local laws and regulations. Compliance with most of the DOE Order 5400.1 requirements was achieved through continued implementation of ongoing monitoring programs required under TDEC and EPA regulations. However, the order did impose additional requirements for monitoring groundwater quality where contamination was most likely to be transported beyond the ORR boundaries.

Exit Pathway Monitoring is implemented under the Y-12 Plant GWPP to comply with the requirements contained in DOE Order 5400.1. In 1993, Exit Pathway Monitoring involved quarterly sampling of groundwater from 10 monitoring wells completed in the Maynardville Limestone and 3 wells located near UEFPC where it passes through a gap in Pine Ridge northeast of the Y-12 Plant (Table 2).

\subsubsection{Grid Well Monitoring}

In 1993, 48 wells were sampled as part of the Grid Well Monitoring program (Table 2). Approximately 20 new and existing wells remain to be incorporated into the program. These wells likely will be added to the active monitoring network during CY 1994 pending project prioritization decisions in conjunction with the Y-12 Plant Environmental Restoration Program.

\subsection{Sampling and Analysis Program}

The following sections describe the groundwater sampling and analyses activities performed during CY 1993. Included are descriptions of the groundwater sampling locations, frequency, and procedures, analytical parameters, quality assurance/quality control (QA/QC) sampling, and data management. 


\subsubsection{Sampling Locations}

Groundwater monitoring during 1993 involved the collection of groundwater samples from 93 monitoring wells (Table 2). Locations of these monitoring wells are shown on Figure 4, and data regarding the elevation of the screened or open hole interval, the aquifer zone, and the bedrock formation monitored by the wells are provided in Table 3. Detailed well construction information is contained in Appendix C.

\subsubsection{Sampling Frequency}

Groundwater samples were collected during each quarter of 1993 from all but two of the monitoring wells. Samples were not collected as planned from wells GW-152 and GW-167 at New Hope Pond because both wells were dry during each sampling event. First through fourth quarter sampling events were performed from January 8 to March 11, April 7 to May 24, July 1 to September 27, and October 12 to December 9, respectively.

\subsubsection{Sample Collection}

Groundwater samples were collected by personnel from the Oak Ridge K-25 Site Sampling and Environmental Support Department. Pre-defined sampling sequences were followed to minimize the potential for cross-contamination, with each quarterly sequence reflecting sampling from least contaminated wells to the most contaminated wells at a site or in a sampling group (a series of monitoring wells grouped for sampling and data-tracking purposes) (Appendix D).

Groundwater sampling was performed in accordance with the most recent version of the Standard Operating Procedure 8102 approved by the Y-12 Plant GWPP Manager. Descriptions of the field methods and procedures used to collect the groundwater samples are contained in Environmental Surveillance Procedures Quality Control Program, Martin Marietta Energy Systems, Inc. (Kimbrough et al. 1987). The EPA (Region IV) and the TDEC have approved these methods and procedures (U.S. Environmental Protection Agency 1988a) 


\subsubsection{Quality Assurance/Quality Control Sampling}

Quality assurance/quality control samples included laboratory blanks, trip blanks, equipment rinsates, and duplicate groundwater samples. A total of 143 laboratory blanks, 197 trip blanks, 47 equipment rinsate samples, and 45 duplicate samples were analyzed during 1993. Similar numbers of QA/QC samples were analyzed during each quarterly sampling event: 32 to 42 laboratory blanks, 46 to 51 trip blanks, 11 to 14 equipment rinsates, and 10 to 14 duplicate samples.

Laboratory blanks were prepared using deionized water and analyzed along with the groundwater samples. Trip blanks were prepared in the laboratory using deionized water and transported unopened in coolers containing groundwater samples for VOC analyses. Equipment rinsates were obtained from the deionized water used to decontaminate the groundwater sampling equipment. These samples were collected after a sampling team had completed sampling at a site or finished a sampling group (typically no more than 10 wells grouped for sampling and data-tracking purposes). If more than one team collected samples at a site or within a sampling group, each collected an equipment rinsate sample. Duplicate groundwater samples were collected at a frequency of at least one per sampling group, or one for every 10 monitoring wells, whichever was greater.

\subsection{Analytical Parameters}

Groundwater samples were analyzed for the suite of parameters and constituents listed in

Table 4. The analytical parameters and constituents included:

trace metals and principal ions (total and dissolved concentrations);

- VOCs;

- gross alpha activity and gross beta activity;

- total suspe: ded solids, total dissolved solids, and turbidity;

- field and laboratory determinations of $\mathrm{pH}$ and specific conductance; and

- field determinations of temperature, dissolved oxygen, and oxidation-reduction potential.

Samples from monitoring wells included in the UST Monitoring program also were analyzed for total petroleum hydrocarbons. The K-25 Analytical Chemistry Department performed most of 
the laboratory analyses; radiochemical analyses were performed by the Oak Ridge National Laboratory (ORNL) Chemistry Division.

All of the laboratory blanks, trip blanks, and equipment rinsate samples were analyzed for the suite of VOCs listed on Table 4. Selected equipment rinsate samples also were analyzed for trace metals (Inductively Coupled Plasma analyses), nitrate (as N), and gross alpha and gross beta activity. Duplicate groundwater samples were analyzed for the constituents and parameters specified for the well from which the duplicate sample was collected.

Groundwater quality data obtained during 1993 are contained in Appendices E through L. Appendix E contains the analytical results for each groundwater sample; results for duplicate groundwater samples are presented in Appendix F. Ion-charge balances for each groundwater sample are presented in Appendix G. Appendix $\mathrm{H}$ contains trace metal results that exceed Maximum Contaminant Levels or background screening levels. Analytical results for the QA/QC samples are summarized in Appendix I. A summary of the validated analytical results for VOCs is provided in Appendix $\mathrm{J}$, and radiochemical data are summarized in Appendix $\mathrm{K}$. Annual average concentrations of nitrate (as $\mathrm{N}$ ) (a primary groundwater contaminant in the western part of the regime) determined for each well are provided in Appendix L.

\subsection{Data Management}

Analytical results obtained by the K-25 and ORNL laboratories were verified, entered into the Y-12 Plant Environmental Monitoring Database (EMD), and electronically processed through several data evaluation procedures. Brief descriptions of the data verification/entry/evaluation procedures follow; details are provided in Data Verification and Evaluation Techniques for Groundwater Monitoring Programs (Mercier and Turner 1990).

Analytical results obtained from each quarterly sampling event were either downloaded directly into the EMD from data files provided by the analytical laboratory or were manually input from hardcopy documentation (laboratory reports and field data sheets). For manually input data, a double-entry procedure was followed whereby the hardcopy data were input twice into separate data files. A computer program then compared the two input data files and identified discrepancies, which were then accounted for and corrected. When all data from the quarterly sampling event were in the EMD, a computer program then verified that the parameter names 
were correct, correct units were reported, and that the results reported for each parameter were within an expected range or were valid numeric values. As a final step in the data entry/verification process, printouts of the data were visually checked against the laboratory reports and field data sheets for completeness and obvious data outliers. The appropriate laboratory was contacted to resolve incomplete data transfers, discrepancies between electronic and hardcopy versions of the data, and questions regarding data outliers.

Upon completion of data verification and entry into the EMD, a computer program was used to scan the monitoring data, flag questionable or suspect data based on pre-defined criteria, and prepare a report for transmittal to the appropriate laboratory. This data evaluation program calculated son-charge balances for each sample, identified changes in analytical detection limits, and validated analytical results for VOCs using the QA/QC sample data in accordance with an EPA validation procedure (U.S. Environmental Protection Agency 1988b). Questionable or suspect data were reported to the appropriate analytical laboratory. Errors in the data were corrected, and questionable results unresolved by the appropriate laboratory were flagged. 


\subsection{HYDROLOGIC FRAMEWORK}

This section contains a general description of the complex hydrogeologic system in the East Fork Regime. It provides sufficient descriptive detail needed for the purposes of RCRA reporting requirements regarding evaluation of groundwater flow directions, and determination of representative rates of groundwater flow and contaminant transport. The description is based on the conceptual framework and associated nomenclature presented in: Status Report - A Hydrologic Framework for the Oak Ridge Reservation (Solomon et al. 1992).

Groundwater in the East Fork Regime occurs in two broad units with fundamentally different hydrologic characteristics: the Knox Aquifer and the ORR Aquitards. The Knox Aquifer is comprised of the Knox Group and the underlying Maynardville Limestone formation of the Conasauga Group. The bulk permeability in the Knox Aquifer, which is due primarily to solution conduits, is ten times greater than that of the ORR Aquitards. The Maynardville Limestone is believed to be a drain for the groundwater flow system in the East Fork Regime and provides the primary pathway for migration of contaminants in groundwater.

The remaining Conasauga Group formations, the underlying Rome Formation, and the Chickamauga Group form the ORR Aquitards. In this report, the term "ORR Aquitards" refers only to the Conasauga Group (excluding the Maynardville Limestone), and the Rome Formation. Flow in the ORR Aquitards is controlled by short, intersecting fractures in a relatively impermeable matrix, and most groundwater flows through a thin zone near the water table. Most of the waste-management sites in the East Fork Regime are underlain by the ORR Aquitards, in

which contaminant migration rates relative to groundwater flow velocities are reduced by diffusive exchange between matrix and fractures (Solomon et al. 1992).

Pine Ridge and Chestnut Ridge are the primary recharge areas for the groundwater system in the East Fork Regime. Annual recharge is greater for the Knox Aquifer (about 2.6 inches) than for the ORR Aquitards (about 0.8 inches), and it is possible that a portion of the recharge to each unit results from delayed unsaturated flow (Solomon et al. 1992). Groundwater in the ORR Aquitards generally flows from the recharge areas toward the Maynardville Limestone, which subcrops in BCV along the base of Chestnut Ridge and underlies UEFPC in much of the regime. Groundwater in the Maynardville Limestone generally flows to the east along geologic strike. 


\subsection{Geology}

The geology in the area of the Y-12 Plant is generally characterized by sequences of southeast-dipping, fractured clastic (primarily shale and siltstone) and carbonate (limestone and dolostone) strata of Lower Cambrian to Lower Ordovician age (Figure 5). Bear Creek Valley is underlain by the interbedded limestone and shale formations of the Conasauga Group. Shale and siltstone beds of the underlying Rome Formation form Pine Ridge to the north and the primarily dolostone strata of the overlying Knox Group form Chestnut Ridge to the south. Strike and dip of bedding in the East Fork Regime are generally $\mathrm{N} 55^{\circ} \mathrm{E}$ and $45^{\circ} \mathrm{SE}$, respectively.

Bedrock in the East Fork Regime is overlain by alluvium, colluvium, man-made fill, fine-grained residuum from the weathering of the bedrock, saprolite (a transitional mixture of fine-grained residuum and bedrock remnants), and weathered bedrock. The overall thickness of these materials in the East Fork Regime is typically less than $40 \mathrm{ft}$. In undeveloped areas, the saprolite retains primary textural features of the unweathered bedrock, including fractures (Solomon et al. 1992).

Several areas of the East Fork Regime contain varying amounts of man-made fill emplaced during construction of the Y-12 Plant and the re-routing of the original channel of UEFPC. Investigations conducted by the U.S. Army Corps of Engineers (1954) showed that two different layers of fill were emplaced during two episodes of plant construction, and that the fill contains many voids and is largely composed of building debris. The non-uniform properties and varying thickness of the fill probably create widely varying hydraulic conditions in the nearsurface.

The Maynardville Limestone subcrops along the axis of BCV at the base of Chestnut Ridge and the underlying formations of the ORR Aquitards subcrop successively to the north toward Pine Ridge (Figure 5). The Maynardville Limestone consists of fine- to coarse-grained, thin-bedded to massive limestone and dolostone (King and Haase 1987). Information obtained during 1992 indicates that the thickness of the Maynardville Limestone varies from about 300 to

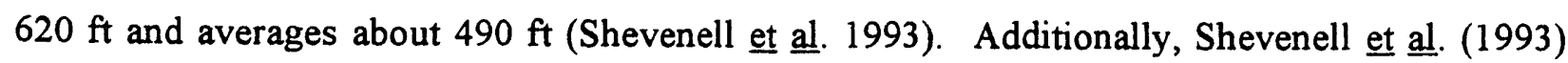
report, based on lithologic information obtained during installation of several exit-pathway monitoring wells, that most of the basal portion of the Maynardville Limestone (the Low Hollow member) is not present in the Bear Creek Regime about 5 miles west of the East Fork Regime. 
This loss of stratigraphic section may reflect a facies change in the Maynardville, as suggested by Shevenell et al. (1993), or may be the result of structural displacement. Further investigation to explain the substantial thinning of the Maynardville Limestone in this area is ongoing.

Underlying formations of the Conasauga Group that comprise the ORR Aquitards are, from youngest to oldest, the Nolichucky Shale, Maryville Limestone, Rogersville Shale, Rutledge Limestone, and Pumpkin Valley Shale (Figure 5). The lithologic compositions of the Nolichucky Shale and Maryville Limestone are similar in the East Fork Regime. These formations consist primarily of between 350 and $550 \mathrm{ft}$ of massive to thin-bedded mudstone with interbedded limestone (King and Haase 1987). Beneath the Maryville Limestone are the massive mudstones of the Rogersville Shale and the interbedded limestone and shale of the Rutledge Limestone. The thickness of each of these formations ranges from 90 to $120 \mathrm{ft}$ (King and Haase 1987). The Pumpkin Valley Shale consists of 260 to $320 \mathrm{ft}$ of massive shale and mudstone with thin beds of siltstone and sandstone (King and Haase 1987).

The most pervasive structural features in the East Fork Regime are extensional, hybrid, and shear fractures (Solomon et al. 1992). Three major joint sets are evident in the East Fork Regime, one that roughly parallels bedding, one steeply dipping set that parallels geologic strike, and one steeply dipping set that trends perpendicular to strike (Drier et al. 1987). Fracture densities ranging from about 1 to 60 per foot have been observed in rock outcrops near the ORNL (Dreier et al. 1987; Sledz and Huff 1981). Most fractures are short, ranging from tenths of inches to a few feet in length (Solomon et al. 1992). Within a fracture, groundwater may flow either downdip, laterally, or in both directions. Changes in flow direction may occur at fracture splits and intersections and groundwater flow paths may locally resemble stairsteps in both plan and sectional views (Moore 1989).

Dissolution of carbonates along fractures, particularly in the Maynardville Limestone, has produced solution-enlarged features ranging from less than an inch to tens of feet in thickness. Shevenell et al. (1993) report that solution cavities occur throughout the Maynardville Limestone, but occur most frequently in the uppermost and lowermost of six stratigraphic zones defined by gamma-log signatures and core data. All water-bearing intervals in the uppermost zone occur at less than $235 \mathrm{ft}$ below the ground surface, and $22 \%$ of these intervals were identified as cavities. Water-bearing intervals in the lowermost zone occur at depths up to about $450 \mathrm{ft}$ below the ground surface, and $19 \%$ of these intervals were identified as cavities (Shevenell et al. 1993). 
An exceptionally large ( $>40 \mathrm{ft}$ ) dissolution feature in the Maynardville Limestone was encountered at a depth of about $60 \mathrm{ft}$ during installation of exit-pathway well GW-734 located about $900 \mathrm{ft}$ southeast of New Hope Pond (Shevenell et al. 1993). Preliminary results of a microgravity survey suggest that this feature is elongated parallel to geologic strike and that the center is west of the well (Shevenell et al . 1993). A pressure transducer was temporarily installed in the well in CY 1993 to continuously monitor water levels. Preliminary data indicate that water levels respond very rapidly to precipitation events, with water level elevation changes of up to $6 \mathrm{ft}$ (Shevenell et al. 1993). Such response patterns indicate that the feature is locally connected to the ground surface, possibly with UEFPC. A $25-\mathrm{ft}$ dissolution feature encountered at a depth of about $100 \mathrm{ft}$ in exit-pathway well GW-722 may be hydraulically connected with the feature in well GW-734 (Shevenell et al. 1993).

\subsection{Groundwater System}

Solomon et al. (1992) divide the groundwater flow system in both the Knox Aquifer and the ORR Aquitards into four subsystems: (1) the stormflow zone, (2) the vadose zone, (3) the groundwater zone (subdivided into water-table, intermediate, and deep intervals), and (4) the aquiclude (Figure 6). The divisions are based on the amount of water transmitted by each subsystem (i.e., flux), which decreases with depth; the largest water flux occurs in the stormflow zone and the smallest in the aquiclude.

The groundwater flow system is vertically gradational with no discreet boundaries separating the subsystems. The stormflow zone occurs in regolith as does much of the vadose zone, although in some areas the vadose zone includes weathered and fresh rock. About $90 \%$ of subsurface flow occurs in the stormflow zone. In the groundwater zone, flow volumes decrease and solute residence times increase sharply with depth. The bulk (98\%) of groundwater flow occurs at shallow depths ( $<100 \mathrm{ft}$ below ground surface), and most $(80 \%)$ of this flow occurs in the water-table interval. Below the water-table interval, groundwater flow occurs along permeable fractures (or possibly fractured regions) within a relatively impermeable matrix. Variations in the number and aperture of the fractures within these regions occur along flow paths, and these differences determine lateral and vertical flow directions. Additionally, the 
vertical spacing between the permeable regions increases with depth, and permeable regions at depth are poorly connected in three dimensions (Solomon et al. 1992).

\subsubsection{Stormflow Zone}

Investigations in Bethel Valley and Melton Valley near ORNL show that groundwater occurs intermittently above the water table in the ORR Aquitards in a shallow "stormflow zone" that extends from ground surface to a depth of about $6 \mathrm{ft}$ (Moore 1989). Construction of the Y-12 Plant probably destroyed the stormflow zone in developed areas of the East Fork Regime. Channels for lateral flow in the stormflow zone include macropores and mesopores, which are connected voids created by various processes, including biochanneling, cracking, and soil particle aggregation (Moore 1989). This zone is thicker and more permeable in forested areas than in grassy or brushy areas, and is more permeable near the land surface than at deeper levels (Moore 1989).

Water is present in the stormflow zone during, and for short periods after, significant rainfall events; some of the water is lost to evapotranspiration and recharge to the water table, and the remaining water discharges at nearby seeps, springs, and streams. Analysis of infiltrometer test results from Bethel Valley and Melton Valley suggest that the hydraulic conductivity of the stormflow zone averages about 29 feet per day ( $\mathrm{ft} / \mathrm{day}$ ) near the top and $0.1 \mathrm{ft} /$ day near the base (Moore 1989). Lateral flow in the stormflow zone is intermittent, lasting a few days to a few weeks after precipitation. Flow is fairly rapid when the zone is full of water but relatively slow when nearly drained (Moore 1989).

\subsubsection{Vadose Zone}

The vadose zone occurs between the stormflow zone and the water table. The geometric mean depth to the water table in the ORR Aquitards is about $13 \mathrm{ft}$ (Moore 1989). The vadose zone in undeveloped areas is primarily composed of regolith, but in much of the East Fork Regime the vadose zone may consist of man-made fill material. Moore (1989) estimates effective porosity (the porosity through which significant advective transport can occur) for regolith of 0.01 to 0.15 , but porosity may be higher in the man-made fill. 
Water is added to the vadose zone by percolation from the stormflow zone and is removed by transpiration and recharge to the water table. The vadose zone is unsaturated except in the capillary fringe above the water table and within wetting fronts during periods of vertical percolation from the stormflow zone (Moore 1989). A geometric mean hydraulic conductivity of about $0.006 \mathrm{ft} /$ day was determined from analysis of infiltrometer tests in the vadose zone in Bethel Valley and Melton Valley (Moore 1989).

\subsubsection{Groundwater Zone}

Solomon et al. (1992) divide the saturated zone in the Knox Aquifer and ORR Aquitards into water-table, intermediate, and deep intervals based on observed changes in hydrologic characteristics and groundwater geochemistry with depth (Figure 6). For the purposes of this report, the above subdivisions have been modified for the East Fork Regime to include a shallow interval between the water-table and intermediate intervals. The boundary between the shallow and intermediate intervals is set at $100 \mathrm{ft}$ below ground surface, which generally corresponds with an aquifer subdivision noted by Moore (1989) in an evaluation of groundwater flow parameters, and is the depth at which a fairly abrupt change from calcium-magnesium-bicarbonate groundwater to sodium-bicarbonate groundwater typically occurs in the ORR Aquitards. Additionally, solution cavities in the Maynardville Limestone occur most frequently at depths less than $100 \mathrm{ft}$ below the ground surface, suggesting that the active karst flow system in the Maynardville Limestone is relatively shallow (Shevenell et al. 1993).

\subsubsection{Water-Table Interval}

A permeable interval 3 to $15 \mathrm{ft}$ thick usually occurs in the transitional horizon between regolith and unweathered bedrock, and the water table often occurs within this permeable interval (Solomon et al. 1992). The saturated thickness of this interval changes with cyclic fluctuations in the water table elevation, and the interval may be nearly drained during seasonal water table declines. It is postulated that in the ORR Aquitards, the water-table interval transmits more than $80 \%$ of the flow that occurs below the stormflow zone. Estimates of the permeability of the water-table zone are provided by results of hydraulic conductivity tests performed in wells 
completed in regolith below the water table. Analysis of these test results indicated a range of hydraulic conductivity of 0.03 to $3.25 \mathrm{ft} /$ day (Connell and Bailey 1989).

Results of geochemical dating studies of groundwater in the ORR Aquitards in Melton Valley generally support the concept of a groundwater zone that is most active near the water table and becomes increasingly sluggish with depth (Solomon ef al. 1992). Analyses of ${ }^{3} \mathrm{H}:{ }^{3} \mathrm{He}$ ratios in groundwater samples collected from 1 to $6 \mathrm{ft}$ below the water table showed ages ranging from 0.09 to 7.23 years, and the presence of large amounts of ${ }^{4} \mathrm{He}$ in groundwater samples from greater depths (45 to $165 \mathrm{ft}$ below ground surface) suggest much greater residence times (Poreda et al. 1988).

\subsubsection{Shallow Interval}

The shallow interval of the groundwater zone extends from the bottom of the water-table interval to depths of about $100 \mathrm{ft}$ in the ORR Aquitards (Figure 6), and from 160 to $325 \mathrm{ft}$ in the Knox Aquifer (Moore 1989). Groundwater in this zone is typically encountered under unconfined conditions in fractures and solution cavities. Enlarged fractures and cavities are the primary water-producing features and solute-transport pathways, and are supplied by seepage through fractures in the rock matrix. Fractures outnumber the cavities, are interconnected, and provide the continuity for groundwater flow paths (Moore 1989).

Analysis of hydraulic conductivity test results for wells completed at shallow depths in the ORR Aquitards and the Knox Aquifer generally shows two populations; an upper population with a geometric mean hydraulic conductivity of about $0.15 \mathrm{ft} /$ day and a lower population with a geometric mean hydraulic conductivity of about $0.0008 \mathrm{ft} /$ day (Moore 1989). The upper population represents more permeable, water-producing intervals and the lower population represents less permeable matrix intervals.

The hydraulic conductivity of the water-producing intervals at shallow depths in the Knox Aquifer is much greater owing to an interconnected network of solution cavities and solution-enlarged fractures. For instance, results of hydraulic conductivity tests for wells less than $100 \mathrm{ft}$ deep in the Maynardville Limestone, as summarized in Geraghty \& Miller, Inc. (1989b), range from 0.13 to $99 \mathrm{ft} /$ day and average about $22 \mathrm{ft} /$ day. Additionally, Shevenell et al . (1993) report that fractures and solution cavities in the Maynardville Limestone encountered 
during installation of several exit-pathway wells yield as much as 200 to 300 gallons per minute (gpm), which is several orders-of-magnitude greater than the typical yield $(0.25 \mathrm{gpm}$; Solomon ef al. 1992) of wells completed in the ORR Aquitards. Results of a dye tracer test in the Maynardville Limestone in the Bear Creek Regime suggested (qualitatively) a conduit flow rate of about $25 \mathrm{ft} /$ day (Geraghty \& Miller, Inc. 1989c).

Results of tracer tests performed in the Bear Creek Regime and calculations of the fracture porosity in bedrock provide estimates of the effective porosity of the ORR Aquitards and the Maynardville Limestone. Tracer tests have yielded effective porosity values ranging from 0.002 to 0.004 (Law Engineering Testing Company 1983; Lozier et al. 1987). Moore (1989) calculated a fracture porosity ranging from 0.001 to 0.002 , based on estimates of fracture density and average fracture aperture for water-producing intervals at shallow depths in the Conasauga Group.

\subsubsection{Intermediate Interval}

Groundwater in the intermediate bedrock interval occurs in relatively permeable fractures (or possibly fractured intervals) within a relatively impermeable matrix. Review of data obtained with an electromagnetic borehole flowmeter suggest that the permeable regions commonly develop within a single layer of rock, and the average thickness of a permeable region (assuming an average dip of $35^{\circ}$ ) is less than $2 \mathrm{ft}$. The vertical spacing between permeable regions in the intermediate interval is believed to be more than $100 \mathrm{ft}$ (Solomon et al. 1992); a vertical spacing of $150 \mathrm{ft}$ is supported by results of matrix diffusion modeling of measured ${ }^{14} \mathrm{C}$ and ${ }^{3} \mathrm{H}$ activities (Toran et al. 1991). Results of straddle packer tests in wells completed in the intermediate interval of the ORR Aquitards at several locations in BCV and Melton Valley show a range of mean hydraulic conductivity of 0.006 to $0.016 \mathrm{ft} /$ day (Solomon et al. 1992).

\subsubsection{Deep Interval}

Identification of the deep bedrock interval is based on hydraulic and geochemical data for several deep (greater than $500 \mathrm{ft}$ ) monitoring wells and coreholes. Solomon et 1ㅣ. (1992) report that the boundary between the intermediate interval and the deep interval in BCV occurs at 
depths of about $325 \mathrm{ft}$ in the ORR Aquitards and $650 \mathrm{ft}$ in the Knox Aquifer The boundary is based on lower hydraulic conductivity and unusually high hydraulic heads (estimated from packer tests), although use of hydraulic conductivity may not always be a reliable indicator (Solomon et al. 1992).

The transition from the intermediate interval to the deep interval in the ORR Aquitards is often indicated by a change from sodium-bicarbonate groundwater to sodium-chloride groundwater accompanied by a general increase in specific conductance and total dissolved solids. This change in groundwater geochemistry probably reflects longer residence times in the deep interval, and usually occurs at depths of more than $400 \mathrm{ft}$ below the ground surface (Solomon et al. 1992). However, no such change in groundwater geochemistry has been observed in the Knox Aquifer; calcium-magnesium-bicarbonate or calcium-sulfate groundwater occurs to depths of $1,000 \mathrm{ft}$ below the ground surface (Dreier et al. 1993).

Evaluation of data obtained from straddle packer tests and slug tests, and slow-recovery analyses, indicates that the geometric mean hydraulic conductivity of the deep interval in BCV is about $0.001 \mathrm{ft} /$ day (Solomon ef al. 1992). Although the mean hydraulic conductivity is comparable to that of the intermediate interval, measured conductivities in the deep interval are as low as $2.83 \times 10^{-6} \mathrm{ft} /$ day (Solomon et al. 1992). The low conductivities may reflect reduced fracture apertures or increased fracture spacings (Solomon et al 1992).

\subsubsection{Aquiclude}

The aquiclude occurs below the deep groundwater interval and is generally marked by the presence of saline water with total dissolved solids of 40,000 to 300,000 milligrams per liter $(\mathrm{mg} / \mathrm{L})$ (Solomon ef al. 1992). The water in the aquiclude is a sodium-, calcium-, and chloride-rich brine that is chemically similar to brines associated with major sedimentary basins (Solomon et al 1992). In Melton Valley, saline water typically occurs at depths of about 600 to $700 \mathrm{ft}$ below the ground surface (Solomon et al. 1992).

Saline groundwater has not been encountered at depth in the East Fork Regime. However, several wells in BCV completed at depths of about 500 to $1,000 \mathrm{ft}$ below the ground surface monitor groundwater with total dissolved solids concentrations of several thousand $\mathrm{mg} / \mathrm{L}$ to several tens of thousand $\mathrm{mg} / \mathrm{L}$. The presence of water with relatively high total dissolved solids 
(as well as increased sodium and chloride concentrations) in the deep groundwater interval in BCV may represent a mixing zone or diffusion zone between the fresh and saline groundwater suggesting that the aquiclude is most likely present at greater depth in the BCV than in Melton Valley (Solomon et al. 1992).

\subsubsection{Groundwater Flow Directions}

Directions of groundwater flow in the East Fork Regime were evaluated from static water level measurements obtained in January and August/September 1993. Water levels were determined in 98 monitoring wells during January 1993 (the seasonally high water table) and in 90 wells in August/September 1993 (the seasonally low water table); depths to water and waterlevel elevations for each well are tabulated in Appendix M. Review of these data show that water level declines averaged about $3 \mathrm{ft}$ in most of the wells, with the largest decreases occurring in shallow wells completed in the water-table interval. Additionally, the pattern of water level decrease was the same in all wells such that there were no significant changes in groundwater flow directions (i.e., no reversal of horizontal hydraulic gradient(s).

Groundwater elevations in the water-table interval and bedrock (shallow and intermediate) intervals during the seasonally high and seasonally low groundwater flow conditions are illustrated on Figures 7 and 8, respectively. As shown, the water table is a subdued replica of surface topography, with steep gradients along the flanks of Pine Ridge and Chestnut Ridge and a gentle slope northeast down the axis of BCV. The horizontal hydraulic gradient averages about 0.046 across BCV (strike-normal) and about 0.012 along the axis of BCV (strike-parallel).

The pattern of groundwater flow in the ORR Aquitards is toward the Maynardville Limestone, suggesting that this formation is a hydrologic drain for the groundwater flow system in the regime. Flow in the Maynardville is generally along strike toward the east end of the Y-12 Plant. At the east end of the regime, flow directions apparently diverge with one component of flow to the north following UEFPC through a water gap in Pine Ridge, and another flow component to the east along strike in the Maynardville Limestone. 


\subsubsection{Contaminant Trausport}

Quantitative and qualitative methods were used to estimate contaminant migration rates. Quantitative rates were estimated only for the water-table and shallow bedrock intervals in the groundwater zone. Migration rates in the stormflow zone were not estimated because this zone has probably been destroyed or substantially altered throughout most of the East Fork Regime. Estimates of migration rates in the intermediate and deep intervals also were not performed, but representative rates are probably as low as a few centimeters per year (Solomon et al 1992). Qualitative transport rates were determined based on the extent of nitrate in the Bear Creek Regime assuming a constant input rate from a single source area. These qualitative rates were assumed to be applicable to the East Fork Regime because of similar geologic and hydrologic conditions.

The wide range of potential contaminant migration rates reported in the following sections demonstrate the uncertainty in determining a representative rate of solute transport for this groundwater flow system. Long term transport of solutes in dual porosity and/or highly heterogeneous media is a complex physical and chemical process that is not fully explained by calculations using the modified Darcy equation or qualitative estimates based on distances from source areas to plume boundaries. It is probable that at various times and under various conditions, migration rates throughout the given range occur; some contaminants such as nitrate move relatively rapidly through the flow system, while other more highly attenuated constituents move much more slowly. Moreover, even the highly mobile compounds will diffuse into smaller pores from the large conduits and later, under a reverse concentration gradient, will slowly diffuse back out. Thus, although the actual transport rates may vary over several orders of magnitude, the relatively limited extent of the contaminant plumes in the East Fork Regime suggests that slower transport rates may dominate and that attenuation processes such as matrix diffusion are an important controlling factor.

\subsubsection{Quantitative Estimates}

Quantitative estimates of contaminant transport were based on the assumption that contaminants move with the groundwater unimpeded by various retardation processes. Factors 
which would reduce transport rates, such as matrix diffusion, degradation, sorption, and chemical precipitation, or factors which may increase transport, such as dispersion, were not explicitly considered. Free product migration, which may occur independent of groundwater flow rates and directions, also was not considered.

Assuming that saturated regolith and fractured bedrock can be treated as an equivalent porous medium, average groundwater flow rates can be estimated using the modified Darcy equation:

$$
\mathbf{v}=\mathbf{K} \mathbf{i} / \mathbf{n}
$$

where $\mathbf{v}$ is the average linear velocity of the groundwater, $\mathbf{K}$ is the hydraulic conductivity of the medium, $\mathbf{i}$ is the hydraulic gradient, and $\mathbf{n}$ is the effective porosity. Although the modified Darcy equation can be used to calculate average flow velocities in fractured rock, the velocities in individual fractures may vary over orders of magnitude, depending on the fracture aperture and wall roughness (Freeze and Cherry 1979).

Using the range of hydraulic conductivities for regolith (0.03 to $3.25 \mathrm{ft} /$ day), effective porosities ranging from 0.001 to 0.15 , an average strike-parallel (down-valley) gradient of 0.011 , and an average strike-normal (cross-valley) gradient of 0.045 , calculated rates of solute transport in the water-table interval range from 0.002 to $146.2 \mathrm{ft} /$ day. However, the bulk of the water mass in the water-table interval resides within porous matrix zones between fractures, and diffusive exchange between matrix and fractures would reduce contaminant migration rates relative to calculated flow rates (Solomon et al. 1992).

Contaminant transport rates for the shallow interval in the ORR Aquitards were calculated using a geometric mean hydraulic conductivity of $0.0008 \mathrm{ft} /$ day for matrix intervals and $0.15 \mathrm{ft} /$ day for water-producing intervals, effective porosity values ranging from 0.001 to 0.004 , and an average strike-normal hydraulic gradient of 0.046 . Using these values, the rate of contaminant migration in the ORR Aquitards ranges from 0.009 to $6.7 \mathrm{ft} /$ day.

Contaminant transport rates for the shallow interval in the Maynardville Limestone were calculated using the average hydraulic conductivity ( $22 \mathrm{ft} /$ day) obtained from well tests, effective porosity values ranging from 0.001 to 0.004 , and an average strike-parallel hydraulic gradient of 0.012 . Using these values, the rate of contaminant migration in the Maynardville Limestone 
ranges from about 66 to $264 \mathrm{ft} /$ day. Relatively high transport rates in the Maynardville Limestone also are indicated by results of a dye-tracer study performed in the Bear Creek Regime which yielded a transport rate of about $200 \mathrm{ft} /$ day (Geraghty \& Miller, Inc. 1989c).

\subsubsection{Qualitative Estimates}

Qualitative estimates of contaminant migration rates in the East Fork Regime were based on the extent of the nitrate plume originating from the S-3 Site observed in the Bear Creek Regime during CY 1990 (HSW Environmental Consultants, Inc. 1991). Unlike most of the other groundwater contaminants in the Bear Creek Regime, many of which have multiple source areas, the S-3 Site is the only significant nitrate source in the regime. Moreover, nitrate salts are highly soluble and generally move with no significant sorption or decay during transport (Geraghty \& Miller, Inc. 1989d).

The nitrate plume originating from the S-3 Site extends westward (along strike) about $3,650 \mathrm{ft}$ in the water-table interval, approximately $2,000 \mathrm{ft}$ at shallow depths in the ORR Aquitards, and about $9,500 \mathrm{ft}$ at shallow depths in the Maynardville Limestone (HSW Environmental Consultants, Inc. 1991). Given the 39 years between initial waste disposal at the S-3 Site in 1951 and the horizontal extent of the nitrate plume observed in 1990, the rate of nitrate transport ( $v=$ distance/time) is about $0.3 \mathrm{ft} /$ day in the water-table interval, about $0.1 \mathrm{ft} /$ day in the ORR Aquitards, and about $0.7 \mathrm{ft} /$ day in the Maynardville Limestone.

\subsection{Surface-Water System}

Upper East Fork Poplar Creek originates near the western edge of the Y-12 Plant as a surface water drainage system. The headwaters are contained in a network of underground collection pipes that extend to near the central area of the Plant, where the above-ground portion of the creek emerges. The creek then flows along the southern boundary of the Plant complex and enters Lake Reality. From the outfall at Lake Reality, UEFPC passes north through a gap in Pine Ridge and enters the City of Oak Ridge.

During construction of the Y-12 Plant, all the north-south tributaries to UEFPC were backfilled or replaced with storm drains. Additionally, UEFPC was altered by construction of 
the storm sewer system at its headwaters and straightening of the original channel along the southeastern portion of the Plant complex (PEER Consultants 1989).

Surface-water runoff from the Y-12 Plant is discharged to UEFPC through an extensive array of storm drains. Flow monitoring data indicate that over $78 \%$ of dry weather flow in UEFPC is attributable to once-through noncontact cooling water, condensate, and cooling tower blowdown, and over $21 \%$ can be attributed to groundwater infiltration (Camp, Dresser \& McKee 1987). Surface-water quality in UEFPC is monitored at Station 17 located downstream of Lake Reality (Figure 3) as part of the ORR Environmental Monitoring Program (U.S. Department of Energy 1993b). 


\subsection{REFERENCES}

Camp, Dresser and McKee, Inc. 1987. Draft Comprehensive Sampling Program for East Fork Poplar Creek, Source Pollution Assessment and Control Plan, Y-12 Plant, Oak Ridge, Tennessee. Camp Dresser and McKee, Inc., Atlanta, Georgia.

Connell, J.F. and Z.C. Bailey. 1989. Statistical and Simulation Analysis of Hydraulic Conductivity Data For Bear Creek and Melton Valleys, Oak Ridge Reservation, Tennessee. U.S. Geological Survey Water-Resources Investigations Report 89-4062.

Dreier, R.B., K.D. Solomon, and C.M. Beaudoin. 1987. Fracture Characterization in the Unsaturated Zone of a Shallow Land Burial Facility, in: Flow and Transport through Fractured Rock, American Geophysical Union Monograph 42.

Dreier, R.B., T.O. Early, and H.L. King. 1993. Results and Interpretations of Groundwater Data Obtained from Multi-port Instrumented Core Holes (GW-131- GW-135) Fiscal Years 1990 and 1991. Martin Marietta Energy Systems, Inc. (Y/TS-803).

Eaton, J.M. and E.M. Ingram. 1991. Site Investigation Report and Corrective Action Plan for the Former Y-12 Fuel Station at Buildings 9754 and 9754-2, Oak Ridge Y-12 Plant, Oak Ridge, Tennessee. Prepared by Science Applications International Corporation for Martin Marietta Energy Systems, Inc. (Y/SUB/91-TJ997C/6).

Eaton J.M. and F.R. Van Ryn. 1991. Site Investigation Report for Underground Storage Tanks 1219-U, 1222-U, 2068-U, and 2082-U at the Rust Garage Facility, Oak Ridge Y-12 Plant, Oak Ridge, Tennessee. Prepared by Science Applications International Corporation for Martin Marietta Energy Systems, Inc. (Y/SUB/91-TJ997C/3).

Evans, R.D. 1955. The Atomic Nucleus. McGraw - Hill, New York, N.Y.

Freeze, R.A. and J.A. Cherry. 1979. Groundwater. Prentice-Hall, Inc., Eaglewood Cliffs, New Jersey, (pp. 408-409).

Geraghty \& Miller, Inc. 1988. Findings of Gasoline Seepage Study at the Rust Garage Area of the Y-12 Plant, Oak Ridge, Tennessee. Prepared for Martin Marietta Energy Systems, Inc. (Letter Report to L.L. McCauley).

Geraghty \& Miller, Inc. 1989a. RCRA Facility Investigation Plan, Abandoned Nitric Acid Pipeline, Oak Ridge Y-12 Plant, Oak Ridge, Tennessee. Prepared for Martin Marietta Energy Systems, Inc. (Y/TS-598).

Geraghty \& Miller, Inc. 1989b. Development of Ground-Water Flow Models for the S-3 Waste Management Area, Y-12 Plant, Oak Ridge, Tennessee. Prepared for Martin Marietta Energy Systems, Inc. (Y/SUB/89-00206C-1). 
Geraghty \& Miller, Inc. 1989c. Tracer Study of the Hydrologic System of Upper Bear Creek, Y-12 Facility, Oak Ridge, Tennessee. Prepared for Martin Marietta Energy Systems, Inc. (Y/SUB/89-00206C/).

veraghty \& Miller, Inc. 1989d. Development of Contaminant Transport Models for Four Constituents at the S-3 Site, Y-12 Plant, Oak Ridge, Tennessee. Prepared for Martin Marietta Energy Systems, Inc. (Y/SUB/89-00206C/C/3).

Geraghty \& Miller, Inc. 1990. Comprehensive Groundwater Monitoring Plan for the Oak Ridge Y-12 Plant. Prepared for Martin Marietta Energy Systems, Inc. (Y/SUB/9000206C/5).

HSW Environmental Consultants, Inc. 1991. Groundwater Quality Assessment for the Bear Creek Hydrogeologic Regime at the Y-12 Plant 1990: Data Interpretations and Proposed Program Modifications. Prepared for Martin Marietta Energy Systems, Inc. (Y/SUB/91-YP507C/1 Part 2).

Haase, C.S. 1987. RCRA Facility Investigation Plan for the S-2 Site (D-103) at the Oak Ridge Y-12 Plant. Martin Marietta Energy Systems, Inc. (Y/TS-349).

Jones, S.B., B.K. Harrington, and S.M. Field. 1993. Updated Subsurface Data Base for Bear Creek Valley, Chestnut Ridge, and Parts of Bethel Valley on the U.S. Department of Energy Oak Ridge Reservation. Martin Marietta Energy Systems, Inc. (Y/TS-881).

Kimbrough, C.W. 1986. Phase I Installation Assessment of Inactive Hazardous Waste Disposal Sites at the Y-12 Plant. Martin Marietta Energy Systems, Inc. (Y/TS-114).

Kimbrough, C.W., L.W. Long, and L.W. McMahon (eds). 1987. Environmental Surveillance Procedures Quality Control Program, Martin Marietta Energy Systems, Inc. Prepared by Advanced Sciences, Inc. for the Environmental and Safety Activities Organization, Martin Marietta Energy Systems Inc. (Y/SUB/87/21706/1 Revision 1).

Kimbrough, C.W. and L.W. McMahon. 1988a. RCRA Appendix IX Sampling and Analysis Project at the Oak Ridge Y-12 Plant: Disposal Basin Field Sampling Plan and Field Data. Martin Marietta Energy Systems, Inc. (Y/SUB/88-97376/1).

Kimbrough, C.W. and L.W. McMahon. 1988b. RCRA Appendix IX Sampling and Analysis Project at the Oak Ridge Y-12 Plant: New Hope Pond Analytical Data Summary. Martin Marietta Energy Systems, Inc. (Y/SUB/88-97376/2).

King, H.L., and C.S. Haase. 1987. Subsurface-Controlled Geological Maps for the Y-12 Plant and Adjacent Areas of Bear Creek Valley. Oak Ridge National Laboratory (ORNL/TM-10112).

Law Engineering Testing Company. 1983. Results of Ground-Water Monitoring Studies. Prepared for Union Carbide Corporation, Nuclear Division (Y/SUB/83-47936/1). 
Lee Wan \& Associates, Inc. 1989 (Revised). Post Closure Permit Application for the New Hope Pond Site at the Y-12 Plant. Prepared for Martin Marietta Energy Systems, Inc. (Draft)

Lind, D.E. and S.H. Welch. 1989. Closure Plan for the Final Closure of the Salvage Yard Oil/Solvent Drum Storage Area (S-020) at the U.S. DOE Y-12 Plant. Martin Marietta Energy Systems, Inc. (Y/TS-382/R2).

Lozier, W.B., C.A. Spiers, and R. Peerson. 1987. Aquifer Pump Tests with Tracers. Prepared for Martin Marietta Energy Systems, Inc. (ORNL/SUB/86-32136).

Martin Marietta Energy Systems, Inc. 1988. Revised Closure Plan for New Hope Pond. Y-12 Plant Environmental Management Department, Health, Safety, Environment and Accountability Division, Y-12 Plant (Y/TS-389).

Mercier, T.M. and R.R. Turner. 1990. Data Verification and Evaluation Techniques for Groundwater Monitoring Programs. Prepared by H\&R Technical Associates, Inc. for Martin Marietta Energy Systems, Inc. (Y/SUB/90-K523C/2).

Moore, G.K. 1989. Groundwater Parameters and Flow System Near Oak Ridge National Laboratory. Environmental Sciences Division, Oak Ridge National Laboratory (ORNL/TM-1 i368).

Murphy, F. 1989. RCRA Facility Investigation Plan, Waste Coolant Processing Facility (T-038), Y-12 Plant, Oak Ridge, Tennessee. Martin Marietta Energy Systems, Inc. (Y/TS-358 RI).

Poreda, L.N., T.E. Cerling, and D.K. Solomon. 1988. Tritium and Helium Isotopes as Hydrologic Tracers in a Shallow Unconfined Aquifer. Journal of Hydrology (103:1-9).

PEER Consultants. 1989. RCRA Facility Investigation Plan for East Fork Poplar Creek and the Oak Ridge Sewer Line Beltway, Oak Ridge Y-12 Plant, Oak Ridge, Tennessee. Prepared for Martin Marietta Energy Systems, Inc. (Y/TS-366 R1).

Saunders, M.B. 1983. Leachability of Samples from New Hope Pond Disposal Basin, U.S. DOE Y-12 Plant, Oak Ridge, TN. Prepared for Union Carbide Corporation, Nuclear Division (Y/DZ81 Rev. 1).

Shevenell, L.A., R.B. Dreier, and W.K. Jago. 1993. Summary of Fiscal Years 1991 and 1992 Construction, Hydrologic, and Geologic Data Obtained from the Maynardville Limestone Exit-Pathway Monitoring Program, Martin Marietta Energy Systems, Inc. (Y/TS-814).

Sledz, J.J., and D.D. Huff. 1981. Computer Model for Determining Fracture Porosity and Permeability in the Conasauga Group. Oak Ridge National Laboratory (ORNL/TM-7695). 
Solomon, D.K., G.K. Moore, L.E. Toran, R.B. Dreier, and W.M. McMaster. 1992. Status Report - A Hydrologic Framework for the Oak Ridge Reservation. Oak Ridge National Laboratory (ORNL/TM-120262).

Stone, J.E. and L.W. McMahon. 1988. Waste Machine Coolant Biodegradation Facility, Summary of Closure Under Rules Governing Hazardous Waste Management in Tennessee. Martin Marietta Energy Systems, Inc. (Y/TS-457/3).

Stone, J.E. 1989a. Initial Site Characterization and Free Product Removal Report for Underground Storage Tanks 2063-U, 2328-U, and 2329-U at the Salvage Yard Drum Deheader, Oak Ridge, Y-12 Plant, Oak Ridge, Tennessee. Martin Marietta Energy Systems, Inc. (Y/TS-566).

Stone, J.E. 1989b. Site Investigation Plan for the former Y-12 Plant Fuel Stations at Buildings 9754 and 9754-2, Oak Ridge Y-12 Plant, Oak Ridge, Tennessee. Martin Marietta Energy Systems, Inc. (Y/TS-588).

Stone, J.E. 1989c. Phase II Free Product Removal Report for Underground Storage Tanks 0439-U and 0440-U at the Building 9754-2 Gasoline Station, Oak Ridge Y-12 Plant, Oak Ridge, Tennessee. Martin Marietta Energy Systems, Inc. (Y/TS-567).

Stone, J.E. 1989d. Free Product Removal Report for Underground Storage Tank 2331-U at Building 9201-1, Oak Ridge Y-12 Plant, Oak Ridge, Tennessee. Martin Marietta Energy Systems, Inc. (Y/TS/569).

Stone, J.E. 1989e. Initial Site Characterization Report for Underground Storage Tank 0134-U, Building 9204-2, Oak Ridge Y-12 Plant, Oak Ridge, Tennessee. Martin Marietta Energy Systems, Inc. (Y/TS-565).

Stone, J.E. 1989f. Free Product Removal Report for Underground Storage Tank 0134-U at Building 9204-2, Oak Ridge Y-12 Plant, Oak Ridge, Tennessee. Martin Marietta Energy Systems, Inc. (Y/TS-593).

Toran, L.E., D.K. Solomon, W.M.McMaster, and C.M. Morrissey. 1991. Matrix Diffusion as a Mechanism to Explain Recent Tritium and old ${ }^{14} \mathrm{C}$ in Groundwater from Fractured Sedimentary Rock. EOS Transactions 72(17):111.

Turner, R.R., Martin Marietta Energy Systems, Inc. 1992. Memo to T. Early, Martin Marietta Energy Systems, Inc., October 27, 1993.

U.S. Army Corps of Engineers. 1954. Foundation Investigation and Analysis - New Steam Plant, Alpha 5 Area. Y-12 Plant.

U.S. Department of Energy. 1992. Oak Ridge Reservation Site Management Plan for the Environmental Restoration Program. U.S. Department of Energy Oak Ridge Field Office (DOE/OR-100/R2). 
U.S. Department of Energy. 1993a. Remedial Investigation Work Plan for Upper East Fork Poplar Creek Operable Unit 3 at the Oak Ridge Y-12 Plant, Oak Ridge, Tennessee. (DOE/OR/01-1151\&D1).

U.S. Department of Energy. 1993b. Environmental Monitoring Plan for the Oak Ridge Reservation. U.S. Department of Energy Oak Ridge Field Office (DOE/OR-1066).

U.S. Environmental Protection Agency. 1988a. Letter from J.H. Scarbrough, Chief of the Region IV RCRA Branch, and T. Tiesler, Director of the Division of Solid Waste Management, Tennessee Department of Health and Environment, to R.J Spence, Y-12 Plant Manager, U.S. Department of Energy - Oak Ridge Operations, November 18, 1988.

U.S. Environmental Protection Agency. 1988b. Laboratory Data Validation Functional Guidelines for Evaluating Organics Analyses. U.S. EPA, Office of Solid Waste.

Welch, S.H. 1986. Salvage Yard Oil/Solvent Drum Storage Area (S-020): Closure Plan (Revised). Oak Ridge Y-12 Plant. Martin Marietta Energy Systems, Inc. (Y/TS-212/1R).

Welch, S.H. 1987. Solid Waste Management Unit Information for Y-12 Plant RCRA 3004(u) Facility Assessment Container Accumulation Areas. Martin Marietta Energy Systems, Inc., Y-12 Plant Environmental Management Department, Health, Safety, Environment and Accountability Division. (Y/TS-273, Supp.2)

Welch, S.H., C.S. Haase, C.W. Kimbrough, and T.M. Mercier. 1987. Solid Waste Management Unit Information for Y-12 Plant RCRA 3004(u) Facility Assessment, Volumes I, II, and III. Martin Marietta Energy Systems, Inc. (Y/TS-273).

Welch, S.H. 1989a. Initial Site Characterization and Free Product Removal Report for Underground Storage Tanks 0439-U and 0440-U at Building 9754-2, Oak Ridge Y-12 Plant, Oak Ridge, Tennessee. Prepared by Science Applications International Corporation for Martin Marietta Energy Systems, Inc. (Y/TS-560).

Welch, S.H. 1989b. Initial Site Characterization and Site Investigation Plan for Underground Storage Tank 2331-U at Building 9201-1, Oak Ridge Y-12 Plant, Oak Ridge, Tennessee. Martin Marietta Energy Systems, Inc. (Y/TS-557/1).

Wiggins, K.D. and S.H. Welch. 1988a. Supplement to Solid Waste Management Unit Information for Y-12 Plant RCRA 3004(u) Facility Assessment. Martin Marietta Energy Systems, Inc., Y-12 Plant Environmental Management Department, Health, Safety, Environment and Accountability Division. (Y/TS-273, Supp.1)

Wiggins, K.D. and S.H. Welch. 1988b. Supplement to Solid Waste Management Unit Information for Y-12 Plant RCRA 3004(u) Facility Assessment Container Accumulation Areas. Martin Marietta Energy Systems, Inc., Y-12 Plant Environmental Management Department, Health, Safety, Environment and Accountability Division. (Y/TS-273, Supp.3) 
Willoughby, M., D.E. Bohrman, and M.W. Sherrill. 1988. Hazardous Waste Storage Area, Southern Portion of Interim Drum Yard (S-030), Summary of Partial Closure Under Rules Governing Hazardous Waste Management in Tennessee. Martin Marietta Energy Systems, Inc. (Y/TS-347/7). 
APPENDIX A

FIGURES 


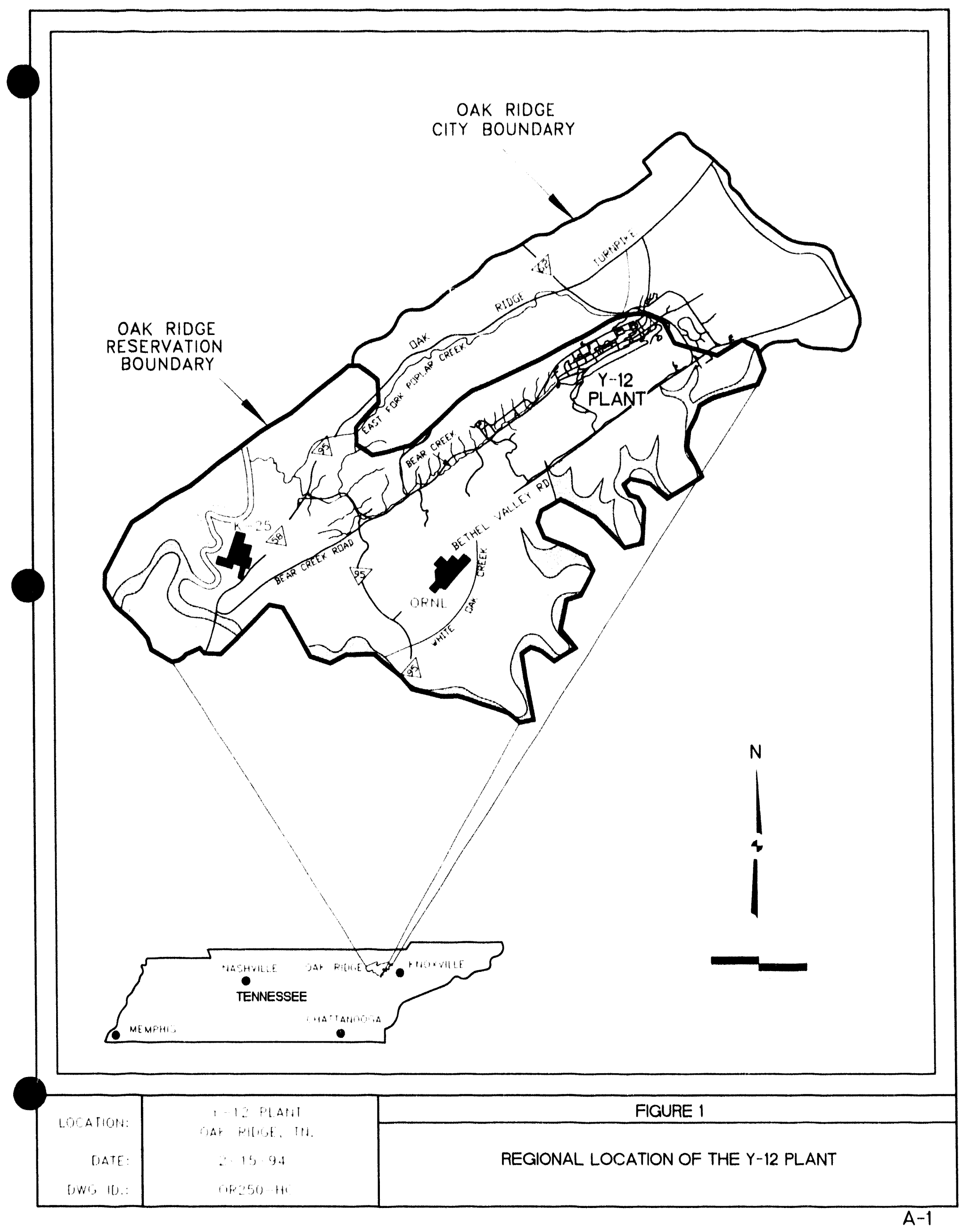




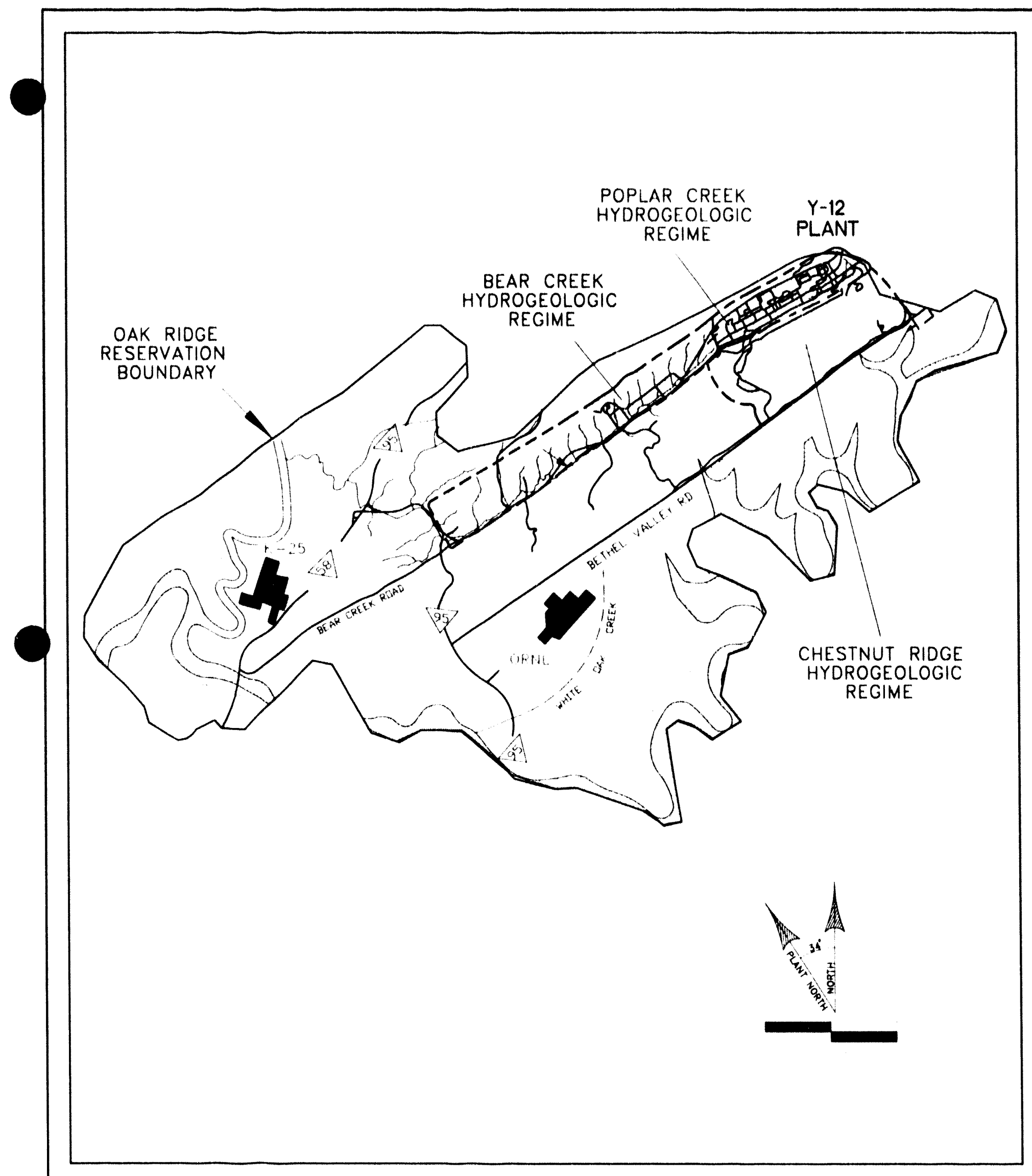

\begin{tabular}{|c|c|c|}
\hline - T & 1 1. HAH & FIGURE 2 \\
\hline $\begin{array}{l}\text { UATE: } \\
\text { WW lo: }\end{array}$ & $\begin{array}{l}3-19-9.1 \\
0+3.1\end{array}$ & $\begin{array}{l}\text { HYDROGEOLOGIC REGIMES } \\
\text { AT THE Y-12 PLANT }\end{array}$ \\
\hline
\end{tabular}




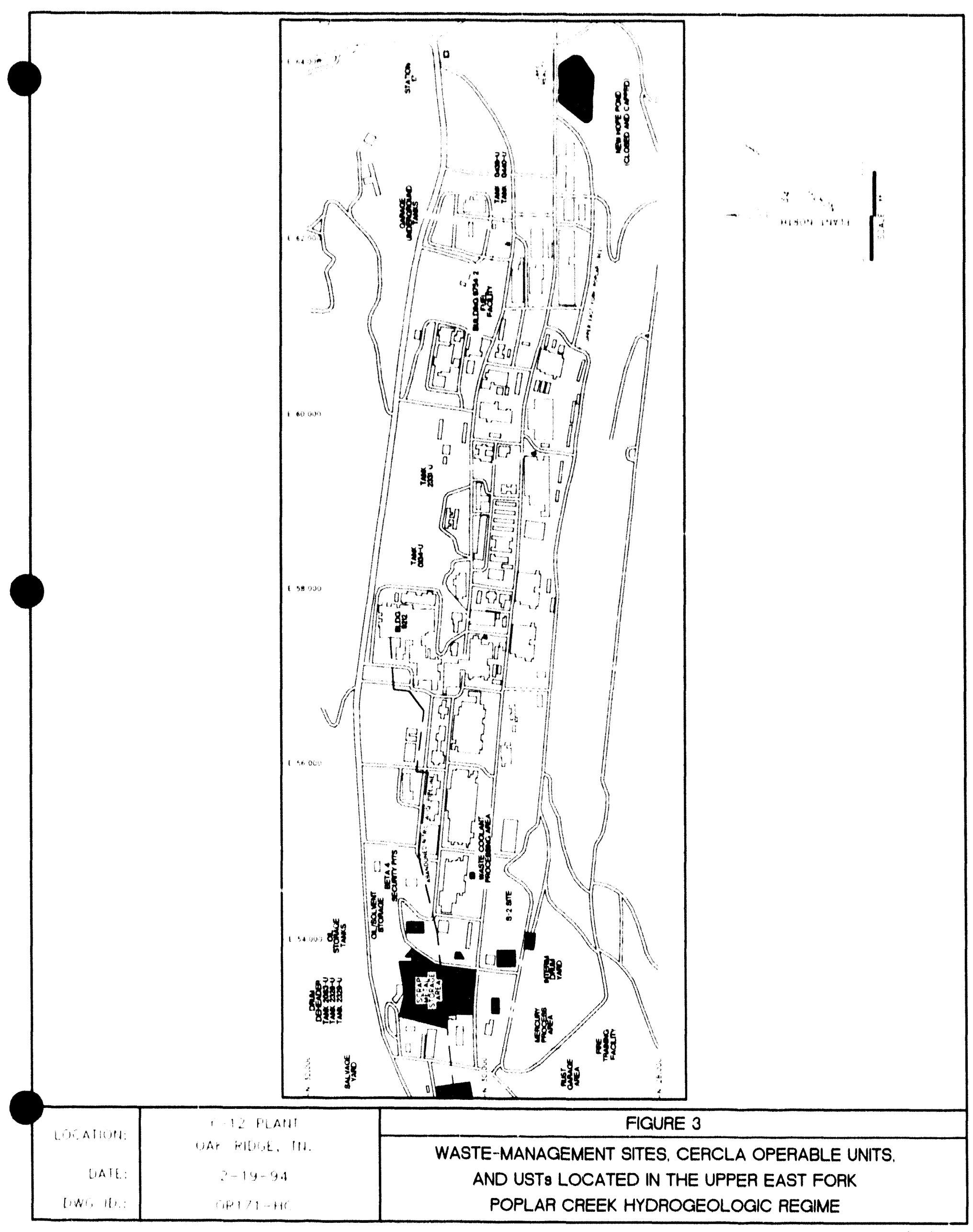




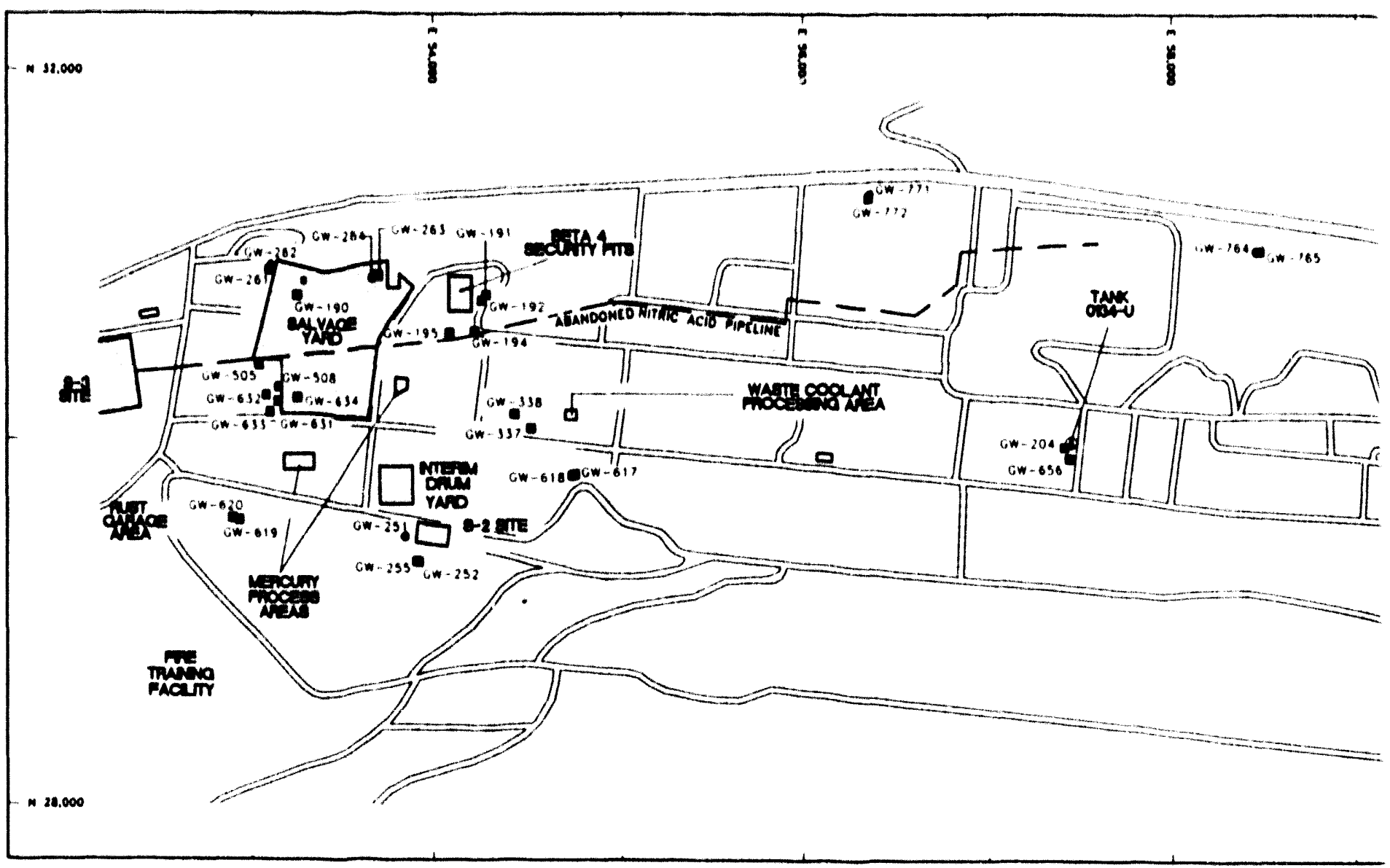

\section{EXPLANATION}

ow 1s: Water-Table Zone Monitori

G. :40 - Bedrock Zone Monitoring

- Boundary of Site 


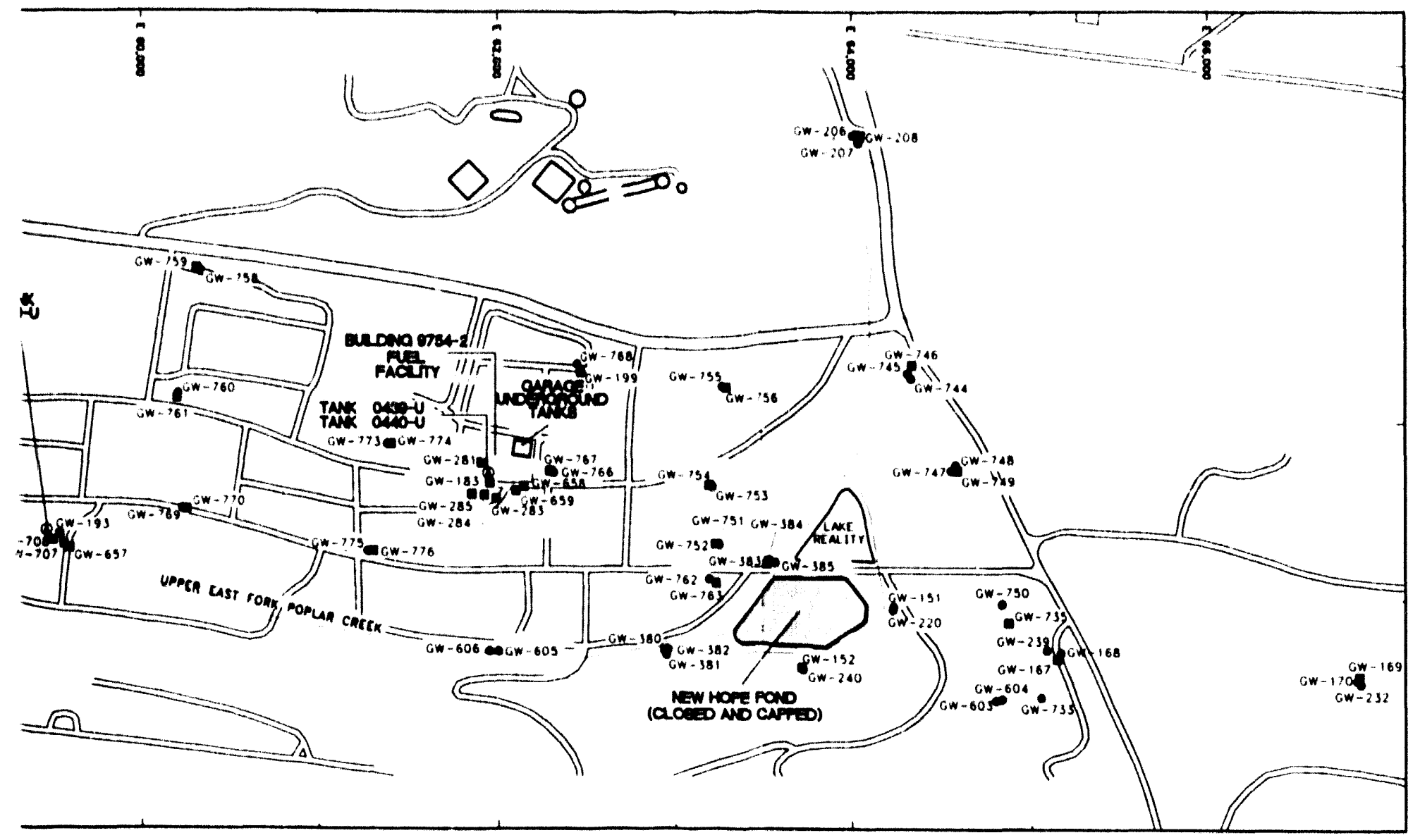

I Well

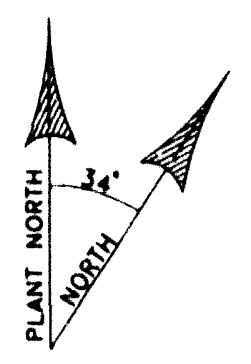

II

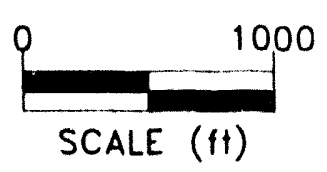

\begin{tabular}{|r|c|}
\hline LOCATION: & $\begin{array}{c}\text { Y-12 PLANT } \\
\text { OAK RIDGE, TN. }\end{array}$ \\
\hline DATE: & $2-19-94$ \\
\hline DWG ID.: & OR306-HC \\
\hline
\end{tabular}

FQURE 4

LOCATION OF MONTORING WELLS SAMPLED DUPING 1993 


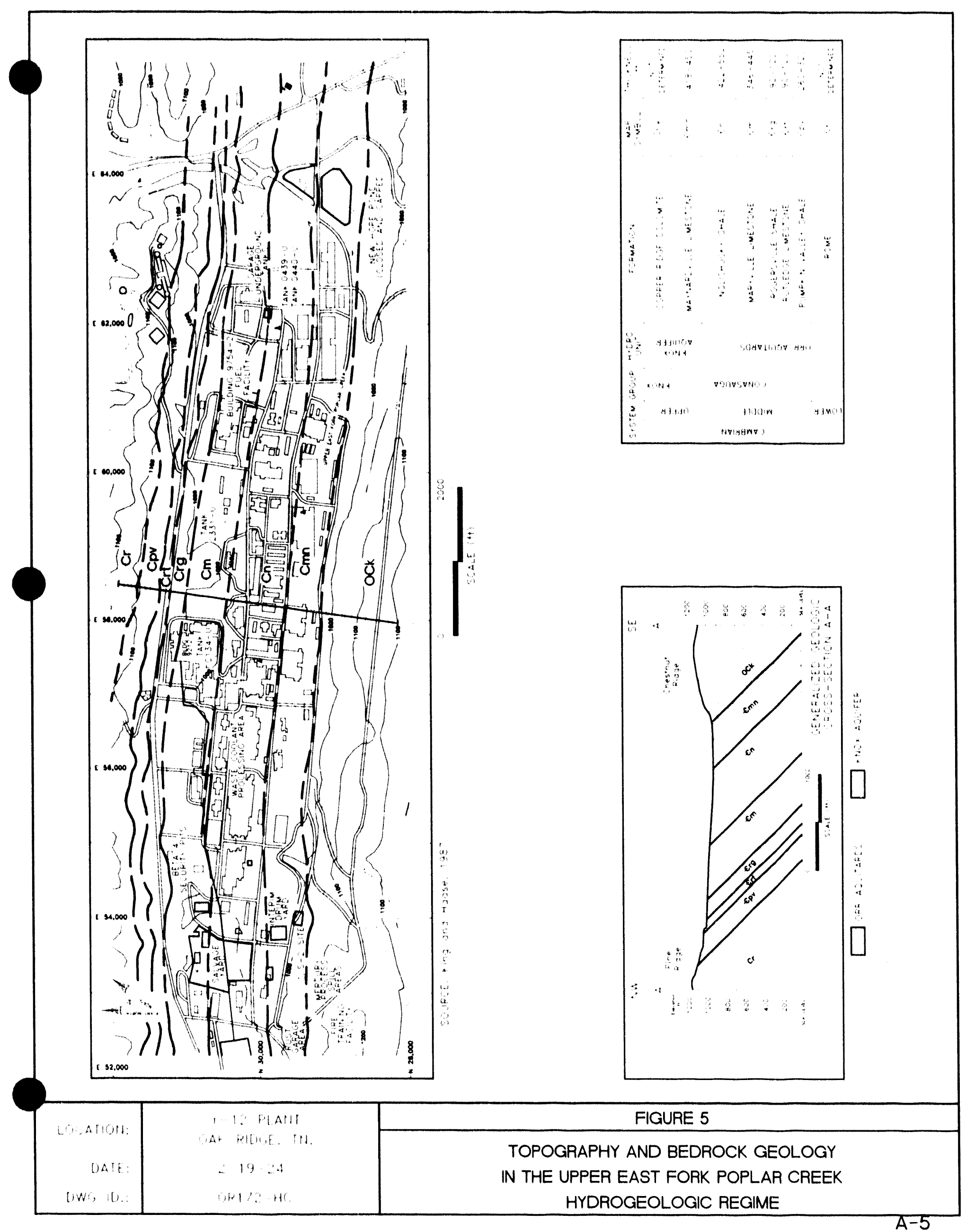




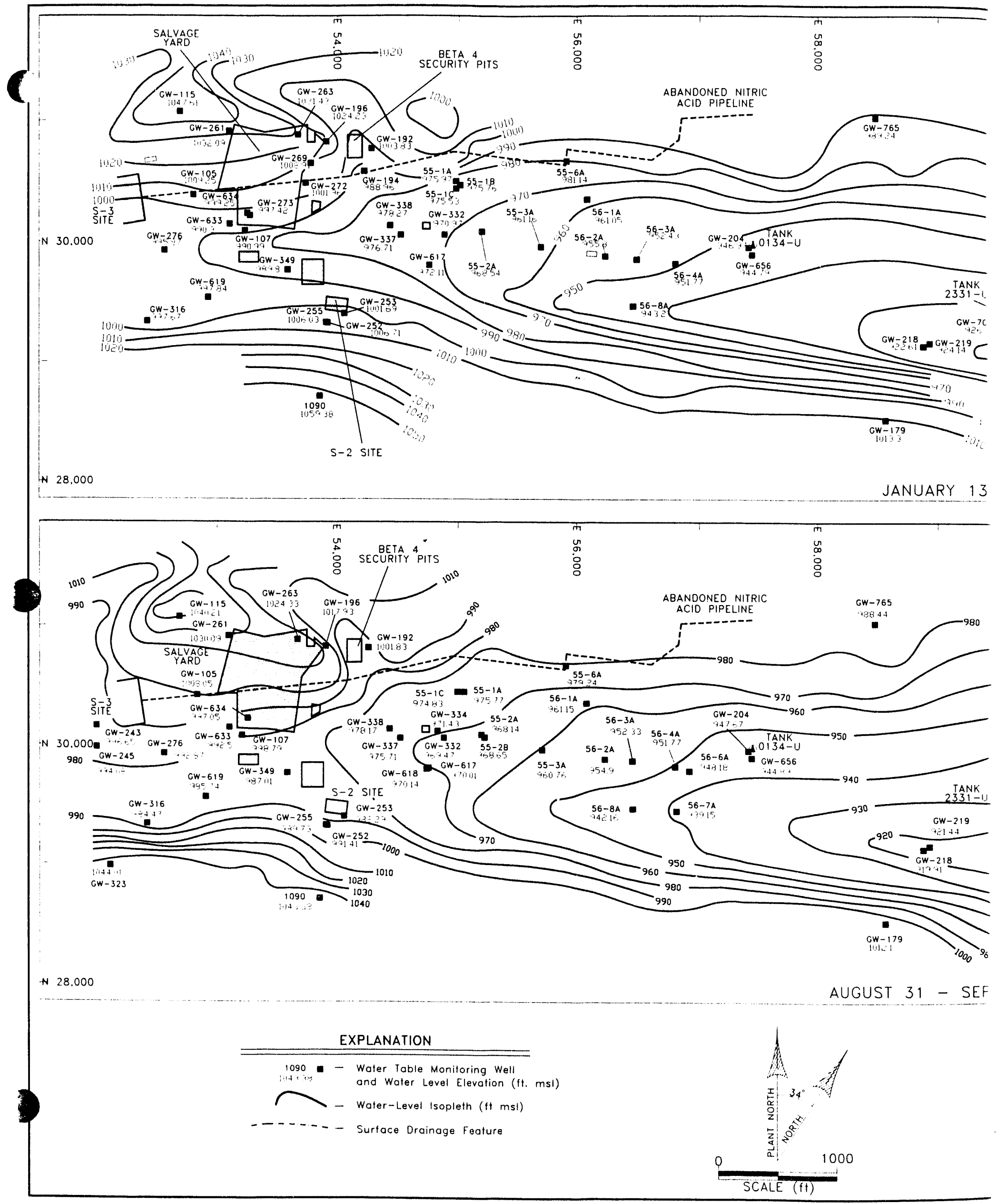



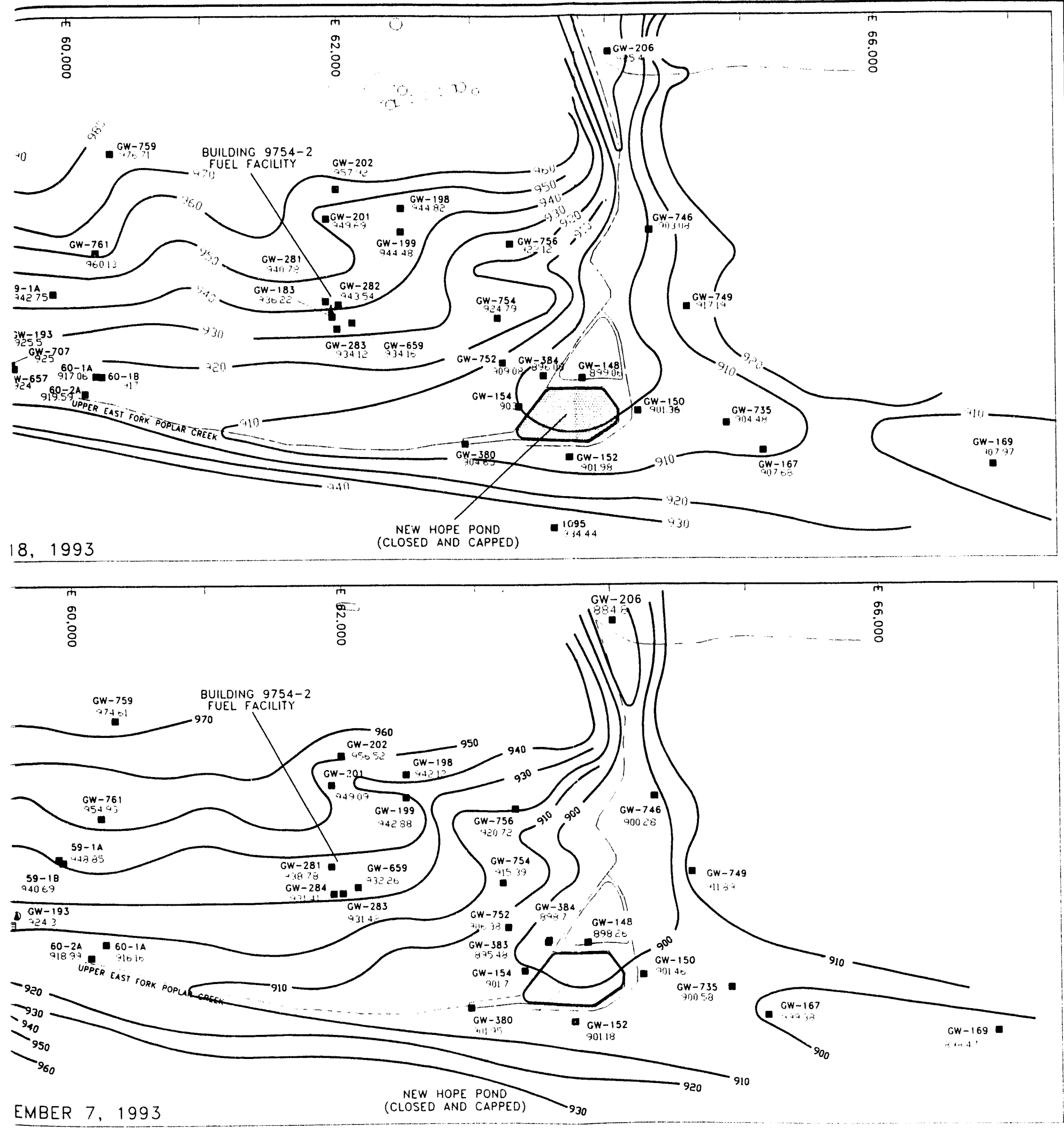

\begin{tabular}{|r|c|c|}
\hline LOCATION: & $\begin{array}{c}Y-12 \text { PLANT } \\
\text { OAK RIDGE, TN. }\end{array}$ & FIGURE 7 \\
\cline { 2 - 3 } DATE: & $2-19-94$ & $\begin{array}{c}\text { GROUNDWATER ELEVATIONS } \\
\text { IN THE WATER-TABLE INTERVAL }\end{array}$ \\
\hline DWG ID.: & OR313-HC & \\
\hline
\end{tabular}




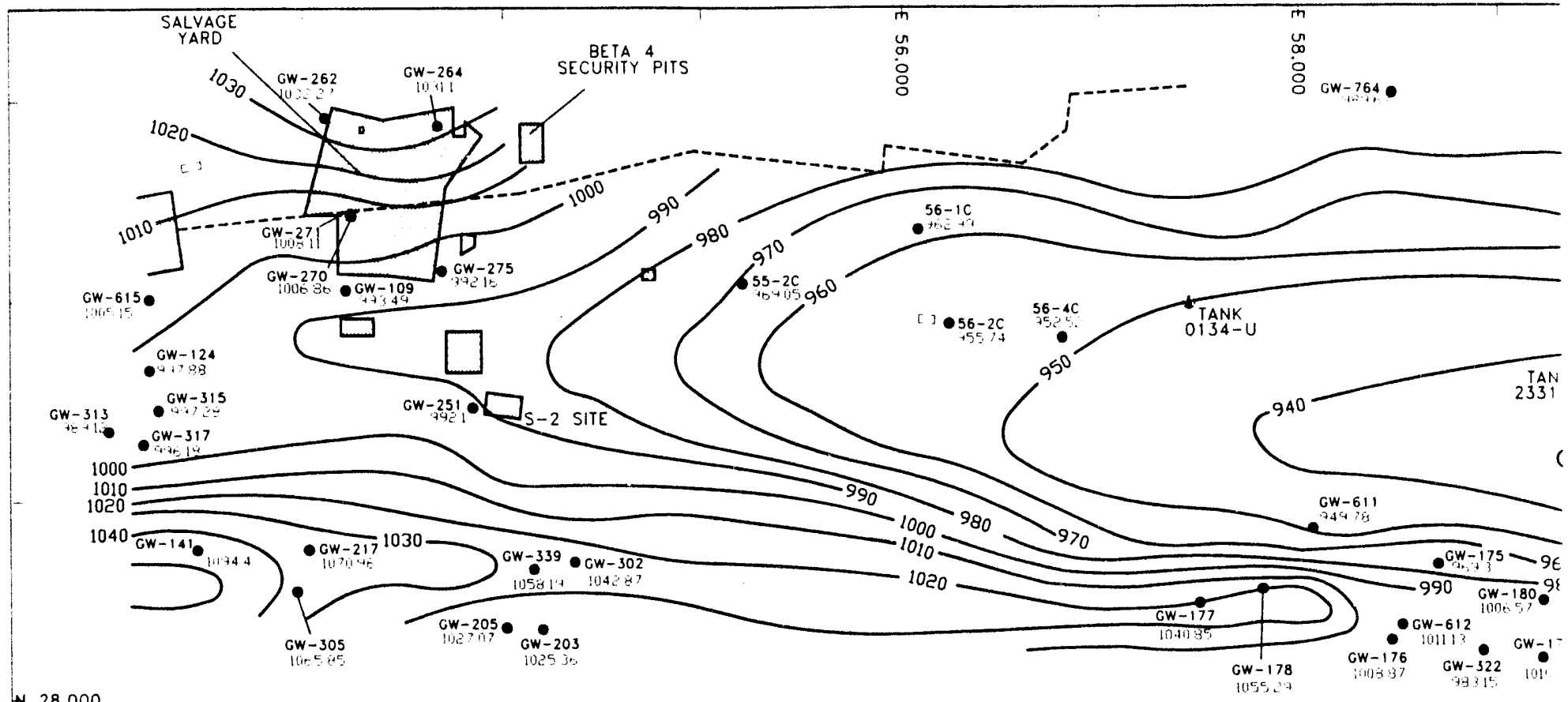

N 28.000

JANUARY 13 .

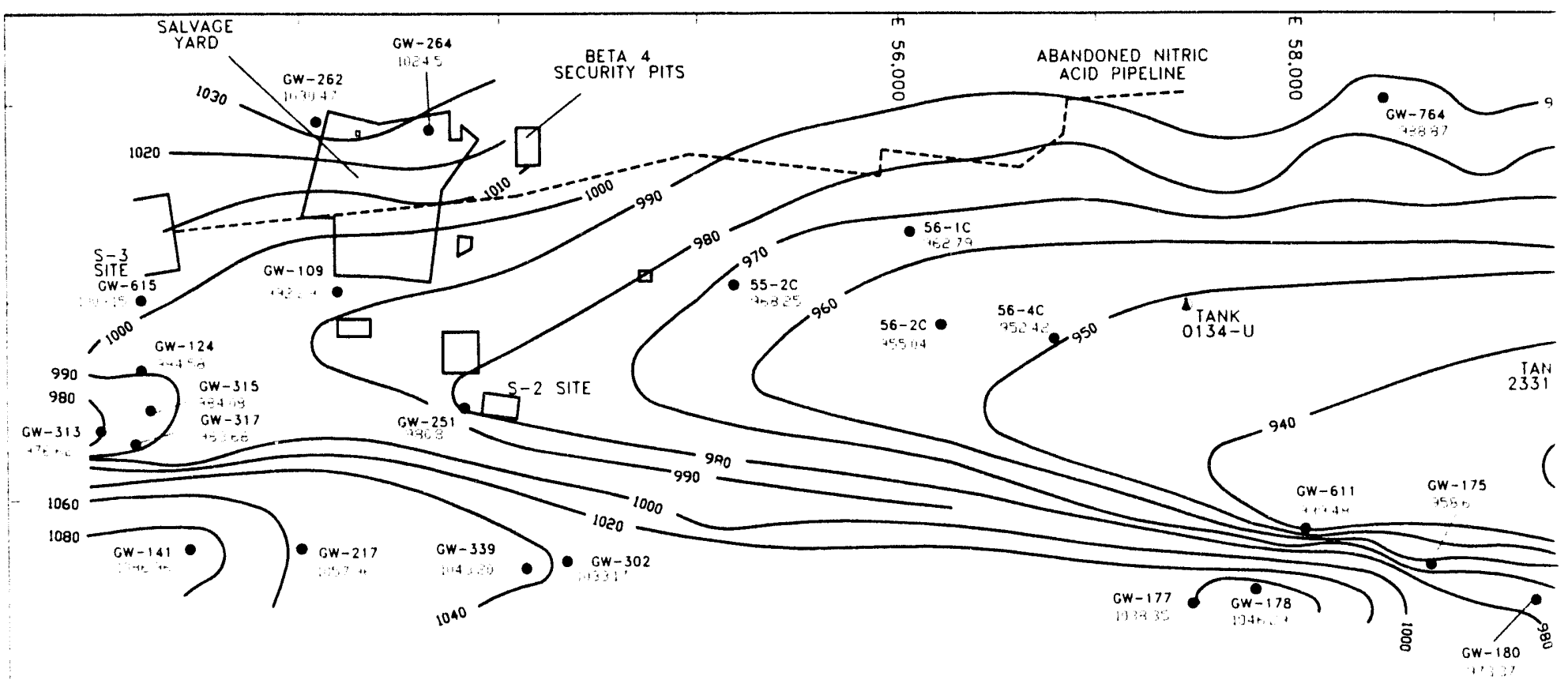

N 28,000

AUGUST 31 - SEPT
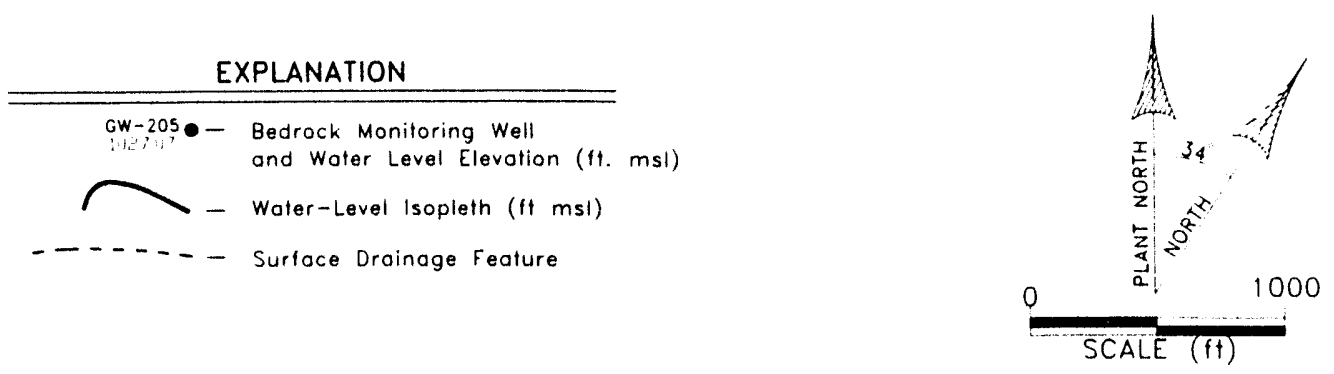


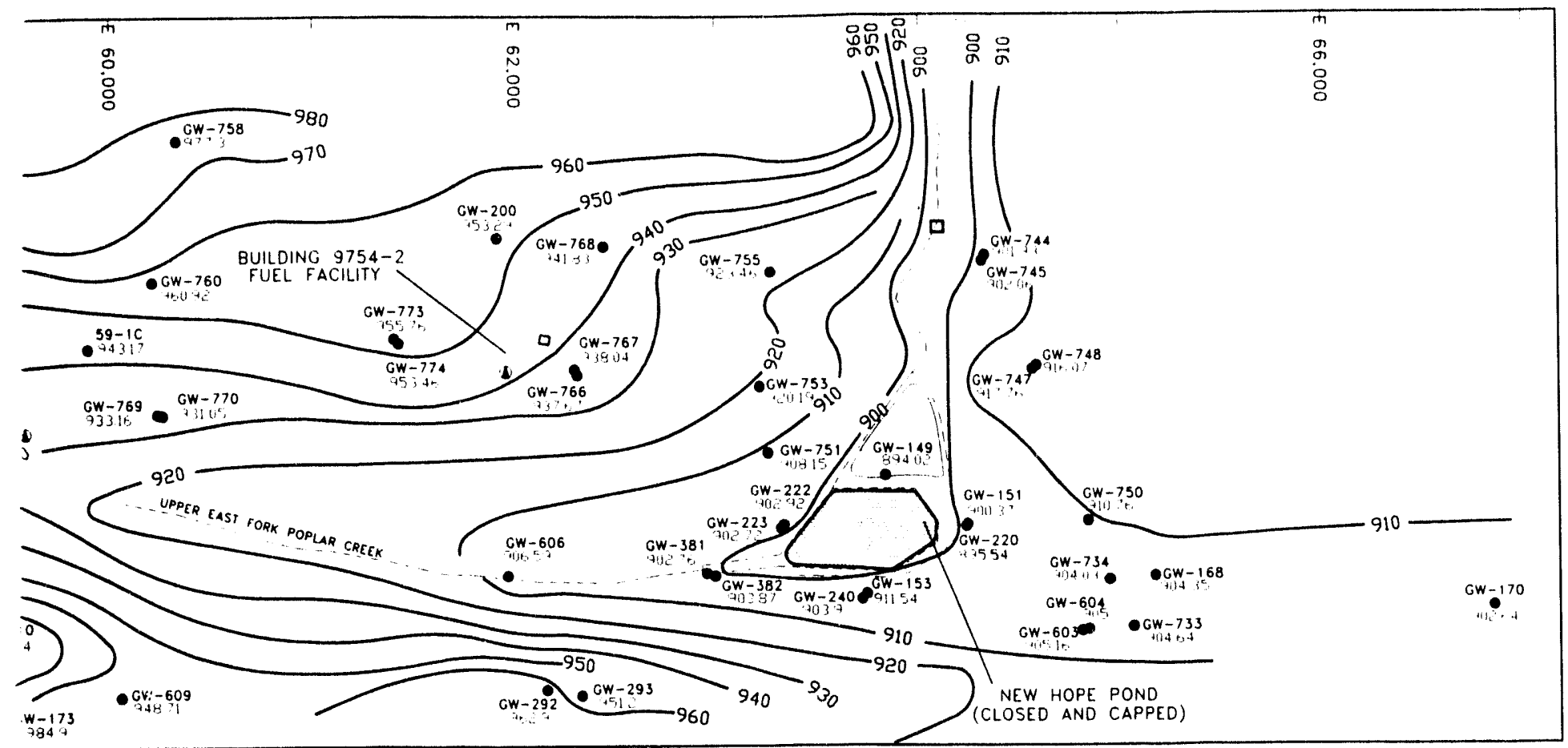

3, 1993

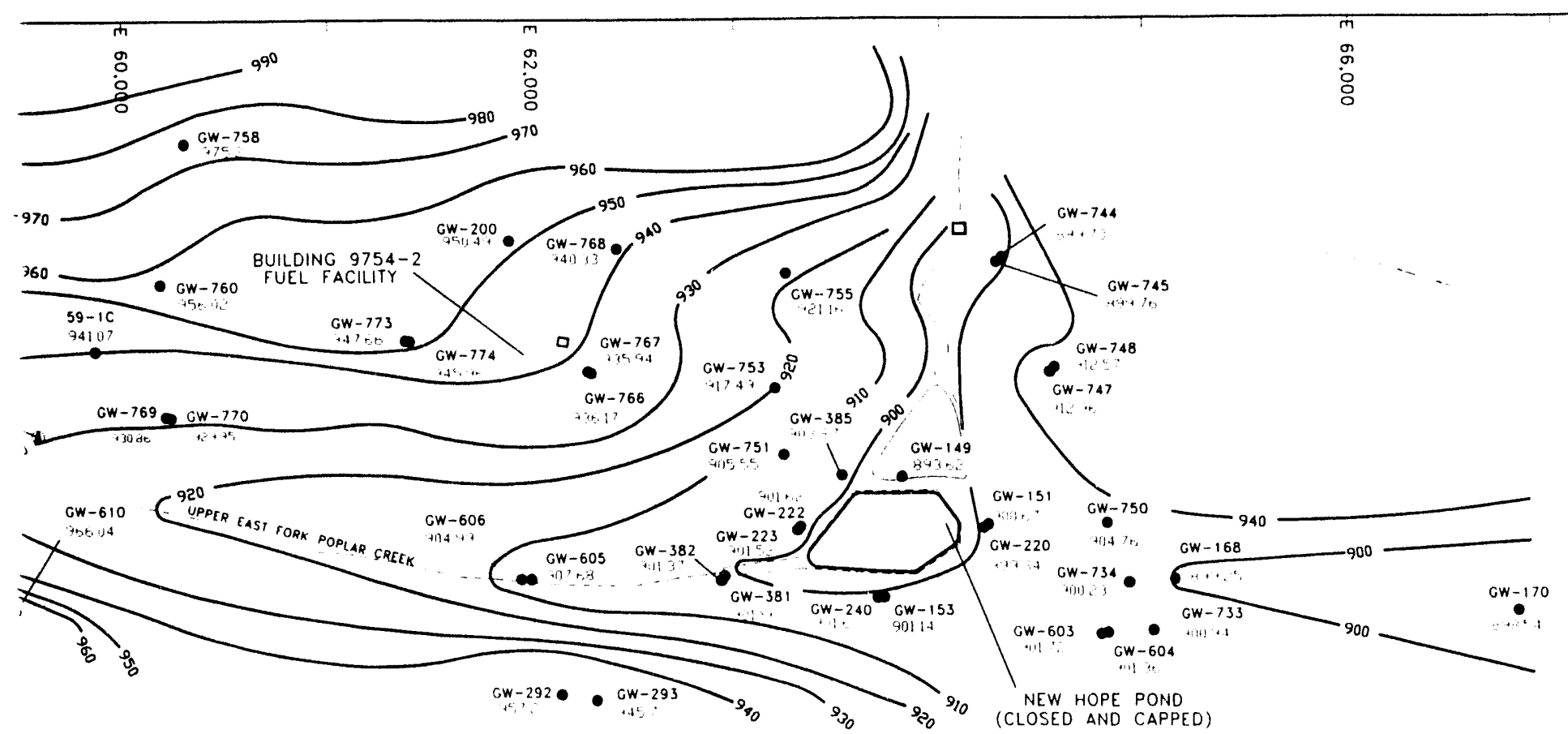

BER 7, 1993

\begin{tabular}{|r|c|c|}
\hline LOCATION: & $\begin{array}{c}\text { Y-12 PLANT } \\
\text { OAK RIDGE, TN. }\end{array}$ & FIGURE 8 \\
\cline { 2 - 3 } DATE: & $2-19-94$ & $\begin{array}{c}\text { GROUNDWATER ELEVATIONS IN THE } \\
\text { DWG ID.: }\end{array}$ \\
\hline
\end{tabular}




\section{APPENDIX B}

TABLES 
Table 1. Waste-Management Sites, CERCLA Operable Units, and Underground Storage Tanks Included in the 1993 Groundwater Protection Program

\begin{tabular}{rcc}
\hline \multicolumn{1}{c}{ Site Name } & \multicolumn{2}{c}{ Regulatory Classification } \\
\cline { 2 - 3 } New Hope Pond & Historical ${ }^{1}$ & Current $^{2}$ \\
Mercury Process Spill Areas & SWMU & TSD Unit \\
Abandoned Nitric Acid Pipeline & SWMU & EFPC OU 01 \\
Salvage Yard Scrap Metal Storage Area & SWMU & EFPC OU 03 \\
Salvage Yard Oil/Solvent Drum Storage Area & SWMU & EFPC OU 03 \\
Salvage Yard Oil Storage & SWMU & EFPC OU 03 \\
Salvage Yard Drum Deheader & SWMU & EFPC OU 03 \\
Tank 2063-U & SWMU & EFPC OU 03 \\
S-2 Site & SWMU & EFPC OU 03 \\
Waste Coolant Processing Area & SWMU & EFPC OU 03 \\
Tank 2328-U & SWMU & Y-12 SA \\
Tank 2329-U & SWMU & Y-12 SA \\
Interim Drum Yard & SWMU & Y-12 SA \\
Beta-4 Security Pits & SWMU & Y-12 SA \\
Tank 2331-U & UST & UST \\
Tank 0134-U & UST & UST \\
Garage Underground Tanks & SWMU/UST & Y-12 SA/UST \\
Rust Garage Area & SWMU/UST & Y-12 SA/UST \\
\hline
\end{tabular}

Notes:

1 Regulatory status before 1992 Federal Facility Agreement.

TSD Unit - RCRA regulated land-based treatment, storage, or disposal unit

SWMU - RCRA regulated Solid Waste Management Unit

UST - Non-RCRA Underground Storage Tank

2 Modified from: Oak Ridge Reservation Site Management Plan for the Environmental Restoration Program. (U.S. Department of Energy 1992)

EFPC OU 01 - East Fork Poplar Creek Operable Unit 01 (Integrator)

EFPC OU 02 - East Fork Poplar Creek Operable Unit 02 (Source Control)

EFPC OU 03 - East Fork Poplar Creek Operable Unit 03 (Source Control)

Y-12 SA - Y-12 Plant Study Area 
Table 2. Monitoring Wells Sampled During 1993

\begin{tabular}{|c|c|c|c|c|}
\hline $\begin{array}{c}\text { Monitoring } \\
\text { Program } \\
\end{array}$ & $\begin{array}{l}\text { Well } \\
\text { No. }\end{array}$ & Location ${ }^{1}$ & $\begin{array}{c}\text { Quarters } \\
\text { Sampled }^{2} \\
\end{array}$ & $\begin{array}{l}\text { Analytical } \\
\text { Parameters } \\
\end{array}$ \\
\hline \multicolumn{5}{|c|}{ RCRA Assessment Monitoing } \\
\hline & GW-151 & NHP & 4 & $\mathbf{S T D}^{3}$ \\
\hline & GW-168 & NHP & 4 & STD \\
\hline & GW-220 & NHP & 4 & STD \\
\hline & GW-239 & NHP & 4 & STD \\
\hline & GW-240 & NHP & 4 & STD \\
\hline & GW-380 & NHP & 4 & STD \\
\hline & GW-381 & NHP & 4 & STD \\
\hline & GW-382 & NHP & 4 & STD \\
\hline & GW-383 & NHP & 4 & STD \\
\hline & GW-384 & NHP & 4 & STD \\
\hline & GW-385 & NHP & 4 & STD \\
\hline & GW-735 & NHP & 4 & STD \\
\hline \multicolumn{5}{|c|}{ Exit Pathway Monitoring } \\
\hline & GW-169 & $\mathrm{CMN}$ & 4 & STD \\
\hline & GW-170 & $\mathrm{CMN}$ & 4 & STD \\
\hline & GW-206 & EXP & 4 & STD \\
\hline & GW-207 & EXP & 4 & STD \\
\hline & GW-208 & EXP & 4 & STD \\
\hline & GW-232 & $\mathrm{CMN}$ & 4 & STD \\
\hline & GW-603 & $\mathrm{CMN}$ & 4 & STD \\
\hline & GW-604 & $\mathrm{CMN}$ & 4 & STD \\
\hline & GW-605 & $\mathrm{CMN}$ & 4 & STD \\
\hline & GW-606 & $\mathrm{CMN}$ & 4 & STD \\
\hline & GW-617 & $\mathrm{CMN}$ & 4 & STD \\
\hline & GW-618 & $\mathrm{CMN}$ & 4 & STD \\
\hline
\end{tabular}


Table 2 (cont'd)

\begin{tabular}{ccccc}
\hline $\begin{array}{c}\text { Monitoring } \\
\text { Program }\end{array}$ & $\begin{array}{l}\text { Well } \\
\text { No. }\end{array}$ & Location $^{1}$ & $\begin{array}{c}\text { Quarters } \\
\text { Sampled }\end{array}$ & $\begin{array}{c}\text { Analytical } \\
\text { Parameters }\end{array}$ \\
\hline
\end{tabular}

Exit Pathway Monitoring (cont'd)

GW-733

CMN

4

STD

Grid Well Monitoring

\begin{tabular}{|c|c|c|c|}
\hline GW-191" & B4 & 4 & STD \\
\hline GW-192" & B4 & 4 & STD \\
\hline GW-194 & B4 & 4 & STD \\
\hline GW-195 & B4 & 4 & STD \\
\hline GW-199 & GRID II & 4 & STD \\
\hline GW-251 & $\mathbf{S} 2$ & 4 & STD \\
\hline$G W-252$ & S2 & 4 & STD \\
\hline GW-255 & S2 & 4 & STD \\
\hline GW-261 & SY & 4 & STD \\
\hline$G W-262^{2}$ & SY & 4 & STD \\
\hline GW-263" & SY & 4 & STD \\
\hline GW-264" & SY & 4 & STD \\
\hline$G W-337$ & WCPA & 4 & STD \\
\hline GW-338 & WCPA & 4 & STD \\
\hline GW-619 & FTF & 4 & STD \\
\hline GW-620 & FTF & 4 & STD \\
\hline GW-744 & GRID Kl & 4 & STD \\
\hline GW-745 & GRID Kl & 4 & STD \\
\hline GW-746 & GRID Kl & 4 & STD \\
\hline GW-747 & GRID K2 & 4 & STD \\
\hline GW-748 & GRID K2 & 4 & STD \\
\hline GW-749 & GRID K2 & 4 & STD \\
\hline $\mathrm{GW}-750$ & GRID K2 & 4 & STD \\
\hline
\end{tabular}


Table 2 (cont'd)

\begin{tabular}{|c|c|c|c|c|}
\hline $\begin{array}{c}\text { Monitoring } \\
\text { Program }\end{array}$ & $\begin{array}{l}\text { Well } \\
\text { No. }\end{array}$ & Location' & $\begin{array}{l}\text { Quarters } \\
\text { Sampled }\end{array}$ & $\begin{array}{l}\text { Analytical } \\
\text { Parameters }\end{array}$ \\
\hline \multicolumn{5}{|c|}{ Grid Well Monitoring (cont'd) } \\
\hline & GW.751 & GRID J3 & 4 & STD \\
\hline & GW-752 & GRID J3 & 4 & STD \\
\hline & GW-753 & GRID J2 & 4 & STD \\
\hline & GW-754 & GRID J2 & 4 & STD \\
\hline & GW-755 & GRID Jl & 4 & STD \\
\hline & GW-756 & GRID Jl & 4 & STD \\
\hline & GW-758 & GRID G1 & 4 & STD \\
\hline & GW-759 & GRID Gl & 4 & STD \\
\hline & GW-760 & GRID G2 & 4 & STD \\
\hline & GW-761 & GRID G2 & 4 & STD \\
\hline & GW-762 & GRID J3 & 4 & STD \\
\hline & GW-763 & GRID J3 & 4 & STD \\
\hline & GW-764 & GRID El & 4 & STD \\
\hline & GW-765 & GRID El & 4 & STD \\
\hline & GW-766 & GRID I2 & 4 & STD, TPH $^{4}$ \\
\hline & GW-767 & GRID I2 & 4 & STD, TPH \\
\hline & GW-768 & GRID II & 4 & STD \\
\hline & GW-769 & GRID G3 & 4 & STD \\
\hline & GW-770 & GRID G3 & 4 & STD \\
\hline & CW-771 & GRID Cl & 4 & STD \\
\hline & GW-772 & GRID Cl & 4 & STD \\
\hline & GW-773 & GRID H2 & 4 & STD \\
\hline & GW-774 & GRID H2 & 4 & STD \\
\hline & GW-775 & GRID H3 & 4 & STD \\
\hline & GW-776 & GRID H3 & 4 & STD \\
\hline
\end{tabular}


Table 2 (cont'd)

\begin{tabular}{|c|c|c|c|c|}
\hline $\begin{array}{c}\text { Monitoring } \\
\text { Program } \\
\end{array}$ & $\begin{array}{l}\text { Well } \\
\text { No. }\end{array}$ & Location' & $\begin{array}{l}\text { Quarters } \\
\text { Sampled } \\
\end{array}$ & $\begin{array}{l}\text { Analytical } \\
\text { Parameters }\end{array}$ \\
\hline \multicolumn{5}{|l|}{ UST Monitoimg } \\
\hline & $G W-183$ & FF & 4 & STD \\
\hline & GW-190 & $\mathbf{R G}$ & 4 & STD \\
\hline & GW-193 & $\mathrm{T} 2331$ & 4 & STD \\
\hline & GW-204 & T0134 & 4 & STD \\
\hline & GW-281" & FF & 4 & STD \\
\hline & GW-283 & FF & 4 & STD \\
\hline & GW-284 & FF & 4 & STD \\
\hline & GW-285 & FF & 4 & STD \\
\hline & GW-505 & $\mathbf{R G}$ & 4 & STD \\
\hline & GW-508 & RG & 4 & STD \\
\hline & GW-631 & RG & 4 & STD \\
\hline & GW-632 & RG & 4 & STD \\
\hline & GW-633 & RG & 4 & STD, TPH \\
\hline & GW-634 & RG & 4 & STD, TPH \\
\hline & GW-656 & T0134 & 4 & STD, TPH \\
\hline & GW-657 & $\mathrm{T} 2331$ & 4 & STD, TPH \\
\hline & GW-658 & FF & 4 & STD, TPH \\
\hline & GW-659 & FF & 4 & STD, TPH \\
\hline & GW-707 & $\mathrm{T} 2331$ & 4 & STD, TPH \\
\hline & GW-708 & $\mathrm{T} 2331$ & 4 & STD, TPH \\
\hline
\end{tabular}

Notes:

$$
\begin{array}{lll}
1 \quad \text { B4 } & - & \text { Beta-4 Security Pits } \\
\text { CMN } & - & \text { Maynardville Limestone } \\
\text { EXP } & - & \text { Exit Pathway }
\end{array}
$$


1 (cont'd)

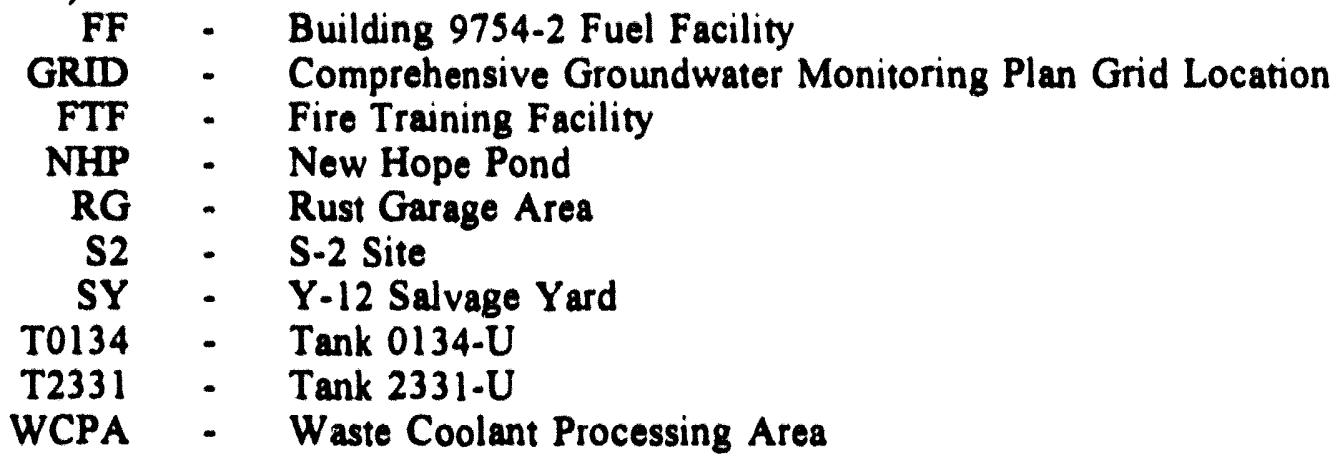

2 The number of quarterly sampling events in which samples were collected during 1993.

3 STD - Standard suite of analytical parameters; includes the following:

- Inductively Coupled Plasma (ICP) Metals

$\begin{array}{lll}\text { Aluminum } & \text { Chromium } & \text { Potassium } \\ \text { Antimony } & \text { Cobalt } & \text { Selenium } \\ \text { Arsenic } & \text { Copper } & \text { Silver } \\ \text { Barium } & \text { Iron } & \text { Sodium } \\ \text { Beryllium } & \text { Magnesium } & \text { Strontium } \\ \text { Boron } & \text { Manganese } & \text { Thorium } \\ \text { Cadmium } & \text { Molybdenum } & \text { Vanadium } \\ \text { Calcium } & \text { Nickel } & \text { Zinc }\end{array}$

Atomic Absorption Spectroscopy Metals

Cadmium

Chromium

Lead

- Other Metals

Mercury (Cold Vapor Atomic Abosrption Spectroscopy)

Uranium (Flourometric Analysis)

- Volatile Organic Compounds

Acetone

Benzene

Bromodichloromethane

Bromoform

Bromomethane

2-Butanone

Carbon disulfide

Carbon tetrachloride

Chlorobenzene

1,2-Dichloropropane cis-1,3-Dichloropropene trans-1,3-Dichloropropene

Ethylbenzene

2-Hexanone

4-Methyl-2-pentanone

Methylene chloride

Styrene

1,1,2,2-Tetrachloroethane 
Table 2 (cont'd)

3 STD - Standard suite of analytical parameters (cont'd)

Chlorodibromomethane

Chloroethane

Chloroform

Chloromethane

1,1-Dichloroethane

1,2-Dichloroethane

1,1-Dichloroethene

1,2-Dichloroethene

Anions

Alkalinity - Carbonate

Alkalinity - Bicarbonate

Chloride

Nitrate (as N)

Fluoride

Sulfate

- Radiological Parameters

Gross Alpha Activity

Gross Beta Activity

- Miscellaneous Parameters

$\mathrm{pH}$

Specific Conductance

Total Suspended Solids (TSS)

Total Dissolved Solids (TDS)

Turbidity

- Field Measurements

Static Water Level

pH

Temperature

Dissolved Oxygen

Specific Conductance

Oxidation - Reduction Potential (REDOX)

4 TPH - Total Petroleum Hydrocarbons

a Background/Upgradient monitoring well 
Table 3. General Construction Information for Monitoring Wells Sampled During 1993

\begin{tabular}{|c|c|c|c|c|c|c|c|c|c|}
\hline \multirow{3}{*}{$\begin{array}{r}\begin{array}{r}\text { Well } \\
\text { No. }\end{array} \\
\text { GW-151 }\end{array}$} & \multirow{3}{*}{$\begin{array}{l}\text { Location }^{2} \\
\text { NHP }\end{array}$} & \multicolumn{2}{|c|}{ Aquifer ${ }^{3}$} & \multicolumn{6}{|c|}{ Monitored Interval' } \\
\hline & & \multirow{2}{*}{$\begin{array}{c}\text { Zone } \\
\text { BDR }\end{array}$} & \multirow{2}{*}{$\frac{\text { Unit }}{\mathrm{Cmn}}$} & \multicolumn{3}{|c|}{ Depths } & \multicolumn{3}{|c|}{ Elevations } \\
\hline & & & & 85.0 & - & 96.5 & 828.1 & - & 816.6 \\
\hline$G W-152$ & NHP & WT & $\mathrm{Cmn}$ & 10.8 & - & 17.3 & 907.6 & - & 901.1 \\
\hline GW-167 & NHP & WT & $\mathrm{Cmn}$ & 25.0 & - & 30.1 & 904.7 & - & 899.6 \\
\hline GW-168" & NHP & BDR & $\mathrm{Cn}$ & 104.0 & - & 135.4 & 825.5 & - & 794.1 \\
\hline GW-169 & EXP & WT & $\mathrm{Cmn}$ & 28.7 & - & 34.7 & 901.3 & - & 895.3 \\
\hline GW-170" & EXP & BDR & $\mathrm{Cmn}$ & 104.0 & - & 156.9 & 826.7 & - & 773.8 \\
\hline GW-183 & FF & WT & $\mathrm{CON}$ & 5.0 & - & 30.6 & 938.0 & - & 912.4 \\
\hline GW-190 & $\mathbf{R G}$ & WT & CON & 8.0 & - & 26.2 & $1,022.2$ & - & $1,004.0$ \\
\hline GW-191" & B4 & WT & $\mathrm{Cm}$ & 45.0 & - & 60.5 & 962.1 & - & 946.6 \\
\hline GW-192' & B4 & WT & $\mathrm{Cm}$ & 6.5 & - & 17.5 & 999.5 & - & 988.5 \\
\hline GW-193 & T2331 & WT & CON & 5.5 & - & 18.4 & 925.6 & - & 912.7 \\
\hline GW-194 & B4 & WT & $\mathrm{Cm}$ & 6.5 & - & 12.6 & 986.0 & - & 979.9 \\
\hline GW-195 & B4 & WT & $\mathrm{Cm}$ & 17.0 & - & 23.0 & 983.2 & - & 977.2 \\
\hline GW-199 & GRIDII & WT & $\mathrm{Crg}$ & 16.5 & - & 22.5 & 941.6 & - & 935.6 \\
\hline GW-204 & T0134 & WT & $\mathrm{CON}$ & 6.5 & - & 17.3 & 949.0 & - & 938.2 \\
\hline GW-206 & EXP & WT & $\mathrm{Cr}$ & 10.0 & - & 16.9 & 884.7 & - & 877.8 \\
\hline GW-207" & EXP & BDR & $\mathrm{Cr}$ & 100.0 & - & 109.6 & 794.7 & - & 785.1 \\
\hline$G W-208^{2}$ & EXP & BDR & $\mathrm{Cr}$ & 404.0 & - & 412.8 & 490.5 & - & 481.7 \\
\hline GW-220 & NHP & BDR & $\mathrm{Cmn}$ & 31.0 & - & 45.2 & 881.7 & - & 867.5 \\
\hline GW-232 & EXP & BDR & $\mathrm{Cmn}$ & 401.0 & - & 411.7 & 528.5 & - & 517.8 \\
\hline GW-239" & NHP & BDR & $\mathrm{Cn}$ & 404.0 & - & 433.3 & 524.5 & - & 495.2 \\
\hline GW-240 & NHP & BDR & $\mathrm{Cmn}$ & 21.0 & - & 29.5 & 898.5 & - & 890.0 \\
\hline GW-25l & $\mathbf{S 2}$ & BDR & $\mathrm{Cmn}$ & 35.0 & - & 51.0 & 966.6 & - & 950.6 \\
\hline GW-252 & S2 & WT & $\mathrm{Cmn}$ & 40.2 & - & 49.0 & 982.5 & - & 973.7 \\
\hline GW-255 & S2 & WT & $\mathrm{Cmn}$ & 66.3 & - & 81.5 & 958.0 & - & 942.8 \\
\hline
\end{tabular}

B-8 
Table 3 (cont'd)

\begin{tabular}{|c|c|c|c|c|c|c|c|c|c|}
\hline \multirow{3}{*}{$\begin{array}{r}\begin{array}{r}\text { Well } \\
\text { No. }\end{array} \\
\text { GW-261' }\end{array}$} & \multirow{3}{*}{$\begin{array}{l}\text { Location }^{2} \\
S Y\end{array}$} & \multicolumn{2}{|c|}{ Aquifer } & \multicolumn{6}{|c|}{ Monitored Interval } \\
\hline & & \multirow{2}{*}{$\frac{\text { Zone }}{\text { WT }}$} & \multirow{2}{*}{$\frac{\text { Unit }}{\mathrm{Cm}}$} & \multicolumn{3}{|c|}{ Depths } & \multicolumn{3}{|c|}{ Elevations } \\
\hline & & & & 16.7 & - & 23.7 & $1,030.9$ & - & $1,023.9$ \\
\hline GW-262 & SY & BDR & $\mathrm{Cm}$ & 57.7 & - & 70.6 & 989.6 & - & 976.7 \\
\hline GW-263' & SY & WT & $\mathrm{Cm}$ & 23.0 & - & 30.8 & $1,032.1$ & - & $1,024.3$ \\
\hline GW-264" & SY & BDR & $\mathrm{Cm}$ & 58.5 & - & 71.0 & 997.0 & - & 984.5 \\
\hline$G W-281^{2}$ & FF & WT & $\mathrm{Cn}$ & 4.0 & - & 15.0 & 942.5 & - & 931.5 \\
\hline$G W-283$ & $\mathrm{FF}$ & WT & $\mathrm{Cn}$ & 4.0 & - & 20.0 & 935.2 & - & 919.2 \\
\hline GW-284 & $\mathrm{FF}$ & WT & $\mathrm{Cn}$ & 2.0 & - & 18.0 & 940.3 & - & 924.3 \\
\hline GW-285 & FF & WT & $\mathrm{Cn}$ & 3.5 & - & 21.0 & 939.4 & - & 921.9 \\
\hline GW-337 & WCPA & WT & $\mathrm{Cn}$ & 15.0 & - & 22.1 & 969.1 & - & 962.0 \\
\hline GW-338 & WCPA & WT & $\mathrm{Cn}$ & 11.2 & - & 17.6 & 972.8 & - & 966.4 \\
\hline GW-380 & NHP & WT & $\mathrm{Cmn}$ & 2.8 & - & 15.5 & 910.9 & - & 898.2 \\
\hline GW-381 & NHP & BDR & Cmn & 49.3 & - & 60.4 & 864.1 & - & 853.0 \\
\hline GW-382 & NHP & BDR & Cmn & 125.0 & - & 173.0 & 788.2 & - & 740.2 \\
\hline GW-383 & NHP & WT & $\mathrm{Cn}$ & 16.6 & - & 23.6 & 889.4 & - & 882.4 \\
\hline GW-384 & NHP & WT & $\mathrm{Cn}$ & 35.5 & - & 55.7 & 869.9 & - & 849.7 \\
\hline GW-385 & NHP & BDR & $\mathrm{Cn}$ & 123.7 & - & 178.7 & 782.2 & - & 727.2 \\
\hline GW-505 & $\mathbf{R G}$ & WT & $\mathrm{Cn}$ & 1.5 & - & 13.5 & $1,010.1$ & - & 998.1 \\
\hline GW-508 & $\mathrm{RG}$ & WT & $\mathrm{Cn}$ & 1.3 & - & 15.0 & $1,008.3$ & $\cdot$ & 994.6 \\
\hline GW-603 & EXP & BDR & $\mathrm{Cmn}$ & 63.4 & - & 75.2 & 896.0 & - & 884.2 \\
\hline$G W-604$ & EXP & BDR & $\mathrm{Cmn}$ & 100.2 & - & 112.4 & 859.3 & - & 847.1 \\
\hline GW-605 & EXP & BDR & $\mathrm{Cmn}$ & 28.2 & - & 39.9 & 888.8 & - & 877.1 \\
\hline GW-606 & EXP & BDR & $\mathrm{Cmn}$ & 155.0 & - & 171.7 & 762.0 & - & 745.3 \\
\hline GW-617 & EXP & WT & $\mathrm{Cmn}$ & 6.8 & - & 18.0 & 975.8 & - & 964.6 \\
\hline GW-618 & $\mathrm{EXP}$ & WT & $\mathrm{Cmn}$ & 26.0 & - & 37.0 & 956.6 & - & 945.6 \\
\hline GW-619 & FTF & WT & $\mathrm{Cmn}$ & 26.8 & - & 40.8 & 985.9 & - & 971.9 \\
\hline
\end{tabular}


Table 3 (cont'd)

\begin{tabular}{|c|c|c|c|c|c|c|c|c|c|}
\hline \multirow{3}{*}{$\begin{array}{r}\text { Well } \\
\text { No. }{ }^{1} \\
\text { GW-620 }\end{array}$} & \multirow{3}{*}{$\begin{array}{l}\text { Location }^{2} \\
\text { FTF }\end{array}$} & \multicolumn{2}{|c|}{ Aquifer ${ }^{3}$} & \multicolumn{6}{|c|}{ Monitored Interval $^{4}$} \\
\hline & & \multirow{2}{*}{$\frac{\text { Zone }}{\text { WT }}$} & \multirow{2}{*}{$\frac{\text { Unit }}{\text { Cmn }}$} & \multicolumn{3}{|c|}{ Depths } & \multicolumn{3}{|c|}{ Elevations } \\
\hline & & & & 61.7 & - & 75.0 & 951.1 & - & 937.8 \\
\hline GW-631 & $\mathrm{RG}$ & WT & $\mathrm{Cn}$ & 4.0 & - & 16.0 & $1,000.1$ & - & 988.1 \\
\hline GW-632 & $\mathbf{R G}$ & WT & $\mathrm{Cn}$ & 3.5 & - & 15.0 & $1,002.5$ & - & 991.0 \\
\hline GW-633 & $\mathbf{R G}$ & WT & $\mathrm{Cn}$ & 3.5 & - & 15.0 & 993.0 & - & 981.5 \\
\hline$G W-634$ & RG & WT & $\mathrm{Cn}$ & 3.5 & - & 15.0 & $1,003.8$ & - & 992.3 \\
\hline GW-656 & T0134 & WT & $\mathrm{Cn}$ & 8.3 & - & 21.5 & 946.6 & - & 933.4 \\
\hline GW-657 & $\mathrm{T} 2331$ & WT & $\mathrm{Cmn}$ & 4.0 & - & 17.1 & 926.8 & - & 913.7 \\
\hline GW-658 & FF & WT & $\mathrm{Cn}$ & 6.9 & - & 19.1 & 935.1 & - & 922.9 \\
\hline GW-659 & FF & WT & $\mathrm{Cn}$ & 4.6 & - & 16.7 & 937.2 & - & 925.1 \\
\hline GW-707 & T2331 & WT & $\mathrm{Cmn}$ & 5.5 & - & 26.0 & 925.7 & - & 905.2 \\
\hline GW-708 & $\mathrm{T} 2331$ & WT & $\mathrm{Cmn}$ & 2.7 & - & 13.7 & 928.3 & - & 917.3 \\
\hline GW-733 & EXP & BDR & $\mathrm{Cmn}$ & 240.1 & - & 256.5 & 715.6 & - & 699.2 \\
\hline GW-735 & NHP & WT & $\mathrm{Cn}$ & 67.5 & - & 79.2 & 853.8 & - & 842.1 \\
\hline GW-744 & GRIDK 1 & BDR & $\mathrm{Cpv}$ & 55.0 & - & 69.5 & 850.1 & - & 835.6 \\
\hline GW-745 & GRIDK1 & BDR & $\mathrm{Cpv}$ & 21.2 & - & 32.8 & 883.1 & - & 871.5 \\
\hline GW-746 & GRIDK1 & WT & $\mathrm{Cpv}$ & 3.2 & - & 15.2 & 901.1 & - & 889.1 \\
\hline GW-747 & GRIDK2 & BDR & $\mathrm{Cm}$ & 67.4 & - & 79.6 & 850.9 & - & 838.7 \\
\hline GW-748 & GRIDK2 & BDR & $\mathrm{Cm}$ & 14.8 & - & 27.2 & 904.1 & - & 891.7 \\
\hline GW-749 & GRIDK2 & WT & $\mathrm{Cm}$ & 4.8 & - & 16.4 & 913.9 & - & 902.3 \\
\hline GW-750 & GRIDK2 & BDR & $\mathrm{Cn}$ & 61.2 & - & 72.7 & 854.8 & - & 843.3 \\
\hline GW-751 & GRIDJ3 & BDR & $\mathrm{Cn}$ & 49.0 & - & 60.6 & 860.4 & - & 848.8 \\
\hline GW-752 & GRIDJ3 & WT & $\mathrm{Cn}$ & 4.8 & - & 15.9 & 905.0 & - & 893.9 \\
\hline GW-753 & GRIDJ2 & BDR & $\mathrm{Cm}$ & 59.6 & - & 70.7 & 865.1 & - & 854.0 \\
\hline GW-754 & GRIDJ2 & WT & $\mathrm{Cn}$ & 13.0 & - & 24.1 & 912.6 & - & 901.5 \\
\hline GW-755 & GRIDJl & BDR & $\mathrm{Crg}$ & 49.2 & - & 60.5 & 878.2 & - & 866.9 \\
\hline
\end{tabular}


Table 3 (cont'd)

\begin{tabular}{|c|c|c|c|c|c|c|c|c|c|}
\hline \multirow{3}{*}{$\begin{array}{r}\begin{array}{r}\text { Well } \\
\text { No. }\end{array} \\
\text { GW-756 }\end{array}$} & \multirow{3}{*}{$\begin{array}{l}\text { Location }^{2} \\
\text { GRIDJ1 }\end{array}$} & \multicolumn{2}{|c|}{ Aquifer ${ }^{3}$} & \multicolumn{6}{|c|}{ Monitored Interval ${ }^{4}$} \\
\hline & & \multirow{2}{*}{$\frac{\text { Zone }}{W T}$} & \multirow{2}{*}{$\frac{\text { Unit }}{\mathrm{Cm}}$} & \multicolumn{3}{|c|}{ Depths } & \multicolumn{3}{|c|}{ Elevations } \\
\hline & & & & 5.3 & - & 16.6 & 919.6 & - & 908.3 \\
\hline GW-758 & GRIDG1 & BDR & $\mathrm{Crt}$ & 38.4 & - & 50.2 & 951.2 & - & 939.5 \\
\hline GW-759 & GRIDG1 & WT & $\mathrm{Crg}$ & 18.0 & - & 30.2 & 973.0 & - & 960.8 \\
\hline GW-760 & GRIDG2 & BDR & $\mathrm{Cm}$ & 48.3 & - & 60.1 & 918.2 & - & 906.4 \\
\hline GW-761 & GRIDG2 & WT & $\mathrm{Cm}$ & 3.6 & - & 15.3 & 961.3 & - & 949.6 \\
\hline GW-762 & GRIDJ3 & BDR & $\mathrm{Cn}$ & 46.4 & - & 58.7 & 865.5 & - & 853.2 \\
\hline$G W-763$ & GRIDJ3 & WT & $\mathrm{Cn}$ & 4.0 & - & 16.0 & 907.4 & - & 895.4 \\
\hline GW-764 & GRIDE 1 & BDR & $\mathrm{Crg}$ & 53.9 & - & 65.0 & 952.8 & - & 941.7 \\
\hline GW-765 & GRIDE 1 & WT & Crg & 19.9 & - & 32.4 & 985.6 & - & 973.1 \\
\hline GW-766 & GRIDI2 & BDR & $\mathrm{Cm}$ & 34.0 & - & 47.6 & 910.7 & - & 897.1 \\
\hline GW-767 & GRIDI2 & WT & $\mathrm{Cm}$ & 6.7 & - & 18.0 & 938.5 & - & 927.2 \\
\hline GW-768 & GRDII & BDR & $\mathrm{Crg}$ & 53.3 & - & 65.0 & 903.4 & - & 891.7 \\
\hline GW-769 & GRIDG3 & BDR & $\mathrm{Cn}$ & 48.2 & - & 60.3 & 893.3 & - & 881.2 \\
\hline GW-770 & GRIDG3 & WT & $\mathrm{Cn}$ & 7.5 & - & 19.0 & 934.2 & - & 922.7 \\
\hline GW-771 & GRIDC 1 & BDR & $\mathrm{Crg}$ & 42.2 & - & 54.4 & 966.1 & - & 953.9 \\
\hline GW-772 & GRIDCl & WT & Crt & 3.8 & - & 17.0 & $1,005.8$ & - & 992.6 \\
\hline GW-773 & GRIDH2 & BDR & $\mathrm{Cm}$ & 46.1 & - & 59.6 & 914.6 & - & 901.1 \\
\hline GW-774 & GRIDH2 & WT & $\mathrm{Cm}$ & 12.5 & - & 26.6 & 947.6 & - & 933.5 \\
\hline GW-775 & GRIDH3 & BDR & $\mathrm{Cn}$ & 45.0 & - & 56.4 & 886.5 & - & 875.1 \\
\hline GW-776 & GRIDH3 & WT & $\mathrm{Cn}$ & 10.6 & - & 23.0 & 920.8 & - & 908.4 \\
\hline
\end{tabular}

Notes:

1 Well construction information compiled from: Updated Subsurface Data Base for Bear Creek Valley, Chestnut Ridge, and Parts of Bethel Valley on the U.S. Department of Energy Oak Ridge Reservation (Jones et al. 1993). 
Table 3 (cont'd)

2

B4 - Beta-4 Security Pits

EXP - Exit-Pathway Monitoring Well

FF - Building 9754-2 Fuel Facility

GRID - Comprehensive Groundwater Monitoring Plan Grid Location

FTF - Fire Training Facility

NHP - New Hope Pond

RG - Rust Garage Area

S2 - S-2 Site

SY - Y-12 Salvage Yard

T0134 - Tank 0134-U

T2331 - Tank 2331-U

WCPA - Waste Coolant Processing Area

3 BDR - Bedrock (Shallow, Intermediate, and Deep Intervals)

WT - Water-Table Interval

CON - Conasauga Group (undifferentiated)

Cmn - Maynardville Limestone

Cn - Nolichucky Shale

$\mathrm{Cm}$ - Maryville Limestone

Crg - Rogersville Shale

Crt - Rutledge Limestone

Cpv - Pumpkin Valley Shale

$\mathrm{Cr} \quad$ - Rome Formation

4 Depth in feet from the ground surface.

Elevation in feet above mean sea level.

a Background/Upgradient monitoring well 
APPENDIX C

MONITORING-WELL CONSTRUCTION DETAIS 


\section{EXPLANATION}

\section{LOCATION:}

$\begin{array}{rll}\text { B4 } & - & \text { Beta-4 Security Pits } \\ \text { EXP } & - & \text { Exit-Pathway Monitoring Well } \\ \text { FF } & - & \text { Building 9754-2 Fuel Facility } \\ \text { FTF } & - & \text { Fire Training Facility } \\ \text { GRID } & - & \text { Comprehensive Groundwater Monitoring Plan Grid Location } \\ \text { NHP } & - & \text { New Hope Pond } \\ \text { RG } & - & \text { Rust Garage Area } \\ \text { S2 } & - & \text { S-2 Site } \\ \text { SY } & - & \text { Y-12 Salvage Yard } \\ \text { T0134 } & - & \text { Underground Storage Tank 0134-U } \\ \text { T2331 } & - & \text { Underground Storage Tank 2331-U } \\ \text { WC } & - & \text { Waste Coolant Processing Area }\end{array}$

\section{CASING MATERIAL:}

$\begin{array}{rll}\text { PVC } & \text { - } & \text { Polyvinyl chloride } \\ \text { SS } & - & \text { Stainless Steel }\end{array}$

\section{MONITORED INTERVAL:}

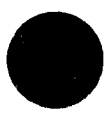

$\begin{array}{rll}\text { BDR } & - & \text { Bedrock Interval } \\ \text { WT } & - & \text { Water-Table Interval } \\ \text { CON } & - & \text { Conasauga Group (undifferentiated) } \\ \text { Cmn } & - & \text { Maynardville Limestone } \\ \text { Cn } & - & \text { Nolichucky Shale } \\ \text { Cm } & - & \text { Maryville Limestone } \\ \text { Crg } & - & \text { Rogersville Shale } \\ \text { Crt } & - & \text { Rutledge Limestone } \\ \text { Cpv } & - & \text { Pumpkin Valley Shale } \\ \text { Cr } & - & \text { Rome Formation } \\ \text { Top } & - & \text { Depth to top of filter pack or open-hole } \\ \text { Bottom } & - & \text { Depth to bottom of filter pack or open-hole } \\ \text { PVC/sl } & - & \text { Slotted } \\ \text { PVC/sl/.01 } & - & \text { Slotted, 0.01-inch slot size } \\ \text { PVC/sw/.01 } & - & \text { Spiral wound, 0.01-inch slot size } \\ \text { SS/s1/.01 } & - & \text { Slotted, 0.01-inch slot size } \\ \text { SS/sw/.01 } & - & \text { Spiral wound, } 0.01 \text {-inch slot size }\end{array}$

\section{NOTES:}

Coordinates - Y-12 Plant grid system

Elevations - Feet above mean sea level

Depths - Feet below ground surface

Casing Diameter - Outside diameter, in inches

Borehole Diameter - Nominal diameter, in inches

Length - Feet

- Not Applicable or not available 
APPENDIX $C$

Monitoring-Well Construction Details

\begin{tabular}{|c|c|c|c|c|c|c|c|}
\hline \multirow{2}{*}{$\begin{array}{l}\text { Well No. } \\
\text { Location }\end{array}$} & GW-151 & GW-152 & GW-167 & GW-168 & GW-169 & GW-170 & GW-183 \\
\hline & NHP & NHP & NHP & NHP & $\operatorname{EXP}$ & EXP & $\mathbf{F F}$ \\
\hline East Coordinate & 64232 & 63718 & 65146 & 65167 & 66855 & 66843 & 61954.52 \\
\hline North Coordinate & 28958 & 28614 & 28661 & 28699 & 28545 & 28545 & 29658.59 \\
\hline ELEVATIONS & - & . & . & . & . & . & . \\
\hline Measuring Point & 915.97 & 921.18 & 931.38 & 930.95 & 931.87 & 932.44 & 946.02 \\
\hline Surface & 913.06 & 918.36 & 929.67 & 929.45 & 929.95 & 930.7 & 943.01 \\
\hline Weathered Rock & 901.06 & 901.06 & • & . & • & . & 941.51 \\
\hline Fresh Rock & 901.06 & • & . & 900.45 & . & 900.7 & • \\
\hline Top Monitored Interval & 828.06 & 907.56 & 904.67 & 825.45 & 901.25 & 826.7 & 938.01 \\
\hline Bottom Monitored Interval & 816.56 & 901.06 & 899.57 & 794.05 & 895.25 & 773.8 & 912.41 \\
\hline SURFACE/CONDUCTOR CASING & . & . & . & . & . & . & . \\
\hline Casing Depth & 12 &. & $0 . \dot{1}$ & 30 & • & 30 & • \\
\hline Casing Diameter & 12.5 & . & 8.625 & 8.625 & . & 8.625 & • \\
\hline Casing Material & $\mathrm{PVC} / \$ 40$ & . & PvC $/ \$ 40$ & $\mathrm{PVC} / \$ 40$ & . & Pvc $/ 40$ & • \\
\hline WELL CASING BORBHOLE & . & . & . & . & . & . & • \\
\hline Borehole Depth & $96 . \dot{5}$ & $17 . \dot{3}$ & 30.1 & 104 & $\dot{42}$ & 104 & 30.6 \\
\hline Borehole Diameter & 11 & 8 & 6.62 & 6.62 & 8 & 6.62 & 8 \\
\hline Weathered Rock - Depth & 12 & 17.3 & • & . & . & . & 1.5 \\
\hline Fresh Rock - Depth & 12 & . & . & 29 & . & 30 & • \\
\hline WELL CASING & . & . & . & . & . & . & • \\
\hline Well Casing Depth & $96 . \dot{5}$ & $17 . \dot{3}$ & $30 . \dot{1}$ & 104 & 34.8 & 104 & 29.8 \\
\hline Well Casing Diameter & 4.5 & 4.5 & 2.37 & 4.38 & 2.37 & 4.38 & 4.5 \\
\hline Well Casing Material & $\mathrm{PVC} / \$ 40$ & PVC $/ \$ 40$ & PVC $/ \$ 40$ & Steel & PVC $/ \$ 40$ & Steel & $S S / \$ 304$ \\
\hline MONITORED INTERVAL & . & $\cdot$ & . & . & . & . & • \\
\hline Aquifer zone & BDR & WT & WT & BDR & WT & BDR & พT \\
\hline Geologic Formation & $C m n$ & $\operatorname{conn}$ & $\operatorname{comn}$ & $\mathrm{Cn}$ & $\mathrm{cmn}$ & comn & CON \\
\hline Screened or Open & Screen & Screen & Screen & Open & Screen & Open & Screen \\
\hline Top - Depth & 85 & 10.8 & 25 & 104 & 28.7 & 104 & 5 \\
\hline Bottom - Depth & 96.5 & 17.3 & 30.1 & 135.4 & 34.7 & 156.9 & 30.6 \\
\hline Screen Materiai & PVC/sw/.01 & $\mathrm{PVC} / \mathrm{sw} / .01$ & PVC/sl & . & PVC/sl & . & $\mathrm{ss} / \mathrm{sw} / .01$ \\
\hline Screen Length & 10.0 & 5.0 & 4.1 & . & 5.0 & . & 20.0 \\
\hline Open-Hole Length & - & . & - & 31.4 & . & 52.9 & . \\
\hline Open-Hole Diameter & . & . & . & 3.875 & . & 3.875 & . \\
\hline
\end{tabular}

(CONTINUED) 
APPENDIX C

Monitoring-Well Construction Details

\begin{tabular}{|c|c|c|c|c|c|c|c|}
\hline Well No. & GW-190 & GW-191 & GW- 192 & GW-193 & GW-194 & GW-195 & GW-199 \\
\hline Iocation & RG & B4 & B4 & T2331 & B4 & B4 & GRIDI 1 \\
\hline Bast Coordinate & 53254.42 & 54279 & 54277 & 59536.17 & 54218 & 54089 & 62464 \\
\hline North Coordinate & 30776.49 & 30780 & 30772 & 29343.93 & 30583 & 30573 & 30283 \\
\hline BIEVATIONS & - & - & - & - & - & - & - \\
\hline Measuring Point & 1033.69 & 1011.14 & 1008.63 & 934 & 995.36 & 1002.94 & 961.08 \\
\hline Surface & 1030.19 & 1007.1 & 1006.04 & 931.11 & 992.47 & 1000.23 & 958.06 \\
\hline Weathered Rock & 1022.19 & • & 988.54 & 928.61 & 979.87 & 977.23 & 957.26 \\
\hline Fresh Rock & & - & • & $\bullet$ & • & • & 940.06 \\
\hline Top Monitored Interval & 1022.19 & 962.1 & 999.54 & 925.61 & 985.97 & 983.23 & 941.56 \\
\hline Bottom Monitored Interval & 1003.99 & 946.6 & 988.54 & 912.71 & 979.87 & 977.23 & 935.56 \\
\hline SURFACE/CONDUCTOR CASING & • & $\bullet$ & • & $\bullet$ & $\cdot$ & • & • \\
\hline Casing Depth & $\cdot$ & 17 & • & • & • & • & • \\
\hline Casing Diameter & - & $12 \cdot 5$ & - & - & - & - & - \\
\hline Casing Material & - & PVC $/ \$ 40$ & - & - & - & - & - \\
\hline WELL CASING BOREHOLE & - & $\cdot$ & . & $\cdot$ & $\cdot$ & $\cdot$ & $\cdot$ \\
\hline Borehole Depth & 26.2 & 60.5 & 17.5 & 18.4 & 12.6 & 23 & 22.5 \\
\hline Borehole Diameter & 8.5 & 11 & 6 & 8 & 8 & 6 & 8 \\
\hline Weathered Rock - Depth & 8 & $\cdot$ & 17.5 & 2.5 & 12.6 & 23 & WT \\
\hline Fresh Rock - Depth & - & $\cdot$ & • & • & • & - & 18 \\
\hline WELL CASING & - & $\cdot$ & $\cdot$ & - & • & - & - \\
\hline Well Casing Depth & 26.2 & 60 & 17.5 & 18.4 & 12.5 & 23 & 22.5 \\
\hline Well Casing Diameter & 4.5 & 4.5 & 4.5 & 4.5 & 4.5 & 4.5 & 4.5 \\
\hline Well Casing Material & ss $/ \$ 304$ & PVC $/ \$ 40$ & PVC $/ \$ 40$ & ss/1304 & $\mathrm{PVC} / \$ 40$ & PVC $/ 40$ & PVC $/ \$ 40$ \\
\hline MONITORED INTERVAI & - & - & $\cdot$ & - & - & - & - \\
\hline Aquifer zone & พT & WT & พT & WT & wT & wr & WT \\
\hline Geologic Formation & $\operatorname{coN}$ & $\mathrm{cm}$ & $\mathrm{cm}$ & CON & $\mathrm{cm}$ & $\mathrm{Cm}$ & $\mathrm{Crg}$ \\
\hline Screened or Open & Screen & Screen & screen & Screen & Screen & Screen & Screen \\
\hline Top - Depth & 8 & 45 & 6.5 & 5.5 & 6.5 & 17 & 16.5 \\
\hline Bottom - Depth & 26.2 & 60.5 & 17.5 & 18.4 & 12.6 & 23 & 22.5 \\
\hline Screen Material & $\mathrm{ss} / \mathrm{sw} / .01$ & PVC/EW/.01 & PVC/sw/.01 & $\mathrm{ss} / \mathrm{sw} / .02$ & PVC/sw/.01 & PVC/sw/.01 & PVC/sw/.01 \\
\hline Screen Length & 16.1 & 10.0 & 10.0 & 10.2 & 5.0 & 6.0 & 5.0 \\
\hline Open-Hole Length & - & $\cdot$ & - & - & - & $\cdot$ & - \\
\hline (S)en-Hole Diameter & - & - & . & - & - & - & - \\
\hline
\end{tabular}


APPENDIX C

Monitoring-Well Construction Details

\begin{tabular}{|c|c|c|c|c|c|c|c|}
\hline Well No. & $G W-204$ & $G W-206$ & GW-207 & $G W-208$ & $G w-220$ & GW-232 & GW-239 \\
\hline Location & T0134 & $\mathbf{E x P}$ & EXP & EXP & NHP & EXP & NHP \\
\hline Bast Coordinate & 57410.93 & 64019 & 64023 & 64007 & 64225 & 66863 & 65088.59 \\
\hline North Coordinate & 29955.91 & 31603 & 31596 & 31612 & 28949 & 28546 & 28714.85 \\
\hline ELEVATIONS & - & . & . & - & - & - & . \\
\hline Measuring Point & 958.57 & 984.97 & 901.81 & 901.3 & 915.64 & 931.22 & 931.5 \\
\hline Surface & 955.47 & 894.65 & 894.66 & 894.52 & 912.74 & 929.52 & 928.51 \\
\hline Weathered Rock & 945.47 & . & $\cdot$ & & $\cdot$ & • & • \\
\hline Fresh Rock & & - & . & 882.52 & 901.74 & - & • \\
\hline Top Monitored Interval & 948.97 & 884.65 & 794.66 & 490.52 & 881.74 & 528.52 & 524.51 \\
\hline Bottom Monitored Interval & 938.17 & 877.75 & 785.06 & 481.72 & 867.54 & 517.82 & 495.21 \\
\hline SURFACE/CONDUCTOR CASING & . & $\cdot$ & • & - & - & - & - \\
\hline Casing Depth & $\bullet$ & $\cdot$ & 17 & 24 & 13 & 33 & 33.5 \\
\hline Casing Diameter & . & . & 8.625 & 8.626 & 12.5 & 8.625 & 8.5 \\
\hline Casing Material & - & . & PvC $/ 140$ & Pvc $/ 140$ & Pvc $/ \$ 40$ & Pvc $/ 140$ & PvC $/ \$ 40$ \\
\hline WELI CASING BOREHOLE & - & - & - & . & . & - & • \\
\hline Borehole Depth & 17.5 & 16.9 & 100 & 404 & $45 . \dot{2}$ & 401 & 404 \\
\hline Borehole Diameter & 6 & 8 & 7.87 & 6.62 & 11 & 6.62 & 6.62 \\
\hline Weathered Rock - Depth & 10 & . & . & • & $\cdot$ & $\cdot$ & • \\
\hline Fresh Rock - Depth & $\cdot$ & $\cdot$ & - & 12 & 11 & - & • \\
\hline WELL CASING & $\cdot$ & $\cdot$ & $\cdot$ & $\cdot$ & $\cdot$ & $\cdot$ & $\cdot$ \\
\hline Well Casing Depth & 17.3 & 16.9 & 100 & ${ }_{404}^{\circ}$ & 45.2 & 401 & 404 \\
\hline Well Casing Diameter & 4.5 & 2.37 & 4.38 & 4.38 & 4.5 & 4.38 & 4.38 \\
\hline Well Casing Material & $\mathrm{sS} / \$ 304$ & $\mathrm{PVC} / 140$ & $\mathrm{PVC} / 140$ & Steel & PVC $/ \$ 40$ & Steel & Steel \\
\hline MONITORED INTERVAI & $\cdot$ & $\dot{\bullet}$ & $\cdot$ & $\cdot$ & $\cdot$ & . & • \\
\hline Aquifer zone & $\dot{\text { wT }}$ & $\dot{\text { WT }}$ & BDR & BDR & BDR & BDR & BDR \\
\hline Geologic Formation & $\cos$ & cr & $\mathrm{cr}$ & $\mathrm{Cr}$ & conn & $\mathrm{cmn}$ & $\mathrm{Cn}$ \\
\hline Screened or Open & Screen & Screen & Open & Open & Screen & Open & Open \\
\hline Top - Depth & 6.5 & 10 & 100 & 404 & 31 & 401 & 404 \\
\hline Bottom - Depth & 17.3 & 16.9 & 109.6 & 412.8 & 45.2 & 411.7 & 433.3 \\
\hline Screen Material. & $\mathrm{sS} / \mathrm{sw} / .01$ & $\mathrm{PVC} / \mathrm{sw} / .01$ & . & . & PVC/sw/.01 & • & • \\
\hline Screen Length & 10.0 & 5.1 & $\cdot$ & - & 10.0 & • & . \\
\hline Oren-Hole Length & . & . & 9.6 & 8.8 & - & 10.7 & 29.3 \\
\hline Open-Hole Diameter & . & . & 3.875 & 3.625 & . & 3.875 & 3.875 \\
\hline
\end{tabular}

(CONTINUED) 
APPBNDIX C

Monltoring-Well Construction Detall

\begin{tabular}{|c|c|c|c|c|c|c|c|}
\hline \multirow{2}{*}{$\begin{array}{l}\text { Well No. } \\
\text { Location }\end{array}$} & $G W-240$ & $6 w-251$ & QW-252 & GW-255 & $G W-261$ & $G W-262$ & GW-263 \\
\hline & NHP & s2 & s2 & s2 & sy & $\mathbf{8 y}$ & $\mathbf{8 Y}$ \\
\hline East Coordinate & 63726 & 53843 & 53920 & 53906 & 53094 & 53106 & 53674 \\
\hline North Coordinate & 28604 & 29467 & 29326 & 29330 & 30914 & 30913 & 30885 \\
\hline BLEVATIONS & $\cdot$ & • & . & - & . & . & - \\
\hline Measuring Point & 922.9 & 1003.6 & 1024.21 & 1027.13 & $1049.99^{\circ}$ & 1049.67 & 1057.73 \\
\hline Surface & 919.5 & 1001.6 & 1022.66 & 1024.27 & 1047.57 & 1047.32 & 1055.13 \\
\hline Weathered Rock & & &. &. & &. & • \\
\hline Fresh Rock & 905.5 & 969.1 & . & . & 1024.57 & 1027.32 & 1025.13 \\
\hline Top Monitorud Interval & 898.5 & 966.6 & 982.46 & 957.97 & 1030.87 & 989.62 & 1032.13 \\
\hline Bottom Monitored Interval & 890 & 950.6 & 973.66 & 942.77 & 1023.87 & 976.72 & 1024.33 \\
\hline SURFACE/CONDUCTOR CASING & . &. &. & . & . & . & $\bullet$ \\
\hline Casing Depth & 14 & . & . & 43 & . & 37.8 & • \\
\hline Casing Diameter & 12.5 &. & . & 10.5 & . & 10.625 & - \\
\hline Casing Matorial & $\mathrm{PvC} / \$ 40$ &. &. & PVC $/ 40$ & . & PvC $/ \$ 40$ & - \\
\hline WELT CASING BOREHOIE & . & . &. & . & . & . & $\bullet$ \\
\hline Borehole Depth & 29.5 & $5 i$ & 49 & 81.5 & 23.7 & 70.6 & $30 . \dot{8}$ \\
\hline Borehole Diameter & 11 & 8.25 & 8.25 & 9.62 & 10 & 10 & 10 \\
\hline Weathered Rock - Depth &. & &. & . &. & . & • \\
\hline Fresh Rock - Depth & 14 & 32.5 & . & - & 23 & 20 & 30 \\
\hline WELI CASING & $\cdot$ & $\cdot$ & $\cdot$ & $\cdot$ & $\cdot$ & $\cdot$ & • \\
\hline Well Casing Depth & $2 \dot{9}$ & $47 . \dot{1}$ & $48 . \dot{8}$ & $81 . \dot{3}$ & 23.7 & 70.6 & $30 . \dot{8}$ \\
\hline Well Casing Diameter & 4.5 & 4.5 & 4.5 & 4.5 & 4.5 & 4.5 & 4.5 \\
\hline Well Casing Material & PVC $/ \$ 40$ & PvC $/ 40$ & PvC $/ \$ 40$ & ss $/ 1304$ & ss $/ 1304$ & ss $/ \$ 304$ & $s s / \$ 304$ \\
\hline MONITORED INTERVAL & $\cdot$ & $\cdot$ & $\cdot$ & $\cdot$ & . & $\cdot$ & • \\
\hline & & $\cdot$ & $\cdot$ & $\cdot$ & $\cdot$ & $\cdot$ & • \\
\hline Aquifer zone & BDR & BDR & wT & WT & WT & BDR & WT \\
\hline Geologic Formation & $\mathrm{cmn}$ & $C m n$ & $\cos n$ & $\operatorname{cons}$ & $\mathrm{cm}$ & $\mathrm{cm}$ & $\mathrm{cm}$ \\
\hline Screened or Open & Screen & screen & screen & Screen & Screen & Screen & screen \\
\hline Top - Depth & 21 & 35 & 40.2 & 66.3 & 16.7 & 57.7 & 23 \\
\hline Bottom - Depth & 29.5 & 51 & 49 & 81.5 & 23.7 & 70.6 & 30.8 \\
\hline Screen Material & $\mathrm{PVC} / \mathrm{sw} / .01$ & $\mathrm{PVC} / \mathrm{sw} / .01$ & $\mathrm{PVC} / \mathrm{sw} / .01$ & Ss/ow/.01 & ss/sw/.01 & ss/sw/.01 & SS/Bw/.01 \\
\hline Screen Length & 5.0 & 9.6 & 5.0 & 10.0 & 5.4 & 10.4 & 5.3 \\
\hline Open-Hole Length & . & . & $\cdot$ & . & . & . & • \\
\hline Open-Hole Diameter & . & . & . & . & . & . & . \\
\hline
\end{tabular}

(CONTINUED) 
APPENDIX C

Monitoring-Well Conatruction Detalle

\begin{tabular}{|c|c|c|c|c|c|c|c|}
\hline \multirow{2}{*}{$\begin{array}{l}\text { Mell Ho. } \\
\text { Location }\end{array}$} & $G W-264$ & $G W-281$ & $0 w-283$ & GW-284 & $G W-285$ & $6 W-337$ & $6 w-338$ \\
\hline & sy & $\mathbf{r r}$ & $\mathbf{F r}$ & $\mathbf{r r}$ & $\mathbf{F}$ & wc & wC \\
\hline Bast Coordinate & 53670 & 61907 & 61990 & 61925 & 61856 & 54519 & 54430 \\
\hline North Coordinate & 30872 & 29771 & 29570 & 29590 & 29595 & 30057 & 30134 \\
\hline ELEVATIONS & $\cdot$ & $\cdot$ & $\cdot$ & $\cdot$ & $\cdot$ & $\cdot$ & - \\
\hline Maasuring Point & 1058.3 & 946.08 & 938.62 & 941.61 & 942.46 & 987.31 & 987.27 \\
\hline surface & 1055.54 & 946.51 & 939.22 & 942.3 & 942.88 & 984.12 & 984.02 \\
\hline Weathered Rook & & 929.01 & 919.22 & 924.3 & 921.88 & $\cdot$ & 978.02 \\
\hline Freah Rock & 1022.54 & $\cdot$ & $\cdot$ & $\cdot$ & $\cdot$ & $\cdot$ & $\cdot$ \\
\hline Top Monitored Interval & 997.04 & 942.51 & 935.22 & 940.3 & 939.38 & 969.12 & 972.82 \\
\hline Bottom Monitored Interval & 984.54 & 931.51 & 919.22 & 924.3 & 921.88 & 962.02 & 966.42 \\
\hline SURPACE/CONDUCTOR CASING & $\cdot$ & $\cdot$ & $\cdot$ & $\cdot$ &. & - & - \\
\hline Casing Depth & 35 & $\cdot$ & $\cdot$ & $\cdot$ & $\cdot$ & . & • \\
\hline Casing Diameter & 10.625 & $\cdot$ & $\cdot$ & $\cdot$ & $\cdot$ & $\cdot$ & • \\
\hline Casing Material & Pve $/ 140$ & $\cdot$ & $\cdot$ & $\cdot$ & $\cdot$ & $\cdot$ & $\cdot$ \\
\hline WELL CASTMG BORRHOLE & $\cdot$ & $\cdot$ & $\cdot$ & $\cdot$ & $\cdot$ & $\cdot$ & • \\
\hline Borahole Depth & $\dot{71}$ & $17 . \dot{5}$ & $\dot{2 n}$ & 18 & 21 & 22.1 & 17.6 \\
\hline Borehole Diameter & 10 & 6 & 6 & 6 & 6 & 10 & 10 \\
\hline Weathered Rock - Depth & $\cdot$ & 17.5 & 20 & 18 & 21 & $\cdot$ & 6 \\
\hline Fresh Rock - Depth & 33 & & $\cdot$ & $\cdot$ & $\cdot$ & - & - \\
\hline WELL NASING & $\cdot$ & $\cdot$ & $\cdot$ & $\cdot$ & $\cdot$ & $\cdot$ & - \\
\hline Well Casing Depth & 70.7 & 15 & 20 & 18 & 21 & 22.1 & 17.6 \\
\hline Well Casing Diameter & 4.5 & 4.5 & 4.5 & 4.5 & 4.5 & 4.5 & 4.5 \\
\hline Nell Casing Material & ss//304 & Ss $/ \$ 304$ & ss/1304 & ss/1304 & $\mathrm{ss} / \$ 304$ & $5 s / 1304$ & $\mathrm{ss} / 1304$ \\
\hline MONITORED INTERVAL & $\cdot$ &. & $\cdot$ & $\cdot$ & $\cdot$ & . & • \\
\hline Aquifer zone & BDR & พT & wT & $\dot{w T}$ & WT & $\dot{w r}$ & $\dot{w T}$ \\
\hline Geologic rormation & $\mathrm{cm}$ & $\mathrm{Cn}$ & $\mathrm{cn}$ & $\mathrm{Cn}$ & $\mathrm{Cn}$ & $\mathrm{cn}$ & $\mathrm{Cn}$ \\
\hline Screened or Open & Screen & Screen & Screen & Screen & Screen & Screen & Screen \\
\hline Top - Depth & 58.5 & 4 & 4 & 2 & 3.5 & 15 & 11.2 \\
\hline Bottom - Depth & 71 & 15 & 20 & 18 & 21 & 22.1 & 17.6 \\
\hline Screen Materlal & ss/sw/.01 & $\mathrm{SS} / \mathrm{s} 1 / .01$ & $\mathrm{ss} / 81 / .01$ & $\mathrm{ss} / 81 / .01$ & $\mathrm{s5} / 81 / .01$ & $\mathrm{ss} / \mathrm{sw} / .01$ & ss/8w/.01 \\
\hline Screen Length & 10.2 & 10.0 & 15.0 & 15.0 & 15.0 & 5.4 & 5.5 \\
\hline Open-Hole Length & $\cdot$ & $\cdot$ & $\cdot$ & $\cdot$ & $\cdot$ & $\cdot$ & • \\
\hline Open-Hole Diameter & $\cdot$ & - & • & $\cdot$ & - & & . \\
\hline
\end{tabular}

(CONTINUED) 
APPENDIX C

Monltoring-Well Conetruction Detalle

\begin{tabular}{|c|c|c|c|c|c|c|c|}
\hline \multirow{2}{*}{$\begin{array}{l}\text { Woll No. } \\
\text { Looution }\end{array}$} & OW- 380 & $a w-381$ & $O W-382$ & OW-383 & $O W-384$ & OW-385 & $6 W-505$ \\
\hline & NHP & MHP & NHP & NHP & NHP & NHP & RG \\
\hline Inet coordinate & 62938 & 62947 & 62956 & 63522 & 63530 & 63526 & 53037 \\
\hline North Coordinate & 28714 & 28725 & 28716 & 29201 & 29216 & 29208 & 30400 \\
\hline ELTVATIONS & - & . & $\cdot$ & . & - & - & - \\
\hline Measuring Point & 913.55 & 913.36 & 913.17 & $908 . \dot{5}$ & 908.38 & 908.87 & 1014.78 \\
\hline surface & 913.66 & 913.4 & 913.16 & 906 & 905.38 & 905.87 & 1011.6 \\
\hline Weathered Rock & 898.16 & 899.9 & 900.46 & 894.5 & 893.38 & 894.87 & • \\
\hline Preah Rock & $\cdot$ & 887.4 & 896.16 & $\cdot$ & • & • & • \\
\hline Top Monitored Interval & 910.86 & 864.1 & 788.16 & 889.4 & 869.88 & 782.17 & 1010.1 \\
\hline Bottom Monitored Interval & 898.16 & 853 & 740.16 & 882.4 & 849.68 & 727.17 & 998.1 \\
\hline SURAACE/CONDUCTOR CASING &. & $\cdot$ & . & - & - & - & • \\
\hline Casing Depth & & 13.5 & $12 . \overline{7}$ & $\dot{5}$ & 16 & 13 & • \\
\hline Caning Diameter & . & 13 & 10.75 & 10.75 & 10.75 & 13 & • \\
\hline Caning Material & - & unknown & unknown & unknown & unknown & unknown & • \\
\hline WEL CASINO BORHOLE & - & $\cdot$ & - & $\cdot$ & - & - & • \\
\hline Borahole Depth & 15.5 & 49.3 & 125 & $24 . \dot{1}$ & 35.5 & 123.7 & 13.5 \\
\hline Borehole Dianoter & 10 & 9.5 & 9.5 & 8.75 & 9.5 & 9.5 & 7 \\
\hline Weathered Rock - Depth & 15.5 & 13.5 & 12.7 & 11.5 & 12 & 11 & $\cdot$ \\
\hline Fresh Rock - Depth & . & 26 & 17 & • & - & - & $\cdot$ \\
\hline MRLL CASING & & $\cdot$ & . & - & - & - & • \\
\hline We11 Casing Depth & 15.5 & 49.3 & 125 & 23.6 & $35 . \dot{5}$ & 123.7 & 13.3 \\
\hline Well Casing Diameter & 4.5 & 6.62 & 6.62 & 4.5 & 6.62 & 6.62 & 2.37 \\
\hline Well Casing Material & ss/1304 & Steel/r25 & Stee1/F25 & $8 s / 1304$ & Steel/F25 & Steel/F25 & Ss/ 1304 \\
\hline MONITORED INTERVAI & $\dot{\bullet}$ & $\cdot$ & $\cdot$ & $\cdot$ & . & • & $\cdot$ \\
\hline Aquifer zone & $\dot{w r}$ & BDR & BDR & $\dot{w T}$ & $\dot{w r}$ & BDR & $\dot{w}$ \\
\hline Ceologic Formation & $\operatorname{cosn}$ & $\operatorname{cmn}$ & $\mathrm{cmn}$ & $\mathrm{cn}$ & $\mathrm{cn}$ & $\mathrm{Cn}$ & $\mathrm{Cn}$ \\
\hline Screened or open & Screen & Open & Open & Screan & Open & Open & Screen \\
\hline Top - Depth & 2.8 & 49.3 & 125 & 16.6 & 35.5 & 123.7 & 1.5 \\
\hline Bottom - Depth & 15.5 & 60.4 & 173 & 23.6 & 55.7 & 178.7 & 13.5 \\
\hline Screen Material & $\mathrm{ss} / \mathrm{sw} / .01$ & - & . & $\mathrm{ss} / \mathrm{sw} / .01$ & . & • & $\mathrm{ss} / \mathrm{sw} / .01$ \\
\hline Screen Length & 5.4 & • & • & 5.0 & - & - & 20.4 \\
\hline Open-Hole Length & • & 11.1 & 48 & . & 20.2 & 55 & • \\
\hline Open-Hole DLameter & - & 6.1 & 6.13 & . & 6 & 6 & . \\
\hline
\end{tabular}

(CONTINUED) 
APPENOIX C

Monltoring-well Construction Detall.

\begin{tabular}{|c|c|c|c|c|c|c|c|}
\hline \multirow{2}{*}{$\begin{array}{l}\text { Woll Ho. } \\
\text { Looation }\end{array}$} & OW-508 & $G N-603$ & OW-604 & $O W-605$ & GW-606 & $G W-617$ & aw-618 \\
\hline & RO & $\mathbf{E x p}$ & $\mathbf{E x p}$ & $\mathbf{E x p}$ & $\mathbf{E x p}$ & $\mathbf{E x p}$ & $\operatorname{mXP}$ \\
\hline Iast Coordinate & 53148 & 64803.26 & 64836.82 & 62001.5 & 61951.42 & 54755.65 & 54738.12 \\
\hline Morth Coordinate & 30281 & 28429.84 & 28436.97 & 28706.83 & 28708.32 & 29806.44 & 29798.46 \\
\hline ELEVATIONS &. & - & . & . & - & . & - \\
\hline Maseuring Point & 1012.5 & 961.32 & 960.86 & 918.88 & 919.39 & 985.11 & $984.94^{\circ}$ \\
\hline surface & 1009.56 & 959.41 & 959.53 & 916.97 & 916.98 & 982.61 & 982.64 \\
\hline Weathered Rock &. & 935.41 & 928.53 &. & • & . & 955.64 \\
\hline Freah Rock & & 899.41 & 904.53 & 907.47 & 906.18 & . & • \\
\hline Top Monltored Interval & 1008.26 & 896.01 & 859.33 & 888.77 & 761.98 & 975.81 & 956.64 \\
\hline Bottom Monitored Interval & 994.56 & 884.21 & 847.13 & 877.07 & 745.28 & 964.61 & 945.64 \\
\hline SURFACE/CONDUCTOR CASINO & $\cdot$ & $\cdot$ & $\cdot$ & $\cdot$ & • & $\dot{\bullet}$ & • \\
\hline Casing Depth & $\dot{.}$ & $31 . \dot{9}$ & $99 . \dot{2}$ & 9.5 & $10 . \dot{8}$ & $\dot{\bullet}$ & 27.5 \\
\hline Casing Dianeter & . & 10.75 & 10.75 & 11.75 & 16 & . & 10.75 \\
\hline Casing Material & - & Steel & steel & steel & steel & $\cdot$ & steal \\
\hline WELL CASIMG BOREGOLE & $\cdot$ & $\dot{.}$ & $\dot{\bullet}$ & $\dot{\bullet}$ & $\dot{\bullet}$ & $\dot{\bullet}$ & • \\
\hline Borehole Depth & $2 \dot{3}$ & $75 . \dot{2}$ & 112.4 & $40 . \dot{5}$ & 66 & $\dot{18}$ & 37 \\
\hline Borahole Diameter & 7 & 9.5 & 9.5 & 10.6 & 9.63 & 9.5 & 9.5 \\
\hline Weathered Rock - Depth & . & 24 & 31 & & . &. & 27 \\
\hline Fresh Rock - Depth & - & 60 & 55 & 9.5 & 10.8 & - & • \\
\hline WELL CASING & $\dot{\bullet}$ & $\dot{\cdot}$ & $\cdot$ & $\cdot$ & $\cdot$ & • & • \\
\hline Well Casing Depth & 13.7 & 75.2 & 112.4 & 39.9 & 64.7 & 18 & 37 \\
\hline Well casing Dianeter & 2.37 & 4.5 & 4.5 & 4.25 & 7 & 4.5 & 4.5 \\
\hline Well Casing Material & $8 s / 1304$ & ss/\$304 & ss $/ \$ 304$ & $58 / 1304$ & steel & ss/1304 & $8 s / 1304$ \\
\hline MONITORED INTERVAI & . & $\dot{\bullet}$ & $\dot{\bullet}$ & • & $\dot{\bullet}$ & • & • \\
\hline Anuter zenes & $\cdot$ & - & $\dot{0}$ & & $\dot{0}$ & $\dot{1}$ & $\dot{0}$ \\
\hline $\begin{array}{l}\text { Aquifer zone } \\
\text { Geologic rormation }\end{array}$ & $\begin{array}{l}\mathrm{WT} \\
\mathrm{Cn}\end{array}$ & $\begin{array}{l}\text { BDR } \\
\mathrm{Cmn}\end{array}$ & BDR & $\begin{array}{l}\text { BDR } \\
\text { Cmn }\end{array}$ & BDR & $\begin{array}{r}\mathrm{WT} \\
\mathrm{Cmn}\end{array}$ & WT \\
\hline $\begin{array}{l}\text { Geologic Formation } \\
\text { Screened or Open }\end{array}$ & Screen & Screen & Screen & Screen & Screan & Screen & $\begin{array}{r}\text { Comn } \\
\text { Screen }\end{array}$ \\
\hline Top - Depth & 1.3 & 63.4 & 100.2 & 28.2 & 155 & 6.8 & 26 \\
\hline Bottom - Depth & 15 & 75.2 & 112.4 & 39.5 & 171.7 & 18 & 37 \\
\hline Screen Material & $\mathrm{ss} / \mathrm{sw} / .01$ & Ss/sw/.01 & Ss/sw/.01 & ss/sw/.01 & $\mathrm{ss} / \mathrm{pp} / .01$ & Ss/sw/.01 & ss/ow/.01 \\
\hline Screen Length & 10.3 & 10.3 & 9.9 & 10.2 & 10.7 & 10.3 & 10.3 \\
\hline Open-Hole Length & - & - & . & . & . &. & • \\
\hline Open-Hole Diameter & . & . & . & . & . & - & . \\
\hline
\end{tabular}

(CONTINUED) 
APPENDIX C

Monitoring-Wedl Construetion Detalla

\begin{tabular}{|c|c|c|c|c|c|c|c|}
\hline \multirow{2}{*}{$\begin{array}{l}\text { Wo . No. } \\
\text { Location }\end{array}$} & $G W-619$ & $a w-620$ & $a w-631$ & GW- 632 & $6 w-633$ & $G W-634$ & ow-656 \\
\hline & PTP & mTr & RO & RG & RG & RO & T0134 \\
\hline Iast Coordinate & 52905.94 & 52894.57 & 53144.93 & 33077.9 & 53100.64 & 53256.62 & 57439.12 \\
\hline Morth Coordinate & 29562.8 & 29564.54 & 30202.77 & 30236.97 & 30144.44 & 30222.38 & 29894.69 \\
\hline ELEVATIONS & . & . & . & - & . & . & . \\
\hline Maasuring Polnt & 1015.24 & 1015.34 & 1003.99 & 1005.73 & 996.5 & 1007.15 & 954.79 \\
\hline Surface & 1012.74 & 1012.84 & 1004.11 & 2005.95 & 996.52 & 1007.31 & 954.9 \\
\hline Weathered Rock & 972.74 & 971.84 & $\cdot$ & 997.45 & 988.02 & 1002.31 & 942.9 \\
\hline Preah Rock & 971.94 & 942.84 & . & • & . & . & • \\
\hline Top Monitored Interval & 985.94 & 951.14 & 1000.11 & 1002.45 & 993.02 & 1003.81 & 946.6 \\
\hline Bottom Monitored Interval & 971.94 & 937.84 & 988.11 & 990.95 & 981.52 & 992.31 & 933.4 \\
\hline SURTACE/COADUCTOR CASIMO & - & . & . & - & • & • & - \\
\hline Casing Dapth & $\cdot$ & 42.5 & $\cdot$ & $\cdot$ & $\cdot$ & $\cdot$ & $\cdot$ \\
\hline Casing Diameter & . & 10.75 & - & - & . & - & • \\
\hline Casing Material & . & steel & - & - & - & - & • \\
\hline WELL CASING BORIHOLE & • & $\cdot$ & • & - & - & - & • \\
\hline Borehole Depth & 40.8 & 75 & $\dot{16}$ & 15 & 15 & $1 \dot{15}$ & 21.5 \\
\hline Borehole Diameter & 9.5 & 9.5 & 10.5 & 10.5 & 10.5 & 10.5 & 9.5 \\
\hline Weathered Rock - Dapth & 40 & 41 & . & 8.5 & 8.5 & 5 & 12 \\
\hline Fresh ROck - Depth & 40.8 & 70 & . & - & - & - & • \\
\hline WELL CASIHO & $\dot{\bullet}$ & $\cdot$ & $\dot{\bullet}$ & $\cdot$ & $\dot{\bullet}$ & $\cdot$ & • \\
\hline Well Casing Depth & $40 . \dot{8}$ & is & $15 . \dot{5}$ & 14.5 & $\dot{15}$ & $\dot{15}$ & 20.9 \\
\hline Well Casing Diameter & 4.5 & 4.5 & 4.5 & 4.5 & 4.5 & 4.5 & 4.5 \\
\hline Well Casing Material & ss/1304 & ss/1304 & Pvc & Pvc & Pvc & pvc & $s s / 1304$ \\
\hline MOHITORED INTERVAI & . & . & - & . & $\cdot$ & $\dot{.}$ & • \\
\hline Aguifer zone & • & $\dot{\omega r}$ & $\dot{v}$ & • & • & $\cdot \dot{r}$ & $\dot{x}$ \\
\hline $\begin{array}{l}\text { Aquifer zone } \\
\text { Geologic Formation }\end{array}$ & $\operatorname{cran}$ & $\operatorname{comn}$ & $\begin{array}{l}\text { WT } \\
\text { Cn }\end{array}$ & $\begin{array}{l}\text { WT } \\
\mathrm{cn}\end{array}$ & $\begin{array}{l}\text { WT } \\
\mathrm{cn}\end{array}$ & $\begin{array}{l}\text { WT } \\
\text { Cn }\end{array}$ & $\mathrm{cn}$ \\
\hline Screened or Open & Screen & Screen & Screen & Screen & Screen & Screen & Screen \\
\hline Top - Depth & 26.8 & 61.7 & 4 & 3.5 & 3.5 & 3.5 & 8.3 \\
\hline Bottom - Depth & 40.8 & 75 & 16 & 15 & 15 & 15 & 21.5 \\
\hline Screen Material & ss/sw/.01 & ss/sw/.01 & PVC $/ \mathrm{sl} / .01$ & $\mathrm{PVC} / \mathrm{s1} / .01$ & PvC/s1/.01 & PVC/81/.01 & ss/ow/.01 \\
\hline Screen Length & 10.8 & 10.8 & 10.0 & 10.0 & 10.0 & 10.0 & 10.0 \\
\hline Open-Hole Length & - & . & - & • & - & . & • \\
\hline Open-Hole Dianeter & . & . & . & • & - & - & . \\
\hline
\end{tabular}

(CONTINUED) 
APPENDIX $C$

Monltoring-Well Conutruction Detail

\begin{tabular}{|c|c|c|c|c|c|c|c|}
\hline \multirow{2}{*}{$\begin{array}{l}\text { Well No. } \\
\text { wocation }\end{array}$} & $6 w-657$ & $G W-658$ & GW-659 & GW-707 & $6 W-708$ & $6 W-733$ & OW-735 \\
\hline & T2331 & $\mathbf{F r}$ & $\mathbf{r r}$ & T2331 & T2331 & $\mathbf{E X P}$ & NHP \\
\hline East Coordinate & 59598.78 & 62146.2 & 62100.46 & 59578.51 & 59529.84 & 65057 & 64872 \\
\hline North Coordinate & 29287.13 & 29638.24 & 29614.54 & 29319.23 & 29335.42 & 28447 & 28867 \\
\hline ELEVATIONS & $\cdot$ & . & $\cdot$ & - & - & - & - \\
\hline Measuring Point & 930.53 & 944.81 & 941.16 & 930.91 & 930.87 & 959.04 & 924.28 \\
\hline surface & 930.8 & 942.04 & 941.79 & 931.16 & 931.03 & 955.69 & 921.34 \\
\hline Weathered Rock & $\cdot$ & 938.54 & 938.39 & 911.16 & $\cdot$ & 944.59 & 902.34 \\
\hline Fresh Rock & $\cdot$ & & $\cdot$ & & $\cdot$ & 913.19 & 843.84 \\
\hline Top Monitored Interval & 926.8 & 935.14 & 937.19 & 925.66 & 928.33 & 715.59 & 853.84 \\
\hline Bottom Monitored Interval & 913.7 & 922.94 & 925.09 & 905.16 & 917.33 & 699.19 & 842.14 \\
\hline SURAACE / CONDUCTOR CASING & $\cdot$ & $\cdot$ & $\cdot$ & $\cdot$ & - & . & • \\
\hline Casing Depth & 3 & 8.8 & 2.9 & 3 & 3 & 51.8 & 25.5 \\
\hline Casing Diameter & 11.75 & 10.75 & 10.75 & 11.75 & 11.75 & 11.75 & 11.75 \\
\hline Caning Material & steel & Steol & steel & steel & steel & $\cdot$ & • \\
\hline MELL CASING BOREHOLE & . & $\cdot$ & $\cdot$ & - & . & - & - \\
\hline Borehole Depth & $17 . \dot{1}$ & $19 . \dot{1}$ & 16.7 & $\dot{26}$ & 13.9 & $240 . \dot{1}$ & 83 \\
\hline Borehole Diameter & 9.5 & 9.5 & 9.5 & 9.5 & 9.5 & 10.6 & 10.6 \\
\hline Weathered Rock - Depth & $\cdot$ & 3.5 & 3.4 & 20 & • & 11.1 & 19 \\
\hline Fresh Rock - Depth & $\cdot$ & $\cdot$ & $\cdot$ & $\cdot$ & - & 42.5 & 77.5 \\
\hline WRLL CASING & $\cdot$ & . & $\cdot$ & . & • & • & • \\
\hline Well Casing Depth & 15.6 & 19.1 & 16.7 & 17.2 & 13.7 & $240 . \dot{1}$ & $78 . \dot{1}$ \\
\hline Well Casing Diameter & 4.5 & 4.5 & 4.5 & 4.5 & 4.5 & 7 & 4.5 \\
\hline Well Casing Material & ss $/ 1304$ & ss/1304 & ss/ $/ 304$ & Ss $/ 1304$ & ss $/ 1304$ & Steel/F25 & $s s / \$ 304$ \\
\hline MONITORED INTERVAL & $\cdot$ & - & $\cdot$ & $\cdot$ & $\cdot$ & • & • \\
\hline Aquifer zone & $\dot{w T}$ & $\dot{w T}$ & $\dot{\omega T}$ & $\dot{w T}$ & $\dot{w T}$ & BDR & $\dot{\text { wI }}$ \\
\hline Geologic Formation & $\mathrm{cmn}$ & $\mathrm{cn}$ & $c_{n}$ & $\mathrm{Cm} n$ & $\operatorname{com}$ & Com & $\mathrm{Cn}$ \\
\hline Screened or Open & Screen & Screen & Screen & Screen & Screen & Open & Screen \\
\hline Top - Depth & 4 & 6.9 & 4.6 & 5.5 & 2.7 & 240.1 & 67.5 \\
\hline Bottom - Depth & 17.1 & 19.1 & 16.7 & 26 & 13.7 & 256.5 & 79.2 \\
\hline Screen Material & ss/sw/.01 & $\mathrm{ss} / \mathrm{sw} / .01$ & ss/sw/.01 & SS/Bw/.01 & SS/sw/.01 & • & $\mathrm{ss} / \mathrm{sw} / .01$ \\
\hline Screen Length & 10.0 & 10.0 & 11.0 & 10.0 & 10.0 & • & 10.2 \\
\hline Open-Hole Length & . & • & $\cdot$ & • & . & 16.4 & • \\
\hline Open-Hole Diameter & . & - & - & - & - & 6.25 & • \\
\hline
\end{tabular}

(CONTINUED) 
APPENDIX $\mathrm{C}$

Monitoring-well Construction Details

\begin{tabular}{|c|c|c|c|c|c|c|c|}
\hline \multirow{2}{*}{$\begin{array}{l}\text { Well No. } \\
\text { Location }\end{array}$} & $G w-744$ & $G W-745$ & GW-746 & GW-747 & GW-748 & GW-749 & $G W-750$ \\
\hline & GRIDKI & GRIDK1 & GRIDK1 & GRIDK2 & GRIDK2 & GRIDK2 & GRIDK2 \\
\hline East Coordinate & 64324 & 64309 & 64312 & 64569.57 & 64579.38 & 64585.74 & 64835.48 \\
\hline North Coordinate & 30282 & 30278 & 30291 & 29729.81 & 29740.86 & 29726.4 & 28974.53 \\
\hline BLEVATIONS & - & . & . & . & $\cdot$ & $\cdot$ & - \\
\hline Measuring Point & 907.43 & 907.06 & 906.88 & 920.96 & 921.17 & 921.19 & 918.86 \\
\hline Surface & 905.05 & 904.28 & 904.26 & 918.33 & 918.89 & 918.69 & 915.96 \\
\hline Weathered Rock & 895.45 & 894.78 & 895.26 & 907.83 & 910.89 & 909.69 & 897.46 \\
\hline Fresh Rock & 890.45 & 891.78 & 893.26 & 906.33 & 907.09 & 907.69 & 891.16 \\
\hline Top Monitored Interval & 850.05 & 883.08 & 901.06 & 850.93 & 904.09 & 913.89 & 854.76 \\
\hline Bottom Monitored Interval & 835.55 & 871.48 & 889.06 & 838.73 & 891.69 & 902.29 & 843.26 \\
\hline SURFACE/CONDUCTOR CASING & $\cdot$ & • & $\bullet$ & • & . &. & • \\
\hline Casing Depth & 27.6 &. & $\cdot$ & 23.8 & . &. & 21.7 \\
\hline C. sing Diameter & 10 & - &. & 10.75 & • & . & 11.75 \\
\hline C.using Material & • & - & . & Steel & - & - & Steel \\
\hline WELL CASING BOREHOLE & $\dot{\bullet}$ & $\cdot \dot{\varphi}$ & $\cdot$ & $\dot{\bullet}$ & • & $\dot{\bullet}$ & • \\
\hline Borehole Depth & 69.5 & 33 & $15 . \dot{2}$ & $23 . \dot{8}$ & $27 . \dot{2}$ & 16.4 & 72.8 \\
\hline Borehole Diameter & 9.87 & 9.87 & 9.87 & 15 & 9.9 & 9.9 & 10.6 \\
\hline Weathered Rock - Depth & 9.6 & 9.5 & 9 & 10.5 & 8 & 9 & 18.5 \\
\hline Fresh Rock - Depth & 14.6 & 12.5 & 11 & 12 & 11.8 & 11 & 24.8 \\
\hline WELI CASING & $\cdot$ & $\dot{.}$ & $\dot{.}$ & $\cdot$ & • & $\cdot$ & • \\
\hline Well Casing Depth & $\dot{67}$ & 32.5 & 14.75 & 69.2 & $\dot{17}$ & $\dot{6}$ & 62.4 \\
\hline Well Casing Diameter & 4.5 & 4.5 & 4.5 & 4.5 & 4.5 & 4.5 & 4.5 \\
\hline Well Casing Material & $\mathrm{SS} / \$ 304$ & $S S / \$ 304$ & $S S / \$ 304$ & $\mathrm{SS} / \$ 304$ & $s S / \$ 304$ & $\mathrm{SS} / \$ 304$ & $S S / \$ 304$ \\
\hline MONITORED INTERVAL & - & $\cdot$ & $\cdot$ & $\cdot$ & $\cdot$ & $\dot{.}$ & • \\
\hline Aquifer zone & BDR & BDR & WT & BDR & $\begin{array}{r}\cdot \\
B D R\end{array}$ & $\dot{\omega T}$ & BDR \\
\hline Geologic Formetion & $\mathrm{Cpv}$ & Cpv & $\mathrm{cpv}$ & $\mathrm{cm}$ & $\mathrm{cm}$ & $\mathrm{cm}$ & $\mathrm{Cn}$ \\
\hline Screened or Open & Screen & Screen & Screen & Screen & Screen & Screen & Scree: \\
\hline Top - Depth & 55 & 21.2 & 3.2 & 67.4 & 14.8 & 4.8 & 61.2 \\
\hline Bottom - Depth & 69.5 & 32.8 & 15.2 & 79.6 & 27.2 & 16.4 & 72.7 \\
\hline Screen Material & $\mathrm{SS} / \mathrm{sw} / .01$ & $\mathrm{ss} / \mathrm{sw} / .01$ & $\mathrm{ss} / \mathrm{sw} / .01$ & $\mathrm{ss} / \mathrm{sw} / .01$ & $\mathrm{ss} / \mathrm{sw} / .01$ & ss/sw/.01 & $\mathrm{ss} / \mathrm{sw} / .01$ \\
\hline Screen Length & 10.0 & 10.0 & 10.3 & 9.9 & 9.9 & 9.9 & 9.9 \\
\hline Open-Hole Length & 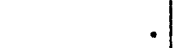 & 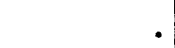 & 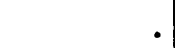 & • & $\cdot$ & - & 。 \\
\hline Open-Hole Diameter & - & - & - & - & - & - & - \\
\hline
\end{tabular}

(CONTINUED) 
Monitoring-Well Const-uction Details

\begin{tabular}{|c|c|c|c|c|c|c|c|}
\hline \multirow{2}{*}{$\begin{array}{l}\text { Well No. } \\
\text { Location }\end{array}$} & $G W-751$ & $G W-752$ & $G W-753$ & GW-754 & GW-755 & GW-756 & GW-758 \\
\hline & GRIDJ3 & GRIDJ3 & GRIDJ 2 & GRIDJ2 & GRIDJI & GRIDJ 1 & GRIDGI \\
\hline Bast Coordinate & 63248.8 & 63226.77 & 63208.59 & 63190.57 & 63266.73 & 63288.31 & 60331.97 \\
\hline North Coordinate & 29310 & 29312.53 & 29638 & 29644.34 & 30203.59 & 30195.42 & 30853.74 \\
\hline EIEVATIONS & - & - & - & - & - & . & • \\
\hline Measuring Point & 912.05 & 912.78 & 927.69 & 928.59 & 930.36 & 927.92 & $992.7^{\circ}$ \\
\hline Surface & 909.38 & 909.76 & 924.73 & 925.63 & 927.41 & 924.89 & 989.65 \\
\hline Weathered Rock & 899.38 & 898.26 & 923.73 & 924.63 & 912.81 & 911.89 & 973.65 \\
\hline Fresh Rock & 897.38 & 897.26 & 902.23 & 908.13 & 905.41 & 908.89 & 970.65 \\
\hline Top Monitored Interval & 860.38 & 904.96 & 865.13 & 912.63 & 878.21 & 919.59 & 951.25 \\
\hline Bottom Monitored Interval & 848.78 & 893.86 & 854.03 & 901.53 & 866.91 & 908.29 & 939.45 \\
\hline SURFACE/CONDUCTOR CASING & & • & $\cdot$ & . & • & . & • \\
\hline Casing Depth & 22.9 & • & 38 & • & 36.1 & • & $\cdot$ \\
\hline Casing Diameter & 10.75 & . & 10.75 & . & 11.75 & • & . \\
\hline Casing Material & Steel & . & Steel & . & steel & - & • \\
\hline WELL CASING BOREHOLE & . & . & . & . & . & . & $\cdot$ \\
\hline Borehole Depth & 60.8 & $\dot{16}$ & 70.7 & $24 . \dot{1}$ & 60.7 & 16.7 & $50 . \dot{2}$ \\
\hline Borehole Diameter & 9.9 & 9.8 & 9.9 & 9.9 & 9.9 & 9.9 & 9.9 \\
\hline Weathered Rock - Depth & 10 & 11.5 & 1 & 1 & 14.6 & 13 & 16 \\
\hline Fresh Rock - Depth & 12 & 12.5 & 22.5 & 17.5 & 22 & 16 & 19 \\
\hline WELI CASING & . & . & . & • & . & • & • \\
\hline Well Casing Depth & 50.1 & 15.5 & 60.4 & $\dot{14}$ & 50 & $\dot{6}$ & 40.2 \\
\hline Well Casing Diameter & 4.5 & 4.5 & 4.5 & 4.5 & 4.5 & 4.5 & 4.5 \\
\hline Well Casing Material & ss $/ \$ 304$ & $S S / \$ 304$ & SS $/ \$ 304$ & $s s / \$ 304$ & ss $/ \$ 304$ & ss $/ \$ 304$ & ss $/ 304$ \\
\hline MONITORED INTERVAL & • & • & • & • & • & • & $\cdot$ \\
\hline Aguifer zone & BDR & $\cdot \dot{ }$ & $\stackrel{\bullet}{B D R}$ & พ & BDR & WT & BDR \\
\hline $\begin{array}{l}\text { Aquifer Zone } \\
\text { Geologic Formation }\end{array}$ & $\mathrm{cn}$ & $\mathrm{Cn}$ & $\mathrm{cm}$ & $\mathrm{Cn}$ & Crg & $\mathrm{cm}$ & Crt \\
\hline Screened or Open & Screen & Screen & Screen & Screen & Screen & Screen & Screen \\
\hline Top - Depth & 49 & 4.8 & 59.6 & 13 & 49.2 & 5.3 & 38.4 \\
\hline Bottom - Depth & 60.6 & 15.9 & 70.7 & 24.1 & 60.5 & 26.6 & 50.2 \\
\hline Screen Material & $\mathrm{ss} / \mathrm{sw} / .01$ & ss/sw/.01 & $\mathrm{SS} / \mathrm{sw} / .01$ & $\mathrm{ss} / \mathrm{sw} / .01$ & $\mathrm{ss} / \mathrm{sw} / .01$ & ss/sw/.01 & Ss/sw/.01 \\
\hline Screen Length & 9.9 & 9.9 & 9.9 & 9.9 & 9.9 & 9.9 & 9.9 \\
\hline Open-Hole Length & - & - & - & - & - & - & . \\
\hline Open-Hole Diameter & - & • & - & . & . & - & . \\
\hline
\end{tabular}


APPENDIX $C$

Monitoring-Well Construction Details

\begin{tabular}{|c|c|c|c|c|c|c|c|}
\hline Well No. & GW-759 & $G W-760$ & GW-761 & GW-762 & $G W-763$ & $G W-764$ & $G W-765$ \\
\hline Iocation & GRIDG1 & GRIDG2 & GRIDG2 & GRIDJ3 & GRIDJ3 & GRIDE I & GRIDE 1 \\
\hline East Coordinate & 60312.58 & 60207.3 & 60201.31 & 63192.53 & 63219.76 & 58461.86 & 58481.71 \\
\hline North Coordinate & 30863.89 & 30160.44 & 30133.05 & 29114.91 & 29117.17 & 31026.68 & 31025.53 \\
\hline BLEVATIONS & - & - & - & $\bullet$ & - & - & • \\
\hline Measuring Point & 994.01 & 970.02 & 968.23 & 915.34 & 914.85 & 1009.87 & 1008.54 \\
\hline Surface & 991.04 & 966.51 & 964.91 & 911.85 & 911.38 & 1006.72 & 1005.53 \\
\hline Weathered Rock & 977.04 & 962.51 & 960.11 & 899.85 & 894.38 & 981.82 & 981.03 \\
\hline Fresh Rock & 971.04 & 954.01 & 953.41 & 897.35 & • & 972.82 & • \\
\hline Top Monitored Interval & 973.04 & 918.21 & 961.31 & 865.45 & 907.38 & 952.82 & 985.63 \\
\hline Bottom Monitored Interval & 960.84 & 906.41 & 949.61 & 853.15 & 895.38 & 941.72 & 973.13 \\
\hline SURFACE/CONDUCTOR CASING & $\cdot$ & $\cdot$ & $\cdot$ & $\cdot$ & . & $\dot{\bullet}$ & • \\
\hline Casing Depth & 15.4 & $\cdot$ & $\dot{.}$ & 11.5 & $\cdot$ & 38 & • \\
\hline Casing Diameter & 10.75 & - & $\cdot$ & 16.75 & - & 10.75 & • \\
\hline Casing Material & Steel & $\cdot$ & - & $\cdot$ & • & - & • \\
\hline WELL CASING BOREHOLE & - & $\cdot$ & $\cdot$ & - & • & . & $\bullet$ \\
\hline Borehole Depth & $30 . \dot{2}$ & $60 . \dot{1}$ & 15.3 & $60 . \dot{2}$ & $\dot{17}$ & $65 . \dot{1}$ & 32.5 \\
\hline Borehole Diameter & 9.9 & 9.9 & 9.9 & 9.9 & 8 & 9.9 & 9.9 \\
\hline Weathered Rock - Depth & 14 & 4 & 4.8 & 12 & 17 & 24.9 & 24.5 \\
\hline F sh Rock - Depth & 20 & 12.5 & 11.5 & 14.5 & • & 33.9 & • \\
\hline .. CASING & $\cdot$ & $\cdot$ & $\cdot$ & • & $\dot{\bullet}$ & • & • \\
\hline Well Casing Depth & 18.9 & 49.7 & 5.3 & $58 . \dot{2}$ & 15.4 & 64.9 & 32.2 \\
\hline Well Casing Diameter & 4.5 & 4.5 & 4.5 & 4.5 & 4.5 & 4.5 & 4.5 \\
\hline Well Casing Material & ss $/ 304$ & ss $/ \$ 304$ & SS $; \$ 304$ & ss $/ \$ 304$ & SS $/ \$ 304$ & ss $/ \$ 304$ & ss $/ \$ 304$ \\
\hline HONITORED INTERVAI & - & - & . & - & - & - & • \\
\hline Aquifer zone & $\dot{\text { WT }}$ & BDR & WT & BDR & $\dot{w T}$ & BDR & WT \\
\hline Geologic Formation & crg & $\mathrm{cm}$ & $\mathrm{cm}$ & $\mathrm{Cn}$ & $\mathrm{Cn}$ & Crg & Crg \\
\hline Screened or Open & Screen & Screen & Screen & Screen & Screen & Screen & Screen \\
\hline Top - Deprh & 18 & 48.3 & 3.6 & 46.4 & 4 & 53.9 & 19.9 \\
\hline Bottom - Depth & 30.2 & 60.1 & 15.3 & 58.7 & 16 & 65 & 32.4 \\
\hline Screen Material & SS/Ew/.01 & ss/sw/.01 & $\mathrm{SS} / \mathrm{sw} / .01$ & $\mathrm{ss} / \mathrm{sw} / .01$ & SS/sw/.01 & $\mathrm{ss} / \mathrm{sw} / .01$ & $\mathrm{ss} / \mathrm{sw} / .01$ \\
\hline Screen Length & 10.1 & 9.9 & 9.6 & 10.0 & 10.3 & 10.0 & 10.3 \\
\hline $\begin{array}{l}\text { Open-Hole Length } \\
\text { pen-Hole Diameter }\end{array}$ & . & & $\cdot$ & . & • & . & • \\
\hline
\end{tabular}

(CONTINUED) 
APPENDIX $C$

Monitoring-Well Construction Details

\begin{tabular}{|c|c|c|c|c|c|c|c|}
\hline \multirow{2}{*}{$\begin{array}{l}\text { Well No. } \\
\text { Location }\end{array}$} & Gw-766 & Gw-767 & GW-768 & GW-769 & GW-770 & GW-771 & GW-772 \\
\hline & GRIDI2 & GRIDI 2 & GRIDII & GRIDG3 & GRIDG3 & GRIDC1 & GRIDCl \\
\hline East Coordinate & 62312.08 & 62294.12 & 62445.04 & 60230.01 & 60255 & 56360.67 & 56356.69 \\
\hline North Coordinate & 29717.66 & 29725.79 & 30330.21 & 29510.42 & 29504.56 & 31331.05 & 31310.43 \\
\hline ELEVATIONS & . & $\cdot$ & $\cdot$ & - & $\cdot$ & - & - \\
\hline Measuring Point & 948.37 & 948.54 & 959.73 & 944.26 & $944.55^{\circ}$ & $1011 . \dot{2}$ & 1012.66 \\
\hline Surface & 944.67 & 945.17 & 956.65 & 941.53 & 941.67 & 1008.27 & 1009.6 \\
\hline Weathered Rock & $\cdot$ & $\cdot$ & $\cdot$ & • & $\cdot$ & $\cdot$ & - \\
\hline Fresh Rock & $\cdot$ & $\cdot$ & $\cdot$ & $\cdot$ & $\cdot$ & $\cdot$ & • \\
\hline Top Monitored Interval & 910.67 & 938.47 & 903.35 & 893.33 & 934.17 & 966.07 & 1005.8 \\
\hline Bottom Monitored Interval & 897.07 & 927.17 & 891.65 & 881.23 & 922.67 & 953.87 & 992.6 \\
\hline SURFACE/CONDUCTOR CASING & $\cdot$ & $\cdot$ & $\cdot$ & . & . & . & - \\
\hline Casing Depth & 24 & $\cdot$ & 32.3 & 14.2 & $\cdot$ & $25 . \dot{1}$ & • \\
\hline Casing Diameter & 10.75 & $\cdot$ & 10.75 & 16.75 & - & 10.75 & • \\
\hline Casing Material & • & $\cdot$ & $\cdot$ & • & - & $\cdot$ & - \\
\hline WEIL CASING BOREHOLE & . &. & . & $\cdot$ & $\cdot$ & - & - \\
\hline Borehole Depth & 47.6 & 18 & 65 & 61.4 & 20 & $54 . \dot{4}$ & 19 \\
\hline Borehole Diameter & 9.87 & 9.87 & 9.62 & 10.62 & 10.62 & 9.87 & 8 \\
\hline Weathered Rock - Depth & $\cdot$ & $\cdot$ & $\cdot$ & $\cdot$ & $\cdot$ & $\cdot$ & - \\
\hline Fresh Rock - Depth & $\cdot$ & $\cdot$ & $\cdot$ & $\cdot$ & $\cdot$ & • & - \\
\hline WELI CASING & $\cdot$ & - & . & • & • & - & - \\
\hline Well Casing Depth & 44.9 & $17 . \dot{8}$ & $64 . \dot{6}$ & $59 . \dot{4}$ & $18 . \dot{5}$ & $53 . \dot{8}$ & $15 . \dot{2}$ \\
\hline Well Casing Diameter & 4.5 & 4.5 & 4.5 & 4.5 & 4.5 & 4.5 & 4.5 \\
\hline Well Casing Material & ss $/ \$ 304$ & SS $/ \$ 304$ & $\mathrm{SS} / 1304$ & $\mathrm{SS} / \$ 304$ & $\mathrm{SS} / \$ 304$ & SS $/ \$ 304$ & SS $/ \$ 304$ \\
\hline MONITORED INTERVAL & . & - & . & - & - & - & • \\
\hline Aquifer Zone & $\begin{array}{r}\cdot \\
\mathrm{BDR}\end{array}$ & พT & BDR & BDR & wr & BDR & WT \\
\hline Geologic Formation & $\mathrm{cm}$ & $\mathrm{cm}$ & $\operatorname{crg}$ & $\mathrm{cn}$ & $\mathrm{Cn}$ & $\operatorname{crg}$ & Crt \\
\hline Screened or Open & Screen & Screen & Screen & Screen & Screen & Screen & Screen \\
\hline Top - Depth & 34 & 6.7 & 53.3 & 48.2 & 7.5 & 42.2 & 3.8 \\
\hline Bottom - Depth & 47.6 & 18 & 65 & 60.3 & 19 & 54.4 & 17 \\
\hline Screen Material & SS/Ew/.01 & $\mathrm{ss} / \mathrm{sw} / .01$ & $\mathrm{ss} / \mathrm{sw} / .01$ & $\mathrm{ss} / \mathrm{sw} / .01$ & $\mathrm{ss} / \mathrm{sw} / .01$ & $\mathrm{ss} / \mathrm{sw} / .01$ & $\mathrm{ss} / \mathrm{sw} / .01$ \\
\hline Screen Length & 10.0 & 10.0 & 9.9 & 10.0 & 10.0 & 14.8 & 10.1 \\
\hline Open-Hole Length & $\cdot$ & • & $\cdot$ & - & $\cdot$ & • & • \\
\hline Open-Hole Diameter & - & - & - & - & - & - & • \\
\hline
\end{tabular}


APPENDIX $C$

Monitoring-Well Construction Details

\begin{tabular}{|c|c|c|c|c|}
\hline Well No. & GW-773 & GW-774 & $G W-775$ & GW-776 \\
\hline Iocation & GRIDH2 & GRIDH2 & GRIDH3 & GRIDH3 \\
\hline Bast Coordinate & 61388.81 & 61408.76 & 61277.82 & 61309.06 \\
\hline North Coordinate & 29878.95 & 29876.52 & 29272.38 & 29271.38 \\
\hline EIEVATIONS & $\cdot$ & - & - & - \\
\hline Measuring Point & 963.76 & 963.16 & 931.35 & 931.45 \\
\hline Surface & 960.72 & 960.09 & 931.48 & 931.44 \\
\hline Weathered Rock & $\cdot$ & • & • & • \\
\hline Fresh Rock & $\bullet$ & $\bullet$ & $\bullet$ & • \\
\hline Top Monltored Interval & 914.62 & 947.59 & 886.48 & 920.84 \\
\hline Bottom Monitored Interval & 901.12 & 933.49 & 875.08 & 908.44 \\
\hline SURPACE/CONDUCTOR CASING & - & - & - & - \\
\hline Casing Depth & 25.5 & - & $16 \cdot 7$ & - \\
\hline Casing Diameter & 10.75 & - & 11.75 & - \\
\hline Casing Material & - & - & - & - \\
\hline WEII CASING BOREHOLE & - & - & - & - \\
\hline Borehole Depth & 59.6 & 26.6 & 60.5 & 24 \\
\hline Borehole Diameter & 9.87 & 9.87 & 10.62 & 9.87 \\
\hline Weathered Rock - Depth & $\bullet$ & $\cdot$ & $\cdot$ & - \\
\hline Fresh Rock - Depth & - & - & - & - \\
\hline WELL CASING & - & - & - & - \\
\hline Well Casing Depth & 58.7 & 25.8 & 56.3 & 22.3 \\
\hline Well Casing Diameter & 4.5 & 4.5 & 4.5 & 4.5 \\
\hline Well Casing Material & $\mathrm{SS} / \$ 304$ & $\mathrm{sS} / \$ 304$ & SS $/ \$ 304$ & $S S / \$ 304$ \\
\hline MONITORED INTERVAL & - & - & - & - \\
\hline Aquifer zone & BDR & WT & BDR & WT \\
\hline Geologic Formation & $\mathrm{Cm}$ & $\mathrm{Cm}$ & $\mathrm{Cn}$ & $\mathrm{Cn}$ \\
\hline Screened or open & Screen & Screen & Screen & Screen \\
\hline Top - Depth & 46.1 & 12.5 & 45 & 10.6 \\
\hline Bottom - Depth & 59.6 & 26.6 & 56.4 & 23 \\
\hline Screen Material & $\mathrm{sS} / \mathrm{sw} / .01$ & SS/EW/.01 & ss/sw/.01 & $\mathrm{ss} / \mathrm{sw} / .01$ \\
\hline Screen Length & 10.1 & 9.8 & 10.0 & 10.0 \\
\hline Open-Hole Iength & • & - & - & - \\
\hline Open-Hole Diameter & - & - & - & - \\
\hline
\end{tabular}


APPENDIX D

SAMPLING SEQUENCE 


\section{EXPLANATION}

\section{LOCATION:}

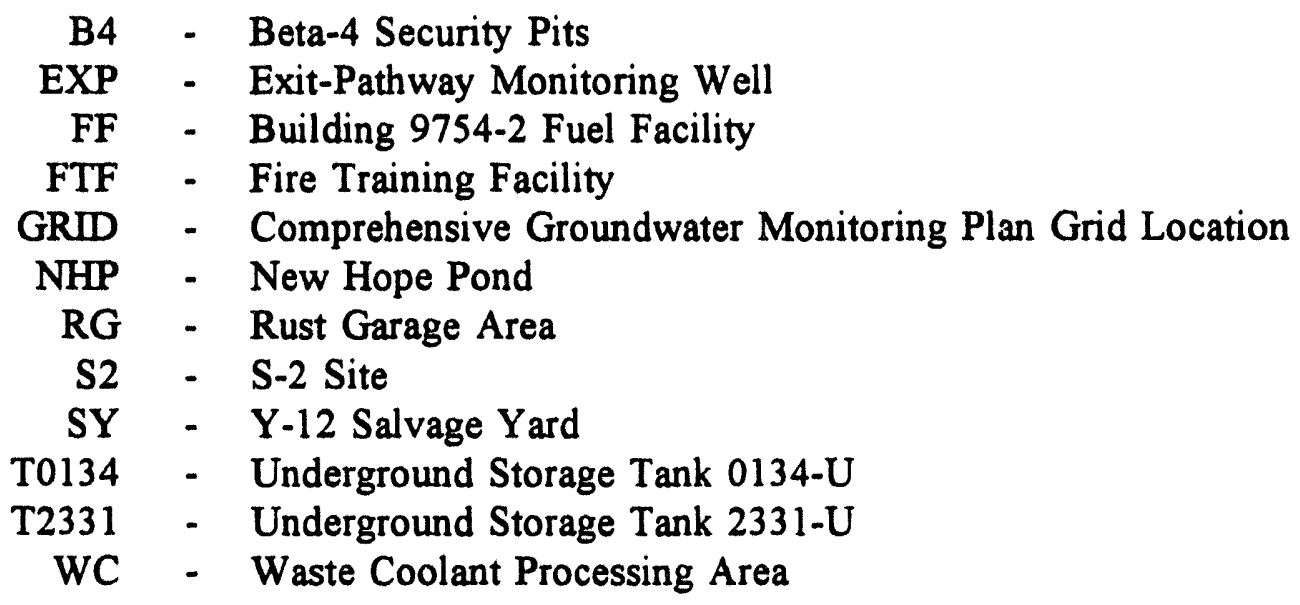


APPENDIX D

Sampling sequence

\begin{tabular}{|c|c|c|c|}
\hline $\begin{array}{l}\text { Sampling } \\
\text { Point }\end{array}$ & Location & Date & Time \\
\hline \multicolumn{4}{|c|}{ First Quarter 1993} \\
\hline$G W-252$ & s2 & $01 / 08 / 93$ & $12: 07$ \\
\hline$G W-255$ & s2 & $01 / 08 / 93$ & $13: 49$ \\
\hline GW-261 & SY & $01 / 08 / 93$ & $14: 30$ \\
\hline$G W-338$ & พC & $01 / 11 / 93$ & $10: 30$ \\
\hline$G W-751$ & GRIDJ 3 & $01 / 11 / 93$ & $10: 42$ \\
\hline GW-262 & SY & $01 / 11 / 93$ & $12: 12$ \\
\hline$G W-752$ & GRIDJ 3 & $01 / 11 / 93$ & $12: 30$ \\
\hline$G W-263$ & SY & $01 / 11 / 93$ & $14: 15$ \\
\hline GW -617 & EXP & $01 / 12 / 93$ & $9: 30$ \\
\hline GW-618 & EXP & $01 / 12 / 93$ & $10: 31$ \\
\hline$G W-264$ & SY & $01 / 12 / 93$ & $10: 45$ \\
\hline GW-191 & B4 & $01 / 12 / 93$ & $13: 50$ \\
\hline$G W-385$ & NHP & $01 / 12 / 93$ & $14: 36$ \\
\hline GW-194 & B4 & $01 / 13 / 93$ & $10: 30$ \\
\hline$G W-384$ & NAP & $01 / 13 / 93$ & $11: 35$ \\
\hline GW-195 & B4 & $01 / 13 / 93$ & $12: 30$ \\
\hline GW-192 & B4 & $01 / 14 / 93$ & $10: 00$ \\
\hline GW-755 & GRIDJ 1 & $01 / 14 / 93$ & $11: 00$ \\
\hline$G W-207$ & EXP & $01 / 14 / 93$ & $11: 13$ \\
\hline GW-756 & GRIDJ 1 & $01 / 15 / 93$ & $10: 10$ \\
\hline$G W-753$ & GRIDJ2 & $01 / 15 / 93$ & $12: 09$ \\
\hline GW-754 & GRIDJ2 & $01 / 18 / 93$ & $11: 00$ \\
\hline$G W-744$ & GRIDK 1 & $01 / 18 / 93$ & $13: 17$ \\
\hline GW -745 & GRIDR 1 & $01 / 19 / 93$ & $9: 55$ \\
\hline$G W-746$ & GRIDK1 & $01 / 19 / 93$ & $11: 40$ \\
\hline GW-206 & EXP & $01 / 19 / 93$ & $11: 45$ \\
\hline$G W-208$ & EXP & $01 / 19 / 93$ & $12: 26$ \\
\hline$G W-747$ & GRIDK2 & $01 / 19 / 93$ & $14: 36$ \\
\hline$G W-251$ & s2 & $01 / 20 / 93$ & $10: 31$ \\
\hline GW-619 & FTF & $01 / 20 / 93$ & $12: 15$ \\
\hline$G W-748$ & GRIDK2 & $01 / 20 / 93$ & $12: 23$ \\
\hline$G W-620$ & FTF & $01 / 20 / 93$ & $15: 00$ \\
\hline$G W-749$ & GRIDK2 & $01 / 21 / 93$ & $9: 40$ \\
\hline$G W-337$ & WC & $01 / 21 / 93$ & $11: 00$ \\
\hline$G W-750$ & GRIDK2 & $01 / 21 / 93$ & $11: 50$ \\
\hline GW-168 & NHP & $01 / 22 / 93$ & $11: 46$ \\
\hline GW-771 & GRIDC1 & $01 / 23 / 93$ & $10: 30$ \\
\hline GW-170 & EXP & $01 / 23 / 93$ & $11: 28$ \\
\hline GW-772 & GRIDC1 & $01 / 23 / 93$ & $11: 33$ \\
\hline$G W-239$ & NHP & $01 / 23 / 93$ & $11: 35$ \\
\hline GW-169 & EXP & $01 / 23 / 93$ & $12: 15$ \\
\hline GW-762 & GRIDJ3 & $01 / 23 / 93$ & $14: 18$ \\
\hline GW-764 & GRIDE 1 & $01 / 23 / 93$ & $14: 40$ \\
\hline GW-763 & GRIDJ3 & $01 / 25 / 93$ & $10: 10$ \\
\hline GW-765 & GRIDE 1 & $01 / 25 / 93$ & $10: 35$ \\
\hline
\end{tabular}


APPENDIX D

Sampling sequence

\begin{tabular}{|c|c|c|c|}
\hline $\begin{array}{l}\text { sampling } \\
\text { point }\end{array}$ & Location & Date & Time \\
\hline$G W-380$ & NEP & $01 / 25 / 93$ & $12: 15$ \\
\hline GW-758 & GRIDG 1 & $01 / 25 / 93$ & $13: 10$ \\
\hline$G W-220$ & NHP & $01 / 25 / 93$ & $14: 20$ \\
\hline GW-759 & GRIDG1 & $01 / 26 / 93$ & $10: 20$ \\
\hline$G W-151$ & NHP & $01 / 26 / 93$ & $11: 17$ \\
\hline$G W-232$ & EXP & $01 / 26 / 93$ & $12: 10$ \\
\hline$G W-383$ & NBP & $01 / 26 / 93$ & $13: 01$ \\
\hline$G W-761$ & GRIDG2 & $01 / 26 / 93$ & $14: 30$ \\
\hline$G W-240$ & NHP & $01 / 27 / 93$ & $9: 26$ \\
\hline GW-769 & GRIDG 3 & $01 / 27 / 93$ & $11: 10$ \\
\hline$G W-381$ & NBP & $01 / 27 / 93$ & $12: 28$ \\
\hline GW-770 & GRIDG 3 & $01 / 27 / 93$ & $12: 58$ \\
\hline GW-760 & GRIDG2 & $01 / 27 / 93$ & $13: 40$ \\
\hline$G W-382$ & NHP & $01 / 28 / 93$ & $12: 34$ \\
\hline GW-199 & GRIDI 1 & $01 / 29 / 93$ & $9: 10$ \\
\hline GW-768 & GRIDI 1 & $01 / 29 / 93$ & $10: 00$ \\
\hline GW-603 & EXP & $01 / 29 / 93$ & $12: 50$ \\
\hline GW-604 & EXP & $01 / 30 / 93$ & $10: 46$ \\
\hline GW-766 & GRIDI2 & $02 / 01 / 93$ & $11: 15$ \\
\hline GW-767 & GRIDI2 & $02 / 02 / 93$ & $9: 15$ \\
\hline GW-281 & $\mathbf{F F}$ & $02 / 02 / 93$ & $10: 30$ \\
\hline$G W-605$ & EXP & $02 / 02 / 93$ & $10: 40$ \\
\hline$G W-284$ & $\mathbf{F F}$ & $02 / 02 / 93$ & $12: 00$ \\
\hline GW-733 & EXP & $02 / 02 / 93$ & $12: 36$ \\
\hline$G W-285$ & $\mathbf{F F}$ & $02 / 02 / 93$ & $13: 45$ \\
\hline GW-735 & NBP & $02 / 02 / 93$ & $14: 43$ \\
\hline GW-283 & $F \mathbf{F}$ & $02 / 03 / 93$ & $11: 15$ \\
\hline$G W-606$ & EXP & $02 / 03 / 93$ & $11: 15$ \\
\hline GW-773 & GRIDH2 & $02 / 03 / 93$ & $14: 15$ \\
\hline GW-774 & GRIDH2 & $02 / 04 / 93$ & $10: 40$ \\
\hline$G W-775$ & GRIDH 3 & $02 / 04 / 93$ & $13: 08$ \\
\hline GW-776 & GRIDH 3 & $02 / 04 / 93$ & $14: 45$ \\
\hline GW-762 & GRIDJ 3 & $02 / 22 / 93$ & $12: 20$ \\
\hline$G W-505$ & RG & $03 / 01 / 93$ & $10: 50$ \\
\hline GW-190 & RG & $03 / 01 / 93$ & $12: 49$ \\
\hline$G W-631$ & RG & $03 / 02 / 93$ & $11: 25$ \\
\hline$G W-634$ & RG & $03 / 02 / 93$ & $14: 45$ \\
\hline$G W-632$ & RG & $03 / 03 / 93$ & $10: 50$ \\
\hline$G W-633$ & RG & $03 / 03 / 93$ & $13: 45$ \\
\hline$G W-508$ & RG & $03 / 05 / 93$ & $9: 45$ \\
\hline GW-708 & T2331 & $03 / 10 / 93$ & $10: 30$ \\
\hline$G W-657$ & T2331 & $03 / 10 / 93$ & $11: 30$ \\
\hline GW-659 & $\mathbf{F F}$ & $03 / 10 / 93$ & $13: 45$ \\
\hline$G W-183$ & $\mathbf{F F}$ & $03 / 10 / 93$ & $15: 15$ \\
\hline$G W-204$ & T0134 & $03 / 11 / 93$ & $9: 15$ \\
\hline$G W-656$ & T0134 & $03 / 11 / 93$ & $11: 00$ \\
\hline GW-193 & T2331 & $03 / 11 / 93$ & $13: 30$ \\
\hline
\end{tabular}


APPENDIX D

sampling sequence

\begin{tabular}{|c|c|c|c|}
\hline $\begin{array}{l}\text { Sampling } \\
\text { Point }\end{array}$ & Location & Date & Time \\
\hline GW-707 & T2331 & $03 / 11 / 93$ & $15: 00$ \\
\hline \multirow[t]{2}{*}{$G W-658$} & $\mathbf{F F}$ & $03 / 11 / 93$ & $16: 30$ \\
\hline & \multicolumn{2}{|c|}{ second Quarter 1993} & \\
\hline GW-199 & GRIDI 1 & $04 / 07 / 93$ & $11: 45$ \\
\hline$G W-768$ & GRIDI 1 & $04 / 07 / 93$ & $12: 15$ \\
\hline$G W-261$ & SY & $04 / 07 / 93$ & $13: 20$ \\
\hline GW-755 & GRIDJ 1 & $04 / 07 / 93$ & $14: 00$ \\
\hline GW-756 & GRIDJ 1 & $04 / 08 / 93$ & $10: 09$ \\
\hline GW-262 & SY & $04 / 08 / 93$ & $10: 35$ \\
\hline$G W-753$ & GRIDJ2 & $04 / 08 / 93$ & $11: 44$ \\
\hline GW-263 & SY & $04 / 08 / 93$ & $13: 30$ \\
\hline GW-754 & GRIDJ2 & $04 / 08 / 93$ & $13: 30$ \\
\hline GW-744 & GRIDK1 & $04 / 12 / 93$ & $9: 38$ \\
\hline$G W-264$ & SY & $04 / 12 / 93$ & $10: 25$ \\
\hline$G W-771$ & GRIDC1 & $04 / 12 / 93$ & $11: 00$ \\
\hline GW -745 & GRIDK1 & $04 / 12 / 93$ & $11: 20$ \\
\hline GW-191 & B4 & $04 / 12 / 93$ & $13: 00$ \\
\hline GW-772 & GRIDC1 & $04 / 12 / 93$ & $13: 00$ \\
\hline$G W-746$ & GRIDK1 & $04 / 12 / 93$ & $13: 10$ \\
\hline GW-194 & $\mathrm{B} 4$ & $04 / 13 / 93$ & $10: 50$ \\
\hline$G W-764$ & GRIDE 1 & $04 / 13 / 93$ & $11: 00$ \\
\hline GW-747 & GRIDK2 & $04 / 13 / 93$ & $11: 05$ \\
\hline GW-195 & B4 & $04 / 13 / 93$ & $12: 20$ \\
\hline GW-765 & GRIDE 1 & $04 / 13 / 93$ & $12: 30$ \\
\hline GW-748 & GRIDK2 & $04 / 13 / 93$ & $12: 45$ \\
\hline$G W-758$ & GRIDG1 & $04 / 13 / 93$ & $13: 48$ \\
\hline GW-192 & B4 & $04 / 13 / 93$ & $13: 50$ \\
\hline$G W-749$ & GRIDK2 & $04 / 14 / 93$ & $14: 00$ \\
\hline GW-750 & GRIDK2 & $04 / 15 / 93$ & $9: 44$ \\
\hline GW-766 & GRIDI2 & $04 / 15 / 93$ & $11: 00$ \\
\hline GW-759 & GRIDG 1 & $04 / 15 / 93$ & $11: 30$ \\
\hline GW-767 & GRIDI2 & $04 / 15 / 93$ & $13: 10$ \\
\hline GW-751 & GRIDJ3 & $04 / 16 / 93$ & $9: 59$ \\
\hline GW-281 & FF & $04 / 16 / 93$ & $10: 30$ \\
\hline GW-752 & GRIDJ 3 & $04 / 16 / 93$ & $10: 36$ \\
\hline GW-760 & GRIDG2 & $04 / 16 / 93$ & $11: 30$ \\
\hline GW-284 & FF & $04 / 16 / 93$ & $12: 15$ \\
\hline $\mathrm{GW}-761$ & GRIDG2 & $04 / 16 / 93$ & $13: 15$ \\
\hline GW-285 & FF & $04 / 19 / 93$ & $11: 00$ \\
\hline GW-769 & GRIDG 3 & $04 / 19 / 93$ & $11: 11$ \\
\hline$G W-770$ & GRIDG 3 & $04 / 19 / 93$ & $11: 41$ \\
\hline$G W-283$ & $\mathbf{F F}$ & $04 / 19 / 93$ & $13: 10$ \\
\hline$G W-385$ & NHP & $04 / 19 / 93$ & $15: 15$ \\
\hline GW-773 & GRIDH2 & $04 / 20 / 93$ & $9: 24$ \\
\hline$G W-774$ & GRIDH2 & $04 / 20 / 93$ & $10: 45$ \\
\hline
\end{tabular}


APPENDIX D

Sampling sequence

\begin{tabular}{|c|c|c|c|}
\hline $\begin{array}{l}\text { Bampling } \\
\text { Point }\end{array}$ & Location & Date & Time \\
\hline$G W-384$ & NHP & $04 / 20 / 93$ & $11: 00$ \\
\hline GW-775 & GRIDH 3 & $04 / 20 / 93$ & $12: 40$ \\
\hline GW-762 & GRIDJ 3 & $04 / 20 / 93$ & $13: 12$ \\
\hline GW -776 & GRIDH3 & $04 / 20 / 93$ & $14: 00$ \\
\hline GW-763 & GRIDJ 3 & $04 / 21 / 93$ & $9: 45$ \\
\hline GW-380 & NHP & $04 / 21 / 93$ & $11: 45$ \\
\hline GW -240 & NHP & $04 / 21 / 93$ & $13: 45$ \\
\hline$G W-220$ & NAP & $04 / 22 / 93$ & $9: 49$ \\
\hline$G W-151$ & NHP & $04 / 22 / 93$ & $11: 59$ \\
\hline$G W-383$ & NHP & $04 / 22 / 93$ & $12: 57$ \\
\hline GW-382 & NAP & $04 / 26 / 93$ & $11: 24$ \\
\hline$G W-381$ & NAP & $04 / 26 / 93$ & $14: 11$ \\
\hline$G W-252$ & $\mathbf{s 2}$ & $05 / 03 / 93$ & $12: 30$ \\
\hline GW-255 & $\mathbf{s 2}$ & $05 / 03 / 93$ & $14: 00$ \\
\hline GW-603 & EXP & $05 / 04 / 93$ & $11: 25$ \\
\hline$G W-338$ & พC & $05 / 04 / 93$ & $12: 00$ \\
\hline$G W-604$ & EXP & $05 / 04 / 93$ & $12: 58$ \\
\hline GW-617 & EXP & $05 / 04 / 93$ & $13: 30$ \\
\hline$G W-207$ & $\operatorname{EXP}$ & $05 / 04 / 93$ & $14: 26$ \\
\hline$G W-618$ & EXP & $05 / 05 / 93$ & $10: 35$ \\
\hline GW -251 & $\mathbf{s 2}$ & $05 / 05 / 93$ & $11: 43$ \\
\hline$G W-619$ & FTF & $05 / 05 / 93$ & $13: 06$ \\
\hline$G W-620$ & FTF & $05 / 06 / 93$ & $9: 45$ \\
\hline GW-208 & EXP & $05 / 06 / 93$ & $13: 36$ \\
\hline$G W-206$ & EXP & $05 / 06 / 93$ & $14: 00$ \\
\hline GW-733 & EXP & $05 / 06 / 93$ & $14: 40$ \\
\hline GW-337 & WC & $05 / 07 / 93$ & $13: 45$ \\
\hline GW-735 & NHP & $05 / 07 / 93$ & $13: 55$ \\
\hline$G W-605$ & : EXP & $05 / 10 / 93$ & $11: 15$ \\
\hline$G W-606$ & EXP & $05 / 10 / 93$ & $15: 10$ \\
\hline GW-239 & NEP & $05 / 13 / 93$ & $11: 15$ \\
\hline$G W-168$ & NHP & $05 / 14 / 93$ & $9: 58$ \\
\hline GW-169 & EXP & $05 / 21 / 93$ & $10: 30$ \\
\hline GW-232 & $\mathbf{E X P}$ & $05 / 21 / 93$ & $11: 11$ \\
\hline GW- 170 & EXP & $05 / 24 / 93$ & $13: 15$ \\
\hline$G W-190$ & RG & $06 / 17 / 93$ & $13: 12$ \\
\hline$G W-708$ & T2331 & $06 / 18 / 93$ & $9: 30$ \\
\hline GW-505 & RG & $06 / 18 / 93$ & $10: 00$ \\
\hline GW-657 & T2331 & $06 / 18 / 93$ & $10: 19$ \\
\hline$G W-631$ & RG & $06 / 18 / 93$ & $11: 30$ \\
\hline GW-634 & RG & $06 / 18 / 93$ & $13: 00$ \\
\hline$G W-659$ & $\mathbf{F F}$ & $06 / 18 / 93$ & $13: 14$ \\
\hline$G W-183$ & FF & $06 / 21 / 93$ & $12: 00$ \\
\hline$G W-632$ & RG & $06 / 21 / 93$ & $12: 30$ \\
\hline$G W-633$ & RG & $06 / 21 / 93$ & $14: 10$ \\
\hline$G W-204$ & T0134 & $06 / 22 / 93$ & $10: 30$ \\
\hline GW -656 & T0134 & $06 / 22 / 93$ & $12: 30$ \\
\hline
\end{tabular}


APPENDIX D

sampling sequence

\begin{tabular}{|c|c|c|c|}
\hline $\begin{array}{l}\text { sampling } \\
\text { point }\end{array}$ & Location & Date & Time \\
\hline GW-193 & T2331 & $06 / 22 / 93$ & $14: 00$ \\
\hline GW-508 & $\mathbf{R G}$ & $06 / 23 / 93$ & $8: 45$ \\
\hline GW-707 & T2331 & $06 / 23 / 93$ & $11: 00$ \\
\hline$G W-658$ & $F \mathbf{F}$ & $06 / 23 / 93$ & $13: 15$ \\
\hline GW-183 & $\mathbf{F F}$ & $06 / 29 / 93$ & $11: 15$ \\
\hline GW-632 & RG & $06 / 29 / 93$ & $12: 40$ \\
\hline$G W-508$ & RG & $06 / 29 / 93$ & $13: 10$ \\
\hline \multirow[t]{2}{*}{$G W-633$} & $\mathbf{R G}$ & $06 / 29 / 93$ & $13: 45$ \\
\hline & \multicolumn{2}{|c|}{ Third Quarter 1993} & \\
\hline$G W-261$ & SY & $07 / 01 / 93$ & $10: 35$ \\
\hline$G W-262$ & SY & $07 / 01 / 93$ & $12: 25$ \\
\hline$G W-263$ & SY & $07 / 01 / 93$ & $14: 45$ \\
\hline$G W-264$ & SY & $07 / 02 / 93$ & $10: 32$ \\
\hline GW-708 & T2331 & $07 / 07 / 93$ & $10: 25$ \\
\hline GW-190 & RG & $07 / 07 / 93$ & $11: 20$ \\
\hline GW-659 & $\mathbf{F F}$ & $07 / 07 / 93$ & $12: 00$ \\
\hline GW-505 & $\mathbf{R G}$ & $07 / 07 / 93$ & $13: 00$ \\
\hline GW-631 & RG & $07 / 07 / 93$ & $13: 00$ \\
\hline$G W-634$ & RG & $07 / 07 / 93$ & $14: 00$ \\
\hline GW-191 & B4 & $07 / 08 / 93$ & $10: 00$ \\
\hline GW-194 & B4 & $07 / 08 / 93$ & $11: 30$ \\
\hline$G W-195$ & B4 & $07 / 08 / 93$ & $12: 30$ \\
\hline GW-192 & B4 & $07 / 08 / 93$ & $13: 30$ \\
\hline$G W-751$ & GRIDJ3 & $08 / 02 / 93$ & $11: 33$ \\
\hline GW-752 & GRIDJ3 & $08 / 02 / 93$ & $13: 23$ \\
\hline GW-199 & GRIDI 1 & $08 / 02 / 93$ & $14: 15$ \\
\hline GW-755 & GRIDJI & $08 / 03 / 93$ & $11: 00$ \\
\hline GW-756 & GRIDJ 1 & $08 / 03 / 93$ & $11: 56$ \\
\hline GW-753 & GRIDJ2 & $08 / 03 / 93$ & $13: 58$ \\
\hline GW-766 & GRIDI2 & $08 / 03 / 93$ & $14: 10$ \\
\hline$G W-385$ & NHP & $08 / 03 / 93$ & $14: 30$ \\
\hline GW-754 & GRIDJ2 & $08 / 04 / 93$ & $10: 00$ \\
\hline GW-768 & GRIDI 1 & $08 / 04 / 93$ & $10: 30$ \\
\hline GW-384 & NAP & $08 / 04 / 93$ & $11: 07$ \\
\hline$G W-744$ & GRIDR 1 & $08 / 04 / 93$ & $12: 02$ \\
\hline GW-767 & GRIDI2 & $08 / 04 / 93$ & $12: 20$ \\
\hline$G W-762$ & GRIDJ 3 & $08 / 04 / 93$ & $13: 12$ \\
\hline$G W-745$ & GRIDKI & $08 / 04 / 93$ & $13: 45$ \\
\hline$G W-281$ & $\mathbf{F F}$ & $08 / 04 / 93$ & $14: 15$ \\
\hline$G W-763$ & GRIDJ 3 & $08 / 05 / 93$ & $9: 50$ \\
\hline$G W-380$ & NHP & $08 / 05 / 93$ & $11: 20$ \\
\hline$G W-284$ & FF & $08 / 05 / 93$ & $13: 00$ \\
\hline$G W-240$ & NAP & $08 / 05 / 93$ & $13: 12$ \\
\hline GW-747 & GRIDK2 & $08 / 05 / 93$ & $13: 28$ \\
\hline$G W-746$ & GRIDKI & $08 / 05 / 93$ & $13: 45$ \\
\hline
\end{tabular}


APPENDIX D

sampling sequence

\begin{tabular}{|c|c|c|c|}
\hline $\begin{array}{l}\text { Sampling } \\
\text { Point }\end{array}$ & Location & Date & Time \\
\hline$G W-285$ & $\mathbf{F F}$ & $08 / 05 / 93$ & $14: 00$ \\
\hline$G W-748$ & GRIDK2 & $08 / 06 / 93$ & $10: 00$ \\
\hline GW -283 & $\mathbf{F F}$ & $08 / 06 / 93$ & $10: 15$ \\
\hline$G W-220$ & NHP & $08 / 06 / 93$ & $10: 38$ \\
\hline ow -773 & GRIDH2 & $08 / 06 / 93$ & $11: 57$ \\
\hline $6 W-749$ & GRIDK2 & $08 / 06 / 93$ & $12: 20$ \\
\hline$G W-151$ & NHP & $08 / 06 / 93$ & $13: 32$ \\
\hline GW-750 & GRIDK2 & $08 / 06 / 93$ & $14: 12$ \\
\hline GW-383 & NHP & $08 / 07 / 93$ & $8: 53$ \\
\hline$G W-382$ & N日P & $08 / 08 / 93$ & $11: 00$ \\
\hline$G W-381$ & NHP & $08 / 08 / 93$ & $13: 47$ \\
\hline GW-774 & GRIDB2 & $08 / 09 / 93$ & $13: 10$ \\
\hline GW-775 & GRIDA3 & $08 / 09 / 93$ & $14: 42$ \\
\hline GW-776 & GRIDH 3 & $08 / 10 / 93$ & $10: 00$ \\
\hline GW-207 & EXP & $08 / 11 / 93$ & $12: 21$ \\
\hline$G W-206$ & $\mathbf{E X P}$ & $08 / 12 / 93$ & $13: 30$ \\
\hline GW-208 & EXP & $08 / 13 / 93$ & $13: 20$ \\
\hline GW-603 & EXP & $08 / 16 / 93$ & $10: 06$ \\
\hline$G W-604$ & EXP & $08 / 16 / 93$ & $11: 28$ \\
\hline GW -733 & $\operatorname{ExP}$ & $08 / 18 / 93$ & $12: 00$ \\
\hline GW-239 & NAP & $08 / 18 / 93$ & $13: 18$ \\
\hline GW-735 & NAP & $08 / 18 / 93$ & $14: 30$ \\
\hline$G W-168$ & NAP & $08 / 19 / 93$ & $11: 13$ \\
\hline$G W-605$ & EXP & $08 / 19 / 93$ & $13: 23$ \\
\hline GW-169 & EXP & $08 / 19 / 93$ & $13: 45$ \\
\hline$G W-606$ & $\mathbf{E X P}$ & $08 / 20 / 93$ & $12: 45$ \\
\hline$G w-232$ & $\mathbf{E X P}$ & $08 / 21 / 93$ & $9: 56$ \\
\hline GW-170 & $\mathbf{E X P}$ & $08 / 21 / 93$ & $13: 42$ \\
\hline GW-771 & GRIDC1 & $09 / 03 / 93$ & $10: 48$ \\
\hline$G W-772$ & GRIDC1 & $09 / 12 / 93$ & $9: 00$ \\
\hline GW-764 & GRIDEI & $09 / 12 / 93$ & $11: 00$ \\
\hline GW-765 & GRIDE 1 & $09 / 12 / 93$ & $12: 40$ \\
\hline GW-758 & GRIDG1 & $09 / 12 / 93$ & $14: 34$ \\
\hline GW-759 & GRIDG 1 & $09 / 13 / 93$ & $12: 15$ \\
\hline GW-761 & GRIDG2 & $09 / 13 / 93$ & $14: 05$ \\
\hline GW -770 & GRIDG3 & $09 / 14 / 93$ & $9: 15$ \\
\hline GW -760 & GRIDG2 & $09 / 14 / 93$ & $11: 30$ \\
\hline GW-769 & GRIDG 3 & $09 / 14 / 93$ & $14: 05$ \\
\hline$G W-505$ & RG & $09 / 15 / 93$ & $10: 30$ \\
\hline GW-252 & s2 & $09 / 15 / 93$ & $12: 00$ \\
\hline GW-190 & RG & $09 / 15 / 93$ & $13: 00$ \\
\hline$G W-255$ & $\mathbf{s 2}$ & $09 / 15 / 93$ & $13: 19$ \\
\hline$G W-631$ & $\mathbf{R G}$ & $09 / 16 / 93$ & $10: 15$ \\
\hline GW-617 & EXP & $09 / 16 / 93$ & $12: 00$ \\
\hline$G W-634$ & $\mathbf{R G}$ & $09 / 16 / 93$ & $12: 15$ \\
\hline$G W-618$ & EXP & $09 / 16 / 93$ & $12: 55$ \\
\hline$G W-632$ & RG & $09 / 16 / 93$ & $14: 00$ \\
\hline
\end{tabular}


APPENDIX D

sampling sequence

\begin{tabular}{|c|c|c|c|}
\hline $\begin{array}{l}\text { sampling } \\
\text { Point }\end{array}$ & Location & Date & Time \\
\hline GW-251 & s2 & $09 / 17 / 93$ & $9: 28$ \\
\hline ow-619 & FTF & $09 / 17 / 93$ & 10,37 \\
\hline OW-620 & FTF & $09 / 17 / 93$ & $12: 10$ \\
\hline GW-337 & wc & $09 / 17 / 93$ & $14: 00$ \\
\hline QW-633 & RG & $09 / 20 / 93$ & $11: 50$ \\
\hline OW-508 & RG & $09 / 21 / 93$ & $9: 30$ \\
\hline GW-708 & T2331 & $09 / 21 / 93$ & $10: 50$ \\
\hline GW-657 & T2331 & $09 / 21 / 93$ & $12: 10$ \\
\hline GW-508 & RG & $09 / 22 / 93$ & $9: 30$ \\
\hline OW-659 & $\mathbf{F F}$ & $09 / 22 / 93$ & $11: 06$ \\
\hline$G w-183$ & $\mathbf{F F}$ & $09 / 22 / 93$ & $12: 59$ \\
\hline$O W-204$ & T0134 & $09 / 23 / 93$ & $10: 30$ \\
\hline OW-656 & T0134 & $09 / 23 / 93$ & $12: 20$ \\
\hline GW-338 & wc & $09 / 23 / 93$ & $12: 45$ \\
\hline$G W-193$ & T2331 & $09 / 23 / 93$ & $14: 10$ \\
\hline 6w-707 & T2331 & $09 / 27 / 93$ & $10: 05$ \\
\hline \multicolumn{4}{|c|}{ Fourth Quarter 1993} \\
\hline OW-199 & GRIDI I & $10 / 12 / 93$ & $11: 30$ \\
\hline$G W-768$ & GRIDI 1 & $10 / 13 / 93$ & $9: 00$ \\
\hline GW-766 & GRIDI2 & $10 / 13 / 93$ & $11: 00$ \\
\hline GW-767 & GRIDI2 & $10 / 13 / 93$ & $13: 00$ \\
\hline GW-281 & $\mathbf{F F}$ & $10 / 14 / 93$ & $9: 15$ \\
\hline GW-284 & $F F$ & $10 / 14 / 93$ & $11: 00$ \\
\hline GW-285 & $\mathbf{F F}$ & $10 / 14 / 93$ & $12: 30$ \\
\hline GW-283 & $\mathbf{F F}$ & $10 / 14 / 93$ & $14: 00$ \\
\hline GW-773 & GRIDH2 & $10 / 15 / 93$ & $10: 34$ \\
\hline GW-774 & GRIDH2 & $10 / 15 / 93$ & $12: 00$ \\
\hline GW-775 & GRIDH3 & $10 / 15 / 93$ & $13: 21$ \\
\hline GW-776 & GRIDH3 & $10 / 18 / 93$ & $11: 00$ \\
\hline GW-755 & GRIDJ 1 & $10 / 21 / 93$ & $11: 00$ \\
\hline GW-756 & GRIDJ 1 & $10 / 21 / 93$ & $11: 36$ \\
\hline GW-753 & GRIDJ2 & $10 / 21 / 93$ & $14: 05$ \\
\hline GW-754 & GRIDJ2 & $10 / 22 / 93$ & $9: 45$ \\
\hline GW-744 & GRIDKI & $10 / 22 / 93$ & $11: 44$ \\
\hline GW-745 & GRIDKI & $10 / 23 / 93$ & $9: 30$ \\
\hline GW-746 & GRIDKI & $10 / 23 / 93$ & $11: 30$ \\
\hline GW- . 47 & GRIDK2 & $10 / 23 / 93$ & $13: 37$ \\
\hline GW-748 & GRIDK2 & $10 / 24 / 93$ & $10: 15$ \\
\hline GW-749 & GRIDK2 & $10 / 24 / 93$ & $12: 45$ \\
\hline GW-750 & GRIDK2 & $10 / 25 / 93$ & $12: 01$ \\
\hline GW-751 & GRIDJ3 & $10 / 26 / 93$ & $10: 20$ \\
\hline GW-752 & GRIDJ3 & $10 / 26 / 93$ & $10: 53$ \\
\hline GW-207 & EXP & $10 / 26 / 93$ & $11: 55$ \\
\hline GW-206 & EXP & $10 / 27 / 93$ & $13: 00$ \\
\hline$G W-385$ & NHP & $10 / 27 / 93$ & $14: 00$ \\
\hline
\end{tabular}


APPENDIX D

sampling sequence

\begin{tabular}{|c|c|c|c|}
\hline $\begin{array}{l}\text { sampling } \\
\text { Point }\end{array}$ & Location & Date & Time \\
\hline$G W-208$ & EXP & $10 / 28 / 93$ & $10: 00$ \\
\hline$G W-603$ & EXP & $10 / 28 / 93$ & $10: 30$ \\
\hline$G W-384$ & NEP & $10 / 28 / 93$ & $11: 02$ \\
\hline$G W-604$ & $\operatorname{EXP}$ & $10 / 28 / 93$ & $12: 10$ \\
\hline GW-762 & GRIDJ 3 & $10 / 28 / 93$ & $12: 53$ \\
\hline GW-763 & GRIDJ 3 & $10 / 29 / 93$ & $10: 10$ \\
\hline GW-735 & NHP & $10 / 29 / 93$ & $10: 58$ \\
\hline GW-380 & NHP & $10 / 29 / 93$ & $13: 25$ \\
\hline GW-239 & NHP & $10 / 30 / 93$ & $12: 30$ \\
\hline$G W-240$ & NHP & $11 / 01 / 93$ & $10: 25$ \\
\hline GW-220 & NHP & $11 / 01 / 93$ & $11: 36$ \\
\hline GW-169 & EXP & $11 / 01 / 93$ & $13: 40$ \\
\hline GW-232 & EXP & $11 / 01 / 93$ & $14: 48$ \\
\hline$G W-151$ & NBP & $11 / 02 / 93$ & $11: 37$ \\
\hline$G W-168$ & NAP & $11 / 02 / 93$ & $11: 38$ \\
\hline$G W-383$ & NEP & $11 / 03 / 93$ & $8: 43$ \\
\hline GW-170 & EXP & $11 / 04 / 93$ & $11: 10$ \\
\hline GW-382 & NHP & $11 / 04 / 93$ & $12: 40$ \\
\hline GW-733 & EXP & $11 / 04 / 93$ & $14: 30$ \\
\hline GW-381 & NHP & $11 / 08 / 93$ & $13: 20$ \\
\hline$G W-605$ & $\operatorname{EXP}$ & $11 / 10 / 93$ & $10: 39$ \\
\hline GW-606 & $\operatorname{EXP}$ & $11 / 11 / 93$ & $11: 04$ \\
\hline GW-771 & GRIDC 1 & $11 / 11 / 93$ & $12: 40$ \\
\hline GW-708 & T2331 & $11 / 12 / 93$ & $9: 20$ \\
\hline GW-772 & GRIDC 1 & $11 / 12 / 93$ & $9: 30$ \\
\hline GW-764 & GRIDE 1 & $11 / 12 / 93$ & $11: 30$ \\
\hline$G W-657$ & T2331 & $11 / 15 / 93$ & $11: 18$ \\
\hline GW-659 & FF & $11 / 15 / 93$ & $13: 50$ \\
\hline$G W-505$ & RG & $11 / 16 / 93$ & $10: 10$ \\
\hline GW-183 & $\mathbf{F F}$ & $11 / 16 / 93$ & $10: 50$ \\
\hline GW-190 & RG & $11 / 16 / 93$ & $12: 23$ \\
\hline$G W-204$ & T0134 & $11 / 16 / 93$ & $14: 05$ \\
\hline$G W-631$ & RG & $11 / 17 / 93$ & $10: 30$ \\
\hline GW-656 & T0134 & $11 / 17 / 93$ & $11: 00$ \\
\hline GW-193 & T2331 & $11 / 18 / 93$ & $10: 45$ \\
\hline$G W-634$ & RG & $11 / 18 / 93$ & $11: 00$ \\
\hline$G W-632$ & RG & $11 / 18 / 93$ & $13: 45$ \\
\hline GW-707 & T2331 & $11 / 18 / 93$ & $13: 56$ \\
\hline$G W-633$ & RG & $11 / 19 / 93$ & $9: 30$ \\
\hline$G W-658$ & $F F$ & $11 / 19 / 93$ & $10: 00$ \\
\hline GW-765 & GRIDE 1 & $11 / 19 / 93$ & $10: 00$ \\
\hline$G W-508$ & RG & $11 / 19 / 93$ & $10: 15$ \\
\hline$G W-758$ & GRIDG 1 & $11 / 19 / 93$ & $11: 12$ \\
\hline GW-759 & GRIDG 1 & $11 / 19 / 93$ & $13: 30$ \\
\hline GW-761 & GRIDG2 & $11 / 29 / 93$ & $12: 00$ \\
\hline GW-770 & GRIDG 3 & $11 / 29 / 93$ & $12: 45$ \\
\hline$G W-760$ & GRIDG2 & $11 / 30 / 93$ & $11: 35$ \\
\hline
\end{tabular}


APPENDIX D

Sampling sequence

\begin{tabular}{|c|c|c|c|}
\hline $\begin{array}{l}\text { Sampling } \\
\text { Point }\end{array}$ & Location & Date & Time \\
\hline GW-769 & GRIDG 3 & $11 / 30 / 93$ & $13: 33$ \\
\hline$G W-261$ & SY & $12 / 07 / 93$ & $10: 35$ \\
\hline$G W-252$ & $\mathbf{s} 2$ & $12 / 07 / 93$ & $11: 30$ \\
\hline$G W-262$ & SY & $12 / 07 / 93$ & $13: 25$ \\
\hline$G W-255$ & $\mathbf{s 2}$ & $12 / 07 / 93$ & $13: 34$ \\
\hline$G W-338$ & WC & $12 / 07 / 93$ & $15: 00$ \\
\hline$G W-263$ & $\mathbf{S Y}$ & $12 / 07 / 93$ & $15: 30$ \\
\hline GW-617 & EXP & $12 / 07 / 93$ & $16: 30$ \\
\hline$G W-618$ & EXP & $12 / 08 / 93$ & $9: 42$ \\
\hline$G W-251$ & s2 & $12 / 08 / 93$ & $11: 53$ \\
\hline GW-619 & FTF & $12 / 08 / 93$ & $12: 47$ \\
\hline$G W-264$ & SY & $12 / 08 / 93$ & $13: 20$ \\
\hline$G W-620$ & FTF & $12 / 09 / 93$ & $10: 30$ \\
\hline GW-191 & B4 & $12 / 09 / 93$ & $11: 00$ \\
\hline GW-194 & 34 & $12 / 09 / 93$ & $12: 25$ \\
\hline$G W-337$ & wC & $12 / 09 / 93$ & $12: 45$ \\
\hline GW-195 & B4 & $12 / 09 / 93$ & $13: 40$ \\
\hline GW-192 & B4 & $12 / 09 / 93$ & $14: 45$ \\
\hline GW-658 & FF & $09 / 27 / 04$ & $12: 15$ \\
\hline
\end{tabular}


APPENDIX E

GROUNDWATER QUALTY DATA 


\section{EXPLANATION}

LOCATION:

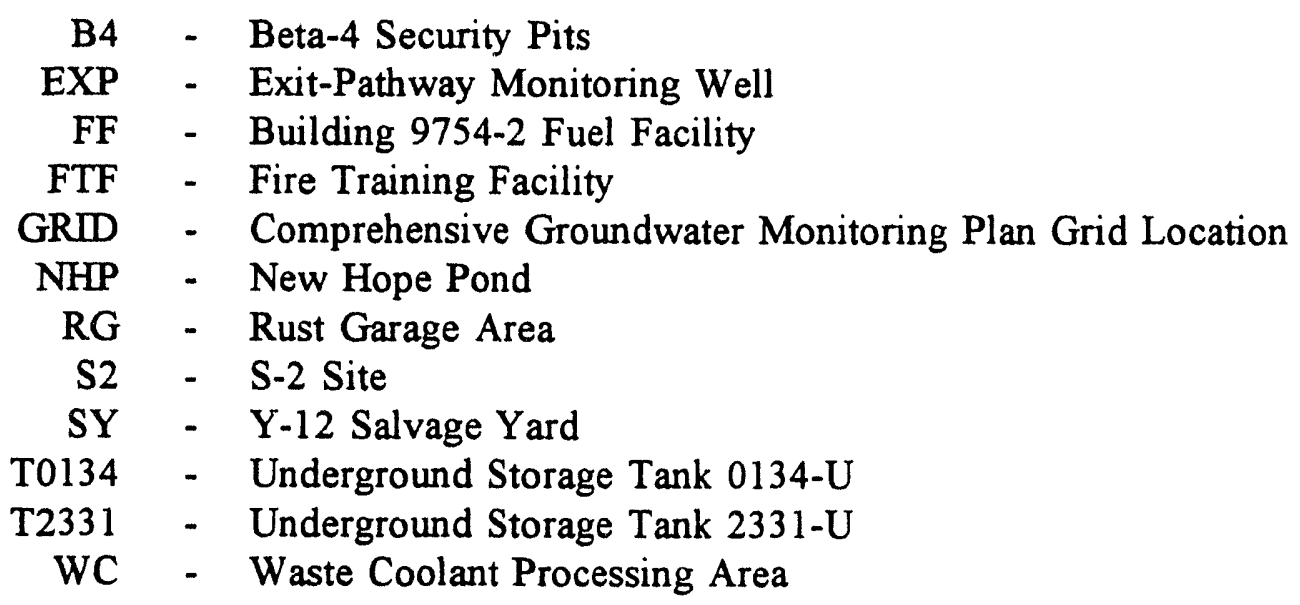

ALL DATA:

- Not Analyzed

VOLATILE ORGANIC COMPOUNDS:

All results in micrograms per Liter (ug/L).

$\mathrm{U}$ - Compound not detected at the reported minimum attainable detection limit.

METALS, MAJOR IONS, MISCELLANEOUS, AND RADIOCHEMICAL PARAMETERS:

All analyses are by Inductively Coupled Plasma (ICP) Spectroscopy unless otherwise noted.

Bicarbonate and carbonate alkalinity reported as $\mathrm{CaCO}_{3}$.

$<\quad$ - Compound not detected at the reported minimum attainable detection limit.

$\mathrm{mg} / \mathrm{L}$ - milligrams per Liter

AAS - Atomic Absorption Spectroscopy

CVAA - Cold Vapor Atomic Absorption

Fluor - Fluorometric

DIS - Dissolved Concentration (Filtered Sample)

TOT - Total Concentration (Unfiltered Sample) 


\section{EXPLANATION (cont'd)}

TPH - Total Petroleum Hydrocarbons

TDS - Total Dissolved Solids

TSS - Total Suspended Solids

Sp. Cond. - Specific Conductance

ppm - parts per million

umho/cm - micromhos per centimeter

NTU - Nephelometric Turbidity Unit

$\mathrm{mV}$ - millivolts

$\mathrm{pCi} / \mathrm{L}$ - picoCuries per liter

$\mathrm{Bq} / \mathrm{L}$ - Becquerels per liter

$\mathrm{CE}+/$ - Counting Error (two standard deviations) 
APPENDIX E.1

TRACE METALS AND MAJOR IONS 
APPENDIX E.I

Groundwater Quality Data, 1993

\begin{tabular}{|c|c|c|c|c|c|c|c|c|}
\hline \multirow{4}{*}{$\begin{array}{l}\text { Sampling Point } \\
\text { - } \\
\text { Location } \\
\text { - } \\
\text { Date Sampled }\end{array}$} & \multicolumn{8}{|c|}{ GW-151 } \\
\hline & \multicolumn{8}{|c|}{ NHP } \\
\hline & \multicolumn{2}{|c|}{$01 / 26 / 93$} & \multicolumn{2}{|c|}{$04 / 22 / 93$} & \multicolumn{2}{|c|}{$08 / 06 / 93$} & \multicolumn{2}{|c|}{$11 / 02 / 93$} \\
\hline & TO'T & DIS & TOT & DIS & TOT & DIS & TOT & DIS \\
\hline METALS (mg/L) & $\cdot$ & - & - & $\cdot$ & - & - & • & • \\
\hline Aluminum & $<0.02$ & $<0.02$ & $<0.02$ & $<0.02$ & 0.034 & $<0.02$ & $<0.02$ & $<0.02$ \\
\hline Antimony & $<0.05$ & $<0.05$ & $<0.05$ & $<0.05$ & $<0.05$ & $<0.05$ & $<0.05$ & $<0.05$ \\
\hline Arsenic & $<0.05$ & $<0.05$ & $<0.05$ & $<0.05$ & $<0.05$ & $<0.05$ & $<0.05$ & $<0.05$ \\
\hline Barium & 0.19 & 0.18 & 0.19 & 0.041 & 0.19 & 0.17 & 0.2 & 0.2 \\
\hline Beryllium & $<0.0003$ & $<0.0003$ & $<0.0003$ & $<0.0003$ & $<0.0003$ & $<0.0003$ & 0.0055 & $<0.0003$ \\
\hline Boron & 0.085 & 0.085 & 0.084 & 0.47 & 0.087 & 0.08 & 0.095 & 0.089 \\
\hline Cadmium (AAS) & $<0.002$ & $<0.002$ & $<0.002$ & $<0.002$ & $<0.002$ & $<0.002$ & $<0.002$ & $<0.002$ \\
\hline Cadmium & $<0.003$ & $<0.003$ & $<0.003$ & $<0.003$ & $<0.003$ & $<0.003$ & 0.0055 & $<0.003$ \\
\hline Chromium (AAS) & $<0.01$ & $<0.01$ & $<0.01$ & $<0.01$ & $<0.01$ & $<0.01$ & $<0.01$ & $<0.01$ \\
\hline Chromium & $<0.01$ & $<0.01$ & $<0.01$ & $<0.01$ & $<0.01$ & $<0.01$ & $<0.01$ & $<0.01$ \\
\hline Cobalt & $<0.005$ & $<0.005$ & $<0.005$ & $<0.005$ & $<0.005$ & $<0.005$ & $<0.005$ & $<0.005$ \\
\hline Copper & $<0.004$ & 0.0055 & $<0.004$ & $<0.004$ & $<0.004$ & $<0.004$ & 0.019 & 0.014 \\
\hline Iron & 0.13 & $<0.005$ & $<0.005$ & 0.039 & 0.036 & $<0.005$ & 0.029 & $<0.005$ \\
\hline Lead (AAS) & $<0.004$ & $<0.004$ & $<0.004$ & $<0.004$ & $<0.004$ & $<0.004$ & $<0.004$ & $<0.004$ \\
\hline Mercury (CVAA) & $<0.0002$ & $<0.0002$ & $<0.0002$ & $<0.0002$ & $<0.0002$ & $<0.0002$ & $<0.0002$ & $<0.0002$ \\
\hline Mol ybdenum & $<0.01$ & $<0.01$ & $<0.01$ & $<0.01$ & $<0.01$ & $<0.01$ & $<0.01$ & $<0.01$ \\
\hline Nickel & $<0.01$ & $<0.01$ & $<0.01$ & $<0.01$ & $<0.01$ & $<0.01$ & $<0.01$ & $<0.01$ \\
\hline Selenium & $<0.05$ & $<0.05$ & $<0.05$ & $<0.05$ & $<0.05$ & $<0.05$ & $<0.05$ & $<0.05$ \\
\hline silver & $<0.006$ & $<0.006$ & $<0.006$ & $<0.006$ & $<0.006$ & $<0.006$ & $<0.006$ & $<0.006$ \\
\hline strontium & 0.53 & 0.52 & 0.52 & 0.069 & 0.53 & 0.48 & 0.58 & 0.58 \\
\hline Thorium & $<0.2$ & $<0.2$ & $<0.2$ & $<0.2$ & $<0.2$ & $<0.2$ & $<0.2$ & $<0.2$ \\
\hline Uranium (Fluor) & $<0.001$ & $<0.001$ & $<0.001$ & $<0.001$ & $<0.001$ & 0.002 & $<0.001$ & $<0.001$ \\
\hline Vanadium & $<0.005$ & $<0.005$ & $<0.005$ & $<0.005$ & $<0.005$ & $<0.005$ & 0.007 & $<0.005$ \\
\hline zinc & 0.0036 & 0.0057 & 0.0021 & 0.012 & 0.0038 & 0.014 & 0.014 & $<0.002$ \\
\hline MAJOR IONS $(\mathrm{mg} / \mathrm{L})$ & $\cdot$ & $\cdot$ & - & - & - & - & - & - \\
\hline Alkalinity-HCO3 & 218 & . & 213 & • & 215 & $\cdot$ & 213 & • \\
\hline Alkalinity- $\mathrm{CO} 3$ & $<1$ & $\cdot$ & $<1$ & • & $<1$ & $\cdot$ & $<1$ & - \\
\hline Calcium & 56 & 54 & 53 & 0.86 & 53 & 49 & 56 & 56 \\
\hline Chloride & 19 & $\cdot$ & 13 & • & 13 & - & 16 & - \\
\hline Fluoride & 0.1 & $\cdot$ & 0.1 & • & 0.1 & - & 0.1 & • \\
\hline Magnesium & 24 & 24 & 24 & 0.21 & 24 & 22 & 25 & 25 \\
\hline Manganese & $<0.001$ & $<0.001$ & $<0.001$ & 0.0057 & 0.0019 & $<0.001$ & 0.0072 & 0.0013 \\
\hline Nitrate-N & 0.7 & $\cdot$ & 0.86 & • & 0.92 & • & 1 & • \\
\hline Potassium & 2.1 & 1.7 & 2.3 & 1.7 & 2.2 & 2 & 2.1 & 2.1 \\
\hline Sodium & 6.8 & 6.9 & 7 & 270 & 7.1 & 6.6 & 6.9 & 6.9 \\
\hline Sulfate & 20 & $\cdot$ & 21 & • & 22 & • & 17 & • \\
\hline
\end{tabular}

(CONTINUED) 
APPENDIX B. 1

Groundwater Quality Data, 1993

\begin{tabular}{|c|c|c|c|c|c|c|c|c|}
\hline \multirow{4}{*}{$\begin{array}{l}\text { Sampling Point } \\
\text { Location } \\
\text { - } \\
\text { Date Sampled }\end{array}$} & \multicolumn{8}{|c|}{$G w-168$} \\
\hline & \multicolumn{8}{|c|}{ NHP } \\
\hline & \multicolumn{2}{|c|}{$01 / 22 / 93$} & \multicolumn{2}{|c|}{$05 / 14 / 93$} & \multicolumn{2}{|c|}{$08 / 19 / 93$} & \multicolumn{2}{|c|}{$11 / 02 / 93$} \\
\hline & TOT & DIS & TOT & DIS & TOT & DIS & TOT & DIS \\
\hline METALS (mg/I) & - & • & - & - & - & - & - & • \\
\hline Aluminum & 0.029 & $<0.02$ & 0.024 & 0.053 & $<0.02$ & $<0.02$ & $<0.02$ & $<0.02$ \\
\hline Antimony & $<0.05$ & $<0.05$ & $<0.05$ & $<0.05$ & $<0.05$ & $<0.05$ & $<0.05$ & $<0.05$ \\
\hline Arsenic & $<0.05$ & $<0.05$ & $<0.05$ & 0.05 & $<0.05$ & $<0.05$ & $<0.05$ & $<0.05$ \\
\hline Barium & 0.12 & 0.12 & 0.12 & 0.12 & 0.13 & 0.13 & 0.12 & 0.12 \\
\hline Beryllium & $<0.0003$ & $<0.0003$ & $<0.0003$ & $<0.0003$ & $<0.0003$ & $<0.0003$ & $<0.0003$ & $<0.0003$ \\
\hline Boron & 0.057 & 0.051 & 0.042 & 0.045 & 0.049 & 0.044 & 0.061 & 0.057 \\
\hline Cadmium (AAS) & $<0.002$ & $<0.002$ & $<0.002$ & $<0.002$ & $<0.002$ & $<0.002$ & $<0.002$ & $<0.002$ \\
\hline Cadmium & $<0.003$ & $<0.003$ & $<0.003$ & $<0.003$ & $<0.003$ & $<0.003$ & $<0.003$ & $<0.003$ \\
\hline Chromium (AAS) & $<0.01$ & $<0.01$ & $<0.01$ & $<0.01$ & $<0.01$ & $<0.01$ & $<0.01$ & $<0.01$ \\
\hline Chromium & 0.03 & $<0.01$ & $<0.01$ & $<0.01$ & $<0.01$ & $<0.01$ & $<0.01$ & $<0.01$ \\
\hline Cobalt & $<0.005$ & $<0.005$ & $<0.005$ & $<0.005$ & $<0.005$ & $<0.005$ & $<0.005$ & $<0.005$ \\
\hline Copper & 0.004 & $<0.004$ & $<0.004$ & $<0.004$ & $<0.004$ & $<0.004$ & $<0.004$ & $<0.004$ \\
\hline Iron & 0.52 & 0.18 & 0.19 & 0.13 & 0.16 & 0.087 & 0.35 & 0.15 \\
\hline Lead (AAS) & $<0.004$ & $<0.004$ & $<0.004$ & $<0.004$ & $<0.004$ & $<0.004$ & $<0.004$ & $<0.004$ \\
\hline Mercury (CVAA) & $<0.0002$ & $<0.0002$ & $<0.0002$ & $<0.0002$ & $<0.0002$ & $<0.0002$ & $<0.0002$ & $<0.0002$ \\
\hline Molybdenum & $<0.01$ & $<0.01$ & $<0.01$ & $<0.01$ & $<0.01$ & $<0.01$ & $<0.01$ & $<0.01$ \\
\hline Nickel & 0.014 & $<0.01$ & $<0.01$ & $<0.01$ & $<0.01$ & $<0.01$ & $<0.01$ & $<0.01$ \\
\hline Selenium & $<0.05$ & $<0.05$ & $<0.05$ & $<0.05$ & $<0.05$ & $<0.05$ & $<0.05$ & $<0.05$ \\
\hline silver & $<0.006$ & $<0.006$ & $<0.006$ & $<0.006$ & $<0.006$ & $<0.006$ & $<0.006$ & $<0.006$ \\
\hline Strontium & 0.48 & 0.49 & 0.45 & 0.45 & 0.51 & 0.51 & 0.53 & 0.52 \\
\hline Thoriun & $<0.2$ & $<0.2$ & $<0.2$ & $<0.2$ & $<0.2$ & $<0,2$ & $<0.2$ & $<0.2$ \\
\hline Uranium (Fluor) & $<0.001$ & 0.001 & $<0.001$ & $<0.001$ & 0.001 & $<0.001$ & $<0.001$ & $<0.001$ \\
\hline Vanadium & $<0.005$ & $<0.005$ & $<0.005$ & $<0.005$ & $<0.005$ & $<0.005$ & $<0.005$ & $<0.005$ \\
\hline $\operatorname{zin}$ & 0.0094 & 0.0072 & 0.0083 & 0.0079 & 0.0073 & 0.0064 & 0.0091 & 0.0061 \\
\hline MAJOR IONS (mg/L) & - & - & $\cdot$ & - & - & - & - & - \\
\hline Alkalinity-HCO3 & 275 & . & 265 & • & 275 & . & 281 & • \\
\hline Alkalinity-CO3 & $<1$ & • & $<1$ & - & $<1$ & - & $<1$ & • \\
\hline Calcium & 76 & 77 & 76 & 75 & 79 & 81 & 81 & 80 \\
\hline Chloride & 21 & - & 13 & - & 12 & - & 13 & - \\
\hline Fluoride & $<0.1$ & • & $<0.1$ & - & 0.1 & - & 0.1 & • \\
\hline Magnesium & 21 & 21 & 20 & 20 & 22 & 22 & 21 & 21 \\
\hline Manganese & 0.0051 & 0.0017 & 0.0012 & 0.0016 & 0.0014 & 0.0017 & 0.001 & $<0.001$ \\
\hline Nitrate-N & 0.51 & • & 0.42 & • & 0.41 & • & 0.5 & • \\
\hline Potassium & 2.4 & 2.4 & 1.5 & 1.6 & 2.2 & 2 & 2 & 2.2 \\
\hline Sodium & 6.4 & 6.5 & 4.5 & 4.5 & 5.9 & 5.9 & 5.7 & 5.8 \\
\hline Sulfate & 12 & • & 11 & • & 8.4 & • & 10 & • \\
\hline
\end{tabular}

( CONTINUED) 
APPENDIX E.

Groundwater Quality Data, 1993

\begin{tabular}{|c|c|c|c|c|c|c|c|c|}
\hline \multirow{4}{*}{$\begin{array}{l}\text { Sampling Point } \\
\text { Iocation } \\
\text { - } \\
\text { Date Sampled }\end{array}$} & \multicolumn{8}{|c|}{ GW-169 } \\
\hline & \multicolumn{8}{|c|}{ EXP } \\
\hline & \multicolumn{2}{|c|}{$01 / 23 / 93$} & \multicolumn{2}{|c|}{$05 / 21 / 93$} & \multicolumn{2}{|c|}{$08 / 19 / 93$} & \multicolumn{2}{|c|}{$11 / 01 / 93$} \\
\hline & TOT & DIS & TOT & DIS & TOT & DIS & TOT & DIS \\
\hline $\operatorname{METALS}(\mathrm{mg} / \mathrm{L})$ & - & - & $\cdot$ & - & - & - & - & - \\
\hline Aluminum & 75 & 0.023 & 14 & 0.041 & 71 & $<0.02$ & 29 & 0.045 \\
\hline Antimony & $<0.05$ & $<0.05$ & $<0.05$ & $<0.05$ & $<0.05$ & $<0.05$ & $<0.05$ & $<0.05$ \\
\hline Arsenic & 0.063 & $<0.05$ & $<0.05$ & $<0.05$ & $<0.05$ & $<0.05$ & $<0.05$ & $<0.05$ \\
\hline Barium & 0.33 & 0.023 & 0.11 & 0.032 & 0.34 & 0.03 & 0.18 & 0.026 \\
\hline Beryllium & 0.0092 & $<0.0003$ & 0.0026 & $<0.0003$ & 0.0092 & $<0.0003$ & 0.005 & $<0.0003$ \\
\hline Boron & 0.036 & 0.022 & 0.05 & 0.028 & 0.036 & $<0.004$ & 0.02 & 0.014 \\
\hline Cadmium (AAS) & 0.0021 & $<0.002$ & $<0.002$ & $<0.002$ & $<0.002$ & $<0.002$ & $<0.002$ & $<0.002$ \\
\hline Cadmium & 0.025 & $<0.003$ & $<0.003$ & $<0.003$ & 0.025 & $<0.003$ & 0.0035 & $<0.003$ \\
\hline Chromium (AAS) & 0.049 & $<0.01$ & 0.024 & $<0.01$ & 0.067 & $<0.01$ & 0.078 & $<0.01$ \\
\hline Chromium & 0.085 & $<0.01$ & 0.016 & $<0.01$ & 0.087 & $<0.01$ & 0.038 & $<0.01$ \\
\hline Cobalt & 0.052 & $<0.005$ & 0.015 & $<0.005$ & 0.054 & $<0.005$ & 0.031 & $<0.005$ \\
\hline Copper & 0.086 & $<0.004$ & 0.028 & $<0.004$ & 0.083 & $<0.004$ & 0.044 & $<0.004$ \\
\hline Iron & 79 & 0.014 & 24 & $<0.005$ & 83 & $<0.005$ & 45 & 0.11 \\
\hline Lead (AAS) & 0.15 & $<0.004$ & 0.042 & $<0.004$ & 0.18 & $<0.004$ & 0.091 & $<0.004$ \\
\hline Mercury (CVAA) & $<0.0002$ & $<0.0002$ & $<0.0002$ & $<0.0002$ & 0.00031 & $<0.0002$ & $<0.0002$ & $<0.0002$ \\
\hline Mol ybdenum & $<0.01$ & $<0.01$ & $<0.01$ & $<0.01$ & $<0.01$ & $<0.01$ & $<0.01$ & $<0.01$ \\
\hline Nickel & 0.11 & $<0.01$ & 0.032 & $<0.01$ & 0.11 & $<0.01$ & 0.054 & $<0.01$ \\
\hline Selenium & $<0.05$ & $<0.05$ & $<0.05$ & $<0.05$ & $<0.05$ & $<0.05$ & $<0.05$ & $<0.05$ \\
\hline Silver & $<0.006$ & $<0.006$ & $<0.006$ & $<0.006$ & $<0.006$ & $<0.006$ & $<0.006$ & $<0.006$ \\
\hline strontium & 0.09 & 0.06 & 0.093 & 0.086 & 0.12 & 0.083 & 0.093 & 0.078 \\
\hline Thorium & $<0.2$ & $<0.2$ & $<0.2$ & $<0.2$ & $<0.2$ & $<0.2$ & $<0.2$ & $<0.2$ \\
\hline Uranium (Fluor) & 0.002 & $<0.001$ & $<0.001$ & $<0.001$ & $<0.001$ & 0.002 & 0.001 & $<0.001$ \\
\hline Vanadium & 0.13 & $<0.005$ & 0.034 & $<0.005$ & 0.13 & $<0.005$ & 0.064 & $<0.005$ \\
\hline Zinc & 0.36 & 0.0056 & 0.12 & 0.0054 & 0.36 & 0.0082 & 0.21 & 0.0046 \\
\hline MAJOR IONS $(\mathrm{mg} / \mathrm{L})$ & . & . & . & . & . & . & . & . \\
\hline Alkalinity-HCO3 & 145 & . & 168 & . & 190 & . & $175^{\circ}$ & . \\
\hline Alkalinity-CO3 & $<1$ & $\cdot$ & $<1$ & $\cdot$ & $<1$ & • & $<1$ & • \\
\hline Calcium & 61 & 50 & 75 & 72 & 81 & 67 & 71 & 63 \\
\hline Chloride & 1.7 & • & 1.7 & • & 1.7 & - & 1.5 & • \\
\hline Fluoride & $<0.1$ & . & $<0.1$ & • & $<0.1$ & . & $<0.1$ & • \\
\hline Magnesium & 9.6 & 2.7 & 5.3 & 3.6 & 11 & 4 & 6.4 & 3.1 \\
\hline Manganese & 3.1 & 0.0026 & 0.85 & 0.0017 & $3 \cdot 3$ & 0.0012 & 1.9 & 0.0053 \\
\hline Nitrate-N & 1.1 & . & 0.79 & . & 0.71 & . & 0.91 & • \\
\hline Potassium & 15 & 2.4 & 5.3 & 2.9 & 14 & 2 & 7.4 & 2.2 \\
\hline Sodium & 1.3 & 1.3 & 1.6 & 1.6 & 1.5 & 1.4 & 1.3 & 1.2 \\
\hline sulfate & 4.9 & $\cdot$ & 7.4 & • & 5.7 & • & 5.9 & • \\
\hline
\end{tabular}

(CONT INUED) 
APPENDIX E.1

Groundwater Quality Data, 1993

\begin{tabular}{|c|c|c|c|c|c|c|c|c|}
\hline \multirow{4}{*}{$\begin{array}{l}\text { Sampling Point } \\
\text { Location } \\
\text { - } \\
\text { Date Sampled }\end{array}$} & \multicolumn{8}{|c|}{ GW- 170} \\
\hline & \multicolumn{8}{|c|}{ EXP } \\
\hline & \multicolumn{2}{|c|}{$01 / 23 / 93$} & \multicolumn{2}{|c|}{$05 / 24 / 93$} & \multicolumn{2}{|c|}{$08 / 21 / 93$} & \multicolumn{2}{|c|}{$11 / 04 / 93$} \\
\hline & TOT & DIS & TOT & DIS & TOT & DIS & TOT & DIS \\
\hline METALS (mg/L) & $\cdot$ & $\cdot$ & - & . & $\cdot$ & - & - & - \\
\hline Aluminum & 0.057 & $<0.02$ & $<0.02$ & $<0.02$ & $<0.02$ & $<0.02$ & $<0.02$ & $<0.02$ \\
\hline Antimony & $<0.05$ & $<0.05$ & $<0.05$ & $<0.05$ & $<0.05$ & $<0.05$ & $<0.05$ & $<0.05$ \\
\hline Arsenic & $<0.05$ & $<0.05$ & $<0.05$ & $<0.05$ & $<0.05$ & $<0.05$ & $<0.05$ & $<0.05$ \\
\hline Barium & 0.091 & 0.089 & 0.086 & 0.084 & 0.099 & 0.098 & 0.092 & 0.093 \\
\hline Beryllium & $<0.0003$ & $<0.0003$ & $<0.0003$ & $<0.0003$ & $<0.0003$ & $<0.0003$ & $<0.0003$ & $<0.0003$ \\
\hline Boron & 0.091 & 0.088 & 0.098 & 0.083 & 0.095 & 0.085 & 0.092 & 0.092 \\
\hline Cadmium (AAS) & $<0.002$ & $<0.002$ & $<0.002$ & $<0.002$ & $<0.002$ & $<0.002$ & $<0.002$ & $<0.002$ \\
\hline Cadmium & $<0.003$ & $<0.003$ & $<0.003$ & $<0.003$ & $<0.003$ & $<0.003$ & $<0.003$ & $<0.003$ \\
\hline Chromium (AAS) & $<0.01$ & $<0.01$ & $<0.01$ & $<0.01$ & $<0.01$ & $<0.01$ & $<0.01$ & $<0.01$ \\
\hline Chromium & $<0.01$ & $<0.01$ & $<0.01$ & $<0.01$ & $<0.01$ & $<0.01$ & $<0.01$ & $<0.01$ \\
\hline Cobalt & $<0.005$ & $<0.005$ & $<0.005$ & $<0.005$ & $<0.005$ & $<0.005$ & $<0.005$ & $<0.005$ \\
\hline Copper & $<0.004$ & $<0.004$ & $<0.004$ & $<0.004$ & 0.0062 & $<0.004$ & $<0.004$ & $<0.004$ \\
\hline Iron & 0.12 & $<0.005$ & 0.057 & $<0.005$ & 0.1 & $<0.005$ & 0.073 & $<0.005$ \\
\hline Lead (AAS) & $<0.004$ & $<0.004$ & $<0.004$ & $<0.004$ & $<0.004$ & $<0.004$ & $<0.004$ & $<0.004$ \\
\hline Mercury (CVAA) & $<0.0002$ & $<0.0002$ & $<0.0002$ & $<0.0002$ & $<0.0002$ & $<0.0002$ & $<0.0002$ & $<0.0002$ \\
\hline Molybdenum & $<0.01$ & $<0.01$ & $<0.01$ & $<0.01$ & $<0.01$ & $<0.01$ & $<0.01$ & $<0.01$ \\
\hline Nickel & $<0.01$ & $<0.01$ & $<0.01$ & $<0.01$ & $<0.01$ & $<0.01$ & $<0.01$ & $<0.01$ \\
\hline Selenium & $<0.05$ & $<0.05$ & $<0.05$ & $<0.05$ & $<0.05$ & $<0.05$ & $<0.05$ & $<0.05$ \\
\hline Silver & $<0.006$ & $<0.006$ & $<0.006$ & $<0.006$ & $<0.006$ & $<0.006$ & $<0.006$ & $<0.006$ \\
\hline strontium & 0.5 & 0.49 & 0.51 & 0.5 & 0.55 & 0.55 & 0.55 & 0.56 \\
\hline Thorium & $<0.2$ & $<0.2$ & $<0.2$ & $<0.2$ & $<0.2$ & $<0.2$ & $<0.2$ & $<0.2$ \\
\hline Uranium (Fluor) & 0.001 & $<0.001$ & 0.001 & 0.001 & $<0.001$ & $<0.001$ & $<0.001$ & $<0.001$ \\
\hline Vanadium & $<0.005$ & $<0.005$ & $<0.005$ & $<0.005$ & $<0.005$ & $<0.005$ & $<0.005$ & $<0.005$ \\
\hline zinc & 0.0076 & 0.0068 & 0.01 & 0.012 & 0.012 & 0.019 & $<0.002$ & 0.004 \\
\hline MAJOR IONS $(\mathrm{mg} / \mathrm{L})$ & . & $\cdot$ & . & . & . & . & . & . \\
\hline Alkalinity- $\mathrm{HCO} 3$ & 211 & . & 209 & . & 216 & . & 213 & . \\
\hline Alkalinity-CO3 & $<1$ & $\cdot$ & $<1$ & . & $<1$ & $\cdot$ & $<1$ & • \\
\hline Calcium & 45 & 45 & 45 & 44 & 49 & 48 & 47 & 48 \\
\hline Chloride & 16.2 & $\cdot$ & 12.1 & $\cdot$ & 14.7 & . & 17 & • \\
\hline Fluoride & 0.2 & $\cdot$ & 0.2 & $\cdot$ & 0.1 & . & 0.2 & • \\
\hline Magnesium & 23 & 23 & 23 & 23 & 25 & 25 & 23 & 24 \\
\hline Manganese & 0.0021 & 0.0018 & $<0.001$ & $<0.001$ & $<0.001$ & $<0.001$ & $<0.001$ & $<0.001$ \\
\hline Nitrate-N & 0.79 & • & 0.66 & $\cdot$ & 0.76 & . & 1.1 & • \\
\hline Potassium & 2.2 & 2.1 & 2 & 1.9 & 1.5 & 1.5 & 1.7 & 1.7 \\
\hline Sodium & 17 & 16 & 14 & 14 & 17 & 17 & 16 & 16 \\
\hline Sulfate & 19.8 & . & 17.6 & $\cdot$ & 18.2 & $\cdot$ & 20 & - \\
\hline
\end{tabular}

( CONTINUED) 
APPENDIX E. 1

Groundwater Quality Data, 1993

\begin{tabular}{|c|c|c|c|c|c|c|c|c|}
\hline \multirow{4}{*}{$\begin{array}{l}\text { Sampling Point } \\
\text { Location } \\
\text { - } \\
\text { Date Sampled }\end{array}$} & \multicolumn{8}{|c|}{ GW-183 } \\
\hline & \multicolumn{8}{|c|}{ FF } \\
\hline & \multicolumn{2}{|c|}{$03 / 10 / 93$} & \multicolumn{2}{|c|}{$06 / 21 / 93$} & \multicolumn{2}{|c|}{$09 / 22 / 93$} & \multicolumn{2}{|c|}{$11 / 16 / 93$} \\
\hline & TOT & DIS & TOT & DIS & TOT & DIS & TOT & DIS \\
\hline MBTALS (mg/L) & - & - & - & - & $\cdot$ & $\cdot$ & . & - \\
\hline Aluminum & 0.71 & $<0.02$ & 0.27 & 0.037 & 1.2 & 0.059 & 0.23 & $<0.02$ \\
\hline Antimony & $<0.05$ & $<0.05$ & $<0.05$ & $<0.05$ & $<0.05$ & $<0.05$ & $<0.05$ & $<0.05$ \\
\hline Arsenic & $<0.05$ & $<0.05$ & $<0.05$ & $<0.05$ & $<0.05$ & $<0.05$ & $<0.05$ & $<0.05$ \\
\hline Barium & 0.23 & 0.22 & 0.22 & 0.22 & 0.24 & 0.23 & 0.21 & 0.22 \\
\hline Beryllium & $<0.0003$ & $<0.0003$ & $<0.0003$ & $<0.0003$ & $<0.0003$ & $<0.0003$ & $<0.0003$ & $<0.0003$ \\
\hline Boron & 0.038 & 0.045 & 0.053 & 0.053 & 0.051 & 0.05 & 0.082 & 0.043 \\
\hline Cadmium (AAS) & $<0.002$ & $<0.002$ & $<0.002$ & $<0.002$ & $<0.002$ & $<0.002$ & $<0.002$ & $<0.002$ \\
\hline Cadmium & $<0.003$ & $<0.003$ & $<0.003$ & $<0.003$ & $<0.003$ & $<0.003$ & $<0.003$ & $<0.003$ \\
\hline Chromium (AAS) & $<0.01$ & $<0.01$ & $<0.01$ & $<0.01$ & $<0.01$ & $<0.01$ & $<0.01$ & $<0.01$ \\
\hline Chromium & $<0.01$ & $<0.01$ & $<0.01$ & $<0.01$ & $<0.01$ & $<0.01$ & $<0.01$ & $<0.01$ \\
\hline Cobalt & $<0.005$ & $<0.005$ & $<0.005$ & $<0.005$ & $<0.005$ & $<0.005$ & $<0.005$ & $<0.005$ \\
\hline Copper & $<0.004$ & $<0.004$ & 0.0047 & $<0.004$ & $<0.004$ & $<0.004$ & $<0.004$ & $<0.004$ \\
\hline Iron & 1.4 & 0.85 & 1.1 & 0.87 & 2.1 & 0.53 & 0.52 & 0.061 \\
\hline Lead (AAS) & $<0.004$ & $<0.004$ & $<0.004$ & $<0.004$ & $<0.004$ & $<0.004$ & $<0.004$ & $<0.004$ \\
\hline Mercury (CVAA) & $<0.0002$ & $<0.0002$ & $<0.0002$ & $<0.0002$ & $<0.0002$ & $<0.0002$ & $<0.0002$ & $<0.0002$ \\
\hline Molybdenum & $<0.01$ & $<0.01$ & $<0.01$ & $<0.01$ & $<0.01$ & $<0.01$ & $<0.01$ & $<0.01$ \\
\hline Nickel & $<0.01$ & $<0.01$ & $<0.01$ & $<0.01$ & $<0.01$ & $<0.01$ & 0.014 & $<0.01$ \\
\hline Selenium & $<0.05$ & $<0.05$ & $<0.05$ & $<0.05$ & $<0.05$ & $<0.05$ & $<0.05$ & $<0.05$ \\
\hline Silver & $<0.006$ & $<0.006$ & $<0.006$ & $<0.006$ & $<0.006$ & $<0.006$ & $<0.006$ & $<0.006$ \\
\hline strontium & 0.68 & 0.64 & 0.65 & 0.65 & 0.73 & 0.72 & 0.7 & 0.71 \\
\hline Thorium & $<0.2$ & $<0.2$ & $<0.2$ & $<0.2$ & $<0.2$ & $<0.2$ & $<0.2$ & $<0.2$ \\
\hline Oranium (Fluor) & 0.001 & 0.002 & 0.002 & 0.002 & $<0.001$ & 0.002 & 0.004 & 0.003 \\
\hline Vanadium & $<0.005$ & $<0.005$ & $<0.005$ & $<0.005$ & $<0.005$ & $<0.005$ & $<0.005$ & $<0.005$ \\
\hline zinc & 0.0057 & 0.0072 & 0.0088 & 0.0046 & 0.0096 & 0.0071 & 0.031 & 0.029 \\
\hline MAJOR IONS (mg/L) & . & - & . & - & . & - & - & - \\
\hline Alkalinity- $\mathrm{HCO} 3$ & 435 & . & 449 & . & 460 & $\cdot$ & 461 & • \\
\hline Alkalinity-CO3 & $<1$ & - & $<1$ & $\cdot$ & $<1$ & • & $<1$ & • \\
\hline Calcium & 140 & 140 & 140 & 140 & 150 & 150 & 150 & 150 \\
\hline Chloride & 35.4 & - & 30 & - & 30 & • & 33 & • \\
\hline Fluoride & $<0.1$ & • & $<0.1$ & $\cdot$ & $<0.1$ & - & $<0.1$ & • \\
\hline Magnesium & 24 & 23 & 24 & 24 & 25 & 24 & 26 & 27 \\
\hline Manganese & 0.49 & 0.51 & 0.31 & 0.35 & 0.18 & 0.17 & 0.23 & 0.25 \\
\hline Nitrate-N & $<0.2$ & • & $<0.2$ & • & $<0.2$ & • & $<0.2$ & • \\
\hline Potassium & 2.1 & 2.9 & 1.9 & 1.6 & $2 \cdot 3$ & 2 & 1.9 & 2.2 \\
\hline Sodium & 27 & 25 & 24 & 24 & 28 & 27 & 25 & 26 \\
\hline Sulfate & 26.6 & . & 29 & - & 29 & • & 25 & • \\
\hline
\end{tabular}

(CONTINUED) 
APPENDIX E.1

Groundwater Quality Data, 1993

\begin{tabular}{|c|c|c|c|c|c|c|c|c|}
\hline \multirow{4}{*}{$\begin{array}{l}\text { Sampling Point } \\
\text { - } \\
\text { Location } \\
\text { Date Sampled }\end{array}$} & \multicolumn{8}{|c|}{ GW-190 } \\
\hline & \multicolumn{8}{|c|}{ RG } \\
\hline & \multicolumn{2}{|c|}{$03 / 01 / 93$} & \multicolumn{2}{|c|}{$06 / 17 / 93$} & \multicolumn{2}{|c|}{$09 / 15 / 93$} & \multicolumn{2}{|c|}{$11 / 16 / 93$} \\
\hline & TOT & DIS & TOT & DIS & TOT & DIS & TOT & DIS \\
\hline MRTALS (mg/I) & - & $\cdot$ & - & $\cdot$ & - & - & - & - \\
\hline Aluminum & 0.13 & 0.044 & 0.65 & $<0.02$ & 0.073 & $<0.02$ & 0.6 & 0.034 \\
\hline Antimony & $<0.04$ & $<0.05$ & $<0.05$ & $<0.05$ & $<0.05$ & $<0.05$ & $<0.05$ & $<0.05$ \\
\hline Arsenic & $<0.04$ & $<0.05$ & $<0.05$ & $<0.05$ & $<0.05$ & $<0.05$ & $<0.05$ & $<0.05$ \\
\hline Barium & 0.092 & 0.12 & 0.13 & 0.11 & 0.13 & 0.12 & 0.18 & 0.14 \\
\hline Beryllium & $<0.00024$ & $<0.0003$ & $<0.0003$ & 0.00031 & 0.0003 & $<0.0003$ & 0.0045 & 0.0022 \\
\hline Boron & 0.011 & 0.014 & 0.013 & 0.049 & 0.015 & $0.0: 4$ & 0.042 & 0.038 \\
\hline Cadmium (AAS) & $<0.002$ & $<0.002$ & $<0.002$ & $<0.002$ & $<0.002$ & $<0.002$ & $<0.002$ & $<0.002$ \\
\hline Cadmium & $<0.0024$ & $<0.003$ & $<0.003$ & $<0.003$ & $<0.003$ & $<0.003$ & $<0.003$ & $<0.003$ \\
\hline Chromium (AAS) & $<0.01$ & $<0.01$ & $<0.01$ & $<0.01$ & $<0.01$ & $<0.01$ & $<0.01$ & $<0.01$ \\
\hline Chromium & $<0.008$ & $<0.01$ & $<0.01$ & $<0.01$ & $<0.01$ & $<0.01$ & 0.013 & 0.011 \\
\hline Cobalt & $<0.004$ & $<0.005$ & $<0.005$ & $<0.005$ & $<0.005$ & $<0.005$ & 0.0054 & $<0.005$ \\
\hline Copper & $<0.0032$ & $<0.004$ & $<0.004$ & $<0.004$ & 0.023 & 0.0067 & 0.0081 & 0.0076 \\
\hline Iron & 0.34 & 0.039 & 0.92 & 0.028 & 0.38 & 0.032 & 2.1 & 0.39 \\
\hline Lead (AAS) & $<0.004$ & 0.0049 & $<0.004$ & $<0.004$ & $<0.004$ & $<0.004$ & $<0.004$ & $<0.004$ \\
\hline Mercury (CVAA) & $<0.0002$ & $<0.0002$ & $<0.0002$ & $<0.0002$ & $<0.0002$ & $<0.0002$ & $<0.0002$ & $<0.0002$ \\
\hline M1ybdenum & $<0.008$ & $<0.01$ & $<0.01$ & $<0.01$ & $<0.01$ & $<0.01$ & $<0.01$ & $<0.01$ \\
\hline Nickel & $<0.008$ & $<0.01$ & $<0.01$ & $<0.01$ & $<0.01$ & 0.01 & 0.012 & $<0.01$ \\
\hline Selenium & $<0.04$ & $<0.05$ & $<0.05$ & $<0.05$ & $<0.05$ & $<0.05$ & $<0.05$ & $<0.05$ \\
\hline silver & $<0.0048$ & $<0.006$ & $<0.006$ & $<0.006$ & $<0.006$ & $<0.006$ & 0.009 & $<0.006$ \\
\hline Strontium & 0.063 & 0.095 & 0.11 & 0.11 & 0.14 & 0.12 & 0.12 & 0.12 \\
\hline Thorium & $<0.16$ & $<0.2$ & $<0.2$ & $<0.2$ & $<0.2$ & $<0.2$ & $<0.2$ & $<0.2$ \\
\hline Uranium (Fluor) & $<0.001$ & $<0.001$ & $<0.001$ & $<0.001$ & $<0.001$ & $<0.001$ & 0.001 & 0.001 \\
\hline Vanadium & $<0.004$ & $<0.005$ & $<0.005$ & $<0.005$ & $<0.005$ & $<0.005$ & 0.0051 & $<0.005$ \\
\hline zinc & 0.016 & 0.043 & 0.037 & 0.021 & 0.036 & 0.021 & 0.018 & 0.014 \\
\hline MAJOR IONS $(\mathrm{mg} / \mathrm{L})$ & . & . & . & . & - & . & . & . \\
\hline Alkalinity- $\mathrm{HCO} 3$ & 14 & . & 112 & . & 112 & $\cdot$ & 121 & • \\
\hline Alkalinity- $\mathrm{CO} 3$ & $<1$ & - & $<1$ & - & $<1$ & . & $<1$ & - \\
\hline Calcium & 17 & 29 & 36 & 36 & 44 & 36 & 32 & 33 \\
\hline Chloride & $5 \cdot 3$ & $\cdot$ & 6.2 & - & 6.2 & $\cdot$ & 5.9 & - \\
\hline Fluoride & 0.2 & $\cdot$ & 0.2 & - & 0.1 & $\cdot$ & 0.2 & - \\
\hline Magnesium & 3.7 & 5.7 & 6.2 & 6.1 & 6.4 & 6.3 & 5.8 & 6 \\
\hline Manganese & 0.48 & 0.42 & 0.39 & 0.18 & 0.27 & 0.35 & 1.1 & 0.96 \\
\hline Nitrate-N & $<0.2$ & . & $<0.2$ & $\cdot$ & 0.26 & $\cdot$ & $<0.2$ & • \\
\hline Potassium & 0.72 & 1.2 & 0.64 & 0.85 & 1.1 & 1.2 & 1.1 & 0.97 \\
\hline Sodium & 4.3 & 6.7 & 8.3 & 8.5 & 8.8 & 8.2 & 8.6 & 8.6 \\
\hline Sulfate & 15.3 & • & 17 & • & 19 & $\cdot$ & 16.3 & • \\
\hline
\end{tabular}

(CONTINUED) 
APPENDIX E.1

Groundwater Quality Data, 1993

\begin{tabular}{|c|c|c|c|c|c|c|c|c|}
\hline \multirow{4}{*}{$\begin{array}{l}\text { Sampling Point } \\
\text { - } \\
\text { Location } \\
\text { - } \\
\text { Date Sampled }\end{array}$} & \multicolumn{8}{|c|}{ GW-191 } \\
\hline & \multicolumn{8}{|c|}{ B4 } \\
\hline & \multicolumn{2}{|c|}{$01 / 12 / 93$} & \multicolumn{2}{|c|}{$04 / 12 / 93$} & \multicolumn{2}{|c|}{$07 / 08 / 93$} & \multicolumn{2}{|c|}{$12 / 09 / 93$} \\
\hline & TOT & DIS & TOT & DIS & TOT & DIS & TOT & DIS \\
\hline NETALS $(\mathrm{mg} / \mathrm{L})$ & - & - & $\cdot$ & . & $\cdot$ & - & - & - \\
\hline Aluminum & 0.83 & 0.022 & 42 & 0.051 & 2.1 & $<0.02$ & 2.9 & $<0.02$ \\
\hline Antimony & $<0.05$ & $<0.05$ & $<0.05$ & $<0.05$ & $<0.05$ & $<0.05$ & $<0.05$ & $<0.05$ \\
\hline Arsenic & $<0.05$ & $<0.05$ & $<0.05$ & $<0.05$ & $<0.05$ & $<0.05$ & $<0.05$ & $<0.05$ \\
\hline Barium & 0.33 & 0.29 & 1.1 & 0.3 & 0.31 & 0.27 & 0.35 & 0.32 \\
\hline Beryllium & $<0.0003$ & $<0.0003$ & 0.0021 & $<0.0003$ & $<0.0003$ & $<0.0003$ & $<0.0003$ & $<0.0003$ \\
\hline Boron & 0.015 & 0.011 & 0.076 & 0.058 & 0.01 & 0.011 & 0.049 & 0.021 \\
\hline Cadmium (AAS) & $<0.002$ & $<0.002$ & $<0.002$ & $<0.002$ & $<0.002$ & $<0.002$ & $<0.002$ & $<0.002$ \\
\hline Cadmium & $<0.003$ & $<0.003$ & $<0.003$ & $<0.003$ & $<0.003$ & $<0.003$ & $<0.003$ & $<0.003$ \\
\hline Chromium (AAS) & $<0.01$ & $<0.01$ & 0.094 & $<0.01$ & $<0.01$ & $<0.01$ & $<0.01$ & $<0.01$ \\
\hline Chromium & $<0.01$ & $<0.01$ & 0.079 & $<0.01$ & $<0.01$ & $<0.01$ & $<0.01$ & $<0.01$ \\
\hline Cobalt & $<0.005$ & $<0.005$ & 0.03 & $<0.005$ & $<0.005$ & $<0.005$ & $<0.005$ & $<0.005$ \\
\hline Copper & $<0.004$ & $<0.004$ & 0.026 & $<0.004$ & 0.0062 & $<0.004$ & $<0.004$ & $<0.004$ \\
\hline Iron & 0.8 & 0.015 & 56 & 0.084 & 2.3 & 0.034 & 3.2 & 0.11 \\
\hline Lead (AAS) & $<0.004$ & $<0.004$ & 0.026 & $<0.004$ & $<0.004$ & $<0.004$ & $<0.004$ & $<0.004$ \\
\hline Mercury (CVAA) & $<0.0002$ & $<0.0002$ & $<0.0002$ & $<0.0002$ & $<0.0002$ & $<0.0002$ & $<0.0002$ & $<0.0002$ \\
\hline Molybdenum & $<0.01$ & $<0.01$ & $<0.01$ & $<0.01$ & $<0.01$ & $<0.01$ & $<0.01$ & $<0.01$ \\
\hline Nickel & $<0.01$ & 0.014 & 0.078 & $<0.01$ & $<0.01$ & $<0.01$ & $<0.01$ & $<0.01$ \\
\hline Selenium & $<0.05$ & $<0.05$ & $<0.05$ & $<0.05$ & $<0.05$ & $<0.05$ & $<0.05$ & $<0.05$ \\
\hline Silver & $<0.006$ & $<0.006$ & $<0.006$ & $<0.006$ & $<0.006$ & $<0.006$ & $<0.006$ & $<0.006$ \\
\hline Strontium & 0.47 & 0.5 & 0.96 & 0.48 & 0.45 & 0.41 & 0.48 & 0.53 \\
\hline Thorium & $<0.2$ & $<0.2$ & $<0.2$ & $<0.2$ & $<0.2$ & $<0.2$ & $<0.2$ & $<0.2$ \\
\hline Oranium (Fluor) & $<0.001$ & $<0.001$ & 0.001 & $<0.001$ & $<0.001$ & 0.001 & $<0.001$ & 0.001 \\
\hline Vanadium & $<0.005$ & $<0.005$ & 0.06 & $<0.005$ & $<0.005$ & $<0.005$ & $<0.005$ & $<0.005$ \\
\hline zine & 0.011 & 0.0056 & 0.2 & 0.0066 & 0.013 & 0.01 & 0.016 & 0.0062 \\
\hline MANOR IONS ( $\mathrm{mg} / \mathrm{L}$ ) & • & $\cdot$ & $\cdot$ & $\cdot$ & $\cdot$ & $\cdot$ & $\cdot$ & • \\
\hline Alkalinity-HCO3 & 239 & • & 233 & $\cdot$ & $\begin{array}{r}\cdot \\
255\end{array}$ & $\cdot$ & $\begin{array}{r}\cdot \dot{ } \\
257\end{array}$ & . \\
\hline Alkalinity-CO3 & $<1$ & - & $<1$ &. & $<1$ & - & $<1$ & - \\
\hline Calcium & 78 & 83 & 220 & 73 & 75 & 69 & 83 & 84 \\
\hline Chloride & 3.4 & • & 3.8 &. & 4.3 & . & 6 & • \\
\hline Fluoride & $<0.1$ & . & 0.1 & . & 0.1 & . & $<0.1$ & . \\
\hline Magnesium & 10 & 11 & 33 & 9.7 & 10 & 9 & 11 & 11 \\
\hline Manganese & 0.042 & 0.047 & 1.4 & 0.041 & 0.069 & 0.016 & 0.095 & 0.027 \\
\hline Nitrate-N & $<0.2$ & • & $<0.2$ & . & $<0.2$ & . & $<0.2$ & • \\
\hline Potassium & 2.4 & 3.2 & 13 & 2 & 3 & 2.6 & 2.9 & 2.6 \\
\hline Sodium & 10 & 8.8 & 13 & 12 & 14 & 14 & 15 & 11 \\
\hline Sulfate & 12 & • & 11.3 & . & 20 & . & 13 & • \\
\hline
\end{tabular}

(CONTINUED) 
APPENDIX E.1

\begin{tabular}{|c|c|c|c|c|c|c|c|c|}
\hline \multirow{4}{*}{$\begin{array}{l}\text { Sampling Point } \\
\text { Location } \\
\text { Date Sampled }\end{array}$} & \multicolumn{8}{|c|}{ GW-192 } \\
\hline & \multicolumn{8}{|c|}{ B4 } \\
\hline & \multicolumn{2}{|c|}{$01 / 14 / 93$} & \multicolumn{2}{|c|}{$04 / 13 / 93$} & \multicolumn{2}{|c|}{$07 / 08 / 93$} & \multicolumn{2}{|c|}{$12 / 09 / 93$} \\
\hline & TOT & DIS & TOT & DIS & TOT & DIS & TOT & DIS \\
\hline METALS (mg/L) & - & - & - & . & - & - & - & • \\
\hline Aluminum & 5.8 & $<0.02$ & 0.077 & 0.029 & 0.18 & 0.038 & 0.21 & 0.022 \\
\hline Antimony & $<0.05$ & $<0.05$ & $<0.05$ & $<0.05$ & $<0.05$ & $<0.05$ & $<0.05$ & $<0.05$ \\
\hline Arsenic & $<0.05$ & $<0.05$ & $<0.05$ & $<0.05$ & $<0.05$ & $<0.05$ & $<0.05$ & $<0.05$ \\
\hline Barium & 0.22 & 0.15 & 0.16 & 0.16 & 0.16 & 0.17 & 0.18 & 0.18 \\
\hline Beryllium & $<0.0003$ & $<0.0003$ & $<0.0003$ & $<0.0003$ & $<0.0003$ & $<0.0003$ & $<0.0003$ & $<0.0003$ \\
\hline Boron & 0.013 & 0.016 & 0.034 & 0.037 & 0.014 & 0.01 & 0.073 & 0.045 \\
\hline Cadmium (AAS) & $<0.002$ & $<0.002$ & $<0.002$ & $<0.002$ & $<0.002$ & $<0.002$ & $<0.002$ & $<0.002$ \\
\hline Cadmium & $<0.003$ & $<0.003$ & $<0.003$ & $<0.003$ & $<0.003$ & $<0.003$ & $<0.003$ & $<0.003$ \\
\hline Chromium (AAS) & 0.016 & $<0.01$ & $<0.01$ & $<0.01$ & $<0.01$ & $<0.01$ & $<0.01$ & $<0.01$ \\
\hline Chromium & $<0.01$ & $<0.01$ & $<0.01$ & $<0.01$ & $<0.01$ & $<0.01$ & $<0.01$ & $<0.01$ \\
\hline Cobalt & 0.0077 & $<0.005$ & 0.0068 & 0.0066 & $<0.005$ & $<0.005$ & $<0.005$ & $<0.005$ \\
\hline Copper & 0.0065 & $<0.004$ & 0.0042 & $<0.004$ & 0.0041 & $<0.004$ & $<0.004$ & $<0.004$ \\
\hline Iron & 18 & 2.7 & 3.3 & 3 & 4.2 & 4.6 & 2.5 & 2.3 \\
\hline Lead (AAS) & 0.0066 & $<0.004$ & $<0.004$ & $<0.004$ & $<0.004$ & $<0.004$ & $<0.004$ & $<0.004$ \\
\hline Mercury (CVAA) & $<0.0002$ & $<0.0002$ & $<0.0002$ & $<0.0002$ & $<0.0002$ & $<0.0002$ & $<0.0002$ & $<0.0002$ \\
\hline Molybdenum & $<0.01$ & $<0.01$ & $<0.01$ & $<0.01$ & $<0.01$ & $<0.01$ & $<0.01$ & $<0.01$ \\
\hline Nickel & 0.011 & $<0.01$ & $<0.01$ & $<0.01$ & $<0.01$ & $<0.01$ & $<0.01$ & $<0.01$ \\
\hline Selenium & $<0.05$ & $<0.05$ & $<0.05$ & $<0.05$ & $<0.05$ & $<0.05$ & $<0.05$ & $<0.05$ \\
\hline silver & $<0.006$ & $<0.006$ & $<0.006$ & $<0.006$ & $<0.006$ & $<0.006$ & $<0.006$ & $<0.006$ \\
\hline Strontium & 0.17 & 0.18 & 0.2 & 0.2 & 0.21 & 0.23 & 0.2 & 0.2 \\
\hline Thorium & $<0.2$ & $<0.2$ & $<0.2$ & $<0.2$ & $<0.2$ & $<0.2$ & $<0.2$ & $<0.2$ \\
\hline Uranium (Fluor) & $<0.001$ & $<0.001$ & $<0.001$ & $<0.001$ & $<0.001$ & $<0.001$ & $<0.001$ & $<0.001$ \\
\hline Vanadium & 0.0084 & $<0.005$ & $<0.005$ & $<0.005$ & $<0.005$ & $<0.005$ & $<0.005$ & $<0.005$ \\
\hline zinc & 0.026 & 0.016 & 0.049 & 0.046 & 0.027 & 0.02 & 0.039 & 0.036 \\
\hline MAJOR IONS $(\mathrm{mg} / \mathrm{L})$ & . & . & . &. & . & . & . & . \\
\hline Alkalinity- $\mathrm{HCO} 3$ & 322 & $\cdot$ & 331 & . & 350 &. & $326^{\circ}$ & • \\
\hline Alkalinity-CO3 & $<1$ & . & $<1$ & . & $<1$ &. & $<1$ & . \\
\hline Calcium & 98 & 110 & 110 & 110 & 110 & 110 & 120 & 110 \\
\hline Chloride & 11 & . & 9.8 & . & 13 & . & 15 & • \\
\hline Fluoride & 0.2 & • & 0.2 & $\cdot$ & 0.2 & . & 0.1 & • \\
\hline Magnesium & 12 & 11 & 12 & 12 & 12 & 13 & 12 & 12 \\
\hline Manganese & 1.8 & 1.9 & 2 & 2 & 2.1 & 2.2 & 2 & 2 \\
\hline Nitrate-N & $<0.2$ & . & $<0.2$ & . & $<0.2$ & . & $<0.2$ & • \\
\hline Potassium & 2.6 & 0.91 & 1.6 & 1.2 & 1.4 & 1.6 & 1.2 & 1.3 \\
\hline Sodium & 6.8 & 6.8 & 7.2 & 7.1 & 7.2 & 7.1 & 8 & 7.8 \\
\hline Sulfate & 4.4 & . & 6.6 & . & 2 & . & 2 & • \\
\hline
\end{tabular}

(CONTINUED) 
APPENDIX E.1

Groundwater Quality Data, 1993

\begin{tabular}{|c|c|c|c|c|c|c|c|c|}
\hline \multirow{4}{*}{$\begin{array}{l}\text { Sampling Point } \\
\text { Location } \\
\text { Date Sampled }\end{array}$} & \multicolumn{8}{|c|}{ GW-193 } \\
\hline & \multicolumn{8}{|c|}{ T2331 } \\
\hline & \multicolumn{2}{|c|}{$03 / 11 / 93$} & \multicolumn{2}{|c|}{$06 / 22 / 93$} & \multicolumn{2}{|c|}{$09 / 23 / 93$} & \multicolumn{2}{|c|}{$11 / 18 / 93$} \\
\hline & TOT & DIS & TOT & DIS & TOT & DIS & TOT & DIS \\
\hline METALS $(\mathrm{mg} / \mathrm{L})$ & $\cdot$ & $\cdot$ & $\cdot$ & - & - & - & • & - \\
\hline Aluminum & 0.62 & 0.028 & 9.3 & $<0.02$ & 0.26 & 0.037 & 0.64 & $<0.02$ \\
\hline Antimony & $<0.05$ & $<0.05$ & $<0.05$ & $<0.05$ & $<0.05$ & $<0.05$ & $<0.05$ & $<0.05$ \\
\hline Arsenic & $<0.05$ & $<0.05$ & $<0.05$ & $<0.05$ & $<0.05$ & $<0.05$ & $<0.05$ & $<0.05$ \\
\hline Barium & 0.15 & 0.14 & 0.21 & 0.17 & 0.29 & 0.28 & 0.25 & 0.26 \\
\hline Beryllium & $<0.0003$ & $<0.0003$ & 0.00064 & $<0.0003$ & $<0.0003$ & $<0.0003$ & $<0.0003$ & $<0.0003$ \\
\hline Boron & 0.073 & 0.068 & 0.13 & 0.068 & 0.15 & 0.15 & 0.13 & 0.12 \\
\hline Cadmium (AAS) & $<0.002$ & $<0.002$ & $<0.002$ & $<0.002$ & $<0.002$ & $<0.002$ & $<0.002$ & $<0.002$ \\
\hline Cadmium & $<0.003$ & $<0.003$ & 0.0032 & $<0.003$ & $<0.003$ & $<0.003$ & $<0.003$ & $<0.003$ \\
\hline Chromium (AAS) & $<0.01$ & $<0.01$ & 0.028 & $<0.01$ & $<0.01$ & $<0.01$ & $<0.01$ & $<0.01$ \\
\hline Chromium & $<0.01$ & $<0.01$ & 0.023 & $<0.01$ & $<0.01$ & $<0.01$ & $<0.01$ & $<0.01$ \\
\hline Cobalt & $<0.005$ & $<0.005$ & 0.0056 & $<0.005$ & $<0.005$ & $<0.005$ & $<0.005$ & $<0.005$ \\
\hline Copper & $<0.004$ & $<0.004$ & 0.009 & 0.0099 & $<0.004$ & $<0.004$ & $<0.004$ & $<0.004$ \\
\hline Iron & 1.4 & 0.36 & 13 & 0.88 & 1.4 & 0.75 & 2.1 & 0.64 \\
\hline Lead (AAS) & $<0.004$ & $<0.004$ & 0.008 & $<0.004$ & $<0.004$ & $<0.004$ & $<0.004$ & $<0.004$ \\
\hline Mercury (CVAA) & $<0.0002$ & $<0.0002$ & $<0.0002$ & $<0.0002$ & $<0.0002$ & $<0.0002$ & $<0.0002$ & $<0.0002$ \\
\hline Molybdenum & $<0.01$ & $<0.01$ & $<0.01$ & $<0.01$ & $<0.01$ & $<0.01$ & $<0.01$ & 0.013 \\
\hline Nickel & 0.011 & $<0.01$ & 0.028 & $<0.01$ & $<0.01$ & $<0.01$ & 0.01 & 0.01 \\
\hline Selenium & $<0.05$ & $<0.05$ & $<0.05$ & $<0.05$ & $<0.05$ & $<0.05$ & $<0.05$ & $<0.05$ \\
\hline Silver & $<0.006$ & $<0.006$ & $<0.006$ & $<0.006$ & $<0.006$ & $<0.006$ & $<0.006$ & $<0.006$ \\
\hline Strontium & 0.38 & 0.38 & 0.47 & 0.46 & 0.6 & 0.59 & 0.57 & 0.58 \\
\hline Thorium & $<0.2$ & $<0.2$ & $<0.2$ & $<0.2$ & $<0.2$ & $<0.2$ & $<0.2$ & $<0.2$ \\
\hline Uranium (Fluor) & 0.017 & 0.018 & 0.009 & 0.017 & 0.086 & 0.077 & 0.08 & 0.08 \\
\hline Vanadium & $<0.005$ & $<0.005$ & 0.019 & $<0.005$ & 0.0064 & $<0.005$ & $<0.005$ & $<0.005$ \\
\hline Zinc & 0.0039 & 0.0028 & 0.024 & 0.005 & 0.011 & 0.012 & 0.0069 & 0.0026 \\
\hline MAJOR IONS (mg/I) & - & - & . & - & - & - & - & • \\
\hline Alkalinity- $\mathrm{HCO} 3$ & 318 & $\cdot$ & 418 & $\cdot$ & 174 & $\cdot$ & 457 & • \\
\hline Alkalinity-CO3 & $<1$ & $\cdot$ & $<1$ & $\cdot$ & $<1$ & - & $<1$ & - \\
\hline Calcium & 94 & 92 & 130 & 120 & 140 & 140 & 140 & 140 \\
\hline Chloride & 6.7 & $\cdot$ & 15 & , & 6.5 & • & 16 & • \\
\hline Fluoride & 0.5 & • & 0.49 & • & 0.6 & - & 0.5 & - \\
\hline Magnesium & 15 & 15 & 20 & 19 & 21 & 21 & 22 & 23 \\
\hline Manganese & 0.69 & 0.67 & 1 & 1.1 & 1.1 & 1.1 & 0.96 & 1 \\
\hline Nitrate-N & $<0.2$ & $\cdot$ & $<0.2$ & • & 1.3 & . & $<0.2$ & • \\
\hline Potassium & 7.8 & 7.4 & 9.9 & 7.5 & 10 & 10 & 11 & 12 \\
\hline Sodium & 7.9 & 7.7 & 11 & 11 & 9.4 & 9.5 & 15 & 16 \\
\hline sulfate & 11 & $\cdot$ & 8.2 & $\cdot$ & 11 & • & 4 & • \\
\hline
\end{tabular}

(CONTINUED) 
APPENDIX E. 1

Groundwater Quality Data, 1993

\begin{tabular}{|c|c|c|c|c|c|c|c|c|}
\hline \multirow{4}{*}{$\begin{array}{l}\text { Sampling Point } \\
\text { Location } \\
\text { Date Sampled }\end{array}$} & \multicolumn{8}{|c|}{ GW-194 } \\
\hline & \multicolumn{8}{|c|}{ B4 } \\
\hline & \multicolumn{2}{|c|}{$01 / 13 / 93$} & \multicolumn{2}{|c|}{$04 / 13 / 93$} & \multicolumn{2}{|c|}{$07 / 08 / 93$} & \multicolumn{2}{|c|}{$12 / 09 / 93$} \\
\hline & TOT & DIS & TOT & DIS & TOT & DIS & TOT & DIS \\
\hline NETALS $(\mathrm{mg} / \mathrm{L})$ & $\cdot$ & $\cdot$ & $\cdot$ & - & - & . & - & - \\
\hline Aluminum & 4.2 & $<0.02$ & 1.5 & 0.022 & 1.4 & $<0.02$ & 0.89 & $<0.02$ \\
\hline Antimony & $<0.05$ & $<0.05$ & $<0.05$ & $<0.05$ & $<0.05$ & $<0.05$ & $<0.05$ & $<0.05$ \\
\hline Arsenic & $<0.05$ & $<0.05$ & $<0.05$ & $<0.05$ & $<0.05$ & $<0.05$ & $<0.05$ & $<0.05$ \\
\hline Barium & 0.27 & 0.22 & 0.3 & 0.27 & 0.29 & 0.27 & 0.33 & 0.29 \\
\hline Beryllium & $<0.0003$ & $<0.0003$ & $<0.0003$ & $<0.0003$ & $<0.0003$ & $<0.0003$ & $<0.0003$ & $<0.0003$ \\
\hline Boron & 0.097 & 0.084 & 0.017 & 0.024 & 0.0092 & 0.0099 & 0.057 & 0.06 \\
\hline Cadmium (AAS) & $<0.002$ & $<0.002$ & $<0.002$ & $<0.002$ & $<0.002$ & $<0.002$ & $<0.002$ & $<0.002$ \\
\hline Cadmium & $<0.003$ & $<0.003$ & $<0.003$ & $<0.003$ & $<0.003$ & $<0.003$ & $<0.003$ & $<0.003$ \\
\hline Chromium (AAS) & $<0.01$ & $<0.01$ & $<0.01$ & $<0.01$ & $<0.01$ & $<0.01$ & $<0.01$ & $<0.01$ \\
\hline Chromium & $<0.01$ & $<0.01$ & $<0.01$ & $<0.01$ & $<0.01$ & $<0.01$ & $<0.01$ & 0.017 \\
\hline Cobalt & $<0.005$ & $<0.005$ & $<0.005$ & $<0.005$ & $<0.005$ & $<0.005$ & $<0.005$ & $<0.005$ \\
\hline Copper & 0.0065 & $<0.004$ & $<0.004$ & $<0.004$ & 0.013 & 0.012 & $<0.004$ & $<0.004$ \\
\hline Iron & 4.2 & 0.0064 & 1.8 & 0.017 & 1.9 & $<0.005$ & 1.3 & 0.062 \\
\hline Lead (AAS) & $<0.004$ & $<0.004$ & $<0.004$ & $<0.004$ & $<0.004$ & $<0.004$ & $<0.004$ & $<0.004$ \\
\hline Mercury (CVAA) & $<0.0002$ & $<0.0002$ & $<0.0002$ & $<0.0002$ & $<0.0002$ & $<0.0002$ & $<0.0002$ & $<0.0002$ \\
\hline Molybdenum & $<0.01$ & $<0.01$ & $<0.01$ & $<0.01$ & $<0.01$ & $<0.01$ & $<0.01$ & $<0.01$ \\
\hline Nickel & 0.01 & $<0.01$ & $<0.01$ & $<0.01$ & $<0.01$ & $<0.01$ & $<0.01$ & $<0.01$ \\
\hline Selenium & $<0.05$ & $<0.05$ & $<0.05$ & $<0.05$ & $<0.05$ & $<0.05$ & $<0.05$ & $<0.05$ \\
\hline silver & $<0.006$ & $<0.006$ & $<0.006$ & $<0.006$ & $<0.006$ & $<0.006$ & $<0.006$ & $<0.006$ \\
\hline Strontium & 0.14 & 0.15 & 0.15 & 0.15 & 0.16 & 0.16 & 0.16 & 0.16 \\
\hline Thorium & $<0.2$ & $<0.2$ & $<0.2$ & $<0.2$ & $<0.2$ & $<0.2$ & $<0.2$ & $<0.2$ \\
\hline Oranium (Fluor) & 0.09 & 0.09 & 0.003 & 0.003 & $<0.001$ & $<0.001$ & 0.007 & 0.009 \\
\hline Vanadium & 0.0064 & $<0.005$ & $<0.005$ & $<0.005$ & $<0.005$ & $<0.005$ & $<0.005$ & $<0.005$ \\
\hline zinc & 0.02 & 0.0072 & 0.027 & 0.023 & 0.036 & 0.045 & 0.029 & 0.027 \\
\hline MAJOR IONS $(\mathrm{mg} / \mathrm{L})$ & - & . & . & - & . & - & - & • \\
\hline Alkalinity-HCO3 & 225 & $\cdot$ & 217 & $\cdot$ & $22 \dot{8}$ & • & $229^{\circ}$ & • \\
\hline Alkalinity- $\mathrm{CO} 3$ & $<1$ & . & $<1$ & . & $<1$ & . & $<1$ & . \\
\hline Calcium & 75 & 74 & 76 & 76 & 77 & 77 & 84 & 80 \\
\hline chloride & 2.2 & . & 3.3 & . & 3.5 & - & 4 & • \\
\hline fluoride & $<0.1$ & • & $<0.1$ & . & $<0.1$ & . & $<0.1$ & • \\
\hline Magnesium & 8.4 & 7.2 & 6.2 & 5.9 & 6.3 & 6 & 6.8 & 6.4 \\
\hline Manganese & 0.57 & 0.064 & 0.48 & 0.12 & 0.78 & 0.34 & 1 & 0.15 \\
\hline Nitrate-N & $<0.2$ & • & $<0.2$ & . & $<0.2$ & • & $<0.2$ & • \\
\hline Potassium & 2.7 & 1.2 & 1.2 & 0.88 & 1.6 & 0.84 & 0.74 & 0.86 \\
\hline Sodium & 4.7 & 5 & 6.7 & 6.7 & 6.7 & 7 & 7.1 & 6.9 \\
\hline Sulfate & 14 & . & 14 & . & 12 & . & 11 & • \\
\hline
\end{tabular}


APPENDIX R.1

Groundwater Quality Data, 1993

\begin{tabular}{|c|c|c|c|c|c|c|c|c|}
\hline \multirow{4}{*}{$\begin{array}{l}\text { Sampling Point } \\
\text { Location } \\
\text { Date Sampled }\end{array}$} & \multicolumn{8}{|c|}{$G W-195$} \\
\hline & \multicolumn{8}{|c|}{ B4 } \\
\hline & \multicolumn{2}{|c|}{$01 / 13 / 93$} & \multicolumn{2}{|c|}{$04 / 13 / 93$} & \multicolumn{2}{|c|}{$07 / 08 / 93$} & \multicolumn{2}{|c|}{$12 / 09 / 93$} \\
\hline & TOT & DIS & TOT & DIS & TOT & DIS & TOT & DIS \\
\hline METALS (mg/L) & . & . & . & . & . & . & . & s \\
\hline Aluminum & 14 & 0.097 & 13 & 0.055 & 12 & $<0.02$ & 9.3 & $<0.02$ \\
\hline Antimony & $<0.05$ & $<0.05$ & $<0.05$ & $<0.05$ & $<0.05$ & $<0.05$ & $<0.05$ & $<0.05$ \\
\hline Arsenic & $<0.05$ & $<0.05$ & $<0.05$ & $<0.05$ & $<0.05$ & $<0.05$ & $<0.05$ & $<0.05$ \\
\hline Barium & 0.49 & 0.18 & 0.45 & 0.18 & 0.46 & 0.17 & 0.37 & 0.19 \\
\hline Beryllium & 0.00076 & $<0.0003$ & 0.00069 & $<0.0003$ & 0.0006 & $<0.0003$ & 0.00042 & $<0.0003$ \\
\hline Boron & 0.026 & 0.019 & 0.054 & 0.031 & 0.026 & 0.02 & 0.058 & 0.038 \\
\hline Cadmium (AAS) & $<0.002$ & $<0.002$ & $<0.002$ & $<0.002$ & $<0.002$ & $<0.002$ & $<0.002$ & $<0.002$ \\
\hline Cadmium & 0.0049 & $<0.003$ & 0.0056 & $<0.003$ & 0.0042 & $<0.003$ & $<0.003$ & $<0.003$ \\
\hline Chromium (AAS) & 0.029 & $<0.01$ & 0.033 & $<0.01$ & 0.041 & $<0.01$ & 0.023 & $<0.01$ \\
\hline Chromium & 0.027 & $<0.01$ & 0.024 & $<0.01$ & 0.024 & $<0.01$ & 0.015 & $<0.01$ \\
\hline Cobalt & 0.02 & $<0.005$ & 0.013 & 0.0053 & 0.015 & $<0.005$ & 0.0098 & $<0.005$ \\
\hline Copper & 0.05 & $<0.004$ & 0.039 & $<0.004$ & 0.041 & $<0.004$ & 0.028 & $<0.004$ \\
\hline Iron & 24 & 0.12 & 17 & 0.04 & 18 & 0.014 & 14 & 0.0095 \\
\hline Lead (AAS) & 0.011 & $<0.004$ & 0.01 & $<0.004$ & 0.0098 & $<0.004$ & 0.0041 & $<0.004$ \\
\hline Mercury (CVAA) & $<0.0002$ & $<0.0002$ & $<0.0002$ & $<0.0002$ & $<0.0002$ & $<0.0002$ & $<0.0002$ & $<0.0002$ \\
\hline Molybdenum & $<0.01$ & $<0.01$ & $<0.01$ & $<0.01$ & $<0.01$ & $<0.01$ & $<0.01$ & $<0.01$ \\
\hline Nickel & 0.026 & 0.017 & 0.024 & $<0.01$ & 0.023 & $<0.01$ & 0.019 & $<0.01$ \\
\hline Selenium & $<0.05$ & $<0.05$ & $<0.05$ & $<0.05$ & $<0.05$ & $<0.05$ & $<0.05$ & $<0.05$ \\
\hline Silver & $<0.006$ & $<0.006$ & $<0.006$ & $<0.006$ & $<0.006$ & $<0.006$ & $<0.006$ & $<0.006$ \\
\hline Strontium & 0.13 & 0.13 & 0.14 & 0.13 & 0.15 & 0.14 & 0.14 & 0.14 \\
\hline Thorium & $<0.2$ & $<0.2$ & $<0.2$ & $<0.2$ & $<0.2$ & $<0.2$ & $<0.2$ & $<0.2$ \\
\hline Uranium (Fluor) & $<0.001$ & 0.002 & $<0.001$ & $<0.001$ & 0.001 & 0.001 & $<0.001$ & 0.001 \\
\hline Vanadium & 0.018 & $<0.005$ & 0.015 & $<0.005$ & 0.018 & $<0.005$ & 0.013 & $<0.005$ \\
\hline zinc & 0.049 & 0.01 & 0.067 & 0.023 & 0.051 & 0.015 & 0.042 & 0.011 \\
\hline MAJOR IONS (mg/L) & . & . & . & . & . & . & . & • \\
\hline Alkalinity-HCO3 & ${ }_{183}$ & $\cdot$ & 179 & . & ${ }_{181}^{\circ}$ & . & ${ }_{180}^{\circ}$ & • \\
\hline Alkalinity- $\mathrm{CO} 3$ & $<1$ & . & $<1$ & . & $<1$ & . & $<1$ & - \\
\hline Calcium & 64 & 63 & 64 & 65 & 66 & 62 & 66 & 65 \\
\hline Chloride & 3.5 & $\cdot$ & 3.5 & . & 3.7 & . & 4 & • \\
\hline Fluoride & $<0.1$ & . & $<0.1$ & . & $<0.1$ & . & $<0.1$ & . \\
\hline Magnesium & 9.4 & 5.7 & 8.5 & 5.7 & 9 & 5.7 & 8.1 & 5.7 \\
\hline Manganese & 1.9 & 0.03 & 1.4 & 0.018 & 1.6 & 0.026 & 1.2 & 0.023 \\
\hline Nitrate-N & $<0.2$ & & $<0.2$ &. & $<0.2$ & • & 0.3 & 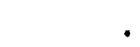 \\
\hline Potassium & 4.9 & 0.75 & 5.4 & $<0.6$ & 5 & 0.84 & 3.7 & 0.88 \\
\hline Sodium & 5.4 & 5.5 & 6 & 6 & 6.2 & 6.2 & 6.3 & 6.2 \\
\hline Sulfate & 14 & . & 15 & . & 14 & . & 12 & • \\
\hline
\end{tabular}

(CONTINURD) 
APPENDIX E. 1

Groundwater Quality Data, 1993

\begin{tabular}{|c|c|c|c|c|c|c|c|c|}
\hline \multirow{4}{*}{$\begin{array}{l}\text { Sampling Point } \\
\text { Location } \\
\text { - } \\
\text { Date Sampled }\end{array}$} & \multicolumn{8}{|c|}{ GW- 199} \\
\hline & \multicolumn{8}{|c|}{ GRIDII } \\
\hline & \multicolumn{2}{|c|}{$01 / 29 / 93$} & \multicolumn{2}{|c|}{$04 / 07 / 93$} & \multicolumn{2}{|c|}{$08 / 02 / 93$} & \multicolumn{2}{|c|}{$10 / 12 / 93$} \\
\hline & TOT & DIS & TOT & DIS & TOT & DIS & TOT & DIS \\
\hline METALS (mg/L) & . & . & . & . & . & . & - & \\
\hline Al uminum & 0.75 & $<0.02$ & 0.5 & 0.031 & 0.28 & $<0.02$ & 0.47 & 0.027 \\
\hline Antimony & $<0.05$ & $<0.05$ & $<0.05$ & $<0.05$ & $<0.05$ & $<0.05$ & $<0.05$ & $<0.05$ \\
\hline Arsenic & $<0.05$ & $<0.05$ & $<0.05$ & $<0.05$ & $<0.05$ & $<0.05$ & $<0.05$ & $<0.05$ \\
\hline Barium & 0.22 & 0.12 & 0.15 & 0.1 & 0.18 & 0.15 & 0.15 & 0.11 \\
\hline Beryllium & $<0.0003$ & $<0.0003$ & $<0.0003$ & $<0.0003$ & $<0.0003$ & $<0.0003$ & $<0.0003$ & $<0.0003$ \\
\hline Boron & 0.073 & 0.06 & 0.062 & 0.052 & 0.052 & 0.049 & 0.05 & 0.048 \\
\hline Cadmium (AAS) & $<0.002$ & $<0.002$ & $<0.002$ & $<0.002$ & $<0.002$ & $<0.002$ & $<0.002$ & $<0.002$ \\
\hline Cadmium & $<0.003$ & $<0.003$ & $<0.003$ & $<0.003$ & $<0.003$ & $<0.003$ & $<0.003$ & $<0.003$ \\
\hline Chromium (AAS) & 0.01 & $<0.01$ & $<0.01$ & $<0.01$ & $<0.01$ & $<0.01$ & $<0.01$ & $<0.01$ \\
\hline Chromium & $<0.01$ & $<0.01$ & $<0.01$ & $<0.01$ & $<0.01$ & $<0.01$ & $<0.01$ & $<0.01$ \\
\hline Cobalt & 0.0063 & $<0.005$ & $<0.005$ & $<0.005$ & $<0.005$ & $<0.005$ & $<0.005$ & $<0.005$ \\
\hline Copper & $<0.004$ & $<0.004$ & $<0.004$ & $<0.004$ & 0.0066 & $<0.004$ & 0.005 & $<0.004$ \\
\hline Iron & 4.9 & 0.047 & 2 & 0.033 & 7.2 & 0.2 & 2.3 & 0.036 \\
\hline Lead (AAS) & 0.034 & $<0.004$ & $<0.004$ & $<0.004$ & $<0.004$ & $<0.004$ & $<0.004$ & $<0.004$ \\
\hline Mercury (CVAA) & $<0.0002$ & $<0.0002$ & $<0.0002$ & $<0.0002$ & $<0.0002$ & $<0.0002$ & $<0.0002$ & $<0.0002$ \\
\hline Molybdenum & $<0.01$ & $<0.01$ & $<0.01$ & $<0.01$ & $<0.01$ & $<0.01$ & $<0.01$ & $<0.01$ \\
\hline Nickel & $<0.01$ & $<0.01$ & $<0.01$ & $<0.01$ & $<0.01$ & $<0.01$ & $<0.01$ & 0.017 \\
\hline Selenium & $<0.05$ & $<0.05$ & $<0.05$ & $<0.05$ & $<0.05$ & $<0.05$ & $<0.05$ & $<0.05$ \\
\hline silver & $<0.006$ & $<0.006$ & $<0.006$ & $<0.006$ & $<0.006$ & $<0.006$ & $<0.006$ & $<0.006$ \\
\hline Strontium & 0.14 & 0.13 & 0.13 & 0.14 & 0.16 & 0.15 & 0.14 & 0.15 \\
\hline Thorium & $<0.2$ & $<0.2$ & $<0.2$ & $<0.2$ & $<0.2$ & $<0.2$ & $<0.2$ & $<0.2$ \\
\hline Uranium (Fluor) & 0.001 & $<0.001$ & $<0.001$ & 0.001 & $<0.001$ & $<0.001$ & $<0.001$ & $<0.001$ \\
\hline Vanadium & $<0.005$ & $<0.005$ & $<0.005$ & $<0.005$ & $<0.005$ & $<0.005$ & $<0.005$ & $<0.005$ \\
\hline Zinc & 0.012 & 0.0034 & 0.0074 & 0.0049 & 0.068 & 0.07 & 0.17 & 0.18 \\
\hline MAJOR IONS $(\mathrm{mg} / \mathrm{L})$ & . &. & . & . & . &. & $\cdot$ & - \\
\hline Alkalinity-HCO3 & 176 & $\cdot$ & $148^{\circ}$ & $\cdot$ & $18 \dot{1}$ & . & $17 \dot{1}$ & • \\
\hline Alkalinity-CO3 & $<1$ & $\cdot$ & $<1$ & . & $<1$ & • & $<1$ & • \\
\hline Calcium & 57 & 55 & 52 & 52 & 58 & 59 & 55 & 55 \\
\hline Chloride & 3 & $\cdot$ & 21 & . & 7.6 & . & 21 & - \\
\hline Fluoride & 0.2 &. & 0.2 & $\cdot$ & 0.2 & . & 0.2 & • \\
\hline Magnesium & 12 & 12 & 12 & 12 & 15 & 15 & 14 & 14 \\
\hline Manganese & 4 & 2.5 & 1.8 & 0.17 & 3.4 & 1.8 & 2 & 0.78 \\
\hline Nitrate-N & 0.3 & $\cdot$ & 0.23 & . & 0.25 & . & 0.4 & - \\
\hline Potassium & 2.5 & 2.4 & 2.2 & 2.2 & 2.9 & 2.9 & 2.6 & 3 \\
\hline Sodium & 6.2 & 6.1 & 6.3 & 7.3 & 6.6 & 6.7 & 6.6 & 6.7 \\
\hline Sulfate & 27 & . & 25 & $\cdot$ & 30 & • & 31 & - \\
\hline
\end{tabular}

(CONTINUED) 
APPENDIX E.1

Groundwater Quality Data, 1993

\begin{tabular}{|c|c|c|c|c|c|c|c|c|}
\hline \multirow{4}{*}{$\begin{array}{l}\text { Sampling Point } \\
\text { Location } \\
\text { Date Sampled }\end{array}$} & \multicolumn{8}{|c|}{ GW-204 } \\
\hline & \multicolumn{8}{|c|}{ T0134 } \\
\hline & \multicolumn{2}{|c|}{$03 / 11 / 93$} & \multicolumn{2}{|c|}{$06 / 22 / 93$} & \multicolumn{2}{|c|}{$09 / 23 / 93$} & \multicolumn{2}{|c|}{$11 / 16 / 93$} \\
\hline & TOT & DIS & TOT & DIS & TOT & DIS & TOT & DIS \\
\hline METALS (mg/L) & . & . & . & . & . & - & . & \\
\hline Aluminum & 0.67 & 0.025 & 0.067 & $<0.02$ & 1.1 & 0.023 & 1.5 & $<0.02$ \\
\hline Antimony & $<0.05$ & $<0.05$ & $<0.05$ & $<0.05$ & $<0.05$ & $<0.05$ & $<0.05$ & $<0.05$ \\
\hline Arsenic & $<0.05$ & $<0.05$ & $<0.05$ & $<0.05$ & $<0.05$ & $<0.05$ & $<0.05$ & $<0.05$ \\
\hline Barium & 0.12 & 0.12 & 0.12 & 0.14 & 0.16 & 0.15 & 0.12 & 0.11 \\
\hline Beryllium & 0.00043 & $<0.0003$ & $<0.0003$ & $<0.0003$ & $<0.0003$ & $<0.0003$ & $<0.0003$ & $<0.0003$ \\
\hline Boron & 0.08 & 0.068 & 0.092 & 0.086 & 0.088 & 0.091 & 0.059 & 0.096 \\
\hline Cadmium (AAS) & $<0.002$ & $<0.002$ & 0.0021 & 0.0022 & $<0.002$ & $<0.002$ & $<0.002$ & $<0.002$ \\
\hline Cadmium & $<0.003$ & $<0.003$ & $<0.003$ & $<0.003$ & $<0.003$ & $<0.003$ & $<0.003$ & $<0.003$ \\
\hline Chromium (AAS) & $<0.01$ & $<0.01$ & $<0.01$ & $<0.01$ & $<0.01$ & $<0.01$ & $<0.01$ & $<0.01$ \\
\hline Chromium & $<0.01$ & $<0.01$ & $<0.01$ & $<0.01$ & $<0.01$ & $<0.01$ & $<0.01$ & $<0.01$ \\
\hline Coba.t.t & $<0.005$ & $<0.005$ & $<0.005$ & 0.0055 & $<0.005$ & $<0.005$ & $<0.005$ & $<0.005$ \\
\hline Copper & $<0.004$ & $<0.004$ & 0.008 & 0.0062 & 0.007 & 0.027 & 0.0077 & $<0.004$ \\
\hline Iron & 0.74 & 0.12 & 0.28 & 0.11 & 0.86 & 0.011 & 1.7 & 0.01 \\
\hline Lead (AAS) & $<0.004$ & $<0.004$ & 0.0057 & $0 . \AA 055$ & $<0.004$ & $<0.004$ & 0.0043 & $<0.004$ \\
\hline Mercury (CVAA) & $<0.0002$ & $<0.0002$ & $<0.0002$ & $<0.0002$ & $<0.0002$ & $<0.0002$ & $<0.0002$ & $<0.0002$ \\
\hline Molybdenum & $<0.01$ & $<0.01$ & $<0.01$ & $<0.01$ & $<0.01$ & $<0.01$ & $<0.01$ & $<0.01$ \\
\hline Nickel & 0.013 & $<0.01$ & $<0.01$ & $<0.01$ & $<0.01$ & $<0.01$ & $<0.01$ & $<0.01$ \\
\hline Selenium & $<0.05$ & $<0.05$ & $<0.05$ & $<0.05$ & $<0.05$ & $<0.05$ & $<0.05$ & $<0.05$ \\
\hline silver & $<0.006$ & $<0.006$ & $<0.006$ & $<0.006$ & $<0.006$ & $<0.006$ & $<0.006$ & $<0.006$ \\
\hline Strontium & 0.16 & 0.15 & 0.16 & 0.16 & 0.22 & 0.21 & 0.18 & 0.17 \\
\hline Thorium & $<0.2$ & $<0.2$ & $<0.2$ & $<0,2$ & $<0.2$ & $<0.2$ & $<0.2$ & $<0.2$ \\
\hline Oranium (Fluor) & 0.075 & 0.068 & 0.21 & 0.185 & 0.126 & 0.12 & 0.094 & 0.108 \\
\hline Vanadium & $<0.005$ & $<0.005$ & $<0.005$ & $<0.005$ & $<0.005$ & $<0.005$ & $<0.005$ & $<0.005$ \\
\hline zinc & 0.021 & 0.017 & 0.072 & 0.086 & 0.03 & 0.082 & 0.035 & 0.0085 \\
\hline MAJOR IONS (mg/L) & . & . &. &. & $\cdot$ & . & • & ${ }^{\circ}$ \\
\hline Alkalinity-HCO3 & 239 & . & $17 \dot{1}$ & $\cdot \dot{ }$ & $18 \dot{6}$ & • & $175^{\circ}$ & - \\
\hline Alkalinity-CO3 & $<1$ & . & $<1$ & . & $<1$ & . & $<1$ & - \\
\hline Calciun & 40 & 39 & 53 & 53 & 66 & 64 & 57 & 56 \\
\hline Chloride & 4.4 & • & 3.8 & . & 3.6 & . & 3.4 & - \\
\hline Fluoride & 1.9 & . & 1 & . & 1.3 & . & 1.4 & • \\
\hline Magnesium & 6.9 & 6.5 & 7 & 7 & 9.9 & 9.3 & 9 & 8.5 \\
\hline Manganese & 2 & 1.9 & 0.91 & 0.89 & 0.57 & 0.59 & 0.33 & 0.18 \\
\hline Nitrate-N & $<0.2$ & • & $<0.2$ & . & 0.26 & • & 0.28 & • \\
\hline Potassium & 2.2 & 1.9 & 3 & 2.9 & 4 & 3.6 & 3.8 & 3.2 \\
\hline Sodium & 51 & 49 & 20 & 20 & 26 & 26 & 16 & 16 \\
\hline Sulfate & 4.3 & • & 50 & . & 46 & • & 31.8 & - \\
\hline
\end{tabular}

(CONTINUED) 
APPENDIX E.1

Groundwater Quality Data, 1993

\begin{tabular}{|c|c|c|c|c|c|c|c|c|}
\hline \multirow{4}{*}{$\begin{array}{l}\text { Sampling point } \\
\text { Location } \\
\text { Date Sampled }\end{array}$} & \multicolumn{8}{|c|}{ GW-206 } \\
\hline & \multicolumn{8}{|c|}{ EXP } \\
\hline & \multicolumn{2}{|c|}{$01 / 19 / 93$} & \multicolumn{2}{|c|}{$05 / 06 / 93$} & \multicolumn{2}{|c|}{$08 / 12 / 93$} & \multicolumn{2}{|c|}{$10 / 27 / 93$} \\
\hline & TOT & Drs & TOT & DIS & TOT & DIS & TOT & DIS \\
\hline METALS (mg/L) & $\cdot$ & $\cdot$ & - & $\cdot$ & . & . & . & . \\
\hline Aluminum & 3.4 & 0.18 & 22 & 0.29 & 49 & 2.3 & 42 & 1 \\
\hline Antimony & $<0.05$ & $<0.05$ & $<0.25$ & $<0.05$ & $<0.05$ & $<0.05$ & $<0.5$ & $<0.05$ \\
\hline Arsenic & $<0.05$ & $<0.05$ & $<0.25$ & $<0.05$ & $<0.05$ & $<0.05$ & $<0.5$ & $<0.05$ \\
\hline Barium & 0.18 & 0.18 & 0.48 & 0.28 & 0.88 & 0.6 & 0.59 & 0.25 \\
\hline Beryllium & $<0.0003$ & $<0.0003$ & 0.0016 & $<0.0003$ & 0.0033 & 0.0011 & $<0.003$ & $<0.0003$ \\
\hline boron & 0.048 & 0.039 & 0.069 & 0.15 & 0.077 & 0.02 & 0.091 & 0.028 \\
\hline Cadraium (AAS) & $<0.002$ & $<0.002$ & $<0.002$ & 0.004 & $<0.002$ & $<0.002$ & $<0.002$ & $<0.002$ \\
\hline Cadnitum & $<0.003$ & $<0.003$ & $<0.015$ & $<0.003$ & 0.019 & $<0.003$ & $<0.03$ & $<0.003$ \\
\hline Chromium (AAS) & 0.01 & $<0.01$ & 0.077 & $<0.01$ & 0.11 & $<0.01$ & 0.2 & $<0.01$ \\
\hline Chromium & $<0.01$ & $<0.01$ & 0.058 & $<0.01$ & 0.12 & $<0.01$ & $<0.1$ & $<0.01$ \\
\hline Cobalt & 0.006 & $<0.005$ & 0.058 & 0.0069 & 0.074 & 0.025 & 0.089 & $<0.005$ \\
\hline Copper & 0.0077 & $<0.004$ & 0.058 & 0.015 & 0.11 & 0.017 & 0.05 & $<0.004$ \\
\hline Iron & 23 & 5.9 & 78 & 3.6 & 120 & 47 & 130 & 19 \\
\hline Lead (AAS) & 0.0092 & $<0.004$ & 0.14 & $<0.004$ & 0.13 & 0.064 & 0.12 & $<0.004$ \\
\hline Mercury (CVAA) & $<0.0002$ & $<0.0002$ & $<0.0002$ & $<0.0002$ & $<0.0002$ & $<0.0002$ & $<0.0002$ & $<0.0002$ \\
\hline Mol ybdenum & $<0.01$ & $<0.01$ & $<0.05$ & $<0.01$ & $<0.01$ & $<0.01$ & $<0.1$ & $<0.01$ \\
\hline Nickel & $<0.01$ & $<0.01$ & 0.075 & 0.012 & 0.088 & 0.095 & 0.13 & $<0.01$ \\
\hline Selenium & $<0.05$ & $<0.05$ & $<0.25$ & $<0.05$ & $<0.05$ & $<0.05$ & $<0.5$ & $<0.05$ \\
\hline silver & $<0.006$ & $<0.006$ & $<0.03$ & $<0.006$ & $<0.006$ & $<0.006$ & $<0.06$ & $<0.006$ \\
\hline Strontium & 0.071 & 0.065 & 0.12 & 0.097 & 0.18 & 0.15 & 0.15 & 0.091 \\
\hline Thoriun & $<0.2$ & $<0.2$ & $<1$ & $<0.2$ & $<0.2$ & $<0.2$ & $<2$ & $<0.2$ \\
\hline Uranium (Fluor) & $<0.001$ & $<0.001$ & $<0.001$ & $<0.001$ & $<0.001$ & 0.001 & 0.001 & $<0.001$ \\
\hline Vanadium & 0.0074 & $<0.005$ & 0.05 & $<0.005$ & 0.1 & 0.021 & 0.087 & $<0.005$ \\
\hline zinc & 0.021 & 0.0034 & 0.15 & 0.053 & 0.15 & 0.027 & 0.21 & 0.0067 \\
\hline MAJOR IONS (mg/L) & . & . & . & . & . & . & . & . \\
\hline Alkalinity-HCO3 & 175 & . & 213 & . & 216 & . & 251 & . \\
\hline Alkalinity-CO3 & $<1$ & $\cdot$ & $<1$ & - & $<1$ & . & $<1$ & . \\
\hline Calcium & 45 & 39 & 99 & 51 & 150 & 120 & 120 & 55 \\
\hline Chloride & 5.2 & $\cdot$ & 3.8 & $\cdot$ & 5.7 & $\cdot$ & 21 & • \\
\hline Fluoride & $<0.1$ & . & $<0.1$ & $\cdot$ & $<0.1$ & $\cdot$ & $<0.1$ & • \\
\hline Magnesium & 17 & 13 & 53 & 19 & 79 & 53 & 73 & 18 \\
\hline Manganese & 1.4 & 0.92 & 4.8 & 1.1 & 7.5 & 5.7 & 7.8 & 1.3 \\
\hline Nitrate-N & $<0.2$ & . & $<0.2$ & $\cdot$ & $<0.2$ & . & $<0.2$ & • \\
\hline Potassium & 5.9 & 4.4 & 13 & 7.3 & 19 & 6.1 & 18 & 6.5 \\
\hline Sodium & 4.7 & 4.6 & 4.5 & 5.7 & 5.1 & 4.8 & 4.9 & 5.5 \\
\hline Sulfate & 10.5 & . & 14 & . & 21 & . & 9 & • \\
\hline
\end{tabular}

(CONTINUED) 
APPENDIX E. 1

Groundwater Quality Data, 1993

\begin{tabular}{|c|c|c|c|c|c|c|c|c|}
\hline \multirow{4}{*}{$\begin{array}{l}\text { Sampling Point } \\
\text { Location } \\
\text { Date Sampled }\end{array}$} & \multicolumn{8}{|c|}{ GW-207 } \\
\hline & \multicolumn{8}{|c|}{ EXP } \\
\hline & \multicolumn{2}{|c|}{$01 / 14 / 93$} & \multicolumn{2}{|c|}{$05 / 04 / 93$} & \multicolumn{2}{|c|}{$08 / 11 / 93$} & \multicolumn{2}{|c|}{$10 / 26 / 93$} \\
\hline & TOT & DIS & TOT & DIS & TOT & DIS & TOT & DIS \\
\hline MRTALS (mg/L) & $\cdot$ & • & - & - & $\cdot$ & - & - & • \\
\hline Aluminum & $<0.02$ & $<0.02$ & 0.04 & $<0.02$ & $<0.02$ & $<0.02$ & $<0.02$ & $<0.02$ \\
\hline Antimony & $<0.05$ & $<0.05$ & $<0.05$ & $<0.05$ & $<0.05$ & $<0.05$ & $<0.05$ & $<0.05$ \\
\hline Arsenic & $<0.05$ & $<0.05$ & $<0.05$ & $<0.05$ & $<0.05$ & $<0.05$ & $<0.05$ & $<0.05$ \\
\hline Barium & 0.045 & 0.045 & 0.044 & 0.043 & 0.049 & 0.049 & 0.048 & 0.048 \\
\hline Bery11ium & $<0.0003$ & $<0.0003$ & $<0.0003$ & $<0.0003$ & $<0.0003$ & $<0.0003$ & $<0.0003$ & $<0.0003$ \\
\hline Boron & 0.1 & 0.11 & 0.12 & 0.12 & 0.12 & 0.11 & 0.11 & 0.11 \\
\hline Cadmium (AAS) & $<0.002$ & $<0.002$ & $<0.002$ & $<0.002$ & $<0.002$ & $<0.002$ & $<0.002$ & $<0.002$ \\
\hline Cadmium & $<0.003$ & $<0.003$ & $<0.003$ & $<0.003$ & $<0.003$ & $<0.003$ & $<0.003$ & $<0.003$ \\
\hline Chromium (AAS) & $<0.01$ & $<0.01$ & $<0.01$ & $<0.01$ & $<0.01$ & $<0.01$ & $<0.01$ & $<0.01$ \\
\hline Chromium & $<0.01$ & $<0.01$ & $<0.01$ & $<0.01$ & $<0.01$ & $<0.01$ & $<0.01$ & $<0.01$ \\
\hline Cobalt & $<0.005$ & $<0.005$ & $<0.005$ & $<0.005$ & $<0.005$ & $<0.005$ & $<0.005$ & $<0.005$ \\
\hline Copper & $<0.004$ & $<0.004$ & $<0.004$ & $<0.004$ & $<0.004$ & $<0.004$ & $<0.004$ & $<0.004$ \\
\hline Iron & 0.2 & 0.2 & 0.23 & 0.2 & 0.22 & 0.2 & 0.22 & 0.21 \\
\hline Lead (AAS) & $<0.004$ & $<0.004$ & $<0.004$ & $<0.004$ & $<0.004$ & $<0.004$ & $<0.004$ & $<0.004$ \\
\hline Mercury (CVaA) & $<0.0002$ & $<0.0002$ & $<0.0002$ & $<0.0002$ & $<0.0002$ & $<0.0002$ & $<0.0002$ & $<0.0002$ \\
\hline Mol ybdenum & $<0.01$ & $<0.01$ & $<0.01$ & $<0.01$ & $<0.01$ & $<0.01$ & $<0.01$ & $<0.0 i$ \\
\hline Nickel & $<0.01$ & $<0.01$ & $<0.01$ & $<0.01$ & $<0.01$ & $<0.01$ & $<0.01$ & $<0.01$ \\
\hline Selenium & $<0.05$ & $<0.05$ & $<0.05$ & $<0.05$ & 0.053 & $<0.05$ & $<0.05$ & $<0.05$ \\
\hline silver & $<0.006$ & $<0.006$ & 0.012 & $<0.006$ & $<0.006$ & $<0.006$ & $<0.006$ & $<0.006$ \\
\hline Strontium & 0.48 & 0.48 & 0.52 & 0.51 & 0.52 & 0.52 & 0.49 & 0.49 \\
\hline Thorium & $<0.2$ & $<0.2$ & $<0.2$ & $<0.2$ & $<0.2$ & $<0.2$ & $<0.2$ & $<0.2$ \\
\hline Uranium (Fluor) & $<0.001$ & $<0.001$ & $<0.001$ & $<0.001$ & $<0.001$ & $<0.001$ & $<0.001$ & $<0.001$ \\
\hline Vanadium & $<0.005$ & $<0.005$ & $<0.005$ & $<0.005$ & $<0.005$ & $<0.005$ & $<0.005$ & $<0.005$ \\
\hline zinc & 0.0052 & $<0.002$ & 0.0037 & 0.0037 & 0.0041 & 0.005 & $<0.002$ & $<0.002$ \\
\hline MAJOR IONS $(\mathrm{mg} / \mathrm{L})$ & - & . & $\cdot$ & • & $\cdot$ & $\cdot$ & . & . \\
\hline Alkalinity-HCO3 & 266 & . & 266 & $\cdot$ & 263 & . & $\begin{array}{r}\cdot \\
267\end{array}$ & . \\
\hline Alkalinity $-\mathrm{CO} 3$ & $<1$ & $\cdot$ & $<1$ & • & $<1$ & . & $<1$ & . \\
\hline Calcium & 55 & 55 & 56 & 55 & 58 & 57 & 58 & 58 \\
\hline chloride & 1.3 & . & 1.2 & • & 1.8 & • & 1 & . \\
\hline Fluoride & 0.2 & . & 0.2 & . & 0.2 & . & 0.2 & . \\
\hline Magnesium & 36 & 36 & 36 & 36 & 38 & 38 & 37 & 37 \\
\hline Manganese & 0.0093 & 0.0096 & 0.0092 & 0.0092 & 0.0099 & 0.01 & 0.01 & 0.01 \\
\hline Nitrate-N & $<0.2$ & • & $<0.2$ & • & $<0.2$ & • & $<0.2$ & . \\
\hline Potassium & 2.8 & 2.7 & 2.8 & 2.7 & 2.5 & 2.4 & 3.1 & 2.9 \\
\hline Sodium & 10 & 9.8 & 9.9 & 9.5 & 11 & 11 & 11 & 9.9 \\
\hline Sulfate & 51 & - & 52 & . & 51 & • & 53 & . \\
\hline
\end{tabular}


APPENDIX E, 1

Groundwater Quality Data, 1993

\begin{tabular}{|c|c|c|c|c|c|c|c|c|}
\hline \multirow{4}{*}{$\begin{array}{l}\text { Sampling point } \\
\text { Location } \\
\text { Date Sampled }\end{array}$} & \multicolumn{8}{|c|}{$G W-208$} \\
\hline & \multicolumn{8}{|c|}{ EXP } \\
\hline & \multicolumn{2}{|c|}{$01 / 19 / 93$} & \multicolumn{2}{|c|}{$05 / 06 / 93$} & \multicolumn{2}{|c|}{$08 / 13 / 93$} & \multicolumn{2}{|c|}{$10 / 28 / 93$} \\
\hline & TOT & DIS & TOT & DIS & TOI & DIS & TOT & DIS \\
\hline METALS (mg/L) & - & - & - & - & - & - & - & - \\
\hline Aluminum & $<0.02$ & $<0.02$ & $<0.02$ & $<0.02$ & 0.021 & $<0.02$ & $<0.02$ & $<0.02$ \\
\hline Antimony & $<0.05$ & $<0.05$ & $<0.05$ & $<0.05$ & $<0.05$ & $<0.05$ & $<0.05$ & $<0.05$ \\
\hline Arsenic & $<0.05$ & $<0.05$ & $<0.05$ & $<0.05$ & $<0.05$ & $<0.05$ & $<0.05$ & $<0.05$ \\
\hline Darium & 0.04 & 0.039 & 0.037 & 0.037 & 0.043 & 0.042 & 0.039 & 0.04 \\
\hline Bery 111 um & $<0.0003$ & $<0.0003$ & $<0.0003$ & $<0.0003$ & $<0.0003$ & $<0.0003$ & $<0.0003$ & $<0.0003$ \\
\hline Boron & 0.21 & 0.23 & 0.23 & 0.21 & 0.21 & 0.22 & 0.21 & 0.21 \\
\hline Cadmium (MAS) & $<0.002$ & $<0.002$ & $<0.002$ & $<0.002$ & $<0.002$ & $<0.002$ & $<0.002$ & $<0.002$ \\
\hline Cadmium & $<0.003$ & $<0.003$ & $<0.003$ & $<0.003$ & $<0.003$ & $<0.003$ & $<0.003$ & $<0.003$ \\
\hline Chromium (MAS) & $<0.01$ & $<0.01$ & $<0.01$ & $<0.01$ & $<0.01$ & $<0.01$ & $<0.01$ & $<0.01$ \\
\hline Chromium & $<0.01$ & $<0.01$ & $<0.01$ & $<0.01$ & $<0.01$ & $<0.01$ & $<0.01$ & $<0.01$ \\
\hline Cobalt & $<0.005$ & $<0.005$ & $<0.005$ & $<0.005$ & $<0.005$ & $<0.005$ & $<0.005$ & $<0.005$ \\
\hline Copper & $<0.004$ & $<0.004$ & 0.0053 & $<0.004$ & 0.0097 & $<0.004$ & $<0.004$ & $<0.004$ \\
\hline Iron & 0.65 & 0.45 & 0.49 & 0.43 & 0.89 & 0.47 & 0.64 & 0.47 \\
\hline Laad (AAS) & $<0.004$ & $<0.004$ & $<0.004$ & $<0.004$ & $<0.004$ & $<0.004$ & $<0.004$ & $<0.004$ \\
\hline Mercury (CVAA) & $<0.0002$ & $<0.0002$ & $<0.0002$ & $<0.0002$ & $<0.0002$ & $<0.0002$ & $<0.0002$ & $<0.0002$ \\
\hline Mol ybdenum & $<0.01$ & $<0.01$ & $<0.01$ & $<0.01$ & $<0.01$ & $<0.01$ & $<0.01$ & $<0.01$ \\
\hline Mickel & $<0.01$ & $<0.01$ & 0.018 & $<0.01$ & $<0.01$ & $<0.01$ & $<0.01$ & $<0.01$ \\
\hline Salenium & $<0.05$ & $<0.05$ & $<0.05$ & $<0.05$ & 0.053 & $<0.05$ & $<0.05$ & $<0.05$ \\
\hline silver & $<0.006$ & $<0.006$ & $<0.006$ & $<0.006$ & $<0.006$ & $<0.006$ & $<0.006$ & $<0.006$ \\
\hline Strontium & 1.3 & 1.3 & 1.3 & 1.3 & 1.5 & 1.5 & 2.3 & 1.3 \\
\hline Thorium & $<0.2$ & $<0.2$ & $<0.2$ & $<0.2$ & $<0.2$ & $<0.2$ & $<0.2$ & $<0.2$ \\
\hline Oranium (Pluor) & $<0.001$ & $<0.001$ & $<0.001$ & $<0.001$ & $<0.001$ & $<0.001$ & $<0.001$ & $<0.001$ \\
\hline Vanadium & $<0.005$ & $<0.005$ & $<0.005$ & $<0.005$ & $<0.005$ & $<0.005$ & $<0.005$ & $<0.005$ \\
\hline Zinc & 1.9 & 1.4 & 1.2 & 0.91 & 2 & 1.3 & 2 & 1.8 \\
\hline MAJOR IONS (mg/L) & . & . & . & . & . & . & . & . \\
\hline Alkalinity-HCO3 & 223 & . & 222 & . & 227 & . & 226 & • \\
\hline Alkalinity-CO3 & $<1$ & - & $<1$ & $\cdot$ & $<1$ & • & $<1$ & • \\
\hline Calcium & 73 & 69 & 72 & 74 & 77 & 76 & 73 & 74 \\
\hline Chloride & 1.4 & $\cdot$ & 1.4 & - & 2.1 & - & 2 & • \\
\hline Fluoride & 0.3 & - & 0.2 & - & 0.3 & - & 0.4 & • \\
\hline Magnesium & 24 & 22 & 24 & 24 & 25 & 25 & 23 & 24 \\
\hline Manganese & 0.015 & 0.014 & 0.014 & 0.014 & 0.016 & 0.025 & 0.016 & 0.016 \\
\hline Nitrate-N & $<0.2$ & $\cdot$ & $<0.2$ & $\cdot$ & $<0.2$ & . & $<0.2$ & • \\
\hline Potass ium & 3.3 & 2.6 & 2.8 & 3.5 & 2.9 & 2.6 & 2.7 & 2.7 \\
\hline Sodium & 15 & 15 & 15 & 15 & 16 & 16 & 14 & 14 \\
\hline sulfate & 106 & $\cdot$ & 96 & $\cdot$ & 103 & - & 88 & • \\
\hline
\end{tabular}

(CONTINUED) 
APPENDIX E. 1

Groundwater Quality Data, 1993

\begin{tabular}{|c|c|c|c|c|c|c|c|c|}
\hline \multirow{4}{*}{$\begin{array}{l}\text { Sampling Point } \\
\text { Location } \\
\text { Date Sampled }\end{array}$} & \multicolumn{8}{|c|}{$G W-220$} \\
\hline & \multicolumn{8}{|c|}{ NHP } \\
\hline & \multicolumn{2}{|c|}{$01 / 25 / 93$} & \multicolumn{2}{|c|}{$04 / 22 / 93$} & \multicolumn{2}{|c|}{$08 / 06 / 93$} & \multicolumn{2}{|c|}{$11 / 01 / 93$} \\
\hline & TOT & DIS & TOT & Drs & TOT & DIS & TOT & Drs \\
\hline Merals $(\mathrm{mg} / \mathrm{L})$ & $\cdot$ & $\cdot$ & - & $\cdot$ & $\cdot$ & - & $\cdot$ & \\
\hline Aluminum & 0.23 & 0.039 & 8.6 & 0.043 & 2.4 & $<0.02$ & 0.39 & 0.023 \\
\hline Antimony & $<0.05$ & $<0.05$ & $<0.05$ & $<0.05$ & $<0.05$ & $<0.05$ & $<0.05$ & $<0.05$ \\
\hline Arsenic & $<0.05$ & $<0.05$ & $<0.05$ & $<0.05$ & $<0.05$ & $<0.05$ & $<0.05$ & $<0.05$ \\
\hline Barium & 0.095 & 0.086 & 0.18 & 0.097 & 0.11 & 0.091 & 0.1 & 0.1 \\
\hline Beryl1ium & $<0.0003$ & $<0.0003$ & 0.00087 & $<0.0003$ & $<0.0003$ & $<0.0003$ & $<0.0003$ & $<0.0003$ \\
\hline Boron & 0.04 & 0.029 & 0.045 & 0.033 & 0.061 & 0.058 & 0.042 & 0.043 \\
\hline Cadmitum (AAS) & $<0.002$ & $<0.002$ & $<0.002$ & $<0.002$ & $<0.002$ & $<0.002$ & $<0.002$ & $<0.002$ \\
\hline Cadmium & $<0.003$ & $<0.003$ & $<0.003$ & $<0.003$ & $<0.003$ & $<0.003$ & $<0.003$ & $<0.003$ \\
\hline Chromiun (AAS) & $<0.01$ & $<0.01$ & 0.021 & $<0.01$ & $<0.01$ & $<0.01$ & $<0.01$ & $<0.01$ \\
\hline Chromiun & $<0.01$ & $<0.01$ & 0.01 & $<0.01$ & $<0.01$ & $<0.01$ & $<0.01$ & $<0.01$ \\
\hline Cobalt & $<0.005$ & $<0.005$ & $<0.005$ & $<0.005$ & 0.0052 & $<0.005$ & $<0.005$ & $<0.005$ \\
\hline Copper & 0.0086 & $<0.004$ & 0.0094 & 0.0043 & 0.0073 & $<0.004$ & $<0.004$ & $<0.004$ \\
\hline Iron & 0.2 & $<0.005$ & 8.5 & 0.023 & 2.8 & $<0.005$ & 0.47 & $<0.005$ \\
\hline Lead (AAS) & $<0.004$ & $<0.004$ & 0.023 & $<0.004$ & 0.0055 & $<0.004$ & $<0.004$ & $<0.004$ \\
\hline Mercury (CVAA) & $<0.0002$ & $<0.0002$ & $<0.0002$ & $<0.0002$ & $<0.0002$ & $<0.0002$ & $<0.0002$ & $<0.0002$ \\
\hline Molybdenum & $<0.01$ & $<0.01$ & $<0.01$ & $<0.01$ & $<0.01$ & $<0.01$ & $<0.01$ & $<0.01$ \\
\hline Mickel & $<0.01$ & $<0.01$ & $<0.01$ & $<0.01$ & $<0.01$ & $<0.01$ & $<0.01$ & $<0.01$ \\
\hline Selenium & $<0.05$ & $<0.05$ & $<0.05$ & $<0.05$ & $<0.05$ & $<0.05$ & $<0.05$ & $<0.05$ \\
\hline silver & $<0.006$ & $<0.006$ & $<0.006$ & $<0.006$ & $<0.006$ & $<0.006$ & $<0.006$ & $<0.006$ \\
\hline Strontiun & 0.41 & 0.38 & 0.47 & 0.41 & 0.37 & 0.36 & 0.41 & 0.4 \\
\hline Thorlum & $<0.2$ & $<0.2$ & $<0.2$ & $<0.2$ & $<0.2$ & $<0.2$ & $<0.2$ & $<0.2$ \\
\hline Oranium (Fluor) & $<0.001$ & $<0.001$ & 0.001 & 0.001 & $<0.001$ & $<0.001$ & 0.001 & $<0.001$ \\
\hline Vanadium & $<0.005$ & $<0.005$ & 0.011 & $<0.005$ & 0.0067 & $<0.005$ & $<0.005$ & $<0.005$ \\
\hline zinc & 0.016 & 0.018 & 0.023 & 0.0041 & 0.02 & 0.0092 & 0.0029 & 0.0064 \\
\hline MAJOR IONS (mg/L) & - & . & . & . & - & . & • & . \\
\hline Alkalinity-HCO3 & 222 &. & 234 &. & 220 &. & $23 \dot{9}$ & . \\
\hline Alkalinity-CO3 & $<1$ &. & $<1$ & . & $<1$ & . & $<1$ & . \\
\hline Calcium & 59 & 54 & 99 & 63 & 63 & 57 & 64 & 63 \\
\hline Chloride & 8.3 & $\cdot$ & 6.9 & . & 8.4 & $\cdot$ & 9.2 & - \\
\hline Fluoride & $<0.1$ & $\cdot$ & $<0.1$ & $\cdot$ & $<0.1$ & . & 0.2 & . \\
\hline Magnesium & 24 & 22 & 26 & 23 & 23 & 22 & 22 & 23 \\
\hline Manganese & 0.014 & 0.0082 & 0.24 & 0.0063 & 0.077 & 0.0028 & 0.019 & 0.0067 \\
\hline Nitrate-N & 0.43 & . & 0.78 & $\cdot$ & 0.85 & $\cdot$ & 0.72 & . \\
\hline Potassium & 2.1 & 2.4 & 4.4 & 2.5 & 3.1 & 2 & 3 & 2.8 \\
\hline Sodium & 3.9 & 3.6 & 3.7 & 3.5 & 4.6 & 4.6 & 4.3 & 4.1 \\
\hline sulfate & 10.2 & $\cdot$ & 17 & . & 19 & $\cdot$ & 12.5 & . \\
\hline
\end{tabular}

(CONTINURD) 
APPBNDIX E.1

\begin{tabular}{|c|c|c|c|c|c|c|c|c|}
\hline \multirow{4}{*}{$\begin{array}{l}\text { Sampling Point } \\
\text { Location } \\
\text { Date Sampled }\end{array}$} & \multicolumn{8}{|c|}{ GW-232 } \\
\hline & \multicolumn{8}{|c|}{ EXP } \\
\hline & \multicolumn{2}{|c|}{$01 / 26 / 93$} & \multicolumn{2}{|c|}{$05 / 21 / 93$} & \multicolumn{2}{|c|}{$08 / 21 / 93$} & \multicolumn{2}{|c|}{$11 / 01 / 93$} \\
\hline & TOT & DIS & TOT & DIS & TOT & DIS & TOT & Drs \\
\hline METALS (mg/L) & . & . & . & . & - & . & . & \\
\hline Aluminum & 0.022 & $<0.02$ & 0.045 & 0.042 & $<0.02$ & $<0.02$ & $<0.02$ & $<0.02$ \\
\hline Antimony & $<0.05$ & $<0.05$ & $<0.05$ & $<0.05$ & $<0.05$ & $<0.05$ & $<0.05$ & $<0.05$ \\
\hline Arsente & $<0.05$ & $<0.05$ & $<0.05$ & 0.071 & $<0.05$ & $<0.05$ & $<0.05$ & $<0.05$ \\
\hline Barium & 0.016 & 0.014 & 0.012 & 0.012 & 0.012 & 0.01 & 0.015 & 0.014 \\
\hline Beryl11um & $<0.0003$ & $<0.0003$ & $<0.0003$ & $<0.0003$ & $<0.0003$ & $<0.0003$ & $<0.0003$ & $<0.0003$ \\
\hline Boron & 1.3 & 1.3 & 1.3 & 1.4 & 1.4 & 1.4 & 1.3 & 1.2 \\
\hline Cadmium (AMS) & $<0.002$ & $<0.002$ & $<0.002$ & $<0.002$ & $<0.002$ & $<0.002$ & $<0.002$ & $<0.002$ \\
\hline Cadmium & $<0.003$ & $<0.003$ & $<0.003$ & $<0.003$ & $<0.003$ & $<0.003$ & $<0.003$ & $<0.003$ \\
\hline Chromium (AAS) & $<0.01$ & $<0.01$ & $<0.01$ & $<0.01$ & $<0.01$ & $<0.01$ & $<0.01$ & $<0.01$ \\
\hline Chromium & $<0.01$ & $<0.01$ & $<0.01$ & $<0.01$ & $<0.01$ & $<0.01$ & $<0.01$ & $<0.01$ \\
\hline Cobalt & $<0.005$ & $<0.005$ & $<0.005$ & $<0.005$ & $<0.005$ & $<0.005$ & $<0.005$ & $<0.005$ \\
\hline Copper & 0.0097 & $<0.004$ & 0.01 & 0.0065 & $<0.004$ & 0.0067 & $<0.004$ & $<0.004$ \\
\hline Iron & 1.2 & 0.032 & 0.073 & 0.052 & 2.6 & 0.12 & 0.8 & 0.02 \\
\hline Lead (AAS) & $<0.004$ & $<0.004$ & $<0.004$ & $<0.004$ & $<0.004$ & $<0.004$ & $<0.004$ & $<0.004$ \\
\hline Mercury (CVAA) & $<0.0002$ & $<0.0002$ & $<0.0002$ & $<0.0002$ & $<0.0002$ & $<0.0002$ & $<0.0002$ & $<0.0002$ \\
\hline Molybdenum & $<0.01$ & $<0.01$ & $<0.01$ & $<0.01$ & $<0.01$ & $<0.01$ & $<0.01$ & $<0.01$ \\
\hline Nickel & $<0.01$ & $<0.01$ & $<0.01$ & $<0.01$ & $<0.01$ & $<0.01$ & $<0.01$ & $<0.01$ \\
\hline Selentum & $<0.05$ & $<0.05$ & $<0.05$ & $<0.05$ & $<0.05$ & $<0.05$ & $<0.05$ & $<0.05$ \\
\hline stlver & $<0.006$ & $<0.006$ & $<0.006$ & $<0.006$ & $<0.006$ & $<0.006$ & $<0.006$ & $<0.006$ \\
\hline strontium & 0.31 & 0.31 & 0.3 & 0.33 & 0.32 & 0.31 & 0.33 & 0.32 \\
\hline Thorium & $<0.2$ & $<0.2$ & $<0.2$ & $<0.2$ & $<0.2$ & $<0.2$ & $<0.2$ & $<0.2$ \\
\hline Uranium (Fluor) & $<0.001$ & $<0.001$ & $<0.001$ & $<0.001$ & 0.001 & $<0.001$ & $<0.001$ & $<0.001$ \\
\hline Vanadium & $<0.005$ & $<0.005$ & $<0.005$ & $<0.005$ & $<0.005$ & $<0.005$ & $<0.005$ & $<0.005$ \\
\hline zinc & 0.039 & 0.0068 & 0.006 & 0.0081 & 0.039 & 0.031 & 0.0095 & 0.0034 \\
\hline MAJOR IONS $(\mathrm{mg} / \mathrm{L})$ & • & - & - & . & . & . & . & • \\
\hline Alkalinity- $\mathrm{HCO} 3$ & 368 & . & $352^{\circ}$ & . & $35 \dot{2}^{\circ}$ &. & 362 & . \\
\hline Alkalinity- $\mathrm{CO} 3$ & 72 & • & 82 & • & $<1$ & $\cdot$ & $<1$ & • \\
\hline Calcium & 1.4 & 1.1 & 1.2 & 1.3 & 2.7 & 2.3 & 1.2 & 1.1 \\
\hline Chloride & 5.5 & • & 5.6 & • & 5.6 & . & 6.7 & • \\
\hline Fluoride & 2.1 & . & 1.9 & . & 2.2 &. & 2 & • \\
\hline Magnesium & 0.93 & 0.91 & 0.81 & 0.89 & 0.96 & 0.94 & 0.88 & 0.84 \\
\hline Manganese & 0.0055 & 0.0012 & 0.0025 & 0.003 & 0.0097 & 0.0023 & 0.0038 & $<0.001$ \\
\hline Nitrate-N & $<0.2$ & • & $<0.2$ & • & $<0.2$ &. & $<0.2$ & 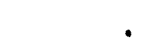 \\
\hline Potassium & 2.8 & 2.6 & 2 & 2.7 & 2.8 & 3.3 & 2.1 & 2.3 \\
\hline Sodtum & 210 & 210 & 210 & 230 & 220 & 220 & 230 & 230 \\
\hline sulfate & 31.3 & • & 30 & . & 26.5 & . & 28.6 & • \\
\hline
\end{tabular}

(CONT INUED) 
APPENDIX R. 1

Groundwater Quality Data, 1993

\begin{tabular}{|c|c|c|c|c|c|c|c|c|}
\hline \multirow{4}{*}{$\begin{array}{l}\text { Sampling Point } \\
\text { Location } \\
\text { Date Sampled }\end{array}$} & \multicolumn{8}{|c|}{ GW-239 } \\
\hline & \multicolumn{8}{|c|}{ NHP } \\
\hline & \multicolumn{2}{|c|}{$01 / 23 / 93$} & \multicolumn{2}{|c|}{$05 / 13 / 93$} & \multicolumn{2}{|c|}{$08 / 18 / 93$} & \multicolumn{2}{|c|}{$10 / 30 / 93$} \\
\hline & TOT & DIS & TOT & DIS & TOT & DIS & TOT & DIS \\
\hline METALS (mg/L) & $\cdot$ & - & - & - & . & . & . & - \\
\hline Aluminum & 0.023 & $<0.02$ & 0.026 & 0.032 & 0.029 & $<0.02$ & $<0.02$ & $<0.02$ \\
\hline Antimony & $<0.05$ & $<0.05$ & $<0.05$ & $<0.05$ & $<0.05$ & $<0.05$ & $<0.05$ & $<0.05$ \\
\hline Arsenic & $<0.05$ & $<0.05$ & $<0.05$ & $<0.05$ & $<0.05$ & $<0.05$ & $<0.05$ & $<0.05$ \\
\hline Barium & 0.051 & 0.048 & 0.049 & 0.047 & 0.045 & 0.043 & 0.069 & 0.051 \\
\hline Beryllium & $<0.0003$ & $<0.0003$ & $<0.0003$ & $<0.0003$ & $<0.0003$ & $<0.0003$ & $<0.0003$ & $<0.0003$ \\
\hline Boron & 1.5 & 1.5 & 1.5 & 1.5 & 1.6 & 1.6 & 1.5 & 1.5 \\
\hline Cadmium (AAS) & $<0.002$ & $<0.002$ & $<0.002$ & $<0.002$ & $<0.002$ & $<0.002$ & $<0.002$ & $<0.002$ \\
\hline Cadmium & $<0.003$ & $<0.003$ & $<0.003$ & $<0.003$ & $<0.003$ & $<0.003$ & $<0.003$ & $<0.003$ \\
\hline Chromium (AAS) & $<0.01$ & $<0.01$ & $<0.01$ & $<0.01$ & $<0.01$ & $<0.01$ & $<0.01$ & $<0.01$ \\
\hline Chromium & $<0.01$ & $<0.01$ & $<0.01$ & $<0.01$ & $<0.01$ & $<0.01$ & $<0.01$ & $<0.01$ \\
\hline Cobalt & $<0.005$ & $<0.005$ & $<0.005$ & $<0.005$ & $<0.005$ & $<0.005$ & $<0.005$ & $<0.005$ \\
\hline Copper & $<0.004$ & $<0.004$ & $<0.004$ & $<0.004$ & $<0.004$ & $<0.004$ & $<0.004$ & $<0.004$ \\
\hline Iron & 2.1 & 0.17 & 1.1 & 0.2 & 3.4 & 0.13 & 16 & 0.37 \\
\hline Lead (AAS) & $<0.004$ & $<0.004$ & $<0.004$ & $<0.004$ & $<0.004$ & $<0.004$ & 0.0045 & $<0.004$ \\
\hline Mercury (CVAA) & $<0.0002$ & $<0.0002$ & $<0.0002$ & $<0.0002$ & $<0.0002$ & $<0.0002$ & $<0.0002$ & $<0.0002$ \\
\hline Mol ybdenum & $<0.01$ & $<0.01$ & $<0.01$ & $<0.01$ & $<0.01$ & $<0.01$ & $<0.01$ & $<0.01$ \\
\hline Nickel & $<0.01$ & $<0.01$ & $<0.01$ & 0.017 & $<0.01$ & $<0.01$ & $<0.01$ & $<0.01$ \\
\hline selenium & $<0.05$ & $<0.05$ & $<0.05$ & $<0.05$ & $<0.05$ & $<0.05$ & $<0.05$ & $<0.05$ \\
\hline silver & $<0.006$ & $<0.006$ & $<0.006$ & $<0.006$ & $<0.006$ & $<0.006$ & $<0.006$ & $<0.006$ \\
\hline Strontium & 0.19 & 0.18 & 0.2 & 0.2 & 0.17 & 0.18 & 0.21 & 0.2 \\
\hline Thorium & $<0.2$ & $<0.2$ & $<0.2$ & $<0.2$ & $<0.2$ & $<0.2$ & $<0.2$ & $<0.2$ \\
\hline Uranium (Fluor) & 0.001 & $<0.001$ & $<0.001$ & $<0.001$ & $<0.001$ & $<0.001$ & $<0.001$ & 0.001 \\
\hline Vanadium & $<0.005$ & $<0.005$ & $<0.005$ & $<0.005$ & $<0.005$ & $<0.005$ & $<0.005$ & $<0.005$ \\
\hline zinc & 0.0043 & 0.004 & 0.005 & 0.0065 & 0.02 & 0.015 & 0.0079 & 0.0027 \\
\hline MAJOR IONS $(\mathrm{mg} / \mathrm{L})$ & . & . & . & . & . & . & . & • \\
\hline Alkalinity-HCO3 & 494 & $\cdot$ & 496 &. & 511. & $\cdot$. & $\begin{array}{r}. \\
505\end{array}$ & . \\
\hline Alkalinity- $\mathrm{CO} 3$ & 44 & . & 56 &. & 40 &. & 50 & . \\
\hline Calcium & 1.7 & 1.6 & 1.7 & 1.7 & 1.5 & 1.5 & 2 & 1.6 \\
\hline Chloride & 280 & . & 281 & . & 256 & . & 229 & • \\
\hline Fluoride & 3 & $\cdot$ & 3 & $\cdot$ & 2.9 & $\cdot$ & 3.6 & • \\
\hline Magnesium & 0.52 & 0.51 & 0.43 & 0.47 & 0.48 & 0.49 & 0.5 & 0.46 \\
\hline Manganese & 0.0076 & 0.0032 & 0.0093 & 0.0068 & 0.013 & 0.0034 & 0.047 & 0.0039 \\
\hline Nitrate-N & $<0.2$ &. & $<0.2$ & $\cdot$ & $<0.2$ &. & $<0.2$ & • \\
\hline Potassium & 4.3 & 3.7 & 3.7 & 3.9 & 4 & 3.8 & 3.2 & 3.3 \\
\hline Sodium & 410 & 410 & 390 & 390 & 410 & 410 & 430 & 430 \\
\hline Sulfate & 47 &. & 44 & . & 43 &. & 45 & . \\
\hline
\end{tabular}

(CONTINUED) 
APPENDIX E. 1

Groundwater Quality Data, 1993

\begin{tabular}{|c|c|c|c|c|c|c|c|c|}
\hline \multirow{4}{*}{$\begin{array}{l}\text { Sampling point } \\
\text { Location } \\
\text { Date Sampled }\end{array}$} & \multicolumn{8}{|c|}{$G W-240$} \\
\hline & \multicolumn{8}{|c|}{ NHP } \\
\hline & \multicolumn{2}{|c|}{$01 / 27 / 93$} & \multicolumn{2}{|c|}{$04 / 21 / 93$} & \multicolumn{2}{|c|}{$08 / 05 / 93$} & \multicolumn{2}{|c|}{$11 / 01 / 93$} \\
\hline & TOT & DIS & TOT & DIS & TOT & Drs & TOT & DIS \\
\hline METALS $(\mathrm{mg} / \mathrm{L})$ & $\cdot$ & $\cdot$ & • & . & . & . & - & • \\
\hline Aluminum & 0.066 & $<0.02$ & 0.027 & $<0.02$ & 0.029 & $<0.02$ & 0.039 & $<0.02$ \\
\hline Antimony & $<0.05$ & $<0.05$ & $<0.05$ & $<0.05$ & $<0.05$ & $<0.05$ & $<0.05$ & $<0.05$ \\
\hline Arsenic & $<0.05$ & $<0.05$ & $<0.05$ & $<0.05$ & $<0.05$ & $<0.05$ & $<0.05$ & $<0.05$ \\
\hline Barium & 0.046 & 0.042 & 0.027 & 0.028 & 0.035 & 0.036 & 0.048 & 0.047 \\
\hline Beryllium & 0.0021 & $<0.0003$ & $<0,0003$ & $<0.0003$ & $<0.0003$ & $<0.0003$ & $<0.0003$ & $<0.0003$ \\
\hline Boron & 0.028 & 0.027 & 0.073 & 0.069 & 0.072 & 0.055 & 0.064 & 0.068 \\
\hline Cadmium (AAS) & $<0.002$ & $<0.002$ & $<0.002$ & $<0.002$ & $<0.002$ & $<0.002$ & $<0.002$ & $<0.002$ \\
\hline Cadmium & 0.0075 & 0.0037 & $<0.003$ & $<0.003$ & $<0.003$ & $<0.003$ & $<0.003$ & $<0.003$ \\
\hline Chromium (AAS) & $<0.01$ & $<0.01$ & $<0.01$ & $<0.01$ & $<0.01$ & $<0.01$ & $<0.01$ & $<0.01$ \\
\hline Chromium & $<0.01$ & $<0.01$ & $<0.01$ & $<0.01$ & $<0.01$ & $<0.01$ & $<0.01$ & $<0.01$ \\
\hline Cobalt & $<0.005$ & $<0.005$ & $<0.005$ & $<0.005$ & $<0.005$ & $<0.005$ & $<0.005$ & $<0.005$ \\
\hline Copper & 0.014 & $<0.004$ & $<0.004$ & $<0.004$ & $<0.004$ & $<0.004$ & $<0.004$ & $<0.004$ \\
\hline Iron & 0.019 & 0.051 & 0.02 & $<0.005$ & 0.012 & $<0.005$ & $<0.005$ & $<0.005$ \\
\hline Lead (AAS) & $<0.004$ & $<0.004$ & $<0.004$ & $<0.004$ & $<0.004$ & $<0.004$ & $<0.004$ & $<0.004$ \\
\hline Mercury (CVAA) & $<0.0002$ & $<0.0002$ & $<0.0002$ & $<0.0002$ & $<0.0002$ & $<0.0002$ & $<0.0002$ & $<0.0002$ \\
\hline Molybdenum & $<0.01$ & $<0.01$ & $<0.01$ & $<0.01$ & $<0.01$ & $<0.01$ & $<0.01$ & $<0.01$ \\
\hline Nickel & 0.017 & $<0.01$ & $<0.01$ & 0.015 & $\therefore 0.01$ & $<0.01$ & $<0.01$ & $<0.01$ \\
\hline Selenium & $<0.05$ & $<0.05$ & $<0.05$ & $<0.05$ & $<0.05$ & 0.063 & $<0.05$ & $<0.05$ \\
\hline Silver & $<0.006$ & $<0.006$ & $<0.006$ & $<0.006$ & $<0.006$ & $<0.006$ & $<0.006$ & $<0.006$ \\
\hline Strontium & 0.061 & 0.058 & 0.044 & 0.047 & 0.057 & 0.059 & 0.076 & 0.076 \\
\hline Thorium & $<0.2$ & $<0.2$ & $<0.2$ & $<0.2$ & $<0.2$ & $<0.2$ & $<0.2$ & $<0.2$ \\
\hline Uranium (Fluor) & 0.004 & 0.005 & 0.003 & 0.003 & 0.005 & 0.004 & 0.007 & 0.001 \\
\hline Vanadium & $<0.005$ & $<0.005$ & $<0.005$ & $<0.005$ & $<0.005$ & $<0.005$ & $<0.005$ & $<0.005$ \\
\hline zine & 0.0079 & $<0.002$ & 0.011 & 0.0077 & 0.0084 & 0.0098 & 0.0027 & 0.0056 \\
\hline MAJOR IONS (mg/L) & $\cdot$ & $\cdot$ & $\cdot$ & . &. & $\cdot$ & $\cdot$ & - \\
\hline Alkalinity-HCO3 & $191^{\circ}$ & $\cdot$ & $182^{\circ}$ & $\cdot$ & 161 & . & $\begin{array}{r}\cdot \\
156\end{array}$ & • \\
\hline Alkalinity-CO3 & $<1$ & . & $<1$ & . & $<1$ & . & $<1$ & . \\
\hline Calcium & 50 & 48 & 38 & 36 & 42 & 45 & 53 & 52 \\
\hline Chloride & 139 & $\cdot$ & 57 & $\cdot$ & 39 & . & 79.6 & • \\
\hline Fluoride & 0.3 & $\cdot$ & 0.2 & $\cdot$ & 0.3 & . & 0.5 & . \\
\hline Magnesium & 18 & 17 & 16 & 15 & 16 & 16 & 17 & 17 \\
\hline Manganese & 0.0054 & 0.0026 & $<0.001$ & 0.0016 & 0.0017 & 0.0011 & 0.0019 & $<0.001$ \\
\hline Nitrate-N & 1.1 & $\cdot$ & 0.85 & $\cdot$ & 2.5 & $\cdot$ & 4 & \\
\hline Potassium & 3 & 4 & 5.2 & 11 & 1.4 & 2.2 & 1.8 & 1.9 \\
\hline Sodium & 68 & 71 & 42 & 42 & 34 & 35 & 51 & 53 \\
\hline Sulfate & 14 & $\cdot$ & 14 & $\cdot$ & 36 & $\cdot$ & 58.1 & 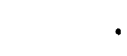 \\
\hline
\end{tabular}

(CONTINUED) 
APPENDIX $\mathrm{E} .1$

Groundwater Quality Data, 1993

\begin{tabular}{|c|c|c|c|c|c|c|c|c|}
\hline \multirow{4}{*}{$\begin{array}{l}\text { Sampling Point } \\
\text { Location } \\
\text { Date Sampled }\end{array}$} & \multicolumn{8}{|c|}{ GW-251 } \\
\hline & \multicolumn{8}{|c|}{ s2 } \\
\hline & \multicolumn{2}{|c|}{$01 / 20 / 93$} & \multicolumn{2}{|c|}{$05 / 05 / 93$} & \multicolumn{2}{|c|}{$09 / 17 / 93$} & \multicolumn{2}{|c|}{$12 / 08 / 93$} \\
\hline & TOT & DIS & TOT & DIS & TOT & DIS & TOT & DIS \\
\hline METALS (mg/L) & $\cdot$ & $\cdot$ & $\cdot$ & $\cdot 1$ & $\cdot$ & - & - & • \\
\hline Aluminum & 0.96 & 0.43 & 4.4 & 0.24 & 17 & 0.033 & 6.7 & 0.067 \\
\hline Antimony & $<0.05$ & $<0.05$ & $<0.05$ & $<0.05$ & $<0.05$ & $<0.05$ & $<0.05$ & $<0.05$ \\
\hline Arsenic & $<0.05$ & $<0.05$ & $<0.05$ & $<0.05$ & $<0.05$ & $<0.05$ & $<0.05$ & $<0.05$ \\
\hline Barium & 0.12 & 0.12 & 0.14 & 0.15 & 0.13 & 0.078 & 0.097 & 0.078 \\
\hline Beryllium & 0.00094 & 0.00054 & 0.0011 & 0.0019 & 0.0026 & $<0.0003$ & 0.00097 & $<0.0003$ \\
\hline Boron & 0.032 & 0.023 & 0.047 & 0.045 & 0.012 & 0.017 & 0.023 & 0.049 \\
\hline Cadmium (AAS) & 0.16 & 0.15 & 0.2 & 0.2 & 0.083 & 0.075 & 0.078 & 0.072 \\
\hline Cadmium & 0.16 & 0.16 & 0.18 & 0.18 & 0.084 & 0.069 & 0.075 & 0.089 \\
\hline Chromium (AAS) & 0.012 & $<0.01$ & 0.014 & $<0.01$ & 0.04 & $<0.01$ & 0.011 & $<0.01$ \\
\hline Chromium & $<0.01$ & $<0.01$ & 0.022 & 0.017 & 0.033 & $<0.01$ & 0.011 & $<0.01$ \\
\hline Cobalt & 0.035 & 0.032 & 0.039 & 0.036 & 0.023 & 0.0063 & 0.015 & 0.013 \\
\hline Copper & 0.58 & 0.42 & 0.65 & 0.46 & 0.95 & 0.17 & 0.39 & 0.15 \\
\hline Iron & 0.75 & 0.26 & 4.9 & 0.12 & 23 & $<0.005$ & 7.6 & 0.0055 \\
\hline Lead (AAS) & 0.033 & $<0.004$ & 0.022 & $<0.004$ & 0.06 & $<0.004$ & 0.033 & $<0.004$ \\
\hline Mercury (CVAA) & 0.00025 & $<0.0002$ & $<0.0002$ & $<0.0002$ & $<0.0002$ & $<0.0002$ & $<0.0002$ & $<0.0002$ \\
\hline Molybdenum & $<0.01$ & $<0.01$ & $<0.01$ & $<0.01$ & $<0.01$ & $<0.01$ & $<0.01$ & $<0.01$ \\
\hline Nickel & 0.059 & 0.049 & 0.067 & 0.061 & 0.051 & 0.014 & 0.018 & $<0.01$ \\
\hline Selenium & $<0.05$ & 0.058 & $<0.05$ & $<0.05$ & $<0.05$ & $<0.05$ & $<0.05$ & $<0.05$ \\
\hline silver & $<0.006$ & $<0.006$ & $<0.006$ & $<0.006$ & $<0.006$ & $<0.006$ & $<0.006$ & $<0.006$ \\
\hline Strontium & 0.19 & 0.19 & 0.23 & 0.26 & 0.14 & 0.14 & 0.13 & 0.13 \\
\hline Thorium & $<0.2$ & $<0.2$ & $<0.2$ & $<0.2$ & $<0.2$ & $<0.2$ & $<0.2$ & $<0.2$ \\
\hline Uranium (Fluor) & 0.006 & 0.005 & 0.007 & 0.006 & 0.01 & 0.004 & 0.004 & 0.003 \\
\hline Vanadium & $<0.005$ & $<0.005$ & $<0.005$ & $<0.005$ & 0.029 & $<0.005$ & 0.0091 & $<0.00$ \\
\hline zinc & 0.11 & 0.081 & 0.11 & 0.11 & 0.12 & 0.028 & 0.067 & 0.033 \\
\hline MAJOR IONS $(\mathrm{mg} / \mathrm{L})$ &. & • & . & . & . & . & . & • \\
\hline Alkalinity-HCO3 & 159 & $\cdot$ & 160 & $\cdot$ & $16 \dot{4}$ & $\cdot$ & $15 \dot{8}$ & • \\
\hline Alkalinity-CO3 & $<1$ & . & $<1$ & . & $<1$ & . & $<1$ & • \\
\hline Calcium & 120 & 110 & 120 & 130 & 98 & 95 & 84 & 110 \\
\hline Chloride & 12 & $\cdot$ & 11 & • & 6 & - & 7.1 & • \\
\hline Fluoride & 1.3 & $\cdot$ & 1.2 & $\cdot$ & 1.6 & . & 1.5 & • \\
\hline Magnesium & 18 & 18 & 19 & 19 & 18 & 17 & 15 & 18 \\
\hline Manganese & 4.7 & 4.6 & 5.4 & 5.2 & 2.9 & 1.8 & 2 & 1.9 \\
\hline Nitrate-N & 82 & . & 83 &. & 244 & • & 42.9 & • \\
\hline Potassium & 3.5 & 3.7 & 3.9 & 3.4 & 5.6 & 2.4 & 3.7 & 2.4 \\
\hline Sodium & 18 & 18 & 20 & 20 & 12 & 12 & 11 & 10 \\
\hline sulfate & 19 & $\cdot$ & 22 & . & 11 & • & 9.1 & • \\
\hline
\end{tabular}

(CONTINUED) 
APPENDIX E.1

Groundwater Quality Jata, 1993

\begin{tabular}{|c|c|c|c|c|c|c|c|c|}
\hline \multirow{4}{*}{$\begin{array}{l}\text { Sampling Point } \\
\text { Location } \\
\text { - } \\
\text { Date Sampled }\end{array}$} & \multicolumn{8}{|c|}{$G w-252$} \\
\hline & \multicolumn{8}{|c|}{ s2 } \\
\hline & \multicolumn{2}{|c|}{$01 / 08 / 93$} & \multicolumn{2}{|c|}{$05 / 03 / 93$} & \multicolumn{2}{|c|}{$09 / 15 / 93$} & \multicolumn{2}{|c|}{$12 / 07 / 93$} \\
\hline & TOT & DIS & TOT & DIS & TOT & DIS & TOS & DIS \\
\hline METALS (mg/I) & $\cdot$ & $\cdot$ & $\cdot$ & $\cdot$ & - & $\cdot$ & - & - \\
\hline Aluminum & 0.27 & 0.021 & 1.6 & $<0.02$ & 16 & $<0.02$ & 0.55 & $<0.02$ \\
\hline Antimony & $<0.05$ & $<0.05$ & $<0.05$ & $<0.05$ & $<0.05$ & $<0.05$ & $<0.05$ & $<0.05$ \\
\hline Arsenic & $<0.05$ & $<0.05$ & $<0.05$ & $<0.05$ & $<0.05$ & $<0.05$ & $<0.05$ & $<0.05$ \\
\hline Barium & 0.01 & 0.0094 & 0.023 & 0.015 & 0.06 & 0.022 & 0.016 & 0.012 \\
\hline Beryllium & $<0.0003$ & $<0.0003$ & $<0.0003$ & $<0.0003$ & 0.0014 & $<0.0003$ & $<0.0003$ & $<0.0003$ \\
\hline Boron & 0.026 & 0.018 & 0.03 & 0.012 & 0.022 & 0.0045 & 0.027 & 0.012 \\
\hline Cadmium (AAS) & $<0.002$ & $<0.002$ & $<0.002$ & $<0.002$ & $<0.002$ & $<0.002$ & 0.0032 & $<0.002$ \\
\hline Cadmium & $<0.003$ & $<0.003$ & $<0.003$ & $<0.003$ & 0.0077 & $<0.003$ & $<0.003$ & $<0.003$ \\
\hline Chromium (AAS) & $<0.01$ & $<0.01$ & 0.018 & $<0.01$ & 0.05 & 0.01 & $<0.01$ & $<0.01$ \\
\hline Chromium & $<0.01$ & $<0.01$ & 0.017 & $<0.01$ & 0.042 & $<0.01$ & $<0.01$ & $<0.01$ \\
\hline Cobalt & $<0.005$ & $<0.005$ & $<0.005$ & $<0.005$ & 0.0085 & $<0.005$ & $<0.005$ & $<0.005$ \\
\hline Copper & $<0.004$ & $<0.004$ & $<0.004$ & $<0.004$ & 0.053 & $<0.004$ & 0.0059 & 0.0044 \\
\hline Iron & 0.4 & $<0.005$ & 1.7 & $<0.005$ & 20 & $<0.005$ & 0.64 & $<0.005$ \\
\hline Lead (AAS) & $<0.004$ & $<0.004$ & 0.0074 & $<0.004$ & 0.067 & $<0.004$ & $<0.004$ & $<0.004$ \\
\hline Mercury (CVAA) & $<0.0002$ & $<0.0002$ & $<0.0002$ & $<0.0002$ & 0.00023 & $<0.0002$ & $<0.0002$ & $<0.0002$ \\
\hline Mlybdenum & $<0.01$ & $<0.01$ & $<0.01$ & $<0.01$ & $<0.01$ & $<0.01$ & $<0.01$ & $<0.01$ \\
\hline Nickel & $<0.01$ & $<0.01$ & 0.011 & $<0.01$ & 0.034 & $<0.01$ & $<0.01$ & $<0.01$ \\
\hline Selenium & $<0.05$ & $<0.05$ & $<0.05$ & $<0.05$ & $<0.05$ & $<0.05$ & $<0.05$ & $<0.05$ \\
\hline silver & $<0.006$ & $<0.006$ & $<0.006$ & $<0.006$ & $<0.006$ & $<0.006$ & $<0.006$ & $<0.006$ \\
\hline strontium & 0.0067 & 0.0058 & 0.018 & 0.016 & 0.021 & 0.018 & 0.0078 & 0.0061 \\
\hline Thorium & $<0.2$ & $<0.2$ & $<0.2$ & $<0.2$ & $<0.2$ & $<0.2$ & $<0.2$ & $<0.2$ \\
\hline Uranium (Fluor) & $<0.001$ & 0.001 & $<0.001$ & $<0.001$ & $<0.001$ & $<0.001$ & $<0.001$ & $<0.001$ \\
\hline Vanadium & $<0.005$ & $<0.005$ & 0.0072 & $<0.005$ & 0.028 & $<0.005$ & $<0.005$ & $<0.005$ \\
\hline zinc & 0.01 & 0.0067 & 0.026 & 0.023 & 0.15 & 0.037 & 0.017 & 0.009 \\
\hline MAJOR IONS $(\mathrm{mg} / \mathrm{L})$ & - & - & - & - & • & - & - & . \\
\hline Alkalinity-HCO3 & 46 & . & 59 & . & 197 & . & 95 & . \\
\hline Alkalinity-CO3 & $<1$ & $\cdot$ & $<1$ & - & $<1$ & $\cdot$ & $<1$ & - \\
\hline Calcium & 7.2 & 5.3 & 31 & 31 & 45 & 39 & 7.9 & 5 \\
\hline Chloride & 1.5 & $\cdot$ & 2.2 & - & 3.5 & • & 1 & - \\
\hline Fluoride & $<0.1$ & $\cdot$ & 0.2 & - & 0.2 & $\cdot$ & $<0.1$ & • \\
\hline Magnesium & 3.9 & 2.7 & 22 & 22 & 28 & 24 & 4.9 & 2.8 \\
\hline Manganese & 0.012 & 0.0024 & 0.075 & 0.0018 & 0.65 & 0.0031 & 0.017 & 0.0018 \\
\hline Nitrate-N & 0.47 & $\cdot$ & 0.87 & • & 2.5 & $\bullet$ & 0.5 & • \\
\hline Potassium & $<0.6$ & $<0.6$ & 1.9 & 1.7 & 3.7 & 1.4 & 0.92 & 0.64 \\
\hline Sodium & 5.2 & 3.4 & 10 & 10 & 10 & 13 & $3 \cdot 4$ & 2.1 \\
\hline Sulfate & 2.7 & $\cdot$ & 9.6 & - & 11 & - & 1 & • \\
\hline
\end{tabular}

(CONTINUED) 
APPENDIX R.1

Groundwater Quality Data, 1993

\begin{tabular}{|c|c|c|c|c|c|c|c|c|}
\hline \multirow{4}{*}{$\begin{array}{l}\text { Sampling Point } \\
\text { Location } \\
\text { Date Sampled }\end{array}$} & \multicolumn{8}{|c|}{ GW-255 } \\
\hline & \multicolumn{8}{|c|}{ s2 } \\
\hline & \multicolumn{2}{|c|}{$01 / 08 / 93$} & \multicolumn{2}{|c|}{$05 / 03 / 93$} & \multicolumn{2}{|c|}{$09 / 15 / 93$} & \multicolumn{2}{|c|}{$12 / 07 / 93$} \\
\hline & TOT & DIS & TOT & DIS & TOT & DIS & TOT & DIS \\
\hline METALS (mg/L) & - & . & . & - & - & . & . & • \\
\hline Aluminum & 2.9 & $<0.02$ & 4 & $<0.02$ & 7.8 & 0.033 & 29 & $<0.02$ \\
\hline Antimony & $<0.05$ & $<0.05$ & $<0.05$ & $<0.05$ & $<0.05$ & $<0.05$ & $<0.05$ & $<0.05$ \\
\hline Arsenic & $<0.05$ & $<0.05$ & $<0.05$ & $<0.05$ & $<0.05$ & $<0.05$ & $<0.05$ & $<0.05$ \\
\hline Barium & 0.04 & 0.032 & 0.045 & 0.03 & 0.079 & 0.041 & 0.22 & 0.04 \\
\hline Beryllium & $<0.0003$ & $<0.0003$ & 0.0003 & $<0.0003$ & 0.0008 & $<0.0003$ & 0.0033 & $<0.0003$ \\
\hline Boron & 0.025 & 0.02 & 0.018 & 0.018 & 0.021 & 0.02 & 0.084 & 0.084 \\
\hline Cadmium (AAS) & $<0.002$ & $<0.002$ & $<0.002$ & $<0.002$ & $<0.002$ & $<0.002$ & 0.0023 & $<0.002$ \\
\hline Cadmium & $<0.003$ & $<0.003$ & $<0.003$ & $<0.003$ & $<0.003$ & $<0.003$ & $<0.003$ & $<0.003$ \\
\hline Chromium (AAS) & $<0.01$ & $<0.01$ & 0.013 & $<0.01$ & 0.018 & $<0.01$ & 0.045 & $<0.01$ \\
\hline Chromium & $<0.01$ & $<0.01$ & $<0.01$ & $<0.01$ & 0.014 & $<0.01$ & 0.027 & $<0.01$ \\
\hline Cobalt & $<0.005$ & $<0.005$ & $<0.005$ & $<0.005$ & 0.0055 & $<0.005$ & 0.03 & $<0.005$ \\
\hline Copper & 0.0071 & $<0.004$ & $<0.004$ & $<0.004$ & 0.039 & $<0.004$ & 0.1 & $<0.004$ \\
\hline Iron & 3.1 & $<0.005$ & 4.5 & 0.011 & 11 & 0.046 & 39 & $<0.005$ \\
\hline Lead (AAS) & 0.0056 & $<0.004$ & 0.0081 & $<0.004$ & 0.018 & $<0.004$ & 0.077 & $<0.004$ \\
\hline Mercury (CVAA) & $<0.0002$ & $<0.0002$ & 0.0002 & $<0.0002$ & $<0.0002$ & $<0.0002$ & 0.00038 & $<0.0002$ \\
\hline Molybdenum & $<0.01$ & $<0.01$ & $<0.01$ & $<0.01$ & $<0.01$ & $<0.01$ & $<0.01$ & $<0.01$ \\
\hline Nickel & $<0.01$ & $<0.01$ & $<0.01$ & $<0.01$ & 0.014 & $<0.01$ & 0.047 & $<0.01$ \\
\hline Selenium & $<0.05$ & $<0.05$ & $<0.05$ & $<0.05$ & $<0.05$ & $<0.05$ & $<0.05$ & $<0.05$ \\
\hline Silver & $<0.006$ & $<0.006$ & $<0.006$ & $<0.006$ & $<0.006$ & $<0.006$ & $<0.006$ & $<0.006$ \\
\hline strontium & 0.028 & 0.028 & 0.026 & 0.025 & 0.035 & 0.034 & 0.04 & 0.032 \\
\hline Thorium & $<0.2$ & $<0.2$ & $<0.2$ & $<0.2$ & $<0.2$ & $<0.2$ & $<0.2$ & $<0.2$ \\
\hline Uranium (Fluor) & 0.001 & 0.001 & 0.001 & $<0.001$ & 0.001 & $<0.001$ & 0.001 & 0.001 \\
\hline Vanadiun & $<0.005$ & $<0.005$ & 0.0055 & $<0.005$ & 0.016 & $<0.005$ & 0.051 & $<0.005$ \\
\hline zinc & 0.023 & 0.0032 & 0.029 & 0.0072 & 0.055 & 0.0049 & 0.19 & $<0.002$ \\
\hline MAJOR IONS $(\mathrm{mg} / \mathrm{L})$ & . & . & . & . & . &. & $\dot{.}$ & . \\
\hline Alkalinity-нCO3 & 236 & • & 237 & . & $239^{\circ}$ & . & 244 & • \\
\hline Alkalinity-CO3 & $<1$ & . & $<1$ & . & $<1$ & . & $<1$ & . \\
\hline Calcium & 56 & 57 & 49 & 48 & 61 & 59 & 67 & 58 \\
\hline Chloride & 5.5 & . & 4.8 & . & 6.1 & . & 6 & • \\
\hline Fluoride & $<0.1$ & . & $<0.1$ & . & $<0.1$ & . & $<0.1$ & • \\
\hline Magnesium & 30 & 30 & 31 & 30 & 33 & 30 & 43 & 31 \\
\hline Manganese & 0.1 & $<0.001$ & 0.24 & 0.0016 & 0.59 & 0.0032 & 2.8 & $<0.001$ \\
\hline Nitrate-N & 1.6 &. & 2.2 & . & 1.8 & . & 3.1 & • \\
\hline Potassium & 1.6 & 0.83 & 1.6 & 1.2 & 3 & 1.2 & 7.1 & 1.3 \\
\hline Sodium & 3.4 & 3.4 & 3.1 & 3.3 & 4.2 & 4.2 & 3.9 & 3.8 \\
\hline Sulfate & 12 & . & 12 & . & 16 & . & 9 & • \\
\hline
\end{tabular}

(CONTINUED) 
APPENDIX E.1

Groundwater Quality Data, 1993

\begin{tabular}{|c|c|c|c|c|c|c|c|c|}
\hline \multirow{4}{*}{$\begin{array}{l}\text { Sampling Point } \\
\text { Location } \\
\text { Date Sampled }\end{array}$} & \multicolumn{8}{|c|}{$G W-261$} \\
\hline & \multicolumn{8}{|c|}{ SY } \\
\hline & \multicolumn{2}{|c|}{$01 / 08 / 93$} & \multicolumn{2}{|c|}{$04 / 07 / 93$} & \multicolumn{2}{|c|}{$07 / 01 / 93$} & \multicolumn{2}{|c|}{$12 / 07 / 93$} \\
\hline & TOT & DIS & TOT & DIS & TOT & DIS & TOT & DIS \\
\hline METALS (mg/L) & - & - & - & - & - & - & - & - \\
\hline Aluminum & 0.06 & $<0.02$ & 0.24 & 0.028 & 0.39 & $<0.02$ & 0.06 & $<0.02$ \\
\hline Antimony & $<0.05$ & $<0.05$ & $<0.05$ & $<0.05$ & $<0.05$ & $<0.05$ & $<0.05$ & $<0.05$ \\
\hline Arsenic & $<0.05$ & $<0.05$ & $<0.05$ & $<0.05$ & $<0.05$ & $<0.05$ & $<0.05$ & $<0.05$ \\
\hline Barium & 0.079 & 0.076 & 0.086 & 0.079 & 0.089 & 0.071 & 0.07 & 0.073 \\
\hline Beryllium & $<0.0003$ & $<0.0003$ & $<0.0003$ & $<0.0003$ & $<0.0003$ & $<0.0003$ & $<0,0003$ & $<0.0003$ \\
\hline Boron & 0.017 & 0.013 & 0.021 & 0.018 & 0.011 & 0.035 & 0.027 & 0.026 \\
\hline Cadmiun (AAS) & $<0.002$ & $<0.002$ & $<0.002$ & $<0.002$ & $<0.002$ & 0.0066 & $<0.002$ & $<0.002$ \\
\hline Cadmium & $<0.003$ & $<0.003$ & $<0.003$ & $<0.003$ & $<0.003$ & 0.0071 & $<0.003$ & $<0.003$ \\
\hline Chromium (AAS) & $<0.01$ & $<0.01$ & $<0.01$ & $<0.01$ & $<0.01$ & $<0.01$ & $<0.01$ & $<0.01$ \\
\hline Chromium & $<0.01$ & $<0.01$ & $<0.01$ & $<0.01$ & $<0.01$ & $<0.01$ & $<0.01$ & $<0.01$ \\
\hline Cobalt & $<0.005$ & $<0.005$ & $<0.005$ & $<0.005$ & $<0.005$ & $<0.005$ & $<0.005$ & $<0.005$ \\
\hline Copper & 0.0056 & $<0.004$ & $<0.004$ & $<0.004$ & 0.0061 & 0.034 & $<0.004$ & $<0.004$ \\
\hline Iron & 0.058 & $<0.005$ & 0.53 & 0.0083 & 0.69 & $<0.005$ & 0.055 & $<0.005$ \\
\hline Lead (AAS) & $<0.004$ & $<0.004$ & $<0.004$ & $<0.004$ & $<0.004$ & $<0.004$ & $<0.004$ & $<0.004$ \\
\hline Mercury (CVAA) & $<0.0002$ & $<0.0002$ & $<0.0002$ & $<0.0002$ & $<0.0002$ & $<0.0002$ & $<0.0002$ & $<0.0002$ \\
\hline Molybdenum & $<0.01$ & $<0.01$ & $<0.01$ & $<0.01$ & $<0.01$ & $<0.01$ & $<0.01$ & $<0.01$ \\
\hline Nickel & $<0.01$ & $<0.01$ & $<0.01$ & $<0.01$ & $<0.01$ & $<0.01$ & $<0.01$ & $<0.01$ \\
\hline Selenium & $<0.05$ & $<0.05$ & $<0.05$ & $<0.05$ & $<0.05$ & $<0.05$ & $<0.05$ & $<0.05$ \\
\hline silver & $<0.006$ & $<0.006$ & $<0.006$ & $<0.006$ & $<0.006$ & $<0.006$ & $<0.006$ & $<0.006$ \\
\hline Strontium & 0.089 & 0.087 & 0.092 & 0.091 & 0.087 & 0.089 & 0.09 & 0.089 \\
\hline Thorium & $<0.2$ & $<0.2$ & $<0.2$ & $<0.2$ & $<0.2$ & $<0.2$ & $<0.2$ & $<0.2$ \\
\hline Uranium (Fluor) & $<0.001$ & $<0.001$ & $<0.001$ & 0.001 & $<0.001$ & $<0.001$ & $<0.001$ & $<0.001$ \\
\hline Vanadium & $<0.005$ & $<0.005$ & $<0.005$ & $<0.005$ & $<0.005$ & $<0.005$ & $<0.005$ & $<0.005$ \\
\hline zinc & 0.014 & 0.0092 & 0.03 & 0.028 & 0.018 & 0.063 & 0.016 & 0.014 \\
\hline MANO: IONS $(\mathrm{mg} / \mathrm{L})$ & • & $\cdot$ & $\cdot$ & $\cdot$ & . & $\cdot$ & $\cdot$ & • \\
\hline Alkalinity-HCO3 & 149 & • & 153 & $\dot{.}$ & 150 & . & ${ }_{153}$ & • \\
\hline Alkalinity-CO3 & $<1$ & . & $<1$ & . & $<1$ & . & $<1$ & . \\
\hline Calcium & 46 & 46 & 49 & 48 & 46 & 48 & 47 & 49 \\
\hline Chloride & 3.5 & . & 3.8 & . & 4.2 & • & 5 & • \\
\hline Fluoride & 0.2 & $\cdot$ & 0.2 & . & 0.3 & . & 0.2 & . \\
\hline Magnesium & 11 & 11 & 12 & 12 & 12 & 12 & 12 & 12 \\
\hline Manganese & 0.013 & 0.0084 & 0.011 & 0.0027 & 0.023 & 0.032 & 0.011 & 0.012 \\
\hline Nitrate-N & $<0.2$ & $\cdot$ & $<0.2$ & . & $<0.2$ & • & $<0.2$ & • \\
\hline Potassium & 1.8 & 1.3 & 1.9 & 1.7 & 1.6 & 2 & 1.2 & 1.7 \\
\hline Sodium & 9.8 & 9.7 & 9.3 & 9.4 & 9 & 9.4 & 10 & 10 \\
\hline Sulfate & 33 & . & 33 & . & 36 & . & 31 & • \\
\hline
\end{tabular}

(CONTINUED) 
APPENDIX E. 1

\begin{tabular}{|c|c|c|c|c|c|c|c|c|}
\hline \multirow{4}{*}{$\begin{array}{l}\text { Sampling Point } \\
\text { Location } \\
\text { Date Sampled }\end{array}$} & \multicolumn{8}{|c|}{$G W-262$} \\
\hline & \multicolumn{8}{|c|}{ SY } \\
\hline & \multicolumn{2}{|c|}{$01 / 11 / 93$} & \multicolumn{2}{|c|}{$04 / 08 / 93$} & \multicolumn{2}{|c|}{$07 / 01 / 93$} & \multicolumn{2}{|c|}{$12 / 07 / 93$} \\
\hline & TOT & DIS & TOT & DIS & TOT & DIS & TOT & DIS \\
\hline MBTALS (mg/L) & • & - & $\cdot$ & $\cdot$ & $\cdot$ & - & $\cdot$ & • \\
\hline Aluminum & 0.061 & 0.025 & 0.042 & 0.16 & 0.81 & 0.027 & 0.94 & $<0.02$ \\
\hline Antimony & $<0.05$ & $<0.05$ & $<0.05$ & $<0.05$ & $<0.05$ & $<0.05$ & $<0.05$ & $<0.05$ \\
\hline Arsenic & $<0.05$ & $<0.05$ & $<0.05$ & $<0.05$ & $<0.05$ & $<0.05$ & $<0.05$ & $<0.05$ \\
\hline Barium & 0.28 & 0.27 & 0.27 & 0.26 & 0.28 & 0.26 & 0.29 & 0.27 \\
\hline Beryllium & $<0.0003$ & $<0.0003$ & $<0.0003$ & $<0.0003$ & $<0.0003$ & $<0.0003$ & $<0.0003$ & $<0.0003$ \\
\hline Boron & 0.021 & 0.019 & 0.019 & 0.016 & 0.027 & 0.036 & 0.045 & 0.037 \\
\hline Cacmium (AAS) & $<0.002$ & $<0.002$ & $<0.002$ & $<0.002$ & $<0.002$ & $<0.002$ & $<0.002$ & $<0.002$ \\
\hline Cadmium & $<0.003$ & $<0.003$ & $<0.003$ & $<0.003$ & $<0.003$ & $<0.003$ & $<0.003$ & $<0.003$ \\
\hline Chromium (AAS) & $<0.01$ & $<0.01$ & $<0.01$ & $<0.01$ & $<0.01$ & $<0.01$ & $<0.01$ & $<0.01$ \\
\hline Chromium & $<0.01$ & $<0.01$ & $<0.01$ & $<0.01$ & $<0.01$ & $<0.01$ & $<0.01$ & $<0.01$ \\
\hline Cobalt & $<0.005$ & $<0.005$ & $<0.005$ & $<0.005$ & $<0.005$ & $<0.005$ & $<0.005$ & $<0.005$ \\
\hline Copper & $<0.004$ & $<0.004$ & $<0.004$ & $<0.004$ & 0.0045 & $<0.004$ & $<0.004$ & $<0.004$ \\
\hline Iron & 0.18 & 0.076 & 0.011 & 0.79 & 1 & $<0.005$ & 0.95 & $<0.005$ \\
\hline Lead (AAS) & $<0.004$ & $<0.004$ & $<0.004$ & $<0.004$ & $<0.004$ & $<0.004$ & $<0.004$ & $<0.004$ \\
\hline Mercury (CVAA) & $<0.0002$ & $<0.0002$ & $<0.0002$ & $<0.0002$ & $<0.0002$ & $<0.0002$ & $<0.0002$ & $<0.0002$ \\
\hline Molybdenum & $<0.01$ & $<0.01$ & $<0.01$ & $<0.01$ & $<0.01$ & $<0.01$ & $<0.01$ & $<0.01$ \\
\hline Nickel & $<0.01$ & 0.012 & $<0.01$ & $<0.01$ & $<0.01$ & $<0.01$ & $<0.01$ & $<0.01$ \\
\hline Selenium & $<0.05$ & $<0.05$ & $<0.05$ & $<0.05$ & $<0.05$ & $<0.05$ & $<0.05$ & $<0.05$ \\
\hline Silver & $<0.006$ & $<0.006$ & $<0.006$ & $<0.006$ & $<0.006$ & $<0.006$ & $<0.006$ & $<0.006$ \\
\hline strontium & 0.15 & 0.15 & 0.15 & 0.15 & 0.15 & 0.14 & 0.15 & 0.15 \\
\hline Thorium & $<0.2$ & $<0.2$ & $<0.2$ & $<0.2$ & $<0.2$ & $<0.2$ & $<0.2$ & $<0.2$ \\
\hline Uranium (Fluor) & 0.001 & $<0.001$ & 0.001 & 0.001 & 0.002 & 0.001 & 0.001 & 0.001 \\
\hline Vanadium & $<0.005$ & $<0.005$ & $<0.005$ & $<0.005$ & $<0.005$ & $<0.005$ & $<0.005$ & $<0.005$ \\
\hline zine & 0.0092 & 0.0055 & 0.0086 & 0.018 & 0.026 & 0.032 & 0.0057 & 0.0024 \\
\hline MAJOR IONS $(\mathrm{mg} / \mathrm{L})$ &. & - &. & - & - & . & - & • \\
\hline Alkalinity-HCO3 & 198 & . & 200 & . & 207 & . & $\begin{array}{r}\cdot \\
204\end{array}$ & • \\
\hline Alkalinity-CO3 & $<1$ & . & $<1$ & . & $<1$ & . & $<1$ & • \\
\hline Calcium & 62 & 61 & 66 & 64 & 64 & 62 & 71 & 64 \\
\hline Chloride & 1.1 & • & 1 & - & $<1$ & • & $<1$ & • \\
\hline Fluoride & 0.1 & . & 0.2 & . & 0.1 & . & 0.1 & . \\
\hline Magnesium & 5.9 & 5.9 & 6.1 & 6.1 & 6.2 & 5.9 & 6.7 & 6.1 \\
\hline Manganese & 0.066 & 0.059 & 0.096 & 0.1 & 0.085 & 0.06 & 0.094 & 0.069 \\
\hline Nitrate-N & $<0.2$ & • & $<0.2$ & . & $<0.2$ & . & $<0.2$ & • \\
\hline Potassium & 1.5 & 1.9 & 1.6 & 1.8 & 2.2 & 1.4 & 1.7 & 1.3 \\
\hline Sodium & 14 & 14 & 15 & 14 & 14 & 15 & 14 & 14 \\
\hline Sulfate & 14 & • & 15 & . & 13 & . & 10 & • \\
\hline
\end{tabular}


APPENDIX 8.1

\begin{tabular}{|c|c|c|c|c|c|c|c|c|}
\hline \multirow{4}{*}{$\begin{array}{l}\text { ampling point } \\
\text { scation } \\
\text { Date sampled }\end{array}$} & \multicolumn{8}{|c|}{$G w-263$} \\
\hline & \multicolumn{8}{|c|}{ SY } \\
\hline & \multicolumn{2}{|c|}{$01 / 11 / 93$} & \multicolumn{2}{|c|}{$04 / 08 / 93$} & \multicolumn{2}{|c|}{$07 / 01 / 93$} & \multicolumn{2}{|c|}{$12 / 07 / 93$} \\
\hline & TOT & DIS & TOT & DIS & Tot & Drs & TOT & DIS \\
\hline Metrals (mg/L) & - & . & $\cdot$ & - & . & • & - & - \\
\hline Aluminum & 13 & 0.063 & 0.3 & 0.042 & 2.7 & $<0.02$ & 0.3 & $<0.02$ \\
\hline Antimony & $<0.05$ & $<0.05$ & $<0.05$ & $<0.05$ & $<0.05$ & $<0.05$ & $<0.05$ & $<0.05$ \\
\hline Arsenic & $<0.05$ & $<0.05$ & $<0.05$ & $<0.05$ & $<0.05$ & $<0.05$ & $<0.05$ & $<0.05$ \\
\hline Barium & 0.45 & 0.17 & 0.14 & 0.13 & 0.14 & 0.13 & 0.17 & 0.16 \\
\hline Beryl11um & 0.00065 & $<0.0003$ & 0.00038 & $<0.0003$ & $<0.0003$ & $<0.0003$ & $<0.0003$ & $<0.0003$ \\
\hline Boron & 0.024 & 0.29 & 0.021 & 0.053 & 0.013 & 0.013 & 0.18 & 0.049 \\
\hline Cadmium (MAS) & $<0.002$ & $<0.002$ & 0.0038 & 0.003 & 0.003 & $<0.002$ & 0.0067 & 0.0025 \\
\hline Cadmium & 0.005 & $<0.003$ & 0.0035 & 0.0031 & $<0.003$ & $<0.003$ & 0.0062 & $<0.003$ \\
\hline Chromium (AAS) & 5.8 & 0.026 & 0.049 & $<0.01$ & 0.17 & $<0.01$ & 0.074 & $<0.01$ \\
\hline Chromium & 5 & 0.021 & 0.036 & $<0.01$ & 0.16 & $<0.01$ & 0.085 & $<0.01$ \\
\hline Cobalt & 0.048 & 0.0091 & $<0.005$ & $<0.005$ & 0.0054 & $<0.005$ & $<0.005$ & $<0.005$ \\
\hline Copper & 0.2 & 0.0046 & $<0.004$ & $<0.004$ & 0.02 & $<0.004$ & 0.0071 & $<0.004$ \\
\hline Iron & 37 & 0.15 & 0.93 & 0.018 & 3.6 & $<0.005$ & 0.8 & $<0.005$ \\
\hline Lead (AAS) & 0.11 & $<0.004$ & $<0.004$ & $<0.004$ & $<0.004$ & $<0.004$ & $<0.004$ & $<0.004$ \\
\hline Mercury (CVAA) & $<0.0002$ & $<0.0002$ & $<0.0002$ & $<0.0002$ & $<0.0002$ & $<0.0002$ & $<0.0002$ & $<0.0002$ \\
\hline Nolybdenum & 0.051 & $<0.01$ & $<0.01$ & $<0.01$ & $<0.01$ & $<0.01$ & $<0.01$ & $<0.01$ \\
\hline Nickel & 2.1 & 0.96 & 0.33 & 0.33 & 0.36 & 0.15 & 0.28 & 0.26 \\
\hline Selenium & $<0.05$ & $<0.05$ & $<0.05$ & $<0.05$ & $<0.05$ & $<0.05$ & $<0.05$ & $<0.05$ \\
\hline Silver & $<0.006$ & $<0.006$ & $<0.006$ & $<0.006$ & $<0.006$ & $<0.006$ & $<0.006$ & $<0.006$ \\
\hline Strontium & 0.18 & 0.16 & 0.14 & 0.14 & 0.13 & 0.14 & 0.16 & 0.16 \\
\hline Thorium & $<0.2$ & $<0.2$ & $<0.2$ & $<0.2$ & $<0.2$ & $<0.2$ & $<0.2$ & $<0.2$ \\
\hline Uranium (Fluor) & 0.001 & $<0.001$ & $<0.001$ & $<0.001$ & 0.001 & $<0.001$ & $<0.001$ & $<0.001$ \\
\hline Vanadium & 0.032 & $<0.005$ & $<0.005$ & $<0.005$ & 0.0057 & $<0.005$ & $<0.005$ & $<0.005$ \\
\hline zinc & 0.075 & $<0.002$ & 0.03 & 0.023 & 0.04 & 0.015 & 0.032 & 0.0041 \\
\hline MAJOR IONS $(\mathrm{mg} / \mathrm{L})$ & - & . & . & . & . & . & . & • \\
\hline Alkalinity- $\mathrm{HCO} 3$ & 190 & . & 163 &. & $157^{\circ}$ & $\cdot$ & 198 & • \\
\hline Alkalinity- $\mathrm{CO} 3$ & $<1$ & . & $<1$ &. & $<1$ & . & $<1$ & . \\
\hline Calcium & 75 & 65 & 61 & 61 & 56 & 59 & 72 & 71 \\
\hline Chloride & 59 & - & 58 & $\cdot$ & 43 & $\cdot$ & 53 & • \\
\hline Fluoride & 0.1 & . & 0.3 & . & 0.2 &. & 0.1 & • \\
\hline Magnesium & 20 & 15 & 15 & 15 & 14 & 14 & 17 & 17 \\
\hline Manganese & 0.75 & 0.21 & 0.043 & 0.023 & 0.11 & 0.023 & 0.039 & 0.018 \\
\hline Nitrate-N & 0.52 & . & 0.39 & . & 0.27 & . & 0.6 & • \\
\hline Potassium & 4.7 & 2.1 & 1.7 & 1.7 & 2.6 & 1.8 & 1.9 & 1.5 \\
\hline Sodium & 21 & 20 & 20 & 19.8 & 20 & 20 & 22 & 21 \\
\hline Sulfate & 26 & . & 19 & . & 16 & . & 16 & • \\
\hline
\end{tabular}

(CONT INUED) 
APPENDIX R.1

Groundwater Quality Data, 1993

\begin{tabular}{|c|c|c|c|c|c|c|c|c|}
\hline \multirow{4}{*}{$\begin{array}{l}\text { Sampling Point } \\
\text { Location } \\
\text { Date Sampled }\end{array}$} & \multicolumn{8}{|c|}{$G W-264$} \\
\hline & \multicolumn{8}{|c|}{ sy } \\
\hline & \multicolumn{2}{|c|}{$01 / 12 / 93$} & \multicolumn{2}{|c|}{$04 / 12 / 93$} & \multicolumn{2}{|c|}{$07 / 02 / 93$} & \multicolumn{2}{|c|}{$12 / 08 / 93$} \\
\hline & TOT & DIS & TOT & DIS & TOT & DIS & TOT & DIS \\
\hline $\operatorname{varuLS}(\mathrm{mg} / \mathrm{L})$ & $\cdot$ & . & . & . & . & . & - & • \\
\hline Aluminum & 0.038 & 0.03 & 0.61 & 0.079 & 0.24 & $<0.02$ & 0.51 & 0.021 \\
\hline Antimony & $<0.05$ & $<0.05$ & $<0.05$ & $<0.05$ & $<0.05$ & $<0.05$ & $<0.05$ & $<0.05$ \\
\hline Armenic & $<0.05$ & $<0.05$ & $<0.05$ & $<0.05$ & $<0.05$ & $<0.05$ & $<0.05$ & $<0.05$ \\
\hline Barium & 0.024 & 0.05 & 0.044 & 0.04 & 0.042 & 0.038 & 0.036 & 0.026 \\
\hline Beryl11um & $<0.0003$ & $<0 \quad 0003$ & $<0.0003$ & $<0.0003$ & $<0.0003$ & $<0.0003$ & $<0.0003$ & $<0.0003$ \\
\hline Boron & 0.028 & 0.028 & 0.12 & 0.052 & 0.022 & 0.017 & 0.06 & 0.075 \\
\hline Cadnium (MAS) & $<0,002$ & $<0.002$ & $<0.002$ & $<0.002$ & $<0.002$ & $<0.002$ & $<0.002$ & $<0.002$ \\
\hline Cadmium & $<0.003$ & $<0.003$ & $<0.003$ & $<0.003$ & $<0.003$ & $<0.003$ & $<0.003$ & $<0.003$ \\
\hline Chromium (AAs) & $<0.01$ & $<0.01$ & $<0.01$ & $<0.01$ & $<0.01$ & $<0.01$ & $<0.01$ & $<0.01$ \\
\hline Chromium & $<0.01$ & $<0.01$ & $<0.01$ & $<0.01$ & $<0.01$ & $<0.01$ & $<0.01$ & $<0.01$ \\
\hline Cobalt & $<0.005$ & $<0.005$ & $<0.005$ & $<0.005$ & $<0.005$ & $<0.005$ & $<0.005$ & $<0.005$ \\
\hline Copper & $<0.004$ & $<0.004$ & $<0.004$ & $<0.004$ & 0.005 & $<0.004$ & $<0.004$ & $<0.004$ \\
\hline Iron & 0.18 & 0.12 & 0.98 & 0.14 & 0.39 & 0.047 & 0.75 & 0.044 \\
\hline Lead (MAS) & $<0.004$ & $<0.004$ & $<0.004$ & $<0.004$ & $<0.004$ & $<0.004$ & $<0.004$ & $<0.004$ \\
\hline Mercury (CVAA) & $<0.0002$ & $<0.0002$ & $<0.0002$ & $<0.0002$ & $<0.0002$ & $<0.0002$ & $<0.0002$ & $<0.0002$ \\
\hline Molybdenum & $<0.01$ & $<0.01$ & $<0.01$ & $<0.01$ & $<0.01$ & $<0.01$ & $<0.01$ & $<0.01$ \\
\hline Nickel & $<0.01$ & 0.027 & 0.011 & $<0.01$ & $<0.01$ & $<0.01$ & $<0.01$ & $<0.01$ \\
\hline Selenium & $<0.05$ & $<0.05$ & $<0.05$ & $<0.05$ & $<0.05$ & $<0.05$ & $<0.05$ & $<0.05$ \\
\hline Silver & $<0.006$ & $<0.006$ & $<0.006$ & $<0.006$ & $<0.006$ & $<0.006$ & $<0.006$ & $<0.006$ \\
\hline strontium & 0.34 & 0.32 & 0.31 & 0.34 & 0.31 & 0.3 & 0.33 & 0.33 \\
\hline Thorium & $<0.2$ & $<0.2$ & $<0.2$ & $<0.2$ & $<0.2$ & $<0.2$ & $<0.2$ & $<0.2$ \\
\hline Uranium (Fluor) & $<0.001$ & 0.001 & $<0.001$ & $<0.001$ & 0.001 & 0.001 & 0.001 & 0.001 \\
\hline Vanadium & $<0.005$ & $<0.005$ & $<0.005$ & $<0.005$ & $<0.005$ & $<0.005$ & $<0.005$ & $<0.005$ \\
\hline zinc & 0.0042 & 0.0033 & 0.012 & 0.0036 & 0.016 & 0.0057 & 0.0066 & $<0.002$ \\
\hline MAJOR IONS $(\mathrm{mg} / \mathrm{L})$ & . & . & . & . & . & . & . & • \\
\hline Alkalinity-HCO3 & $453^{\circ}$ & $\cdot$ & 398 & $\cdot$ & 407 & $\dot{.}$ & 424 & . \\
\hline Alkalinity-CO3 & $<1$ & $\cdot$ & $<1$ & . & $<1$ & . & $<1$ & • \\
\hline Calcium & 140 & 140 & 130 & 150 & 130 & 120 & 160 & 150 \\
\hline Chloride & 16 & $\cdot$ & 15.7 & $\cdot$ & 20 & • & 11.8 & - \\
\hline Fluoride & $<0.1$ & $\cdot$ & 0.1 & $\cdot$ & 0.1 & • & $<0.1$ & • \\
\hline Magnesium & 40 & 39 & 37 & 41 & 35 & 34 & 42 & 39 \\
\hline Manganese & 0.025 & 0.032 & 0.045 & 0.043 & 0.042 & 0.037 & 0.049 & 0.041 \\
\hline Nitrate-N & $<0.2$ & . & $<0.2$ & . & $<0.2$ & • & $<0.2$ & 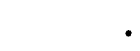 \\
\hline Potassium & 3.1 & 3.7 & 3.5 & 3.8 & 3.1 & 3.5 & 3.7 & 3.7 \\
\hline Sodium & 27 & 26 & 28 & 30 & 27 & 27 & 29 & 28 \\
\hline Sulfate & 141 &. & 110 & . & 113 & • & 141 & • \\
\hline
\end{tabular}

(CONTINUED) 
APPENDIX $\mathrm{E} .1$

Groundwater Quallty Data, 1993

\begin{tabular}{|c|c|c|c|c|c|c|c|c|}
\hline \multirow{4}{*}{$\begin{array}{l}\text { Sampling Point } \\
\text { Location } \\
\text { Date samplad }\end{array}$} & \multicolumn{8}{|c|}{$O W-281$} \\
\hline & \multicolumn{8}{|c|}{$\mathbf{r}$} \\
\hline & \multicolumn{2}{|c|}{$02 / 02 / 93$} & \multicolumn{2}{|c|}{$04 / 16 / 93$} & \multicolumn{2}{|c|}{$08 / 04 / 93$} & \multicolumn{2}{|c|}{$10 / 14 / 93$} \\
\hline & TOT & DIS & TOT & D1s & Tor & DIs & TOT & DIs \\
\hline MeTALs (mg/L) & . & . & . & - & . & • & . & • \\
\hline Al uminum & 1.1 & $<0.02$ & 3.5 & $<0.02$ & 18 & $<0.02$ & 0.49 & 0.023 \\
\hline Ant Imony & $<0.05$ & $<0.05$ & $<0.05$ & $<0.05$ & $<0.05$ & $<0.05$ & $<0.05$ & $<0.05$ \\
\hline Araenic & $<0.05$ & $<0.05$ & $<0.05$ & $<0.05$ & $<0.05$ & $<0.05$ & $<0.05$ & $<0.05$ \\
\hline Barium & 0.11 & 0.084 & 0.084 & 0.054 & 0.21 & 0.14 & 0.095 & 0.048 \\
\hline Bery 11 iun & $<0.0003$ & $<0.0003$ & $<0.0003$ & $<0.0003$ & 0.00064 & $<0.0003$ & $<0.0003$ & $<0.0003$ \\
\hline Boron & 0.03 & 0.024 & 0.044 & 0.037 & 0.079 & 0.045 & 0.034 & 0.033 \\
\hline Cadmium (MAs) & $<0.002$ & $<0.002$ & $<0.002$ & $<0.002$ & $<0.002$ & $<0.002$ & $<0.002$ & $<0,002$ \\
\hline Cadmium & $<0.003$ & $<0.003$ & $<0.003$ & $<0.003$ & 0.0064 & $<0.003$ & $<0.003$ & $<0.003$ \\
\hline Chromium (MAs) & $<0.01$ & $<0.01$ & 0.013 & $<0.01$ & 0.026 & $<0.01$ & $<0.01$ & $<0.01$ \\
\hline Chromiun & $<0.01$ & $<0.01$ & $<0.01$ & $<0.01$ & 0.037 & $<0.01$ & $<0.01$ & $<0.01$ \\
\hline Cobalt & $<0.005$ & $<0.005$ & $<0.005$ & $<0.003$ & 0.014 & $<0.003$ & $<0.005$ & $<0.005$ \\
\hline Copper & $<0.004$ & $<0.004$ & 0.0057 & 0.01 & 0.038 & $<0.004$ & $<0.004$ & $<0.004$ \\
\hline Iron & 1.8 & 0.0066 & 6.9 & $<0.005$ & 23 & 1.8 & 0.82 & 0.052 \\
\hline Lead (MS) & $<0.004$ & $<0.004$ & 0.0056 & $<0.004$ & 0.0086 & $<0.004$ & $<0.004$ & $<0.004$ \\
\hline Mercury (CVMA) & 0.00041 & $<0.0002$ & 0.00065 & $<0.0002$ & 0.00096 & $<0.0002$ & $<0.0002$ & $<0.0002$ \\
\hline Mol ybdenum & $<0.01$ & $<0.01$ & $<0.01$ & $<0.01$ & $<0.01$ & $<0.01$ & $<0.01$ & $<0.01$ \\
\hline HLckel & $<0.01$ & $<0.01$ & $<0.01$ & $<0.01$ & 0.036 & $<0.01$ & 0.011 & $<0.01$ \\
\hline Selenium & $<0.05$ & $<0.05$ & $<0.05$ & $<0.05$ & $<0.05$ & $<0.05$ & $<0.05$ & $<0.05$ \\
\hline sliver & $<0.006$ & $<0.006$ & $<0.006$ & $<0.006$ & $<0.006$ & $<0.006$ & $<0.006$ & $<0.006$ \\
\hline strontium & 0.26 & 0.23 & 0.17 & 0.16 & 0.33 & 0.3 & 0.27 & 0.17 \\
\hline Thorium & $<0.2$ & $<0.2$ & $<0.2$ & $<0.2$ & $<0.2$ & $<0.2$ & $<0.2$ & $<0.2$ \\
\hline Uranium (Fluor) & 0.003 & 0.003 & 0.005 & 0.006 & 0.002 & 0.003 & 0.002 & 0.001 \\
\hline Vanadiur & $<0.005$ & $<0.005$ & 0.0056 & $<0.005$ & 0.021 & $<0.005$ & $<0.005$ & $<0.005$ \\
\hline 2inc & 0.0099 & 0.0048 & 0.024 & 0.012 & 0.63 & 0.009 & 0.017 & 0.03 \\
\hline MAJOR ION $\mathrm{mg} / \mathrm{L}$ ) & . & . & . & . & . & . & . & . \\
\hline Alkalinity-HCO3 & $176^{\circ}$ & $\cdot$ & 123 & $\cdot$ & 190 & . & $16 \dot{2}$ & • \\
\hline Alkalinity-CO3 & $<1$ & . & $<1$ & . & $<1$ & . & $<1$ & . \\
\hline Calcium & 58 & 53 & 43 & 43 & 62 & 60 & 50 & 38 \\
\hline Chloride & 5.2 & . & 9.5 & . & 7.4 & - & 6 & • \\
\hline Fluoride & 0.2 & . & 0.1 & • & 0.2 & - & 0.2 & • \\
\hline Magnesium & 10 & 8.9 & 7.1 & 6.6 & 15 & 11 & 10 & 7.5 \\
\hline Manganese & 0.61 & 0.5 & 0.2 & 0.051 & 1.2 & 1.6 & 0.52 & 0.38 \\
\hline Nitrate-N & $<0.2$ & . & 0.44 & . & 0.45 & - & $<0.2$ & • \\
\hline Potansium & 1.8 & 1 & 2.6 & 1.8 & 6.4 & 2 & 2.3 & 2.5 \\
\hline Sodium & 7.3 & 7.1 & 8.2 & 8.6 & 8.7 & $\theta .5$ & 7.2 & 5.5 \\
\hline Sulfate & 15 & . & 21 & • & 18 & - & 16 & • \\
\hline
\end{tabular}

( CONTINUED) 
APPENDIX $\mathbf{E} .1$

Groundwater Quality Data, 1993

\begin{tabular}{|c|c|c|c|c|c|c|c|c|}
\hline \multirow{4}{*}{$\begin{array}{l}\text { 8anpling Point } \\
\text { Location } \\
\text { Date sampled }\end{array}$} & \multicolumn{8}{|c|}{ OW- 283} \\
\hline & \multicolumn{8}{|c|}{$\boldsymbol{r r}$} \\
\hline & \multicolumn{2}{|c|}{$02 / 03 / 93$} & \multicolumn{2}{|c|}{$04 / 19 / 93$} & \multicolumn{2}{|c|}{$08 / 06 / 93$} & \multicolumn{2}{|c|}{$10 / 14 / 93$} \\
\hline & TOT & DIs & $\operatorname{Tox}$ & Dis & TOT & DIs & TOT & D18 \\
\hline NeThLs (mg/t) & $\cdot$ & - & $\cdot$ & $\cdot$ & $\cdot$ & . & . & . \\
\hline Aluminum & 1.7 & 0.047 & 2.1 & $<0.02$ & 1.2 & 0.028 & 1.1 & $<0.02$ \\
\hline Antimony & $<0.05$ & $<0.05$ & $<0.05$ & $<0.05$ & $<0.05$ & $<0.05$ & $<0.05$ & $<0.05$ \\
\hline Areanio & $<0.05$ & $<0.05$ & $<0.05$ & $<0.05$ & $<0.05$ & $<0.05$ & $<0.05$ & $<0.05$ \\
\hline Bartum & 0.54 & 0.49 & 0.48 & 0.45 & 0.57 & 0.36 & 0.57 & 0.52 \\
\hline Bery111um & $<0.0003$ & $<0.0003$ & $<0.0003$ & $<0.0003$ & $<0.0003$ & $<0.0003$ & $<0.0003$ & $<0.0003$ \\
\hline Boron & 0.05 & 0.032 & 0.033 & 0.02 & 0.026 & 0.028 & 0.03 & 0.029 \\
\hline Cadniun (MAs) & $<0.002$ & $<0.002$ & $<0.002$ & $<0.002$ & $<0.002$ & $<0.002$ & $<0.002$ & $<0.002$ \\
\hline Cadmiun & $<0.003$ & 80.003 & $<0.003$ & $<0.003$ & $<0.003$ & $<0.003$ & $<0.003$ & $<0.003$ \\
\hline Chromlun (MAs) & 0.018 & $<0.01$ & 0.02 & $<0.01$ & 0.01 & $<0.01$ & 0.026 & $<0.01$ \\
\hline Chromilum & $<0.01$ & $<0.01$ & 0.023 & $<0.01$ & 0.011 & $<0.01$ & 0.026 & $<0.01$ \\
\hline Cobalt & 0.0067 & $<0.005$ & $<0.005$ & $<0.005$ & $<0.005$ & $<0.005$ & $<0.005$ & $<0.005$ \\
\hline Copper & $<0.004$ & $<0.004$ & 0.0043 & $<0.004$ & 0.011 & 0.0079 & $<0.004$ & $<0.004$ \\
\hline Iron & 2.2 & 0.12 & 2.5 & 0.025 & 1.6 & 0.027 & 1.9 & 0.14 \\
\hline Land (MS) & $<0.004$ & $<0.004$ & $<0.004$ & $<0.004$ & $<0.004$ & $<0.004$ & $<0.004$ & $<0.004$ \\
\hline Mercury (CVNA) & $<0.0002$ & $<0.0002$ & $<0.0002$ & $<0.0002$ & $<0.0002$ & $<0.0002$ & $<0.0002$ & $<0.0002$ \\
\hline Mol ybdenum & $<0.01$ & $<0.01$ & $<0.01$ & $<0.01$ & $<0.01$ & $<0.01$ & $<0.01$ & $<0.01$ \\
\hline Mlckel & 0.15 & 0.14 & 0.12 & 0.11 & 0.15 & 0.15 & 0.16 & 0.25 \\
\hline Selenium & $<0.05$ & $<0.05$ & $<0.05$ & $<0.05$ & $<0.05$ & 0.071 & $<0.05$ & $<0.05$ \\
\hline sllver & $<0.006$ & $<0.006$ & $<0.006$ & $<0.006$ & $<0.006$ & $<0.006$ & $<0.006$ & $<0.006$ \\
\hline strontium & 0.58 & 0.56 & 0.5 & 0.49 & 0.59 & 0.59 & 0.59 & 0.56 \\
\hline Thorium & $<0.2$ & $<0.2$ & $<0.2$ & $<0.2$ & $<0.2$ & $<0.2$ & $<0.2$ & $<0.2$ \\
\hline Oraniur (Pluor) & 0.001 & 0.001 & 0.001 & 0.001 & 0.001 & 0.001 & 0.001 & 0.001 \\
\hline Vanadium & $<0.005$ & $<0.005$ & $<0.005$ & $<0.005$ & $<0.005$ & $<0.005$ & $<0.005$ & $<0.005$ \\
\hline $2 \operatorname{inc}$ & 0.025 & 0.02 & 0.016 & 0.01 & 0.047 & 0.073 & 0.041 & 0.029 \\
\hline MnJOR IONS $(\mathrm{mg} / \mathrm{L})$ & . & . & . & . & . & . & . & . \\
\hline Alkalinity-uco3 & 339 &. & 350 & $\cdot$ & $38 \dot{3}$ & . & 380 & • \\
\hline Alkalinity-CO3 & $<1$ &. & $<1$ & . & $<1$ & . & $<1$ & . \\
\hline Calcium & 160 & 160 & 150 & 150 & 170 & 170 & 160 & 150 \\
\hline Chloride & 122 &. & 90 & . & 94 & . & 439 & . \\
\hline ruoride & $<0.1$ & . & $<0.1$ &. & $<0.1$ & . & $<0.1$ & . \\
\hline Magnesium & 17 & 17 & 16 & 15 & 18 & 18 & 18 & 17 \\
\hline Manganese & 1.4 & 1.3 & 1.1 & 1 & 0.92 & 0.88 & 0.88 & 1.1 \\
\hline Nitrate-n & $<0.2$ & . & $<0.2$ & . & $<0.2$ & . & $<0.2$ & • \\
\hline Potaseium & 3.4 & 2.9 & 3.6 & 2.7 & 3.5 & 2.9 & 3.2 & 2.8 \\
\hline sodium & 12 & 12 & 11 & 11 & 9.3 & 9.4 & 9.1 & 11 \\
\hline sulfate & 12 & . & 10.5 & . & 13 & . & 8 & • \\
\hline
\end{tabular}

(CONTIMUBD) 
Appender 2.1

Groundwater qualty bata, 1993

\begin{tabular}{|c|c|c|c|c|c|c|c|c|}
\hline \multirow{4}{*}{$\begin{array}{l}\text { sampling Polnt } \\
\text { Location } \\
\text { Date sampled }\end{array}$} & \multicolumn{8}{|c|}{$O w-284$} \\
\hline & \multicolumn{8}{|c|}{$\mathbf{P r}$} \\
\hline & \multicolumn{2}{|c|}{$02 / 02 / 93$} & \multicolumn{2}{|c|}{$04 / 16 / 93$} & \multicolumn{2}{|c|}{$08 / 05 / 93$} & \multicolumn{2}{|c|}{$10 / 14 / 93$} \\
\hline & TOT & DIs & ror & DIs & $\operatorname{Tor}$ & D18 & not & DI8 \\
\hline MrNLs $(\mathrm{mg} / \mathrm{L})$ & $\cdot$ & - & $\cdot$ & . & . & - & - & - \\
\hline Alund num & 0.23 & $<0.02$ & 0.44 & $<0.02$ & 14 & $<0.02$ & 0.5 & 0.033 \\
\hline Ant Lmony & $<0.05$ & $<0.0 s$ & $<0.05$ & $<0.05$ & $<0.0 s$ & $<0.05$ & $<0.05$ & $<0.05$ \\
\hline Areende & $<0.05$ & $<0.05$ & $<0.05$ & $<0.05$ & $<0.05$ & $<0.05$ & $<0.0 s$ & $<0.05$ \\
\hline Dariun & 0.36 & 0.35 & 0.39 & 0.35 & 0.6 & 0.44 & 0.45 & 0.44 \\
\hline Bery111um & $<0.0003$ & $<0.0003$ & $<0.0003$ & $<0.0003$ & $0.0005 \theta$ & $<0.0003$ & $<0.0003$ & $<0.0003$ \\
\hline Doron & 0.009 & 0.015 & 0.03 & 0.024 & 0.039 & 0.032 & 0.017 & 0.017 \\
\hline Cadmalum (MB) & $<0.002$ & $<0.002$ & $<0.002$ & $<0.002$ & $<0.002$ & $<0.002$ & $<0.002$ & $<0.002$ \\
\hline Codintum & $<0.003$ & $<0.003$ & $<0.003$ & $<0.003$ & 0.0056 & $<0.003$ & $<0.003$ & $<0.003$ \\
\hline Chrondum (NAs) & 0.64 & $<0.01$ & 0.16 & $<0.01$ & 1.9 & $<0.01$ & 0.12 & $<0.01$ \\
\hline Chrondun & 0.16 & $<0.01$ & 0.16 & $<0.01$ & 1.7 & $<0.01$ & 0.12 & $<0.01$ \\
\hline Cobelt & 0.0086 & 0.006 & 0.0056 & 0.0057 & 0.064 & 0.016 & 0.0097 & 0.016 \\
\hline Coppor & $<0.004$ & 0.0057 & 0.0063 & 0.0042 & 0.035 & 0.0049 & $<0.004$ & $<0.004$ \\
\hline Iron & 2.2 & $<0.005$ & 1.6 & 0.12 & 35 & 0.0063 & $2 \cdot 3$ & 0.32 \\
\hline Land (MS) & $<0.004$ & $<0.004$ & $<0.004$ & $<0.004$ & 0.024 & $<0.004$ & $<0.004$ & $<0.004$ \\
\hline Marcury (CVNA) & $<0.0002$ & $<0.0002$ & $<0.0002$ & $<0.0002$ & $<0.0002$ & $<0.0002$ & $<0.0002$ & $<0.0002$ \\
\hline Nolybdenum & $<0.01$ & $<0.01$ & $<0.01$ & $<0.01$ & 0.022 & $<0.01$ & $<0.01$ & $<0.01$ \\
\hline Nickel & 0.96 & 0.92 & 0.8 & 1.4 & 1.0 & 0.98 & 0.59 & 0.96 \\
\hline Selentum & 0.077 & $<0.05$ & $<0.05$ & $<0.05$ & 0.08 & $<0.05$ & $<0.05$ & $<0.05$ \\
\hline s11ver & $<0.006$ & $<0.006$ & $<0.006$ & $<0.006$ & $<0.006$ & $<0.006$ & $<0.006$ & $<0.006$ \\
\hline strontlum & 0.56 & 0.36 & 0.6 & 0.68 & 0.67 & 0.68 & 0.6 & 0.68 \\
\hline Thortum & $<0.2$ & $<0.2$ & $<0.2$ & $<0.2$ & $<0.2$ & $<0.2$ & $<0.2$ & $<0.2$ \\
\hline Uranium (Pluor) & 0.01 & 0.01 & 0.005 & 0.007 & 0.008 & 0.007 & 0.003 & 0.004 \\
\hline Vanadlum & $<0.005$ & $<0.005$ & $<0.005$ & $<0.005$ & 0.029 & $<0.00 s$ & $<0.005$ & $<0.005$ \\
\hline elnc & 0.0058 & 0.0061 & 0.014 & 0.0097 & 0.11 & 0.049 & 0.063 & 0.08 \\
\hline MuJOR IOHS (mg/L) & - & . &. & - & - & - & - & - \\
\hline Mkalinity-uCO3 & 285 &. & 263 &. & 302 & . & 311 & . \\
\hline Alkal In 1 ty-CO3 & $<1$ & $\cdot$ & $<1$ & . & $<1$ & - & $<1$ & - \\
\hline Calctum & 190 & 190 & 190 & 180 & 210 & 200 & 190 & 190 \\
\hline Chlorlde & 212 & . & 238 & . & 220 & . & 249 & • \\
\hline Iuorlde & $<0.1$ & $\cdot$ & $<0.1$ & . & $<0.1$ & - & $<0.1$ & - \\
\hline Megnes Lum & 18 & 19 & 19 & 26 & 25 & 22 & 19 & 22 \\
\hline Manganese & 0.64 & 0.56 & 0.77 & 0.099 & 1.8 & 1.2 & 1.6 & 1.4 \\
\hline Nitrate-n & $<0.2$ & $\cdot$ & $<0.2$ & . & $<0.2$ & - & $<0.2$ & • \\
\hline Potase 1 un & 1.5 & 1.9 & $2 \cdot 3$ & 1.6 & 5.5 & 1.7 & 1.9 & 1.6 \\
\hline sodiun & 26 & 26 & 29 & 32 & 29 & 28 & 33 & 32 \\
\hline sulfate & 32 & - & 23 & - & 28 & • & 30 & • \\
\hline
\end{tabular}

(CONTINULD) 
APPENDIX 8.1

Groundwater Quality Data, 1993

\begin{tabular}{|c|c|c|c|c|c|c|c|c|}
\hline \multirow{4}{*}{$\begin{array}{l}\text { Bampling Polnt } \\
\text { Location } \\
\text { Date sumpled }\end{array}$} & \multicolumn{8}{|c|}{$0 W-285$} \\
\hline & \multicolumn{8}{|c|}{$r$} \\
\hline & \multicolumn{2}{|c|}{$02 / 02 / 93$} & \multicolumn{2}{|c|}{$04 / 19 / 93$} & \multicolumn{2}{|c|}{$08 / 05 / 93$} & \multicolumn{2}{|c|}{$10 / 14 / 93$} \\
\hline & $\operatorname{tot}$ & DIs & TOT & DIs & TOT & D18 & TOT & Drs \\
\hline Maruss (mg/L) & $\cdot$ & - & - & . & . & - & . & \\
\hline Alunlnum & 0.28 & 0.085 & 3.2 & $<0.02$ & 0.91 & 0.054 & 0.33 & $<0.02$ \\
\hline Ane teony & $<0.05$ & $<0.05$ & $<0.03$ & $<0.05$ & $<0.05$ & $<0.05$ & $<0.05$ & $<0.05$ \\
\hline Areente & $<0.05$ & $<0.05$ & $<0.05$ & $<0.05$ & $<0.03$ & $<0.05$ & $<0.05$ & $<0.05$ \\
\hline Barlum & 0.21 & 0.12 & 0.15 & 0.11 & 0.15 & 0.14 & 0.14 & 0.13 \\
\hline Deryldium & $<0.0003$ & 0.0088 & $<0.0003$ & $<0,0003$ & $<0.0003$ & $<0.0003$ & $<0.0003$ & $<0.0003$ \\
\hline Boron & 0.018 & 0.033 & 0.044 & 0.028 & 0.015 & 0.039 & 0.021 & 0.018 \\
\hline Cadnium (Ms) & $<0.002$ & $<0.002$ & $<0.002$ & $<0.002$ & $<0.002$ & $<0.002$ & $<0.002$ & $<0.002$ \\
\hline Cadniun & $<0.003$ & 0.01 & $<0.003$ & $<0.003$ & $<0.003$ & $<0.003$ & $<0.003$ & $<0.003$ \\
\hline Chromiun (MAs) & 0.047 & $<0.01$ & 0.76 & $<0.01$ & 0.076 & $<0.01$ & 0.098 & $<0.01$ \\
\hline Chromium & $<0.01$ & $<0.01$ & 0.28 & $<0.01$ & 0.081 & $<0.01$ & 0.093 & $<0.01$ \\
\hline Cobalt & 0.0056 & 0.016 & 0.011 & $<0.005$ & 0.017 & 0.013 & 0.013 & 0.013 \\
\hline Copper & $<0.004$ & 0.011 & 0.017 & $<0.004$ & 0.013 & 0.0085 & $<0.004$ & $<0.004$ \\
\hline Iron & 0.73 & 0.056 & 0.5 & 0.17 & 2 & 0.14 & 2.2 & 0.23 \\
\hline Lead (MS) & $<0.004$ & $<0.004$ & 0.011 & $<0.004$ & $<0.004$ & $<0.004$ & $<0.004$ & $<0.004$ \\
\hline Marcury (CVAA) & $<0.0002$ & $<0.0002$ & $<0.0002$ & $<0.0002$ & $<0.0002$ & $<0.0002$ & $<0.0002$ & $<0.0002$ \\
\hline Nol ybdenun & $<0.01$ & $<0.01$ & $<0.01$ & $<0.01$ & $<0.01$ & $<0.01$ & $<0.01$ & $<0.01$ \\
\hline Nickel & 0.58 & 0.58 & 0.91 & 0.58 & 0.5 & 0.45 & 0.96 & 0.93 \\
\hline selenium & $<0.05$ & $<0.05$ & $<0.05$ & $<0.05$ & $<0.05$ & $<0.05$ & $<0.05$ & $<0.05$ \\
\hline silver & $<0.006$ & 0.011 & $<0.006$ & $<0.006$ & $<0.006$ & $<0.006$ & $<0.006$ & $<0.006$ \\
\hline strontiun & 0.18 & 0.19 & 0.17 & 0.17 & 0.18 & 0.18 & 0.18 & 0.18 \\
\hline Thoriun & $<0.2$ & $<0.2$ & $<0.2$ & $<0.2$ & $<0.2$ & $<0.2$ & $<0.2$ & $<0.2$ \\
\hline Uranium (Fluor) & 0.007 & 0.006 & 0.007 & 0.005 & 0.003 & 0.002 & 0.002 & 0.002 \\
\hline Vanadium & $<0.005$ & 0.013 & 0.0051 & $<0.005$ & $<0.005$ & $<0.005$ & $<0.005$ & $<0.005$ \\
\hline zine & 0.016 & 0.038 & 0.022 & 0.013 & 0.076 & 0.13 & 0.052 & 0.066 \\
\hline MUOR IONS (mg/L) & $\cdot$ & • & $\cdot$ & $\cdot$ & $\cdot$ & $\cdot$ & $\cdot$ & • \\
\hline Alkalinity-HCO3 & 181 & 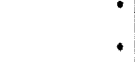 & 191 & $\cdot$ & 185 & $\dot{.}$ & $17 \dot{8}$ & 。 \\
\hline Alkadindty-co3 & $<1$ & - & $<1$ & $\cdot$ & $<1$ & $\cdot$ & $<1$ & • \\
\hline Calcium & 72 & 73 & 74 & 71 & 75 & 74 & 65 & 65 \\
\hline Chloride & 20 & - & 22.9 & . & 22 & $\cdot$ & 47 & - \\
\hline Fluoride & 0.2 & . & 0.1 & $\cdot$ & $<0.1$ & $\cdot$ & 0.1 & • \\
\hline Magnesium & 6.8 & 6.8 & 7.2 & 6.1 & 6.8 & 6.8 & 7.2 & 7.1 \\
\hline Mangareue & 0.073 & 0.069 & 0.27 & 0.087 & 0.52 & 0.42 & 0.36 & 0.33 \\
\hline Nitrate-N & 0.58 & • & 0.3 & $\cdot$ & $<0.2$ & $\cdot$ & $<0.2$ & 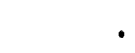 \\
\hline Potaseiun & 1.7 & 1.8 & 2.7 & 1.3 & 2.4 & 1.8 & 1.8 & 1.7 \\
\hline Sodium & 9.3 & 9.3 & 8.6 & 7.6 & 7.6 & 8.1 & 11 & 12 \\
\hline sulfate & 45 & - & 19.4 &. & 7.2 & $\cdot$ & 14 & 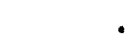 \\
\hline
\end{tabular}

(CONTINUSD) 
APPEND1X $\geq .1$

Groundwater Quellty Data, 1993

\begin{tabular}{|c|c|c|c|c|c|c|c|c|}
\hline \multirow{4}{*}{$\begin{array}{l}\text { sampling Polnt } \\
\text { Location } \\
\text { Date sampled }\end{array}$} & \multicolumn{8}{|c|}{ aw-337 } \\
\hline & \multicolumn{8}{|c|}{ wc } \\
\hline & \multicolumn{2}{|c|}{$01 / 21 / 93$} & \multicolumn{2}{|c|}{$05 / 07 / 93$} & \multicolumn{2}{|c|}{$09 / 17 / 93$} & \multicolumn{2}{|c|}{$12 / 09 / 93$} \\
\hline & TOT & DIs & TOT & D18 & TOT & DIS & TOT & DI8 \\
\hline Mrats (mg/L) & $\cdot$ & - & $\cdot$ & - & - & $\cdot$ & - & • \\
\hline Aluminum & 5.3 & 0.091 & 0.46 & $<0.02$ & 6.2 & $<0.02$ & 0.2 & 0.026 \\
\hline Ant Lmony & $<0.03$ & $<0.05$ & $<0.08$ & $<0.05$ & $<0.05$ & $<0.05$ & $<0,05$ & $<0.05$ \\
\hline Araende & $<0.05$ & $<0.05$ & $<0.05$ & $<0.05$ & $<0.05$ & $<0.05$ & $<0.05$ & $<0.05$ \\
\hline Barium & 0.24 & 0.16 & 0.19 & 0.18 & 0.33 & 0.21 & 0.21 & 0.21 \\
\hline Beryll1um & 0.00069 & $<0.0003$ & $<0.0003$ & $<0.0003$ & 0.001 & $<0.0003$ & $<0.0003$ & $<0.0003$ \\
\hline Boron & 0.034 & 0.023 & 0.06 & 0.048 & 0.038 & 0.031 & 0.034 & 0.03 \\
\hline Cadmiun (MB) & 0.0069 & 0.0051 & $<0.002$ & $<0,002$ & $<0.002$ & $<0.002$ & 0.0048 & 0.0068 \\
\hline cadntum & 0.0078 & 0.0063 & $<0.003$ & $<0.003$ & 0.0044 & $<0.003$ & 0.0036 & 0.0055 \\
\hline Chromium (MS) & $<0.01$ & $<0.01$ & $<0.01$ & $<0.01$ & 0.028 & $<0.01$ & $<0.01$ & 0.013 \\
\hline Chromiun & 0.013 & $<0.01$ & $<0.01$ & $<0.01$ & 0.026 & $<0.01$ & $<0.01$ & $<0.01$ \\
\hline Cobalt & $<0.005$ & $<0.005$ & $<0.005$ & $<0.00 s$ & 1.0094 & $<0.005$ & $<0.005$ & $<0.005$ \\
\hline Copper & 0.021 & 0.0072 & $<0.004$ & $<0.004$ & 0.027 & $<0.004$ & 0.013 & 0.012 \\
\hline Iron & 7.9 & 0.1 & 0.63 & 0.0072 & 14 & $<0.005$ & 0.28 & $<0.005$ \\
\hline Lead (MAs) & 0.004 & $<0.004$ & $<0.004$ & $<0.004$ & 0.0087 & $<0.004$ & $<0.004$ & $<0.004$ \\
\hline Nercury (CVMA) & $<0.0002$ & $<0.0002$ & $<0.0002$ & $<0.0002$ & $<0.0002$ & $<0.0002$ & $<0.0002$ & $<0.0002$ \\
\hline Holybdenum & $<0.01$ & $<0.01$ & $<0.01$ & $<0.01$ & $<0.01$ & $<0.01$ & $<0.01$ & $<0.01$ \\
\hline Nickel & 0.014 & $<0.01$ & 0.011 & $<0.01$ & 0.033 & 0.01 & $<0.01$ & $<0.01$ \\
\hline Selentum & $<0.05$ & 0.055 & $<0.05$ & $<0.05$ & $<0.05$ & $<0.05$ & $<0.05$ & $<0.05$ \\
\hline silver & $<0.006$ & $<0.006$ & $<0.006$ & $<0.006$ & $<0.006$ & $<0.006$ & $<0.006$ & $<0.006$ \\
\hline strontiun & 0.22 & 0.19 & 0.23 & 0.22 & 0.25 & 0.24 & 0.23 & 0.17 \\
\hline Thorium & $<0.2$ & $<0.2$ & $<0.2$ & $<0.2$ & $<0.2$ & $<0.2$ & $<0.2$ & $<0.2$ \\
\hline orantum (rluor) & 0.002 & 0.001 & 0.001 & 0.001 & 0.002 & 0.001 & 0.001 & 0.001 \\
\hline Vanadiun & 0.0085 & $<0.005$ & $<0.005$ & $<0.005$ & 0.011 & $<0.005$ & $<0.005$ & $<0.005$ \\
\hline zine & 0.14 & 0.12 & 0.0099 & 0.0089 & 0.052 & 0.018 & 0.05 & 0.091 \\
\hline MNOR IONS (mg/L) & . & . & $\cdot$ & $\dot{.}$ & • & $\cdot$ & • & • \\
\hline Alkadinity-HCO3 & $25 i^{\circ}$ & . & 236 & . & 251 & $\cdot$ & 240 & • \\
\hline Alkalindty-co3 & $<1$ & . & $<1$ & $\cdot$ & $<1$ & $\dot{.}$ & $<1$ & • \\
\hline Calcium & 97 & 88 & 86 & 84 & 100 & 97 & 93 & 68 \\
\hline Chloride & 35 &. & 24 &. & 22 &. & 18.9 & 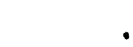 \\
\hline rluoride & $<0.1$ & . & $<0.1$ & . & $<0.1$ & . & $<0.1$ & . \\
\hline Magnosium & 11 & 8.5 & 8.3 & 8.1 & 12 & 9.6 & 9.1 & 8.6 \\
\hline Manganese & 0.39 & 0.097 & 0.097 & 0.073 & 0.73 & 0.13 & 0.11 & 0.11 \\
\hline Nitrate-N & $<0.2$ & . & $<0.2$ &. & $<0.2$ &. & $<0.2$ & • \\
\hline Potanesum & 3.8 & 2.6 & 1.6 & 1.5 & 5 & 1.9 & 1.9 & 3.1 \\
\hline Sodium & 3.8 & 3.7 & 3.9 & 3.8 & 4.3 & 4.2 & 4 & 4.2 \\
\hline Sulfate & 7.5 & $\cdot$ & 7 & $\cdot$ & 8 & . & 6.2 & • \\
\hline
\end{tabular}

(CONT INUED) 
APPBNDIX $\mathrm{E} .1$

Groundwater Quality Data, 1993

\begin{tabular}{|c|c|c|c|c|c|c|c|c|}
\hline \multirow{4}{*}{$\begin{array}{l}\text { Sampling Point } \\
\text { Location } \\
\text { Date Sampled }\end{array}$} & \multicolumn{8}{|c|}{$G W-338$} \\
\hline & \multicolumn{8}{|c|}{ WC } \\
\hline & \multicolumn{2}{|c|}{$01 / 11 / 93$} & \multicolumn{2}{|c|}{$05 / 04 / 93$} & \multicolumn{2}{|c|}{$09 / 23 / 93$} & \multicolumn{2}{|c|}{$12 / 07 / 93$} \\
\hline & TOT & DIS & TOT & DIS & TOT & DIS & TOT & DIS \\
\hline Mernis (mg/L) & $\cdot$ & - & $\cdot$ & $\cdot$ & $\cdot$ & $\cdot$ & $\cdot$ & - \\
\hline Aluminum & 0.81 & $<0.02$ & 1.8 & 0.049 & 0.33 & $<0.02$ & 0.34 & 0.031 \\
\hline Nutimony & $<0.05$ & $<0.05$ & $<0.05$ & $<0.05$ & $<0.05$ & $<0.05$ & $<0.05$ & $<0.05$ \\
\hline Areenic & $<0.05$ & $<0.05$ & $<0.05$ & $<0.05$ & $<0.05$ & $<0.05$ & $<0.05$ & $<0.05$ \\
\hline Barium & 0.29 & 0.25 & 0.35 & 0.32 & 0.37 & 0.35 & 0.35 & 0.34 \\
\hline Baryl11um & $<0.0003$ & $<0.0003$ & $<0.0003$ & $<0.0003$ & $<0.0003$ & $<0.0003$ & $<0.0003$ & $<0.0003$ \\
\hline Boron & 0.025 & 0.048 & 0.028 & 0.017 & 0.025 & 0.017 & 0.091 & 0.062 \\
\hline Cadintun (MAs) & $<0.002$ & $<0.002$ & $<0.002$ & $<0.002$ & $<0.002$ & $<0.002$ & $<0.002$ & $<0.002$ \\
\hline Cadniun & $<0.003$ & $<0.003$ & $<0.003$ & $<0.003$ & $<0.003$ & $<0.003$ & $<0.003$ & 0.0038 \\
\hline Chromium (AAS) & $<0.01$ & $<0.01$ & $<0.01$ & $<0.01$ & $<0.01$ & $<0.01$ & $<0.01$ & $<0.01$ \\
\hline Chromiun & $<0.01$ & $<0.01$ & $<0.01$ & $<0.01$ & $<0.01$ & $<0.01$ & $<0.01$ & $<0.01$ \\
\hline Cobalt & $<0.005$ & $<0.005$ & 0.0056 & $<0.005$ & $<0.005$ & $<0.005$ & $<0.005$ & $<0.005$ \\
\hline Copper & $<0.004$ & $<0.004$ & 0.0058 & $<0.004$ & 0.0067 & $<0.004$ & 0.0049 & 0.0045 \\
\hline Iron & 1.2 & $<0.005$ & 1.6 & 0.0096 & 0.35 & $<0.005$ & 0.32 & $<0.005$ \\
\hline Lead (MAS) & $<0.004$ & $<0.004$ & $<0.004$ & $<0.004$ & $<0.004$ & $<0.004$ & $<0.004$ & $<0.004$ \\
\hline Marcury (CVMA) & $<0.0002$ & $<0.0002$ & $<0.0002$ & $<0.0002$ & $<0.0002$ & $<0.0002$ & $<0.0002$ & $<0.0002$ \\
\hline Mol ybdenum & $<0.01$ & $<0.01$ & $<0.01$ & $<0.01$ & $<0.01$ & $<0.01$ & $<0.01$ & $<0.01$ \\
\hline Nickel & $<0.01$ & $<0.01$ & $<0.01$ & $<0.01$ & $<0.01$ & $<0.01$ & $<0.01$ & $<0.01$ \\
\hline seleniun & $<0.05$ & $<0.05$ & $<0.05$ & $<0.05$ & $<0.05$ & $<0.05$ & $<0.05$ & $<0.05$ \\
\hline silver & $<0.006$ & $<0.006$ & $<0.006$ & $<0.006$ & $<0.006$ & $<0.006$ & $<0.006$ & $<0.006$ \\
\hline strontium & 0.23 & 0.22 & 0.26 & 0.24 & 0.23 & 0.23 & 0.26 & 0.25 \\
\hline Thorium & $<0.2$ & $<0.2$ & $<0.2$ & $<0.2$ & $<0.2$ & $<0.2$ & $<0.2$ & $<0.2$ \\
\hline Uranium (Fluor) & 0.001 & 0.001 & $<0.001$ & $<0.001$ & $<0.001$ & $<0.001$ & 0.001 & 0.001 \\
\hline Vanadium & $<0.005$ & $<0.005$ & $<0.005$ & $<0.005$ & $<0.005$ & $<0.005$ & $<0.005$ & $<0.005$ \\
\hline zinc & 0.025 & 0.025 & 0.012 & 0.025 & 0.017 & 0.018 & 0.014 & 0.0093 \\
\hline MNJOR IONS $(\mathrm{mg} / \mathrm{L})$ & . & . & . & . & . & . & . & . \\
\hline Alkalinity-HCO3 & 233 & $\dot{.}$ & 234 &. & 252 & $\cdot$ & 245 & . \\
\hline Alkalinity-CO3 & $<1$ & - & $<1$ & $\cdot$ & $<1$ & $\cdot$ & $<1$ & • \\
\hline Calcium & 87 & 85 & 86 & 83 & 90 & 89 & 91 & 89 \\
\hline Chloride & 6.1 & - & 6.5 & - & 5.8 & . & 7 & • \\
\hline Fluoride & $<0.1$ & • & $<0.1$ & $\cdot$ & 0.1 & $\cdot$ & $<0.1$ & • \\
\hline Magnesium & 9.7 & 9.4 & 9.5 & 8.8 & 9.7 & 9.6 & 10 & 9.8 \\
\hline Manganese & 0.3 & 0.047 & 0.3 & 0.035 & 0.29 & 0.3 & 0.11 & 0.032 \\
\hline Nitrate-N & 0.51 & • & 0.41 & . & 0.31 & . & 0.6 & • \\
\hline Potassium & 1.8 & 1 & 2.8 & 1.9 & 2.1 & 1.8 & 2.2 & 2.2 \\
\hline Sodium & 5.1 & 5.4 & 5.1 & 5.1 & 5.9 & 6.1 & 5.9 & 6.2 \\
\hline sulfate & 15 & - & 14 & - & 18 & - & 12 & • \\
\hline
\end{tabular}

(CONTINUED) 
APPENDIX R.1

Groundwatel Quality Data, 1993

\begin{tabular}{|c|c|c|c|c|c|c|c|c|}
\hline \multirow{4}{*}{$\begin{array}{l}\text { Sampling point } \\
\text { Location } \\
\text { Date sampled }\end{array}$} & \multicolumn{8}{|c|}{$G W-380$} \\
\hline & \multicolumn{8}{|c|}{ NHP } \\
\hline & \multicolumn{2}{|c|}{$01 / 25 / 93$} & \multicolumn{2}{|c|}{$04 / 21 / 93$} & \multicolumn{2}{|c|}{$08 / 05 / 93$} & \multicolumn{2}{|c|}{$10 / 29 / 93$} \\
\hline & TOT & DIS & TOT & DIS & TOT & DIS & TOT & DIS \\
\hline NETALS (mg/L) & - & - & $\cdot$ & - & . & $\cdot$ & $\cdot$ & • \\
\hline Aluminum & 0.66 & $<0.02$ & 0.23 & 0.021 & 0.27 & $<0.02$ & 0.19 & $<0.02$ \\
\hline Ant imony & $<0.05$ & $<0.05$ & $<0.05$ & $<0.05$ & $<0.05$ & $<0.05$ & $<0.05$ & $<0.05$ \\
\hline Arsenic & $<0.05$ & $<0.05$ & $<0.05$ & $<0.05$ & $<0.05$ & $<0.05$ & $<0.05$ & $<0.05$ \\
\hline Barium & 0.054 & 0.047 & 0.029 & 0.028 & 0.056 & 0.033 & 0.04 & 0.07 \\
\hline Beryllium & $<0.0003$ & $<0.0003$ & $<0.0003$ & $<0.0003$ & $<0.0003$ & $<0.0003$ & $<0.0003$ & $<0.0003$ \\
\hline Boron & 0.034 & 0.022 & 0.061 & 0.13 & 0.045 & 0.047 & 0.06 & 0.059 \\
\hline Cadmium (AAS) & $<0.002$ & $<0.002$ & 0.0037 & 0.0033 & 0.0065 & 0.0041 & $<0.002$ & $<0.002$ \\
\hline Cadmium & $<0.003$ & $<0.003$ & 0.004 & 0.0034 & 0.0069 & 0.0038 & $<0.003$ & $<0.003$ \\
\hline Chromium (AAS) & 0.99 & $<0.01$ & 0.27 & $<0.01$ & 4 & 0.032 & 0.82 & 0.017 \\
\hline Chromium & 0.75 & $<0.01$ & 0.18 & $<0.01$ & 2.5 & 0.031 & 0.75 & 0.011 \\
\hline Cobalt & $<0.005$ & $<0.005$ & $<0.005$ & $<0.005$ & 0.074 & 0.011 & 0.0063 & 0.073 \\
\hline Copper & 0.015 & $<0.004$ & 0.011 & 0.0093 & 0.074 & $<0.004$ & 0.0056 & $<0.004$ \\
\hline Iron & 3.3 & 0.05 & 1.3 & 0.045 & 28 & 2.2 & 2.5 & 14 \\
\hline Lead (AAS) & $<0.004$ & $<0.004$ & $<0.004$ & $<0.004$ & $<0.004$ & $<0.004$ & $<0.004$ & $<0.004$ \\
\hline Mercury (CVAA) & $<0.0002$ & $<0.0002$ & $<0.0002$ & $<0.0002$ & $<0.0002$ & $<0.0002$ & $<0,0002$ & $<0.0002$ \\
\hline Mol ybdenum & $<0.01$ & $<0.01$ & $<0.01$ & $<0.01$ & 0.011 & $<0.01$ & 0.01 & $<0.01$ \\
\hline Nickel & 0.16 & 0.12 & 0.12 & 0.094 & 3.9 & 0.54 & 0.39 & 4 \\
\hline Selenium & $<0.05$ & $<0.05$ & $<0.05$ & $<0.05$ & $<0.05$ & $<0.05$ & $<0.05$ & $<0.05$ \\
\hline silver & $<0.006$ & $<0.006$ & $<0.006$ & $<0.006$ & $<0.006$ & $<0.006$ & $<0.006$ & $<0.006$ \\
\hline strontium & 0.057 & 0.057 & 0.032 & 0.029 & 0.04 & 0.033 & 0.04 & 0.059 \\
\hline Thorium & $<0.2$ & $<0.2$ & $<0.2$ & $<0.2$ & $<0.2$ & $<0.2$ & $<0.2$ & $<0.2$ \\
\hline Uranium (Fluor) & 0.001 & 0.001 & 0.001 & 0.001 & 0.001 & $<0.001$ & 0.002 & $<0.001$ \\
\hline Vanadium & $<0.005$ & $<0.005$ & $<0.005$ & $<0.005$ & $<0.005$ & $<0.005$ & $<0.005$ & $<0.005$ \\
\hline $2 \pm n c$ & 0.026 & 0.04 & 0.031 & 0.031 & 0.026 & 0.013 & 0.016 & 0.18 \\
\hline MAJOR IONS $(\mathrm{mg} / \mathrm{L})$ & . & $\cdot$ & . & . & . & - & . & • \\
\hline Alkalinity-HCO3 & 164 & $\cdot$ & $17 \dot{7}$ & . & 78 & $\cdot$ & $17 \dot{9}$ & • \\
\hline Alkalinity-CO3 & $<1$ & . & $<1$ & . & $<1$ &. & $<1$ & • \\
\hline Calcium & 50 & 51 & 30 & 27 & 33 & 29 & 35 & 49 \\
\hline Chloride & 250 & . & 140 & . & 364 & . & 47 & • \\
\hline Fluoride & 0.1 & . & 0.2 & . & $<0.1$ & . & 0.2 & . \\
\hline Magnesium & 21 & 21 & 12 & 11 & 12 & 12 & 13 & 15 \\
\hline Manganese & 0.052 & 0.024 & 0.023 & 0.0074 & 0.81 & 0.15 & 0.17 & 1 \\
\hline Nitrate-N & 1.4 & . & 1.1 & • & 1.8 &. & 3.6 & . \\
\hline Potassium & 1.4 & 1 & 0.86 & 0.96 & 1.4 & 0.97 & 1.5 & 1.5 \\
\hline Sodium & 130 & 130 & 100 & 98 & 81 & 78 & 77 & 93 \\
\hline Sulfate & 20.7 & . & 13 & . & 31 & $\cdot$ & 44 & • \\
\hline
\end{tabular}

(CONTINUED) 
APPENDIX E.1

Groundwater Quality Data, 1993

\begin{tabular}{|c|c|c|c|c|c|c|c|c|}
\hline \multirow{4}{*}{$\begin{array}{l}\text { Sampling Point } \\
\text { - } \\
\text { Location } \\
\text { Date Sampled }\end{array}$} & \multicolumn{8}{|c|}{$G W-381$} \\
\hline & \multicolumn{8}{|c|}{ NHP } \\
\hline & \multicolumn{2}{|c|}{$01 / 27 / 93$} & \multicolumn{2}{|c|}{$04 / 26 / 93$} & \multicolumn{2}{|c|}{$08 / 08 / 93$} & \multicolumn{2}{|c|}{$11 / 08 / 93$} \\
\hline & TOT & DIS & TOT & DIS & TOT & DIS & TOT & DIS \\
\hline METALS (mg/L) & - & - & - & - & - & - & - & . \\
\hline Aluminum & 2.5 & $<0.02$ & 0.86 & $<0.02$ & 0.16 & $<0.02$ & 0.26 & $<0.02$ \\
\hline Antimony & $<0.05$ & $<0.05$ & $<0.05$ & $<0.05$ & $<0.05$ & $<0.05$ & $<0.05$ & $<0.05$ \\
\hline Arsenic & $<0.05$ & $<0.05$ & $<0.05$ & $<0.05$ & $<0.05$ & $<0.05$ & $<0.05$ & $<0.05$ \\
\hline Barium & 0.2 & 0.2 & 0.2 & 0.18 & 0.22 & 0.2 & 0.24 & 0.23 \\
\hline Beryllium & 0.00092 & $<0.0003$ & $<0.0003$ & $<0.0003$ & $<0.0003$ & $<0.0003$ & $<0.0003$ & $<0.0003$ \\
\hline Boron & 0.042 & 0.17 & 0.011 & 0.019 & 0.018 & 0.012 & 0.036 & 0.034 \\
\hline Cadmium (AAS) & $<0.002$ & $<0.002$ & $<0.002$ & $<0.002$ & $<0.002$ & $<0.002$ & $<0.002$ & $<0.002$ \\
\hline Cadmium & 0.0043 & $<0.003$ & $<0.003$ & $<0.003$ & $<0.003$ & $<0.003$ & $<0.003$ & $<0.003$ \\
\hline Chromium (AAS) & $<0.01$ & $<0.01$ & $<0.01$ & $<0.01$ & $<0.01$ & $<0.01$ & $<0.01$ & $<0.01$ \\
\hline Chromium & $<0.01$ & $<0.01$ & $<0.01$ & $<0.01$ & $<0.01$ & $<0.01$ & $<0.01$ & $<0.01$ \\
\hline Cobalt & $<0.005$ & $<0.005$ & $<0.005$ & $<0.005$ & $<0.005$ & $<0.005$ & $<0.005$ & $<0.005$ \\
\hline Copper & 0.01 & $<0.004$ & $<0.004$ & $<0.004$ & $<0.004$ & $<0.004$ & 0.0064 & 0.0076 \\
\hline Iron & 4.2 & 0.35 & 1.8 & 0.18 & 0.69 & 0.22 & 0.58 & 0.22 \\
\hline Lead (AAS) & $<0.004$ & $<0.004$ & $<0.004$ & $<0.004$ & $<0.004$ & $<0.004$ & $<0.004$ & $<0.004$ \\
\hline Mercury (CVAA) & $<0.0002$ & $<0.0002$ & $<0.0002$ & $<0.0002$ & $<0.0002$ & $<0.0002$ & $<0.0002$ & $<0.0002$ \\
\hline Mol ybdenum & $<0.01$ & $<0.01$ & $<0.01$ & $<0.01$ & $<0.01$ & $<0.01$ & $<0.01$ & $<0.01$ \\
\hline Nickel & $<0.01$ & $<0.01$ & $<0.01$ & $<0.01$ & $<0.01$ & $<0.01$ & $<0.01$ & $<0.01$ \\
\hline Selenium & $<0.05$ & $<0.05$ & $<0.05$ & $<0.05$ & $<0.05$ & $<0.05$ & $<0.05$ & $<0.05$ \\
\hline Silver & $<0.006$ & $<0.006$ & $<0.006$ & $<0.006$ & $<0.006$ & $<0.006$ & $<0.006$ & $<0.006$ \\
\hline Strontium & 0.13 & 0.14 & 0.13 & 0.13 & 0.15 & 0.13 & 0.18 & 0.17 \\
\hline Thorium & $<0.2$ & $<0.2$ & $<0.2$ & $<0.2$ & $<0.2$ & $<0.2$ & $<0.2$ & $<0.2$ \\
\hline Uranium (Fluor) & $<0.001$ & $<0.001$ & 0.001 & 0.001 & 0.001 & 0.001 & $<0.001$ & 0.004 \\
\hline Vanadium & $<0.005$ & $<0.005$ & $<0.005$ & $<0.005$ & $<0.005$ & $<0.005$ & $<0.005$ & $<0.005$ \\
\hline zinc & 0.012 & 0.0052 & 0.012 & 0.0059 & 0.01 & 0.011 & 0.0035 & 0.0031 \\
\hline MAJOR IONS $(\mathrm{mg} / \mathrm{L})$ & $\cdot$ & • & $\dot{\bullet}$ & • & $\cdot$ & . & • & 。 \\
\hline Alkalinity-HCO3 & $23 \dot{2}$ & $\dot{\bullet}$ & $21 \dot{5}$ & • & $24 \dot{5}$ & . & $256^{\circ}$ & • \\
\hline Alkalinity- $\mathrm{CO} 3$ & $<1$ & - & $<1$ & . & $<1$ & . & $<1$ & . \\
\hline Calcium & 72 & 75 & 72 & 70 & 78 & 71 & 88 & 83 \\
\hline Chloride & 76 & • & 83 & $\cdot$ & 66 & $\cdot$ & 68 & $\cdot$ \\
\hline Fluoride & $<0.1$ & - & 0.1 & . & $<0.1$ & . & 0.1 & • \\
\hline Magnesium & 19 & 19 & 20 & 20 & 22 & 20 & 24 & 23 \\
\hline Manganese & 0.28 & 0.21 & 0.25 & 0.18 & 0.27 & 0.21 & 0.29 & 0.25 \\
\hline Nitrate-N & $<0.2$ & • & 0.34 & . & $<0.2$ & . & $<0.2$ & • \\
\hline Potassium & 2.4 & 2 & 1.7 & 1.6 & 2 & 1.3 & 2.4 & 2.3 \\
\hline Sodium & 23 & 25 & 29 & 28 & 23 & 21 & 20 & 20 \\
\hline Sulfate & 7.3 & • & 5.3 & . & 6.5 & - & 6.3 & • \\
\hline
\end{tabular}

(CONTINUED) 
APPENDTX E.1

Groundwater Quality Data, 1993

\begin{tabular}{|c|c|c|c|c|c|c|c|c|}
\hline \multirow{4}{*}{$\begin{array}{l}\text { Sampling Point } \\
\text { Iocation } \\
\text { Date Sampled }\end{array}$} & \multicolumn{8}{|c|}{$G W-382$} \\
\hline & \multicolumn{8}{|c|}{ NHP } \\
\hline & \multicolumn{2}{|c|}{$01 / 28 / 93$} & \multicolumn{2}{|c|}{$04 / 26 / 93$} & \multicolumn{2}{|c|}{$08 / 08 / 93$} & \multicolumn{2}{|c|}{$11 / 04 / 93$} \\
\hline & TOT & DIS & TOT & DIS & TOT & DIS & TOT & DIS \\
\hline METALS (mg/L) & - & . & - & . & - & . & . & . \\
\hline Aluminum & 0.047 & $<0.02$ & $<0.02$ & $<0.02$ & $<0.02$ & $<0.02$ & $<0.02$ & $<0.02$ \\
\hline Antimony & $<0.05$ & $<0.05$ & $<0.05$ & $<0.05$ & $<0.05$ & $<0.05$ & $<0.05$ & $<0.05$ \\
\hline Arsenic & $<0.05$ & $<0.05$ & $<0.05$ & $<0.05$ & $<0.05$ & $<0.05$ & $<0.05$ & $<0.05$ \\
\hline Barium & 0.11 & 0.11 & 0.14 & 0.14 & 0.12 & 0.11 & 0.12 & 0.12 \\
\hline Beryllium & $<0.0003$ & $<0.0003$ & $<0.0003$ & $<0.0003$ & $<0.0003$ & $<0.0003$ & $<0.0003$ & $<0.0003$ \\
\hline Boron & 0.26 & 0.07 & 0.14 & 0.043 & 0.057 & 0.072 & 0.064 & 0.061 \\
\hline Cadmium (AAS) & $<0.002$ & $<0.002$ & $<0.002$ & $<0.002$ & $<0.002$ & $<0.002$ & $<0.002$ & $<0.002$ \\
\hline Cadmium & $<0.003$ & $<0.003$ & $<0.003$ & $<0.003$ & $<0.003$ & $<0.003$ & $<0.003$ & $<0.003$ \\
\hline Chromium (AAS) & $<0.01$ & $<0.01$ & $<0.01$ & $<0.01$ & $<0.01$ & $<0.01$ & $<0.01$ & $<0.01$ \\
\hline Chromium & $<0.01$ & $<0.01$ & $<0.01$ & $<0.01$ & $<0.01$ & $<0.01$ & 0.024 & $<0.01$ \\
\hline Cobalt & $<0.005$ & $<0.005$ & $<0.005$ & $<0.005$ & $<0.005$ & $<0.005$ & $<0.005$ & $<0.005$ \\
\hline Copper & $<0.004$ & $<0.004$ & $<0.004$ & $<0.004$ & $<0.004$ & $<0.004$ & 0.012 & 0.0076 \\
\hline Iron & 1 & 0.34 & 1.3 & 0.36 & 1 & 0.12 & 0.36 & 0.12 \\
\hline Lead (AAS) & $<0.004$ & $<0.004$ & $<0.004$ & $<0.004$ & $<0.004$ & $<0.004$ & $<0.004$ & $<0.004$ \\
\hline Mercury (CVAA) & $<0.0002$ & $<0.0002$ & $<0.0002$ & $<0.0002$ & $<0.0002$ & $<0.0002$ & $<0.0002$ & $<0.0002$ \\
\hline Mol ybdenum & $<0.01$ & $<0.01$ & $<0.01$ & $<0.01$ & $<0.01$ & $<0.01$ & $<0.01$ & $<0.01$ \\
\hline Nickel & $<0.01$ & 0.015 & $<0.01$ & $<0.01$ & $<0.01$ & $<0.01$ & 0.32 & $<0.01$ \\
\hline Selenium & $<0.05$ & $<0.05$ & $<0.05$ & $<0.05$ & $<0.05$ & $<0.05$ & $<0.05$ & $<0.05$ \\
\hline Silver & $<0.006$ & $<0.006$ & $<0.006$ & $<0.006$ & $<0.006$ & $<0.006$ & $<0.006$ & $<0.006$ \\
\hline Strontium & 0.39 & 0.39 & 0.36 & 0.36 & 0.4 & 0.4 & 0.45 & 0.44 \\
\hline Thorium & $<0.2$ & $<0.2$ & $<0.2$ & $<0.2$ & $<0.2$ & $<0.2$ & $<0.2$ & $<0.2$ \\
\hline Uranium (Fluor) & 0.001 & 0.001 & $<0.001$ & 0.001 & $<0.001$ & $<0.001$ & $<0.001$ & 0.001 \\
\hline Vanadium & $<0.005$ & $<0.005$ & $<0.005$ & $<0.005$ & $<0.005$ & $<0.005$ & $<0.005$ & $<0.005$ \\
\hline zinc & 0.0021 & $<0.002$ & 0.0035 & 0.004 & 0.0068 & 0.0078 & $<0.002$ & 0.0043 \\
\hline MAJOR IONS $(\mathrm{mg} / \mathrm{I})$ & . & . & . & . & . & . & . & • \\
\hline Alkalinity-HCO3 & 206 &. & 204 & $\cdot$ & 207 & . & 206 & • \\
\hline Alkalinity- $\mathrm{CO} 3$ & $<1$ & . & $<1$ & . & $<1$ & . & $<1$ & . \\
\hline Calcium & 50 & 50 & 51 & 51 & 51 & 50 & 54 & 53 \\
\hline Chloride & 22 & . & 19.6 & . & 19.5 & - & 20 & - \\
\hline Fluoride & 0.1 & . & 0.2 & . & 0.2 & . & 0.2 & . \\
\hline Magnesium & 25 & 25 & 25 & 25 & 27 & 27 & 28 & 28 \\
\hline Manganese & 0.006 & 0.006 & 0.014 & 0.012 & 0.0044 & 0.0047 & 0.0085 & 0.0036 \\
\hline Nitrate-N & 2.8 & . & 2.08 & . & 3.45 & . & 3.6 & • \\
\hline Potassium & 1.3 & 1.3 & 1.2 & 0.7 & 1.2 & 1.3 & 1.2 & 1.3 \\
\hline Sodium & 6.5 & 6.1 & 6.1 & 6 & 6.6 & 6.6 & 6.7 & 6.8 \\
\hline Sulfate & 17 &. & 15 & . & 13.8 & . & 16 & . \\
\hline
\end{tabular}

(CONTINUED) 
APPENDIX E. 1

Groundwater Quality Data, 1993

\begin{tabular}{|c|c|c|c|c|c|c|c|c|}
\hline \multirow{4}{*}{$\begin{array}{l}\text { Sampling Point } \\
\text { Location } \\
\text { Date Sampled }\end{array}$} & \multicolumn{8}{|c|}{ GW-383 } \\
\hline & \multicolumn{8}{|c|}{ NHP } \\
\hline & \multicolumn{2}{|c|}{$01 / 26 / 93$} & \multicolumn{2}{|c|}{$04 / 22 / 93$} & \multicolumn{2}{|c|}{$08 / 07 / 93$} & \multicolumn{2}{|c|}{$11 / 03 / 93$} \\
\hline & TOT & DIS & TOT & DIS & TOT & DIS & TOT & DIS \\
\hline METALS (mg/I) & . & $\cdot$ & . & . & . & - & . & • \\
\hline Aluminum & 0.022 & $<0.02$ & 0.031 & 0.02 & $<0.02$ & $<0.02$ & $<0.02$ & $<0.02$ \\
\hline Antimony & $<0.05$ & $<0.05$ & $<0.05$ & $<0.05$ & $<0.05$ & $<0.05$ & $<0.05$ & $<0.05$ \\
\hline Arsenic & $<0.05$ & $<0.05$ & $<0.05$ & $<0.05$ & $<0.05$ & $<0.05$ & $<0.05$ & $<0.05$ \\
\hline Barium & 0.55 & 0.54 & 0.53 & 0.53 & 0.55 & 0.54 & 0.6 & 0.59 \\
\hline Beryllium & $<0.0003$ & $<0.0003$ & $<0.0003$ & $<0.0003$ & $<0.0003$ & $<0.0003$ & 0.0049 & $<0.0003$ \\
\hline Boron & 0.12 & 0.06 & 0.11 & 0.098 & 0.11 & 0.11 & 0.13 & 0.12 \\
\hline Cadmium (AAS) & $<0.002$ & $<0.002$ & $<0.002$ & $<0.002$ & $<0.002$ & $<0.002$ & $<0.002$ & $<0.002$ \\
\hline Cadmium & $<0.003$ & $<0.003$ & $<0.003$ & $<0.003$ & $<0.003$ & $<0.003$ & 0.007 & $<0.003$ \\
\hline Chromium (AAS) & $<0.01$ & $<0.01$ & $<0.01$ & $<0.01$ & $<0.01$ & $<0.01$ & $<0.01$ & $<0.01$ \\
\hline Chromium & $<0.01$ & $<0.01$ & $<0.01$ & $<0.01$ & $<0.01$ & $<0.01$ & $<0.01$ & $<0.01$ \\
\hline Cobalt & $<0.005$ & $<0.005$ & $<0.005$ & $<0.005$ & $<0.005$ & $<0.005$ & 0.0064 & $<0.005$ \\
\hline Copper & $<0.004$ & $<0.004$ & $<0.004$ & $<0.004$ & $<0.004$ & $<0.004$ & 0.016 & 0.091 \\
\hline Iron & 0.67 & 0.57 & 0.77 & 0.62 & 0.86 & 0.77 & 0.83 & 0.77 \\
\hline Lead (AAS) & $<0.004$ & $<0.004$ & $<0.004$ & $<0.004$ & $<0.004$ & $<0.004$ & $<0.004$ & $<0.004$ \\
\hline Mercury (CVAA) & $<0.0002$ & $<0.0002$ & 0.00036 & $<0.0002$ & $<0.0002$ & $<0.0002$ & $<0.0002$ & $<0.0002$ \\
\hline Molybdenum & $<0.01$ & $<0.01$ & $<0.01$ & $<0.01$ & $<0.01$ & $<0.01$ & $<0.01$ & $<0.01$ \\
\hline Nickel & $<0.01$ & $<0.01$ & $<0.01$ & $<0.01$ & $<0.01$ & $<0.01$ & $<0.01$ & $<0.01$ \\
\hline Selenium & $<0.05$ & $<0.05$ & $<0.05$ & $<0.05$ & $<0.05$ & 0.072 & $<0.05$ & $<0.05$ \\
\hline Silver & $<0.006$ & $<0.006$ & $<0.006$ & $<0.006$ & $<0.006$ & $<0.006$ & $<0.006$ & $<0.006$ \\
\hline Strontium & 0.4 & 0.39 & 0.37 & 0.38 & 0.39 & 0.38 & 0.45 & 0.43 \\
\hline Thorium & $<0.2$ & $<0.2$ & $<0.2$ & $<0.2$ & $<0.2$ & $<0.2$ & $<0.2$ & $<0.2$ \\
\hline Uranium (Fluor) & $<0.001$ & $<0.001$ & $<0.001$ & $<0.001$ & $<0.001$ & $<0.001$ & $<0.001$ & $<0.001$ \\
\hline Vanadium & $<0.005$ & $<0.005$ & $<0.005$ & $<0.005$ & $<0.005$ & $<0.005$ & 0.0072 & $<0.005$ \\
\hline zinc & 0.01 & 0.015 & 0.016 & 0.01 & 0.0085 & 0.0085 & 0.013 & 0.011 \\
\hline MAJOR IONS $(\mathrm{mg} / \mathrm{I})$ & . & . & . & $\cdot$ & • & . & . & • \\
\hline Alkalinity-HCO3 & 219 & $\cdot$ & 213 & $\cdot$ & $222^{\circ}$ & $\cdot$ & 220 & . \\
\hline Alkalinity- $\mathrm{CO} 3$ & $<1$ &. & $<1$ &. & $<1$ & . & $<1$ & . \\
\hline Calcium & 80 & 76 & 74 & 75 & 75 & 73 & 87 & 82 \\
\hline Chloride & 34 & $\cdot$ & 27 & $\cdot$ & 24 & $\cdot$ & 30 & • \\
\hline Fluoride & $<0.1$ & $\cdot$ & $<0.1$ & $\cdot$ & $<0.1$ & . & 0.1 & · \\
\hline Magnesium & 10 & 9.9 & 9.8 & 10 & 10 & 10 & 11 & 11 \\
\hline Manganese & 0.17 & 0.16 & 0.14 & 0.14 & 0.16 & 0.16 & 0.17 & 0.15 \\
\hline Nitrate-N & $<0.2$ & $\cdot$ & $<0.2$ & . & $<0.2$ & $\cdot$ & $<0.2$ & • \\
\hline Potassium & 2.8 & 2.5 & 3 & 2.7 & 2.4 & 2.7 & 3 & 2.8 \\
\hline Sodium & 17 & 16 & 15 & 16 & 17 & 18 & 16 & 16 \\
\hline Sulfate & 19.1 & . & 21 & $\cdot$ & 22 &. & 17 & . \\
\hline
\end{tabular}


APPENDIX E.1

Groundwater Quality Data, 1993

\begin{tabular}{|c|c|c|c|c|c|c|c|c|}
\hline \multirow{4}{*}{$\begin{array}{l}\text { Sampling Point } \\
\text { Iocation } \\
\text { - } \\
\text { Date Sampled }\end{array}$} & \multicolumn{8}{|c|}{$G W-384$} \\
\hline & \multicolumn{8}{|c|}{ NHP } \\
\hline & \multicolumn{2}{|c|}{$01 / 13 / 93$} & \multicolumn{2}{|c|}{$04 / 20 / 93$} & \multicolumn{2}{|c|}{$08 / 04 / 93$} & \multicolumn{2}{|c|}{$10 / 28 / 93$} \\
\hline & TOT & DIS & TOT & DIS & TOT & DIS & TOT & DIS \\
\hline MBTALS (mg/L) & - & - & - & - & - & - & - & - \\
\hline Aluminum & $<0.02$ & 0.045 & $<0.02$ & $<0.02$ & 0.023 & $<0.02$ & $<0.02$ & $<0.02$ \\
\hline Antimony & $<0.05$ & $<0.05$ & $<0.05$ & $<0.05$ & $<0.05$ & $<0.05$ & $<0.05$ & $<0.05$ \\
\hline Arsenic & $<0.05$ & $<0.05$ & $<0.05$ & $<0.05$ & $<0.05$ & $<0.05$ & $<0.05$ & $<0.05$ \\
\hline Barium & 0.44 & 0.45 & 0.45 & 0.45 & 0.46 & 0.46 & 0.47 & 0.48 \\
\hline Beryllium & 0.00031 & 0.00046 & $<0.0003$ & $<0.0003$ & $<0.0003$ & $<0.0003$ & $<0.0003$ & $<0.0003$ \\
\hline Boron & 0.079 & 0.086 & 0.066 & 0.074 & 0.13 & 0.087 & 0.1 & 0.12 \\
\hline Cadmium (AAS) & $<0.002$ & $<0.002$ & $<0.002$ & $<0.002$ & $<0.002$ & $<0.002$ & $<0.002$ & $<0.002$ \\
\hline Cadmium & $<0.003$ & $<0.003$ & $<0.003$ & $<0.003$ & $<0.003$ & $<0.003$ & $<0.003$ & $<0.003$ \\
\hline Chromium (AAS) & $<0.01$ & $<0.01$ & $<0.01$ & $<0.01$ & $<0.01$ & $<0.01$ & $<0.01$ & $<0.01$ \\
\hline Chromium & $<0.01$ & $<0.01$ & $<0.01$ & $<0.01$ & $<0.01$ & $<0.01$ & $<0.01$ & $<0.01$ \\
\hline Cobalt & $<0.005$ & $<0.005$ & $<0.005$ & $<0.005$ & $<0.005$ & $<0.005$ & $<0.005$ & $<0.005$ \\
\hline Copper & $<0.004$ & 0.0044 & $<0.004$ & $<0.004$ & $<0.004$ & $<0.004$ & $<0.004$ & $<0.004$ \\
\hline Iron & 1.2 & 1.7 & 1.1 & 0.96 & 1.5 & 1.3 & 1.1 & 1 \\
\hline Lead (AAS) & $<0.004$ & $<0.004$ & $<0.004$ & $<0.004$ & $<0.004$ & $<0.004$ & $<0.004$ & $<0.004$ \\
\hline Mercury (CVAA) & $<0.0002$ & $<0.0002$ & $<0.0002$ & $<0.0002$ & $<0.0002$ & $<0.0002$ & $<0.0002$ & $<0.0002$ \\
\hline Molybdenum & $<0.01$ & $<0.01$ & $<0.01$ & $<0.01$ & $<0.01$ & $<0.01$ & $<0.01$ & $<0.01$ \\
\hline Nickel & $<0.01$ & $<0.01$ & $<0.01$ & $<0.01$ & $<0.01$ & $<0.01$ & $<0.01$ & $<0.01$ \\
\hline Selenium & $<0.05$ & $<0.05$ & $<0.05$ & $<0.05$ & $<0.05$ & $<0.05$ & $<0.05$ & $<0.05$ \\
\hline silver & $<0.006$ & $<0.006$ & $<0.006$ & $<0.006$ & $<0.006$ & $<0.006$ & $<0.006$ & $<0.006$ \\
\hline strontium & 0.49 & 0.5 & 0.48 & 0.48 & 0.49 & 0.49 & 0.5 & 0.5 \\
\hline Thorium & $<0.2$ & $<0.2$ & $<0.2$ & $<0.2$ & $<0.2$ & $<0.2$ & $<0.2$ & $<0.2$ \\
\hline Uranium (Fluor) & $<0.001$ & 0.002 & $<0.001$ & $<0.001$ & 0.003 & $<0.001$ & $<0.001$ & $<0.001$ \\
\hline Vanadium & $<0.005$ & $<0.005$ & $<0.005$ & $<0.005$ & $<0.005$ & $<0.005$ & $<0.005$ & $<0.005$ \\
\hline zine & 0.0031 & 0.0042 & 0.0022 & 0.0023 & 0.0037 & 0.0074 & 0.0033 & $<0.002$ \\
\hline MAJOR IONS $(\mathrm{mg} / \mathrm{L})$ & - & - & . & - & - & . & . & - \\
\hline Alkalinity-HCO3 & 270 & $\cdot$ & $277^{\circ}$ & . & 279 & $\cdot$ & 281 & . \\
\hline Alkalinity-CO3 & $<1$ & . & $<1$ & . & $<1$ & . & $<1$ & - \\
\hline Calcium & 95 & 97 & 97 & 97 & 94 & 94 & 97 & 97 \\
\hline Chloride & 30 & $\cdot$ & 27.7 & - & 26 & - & 30 & - \\
\hline Fluoride & $<0.1$ & . & 0.1 & - & 0.1 & - & $<0.1$ & - \\
\hline Magnesium & 12 & 12 & 12 & 12 & 12 & 12 & 12 & 12 \\
\hline Manganese & 0.44 & 0.45 & 0.44 & 0.44 & 0.45 & 0.45 & 0.46 & 0.47 \\
\hline Nitrate-N & $<0.2$ & • & $<0.2$ & • & $<0.2$ & $\cdot$ & $<0.2$ & • \\
\hline Potassium & 2.7 & 2.6 & 2.5 & 2.2 & 3 & 2.8 & 2.9 & 2.9 \\
\hline Sodium & 14 & 14 & 13 & 13 & 15 & 15 & 16 & 16 \\
\hline Sulfate & 14 & - & 15.4 & - & 18 & • & 12 & \\
\hline
\end{tabular}

(CONTINUED) 
Groundwater Quality Data, 1993

\begin{tabular}{|c|c|c|c|c|c|c|c|c|}
\hline \multirow{4}{*}{$\begin{array}{l}\text { Sampling Point } \\
\text { Location } \\
\text { - } \\
\text { Date Sampled }\end{array}$} & \multicolumn{8}{|c|}{$G W-385$} \\
\hline & \multicolumn{8}{|c|}{ NHP } \\
\hline & \multicolumn{2}{|c|}{$01 / 12 / 93$} & \multicolumn{2}{|c|}{$04 / 19 / 93$} & \multicolumn{2}{|c|}{$08 / 03 / 93$} & \multicolumn{2}{|c|}{$10 / 27 / 93$} \\
\hline & TOT & DIS & TOT & DIS & TOT & DIS & TOT & DIS \\
\hline MBTALS $(m g / L)$ & . & $\cdot$ & - & $\cdot$ & $\cdot$ & - & . & . \\
\hline Aluminum & 0.12 & 0.022 & 0.12 & $<0.02$ & 0.65 & 0.023 & 0.58 & $<0.02$ \\
\hline Antimony & $<0.05$ & $<0.05$ & $<0.05$ & $<0.05$ & $<0.05$ & $<0.05$ & $<0.05$ & $<0.05$ \\
\hline Arsenic & $<0.05$ & 0.056 & $<0.05$ & $<0.05$ & $<0.05$ & $<0.05$ & $<0.05$ & $<0.05$ \\
\hline Barium & 0.047 & 0.036 & 0.043 & 0.039 & 0.052 & 0.045 & 0.054 & 0.046 \\
\hline Beryllium & 0.0006 & 0.0003 & $<0.0003$ & $<0.0003$ & $<0.0003$ & $<0.0003$ & $<0.0003$ & $<0.0003$ \\
\hline Boron & 0.83 & 0.88 & 0.77 & 0.75 & 0.85 & 0.87 & 0.89 & 0.89 \\
\hline Cadmium (AAS) & $<0.002$ & $<0.002$ & $<0.002$ & $<0.002$ & $<0.002$ & $<0.002$ & $<0.002$ & $<0.002$ \\
\hline Cadmium & $<0.003$ & $<0.003$ & $<0.003$ & $<0.003$ & $<0.003$ & $<0.003$ & $<0.003$ & $<0.003$ \\
\hline Chromiun (AAS) & $<0.01$ & $<0.01$ & $<0.01$ & $<0.01$ & $<0.01$ & $<0.01$ & $<0.01$ & $<0.01$ \\
\hline Chromium & $<0.01$ & $<0.01$ & $<0.01$ & $<0.01$ & $<0.01$ & $<0.01$ & $<0.01$ & $<0.01$ \\
\hline Cobalt & $<0.005$ & $<0.005$ & $<0.005$ & $<0.005$ & $<0.005$ & $<0.005$ & $<0.005$ & $<0.005$ \\
\hline Copper & $<0.004$ & $<0.004$ & $<0.004$ & $<0.004$ & $<0.004$ & $<0.004$ & $<0.004$ & $<0.004$ \\
\hline Iron & 0.48 & 0.015 & 0.15 & 0.016 & 0.75 & 0.013 & 0.79 & $<0.005$ \\
\hline Lead (AAS) & $<0.004$ & $<0.004$ & $<0.004$ & $<0.004$ & $<0.004$ & $<0.004$ & $<0.004$ & $<0.004$ \\
\hline Mercury (CVAA) & $<0.0002$ & $<0.0002$ & $<0.0002$ & $<0.0002$ & $<0.0002$ & $<0.0002$ & $<0.0002$ & $<0.0002$ \\
\hline Mol ybdenum & $<0.01$ & $<0.01$ & $<0.01$ & $<0.01$ & $<0.01$ & $<0.01$ & $<0.01$ & $<0.01$ \\
\hline Nickel & $<0.01$ & $<0.01$ & $<0.01$ & $<0.01$ & $<0.01$ & $<0.01$ & $<0.01$ & $<0.01$ \\
\hline Selenium & $<0.05$ & $<0.05$ & $<0.05$ & $<0.05$ & $<0.05$ & $<0.05$ & $<0.05$ & $<0.05$ \\
\hline Silver & $<0.006$ & $<0.006$ & $<0.006$ & $<0.006$ & $<0.006$ & $<0.006$ & $<0.006$ & $<0.006$ \\
\hline Strontium & 0.088 & 0.083 & 0.082 & 0.08 & 0.088 & 0.083 & 0.092 & 0.086 \\
\hline Thorium & $<0.2$ & $<0.2$ & $<0.2$ & $<0.2$ & $<0.2$ & $<0.2$ & $<0.2$ & $<0.2$ \\
\hline Uranium (Fluor) & $<0.001$ & $<0.001$ & $<0.001$ & $<0.001$ & $<0.001$ & $<0.001$ & $<0.001$ & $<0.001$ \\
\hline Vanadium & $<0.005$ & $<0.005$ & $<0.005$ & $<0.005$ & $<0.005$ & $<0.005$ & $<0.005$ & $<0.005$ \\
\hline 2 inc & 0.0057 & 0.0023 & 0.0048 & 0.0063 & 0.0057 & 0.0029 & 0.0057 & $<0.002$ \\
\hline MAJOR IONS (mg/L) & . &. & . & - & . & . & . & - \\
\hline Alkalinity-HCO3 & 318 & $\cdot$ & 295 & $\cdot$ & 317 & $\cdot$ & 330 & • \\
\hline Alkalinity-CO3 & 46 & $\cdot$ & 48 &. & 50 & . & 54 & • \\
\hline Calcium & 1.1 & 1 & 1.2 & 0.95 & 1.3 & 0.99 & 1.5 & 1 \\
\hline Chloride & 2 & $\cdot$ & 2.4 & . & 3 & $\cdot$ & 3 & • \\
\hline Fluoride & 2.3 & $\cdot$ & 3.1 & $\cdot$ & 3.1 & . & 3.2 & • \\
\hline Magnesium & 0.23 & 0.2 & 0.26 & 0.21 & 0.45 & 0.25 & 0.39 & 0.23 \\
\hline Mangarese & 0.0061 & $<0,001$ & 0.0055 & 0.0047 & 0.0064 & 0.0012 & 0.0086 & $<0.001$ \\
\hline Nitrate-N & $<0.2$ & $\cdot$ & $<0.2$ & $\cdot$ & $<0.2$ & $\cdot$ & $<0.2$ & • \\
\hline Potassium & 2.2 & 2 & 2.5 & 1.9 & 2.5 & 2.6 & 2.5 & 2.1 \\
\hline Sodium & 170 & 180 & 160 & 160 & 170 & 170 & 180 & 180 \\
\hline Sulfate & 18 & . & 18.6 & . & 23 & . & 17 & • \\
\hline
\end{tabular}


APPENDIX E.1

Groundwater Quality Data, 1993

\begin{tabular}{|c|c|c|c|c|c|c|c|c|}
\hline \multirow{4}{*}{$\begin{array}{l}\text { Sampling Point } \\
\text { Location } \\
\text { Date Sampled }\end{array}$} & \multicolumn{8}{|c|}{ GW- 505} \\
\hline & \multicolumn{8}{|c|}{ RG } \\
\hline & \multicolumn{2}{|c|}{$03 / 01 / 93$} & \multicolumn{2}{|c|}{$06 / 18 / 93$} & \multicolumn{2}{|c|}{$09 / 15 / 93$} & \multicolumn{2}{|c|}{$11 / 16 / 93$} \\
\hline & TOT & DIS & TOT & DIS & TOT & DIS & TOT & DIS \\
\hline METALS (mg/L) & $\cdot$ & $\cdot$ & . & $\cdot$ & $\cdot$ & - & - & - \\
\hline Aluminum & 6.3 & $<0.02$ & 6.2 & 0.036 & 3.4 & 0.062 & 7.1 & 0.5 \\
\hline Antimony & $<0.05$ & $<0.05$ & $<0.05$ & $<0.05$ & $<0.05$ & $<0.05$ & $<0.05$ & $<0.05$ \\
\hline Areenic & $<0.05$ & $<0.05$ & $<0.05$ & $<0.05$ & $<0.05$ & $<0.05$ & $<0.05$ & $<0.05$ \\
\hline Barium & 0.078 & 0.044 & 0.1 & 0.065 & 0.097 & 0.072 & 0.11 & 0.067 \\
\hline Beryl1ium & $<0.0003$ & $<0.0003$ & 0.00043 & $<0.0003$ & 0.00074 & $<0.0003$ & 0.005 & 0.00058 \\
\hline Boron & 0.037 & 0.04 & 0.04 & 0.039 & 0.05 & 0.041 & 0.05 & 0.044 \\
\hline Cadmium (AMS) & $<0.002$ & $<0.002$ & 0.0028 & $<0.002$ & 0.0023 & 0.002 & $<0.002$ & $<0.002$ \\
\hline Cacmium & 0.0063 & $<0.003$ & 0.0056 & $<0.003$ & $<0.003$ & 0.0034 & 0.0046 & $<0.003$ \\
\hline Chromium (AAS) & 0.027 & $<0.01$ & 0.032 & $<0.01$ & 0.39 & $<0.01$ & 0.3 & $<0.01$ \\
\hline Chromium & 0.019 & $<0.01$ & 0.034 & $<0.01$ & 0.48 & $<0.01$ & 0.35 & 0.012 \\
\hline Cobalt & 0.0074 & $<0.005$ & $<0.005$ & $<0.005$ & 0.014 & $<0.005$ & 0.021 & $<0.005$ \\
\hline Copper & $<0.004$ & $<0.004$ & 0.0096 & $<0.004$ & 0.0076 & $<0.004$ & 0.017 & $<0.004$ \\
\hline Iron & 4.1 & $<0.005$ & 5 & 0.016 & 4.1 & $<0.005$ & 6.2 & 0.39 \\
\hline Lead (MAS) & 0.0048 & $<0.004$ & 0.0063 & $<0.004$ & 0.011 & $<0.004$ & 0.007 & $<0.004$ \\
\hline Mercury (CVAA) & $<0.0002$ & $<0.0002$ & $<0.0002$ & $<0.0002$ & $<0.0002$ & $<0.0002$ & $<0.0002$ & $<0.0002$ \\
\hline Mol ybdenum & $<0.01$ & $<0.01$ & $<0.01$ & $<0.01$ & 0.017 & $<0.01$ & 0.016 & $<0.01$ \\
\hline Nickel & 0.035 & 0.02 & 0.039 & 0.021 & 0.12 & 0.072 & 0.087 & 0.023 \\
\hline Selenium & $<0.05$ & $<0.05$ & $<0.05$ & $<0.05$ & $<0.05$ & $<0.05$ & $<0.05$ & $<0.05$ \\
\hline silver & $<0.006$ & $<0.006$ & $<0.006$ & $<0.006$ & $<0.006$ & $<0.006$ & 0.0075 & $<0.006$ \\
\hline Strontium & 0.088 & 0.084 & 0.08 & 0.19 & 0.07 & 0.063 & 0.12 & 0.13 \\
\hline Thorium & $<0.2$ & $<0.2$ & $<0.2$ & $<0.2$ & $<0.2$ & $<0.2$ & $<0.2$ & $<0.2$ \\
\hline Uranium (Fluor) & 0.012 & 0.007 & 0.009 & 0.003 & 0.007 & 0.002 & 0.014 & 0.014 \\
\hline Vanadium & $<0.005$ & $<0.005$ & 0.0072 & $<0.005$ & 0.0066 & $<0.005$ & 0.015 & $<0.005$ \\
\hline zinc & 0.018 & 0.0031 & 0.026 & 0.01 & 0.027 & 0.014 & 0.026 & 0.0056 \\
\hline MAJOR IONS (mg/L) & . & . & - & - & . & $\cdot$ & • & • \\
\hline Alkalinity- $\mathrm{HCO}_{3}$ & $\dot{52}$ & $\cdot$ & 37 & $\cdot$ & 30 & $\cdot$ & & • \\
\hline Alkalinity- $\mathrm{CO} 3$ & $<1$ &. & $<1$ & . & $<1$ & . & $<1$ & • \\
\hline Calcium & 27 & 27 & 24 & 22 & 18 & 17 & 33 & 39 \\
\hline Chloride & 27.5 & $\cdot$ & 24 & • & 24 & $\cdot$ & 21.4 & • \\
\hline Fluoride & 0.3 & . & 0.2 & . & 0.2 & • & 0.3 & • \\
\hline Magnesium & 6.2 & 5.5 & 6.1 & 5.6 & 5.2 & 4.6 & 7.5 & 7.8 \\
\hline Manganese & 0.37 & 0.022 & 0.5 & 0.037 & 0.75 & 0.12 & 0.75 & 0.051 \\
\hline Nitrate-N & 1.2 & $\cdot$ & 1.4 & . & 1 & . & 0.93 & • \\
\hline Potassium & 2.7 & 1.3 & 2.6 & 1.7 & 2.7 & 1.6 & 3.6 & 1.9 \\
\hline Sodium & 6.8 & 6.7 & 7.3 & 7.3 & 7.5 & 7.3 & 7 & 7.1 \\
\hline Sulfate & 20.2 & . & 15 & . & 21 & . & 29.4 & • \\
\hline
\end{tabular}

(CONTINUED) 
APPENDIX E.1

Groundwater Quallty Data, 1993

\begin{tabular}{|c|c|c|c|c|c|c|c|c|}
\hline \multirow{4}{*}{$\begin{array}{l}\text { Sampling Point } \\
\text { Location } \\
\text { Date Sampled }\end{array}$} & \multicolumn{8}{|c|}{ GW-508 } \\
\hline & \multicolumn{8}{|c|}{$\mathbf{R G}$} \\
\hline & \multicolumn{2}{|c|}{$03 / 05 / 93$} & \multicolumn{2}{|c|}{$06 / 23 / 93$} & \multirow{2}{*}{\begin{tabular}{|c|}
$09 / 21 / 93$ \\
TOT
\end{tabular}} & \multirow{2}{*}{\begin{tabular}{|c|}
$09 / 22 / 93$ \\
DIs
\end{tabular}} & \multicolumn{2}{|c|}{$11 / 19 / 93$} \\
\hline & TOT & D1S & TOT & DIS & & & TOT & DI8 \\
\hline Merals (mg/L) & . & - & . & - & - & - & - & - \\
\hline Aluminum & 0.81 & 0.37 & 1.1 & 1.2 & 2.6 & 2 & 1.7 & 1.1 \\
\hline Antimony & $<0.05$ & $<0.05$ & $<0.05$ & $<0.05$ & $<0.05$ & $<0.05$ & $<0.05$ & $<0.05$ \\
\hline Arsenic & $<0.05$ & $<0.05$ & $<0.05$ & $<0.05$ & $<0.05$ & $<0.05$ & $<0.05$ & $<0.05$ \\
\hline Barium & 0.33 & 0.31 & 0.39 & 0.54 & 0.64 & 0.74 & 0.46 & 0.45 \\
\hline Beryllium & 0.0013 & 0.0011 & 0.0021 & 0.0029 & 0.0042 & 0.0042 & 0.0025 & 0.0061 \\
\hline Boron & 0.074 & 0.075 & 0.086 & 0.07 & 0.088 & 0.093 & 0.075 & 0.086 \\
\hline Cadmium (MAS) & $<0.002$ & $<0.002$ & $<0.002$ & $<0.002$ & $<0.002$ & $<0.002$ & $<0.002$ & $<0.002$ \\
\hline Cactoium & $<0.003$ & $<0.003$ & $<0.003$ & $<0.003$ & $<0.003$ & $<0.003$ & $<0.003$ & 0.0044 \\
\hline Chromium (AAS) & $<0.01$ & $<0.01$ & $<0.01$ & $<0.01$ & $<0.01$ & $<0.01$ & $<0.01$ & $<0.01$ \\
\hline Chromium & $<0.01$ & $<0.01$ & $<0.01$ & $<0.01$ & $<0.01$ & $<0.01$ & $<0.01$ & $<0.01$ \\
\hline Cobalt & 0.13 & 0.11 & 0.15 & 0.21 & 0.22 & 0.25 & 0.15 & 0.15 \\
\hline Copper & 0.0063 & $<0.004$ & 0.0051 & 0.0052 & $<0.004$ & 0.0059 & $<0.004$ & 0.0049 \\
\hline Iron & 0.21 & 0.01 & 0.04 & $<0.005$ & 0.77 & $<0.005$ & 0.52 & 0.017 \\
\hline Lead (MAS) & $<0.004$ & $<0.004$ & $<0.004$ & $<0.004$ & $<0.004$ & $<0.004$ & $<0.004$ & $<0.004$ \\
\hline Mercury (CVMA) & 0.00022 & $<0.0002$ & $<0.0002$ & $<0.0002$ & $<0.0002$ & $<0.0002$ & 0.00021 & $<0.0002$ \\
\hline Molybdenum & $<0.01$ & $<0.01$ & $<0.01$ & $<0.01$ & $<0.02$ & $<0.01$ & $<0.01$ & $<0.01$ \\
\hline Nickel & 0.068 & 0.058 & 0.072 & 0.11 & 0.12 & 0.11 & 0.075 & 0.073 \\
\hline Selenium & $<0.05$ & $<0.05$ & $<0.05$ & $<0.05$ & $<0.05$ & $<0.05$ & $<0.05$ & $<0.05$ \\
\hline silver & $<0.006$ & $<0.006$ & $<0.006$ & $<0.006$ & $<0.006$ & $<0.006$ & $<0.006$ & 0.0072 \\
\hline strontium & 0.039 & 0.04 & 0.041 & 0.1 & 0.063 & 0.076 & 0.047 & 0.053 \\
\hline Thorium & $<0.2$ & $<0.2$ & $<0.2$ & $<0.2$ & $<0.2$ & $<0.2$ & $<0.2$ & $<0.2$ \\
\hline Oranium (Fluor) & $<0.001$ & $<0.001$ & $<0.001$ & 0.002 & $<0.001$ & $<0.001$ & 0.001 & 0.001 \\
\hline Vanadium & $<0.005$ & $<0.005$ & $<0.005$ & $<0.005$ & $<0.005$ & $<0.005$ & $<0.005$ & $<0.005$ \\
\hline zinc & 0.097 & 0.069 & 0.084 & 0.12 & 0.15 & 0.16 & 0.1 & 0.1 \\
\hline MAJOR IONS $(\mathrm{mg} / \mathrm{L})$ & . & . & - & . & - & - & - & • \\
\hline Alkalinity-HCO3 & $\dot{17}$ & . & 5 & . & $\dot{3}$ & . & $\dot{2}$ & • \\
\hline Alkalinity-CO3 & $<1$ & . & $<1$ & . & $<1$ & . & $<1$ & . \\
\hline Calcium & 6.2 & 8.5 & 4.9 & 7.2 & 4.5 & 6 & 4.4 & 5.6 \\
\hline Chloride & 127 & . & 68 & $\cdot$ & 66 & . & 134 & • \\
\hline Fluoride & 0.2 & . & 0.2 & . & 0.2 & . & 0.2 & • \\
\hline Magnesi um & 9.8 & 9.4 & 11 & 15 & 15 & 18 & 11 & 10 \\
\hline Manganese & 5.6 & 5.7 & 5.4 & 6.8 & 6.6 & 7.8 & 5 & 4.8 \\
\hline Nitrate-N & $<0.2$ & . & $<0.2$ &. & $<0.2$ & • & $<0.2$ & • \\
\hline Potassium & 0.85 & 1 & 0.91 & 0.99 & 1.3 & 1.2 & 0.99 & 0.81 \\
\hline Sodium & 23 & 25 & 22 & 26 & 28 & 32 & 23 & 23 \\
\hline Sulfate & 10 & . & 13 & . & 7.6 & • & 3 & • \\
\hline
\end{tabular}

(CONTINUED) 
APPENDIX K.1

Groundwater Quallty Data, 1993

\begin{tabular}{|c|c|c|c|c|c|c|c|c|}
\hline \multirow{4}{*}{$\begin{array}{l}\text { Sampiling Polnt } \\
\text { Location } \\
\text { Date sampled }\end{array}$} & \multicolumn{8}{|c|}{$O N-603$} \\
\hline & \multicolumn{8}{|c|}{$\mathbf{E X P}$} \\
\hline & \multicolumn{2}{|c|}{$01 / 29 / 93$} & \multicolumn{2}{|c|}{$05 / 04 / 93$} & \multicolumn{2}{|c|}{$08 / 16 / 93$} & \multicolumn{2}{|c|}{$10 / 28 / 93$} \\
\hline & TOT & DIS & TOT & DIs & TOT & DI8 & Tor & Drs \\
\hline neturs (mg/L) & - & $\cdot$ & . & $\cdot$ & - & . & . & - \\
\hline Mluminum & 0.54 & $<0.02$ & 3.5 & 0.038 & 1.1 & $<0.02$ & 0.73 & $<0.02$ \\
\hline Antimony & $<0.05$ & $<0.05$ & $<0.05$ & $<0.05$ & $<0.05$ & $<0.05$ & $<0.05$ & $<0.05$ \\
\hline Areenic & $<0.05$ & $<0.05$ & $<0.05$ & $<0.05$ & $<0.05$ & $<0.05$ & $<0.05$ & $<0.05$ \\
\hline Darium & 0.12 & 0.12 & 0.14 & 0.13 & 0.15 & 0.14 & 0.14 & 0.14 \\
\hline Bery111um & $<0.0003$ & $<0.0003$ & 0.00058 & $<0.0003$ & $<0.0003$ & $<0.0003$ & $<0.0003$ & $<0.0003$ \\
\hline Boron & 0.0069 & 0.027 & 0.042 & 0.026 & 0.0061 & 0.013 & 0.014 & 0.018 \\
\hline Cadmium (MNS) & $<0.002$ & $<0.002$ & $<0.002$ & $<0.002$ & $<0.002$ & $<0.002$ & $<0.002$ & $<0.002$ \\
\hline Cadmium & $<0.003$ & $<0.003$ & $<0.003$ & $<0.003$ & $<0.003$ & $<0.003$ & $<0.003$ & $<0.003$ \\
\hline Chromiun (MS) & $<0.01$ & $<0.01$ & 0.08 & $<0.01$ & 0.089 & $<0.01$ & 0.054 & $<0.01$ \\
\hline Chromium & $<0.01$ & $<0.01$ & 0.057 & $<0.01$ & 0.11 & $<0.01$ & 0.04 & $<0.01$ \\
\hline Cobalt & $<0.005$ & 0.0051 & 0.009 & $<0.005$ & $<0.005$ & $<0.005$ & $<0.005$ & $<0.005$ \\
\hline Copper & $<0.004$ & $<0.004$ & 0.012 & $<0.004$ & 0.0081 & $<0.004$ & 0.0042 & $<0.004$ \\
\hline Iron & 0.49 & $<0.005$ & 3.7 & 0.011 & 1.7 & $<0.005$ & 0.78 & $<0.005$ \\
\hline Laad (MAs) & $<0.004$ & $<0.004$ & 0.011 & $<0.004$ & $<0.004$ & $<0.004$ & $<0.004$ & $<0.004$ \\
\hline Harcury (CVAM) & $<0.0002$ & $<0.0002$ & $<0.0002$ & $<0.0002$ & $<0.0002$ & $<0.0002$ & $<0,0002$ & $<0.0002$ \\
\hline Molybdanum & $<0.01$ & $<0.01$ & $<0.01$ & $<0.01$ & $<0.01$ & $<0.01$ & $<0.01$ & $<0.01$ \\
\hline H1ckel & 0.082 & 0.084 & 0.26 & 0.2 & 0.37 & 0.3 & 0.17 & 0.13 \\
\hline selenium & $<0.05$ & $<0.05$ & $<0.05$ & $<0.05$ & $<0.05$ & $<0.05$ & $<0.05$ & $<0.05$ \\
\hline silver & $<0.006$ & $<0.006$ & $<0.006$ & $<0.006$ & $<0.006$ & $<0.006$ & $<0.006$ & $<0.006$ \\
\hline strontlum & 0.089 & 0.086 & 0.11 & 0.11 & 0.1 & 0.1 & 0.099 & 0.098 \\
\hline Thorium & $<0.2$ & $<0.2$ & $<0.2$ & $<0.2$ & $<0.2$ & $<0.2$ & $<0.2$ & $<0.2$ \\
\hline Uranium (Fluor) & 0.001 & 0.001 & $<0.001$ & $<0.001$ & $<0.001$ & $<0.001$ & $<0.001$ & $<0.001$ \\
\hline Vanadium & $<0.005$ & $<0.005$ & 0.0061 & $<0.005$ & $<0.005$ & $<0.005$ & $<0.005$ & $<0.005$ \\
\hline zinc & 0.015 & 0.0088 & 0.035 & 0.0096 & 0.029 & 0.014 & 0.043 & 0.0095 \\
\hline MAJOR IONS $(\mathrm{mg} / \mathrm{L})$ &. &. & $\cdot$ & 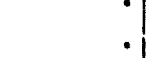 & $\cdot$ & $\cdot$ & . & • \\
\hline Alkalinity-HCO3 & 283 &. & $29 \dot{1}$ & . & 288 &. & 303 & . \\
\hline Alkalinity-CO3 & $<1$ & . & $<1$ &. & $<1$ & . & $<1$ & • \\
\hline Calcium & 73 & 71 & 81 & 79 & 90 & 85 & 82 & 80 \\
\hline Chloride & 5.3 &. & 6.8 & $\cdot$ & 6.4 &. & 5 & • \\
\hline Fluorida & 0.2 &. & 0.1 & . & 0.2 & . & 0.2 & . \\
\hline Magnesium & 24 & 23 & 30 & 26 & 29 & 27 & 26 & 25 \\
\hline Manganese & 0.02 & 0.0075 & 0.11 & 0.026 & 0.16 & 0.1 & 0.064 & 0.031 \\
\hline Nitrate-N & 0.73 &. & 0.69 & . & $0 . ?$ & . & 0.9 & • \\
\hline Potassium & 5.9 & 5.6 & 6 & 5.7 & 7.5 & 7.5 & 6.7 & 6.5 \\
\hline Sodium & 3.6 & 3.6 & 3.5 & 3.6 & 4 & 4 & 3.9 & 3.8 \\
\hline sulfate & 14 &. & 18 &. & 12.7 & . & 12 & • \\
\hline
\end{tabular}

(CONTINURD) 
APPEMDIX $\approx .1$

Groundwater Quallty Data, 1993

\begin{tabular}{|c|c|c|c|c|c|c|c|c|}
\hline \multirow{4}{*}{$\begin{array}{l}\text { Sampling point } \\
\text { Location } \\
\text { Date sampled }\end{array}$} & \multicolumn{8}{|c|}{ GW-604 } \\
\hline & \multicolumn{8}{|c|}{$\operatorname{Exp}$} \\
\hline & \multicolumn{2}{|c|}{$01 / 30 / 93$} & \multicolumn{2}{|c|}{$05 / 04 / 93$} & \multicolumn{2}{|c|}{$08 / 16 / 93$} & \multicolumn{2}{|c|}{$10 / 28 / 93$} \\
\hline & TOT & D18 & TOT & oxs & Tor & DIs & $\operatorname{Tot}$ & Drs \\
\hline mexals $(\mathrm{mg} / L)$ & . & $\cdot$ & . & . & - & . & - & $\cdot$ \\
\hline Numinum & 1.1 & 0.054 & 0.15 & $<0.02$ & 0.65 & $<0.02$ & $<0.02$ & $<0.02$ \\
\hline Antimony & $<0.05$ & $<0.05$ & $<0.05$ & $<0.05$ & $<0.05$ & $<0.05$ & $<0.05$ & $<0.05$ \\
\hline Areende & $<0.05$ & $<0.03$ & $<0.05$ & $<0.05$ & $<0.05$ & $<0.05$ & $<0.05$ & $<0.05$ \\
\hline Barium & 0.037 & 0.034 & 0.031 & 0.03 & 0.033 & 0.031 & 0.026 & 0.03 \\
\hline Bery111um & $<0.0003$ & $<0.0003$ & $<0.0003$ & $<0.0003$ & $<0.0003$ & $<0.0003$ & $<0.0003$ & $<0.0003$ \\
\hline Boron & 0.028 & 0.022 & 0.025 & 0.021 & $<0.004$ & 0.0095 & 0.017 & 0.0049 \\
\hline Cadmium (MS) & $<0.002$ & $<0.002$ & $<0.002$ & $<0.002$ & $<0.002$ & $<0.002$ & $<0.002$ & $<0.002$ \\
\hline Cadmiun & $<0.003$ & 0.0031 & $<0.003$ & $<0.003$ & $<0.003$ & $<0.003$ & $<0.003$ & $<0.003$ \\
\hline Chromium (MAs) & $<0.01$ & $<0.01$ & $<0.01$ & $<0.01$ & $<0.01$ & $<0.01$ & $<0.01$ & $<0.01$ \\
\hline Chromium & $<0.01$ & $<0.01$ & $<0.01$ & $<0.01$ & $<0.01$ & $<0.01$ & $<0.01$ & $<0.01$ \\
\hline Cobalt & $<0.005$ & $<0.003$ & $<0.005$ & $<0.005$ & $<0.005$ & $<0.005$ & $<0.005$ & $<0.005$ \\
\hline Copper & $<0.004$ & $<0.004$ & $<0.004$ & $<0.004$ & $<0.004$ & $<0.004$ & $<0.004$ & $<0.004$ \\
\hline Iron & 0.79 & $<0.005$ & 0.27 & 0.0071 & 0.79 & $<0.005$ & 0.024 & $<0.00 s$ \\
\hline Laed (MAS) & $<0.004$ & $<0.004$ & $<0.004$ & $<0.004$ & $<0.004$ & $<0.004$ & $<0.004$ & $<0.004$ \\
\hline Marcury (CVM) & $<0.0002$ & $<0.0002$ & $<0.0002$ & $<0.0002$ & $<0.0002$ & $<0.0002$ & $<0.0002$ & $<0.0002$ \\
\hline Molybdenum & $<0.01$ & $<0.01$ & $<0.01$ & $<0.01$ & $<0.01$ & $<0.01$ & $<0.01$ & $<0.01$ \\
\hline Mlckel & $<0.01$ & $<0.01$ & 0.014 & 0.011 & $<0.01$ & $<0.01$ & $<0.01$ & $<0.01$ \\
\hline selenium & $<0.05$ & $<0.05$ & $<0.05$ & $<0.05$ & $<0.05$ & $<0.05$ & $<0.05$ & $<0.05$ \\
\hline silver & $<0.006$ & $<0.006$ & $<0.006$ & $<0.006$ & $<0.006$ & $<0.006$ & $<0.006$ & $<0.006$ \\
\hline Strontium & 0.042 & 0.041 & 0.04 & 0.039 & 0.037 & 0.036 & 0.13 & 0.033 \\
\hline Thorium & $<0.2$ & $<0.2$ & $<0.2$ & $<0.2$ & $<0.2$ & $<0.2$ & $<0.2$ & $<0.2$ \\
\hline Uranium (Pluor) & $<0.001$ & $<0.001$ & $<0.001$ & $<0.001$ & $<0.001$ & $<0.001$ & $<0.001$ & $<0.001$ \\
\hline Vanadium & $<0.005$ & $<0.005$ & $<0.005$ & $<0.005$ & $<0.005$ & $<0.005$ & $<0.005$ & $<0.005$ \\
\hline zinc & 0.026 & 0.0091 & 0.021 & 0.011 & 0.028 & 0.013 & $<0.002$ & 0.011 \\
\hline MAJOR IONS $(\mathrm{mg} / \mathrm{L})$ & • & $\cdot$ & . & $\dot{\cdot}$ & . & $\dot{\bullet}$ & • & • \\
\hline Alkalinity-taco3 & 208 & . & 183 & . & $\begin{array}{r}.0 \\
167\end{array}$ & . & 160 & $\cdot$ \\
\hline Alkalinity-CO3 & $<1$ & . & $<1$ & . & $<1$ & . & $<1$ & - \\
\hline Calcium & 53 & 54 & 46 & 46 & 47 & 48 & 12 & 41 \\
\hline Chloride & 2.8 & . & 2.5 & . & 2.2 & - & 2 & • \\
\hline Fluoride & 0.1 & . & 0.1 & . & 0.2 & • & 0.2 & • \\
\hline Magnesium & 10 & 18 & 17 & 17 & 17 & 17 & 17 & 16 \\
\hline Manganese & 0.0098 & $<0.001$ & 0.0053 & 0.0014 & 0.0087 & $<0.001$ & 0.012 & $<0.001$ \\
\hline Nitrata-N & 0.62 & . & 0.45 & $\cdot$ & 0.5 & • & 0.5 & 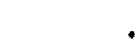 \\
\hline Potann I um & 1.6 & 1.3 & 1.4 & 1.4 & 1.4 & 1.2 & 2 & 1.2 \\
\hline sodium & 2 & 2 & 1.7 & 1.7 & 1.4 & 1.4 & 6 & 1.9 \\
\hline Sulfate & 6.9 & . & 5.7 & $\cdot$ & 4.5 & • & 5 & • \\
\hline
\end{tabular}

(CONTINUED) 
APPENDIX 8.1

Groundwater Quality Date, 1993

\begin{tabular}{|c|c|c|c|c|c|c|c|c|}
\hline \multirow{4}{*}{$\begin{array}{l}\text { Bampling polnt } \\
\text { Location } \\
\text { Date sampled }\end{array}$} & \multicolumn{8}{|c|}{$O N-605$} \\
\hline & \multicolumn{8}{|c|}{$\mathbf{E x p}$} \\
\hline & \multicolumn{2}{|c|}{$02 / 02 / 93$} & \multicolumn{2}{|c|}{$05 / 10 / 93$} & \multicolumn{2}{|c|}{$08 / 19 / 93$} & \multicolumn{2}{|c|}{$11 / 10 / 93$} \\
\hline & Tor & DI8 & Tor & DI8 & Tor & D1s & TOT & Dis \\
\hline netals $(\mathrm{mg} / \mathrm{L})$ & - & - & - & - & - & - & $\cdot$ & - \\
\hline Nuninum & 0.034 & $<0.02$ & 0.027 & $<0.02$ & 0.014 & $<0.02$ & 0.026 & $<0.02$ \\
\hline Antimony & $<0.05$ & $<0.05$ & $<0.05$ & $<0.05$ & $<0.05$ & $<0.05$ & $<0.05$ & $<0.05$ \\
\hline Arsenic & $<0.05$ & $<0.05$ & $<0.05$ & $<0.05$ & $<0.03$ & $<0.05$ & $<0.05$ & $<0.05$ \\
\hline Darlun & 0.065 & 0.065 & 0.068 & 0.066 & 0.063 & 0.059 & 0.075 & 0.074 \\
\hline Dery111um & $<0.0003$ & $<0.0003$ & $<0.0003$ & $<0.0003$ & $<0.0003$ & $<0.0003$ & $<0.0003$ & $<0.0003$ \\
\hline Boron & 0.16 & 0.16 & 0.17 & 0.17 & 0.063 & 0.087 & 0.18 & 0.18 \\
\hline Cadniun (MB) & $<0.002$ & $<0.002$ & $<0.002$ & $<0.002$ & $<0.002$ & $<0.002$ & $<0.002$ & $<0.002$ \\
\hline Cadnium & $<0.003$ & $<0.003$ & $<0.003$ & $<0.003$ & $<0.003$ & $<0.003$ & $<0.003$ & $<0.003$ \\
\hline Chromium (MAs) & $<0.01$ & $<0.01$ & $<0.01$ & $<0.01$ & $<0.01$ & $<0.01$ & $<0.01$ & $<0.01$ \\
\hline Chromium & $<0.01$ & $<0.01$ & $<0.01$ & $<0.01$ & 0.01 & $<0.01$ & $<0.01$ & $<0.01$ \\
\hline Cobalt & $<0.00 s$ & $<0.005$ & $<0.00 s$ & $<0.005$ & $<0.005$ & $<0.003$ & $<0.005$ & $<0.005$ \\
\hline Copper & 0.0068 & $<0.004$ & $<0.004$ & 0.004 & 0.01 & $<0.004$ & $<0.004$ & $<0.004$ \\
\hline Iron & 0.03 & $<0.005$ & 0.08 & 0.0078 & 0.97 & $<0.005$ & 0.033 & $<0.005$ \\
\hline Laed (MAs) & $<0.004$ & $<0.004$ & $<0.004$ & $<0.004$ & $<0.004$ & $<0.004$ & $<0.004$ & $<0.004$ \\
\hline Moroury (CVMA) & $<0.0002$ & $<0.0002$ & $<0.0002$ & $<0.0002$ & $<0.0002$ & $<0.0002$ & $<0.0002$ & $<0.0002$ \\
\hline Mlybdenum & $<0.01$ & $<0.01$ & $<0.01$ & $<0.01$ & $<0.01$ & $<0.01$ & $<0.01$ & $<0.01$ \\
\hline aickel & $<0.01$ & $<0.01$ & $<0.01$ & $<0.01$ & $<0.01$ & $<0.01$ & $<0.01$ & $<0.01$ \\
\hline selenium & $<0.05$ & $<0.05$ & $<0.05$ & $<0.05$ & $<0.05$ & $<0.05$ & $<0.05$ & $<0.05$ \\
\hline silver & $<0.006$ & $<0.006$ & $<0.006$ & $<0.006$ & $<0.006$ & $<0.006$ & $<0.006$ & $<0.006$ \\
\hline strontiun & 0.15 & 0.15 & 0.17 & 0.17 & 0.16 & 0.15 & 0.17 & 0.17 \\
\hline Thorium & $<0.2$ & $<0.2$ & $<0.2$ & $<0.2$ & $<0.2$ & $<0.2$ & $<0.2$ & $<0.2$ \\
\hline Uranium (rluor) & 0.149 & 0.152 & 0.189 & 0.294 & $<0.001$ & 0.098 & 0.19 & 0.16 \\
\hline Vanadium & $<0.005$ & $<0.005$ & $<0.005$ & $<0.005$ & $<0.005$ & $<0.005$ & $<0.005$ & $<0.005$ \\
\hline zine & 0.01 & 0.015 & 0.0089 & 0.015 & 0.03 & 0.0067 & 0.014 & 0.0035 \\
\hline MWOR IONS $(\mathrm{mg} / \mathrm{L})$ & - & - & . & $\cdot$ & - & - & - & • \\
\hline Akallnity-HCO3 & 256 & . & 252 & . & $255^{\circ}$ & . & 260 & • \\
\hline Alkalinity-CO3 & $<1$ & . & $<1$ & . & $<1$ & . & $<1$ & . \\
\hline Calcium & 82 & 82 & 85 & 84 & 100 & 98 & 87 & 88 \\
\hline Chloride & 22 & $\cdot$ & 26 & $\cdot$ & 23 & . & 26.6 & • \\
\hline rivoride & $<0.1$ & . & $<0.1$ & . & $<0.1$ & . & 0.1 & • \\
\hline Magnesium & 25 & 15 & 15 & 15 & 17 & 16 & 17 & 17 \\
\hline Manganese & 0.42 & 0.41 & 0.49 & 0.48 & 0.45 & 0.28 & 0.54 & 0.54 \\
\hline Mitrate-N & $<0.2$ & . & 0.38 & . & $<0.2$ & . & 0.25 & • \\
\hline Potasuium & 2.5 & 3.2 & 2.6 & 2.5 & 5.6 & 4.9 & 3.4 & 3.4 \\
\hline Sodium & 12 & 12 & 13 & 13 & 8.8 & 8.5 & 15 & 15 \\
\hline sulfate & 24 & $\cdot$ & 26 & $\cdot$ & 16 & • & 25.3 & • \\
\hline
\end{tabular}

(CONTINUED) 
APPENDIX ะ.1

Groundwater Quallty Data, 1993

\begin{tabular}{|c|c|c|c|c|c|c|c|c|}
\hline \multirow{4}{*}{$\begin{array}{l}\text { Sampling Point } \\
\text { Location } \\
\text { Date sampled }\end{array}$} & \multicolumn{8}{|c|}{$O N-606$} \\
\hline & \multicolumn{8}{|c|}{$\exp$} \\
\hline & \multicolumn{2}{|c|}{$02 / 03 / 93$} & \multicolumn{2}{|c|}{$05 / 10 / 93$} & \multicolumn{2}{|c|}{$08 / 20 / 93$} & \multicolumn{2}{|c|}{$11 / 11 / 93$} \\
\hline & Tot & DIs & Tot & DIs & $\operatorname{rot}$ & DIs & $20 T$ & D18 \\
\hline ments (mg/L) & $\cdot$ & $\cdot$ & $\cdot$ & $\cdot$ & · & $\cdot$ & $\cdot$ & • \\
\hline Muninum & $<0.02$ & $<0.02$ & $<0.02$ & $<0.02$ & 0.055 & $<0.02$ & $<0.02$ & 0.041 \\
\hline Ant Lmony & $<0.03$ & $<0.05$ & $<0.05$ & $<0.05$ & $<0.05$ & $<0.05$ & $<0.0 s$ & $<0.05$ \\
\hline Araenic & $<0.05$ & $<0.05$ & $<0.05$ & $<0.05$ & $<0.05$ & $<0.05$ & $<0.05$ & $<0.03$ \\
\hline Barlum & 0.005 & 0.084 & 0.08 & 0.08 & 0.095 & 0.095 & 0.11 & 0.1 \\
\hline Boryl11um & $<0.0003$ & $<0.0003$ & $<0.0003$ & $<0.0003$ & $<0.0003$ & $<0.0003$ & $<0.0003$ & $<0.0003$ \\
\hline Boron & 0.026 & 0.023 & 0.048 & 0.053 & 0.034 & 0.031 & 0.031 & 0.033 \\
\hline Cadmiun (MAs) & $<0.002$ & $<0.002$ & $<0.002$ & $<0.002$ & $<0.002$ & $<0.002$ & $<0.002$ & $<0.002$ \\
\hline Cadniun & $<0,003$ & $<0.003$ & $<0.003$ & $<0.003$ & $<0.003$ & $<0.003$ & $<0.003$ & $<0.003$ \\
\hline Chromiun (MAs) & $<0.01$ & $<0.01$ & $<0.01$ & $<0.01$ & $<0.01$ & $<0.01$ & $<0.01$ & $<0.01$ \\
\hline Chromiun & $<0.01$ & $<0.01$ & $<0.01$ & $<0.01$ & $<0.01$ & $<0.01$ & $<0.01$ & $<0.01$ \\
\hline Cobalt & $<0.005$ & $<0.005$ & $<0.005$ & $<0.005$ & $<0.00 s$ & $<0.005$ & $<0.005$ & $<0.005$ \\
\hline Copper & $<0.004$ & $<0.004$ & $<0.004$ & $<0.004$ & $<0.004$ & $<0.004$ & $<0.004$ & $<0.004$ \\
\hline Iron & 0.028 & $<0.005$ & 0.049 & $<0.005$ & 0.017 & $<0.005$ & 0.018 & $<0.005$ \\
\hline Lad (MAS) & $<0.004$ & $<0.004$ & $<0.004$ & $<0.004$ & $<0.004$ & $<0.004$ & $<0.004$ & $<0.004$ \\
\hline Mercury (CVMA) & $<0.0002$ & $<0.0002$ & $<0.0002$ & $<0.0002$ & $<0.0002$ & $<0.0002$ & $<0.0002$ & $<0.0002$ \\
\hline Mol ybdanum & $<0.01$ & $<0.01$ & $<0.01$ & $<0.01$ & $<0.01$ & $<0.01$ & $<0.01$ & $<0.01$ \\
\hline Mickel & $<0.01$ & $<0.01$ & $<0.01$ & 0.024 & $<0.01$ & $<0.01$ & $<0.01$ & $<0.01$ \\
\hline selentum & 0.056 & $<0.05$ & $<0.05$ & $<0.05$ & $<0.05$ & $<0.05$ & $<0.05$ & $<0.05$ \\
\hline stlver & $<0.006$ & $<0.006$ & $<0.006$ & $<0.006$ & $<0.006$ & $<0.006$ & $<0.006$ & $<0.006$ \\
\hline serontiun & 0.43 & 0.43 & 0.45 & 0.16 & 0.5 & 0.51 & 0.5 & 0.48 \\
\hline Thoriun & $<0.2$ & $<0.2$ & $<0.2$ & $<0.2$ & $<0.2$ & $<0.2$ & $<0.2$ & $<0.2$ \\
\hline Uranium (Pluor) & 0.006 & 0.007 & 0.007 & 0.009 & 0.001 & 0.008 & 0.007 & 0.007 \\
\hline Vanadium & $<0.005$ & $<0.005$ & $<0.005$ & $<0.025$ & $<0.005$ & $<0.005$ & $<0.005$ & $<0.005$ \\
\hline $2 \ln c$ & 0.0076 & 0.01 & 0.013 & 0.016 & 0.0049 & 0.012 & 0.0087 & 0.0094 \\
\hline MuJOR IONS (mg/L) & $\cdot$ & $\cdot$ & $\cdot$ & • & $\cdot$ & • & $\cdot$ & $\cdot$ \\
\hline AlkalinLtey-HCO3 & 207 & . & $21 \dot{8}$ &. & $22 \dot{ }$ & . & 243 & . \\
\hline Alkallnity-CO3 & $<1$ & . & $<1$ & . & $<1$ & . & $<1$ & . \\
\hline Calcium & 60 & 60 & 63 & 64 & 74 & 77 & 69 & 69 \\
\hline Chloride & 45 & $\cdot$ & 43 & - & 46 & . & 51 & • \\
\hline rluoride & 0.1 & $\cdot$ & 0.1 & . & 0.1 & . & 0.2 & • \\
\hline Magnesium & 31 & 31 & 31 & 31 & 36 & 37 & 35 & 35 \\
\hline Manganese & 0.021 & 0.02 & 0.018 & 0.018 & 0.02 & 0.021 & 0.022 & 0.022 \\
\hline NLtrate-N & 0.73 & • & 0.87 & • & 0.64 & • & 0.95 & • \\
\hline Potase iun & 4.1 & 3.9 & 3.2 & 3.1 & 3.3 & 3.3 & 3.8 & 3.7 \\
\hline Bodium & 4.7 & 4.6 & 4.5 & 4.4 & 5 & 5 & 4.9 & 4.8 \\
\hline suleate & 34 & . & 31 & . & 28 & . & 33.8 & • \\
\hline
\end{tabular}

(CONT INUSD) 
APPENDIX $\geq .1$

\begin{tabular}{|c|c|c|c|c|c|c|c|c|}
\hline \multirow{4}{*}{$\begin{array}{l}\text { Sampling Polnt } \\
\text { Location } \\
\text { Date sampled }\end{array}$} & \multicolumn{8}{|c|}{$0 W-617$} \\
\hline & \multicolumn{8}{|c|}{$\mathbf{E x p}$} \\
\hline & \multicolumn{2}{|c|}{$01 / 12 / 93$} & \multicolumn{2}{|c|}{$05 / 04 / 93$} & \multicolumn{2}{|c|}{$09 / 16 / 93$} & \multicolumn{2}{|c|}{$12 / 07 / 93$} \\
\hline & Tor & DIs & Tot & Dis & Tot & DIS & TOT & DIs \\
\hline Naras (mg/L) & $\cdot$ & - & - & - & - & - & - & • \\
\hline Aluminum & 0.43 & $<0.02$ & 0.069 & 0.032 & 0.31 & $<0.02$ & 6.7 & $<0.02$ \\
\hline Ant Leny & $<0.05$ & $<0.05$ & $<0.05$ & $<0.05$ & $<0.05$ & $<0.05$ & $<0.05$ & $<0.05$ \\
\hline Areenio & $<0.05$ & $<0.05$ & $<0.05$ & $<0.03$ & $<0.05$ & $<0.05$ & $<0.03$ & $<0.05$ \\
\hline Bariun & 0.031 & 0.03 & 0.035 & 0.033 & 0.057 & 0.049 & 0.089 & 0.06 \\
\hline Bery 111 um & $<0.0003$ & $<0.0003$ & $<0.0003$ & $<0.0003$ & 0.001 & $<0.0003$ & $<0.0003$ & $<0.0003$ \\
\hline Boron & 0.22 & 0.24 & 0.16 & 0.16 & 0.11 & 0.1 & 0.16 & 0.17 \\
\hline Cadadiun (iAs) & $<0.002$ & $<0.002$ & $<0.002$ & $<0.002$ & 0.0054 & 0.0043 & 0.0044 & 0.022 \\
\hline Codinl un & $<0.003$ & $<0.003$ & $<0,003$ & $<0.003$ & 0.0061 & 0.0042 & 0.0051 & 0.022 \\
\hline Chronitum (MAs) & $<0.01$ & $<0.01$ & $<0.01$ & $<0.01$ & $<0.01$ & $<0.01$ & 0.014 & $<0.01$ \\
\hline Chromilum & $<0.01$ & $<0.01$ & $<0.01$ & $<0.01$ & $<0.01$ & $<0.01$ & $<0.01$ & $<0.01$ \\
\hline Cobalt & $<0.005$ & $<0.005$ & 0.012 & 0.0088 & 0.009 & 0.008 & 0.0091 & 0.0071 \\
\hline Copper & $<0.004$ & $<0.004$ & $<0.004$ & 0.01 & 0.01 & 0.0041 & 0.015 & $<0.004$ \\
\hline Iron & 0.81 & $<0.005$ & 1.2 & 0.55 & 1.7 & 0.52 & 11 & 1.1 \\
\hline Lead (MAS) & $<0.004$ & $<0.004$ & $<0.004$ & $<0.004$ & $<0.004$ & $<0.004$ & 0.0087 & $<0.004$ \\
\hline Mercury (CVMA) & $<0.0002$ & $<0.0002$ & $<0.0002$ & $<0.0002$ & $<0.0002$ & $<0.0002$ & $<0.0002$ & $<0.0002$ \\
\hline nolybdenum & $<0.01$ & $<0.01$ & $<0.01$ & $<0.01$ & $<0.01$ & $<0.01$ & $<0.01$ & $<0.01$ \\
\hline nlckel & $<0.01$ & $<0.01$ & 0.012 & 0.01 & $<0.01$ & 0.01 & $<0.01$ & $<0.01$ \\
\hline solentur & $<0.05$ & $<0.05$ & $<0.05$ & $<0.05$ & $<0.05$ & $<0.05$ & $<0.05$ & $<0.05$ \\
\hline 811ver & $<0.006$ & $<0.006$ & $<0.006$ & $<0.006$ & $<0.006$ & $<0.006$ & $<0.006$ & $<0.006$ \\
\hline serontium & 0.036 & 0.037 & 0.038 & 0.035 & 0.045 & 0.039 & 0.062 & 0.054 \\
\hline Thorium & $<0.2$ & $<0.2$ & $<0.2$ & $<0.2$ & $<0.2$ & $<0.2$ & $<0.2$ & $<0.2$ \\
\hline Oranium (rluor) & $<0.001$ & $<0.001$ & $<0.001$ & $<0.001$ & $<0.001$ & $<0.001$ & $<0.001$ & $<0.001$ \\
\hline Vanadium & $<0.005$ & $<0.005$ & $<0.005$ & $<0.005$ & $<0.005$ & $<0.005$ & 0.01 & $<0.005$ \\
\hline $2 \ln c$ & 0.021 & 0.039 & 0.027 & 0.048 & 0.029 & 0.032 & 0.15 & 0.19 \\
\hline MUOR IONS $(\mathrm{mg} / \mathrm{L})$ & . & - & . & . & - & - & - & • \\
\hline Alkalinity-HCO3 & 34 & . & 36 & $\cdot$ & 67 & $\cdot$ & ${ }_{76}$ & • \\
\hline Alkalinity-CO3 & $<1$ & . & $<1$ & . & $<1$ & - & $<1$ & • \\
\hline Calcium & 22 & 23 & 18 & 17 & 22 & 19 & 33 & 25 \\
\hline Chloride & 3.3 & . & 4.7 &. & 11 & . & 14 & • \\
\hline Inoride & $<0.1$ & . & $<0.1$ & . & $<0.1$ & . & $<0.1$ & . \\
\hline Magnesi um & 5.3 & 5.1 & 4.7 & 4.4 & 5.7 & 5 & 6.4 & 6 \\
\hline Manganese & 0.27 & 0.47 & 1.4 & 1.2 & 4.2 & 3.9 & 3.2 & 2.7 \\
\hline Nitrate-n & 2.4 & . & 1.5 & . & 0.51 & . & 0.8 & • \\
\hline Potaxaium & $<0.6$ & $<0.6$ & 1 & 0.92 & 1.1 & 1.3 & 2.9 & 1.7 \\
\hline Sodiun & 4 & 3.9 & 3.6 & 3.3 & 4.8 & 3.9 & 7 & 6 \\
\hline sultate & 37 & . & 31 & . & 23 & • & 29 & • \\
\hline
\end{tabular}

(CONTINUED) 
APPENDIX R.1

Groundwater Quality Data, 1993

\begin{tabular}{|c|c|c|c|c|c|c|c|c|}
\hline \multirow{4}{*}{$\begin{array}{l}\text { Sampling Point } \\
\text { Location } \\
\text { Date sampled }\end{array}$} & \multicolumn{8}{|c|}{$G W-618$} \\
\hline & \multicolumn{8}{|c|}{$\operatorname{Exp}$} \\
\hline & \multicolumn{2}{|c|}{$01 / 12 / 93$} & \multicolumn{2}{|c|}{$05 / 05 / 93$} & \multicolumn{2}{|c|}{$09 / 16 / 93$} & \multicolumn{2}{|c|}{$12 / 08 / 93$} \\
\hline & TOT & DIs & TOT & DIS & TOT & DIS & TOT & DIS \\
\hline mexals (mg/L) & $\cdot$ & $\cdot$ & $\cdot$ & $\cdot$ & $\cdot$ & $\cdot$ & . & - \\
\hline Aluminum & 0.032 & $<0.02$ & 0.037 & 0.066 & 0.16 & $<0.02$ & 0.028 & $<0.02$ \\
\hline Antimony & $<0.05$ & $<0.05$ & $<0.05$ & $<0.05$ & $<0.05$ & $<0.05$ & $<0.05$ & 0.097 \\
\hline Arsanic & $<0.05$ & $<0.05$ & $<0.05$ & $<0.05$ & $<0.05$ & $<0.05$ & $<0.05$ & $<0.05$ \\
\hline Barium & 0.053 & 0.053 & 0.055 & 0.055 & 0.06 & 0.061 & 0.06 & 0.06 \\
\hline Beryllium & $<0.0003$ & $<0.0003$ & $<0.0003$ & $<0.0003$ & $<0.0003$ & $<0.0003$ & $<0.0003$ & $<0.0003$ \\
\hline Boron & 0.13 & 0.13 & 0.11 & 0.13 & 0.14 & 0.15 & 0.17 & 0.17 \\
\hline Cadmium (MAS) & 0.014 & $<0.002$ & 0.02 & $<0.002$ & 0.03 & $<0.002$ & 0.02 & 0.0053 \\
\hline Cadmiun & 0.014 & $<0.003$ & 0.017 & $<0.003$ & 0.027 & $<0.003$ & 0.02 & 0.0054 \\
\hline Chromium (AMS) & $<0.01$ & $<0.01$ & $<0.01$ & $<0.01$ & $<0.01$ & $<0.01$ & $<0.01$ & $<0.01$ \\
\hline Chromium & $<0.01$ & $<0.01$ & $<0.01$ & $<0.01$ & $<0.01$ & $<0.01$ & $<0.01$ & $<0.01$ \\
\hline Cobalt & $<0.005$ & $<0.005$ & $<0.005$ & $<0.005$ & $<0.005$ & $<0.005$ & $<0.005$ & $<0.005$ \\
\hline Copper & $<0.004$ & $<0.004$ & $<0.004$ & $<0.004$ & $<0.004$ & 0.0047 & 0.0045 & $<0.004$ \\
\hline Iron & 0.43 & 0.36 & 0.4 & 0.19 & 0.15 & 0.13 & 0.16 & 0.15 \\
\hline Lead (MS) & $<0.004$ & $<0.004$ & $<0.004$ & $<0.004$ & $<0.004$ & $<0.004$ & $<0.004$ & $<0.004$ \\
\hline Mercury (CVMA) & $<0.0002$ & $<0.0002$ & $<0.0002$ & $<0.0002$ & $<0.0002$ & $<0.0002$ & $<0.0002$ & $<0.0002$ \\
\hline Mol ybdenum & $<0.01$ & $<0.01$ & $<0.01$ & $<0.01$ & $<0.01$ & $<0.01$ & $<0.01$ & $<0.01$ \\
\hline Mickel & $<0.01$ & $<0.01$ & $<0.01$ & $<0.01$ & $<0.01$ & $<0.01$ & $<0.01$ & $<0.01$ \\
\hline selenium & $<0.05$ & $<0.05$ & $<0.05$ & $<0.05$ & $<0.05$ & 0.061 & $<0.05$ & $<0.05$ \\
\hline silver & $<0.006$ & $<0.006$ & $<0.006$ & $<0.006$ & $<0.006$ & $<0.006$ & $<0.006$ & $<0.006$ \\
\hline strontium & 0.16 & 0.16 & 0.18 & 0.18 & 0.18 & 0.18 & 0.18 & 0.18 \\
\hline Thorium & $<0.2$ & $<0.2$ & $<0.2$ & $<0.2$ & $<0.2$ & $<0.2$ & $<0.2$ & $<0.2$ \\
\hline Oranium (Fluor) & 0.001 & $<0.001$ & 0.001 & $<0.001$ & $<0.001$ & $<0.001$ & $<0.001$ & $<0.001$ \\
\hline Vanadium & $<0.005$ & $<0.005$ & $<0.005$ & $<0.005$ & $<0.005$ & $<0.005$ & $<0.005$ & $<0.005$ \\
\hline zinc & 0.0062 & 0.0055 & 0.018 & 0.013 & 0.01 & 0.0049 & 0.005 & 0.0059 \\
\hline MAJOR IONS $(\mathrm{mg} / \mathrm{L})$ & . & . & . &. & - & $\cdot$ & . & • \\
\hline Alkalinity-HCO3 & 271 & $\cdot$ & 266 &. & 279 & . & $28 \dot{ }$ & • \\
\hline Alkalinity-CO3 & $<1$ & . & $<1$ & . & $<1$ & . & $<1$ & • \\
\hline Calcium & 100 & 99 & 95 & 95 & 100 & 100 & 100 & 100 \\
\hline Chloride & 30 & . & 23 & $\cdot$ & 27 & $\cdot$ & 23.5 & • \\
\hline Fluoride & 0.2 & . & 0.2 & . & 0.2 & $\cdot$ & 0.3 & • \\
\hline Magnesium & 9.2 & 9.1 & 8.7 & 8.8 & 9.3 & 9.4 & 9.3 & 9.3 \\
\hline Manganese & 1.7 & 2.7 & 1.8 & 1.8 & 1.9 & 1.9 & 1.8 & 1.8 \\
\hline Nitrate-N & 1.2 & . & 1.6 & . & 1.8 & . & 1.11 & • \\
\hline Potaseiun & 3.6 & 3.1 & 3.3 & 3.4 & 3.3 & 3.5 & 3.6 & 3.7 \\
\hline Sodium & 18 & 18 & 18 & 17 & 18 & 18 & 18 & 18 \\
\hline Sulfate & 24 & $\cdot$ & 21 & $\cdot$ & 22 & $\cdot$ & 20.8 & · \\
\hline
\end{tabular}

(CONTINUED) 
APPENDIX E.1

\begin{tabular}{|c|c|c|c|c|c|c|c|c|}
\hline \multirow{4}{*}{$\begin{array}{l}\text { Sampling Point } \\
\text { Location } \\
\text { - } \\
\text { Date Samplad }\end{array}$} & \multicolumn{8}{|c|}{$G W-619$} \\
\hline & \multicolumn{8}{|c|}{ MTr } \\
\hline & \multicolumn{2}{|c|}{$01 / 20 / 93$} & \multicolumn{2}{|c|}{$05 / 05 / 93$} & \multicolumn{2}{|c|}{$09 / 17 / 93$} & \multicolumn{2}{|c|}{$12 / 08 / 93$} \\
\hline & TOT & DIS & TOT & DIS & TOT & DIS & TOT & DIs \\
\hline METALS (mg/L) & - & $\cdot$ & - & $\cdot$ & - & - & - & - \\
\hline Aluminum & 1.8 & 1.7 & 3.7 & 3.3 & 1.8 & 1.9 & 1.5 & 1,1 \\
\hline Ant Imony & $<0.05$ & $<0.05$ & $<0.05$ & $<0.05$ & $<0.05$ & $<0.05$ & $<0.05$ & $<0.05$ \\
\hline Arsenic & $<0.05$ & $<0.05$ & $<0.05$ & $<0.05$ & $<0.05$ & $<0.05$ & $<0.05$ & 0.057 \\
\hline Barium & 0.0069 & 0.0067 & 0.0072 & 0.0068 & 0.0087 & 0.0084 & 0.0082 & 0.0078 \\
\hline Bery 111 um & $<0.0003$ & $<0.0003$ & $<0,0003$ & $<0.0003$ & $<0.0003$ & $<0.0003$ & $<0.0003$ & $<0.0003$ \\
\hline Boron & 0.026 & 0.026 & 0.022 & 0.019 & 0.035 & 0.041 & 0.019 & 0.024 \\
\hline Cadmium (AAS) & $<0.002$ & $<0.002$ & $<0.002$ & $<0.002$ & $<0.002$ & $<0.002$ & $<0.002$ & $<0.002$ \\
\hline Cadmium & $<0.003$ & $<0.003$ & $<0.003$ & $<0.003$ & $<0.003$ & $<0.003$ & $<0.003$ & $<0.003$ \\
\hline Chromium (MAs) & $<0.01$ & $<0.01$ & 0.013 & 0.011 & $<0,01$ & $<0.01$ & $<0.01$ & $<0.01$ \\
\hline Chromtum & $<0.01$ & $<0.01$ & 0.011 & $<0.01$ & $<0.01$ & $<0.01$ & $<0.01$ & $<0.01$ \\
\hline Cobalt & $<0.005$ & $<0.005$ & $<0.005$ & $<0.005$ & $<0.005$ & $<0.005$ & $<0.005$ & $<0.005$ \\
\hline Copper & $<0,004$ & $<0.004$ & 0.0064 & $<0.004$ & 0.0067 & $<0.004$ & $<0.004$ & $<0.004$ \\
\hline Iron & $<0.005$ & $<0.005$ & 0.037 & 0.012 & 0.031 & $<0.005$ & 0.03 & $<0.005$ \\
\hline Lead (MAS) & $<0.004$ & $<0.004$ & $<0.004$ & $<0.004$ & $<0.004$ & $<0.004$ & $<0.004$ & $<0.004$ \\
\hline Marcury (CVAA) & $<0.0002$ & $<0.0002$ & $<0.0002$ & $<0.0002$ & $<0.0002$ & $<0.0002$ & $<0.0002$ & $<0.0002$ \\
\hline Molybdenum & $<0.01$ & $<0.01$ & $<0.01$ & $<0.01$ & $<0.01$ & $<0.01$ & $<0.01$ & $<0.01$ \\
\hline Nickel & $<0.01$ & $<0.01$ & 0.01 & $<0.01$ & $<0.01$ & $<0.01$ & $<0.01$ & $<0.01$ \\
\hline selentum & $<0.05$ & $<0.05$ & $<0.05$ & $<0.05$ & $<0.05$ & $<0.05$ & $<0.05$ & $<0.05$ \\
\hline Silver & $<0.006$ & $<0.006$ & $<0.006$ & $<0.006$ & $<0.006$ & $<0.006$ & $<0.006$ & $<0.006$ \\
\hline strontium & 0.18 & 0.17 & 0.26 & 0.25 & 0.19 & 0.19 & 0.17 & 0.17 \\
\hline Thorium & $<0.2$ & $<0.2$ & $<0.2$ & $<0.2$ & $<0.2$ & $<0.2$ & $<0.2$ & $<0.2$ \\
\hline Uranium (Fluor) & 0.001 & 0.001 & $<0.001$ & $<0.001$ & 0.001 & 0.001 & 0.001 & 0.001 \\
\hline Vanad1um & 0.0052 & $<0.005$ & 0.0063 & $<0.005$ & 0.0055 & 0.0052 & $<0.005$ & $<0.005$ \\
\hline Zinc & 0.0056 & 0.004 & 0.018 & 0.0078 & 0.01 & 0.0049 & $<0.002$ & $<0.002$ \\
\hline MAJOR IONS (mg/L) & $\cdot$ & . & $\cdot$ & $\cdot$ & $\cdot$ & . & . & - \\
\hline Alkalinity-HCO3 & 37 & - & $<1$ & . & 62 & . & 59 & - \\
\hline Alkalinity-co3 & 8 & - & 28 & $\cdot$ & 12 & $\cdot$ & 8 & - \\
\hline Calcium & 51 & 49 & 57 & 53 & 54 & 53 & 44 & 44 \\
\hline Chloride & 2.1 & $\cdot$ & 1.7 & - & 3 & $\cdot$ & 1.9 & - \\
\hline Fluoride & $<0.1$ & $\cdot$ & 0.1 & $\cdot$ & $<0.1$ & $\cdot$ & 0.2 & • \\
\hline Magnesium & 1.3 & 1.3 & 0.68 & 0.78 & 1.8 & 1.7 & 1.4 & 1.4 \\
\hline Manganese & $<0.001$ & $<0.001$ & 0.0052 & 0.0014 & 0.0038 & 0.0033 & 0.0014 & $<0.001$ \\
\hline Nitrate-N & 0.71 & $\cdot$ & 0.49 & . & 2 & $\cdot$ & 0.77 & • \\
\hline Potassium & 18 & 17 & 28 & 27 & 18 & 19 & 16 & 16 \\
\hline Sodium & $3 \cdot 3$ & 3.3 & 4.9 & 4.6 & 3.7 & 3.8 & 2.7 & 2.8 \\
\hline Sulfate & 3 & $\cdot$ & 3 & $\cdot$ & 6 & $\cdot$ & 2.8 & • \\
\hline
\end{tabular}

(CONT INUED) 
APPENDIX E.1

Groundwater Quality Data, 1993

\begin{tabular}{|c|c|c|c|c|c|c|c|c|}
\hline \multirow{4}{*}{$\begin{array}{l}\text { Sampling Point } \\
\text { Location } \\
\text { Date Sampled }\end{array}$} & \multicolumn{8}{|c|}{$G W-620$} \\
\hline & \multicolumn{8}{|c|}{ FTF } \\
\hline & \multicolumn{2}{|c|}{$01 / 20 / 93$} & \multicolumn{2}{|c|}{$05 / 06 / 93$} & \multicolumn{2}{|c|}{$09 / 17 / 93$} & \multicolumn{2}{|c|}{$12 / 09 / 93$} \\
\hline & TOT & DIS & TOT & DIS & TOT & DIS & TOT & DIS \\
\hline METALS $(\mathrm{mg} / \mathrm{L})$ & - & - & . & - & - & - & - & . \\
\hline Aluminum & 0.2 & 0.11 & 0.54 & 0.23 & 0.14 & 0.032 & 0.14 & 0.22 \\
\hline Antimony & $<0.05$ & $<0.05$ & $<0.05$ & $<0.05$ & $<0.05$ & $<0.05$ & $<0.05$ & $<0.05$ \\
\hline Arsenic & $<0.05$ & $<0.05$ & $<0.05$ & $<0.05$ & $<0.05$ & $<0.05$ & $<0.05$ & $<0.05$ \\
\hline Barium & 0.027 & 0.018 & 0.061 & 0.055 & 0.035 & 0.032 & 0.038 & 0.036 \\
\hline Beryllium & $<0.0003$ & $<0.0003$ & $<0.0003$ & $<0.0003$ & $<0.0003$ & $<0.0003$ & $<0.0003$ & $<0.0003$ \\
\hline Boron & 0.084 & 0.056 & 0.028 & 0.037 & 0.053 & 0.049 & 0.068 & 0.08 \\
\hline Cadmium (AAS) & $<0.002$ & $<0.002$ & $<0.002$ & $<0.002$ & $<0.002$ & $<0.002$ & $<0.002$ & $<0.002$ \\
\hline Cadmium & $<0.003$ & $<0.003$ & $<0.003$ & $<0.003$ & $<0.003$ & $<0.003$ & $<0.003$ & $<0.003$ \\
\hline Chromium (AAS) & $<0.01$ & $<0.01$ & $<0.01$ & $<0.01$ & $<0.01$ & $<0.01$ & $<0.01$ & $<0.01$ \\
\hline Chromium & $<0.01$ & $<0.01$ & $<0.01$ & $<0.01$ & $<0.01$ & $<0.01$ & $<0.01$ & $<0.01$ \\
\hline Cobalt & $<0.005$ & $<0.005$ & $<0.005$ & $<0.005$ & $<0.005$ & $<0.005$ & $<0.005$ & $<0.005$ \\
\hline Copper & 0.0044 & $<0.004$ & 0.0052 & $<0.004$ & $<0.004$ & $<0.004$ & $<0.004$ & 0.0046 \\
\hline Iron & 0.022 & $<0.005$ & 0.013 & $<0.005$ & 0.11 & 0.0051 & 0.054 & $<0.005$ \\
\hline Lead (AAS) & $<0.004$ & $<0.004$ & $<0.004$ & $<0.004$ & $<0.004$ & $<0.004$ & $<0.004$ & $<0.004$ \\
\hline Mercury (CVAA) & $<0.0002$ & $<0.0002$ & $<0.0002$ & $<0.0002$ & $<0.0002$ & $<0.0002$ & $<0.0002$ & $<0.0002$ \\
\hline Molybdenum & $<0.01$ & $<0.01$ & $<0.01$ & $<0.01$ & $<0.01$ & $<0.01$ & $<0.01$ & $<0.01$ \\
\hline Nickel & $<0.01$ & $<0.01$ & $<0.01$ & $<0.01$ & $<0.01$ & $<0.01$ & $<0.01$ & $<0.01$ \\
\hline Selenium & $<0.05$ & $<0.05$ & $<0.05$ & $<0.05$ & $<0.05$ & $<0.05$ & $<0.05$ & $<0.05$ \\
\hline Silver & $<0.006$ & $<0.006$ & $<0.006$ & $<0.006$ & $<0.006$ & $<0.006$ & $<0.006$ & $<0.006$ \\
\hline Strontium & 0.13 & 0.11 & 0.6 & 0.57 & 0.13 & 0.12 & 0.21 & 0.38 \\
\hline Thorium & $<0.2$ & $<0.2$ & $<0.2$ & $<0.2$ & $<0.2$ & $<0.2$ & $<0.2$ & $<0.2$ \\
\hline Uranium (Fluor) & $<0.001$ & $<0.001$ & $<0.001$ & $<0.001$ & 0.001 & $<0.001$ & $<0.001$ & $<0.001$ \\
\hline Vanadium & $<0.005$ & $<0.005$ & $<0.005$ & $<0.005$ & $<0.005$ & $<0.005$ & $<0.005$ & $<0.005$ \\
\hline zinc & 0.022 & 0.0052 & 0.0022 & $<0.002$ & 0.0044 & 0.0068 & $<0.002$ & 0.0025 \\
\hline MAJOR IONS (mg/L) & • & . & . & . & . & - & - & - \\
\hline Alkalinity-HCO3 & $<1$ & . & $<1$ & $\cdot$ & $<1$ & • & $138^{\circ}$ & • \\
\hline Alkalinity- $\mathrm{CO} 3$ & 10 & • & 40 & • & 237 & • & $<1$ & • \\
\hline Calcium & 53 & 36 & 200 & 180 & 56 & 48 & 75 & 88 \\
\hline Chloride & 3.1 & • & 2.7 & • & 3 & • & 3.2 & • \\
\hline Fluoride & $<0.1$ & • & 0.1 & $\cdot$ & 0.2 & • & $<0.1$ & • \\
\hline Magnesium & 6.6 & 5.9 & 1.8 & 0.03 & 9 & 8.5 & 7.3 & 0.11 \\
\hline Manganese & $<0.001$ & $<0.001$ & $<0.001$ & $<0.001$ & 0.0014 & $<0.001$ & $<0.001$ & $<0.001$ \\
\hline Nitrate-N & 1.9 & • & 1.3 & • & 1.1 & • & 2.31 & 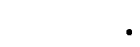 \\
\hline Potassium & 9.8 & 9.4 & 32 & 32 & 4.4 & 4.9 & 9.1 & 27 \\
\hline Sodiurn & 3.2 & 3 & 6 & 5.9 & 2.5 & 2.5 & 2.8 & 5.3 \\
\hline Sulfate & 6.9 & . & 5.7 & . & 4 & . & 9.1 & - \\
\hline
\end{tabular}

(CONTINUED) 
APPENDIX E.1

Groundwater Quality Data, 1993

\begin{tabular}{|c|c|c|c|c|c|c|c|c|}
\hline \multirow{4}{*}{$\begin{array}{l}\text { Sampling Point } \\
\text { L } \\
\text { - } \\
\text { Dacation Sampled }\end{array}$} & \multicolumn{8}{|c|}{ GW-631 } \\
\hline & \multicolumn{8}{|c|}{ RG } \\
\hline & \multicolumn{2}{|c|}{$03 / 02 / 93$} & \multicolumn{2}{|c|}{$06 / 18 / 93$} & \multicolumn{2}{|c|}{$09 / 16 / 93$} & \multicolumn{2}{|c|}{$11 / 17 / 93$} \\
\hline & TOT & DIS & TOT & DIS & TOT & DIS & TOT & DIS \\
\hline METALS (mg/L) & - & - & - & - & - & . & - & - \\
\hline Aluminum & 0.32 & 0.14 & 0.25 & 0.2 & 0.44 & 0.22 & 5.9 & 0.16 \\
\hline Antimony & $<0.05$ & $<0.05$ & $<0.05$ & $<0.05$ & $<0.05$ & $<0.05$ & $<0.05$ & $<0.05$ \\
\hline Arsenic & $<0.05$ & $<0.05$ & $<0.05$ & $<0.05$ & $<0.05$ & $<0.05$ & $<0.05$ & $<0.05$ \\
\hline Barium & 0.26 & 0.33 & 0.28 & 0.23 & 0.2 & 0.2 & 0.25 & 0.2 \\
\hline Beryllium & 0.00082 & 0.00041 & 0.001 & 0.00085 & 0.0015 & 0.0013 & 0.00071 & 0.00091 \\
\hline Boron & 0.041 & 0.033 & 0.056 & 0.029 & 0.058 & 0.05 & 0.093 & 0.052 \\
\hline Cadmium (AAS) & $<0.002$ & $<0.002$ & $<0.002$ & $<0.002$ & $<0.002$ & $<0.002$ & $<0.002$ & $<0.002$ \\
\hline Cadmium & $<0.003$ & $<0.003$ & $<0.003$ & $<0.003$ & $<0.003$ & $<0.003$ & $<0.003$ & $<0.003$ \\
\hline Chromium (AAS) & $<0.01$ & $<0.01$ & $<0.01$ & $<0.01$ & $<0.01$ & $<0.01$ & 0.026 & $<0.01$ \\
\hline Chromium & $<0.01$ & $<0.01$ & $<0.01$ & $<0.01$ & $<0.01$ & $<0.01$ & 0.011 & $<0.01$ \\
\hline Cobalt & 0.035 & 0.027 & 0.028 & 0.032 & 0.034 & 0.034 & 0.025 & 0.028 \\
\hline Copper & $<0.004$ & $<0.004$ & 0.0058 & 0.007 & 0.011 & 0.006 & 0.011 & $<0.004$ \\
\hline Iron & 0.07 & 0.23 & 0.077 & 0.036 & 0.27 & 0.0055 & 6.1 & 0.069 \\
\hline Lead (AAS) & $<0.004$ & $<0.004$ & $<0.004$ & $<0.004$ & $<0.004$ & $<0.004$ & 0.0043 & $<0.004$ \\
\hline Mercury (CVAA) & 0.00023 & $<0.0002$ & $<0.0002$ & $<0.0002$ & $<0.0002$ & $<0.0002$ & $<0.0002$ & $<0.0002$ \\
\hline Molybdenum & $<0.01$ & $<0.01$ & $<0.01$ & $<0.01$ & $<0.01$ & $<0.01$ & $<0.01$ & $<0.01$ \\
\hline Nickel & 0.045 & 0.07 & 0.033 & 0.027 & 0.036 & 0.025 & 0.034 & 0.027 \\
\hline Selenium & $<0.05$ & $<0.05$ & $<0.05$ & $<0.05$ & $<0.05$ & $<0.05$ & $<0.05$ & $<0.05$ \\
\hline Silver & $<0.006$ & $<0.006$ & $<0.006$ & $<0.006$ & $<0.006$ & $<0.006$ & $<0.006$ & $<0.006$ \\
\hline Strontium & 0.069 & 0.11 & 0.07 & 0.054 & 0.044 & 0.055 & 0.065 & 0.051 \\
\hline Thorium & $<0.2$ & $<0.2$ & $<0.2$ & $<0.2$ & $<0.2$ & $<0.2$ & $<0.2$ & $<0.2$ \\
\hline Uranium (Fluor) & $<0.001$ & $<0.001$ & $<0.001$ & $<0.001$ & $<0.001$ & $<0.001$ & $<0.001$ & $<0.001$ \\
\hline Vanadium & $<0.005$ & $<0.005$ & $<0.005$ & $<0.005$ & $<0.005$ & $<0.005$ & 0.01 & $<0.005$ \\
\hline zinc & 0.07 & 0.24 & 0.063 & 0.056 & 0.048 & 0.052 & 0.23 & 0.066 \\
\hline MAJOR IONS (mg/L) & . & . &. &. & . & . & . & . \\
\hline Alkalinity- $\mathrm{HCO} 3$ & 11 & . & 11 & . & 7 & . & 10 & . \\
\hline Alkalinity- $\mathrm{CO} 3$ & $<1$ & . & $<1$ &. & $<1$ & . & $<1$ & . \\
\hline Calcium & 9.2 & 16 & 9.5 & 7 & 5 & 7.2 & 9.6 & 7.3 \\
\hline Chloride & 48 & . & 47 & $\cdot$ & 59 & • & 45 & • \\
\hline Fluoride & $<0.1$ & . & $<0.1$ & . & $<0.1$ & . & $<0.1$ & . \\
\hline Magnesium & 7.1 & 9.6 & 7.7 & 6.4 & 5 & 5.8 & 6.9 & 5.3 \\
\hline Manganese & 1 & 0.94 & 0.9 & 0.92 & 0.94 & 1 & 1 & 0.88 \\
\hline Nitrate-N & 2.8 & $\cdot$ & 14 & . & 1.1 & . & 4.4 & • \\
\hline Potassium & 2.2 & 2.4 & 1.6 & 2 & 2.5 & 2.5 & 4.2 & 2.3 \\
\hline Sodium & 16 & 16 & 17 & 16 & 15 & 17 & 16 & 17 \\
\hline Sulfate & 4.4 & • & 2 & $\cdot$ & 4 & . & 4 & • \\
\hline
\end{tabular}

(CONTINUED) 
APPENDIX E.1

Groundwater Quality Data, 1993

\begin{tabular}{|c|c|c|c|c|c|c|c|c|}
\hline \multirow{4}{*}{$\begin{array}{l}\text { Sampling Point } \\
\text { Location } \\
\text { - } \\
\text { Date Sampled }\end{array}$} & \multicolumn{8}{|c|}{ GW- 632} \\
\hline & \multicolumn{8}{|c|}{ RG } \\
\hline & \multicolumn{2}{|c|}{$03 / 03 / 93$} & \multicolumn{2}{|c|}{$06 / 21 / 93$} & \multicolumn{2}{|c|}{$09 / 16 / 93$} & \multicolumn{2}{|c|}{$11 / 18 / 93$} \\
\hline & TOT & DIS & TOT & DIS & TOT & DIS & TOT & DIS \\
\hline METALS (mg/L) & . & . & . & . & - & . & . & . \\
\hline Aluminum & 0.35 & $<0.02$ & 0.25 & $<0.02$ & 6.9 & 0.024 & 0.51 & 0.056 \\
\hline Antimony & $<0.05$ & $<0.05$ & $<0.05$ & $<0.05$ & $<0.05$ & $<0.05$ & $<0.05$ & $<0.05$ \\
\hline Arsenic & $<0.05$ & $<0.05$ & $<0.05$ & $<0.05$ & $<0.05$ & $<0.05$ & $<0.05$ & $<0.05$ \\
\hline Barium & 0.18 & 0.2 & 0.18 & 0.18 & 0.27 & 0.17 & 0.22 & 0.23 \\
\hline Beryllium & $<0.0003$ & $<0.0003$ & $<0.0003$ & 0.00041 & 0.001 & 0.00044 & 0.00042 & 0.00058 \\
\hline Boron & 0.025 & 0.022 & 0.028 & 0.017 & 0.025 & 0.025 & 0.016 & 0.018 \\
\hline Cadmium (AAS) & $<0.002$ & $<0.002$ & $<0.002$ & $<0.002$ & $<0.002$ & $<0.002$ & $<0.002$ & $<0.002$ \\
\hline Cadmium & $<0.003$ & $<0.003$ & $<0.003$ & $<0.003$ & $<0.003$ & 0.0033 & $<0.003$ & $<0.003$ \\
\hline Chromium (AAS) & $<0.01$ & $<0.01$ & $<0.01$ & $<0.01$ & 0.022 & $<0.01$ & $<0.01$ & $<0.01$ \\
\hline Chromium & $<0.01$ & 0.01 & $<0.01$ & $<0.01$ & 0.02 & $<0.01$ & $<0.01$ & $<0.01$ \\
\hline Cobalt & 0.071 & 0.044 & 0.08 & 0.08 & 0.11 & 0.055 & 0.084 & 0.11 \\
\hline Copper & $<0.004$ & $<0.004$ & $<0.004$ & $<0.004$ & 0.0077 & $<0.004$ & $<0.004$ & $<0.004$ \\
\hline Iron & 0.24 & $<0.005$ & 0.47 & 0.1 & 7.1 & 0.041 & 0.48 & 0.095 \\
\hline Lead (AAS) & $<0.004$ & $<0.004$ & $<0.004$ & $<0.004$ & 0.01 & $<0.004$ & $<0.004$ & $<0.004$ \\
\hline Mercury (CVAA) & $<0.0002$ & $<0.0002$ & $<0.0002$ & $<0.0002$ & $<0.0002$ & $<0.0002$ & $<0.0002$ & $<0.0002$ \\
\hline Molybdenum & $<0.01$ & $<0.01$ & $<0.01$ & $<0.01$ & $<0.01$ & $<0.01$ & $<0.01$ & $<0.01$ \\
\hline Nickel & 0.024 & 0.018 & 0.026 & 0.027 & 0.039 & 0.03 & 0.027 & 0.032 \\
\hline Selenium & $<0.05$ & $<0.05$ & $<0.05$ & $<0.05$ & $<0.05$ & $<0.05$ & $<0.05$ & $<0.05$ \\
\hline Silver & $<0.006$ & $<0.006$ & $<0.006$ & $<0.006$ & $<0.006$ & $<0.006$ & $<0.006$ & $<0.006$ \\
\hline Strontium & 0.092 & 0.14 & 0.082 & 0.077 & 0.097 & 0.1 & 0.098 & 0.077 \\
\hline Thorium & $<0.2$ & $<0.2$ & $<0.2$ & $<0.2$ & $<0.2$ & $<0.2$ & $<0.2$ & $<0.2$ \\
\hline Uranium (Fluor) & $<0.001$ & $<0.001$ & $<0.001$ & $<0.001$ & $<0.001$ & $<0.001$ & 0.001 & 0.001 \\
\hline Vanadium & $<0.005$ & $<0.005$ & $<0.005$ & $<0.005$ & 0.0074 & $<0.005$ & $<0.005$ & $<0.005$ \\
\hline Zinc & 0.043 & 0.066 & 0.067 & 0.06 & 0.087 & 0.04 & 0.058 & 0.066 \\
\hline MAJOR IONS (mg/L) & . & - & . & - & - & - & - & - \\
\hline Alkalinity-HCO3 & ${ }_{51}$ & . & 138 & $\cdot$ & $120^{\circ}$ & . & $\begin{array}{r}\circ \\
147\end{array}$ & $\cdot$ \\
\hline Alkalinity- $\mathrm{CO} 3$ & $<1$ & . & $<1$ & . & $<1$ & . & $<1$ & - \\
\hline Calcium & 48 & 78 & 40 & 37 & 43 & 54 & 42 & 28 \\
\hline Chloride & 15 & . & 18 & . & 16 & . & 21 & - \\
\hline Fluoride & $<0.1$ & . & 0.1 & . & $<0.1$ & . & $<0.1$ & . \\
\hline Magnesium & 8.9 & 11 & 8.8 & 8.3 & 10 & 8.6 & 9.2 & 7.9 \\
\hline Manganese & 6.1 & 5.4 & 6.5 & 6.2 & 5.8 & 4.4 & 5.2 & 4.9 \\
\hline Nitrate-N & 11 & . & 11 & . & 13.9 & . & 12.3 & - \\
\hline Potassium & 1.6 & 1.8 & 1.8 & 1.3 & 3.5 & 1.7 & 1.8 & 1.8 \\
\hline Sodium & 4.2 & 4.5 & 4.1 & 4 & 4.5 & 5 & 4.7 & 4.4 \\
\hline Sulfate & $<1$ & . & $<1$ & . & 7 & . & 2 & \\
\hline
\end{tabular}

(CONTINUED) 
APPENDIX E. 1

Groundwater Quality Data, 1993

\begin{tabular}{|c|c|c|c|c|c|c|c|c|}
\hline \multirow{4}{*}{$\begin{array}{l}\text { Sampling Point } \\
\text { Iocation } \\
\text { - } \\
\text { Date Sampled }\end{array}$} & \multicolumn{8}{|c|}{ GW-633 } \\
\hline & \multicolumn{8}{|c|}{ RG } \\
\hline & \multicolumn{2}{|c|}{$03 / 03 / 93$} & \multicolumn{2}{|c|}{$06 / 21 / 93$} & \multicolumn{2}{|c|}{$09 / 20 / 93$} & \multicolumn{2}{|c|}{$11 / 19 / 93$} \\
\hline & TOT & DIS & TOT & DIS & TOT & DIS & TOT & DIS \\
\hline METALS (mg/L) & - & - & - & - & - & - & - & • \\
\hline Al uminum & 11 & $<2$ & 5.5 & $<1$ & 3.6 & 0.58 & 0.85 & 0.061 \\
\hline Antimony & $<5$ & $<5$ & $<2.5$ & $<2.5$ & $<1$ & $<0.5$ & $<0.05$ & $<0.05$ \\
\hline Arsenic & $<5$ & $<5$ & $<2.5$ & $<2.5$ & $<1$ & $<0.5$ & $<0.05$ & $<0.05$ \\
\hline Barium & 33 & 37 & 22 & 21 & 22 & 24 & 0.87 & 0.92 \\
\hline Bery11ium & $<0.03$ & $<0.03$ & $<0.015$ & $<0.015$ & $<0.006$ & $<0.003$ & $<0.0003$ & $<0.0003$ \\
\hline Boron & $<0.4$ & $<0.4$ & $<0.2$ & $<0.2$ & 0.22 & 0.21 & 0.043 & 0.055 \\
\hline Cadmium (AAS) & $<0.002$ & $<0.002$ & $<0.002$ & $<0.002$ & $<0.002$ & $<0.002$ & $<0.002$ & $<0.002$ \\
\hline Cadmium & $<0.3$ & $<0.3$ & $<0.15$ & $<0.15$ & $<0.06$ & $<0.03$ & $<0.003$ & $<0.003$ \\
\hline Chromium (AAS) & 0.046 & $<0.01$ & 0.013 & $<0.01$ & 0.024 & $<0.01$ & $<0.01$ & $<0.01$ \\
\hline Chromium & $<1$ & $<1$ & $<0.5$ & $<0.5$ & $<0.2$ & $<0.1$ & 0.01 & $<0.01$ \\
\hline Cobalt & $<0.5$ & $<0.5$ & $<0.25$ & $<0.25$ & $<0.1$ & 0.086 & $<0.005$ & 0.0069 \\
\hline Copper & $<0.4$ & $<0.4$ & $<0.2$ & $<0.2$ & $<0.08$ & $<0.04$ & 0.0045 & $<0.004$ \\
\hline Iron & 14 & $<0.5$ & 7.9 & 0.9 & 6.8 & 2.8 & 0.8 & 0.033 \\
\hline Lead (AAS) & 0.011 & $<0.004$ & $<0.004$ & $<0.004$ & 0.0046 & $<0.004$ & $<0.004$ & $<0.004$ \\
\hline Mercury (CVAA) & $<0.0002$ & $<0.0002$ & $<0.0002$ & $<0.0002$ & $<0.0002$ & $<0.0002$ & $<0.0002$ & $<0.0002$ \\
\hline Molybdenum & $<1$ & $<1$ & $<0.5$ & $<0.5$ & $<0.2$ & $<0.1$ & $<0.01$ & $<0.01$ \\
\hline Nickel & $<1$ & $<1$ & $<0.5$ & $<0.5$ & $<0.2$ & 0.28 & 0.029 & 0.026 \\
\hline Selenium & $<5$ & $<5$ & $<2.5$ & $<2.5$ & $<1$ & $<0.5$ & $<0.05$ & $<0.05$ \\
\hline Silver & $<0.6$ & $<0.6$ & $<0.3$ & $<0.3$ & $<0.12$ & $<0.06$ & $<0.006$ & $<0.006$ \\
\hline strontium & 15 & 17 & 11 & 10 & 11 & 12 & 0.68 & 0.74 \\
\hline Thorium & $<20$ & $<20$ & $<10$ & $<10$ & $<4$ & $<2$ & $<0.2$ & $<0.2$ \\
\hline Uranium (Fluor) & 0.003 & 0.002 & 0.003 & 0.003 & 0.002 & 0.002 & 0.003 & 0.002 \\
\hline Vanadium & $<0.5$ & $<0.5$ & $<0.25$ & $<0.25$ & $<0.1$ & $<0.05$ & $<0.005$ & $<0.005$ \\
\hline zinc & $<0.2$ & $<0.2$ & $<0.1$ & 0.26 & 0.11 & 0.078 & 0.062 & 0.069 \\
\hline MAJOR IONS (mg/L) & - & - & . & . & . & . & . & . \\
\hline Alkalinity-HCO3 & 265 & • & 273 & . & 261 & • & 97 & • \\
\hline Alkalinity-CO3 & $<1$ & - & $<1$ & - & $<1$ & - & $<1$ & • \\
\hline Calcium & 6600 & 7100 & 5000 & 4700 & 4600 & 4900 & 230 & 240 \\
\hline Chloride & $<100$ & • & $<100$ & • & $<100$ & • & 8 & • \\
\hline Fluoride & $<0.1$ & • & $<0.1$ & - & $<0.1$ & - & $<0.1$ & • \\
\hline Magnesium & 430 & 460 & 350 & 330 & 310 & 330 & 21 & 22 \\
\hline Manganese & 1.8 & 1.7 & 5.5 & 7.2 & 11 & 13 & 0.76 & 0.89 \\
\hline Nitrate-N & 3874 & • & 4300 & • & 5501 & • & 841 & • \\
\hline Potassium & $<60$ & $<60$ & $<30$ & $<30$ & $<12$ & 14 & 5 & 4.9 \\
\hline Sodium & 63 & 72 & 47 & 47 & 55 & 61 & 8.3 & 8.7 \\
\hline Sulfate & $<100$ & . & $<2000$ & • & 305 & • & 29 & • \\
\hline
\end{tabular}

(CONTINUED) 
APRENDIX R.1

Groundwater Quallty Data, 1993

\begin{tabular}{|c|c|c|c|c|c|c|c|c|}
\hline \multirow{4}{*}{$\begin{array}{l}\text { Sampling Point } \\
\text { Location } \\
\text { Date Sampled }\end{array}$} & \multicolumn{8}{|c|}{$G W-634$} \\
\hline & \multicolumn{8}{|c|}{$\mathbf{R G}$} \\
\hline & \multicolumn{2}{|c|}{$03 / 02 / 93$} & \multicolumn{2}{|c|}{$06 / 18 / 93$} & \multicolumn{2}{|c|}{$09 / 16 / 93$} & \multicolumn{2}{|c|}{$11 / 18 / 93$} \\
\hline & TOT & DIS & TOT & DIS & TOT & DIS & TOT & DIS \\
\hline METAIS (mg/L) & - & . & . & . & . & . & . & . \\
\hline Aluminum & 0.071 & 0.025 & 0.056 & 0.031 & 0.11 & 0.041 & 0.03 & $<0.02$ \\
\hline Antimony & $<0.05$ & $<0.05$ & $<0.05$ & $<0.05$ & $<0.05$ & $<0.05$ & $<0.05$ & $<0.05$ \\
\hline Arsenic & $<0.05$ & $<0.05$ & $<0.05$ & $<0.05$ & $<0.05$ & $<0.05$ & $<0.05$ & $<0.05$ \\
\hline Barium & 0.065 & 0.067 & 0.065 & 0.063 & 0.063 & 0.062 & 0.066 & 0.061 \\
\hline Beryllium & $<0.0003$ & $<0.0003$ & $<0.0003$ & $<0.0003$ & 0.00044 & $<0.0003$ & $<0.0003$ & $<0.0003$ \\
\hline Boron & 0.036 & 0.033 & 0.044 & 0.034 & 0.047 & 0.048 & 0.054 & 0.053 \\
\hline Cadmium (AAS) & $<0.002$ & $<0.002$ & $<0.002$ & $<0.002$ & $<0.002$ & $<0.002$ & $<0.002$ & $<0.002$ \\
\hline Cadmium & $<0.003$ & $<0.003$ & $<0.003$ & $<0.003$ & $<0.003$ & $<0.003$ & $<0.003$ & $<0.003$ \\
\hline Chromium (AAS) & $<0.01$ & $<0.01$ & $<0.01$ & $<0.01$ & $<0.01$ & $<0.01$ & $<0.01$ & $<0.01$ \\
\hline Chromium & $<0.01$ & $<0.01$ & $<0.01$ & $<0.01$ & $<0.01$ & $<0.01$ & $<0.01$ & $<0.01$ \\
\hline Cobalt & 0.046 & 0.047 & 0.052 & 0.052 & 0.051 & 0.05 & 0.051 & 0.048 \\
\hline Copper & $<0.004$ & $<0.004$ & $<0.004$ & $<0.004$ & $<0.004$ & $<0.004$ & $<0.004$ & $<0.004$ \\
\hline Iron & 1.5 & 1.9 & 1.2 & 1.2 & 1.2 & 2.8 & 1.2 & 1.2 \\
\hline Lead (AAS) & $<0.004$ & $<0.004$ & $<0.004$ & $<0.004$ & 0.0044 & $<0.004$ & $<0.004$ & $<0.004$ \\
\hline Mercury (CVAA) & $<0.0002$ & $<0.0002$ & $<0.0002$ & $<0.0002$ & $<0.0002$ & $<0.0002$ & $<0.0002$ & $<0.0002$ \\
\hline Mol ybdenum & $<0.01$ & $<0.01$ & $<0.01$ & $<0.01$ & $<0.01$ & $<0.01$ & $<0.01$ & $<0.01$ \\
\hline Nickel & 0.019 & 0.016 & $<0.01$ & 0.012 & 0.025 & 0.019 & 0.014 & 0.01 \\
\hline Selenium & $<0.05$ & $<0.05$ & $<0.05$ & $<0.05$ & $<0.05$ & $<0.05$ & $<0.05$ & $<0.05$ \\
\hline Silver & $<0.006$ & $<0.006$ & $<0.006$ & $<0.006$ & $<0.006$ & $<0.006$ & $<0.006$ & $<0.006$ \\
\hline Strontium & 0.012 & 0.012 & 0.011 & 0.011 & 0.011 & 0.011 & 0.011 & 0.011 \\
\hline Thorium & $<0.2$ & $<0.2$ & $<0.2$ & $<0.2$ & $<0.2$ & $<0.2$ & $<0.2$ & $<0.2$ \\
\hline Uranium (Fluor) & $<0.001$ & $<0.001$ & $<0.001$ & 0.001 & $<0.001$ & $<0.001$ & $<0.001$ & $<0.001$ \\
\hline Vanadium & $<0.005$ & $<0.005$ & $<0.005$ & $<0.005$ & $<0.005$ & $<0.005$ & $<0.005$ & $<0.005$ \\
\hline zinc & 0.029 & 0.051 & 0.036 & 0.03 & 0.036 & 0.033 & 0.064 & 0.057 \\
\hline MAJOR IONS (mg/L) & - & . & . & . & - & . & . & • \\
\hline Alkalinity-HCO3 & $\dot{21}$ & . & $\dot{12}$ & . & $\begin{array}{r}\dot{1} \\
\end{array}$ & . & $\dot{19}$ & • \\
\hline Alkalinity-CO3 & $<1$ & . & $<1$ & . & $<1$ & . & $<1$ & . \\
\hline Calcium & 2.2 & 2.4 & 1.9 & 1.8 & 1.7 & 1.7 & 1.7 & 1.8 \\
\hline Chloride & 7.9 & $\cdot$ & 9.1 & . & 9 & . & 8 & • \\
\hline Fluoride & $<0.1$ & . & $<0.1$ & . & $<0,1$ & . & $<0.1$ & . \\
\hline Magnesium & 1.9 & 1.9 & 2.2 & 2.2 & 1.9 & 1.9 & 1.9 & 1.8 \\
\hline Manganese & 3.6 & 3.7 & 4.3 & 4.2 & 3.9 & 3.8 & 3.9 & 3.7 \\
\hline Nitrate-N & $<0.2$ & . & $<0.2$ &. & $<0.2$ &. & $<0.2$ & • \\
\hline Potassium & 0.93 & 0.9 & $<0.6$ & 0.65 & 1.2 & 1.2 & 0.99 & 0.99 \\
\hline Sodium & 3.5 & 3.4 & 3.6 & 3.6 & 3.6 & 3.6 & 3.9 & 4 \\
\hline Sulfate & $<1$ & . & 1.8 & . & 1 & . & $<1$ & . \\
\hline
\end{tabular}

(CONTINUED) 
APPENDIX E.1

Groundwater Quallty Data, 1993

\begin{tabular}{|c|c|c|c|c|c|c|c|c|}
\hline \multirow{4}{*}{$\begin{array}{l}\text { Sampling Point } \\
\text { Location } \\
\text { Date Sampled }\end{array}$} & \multicolumn{8}{|c|}{$O W-656$} \\
\hline & \multicolumn{8}{|c|}{ T0134 } \\
\hline & \multicolumn{2}{|c|}{$03 / 11 / 93$} & \multicolumn{2}{|c|}{$06 / 22 / 93$} & \multicolumn{2}{|c|}{$09 / 23 / 93$} & \multicolumn{2}{|c|}{$11 / 17 / 93$} \\
\hline & TOT & DIS & TOT & DIs & TOT & DIS & TOT & DIS \\
\hline $\operatorname{METALS}(\mathrm{mg} / \mathrm{L})$ & • & - & $\cdot$ & - & - & $\cdot$ & - & • \\
\hline Al uminum & 2.6 & 0.045 & 2.4 & $<0.02$ & 1.3 & 0.035 & 14 & 0.022 \\
\hline Antimony & $<0.05$ & $<0.05$ & $<0.05$ & $<0.05$ & $<0.05$ & $<0.05$ & $<0.05$ & $<0.05$ \\
\hline Arsenic & $<0.05$ & $<0.05$ & $<0.05$ & $<0.05$ & $<0.05$ & $<0.05$ & $<0.05$ & $<0.05$ \\
\hline Barium & 0.21 & 0.19 & 0.19 & 0.18 & 0.2 & 0.19 & 0.37 & 0.18 \\
\hline Beryl11um & $<0.0003$ & $<0.0003$ & $<0.0003$ & $<0.0003$ & $<0.0003$ & $<0.0003$ & 0.0013 & $<0.0003$ \\
\hline Boron & 0.017 & 0.013 & 0.091 & 0.011 & 0.017 & 0.021 & 0.032 & 0.017 \\
\hline Cadmium (AAS) & $<0.002$ & $<0.002$ & $<0.002$ & $<0.002$ & $<0.002$ & $<0.002$ & $<0.002$ & $<0.002$ \\
\hline Cadmium & $<0.003$ & $<0.003$ & $<0.003$ & $<0.003$ & $<0.003$ & $<0.003$ & $<0.003$ & $<0.003$ \\
\hline Chromium (AAS) & 0.011 & $<0.01$ & 0.017 & $<0.01$ & 0.07 & $<0.01$ & 0.072 & $<0.01$ \\
\hline Chromium & $<0.01$ & $<0.01$ & 0.016 & $<0.01$ & 0.063 & $<0.01$ & 0.069 & $<0.01$ \\
\hline Cobalt & $<0.005$ & $<0.005$ & $<0.005$ & $<0.005$ & $<0.005$ & $<0.005$ & 0.022 & $<0.005$ \\
\hline Copper & 0.0054 & $<0.004$ & $<0.004$ & $<0.004$ & 0.0093 & $<0.004$ & 0.035 & $<0.004$ \\
\hline Iron & 5.2 & 1.1 & 7.1 & 1.2 & 4.9 & 0.79 & 41 & 0.12 \\
\hline Lead (AAS) & $<0.004$ & $<0.004$ & $<0.004$ & $<0.004$ & $<0.004$ & $<0.004$ & 0.013 & $<0.004$ \\
\hline Mercury (CVAA) & $<0.0002$ & $<0.0002$ & $<0.0002$ & $<0.0002$ & $<0.0002$ & $<0.0002$ & $<0.0002$ & $<0.0002$ \\
\hline Molybdenum & $<0.01$ & $<0.01$ & $<0.01$ & $<0.01$ & $<0.01$ & $<0.01$ & $<0.01$ & $<0.01$ \\
\hline Nickel & 0.029 & 0.017 & 0.054 & 0.029 & 0.043 & 0.02 & 0.078 & 0.023 \\
\hline Selentum & $<0.05$ & $<0.05$ & $<0.05$ & $<0.05$ & $<0.05$ & $<0.05$ & $<0.05$ & $<0.05$ \\
\hline Silver & $<0.006$ & $<0.006$ & $<0.006$ & $<0.006$ & $<0.006$ & $<0,006$ & $<0.006$ & 0.0075 \\
\hline strontium & 0.21 & 0.2 & 0.19 & 0.19 & 0.23 & 0.23 & 0.23 & 0.21 \\
\hline Thorium & $<0.2$ & $<0.2$ & $<0.2$ & $<0.2$ & $<0.2$ & $<0.2$ & $<0.2$ & $<0.2$ \\
\hline oranium (Fluor) & $<0.001$ & $<0.001$ & $<0.001$ & $<0.001$ & $<0.001$ & $<0.001$ & 0.004 & 0.004 \\
\hline Vanadium & $<0.005$ & $<0.005$ & 0.0058 & $<0.005$ & $<0.005$ & $<0.005$ & 0.025 & $<0.005$ \\
\hline zinc & 0.026 & 0.017 & 0.25 & 0.2 & 0.038 & 0.035 & 0.19 & 0.078 \\
\hline MAJOR IONS (mg/L) & . & . & . & . & . & . & . & • \\
\hline Alkalinity-HCO3 & 159 & $\cdot$ & 155 & $\cdot$ & 178 & $\cdot$ & $17 \dot{5}$ & . \\
\hline Alkalinity- $\mathrm{CO} 3$ & $<1$ & . & $<1$ &. & $<1$ & . & $<1$ & . \\
\hline Calcium & 100 & 100 & 110 & 100 & 120 & 120 & 120 & 110 \\
\hline Chloride & 69.6 & $\cdot$ & 66 & $\cdot$ & 68 & $\cdot$ & 64 & • \\
\hline Fluoride & $<0.1$ & . & 0.1 & - & $<0.1$ & • & $<0.1$ & • \\
\hline Magnesium & 6.4 & 5.9 & 6.6 & 6 & 6.4 & 6.2 & 10 & 6.1 \\
\hline Manganese & 0.69 & 0.59 & 0.99 & 0.75 & 0.88 & 0.81 & 1.8 & 0.75 \\
\hline Nitrate-N & $<0.2$ & . & 0.33 & . & $<0.2$ & . & $<0.2$ & • \\
\hline Potassium & 2.5 & 2.8 & 3 & 1.8 & 3.1 & 2.4 & 8.2 & 2.3 \\
\hline Sodium & 8 & 7.5 & 7.2 & 7 & 7.9 & 8.1 & 8.1 & 8.1 \\
\hline Sulfate & 62.2 & . & 61 & . & 65 & . & 67 & • \\
\hline
\end{tabular}

(CONTINUED) 
APPENDIX R.1

Groundwater Quality Data, 1993

\begin{tabular}{|c|c|c|c|c|c|c|c|c|}
\hline \multirow{4}{*}{$\begin{array}{l}\text { Sampling point } \\
\text { Location } \\
\text { Date Sampled }\end{array}$} & \multicolumn{8}{|c|}{$G W-657$} \\
\hline & \multicolumn{8}{|c|}{ T2331 } \\
\hline & \multicolumn{2}{|c|}{$03 / 10 / 93$} & \multicolumn{2}{|c|}{$06 / 18 / 93$} & \multicolumn{2}{|c|}{$09 / 21 / 93$} & \multicolumn{2}{|c|}{$11 / 15 / 93$} \\
\hline & TOT & DIS & TOT & DIS & тот & DIS & TOT & DIS \\
\hline MeThLs (mg/L) & . & . &. &. &. &. & . & . \\
\hline Aluminum & 0.033 & $<0.02$ & $<0.02$ & $<0.02$ & 0.047 & 0.03 & $<0.02$ & $<0.02$ \\
\hline Ant imony & $<0.05$ & $<0.05$ & $<0.05$ & $<0.05$ & $<0.05$ & $<0.05$ & $<0.05$ & $<0.05$ \\
\hline Areanic & $<0.05$ & $<0.05$ & $<0.05$ & $<0.05$ & $<0.05$ & $<0.05$ & $<0.05$ & $<0.05$ \\
\hline Barium & 0.05 & 0.049 & 0.055 & 0.055 & 0.063 & 0.062 & 0.061 & 0.061 \\
\hline Beryl11um & $<0.0003$ & $<0.0003$ & $<0.0003$ & $<0.0003$ & 0.0004 & $<0.0003$ & $<0.0003$ & $<0.0003$ \\
\hline Boron & 0.027 & 0.027 & 0.031 & 0.061 & 0.035 & 0.051 & 0.049 & 0.043 \\
\hline Cadmium (MAS) & $<0.002$ & $<0.002$ & $<0.002$ & $<0.002$ & $<0.002$ & $<0.002$ & $<0.002$ & $<0.002$ \\
\hline Cadmium & $<0.003$ & $<0.003$ & $<0.003$ & $<0.003$ & $<0.003$ & $<0.003$ & 0.0032 & $<0.003$ \\
\hline Chromium (ANS) & $<0.01$ & $<0.01$ & $<0.01$ & $<0.01$ & $<0.01$ & $<0.01$ & $<0.01$ & $<0.01$ \\
\hline Chromiurn & $<0.01$ & $<0.01$ & $<0.01$ & $<0.01$ & $<0.01$ & $<0.01$ & $<0.01$ & $<0.01$ \\
\hline Cobalt & $<0.005$ & $<0.005$ & $<0.005$ & $<0.005$ & $<0.005$ & $<0.005$ & $<0.005$ & $<0.005$ \\
\hline Copper & $<0.004$ & $<0.004$ & $<0.004$ & $<0.004$ & $<0.004$ & $<0.004$ & $<0.004$ & $<0.004$ \\
\hline Iron & 0.2 & 0.13 & 2 & 2.2 & 0.84 & 0.81 & 0.13 & 0.066 \\
\hline Lead (MAS) & $<0.004$ & $<0.004$ & $<0.004$ & $<0.004$ & $<0.004$ & $<0.004$ & $<0.004$ & $<0.004$ \\
\hline Mercury (CVMA) & $<0.0002$ & $<0.0002$ & $<0.0002$ & $<0.0002$ & $<0.0002$ & $<0.0002$ & $<0.0002$ & $<0.0002$ \\
\hline Molybdenum & $<0.01$ & $<0.01$ & $<0.01$ & $<0.01$ & $<0.01$ & $<0.01$ & 0.013 & 0.01 \\
\hline Nickel & $<0.01$ & $<0.01$ & $<0.01$ & $<0.01$ & $<0.01$ & $<0.01$ & $<0.01$ & $<0.01$ \\
\hline Selenium & $<0.05$ & $<0.05$ & $<0.05$ & $<0.05$ & $<0.05$ & $<0.05$ & $<0.05$ & $<0.05$ \\
\hline silver & $<0.006$ & $<0.006$ & $<0.006$ & $<0.006$ & $<0.006$ & $<0.006$ & $<0.006$ & $<0.006$ \\
\hline Strontium & 0.17 & 0.16 & 0.16 & 0.17 & 0.2 & 0.2 & 0.19 & 0.2 \\
\hline Thorium & $<0.2$ & $<0.2$ & $<0.2$ & $<0.2$ & $<0.2$ & $<0.2$ & $<0.2$ & $<0.2$ \\
\hline Oranium (Fluor) & 0.005 & 0.005 & 0.002 & 0.002 & 0.001 & 0.001 & 0.006 & 0.005 \\
\hline Vanadium & $<0.005$ & $<0.005$ & $<0.005$ & $<0.005$ & $<0.005$ & $<0.005$ & $<0.005$ & $<0.005$ \\
\hline zinc & 0.03 & 0.028 & 0.01 & 0.0071 & 0.018 & 0.0064 & 0.032 & 0.03 \\
\hline MAJOR IONS (mg/L) & $\cdot$ & $\cdot \dot{ }$ & $\cdot$ & $\cdot$ & $\cdot$ & $\dot{\bullet}$ & $\dot{.}$ & • \\
\hline Alkalinity-HCO3 & 195 & $\cdot$ & 221 & $\cdot \dot{ }$ & 220 &. & $21 \dot{ }$ & . \\
\hline Alkalinity- $\mathrm{CO} 3$ & $<1$ &. & $<1$ &. & $<1$ &. & $<1$ & . \\
\hline Calcium & 70 & 70 & 68 & 70 & 79 & 78 & 78 & 81 \\
\hline Chloride & 25 &. & 22 &. & 15 & . & 18.6 & . \\
\hline Fluoride & 0.4 & $\cdot$ & 0.5 &. & 0.5 &. & 0.4 & . \\
\hline Magnesium & 8.1 & 8.1 & 8.6 & 8.9 & 9.1 & 9 & 9.1 & 9.6 \\
\hline Manganese & 0.56 & 0.54 & 0.55 & 0.58 & 1.1 & 1.1 & 1.1 & 1.2 \\
\hline Nitrate-n & $<0.2$ &. & $<0.2$ &. & $<0.2$ &. & 0.26 & • \\
\hline Potassium & 5.6 & 5.4 & 6.4 & 6 & 5.7 & 5.8 & 4.8 & 5.3 \\
\hline Sodium & 13 & 13 & 15 & 15 & 12 & 12 & 11 & 12 \\
\hline Sulfate & 30.9 &. & 22 & . & 30 &. & 37.4 & • \\
\hline
\end{tabular}

(CONTINUED) 
APPENDIX $\mathbf{E} \cdot 1$

Groundwater Quality Data, 1993

\begin{tabular}{|c|c|c|c|c|c|c|c|c|}
\hline \multirow{4}{*}{$\begin{array}{l}\text { 8ampling Polnt } \\
\text { L } \\
\text { Location } \\
\text { Date sampled }\end{array}$} & \multicolumn{8}{|c|}{$a w-658$} \\
\hline & \multicolumn{8}{|c|}{$\mathbf{F}$} \\
\hline & \multicolumn{2}{|c|}{$03 / 11 / 93$} & \multicolumn{2}{|c|}{$06 / 23 / 93$} & \multicolumn{2}{|c|}{$11 / 19 / 93$} & \multicolumn{2}{|c|}{$09 / 27 / 04$} \\
\hline & $\operatorname{TOI}$ & DI8 & TOT & DIS & TOT & DI 3 & $\operatorname{tot}$ & DI8 \\
\hline MrAxs (mg/L) & - & - & . & . & - & - & - & - \\
\hline Aluminum & 0.58 & $<0.04$ & 0.71 & $<0.04$ & 1.6 & 0.023 & 0.63 & 0.032 \\
\hline Ant imony & $<0.1$ & $<0.1$ & $<0.1$ & $<0.1$ & $<0.05$ & $<0.05$ & $<0.05$ & $<0.05$ \\
\hline Areenic & $<0.1$ & $<0.1$ & $<0.1$ & $<0.1$ & $<0.05$ & $<0.05$ & $<0.05$ & $<0.05$ \\
\hline Bartum & 0.14 & 0.14 & 0.13 & 0.13 & 0.18 & 0.17 & 0.17 & 0.16 \\
\hline Bery $111 \mathrm{um}$ & 0.0009 & $<0.0006$ & $<0.0006$ & $<0.0006$ & $<0.0003$ & $<0.0003$ & $<0.0003$ & $<0.0003$ \\
\hline Boron & $<0.008$ & 0.023 & $<0.008$ & $<0.008$ & 0.019 & 0.013 & 0.024 & 0.018 \\
\hline Cadmium ( $\mathbf{M g}$ ) & $<0.002$ & $<0.002$ & $<0.002$ & $<0.002$ & $<0.002$ & $<0.002$ & $<0.002$ & $<0.002$ \\
\hline Cadmitum & $<0.006$ & $<0.006$ & $<0.006$ & $<0.006$ & $<0.003$ & $<0.003$ & 0.0032 & 0.0031 \\
\hline Chromium (MAS) & $<0.01$ & $<0.01$ & $<0.01$ & $<0.01$ & $<0.01$ & $<0.01$ & $<0.01$ & $<0.01$ \\
\hline Chromium & 0.05 & 0.047 & $<0.02$ & $<0.02$ & 0.013 & $<0.01$ & $<0.01$ & $<0.01$ \\
\hline Cobalt & $<0.01$ & $<0.01$ & $<0.01$ & $<0.01$ & 0.0093 & 0.0062 & 0.0068 & 0.0054 \\
\hline Copper & $<0.008$ & $<0.008$ & $<0.008$ & $<0.008$ & $<0.004$ & $<0.004$ & 0.0073 & $<0.004$ \\
\hline Iron & 19 & 18 & 13 & 18 & 6.2 & 4.3 & 16 & 14 \\
\hline Lead (MAS) & $<0.004$ & $<0.004$ & $<0.004$ & $<0.004$ & $<0.004$ & $<0.004$ & $<0.004$ & $<0.004$ \\
\hline Mercury (CVAA) & $<0.0002$ & $<0.0002$ & $<0.0002$ & $<0.0002$ & $<0.0002$ & $<0.0002$ & $<0.0002$ & $<0.0002$ \\
\hline Molybdenum & $<0.02$ & $<0.02$ & $<0.02$ & $<0.02$ & $<0.01$ & $<0.01$ & $<0.01$ & $<0.01$ \\
\hline rickel & $<0.02$ & $<0.02$ & $<0.02$ & $<0.02$ & $<0.01$ & $<0.01$ & $<0.01$ & $<0.01$ \\
\hline selenium & $<0.1$ & $<0.1$ & $<0.1$ & $<0.1$ & $<0.05$ & $<0.05$ & $<0.05$ & $<0.05$ \\
\hline sllver & $<0.012$ & $<0.012$ & $<0.012$ & $<0.012$ & $<0.006$ & $<0.006$ & $<0.006$ & $<0.006$ \\
\hline strontium & 0.14 & 0.14 & 0.13 & 0.13 & 0.25 & 0.23 & 0.17 & 0.17 \\
\hline Thorium & $<0.4$ & $<0.4$ & $<0.4$ & $<0.4$ & $<0.2$ & $<0.2$ & $<0.2$ & $<0.2$ \\
\hline Uranium (Fluor) & $<0.001$ & $<0.001$ & $<0.001$ & $<0.001$ & 0.001 & 0.002 & $<0.001$ & $<0.001$ \\
\hline Vanadium & $<0.01$ & $<0.01$ & $<0.01$ & $<0.01$ & $<0.005$ & $<0.005$ & $<0.005$ & $<0.005$ \\
\hline Zinc & 0.005 & $<0.004$ & $<0.004$ & $<0.004$ & 0.052 & 0.016 & 0.023 & 0.017 \\
\hline MANOR IONS (mg/L) & $\cdot$ & - & - & $\cdot$ & • & - & - & - \\
\hline Alkalinity-hCO3 & 219 & . & 241 & $\cdot$ & 270 & . & $259^{\circ}$ & • \\
\hline Alkallnity-CO3 & $<1$ & • & $<1$ & $\cdot$ & $<1$ & - & $<1$ & - \\
\hline Calcium & 45 & 46 & 48 & 47 & 83 & 75 & 56 & 56 \\
\hline Chloride & 19 & $\cdot$ & 14 & $\cdot$ & 27 & • & 26 & • \\
\hline Fluoride & 0.1 & $\cdot$ & $<0.1$ & . & 0.1 & - & $<0.1$ & • \\
\hline Magnesium & 13 & 12 & 14 & 14 & 15 & 14 & 13 & 13 \\
\hline Manganese & 22 & 22 & 29 & 28 & 8.4 & 8.5 & 24 & 23 \\
\hline Nitrate-N & $<0.2$ & • & $<0.2$ & $\cdot$ & $<0.2$ & • & $<0.2$ & • \\
\hline Potassium & $<1.2$ & $<1.2$ & $<1.2$ & $<1.2$ & 1.8 & 1.2 & 1.2 & 0.95 \\
\hline Sodium & 16 & 16 & 13 & 13 & 15 & 16 & 16 & 16 \\
\hline Sulfate & 16.5 & • & 7.5 & • & 2 & • & $<1$ & - \\
\hline
\end{tabular}

(CONTINUED) 
APPEND $1 \times$ Q.1

Groundwater Quality Data, 1993

\begin{tabular}{|c|c|c|c|c|c|c|c|c|}
\hline \multirow{4}{*}{$\begin{array}{l}\text { Sampling Point } \\
\text { Location } \\
\text { Date sampled }\end{array}$} & \multicolumn{8}{|c|}{ OW-659 } \\
\hline & \multicolumn{8}{|c|}{$\mathbf{r F}$} \\
\hline & \multicolumn{2}{|c|}{$03 / 10 / 93$} & \multicolumn{2}{|c|}{$06 / 18 / 93$} & \multicolumn{2}{|c|}{$09 / 22 / 93$} & \multicolumn{2}{|c|}{$11 / 15 / 93$} \\
\hline & TOT & DIs & TOT & DIs & TOT & DIS & TOT & DIS \\
\hline horducs $(\mathrm{mg} / \mathrm{L})$ & $\cdot$ & $\cdot$ & $\cdot$ & . & . & . & - & • \\
\hline Aluminum & 3.8 & 0.025 & 23 & 0.03 & 4.4 & $<0.02$ & 14 & 0.17 \\
\hline Antimony & $<0.05$ & $<0.05$ & $<0.05$ & $<0.05$ & $<0.05$ & $<0.05$ & $<0.05$ & $<0.05$ \\
\hline Areenic & $<0.05$ & $<0.05$ & $<0.05$ & $<0.05$ & $<0.05$ & $<0.05$ & $<0.05$ & $<0.05$ \\
\hline Bartum & 0.27 & 0.27 & 0.13 & 0.041 & 0.07 & 0.074 & 0.094 & 0.016 \\
\hline Beryl11um & $<0.0003$ & $<0.0003$ & 0.0013 & $<0.0003$ & 0.00063 & $<0.0003$ & 0.0065 & $<0.0003$ \\
\hline Boron & 0.015 & 0.016 & 0.049 & 0.055 & 0.02 & 0.022 & 0.024 & 0.016 \\
\hline Cadmium (Ans) & 0.002 & $<0.002$ & 0.0067 & $<0.002$ & 0.0026 & $<0.002$ & 0.005 & $<0.002$ \\
\hline Cadmiun & 0.0045 & $<0.003$ & 0.014 & $<0.003$ & 0.0046 & 0.0042 & 0.013 & $<0.003$ \\
\hline Chromium (AAs) & 0.023 & $<0.01$ & 0.18 & $<0.01$ & 0.13 & 0.011 & 0.24 & $<0.01$ \\
\hline Chromium & 0.015 & $<0.01$ & 0.19 & $<0.01$ & 0.16 & $<0.01$ & 0.21 & $<0.01$ \\
\hline Cobalt & 0.015 & 0.011 & 0.025 & 0.0063 & 0.013 & 0.029 & 0.019 & $<0.005$ \\
\hline Copper & 0.0073 & $<0.004$ & 0.032 & $<0.004$ & 0.0094 & $<0.004$ & 0.04 & $<0.004$ \\
\hline Iron & 3.5 & 0.049 & 27 & 0.45 & 6.4 & 13 & 18 & 0.27 \\
\hline Lead (MAS) & 0.0056 & $<0.004$ & 0.017 & $<0.004$ & 0.0044 & $<0.004$ & 0.034 & $<0.004$ \\
\hline Mercury (CVAA) & $<0.0002$ & $<0.0002$ & $<0.0002$ & $<0.0002$ & $<0.0002$ & $<0.0002$ & 0.00026 & $<0.0002$ \\
\hline Hol ybdenum & $<0.01$ & $<0.01$ & $<0.01$ & $<0.01$ & $<0.01$ & $<0.01$ & 0.014 & $<0.01$ \\
\hline Hickel & 0.058 & 0.055 & 0.23 & 0.13 & 0.12 & 1.3 & 0.062 & 0.016 \\
\hline selentum & $<0.05$ & $<0.05$ & $<0.05$ & $<0.05$ & $<0.05$ & $<0.05$ & $<0.05$ & $<0.05$ \\
\hline stiver & $<0.006$ & $<0.006$ & $<0.006$ & $<0.006$ & $<0.006$ & $<0.006$ & $<0.006$ & $<0.006$ \\
\hline strontium & 0.14 & 0.15 & 0.036 & 0.027 & 0.031 & 0.023 & 0.048 & 0.024 \\
\hline Thorium & $<0.2$ & $<0.2$ & $<0.2$ & $<0.2$ & $<0.2$ & $<0.2$ & $<0.2$ & $<0.2$ \\
\hline Uranium (Fluor) & $<0.001$ & $<0.001$ & 0.002 & $<0.001$ & 0.001 & $<0.001$ & 0.002 & $<0.001$ \\
\hline Vanadium & 0.0086 & $<0.005$ & 0.046 & $<0.005$ & 0.012 & $<0.005$ & 0.032 & $<0.005$ \\
\hline Zine & 0.076 & 0.07 & 0.15 & 0.04 & 0.054 & 0.084 & 0.19 & 0.012 \\
\hline MANOR IONS (mg/L) & . & . & $\cdot$ & $\cdot$ & $\cdot$ & $\cdot$ & $\cdot$ & • \\
\hline Alkalinity-HCO3 & $\dot{33}$ & $\cdot$ & $\dot{72}$ & $\cdot$ & 63 & . & 37 & • \\
\hline Alkalinity-CO3 & $<1$ & . & $<1$ & . & $<1$ & . & $<1$ & • \\
\hline Calcium & 47 & 49 & 14 & 11 & 10 & 7.8 & 16 & 11 \\
\hline Chloride & 578 &. & 238 &. & 50 & • & 3.2 & • \\
\hline Fluoride & $<0.1$ &. & $<0.1$ &. & 0.1 & . & 0.1 & $\bullet$ \\
\hline Magnesium & 6.2 & 5.7 & 4.6 & 1.3 & 2 & 1.3 & 3.4 & 0.75 \\
\hline Manganese & 3.6 & 3.6 & 1 & 0.75 & 0.84 & 1.2 & 0.32 & 0.079 \\
\hline Nitrate-N & $<2.26$ &. & $<0.2$ &. & $<0.2$ & . & 0.33 & • \\
\hline Potassium & 5.9 & 4.5 & 8.5 & 2.2 & 3.7 & 1.9 & 3.6 & 1.3 \\
\hline Sodium & 260 & 300 & 140 & 130 & 54 & 82 & 3.9 & 6.4 \\
\hline Sulfate & 19.6 &. & 10 &. & 11 & . & 5.3 & 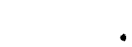 \\
\hline
\end{tabular}

(CONTINUED) 
APPENDIX 8.1

Groundwater Qualley Data, 1993

\begin{tabular}{|c|c|c|c|c|c|c|c|c|}
\hline \multirow{4}{*}{$\begin{array}{l}\text { Sampling point } \\
\text { Location } \\
\text { Date sampled }\end{array}$} & \multicolumn{8}{|c|}{$a W-707$} \\
\hline & \multicolumn{8}{|c|}{ T2331 } \\
\hline & \multicolumn{2}{|c|}{$03 / 11 / 93$} & \multicolumn{2}{|c|}{$06 / 23 / 93$} & \multicolumn{2}{|c|}{$09 / 27 / 93$} & \multicolumn{2}{|c|}{$11 / 18 / 93$} \\
\hline & TOT & Dis & TOT & DIs & TOT & DIs & $20 x$ & Dr8 \\
\hline narhls (mg/L) & - & - & - & - & - & - & $\cdot$ & • \\
\hline Aluminum & 0.11 & 0.053 & $<0.02$ & $<0.02$ & 0.11 & 0.025 & 0.065 & $<0.02$ \\
\hline Ant Lmony & $<0.03$ & $<0.05$ & $<0.05$ & $<0.05$ & $<0.05$ & $<0.05$ & $<0.05$ & $<0.05$ \\
\hline Arsenic & $<0.05$ & $<0.03$ & $<0.05$ & $<0.05$ & $<0.05$ & $<0.05$ & $<0.05$ & $<0.05$ \\
\hline Barlum & 0.036 & 0.014 & 0.048 & 0.056 & 0.056 & 0.06 & 0.042 & 0.039 \\
\hline Beryl1ium & $<0.0003$ & $<0.0003$ & $<0.0003$ & $<0.0003$ & $<0.0003$ & $<0.0003$ & $<0.0003$ & $<0.0003$ \\
\hline Boron & 0.011 & 0.0079 & 0.029 & 0.026 & 0.037 & 0.042 & 0.026 & 0.028 \\
\hline Cadmium (MS) & $<0.002$ & $<0.002$ & 0.0061 & $<0.002$ & $<0.002$ & $<0.002$ & $<0.002$ & $<0.002$ \\
\hline Cadmitum & $<0.003$ & $<0.003$ & 0.0065 & $<0.003$ & $<0.003$ & $<0.003$ & $<0.003$ & $<0.003$ \\
\hline Chromium (MAS) & $<0.01$ & $<0.01$ & $<0.01$ & $<0.01$ & $<0.01$ & $<0.01$ & $<0.01$ & $<0.01$ \\
\hline Chromium & $<0.01$ & $<0.01$ & $<0.01$ & $<0.01$ & $<0.01$ & $<0.01$ & $<0.01$ & $<0.01$ \\
\hline Cobalt & $<0.003$ & $<0.005$ & $<0.005$ & $<0.005$ & $<0.005$ & $<0.005$ & $<0.005$ & $<0.005$ \\
\hline Copper & 0.011 & $<0.004$ & 0.0064 & $<0.004$ & 0.0052 & $<0.004$ & $<0.004$ & $<0.004$ \\
\hline Iron & 0.43 & 0.011 & 0.11 & 0.038 & 0.31 & 0.031 & 0.22 & 0.024 \\
\hline Lead (MAS) & $<0.004$ & $<0.004$ & $<0.004$ & $<0.004$ & $<0.004$ & $<0.004$ & $<0.004$ & $<0.004$ \\
\hline Mercury (CVMA) & $<0.0002$ & $<0.0002$ & $<0.0002$ & $<0.0002$ & $<0.0002$ & $<0.0002$ & $<0.0002$ & $<0.0002$ \\
\hline Holybdenum & $<0.01$ & $<0.01$ & $<0.01$ & $<0.01$ & 0.02 & 0.021 & 0.028 & 0.025 \\
\hline Wiekel & $<0.01$ & $<0.01$ & $<0.01$ & $<0.01$ & $<0.03$ & $<0.01$ & $<0.01$ & $<0.01$ \\
\hline selentum & $<0.05$ & $<0.05$ & $<0.05$ & $<0.05$ & $<0.05$ & $<0.05$ & $<0.05$ & $<0.05$ \\
\hline silver & $<0.006$ & $<0.006$ & $<0.006$ & $<0.006$ & $<0.006$ & $<0.006$ & $<0.006$ & $<0.006$ \\
\hline strontium & 0.12 & 0.077 & 0.14 & 0.15 & 0.2 & 0.19 & 0.17 & 0.25 \\
\hline Thorium & $<0.2$ & $<0.2$ & $<0.2$ & $<0.2$ & $<0.2$ & $<0.2$ & $<0.2$ & $<0.2$ \\
\hline Uraniun (rluor) & 0.002 & 0.001 & 0.003 & 0.004 & 0.006 & 0.005 & 0.003 & 0.003 \\
\hline Vanadium & $<0.005$ & $<0.005$ & $<0.005$ & 0.0053 & 0.014 & 0.017 & $<0.005$ & $<0.005$ \\
\hline $\operatorname{linc}$ & 0.032 & 0.021 & 0.043 & 0.019 & 0.067 & 0.026 & 0.05 & 0.021 \\
\hline MUOR IOWS $(\mathrm{mg} / \mathrm{L})$ &. & $\dot{.}$ & $\cdot$ & $\cdot$ & . & 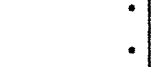 & $\dot{\bullet}$ & • \\
\hline Alkallnity-HCO3 & $13 \dot{1}$ & $\cdot$ & $15 \dot{1}$ & . & $200^{\circ}$ & • & $15 \dot{5}$ & • \\
\hline Alkalinity-CO3 & $<1$ & . & $<1$ & - & $<1$ & . & $<1$ & . \\
\hline Calcium & 44 & 26 & 41 & 48 & 70 & 73 & 58 & 56 \\
\hline Chloride & 17 & $\cdot$ & 202 & • & 82 & • & 23 & • \\
\hline Fluoride & 0.3 & • & 0.3 & • & 0.5 & - & 0.5 & . \\
\hline Magnesium & 5 & 2.2 & 5.1 & 6.3 & 9.6 & 10 & 7.5 & 7.2 \\
\hline Manganese & 0.097 & 0.039 & 0.2 & 0.23 & 0.29 & 0.31 & 0.17 & 0.15 \\
\hline Nitrate-N & 0.31 & . & $<0.2$ & • & 0.23 & . & 0.3 & • \\
\hline Potasuium & 6 & 4.6 & 8.8 & 8.9 & 7.7 & 7.7 & 5.3 & 5.2 \\
\hline Sodium & 9.2 & 4 & 29 & 26 & 27 & 19 & 13 & 12 \\
\hline sulfate & 21.2 & . & 32 & • & 45.2 & • & 38 & • \\
\hline
\end{tabular}

(CONT INURD ) 
APPENDIX 2.1

Oroundwater Quallty Data, 1993

\begin{tabular}{|c|c|c|c|c|c|c|c|c|}
\hline \multirow{4}{*}{$\begin{array}{l}\text { Sampling Polnt } \\
\text { Location } \\
\text { Date sampled }\end{array}$} & \multicolumn{8}{|c|}{ OH-708 } \\
\hline & \multicolumn{8}{|c|}{22331} \\
\hline & \multicolumn{2}{|c|}{$03 / 10 / 93$} & \multicolumn{2}{|c|}{$06 / 18 / 93$} & \multicolumn{2}{|c|}{$09 / 21 / 93$} & \multicolumn{2}{|c|}{$11 / 12 / 93$} \\
\hline & TOT & Drs & Tor & DIs & TOT & DIs & TOT & DI8 \\
\hline $\operatorname{menzs}(\operatorname{mg} / 2)$ & $\cdot$ & $\cdot$ & - & $\cdot$ & $\cdot$ & $\cdot$ & - & - \\
\hline Aluminum & 0.14 & $<0.02$ & 0.038 & 0.025 & 0.037 & $<0.02$ & $<0.02$ & 0.023 \\
\hline Antimony & $<0.05$ & $<0.05$ & $<0.03$ & $<0.05$ & $<0.05$ & $<0.05$ & $<0.05$ & $<0.05$ \\
\hline Arsenico & $<0.05$ & $<0.05$ & $<0.05$ & $<0.05$ & $<0.05$ & $<0.05$ & $<0.05$ & $<0.05$ \\
\hline Barlum & 0.18 & 0.19 & 0.27 & 0.28 & 0.44 & 0.45 & 0.6 & 0.58 \\
\hline Beryl11um & $<0.0003$ & $<0.0003$ & $<0.0003$ & $<0.0003$ & $<0.0003$ & $<0.0003$ & 0.0047 & 0.00054 \\
\hline Boron & 0.028 & 0.028 & 0.068 & 0.12 & 0.1 & 0.11 & 0.086 & 0.11 \\
\hline Cadmiun (MAS) & $<0.002$ & $<0.002$ & $<0.002$ & $<0.002$ & $<0.002$ & $<0.002$ & $<0.002$ & $<0.002$ \\
\hline Cactulium & $<0.003$ & $<0.003$ & $<0.003$ & $<0.003$ & $<0.003$ & $<0.003$ & 0.0045 & $<0.003$ \\
\hline Chromiun (MAS) & $<0.01$ & $<0.01$ & $<0.01$ & $<0.02$ & $<0.01$ & $<0.01$ & $<0.01$ & $<0.01$ \\
\hline Chromilum & $<0.01$ & $<0.01$ & $<0.01$ & $<0.01$ & $<0.01$ & $<0.01$ & $<0.01$ & $<0.01$ \\
\hline Cobalt & $<0.005$ & $<0.005$ & $<0.005$ & $<0.005$ & $<0.005$ & $<0.005$ & $<0.005$ & $<0.005$ \\
\hline Copper & $<0.004$ & $<0.004$ & $<0.001$ & $<0.004$ & 0.0097 & $<0.004$ & $<0.004$ & $<0.004$ \\
\hline Iron & 4.2 & 4.1 & 5.4 & 5.6 & 0.1 & 7.7 & 6.2 & 6 \\
\hline Lend (MAs) & $<0.004$ & $<0.004$ & $<0.004$ & $<0.004$ & $<0.004$ & $<0.004$ & $<0.004$ & $<0.004$ \\
\hline Marcury (CVMA) & $<0.0002$ & $<0.0002$ & $<0.0002$ & $<0.0002$ & $<0.0002$ & $<0.0002$ & $<0.0002$ & $<0.0002$ \\
\hline nolybdenum & $<0.01$ & $<0.01$ & $<0.01$ & $<0.01$ & $<0.01$ & $<0.01$ & $<0.01$ & $<0.01$ \\
\hline Nickel & $<0.01$ & $<0.01$ & $<0.01$ & $<0.01$ & $<0.01$ & $<0.01$ & $<0.01$ & $<0.01$ \\
\hline Solenium & $<0.05$ & $<0.05$ & 0.057 & $<0.05$ & $<0.05$ & $<0.05$ & $<0.05$ & $<0.05$ \\
\hline silver & $<0.006$ & $<0.006$ & $<0.006$ & $<0.006$ & $<0.006$ & $<0.006$ & $<0.006$ & $<0.006$ \\
\hline strontium & 0.28 & 0.3 & 0.37 & 0.38 & 0.51 & 0.51 & 0.5 & 0.49 \\
\hline Thorlum & $<0.2$ & $<0.2$ & $<0.2$ & $<0.2$ & $<0.2$ & $<0.2$ & $<0.2$ & $<0.2$ \\
\hline Uranium (rluor) & $<0.001$ & $<0.001$ & $<0.001$ & $<0.001$ & $<0.001$ & $<0.001$ & 0.001 & 0.001 \\
\hline Vanadium & $<0.00 \mathrm{~s}$ & $<0.005$ & $<0.005$ & $<0.005$ & $<0.005$ & $<0.005$ & 0.0056 & $<0.005$ \\
\hline $\operatorname{zinc}$ & 0.0082 & 0.0051 & 0.0053 & 0.0044 & 0.0095 & 0.0089 & 0.012 & 0.0048 \\
\hline MUNOR IONS (mg/L) & . & . & $\cdot$ & $\cdot$ &. & $\cdot$ & $\cdot$ & $\cdot$ \\
\hline Alkalinity-HCO3 & $264^{\circ}$ & $\cdot$ & 372 & $\cdot$ & 393 & $\cdot$ & 422 & . \\
\hline Akalinity-CO3 & $<1$ & $\cdot$ & $<1$ & $\cdot$ & $<1$ & $\cdot$ & $<1$ & . \\
\hline Calcium & 93 & 96 & 120 & 120 & 140 & 140 & 210 & 140 \\
\hline Chloride & 3.3 & $\cdot$ & 6.1 & $\cdot$ & 5.2 & $\cdot$ & 6 & • \\
\hline Fluoride & 0.2 & $\cdot$ & 0.4 & . & 0.5 & $\cdot$ & 0.5 & • \\
\hline Magnesium & 8.9 & 9.6 & 13 & 13 & 15 & 16 & 18 & 18 \\
\hline Manganese & 0.44 & 0.44 & 0.6 & 0.6 & 0.63 & 0.62 & 0.55 & 0.56 \\
\hline Nitrate-N & $<0.2$ & $\cdot$ & $<0.2$ & $\cdot$ & $<0.2$ & $\cdot$ & $<0.2$ & • \\
\hline Potasalum & 5.8 & 5.9 & 8.8 & 8.5 & 11 & 11 & 7.1 & 7.1 \\
\hline Sodium & 2.3 & 2.4 & 3.5 & 3.6 & 4.4 & 4.4 & 4.5 & 4.2 \\
\hline sulfate & 3.7 & . & $<1$ & $\cdot$ & 5.7 & $\cdot$ & 4 & • \\
\hline
\end{tabular}

(CONTINUED) 
APPENDIX E.1

Groundwater Quality Data, 1993

\begin{tabular}{|c|c|c|c|c|c|c|c|c|}
\hline \multirow{4}{*}{$\begin{array}{l}\text { Sempling Point } \\
\text { Loetion } \\
\text { Date sempled }\end{array}$} & \multicolumn{8}{|c|}{ ON-733 } \\
\hline & \multicolumn{8}{|c|}{$\mathbf{E x p}$} \\
\hline & \multicolumn{2}{|c|}{$02 / 02 / 93$} & \multicolumn{2}{|c|}{$05 / 06 / 93$} & \multicolumn{2}{|c|}{$08 / 18 / 93$} & \multicolumn{2}{|c|}{$11 / 04 / 93$} \\
\hline & TOT & DI8 & TOT & DI8 & TOT & DIs & rot & Dr8 \\
\hline Mrazs $(a g / L)$ & $\cdot$ & $\cdot$ & - & $\cdot$ & - & $\cdot$ & $\cdot$ & - \\
\hline Muminum & $<0.02$ & $<0.02$ & 0.082 & $<0.02$ & 0.02 & $<0.02$ & 0.044 & 0.41 \\
\hline Antimony & $<0.05$ & $<0.05$ & $<0.05$ & $<0.05$ & $<0.05$ & $<0.05$ & $<0.05$ & $<0.05$ \\
\hline Araente & $<0.05$ & $<0.03$ & $<0.05$ & $<0.05$ & $<0.05$ & $<0.05$ & $<0.05$ & $<0.05$ \\
\hline Bardum & 0.022 & 0.021 & 0.022 & 0.022 & 0.023 & 0.021 & 0.026 & 0.031 \\
\hline Bery111um & $<0.0003$ & $<0.0003$ & $<0.0003$ & $<0.0003$ & $<0.0003$ & $<0.0003$ & $<0.0003$ & $<0.0003$ \\
\hline Boron & 0.025 & 0.02 & 0.024 & 0.03 & 0.021 & 0.022 & 0.017 & 0.016 \\
\hline Cactalum (MAs) & $<0.002$ & $<0.002$ & $<0.002$ & $<0.002$ & $<0.002$ & $<0.002$ & $<0.002$ & $<0.002$ \\
\hline Cadaium & 0.0034 & 0.0034 & $<0.003$ & $<0.003$ & $<0.003$ & $<0.003$ & $<0.003$ & $<0.003$ \\
\hline Chromium (MS) & $<0.01$ & $<0.01$ & $<0.01$ & $<0.01$ & $<0.01$ & $<0.01$ & $<0.01$ & $<0.01$ \\
\hline Chromiun & $<0.01$ & $<0.01$ & $<0.01$ & $<0.01$ & $<0.01$ & $<0.01$ & $<0.01$ & $<0.01$ \\
\hline Cobalt & $<0.003$ & $<0.005$ & $<0.005$ & $<0.005$ & $<0.005$ & $<0.005$ & $<0.005$ & $<0.005$ \\
\hline Copper & $<0.004$ & $<0.004$ & $<0.004$ & $<0.004$ & 0.0088 & $<0.004$ & 0.0051 & $<0.004$ \\
\hline Iron & 4.7 & 0.26 & 2 & 0.02 & 16 & 0.034 & 1.6 & 0.3 \\
\hline Load (MS) & $<0.004$ & $<0.004$ & $<0.004$ & $<0.004$ & $<0.004$ & $<0.004$ & $<0.004$ & $<0.004$ \\
\hline Marcury (CVMA) & $<0.0002$ & $<0.0002$ & $<0.0002$ & $<0.0002$ & $<0.0002$ & $<0.0002$ & $<0.0002$ & $<0.0002$ \\
\hline Molybdenum & $<0.01$ & $<0.01$ & $<0.01$ & $<0.01$ & $<0.01$ & $<0.01$ & $<0.01$ & $<0.01$ \\
\hline nickel & $<0.01$ & $<0.01$ & $<0.01$ & 0.012 & 0.012 & $<0.01$ & $<0.01$ & $<0.01$ \\
\hline Selenium & $<0.05$ & $<0.05$ & $<0.05$ & $<0.05$ & $<0.05$ & $<0.05$ & $<0.05$ & $<0.05$ \\
\hline 811ver & $<0.006$ & $<0.006$ & $<0.006$ & $<0.006$ & $<0.006$ & $<0.006$ & $<0.006$ & $<0.006$ \\
\hline strontium & 0.11 & 0.11 & 0.098 & 0.11 & 0.13 & 0.13 & 0.12 & 0.033 \\
\hline Thorium & $<0.2$ & $<0.2$ & $<0.2$ & $<0.2$ & $<0.2$ & $<0.2$ & $<0.2$ & $<0.2$ \\
\hline Uranium (rivor) & 0.001 & 0.002 & $<0.001$ & $<0.001$ & 0.001 & 0.001 & $<0.001$ & 0.001 \\
\hline Vanadium & $<0.005$ & $<0.005$ & $<0.005$ & $<0.005$ & $<0.005$ & $<0.005$ & $<0.005$ & $<0.005$ \\
\hline zinc & $<0.002$ & $<0.002$ & 0.0059 & 0.0094 & 0.027 & 0.014 & 0.012 & 0.043 \\
\hline MUJOR IONS (mg/L) & $\cdot$ & $\cdot$ & $\cdot$ & $\cdot$ & - & - & - & 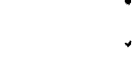 \\
\hline Alkalinity-HCO3 & 149 & $\cdot$ & ${ }_{151}$ & $\cdot$ & 158 &. & 158 & • \\
\hline Alkalinity-CO3 & $<1$ &. & $<1$ &. & $<1$ &. & $<1$ & . \\
\hline Calcium & 39 & 39 & 10 & 38 & 49 & 50 & 40 & 41 \\
\hline Chlordde & 17 & $\cdot$ & 13 & $\cdot$ & 39 & $\cdot$ & 13 & • \\
\hline Fluoride & 0.3 & $\cdot$ & 0.2 & $\cdot$ & 0.2 & $\cdot$ & 0.3 & • \\
\hline Magnesium & 15 & 15 & 16 & 15 & 18 & 18 & 16 & 16 \\
\hline Manganese & 0.053 & 0.036 & 0.021 & 0.0094 & 0.15 & 0.066 & 0.016 & 0.0036 \\
\hline Nitrate-N & $<0.2$ & $\cdot$ & $<0.2$ & $\cdot$ & $<0.2$ & $\cdot$ & 0.2 & • \\
\hline Potaseivm & 1.5 & 1.5 & 1.8 & 1.6 & 1.7 & 1.8 & 2 & 1.8 \\
\hline Sodium & 4.5 & 4.5 & 4 & 4.2 & 5.3 & 5.2 & 5.8 & 1.8 \\
\hline sultate & 12 & $\cdot$ & 12 &. & 11 & . & 10 & • \\
\hline
\end{tabular}

(CONT INUED) 
APPENDIX 8.1

Groundwater Quailty Data, 1993

\begin{tabular}{|c|c|c|c|c|c|c|c|c|}
\hline \multirow{4}{*}{$\begin{array}{l}\text { Sampling Point } \\
\text { Location } \\
\text { Date sampled }\end{array}$} & \multicolumn{8}{|c|}{$G W-735$} \\
\hline & \multicolumn{8}{|c|}{ NHP } \\
\hline & \multicolumn{2}{|c|}{$02 / 02 / 93$} & \multicolumn{2}{|c|}{$05 / 07 / 93$} & \multicolumn{2}{|c|}{$08 / 18 / 93$} & \multicolumn{2}{|c|}{$10 / 29 / 93$} \\
\hline & TOT & DIS & TOT & Drs & TOT & DIS & TOT & Drs \\
\hline HTALs (mg/t) & $\cdot$ & $\cdot$ & $\cdot$ & $\cdot 1$ & $\cdot$ & $\cdot$ & $\cdot$ & - \\
\hline Aluminum & 0.69 & $<0.02$ & 0.38 & 0.039 & 0.06 & $<0.02$ & 0.63 & $<0.02$ \\
\hline Antimony & $<0,05$ & $<0.05$ & $<0.05$ & $<0.05$ & $<0.05$ & $<0.05$ & $<0.05$ & $<0.05$ \\
\hline Arwenic & $<0.05$ & $<0.05$ & $<0.05$ & $<0.05$ & $<0.05$ & $<0.05$ & $<0.05$ & $<0.05$ \\
\hline Barlum & 0.29 & 0.27 & 0.28 & 0.27 & 0.33 & 0.33 & 0.3 & 0.3 \\
\hline Beryl11um & 0.0027 & $<0.0003$ & $<0.0003$ & $<0.0003$ & $<0.0003$ & $<0.0003$ & $<0.0003$ & $<0.0003$ \\
\hline Boron & 0.042 & 0.025 & 0.051 & 0.051 & 0.027 & 0.029 & 0.027 & 0.033 \\
\hline Cadmium (MS) & $<0.002$ & $<0.002$ & $<0.002$ & $<0.002$ & $<0.002$ & $<0.002$ & $<0.002$ & $<0.002$ \\
\hline Cadmi um & $<0.003$ & $<0.003$ & $<0.003$ & $<0.003$ & $<0.003$ & $<0.003$ & $<0.003$ & $<0 . \cup \times 3$ \\
\hline Chromium (AAS) & $<0.01$ & $<0.01$ & $<0.01$ & $<0.01$ & $<0.01$ & $<0.01$ & $<0.01$ & $<0.01$ \\
\hline Chromiun & $<0.01$ & $<0.01$ & $<0.01$ & $<0.01$ & $<0.01$ & $<0.01$ & $<0.01$ & $<0.01$ \\
\hline Cobalt & 0.0054 & $<0.005$ & $<0.005$ & $<0.005$ & $<0.005$ & $<0.005$ & $<0.005$ & $<0.005$ \\
\hline Copper & 0.0098 & 0.008 & $<0.004$ & $<0.004$ & 0.0042 & $<0.004$ & 0.0052 & $<0.004$ \\
\hline Iron & 0.87 & 0.03 & 0.61 & 0.038 & 0.17 & 0.046 & 0.75 & 0.027 \\
\hline Lead (AAS) & $<0.004$ & $<0.004$ & $<0.004$ & $<0.004$ & $<0.004$ & $<0.004$ & $<0.004$ & $<0.004$ \\
\hline Marcury (CVAA) & $<0.0002$ & $<0.0002$ & $<0.0002$ & $<0.0002$ & $<0.0002$ & $<0.0002$ & $<0.0002$ & $<0.0002$ \\
\hline Nolybdenum & $<0.01$ & $<0.01$ & $<0.01$ & $<0.01$ & $<0.01$ & $<0.01$ & $<0.01$ & $<0.01$ \\
\hline Nlckel & $<0.01$ & $<0.01$ & $<0.01$ & $<0.01$ & $<0.01$ & $<0.01$ & $<0.01$ & $<0.01$ \\
\hline Selenium & $<0.05$ & $<0.05$ & $<0.05$ & $<0.05$ & $<0.05$ & $<0.05$ & $<0.05$ & $<0.05$ \\
\hline silvar & $<0.006$ & $<0.006$ & $<0.006$ & $<0.006$ & $<0.006$ & $<0.006$ & $<0.006$ & $<0.006$ \\
\hline Strontium & 0.26 & 0.24 & 0.27 & 0.26 & 0.28 & 0.28 & 0.25 & 0.25 \\
\hline Thoriun & $<0.2$ & $<0.2$ & $<0.2$ & $<0.2$ & $<0.2$ & $<0.2$ & $<0.2$ & $<0.2$ \\
\hline Uranium (Fluor) & $<0.001$ & $<0.001$ & $<0.001$ & $<0.001$ & $<0.001$ & $<0.001$ & $<0.001$ & $<0.001$ \\
\hline Vanadium & $<0.005$ & $<0.005$ & $<0.005$ & $<0.005$ & $<0.005$ & $<0.005$ & $<0.005$ & $<0.005$ \\
\hline $2 \operatorname{lnc}$ & 0.024 & 0.0065 & 0.013 & 0.0068 & 0.017 & 0.018 & 0.0059 & 0.01 \\
\hline MAJOR IONS (mg/L) & $\cdot$ & $\cdot$ & $\cdot$ & $\cdot$ & $\cdot$ & $\cdot$ & $\cdot$ & . \\
\hline Alkalinity-HCO3 & 290 & $\cdot$ & 281 & $\cdot$ & 286 & $\cdot$ & 290 & • \\
\hline Alkalinity-CO3 & $<1$ & $\cdot$ & $<1$ & $\cdot$ & $<1$ & . & $<1$ & - \\
\hline Calcium & 110 & 110 & 110 & 110 & 120 & 120 & 110 & 110 \\
\hline Chloride & 12 &. & 15 &. & 9.4 &. & 11 & 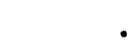 \\
\hline Fluoride & $<0.1$ & . & $<0.1$ & . & $<0.1$ & $\cdot$ & $<0.1$ & - \\
\hline Magnesium & 8.3 & 8.2 & 8.1 & 7.9 & 9.2 & 9.1 & 8.6 & 8.5 \\
\hline Manganese & 0.24 & 0.22 & 0.25 & 0.23 & 0.32 & 0.31 & 0.26 & 0.25 \\
\hline Nitrate-N & $<0.2$ &. & $<0.2$ &. & $<0.2$ &. & $<0.2$ & . \\
\hline Potassium & 2.5 & 2.6 & 2.2 & 2.2 & 2.6 & 2.5 & 2.5 & 2.8 \\
\hline Sodium & 3.5 & 3.6 & 3.4 & 3.3 & 3.6 & 3.6 & 3.8 & 3.8 \\
\hline Sulfate & 21 & $\cdot$ & 19 & $\cdot$ & 18 & $\cdot$ & 21 & • \\
\hline
\end{tabular}

(CONTINUED) 
APPENDIX $\mathbf{E} .1$

Groundwater Quality Data, 1993

\begin{tabular}{|c|c|c|c|c|c|c|c|c|}
\hline \multirow{4}{*}{$\begin{array}{l}\text { sampling point } \\
\text { location } \\
\text { Date sampled }\end{array}$} & \multicolumn{8}{|c|}{$G w-744$} \\
\hline & \multicolumn{8}{|c|}{ GRIDKI } \\
\hline & \multicolumn{2}{|c|}{$01 / 18 / 93$} & \multicolumn{2}{|c|}{$04 / 12 / 93$} & \multicolumn{2}{|c|}{$08 / 04 / 93$} & \multicolumn{2}{|c|}{$10 / 22 / 93$} \\
\hline & TOT & DIS & TOT & DIS & TOT & DIS & TOT & DIS \\
\hline MeTALs $(m g / \tau)$ & $\cdot$ & $\cdot$ & $\cdot$ & $\cdot$ & • & $\cdot$ & $\cdot$ & - \\
\hline Aluminum & $<0.02$ & $<0.02$ & 0.021 & 0.021 & $<0.02$ & $<0.02$ & 0.027 & $<0.02$ \\
\hline Antimony & $<0.05$ & $<0.05$ & $<0.05$ & $<0.05$ & $<0.05$ & $<0.05$ & $<0.05$ & $<0.05$ \\
\hline Areanic & $<0.05$ & $<0.05$ & $<0.05$ & $<0.05$ & $<0.05$ & $<0.05$ & $<0.05$ & $<0.05$ \\
\hline Barium & 0.16 & 0.16 & 0.17 & 0.17 & 0.18 & 0.18 & 0.2 & 0.2 \\
\hline Bery111um & $<0.0003$ & $<0.0003$ & $<0.0003$ & $<0.0003$ & $<0.0003$ & $<0.0003$ & $<0.0003$ & $<0.0 \quad 3$ \\
\hline Boron & 0.065 & 0.053 & 0.094 & 0.072 & 0.062 & 0.067 & 0.058 & 0.059 \\
\hline Cadmium (MAS) & $<0.002$ & $<0.002$ & $<0.002$ & $<0.002$ & $<0.002$ & $<0.002$ & $<0.002$ & $<0.002$ \\
\hline Cadmium & $<0.003$ & $<0.003$ & $<0.003$ & $<0.003$ & $<0.003$ & $<0.003$ & $<0.003$ & $<0.003$ \\
\hline Chromium (AMS) & $<0.01$ & $<0.01$ & $<0.01$ & $<0.01$ & $<0.01$ & $<0.01$ & $<0.01$ & $<0.01$ \\
\hline Chromiun & $<0.01$ & $<0.01$ & $<0.01$ & $<0.01$ & $<0.01$ & $<0.01$ & $<0.01$ & $<0.01$ \\
\hline Cobalt & $<0.005$ & $<0.005$ & $<0.005$ & $<0.005$ & $<0.005$ & $<0.005$ & $<0.005$ & $<0.005$ \\
\hline Copper & $<0.004$ & $<0.004$ & $<0.004$ & $<0.004$ & 0.0092 & $<0.004$ & 0.0061 & $<0.004$ \\
\hline Iron & 0.23 & 0.22 & 0.24 & 0.23 & 0.27 & 0.24 & 0.28 & 0.27 \\
\hline Iaad (MAS) & $<0.004$ & $<0.004$ & $<0.004$ & $<0.004$ & $<0.004$ & $<0.004$ & $<0.004$ & $<0.004$ \\
\hline Mercury (CVMA) & $<0.0002$ & $<0.0002$ & $<0.0002$ & $<0.0002$ & $<0.0002$ & $<0.0002$ & $<0.0002$ & $<0.0002$ \\
\hline Nol ybdenum & $<0.01$ & $<0.01$ & $<0.01$ & $<0.01$ & $<0.01$ & $<0.01$ & $<0.01$ & $<0.01$ \\
\hline Nickel & $<0.01$ & $<0.01$ & $<0.01$ & $<0.01$ & $<0.01$ & $<0.01$ & $<0.01$ & $<0.01$ \\
\hline selenium & $<0.05$ & $<0.05$ & $<0.05$ & $<0.05$ & 0.072 & $<0.05$ & $<0.05$ & $<0.05$ \\
\hline silver & $<0.006$ & $<0.006$ & $<0.006$ & $<0,006$ & $<0.006$ & $<0.006$ & $<0.006$ & $<0.006$ \\
\hline "ront1um & 0.84 & 0.85 & 0.87 & 0.88 & 0.94 & 0.94 & 1.1 & 1.1 \\
\hline Thorium & $<0.2$ & $<0.2$ & $<0.2$ & $<0.2$ & $<0.2$ & $<0.2$ & $<0.2$ & $<0.2$ \\
\hline Uranium (Fluor) & 0.001 & $<0.001$ & $<0.001$ & $<0.001$ & $<0.001$ & 0.001 & $<0.001$ & $<0.001$ \\
\hline Vanadium & $<0.005$ & $<0.005$ & $<0.005$ & $<0.005$ & $<0.005$ & $<0.005$ & $<0.005$ & $<0.005$ \\
\hline Zine & 0.008 & 0.01 & 0.004 & 0.0025 & 0.022 & 0.0073 & 0.0057 & 0.0065 \\
\hline MANOR IONS (mg/L) &. & . & . & . & . & . &. & • \\
\hline Alkalinity-HCO3 & 199 & $\cdot$ & 192 & $\cdot$ & 195 & $\cdot$ & $199^{\circ}$ & • \\
\hline Alkalinity-CO3 & $<1$ & . & $<1$ & . & $<1$ &. & $<1$ & . \\
\hline calcium & 50 & 51 & 48 & 48 & 50 & 50 & 52 & 54 \\
\hline Chloride & 3.3 & $\cdot$ & 3.2 & $\cdot$ & 3.7 & $\cdot$ & 4 & • \\
\hline Fluoride & 0.1 & . & 0.1 & $\cdot$ & 0.1 & . & 0.1 & . \\
\hline Magnesium & 8 & 8.1 & 7.9 & 7.9 & 8.3 & 8.4 & 8.9 & 9.1 \\
\hline Manganese & 0.15 & 0.15 & 0.15 & 0.15 & 0.16 & 0.16 & 0.18 & 0.18 \\
\hline Nitrate-N & $<0.2$ & $\cdot$ & $<0.2$ & $\cdot$ & $<0.2$ & . & $<0.2$ & • \\
\hline Potassium & 2.9 & 2.5 & 3 & 3 & 3.7 & 3.3 & 3.1 & 3 \\
\hline Sodiur & 28 & 29 & 29 & 29 & 29 & 29 & 30 & 30 \\
\hline Sulfate & 22.8 & $\cdot$ & 21.8 & . & 25 & . & 20 & • \\
\hline
\end{tabular}

(CONTINURD) 
APPENDIX E. 1

Groundwater Quality Data, 1993

\begin{tabular}{|c|c|c|c|c|c|c|c|c|}
\hline \multirow{4}{*}{$\begin{array}{l}\text { Sampling Point } \\
\text { Location } \\
\text { Date Sampled }\end{array}$} & \multicolumn{8}{|c|}{$G W-745$} \\
\hline & \multicolumn{8}{|c|}{ GRIDKI } \\
\hline & \multicolumn{2}{|c|}{$01 / 19 / 93$} & \multicolumn{2}{|c|}{$04 / 12 / 93$} & \multicolumn{2}{|c|}{$08 / 04 / 93$} & \multicolumn{2}{|c|}{$10 / 23 / 93$} \\
\hline & TOT & DIS & TOT & DIS & TOT & DIS & TOT & DIS \\
\hline METALS (mg/L) & $\cdot$ & $\cdot$ & $\cdot$ & • & $\cdot$ & - & - & \\
\hline Aluminum & 0.026 & $<0.02$ & 0.22 & 0.03 & 0.04 & $<0.02$ & 0.029 & 0.021 \\
\hline Antimony & $<0.05$ & $<0.05$ & $<0.05$ & $<0.05$ & $<0.05$ & $<0.05$ & $<0.05$ & $<0.05$ \\
\hline Arsenic & $<0.05$ & $<0.05$ & $<0.05$ & $<0.05$ & $<0.05$ & $<0.05$ & $<0.05$ & $<0.05$ \\
\hline Barium & 0.12 & 0.11 & 0.15 & 0.13 & 0.15 & 0.16 & 0.15 & 0.15 \\
\hline Beryllium & $<0.0003$ & $<0.0003$ & $<0.0003$ & $<0.0003$ & $<0.0003$ & $<0.0003$ & $<0.0003$ & $<0.0003$ \\
\hline Boron & 0.057 & 0.06 & 0.046 & 0.042 & 0.048 & 0.066 & 0.071 & 0.056 \\
\hline Cadmium (AAS) & $<0.002$ & $<0.002$ & $<0.002$ & $<0.002$ & $<0.002$ & $<0.002$ & $<0.002$ & $<0.002$ \\
\hline Cadmium & $<0.003$ & $<0.003$ & $<0.003$ & $<0.003$ & $<0.003$ & $<0.003$ & 0.018 & $<0.003$ \\
\hline Chromium (AAS) & $<0.01$ & $<0.01$ & $<0.01$ & $<0.01$ & $<0.01$ & $<0.01$ & $<0.01$ & $<0.01$ \\
\hline Chromium & $<0.01$ & $<0.01$ & $<0.01$ & $<0.01$ & $<0.01$ & $<0.01$ & $<0.01$ & $<0.01$ \\
\hline Cobalt & $<0.005$ & $<0.005$ & $<0.005$ & $<0.005$ & $<0.005$ & $<0.005$ & 0.019 & $<0.005$ \\
\hline Copper & $<0.004$ & $<0.004$ & 0.018 & $<0.004$ & 0.008 & $<0.004$ & $<0.004$ & $<0.004$ \\
\hline Iron & 0.11 & $<0.005$ & 0.34 & 0.069 & 0.22 & 0.019 & 0.21 & 0.2 \\
\hline Lead (AAS) & $<0.004$ & $<0.004$ & $<0.004$ & $<0.004$ & $<0.004$ & $<0.004$ & $<0.004$ & $<0.004$ \\
\hline Mercury (CVAA) & $<0.0002$ & $<0.0002$ & $<0.0002$ & $<0.0002$ & $<0.0002$ & $<0.0002$ & $<0.0002$ & $<0.0002$ \\
\hline Mol ybdenum & $<0.01$ & $<0.01$ & $<0.01$ & $<0.01$ & $<0.01$ & $<0.01$ & 0.019 & $<0.01$ \\
\hline Nickel & $<0.01$ & $<0.01$ & $<0.01$ & $<0.01$ & 0.013 & $<0.01$ & $<0.01$ & $<0.01$ \\
\hline Selenium & $<0.05$ & $<0.05$ & $<0.05$ & $<0.05$ & 0.07 & $<0.05$ & $<0.05$ & $<0.05$ \\
\hline Silver & $<0.006$ & $<0.006$ & $<0.006$ & $<0.006$ & $<0.006$ & $<0.006$ & $<0.006$ & $<0.006$ \\
\hline Strontium & 0.55 & 0.54 & 0.57 & 0.55 & 0.64 & 0.65 & 0.75 & 0.74 \\
\hline Thorium & $<0.2$ & $<0.2$ & $<0.2$ & $<0.2$ & $<0.2$ & $<0.2$ & $<0.2$ & $<0.2$ \\
\hline Uranium (Fluor) & $<0.001$ & $<0.001$ & 0.001 & $<0.001$ & $<0.001$ & 0.001 & $<0.001$ & $<0.001$ \\
\hline Vanadium & $<0.005$ & $<0.005$ & $<0.005$ & $<0.005$ & $<0.005$ & $<0.005$ & $<0.005$ & $<0.005$ \\
\hline zinc & 0.052 & 0.05 & 0.056 & 0.043 & 0.024 & 0.014 & 0.046 & 0.026 \\
\hline MAJOR IONS $(\mathrm{mg} / \mathrm{I})$ & $\cdot$ & $\cdot$ & $\cdot$ & $\cdot$ & $\cdot$ & . & $\cdot$ & • \\
\hline Alkalinity- $\mathrm{HCO} 3$ & 178 & $\cdot$ & 172 & - & $\begin{array}{r}\cdot \\
186\end{array}$ & $\cdot$ & $\begin{array}{r}\cdot \\
183\end{array}$ & - \\
\hline Alkalinity-CO3 & $<1$ & . & $<1$ &. & $<1$ & . & $<1$ & - \\
\hline Calcium & 51 & 49 & 56 & 53 & 58 & 59 & 60 & 59 \\
\hline Chloride & 9.9 & $\cdot$ & 9.7 & $\cdot$ & 9.7 & $\cdot$ & 16 & • \\
\hline Fluoride & $<0.1$ & $\cdot$ & 0.2 & $\cdot$ & 0.1 & $\cdot$ & $<0.1$ & • \\
\hline Magnesium & 8.6 & 8.4 & 9.1 & 8.8 & 9.7 & 9.8 & 10 & 10 \\
\hline Manganese & 0.57 & 0.54 & 0.17 & 0.52 & 0.38 & 0.25 & 0.78 & 0.78 \\
\hline Nitrate-N & $<0.2$ & $\cdot$ & $<0.2$ & $\cdot$ & $<0.2$ & . & $<0.2$ & - \\
\hline Potassium & 2.7 & 2.5 & 3.6 & 3.3 & 3 & 3.6 & 3 & 3 \\
\hline Sodium & 18 & 18 & 20 & 19 & 21 & 21 & 21 & 21 \\
\hline Sulfate & 30 & $\cdot$ & 29.7 & . & 29 & . & 26 & • \\
\hline
\end{tabular}


APPENDIX E.1

Groundwater Quality Data, 1993

\begin{tabular}{|c|c|c|c|c|c|c|c|c|}
\hline \multirow{4}{*}{$\begin{array}{l}\text { Sampling point } \\
\text { - } \\
\text { - } \\
\text { Date Sampled }\end{array}$} & \multicolumn{8}{|c|}{$G W-746$} \\
\hline & \multicolumn{8}{|c|}{ GRIDK1 } \\
\hline & \multicolumn{2}{|c|}{$01 / 19 / 93$} & \multicolumn{2}{|c|}{$04 / 12 / 93$} & \multicolumn{2}{|c|}{$08 / 05 / 93$} & \multicolumn{2}{|c|}{$10 / 23 / 93$} \\
\hline & TOT & DIS & TOI & DIS & TOT & DIS & TOT & DIS \\
\hline MBTALS (mg/L) & - & - & - & - & $\cdot$ & - & - & - \\
\hline Aluminum & 0.39 & $<0.02$ & 0.27 & 0.039 & 20 & 1.3 & 2.8 & $<0.02$ \\
\hline Antimony & $<0.05$ & $<0.05$ & $<0.05$ & $<0.05$ & $<0.05$ & $<0.05$ & $<0.05$ & $<0.05$ \\
\hline Arsenic & $<0.05$ & $<0.05$ & $<0.05$ & $<0.05$ & $<0.05$ & $<0.05$ & $<0.05$ & $<0.05$ \\
\hline Barium & 0.091 & 0.091 & 0.079 & 0.079 & 0.22 & 0.1 & 0.15 & 0.095 \\
\hline Beryl11um & $<0.0003$ & $<0.0003$ & $<0.0003$ & $<0.0003$ & 0.001 & $<0.0003$ & 0.00046 & $<0.0003$ \\
\hline Boron & 0.011 & 0.031 & 0.0086 & 0.017 & 0.027 & $0 . C 25$ & 0.024 & 0.017 \\
\hline Cadmium (AAS) & $<0.002$ & $<0.002$ & $<0.002$ & $<0.002$ & $<0.002$ & $<0.002$ & $<0.002$ & $<0.002$ \\
\hline Cadmium & $<0.003$ & $<0.003$ & $<0.003$ & $<0.003$ & 0.0076 & $<0.003$ & $<0.003$ & $<0.003$ \\
\hline Chromium (AAS) & $<0.01$ & $<0.01$ & 0.013 & $<0.01$ & 0.2 & $<0.01$ & 0.04 & $<0.01$ \\
\hline Chromium & $<0.01$ & $<0.01$ & $<0.01$ & $<0.01$ & 0.18 & $<0.01$ & 0.036 & $<0.01$ \\
\hline Cobalt & $<0.005$ & $<0.005$ & $<0.005$ & $<0.005$ & 0.014 & $<0.005$ & $<0.005$ & $<0.005$ \\
\hline Copper & 0.0052 & 0.0044 & $<0.004$ & $<0.004$ & 0.048 & 0.014 & 0.012 & 0.0084 \\
\hline Iron & 0.49 & 0.015 & 0.21 & $<0.005$ & 18 & 0.86 & 6.2 & 0.011 \\
\hline Lead (AAS) & $<0.004$ & $<0.004$ & $<0.004$ & $<0.004$ & 0.01 & $<0.004$ & $<0.004$ & $<0.004$ \\
\hline Mercury (CVAA) & $<0.0002$ & $<0.0002$ & $<0.0002$ & $<0.00 \ldots$ & $<0.0002$ & $<0.0002$ & $<0.0002$ & $<0.0002$ \\
\hline Mol ybdenum & $<0.01$ & $<0.01$ & $<0.01$ & $<0.01$ & $<0.01$ & $<0.01$ & $<0.01$ & $<0.01$ \\
\hline Nickel & $<0.01$ & $<0.01$ & $<0.01$ & $<0.01$ & 0.044 & 0.028 & 0.033 & 0.028 \\
\hline Selenium & $<0.05$ & $<0.05$ & $<0.05$ & $<0.05$ & $<0.05$ & $<0.05$ & $<0.05$ & $<0.05$ \\
\hline Silver & $<0.006$ & $<0.006$ & $<0.006$ & $<0.006$ & $<0.006$ & $<0.006$ & $<0.006$ & $<0.006$ \\
\hline Strontium & 0.052 & 0.051 & 0.046 & 0.05 & 0.1 & 0.079 & 0.091 & 0.062 \\
\hline Thorium & $<0.2$ & $<0.2$ & $<0.2$ & $<0.2$ & $<0.2$ & $<0.2$ & $<0.2$ & $<0.2$ \\
\hline Oranium (Fluor) & $<0.001$ & $<0.001$ & $<0.001$ & $<0.001$ & 0.002 & $<0.001$ & $<0.001$ & $<0.001$ \\
\hline Vanadium & $<0.005$ & $<0.005$ & $<0.005$ & $<0.005$ & 0.029 & $<0.005$ & 0.01 & $<0.005$ \\
\hline zinc & 0.078 & 0.058 & 0.055 & 0.083 & 0.044 & 0.026 & 0.099 & 0.044 \\
\hline MAJOR IONS $(\mathrm{mg} / \mathrm{I})$ & $\cdot$ & - & - & - & - & - & • & - \\
\hline Alkalinity-hCO3 & 32 & $\cdot$ & 33 & • & 38 & •. & 40 & • \\
\hline Alkalinity-CO3 & $<1$ & $\cdot$ & $<1$ & - & $<1$ & • & $<1$ & - \\
\hline Calcium & 13 & 13 & 10 & 12 & 23 & 18 & 19 & 13 \\
\hline Chloride & 6.8 & $\cdot$ & 10.3 & • & 7.3 & - & 8 & • \\
\hline Fluoride & $<0.1$ & $\cdot$ & $<0.1$ & • & $<0.1$ & • & $<0.1$ & • \\
\hline Magnesium & 4.6 & 4.3 & 3.8 & 4.1 & 9.5 & 5.5 & 6.9 & 4.7 \\
\hline Manganese & 0.04 & 0.033 & 0.033 & 0.029 & 0.16 & 0.035 & 0.1 & 0.034 \\
\hline Nitrate-N & 0.61 & $\cdot$ & 0.46 & • & 0.5 & • & 0.4 & • \\
\hline Potassium & 1.6 & $1 \cdot 3$ & 1.2 & 1.1 & 3.7 & 2 & 2.5 & 1.7 \\
\hline Sodium & 5.8 & 5.8 & 6 & 5.9 & 6.4 & 8.1 & 6.9 & 7 \\
\hline Sulfate & 25 & - & 18.4 & • & 24 & • & 26 & - \\
\hline
\end{tabular}

(CONTINUED) 
APPENDIX E. 1

Groundwater Quality Data, 1993

\begin{tabular}{|c|c|c|c|c|c|c|c|c|}
\hline \multirow{4}{*}{$\begin{array}{l}\text { Sampling Point } \\
\text { Location } \\
\text { Date Sampled }\end{array}$} & \multicolumn{8}{|c|}{$G W-747$} \\
\hline & \multicolumn{8}{|c|}{ GRIDK2 } \\
\hline & \multicolumn{2}{|c|}{$01 / 19 / 93$} & \multicolumn{2}{|c|}{$04 / 13 / 93$} & \multicolumn{2}{|c|}{$08 / 05 / 93$} & \multicolumn{2}{|c|}{$10 / 23 / 93$} \\
\hline & TOT & DIS & TOT & DIS & TOT & DIS & TOT & DIS \\
\hline MRTALS (mg/L) & - & - & $\cdot$ & - & $\cdot$ & - & . & - \\
\hline Al uminum & $<0.02$ & $<0.02$ & 0.032 & $<0.02$ & $<0.02$ & $<0.02$ & 0.021 & $<0.02$ \\
\hline Antimony & $<0.05$ & $<0.05$ & $<0.05$ & $<0.05$ & $<0.05$ & $<0.05$ & $<0.05$ & $<0.05$ \\
\hline Arsenic & $<0.05$ & $<0.05$ & $<0.05$ & $<0.05$ & $<0.05$ & $<0.05$ & $<0.05$ & $<0.05$ \\
\hline Barium & 0.13 & 0.13 & 0.13 & 0.13 & 0.14 & 0.14 & 0.16 & 0.16 \\
\hline Beryllium & $<0.0003$ & $<0.0003$ & $<0.0003$ & $<0.0003$ & $<0.0003$ & $<0.0003$ & $<0.0003$ & $<0.0003$ \\
\hline Boron & 0.064 & 0.062 & 0.065 & 0.063 & 0.064 & 0.067 & 0.073 & 0.071 \\
\hline Cadnium (AAS) & $<0.002$ & $<0.002$ & $<0.002$ & $<0.002$ & $<0.002$ & $<0.002$ & $<0.002$ & $<0.002$ \\
\hline Cadmium & $<0.003$ & $<0.003$ & $<0.003$ & $<0.003$ & $<0.003$ & $<0.003$ & $<0.003$ & $<0.003$ \\
\hline Chromium (AAS) & $<0.01$ & $<0.01$ & $<0.01$ & $<0.01$ & $<0.01$ & $<0.01$ & $<0.01$ & $<0.01$ \\
\hline Chromiun & $<0.01$ & $<0.01$ & $<0.01$ & $<0.01$ & $<0.01$ & $<0.01$ & $<0.01$ & $<0.01$ \\
\hline Cobalt & $<0.005$ & $<0.005$ & $<0.005$ & $<0.005$ & $<0.005$ & $<0.005$ & $<0.005$ & $<0.005$ \\
\hline Copper & $<0.004$ & $<0.004$ & $<0.004$ & $<0.004$ & $<0.004$ & $<0.004$ & 0.0062 & 0.0048 \\
\hline Iron & 0.11 & 0.093 & 0.11 & 0.087 & 0.42 & 0.087 & 0.11 & 0.1 \\
\hline Lead (AAS) & $<0.004$ & $<0.004$ & $<0.004$ & $<0.004$ & $<0.004$ & $<0.004$ & $<0.004$ & $<0.004$ \\
\hline Mercury (CVAA) & $<0.0002$ & $<0.0002$ & $<0.0002$ & $<0.0002$ & $<0.0002$ & $<0.0002$ & $<0.0002$ & $<0.0002$ \\
\hline Molybdenum & $<0.01$ & $<0.01$ & $<0.01$ & $<0.01$ & $<0.01$ & $<0.01$ & $<0.01$ & $<0.01$ \\
\hline Nickel & $<0.01$ & $<0.01$ & $<0.01$ & $<0.01$ & $<0.01$ & $<0.01$ & $<0.01$ & $<0.01$ \\
\hline Selenium & $<0.05$ & $<0.05$ & $<0.05$ & $<0.05$ & 0.056 & 0.067 & $<0.05$ & $<0.05$ \\
\hline Silver & $<0.006$ & $<0.006$ & $<0.006$ & $<0.006$ & $<0.006$ & $<0.006$ & $<0.006$ & $<0.006$ \\
\hline Strontium & 0.58 & 0.59 & 0.57 & 0.58 & 0.59 & 0.59 & 0.7 & 0.7 \\
\hline Thoriun & $<0.2$ & $<0.2$ & $<0.2$ & $<0.2$ & $<0.2$ & $<0.2$ & $<0.2$ & $<0.2$ \\
\hline Uranium (Fluor) & $<0.001$ & $<0.001$ & $<0.001$ & $<0.001$ & $<0.001$ & $<0.001$ & $<0.001$ & 0.002 \\
\hline Vanadium & $<0.005$ & $<0.005$ & $<0.005$ & $<0.005$ & $<0.005$ & $<0.005$ & $<0.005$ & $<0.005$ \\
\hline zinc & 0.0075 & 0.0044 & $<0.002$ & $<0.002$ & 0.0088 & 0.0055 & 0.008 & 0.0063 \\
\hline MAJOR IONS $(\mathrm{mg} / \mathrm{L})$ & - & - &. & . & . & . & . & • \\
\hline Alkalinity-HCO3 & 207 & $\cdot$ & 200 & $\cdot$ & 207 & $\cdot$ & 203 & • \\
\hline Alkalinity- $\mathrm{CO} 3$ & $<1$ & . & $<1$ & . & $<1$ & . & $<1$ & . \\
\hline Calcium & 51 & 53 & 52 & 53 & 55 & 54 & 54 & 55 \\
\hline Chloride & 6.5 & - & 5.6 & • & 4.5 & - & 4 & • \\
\hline Fluoride & $<0.1$ & . & 0.2 & . & 0.1 & . & 0.2 & - \\
\hline Magnesium & 11 & 11 & 10 & 10 & 11 & 11 & 11 & 11 \\
\hline Manganese & 0.014 & 0.014 & 0.013 & 0.013 & 0.015 & 0.015 & 0.013 & 0.012 \\
\hline Nitrate-N & $<0.2$ & . & $<0.2$ & . & $<0.2$ & . & $<0.2$ & • \\
\hline Potassium & 1.6 & 1.9 & 2 & 1.8 & 2.1 & 2.4 & 2 & 2.1 \\
\hline Sodium & 23 & 23 & 24 & 25 & 27 & 27 & 30 & 30 \\
\hline Sulfate & 28 & . & 24.1 & - & 24 & • & 22 & • \\
\hline
\end{tabular}

(CONTINUED) 
APPENDIX $\mathrm{E} \cdot 1$

Groundwater Quality Data, 1993

\begin{tabular}{|c|c|c|c|c|c|c|c|c|}
\hline \multirow{4}{*}{$\begin{array}{l}\text { Sampling Point } \\
\text { Location } \\
\text { Date Sampled }\end{array}$} & \multicolumn{8}{|c|}{ GW-748 } \\
\hline & \multicolumn{8}{|c|}{ GRIDK2 } \\
\hline & \multicolumn{2}{|c|}{$01 / 20 / 93$} & \multicolumn{2}{|c|}{$04 / 13 / 93$} & \multicolumn{2}{|c|}{$08 / 06 / 93$} & \multicolumn{2}{|c|}{$10 / 24 / 93$} \\
\hline & TOT & DIS & TOT & DIS & TOT & DIS & TOT & DIS \\
\hline METALS (mg/L) & . & . & . & . & . & . & . & - \\
\hline Aluminum & 3.5 & $<0.02$ & 6.3 & 0.025 & 0.77 & $<0.02$ & 0.92 & 0.034 \\
\hline Antimony & $<0.05$ & $<0.05$ & $<0.05$ & $<0.05$ & $<0.05$ & $<0.05$ & $<0.05$ & $<0.05$ \\
\hline Arsenic & $<0.05$ & $<0.05$ & $<0.05$ & $<0.05$ & $<0.05$ & $<0.05$ & $<0.05$ & $<0.05$ \\
\hline Barium & 0.15 & 0.13 & 0.16 & 0.13 & 0.14 & 0.12 & 0.17 & 0.17 \\
\hline Beryllium & $<0.0003$ & $<0.0003$ & $<0.0003$ & $<0.0003$ & $<0.0003$ & $<0.0003$ & $<0.0003$ & $<0.0003$ \\
\hline Boron & 0.038 & 0.059 & 0.027 & 0.036 & 0.025 & 0.037 & 0.02 & 0.021 \\
\hline Cadmium (AAS) & $<0.002$ & $<0.002$ & $<0.002$ & $<0.002$ & $<0.002$ & $<0.002$ & $<0.002$ & $<0.002$ \\
\hline Cadmium & $<0.003$ & $<0.003$ & $<0.003$ & $<0.003$ & $<0.003$ & $<0.003$ & $<0.003$ & $<0.003$ \\
\hline Chromium (AAS) & $<0.01$ & $<0.01$ & 0.014 & $<0.01$ & $<0.01$ & $<0.01$ & $<0.01$ & $<0.01$ \\
\hline Chromiun & $<0.01$ & $<0.01$ & 0.014 & $<0.01$ & $<0.01$ & $<0.01$ & $<0.01$ & $<0.01$ \\
\hline Cobalt & 0.006 & $<0.005$ & $<0.005$ & $<0.005$ & $<0.005$ & $<0.005$ & $<0.005$ & $<0.005$ \\
\hline Copper & 0.021 & 0.0079 & 0.041 & 0.018 & 0.006 & $<0.004$ & 0.0061 & $<0.004$ \\
\hline Iron & 3.4 & $<0.005$ & 5.1 & $<0.005$ & 1.2 & $<0.005$ & 1.3 & $<0.005$ \\
\hline Lead (AAS) & $<0.004$ & $<0.004$ & $<0.004$ & $<0.004$ & $<0.004$ & $<0.004$ & $<0.004$ & $<0.004$ \\
\hline Mercury (CVAA) & $<0.0002$ & $<0.0002$ & $<0.0002$ & $<0.0002$ & $<0.0002$ & $<0.0002$ & $<0.0002$ & $<0.0002$ \\
\hline Molybdenum & $<0.01$ & $<0.01$ & $<0.01$ & $<0.01$ & $<0.01$ & $<0.01$ & $<0.01$ & $<0.01$ \\
\hline Nickel & $<0.01$ & $<0.01$ & $<0.01$ & $<0.01$ & $<0.01$ & $<0.01$ & $<0.01$ & $<0.01$ \\
\hline Selenium & 0.053 & $<0.05$ & $<0.05$ & $<0.05$ & $<0.05$ & $<0.05$ & $<0.05$ & $<0.05$ \\
\hline Silver & $<0.006$ & $<0.006$ & $<0.006$ & $<0.006$ & $<0.006$ & $<0.006$ & $<0.006$ & $<0.006$ \\
\hline strontium & 0.21 & 0.2 & 0.21 & 0.2 & 0.22 & 0.22 & 0.28 & 0.28 \\
\hline Thorium & $<0.2$ & $<0.2$ & $<0.2$ & $<0.2$ & $<0.2$ & $<0.2$ & $<0.2$ & $<0.2$ \\
\hline Uranium (Fluor) & $<0.001$ & 0.001 & $<0.001$ & $<0.001$ & $<0.001$ & $<0.001$ & $<0.001$ & $<0.001$ \\
\hline Vanadium & 0.0089 & $<0.005$ & 0.013 & $<0.005$ & 0.0052 & $<0.005$ & $<0.005$ & $<0.005$ \\
\hline zinc & 0.043 & 0.029 & 0.091 & 0.051 & 0.013 & 0.013 & 0.02 & 0.014 \\
\hline MAJOR IONS (mg/L) & • & $\cdot$ & $\cdot$ & $\cdot$ & $\cdot \dot{ }$ & $\dot{\bullet}$ & • & • \\
\hline Alkalinity-HCO3 & 203 & $\cdot$ & 197 & $\cdot$ & 219 & . & $22 \dot{ }$ & • \\
\hline Alkalinity- $\mathrm{CO} 3$ & $<1$ & . & $<1$ & . & $<1$ & . & $<1$ & . \\
\hline Calcium & 77 & 74 & 79 & 77 & 83 & 85 & 93 & 91 \\
\hline Chloride & 23 & . & 9.5 & . & 15 &. & 18.5 & . \\
\hline Fluoride & $<0.1$ & . & 0.1 & . & 0.1 &. & $<0.1$ & - \\
\hline Magnesium & 9.1 & 8.2 & 9.4 & 8.2 & 9.7 & 9.6 & 10 & 10 \\
\hline Manganese & 0.089 & 0.039 & 0.11 & 0.032 & 0.11 & 0.063 & 0.092 & 0.072 \\
\hline Nitrate-N & $<0.2$ & . & $<0.2$ & . & $<0.2$ &. & $<0.2$ & • \\
\hline Potassium & 2.2 & 0.78 & 3 & 1.2 & 0.95 & 0.74 & 1.4 & 0.92 \\
\hline odium & 9.3 & 9.1 & 9.6 & 9.6 & 9.6 & 9.6 & 10 & 11 \\
\hline ulfate & 39 & • & 27.1 & • & 29 &. & 32.4 & • \\
\hline
\end{tabular}

(CONTINUED) 
APPENDIX E. 1

Groundwater Quality Data, 1993

\begin{tabular}{|c|c|c|c|c|c|c|c|c|}
\hline \multirow{4}{*}{$\begin{array}{l}\text { Sampling Point } \\
\text { Location } \\
\text { Date Sampled }\end{array}$} & \multicolumn{8}{|c|}{ GW-749 } \\
\hline & \multicolumn{8}{|c|}{ GRIDK2 } \\
\hline & \multicolumn{2}{|c|}{$01 / 21 / 93$} & \multicolumn{2}{|c|}{$04 / 14 / 93$} & \multicolumn{2}{|c|}{$08 / 06 / 93$} & \multicolumn{2}{|c|}{$10 / 24 / 93$} \\
\hline & TOT & DIS & TOT & DIS & TOT & DIS & TOT & DIS \\
\hline MRTALS (mg/L) & . & . & . & . & . & . & . & • \\
\hline Aluminum & 0.12 & $<0.02$ & 0.92 & 0.026 & 0.33 & $<0.02$ & 0.075 & $<0.02$ \\
\hline Antimony & $<0.05$ & $<0.05$ & $<0.05$ & $<0.05$ & $<0.05$ & $<0.05$ & $<0.05$ & $<0.05$ \\
\hline Arsenic & $<0.05$ & $<0.05$ & $<0.05$ & $<0.05$ & $<0.05$ & $<0.05$ & $<0.05$ & $<0.05$ \\
\hline Barium & 0.14 & 0.14 & 0.12 & 0.11 & 0.21 & 0.2 & 0.23 & 0.27 \\
\hline Beryllium & $<0.0003$ & $<0.0003$ & $<0.0003$ & $<0.0003$ & $<0.0003$ & $<0.0003$ & $<0.0003$ & $<0.0003$ \\
\hline Boron & 0.025 & 0.04 & 0.019 & 0.01 & 0.023 & 0.032 & 0.019 & 0.023 \\
\hline Cadmium (AAS) & $<0.002$ & $<0.002$ & $<0.002$ & $<0.002$ & $<0.002$ & $<0.002$ & $<0.002$ & $<0.002$ \\
\hline Cadmium & $<0.003$ & $<0.003$ & $<0.003$ & $<0.003$ & $<0.003$ & $<0.003$ & $<0.003$ & $<0.003$ \\
\hline Chromium (AAS) & $<0.01$ & $<0.01$ & $<0.01$ & $<0.01$ & $<0.01$ & $<0.01$ & $<0.01$ & $<0.01$ \\
\hline Chromium & $<0.01$ & $<0.01$ & $<0.01$ & $<0.01$ & $<0.01$ & $<0.01$ & $<0.01$ & $<0.01$ \\
\hline Cobalt & $<0.005$ & $<0.005$ & $<0.005$ & $<0.005$ & 0.0058 & $<0.005$ & $<0.005$ & $<0.005$ \\
\hline Copper & $<0.004$ & $<0.004$ & 0.0075 & 0.0079 & 0.0078 & 0.006 & $<0.004$ & $<0.004$ \\
\hline Iron & 0.12 & $<0.005$ & 1 & 0.012 & 0.67 & 0.11 & 0.59 & 0.14 \\
\hline Lead (AAS) & $<0.004$ & $<0.004$ & $<0.004$ & $<0.004$ & $<0.004$ & $<0.004$ & $<0.004$ & $<0.004$ \\
\hline Mercury (CVAA) & $<0.0002$ & $<0.0002$ & $<0.0002$ & $<0.0002$ & $<0.0002$ & $<0.0002$ & $<0.0002$ & $<0.0002$ \\
\hline Molybdenum & $<0.01$ & $<0.01$ & $<0.01$ & $<0.01$ & $<0.01$ & $<0.01$ & $<0.01$ & $<0.01$ \\
\hline Nickel & $<0.01$ & $<0.01$ & $<0.01$ & $<0.01$ & $<0.01$ & $<0.01$ & $<0.01$ & $<0.01$ \\
\hline Selenium & $<0.05$ & $<0.05$ & $<0.05$ & $<0.05$ & $<0.05$ & $<0.05$ & $<0.05$ & $<0.05$ \\
\hline silver & $<0.006$ & $<0.006$ & $<0.006$ & $<0.006$ & $<0.006$ & $<0.006$ & $<0.006$ & $<0.006$ \\
\hline Strontium & 0.2 & 0.2 & 0.18 & 0.18 & 0.25 & 0.25 & 0.27 & 0.27 \\
\hline Thorium & $<0.2$ & $<0.2$ & $<0.2$ & $<0.2$ & $<0.2$ & $<0.2$ & $<0.2$ & $<0.2$ \\
\hline Oranium (Fluor) & 0.003 & 0.003 & 0.004 & 0.003 & 0.002 & 0.001 & $<0.001$ & 0.001 \\
\hline Vanadium & $<0.005$ & $<0.005$ & $<0.005$ & $<0.005$ & $<0.005$ & $<0.005$ & $<0.005$ & $<0.005$ \\
\hline zinc & 0.085 & 0.079 & 0.025 & 0.023 & 0.031 & 0.039 & 0.022 & 0.0093 \\
\hline MAJOR IONS $(\mathrm{mg} / \mathrm{L})$ & $\cdot$ & $\cdot$ & $\cdot$ & $\cdot$ & $\cdot$ & $\cdot$ & $\cdot$ & • \\
\hline Alkalinity-HCO3 & 209 & $\cdot$ & 202 & $\cdot$ & 206 & $\cdot$ & 271 & . \\
\hline Alkalinity-CO3 & $<1$ &. & $<1$ & . & $<1$ & . & $<1$ & . \\
\hline Calcium & 85 & 88 & 79 & 80 & 110 & 110 & 110 & 110 \\
\hline Chloride & 20 & . & 14 & . & 16 &. & 38.3 & . \\
\hline Fluoride & $<0.1$ & . & 0.2 &. & 0.2 &. & 0.1 & . \\
\hline Magnesium & 8.9 & 8.9 & 7.7 & 7.6 & 9.8 & 9.7 & 9.6 & 9.3 \\
\hline Manganese & 0.3 & 0.21 & 1.2 & 0.93 & 0.77 & 0.81 & 1.4 & 0.89 \\
\hline Nitrate-N & 0.45 & . & $<0.2$ & • & 7.1 & . & $<0.2$ & • \\
\hline Potassium & 3.9 & 4 & 3.2 & 3.2 & 1.3 & 1.3 & 1.8 & 1.4 \\
\hline Sodium & 6.4 & 6.3 & 7.2 & 7.2 & 12 & 12 & 12 & 13 \\
\hline Sulfate & 54 & . & 38 &. & 81 &. & 47.9 & • \\
\hline
\end{tabular}


APPENDIX E.1

Groundwater Quality Data, 1993

\begin{tabular}{|c|c|c|c|c|c|c|c|c|}
\hline \multirow{4}{*}{$\begin{array}{l}\text { Sampling Point } \\
\text { Location } \\
\text { Date Sampled }\end{array}$} & \multicolumn{8}{|c|}{ GW-750 } \\
\hline & \multicolumn{8}{|c|}{ GRIDK2 } \\
\hline & \multicolumn{2}{|c|}{$01 / 21 / 93$} & \multicolumn{2}{|c|}{$04 / 15 / 93$} & \multicolumn{2}{|c|}{$08 / 06 / 93$} & \multicolumn{2}{|c|}{$10 / 25 / 93$} \\
\hline & TOT & DIS & TOT & DIS & TOT & DIS & TOT & Drs \\
\hline METALS (mg/L) & $\cdot$ & $\cdot$ & $\cdot$ & • & . & . & . & \\
\hline Aluminum & $<0.02$ & $<0.02$ & $<0.02$ & 0.025 & 0.073 & $<0.02$ & 0.022 & 0.022 \\
\hline Antimony & $<0.05$ & $<0.05$ & $<0.05$ & $<0.05$ & $<0.05$ & $<0.05$ & $<0.05$ & $<0.05$ \\
\hline Arsenic & $<0.05$ & $<0.05$ & $<0.05$ & $<0.05$ & $<0.05$ & $<0.05$ & $<0.05$ & $<0.05$ \\
\hline Barium & 0.72 & 0.7 & 0.69 & 0.68 & 0.8 & 0.79 & 0.81 & 0.78 \\
\hline Beryllium & $<0.0003$ & $<0.0003$ & $<0.0003$ & $<0.0003$ & $<0.0003$ & $<0.0003$ & $<0.0003$ & $<0.0003$ \\
\hline Boron & 0.18 & 0.1 & 0.08 & 0.085 & 0.066 & 0.1 & 0.1 & 0.1 \\
\hline Cadmium (AAS) & $<0.002$ & $<0.002$ & $<0.002$ & $<0.002$ & $<0.002$ & $<0.002$ & $<0.002$ & $<0.002$ \\
\hline Cadmium & $<0.003$ & $<0.003$ & $<0.003$ & $<0.003$ & $<0.003$ & $<0.003$ & $<0.003$ & $<0.003$ \\
\hline Chromium (AAS) & $<0.01$ & $<0.01$ & $<0.01$ & $<0.01$ & $<0.01$ & $<0.01$ & $<0.01$ & $<0.01$ \\
\hline Chromium & $<0.01$ & $<0.01$ & $<0.01$ & $<0.01$ & $<0.01$ & $<0.01$ & $<0.01$ & $<0.01$ \\
\hline Cobalt & $<0.005$ & $<0.005$ & $<0.005$ & $<0.005$ & $<0.005$ & $<0.005$ & $<0.005$ & $<0.005$ \\
\hline Copper & $<0.004$ & $<0.004$ & $<0.004$ & 0.005 & 0.0096 & $<0.004$ & $<0.004$ & $<0.004$ \\
\hline Iron & 0.23 & 0.22 & 0.28 & 0.26 & 0.96 & 0.21 & 0.22 & 0.23 \\
\hline Lead (AAS) & $<0.004$ & $<0.004$ & $<0.004$ & $<0.004$ & $<0.004$ & $<0.004$ & $<0.004$ & $<0.004$ \\
\hline Mercury (CVAA) & $<0.0002$ & $<0.0002$ & $<0.0002$ & $<0.0002$ & $<0.0002$ & $<0.0002$ & $<0.0002$ & $<0.0002$ \\
\hline Molybdenum & $<0.01$ & $<0.01$ & $<0.01$ & $<0.01$ & $<0.01$ & $<0.01$ & $<0.01$ & $<0.01$ \\
\hline Nickel & $<0.01$ & $<0.01$ & $<0.01$ & $<0.01$ & $<0.01$ & $<0.01$ & $<0.01$ & $<0.01$ \\
\hline Selenium & $<0.05$ & $<0.05$ & $<0.05$ & 0.053 & $<0.05$ & $<0.05$ & $<0.05$ & $<0.05$ \\
\hline silver & $<0.006$ & $<0.006$ & $<0.006$ & $<0.006$ & $<0.006$ & $<0.006$ & $<0.006$ & $<0.006$ \\
\hline Strontium & 0.7 & 0.69 & 0.61 & 0.6 & 0.79 & 0.79 & 0.84 & 0.82 \\
\hline Thorium & $<0.2$ & $<0.2$ & $<0.2$ & $<0.2$ & $<0.2$ & $<0.2$ & $<0.2$ & $<0.2$ \\
\hline Uranium (Fluor) & $<0.001$ & $<0.001$ & $<0.001$ & $<0.001$ & $<0.001$ & $<0.001$ & 0.002 & $<0.001$ \\
\hline Vanadium & $<0.005$ & $<0.005$ & $<0.005$ & $<0.005$ & $<0.005$ & $<0.005$ & $<0.005$ & $<0.005$ \\
\hline Zinc & 0.0089 & 0.0077 & 0.0042 & 0.0056 & 0.016 & 0.0082 & 0.0095 & 0.0053 \\
\hline MAJOR IONS $(\mathrm{mg} / \mathrm{L})$ & . & . & . & . & . & . & . & \\
\hline Alkalinity- $\mathrm{HCO} 3$ & 234 & . & 229 & . & 241 & . & 241 & \\
\hline Alkalinity-CO3 & $<1$ & . & $<1$ & . & $<1$ & . & $<1$ & • \\
\hline Calcium & 72 & 72 & 78 & 76 & 74 & 75 & 74 & 74 \\
\hline Chloride & 4.3 & $\cdot$ & 3.4 & $\cdot$ & 4.2 & • & 4.1 & - \\
\hline Fluoride & $<0.1$ & . & $<0.1$ & . & $<0.1$ & . & $<0.1$ & \\
\hline Magnesium & 11 & 11 & 11 & 10 & 12 & 13 & 12 & 12 \\
\hline Manganese & 0.045 & 0.044 & 0.048 & 0.047 & 0.097 & 0.045 & 0.043 & 0.043 \\
\hline Nitrate-N & $<0.2$ & . & $<0.2$ & . & $<0.2$ & • & $<0.2$ & \\
\hline Potassium & 4.6 & 4.7 & 3.7 & 4.4 & 5 & 4.7 & 5.5 & 5.7 \\
\hline Sodium & 5.4 & 5.1 & 4.6 & 4.6 & 6.3 & 6.3 & 6.2 & 6 \\
\hline Sulfate & 19 & . & 19 &. & 21 & • & 16.5 & \\
\hline
\end{tabular}

(CONTINUED) 
APPENDIX E.1

Groundwater Quality Data, 1993

\begin{tabular}{|c|c|c|c|c|c|c|c|c|}
\hline \multirow{4}{*}{$\begin{array}{l}\text { Sampling Point } \\
\text { Location } \\
\text { Date Sampled }\end{array}$} & \multicolumn{8}{|c|}{ GW-751 } \\
\hline & \multicolumn{8}{|c|}{ GRIDJ3 } \\
\hline & \multicolumn{2}{|c|}{$01 / 11 / 93$} & \multicolumn{2}{|c|}{$04 / 16 / 93$} & \multicolumn{2}{|c|}{$08 / 02 / 93$} & \multicolumn{2}{|c|}{$10 / 26 / 93$} \\
\hline & TOT & DIS & TOT & DIS & TOT & DIS & TOT & DIS \\
\hline METALS (mg/L) & $\cdot$ & $\cdot$ & $\cdot$ & . & . & - & . & - \\
\hline Aluminum & 0.041 & 0.057 & 0.027 & $<0.02$ & 0.053 & $<0.02$ & 0.2 & $<0.02$ \\
\hline Antimony & $<0.05$ & $<0.05$ & $<0.05$ & $<0.05$ & $<0.05$ & $<0.05$ & $<0.05$ & $<0.05$ \\
\hline Arsenic & $<0.05$ & $<0.05$ & $<0.05$ & $<0.05$ & $<0.05$ & $<0.05$ & $<0.05$ & $<0.05$ \\
\hline Barium & 0.53 & 0.54 & 0.5 & 0.5 & 0.52 & 0.5 & 0.57 & 0.56 \\
\hline Beryllium & 0.00076 & $<0.0003$ & $<0.0003$ & $<0.0003$ & $<0.0003$ & $<0.0003$ & $<0.0003$ & $<0.0003$ \\
\hline Boron & 0.045 & 0.033 & 0.032 & 0.035 & 0.036 & 0.03 & 0.041 & 0.042 \\
\hline Cadmium (AAS) & $<0.002$ & $<0.002$ & $<0.002$ & $<0.002$ & $<0.002$ & $<0.002$ & $<0.002$ & $<0.002$ \\
\hline Cadmium & $<0.003$ & $<0.003$ & $<0.003$ & $<0.003$ & $<0.003$ & $<0.003$ & $<0.003$ & $<0.003$ \\
\hline Chromium (AAS) & $<0.01$ & $<0.01$ & $<0.01$ & $<0.01$ & $<0.01$ & $<0.01$ & $<0.01$ & $<0.01$ \\
\hline Chromium & $<0.01$ & $<0.01$ & $<0.01$ & $<0.01$ & $<0.01$ & $<0.01$ & $<0.01$ & $<0.01$ \\
\hline Cobalt & $<0.005$ & $<0.005$ & $<0.005$ & $<0.005$ & $<0.005$ & $<0.005$ & $<0.005$ & $<0.005$ \\
\hline Copper & $<0.004$ & $<0.004$ & $<0.004$ & $<0.004$ & 0.005 & $<0.004$ & $<0.004$ & $<0.004$ \\
\hline Iron & 0.2 & 0.16 & 0.18 & 0.16 & 0.24 & 0.21 & 0.21 & 0.12 \\
\hline Lead (AAS) & $<0.004$ & $<0.004$ & $<0.004$ & $<0.004$ & $<0.004$ & $<0.004$ & $<0.004$ & $<0.004$ \\
\hline Mercury (CVAA) & $<0.0002$ & $<0.0002$ & $<0.0002$ & $<0.0002$ & $<0.0002$ & $<0.0002$ & $<0.0002$ & $<0.0002$ \\
\hline Molybdenum & $<0.01$ & $<0.01$ & $<0.01$ & $<0.01$ & $<0.01$ & $<0.01$ & $<0.01$ & $<0.01$ \\
\hline Nickel & $<0.01$ & $<0.01$ & $<0.01$ & $<0.01$ & $<0.01$ & $<0.01$ & $<0.01$ & $<0.01$ \\
\hline Selenium & $<0.05$ & $<0.05$ & $<0.05$ & $<0.05$ & $<0.05$ & $<0.05$ & $<0.05$ & $<0.05$ \\
\hline silver & $<0.006$ & $<0.006$ & $<0.006$ & $<0.006$ & $<0.006$ & $<0.006$ & $<0.006$ & $<0.006$ \\
\hline strontium & 0.46 & 0.47 & 0.42 & 0.42 & 0.44 & 0.42 & 0.46 & 0.46 \\
\hline Thorium & $<0.2$ & $<0.2$ & $<0.2$ & $<0.2$ & $<0.2$ & $<0.2$ & $<0.2$ & $<0.2$ \\
\hline Uranium (F1uor) & $<0.001$ & $<0.001$ & $<0.001$ & $<0.001$ & $<0.001$ & 0.001 & 0.001 & 0.001 \\
\hline Vanadium & $<0.005$ & $<0.005$ & $<0.005$ & $<0.005$ & $<0.005$ & $<0.005$ & $<0.005$ & $<0.005$ \\
\hline zinc & 0.0073 & 0.0071 & 0.0055 & 0.0048 & 0.029 & 0.013 & $<0.002$ & $<0.002$ \\
\hline MAJOR IONS $(\mathrm{mg} / \mathrm{L})$ & . & . & . & . & . & . &. & . \\
\hline Alkalinity-HCO3 & 181 & $\cdot$ & $177^{\circ}$ &. & $181^{\circ}$ & . & 182 & • \\
\hline Alkalinity- $\mathrm{CO} 3$ & $<1$ & . & $<1$ & . & $<1$ & . & $<1$ & . \\
\hline Calcium & 55 & 57 & 53 & 52 & 52 & 50 & 56 & 55 \\
\hline Chloride & 9.1 & - & 8.9 & • & 8 & . & 10 & • \\
\hline Fluoride & $<0.1$ & . & $<0.1$ & . & $<0.1$ & . & $<0.1$ & . \\
\hline Magnesium & 9.5 & 9.7 & 8.9 & 8.8 & 9.4 & 9.1 & 9.8 & 9.6 \\
\hline Manganese & 0.014 & 0.014 & 0.011 & 0.011 & 0.013 & 0.012 & 0.014 & 0.013 \\
\hline Nitrate-N & $<0.2$ & . & $<0.2$ & . & $<0.2$ & . & $<0.2$ & • \\
\hline Potassium & 2.9 & 3 & 3 & 3 & 3.4 & 3 & 3.2 & 3.2 \\
\hline Sodium & 8.2 & 8.4 & 7.8 & 7.8 & 8.7 & 8.4 & 9 & 9 \\
\hline Sulfate & 6.2 & . & 9.1 & . & 3.9 & . & 4 & . \\
\hline
\end{tabular}

(CONTINUED) 
APPENDIX E.1

Groundwater Quality Data, 1993

\begin{tabular}{|c|c|c|c|c|c|c|c|c|}
\hline \multirow{4}{*}{$\begin{array}{l}\text { Sampling point } \\
\text { - } \\
\text { Location } \\
\text { Date sampled }\end{array}$} & \multicolumn{8}{|c|}{$O W-752$} \\
\hline & \multicolumn{8}{|c|}{ GRIDJ 3} \\
\hline & \multicolumn{2}{|c|}{$01 / 11 / 93$} & \multicolumn{2}{|c|}{$04 / 16 / 93$} & \multicolumn{2}{|c|}{$08 / 02 / 93$} & \multicolumn{2}{|c|}{$10 / 26 / 93$} \\
\hline & TOT & DIS & TOT & DIS & TOT & DIS & TOT & DIS \\
\hline METALS (mg/L) & - & $\cdot$ & - & - & - & - & - & - \\
\hline Al uminum & 0.15 & 0.038 & 0.47 & $<0.02$ & 0.91 & 0.028 & 0.22 & $<0.02$ \\
\hline Antimony & $<0.05$ & $<0.05$ & $<0.05$ & $<0.05$ & $<0.05$ & $<0.05$ & $<0.05$ & $<0.05$ \\
\hline Araentc & $<0.05$ & $<0.05$ & $<0.05$ & $<0.05$ & $<0.05$ & $<0.05$ & $<0.05$ & $<0.05$ \\
\hline Barium & 0.12 & 0.12 & 0.12 & 0.11 & 0.14 & 0.13 & 0.13 & 0.13 \\
\hline Beryll1um & 0.00062 & 0.00061 & $<0.0003$ & $<0.0003$ & $<0.0003$ & $<0.0003$ & $<0.0003$ & $<0.0003$ \\
\hline Boron & 0.017 & 0.012 & 0.0097 & 0.019 & 0.03 & 0.015 & 0.038 & 0.037 \\
\hline Cadmium (AAS) & $<0.002$ & $<0.002$ & $<0.002$ & $<0.002$ & $<0.002$ & $<0.002$ & $<0.002$ & $<0.002$ \\
\hline Cadmium & $<0.003$ & $<0.003$ & $<0.003$ & $<0.003$ & $<0.003$ & $<0.003$ & $<0.003$ & $<0.003$ \\
\hline Chromium (AAS) & $<0.01$ & $<0.01$ & $<0.01$ & $<0.01$ & $<0.01$ & $<0.01$ & $<0.01$ & $<0.01$ \\
\hline Chromium & 0.011 & $<0.01$ & $<0.01$ & $<0.01$ & $<0.01$ & $<0.01$ & $<0.01$ & $<0.01$ \\
\hline Cobalt & 0.0066 & 0.0073 & $<0.005$ & 0.0067 & $<0.005$ & 0.0078 & 0.0051 & $<0.005$ \\
\hline Copper & $<0.004$ & $<0.004$ & $<0.004$ & $<0.004$ & 0.0061 & $<0.004$ & $<0.004$ & $<0.004$ \\
\hline Iron & 0.97 & 0.93 & 1.6 & 0.58 & 1.6 & 0.69 & 0.59 & 0.37 \\
\hline Lead (MAs) & $<0.004$ & $<0.004$ & $<0.004$ & $<0.004$ & $<0.004$ & $<0.004$ & $<0.004$ & $<0.004$ \\
\hline Mercury (CVAA) & $<0.0002$ & $<0.0002$ & $<0.0002$ & $<0.0002$ & $<0.0002$ & $<0.0002$ & $<0.0002$ & $<0.0002$ \\
\hline Molybdenum & $<0.01$ & $<0.01$ & $<0.01$ & $<0.01$ & $<0.01$ & $<0.01$ & $<0.01$ & $<0.01$ \\
\hline Rickel & $<0.01$ & $<0.01$ & 0.021 & 0.013 & 0.029 & 0.02 & 0.025 & 0.025 \\
\hline Selenium & $<0.05$ & $<0.05$ & $<0.05$ & $<0.05$ & $<0.05$ & $<0.05$ & $<0.05$ & $<0.05$ \\
\hline Silver & $<0.006$ & $<0.006$ & $<0.006$ & $<0.006$ & $<0.006$ & $<0.006$ & $<0.006$ & $<0.006$ \\
\hline Strontium & 0.079 & 0.079 & 0.077 & 0.075 & 0.081 & 0.079 & 0.077 & 0.076 \\
\hline Thorium & $<0.2$ & $<0.2$ & $<0.2$ & $<0.2$ & $<0.2$ & $<0.2$ & $<0.2$ & $<0.2$ \\
\hline Oranium (Fluor) & $<0.001$ & $<0.001$ & $<0.001$ & $<0.001$ & $<0.001$ & $<0.001$ & $<0.001$ & $<0.001$ \\
\hline Vanadium & $<0.005$ & $<0.005$ & $<0.005$ & $<0.005$ & $<0.005$ & $<0.005$ & $<0.005$ & $<0.005$ \\
\hline ainc & 0.013 & 0.012 & 0.0076 & 0.0085 & 0.018 & 0.014 & 0.0075 & 0.0076 \\
\hline MAJOR IONS (mg/L) & - & - & - & - & - & - & - & - \\
\hline Alkalinity-HCO3 & 58 & . & 54 & . & 57 & • & 45 & • \\
\hline Alkalinity-CO3 & $<1$ & $\cdot$ & $<1$ & $\cdot$ & $<1$ & $\cdot$ & $<1$ & • \\
\hline Calcium & 29 & 30 & 30 & 29 & 30 & 29 & 28 & 28 \\
\hline Chloride & 52 & • & 40 & - & 54 & • & 39 & • \\
\hline Fluoride & $<0.1$ & . & $<0.1$ & • & $<0.1$ & • & $<0.1$ & • \\
\hline Magnesium & 3.5 & 3.5 & 3.6 & 3.4 & 3.9 & 3.7 & 3.4 & 3.4 \\
\hline Manganese & 1.9 & 1.9 & 1.7 & 1.6 & 1.8 & 1.7 & 1.7 & 1.7 \\
\hline Nitrate-N & $<0.2$ & . & $<0.2$ & • & $<0.2$ & - & $<0.2$ & • \\
\hline Po:assium & 1.6 & 1.5 & 1.5 & 1.6 & 2.2 & 2.4 & $2 \cdot 3$ & 2.2 \\
\hline Sudium & 8.1 & 8 & 8.2 & 8.1 & 9.2 & 8.9 & 8.8 & 8.9 \\
\hline Sulfate & 4.7 & - & 8.3 & . & 4.9 & • & 5 & • \\
\hline
\end{tabular}

(CONTINUED) 
APPENDIX E.1

Groundwater Quality Data, 1993

\begin{tabular}{|c|c|c|c|c|c|c|c|c|}
\hline \multirow{4}{*}{$\begin{array}{l}\text { Sampling polnt } \\
\text { Location } \\
\text { Date Sampled }\end{array}$} & \multicolumn{8}{|c|}{ GW-753 } \\
\hline & \multicolumn{8}{|c|}{ GRIDJ2 } \\
\hline & \multicolumn{2}{|c|}{$01 / 15 / 93$} & \multicolumn{2}{|c|}{$04 / 08 / 93$} & \multicolumn{2}{|c|}{$08 / 03 / 93$} & \multicolumn{2}{|c|}{$10 / 21 / 93$} \\
\hline & TOT & DIS & TOT & DIS & TOT & DIS & Tot & DIs \\
\hline MRTALs (mg/L) & $\cdot$ & $\cdot$ & $\cdot$ & • & - & . & . & • \\
\hline Aluminum & $<0.02$ & $<0.02$ & 0.031 & $<0.02$ & $<0.02$ & $<0.02$ & $<0.02$ & 0.024 \\
\hline Antimony & $<0.05$ & $<0.05$ & $<0.05$ & $<0.05$ & $<0.05$ & $<0.05$ & $<0.05$ & $<0.05$ \\
\hline Arsenic & $<0.05$ & $<0.05$ & $<0.05$ & $<0.05$ & $<0.05$ & $<0.05$ & $<0.05$ & $<0.05$ \\
\hline Barium & 0.2 & 0.2 & 0.21 & 0.21 & 0.22 & 0.22 & 0.24 & 0.24 \\
\hline Beryllium & $<0.0003$ & $<0.0003$ & $<0.0003$ & $<0.0003$ & $<0.0003$ & $<0.0003$ & $<0.0003$ & $<0.0003$ \\
\hline Boron & 0.032 & 0.037 & 0.03 & 0.031 & 0.038 & 0.028 & 0.037 & 0.041 \\
\hline Cactmium (AAS) & $<0.002$ & $<0.002$ & $<0.002$ & $<0.002$ & $<0.002$ & $<0.002$ & $<0.002$ & $<0.002$ \\
\hline Cadmium & $<0.003$ & $<0.003$ & $<0.003$ & $<0.003$ & $<0.003$ & $<0.003$ & $<0.003$ & $<0.003$ \\
\hline Chromium (AAS) & $<0.01$ & $<0.01$ & $<0.01$ & $<0.01$ & $<0.01$ & $<0.01$ & $<0.01$ & $<0.01$ \\
\hline Chromium & $<0.01$ & $<0.01$ & $<0.01$ & $<0.01$ & $<0.01$ & $<0.01$ & $<0.01$ & $<0.01$ \\
\hline Cobalt & $<0.005$ & $<0.005$ & $<0.005$ & $<0.005$ & $<0.005$ & $<0.005$ & $<0.005$ & $<0.005$ \\
\hline Copper & $<0.004$ & 0.0057 & $<0.004$ & $<0.004$ & $<0.004$ & $<0.004$ & 0.013 & 0.014 \\
\hline Iron & 0.16 & 0.44 & 0.17 & 0.15 & 0.22 & 0.15 & 0.17 & 0.16 \\
\hline Lead (AAS) & $<0.004$ & $<0.004$ & $<0.004$ & $<0.004$ & $<0.004$ & $<0.004$ & $<0.004$ & $<0.004$ \\
\hline Mercury (CVAA) & $<0.0002$ & $<0.0002$ & $<0.0002$ & $<0.0002$ & $<0.0002$ & $<0.0002$ & $<0.0002$ & $<0.0002$ \\
\hline Mol ybdenum & $<0.01$ & $<0.01$ & $<0.01$ & $<0.01$ & $<0.01$ & $<0.01$ & $<0.01$ & $<0.01$ \\
\hline Nickel & $<0.01$ & $<0.01$ & $<0.01$ & $<0.01$ & $<0.01$ & $<0.01$ & $<0.01$ & $<0.01$ \\
\hline Selenium & $<0.05$ & $<0.05$ & $<0.05$ & $<0.05$ & 0.068 & $<0.05$ & $<0.05$ & $<0.05$ \\
\hline Silver & $<0.006$ & $<0.006$ & $<0.006$ & $<0.006$ & $<0.006$ & $<0.006$ & $<0.006$ & $<0.006$ \\
\hline Strontium & 0.33 & 0.33 & 0.37 & 0.37 & 0.36 & 0.36 & 0.43 & 0.43 \\
\hline Thorium & $<0.2$ & $<0.2$ & $<0.2$ & $<0.2$ & $<0.2$ & $<0.2$ & $<0.2$ & $<0.2$ \\
\hline Oranium (Fluor) & $<0.001$ & $<0.001$ & $<0.001$ & $<0.001$ & $<0.001$ & $<0.001$ & $<0.001$ & $<0.001$ \\
\hline Vanadium & $<0.005$ & $<0.005$ & $<0.005$ & $<0.005$ & $<0.005$ & $<0.005$ & $<0.005$ & $<0.005$ \\
\hline zinc & 0.006 & 0.0064 & 0.0046 & 0.011 & 0.0036 & 0.0078 & 0.0082 & 0.0079 \\
\hline MAJOR IONS $(\mathrm{mg} / \mathrm{L})$ & . & . & . & - & . & • & - & • \\
\hline Alkalinity-HCO3 & 199 & $\cdot$ & 193 & $\cdot$ & $20 \dot{0}$ & . & 196 & • \\
\hline Alkalinity- $\mathrm{CO} 3$ & $<1$ & . & $<1$ & . & $<1$ & . & $<1$ & . \\
\hline Calcium & 61 & 61 & 59 & 58 & 58 & 58 & 61 & 60 \\
\hline Chloride & 2.6 & . & 2.6 &. & 3 & . & 3 & • \\
\hline Fluoride & $<0.1$ & - & 0.1 & . & $<0.1$ & - & $<0.1$ & • \\
\hline Magnesium & 8.9 & 9 & 8.9 & 8.8 & 9 & 9 & 9.7 & 9.6 \\
\hline Manganese & 0.033 & 0.034 & 0.032 & 0.032 & 0.034 & 0.034 & 0.035 & 0.036 \\
\hline Nitrate-N & $<0.2$ & • & $<0.2$ & $\cdot$ & $<0.2$ & $\cdot$ & $<0.2$ & • \\
\hline Potassium & 2.1 & 2.1 & 1.6 & 1.6 & 1.9 & 2 & 1.8 & 2 \\
\hline Sodium & 10 & 10 & 11 & 11 & 11 & 11 & 12 & 12 \\
\hline Sulfate & 12 & • & 11 & . & 15 & . & 9 & • \\
\hline
\end{tabular}

(CONTINUED) 
APPENDIX $\mathbf{2} .1$

Groundwatar Qual1ty Data, 1993

\begin{tabular}{|c|c|c|c|c|c|c|c|c|}
\hline \multirow{4}{*}{$\begin{array}{l}\text { 8ampling Point } \\
\text { Loontion } \\
\text { - } \\
\text { Date gampled }\end{array}$} & \multicolumn{8}{|c|}{$O N-754$} \\
\hline & \multicolumn{8}{|c|}{ GRIDJ2 } \\
\hline & \multicolumn{2}{|c|}{$01 / 18 / 93$} & \multicolumn{2}{|c|}{$04 / 08 / 93$} & \multicolumn{2}{|c|}{$08 / 04 / 93$} & \multicolumn{2}{|c|}{$10 / 22 / 93$} \\
\hline & TOS & DIS & TOT & DIs & TOT & DIS & TOT & DIs \\
\hline MTNRs (mg/I) & $\cdot$ & - & - & - & - & - & - & - \\
\hline Muminum & 1 & $<0.02$ & 4.2 & 0.039 & 2 & $<0.02$ & 0.26 & 0.032 \\
\hline Antimony & $<0.05$ & $<0.05$ & $<0.05$ & $<0.05$ & $<0.05$ & $<0.05$ & $<0.05$ & $<0.05$ \\
\hline Areente & $<0.05$ & $<0.05$ & $<0.05$ & $<0.05$ & $<0.05$ & $<0.05$ & $<0.05$ & $<0.05$ \\
\hline Barium & 0.23 & 0.19 & 0.31 & 0.25 & 0.31 & 0.26 & 0.27 & 0.26 \\
\hline Baryl11um & $<0.0003$ & $<0.0003$ & $<0.0003$ & $<0.0003$ & $<0.0003$ & $<0.0003$ & 0.00034 & $<0.0003$ \\
\hline Boron & 0.027 & 0.032 & 0.041 & 0.024 & 0.017 & 0.033 & 0.031 & 0.031 \\
\hline Cadmium (MAS) & $<0.002$ & $<0.002$ & $<0.002$ & $<0.002$ & $<0.002$ & $<0.002$ & $<0.002$ & $<0.002$ \\
\hline Cadmium & $<0.003$ & $<0.003$ & $<0.003$ & $<0.003$ & $<0.003$ & $<0,003$ & $<0.003$ & $<0.003$ \\
\hline Chromium (MAs) & $<0.01$ & $<0.01$ & $<0.01$ & $<0.01$ & $<0.01$ & $<0.01$ & $<0.01$ & $<0.01$ \\
\hline Chromium & $<0.01$ & $<0.01$ & $<0.01$ & $<0.01$ & $<0.01$ & $<0.01$ & $<0.01$ & $<0.01$ \\
\hline Cobalt & $<0.005$ & $<0.005$ & $<0.005$ & $<0.005$ & $<0.005$ & $<0.005$ & $<0.005$ & $<0.005$ \\
\hline Copper & 0.0053 & $<0.004$ & $<0.004$ & $<0.004$ & $<0.004$ & $<0.004$ & 0.0066 & $<0.004$ \\
\hline Iron & 1.6 & 0.35 & 5 & 0.1 & 2.2 & 0.082 & 0.44 & 0.077 \\
\hline Lead (MAS) & $<0.004$ & $<0.004$ & $<0.004$ & $<0.004$ & $<0.004$ & $<0.004$ & $<0.004$ & $<0.004$ \\
\hline Mercury (CVMA) & $<0.0002$ & $<0.0002$ & $<0.0002$ & $<0.0002$ & $<0.0002$ & $<0.0002$ & $<0.0002$ & $<0.0002$ \\
\hline Molybdenum & $<0.01$ & $<0.01$ & $<0.01$ & $<0.01$ & $<0.01$ & $<0.01$ & $<0.01$ & $<0.01$ \\
\hline Nickel & $<0.01$ & $<0.01$ & $<0.01$ & $<0.01$ & 0.012 & $<0.01$ & $<0.01$ & $<0.01$ \\
\hline Selentum & $<0.05$ & $<0.05$ & $<0.05$ & $<0.05$ & 0.083 & $<0.05$ & $<0.05$ & $<0.05$ \\
\hline silver & $<0.006$ & $<0.006$ & $<0.006$ & $<0.006$ & $<0.006$ & $<0.006$ & $<0.006$ & $<0.006$ \\
\hline Strontium & 0.12 & 0.11 & 0.15 & 0.16 & 0.16 & 0.16 & 0.17 & 0.19 \\
\hline Thorium & $<0.2$ & $<0.2$ & $<0.2$ & $<0.2$ & $<0.2$ & $<0.2$ & $<0.2$ & $<0.2$ \\
\hline Uranium (Fluor) & $<0.001$ & 0.001 & 0.001 & $<0.001$ & 0.001 & $<0.001$ & $<0.001$ & $<0.001$ \\
\hline Vanadium & $<0.005$ & $<0.005$ & $<0.005$ & $<0.005$ & $<0.005$ & $<0.005$ & $<0.005$ & $<0.005$ \\
\hline 2inc & 0.021 & 0.015 & 0.024 & 0.011 & 0.013 & 0.0087 & 0.013 & 0.012 \\
\hline MAJOR IONS (mg/L) & - & . & $\cdot$ & • & . & . & . & • \\
\hline Alkalinity- $\mathrm{KCO} 3$ & 130 & - & 91 & . & 144 & - & 142 & - \\
\hline Alkalinlty-CO3 & $<1$ & - & $<1$ & - & $<1$ & - & $<1$ & - \\
\hline Calcium & 41 & 38 & 47 & 51 & 52 & 53 & 49 & 54 \\
\hline Chloride & 4.1 & • & 3.2 & • & 4.5 & - & 4 & - \\
\hline Fluoride & $<0.1$ & - & $<0.1$ & • & $<0.1$ & • & $<0.1$ & • \\
\hline Magnesium & 8.8 & 8.1 & 9.5 & 8.9 & 10 & 9.5 & 9.3 & 9.9 \\
\hline Manganese & 1 & 0.88 & 0.77 & 0.87 & 0.67 & 0.63 & 0.8 & 0.67 \\
\hline Nitrate-N & $<0.2$ & • & $<0.2$ & • & $<0.2$ & • & $<0.2$ & • \\
\hline Potassium & 1.9 & 1.9 & 2.1 & 1.6 & $2 \cdot 3$ & 1.6 & 1.7 & 1.6 \\
\hline Sodium & 4.8 & 4.8 & 4.6 & 4.7 & 5 & 4.6 & 5 & $5 \cdot 1$ \\
\hline Sulfate & 12.9 & • & 17 & • & 14 & • & 11 & - \\
\hline
\end{tabular}

(CONTINUED) 
APPENDIX E.1

Groundwater Quadity Data, 1993

\begin{tabular}{|c|c|c|c|c|c|c|c|c|}
\hline \multirow{4}{*}{$\begin{array}{l}\text { Sampling Point } \\
\text { Location } \\
\text { Date sampled }\end{array}$} & \multicolumn{8}{|c|}{$6 W-755$} \\
\hline & \multicolumn{8}{|c|}{ GRIDJI } \\
\hline & \multicolumn{2}{|c|}{$01 / 14 / 93$} & \multicolumn{2}{|c|}{$04 / 07 / 93$} & \multicolumn{2}{|c|}{$08 / 03 / 93$} & \multicolumn{2}{|c|}{$10 / 21 / 93$} \\
\hline & TOT & DIS & TOT & Drs & TOT & DIS & TOT & Drs \\
\hline HeTALs (mg/L) & . & • & $\cdot$ & - & - & - & • & . \\
\hline Aluminum & 0.35 & $<0.02$ & 2.5 & 0.1 & 0.89 & $<0.02$ & $<0.02$ & $<0.02$ \\
\hline Antimony & $<0.05$ & $<0.05$ & $<0.05$ & $<0.05$ & $<0.05$ & $<0.05$ & $<0.05$ & $<0.05$ \\
\hline Arsente & $<0.05$ & $<0.08$ & $<0.05$ & $<0.05$ & $<0.05$ & $<0.05$ & $<0.05$ & $<0.05$ \\
\hline Barium & 0.24 & 0.13 & 0.22 & 0.096 & 0.19 & 0.17 & 0.2 & 0.2 \\
\hline Beryllium & $<0.0003$ & $<0.0003$ & $<0.0003$ & $<0.0003$ & $<0.0003$ & $<0.0003$ & 0.00034 & $<0.0003$ \\
\hline Boron & 0.12 & 0.11 & 0.11 & 0.12 & 0.13 & 0.12 & 0.13 & 0.12 \\
\hline Cadmiun (MAs) & $<0.002$ & $<0.002$ & $<0.002$ & $<0.002$ & $<0.002$ & $<0.002$ & $<0.002$ & $<0.002$ \\
\hline Cadmium & $<0.003$ & $<0.003$ & $<0.003$ & $<0.003$ & $<0.003$ & $<0.003$ & $<0.003$ & $<0.003$ \\
\hline Chromium (MAs) & $<0.01$ & $<0.01$ & 0.012 & $<0.01$ & $<0.01$ & $<0.01$ & $<0.01$ & $<0.01$ \\
\hline Chromium & $<0.01$ & $<0.01$ & $<0.01$ & $<0.01$ & $<0.01$ & $<0.01$ & $<0.01$ & $<0.01$ \\
\hline Cobalt & $<0.005$ & $<0.005$ & $<0.005$ & $<0.005$ & $<0.005$ & $<0.005$ & $<0.005$ & $<0.005$ \\
\hline Copper & 0.0073 & $<0.004$ & $<0.004$ & $<0.004$ & 0.0074 & $<0.004$ & 0.01 & 0.011 \\
\hline Iron & 1.1 & $<0.005$ & 6 & 0.012 & 2.3 & $<0.005$ & 0.049 & 0.018 \\
\hline Lead (MAS) & $<0.004$ & $<0.004$ & 0.0045 & $<0.004$ & $<0.004$ & $<0.004$ & $<0.004$ & $<0.004$ \\
\hline Mercury (CVAA) & $<0.0002$ & $<0.0002$ & $<0.0002$ & $<0.0002$ & $<0.0002$ & $<0.0002$ & $<0.0002$ & $<0.0002$ \\
\hline Mol ybdenum & $<0.01$ & $<0.01$ & $<0.01$ & $<0.01$ & $<0.01$ & $<0.01$ & $<0.01$ & $<0.01$ \\
\hline Nickel & $<0.01$ & $<0.01$ & $<0.01$ & $<0.01$ & $<0.01$ & $<0.01$ & $<0.01$ & $<0.01$ \\
\hline Selentum & $<0.05$ & $<0.05$ & $<0.05$ & $<0.05$ & $<0.05$ & $<0.05$ & $<0.05$ & $<0.05$ \\
\hline siluer & $<0.006$ & $<0.006$ & $<0.006$ & $<0.006$ & $<0.006$ & $<0.006$ & $<0.006$ & $<0.006$ \\
\hline strontium & 0.55 & 0.59 & 0.91 & 0.55 & 0.7 & 0.72 & 0.9 & 0.93 \\
\hline Thorium & $<0.2$ & $<0.2$ & $<0.2$ & $<0.2$ & $<0.2$ & $<0.2$ & $<0.2$ & $<0.2$ \\
\hline Uranium (Fluor) & $<0.001$ & 0.001 & 0.001 & 0.001 & $<0.001$ & $<0.001$ & $<0.001$ & $<0.001$ \\
\hline Vanadiun & $<0.005$ & $<0.005$ & $<0.005$ & $<0.005$ & $<0.005$ & $<0.005$ & $<0.005$ & $<0.005$ \\
\hline zinc & 0.016 & 0.0049 & 0.032 & 0.0042 & 0.019 & 0.0074 & 0.014 & 0.011 \\
\hline MAJOR IONS (mg/L) &. & . &. & $\cdot$ & . & - & . & • \\
\hline Alkalinity-HCO3 & 212 & $\cdot$ & 217 & $\cdot$ & 215 & $\cdot$ & 216 & • \\
\hline Alkalinity- $\mathrm{CO} 3$ & $<1$ & $\cdot$ & 2 & $\cdot$ & $<1$ & . & $<1$ & • \\
\hline Calcium & 22 & 21 & 54 & 19 & 25 & 24 & 24 & 27 \\
\hline Chloride & 6 & $\cdot$ & 9.5 & $\cdot$ & 8.2 & $\cdot$ & 14 & • \\
\hline Fluoride & 0.6 & . & 0.4 & $\cdot$ & 0.7 & - & 0.5 & • \\
\hline Magnesium & 4.3 & 4.7 & 7.1 & 4 & 5.5 & 5.4 & 6.1 & 6.3 \\
\hline Manganese & 0.033 & 0.011 & 0.14 & 0.006 & 0.056 & 0.017 & 0.02 & 0.019 \\
\hline Nitrate-N & $<0.2$ & . & $<0.2$ & $\cdot$ & $<0.2$ & . & $<0.2$ & • \\
\hline Potassium & 2.7 & 2.2 & 3.2 & 2.2 & 3.5 & 3.5 & 3.1 & 3.2 \\
\hline Sodium & 76 & 68 & 69 & 76 & 77 & 74 & 81 & 79 \\
\hline Sulfate & 19 & $\cdot$ & 20 & . & 21 & • & 16 & • \\
\hline
\end{tabular}

(CONTINUED) 
APPENDIX $\mathrm{R} .1$

Oroundwater Quadity Data, 1993

\begin{tabular}{|c|c|c|c|c|c|c|c|c|}
\hline \multirow{4}{*}{$\begin{array}{l}\text { Sampling Point } \\
\text { Location } \\
\text { Date sampled }\end{array}$} & \multicolumn{8}{|c|}{ GW-756 } \\
\hline & \multicolumn{8}{|c|}{ GRIDJ1 } \\
\hline & \multicolumn{2}{|c|}{$01 / 15 / 93$} & \multicolumn{2}{|c|}{$04 / 08 / 93$} & \multicolumn{2}{|c|}{$08 / 03 / 93$} & \multicolumn{2}{|c|}{$10 / 21 / 93$} \\
\hline & TOT & DIS & TOT & DIS & TOT & DIs & TOT & DIs \\
\hline Mancs (mg/L) & . & - & . & - & - & - & - & . \\
\hline Aluminum & 47 & 0.028 & 7.3 & $<0.02$ & 16 & $<0.02$ & 4.8 & $<0.02$ \\
\hline Antimony & $<0.05$ & $<0.05$ & $<0.05$ & $<0.05$ & $<0.05$ & $<0.05$ & $<0.05$ & $<0.05$ \\
\hline Arsonic & $<0.05$ & $<0.05$ & $<0.05$ & $<0.05$ & $<0.05$ & $<0.05$ & $<0.05$ & $<0.05$ \\
\hline Barium & 0.55 & 0.075 & 0.14 & 0.079 & 0.22 & 0.083 & 0.14 & $n .1$ \\
\hline Bory111um & 0.0026 & $<0.0003$ & 0.00032 & $<0,0003$ & 0.00036 & $<0.0003$ & 0.00046 & $<0.0003$ \\
\hline Boron & 0.049 & 0.031 & 0.023 & 0.023 & 0.046 & 0.018 & 0.03 & 0.023 \\
\hline Cactulum (MS) & $<0.002$ & $<0.002$ & $<0.002$ & $<0.002$ & $<0.002$ & $<0.002$ & $<0.002$ & $<0.002$ \\
\hline Cadmitum & 0.019 & $<0.003$ & $<0.003$ & $<0.003$ & 0.0063 & $<0.003$ & $<0.003$ & $<0.003$ \\
\hline Chromiun (MAs) & 0.12 & $<0.01$ & 0.018 & $<0.01$ & $<0.01$ & $<0.01$ & 0.016 & $<0.01$ \\
\hline Chromium & 0.093 & $<0.01$ & $<0.01$ & $<0.01$ & 0.051 & $<0.01$ & $<0.01$ & $<0.01$ \\
\hline :obalt & 0.048 & $<0.005$ & 0.0061 & $<0.005$ & 0.025 & 0.015 & 0.013 & 0.0093 \\
\hline ¿opper & 0.061 & $<0.004$ & $<0.004$ & $<0.004$ & 0.022 & $<0.004$ & 0.013 & 0.011 \\
\hline Iron & 110 & 0.12 & 13 & 0.071 & 30 & 1.3 & 9.7 & 0.54 \\
\hline Lead (MAs) & 0.042 & $<0.004$ & 0.0047 & $<0.004$ & 0.01 & $<0.004$ & $<0.004$ & $<0.004$ \\
\hline Marcury (CVM) & $<0.0002$ & $<0.0002$ & $<0.0002$ & $<0.0002$ & $<0.0002$ & $<0.0002$ & $<0.0002$ & $<0.0002$ \\
\hline Mol ybdenum & $<0.01$ & $<0.01$ & $<0.01$ & $<0.01$ & $<0.01$ & $<0.01$ & $<0.01$ & $<0.01$ \\
\hline NLckel & 0.079 & $<0.01$ & 0.018 & $<0.01$ & 0.07 & 0.019 & $<0.01$ & $<0.01$ \\
\hline selentum & $<0.05$ & $<0.05$ & $<0.05$ & $<0.05$ & $<0.05$ & $<0.05$ & $<0.05$ & $<0.05$ \\
\hline stlver & $<0.006$ & $<0.006$ & $<0.006$ & $<0.006$ & $<0.006$ & $<0.006$ & $<0.006$ & $<0.006$ \\
\hline stront Ium & 0.12 & 0.09 & 0.098 & 0.098 & 0.09 & 0.08 & 0.1 & 0.1 \\
\hline norium & $<0.2$ & $<0.2$ & $<0.2$ & $<0.2$ & $<0.2$ & $<0.2$ & $<0.2$ & $<0.2$ \\
\hline Uranium (Pluor) & 0.002 & $<0.001$ & $<0.001$ & $<0.001$ & $<0.001$ & $<0.001$ & $<0.001$ & $<0.001$ \\
\hline Vanadium & 0.087 & $<0.005$ & 0.011 & $<0.005$ & 0.028 & $<0.005$ & 0.0095 & $<0.005$ \\
\hline zinc & 0.15 & 0.016 & 0.026 & 0.0091 & 0.08 & 0.014 & 0.034 & 0.019 \\
\hline MuJOR IONS (mg/L) & . & . & . & . & . & . & $\dot{.}$ & · \\
\hline Alkalinity-HCO3 & 62 & $\cdot$ & 56 & . & 54 & . & 54 & . \\
\hline Alkalinity-CO3 & $<1$ & . & $<1$ & . & $<1$ & . & $<1$ & . \\
\hline Calcium & 35 & 29 & 27 & 27 & 30 & 28 & 30 & 29 \\
\hline Chloride & 39 & . & 29 & • & 42 & . & 45 & • \\
\hline Fluoride & $<0.1$ & . & 0.1 & . & $<0.1$ & . & $<0.1$ & . \\
\hline Magnesium & 14 & 5.1 & 6.4 & 5.4 & 8.5 & 6.1 & 7.1 & 6.3 \\
\hline Manganese & 1.1 & 0.67 & 0.99 & 1 & 3 & 3 & 2.5 & 2.4 \\
\hline Nitrate-N & $<0.2$ & . & $<0.2$ & . & $<0.2$ & . & $<0.2$ & • \\
\hline Potaseium & 9.2 & 1.1 & 3.1 & 1 & 5.9 & 1.9 & 3 & 1.5 \\
\hline Sodium & 16 & 16 & 17 & 18 & 21 & 21 & 22 & 22 \\
\hline sulfate & 32 & . & 36 & - & 36 & . & 32 & . \\
\hline
\end{tabular}

(CONTINUED) 
APPENDIX E.1

Groundwater Quality Data, 1993

\begin{tabular}{|c|c|c|c|c|c|c|c|c|}
\hline \multirow{4}{*}{$\begin{array}{l}\text { Sampling Polnt } \\
\text { Location } \\
\text { Date sampled }\end{array}$} & \multicolumn{8}{|c|}{$a W-758$} \\
\hline & \multicolumn{8}{|c|}{ GRIDGI } \\
\hline & \multicolumn{2}{|c|}{$01 / 25 / 93$} & \multicolumn{2}{|c|}{$04 / 23 / 93$} & \multicolumn{2}{|c|}{$09 / 12 / 93$} & \multicolumn{2}{|c|}{$11 / 19 / 93$} \\
\hline & TOT & DIs & TOT & DIs & TOT & DIs & TOT & DIS \\
\hline HETALS (mg/L) & $\cdot$ & $\cdot$ & $\cdot$ & - & • & - & $\cdot 1$ & - \\
\hline Muminum & $<0.02$ & 0.05 & 0.078 & $<0.02$ & 0.023 & $<0.02$ & 0.037 & $<0.02$ \\
\hline Antimony & $<0.05$ & $<0.05$ & $<0.05$ & $<0.05$ & $<0.05$ & $<0.05$ & $<0.05$ & $<0.05$ \\
\hline Areanic & $<0.05$ & $<0.05$ & $<0.05$ & $<0.05$ & $<0.05$ & $<0.05$ & $<0.05$ & $<0.05$ \\
\hline Barium & 0.16 & 0.12 & 0.11 & 0.11 & 0.12 & 0.12 & 0.12 & 0.12 \\
\hline Beryllium & $<0.0003$ & $<0.0003$ & $<0.0003$ & $<0.0003$ & $<0.0003$ & $<0,0003$ & $<0.0003$ & $<0.0003$ \\
\hline Boron & 0.02 & 0.043 & 0.085 & 0.049 & 0.049 & 0.037 & 0.045 & 0.042 \\
\hline Cadmium (MAS) & $<0.002$ & $<0.002$ & $<0.002$ & $<0.002$ & $<0.002$ & $<0.002$ & $<0.002$ & $<0.002$ \\
\hline Cadmi un & $<0.003$ & $<0.003$ & $<0.003$ & $<0.003$ & $<0.003$ & $<0.003$ & $<0.003$ & $<0.003$ \\
\hline Chromilum (MAS) & $<0.01$ & $<0.01$ & $<0.01$ & $<0.01$ & $<0.01$ & $<0.01$ & $<0.01$ & $<0.01$ \\
\hline Chromium & $<0.01$ & $<0.01$ & $<0.01$ & $<0.01$ & $<0.01$ & $<0.01$ & $<0.01$ & $<0.01$ \\
\hline Cobalt & $<0.005$ & $<0.005$ & $<0.005$ & $<0.005$ & $<0.005$ & $<0.005$ & $<0.005$ & $<0.005$ \\
\hline Copper & $<0.004$ & $<0.004$ & $<0.004$ & $<0.004$ & $<0.004$ & $<0.004$ & $<0.004$ & $<0.004$ \\
\hline Iron & 0.02 & 0.34 & 0.25 & 0.19 & 0.46 & 0.19 & 0.46 & 0.28 \\
\hline Lead (MAS) & $<0.004$ & $<0.004$ & $<0.004$ & $<0.004$ & $<0.004$ & $<0.004$ & $<0.004$ & $<0.004$ \\
\hline Mercury (CVMA) & $<0.0002$ & $<0.0002$ & $<0.0002$ & $<0.0002$ & $<0.0002$ & $<0.0002$ & $<0.0002$ & $<0.0002$ \\
\hline Nolybdenum & $<0.02$ & $<0.01$ & $<0.01$ & $<0.01$ & $<0.01$ & $<0.01$ & $<0.01$ & $<0.01$ \\
\hline Hickel & $<0.01$ & 0.011 & $<0.01$ & $<0.01$ & $<0.01$ & $<0.01$ & $<0.01$ & $<0.01$ \\
\hline selenium & $<0.05$ & $<0.05$ & $<0.05$ & $<0.05$ & $<0.05$ & $<0.05$ & $<0.05$ & $<0.05$ \\
\hline silver & $<0.006$ & $<0.006$ & $<0.006$ & $<0.006$ & $<0.006$ & $<0.006$ & $<0.006$ & $<0.006$ \\
\hline Strontium & 0.14 & 0.46 & 0.43 & 0.43 & 0.46 & 0.46 & 0.49 & 0.51 \\
\hline Thorium & $<0.2$ & $<0.2$ & $<0.2$ & $<0.2$ & $<0.2$ & $<0.2$ & $<0.2$ & $<0.2$ \\
\hline Uranium (Pluor) & $<0.001$ & $<0.001$ & $<0.001$ & $<0.001$ & $<0.001$ & $<0.001$ & $<0.001$ & $<0.001$ \\
\hline Vanadium & $<0.005$ & $<0.005$ & $<0.005$ & $<0.005$ & $<0.005$ & $<0.005$ & $<0.005$ & $<0.005$ \\
\hline Zine & 0.0079 & 0.0037 & 0.0033 & $<0.002$ & 0.0065 & 0.0042 & 0.0022 & 0.0033 \\
\hline MNOR IONS (mg/L) & . &. & . &. & - & $\cdot$ & $\cdot$ & - \\
\hline Alkalinity-HCO3 & 192 & $\cdot$ & 287 & $\cdot$ & 200 & . & 201 & . \\
\hline Alkalinity- $\mathrm{CO} 3$ & $<1$ & $\cdot$ & $<1$ & $\cdot$ & $<1$ & . & $<1$ & . \\
\hline Calcium & 96 & 68 & 63 & 63 & 72 & 72 & 66 & 73 \\
\hline Chloride & 16.6 & $\cdot$ & 14 & $\cdot$ & 15.4 & . & 19 & . \\
\hline Fluoride & 0.1 & $\cdot$ & 0.1 & . & 0.1 & . & 0.2 & . \\
\hline Magnesium & 9.5 & 8.8 & 8 & 8 & 9.1 & 9.1 & 8.7 & 9.4 \\
\hline Manganese & 0.22 & 0.21 & 0.19 & 0.19 & 0.25 & 0.25 & 0.21 & 0.23 \\
\hline Nitrate-N & $<0.2$ & . & $<0.2$ &. & $<0.2$ & . & $<0.2$ & . \\
\hline Potassium & 1 & 2.5 & 2.3 & 2.7 & 2 & 2.4 & 2.4 & 2.6 \\
\hline Sodium & 8.5 & 15 & 14 & 14 & 13 & 14 & 15 & 14 \\
\hline sulfate & 19.8 & $\cdot$ & 20 &. & 20.1 & - & 16 & . \\
\hline
\end{tabular}

(CONTINUED) 
APPENDIX $\mathbf{R}, 1$

Groundwater Quallty Data, 1993

\begin{tabular}{|c|c|c|c|c|c|c|c|c|}
\hline \multirow{4}{*}{$\begin{array}{l}\text { 8umpling Point } \\
\text { Iocation } \\
\text { - } \\
\text { Date Sampled }\end{array}$} & \multicolumn{8}{|c|}{ OW-759 } \\
\hline & \multicolumn{8}{|c|}{ GRIDOL } \\
\hline & \multicolumn{2}{|c|}{$01 / 26 / 93$} & \multicolumn{2}{|c|}{$04 / 15 / 93$} & \multicolumn{2}{|c|}{$09 / 13 / 93$} & \multicolumn{2}{|c|}{$11 / 19 / 93$} \\
\hline & TOT & DIS & TOT & DIs & TOT & DIs & TOT & DIS \\
\hline VETALS (mg/t) & - & - & - & - & $\cdot$ & . & - & - \\
\hline Aluminum & 1.9 & 0.031 & 0.076 & $<0.02$ & 12 & 0.03 & 0.24 & $<0.02$ \\
\hline Ant Lmony & $<0.05$ & $<0.05$ & $<0.05$ & $<0.05$ & $<0.05$ & $<0.05$ & $<0.05$ & $<0.05$ \\
\hline Areenic & $<0.05$ & $<0.05$ & $<0.05$ & $<0.05$ & $<0.05$ & $<0.05$ & 0.08 & $<0.05$ \\
\hline Barium & 0.13 & 0.072 & 0.1 & 0.1 & 0.29 & 0.18 & 0.11 & 0.099 \\
\hline Bery111um & $<0.0003$ & $<0.0003$ & 0.0006 & $<0.0003$ & 0.00072 & $<0.0003$ & $<0.0003$ & $<0.0003$ \\
\hline Boron & 0.054 & 0.032 & 0.027 & 0.034 & 0.022 & 0.048 & 0.033 & 0.071 \\
\hline Cudmium (MS) & $<0.002$ & $<0.002$ & $<0.002$ & $<0.002$ & $<0.002$ & $<0.002$ & $<0.002$ & $<0.002$ \\
\hline Cadmium & $<0.003$ & $<0.003$ & $<0.003$ & $<0.003$ & 0.0046 & $<0.003$ & $<0.003$ & $<0.003$ \\
\hline Chromlum (MAs) & $<0.01$ & $<0.01$ & $<0.01$ & $<0.01$ & 0.036 & $<0.01$ & $<0.01$ & $<0.01$ \\
\hline Chromium & 0.01 & $<0.01$ & $<0.01$ & $<0.01$ & 0.052 & $<0.01$ & $<0.01$ & $<0.02$ \\
\hline Cobalt & $<0.005$ & $<0.005$ & $<0.005$ & $<0.005$ & 0.012 & $<0.005$ & $<0.005$ & $<0.005$ \\
\hline copper & $<0.004$ & $<0.004$ & $<0.004$ & $<0.004$ & 0.038 & $<0.004$ & $<0.004$ & $<0.004$ \\
\hline Iron & 2.7 & $<0.005$ & 0.28 & 0.068 & 24 & 0.055 & 0.77 & 0.078 \\
\hline Laad (MAS) & $<0.004$ & $<0.004$ & $<0.004$ & $<0.004$ & 0.014 & $<0.004$ & $<0.004$ & $<0.004$ \\
\hline Mercury (CVMA) & $<0.0002$ & $<0.0002$ & $<0.0002$ & $<0.0002$ & $<0.0002$ & $<0.0002$ & $<0.0002$ & $<0.0002$ \\
\hline Nol ybdenum & $<0.01$ & $<0.01$ & $<0.01$ & $<0.01$ & $<0.01$ & $<0.01$ & $<0.01$ & $<0.01$ \\
\hline Nickel & 0.024 & 0.012 & $<0.01$ & 0.012 & 0.061 & $<0.01$ & $<0.01$ & 0.013 \\
\hline selentum & $<0.05$ & $<0.05$ & $<0.05$ & $<0.05$ & $<0.05$ & $<0.05$ & $<0.05$ & $<0.05$ \\
\hline silver & $<0.006$ & $<0.006$ & $<0.006$ & $<0.006$ & $<0.006$ & $<0.006$ & $<0.006$ & $<0.006$ \\
\hline strontium & 0.2 & 0.052 & 0.18 & 0.18 & 0.22 & 0.21 & 0.22 & 0.21 \\
\hline Thorium & $<0.2$ & $<0.2$ & $<0.2$ & $<0.2$ & $<0.2$ & $<0.2$ & $<0.2$ & $<0.2$ \\
\hline Uranium (Fluor) & $<0.001$ & $<0.001$ & $<0.001$ & $<0.001$ & 0.001 & 0.001 & $<0.001$ & $<0.001$ \\
\hline Vanadium & $<0.005$ & $<0.005$ & $<0.005$ & $<0.005$ & 0.032 & $<0.005$ & $<0.005$ & $<0.005$ \\
\hline Zinc & 0.015 & 0.0088 & 0.02 & 0.022 & 0.058 & 0.005 & 0.0097 & $<0.002$ \\
\hline MAJOR IONS (mg/L) & - & - & - &. & . & . & - & - \\
\hline Alkal InItty-HCO3 & 191 & . & 186 & $\cdot$ & 195 & $\cdot$ & $198^{\circ}$ & . \\
\hline Alkalinity-co3 & $<1$ & - & $<1$ & $\cdot$ & $<1$ & - & $<1$ & - \\
\hline Calcium & 69 & 32 & 67 & 66 & 87 & 75 & 70 & 69 \\
\hline Chloride & 67 & - & 21 & $\cdot$ & 17.8 & $\cdot$ & 21 & - \\
\hline Fluoride & 0.1 & . & 0.1 & $\cdot$ & $<0.1$ & $\cdot$ & 0.1 & - \\
\hline Magnesium & 9.8 & 2.9 & 8.9 & 8.8 & 16 & 10 & 9.6 & 9.5 \\
\hline Manganese & 0.25 & 0.0029 & 0.18 & 0.15 & 1.2 & 0.35 & 0.24 & 0.24 \\
\hline Nitrate-N & $<0.2$ & • & $<0.2$ & . & $<0.2$ & $\cdot$ & $<0.2$ & • \\
\hline Potassium & 2.4 & $<0.6$ & 1.7 & 1.5 & 4.7 & 1.4 & 2 & 2 \\
\hline Sodium & 12 & 6.5 & 13 & 13 & 12 & 12 & 12 & 11 \\
\hline sulfate & 20 & • & 17 & $\cdot$ & 21 & . & 18 & - \\
\hline
\end{tabular}

(CONT INUED) 
APPENDIX E.1

Groundwater Quality Data, 1993

\begin{tabular}{|c|c|c|c|c|c|c|c|c|}
\hline \multirow{4}{*}{$\begin{array}{l}\text { Sampling Point } \\
\text { - } \\
\text { Location } \\
\text { - } \\
\text { Date Sampled }\end{array}$} & \multicolumn{8}{|c|}{$G W-760$} \\
\hline & \multicolumn{8}{|c|}{ GRIDG2 } \\
\hline & \multicolumn{2}{|c|}{$01 / 27 / 93$} & \multicolumn{2}{|c|}{$04 / 16 / 93$} & \multicolumn{2}{|c|}{$09 / 14 / 93$} & \multicolumn{2}{|c|}{$11 / 30 / 93$} \\
\hline & TOT & DIS & TOT & DIS & TOT & DIS & TOT & DIS \\
\hline METALS (mg/L) & $\cdot$ & $\cdot$ & - & • & - & - & $\cdot$ & • \\
\hline Aluminum & 0.16 & 0.11 & 0.027 & $<0.02$ & 0.1 & $<0.02$ & 0.021 & $<0.02$ \\
\hline Antimony & $<0.05$ & $<0.05$ & $<0.05$ & $<0.05$ & $<0.05$ & $<0.05$ & $<0.05$ & $<0.05$ \\
\hline Arsenic & $<0.05$ & $<0.05$ & $<0.05$ & $<0.05$ & $<0.05$ & $<0.05$ & $<0.05$ & $<0.05$ \\
\hline Barium & 0.43 & 0.42 & 0.42 & 0.4 & 0.47 & 0.47 & 0.41 & 0.41 \\
\hline Beryllium & $<0.0003$ & $<0.0003$ & $<0.0003$ & $<0.0003$ & $<0.0003$ & $<0.0003$ & $<0.0003$ & $<0.0003$ \\
\hline Boron & 0.036 & 0.031 & 0.019 & 0.014 & 0.035 & 0.03 & 0.017 & 0.025 \\
\hline Cadmium (AAS) & $<0.002$ & $<0.002$ & 0.0087 & 0.014 & 0.02 & 0.013 & $<0.002$ & $<0.002$ \\
\hline Cadmium & $<0.003$ & $<0.003$ & 0.0069 & 0.01 & 0.02 & 0.012 & $<0.003$ & $<0.003$ \\
\hline Chromium (AAS) & 0.15 & $<0.01$ & $<0.01$ & $<0.01$ & 0.051 & $<0.01$ & 0.015 & $<0.01$ \\
\hline Chromium & 0.061 & $<0.01$ & $<0.01$ & $<0.01$ & 0.081 & $<0.01$ & 0.014 & $<0.01$ \\
\hline Cobalt & $<0.005$ & $<0.005$ & $<0.005$ & $<0.005$ & $<0.005$ & $<0.005$ & $<0.005$ & $<0.005$ \\
\hline Copper & $<0.004$ & $<0.004$ & 0.0047 & $<0.004$ & $<0.004$ & $<0.004$ & $<0.004$ & $<0.004$ \\
\hline Iron & 0.4 & 0.0078 & 0.058 & $<0.005$ & 0.64 & 0.013 & 0.18 & $<0.005$ \\
\hline Lead (AAS) & $<0.004$ & $<0.004$ & $<0.004$ & $<0.004$ & $<0.004$ & $<0.004$ & $<0.004$ & $<0.004$ \\
\hline Mercury (CVAA) & $<0.0002$ & $<0.0002$ & $<0.0002$ & $<0.0002$ & $<0.0002$ & $<0.0002$ & $<0.0002$ & $<0.0002$ \\
\hline Nolybdenum & $<0.01$ & $<0.01$ & $<0.01$ & $<0.01$ & $<0.01$ & $<0.01$ & $<0.01$ & $<0.01$ \\
\hline Nickel & 0.059 & 0.052 & 0.024 & 0.025 & 0.08 & 0.047 & 0.045 & 0.027 \\
\hline Selenium & $<0.05$ & $<0.05$ & $<0.05$ & $<0.05$ & $<0.05$ & $<0.05$ & $<0.05$ & $<0.05$ \\
\hline silver & $<0.006$ & $<0.006$ & $<0.006$ & $<0.006$ & $<0.006$ & $<0.006$ & $<0.006$ & $<0.006$ \\
\hline strontium & 0.23 & 0.23 & 0.24 & 0.23 & 0.27 & 0.26 & 0.25 & 0.25 \\
\hline Thorium & $<0.2$ & $<0.2$ & $<0.2$ & $<0.2$ & $<0.2$ & $<0.2$ & $<0.2$ & $<0.2$ \\
\hline Oranium (Fluor) & $<0.001$ & $<0.001$ & $<0.001$ & 0.001 & $<0.001$ & $<0.001$ & $<0.001$ & $<0.001$ \\
\hline Vanadium & $<0.005$ & $<0.005$ & $<0.005$ & $<0.005$ & $<0.005$ & $<0.005$ & $<0.005$ & $<0.005$ \\
\hline Zine & 0.013 & 0.0058 & 0.013 & 0.014 & 0.015 & 0.014 & 0.014 & 0.0046 \\
\hline MAJOR IONS $(\mathrm{mg} / \mathrm{I})$ & $\cdot$ & $\cdot$ & $\cdot$ & - & $\cdot$ & • & $\cdot$ & - \\
\hline Alkalinity-HCO3 & 154 & $\cdot$ & 152 & - & 166 & • & 162 & • \\
\hline Alkalinity-CO3 & $<1$ & $\cdot$ & $<1$ & . & $<1$ & - & $<1$ & - \\
\hline Calcium & 110 & 110 & 100 & 100 & 130 & 130 & 120 & 110 \\
\hline Chloride & 173 & $\cdot$ & 122 & $\cdot$ & 76 & $\cdot$ & 122 & - \\
\hline Fluoride & $<0.1$ & $\cdot$ & $<0.1$ & $\cdot$ & $<0.1$ & • & $<0.1$ & - \\
\hline Magnesium & 12 & 11 & 11 & 11 & 14 & 14 & 13 & 13 \\
\hline Manganese & 0.045 & 0.04 & 0.026 & 0.023 & 0.072 & 0.063 & 0.017 & 0.015 \\
\hline Nitrate-N & 0.38 & $\cdot$ & 0.54 & . & $<0.2$ & . & 0.4 & • \\
\hline Potassium & 2 & 2 & 1.7 & 1.6 & 1.9 & 1.3 & 1.3 & 1.4 \\
\hline Sodium & 8.9 & 8.8 & 10 & 10 & 11 & 11 & 11 & 11 \\
\hline Sulfate & 21 & $\cdot$ & 21 & • & 21 & - & 18 & • \\
\hline
\end{tabular}

(CONTINUED) 
APPENDIX E.1

Groundwater Quality Data, 1993

\begin{tabular}{|c|c|c|c|c|c|c|c|c|}
\hline \multirow{4}{*}{$\begin{array}{l}\text { Sampling Point } \\
\text { - } \\
\text { Location } \\
\text { Date Sampled }\end{array}$} & \multicolumn{8}{|c|}{$G W-761$} \\
\hline & \multicolumn{8}{|c|}{ GRIDG2 } \\
\hline & \multicolumn{2}{|c|}{$01 / 26 / 93$} & \multicolumn{2}{|c|}{$04 / 16 / 93$} & \multicolumn{2}{|c|}{$09 / 13 / 93$} & \multicolumn{2}{|c|}{$11 / 29 / 93$} \\
\hline & TOT & DIS & TOT & DIS & TOT & DIS & TOT & DIS \\
\hline METALS (mg/L) & - & - & . & - & . & . & - & • \\
\hline Aluminum & 0.71 & 0,046 & 1.5 & $<0.02$ & 0.66 & $<0.02$ & 3.6 & 0.026 \\
\hline Antimony & $<0.05$ & $<0.05$ & $<0.05$ & $<0.05$ & $<0.05$ & $<0.05$ & $<0.05$ & $<0.05$ \\
\hline Arsenic & $<0.05$ & $<0.05$ & $<0.05$ & $<0.05$ & $<0.05$ & $<0.05$ & $<0.05$ & $<0.05$ \\
\hline Barium & 0.077 & 0.12 & 0.076 & 0.065 & 0.078 & 0.071 & 0.11 & 0.066 \\
\hline Beryllium & 0.00031 & $<0.0003$ & $<0.0003$ & $<0.0003$ & $<0.0003$ & $<0.0003$ & 0.006 & 0.00065 \\
\hline Boron & 0.019 & 0.037 & 0.019 & 0.022 & 0.044 & 0.012 & 0.029 & 0.023 \\
\hline Cadmium (AAS) & $<0.002$ & $<0.002$ & $<0.002$ & $<0.002$ & $<0.002$ & $<0.002$ & $<0.002$ & $<0.002$ \\
\hline Cadmium & $<0.003$ & $<0.003$ & $<0.003$ & $<0.003$ & $<0.003$ & $<0.003$ & 0.0059 & $<0.003$ \\
\hline Chromium (AAS) & 0.012 & $<0.01$ & 0.013 & $<0.01$ & $<0.01$ & $<0.01$ & 0.063 & $<0.01$ \\
\hline Chromium & $<0.01$ & $<0.01$ & 0.014 & $<0.01$ & $<0.01$ & $<0.01$ & 0.065 & $<0.01$ \\
\hline Cobalt & $<0.005$ & $<0.005$ & $<0.005$ & $<0.005$ & $<0.005$ & $<0.005$ & 0.015 & $<0.005$ \\
\hline Copper & $<0.004$ & $<0.004$ & $<0.004$ & $<0.004$ & $<0.004$ & $<0.004$ & 0.0095 & $<0.004$ \\
\hline Iron & 0.84 & 0.28 & 1.7 & 0.0078 & 0.93 & 0.019 & 5.2 & 0.022 \\
\hline Lead (AAS) & $<0.004$ & $<0.004$ & $<0.004$ & $<0.004$ & $<0.004$ & $<0.004$ & $<0.004$ & $<0.004$ \\
\hline Mercury (CVAA) & $<0.0002$ & $<0.0002$ & $<0.0002$ & $<0.0002$ & $<0.0002$ & $<0.0002$ & $<0.0002$ & $<0.0002$ \\
\hline Molybdenum & $<0.01$ & $<0.01$ & $<0.01$ & $<0.01$ & $<0.01$ & $<0.01$ & $<0.01$ & $<0.01$ \\
\hline Nickel & 0.017 & $<0.01$ & $<0.01$ & 0.012 & 0.011 & $<0.01$ & 0.049 & $0.03:$ \\
\hline Selenium & $<0.05$ & $<0.05$ & $<0.05$ & $<0.05$ & $<0.05$ & $<0.05$ & $<0.05$ & $<0.05$ \\
\hline Silver & $<0.006$ & $<0.006$ & $<0.006$ & $<0.006$ & $<0.006$ & $<0.006$ & 0.0082 & $<0.006$ \\
\hline Strontium & 0.049 & 0.46 & 0.045 & 0.043 & 0.048 & 0.047 & 0.056 & 0.047 \\
\hline Thorium & $<0.2$ & $<0.2$ & $<0.2$ & $<0.2$ & $<0.2$ & $<0.2$ & $<0.2$ & $<0.2$ \\
\hline Oranium (Fluor) & $<0.001$ & $<0.001$ & $<0.001$ & $<0.001$ & $<0.001$ & $<0.001$ & $<0.001$ & $<0.001$ \\
\hline Vanadium & $<0.005$ & $<0.005$ & $<0.005$ & $<0.005$ & $<0.005$ & $<0.005$ & 0.014 & $<0.005$ \\
\hline zinc & 0.013 & 0.0043 & 0.022 & 0.024 & 0.036 & 0.026 & 0.035 & 0.013 \\
\hline MAJOR IONS (mg/L) & . & . & • & $\cdot$ & . & $\cdot$ & - & • \\
\hline Alkalinity-HCO3 & ${ }_{72}$ & $\cdot$ & ${ }_{74}^{\circ}$ & $\cdot$ & ${ }_{84}^{\circ}$ &. & 78 & • \\
\hline Alkalinity- $\mathrm{CO} 3$ & $<1$ &. & $<1$ & . & $<1$ & . & $<1$ & . \\
\hline Calcium & 30 & 68 & 29 & 28 & 33 & 33 & 30 & 29 \\
\hline Chloride & 9.8 & $\cdot$ & 7.2 & . & 7.3 & . & 7 & • \\
\hline Fluoride & 0.1 & . & 0.1 & $\cdot$ & $<0.1$ & . & 0.1 & • \\
\hline Magnesium & 2.9 & 8.8 & 2.9 & 2.5 & 3.1 & 2.9 & 3.7 & 2.6 \\
\hline Manganese & 0.065 & 0.21 & 0.093 & 0.0039 & 0.053 & 0.0057 & 0.38 & 0.013 \\
\hline Nitrate-N & $<0.2$ & . & 0.26 & . & $<0.2$ & . & 0.2 & • \\
\hline Potassium & 0.72 & 2.5 & 1.2 & 0.78 & 0.96 & $<0.6$ & 1.6 & $<0.6$ \\
\hline Sodium & 6.2 & 15 & 5.9 & 5.7 & 6.1 & 6.1 & 6.2 & 6.1 \\
\hline Sulfate & 13 & . & 16 & . & 11.9 & . & 11 & • \\
\hline
\end{tabular}

(CONTINUED) 
APPENDIX E. 1

Groundwater Quality Data, 1993

\begin{tabular}{|c|c|c|c|c|c|c|c|c|}
\hline \multirow{4}{*}{$\begin{array}{l}\text { Sampling Point } \\
\text { Location } \\
\text { - } \\
\text { Date Sampled }\end{array}$} & \multicolumn{8}{|c|}{ GW-762 } \\
\hline & \multicolumn{8}{|c|}{ GRIDJ3 } \\
\hline & \multicolumn{2}{|c|}{$01 / 23 / 93$} & \multicolumn{2}{|c|}{$04 / 20 / 93$} & \multicolumn{2}{|c|}{$08 / 04 / 93$} & \multicolumn{2}{|c|}{$10 / 28 / 93$} \\
\hline & TOT & DIS & TOT & DIS & TOT & DIS & TOT & DIS \\
\hline METALS (mg/L) & $\cdot$ & - & $\cdot$ & • & $\cdot$ & - & $\cdot$ & - \\
\hline Aluminum & 0.24 & 0.033 & 0.89 & $<0.02$ & 0.95 & $<0.02$ & 0.5 & 0.021 \\
\hline Antimony & $<0.05$ & $<0.05$ & $<0.05$ & $<0.05$ & $<0.05$ & $<0.05$ & $<0.05$ & $<0.05$ \\
\hline Arsenic & $<0.05$ & $<0.05$ & $<0.05$ & $<0.05$ & $<0.05$ & $<0.05$ & $<0.05$ & $<0.05$ \\
\hline Barium & 0.61 & 0.59 & 0.58 & 0.56 & 0.59 & 0.55 & 0.64 & 0.64 \\
\hline Beryllium & 0.00031 & $<0.0003$ & $<0.0003$ & $<0.0003$ & $<0.0003$ & $<0.0003$ & $<0.0003$ & $<0.0003$ \\
\hline Boron & 0.11 & 0.1 & 0.095 & 0.083 & 0.09 & 0.12 & 0.11 & 0.11 \\
\hline Cadmium (AAS) & $<0.002$ & $<0.002$ & $<0.002$ & $<0.002$ & $<0.002$ & $<0.002$ & $<0.002$ & $<0.002$ \\
\hline Cadmium & $<0.003$ & 0.0032 & $<0.003$ & $<0.003$ & $<0.003$ & $<0.003$ & $<0.003$ & $<0.003$ \\
\hline Chromium (AAS) & $<0.01$ & $<0.01$ & $<0.01$ & $<0.01$ & $<0.01$ & $<0.01$ & $<0.01$ & $<0.01$ \\
\hline Chromium & $<0.01$ & $<0.01$ & $<0.01$ & $<0.01$ & $<0.01$ & $<0.01$ & $<0.01$ & $<0.01$ \\
\hline Cobalt & $<0.005$ & $<0.005$ & $<0.005$ & $<0.005$ & $<0.005$ & $<0.005$ & $<0.005$ & $<0.005$ \\
\hline Copper & $<0.004$ & 0.023 & $<0.004$ & 0.0068 & 0.011 & $<0.004$ & $<0.004$ & $<0.004$ \\
\hline Iron & 0.82 & 0.12 & 0.69 & 0.13 & 1.1 & 0.041 & 0.58 & 0.14 \\
\hline Lead (AAS) & 0.004 & $<0.004$ & $<0.004$ & $<0.004$ & 0.0043 & $<0.004$ & $<0.004$ & $<0.004$ \\
\hline Mercury (CVAA) & $<0.0002$ & $<0.0002$ & $<0.0002$ & $<0.0002$ & $<0.0002$ & $<0.0002$ & $<0.0002$ & $<0.0002$ \\
\hline Molybdenum & $<0.01$ & $<0.01$ & $<0.01$ & $<0.01$ & $<0.01$ & $<0.01$ & $<0.01$ & $<0.01$ \\
\hline wickel & $<0.01$ & $<0.01$ & $<0.01$ & $<0.01$ & $<0.01$ & $<0.01$ & $<0.01$ & $<0.01$ \\
\hline Selenium & $<0.05$ & $<0.05$ & $<0.05$ & $<0.05$ & $<0.05$ & $<0.05$ & $<0.05$ & $<0.05$ \\
\hline Silver & $<0.006$ & $<0.006$ & $<0.006$ & $<0.006$ & $<0.006$ & $<0.006$ & $<0.006$ & $<0.006$ \\
\hline Strontium & 0.75 & 0.72 & 0.72 & 0.7 & 0.73 & 0.7 & 0.82 & 0.83 \\
\hline Thorium & $<0.2$ & $<0.2$ & $<0.2$ & $<0.2$ & $<0.2$ & $<0.2$ & $<0.2$ & $<0.2$ \\
\hline Uranium (Fluor) & $<0.001$ & $<0.001$ & $<0.001$ & $<0.001$ & $<0.001$ & $<0.001$ & $<0.001$ & $<0.001$ \\
\hline Vanadium & $<0.005$ & $<0.005$ & $<0.005$ & $<0.005$ & $<0.005$ & $<0.005$ & $<0.005$ & $<0.005$ \\
\hline zinc & 0.01 & 0.012 & 0.0052 & 0.0076 & 0.011 & 0.0088 & $<0.002$ & $<0.002$ \\
\hline MAJOR IONS (mg/L) & . & - &. & . & • & . & $\dot{.}$ & • \\
\hline Alkalinity- $\mathrm{HCO} 3$ & 264 & . & 260 & $\cdot$ & 281 & • & 281 & • \\
\hline Alkalinity- $\mathrm{CO} 3$ & $<1$ & • & $<1$ & $\cdot$ & $<1$ & . & $<1$ & • \\
\hline Calciun & 75 & 73 & 74 & 71 & 73 & 69 & 81 & 82 \\
\hline Chloride & 25 & . & 25.4 & . & 24 & • & 28 & • \\
\hline Fluoride & 0.1 & . & $<0.1$ & $\cdot$ & $<0.1$ & - & $<0.1$ & . \\
\hline Magnesium & 24 & 23 & 23 & 22 & 23 & 23 & 25 & 26 \\
\hline Manganese & 0.39 & 0.36 & 0.29 & 0.27 & 0.39 & 0.15 & 0.2 & 0.2 \\
\hline Nitrate-N & $<0.2$ & • & $<0.2$ & . & $<0.2$ & • & $<0.2$ & • \\
\hline Potassium & 3.8 & 3.4 & 3.8 & 3 & 4.9 & 4.7 & 4.2 & 3.9 \\
\hline Sodium & 8.6 & 9.7 & 8.2 & 8 & 8.3 & 8.1 & 9 & 8.9 \\
\hline Sulfate & 15.7 & • & 12.2 & $\cdot$ & 18 & • & 13 & • \\
\hline
\end{tabular}


APPENDIX E.1

Groundwater Quality Data, 1993

\begin{tabular}{|c|c|c|c|c|c|c|c|c|}
\hline \multirow{4}{*}{$\begin{array}{l}\text { Sampling Point } \\
\text { Location } \\
\text { - } \\
\text { Date Sampled }\end{array}$} & \multicolumn{8}{|c|}{ GW-763 } \\
\hline & \multicolumn{8}{|c|}{ GRIDJ3 } \\
\hline & \multicolumn{2}{|c|}{$01 / 25 / 93$} & \multicolumn{2}{|c|}{$04 / 21 / 93$} & \multicolumn{2}{|c|}{$08 / 05 / 93$} & \multicolumn{2}{|c|}{$10 / 29 / 93$} \\
\hline & TOT & DIS & TOT & DIS & TOT & DIS & TOT & DIS \\
\hline MBTALS (mg/L) & - & - & - & - & - & - & $\cdot$ & - \\
\hline Aluminum & 0.49 & $<0.02$ & $<0.02$ & 0.13 & 0.82 & $<0.02$ & 0.31 & 0.021 \\
\hline Antimony & $<0.05$ & $<0.05$ & $<0.05$ & $<0.05$ & $<0.05$ & $<0.05$ & $<0.05$ & $<0.05$ \\
\hline Arsenic & $<0.05$ & $<0.05$ & $<0.05$ & $<0.05$ & $<0.05$ & $<0.05$ & $<0.05$ & $<0.05$ \\
\hline Barium & 0.081 & 0.072 & 0.065 & 0.066 & 0.095 & 0.094 & 0.11 & 0.11 \\
\hline Beryllium & $<0.0003$ & $<0.0003$ & $<0.0003$ & $<0.0003$ & $<0.0003$ & $<0.0003$ & $<0.0003$ & $<0.0003$ \\
\hline Boron & 0.027 & 0.028 & 0.01 & 0.014 & 0.038 & 0.019 & 0.032 & 0.036 \\
\hline Cadmium (AAS) & $<0.002$ & $<0.002$ & $<0.002$ & $<0.002$ & $<0.002$ & $<0.002$ & $<0.002$ & $<0.002$ \\
\hline Cadmium & $<0.003$ & $<0.003$ & $<0.003$ & $<0.003$ & $<0.003$ & $<0.003$ & $<0.003$ & $<0.003$ \\
\hline Chromium (AAS) & $<0.01$ & $<0.01$ & $<0.01$ & $<0.01$ & $<0.01$ & $<0.01$ & $<0.01$ & $<0.01$ \\
\hline Chromium & $<0.01$ & $<0.01$ & $<0.01$ & $<0.01$ & $<0.01$ & $<0.01$ & $<0.01$ & $<0.01$ \\
\hline Cobalt & $<0.005$ & $<0.005$ & $<0.005$ & $<0.005$ & $<0.005$ & $<0.005$ & $<0.005$ & $<0.005$ \\
\hline Copper & $<0.004$ & 0.0047 & $<0.004$ & $<0.004$ & $<0.004$ & $<0.004$ & $<0.004$ & $<0.004$ \\
\hline Iron & 20 & 21 & 22 & 23 & 18 & 12 & 16 & 16 \\
\hline Lead (AAS) & $<0.004$ & $<0.004$ & $<0.004$ & $<0.004$ & $<0.004$ & $<0.004$ & $<0.004$ & $<0.004$ \\
\hline Mercury (CVAA) & $<0.0002$ & $<0.0002$ & $<0.0002$ & $<0.0002$ & $<0.0002$ & $<0.0002$ & $<0.0002$ & $<0.0002$ \\
\hline Molybdenum & $<0.01$ & $<0.01$ & $<0.01$ & $<0.01$ & $<0.01$ & $<0.01$ & $<0.01$ & $<0.01$ \\
\hline Nickel & $<0.01$ & $<0.01$ & $<0.01$ & $<0.01$ & $<0.01$ & $<0.01$ & $<0.01$ & $<0.01$ \\
\hline Selenium & $<0.05$ & $<0.05$ & $<0.05$ & $<0.05$ & $<0.05$ & $<0.05$ & $<0.05$ & $<0.05$ \\
\hline Silver & $<0.006$ & $<0.006$ & $<0.006$ & $<0.006$ & $<0.006$ & $<0.006$ & $<0.006$ & $<0.006$ \\
\hline Strontium & 0.22 & 0.21 & 0.2 & 0.2 & 0.22 & 0.23 & 0.24 & 0.24 \\
\hline Thorium & $<0.2$ & $<0.2$ & $<0.2$ & $<0.2$ & $<0.2$ & $<0.2$ & $<0.2$ & $<0.2$ \\
\hline Uranium (Fluor) & $<0.001$ & $<0.001$ & $<0.001$ & $<0.001$ & $<0.001$ & $<0.001$ & $<0.001$ & $<0.001$ \\
\hline Vanadium & $<0.005$ & $<0.005$ & $<0.005$ & $<0.005$ & $<0.005$ & $<0.005$ & $<0.005$ & $<0.005$ \\
\hline zine & 0.035 & 0.028 & 0.016 & 0.019 & 0.0091 & 0.01 & 0.0092 & 0.0083 \\
\hline MAJOR IONS (mg/L) & . & . & . & . & . & . & . & • \\
\hline Alkalinity-HCO3 & 310 & . & 318 & . & 323 & . & 323 & . \\
\hline Alkalinity-CO3 & $<1$ & • & $<1$ & • & $<1$ & - & $<1$ & - \\
\hline Calcium & 110 & 110 & 100 & 100 & 110 & 110 & 120 & 120 \\
\hline Chloride & 46.8 & . & 41 & • & 36 & • & 39 & • \\
\hline Fluoride & 0.2 & $\cdot$ & 0.3 & - & 0.2 & - & 0.2 & • \\
\hline Magnesium & 13 & 13 & 12 & 12 & 11 & 12 & 12 & 13 \\
\hline Manganese & 1.4 & $1 \cdot 3$ & 1.4 & 1.4 & 1.1 & 1.1 & 1.2 & 1.2 \\
\hline Nitrate-N & $<0.2$ & $\cdot$ & $<0.2$ & • & $<0.2$ & . & $<0.2$ & • \\
\hline Potassium & 0.94 & 0.88 & $<0.6$ & 0.73 & 2 & 1.8 & 1.6 & 1.3 \\
\hline Sodium & 11 & 11 & 9.3 & 9.5 & 8 & 8.5 & 10 & 9.5 \\
\hline Sulfate & $<1$ & • & 7 & . & 8.2 & • & 2 & • \\
\hline
\end{tabular}

(CONTINUED) 
APPENDIX E.1

Groundwater Quality Data, 1993

\begin{tabular}{|c|c|c|c|c|c|c|c|c|}
\hline \multirow{4}{*}{$\begin{array}{l}\text { Sampling Point } \\
\text { Location } \\
\text { Date Sampled }\end{array}$} & \multicolumn{8}{|c|}{ GW-764 } \\
\hline & \multicolumn{8}{|c|}{ GRIDE 1} \\
\hline & \multicolumn{2}{|c|}{$01 / 23 / 93$} & \multicolumn{2}{|c|}{$04 / 13 / 93$} & \multicolumn{2}{|c|}{$09 / 12 / 93$} & \multicolumn{2}{|c|}{$11 / 12 / 93$} \\
\hline & TOT & DIS & TOT & DIS & TOT & DrS & TOT & DIS \\
\hline METALS (mg/L) & - & $\cdot$ & - & . & - & - & - & \\
\hline Aluminum & 0.066 & 0.042 & 0.022 & $<0.02$ & 0.17 & 0.067 & 1.5 & 0.022 \\
\hline Antimony & $<0.05$ & $<0.05$ & $<0.05$ & $<0.05$ & $<0.05$ & $<0.05$ & $<0.05$ & $<0.05$ \\
\hline Arsenic & $<0.05$ & $<0.05$ & $<0.05$ & $<0.05$ & $<0.05$ & $<0.05$ & $<0.05$ & $<0.05$ \\
\hline Barium & 0.3 & 0.3 & 0.27 & 0.27 & 0.32 & 0.31 & 0.36 & 0.3 \\
\hline Beryllium & $<0.0003$ & $<0.0003$ & $<0.0003$ & $<0.0003$ & $<0.0003$ & $<0.0003$ & $<0.0003$ & $<0.0003$ \\
\hline Boron & 0.057 & 0.076 & 0.059 & 0.044 & 0.087 & 0.079 & 0.085 & 0.066 \\
\hline Cadmium (AAS) & $<0.002$ & $<0.002$ & $<0.002$ & $<0.002$ & $<0.002$ & $<0.002$ & $<0.002$ & $<0.002$ \\
\hline Cadmium & $<0.003$ & $<0.003$ & $<0.003$ & $<0.003$ & $<0.003$ & $<0.003$ & $<0.003$ & $<0.003$ \\
\hline Chromium (AAS) & $<0.01$ & $<0.01$ & $<0.01$ & $<0.01$ & $<0.01$ & $<0.01$ & $<0.01$ & $<0.01$ \\
\hline Chromium & $<0.01$ & $<0.01$ & $<0.01$ & $<0.01$ & $<0.01$ & $<0.01$ & 0.013 & $<0.01$ \\
\hline Cobalt & $<0.005$ & $<0.005$ & $<0.005$ & $<0.005$ & $<0.005$ & $<0.005$ & $<0.005$ & $<0.005$ \\
\hline Copper & $<0.004$ & $<0.004$ & $<0.004$ & 0.013 & $<0.004$ & $<0.004$ & $<0.004$ & $<0.004$ \\
\hline Iron & 0.19 & 0.15 & 0.11 & 0.096 & 0.4 & 0.21 & 2.8 & 0.16 \\
\hline Lead (AAS) & $<0.004$ & $<0.004$ & $<0.004$ & $<0.004$ & $<0.004$ & $<0.004$ & $<0.004$ & $<0.004$ \\
\hline Mercury (CVAA) & $<0.0002$ & $<0.0002$ & $<0.0002$ & $<0.0002$ & $<0.0002$ & $<0.0002$ & $<0.0002$ & $<0.0002$ \\
\hline Molybdenum & $<0.01$ & $<0.01$ & $<0.01$ & $<0.01$ & $<0.01$ & $<0.01$ & $<0.01$ & $<0.01$ \\
\hline Nickel & $<0.01$ & $<0.01$ & $<0.01$ & $<0.01$ & $<0.01$ & $<0.01$ & $<0.01$ & $<0.01$ \\
\hline Selenium & $<0.05$ & $<0.05$ & $<0.05$ & $<0.05$ & 0.061 & $<0.05$ & $<0.05$ & $<0.05$ \\
\hline Silver & $<0.006$ & $<0.006$ & $<0.006$ & $<0.006$ & $<0.006$ & $<0.006$ & $<0.006$ & $<0.006$ \\
\hline Strontium & 0.45 & 0.45 & 0.39 & 0.39 & 0.46 & 0.45 & 0.52 & 0.46 \\
\hline Thorium & $<0.2$ & $<0.2$ & $<0.2$ & $<0.2$ & $<0.2$ & $<0.2$ & $<0.2$ & $<0.2$ \\
\hline Uranium (Fluor) & $<0.001$ & $<0.001$ & $<0.001$ & $<0.001$ & $<0.001$ & $<0.001$ & 0.001 & 0.001 \\
\hline Vanadium & $<0.005$ & $<0.005$ & $<0.005$ & $<0.005$ & $<0.005$ & $<0.005$ & $<0.005$ & $<0.005$ \\
\hline Zinc & 0.013 & 0.0076 & 0.015 & 0.018 & 0.0077 & 0.0078 & 0.026 & $<0.002$ \\
\hline MAJOR IONS $(\mathrm{mg} / \mathrm{L})$ & . & . & . & . & . & . & . & . \\
\hline Alkalinity- $\mathrm{HCO} 3$ & $18 \dot{6}$ & $\cdot$ & $17 \dot{9}$ & $\cdot$ & 189 & . & $\begin{array}{r}\dot{5}\end{array}$ & • \\
\hline Alkalinity-CO3 & $<1$ &. & $<1$ & . & $<1$ & . & $<1$ & • \\
\hline Calcium & 48 & 48 & 43 & 45 & 52 & 51 & 62 & 46 \\
\hline Chloride & 3 & $\cdot$ & 2.8 & $\cdot$ & 3 & . & 3 & $\cdot$ \\
\hline Fluoride & 0.1 & . & 0.1 & . & 0.1 & . & 0.1 & • \\
\hline Magnesium & 6 & 6 & 5.4 & 5.5 & 6.4 & 6.3 & 7 & 5.9 \\
\hline Manganese & 0.093 & 0.085 & 0.069 & 0.069 & 0.094 & 0.086 & 0.21 & 0.08 \\
\hline Nitrate-N & $<0.2$ & . & $<0.2$ & . & $<0.2$ & . & $<0.2$ & • \\
\hline Potassium & 2.3 & 2.1 & 2.2 & 2.2 & 1.7 & 1.5 & 2.4 & 2 \\
\hline Sodium & 27 & 26 & 24 & 24 & 27 & 27 & 28 & 27 \\
\hline Sulfate & 9.9 & . & 13 & $\cdot$ & 9.7 & • & 10 & • \\
\hline
\end{tabular}

(CONTINUED) 
APPENDIX E.1

Groundwater Quality Data, 1993

\begin{tabular}{|c|c|c|c|c|c|c|c|c|}
\hline \multirow{4}{*}{$\begin{array}{l}\text { Sampling Point } \\
\text { Location } \\
\text { Date Sampled }\end{array}$} & \multicolumn{8}{|c|}{ GW-765 } \\
\hline & \multicolumn{8}{|c|}{ GRIDE 1} \\
\hline & \multicolumn{2}{|c|}{$01 / 25 / 93$} & \multicolumn{2}{|c|}{$04 / 13 / 93$} & \multicolumn{2}{|c|}{$09 / 12 / 93$} & \multicolumn{2}{|c|}{$11 / 19 / 93$} \\
\hline & TOT & DIS & TOT & DIS & TOT & DIS & TOT & DIS \\
\hline METALS $(\mathrm{mg} / \mathrm{L})$ & $\cdot$ & . & $\cdot$ & $\cdot$ & . & . & . & • \\
\hline Aluminum & 21 & 0.19 & 2.9 & 0.031 & 14 & $<0.02$ & 6.5 & 0.083 \\
\hline Antimony & $<0.05$ & $<0.05$ & $<0.05$ & $<0.05$ & $<0.05$ & $<0.05$ & $<0.05$ & $<0.05$ \\
\hline Arsenic & $<0.05$ & $<0.05$ & $<0.05$ & $<0.05$ & $<0.05$ & $<0.05$ & $<0.05$ & $<0.05$ \\
\hline Barium & 0.4 & 0.056 & 0.18 & 0.14 & 0.32 & 0.15 & 0.23 & 0.16 \\
\hline Beryllium & 0.00058 & $<0.0003$ & $<0.0003$ & $<0.0003$ & 0.00084 & $<0.0003$ & 0.00031 & $<0.0003$ \\
\hline Boron & 0.039 & 0.033 & 0.02 & 0.012 & 0.033 & 0.035 & 0.064 & 0.048 \\
\hline Cadmium (AAS) & $<0.002$ & $<0.002$ & $<0.002$ & $<0.002$ & $<0.002$ & $<0.002$ & $<0.002$ & $<0.002$ \\
\hline Cadmium & $<0.003$ & $<0.003$ & $<0.003$ & $<0.003$ & 0.005 & $<0.003$ & $<0.003$ & $<0.003$ \\
\hline Chromium (AAS) & 0.068 & $<0.01$ & $<0.01$ & $<0.01$ & 0.024 & $<0.01$ & 0.022 & $<0.01$ \\
\hline Chromium & 0.047 & $<0.01$ & $<0.01$ & $<0.01$ & 0.025 & $<0.01$ & 0.014 & $<0.01$ \\
\hline Cobalt & 0.012 & $<0.005$ & $<0.005$ & $<0.005$ & 0.011 & $<0.005$ & $<0.005$ & $<0.005$ \\
\hline Copper & $<0.004$ & $<0.004$ & 0.0044 & $<0.004$ & 0.015 & $<0.004$ & $<0.004$ & $<0.004$ \\
\hline Iron & 27 & 0.22 & 3.7 & 0.012 & 24 & $<0.005$ & 10 & 0.073 \\
\hline Lead (AAS) & 0.02 & $<0.004$ & $<0.004$ & $<0.004$ & 0.0088 & $<0.004$ & 0.0041 & $<0.004$ \\
\hline Mercury (CVAA) & $<0.0002$ & $<0.0002$ & $<0.0002$ & $<0.0002$ & $<0.0002$ & $<0.0002$ & $<0.0002$ & $<0.0002$ \\
\hline Molybdenum & $<0.01$ & $<0.01$ & $<0.01$ & $<0.01$ & $<0.01$ & $<0.01$ & $<0.01$ & $<0.01$ \\
\hline Nickel & 0.031 & $<0.01$ & $<0.01$ & $<0.01$ & 0.02 & $<0.01$ & $<0.01$ & $<0.01$ \\
\hline Selenium & $<0.05$ & $<0.05$ & $<0.05$ & $<0.05$ & $<0.05$ & $<0.05$ & $<0.05$ & $<0.05$ \\
\hline Silver & $<0.006$ & $<0.006$ & $<0.006$ & $<0.006$ & $<0.006$ & $<0.006$ & $<0.006$ & $<0.006$ \\
\hline Strontium & 0.16 & 0.069 & 0.13 & 0.13 & 0.25 & 0.14 & 0.16 & 0.15 \\
\hline Thorium & $<0.2$ & $<0.2$ & $<0.2$ & $<0.2$ & $<0.2$ & $<0.2$ & $<0.2$ & $<0.2$ \\
\hline Oranium (Fluor) & $<0.001$ & $<0.001$ & $<0.001$ & $<0.001$ & $<0.001$ & $<0.001$ & $<0.001$ & 0.001 \\
\hline Vanadium & 0.027 & $<0.005$ & $<0.005$ & $<0.005$ & 0.021 & $<0.005$ & 0.0082 & $<0.005$ \\
\hline zinc & 0.058 & 0.007 & 0.023 & 0.017 & 0.043 & 0.005 & 0.019 & $<0.002$ \\
\hline MAJOR IONS (mg/L) & . & • & • & $\cdot$ & $\cdot$ & • & • & $\bullet$ \\
\hline Alkalinity-HCO3 & $26 \dot{8}$ & . & 268 & $\cdot$ & 285 & . & 284 & • \\
\hline Alkalinity- $\mathrm{CO} 3$ & $<1$ & - & $<1$ & . & $<1$ & . & $<1$ & • \\
\hline Calcium & 99 & 54 & 96 & 89 & 100 & 100 & 98 & 99 \\
\hline Chloride & 8.3 & • & 6.9 &. & 9.1 & . & 9 & • \\
\hline Fluoride & 0.1 & - & 0.1 & . & 0.1 & . & 0.1 & . \\
\hline Magnesium & 12 & 3.5 & 9.7 & 8.8 & 12 & 10 & 11 & 9.9 \\
\hline Manganese & 0.82 & 0.027 & 0.29 & 0.17 & 0.64 & 0.37 & 0.36 & 0.27 \\
\hline Nitrate-N & $<0.2$ & • & $<0.2$ & . & $<0.2$ & • & $<0.2$ & • \\
\hline Potassium & 6.4 & 2.2 & 2 & $<0.6$ & 4.4 & $<0.6$ & 3 & 1.1 \\
\hline Sodium & 8.8 & 5.1 & 8.3 & 8 & 9 & 9 & 9.7 & 9.6 \\
\hline Sulfate & 11.8 & • & 13 & . & 10.3 & . & 10 & • \\
\hline
\end{tabular}

(CONTINUED) 
APPENDIX E. 1

Groundwater Quality Data, 1993

\begin{tabular}{|c|c|c|c|c|c|c|c|c|}
\hline \multirow{4}{*}{$\begin{array}{l}\text { Sampling Point } \\
\text { Location } \\
\text { - } \\
\text { Date Sampled }\end{array}$} & \multicolumn{8}{|c|}{ GW-766 } \\
\hline & \multicolumn{8}{|c|}{ GRIDI2 } \\
\hline & \multicolumn{2}{|c|}{$02 / 01 / 93$} & \multicolumn{2}{|c|}{$04 / 15 / 93$} & \multicolumn{2}{|c|}{$08 / 03 / 93$} & \multicolumn{2}{|c|}{$10 / 13 / 93$} \\
\hline & TOT & DIS & TOT & DIS & TOT & DIS & TOT & DIS \\
\hline METALS (mg/I) & . & $\cdot$ & $\cdot$ & $\cdot$ & - & $\cdot$ & - & - \\
\hline Aluminum & 0.12 & 0.072 & 0.049 & 0.025 & 0.029 & 0.032 & 0.063 & 0.039 \\
\hline Antimony & $<0.05$ & $<0.05$ & $<0.05$ & $<0.05$ & $<0.05$ & $<0.05$ & $<0.05$ & $<0.05$ \\
\hline Arsenic & $<0.05$ & $<0.05$ & $<0.05$ & $<0.05$ & $<0.05$ & $<0.05$ & $<0.05$ & $<0.05$ \\
\hline Barium & 0.25 & 0.22 & 0.22 & 0.22 & 0.23 & 0.23 & 0.25 & 0.14 \\
\hline Beryllium & $<0.0003$ & $<0.0003$ & $<0.0003$ & $<0.0003$ & $<0.0003$ & $<0.0003$ & $<0.0003$ & $<0.0003$ \\
\hline Boron & 0.044 & 0.04 & 0.034 & 0.032 & 0.043 & 0.033 & 0.03 & 0.031 \\
\hline Cadmium (AAS) & $<0.002$ & $<0.002$ & $<0.002$ & $<0.002$ & $<0.002$ & $<0.002$ & $<0.002$ & $<0.002$ \\
\hline Cadmium & $<0.003$ & $<0.003$ & $<0.003$ & $<0.003$ & $<0.003$ & $<0.003$ & $<0.003$ & $<0.003$ \\
\hline Chromium (AAS) & $<0.01$ & $<0.01$ & $<0.01$ & $<0.01$ & $<0.01$ & $<0.01$ & $<0.01$ & $<0.01$ \\
\hline Chromium & $<0.01$ & $<0.01$ & $<0.01$ & $<0.01$ & $<0.01$ & $<0.01$ & $<0.01$ & $<0.01$ \\
\hline Cobalt & $<0.005$ & $<0.005$ & $<0.005$ & $<0.005$ & $<0.005$ & $<0.005$ & $<0.005$ & $<0.005$ \\
\hline Copper & $<0.004$ & $<0.004$ & $<0.004$ & $<0.004$ & 0.008 & $<0.004$ & $<0.004$ & $<0.004$ \\
\hline Iron & 0.017 & $<0.005$ & 0.044 & 0.0086 & 0.049 & 0.015 & 0.055 & 0.0074 \\
\hline Lead (AAS) & $<0.004$ & $<0.004$ & $<0.004$ & $<0.004$ & $<0.004$ & $<0.004$ & $<0.004$ & $<0.004$ \\
\hline Mercury (CVAA) & $<0.0002$ & $<0.0002$ & $<0.0002$ & $<0.0002$ & $<0.0002$ & $<0.0002$ & $<0.0002$ & $<0.0002$ \\
\hline Molybdenum & $<0.01$ & $<0.01$ & $<0.01$ & $<0.01$ & $<0.01$ & $<0.01$ & $<0.01$ & $<0.01$ \\
\hline Nickel & $<0.01$ & $<0.01$ & $<0.01$ & $<0.01$ & $<0.01$ & $<0.01$ & $<0.01$ & $<0.01$ \\
\hline selenium & $<0.05$ & $<0.05$ & $<0.05$ & $<0.05$ & $<0.05$ & $<0.05$ & $<0.05$ & $<0.05$ \\
\hline Silver & $<0.006$ & $<0.006$ & $<0.006$ & $<0.006$ & $<0.006$ & $<0.006$ & $<0.006$ & $<0.006$ \\
\hline Strontium & 0.53 & 0.5 & 0.38 & 0.38 & 0.43 & 0.42 & 0.46 & 0.49 \\
\hline Thorium & $<0.2$ & $<0.2$ & $<0.2$ & $<0.2$ & $<0.2$ & $<0.2$ & $<0.2$ & $<0.2$ \\
\hline Uranium (Fluor) & $<0.001$ & $<0.001$ & $<0.001$ & 0.001 & $<0.001$ & 0.001 & $<0.001$ & $<0.001$ \\
\hline Vanadium & $<0.005$ & $<0.005$ & $<0.005$ & $<0.005$ & $<0.005$ & $<0.005$ & $<0.005$ & $<0.005$ \\
\hline zinc & 0.0043 & 0.0055 & 0.0046 & 0.0024 & 0.039 & 0.026 & $<0.002$ & $<0.002$ \\
\hline MAJOR IONS (mg/L) & $\cdot$ & . & . & - & - & - & $\cdot$ & - \\
\hline Alkalinity- $\mathrm{HCO} 3$ & 51 & . & 109 & . & 126 & - & 123 & . \\
\hline Alkalinity-CO3 & 26 & $\cdot$ & 16 & - & 4 & - & 10 & - \\
\hline Calcium & 46 & 42 & 34 & 35 & 35 & 33 & 35 & 22 \\
\hline Chloride & 2.3 & $\cdot$ & 2.5 & • & 3.9 & - & 4 & - \\
\hline Fluoride & $<0.1$ & $\cdot$ & 0.1 & . & $<0.1$ & . & 0.1 & - \\
\hline Magnesium & 6.8 & 6.4 & 6.2 & 6.4 & 7.3 & 7.1 & 6.3 & 4.7 \\
\hline Manganese & 0.014 & 0.0071 & 0.0049 & 0.0032 & 0.0065 & 0.0052 & 0.0067 & $<0.001$ \\
\hline Nitrate-N & $<0.2$ & $\cdot$ & $<0.2$ & $\bullet$ & $<0.2$ & $\cdot$ & $<0.2$ & • \\
\hline Potassium & 4.6 & 5 & 3.8 & 3.8 & 4.4 & 3.6 & 3.9 & 5.4 \\
\hline Sodium & 10 & 10 & 12 & 12 & 12 & 12 & 12 & 13 \\
\hline sulfate & 10.5 & . & 8.7 & • & 14 & . & 9 & • \\
\hline
\end{tabular}

(CONTINUED) 
APPENDIX E.1

Groundwater Quality Data, 1993

\begin{tabular}{|c|c|c|c|c|c|c|c|c|}
\hline \multirow{4}{*}{$\begin{array}{l}\text { Sampling Point } \\
\text { Location } \\
\text { Date Sampled }\end{array}$} & \multicolumn{8}{|c|}{ GW-767 } \\
\hline & \multicolumn{8}{|c|}{ GRIDI2 } \\
\hline & \multicolumn{2}{|c|}{$02 / 02 / 93$} & \multicolumn{2}{|c|}{$04 / 15 / 93$} & \multicolumn{2}{|c|}{$08 / 04 / 93$} & \multicolumn{2}{|c|}{$10 / 13 / 93$} \\
\hline & TOT & DIS & TOT & DIS & TOT & DIS & TOT & DIS \\
\hline METALS $(m g / L)$ & - & • & $\cdot$ & - & . & • & - & $\cdot$ \\
\hline Aluminum & 0.24 & 0.039 & 0.039 & 0.092 & 0.46 & $<0.02$ & 0.027 & $<0.02$ \\
\hline Antimony & $<0.05$ & $<0.05$ & $<0.05$ & $<0.05$ & $<0.05$ & $<0.05$ & $<0.05$ & $<0.05$ \\
\hline Arsenic & $<0.05$ & $<0.05$ & $<0.05$ & $<0.05$ & $<0.05$ & $<0.05$ & $<0.05$ & $<0.05$ \\
\hline Barium & 0.062 & 0.05 & 0.048 & 0.047 & 0.15 & 0.14 & 0.06 & 0.083 \\
\hline Beryllium & $<0.0003$ & $<0.0003$ & $<0.0003$ & $<0.0003$ & $<0.0003$ & $<0.0003$ & $<0.0003$ & $<0.0003$ \\
\hline Boron & 0.019 & 0.024 & 0.014 & 0.016 & 0.0086 & 0.017 & 0.012 & 0.014 \\
\hline Cadmium (AAS) & $<0.002$ & $<0.002$ & $<0.002$ & $<0.002$ & $<0.002$ & $<0.002$ & $<0.002$ & $<0.002$ \\
\hline Cadmium & $<0.003$ & $<0.003$ & $<0.003$ & $<0.003$ & $<0.003$ & $<0.003$ & $<0.003$ & $<0.003$ \\
\hline Chromium (AAS) & 0.017 & $<0.01$ & 0.047 & $<0.01$ & $<0.01$ & $<0.01$ & $<0.01$ & $<0.01$ \\
\hline Chromium & $<0.01$ & $<0.01$ & $<0.01$ & $<0.01$ & $<0.01$ & $<0.01$ & $<0.01$ & $<0.01$ \\
\hline Cobalt & 0.0065 & $<0.005$ & $<0.005$ & $<0.005$ & $<0.005$ & $<0.005$ & $<0.005$ & $<0.005$ \\
\hline Copper & $<0.004$ & $<0.004$ & 0.006 & $<0.004$ & 0.0073 & 0.014 & 0.005 & $<0.004$ \\
\hline Iron & 0.53 & $<0.005$ & 0.051 & 0.028 & 1.3 & 0.016 & 0.024 & 0.014 \\
\hline Lead (AAS) & $<0.004$ & $<0.004$ & $<0.004$ & $<0.004$ & $<0.004$ & $<0.004$ & $<0.004$ & $<0.004$ \\
\hline Mercury (CVAA) & $<0.0002$ & $<0.0002$ & $<0.0002$ & $<0.0002$ & $<0.0002$ & $<0.0002$ & $<0.0002$ & $<0.0002$ \\
\hline Molybdenum & $<0.01$ & $<0.01$ & $<0.01$ & $<0.01$ & $<0.01$ & $<0.01$ & $<0.01$ & $<0.01$ \\
\hline Nickel & $<0.01$ & 0.028 & 0.013 & 0.013 & 0.016 & 0.015 & 0.011 & 0.015 \\
\hline Selenium & $<0.05$ & $<0.05$ & $<0.05$ & $<0.05$ & $<0.05$ & $<0.05$ & $<0.05$ & $<0.05$ \\
\hline Silver & $<0.006$ & $<0.006$ & $<0.006$ & $<0.006$ & $<0.006$ & $<0.006$ & $<0.006$ & $<0.006$ \\
\hline strontium & 0.013 & 0.022 & 0.011 & 0.011 & 0.021 & 0.016 & 0.013 & 0.019 \\
\hline Thorium & $<0.2$ & $<0.2$ & $<0.2$ & $<0.2$ & $<0.2$ & $<0.2$ & $<0.2$ & $<0.2$ \\
\hline Uranium (Fluor) & $<0.001$ & $<0.001$ & $<0.001$ & $<0.001$ & $<0.001$ & $<0.001$ & $<0.001$ & $<0.001$ \\
\hline Vanadium & $<0.005$ & $<0.005$ & $<0.005$ & $<0.005$ & $<0.005$ & $<0.005$ & $<0.005$ & $<0.005$ \\
\hline zinc & 0.017 & 0.012 & 0.029 & 0.036 & 0.16 & 0.18 & 0.08 & 0.2 \\
\hline MAJOR IONS (mg/L) & $\cdot$ & $\cdot$ & $\cdot$ & $\cdot$ & $\dot{\bullet}$ & $\cdot$ & $\cdot$ & • \\
\hline Alkalinity-HCO3 & $\dot{8}$ & $\cdot$ & $\dot{7}$ & . & $\dot{13}$ & • & $\begin{array}{r}\cdot \\
13\end{array}$ & $\cdot$ \\
\hline Alkalinity-CO3 & $<1$ & . & $<1$ & . & $<1$ & - & $<1$ & . \\
\hline Calcium & 2.6 & 5.7 & 2.3 & 2.4 & 3.2 & 2.9 & 3.1 & 3.6 \\
\hline Chloride & 3.2 & . & 2.5 & • & 5.4 & $\cdot$ & 4 & • \\
\hline Fluoride & $<0.1$ & . & $<0.1$ & . & $<0.1$ & . & $<0.1$ & . \\
\hline Magnesium & 1.1 & 1.4 & 1 & 1.1 & 1.4 & 1.2 & 1 & 1.1 \\
\hline Manganese & 0.13 & 0.099 & 0.071 & 0.072 & 0.15 & 0.094 & 0.036 & 0.048 \\
\hline Nitrate-N & $<0.2$ &. & $<0.2$ & . & 0.25 &. & $<0.2$ & • \\
\hline Potassium & 0.95 & 0.85 & $<0.6$ & $<0.6$ & 1.1 & 1.3 & 0.8 & 0.85 \\
\hline Sodium & 2.6 & 3 & 2.4 & 2.3 & 2.9 & 2.8 & 3 & 3 \\
\hline Sulfate & 4.2 & . & 3.4 & . & 9 & $\cdot$ & 3 & . \\
\hline
\end{tabular}

(CONTINUED) 
APPENDIX E. 1

\begin{tabular}{|c|c|c|c|c|c|c|c|c|}
\hline \multirow{4}{*}{$\begin{array}{l}\text { Sampling Point } \\
\text { Location } \\
\text { Date Sampled }\end{array}$} & \multicolumn{8}{|c|}{ GW-768 } \\
\hline & \multicolumn{8}{|c|}{ GRIDI 1} \\
\hline & \multicolumn{2}{|c|}{$01 / 29 / 93$} & \multicolumn{2}{|c|}{$04 / 07 / 93$} & \multicolumn{2}{|c|}{$08 / 04 / 93$} & \multicolumn{2}{|c|}{$10 / 13 / 93$} \\
\hline & TOT & DIS & TOT & DIS & TOT & DIS & TOT & DIS \\
\hline METAIS (mg/L) & - & $\cdot$ & $\cdot 1$ & . & - & . & - & • \\
\hline Aluminum & 0.079 & $<0.02$ & 0.026 & 0.031 & 0.038 & $<0.02$ & 0.021 & $<0.02$ \\
\hline Antimony & $<0.05$ & $<0.05$ & $<0.05$ & $<0.05$ & $<0.05$ & $<0.05$ & $<0.05$ & $<0.05$ \\
\hline Arsenic & $<0.05$ & $<0.05$ & $<0.05$ & $<0.05$ & $<0.05$ & $<0.05$ & $<0.05$ & $<0.05$ \\
\hline Barium & 0.25 & 0.25 & 0.24 & 0.25 & 0.28 & 0.27 & 0.27 & 0.24 \\
\hline Beryllium & 0.00094 & 0.0015 & $<0.0003$ & $<0.0003$ & $<0.0003$ & $<0.0003$ & $<0.0003$ & $<0.0003$ \\
\hline Boron & 0.1 & 0.1 & 0.11 & 0.098 & 0.11 & 0.1 & 0.096 & 0.095 \\
\hline Cadmium (AAS) & $<0.002$ & $<0.002$ & $<0.002$ & $<0.002$ & $<0.002$ & $<0.002$ & $<0.002$ & $<0.002$ \\
\hline Cadmium & $<0.003$ & $<0.003$ & $<0.003$ & $<0.003$ & $<0.003$ & $<0.003$ & $<0.003$ & $<0.003$ \\
\hline Chromium (AAS) & $<0.01$ & $<0.01$ & $<0.01$ & $<0.01$ & $<0.01$ & $<0.01$ & $<0.01$ & $<0.01$ \\
\hline Chromium & $<0.01$ & $<0.01$ & $<0.01$ & $<0.01$ & $<0.01$ & $<0.01$ & $<0.01$ & $<0.01$ \\
\hline Cobalt & $<0.005$ & $<0.005$ & $<0.005$ & $<0.005$ & $<0.005$ & $<0.005$ & $<0.005$ & $<0.005$ \\
\hline Copper & $<0.004$ & $<0.004$ & $<0.004$ & $<0.004$ & 0.0067 & 0.0061 & $<0.004$ & $<0.004$ \\
\hline Iron & 0.13 & $<0.005$ & 0.06 & 0.0095 & 0.062 & 0.0051 & 0.069 & $<0.005$ \\
\hline Lead (AAS) & $<0.004$ & $<0.004$ & $<0.004$ & $<0.004$ & $<0.004$ & $<0.004$ & $<0.004$ & $<0.004$ \\
\hline Mercury (CVAA) & $<0.0002$ & $<0.0002$ & $<0.0002$ & $<0.0002$ & $<0.0002$ & $<0.0002$ & $<0.0002$ & $<0.0002$ \\
\hline Molybdenum & $<0.01$ & $<0.01$ & $<0.01$ & $<0.01$ & $<0.01$ & $<0.01$ & $<0.01$ & $<0.01$ \\
\hline Nickel & 0.012 & $<0.01$ & $<0.01$ & $<0.01$ & $<0.01$ & $<0.01$ & $<0.01$ & $<0.01$ \\
\hline Selentum & $<0.05$ & $<0.05$ & $<0.05$ & $<0.05$ & $<0.05$ & $<0.05$ & $<0.05$ & $<0.05$ \\
\hline Silver & $<0.006$ & $<0.006$ & $<0.006$ & $<0.006$ & $<0.006$ & $<0.006$ & $<0.006$ & $<0.006$ \\
\hline Strontium & 0.61 & 0.62 & 0.63 & 0.64 & 0.68 & 0.67 & 0.67 & 0.67 \\
\hline Thorium & $<0.2$ & $<0.2$ & $<0.2$ & $<0.2$ & $<0.2$ & $<0.2$ & $<0.2$ & $<0.2$ \\
\hline Oranium (Fluor) & 0.001 & $<0.001$ & 0.001 & 0.001 & $<0.001$ & 0.001 & 0.001 & $<0.001$ \\
\hline Vanadium & $<0.005$ & $<0.005$ & $<0.005$ & $<0.005$ & $<0.005$ & $<0.005$ & $<0.005$ & $<0.005$ \\
\hline zinc & 0.0066 & 0.0084 & 0.0072 & 0.0085 & 0.017 & 0.0085 & 0.003 & $<0.002$ \\
\hline MAJOR IONS (mg/L) & . & . & . & $\cdot$ & . & . & . & . \\
\hline Alkalinity-HCO3 & $25 \dot{0}$ & $\cdot$ & 253 &. & 257 & . & $2 \dot{255}$ & . \\
\hline Alkalinity-CO3 & $<1$ & . & $<1$ & $\cdot$ & $<1$ & . & $<1$ & . \\
\hline Calcium & 36 & 36 & 36 & 36 & 40 & 39 & 37 & 36 \\
\hline Chloride & 3.2 &. & 2.9 &. & 3.5 & . & 3 & . \\
\hline Fluoride & 0.4 & . & 0.3 &. & 0.4 & . & 0.4 & . \\
\hline Magnesium & 6.5 & 6.6 & 6.7 & 6.7 & 7.7 & 7.5 & 6.6 & 6.6 \\
\hline Manganese & 0.024 & 0.024 & 0.016 & 0.014 & 0.024 & 0.022 & 0.017 & 0.012 \\
\hline Nitrate-N & $<0.2$ & . & $<0.2$ & $\cdot$ & $<0.2$ & • & $<0.2$ & - \\
\hline Potassium & 2.6 & 2.5 & 2.1 & 2.1 & 2.8 & 2.8 & 2.8 & 2.8 \\
\hline Sodium & 67 & 66 & 66 & 67 & 70 & 69 & 71 & 69 \\
\hline Sulfate & 13 & . & 13 &. & 17 & . & 12 & • \\
\hline
\end{tabular}


APPEYDIX R.1

Groundwater Quality Data, 1993

\begin{tabular}{|c|c|c|c|c|c|c|c|c|}
\hline \multirow{4}{*}{$\begin{array}{l}\text { Sampling Point } \\
\text { Location } \\
\text { Date Sampled }\end{array}$} & \multicolumn{8}{|c|}{ GW-769 } \\
\hline & \multicolumn{8}{|c|}{ GRIDG3 } \\
\hline & \multicolumn{2}{|c|}{$01 / 27 / 93$} & \multicolumn{2}{|c|}{$04 / 19 / 93$} & \multicolumn{2}{|c|}{$09 / 14 / 93$} & \multicolumn{2}{|c|}{$11 / 30 / 93$} \\
\hline & TOT & DIS & TOT & DIS & TOT & DIS & TOT & DIS \\
\hline MRTALS (mg/L) & $\cdot$ & - & - & . & . & . & . & $\cdot$ \\
\hline Aluminum & 0.023 & 0.037 & $<0.02$ & $<0.02$ & $<0.02$ & $<0.02$ & $<0.02$ & $<0.02$ \\
\hline Antimony & $<0.05$ & $<0.05$ & $<0.05$ & $<0.05$ & $<0.05$ & $<0.05$ & $<0.05$ & $<0.05$ \\
\hline Arsenic & $<0.05$ & $<0.05$ & $<0.05$ & $<0.05$ & $<0.05$ & $<0.05$ & $<0.05$ & $<0.05$ \\
\hline Barium & 0.47 & 0.47 & 0.42 & 0.41 & 0.48 & 0.48 & 0.46 & 0.46 \\
\hline Beryllium & $<0.0003$ & $<0.0003$ & $<0.0003$ & $<0.0003$ & $<0.0003$ & $<0.0003$ & $<0.0003$ & $<0.0003$ \\
\hline Boron & 0.05 & 0.065 & 0.054 & 0.047 & 0.073 & 0.068 & 0.074 & 0.061 \\
\hline Cadmiun (AAS) & $<0.002$ & $<0.002$ & $<0.002$ & $<0.002$ & $<0.002$ & $<0.002$ & $<0.002$ & $<0.002$ \\
\hline Cadmium & 0.0041 & $<0.003$ & $<0.003$ & $<0.003$ & $<0.003$ & $<0.003$ & $<0.003$ & $<0.003$ \\
\hline Chromium (AAS) & $<0.01$ & $<0.01$ & $<0.01$ & $<0.01$ & $<0.01$ & $<0.01$ & $<0.01$ & $<0.01$ \\
\hline Chromium & $<0.01$ & $<0.01$ & $<0.01$ & $<0.01$ & $<0.01$ & $<0.01$ & $<0.01$ & $<0.01$ \\
\hline Cobalt & $<0.005$ & $<0.005$ & $<0.005$ & $<0.005$ & $<0.005$ & $<0.005$ & $<0.005$ & $<0.005$ \\
\hline Copper & $<0.004$ & $<0.004$ & $<0.004$ & $<0.004$ & $<0.004$ & $<0.004$ & $<0.004$ & $<0.004$ \\
\hline Iron & 0.19 & 0.14 & 0.12 & 0.12 & 0.17 & 0.13 & 0.19 & 0.11 \\
\hline Lead (AAS) & $<0.004$ & $<0.004$ & $<0.004$ & $<0.004$ & $<0.004$ & $<0.004$ & $<0.004$ & $<0.004$ \\
\hline Mercury (CVAA) & $<0.0002$ & $<0.0002$ & $<0.0002$ & $<0.0002$ & $<0.0002$ & $<0.0002$ & $<0.0002$ & $<0.0002$ \\
\hline Mol ybdenum & $<0.01$ & $<0.01$ & $<0.01$ & $<0.01$ & $<0.01$ & $<0.01$ & $<0.01$ & $<0.01$ \\
\hline Nickel & 0.011 & $<0.01$ & $<0.01$ & $<0.01$ & 0.012 & $<0.01$ & $<0.01$ & $<0.01$ \\
\hline Selenium & $<0.05$ & $<0.05$ & $<0.05$ & $<0.05$ & $<0.05$ & $<0.05$ & $<0.05$ & $<0.05$ \\
\hline silver & $<0.006$ & $<0.006$ & $<0.006$ & $<0.006$ & $<0.006$ & $<0.006$ & $<0.006$ & $<0.006$ \\
\hline Strontium & 0.44 & 0.44 & 0.39 & 0.39 & 0.44 & 0.43 & 0.44 & 0.45 \\
\hline Thorium & $<0.2$ & $<0.2$ & $<0.2$ & $<0.2$ & $<n .2$ & $<0.2$ & $<0.2$ & $<0.2$ \\
\hline Uranlum (Fluor) & $<0.001$ & $<0.001$ & $<0.001$ & $<0.001$ & 0.001 & $<0.001$ & $<0.001$ & $<0.001$ \\
\hline Vanadium & $<0.005$ & $<0.005$ & $<0.005$ & $<0.005$ & $<0.005$ & $<0.005$ & $<0.005$ & $<0.005$ \\
\hline zinc & 0.0051 & 0.0058 & 0.0059 & 0.0055 & $<0.002$ & 0.0053 & 0.0074 & 0.0035 \\
\hline MAJOR IONS $(\mathrm{mg} / \mathrm{L})$ & $\dot{.}$ & . & $\dot{\bullet}$ & $\cdot$ & • & • & $\dot{\bullet}$ & $\cdot$ \\
\hline Alkalinity-HCO3 & $19 \dot{9}$ & . & $191^{\circ}$ & $\cdot$ & 205 & • & 207 & . \\
\hline Alkalinity- $\mathrm{CO} 3$ & $<1$ & . & $<1$ & . & $<1$ & • & $<1$ & . \\
\hline Calcium & 75 & 74 & 68 & 68 & 80 & 79 & 77 & 76 \\
\hline Chloride & 21 & • & 15.6 & - & 18 & - & 21 & . \\
\hline Fluoride & $<0.1$ & • & $<0.1$ & • & $<0.1$ & - & $<0.1$ & . \\
\hline Magnesium & 10 & 10 & 9.2 & 9.2 & 21 & 11 & 11 & 11 \\
\hline Manganese & 0.019 & 0.02 & 0.015 & 0.016 & 0.017 & 0.017 & 0.018 & 0.016 \\
\hline Nitrate-N & $<0.2$ & • & $<0.2$ & • & 0.25 & • & $<0.2$ & . \\
\hline Potassium & 3.1 & 3 & 3.3 & 3.1 & 2.7 & 2.9 & 2.8 & 2.9 \\
\hline Sodium & 6.5 & 6.4 & 6.1 & 6 & 6.7 & 6.6 & 6.9 & 7 \\
\hline Sulfate & 19 & • & 18.1 & . & 21 & . & 17 & . \\
\hline
\end{tabular}

(CONTINUED) 
APPENDIX $\mathrm{B} .1$

\begin{tabular}{|c|c|c|c|c|c|c|c|c|}
\hline \multirow{4}{*}{$\begin{array}{l}\text { Sampling Point } \\
\text { Location } \\
\text { Date sampled }\end{array}$} & \multicolumn{8}{|c|}{$6 w-770$} \\
\hline & \multicolumn{8}{|c|}{ GRIDG3 } \\
\hline & \multicolumn{2}{|c|}{$01 / 27 / 93$} & \multicolumn{2}{|c|}{$04 / 19 / 93$} & \multicolumn{2}{|c|}{$09 / 14 / 93$} & \multicolumn{2}{|c|}{$11 / 29 / 93$} \\
\hline & TOT & DIS & TOT & DIS & TOT & DIS & TOT & DIS \\
\hline METALS (mg/L) & $\cdot$ & $\cdot$ & $\cdot$ & $\cdot$ & - & $\cdot$ & $\cdot$ & • \\
\hline Aluminum & $<0.02$ & 0.065 & 0.9 & $<0.02$ & 0.43 & $<0.02$ & 0.061 & $<0.02$ \\
\hline Antimony & $<0.05$ & $<0.05$ & $<0.05$ & $<0.05$ & $<0.05$ & $<0.05$ & $<0.05$ & $<0.05$ \\
\hline Arsenic & $<0.05$ & $<0.05$ & $<0.05$ & $<0.05$ & $<0.05$ & $<0.05$ & $<0.05$ & $<0.05$ \\
\hline Barium & 0.053 & 0.12 & 0.05 & 0.042 & 0.063 & 0.057 & 0.059 & 0.055 \\
\hline Berglilum & $<0.0003$ & $<0.0003$ & $<0.0003$ & $<0.0003$ & 0.00079 & $<0.0003$ & $<0.0003$ & $<0.0003$ \\
\hline Boron & 0.029 & 0.032 & 0.032 & 0.039 & 0.045 & 0.039 & 0.074 & 0.038 \\
\hline Cadmium (AAS) & $<0,002$ & $<0.002$ & $<0.002$ & $<0,002$ & $<0.002$ & $<0.002$ & $<0.002$ & $<0.002$ \\
\hline Cadmium & $<0.003$ & $<0.003$ & $<0.003$ & $<0.003$ & $<0.003$ & $<0.003$ & $<0.003$ & $<0.003$ \\
\hline Chromium (AAS) & $<0.01$ & $<0.01$ & $<0.01$ & $<0.01$ & $<0.01$ & $<0.01$ & $<0.01$ & $<0.01$ \\
\hline Chromium & $<0.01$ & $<0.01$ & $<0.01$ & $<0.01$ & $<0.01$ & $<0.01$ & $<0.01$ & $<0.01$ \\
\hline Cobalt & $<0.005$ & $<0.005$ & $<0.005$ & $<0.005$ & $<0.005$ & $<0.005$ & $<0.005$ & $<0.005$ \\
\hline Copper & $<0.004$ & $<0.004$ & $<0.004$ & $<0.004$ & 0.16 & $<0.004$ & $<0.004$ & $<0.004$ \\
\hline Iron & $<0.005$ & 0.19 & 1 & $<0.005$ & 0.59 & $<0.005$ & 0.09 & $<0.005$ \\
\hline Laad (AAS) & $<0.004$ & $<0.004$ & $<0.004$ & $<0.004$ & $<0.004$ & $<0.004$ & $<0.004$ & $<0.004$ \\
\hline Mercury (CVAA) & $<0.0002$ & $<0.0002$ & $<0.0002$ & $<0.0002$ & $<0.0002$ & $<0.0002$ & $<0.0002$ & $<0.0002$ \\
\hline Molybdenum & $<0.01$ & $<0.01$ & $<0.01$ & $<0.01$ & $<0.01$ & $<0.01$ & $<0.01$ & $<0.01$ \\
\hline Nickel & $<0.01$ & $<0.01$ & $<0.01$ & $<0.01$ & $<0.01$ & $<0.01$ & $<0.01$ & $<0.01$ \\
\hline Selenium & $<0.05$ & $<0.05$ & $<0.05$ & $<0.05$ & $<0.05$ & $<0.05$ & $<0.05$ & $<0.05$ \\
\hline silver & $<0.006$ & $<0.006$ & $<0.006$ & $<0.006$ & $<0.006$ & $<0.006$ & $<0.006$ & $<0.006$ \\
\hline Strontium & 0.07 & 0.2 & 0.061 & 0.06 & 0.074 & 0.073 & 0.075 & 0.072 \\
\hline Thorium & $<0.2$ & $<0.2$ & $<0.2$ & $<0.2$ & $<0.2$ & $<0.2$ & $<0.2$ & $<0.2$ \\
\hline Uranium (Fluor) & $<0.001$ & $<0.001$ & $<0.001$ & $<0.001$ & $<0.001$ & 0.002 & $<0.001$ & $<0.001$ \\
\hline Vanadium & $<0.005$ & $<0.005$ & $<0.005$ & $<0.005$ & $<0.005$ & $<0.005$ & $<0.005$ & $<0.005$ \\
\hline zinc & 0.0061 & 0.0089 & 0.0092 & 0.0078 & 0.029 & 0.019 & 0.015 & 0.014 \\
\hline MAJOR IONS $(\mathrm{mg} / \mathrm{L})$ & $\dot{.}$ & $\cdot$ & $\cdot$ & $\cdot$ & $\cdot$ & $\cdot$ & $\dot{\bullet}$ & $\dot{\bullet}$ \\
\hline Alkalinity-HCO3 & 133 & $\cdot$ & $11 \dot{2}$ & $\cdot$ & 134 & . & ${ }_{141}^{\circ}$ & • \\
\hline Alkalinity-CO3 & $<1$ &. & $<1$ & . & $<1$ & . & $<1$ & . \\
\hline Calcium & 54 & 70 & 44 & 43 & 63 & 60 & 58 & 54 \\
\hline Chloride & 4.4 &. & 4.4 & . & 5.2 & . & 6 & • \\
\hline Fluoride & 0.2 &. & 0.2 & . & 0.2 & . & 0.1 & • \\
\hline Magnesium & 3.5 & 9.5 & 3.3 & 3.2 & 4.1 & 4 & 3.7 & 3.6 \\
\hline Manganese & $<0.001$ & 0.21 & 0.061 & $<0.001$ & 0.023 & $<0.001$ & 0.0024 & $<0.001$ \\
\hline Nitrate-N & 0.46 & . & 0.51 & . & 0.77 & . & 0.6 & • \\
\hline Potassium & 2 & 1.8 & 2.2 & 2 & 2.9 & 2.3 & 2.1 & 2.1 \\
\hline Sodium & 5.1 & 12 & 4.5 & 4.5 & 5.6 & 5.4 & 5.9 & 5.8 \\
\hline Sulfate & 22 & . & 21.7 & . & 25 & . & 20 & • \\
\hline
\end{tabular}


APPENDIX $\mathrm{E} .1$

Groundwater Quallty Data, 1993

\begin{tabular}{|c|c|c|c|c|c|c|c|c|}
\hline \multirow{4}{*}{$\begin{array}{l}\text { Sampling point } \\
\text { Location } \\
\text { Date sumpled }\end{array}$} & \multicolumn{8}{|c|}{$0 W-771$} \\
\hline & \multicolumn{8}{|c|}{ GRIDCl } \\
\hline & \multicolumn{2}{|c|}{$01 / 23 / 93$} & \multicolumn{2}{|c|}{$04 / 12 / 93$} & \multicolumn{2}{|c|}{$09 / 03 / 93$} & \multicolumn{2}{|c|}{$11 / 11 / 93$} \\
\hline & TOT & DIS & TOT & DIs & TOT & DIs & TOT & DIS \\
\hline IRTALs (mg/L) & - & - & - & - & - & . & . & - \\
\hline Aluminum & 0.039 & 0.023 & 0.068 & 0.039 & 0.18 & $<0.02$ & 0.58 & 0.048 \\
\hline Antimony & $<0.05$ & $<0.05$ & $<0.05$ & $<0.05$ & $<0.05$ & $<0.05$ & $<0.05$ & $<0.05$ \\
\hline Arsenic & $<0.05$ & $<0.05$ & $<0.05$ & $<0.05$ & $<0.05$ & $<0.05$ & $<0.05$ & $<0.05$ \\
\hline Barium & 0.14 & 0.15 & 0.15 & 0.14 & 0.16 & 0.15 & 0.17 & 0.16 \\
\hline Beryllium & $<0.0003$ & $<0.0003$ & $<0.0003$ & $<0.0003$ & $<0.0003$ & $<0.0003$ & 0.0052 & 0.0051 \\
\hline Boron & 0.035 & 0.015 & 0.026 & 0.0095 & 0.017 & 0.055 & 0.076 & 0.054 \\
\hline Cadmium (MAS) & $<0.002$ & $<0.002$ & $<0.002$ & $<0.002$ & $<0.002$ & $<0.002$ & $<0.002$ & $<0.002$ \\
\hline Cadmium & $<0.003$ & $<0.003$ & $<0.003$ & $<0.003$ & $<0.003$ & $<0.003$ & 0.0046 & 0.0051 \\
\hline Chromium (AAs) & $<0.01$ & $<0.01$ & $<0.01$ & $<0.01$ & $<0.01$ & $<0.01$ & $<0.01$ & $<0.01$ \\
\hline Chromium & $<0.01$ & $<0.01$ & $<0.01$ & $<0.01$ & $<0.01$ & $<0.01$ & 0.011 & 0.011 \\
\hline Cobalt & $<0.005$ & $<0.005$ & $<0.005$ & $<0.005$ & $<0.005$ & $<0.005$ & $<0.005$ & 0.0054 \\
\hline Copper & $<0.004$ & $<0.004$ & $<0.004$ & $<0.004$ & $<0.004$ & $<0.004$ & 0.012 & 0.013 \\
\hline Iron & 0.15 & 0.19 & 0.18 & 0.061 & 0.35 & 0.019 & 0.96 & 0.037 \\
\hline Lead (MS) & 0.015 & 0.0042 & $<0.004$ & $<0.004$ & $<0.004$ & $<0.004$ & $<0.004$ & $<0.004$ \\
\hline Mercury (CVMA) & $<0.0002$ & $<0.0002$ & $<0.0002$ & $<0.0002$ & $<0.0002$ & $<0.0002$ & $<0.0002$ & $<0.0002$ \\
\hline Molybdenum & $<0.01$ & $<0.01$ & $<0.01$ & $<0.01$ & $<0.01$ & $<0.01$ & $<0.01$ & $<0.01$ \\
\hline Nickel & $<0.01$ & $<0.01$ & $<0.01$ & $<0.01$ & $<0.01$ & $<0.01$ & 0.011 & $<0.01$ \\
\hline Selenium & $<0.05$ & $<0.05$ & $<0.05$ & $<0.05$ & $<0.05$ & $<0.05$ & $<0.05$ & $<0.05$ \\
\hline silver & $<0.006$ & $<0.006$ & $<0.006$ & $<0.006$ & $<0.006$ & $<0.006$ & 0.0091 & 0.0096 \\
\hline Strontium & 0.14 & 0.16 & 0.15 & 0.15 & 0.16 & 0.15 & 0.16 & 0.16 \\
\hline Thorium & $<0.2$ & $<0.2$ & $<0.2$ & $<0.2$ & $<0.2$ & $<0.2$ & $<0.2$ & $<0.2$ \\
\hline Oranium (Fluor) & $<0.001$ & $<0.001$ & $<0.001$ & $<0.001$ & 0.001 & $<0.001$ & $<0.001$ & $<0.001$ \\
\hline Vanadium & $<0.005$ & $<0.005$ & $<0.005$ & $<0.005$ & $<0.005$ & $<0.005$ & 0.0062 & $<0.005$ \\
\hline 2inc & 0.0063 & 0.0092 & 0.012 & 0.012 & 0.0041 & 0.0056 & 0.012 & 0.0055 \\
\hline MAJOR IONS (mg/L) & . &. & . & . & - & - & - & • \\
\hline Alkalinity-HCO3 & 187 &. & $188^{\circ}$ & $\cdot$ & ${ }_{194}$ & • & 202 & • \\
\hline Alkalinity- $\mathrm{CO} 3$ & $<1$ & . & $<1$ & . & $<1$ & . & $<1$ & • \\
\hline Calcium & 66 & 73 & 71 & 70 & 77 & 77 & 72 & 70 \\
\hline Chloride & 9.9 & $\cdot$ & 9.4 & $\cdot$ & 9 & • & 10.1 & • \\
\hline Fluoride & 0.1 & $\cdot$ & 0.1 & $\cdot$ & 0.1 & . & $<0.1$ & • \\
\hline Magnesium & 6.6 & 7.2 & 6.9 & 6.8 & 7.5 & 7.5 & 7.3 & 7.1 \\
\hline Manganese & 0.2 & 0.24 & 0.23 & 0.21 & 0.24 & 0.24 & 0.28 & 0.25 \\
\hline Nitrate-N & $<0.2$ & . & $<0.2$ & . & $<0.2$ & • & $<0.2$ & • \\
\hline Potassium & 1.6 & 1.1 & 1.2 & 1.1 & 0.68 & 1.2 & 1.2 & 1.3 \\
\hline Sodium & 5.1 & 5.5 & s & 5.1 & 5.4 & 5.4 & 5.4 & 5.4 \\
\hline Sulfate & 16.4 & . & 13.3 & . & 15 & • & 11.7 & • \\
\hline
\end{tabular}

(CONTINUED) 
APPENDIX R.1

Orounanater Quality Data, 1993

\begin{tabular}{|c|c|c|c|c|c|c|c|c|}
\hline \multirow{4}{*}{$\begin{array}{l}\text { Sampling Point } \\
\text { Location } \\
\text { Date sampled }\end{array}$} & \multicolumn{8}{|c|}{$a w-772$} \\
\hline & \multicolumn{8}{|c|}{ GRIDC1 } \\
\hline & \multicolumn{2}{|c|}{$01 / 23 / 93$} & \multicolumn{2}{|c|}{$04 / 12 / 93$} & \multicolumn{2}{|c|}{$09 / 12 / 93$} & \multicolumn{2}{|c|}{$11 / 12 / 93$} \\
\hline & TOT & DIS & TOT & DIS & TOT & DIS & TOT & DIS \\
\hline Mardes $(m g / L)$ & $\cdot$ & $\cdot$ & $\cdot$ & $\cdot$ & $\cdot$ & $\cdot$ & $\cdot$ & - \\
\hline Aluminum & 0.024 & 0.032 & $<0.2$ & $<0.2$ & 0.057 & $<0.02$ & $<0.02$ & $<0.02$ \\
\hline Antimony & $<0.05$ & $<0.05$ & $<0.5$ & $<0.5$ & $<0.05$ & $<0.05$ & $<0.05$ & $<0.05$ \\
\hline Arsenic & $<0.05$ & $<0.05$ & $<0.5$ & $<0.5$ & $<0.05$ & $<0.05$ & $<0.05$ & $<0.05$ \\
\hline Barium & 0.096 & 0.094 & 0.093 & 0.083 & 0.084 & 0.083 & 0.086 & 0.086 \\
\hline Beryllium & 0.0079 & $<0.0003$ & 0.0074 & $<0.003$ & $<0.0003$ & $<0.0003$ & 0.00047 & $<0.0003$ \\
\hline Boron & 0.05 & 0.033 & $<0.04$ & $<0.04$ & 0.059 & 0.039 & 0.028 & 0.11 \\
\hline Cadnitum (MAS) & $<0.002$ & $<0.002$ & $<0.002$ & $<0.002$ & $<0.002$ & $<0.002$ & $<0.002$ & $<0.002$ \\
\hline Cadmium & 0.01 & $<0.003$ & $<0.03$ & $<0.03$ & $<0.003$ & $<0.003$ & $<0.003$ & $<0.003$ \\
\hline Chromium (MAS) & $<0.01$ & $<0.01$ & $<0.01$ & $<0.01$ & $<0.01$ & $<0.01$ & $<0.01$ & $<0.01$ \\
\hline Chromi um & 0.038 & 0.037 & $<0.1$ & $<0.1$ & $<0.01$ & $<0.01$ & $<0.01$ & $<0.01$ \\
\hline Cobalt & 0.069 & 0.063 & 0.056 & 0.051 & 0.056 & 0.06 & 0.056 & 0.055 \\
\hline Copper & $<0.004$ & $<0.004$ & $<0.04$ & $<0.04$ & $<0.004$ & $<0.004$ & $<0.004$ & $<0.004$ \\
\hline Iron & 36 & 37 & 37 & 36 & 34 & 32 & 34 & 34 \\
\hline Lead (MAS) & $<0.004$ & $<0.004$ & $<0.004$ & $<0.004$ & $<0.004$ & $<0.004$ & $<0.004$ & $<0.004$ \\
\hline Mercury (CVAA) & $<0.0002$ & $<0.0002$ & $<0.0002$ & $<0.0002$ & $<0.0002$ & $<0.0002$ & $<0.0002$ & $<0.0002$ \\
\hline Molybdenum & $<0.01$ & $<0.01$ & $<0.1$ & $<0.1$ & $<0.01$ & $<0.01$ & $<0.01$ & $<0.01$ \\
\hline Nickel & 0.013 & $<0.01$ & $<0.1$ & $<0.1$ & $<0.01$ & $<0.01$ & $<0.01$ & $<0.01$ \\
\hline Selenium & $<0.05$ & $<0.05$ & $<0.5$ & $<0.5$ & $<0.05$ & $<0.05$ & $<0.05$ & $<0.05$ \\
\hline silver & 0.0082 & $<0.006$ & $<0.06$ & $<0.06$ & $<0.006$ & $<0.006$ & $<0.006$ & $<0.006$ \\
\hline strontium & 0.047 & 0.041 & 0.047 & 0.044 & 0.035 & 0.035 & 0.04 & 0.039 \\
\hline Thorium & $<0.2$ & $<0.2$ & $<2$ & $<2$ & $<0.2$ & $<0.2$ & $<0.2$ & $<0.2$ \\
\hline Oranium (Fluor) & $<0.001$ & $<0.001$ & $<0.001$ & $<0.001$ & $<0.001$ & $<0,001$ & 0.001 & $<0.001$ \\
\hline Vanadium & 0.0065 & $<0.005$ & $<0.05$ & $<0.05$ & $<0.005$ & $<0.005$ & $<0.005$ & $<0.005$ \\
\hline ine & 0.033 & 0.055 & 0.047 & 0.039 & 0.011 & 0.0064 & 0.05 & 0.055 \\
\hline MAJOR IONS (mg/L) & . & $\cdot$ & $\cdot$ & $\cdot$ & $\cdot$ & . & . & - \\
\hline Alkalinity-HCO3 & 108 & $\cdot$ & 116 & $\cdot$ & $\begin{array}{r}\cdot \\
128\end{array}$ & . & $11 \dot{9}$ & • \\
\hline Alkalinity-CO3 & $<1$ & $\cdot$ & $<1$ & $\cdot$ & $<1$ & $\cdot$ & $<1$ & • \\
\hline Calcium & 12 & 12 & 12 & 12 & 11 & 11 & 13 & 13 \\
\hline Chloride & 55.6 & $\cdot$ & 44.7 & $\cdot$ & 26.7 & $\cdot$ & 29 & • \\
\hline Fluoride & 0.1 & $\cdot$ & 0.1 & $\cdot$ & 0.1 & $\cdot$ & 0.1 & • \\
\hline Magnesium & 5.4 & 5.4 & 5.9 & 6.1 & 5 & 5 & 5.1 & 5.1 \\
\hline Manganese & 13 & 13 & 13 & 12 & 12 & 12 & 12 & 13 \\
\hline Nitrate-N & $<0.2$ & $\cdot$ & $<0.2$ & $\cdot$ & $<0.2$ & $\cdot$ & $<0.2$ & • \\
\hline Potassium & 1.3 & 1.3 & $<6$ & $<6$ & 0.81 & 1.5 & 1.1 & 1.3 \\
\hline Sodiun & 21 & 21 & 22 & 22 & 20 & 20 & 21 & 21 \\
\hline Sulfate & 4.9 & $\cdot$ & 7.3 & $\cdot$ & 2.4 & $\cdot$ & 2 & • \\
\hline
\end{tabular}

(CONT INUED) 
APPENDIX $\mathbf{8} .1$

Groundwater Quallty Data, 1993

\begin{tabular}{|c|c|c|c|c|c|c|c|c|}
\hline \multirow{4}{*}{$\begin{array}{l}\text { Sampling point } \\
\text { Looation } \\
\text { - } \\
\text { Date sampled }\end{array}$} & \multicolumn{8}{|c|}{$6 W-773$} \\
\hline & \multicolumn{8}{|c|}{ GRIDH2 } \\
\hline & \multicolumn{2}{|c|}{$02 / 03 / 93$} & \multicolumn{2}{|c|}{$04 / 20 / 93$} & \multicolumn{2}{|c|}{$08 / 06 / 93$} & \multicolumn{2}{|c|}{$10 / 15 / 93$} \\
\hline & TOT & DIS & TOT & DIs & TOT & DIS & $\operatorname{sor}$ & DIs \\
\hline METALS (mg/L) & - & - & - & • & - & - & - & - \\
\hline Aluninum & 0.023 & 0.02 & $<0.02$ & $<0.02$ & 0.12 & $<0.02$ & $<0.02$ & $<0.02$ \\
\hline Antimony & $<0.05$ & $<0.05$ & $<0.05$ & $<0.05$ & $<0.05$ & $<0.05$ & $<0.05$ & $<0.05$ \\
\hline areente & $<0.05$ & $<0.05$ & $<0.05$ & $<0.05$ & $<0.05$ & $<0.05$ & $<0.05$ & $<0.05$ \\
\hline Barlum & 0.36 & 0.36 & 0.36 & 0.36 & 0.39 & 0.38 & 0.38 & 0.37 \\
\hline Beryl11um & $<0.0003$ & 0.0015 & $<0.0003$ & $<0.0003$ & $<0.0003$ & $<0.0003$ & $<0.0003$ & $<0.0003$ \\
\hline Boron & 0.019 & 0.023 & 0.011 & 0.0092 & 0.026 & 0.013 & 0.015 & 0.041 \\
\hline Cadmium (NAS) & $<0.002$ & $<0.002$ & $<0.002$ & $<0.002$ & $<0.002$ & $<0.002$ & $<0.002$ & $<0.002$ \\
\hline Cadmium & $<0.003$ & $<0.003$ & $<0,003$ & $<0.003$ & $<0.003$ & $<0.003$ & $<0.003$ & $<0.003$ \\
\hline Chromium (AAs) & $<0.01$ & $<0.01$ & $<0.01$ & $<0.01$ & $<0.01$ & $<0.01$ & $<0.01$ & $<0.01$ \\
\hline Chromiun & $<0.01$ & $<0.01$ & $<0.01$ & $<0.01$ & $<0.01$ & $<0.01$ & $<0.01$ & $<0.01$ \\
\hline Cobalt & $<0.005$ & $<0.005$ & $<0.005$ & $<0.005$ & $<0.005$ & $<0.005$ & $<0.005$ & $<0.005$ \\
\hline Copper & $<0.004$ & $<0.004$ & $<0.004$ & $<0.004$ & 0.0067 & $<0.004$ & $<0.004$ & $<0.004$ \\
\hline Iron & 0.11 & 0.085 & 0.048 & 0.036 & 0.15 & 0.046 & 0.066 & 0.054 \\
\hline Lead (MAS) & $<0.004$ & $<0.004$ & $<0.004$ & $<0.004$ & $<0.004$ & $<0.004$ & $<0.004$ & 0.004 \\
\hline Mercury (CVAA) & $<0.0002$ & $<0.0002$ & $<0.0002$ & $<0.0002$ & $<0.0002$ & $<0.0002$ & $<0.0002$ & $<0.0002$ \\
\hline Molybdenum & $<0.01$ & $<0.01$ & $<0.01$ & $<0.01$ & $<0.01$ & $<0.01$ & $<0.01$ & $<0.01$ \\
\hline Nickel & $<0.01$ & $<0.01$ & $<0.01$ & $<0.02$ & $<0.01$ & $<0.01$ & $<0.01$ & $<0.01$ \\
\hline Selentum & $<0.05$ & $<0.05$ & $<0.05$ & $<0.05$ & $<0.05$ & $<0.05$ & $<0.05$ & $<0.05$ \\
\hline silver & $<0.006$ & $<0.006$ & $<0.006$ & $<0.006$ & $<0.006$ & $<0.006$ & $<0.006$ & $<0.006$ \\
\hline strontium & 0.14 & 0.15 & 0.13 & 0.13 & 0.14 & 0.14 & 0.14 & 0.14 \\
\hline Thorium & $<0.2$ & $<0.2$ & $<0.2$ & $<0.2$ & $<0.2$ & $<0.2$ & $<0.2$ & $<0.2$ \\
\hline urantum (Fluor) & $<0.001$ & $<0.001$ & $<0.001$ & $<0.001$ & $<0.001$ & $<0.002$ & $<0.001$ & $<0.001$ \\
\hline Vanadium & $<0.005$ & $<0.005$ & $<0.005$ & $<0,005$ & $<0.005$ & $<0.005$ & $<0.005$ & $<0.005$ \\
\hline $2 \ln c$ & 0.0063 & 0.0074 & 0.0028 & 0.0028 & 0.23 & 0.0098 & 0.0073 & 0.0079 \\
\hline MUOR IONS $(\mathrm{mg} / \mathrm{L})$ & - & • & - & - & - & - & - & . \\
\hline Alkalinity-4co3 & 162 & . & 165 & . & 168 & . & 169 & • \\
\hline Alkalinity-CO3 & $<1$ & - & $<1$ & - & $<1$ & - & $<1$ & . \\
\hline Calcium & 51 & 52 & 51 & 52 & 55 & 54 & 55 & 53 \\
\hline Chloride & 2.8 & • & 3.1 & $\cdot$ & 3.1 & - & 3 & - \\
\hline Pluoride & $<0.1$ & • & 0.1 & • & $<0.1$ & $\cdot$ & 0.1 & • \\
\hline Magnesium & 6.7 & 6.8 & 6.4 & 6.4 & 7.5 & 7.2 & 6.8 & 6.5 \\
\hline Manganese & 0.028 & 0.028 & 0.027 & 0.027 & 0.031 & 0.028 & 0.032 & 0.031 \\
\hline Nitrate-N & $<0.2$ & . & $<0.2$ & • & $<0.2$ & , & $<0.2$ & • \\
\hline Potarisium & 1.1 & 1.2 & 0.87 & $<0.6$ & 1.5 & 1.1 & 1.3 & 1.3 \\
\hline Sod.um & 4.6 & 4.5 & 4.1 & 4.2 & 4.6 & 4.6 & 4.4 & 4.4 \\
\hline Sulliate & 5.5 & . & 8.6 & • & 11 & • & 5 & • \\
\hline
\end{tabular}

( CONTINUES; 
APPENDIX E. 1

Groundwater Quality Data, 1993

\begin{tabular}{|c|c|c|c|c|c|c|c|c|}
\hline \multirow{4}{*}{$\begin{array}{l}\text { Sampling Point } \\
\text { Location } \\
\text { - } \\
\text { Date Sampled }\end{array}$} & \multicolumn{8}{|c|}{$G W-774$} \\
\hline & \multicolumn{8}{|c|}{ GRIDH2 } \\
\hline & \multicolumn{2}{|c|}{$02 / 04 / 93$} & \multicolumn{2}{|c|}{$04 / 20 / 93$} & \multicolumn{2}{|c|}{$08 / 09 / 93$} & \multicolumn{2}{|c|}{$10 / 15 / 93$} \\
\hline & TOT & DIS & TOT & DIS & TOT & DIS & TOT & DIS \\
\hline METALS (mg/r) & $\cdot$ & - & $\cdot$ & - & $\cdot$ & $\cdot$ & $\cdot$ & - \\
\hline Aluminum & 24 & 0.27 & 0.14 & $<0.02$ & 1 & 0.024 & 0.2 & $<0.02$ \\
\hline Antimony & $<0.05$ & $<0.05$ & $<0.05$ & $<0.05$ & $<0.05$ & $<0.05$ & $<0.05$ & $<0.05$ \\
\hline Arsenic & $<0.05$ & $<0.05$ & $<0.05$ & $<0.05$ & $<0.05$ & $<0.05$ & $<0.05$ & $<0.05$ \\
\hline Barium & 1.1 & 0.4 & 0.39 & 0.38 & 0.44 & 0.42 & 0.42 & 0.41 \\
\hline Beryllium & 0.0016 & 0.00046 & $<0.0003$ & $<0.0003$ & $<0.0003$ & $<0.0003$ & $<0.0003$ & $<0.0003$ \\
\hline Boron & 0.056 & 0.017 & 0.023 & 0.0076 & 0.012 & 0.028 & 0.01 & 0.01 \\
\hline Cadmium (AAS) & $<0.002$ & $<0.002$ & $<0.002$ & $<0.002$ & $<0.002$ & $<0.002$ & $<0.002$ & $<0.002$ \\
\hline Cadmium & $<0.003$ & $<0.003$ & $<0.003$ & $<0.003$ & $<0.003$ & $<0.003$ & $<0.003$ & $<0.003$ \\
\hline Chromium (AAS) & 0.089 & $<0.01$ & $<0.01$ & $<0.01$ & $<0.01$ & $<0.01$ & $<0.01$ & $<0.01$ \\
\hline Chromium & 0.067 & $<0.01$ & $<0.01$ & $<0.01$ & $<0.01$ & $<0.01$ & $<0.01$ & $<0.01$ \\
\hline Cobalt & 0.023 & $<0.005$ & $<0.005$ & $<0.005$ & 0.0077 & $<0.005$ & $<0.005$ & $<0.005$ \\
\hline Copper & 0.051 & $<0.004$ & 0.0044 & 0.0074 & 0.012 & 0.0054 & $<0.004$ & $<0.004$ \\
\hline Iron & 32 & 0.34 & 0.19 & $<0.005$ & 2.4 & $<0.005$ & 0.28 & $<0.005$ \\
\hline Lead (AAS) & 0.021 & $<0.004$ & $<0.004$ & $<0.004$ & $<0.004$ & $<0.004$ & $<0.004$ & $<0.004$ \\
\hline Mercury (CVAA) & $<0.0002$ & $<0.0002$ & $<0.0002$ & $<0.0002$ & $<0.0002$ & $<0.0002$ & $<0.0002$ & $<0.0002$ \\
\hline Molybdenum & $<0.01$ & $<0.01$ & $<0.01$ & $<0.01$ & $<0.01$ & $<0.01$ & $<0.01$ & $<0.01$ \\
\hline Nickel & 0.031 & $<0.01$ & $<0.01$ & $<0.01$ & $<0.01$ & $<0.01$ & $<0.01$ & $<0.01$ \\
\hline Selenium & $<0.05$ & $<0.05$ & $<0.05$ & $<0.05$ & $<0.05$ & $<0.05$ & $<0.05$ & $<0.05$ \\
\hline silver & $<0.006$ & $<0.006$ & $<0.006$ & $<0.006$ & $<0.006$ & $<0.006$ & $<0.006$ & $<0.006$ \\
\hline Strontium & 0.14 & 0.12 & 0.1 & 0.1 & 0.11 & 0.11 & 0.11 & 0.12 \\
\hline Thorium & $<0.2$ & $<0.2$ & $<0.2$ & $<0.2$ & $<0.2$ & $<0.2$ & $<0.2$ & $<0.2$ \\
\hline Oranium (Fluor) & 0.001 & $<0.001$ & 0.001 & $<0.002$ & $<0.001$ & $<0.001$ & $<0.001$ & $<0.001$ \\
\hline Vanadium & 0.038 & $<0.005$ & $<0.005$ & $<0.005$ & $<0.005$ & $<0.005$ & $<0.005$ & $<0.005$ \\
\hline zinc & 0.075 & 0.011 & 0.007 & 0.0074 & 0.042 & 0.02 & 0.028 & 0.022 \\
\hline MAJOR IONS $(\mathrm{mg} / \mathrm{I})$ &. &. & . &. & $\cdot$ & $\cdot$ & - & • \\
\hline Alkalinity-HCO3 & 194 & $\cdot$ & 199 & $\cdot$ & 196 & $\cdot$ & 195 & . \\
\hline Alkalinity-CO3 & $<1$ & . & $<1$ & . & $<1$ & . & $<1$ & . \\
\hline Calcium & 74 & 68 & 65 & 64 & 67 & 67 & 69 & 69 \\
\hline Chloride & 3.8 & $\cdot$ & 4.1 & $\cdot$ & 3.9 & • & 4 & • \\
\hline Fluoride & $<0.1$ & $\cdot$ & 0.1 & . & $<0.1$ & . & 0.1 & . \\
\hline Magnesium & 13 & 6.1 & 5.8 & 5.7 & 6.5 & 6.3 & 6 & 6.2 \\
\hline Manganese & 1.8 & 0.054 & 0.034 & 0.017 & 0.1 & 0.026 & 0.041 & 0.027 \\
\hline Nitrate-N & $<0.2$ & $\cdot$ & $<0.2$ & . & $<0.2$ & • & $<0.2$ & • \\
\hline Potassium & 8.4 & 2 & 1.9 & 1.9 & 2.9 & 3 & 2.2 & 2 \\
\hline Sodium & 4.9 & 4.4 & 4.3 & 4.3 & 4.5 & 4.5 & 4.3 & 4.3 \\
\hline Sulfate & 10 &. & 9.9 &. & 13 &. & 6 & • \\
\hline
\end{tabular}


APPENDIX E. 1

Groundwater Quality Data, 1993

\begin{tabular}{|c|c|c|c|c|c|c|c|c|}
\hline \multirow{4}{*}{$\begin{array}{l}\text { Sampling Point } \\
\text { Location } \\
\text { Date Sampled }\end{array}$} & \multicolumn{8}{|c|}{$\mathrm{GW}-775$} \\
\hline & \multicolumn{8}{|c|}{ GRIDH3 } \\
\hline & \multicolumn{2}{|c|}{$02 / 04 / 93$} & \multicolumn{2}{|c|}{$04 / 20 / 93$} & \multicolumn{2}{|c|}{$08 / 09 / 93$} & \multicolumn{2}{|c|}{$10 / 15 / 93$} \\
\hline & TOT & DIS & TOT & DIS & TOT & DIS & TOT & DIS \\
\hline METALS (mg/L) & • & - & - & $\cdot$ & - & - & • & - \\
\hline Aluminum & 0.06 & 0.057 & 0.032 & $<0.02$ & 0.025 & $<0.02$ & $<0.02$ & 0.027 \\
\hline Antimony & $<0.05$ & $<0.05$ & $<0.05$ & $<0.05$ & $<0.05$ & $<0.05$ & $<0.05$ & $<0.05$ \\
\hline Arsenic & $<0.05$ & $<0.05$ & $<0.05$ & $<0.05$ & $<0.05$ & $<0.05$ & $<0.05$ & $<0.05$ \\
\hline Barium & 0.16 & 0.16 & 0.16 & 0.15 & 0.16 & 0.16 & 0.17 & 0.16 \\
\hline Beryllium & $<0.0003$ & $<0.0003$ & $<0.0003$ & $<0.0003$ & $<0.0003$ & $<0.0003$ & $<0.0003$ & $<0.0003$ \\
\hline Boron & 0.047 & 0.027 & 0.018 & 0.024 & 0.03 & 0.026 & 0.027 & 0.04 \\
\hline Cadmium (AAS) & $<0.002$ & $<0.002$ & $<0.002$ & $<0.002$ & $<0.002$ & $<0.002$ & $<0.002$ & $<0.002$ \\
\hline Cadmium & $<0.003$ & $<0.003$ & $<0.003$ & $<0.003$ & $<0.003$ & $<0.003$ & $<0.003$ & $<0.003$ \\
\hline Chromium (AAS) & $<0.01$ & $<0.01$ & $<0.01$ & $<0.01$ & $<0.01$ & $<0.01$ & $<0.01$ & $<0.01$ \\
\hline Chromium & $<0.01$ & $<0.01$ & $<0.01$ & $<0.01$ & $<0.01$ & $<0.01$ & $<0.01$ & $<0.01$ \\
\hline Cobalt & $<0.005$ & $<0.005$ & $<0.005$ & $<0.005$ & $<0.005$ & $<0.005$ & $<0.005$ & $<0.005$ \\
\hline Copper & $<0.004$ & 0.0072 & $<0.004$ & $<0.004$ & $<0.004$ & $<0.004$ & $<0.004$ & $<0.004$ \\
\hline Iron & 0.051 & 0.23 & 0.027 & $<0.005$ & 0.17 & $<0.005$ & 0.018 & $<0.005$ \\
\hline Lead (AAS) & $<0.004$ & $<0.004$ & $<0.004$ & $<0.004$ & $<0.004$ & $<0.004$ & $<0.004$ & $<0.004$ \\
\hline Mercury (CVAA) & $<0.0002$ & $<0.0002$ & $<0.0002$ & $<0.0002$ & $<0.0002$ & $<0.0002$ & $<0.0002$ & $<0.0002$ \\
\hline Molybdenum & $<0.01$ & $<0.01$ & $<0.01$ & $<0.01$ & $<0.01$ & $<0.01$ & $<0.01$ & $<0.01$ \\
\hline Nickel & $<0.01$ & $<0.01$ & $<0.01$ & $<0.01$ & $<0.01$ & $<0.01$ & $<0.01$ & $<0.01$ \\
\hline Selenium & $<0.05$ & $<0.05$ & $<0.05$ & $<0.05$ & $<0.05$ & $<0.05$ & $<0.05$ & $<0.05$ \\
\hline Silver & $<0.006$ & $<0.006$ & $<0.006$ & $<0.006$ & $<0.006$ & $<0.006$ & $<0.006$ & $<0.006$ \\
\hline Strontium & 0.2 & 0.19 & 0.19 & 0.18 & 0.2 & 0.2 & 0.2 & 0.2 \\
\hline Thorium & $<0.2$ & $<0.2$ & $<0.2$ & $<0.2$ & $<0.2$ & $<0.2$ & $<0.2$ & $<0.2$ \\
\hline Oranium (Fluor) & $<0.001$ & $<0.001$ & $<0.001$ & 0.001 & $<0.001$ & $<0.001$ & $<0.001$ & 0.002 \\
\hline Vanadium & $<0.005$ & $<0.005$ & $<0.005$ & $<0.005$ & $<0.005$ & $<0.005$ & $<0.005$ & $<0.005$ \\
\hline Zine & 0.012 & 0.0053 & 0.0032 & 0.0036 & 0.0093 & 0.011 & $<0.002$ & $<0.002$ \\
\hline MAJOR IONS (mg/L) & - & - & $\cdot$ & $\cdot$ & - & - & - & • \\
\hline Alkalinity-HCO3 & $157^{\circ}$ & $\cdot$ & 159 & $\cdot$ & $16 \dot{4}$ & . & $168^{\circ}$ & • \\
\hline Alkalinity-CO3 & $<1$ & . & $<1$ & . & $<1$ & . & $<1$ & . \\
\hline Calcium & 71 & 68 & 72 & 71 & 73 & 72 & 70 & 69 \\
\hline Chloride & 22 & . & 27 & $\cdot$ & 22 & • & 41 & • \\
\hline Fluoride & $<0.1$ & . & $<0.1$ & $\cdot$ & $<0.1$ & • & $<0.1$ & . \\
\hline Magnesium & 6.1 & 5.8 & 6 & 5.9 & 6.7 & 6.6 & 6.6 & 6.6 \\
\hline Manganese & 0.0083 & 0.016 & 0.0033 & 0.0025 & 0.0076 & 0.0076 & 0.0051 & 0.0048 \\
\hline Nitrate-N & 0.35 & • & 0.36 & $\cdot$ & 0.44 & • & 0.4 & • \\
\hline Potassium & 3.8 & 3.9 & 2.2 & 2.1 & 2.7 & 2.5 & 2.6 & 2.5 \\
\hline Sodium & 4 & 3.8 & 3.8 & 3.7 & 3.9 & 4 & 4 & 4.1 \\
\hline Sulfate & 29 & • & 26.9 & . & 27 & . & 25 & • \\
\hline
\end{tabular}

(CONTINUED) 
APPENDIX E. 1

Groundwater Quality Data, 1993

\begin{tabular}{|c|c|c|c|c|c|c|c|c|}
\hline \multirow{4}{*}{$\begin{array}{l}\text { Sampling Point } \\
\text { Location } \\
\text { - } \\
\text { Date Sampled }\end{array}$} & \multicolumn{8}{|c|}{ GW-776 } \\
\hline & \multicolumn{8}{|c|}{ GRIDH 3} \\
\hline & \multicolumn{2}{|c|}{$02 / 04 / 93$} & \multicolumn{2}{|c|}{$04 / 20 / 93$} & \multicolumn{2}{|c|}{$08 / 10 / 93$} & \multicolumn{2}{|c|}{$10 / 18 / 93$} \\
\hline & TOT & DIS & TOT & DIS & TOT & DIS & TOT & DrS \\
\hline METALS (mg/L) & $\cdot$ & $\cdot$ & $\cdot$ & $\cdot$ & - & - & $\cdot$ & - \\
\hline Aluminum & 0.42 & $<0.02$ & 0.67 & $<0.02$ & 0.074 & 0.036 & 0.91 & $<0.02$ \\
\hline Antimony & $<0.05$ & $<0.05$ & $<0.05$ & $<0.05$ & $<0.05$ & $<0.05$ & $<0.05$ & $<0.05$ \\
\hline Arsenic & $<0.05$ & $<0.05$ & $<0.05$ & $<0.05$ & $<0.05$ & $<0.05$ & $<0.05$ & $<0.05$ \\
\hline Barium & 0.074 & 0.067 & 0.073 & 0.066 & 0.076 & 0.076 & 0.087 & 0.076 \\
\hline Beryllium & 0.00059 & 0.00046 & $<0.0003$ & $<0.0003$ & $<0.0003$ & $<0.0003$ & $<0.0003$ & $<0.0003$ \\
\hline Boron & 0.029 & 0.028 & 0.028 & 0.011 & 0.014 & 0.037 & 0.02 & 0.018 \\
\hline Cadmium (AAS) & $<0.002$ & $<0.002$ & $<0.002$ & $<0.002$ & $<0.002$ & $<0.002$ & $<0.002$ & $<0.002$ \\
\hline Cadmium & $<0.003$ & $<0.003$ & $<0.003$ & $<0.003$ & $<0.003$ & $<0.003$ & $<0.003$ & $<0.003$ \\
\hline Chromium (AAS) & $<0.01$ & $<0.01$ & 0.16 & $<0.01$ & 0.018 & $<0.01$ & 0.31 & $<0.01$ \\
\hline Chromium & $<0.01$ & $<0.01$ & 0.13 & $<0.01$ & 0.019 & $<0.01$ & 0.31 & $<0.01$ \\
\hline Cobalt & $<0.005$ & $<0.005$ & 0.006 & $<0.005$ & 0.0089 & 0.0083 & 0.0069 & $<0.005$ \\
\hline Copper & 0.008 & $<0.004$ & 0.009 & $<0.004$ & 0.019 & 0.013 & 0.0063 & $<0.004$ \\
\hline Iron & 0.69 & 0.0075 & 2.2 & 0.033 & 0.74 & 0.029 & 2.8 & $<0.005$ \\
\hline Lead (AAS) & $<0.004$ & $<0.004$ & $<0.004$ & $<0.004$ & $<0.004$ & $<0.004$ & $<0.004$ & $<0.004$ \\
\hline Mercury (CVAA) & $<0.0002$ & $<0.0002$ & $<0.0002$ & $<0.0002$ & $<0.0002$ & $<0.0002$ & $<0.0002$ & $<0.0002$ \\
\hline Molybdenum & $<0.01$ & 0.012 & $<0.01$ & 0.011 & $<0.01$ & $<0.01$ & $<0.01$ & $<0.01$ \\
\hline Nickel & 0.15 & 0.13 & 0.19 & 0.15 & 0.33 & 0.32 & 0.24 & 0.22 \\
\hline Selenium & $<0.05$ & $<0.05$ & $<0.05$ & $<0.05$ & $<0.05$ & $<0.05$ & $<0.05$ & $<0.05$ \\
\hline Silver & $<0.006$ & $<0.006$ & $<0.006$ & $<0.006$ & $<0.006$ & $<0.006$ & $<0.006$ & $<0.006$ \\
\hline Strontium & 0.16 & 0.16 & 0.14 & 0.14 & 0.15 & 0.15 & 0.15 & 0.15 \\
\hline Thorium & $<0.2$ & $<0.2$ & $<0.2$ & $<0.2$ & $<0.2$ & $<0.2$ & $<0.2$ & $<0.2$ \\
\hline Uranium (Fluor) & 0.001 & 0.001 & 0.001 & $<0.001$ & $<0.001$ & $<0.001$ & $<0.001$ & $<0.001$ \\
\hline Vanadium & $<0.005$ & $<0.005$ & $<0.005$ & $<0.005$ & $<0.005$ & $<0.005$ & $<0.005$ & $<0.005$ \\
\hline zinc & 0.033 & 0.027 & 0.013 & 0.014 & 0.05 & 0.064 & 0.038 & 0.029 \\
\hline MAJOR IONS $(\mathrm{mg} / \mathrm{I})$ & $\cdot$ & $\cdot$ & $\cdot$ & $\cdot$ & $\dot{\bullet}$ & $\cdot$ & $\cdot$ & $\dot{\bullet}$ \\
\hline Alkalinity-HCO3 & 198 &. & 191 & • & $\begin{array}{r}\circ \\
197\end{array}$ & • & $\begin{array}{r}\circ \\
198\end{array}$ & $\begin{array}{l}\cdot \\
.\end{array}$ \\
\hline Alkalinity- $\mathrm{CO} 3$ & $<1$ &. & $<1$ & . & $<1$ & . & $<1$ & . \\
\hline Calcium & 92 & 89 & 89 & 88 & 92 & 92 & 94 & 91 \\
\hline Chloride & 27 & - & 41.3 & - & 29 & - & 37.4 & • \\
\hline Fluoride & $<0.1$ &. & $<0.1$ & . & $<0.1$ & . & $<0.1$ & . \\
\hline Magnesium & 5.2 & 5 & 4.6 & 4.4 & 5 & 5.1 & 5 & 4.7 \\
\hline Manganese & 0.037 & 0.012 & 0.066 & 0.0093 & 0.038 & 0.021 & 0.099 & 0.019 \\
\hline Nitrate-N & 1.1 & . & 1.11 & • & 1.3 & • & 1.4 & • \\
\hline Potassium & 3.2 & 3.1 & 2.7 & 2.7 & 3.5 & 3.1 & 3 & 2.8 \\
\hline Sodium & 11 & 11 & 11 & 11 & 11 & 11 & 11 & 10 \\
\hline Sulfate & 40 & . & 40.1 & . & 38 & • & 34.5 & • \\
\hline
\end{tabular}


APPENDIX E.2

VOLATILE ORGANIC COMPOUNDS 
APPENDIX E.2

VOC Data Summary

\begin{tabular}{|c|c|c|c|c|c|}
\hline \multirow{2}{*}{ Compound } & \multicolumn{4}{|c|}{ Number of Samples } & \multirow[b]{2}{*}{ Total } \\
\hline & $\begin{array}{r}\text { First } \\
\text { Quarter }\end{array}$ & $\begin{array}{l}\text { Second } \\
\text { Quarter }\end{array}$ & $\begin{array}{l}\text { Third } \\
\text { Quarter }\end{array}$ & $\begin{array}{l}\text { Fourth } \\
\text { Quarter }\end{array}$ & \\
\hline Total Samples & 94 & 93 & 93 & 93 & 373 \\
\hline Not Detected & 29 & 36 & 50 & 25 & 140 \\
\hline VOCs Detected & 65 & 57 & 43 & 68 & 233 \\
\hline Methylene chloride & 20 & 22 & 10 & 44 & 96 \\
\hline Tetrachloroethene & 24 & 24 & 24 & 24 & 96 \\
\hline Trichloroethene & 24 & 23 & 21 & 22 & 90 \\
\hline Chloroform & 16 & 17 & 17 & 17 & 67 \\
\hline 1,2-Dichloroethene & 14 & 14 & 14 & 15 & 57 \\
\hline 4-Methyl-2-pentanone & 36 & 9 & 1 & 7 & 53 \\
\hline Carbon tetrachloride & 11 & 11 & 11 & 12 & 45 \\
\hline Benzene & 10 & 10 & 11 & 10 & 41 \\
\hline Xylenes & 9 & 9 & 10 & 10 & 38 \\
\hline Acetone & 17 & 2 & 3 & 15 & 37 \\
\hline Toluene & 8 & 8 & 10 & 8 & 34 \\
\hline Ethylbenzene & 9 & 7 & 9 & 8 & 33 \\
\hline 2-Butanone & 4 & 1 & 1 & 16 & 22 \\
\hline 1,1-Dichloroethene & 5 & 5 & 5 & 5 & 20 \\
\hline 1,1-Dichloroethane & 4 & 3 & 4 & 3 & 14 \\
\hline Vinyl chloride & 2 & 3 & 4 & 3 & 12 \\
\hline 1,1,1-Trichloroethane & 1 & 2 & 2 & 1 & 6 \\
\hline 1,2-Dichloroethane & 2 & 1 & 1 & 1 & 5 \\
\hline Vinyl acetate & 3 & 0 & 1 & 0 & 4 \\
\hline Bromoform & $l$ & 0 & 1 & 0 & 2 \\
\hline Carbon disulfide & 0 & 0 & 1 & 1 & 2 \\
\hline Chloromethane & 2 & 0 & 0 & 0 & 2 \\
\hline Styrene & 2 & 0 & 0 & 0 & 2 \\
\hline Bromodichloromethane & 0 & 0 & 0 & 0 & 0 \\
\hline Bromomethane & 0 & 0 & 0 & 0 & 0 \\
\hline Chlorobenzene & 0 & 0 & 0 & 0 & 0 \\
\hline Chlorodibromomethane & 0 & 0 & 0 & 0 & 0 \\
\hline Chloroethane & 0 & 0 & 0 & 0 & 0 \\
\hline 1,2-Dichloropropane & 0 & 0 & 0 & 0 & 0 \\
\hline cis-1,3-Dichloropropene & 0 & 0 & 0 & 0 & 0 \\
\hline trans-1,3-Dichloropropene & 0 & 0 & 0 & 0 & 0 \\
\hline 2-Hexanone & 0 & 0 & 0 & 0 & 0 \\
\hline 1,1,2,2-Tetrachloroethane & 0 & 0 & 0 & 0 & 0 \\
\hline 1,1,2-Trichloroethane & 0 & 0 & 0 & 0 & 0 \\
\hline
\end{tabular}


APPENDIX E. 2

\begin{tabular}{|c|c|c|c|c|c|c|c|c|}
\hline \multirow{3}{*}{$\begin{array}{l}\text { Sampling point } \\
\text { Location } \\
\text { - } \\
\text { Date Sampled }\end{array}$} & \multicolumn{4}{|c|}{ GW-151 } & \multicolumn{4}{|c|}{ GW-168 } \\
\hline & \multicolumn{4}{|c|}{ NHP } & \multicolumn{4}{|c|}{ NHP } \\
\hline & $01 / 26 / 93$ & $04 / 22 / 93$ & $08 / 06 / 93$ & $11 / 02 / 93$ & $01 / 22 / 93$ & $05 / 14 / 93$ & $08 / 19 / 93$ & $11 / 02 / 93$ \\
\hline VOLATILE ORGANICS (ug/L) & - & - & $\cdot$ & $\cdot$ & - & - & $\cdot$ & - \\
\hline Acetone & 200 & 200 & 200 & 400 & 1 & 100 & 100 & 100 \\
\hline Benzene & 100 & 100 & 100 & 200 & su & $5 U$ & 50 & $5 U$ \\
\hline Bromodichloromethane & $10 \%$ & 100 & 100 & 200 & 5u & $5 U$ & 50 & $5 U$ \\
\hline Bromoform & 100 & 100 & 100 & 200 & $5 v$ & 50 & 50 & $5 U$ \\
\hline Bromomethane & 200 & $20 \mathrm{U}$ & 200 & 400 & $10 u$ & $10 \mathrm{U}$ & 100 & $10 \mathrm{U}$ \\
\hline 2-Butanone & 200 & 200 & 200 & $40 U$ & 100 & 100 & 100 & 100 \\
\hline Carbon disulfide & $10 \mathrm{U}$ & $10 \mathrm{U}$ & 100 & 200 & $5 U$ & $5 \mathrm{U}$ & $5 u$ & $5 \mathrm{U}$ \\
\hline Carbon tetrachloride & 220 & 330 & 520 & 610 & 50 & 50 & $5 U$ & 50 \\
\hline Chlorobenzene & 100 & 100 & 100 & 200 & 50 & $5 U$ & 50 & $5 U$ \\
\hline Chlorodibromomethane & 100 & $10 \mathrm{U}$ & $10 \mathrm{U}$ & 200 & $5 \mathrm{U}$ & $5 U$ & $5 v$ & $5 U$ \\
\hline Chloroethane & $20 v$ & $20 U$ & 200 & 400 & 100 & 100 & $10 \mathrm{U}$ & 100 \\
\hline Chloroform & 9 & 15 & 19 & 21 & 50 & $5 U$ & $5 U$ & $5 U$ \\
\hline Chloromethane & 200 & 200 & 200 & 400 & 100 & 100 & $10 \mathrm{U}$ & $1.0 \mathrm{U}$ \\
\hline 1,1-Dichloroethane & 100 & 100 & 100 & 200 & $5 u$ & $5 U$ & $5 U$ & 50 \\
\hline 1,2-Dichloroethane & 100 & 100 & 100 & 200 & $5 v$ & $5 U$ & $5 U$ & $5 U$ \\
\hline 1,1-Dichloroethene & 100 & 100 & 100 & 200 & $5 \mathrm{U}$ & $5 v$ & $5 U$ & 50 \\
\hline 1,2-Dichloroethene & 100 & 100 & 100 & 200 & $5 \mathrm{v}$ & 50 & $5 \mathrm{v}$ & 50 \\
\hline 1,2-Dichloropropane & 100 & 100 & 100 & 200 & $5 \mathrm{v}$ & $5 U$ & su & 50 \\
\hline cis-1,3-Dichloropropene & 100 & 100 & 100 & 200 & $5 v$ & $5 U$ & $5 \mathbf{v}$ & 50 \\
\hline trans-1,3-Dichloropropene & 100 & 100 & 100 & 200 & $5 \mathrm{u}$ & 50 & $5 v$ & 50 \\
\hline Bthylbenzene & 100 & 100 & 100 & 200 & $5 u$ & $5 \mathrm{U}$ & $5 u$ & 50 \\
\hline 2-Hexanone & 200 & 200 & 200 & $40 U$ & $10 \mathrm{U}$ & 100 & 100 & 100 \\
\hline 4-Methyl-2-pentanone & 200 & 200 & 200 & $40 v$ & 2 & 100 & 100 & 100 \\
\hline Methylene chloride & 100 & 100 & 100 & 200 & $5 \mathrm{U}$ & 1. & $5 u$ & $5 \mathrm{U}$ \\
\hline Styrene & 100 & 100 & 100 & 200 & su & $5 u$ & 50 & $5 U$ \\
\hline $1,1,2,2$-Tetrachloroethane & 100 & 100 & 100 & 200 & $5 \mathrm{u}$ & $5 \mathrm{U}$ & $5 \mathbf{u}$ & $5 U$ \\
\hline Tetrachloroethene & 12 & 19 & 21 & 27 & $5 \mathrm{v}$ & $5 \mathrm{U}$ & $5 \mathbf{u}$ & 50 \\
\hline Toluene & 100 & 100 & 100 & 200 & 50 & $5 \mathrm{v}$ & $5 U$ & 50 \\
\hline $1,1,1$-Trichloroethane & 100 & 100 & 100 & 200 & $5 \mathrm{u}$ & 50 & $5 U$ & 50 \\
\hline 1,1,2-Trichloroethane & 100 & 100 & 100 & 200 & 50 & $5 U$ & $5 U$ & SU \\
\hline Trichloroethene & 2 & 3 & $10 \mathrm{v}$ & 4 & $5 u$ & $5 U$ & 5u & $5 U$ \\
\hline vinyl acetate & 200 & 200 & 200 & 200 & 100 & 100 & 100 & 100 \\
\hline vinyl chloride & $20 \mathrm{U}$ & 200 & 200 & 400 & 100 & $10 \mathrm{U}$ & 100 & 100 \\
\hline xylenes & 100 & 100 & 100 & 200 & $5 U$ & $5 U$ & $5 U$ & $5 \mathrm{U}$ \\
\hline
\end{tabular}

(CONTINUED) 
APPENDIX E. 2

Groundwater Quality Data, 1993

\begin{tabular}{|c|c|c|c|c|c|c|c|c|}
\hline \multirow{3}{*}{$\begin{array}{l}\text { Sampling Point } \\
\text { Location } \\
\text { Date Sampled }\end{array}$} & \multicolumn{4}{|c|}{ GW-169 } & \multicolumn{4}{|c|}{$G W-170$} \\
\hline & \multicolumn{4}{|c|}{ EXP } & \multicolumn{4}{|c|}{ EXP } \\
\hline & $01 / 23 / 93$ & $05 / 21 / 93$ & $08 / 19 / 93$ & $11 / 01 / 93$ & $01 / 23 / 93$ & $05 / 24 / 93$ & $08 / 21 / 93$ & $11 / 04 / 93$ \\
\hline VOLATILE ORGANICS $(\mathrm{ug} / \mathrm{L})$ & - & - & - & - & - & - & - & - \\
\hline Acetone & 100 & 100 & 100 & 100 & $10 u$ & 100 & 100 & 100 \\
\hline Benzene & su & $5 \mathrm{U}$ & 50 & $5 \mathrm{U}$ & $5 U$ & $5 U$ & 5U & 50 \\
\hline Bromodich loromethane & $5 u$ & su & 50 & 50 & $5 u$ & $5 U$ & $5 U$ & $5 \mathrm{U}$ \\
\hline Bromoform & $5 U$ & $5 U$ & 50 & 50 & su & $5 \mathrm{U}$ & $5 U$ & $5 \mathrm{U}$ \\
\hline Bromomethane & 100 & 100 & 100 & 100 & 100 & 100 & 100 & 100 \\
\hline 2-Butanone & $10 \mathrm{U}$ & 100 & 100 & 100 & 100 & 3 & 100 & 7 \\
\hline Carbon disulfide & 50 & $5 \mathrm{U}$ & $5 \mathrm{U}$ & 50 & 50 & $5 U$ & $5 U$ & $5 \mathrm{U}$ \\
\hline Carbon tetrachloride & 50 & su & 50 & 50 & 1 & $5 \mathrm{U}$ & 16 & 39 \\
\hline Chlorobenzene & $5 U$ & su & 50 & 50 & $5 U$ & $5 U$ & $5 U$ & $5 \mathrm{U}$ \\
\hline Chlorodibromomethane & $5 U$ & SU & 50 & 50 & 50 & 50 & 5u & $5 u$ \\
\hline Chloroethane & 100 & 100 & 100 & 100 & 100 & 100 & 100 & 100 \\
\hline Chloroform & $5 U$ & $5 U$ & $5 \mathrm{U}$ & 50 & 57 & 59 & 43 & 41 \\
\hline Chloromethane & 100 & 100 & 100 & 100 & 100 & 100 & 200 & 100 \\
\hline 1,1-Dichloroethane & $5 u$ & su & 5u & 50 & $5 U$ & $5 \mathrm{U}$ & 5u & 50 \\
\hline 1,2-Dichloroethane & 50 & $5 U$ & $5 U$ & 50 & 50 & 50 & $5 U$ & 50 \\
\hline 1,1-Dichloroethene & SU & 5u & 50 & 50 & SU & $5 U$ & $5 U$ & su \\
\hline 1,2-Dichloroethene & 50 & su & 50 & 50 & $5 u$ & $5 U$ & $5 U$ & 1 \\
\hline 1,2-Dichloropropane & 50 & 50 & 50 & 5u & 50 & 50 & $5 U$ & 50 \\
\hline cis-1,3-Dichloropropene & 50 & $5 \mathrm{U}$ & 50 & $5 U$ & $5 U$ & $5 U$ & $5 U$ & $5 \mathrm{U}$ \\
\hline trans-1,3-Dich loropropene & su & $5 U$ & 50 & 50 & $5 U$ & $5 U$ & su & 50 \\
\hline Ethylbenzene & su & $5 \mathrm{U}$ & $5 \mathrm{U}$ & 50 & 50 & $5 U$ & 50 & 50 \\
\hline 2-Hexanone & 100 & 100 & 100 & 100 & 100 & $10 \mathrm{U}$ & 100 & 100 \\
\hline 4-Methyl-2-pentanone & 1 & 100 & 100 & 100 & 1 & 100 & 100 & 100 \\
\hline Methylene chloride & 50 & su & 50 & 50 & 2 & 2 & 3 & 1 \\
\hline Styrene & $5 U$ & 5u & 50 & 50 & 50 & 50 & $5 U$ & $5 U$ \\
\hline $1,1,2,2$-Tetrachloroethane & $5 U$ & su & 50 & 50 & $5 u$ & 50 & 50 & 50 \\
\hline Tetrachloroethene & 3 & 3 & 3 & 3 & 7 & 6 & 6 & 6 \\
\hline Tolvene & 50 & $5 U$ & 50 & 50 & 50 & 50 & su & $5 \mathrm{U}$ \\
\hline $1,1,1$-Trichloroethane & 50 & 50 & 50 & 50 & $5 U$ & 50 & $5 \mathrm{U}$ & $5 U$ \\
\hline $1,1,2-\operatorname{Tr} 1$ chloroethane & 50 & $5 U$ & 50 & 50 & 50 & $5 U$ & 50 & $5 U$ \\
\hline Trichloroethene & 3 & 3 & 2 & 2 & 2 & 2 & 3 & 2 \\
\hline Vinyl acetate & $10 \mathrm{U}$ & 100 & 100 & 100 & 100 & 100 & $10 U$ & 100 \\
\hline Vinyl chloride & 100 & 100 & 100 & 100 & 100 & 100 & 100 & 100 \\
\hline Xylenes & 50 & 50 & 50 & 50 & $5 U$ & $5 U$ & $5 U$ & 50 \\
\hline
\end{tabular}

(CONTINUED) 
APPENDIX E.2

\begin{tabular}{|c|c|c|c|c|c|c|c|c|}
\hline \multirow{3}{*}{$\begin{array}{l}\text { Sampling Point } \\
\text { - } \\
\text { Location } \\
\text { Date Sampled }\end{array}$} & \multicolumn{4}{|c|}{ GW-183 } & \multicolumn{4}{|c|}{ GW-190 } \\
\hline & \multicolumn{4}{|c|}{ FF } & \multicolumn{4}{|c|}{ RG } \\
\hline & $03 / 10 / 93$ & $06 / 21 / 93$ & $09 / 22 / 93$ & $11 / 16 / 93$ & $03 / 01 / 93$ & $06 / 17 / 93$ & $09 / 15 / 93$ & $11 / 16 / 93$ \\
\hline VOIATILE ORGANICS (ug/L) & • & - & - & - & $\cdot$ & $\cdot$ & - & - \\
\hline Acetone & 20 & $10 u$ & 100 & 100 & 100 & $10 \mathrm{v}$ & $20 U$ & 100 \\
\hline Benzene & 350 & 32 & 57 & 7 & $5 \mathrm{U}$ & $5 \mathrm{u}$ & 100 & 50 \\
\hline Bromodichloromethane & 50 & 50 & $5 U$ & $5 U$ & $5 U$ & 50 & $10 \mathrm{U}$ & $5 U$ \\
\hline Bromoform & $5 \mathrm{U}$ & $5 U$ & $5 \mathrm{U}$ & $5 \mathbf{v}$ & $5 U$ & $5 \mathrm{U}$ & 100 & 50 \\
\hline Bromomethane & 100 & 100 & 100 & 100 & 100 & 100 & 200 & 100 \\
\hline 2-Butanone & 100 & 100 & 100 & 100 & 100 & 100 & 200 & 100 \\
\hline Carbon disulfide & $5 u$ & 50 & 50 & $5 \mathbf{u}$ & $5 U$ & 50 & 100 & $5 U$ \\
\hline Carbon tetrachloride & 50 & 50 & 50 & $5 U$ & $5 U$ & 50 & $10 \mathrm{v}$ & $5 \mathbf{u}$ \\
\hline Chlorobenzene & $5 \mathbf{u}$ & 5u & su & 50 & su & su & 100 & 50 \\
\hline Chlorodibromomethane & $5 u$ & 50 & 50 & 50 & 5u & $5 \mathrm{U}$ & 100 & $5 U$ \\
\hline Chloroethane & 100 & 100 & 100 & 100 & 100 & $10 \mathrm{U}$ & 200 & 100 \\
\hline Chloroform & 5u & 50 & 50 & 50 & 50 & $5 v$ & 100 & 50 \\
\hline Chloromethane & 100 & 100 & 100 & 100 & 100 & 100 & 200 & $10 \mathrm{U}$ \\
\hline 1,1-Dichloroethane & $5 \mathbf{u}$ & $5 U$ & $5 \mathrm{U}$ & $5 \mathbf{U}$ & $5 \mathrm{U}$ & 6 & 170 & 5u \\
\hline 1,2-Dichloroethane & 7 & $5 v$ & 5U & 50 & 50 & 50 & 100 & $5 U$ \\
\hline 1,1-Dichloroethene & $5 u$ & 50 & $5 \mathrm{U}$ & 50 & 5U & 50 & 5 & $5 U$ \\
\hline 1,2-Dichloroethene & $5 u$ & $5 \mathrm{U}$ & $5 u$ & $5 \mathbf{U}$ & 50 & 220 & $10 \mathrm{U}$ & 7 \\
\hline 1,2-Dichloropropane & $5 U$ & 50 & 5U & $5 \mathbf{U}$ & 50 & $5 U$ & 100 & 50 \\
\hline cis-1,3-Dichloropropene & 50 & 50 & 50 & $5 \mathbf{v}$ & $5 v$ & $5 U$ & 100 & 50 \\
\hline trans-1,3-Dichloroprope - & 50 & 50 & $5 \mathbf{v}$ & 50 & 50 & 50 & 100 & 50 \\
\hline Ethylbenzene & 22 & 2 & 5 & 1 & 50 & $5 U$ & 100 & 50 \\
\hline 2-Hexanone & $10 \mathrm{U}$ & 100 & 100 & 100 & 100 & 100 & 200 & 100 \\
\hline 4-Methyl-2-pentanone & 100 & 100 & 100 & 100 & 100 & 100 & 200 & 100 \\
\hline Methylene chloride & 2 & 0.9 & $5 \mathrm{v}$ & 1 & 50 & 50 & 100 & 1 \\
\hline Styrene & 1 & 50 & $5 \mathbf{u}$ & 50 & 50 & $5 U$ & 100 & $5 \mathbf{5}$ \\
\hline $1,1,2,2$-Tetrachloroethane & $5 u$ & $5 \mathrm{U}$ & 50 & 50 & $5 u$ & $5 \mathrm{U}$ & 100 & $5 \mathbf{U}$ \\
\hline Tetrachloroethene & $5 \mathbf{v}$ & $5 U$ & 50 & 50 & 110 & 61 & 89 & 49 \\
\hline Toluene & 180 & 25 & 38 & 6 & 50 & $5 U$ & $10 \mathrm{~J}$ & 50 \\
\hline $1,1,1$-Trichloroethane & 50 & $5 \mathrm{U}$ & 50 & 50 & $5 \mathrm{U}$ & 5U & 100 & 50 \\
\hline $1,1,2$-Trichloroethane & $5 \mathrm{v}$ & $5 \mathrm{U}$ & $5 \mathrm{U}$ & 5u & $5 \mathbf{u}$ & $5 U$ & $10 \mathrm{U}$ & $5 U$ \\
\hline Trichloroethene & $5 U$ & 50 & $5 \mathrm{U}$ & $5 v$ & 11 & 67 & 39 & 7 \\
\hline Vinyl acetate & 63 & 100 & 100 & 100 & $10 \mathrm{U}$ & 100 & 200 & 100 \\
\hline Vinyl chloride & 100 & 100 & 100 & 100 & 100 & 57 & 50 & 2 \\
\hline xylenes & 92 & 11 & 21 & 5 & $5 U$ & 50 & 100 & $5 \mathrm{U}$ \\
\hline
\end{tabular}

(CONTINUED) 
APPENDIX E.2

Groundwater Quality Data, 1993

\begin{tabular}{|c|c|c|c|c|c|c|c|c|}
\hline \multirow{3}{*}{$\begin{array}{l}\text { Sampling Point } \\
\text { Location } \\
\text { Date Sampled }\end{array}$} & \multicolumn{4}{|c|}{ GW-191 } & \multicolumn{4}{|c|}{ GW-192 } \\
\hline & \multicolumn{4}{|c|}{ B4 } & \multicolumn{4}{|c|}{ B4 } \\
\hline & $01 / 12 / 93$ & $04 / 12 / 93$ & $07 / 08 / 93$ & $12 / 09 / 93$ & $01 / 14 / 93$ & $04 / 13 / 93$ & $07 / 08 / 93$ & $12 / 09 / 93$ \\
\hline VOLATILE ORGANICS (ug/L) & - & - & - & - & - & - & • & - \\
\hline Acetone & 100 & 100 & 100 & 4 & $10 \mathrm{U}$ & 100 & 100 & 4 \\
\hline Benzene & $5 \mathrm{U}$ & $5 U$ & 50 & $5 \mathrm{U}$ & $5 \mathbf{U}$ & $5 v$ & $5 \mathbf{v}$ & 5U \\
\hline Bromodichloromethane & 50 & $5 v$ & 50 & 50 & 50 & $5 U$ & 50 & 50 \\
\hline Bromoform & $5 \mathrm{U}$ & $5 U$ & 5u & $5 v$ & 50 & $5 \mathrm{v}$ & su & $5 U$ \\
\hline Bromomethane & 100 & 100 & 100 & 100 & 100 & 100 & 100 & 100 \\
\hline 2-Butanone & 100 & 100 & 100 & 9 & 100 & 100 & 100 & 8 \\
\hline Carbon disulfide & 50 & $5 \mathbf{U}$ & 50 & $5 \mathbf{U}$ & 50 & $5 \mathrm{U}$ & $5 u$ & $5 U$ \\
\hline Carbon tetrachloride & 50 & 50 & 50 & 50 & 50 & $5 u$ & $5 U$ & 50 \\
\hline Chlorobenzene & $5 \mathrm{U}$ & 50 & $5 \mathrm{U}$ & 50 & 50 & 50 & $5 \mathrm{U}$ & 50 \\
\hline Chlorodibromomethane & $5 U$ & 50 & $5 v$ & $5 \mathrm{U}$ & 5IJ & $5 \mathrm{U}$ & $5 \mathrm{u}$ & 50 \\
\hline Chloroethane & 100 & 100 & 100 & 100 & 100 & 100 & 100 & 100 \\
\hline Chloroform & 50 & $5 \mathbf{u}$ & $5 U$ & 50 & $5 \mathbf{U}$ & 50 & 50 & 50 \\
\hline Chloromethane & 100 & 100 & 100 & 100 & 100 & 100 & 100 & 100 \\
\hline 1,1-Dichloroethane & $5 \mathrm{v}$ & $5 U$ & 50 & $5 \mathrm{U}$ & 1 & $5 \mathrm{v}$ & $5 u$ & 1 \\
\hline 1,2-Dichloroethane & 50 & 50 & 50 & $5 u$ & $5 \mathrm{U}$ & $5 U$ & $5 \mathrm{U}$ & $\mathbf{5 U}$ \\
\hline 1,1-Dichloroethene & $5 \mathrm{U}$ & 5u & $5 v$ & 50 & $5 u$ & 50 & 50 & 50 \\
\hline 1,2-Dichloroethene & 50 & 50 & 50 & $5 \mathrm{U}$ & 19 & 18 & 9 & 16 \\
\hline 1,2-Dichloropropane & $5 U$ & $5 u$ & 50 & $5 \mathrm{U}$ & 50 & $5 \mathbf{U}$ & 50 & 50 \\
\hline cis-1,3-Dichloropropene & $5 \mathrm{v}$ & $5 v$ & $5 U$ & 50 & $5 \mathrm{U}$ & $5 \mathbf{v}$ & 50 & 50 \\
\hline $\operatorname{trans}-1,3-$ Dichloropropene & $5 \mathrm{U}$ & $5 \mathrm{u}$ & 50 & $5 \mathbf{U}$ & 50 & 50 & 5u & 50 \\
\hline Ethylbenzene & 50 & 50 & 50 & $5 \mathrm{U}$ & 50 & 50 & 50 & 50 \\
\hline 2-Hexanone & $10 \mathrm{U}$ & 100 & 100 & 100 & 100 & 100 & 100 & 100 \\
\hline 4-Methy1-2-pentanone & $10 u$ & 100 & 100 & 1 & 2 & 100 & 100 & 1 \\
\hline Methylene chloride & 50 & $5 v$ & 50 & 2 & $5 \mathrm{U}$ & 50 & 50 & 2 \\
\hline Styrene & su & $5 \mathrm{v}$ & 50 & 50 & 50 & $5 \mathbf{U}$ & $5 v$ & 50 \\
\hline $1,1,2,2$-Tetrachloroethane & 50 & $5 \mathrm{U}$ & 50 & 50 & $5 \mathrm{U}$ & 50 & $5 \mathrm{U}$ & $5 \mathbf{U}$ \\
\hline Tetrachloroethene & 50 & 50 & $5 U$ & $5 U$ & 1 & 1 & 50 & 1 \\
\hline Toluene & 50 & 50 & 50 & $5 U$ & 50 & 50 & $5 \mathrm{U}$ & $5 \mathrm{U}$ \\
\hline $1,1,1$-Trichloroethane & $5 u$ & 50 & 50 & $5 U$ & 50 & $5 \mathrm{U}$ & $5 \mathrm{U}$ & $5 U$ \\
\hline $1,1,2$-Trichloroethane & $5 \mathrm{U}$ & 50 & 50 & $5 U$ & 50 & 50 & $5 U$ & $5 U$ \\
\hline Trichloroethene & $5 \mathrm{U}$ & $5 U$ & $5 U$ & $5 U$ & 3 & 3 & $5 U$ & 3 \\
\hline vinyl acetate & $10 \mathrm{U}$ & 100 & 100 & 100 & 100 & 100 & 100 & 100 \\
\hline vinyl chloride & 100 & 100 & 100 & $10 \mathrm{U}$ & 100 & 100 & 100 & 100 \\
\hline xylenes & $5 U$ & $5 \mathrm{U}$ & $5 U$ & $5 U$ & $5 \mathrm{U}$ & $5 U$ & $5 U$ & SU \\
\hline
\end{tabular}

(CONTINUED) 
APPENDIX E. 2

Groundwater Quality Data, 1993

\begin{tabular}{|c|c|c|c|c|c|c|c|c|}
\hline \multirow{3}{*}{$\begin{array}{l}\text { Sampling Point } \\
\text { Location } \\
\text { Date Sampled }\end{array}$} & \multicolumn{4}{|c|}{ GW-193 } & \multicolumn{4}{|c|}{ GW- 194} \\
\hline & \multicolumn{4}{|c|}{ T2331 } & \multicolumn{4}{|c|}{ B4 } \\
\hline & $03 / 11 / 93$ & $06 / 22 / 93$ & $09 / 23 / 93$ & $11 / 18 / 93$ & $01 / 13 / 93$ & $04 / 13 / 93$ & $07 / 08 / 93$ & $12 / 09 / 93$ \\
\hline VOLATILE ORGANICS (ug/L) & $\cdot$ & $\cdot$ & - & $\cdot$ & - & - & $\cdot$ & - \\
\hline Acetone & 2500 & 2500 & 1000 & 1000 & 100 & $10 \mathrm{U}$ & 100 & 2 \\
\hline Benzene & 2200 & 1400 & 1200 & 2800 & 50 & SU & $5 \mathrm{U}$ & $5 \mathbf{U}$ \\
\hline Bromodichlorome thane & 1300 & $130 \mathrm{U}$ & $50 U$ & 500 & $5 \mathbf{v}$ & $5 \mathbf{U}$ & $5 \mathrm{U}$ & $5 U$ \\
\hline Bromoform & 1300 & 1300 & $50 U$ & 500 & 50 & $5 \mathbf{U}$ & 50 & $5 U$ \\
\hline Bromomethane & 2500 & 2500 & $100 \mathrm{U}$ & 1000 & 100 & $10 \mathrm{u}$ & 100 & $10 \mathrm{U}$ \\
\hline 2-Butanone & 2500 & $250 \mathrm{U}$ & $100 \mathrm{U}$ & 1000 & 100 & 100 & 100 & 8 \\
\hline Carbon disulfide & 1300 & 1300 & 500 & $50 \mathrm{U}$ & 50 & 50 & $5 \mathbf{v}$ & $5 U$ \\
\hline Carbon tetrachloride & 1300 & 1300 & $50 \mathrm{U}$ & 500 & 50 & 50 & 50 & 50 \\
\hline Chlorobenzene & 1300 & 1300 & 500 & 500 & 50 & $5 \mathbf{v}$ & 50 & 50 \\
\hline Chlorodibromomethane & 1300 & 1300 & 500 & $50 \mathrm{U}$ & 50 & so & $5 \mathbf{U}$ & $5 \mathbf{U}$ \\
\hline Chloroethane & 2500 & 2500 & $100 \mathrm{U}$ & 1000 & $10 U$ & 100 & 100 & 100 \\
\hline Chloroform & 1300 & 1300 & 500 & 500 & $5 \mathrm{U}$ & 50 & 50 & $5 U$ \\
\hline Chloromethane & 2500 & 2500 & 1000 & 1000 & $10 \mathrm{U}$ & 100 & 100 & 100 \\
\hline 1,1-Dichloroethane & $130 \mathrm{U}$ & 1300 & 500 & 500 & su & 50 & $5 \mathbf{v}$ & 50 \\
\hline 1,2-Dichloroethane & $130 \mathrm{U}$ & 1300 & 500 & 500 & $5 u$ & su & 5u & 50 \\
\hline 1,1-Dichloroethene & 1300 & 1300 & 500 & 500 & 50 & 50 & $5 \mathbf{v}$ & 50 \\
\hline 1,2-Dichloroethene & 1300 & 1300 & 500 & 500 & $5 \mathbf{v}$ & 50 & 50 & 50 \\
\hline 1,2-Dichloropropane & 1300 & 1300 & 500 & 500 & $5 \mathbf{v}$ & $5 \mathbf{v}$ & 5u & 50 \\
\hline cis-1,3-Dichloropropene & 1300 & 1300 & 500 & $50 \mathrm{U}$ & $5 \mathbf{U}$ & $5 \mathbf{v}$ & $5 \mathrm{U}$ & $5 \mathbf{U}$ \\
\hline trans-1,3-Dichloropropene & $130 \mathrm{U}$ & 1300 & 500 & 500 & $5 \mathbf{u}$ & $5 \mathbf{v}$ & $5 \mathbf{v}$ & 50 \\
\hline Bthylbenzene & 790 & 740 & 520 & 450 & 50 & 50 & $5 U$ & $5 \mathbf{v}$ \\
\hline 2-Hexanone & 2500 & 2500 & 1000 & 1000 & 100 & 100 & 100 & 100 \\
\hline 4-Methyl-2-pentanone & 2500 & 2500 & 1000 & 1000 & 100 & 100 & 100 & 1 \\
\hline Methylene chloride & 34 & 1300 & $50 \mathrm{U}$ & 500 & 50 & $5 \mathbf{u}$ & $5 \mathbf{u}$ & 2 \\
\hline Styrene & 1300 & 1300 & $50 \mathrm{U}$ & 500 & $5 \mathbf{v}$ & $5 \mathbf{v}$ & 50 & 50 \\
\hline $1,1,2,2$-Tetrachloroethane & 1300 & 1300 & 500 & $50 \mathrm{U}$ & $5 \mathrm{u}$ & su & $5 \mathbf{v}$ & SU \\
\hline Tetrachloroethene & 1300 & $130 \mathrm{u}$ & 500 & 500 & 50 & 50 & 50 & 50 \\
\hline Toluene & 370 & 75 & 100 & 700 & $5 v$ & $5 \mathbf{u}$ & $5 \mathbf{v}$ & 50 \\
\hline 1,1,1-Trichloroethane & 1300 & 1300 & $50 \mathrm{U}$ & 500 & $5 v$ & 50 & $5 \mathbf{U}$ & 50 \\
\hline 1,1,2-Trichloroethane & 1300 & 1300 & 500 & $50 u$ & su & $5 \mathbf{U}$ & $5 \mathbf{U}$ & 50 \\
\hline Trichloroethene & 1300 & 1300 & 500 & $50 U$ & $5 U$ & $5 U$ & 50 & 50 \\
\hline Vinyl acetate & 2500 & 2500 & 1000 & $100 \mathrm{U}$ & $10 \mathrm{U}$ & 100 & 100 & 100 \\
\hline Vinyl chloride & 2500 & 2500 & $100 \mathrm{U}$ & $100 \mathrm{U}$ & $10 \mathrm{U}$ & $10 \mathrm{U}$ & 100 & 100 \\
\hline Xylenes & 680 & 320 & 300 & 580 & $5 u$ & $5 \mathrm{v}$ & 50 & 50 \\
\hline
\end{tabular}

(CONTINUED) 
APPENDIX E. 2

Groundwater Quality Data, 1993

\begin{tabular}{|c|c|c|c|c|c|c|c|c|}
\hline \multirow{3}{*}{$\begin{array}{l}\text { Sampling point } \\
\text { - } \\
\text { Location } \\
\text { - } \\
\text { Date sampled }\end{array}$} & \multicolumn{4}{|c|}{ GW-195 } & \multicolumn{4}{|c|}{ GW-199 } \\
\hline & \multicolumn{4}{|c|}{ B4 } & \multicolumn{4}{|c|}{ GRIDI1 } \\
\hline & $01 / 13 / 93$ & $04 / 13 / 93$ & $07 / 08 / 93$ & $12 / 09 / 93$ & $01 / 29 / 93$ & $04 / 07 / 93$ & $08 / 02 / 93$ & $10 / 12 / 93$ \\
\hline VOLATILE ORGANICS (ug/L) & $\cdot$ & • & - & $\cdot$ & • & - & • & - \\
\hline Acetone & 100 & $10 U$ & 100 & 2 & 100 & 100 & 100 & 100 \\
\hline Benzene & 50 & $5 U$ & $5 u$ & 50 & $5 U$ & $5 U$ & $5 U$ & $5 \mathrm{U}$ \\
\hline Bromodichloromethane & $5 U$ & $5 U$ & $5 U$ & 50 & $5 U$ & $5 \mathrm{U}$ & $5 U$ & 50 \\
\hline Bromoform & $5 v$ & $5 \mathbf{u}$ & $5 U$ & 50 & $5 \mathrm{U}$ & $5 U$ & $5 U$ & 50 \\
\hline Bromomethane & 100 & 100 & 100 & 100 & $10 U$ & $10 U$ & $10 \mathrm{U}$ & 100 \\
\hline 2-Butanone & 100 & 100 & 100 & 6 & $10 U$ & $10 U$ & $10 \mathrm{U}$ & 100 \\
\hline Carbon disulfide & $5 \mathbf{v}$ & $5 \mathbf{v}$ & $5 U$ & $5 U$ & $5 U$ & $5 U$ & $5 U$ & 50 \\
\hline Carbon tetrachloride & 50 & $5 v$ & $5 v$ & 50 & $5 U$ & $5 \mathrm{U}$ & $5 \mathrm{U}$ & $5 U$ \\
\hline Chlorobenzene & 50 & $5 U$ & $5 U$ & 50 & 50 & 50 & $5 U$ & $5 U$ \\
\hline Chlorodibromomethane & $5 \mathrm{U}$ & $5 \mathrm{u}$ & su & 50 & $5 U$ & $5 \mathrm{U}$ & 50 & 50 \\
\hline Chloroethane & 100 & $10 U$ & 100 & 100 & 100 & 100 & 100 & 100 \\
\hline Chloroform & $5 U$ & 50 & $5 U$ & 50 & $5 \mathbf{U}$ & 50 & $5 U$ & $5 U$ \\
\hline Chloromethane & 100 & 100 & 100 & 100 & 100 & 100 & 100 & $10 \mathrm{U}$ \\
\hline 1,1-Dichloroethane & 50 & 50 & $5 U$ & $5 U$ & $5 U$ & 50 & $5 \mathbf{U}$ & 50 \\
\hline 1,2-Dichloroethane & $5 U$ & $5 \mathrm{U}$ & $5 \mathrm{U}$ & $5 \mathbf{U}$ & $5 U$ & 50 & $5 \mathbf{u}$ & 50 \\
\hline 1,1-Dichloroethene & $5 U$ & $5 v$ & $5 U$ & $5 \mathrm{U}$ & $5 \mathrm{U}$ & $5 \mathrm{v}$ & $5 U$ & 5U \\
\hline 1,2-Dichloroethene & 50 & 50 & $5 v$ & 50 & 50 & 50 & $5 v$ & 50 \\
\hline 1,2-Dichloropropane & $5 \mathrm{U}$ & $5 \mathbf{v}$ & $5 U$ & $5 \mathbf{v}$ & $5 U$ & $5 \mathrm{U}$ & $5 U$ & $5 U$ \\
\hline cis-1,3-Dichloropropene & $5 U$ & $5 \mathbf{v}$ & $5 \mathbf{U}$ & $5 \mathbf{u}$ & 50 & 50 & $5 U$ & 50 \\
\hline $\operatorname{trans}-1,3-D i c h$ loropropene & $5 \mathrm{U}$ & $5 \mathrm{U}$ & $5 \mathbf{U}$ & 5u & 50 & 50 & 50 & $5 u$ \\
\hline Ethylbenzene & $5 U$ & $5 \mathrm{U}$ & 50 & $5 U$ & $5 \mathrm{U}$ & 50 & $5 u$ & $5 \mathbf{v}$ \\
\hline 2-Hexanone & 100 & 100 & 100 & 100 & 100 & 100 & 100 & 100 \\
\hline 4-Methy1-2-pentanone & 100 & $10 \mathrm{U}$ & 100 & 100 & 100 & 100 & 100 & 100 \\
\hline Methylene chloride & $5 \mathbf{U}$ & 50 & $5 \mathbf{v}$ & 2 & $5 \mathrm{U}$ & $5 \mathrm{U}$ & 0.9 & $5 U$ \\
\hline Styrene & 50 & 50 & $5 \mathbf{5}$ & 5u & $5 U$ & $5 \mathbf{U}$ & 50 & 50 \\
\hline $1,1,2,2$-Tetrachloroethane & 50 & 50 & $5 U$ & $5 \mathbf{u}$ & $5 U$ & $5 v$ & $5 \mathbf{5 u}$ & 50 \\
\hline Tetrachloroethene & 50 & $5 \mathrm{v}$ & $5 U$ & $5 \mathbf{U}$ & $5 \mathrm{U}$ & $5 \mathrm{U}$ & $5 U$ & $5 \mathbf{v}$ \\
\hline Toluene & 50 & $5 \mathrm{U}$ & $5 \mathbf{U}$ & $5 \mathbf{v}$ & $5 \mathrm{U}$ & 50 & $5 U$ & su \\
\hline $1,1,1$-Trichloroethane & $5 v$ & $5 U$ & $5 U$ & 50 & $5 U$ & 50 & 50 & 50 \\
\hline $1,1,2$-Trichloroethane & $5 U$ & $5 \mathrm{U}$ & $5 U$ & 50 & $5 U$ & 50 & 50 & 50 \\
\hline Trichloroethene & $5 \mathbf{5}$ & $5 \mathrm{U}$ & $5 U$ & 50 & 50 & $5 U$ & $5 U$ & $5 \mathbf{U}$ \\
\hline Vinyl acetate & 100 & 100 & 100 & 100 & 100 & $10 \mathrm{U}$ & $10 \mathrm{U}$ & 100 \\
\hline Vinyl chloride & 100 & 100 & 100 & 100 & 100 & $10 \mathrm{U}$ & $10 \mathrm{U}$ & 100 \\
\hline xylenes & $5 U$ & $5 \mathbf{U}$ & 50 & $5 \mathbf{U}$ & $5 U$ & $5 \mathrm{U}$ & $5 \mathrm{U}$ & $5 U$ \\
\hline
\end{tabular}

(CONTINUED) 
APPENDIX E. 2

Groundwater Quality Data, 1993

\begin{tabular}{|c|c|c|c|c|c|c|c|c|}
\hline \multirow{3}{*}{$\begin{array}{l}\text { Sampling Point } \\
\text { Location } \\
\text { - } \\
\text { Date Sampled }\end{array}$} & \multicolumn{4}{|c|}{ GW-204 } & \multicolumn{4}{|c|}{ GW-206 } \\
\hline & \multicolumn{4}{|c|}{ T0134 } & \multicolumn{4}{|c|}{ EXP } \\
\hline & $03 / 11 / 93$ & $06 / 22 / 93$ & $09 / 23 / 93$ & $11 / 16 / 93$ & $01 / 19 / 93$ & $05 / 06 / 93$ & $08 / 12 / 93$ & $10 / 27 / 93$ \\
\hline VOLATIHE ORGANICS (Ug/L) & $\cdot$ & $\cdot$ & $\cdot$ & - & - & - & $\cdot$ & - \\
\hline Acetone & $10 \mathrm{U}$ & 100 & 100 & 100 & 100 & $10 \mathrm{U}$ & 100 & 100 \\
\hline Benzene & $5 \mathbf{v}$ & su & 50 & su & $5 \mathbf{v}$ & $\mathbf{5 u}$ & $\mathbf{5 U}$ & su \\
\hline Bromodichloromethane & $5 v$ & $5 U$ & ovi & $5 v$ & $5 \mathbf{v}$ & su & 50 & $5 \mathbf{u}$ \\
\hline Bromoform & $5 v$ & 50 & 50 & sv & su & $5 u$ & $5 \mathbf{v}$ & $\mathbf{5 u}$ \\
\hline Bromomethane & 100 & 100 & 100 & 100 & 100 & 100 & 100 & 200 \\
\hline 2-Butanone & 100 & 100 & 100 & 100 & 100 & 100 & 100 & 100 \\
\hline Carbon disulfide & $5 \mathbf{5}$ & su & 50 & 50 & 50 & $5 U$ & $5 \mathbf{u}$ & su \\
\hline Carbon tetrachloride & 50 & su & 50 & 50 & 50 & 50 & $5 \mathrm{U}$ & 50 \\
\hline Chlorobenzene & 50 & $5 \mathrm{U}$ & 50 & 50 & 5v & 50 & $5 \mathbf{U}$ & $5 \mathbf{v}$ \\
\hline Chlorodibromomethane & $5 \mathbf{u}$ & $5 \mathrm{U}$ & 50 & 50 & $5 \mathbf{U}$ & SU & $5 \mathbf{5}$ & $\mathbf{5 u}$ \\
\hline Chloroethane & 100 & 100 & 100 & 100 & 100 & 100 & 100 & 100 \\
\hline Chloroform & 50 & 50 & $\mathbf{5 U}$ & $\mathbf{5 u}$ & 50 & 50 & 50 & $\mathbf{5 u}$ \\
\hline Chloromethane & 100 & 100 & 100 & 100 & 100 & 100 & 100 & 100 \\
\hline 1,1-Dichloroethane & 50 & 50 & su & 50 & $5 n$ & 50 & su & 50 \\
\hline 1,2-Dichloroethane & 50 & 50 & 50 & 50 & $5 \mathbf{v}$ & so & 50 & 50 \\
\hline 1,1-Dichloroethene & $5 u$ & 50 & 50 & 50 & 50 & $5 v$ & 50 & 50 \\
\hline 1,2-Dichloroethene & 50 & $5 \mathrm{U}$ & 50 & $5 \mathbf{5}$ & 50 & $5 v$ & $5 \mathbf{u}$ & 50 \\
\hline 1,2-Dichloropropane & $5 \mathbf{u}$ & 50 & 50 & $\mathbf{5 u}$ & 50 & $5 v$ & $\mathbf{5 u}$ & 50 \\
\hline c18-1,3-Dichloropropene & $5 \mathbf{u}$ & 50 & 50 & $5 \mathbf{U}$ & $5 \mathbf{5 u}$ & 50 & 50 & 50 \\
\hline trans-1, 3-Dichloropropene & $5 u$ & $5 \mathbf{v}$ & $5 v$ & 50 & 5u & 50 & 50 & $5 \mathbf{v}$ \\
\hline Ethylbenzene & 2 & 50 & 50 & 2 & 50 & 50 & $5 U$ & 50 \\
\hline 2-Hexanone & 100 & 100 & 100 & 100 & 100 & 100 & 100 & 100 \\
\hline 4-Methy1-2-pentanone & 100 & 100 & 100 & 100 & 100 & 1 & 100 & 100 \\
\hline Methylene chloride & 1 & $5 \mathbf{u}$ & $5 \mathbf{U}$ & 1 & su & 2 & 5u & $\mathbf{5 0}$ \\
\hline styrene & 50 & 50 & 50 & SU & 5v & 50 & 50 & 5v \\
\hline $1,1,2,2$-Tetrachloroethane & 50 & 50 & 50 & SU & 50 & 50 & 50 & 50 \\
\hline Tetrachloroethene & $5 \mathrm{U}$ & 50 & $5 U$ & 50 & 50 & 50 & $\mathbf{5 u}$ & $5 v$ \\
\hline Toluene & $5 u$ & 50 & $5 \mathrm{U}$ & $5 U$ & $5 U$ & 50 & $5 U$ & 50 \\
\hline $1,1,1$-Trichloroethane & $5 \mathrm{U}$ & $5 U$ & $5 \mathbf{U}$ & 50 & 50 & su & 50 & 50 \\
\hline $1,1,2$-Trichloroethane & $5 U$ & $5 \mathrm{U}$ & $5 u$ & su & 50 & 50 & $5 U$ & $5 v$ \\
\hline Trichloroethene & 5U & 50 & 50 & $5 U$ & 50 & 50 & $5 v$ & 5u \\
\hline vinyl acetate & 100 & 100 & 100 & 100 & 100 & 100 & 100 & 100 \\
\hline Vinyl chloride & $10 U$ & 100 & 100 & $10 \mathrm{U}$ & 100 & 100 & 100 & 100 \\
\hline xylenes & $5 U$ & $5 U$ & $5 U$ & 2 & $5 U$ & $5 U$ & su & su \\
\hline
\end{tabular}

(CONTINUED) 
APPENDIX E.2

Groundwater Quality Data, 1993

\begin{tabular}{|c|c|c|c|c|c|c|c|c|}
\hline \multirow{3}{*}{$\begin{array}{l}\text { Sampling Point } \\
\text { Location } \\
\text { Date Sampled }\end{array}$} & \multicolumn{4}{|c|}{ GW-207 } & \multicolumn{4}{|c|}{$G W-208$} \\
\hline & \multicolumn{4}{|c|}{$\operatorname{EXP}$} & \multicolumn{4}{|c|}{$\mathbf{E X P}$} \\
\hline & $01 / 14 / 93$ & $05 / 04 / 93$ & $08 / 11 / 93$ & $10 / 26 / 93$ & $01 / 19 / 93$ & $05 / 06 / 93$ & $08 / 13 / 93$ & $10 / 28 / 93$ \\
\hline VOLATILE ORGANICS (ug/L) & - & - & $\cdot$ & - & - & - & - & - \\
\hline Acetone & 100 & 100 & 100 & $\dot{3}$ & $10 \dot{0}$ & $10 \dot{U}$ & $10 \dot{0}$ & $10 \dot{0}$ \\
\hline Benzene & $5 v$ & 50 & $5 \mathrm{U}$ & $5 u$ & 5u & $5 \mathbf{U}$ & 50 & su \\
\hline Bromodichloromethane & 50 & 50 & 50 & 50 & 50 & $5 U$ & 50 & $5 U$ \\
\hline Bromoform & 5u & 50 & 50 & 5u & 50 & $5 U$ & 50 & 50 \\
\hline Bromomethane & 100 & 100 & 100 & 100 & 100 & 100 & 100 & 100 \\
\hline 2-Butanone & 100 & 100 & 100 & 8 & 100 & 100 & 100 & 100 \\
\hline Carbon disulfide & $5 v$ & $5 u$ & 50 & 50 & 50 & $5 u$ & 50 & su \\
\hline Carbon tetrachloride & sv & 50 & 50 & 50 & 50 & 50 & 50 & $5 U$ \\
\hline Chlorobenzene & 5u & 5u & 50 & 5u & 50 & $5 u$ & su & 50 \\
\hline Chlorodibromomethane & $5 \mathrm{v}$ & 50 & $5 u$ & 50 & 50 & 50 & 50 & 50 \\
\hline Chloroethane & 100 & 100 & 100 & $10 U$ & 100 & 100 & 100 & 100 \\
\hline Chloroform & 50 & 50 & $5 \mathrm{U}$ & $5 U$ & 50 & 50 & 50 & 5U \\
\hline Chloromethane & 100 & 100 & 100 & 100 & 100 & 100 & 100 & 100 \\
\hline 1,1-Dichloroethane & 5u & 50 & 5u & 50 & 50 & $5 \mathbf{u}$ & 50 & 50 \\
\hline 1,2-Dichloroethane & 50 & 50 & 50 & 5U & 50 & 5u & 50 & 50 \\
\hline 1,1-Dichloroethene & 50 & 50 & 50 & 50 & 50 & 50 & $5 v$ & $5 \mathbf{U}$ \\
\hline 1,2-Dichloroethene & 50 & 50 & 50 & 50 & 50 & 50 & 50 & $5 \mathbf{v}$ \\
\hline 1,2-Dichloropropane & 5u & 50 & 50 & 50 & 50 & 50 & 50 & 50 \\
\hline cis-1,3-Dichloropropene & 50 & 5U & 50 & 5u & 50 & 50 & 50 & 50 \\
\hline trans-1, 3-Dichloropropene & $5 \mathrm{u}$ & 50 & 50 & $5 U$ & 50 & 50 & 50 & 50 \\
\hline Ethylbenzene & 5u & 50 & $5 v$ & 5u & 50 & 50 & 50 & 5u \\
\hline 2-Hexanone & 100 & 100 & 100 & 100 & 100 & 100 & 100 & 100 \\
\hline 4-Methy1-2-pentanone & 1 & 100 & 100 & 100 & 100 & 100 & 100 & 100 \\
\hline Methylene chloride & $5 v$ & 50 & 50 & 2 & 5u & 50 & 50 & 50 \\
\hline Styrene & 50 & $5 v$ & 50 & 50 & 5U & 50 & 50 & 50 \\
\hline $1,1,2,2$-Tetrachloroethane & 5u & $5 \mathrm{U}$ & 50 & 50 & 50 & 50 & 50 & Su \\
\hline Tetrachloroethene & 5u & 50 & 50 & 5u & 50 & 50 & 50 & 50 \\
\hline Toluene & $5 \mathrm{u}$ & 50 & 50 & 50 & 50 & 50 & 50 & 50 \\
\hline 1,1,1-Trichloroethane & 50 & 50 & 50 & 50 & 50 & 50 & 50 & 50 \\
\hline 1,1,2-Trichloroethane & 50 & 50 & 50 & 50 & 50 & 50 & $5 \mathbf{U}$ & 50 \\
\hline Trichloroethene & su & 50 & 50 & $5 \mathrm{u}$ & $5 \mathrm{U}$ & 50 & $5 \mathrm{U}$ & su \\
\hline vinyl acetate & 100 & 100 & 100 & $10 u$ & 100 & 100 & 100 & 100 \\
\hline vinyl chloride & 100 & 100 & 100 & 100 & 100 & 100 & 100 & 100 \\
\hline xylenes & su & 50 & $5 \mathrm{v}$ & $5 U$ & $5 U$ & 50 & 50 & 5u \\
\hline
\end{tabular}

(CONTINUED) 
APPENDIX E.2

Groundwater Quality Data, 1993

\begin{tabular}{|c|c|c|c|c|c|c|c|c|}
\hline \multirow{3}{*}{$\begin{array}{l}\text { Sampling Point } \\
\text { Location } \\
\text { - } \\
\text { Date Sampled }\end{array}$} & \multicolumn{4}{|c|}{$G W-220$} & \multicolumn{4}{|c|}{$G W-232$} \\
\hline & \multicolumn{4}{|c|}{ NHP } & \multicolumn{4}{|c|}{ EXP } \\
\hline & $01 / 25 / 93$ & $04 / 22 / 93$ & $08 / 06 / 93$ & $11 / 01 / 93$ & $01 / 26 / 93$ & $05 / 21 / 93$ & $08 / 21 / 93$ & $11 / 01 / 93$ \\
\hline VOIATILE ORGANICS (ug/L) & - & - & - & $\cdot$ & - & - & - & - \\
\hline Acetone & 3 & 200 & 200 & 500 & 100 & $10 \mathrm{U}$ & 100 & 100 \\
\hline Benzene & 100 & 100 & 100 & 250 & 50 & 50 & 5u & 50 \\
\hline Bromodichloromethane & 100 & 100 & 100 & 250 & 50 & 50 & 50 & $5 U$ \\
\hline Bromoform & 100 & 100 & 100 & 250 & 5U & 50 & 5U & 50 \\
\hline Bromomethane & 200 & 200 & 200 & 500 & 100 & 100 & 100 & 100 \\
\hline 2-Butanone & 200 & 200 & 200 & 500 & 100 & 100 & 100 & 100 \\
\hline Carbon disulfide & 100 & 100 & 100 & 250 & $5 U$ & 50 & 5v & 50 \\
\hline Carbon tetrachloride & 320 & 280 & 490 & 400 & 50 & 50 & su & 50 \\
\hline Chlorobenzene & 100 & 100 & 100 & 250 & 50 & 50 & 50 & 50 \\
\hline Chlorodibromomethane & 100 & 100 & 100 & 250 & 50 & $5 U$ & 5U & 50 \\
\hline Chloroethane & 200 & 200 & 200 & 500 & 100 & 100 & 100 & 100 \\
\hline Chloroform & 17 & 14 & 23 & 21 & 50 & 50 & $5 \mathrm{U}$ & 50 \\
\hline Chloromethane & 200 & 200 & 200 & 500 & 100 & 100 & 100 & 100 \\
\hline 1,1-Dichloroethane & 100 & 100 & 100 & 250 & 50 & $5 v$ & 5u & $5 \mathbf{U}$ \\
\hline 1,2-Dichloroethane & 100 & 100 & 100 & 250 & 50 & 50 & 50 & $5 \mathbf{U}$ \\
\hline 1,1-Dichloroethene & 100 & 100 & 100 & 250 & 50 & 50 & 50 & 50 \\
\hline 1,2-Dichloroethene & 100 & 100 & 100 & 250 & 50 & 50 & 50 & 50 \\
\hline 1,2-Dichloropropane & 100 & 100 & 100 & 250 & 50 & $5 U$ & 50 & 50 \\
\hline cis-1,3-Dichloropropene & 100 & 100 & 100 & 250 & 50 & $5 U$ & 50 & 5u \\
\hline trans-1,3-Dichloropropene & 100 & 100 & 100 & 250 & 5U & 50 & 50 & 50 \\
\hline Rthylbenzene & 100 & 100 & 100 & 250 & 50 & 50 & 50 & 50 \\
\hline 2-Hexanone & 200 & 200 & 200 & 500 & 100 & 100 & 100 & 100 \\
\hline 4-Methy1-2-pentanone & 3 & 200 & 200 & 500 & 2 & 100 & 100 & 100 \\
\hline Methylene chloride & 100 & 100 & 100 & 8 & 50 & 5u & 5u & 2 \\
\hline Styrene & 100 & 100 & 100 & 250 & 50 & 50 & 50 & 50 \\
\hline $1,1,2,2$-Tetrachloroethane & 100 & 100 & 100 & 250 & su & $5 U$ & 50 & 50 \\
\hline Tetrachloroethene & 19 & 23 & 32 & 39 & 50 & su & $5 \mathbf{U}$ & $5 U$ \\
\hline Toluene & 100 & 100 & 100 & 250 & 50 & 50 & 50 & 50 \\
\hline $1,1,1$-Trichloroethane & 100 & 200 & 100 & $25 U$ & 50 & 50 & 50 & 50 \\
\hline $1,1,2$-Trichloroethane & 100 & 100 & 100 & 250 & 50 & 50 & 50 & 5u \\
\hline Trichloroethene & 4 & 4 & 6 & 7 & 50 & 50 & 50 & $5 \mathrm{U}$ \\
\hline vinyl acetate & 200 & 200 & 200 & $50 u$ & 100 & $10 \mathrm{U}$ & 100 & $10 \mathrm{U}$ \\
\hline vinyl chloride & 200 & 200 & 200 & 500 & 100 & 100 & 100 & 100 \\
\hline xylenes & 100 & 100 & 100 & $25 \mathrm{U}$ & 50 & $5 U$ & 50 & $\mathbf{5 u}$ \\
\hline
\end{tabular}

(CONTINUED) 
APRENDIX E. 2

\begin{tabular}{|c|c|c|c|c|c|c|c|c|}
\hline \multirow{3}{*}{$\begin{array}{l}\text { Sampling Point } \\
\text { Location } \\
\text { - } \\
\text { Date Sampled }\end{array}$} & \multicolumn{4}{|c|}{ GW-239 } & \multicolumn{4}{|c|}{$G W-240$} \\
\hline & \multicolumn{4}{|c|}{ NHP } & \multicolumn{4}{|c|}{ NHP } \\
\hline & $01 / 23 / 93$ & $05 / 13 / 93$ & $08 / 18 / 93$ & $10 / 30 / 93$ & $01 / 27 / 93$ & $04 / 21 / 93$ & $08 / 05 / 93$ & $11 / 01 / 93$ \\
\hline VOIATILE ORGANICS (ug/L) & - & - & $\cdot$ & $\cdot$ & $\cdot$ & - & - & - \\
\hline Acetone & 100 & 100 & 100 & 100 & $\dot{1}$ & 100 & 100 & 100 \\
\hline Benzene & $5 U$ & 50 & $5 \mathrm{U}$ & $5 U$ & $5 v$ & $5 U$ & $5 \mathbf{u}$ & 50 \\
\hline Bromodichloromethane & 50 & 50 & $5 U$ & $5 \mathrm{U}$ & $5 \mathbf{v}$ & 50 & 50 & 50 \\
\hline Bromoform & 5u & 50 & su & 50 & 50 & 50 & 50 & 50 \\
\hline Bromomethane & 100 & 100 & $10 \mathrm{U}$ & 100 & 100 & 100 & 100 & 100 \\
\hline 2-Butanone & 100 & 100 & 100 & 100 & 100 & 100 & 100 & 100 \\
\hline Carbon disulfide & $5 U$ & $5 \mathbf{v}$ & $5 U$ & 50 & $5 U$ & 50 & $5 U$ & $5 U$ \\
\hline Carbon tetrachloride & 50 & 50 & 50 & 50 & 10 & 8 & 10 & 15 \\
\hline Chlorobenzene & $5 \mathbf{U}$ & 50 & 50 & $5 U$ & $5 \mathrm{U}$ & 50 & $5 \mathbf{U}$ & $5 U$ \\
\hline Chlorodibromomethane & $5 \mathbf{u}$ & 50 & 50 & 50 & $5 U$ & 50 & $5 \mathrm{U}$ & 50 \\
\hline Chloroethane & 100 & 100 & 100 & 100 & 100 & 100 & 100 & 100 \\
\hline Chloroform & $5 U$ & 50 & 50 & 50 & 1 & 0.8 & 2 & 2 \\
\hline Chloromethane & $10 \mathrm{U}$ & $10 \mathrm{u}$ & 100 & 100 & 100 & 100 & 100 & 100 \\
\hline 1,1-Dichloroethane & 50 & $5 \mathrm{U}$ & 50 & 50 & $5 U$ & $5 \mathbf{v}$ & $5 U$ & $\mathbf{5 U}$ \\
\hline 1,2-Dichloroethane & 50 & 50 & 50 & 50 & su & $5 \mathrm{U}$ & $5 \mathrm{U}$ & $5 \mathbf{U}$ \\
\hline 1,1-Dichloroethene & 50 & su & 50 & 50 & 50 & $5 \mathbf{v}$ & 50 & 50 \\
\hline 1,2-Dichloroethene & 50 & $5 \mathrm{U}$ & $5 U$ & 50 & 50 & $5 \mathbf{U}$ & 50 & $5 U$ \\
\hline 1,2-Dichloropropane & 50 & $5 U$ & 50 & 50 & 50 & 50 & 50 & $5 U$ \\
\hline cis-1,3-Dichloropropene & $5 v$ & 50 & $5 v$ & 50 & 50 & 50 & 50 & $5 \mathbf{U}$ \\
\hline trans-1,3-Dichloropropene & $5 \mathbf{U}$ & 50 & 50 & $5 \mathbf{u}$ & $5 \mathbf{u}$ & 50 & 50 & $5 \mathbf{U}$ \\
\hline Ethylbenzene & 50 & $5 u$ & 50 & 50 & 50 & 50 & 50 & $5 U$ \\
\hline 2-Hexanone & 100 & 100 & 100 & 100 & 100 & 100 & 100 & 100 \\
\hline 4-Methyl-2-pentanone & 1 & 100 & 100 & 100 & 2 & 100 & 100 & 100 \\
\hline Methylene chloride & 50 & 2 & 50 & 1 & $5 \mathbf{v}$ & $5 \mathbf{v}$ & $5 U$ & 1 \\
\hline styrene & 50 & 5u & $5 v$ & 50 & 50 & 50 & $5 \mathbf{v}$ & 50 \\
\hline $1,1,2,2$-Tetrachloroethane & 5u & 50 & 50 & 50 & 50 & $5 \mathbf{U}$ & $5 \mathbf{v}$ & 50 \\
\hline Tetrachloroethene & $5 \mathbf{v}$ & 50 & $5 u$ & $5 v$ & 50 & 0.6 & 50 & 1 \\
\hline Toluene & 50 & 50 & 50 & $5 \mathrm{U}$ & $5 \mathbf{U}$ & 50 & 50 & $5 U$ \\
\hline $1,1,1$-Trichloroethane & 50 & 50 & $5 v$ & $5 v$ & 50 & $5 \mathrm{U}$ & 50 & 50 \\
\hline $1,1,2-$ rrichloroethane & 50 & 50 & 50 & $5 \mathrm{U}$ & $5 \mathrm{U}$ & 50 & 50 & $5 U$ \\
\hline Trichloroethene & $5 U$ & $5 v$ & 50 & $5 \mathrm{U}$ & 50 & $5 v$ & $5 \mathbf{v}$ & 50 \\
\hline vinyl acetate & 100 & 100 & 100 & 100 & 100 & 100 & 100 & 100 \\
\hline vinyl chloride & 100 & 100 & 100 & 100 & 100 & 100 & 100 & 100 \\
\hline xylenes & $5 \mathrm{U}$ & $5 \mathrm{U}$ & $5 U$ & $5 U$ & $5 U$ & 50 & 50 & 50 \\
\hline
\end{tabular}

(CONTINUED) 
APPENDIX E. 2

\begin{tabular}{|c|c|c|c|c|c|c|c|c|}
\hline \multirow{3}{*}{$\begin{array}{l}\text { Sampling point } \\
\text { Location } \\
\text { - } \\
\text { Date Sampled }\end{array}$} & \multicolumn{4}{|c|}{ GW-251 } & \multicolumn{4}{|c|}{ GW-252 } \\
\hline & \multicolumn{4}{|c|}{ s2 } & \multicolumn{4}{|c|}{ s2 } \\
\hline & $01 / 20 / 93$ & $05 / 05 / 93$ & $09 / 17 / 93$ & $12 / 08 / 93$ & $01 / 08 / 93$ & $05 / 03 / 93$ & $09 / 15 / 93$ & $12 / 07 / 93$ \\
\hline VOLATIIE ORGANICS (ug/L) & - & • & $\cdot$ & - & $\cdot$ & $\cdot$ & $\cdot$ & - \\
\hline Acetone & 100 & 100 & 100 & 100 & 100 & 100 & $10 \mathrm{U}$ & 6 \\
\hline Benzene & 50 & $5 U$ & 50 & so & 50 & $5 U$ & 50 & $5 U$ \\
\hline Bromodichloromethane & 50 & 50 & 50 & 50 & 50 & $5 \mathrm{U}$ & $5 \mathbf{U}$ & 50 \\
\hline Bromoform & 50 & $5 U$ & $5 \mathrm{U}$ & 50 & 50 & $5 U$ & 50 & $5 U$ \\
\hline Bromomethane & 100 & 100 & 100 & 100 & 100 & 100 & 100 & $10 \mathrm{U}$ \\
\hline 2-Butanone & 100 & 100 & 100 & 100 & 100 & 100 & 100 & 8 \\
\hline Carbon disulfide & $5 U$ & 50 & 50 & 50 & 50 & 50 & 50 & 50 \\
\hline Carbon tetrachloride & 50 & 11 & 2 & 1 & $5 u$ & 50 & 50 & 50 \\
\hline Chlorobenzene & $5 \mathrm{U}$ & 50 & $5 \mathrm{U}$ & 50 & 50 & 50 & 50 & 50 \\
\hline Chlorodibromomethane & 50 & 50 & 50 & $5 U$ & $5 U$ & $5 U$ & 50 & 50 \\
\hline Chloroethane & $10 \mathrm{U}$ & $10 \mathrm{U}$ & 100 & 100 & $10 \mathrm{U}$ & 100 & 100 & 100 \\
\hline Chloroform & 2 & 8 & 6 & 4 & $5 U$ & 50 & $5 U$ & $5 \mathbf{v}$ \\
\hline Chloromethane & 100 & 100 & 100 & 100 & 100 & $10 \mathrm{u}$ & 100 & 100 \\
\hline 1,1-Dichloroethane & $5 \mathrm{U}$ & 50 & 50 & 50 & su & 50 & 50 & 50 \\
\hline 1,2-Dichloroethane & 50 & su & 50 & 50 & 50 & 50 & $5 \mathbf{U}$ & $5 U$ \\
\hline 1,1-Dichloroethene & $5 \mathrm{U}$ & 50 & 50 & $5 U$ & $5 U$ & 50 & 50 & 50 \\
\hline 1,2-Dichloroethene & $5 \mathbf{v}$ & 7 & $5 v$ & $5 U$ & $5 U$ & 50 & $5 U$ & 50 \\
\hline 1,2-Dichloropropane & 50 & 50 & 50 & 50 & 50 & 5u & 50 & $5 U$ \\
\hline cis-1,3-Dichloropropene & $5 \mathbf{v}$ & $5 v$ & 50 & 50 & $5 U$ & 50 & 50 & 50 \\
\hline trans-1,3-Dichloropropene & 50 & 50 & $5 \mathbf{U}$ & $5 \mathbf{U}$ & $5 \mathbf{v}$ & $5 \mathbf{U}$ & $5 \mathrm{U}$ & 50 \\
\hline Ethylbenzene & $5 \mathbf{5}$ & 50 & 50 & 50 & 50 & 50 & 50 & $\mathbf{5 U}$ \\
\hline 2-Hexanone & 100 & 100 & 100 & 100 & $10 \mathrm{U}$ & 100 & $10 \mathrm{U}$ & 100 \\
\hline 4-Methy1-2-pentanone & $10 U$ & 100 & 100 & 100 & 100 & 1 & 100 & 1 \\
\hline Methylene chloride & $5 U$ & 50 & $5 \mathbf{U}$ & 50 & 2 & 2 & $5 \mathbf{v}$ & 2 \\
\hline Styrene & 50 & $5 \mathrm{U}$ & 50 & 50 & 50 & 50 & 50 & su \\
\hline $1,1,2,2$-Tetrachloroethane & $5 U$ & $5 U$ & 50 & $5 U$ & 50 & 50 & $5 \mathrm{v}$ & $5 \mathbf{U}$ \\
\hline Tetrachloroethene & 24 & 210 & 47 & 31 & 50 & $5 \mathbf{U}$ & 50 & 50 \\
\hline Toluene & $5 \mathrm{U}$ & $5 U$ & 50 & 50 & $5 U$ & 50 & 50 & 50 \\
\hline 1,1,1-Trichloroethane & $5 U$ & $5 U$ & 50 & 50 & $5 U$ & su & 50 & 50 \\
\hline 1,1,2-Trichloroethane & su & 50 & 50 & 50 & 50 & 50 & 50 & 50 \\
\hline Trichloroethene & 7 & 82 & 18 & 10 & $5 U$ & 50 & 5u & $5 \mathbf{v}$ \\
\hline Vinyl acetate & 100 & 100 & 100 & 100 & 100 & 100 & 100 & 100 \\
\hline Vinyl chloride & 100 & $10 \mathrm{U}$ & 100 & $10 U$ & 100 & $20 \mathrm{U}$ & 100 & 100 \\
\hline xylenes & 50 & 50 & 50 & $5 U$ & 50 & 50 & $5 U$ & $5 U$ \\
\hline
\end{tabular}

(CONTINUED) 
APPENDIX E. 2

\begin{tabular}{|c|c|c|c|c|c|c|c|c|}
\hline \multirow{3}{*}{$\begin{array}{l}\text { Sampling Point } \\
\text { Location } \\
\text { - } \\
\text { Date Sampled }\end{array}$} & \multicolumn{4}{|c|}{ GW-255 } & \multicolumn{4}{|c|}{$G W-261$} \\
\hline & \multicolumn{4}{|c|}{ S2 } & \multicolumn{4}{|c|}{ SY } \\
\hline & $01 / 08 / 93$ & $05 / 03 / 93$ & $09 / 15 / 93$ & $12 / 07 / 93$ & $01 / 08 / 93$ & $04 / 07 / 93$ & $07 / 01 / 93$ & $12 / 07 / 93$ \\
\hline VOLATILE CRGANICS (ug/L) & - & - & - & - & - & - & - & - \\
\hline Acetone & 100 & 11 & 100 & 100 & 100 & 100 & 100 & 100 \\
\hline Benzene & $5 U$ & Su & $5 U$ & 50 & 50 & 50 & $5 \mathrm{U}$ & 50 \\
\hline Bromodichloromethane & $5 \mathbf{U}$ & 50 & 50 & 50 & 50 & $5 u$ & 50 & $5 U$ \\
\hline Bromoform & $5 U$ & $5 U$ & $5 U$ & $5 \mathrm{U}$ & $5 U$ & 50 & $5 u$ & $5 U$ \\
\hline Bromomethane & 100 & 100 & 100 & 100 & $10 \mathrm{U}$ & 100 & 100 & 100 \\
\hline 2-Butanone & 100 & 100 & 100 & 100 & 100 & 100 & 100 & 100 \\
\hline Carbon disulfide & $5 U$ & $5 U$ & $5 \mathbf{v}$ & 50 & 50 & 50 & 50 & $5 U$ \\
\hline Carbon tetrachloride & $5 \mathbf{U}$ & $5 \mathrm{U}$ & $5 U$ & $5 \mathbf{v}$ & $5 \mathbf{v}$ & $5 \mathrm{U}$ & $5 u$ & $5 U$ \\
\hline Chlorobenzene & 50 & $5 v$ & 50 & sv & $5 \mathbf{v}$ & 50 & 50 & 50 \\
\hline Chlorodibromomethane & $5 U$ & $5 \mathrm{U}$ & $5 \mathbf{v}$ & $5 U$ & $5 U$ & $5 \mathrm{U}$ & $5 U$ & $5 U$ \\
\hline Chloroethane & 100 & 100 & 100 & 100 & 100 & 100 & 100 & 100 \\
\hline Chloroform & 1 & 1 & 1 & 1 & 50 & $5 U$ & $5 \mathbf{s}$ & 50 \\
\hline Chloromethane & 100 & 100 & 100 & 100 & $10 \mathrm{U}$ & 100 & 100 & 100 \\
\hline 1,1-Dichloroethane & 50 & $5 U$ & $5 \mathbf{0}$ & 50 & 50 & $5 U$ & $5 \mathbf{v}$ & 50 \\
\hline 1,2-Dichloroethane & 50 & $5 \mathrm{U}$ & 50 & 50 & $5 U$ & 50 & $5 \mathbf{u}$ & $5 U$ \\
\hline 1,1-Dichloroethene & 50 & $5 \mathrm{U}$ & $5 U$ & $5 U$ & $5 \mathbf{U}$ & 50 & $5 v$ & $5 U$ \\
\hline 1,2-Dichloroethene & $5 \mathbf{v}$ & $5 \mathbf{u}$ & $5 \mathbf{v}$ & 50 & $5 \mathbf{v}$ & 50 & $5 U$ & 50 \\
\hline 1,2-Dichloropropane & $5 \mathbf{v}$ & $5 \mathbf{5}$ & 50 & 50 & 50 & 50 & $5 U$ & $\mathbf{5 U}$ \\
\hline cis-1,3-Dichloropropene & 50 & 50 & $5 \mathrm{U}$ & $5 U$ & 50 & su & $5 \mathrm{U}$ & $5 \mathbf{s}$ \\
\hline $\operatorname{trans-1,3-Dichloropropene~}$ & 50 & $5 \mathbf{5 U}$ & 50 & $5 \mathbf{u}$ & 50 & $5 \mathbf{v}$ & $5 U$ & $5 \mathbf{U}$ \\
\hline Bthylbenzene & 50 & 50 & $5 \mathbf{U}$ & 50 & 50 & $5 \mathrm{U}$ & $5 U$ & $5 U$ \\
\hline 2-Hexanone & 100 & 100 & 100 & 100 & 100 & 100 & $10 u$ & 100 \\
\hline 4-Methyl-2-pentanone & 1 & 100 & 100 & $10 \mathrm{U}$ & 100 & 100 & 100 & 100 \\
\hline Methylene chloride & 50 & $\mathbf{5 u}$ & 50 & 1 & 2 & 1 & $5 U$ & 1 \\
\hline Styrene & $5 U$ & 50 & $5 U$ & $5 U$ & 50 & 50 & $5 u$ & 50 \\
\hline $1,1,2,2$-Tetrachloroethane & $5 v$ & 50 & 50 & $5 \mathrm{U}$ & $5 \mathbf{U}$ & $5 \mathbf{U}$ & $5 U$ & $5 \mathbf{v}$ \\
\hline Tetrachloroethene & $5 \mathbf{U}$ & $5 \mathbf{U}$ & $5 \mathbf{U}$ & $5 U$ & 50 & 50 & $5 U$ & 50 \\
\hline Toluene & $5 U$ & $5 U$ & $5 U$ & $5 U$ & 50 & $5 U$ & $5 U$ & 50 \\
\hline $1,1,1$-Trichloroethane & 50 & $5 \mathrm{U}$ & $5 U$ & $5 v$ & 50 & 5U & $5 U$ & $5 U$ \\
\hline $1,1,2-T r i c h l o r o e t h a n e$ & $5 U$ & $5 \mathbf{v}$ & 50 & $5 U$ & 50 & $5 v$ & $5 U$ & 50 \\
\hline Trichloroethene & $5 U$ & 50 & $5 \mathrm{U}$ & 513 & 50 & $5 U$ & $5 \mathbf{U}$ & 50 \\
\hline vinyl acetate & $10 \mathrm{U}$ & $10 \mathrm{U}$ & 100 & $10 U$ & 100 & 100 & 100 & 100 \\
\hline vinyl chloride & $10 U$ & 100 & 100 & 100 & 100 & 100 & 100 & 100 \\
\hline xylenes & 50 & 50 & $5 \mathrm{U}$ & $5 U$ & 50 & $5 U$ & 50 & $5 U$ \\
\hline
\end{tabular}

(CONTINUED) 
APPENDIX E. 2

\begin{tabular}{|c|c|c|c|c|c|c|c|c|}
\hline \multirow{3}{*}{$\begin{array}{l}\text { Sampling Point } \\
\text { Location } \\
\text { - } \\
\text { Date Sampled }\end{array}$} & \multicolumn{4}{|c|}{$G W-262$} & \multicolumn{4}{|c|}{ Gw-263 } \\
\hline & \multicolumn{4}{|c|}{ SY } & \multicolumn{4}{|c|}{ SY } \\
\hline & $01 / 11 / 93$ & $04 / 08 / 93$ & $07 / 01 / 93$ & $12 / 07 / 93$ & $01 / 11 / 93$ & $04 / 08 / 93$ & $07 / 01 / 93$ & $12 / 07 / 93$ \\
\hline VOIATIIE ORGANICS (ug/I) & - & - & - & - & - & - & $\cdot$ & - \\
\hline Acetone & 100 & 100 & 100 & 100 & $10 \mathrm{U}$ & $10 \mathrm{U}$ & 32 & 100 \\
\hline Benzene & 50 & 50 & $5 U$ & $5 U$ & $5 U$ & $5 \mathbf{v}$ & 50 & 50 \\
\hline Bromodichloromethane & $5 U$ & 50 & 50 & 50 & 50 & $5 \mathbf{U}$ & 50 & 50 \\
\hline Bromoform & $5 v$ & 50 & 50 & $5 \mathbf{v}$ & $5 \mathbf{v}$ & 50 & $5 \mathbf{u}$ & 50 \\
\hline Bromomethane & 100 & 100 & $10 \mathrm{U}$ & 100 & 200 & 100 & $10 \mathrm{U}$ & 100 \\
\hline 2-Butanone & 100 & 100 & 100 & 100 & 100 & 100 & 100 & 100 \\
\hline Carbon disulfide & $5 \mathbf{U}$ & $\mathbf{s u}$ & 50 & $5 \mathbf{u}$ & $5 U$ & $5 \mathbf{U}$ & $5 \mathrm{U}$ & 50 \\
\hline Carbon tetrachloride & 50 & $5 U$ & 50 & 50 & 50 & $\mathbf{5 u}$ & 50 & 50 \\
\hline Chlorobenzene & $5 u$ & $5 U$ & $5 u$ & $5 u$ & $5 \mathbf{s}$ & $5 u$ & 50 & 50 \\
\hline Chlorodibromomethane & $\mathbf{5 u}$ & 50 & 50 & $5 v$ & $5 \mathrm{U}$ & $\mathbf{5 u}$ & 5u & 50 \\
\hline Chloroethane & 100 & 100 & 100 & 100 & 100 & 100 & 100 & 100 \\
\hline Chloroform & $5 \mathbf{v}$ & $\mathbf{5 u}$ & $5 \mathbf{U}$ & $5 U$ & $5 U$ & 50 & $5 \mathbf{U}$ & $5 U$ \\
\hline Chloromethane & 100 & 100 & 100 & 100 & 100 & 100 & 100 & 100 \\
\hline 1,1-Dichloroethane & $5 \mathbf{v}$ & 5u & $5 U$ & $5 \mathbf{S}$ & $5 \mathbf{u}$ & su & $5 \mathbf{v}$ & $5 \mathbf{U}$ \\
\hline 1,2-Dichloroethane & $5 v$ & 50 & $5 U$ & su & $5 u$ & $5 \mathbf{v}$ & 50 & SU \\
\hline 1,1-Dichloroethene & $5 \mathbf{U}$ & 50 & $5 \mathrm{U}$ & $5 \mathbf{U}$ & 50 & 50 & 50 & 5U \\
\hline 1,2-Dichloroethene & $5 v$ & 50 & $5 \mathbf{U}$ & SU & $5 U$ & 50 & 50 & su \\
\hline 1,2-Dichloropropane & $5 U$ & su & $5 \mathbf{v}$ & $5 v$ & 5u & $5 U$ & $5 v$ & 5U \\
\hline cis-1,3-Dichloropropene & $5 \mathbf{U}$ & 50 & $5 U$ & $5 \mathbf{u}$ & 50 & 50 & $5 \mathbf{v}$ & 50 \\
\hline trans-1,3-Dichloropropene & $5 \mathbf{U}$ & 50 & su & $5 \mathrm{U}$ & 50 & $5 U$ & 50 & 50 \\
\hline Ethylbenzene & 50 & $5 \mathbf{v}$ & $5 \mathbf{U}$ & $5 U$ & $5 \mathbf{u}$ & 50 & 50 & $5 U$ \\
\hline 2-Hexanone & 100 & 100 & 100 & 100 & 100 & 100 & 100 & 100 \\
\hline 4-Methyl-2-pentanone & 2 & $10 U$ & $10 U$ & 100 & 2 & 100 & 100 & 100 \\
\hline Methylene chloride & $5 \mathbf{v}$ & 50 & $5 U$ & 1 & 50 & 5u & 50 & 1 \\
\hline Styrene & 50 & su & $5 \mathrm{u}$ & $5 \mathrm{U}$ & 50 & 50 & $5 \mathbf{u}$ & 50 \\
\hline $1,1,2,2$-Tetrachloroettiane & 50 & $5 \mathbf{u}$ & 50 & 50 & 50 & 50 & 5u & 50 \\
\hline Tetrachloroethene & $5 U$ & $5 U$ & 50 & 50 & $5 v$ & 50 & 50 & 50 \\
\hline Toluene & $5 U$ & 50 & 50 & $5 \mathrm{U}$ & 50 & $5 U$ & $5 u$ & 5U \\
\hline $1,1,1$-Trichloroethane & $5 \mathbf{s}$ & 50 & 50 & $5 \mathbf{v}$ & 50 & $5 v$ & 50 & $5 \mathrm{U}$ \\
\hline 1,1,2-Trichloroethane & 50 & 50 & $5 \mathrm{U}$ & 50 & 50 & 50 & $5 \mathrm{U}$ & $5 U$ \\
\hline Trichloroethene & $5 U$ & 50 & $5 \mathbf{v}$ & su & $5 \mathbf{U}$ & $5 v$ & 50 & $5 \mathbf{U}$ \\
\hline Vinyl acetate & 100 & 100 & 100 & 100 & 100 & 100 & 100 & 100 \\
\hline vinyl chloride & 200 & 100 & 100 & 100 & 100 & 100 & 100 & 100 \\
\hline Xylenes & $5 \mathbf{U}$ & $5 \mathrm{U}$ & $5 \mathrm{v}$ & $5 \mathrm{U}$ & 50 & $5 U$ & 50 & $5 U$ \\
\hline
\end{tabular}

(CONTINUED) 
APPENDIX E. 2

Groundwater Quality Data, 1993

\begin{tabular}{|c|c|c|c|c|c|c|c|c|}
\hline \multirow{3}{*}{$\begin{array}{l}\text { Sampling Point } \\
\text { L } \\
\text { Location } \\
\text { Date Sampled }\end{array}$} & \multicolumn{4}{|c|}{ GW-264 } & \multicolumn{4}{|c|}{ GW-281 } \\
\hline & \multicolumn{4}{|c|}{ sx } & \multicolumn{4}{|c|}{$\mathbf{F F}$} \\
\hline & $01 / 12 / 93$ & $04 / 12 / 93$ & $07 / 02 / 93$ & $12 / 08 / 93$ & $02 / 02 / 93$ & $04 / 16 / 93$ & $08 / 04 / 93$ & $10 / 14 / 93$ \\
\hline VOLATILE ORGANICS (Ug/L) & . &. & . & . & . & . & . & - \\
\hline Acetone & 100 & 100 & 100 & 100 & 1 & 100 & 100 & 2 \\
\hline Benzene & $5 \mathrm{U}$ & 50 & $5 u$ & 50 & 50 & $5 u$ & $5 u$ & 5u \\
\hline Bromodichloromethane & $5 \mathrm{v}$ & 50 & 5u & 50 & 50 & 50 & $5 v$ & su \\
\hline Bromoform & $5 v$ & 50 & $5 u$ & 5u & su & 50 & 50 & 50 \\
\hline Bromomethane & 100 & 100 & 100 & 100 & 100 & 100 & 100 & 100 \\
\hline 2-Butanone & 100 & 100 & 100 & 100 & 5 & 100 & 100 & 9 \\
\hline Carbon disulfide & 50 & 50 & 50 & $5 u$ & 50 & 50 & 50 & 50 \\
\hline Carbon tetrachloride & $5 \mathrm{u}$ & 50 & 5U & 50 & 50 & su & 5u & 50 \\
\hline Chlorobenzene & 50 & 50 & 50 & 50 & 50 & 50 & su & 50 \\
\hline Chlorodibromomethane & 50 & 50 & 50 & 50 & 50 & 50 & 5u & $5 v$ \\
\hline Chloroethane & 100 & 100 & 100 & 100 & 100 & 100 & 100 & 100 \\
\hline Chloroform & 50 & 50 & 5u & su & 5u & $5 \mathrm{U}$ & 50 & su \\
\hline Chloromethane & 100 & 100 & 100 & 100 & 100 & 100 & 100 & 100 \\
\hline 1,1-Dichloroethane & $5 v$ & 50 & 50 & su & 50 & $5 u$ & 50 & 5u \\
\hline 1,2-Dichloroethane & 50 & su & 5u & 50 & 50 & 50 & $5 u$ & 50 \\
\hline 1,1-Dichloroethene & 50 & 5u & $5 U$ & 50 & 50 & 50 & 50 & 50 \\
\hline 1,2-Dichloroethene & 50 & 5u & 5u & 50 & 50 & 50 & 50 & 50 \\
\hline 1,2-Dichloropropane & 50 & 50 & su & 50 & 50 & 50 & 50 & 50 \\
\hline cis-1,3-Dichloropropene & 50 & 50 & 50 & 50 & 50 & 50 & 50 & 50 \\
\hline $\operatorname{trans}-1,3-D i c h$ loropropene & 50 & 50 & 50 & 50 & 50 & 5u & su & $5 v$ \\
\hline Ethylbenzene & 50 & 50 & 50 & 5u & 50 & 5v & 50 & su \\
\hline 2-Hexanone & 100 & 100 & 100 & 100 & 100 & 100 & 100 & 100 \\
\hline 4-Methy1-2-pentanone & 100 & 100 & 100 & 100 & 2 & 100 & 100 & 200 \\
\hline Methylene chloride & 50 & 1 & 50 & su & 50 & 50 & 50 & 2 \\
\hline Styrene & 5u & su & 50 & 50 & 50 & 50 & 50 & 50 \\
\hline 1,1,2,2-Tetrachloroethane & 50 & su & 50 & 50 & 5u & 5u & $5 u$ & 50 \\
\hline Tetrachloroethene & 50 & su & 50 & $5 U$ & 50 & 5u & 5U & 50 \\
\hline Toluene & 50 & su & 50 & 50 & 50 & $5 \mathrm{U}$ & 50 & 50 \\
\hline $1,1,1$-Trichloroethane & 50 & 50 & 50 & $5 u$ & 5u & 50 & 50 & 50 \\
\hline 1,1,2-Trichloroethane & 50 & $5 \mathrm{u}$ & $5 U$ & 50 & 50 & su & $5 U$ & 50 \\
\hline Trichloroethene & su & su & $5 \mathrm{U}$ & $5 U$ & $5 \mathrm{U}$ & 50 & $5 U$ & 50 \\
\hline Vinyl acetate & 100 & 100 & 100 & 100 & 100 & 100 & 100 & 100 \\
\hline Vinyl chloride & 100 & 100 & 100 & 100 & 100 & 100 & 100 & 100 \\
\hline xylenes & 50 & $5 U$ & su & 50 & 5u & $5 U$ & 5u & $5 u$ \\
\hline
\end{tabular}

(CONTINOED) 
APPENDIX E. 2

\begin{tabular}{|c|c|c|c|c|c|c|c|c|}
\hline \multirow{3}{*}{$\begin{array}{l}\text { Sampling Point } \\
\text { Location } \\
\text { - } \\
\text { Date Sampled }\end{array}$} & \multicolumn{4}{|c|}{$G W-283$} & \multicolumn{4}{|c|}{ GW-284 } \\
\hline & \multicolumn{4}{|c|}{ FF } & \multicolumn{4}{|c|}{ FF } \\
\hline & $02 / 03 / 93$ & $04 / 19 / 93$ & $08 / 06 / 93$ & $10 / 14 / 93$ & $02 / 02 / 93$ & $04 / 16 / 93$ & $08 / 05 / 93$ & $10 / 14 / 93$ \\
\hline VOLATILE ORGANICS (Ug/L) & - & $\cdot$ & $\cdot$ & $\cdot$ & $\cdot$ & - & - & - \\
\hline Acetone & 100 & 100 & 100 & 3 & 100 & 100 & 100 & 100 \\
\hline Benzene & $5 \mathrm{U}$ & $5 v$ & $5 \mathrm{U}$ & 50 & $5 \mathbf{U}$ & $5 \mathrm{U}$ & $5 U$ & $5 \mathbf{U}$ \\
\hline Bromodichloromethane & $5 \mathrm{U}$ & $5 \mathrm{U}$ & $5 u$ & su & $5 \mathbf{v}$ & su & 50 & $5 \mathbf{5}$ \\
\hline Bromoform & $5 \mathbf{u}$ & 50 & 50 & $5 v$ & $5 \mathbf{u}$ & $5 U$ & $5 u$ & $\mathbf{5 u}$ \\
\hline Bromomethane & 200 & 100 & 100 & 100 & 100 & 100 & $10 \mathrm{U}$ & 100 \\
\hline 2-Butanone & 100 & 100 & 100 & 9 & 4 & 100 & 200 & 100 \\
\hline Carbon disulfide & su & su & $5 \mathbf{u}$ & 50 & $5 v$ & $5 U$ & $5 \mathbf{v}$ & $5 \mathbf{v}$ \\
\hline Carbon tetrachloride & 50 & $5 U$ & 50 & $5 U$ & 50 & 50 & 5u & $5 \mathbf{u}$ \\
\hline Chlorobenzene & $5 v$ & su & 50 & $5 u$ & $5 u$ & su & 50 & 50 \\
\hline Chlorodibromomethane & 50 & 50 & $5 v$ & 50 & $5 u$ & 50 & $5 u$ & 5u \\
\hline Chloroethane & 100 & 100 & $10 \mathrm{U}$ & 100 & 100 & 100 & 100 & 100 \\
\hline Chloroform & 50 & 50 & $5 \mathbf{v}$ & 50 & $5 \mathbf{U}$ & $5 \mathrm{U}$ & 50 & 5u \\
\hline Chloromethane & 100 & 100 & 100 & 100 & 100 & 100 & $10 u$ & 100 \\
\hline 1,1-Dichloroethane & 50 & $5 \mathbf{v}$ & $5 U$ & 50 & $5 \mathbf{u}$ & 50 & $5 U$ & $\mathbf{5 u}$ \\
\hline 1,2-Dichloroethane & $5 \mathbf{v}$ & $5 v$ & $5 v$ & 50 & $5 u$ & 50 & $5 U$ & $5 v$ \\
\hline 1,1-Dichloroethene & $5 \mathrm{U}$ & 50 & 50 & 50 & 50 & 50 & 50 & 50 \\
\hline 1,2-Dichloroethene & 50 & $5 \mathrm{v}$ & 50 & 50 & $5 \mathrm{U}$ & 50 & su & 50 \\
\hline 1,2-Dichloropropane & $5 u$ & $5 v$ & $5 v$ & 50 & $5 U$ & $5 u$ & 50 & 50 \\
\hline cis-1,3-Dichloropropene & $5 \mathrm{U}$ & 50 & $5 v$ & 50 & 50 & 50 & $5 u$ & 50 \\
\hline trans-1,3-Dichloropropene & su & 50 & 50 & 50 & 50 & su & $5 u$ & 50 \\
\hline Ethylbenzene & $5 \mathbf{u}$ & 50 & $5 \mathrm{U}$ & $\mathbf{5 u}$ & 50 & $5 U$ & $5 v$ & 5u \\
\hline 2-Hexanone & 100 & 100 & 100 & 100 & 100 & 100 & 100 & 100 \\
\hline 4-Methyl-2-pentanone & 2 & 100 & 100 & 100 & 2 & 100 & $10 \mathrm{u}$ & 100 \\
\hline Methylene chloride & 2 & $5 \mathbf{v}$ & 50 & 2 & 50 & 50 & $5 U$ & $\mathbf{5 v}$ \\
\hline Styrene & $5 \mathrm{v}$ & $5 v$ & 50 & $5 \mathbf{v}$ & $5 u$ & su & su & $5 \mathbf{U}$ \\
\hline $1,1,2,2$-Tetrachloroethane & su & 5u & 50 & 50 & 50 & 50 & 5u & 50 \\
\hline Tetrachloroethene & sv & $5 v$ & 50 & 50 & $5 u$ & 50 & $5 v$ & 50 \\
\hline Toluene & 50 & $5 u$ & $5 v$ & $5 \mathbf{u}$ & $5 \mathrm{U}$ & $5 u$ & $5 u$ & 50 \\
\hline 1,1,1-Trichloroethane & $5 u$ & 5u & $5 v$ & 50 & $5 u$ & $5 U$ & su & 5u \\
\hline $1,1,2$-Trichloroethane & su & $5 u$ & $5 \mathrm{v}$ & $5 \mathrm{u}$ & su & su & $5 u$ & $5 \mathbf{v}$ \\
\hline Trichloroethene & 50 & 2 & $5 v$ & $5 u$ & $5 \mathrm{U}$ & 50 & $5 u$ & $5 v$ \\
\hline Vinyl acetate & 100 & 100 & 100 & 100 & 100 & 100 & 100 & 100 \\
\hline vinyl chloride & 100 & 100 & 100 & 100 & 100 & 100 & 100 & 100 \\
\hline xylenes & $5 \mathbf{u}$ & $5 U$ & $5 v$ & 50 & $5 U$ & 50 & $5 \mathbf{v}$ & $5 U$ \\
\hline
\end{tabular}

(CONTINUED) 
APPENDIX E. 2

Groundwater Quality Data, 1993

\begin{tabular}{|c|c|c|c|c|c|c|c|c|}
\hline \multirow{3}{*}{$\begin{array}{l}\text { Sampling Point } \\
\text { Iocation } \\
\text { - } \\
\text { Date Sampled }\end{array}$} & \multicolumn{4}{|c|}{$G W-285$} & \multicolumn{4}{|c|}{ GW-337 } \\
\hline & \multicolumn{4}{|c|}{$\mathbf{F F}$} & \multicolumn{4}{|c|}{ wC } \\
\hline & $02 / 02 / 93$ & $04 / 19 / 93$ & $08 / 05 / 93$ & $10 / 14 / 93$ & $01 / 21 / 93$ & $05 / 07 / 93$ & $09 / 17 / 93$ & $12 / 09 / 93$ \\
\hline VOLATILE ORGANICS (ug/L) & - & . & - & - & - & • & $\cdot$ & - \\
\hline Acetone & 100 & 100 & 100 & 2 & $1000 \mathrm{U}$ & 5000 & $500 \mathrm{U}$ & 240 \\
\hline Benzene & 5u & $5 \mathbf{U}$ & $5 \mathbf{v}$ & $5 \mathrm{U}$ & $500 \mathrm{U}$ & 2500 & 2500 & 3000 \\
\hline Bromodichloromethane & $5 U$ & $5 \mathrm{v}$ & 50 & su & 5000 & 2500 & 2500 & 3000 \\
\hline Bromoform & $5 \mathbf{u}$ & 5u & $5 v$ & $5 \mathbf{v}$ & 5000 & 2500 & 2500 & $300 \mathrm{U}$ \\
\hline Bromomethane & 100 & 100 & 100 & 100 & 10000 & 5000 & 5000 & 5000 \\
\hline 2-Butanone & 4 & 100 & 100 & 10 & $1000 \mathrm{U}$ & 5000 & 5000 & 530 \\
\hline Carbon disulfide & 50 & 50 & $5 v$ & su & $500 \mathrm{U}$ & $250 \mathrm{U}$ & 2500 & 3000 \\
\hline Carbon tetrachloride & 50 & su & $5 \mathrm{u}$ & $5 v$ & 5000 & 2500 & 2500 & 3000 \\
\hline Chlorobenzene & 50 & $5 v$ & $5 u$ & $5 U$ & $500 \mathrm{U}$ & 2500 & 2500 & $300 v$ \\
\hline Chlorodibromomethane & 50 & 50 & $5 v$ & $\mathbf{5 u}$ & 5000 & 2500 & 2500 & 3000 \\
\hline Chloroethane & $10 \mathrm{u}$ & 100 & 100 & 100 & 10000 & 5000 & 5000 & 5000 \\
\hline Chloroform & 50 & 50 & 50 & 50 & 5000 & 2500 & 2500 & 3000 \\
\hline Chloromethane & $10 \mathrm{U}$ & 100 & $10 \mathrm{U}$ & 100 & 10000 & 5000 & 5000 & 5000 \\
\hline 1,1-Dichloroethane & $5 u$ & 50 & $5 \mathbf{v}$ & 5u & 190 & 170 & 160 & 170 \\
\hline 1,2-Dichloroethane & 5u & 50 & 5u & $5 v$ & 5000 & 2500 & 2500 & 3000 \\
\hline 1,1-Dichloroethene & 50 & $5 U$ & $5 \mathrm{U}$ & 50 & 230 & 210 & 210 & 230 \\
\hline 1,2-Dichloroethene & su & 50 & $5 u$ & 1 & 9300 & 8200 & 8100 & 8400 \\
\hline 1,2-Dichloropropane & $5 u$ & 50 & 50 & $5 v$ & 5000 & 2500 & 2500 & 3000 \\
\hline cis-1,3-Dichloropropene & Su & $5 \mathbf{U}$ & su & su & $500 u$ & 2500 & 2500 & 3000 \\
\hline trans-1,3-Dichloropropene & 50 & $\mathbf{5 U}$ & su & 5u & 5000 & 2500 & 2500 & 3000 \\
\hline Ethylbenzene & sv & $\mathbf{5 u}$ & $5 \mathbf{u}$ & 50 & 5000 & 2500 & 2500 & 3000 \\
\hline 2-Hexanone & 100 & 100 & 100 & 100 & 10000 & 5000 & 5000 & 5000 \\
\hline 4-Methy 1-2-pentanone & 2 & 100 & 100 & 100 & $1000 \mathrm{U}$ & 5000 & 5000 & 61 \\
\hline Methylene chloride & 50 & $\mathbf{5 u}$ & $5 \mathbf{u}$ & 3 & 5000 & 60 & 2500 & 110 \\
\hline Styrene & 50 & 50 & $5 u$ & 50 & 5000 & 2500 & 2500 & 3000 \\
\hline $1,1,2,2$-Tetrachloroethane & $5 \mathrm{u}$ & 50 & su & $5 \mathbf{u}$ & 5000 & 2500 & 2500 & 3000 \\
\hline Tetrachloroethene & 50 & $5 \mathrm{u}$ & su & 50 & 870 & 760 & 760 & 910 \\
\hline Toluene & 50 & 50 & $5 v$ & 5u & 5000 & 2500 & 2500 & 3000 \\
\hline 1,1,1-Trichloroethane & 50 & $5 \mathrm{U}$ & su & $5 v$ & 360 & 360 & 360 & 330 \\
\hline 1,1,2-Trichloroethane & $5 U$ & $5 U$ & 50 & 50 & $500 u$ & 2500 & 2500 & $300 \mathrm{U}$ \\
\hline Trichloroethene & 50 & 2 & 50 & $5 u$ & 960 & 980 & 1000 & 1000 \\
\hline Vinyl acetate & 100 & $10 u$ & 100 & 100 & 10000 & 5000 & 5000 & 5000 \\
\hline vinyl chloride & 100 & 100 & 100 & 100 & 340 & 280 & 230 & 170 \\
\hline xylenes & 50 & $5 \mathbf{U}$ & 50 & 50 & 5000 & 2500 & 2500 & 3000 \\
\hline
\end{tabular}

(CONTINUED) 
APPENDIX E. 2

\begin{tabular}{|c|c|c|c|c|c|c|c|c|}
\hline \multirow{3}{*}{$\begin{array}{l}\text { Sampling point } \\
\text { Location } \\
\text { - } \\
\text { Date Sampled }\end{array}$} & \multicolumn{4}{|c|}{$G W-338$} & \multicolumn{4}{|c|}{$G W-380$} \\
\hline & \multicolumn{4}{|c|}{ wc } & \multicolumn{4}{|c|}{ NHP } \\
\hline & $01 / 11 / 93$ & $05 / 04 / 93$ & $09 / 23 / 93$ & $12 / 07 / 93$ & $01 / 25 / 93$ & $04 / 21 / 93$ & $08 / 05 / 93$ & $10 / 29 / 93$ \\
\hline VOLATILB ORGANICS (ug/L) & - & $\cdot$ & - & . & . & $\cdot$ & . & - \\
\hline Acetone & 100 & 100 & 100 & 100 & 1 & 100 & 100 & 100 \\
\hline Benzene & 50 & 50 & $5 v$ & 50 & su & 50 & $5 u$ & 5u \\
\hline Bromodichloromethane & 5u & 5u & 50 & $5 u$ & $5 u$ & 5u & su & su \\
\hline Bromoform & 5u & su & $5 v$ & 5u & 5u & 5u & su & 50 \\
\hline Bromomethane & 100 & 100 & 100 & 100 & 100 & 100 & 100 & 100 \\
\hline 2-Butanone & 100 & 100 & 100 & 100 & 100 & 100 & 100 & 100 \\
\hline Carbon disulfide & su & 5u & su & 50 & su & 50 & $\mathbf{5 u}$ & 50 \\
\hline Carbon tetrachloride & su & 50 & 50 & 50 & 5u & su & su & su \\
\hline Chlorobenzene & 5u & 50 & so & 5u & 50 & 50 & 5u & 50 \\
\hline Chlorodibromomethane & $5 u$ & 5u & so & su & 50 & 50 & $5 \mathbf{v}$ & so \\
\hline Chloroethane & 100 & 100 & 100 & 100 & 100 & 100 & 100 & 100 \\
\hline Chloroform & su & 50 & 5u & 50 & 5u & $5 v$ & so & so \\
\hline Chloromethane & 100 & 100 & 100 & 100 & 100 & 100 & 100 & 100 \\
\hline 1,1-Dichloroethane & su & so & $5 \mathbf{v}$ & 5u & 5v & 50 & su & su \\
\hline 1,2-Dichloroethane & 5u & 5u & 5u & 5u & 5u & 50 & 5u & 50 \\
\hline 1,1-Dichloroethene & 50 & so & 50 & 5u & su & 50 & 5u & 50 \\
\hline 1,2-Dichloroethene & 50 & 50 & 50 & 50 & 50 & 50 & 5u & 50 \\
\hline 1,2-Dichloropropane & 50 & so & 50 & 50 & 5u & so & 5u & su \\
\hline cls-1,3-Dichloropropene & 50 & 50 & 50 & 50 & 50 & 50 & $5 \mathrm{u}$ & 5U \\
\hline trans-1, 3-Dichloropropene & 5u & 5u & 50 & 5u & 50 & 50 & 5u & 5U \\
\hline Ethylbenzene & 5u & 50 & 50 & 5v & 5u & 5u & 50 & su \\
\hline 2-Hexanone & 100 & 100 & 100 & 100 & 100 & 100 & 100 & 100 \\
\hline 4-Methy1-2-pentanone & 100 & 100 & 100 & 100 & 2 & 100 & 100 & 100 \\
\hline Methylene chloride & $5 v$ & $5 v$ & 50 & 1 & 5u & $5 \mathrm{U}$ & 5v & 1 \\
\hline Styrene & 50 & 50 & 50 & 5u & 50 & 5u & 5u & 50 \\
\hline 1,1,2,2-Tetrachloroethane & 5u & 50 & 5u & 50 & 50 & 5u & so & 50 \\
\hline Tetrachloroethene & su & 50 & 50 & 5u & 5u & 5u & 5u & su \\
\hline Toluene & 50 & 50 & su & su & 50 & 5u & 50 & 5u \\
\hline $1,1,1$-Trichloroethane & 50 & 50 & su & 50 & 50 & 5u & 50 & 50 \\
\hline 1,1,2-Trichloroethane & su & 50 & 5u & 5u & 50 & so & 5u & so \\
\hline Trichloroethene & $5 v$ & 50 & su & su & 5u & 5u & 50 & 5u \\
\hline Vinyl acetate & 100 & 100 & 100 & 100 & 100 & 100 & 100 & 100 \\
\hline vinyl chloride & 100 & 100 & 100 & 100 & 100 & 100 & 100 & 100 \\
\hline xylenes & $5 v$ & 50 & $5 \mathbf{v}$ & su & 50 & su & 5u & su \\
\hline
\end{tabular}

(CONTINUED) 
APPENDIX E.2

Groundwater Quallty Data, 1993

\begin{tabular}{|c|c|c|c|c|c|c|c|c|}
\hline \multirow{3}{*}{$\begin{array}{l}\text { Sampling Point } \\
\text { Iocation } \\
\text { Date Sampled }\end{array}$} & \multicolumn{4}{|c|}{ GW-381 } & \multicolumn{4}{|c|}{$G W-382$} \\
\hline & \multicolumn{4}{|c|}{ NHP } & \multicolumn{4}{|c|}{ NHP } \\
\hline & $01 / 27 / 93$ & $04 / 26 / 93$ & $08 / 08 / 93$ & $11 / 08 / 93$ & $01 / 28 / 93$ & $04 / 26 / 93$ & $08 / 08 / 93$ & $11 / 04 / 93$ \\
\hline VOLATILB ORGANICS (ug/L) & $\cdot$ & . & $\cdot$ & . & - & . & . & • \\
\hline Acetone & 52 & 5000 & 5000 & 5000 & 800 & 1000 & 5000 & 5000 \\
\hline Benzene & 2500 & 2500 & 2500 & 2500 & $40 U$ & 500 & 2500 & 2500 \\
\hline Bromodichloromethane & 2500 & 2500 & 2500 & 2500 & 400 & 500 & 2500 & 2500 \\
\hline Bromoform & 2500 & 2500 & 2500 & $250 u$ & $40 U$ & 500 & $250 u$ & 2500 \\
\hline Bromomethane & 5000 & 5000 & 5000 & 5000 & 800 & 1000 & 5000 & 5000 \\
\hline 2-Butanone & 5000 & 5000 & 5000 & $500 u$ & $80 \mathrm{U}$ & 1000 & 5000 & 5000 \\
\hline Carbon disulfide & 2500 & 2500 & 2500 & 2500 & 400 & 500 & 2500 & 2500 \\
\hline Carbon tetrachloride & 6400 & 4800 & 6600 & 5500 & 1500 & 5500 & 6500 & 7000 \\
\hline Chlorobenzene & 2500 & 2500 & 2500 & 2500 & 400 & 500 & 2500 & 2500 \\
\hline Chlorodibromomethane & 2500 & 2500 & 2500 & 2500 & 400 & 500 & 2500 & 2500 \\
\hline Chloroethane & 5000 & 5000 & 5000 & 5000 & 800 & 1000 & 5000 & 5000 \\
\hline Chloroform & 520 & 380 & 570 & 440 & 950 & 380 & 330 & 220 \\
\hline Chloromethane & 5000 & 5000 & 5000 & $500 u$ & 800 & 1000 & 5000 & 5000 \\
\hline 1,1-Dichloroethane & 2500 & 2500 & 2500 & 2500 & 400 & 500 & 2500 & 2500 \\
\hline 1,2-Dichloroethane & 2500 & 2500 & 2500 & 2500 & 400 & 500 & 2500 & 2500 \\
\hline 1,1-Dichloroethene & 2500 & 2500 & 2500 & 2500 & 400 & 500 & 2500 & 2500 \\
\hline 1,2-Dichloroethene & 2500 & 2500 & 2500 & 2500 & 400 & 500 & 2500 & 2500 \\
\hline 1,2-Dichloropropane & 2500 & 2500 & 2500 & 2500 & 400 & 500 & 2500 & 2500 \\
\hline cis-1, 3-Dichloropropene & 2500 & 2500 & 2500 & 2500 & 400 & 500 & 2500 & 2500 \\
\hline trans-1, 3-Dichloropropene & 2500 & 2500 & 2500 & 2500 & 400 & 500 & 2500 & 2500 \\
\hline Ethylbenzene & 2500 & 2500 & 2500 & 2500 & 400 & 500 & 2500 & 2500 \\
\hline 2-Hexanone & 5000 & 5000 & 5000 & 5000 & 800 & 1000 & 5000 & 5000 \\
\hline 4-Methy1-2-pentanone & 78 & 5000 & 5000 & 5000 & 800 & 1000 & 5000 & 5000 \\
\hline Methylene chloride & 2500 & 2500 & 2500 & 2500 & 24 & 500 & 2500 & 80 \\
\hline Styrene & 2500 & 2500 & 2500 & 2500 & 400 & 500 & 2500 & 2500 \\
\hline $1,1,2,2$-Tetrachloroethane & 2500 & 2500 & 2500 & 2500 & 400 & 500 & 2500 & 2500 \\
\hline Tetrachloroethene & 2500 & 2500 & 2500 & 2500 & 130 & 250 & 230 & 260 \\
\hline Toluene & 2500 & 2500 & 2500 & 2500 & 400 & 500 & 2500 & 2500 \\
\hline 1,1,1-Trichloroethane & 2500 & 2500 & 2500 & 2500 & 400 & 500 & 2500 & 2500 \\
\hline $1,1,2$-Trichloroethane & 2500 & 2500 & 2500 & 2500 & 400 & 500 & 2500 & 2500 \\
\hline Trichloroethene & 2500 & 2500 & 2500 & 2500 & 20 & 34 & 2500 & 2500 \\
\hline Vinyl acetate & 5000 & 5000 & 5000 & 5000 & 800 & 1000 & 5000 & 5000 \\
\hline vinyl chloride & 5000 & 5000 & 5000 & 5000 & 800 & 1000 & 5000 & 5000 \\
\hline xylenes & 2500 & 2500 & 2500 & 2500 & 400 & $50 u$ & 2500 & 2500 \\
\hline
\end{tabular}

(CONT INUED) 
APPENDIX R.2

\begin{tabular}{|c|c|c|c|c|c|c|c|c|}
\hline \multirow{3}{*}{$\begin{array}{l}\text { Sampling Point } \\
\text { - } \\
\text { Location } \\
\text { - } \\
\text { Date Sampled }\end{array}$} & \multicolumn{4}{|c|}{ GW-383 } & \multicolumn{4}{|c|}{$G W-384$} \\
\hline & \multicolumn{4}{|c|}{ NHP } & \multicolumn{4}{|c|}{ NHP } \\
\hline & $01 / 26 / 93$ & $04 / 22 / 93 \mid$ & $08 / 07 / 93$ & $11 / 03 / 93$ & $01 / 13 / 93$ & $04 / 20 / 93$ & $08 / 04 / 93$ & $10 / 28 / 93$ \\
\hline VOLATILE ORGANICS (ug/L) & - & - & - & - & - & - & - & - \\
\hline Acetone & 200 & 200 & 400 & 200 & 100 & $10 \mathrm{U}$ & 100 & 100 \\
\hline Benzene & 100 & 100 & 200 & 100 & 5u & 50 & $5 U$ & $5 U$ \\
\hline Bromodichloromethane & 100 & 100 & 200 & 100 & 50 & 50 & $5 U$ & 50 \\
\hline Bromoform & 100 & 100 & 200 & 100 & 50 & $5 \mathbf{U}$ & $5 \mathbf{u}$ & 50 \\
\hline Bromomethane & 200 & 200 & 400 & 200 & 100 & 100 & 100 & 100 \\
\hline 2-Butanone & 200 & 200 & 400 & 200 & 100 & 100 & 100 & 100 \\
\hline Carbon dieulfide & 100 & 100 & 200 & 100 & 50 & 50 & $5 U$ & 50 \\
\hline Carbon tetrachloride & 3 & 4 & 200 & 100 & 50 & 50 & $5 \mathbf{U}$ & 50 \\
\hline Chlorobenzene & 100 & 100 & 200 & 100 & su & 50 & 50 & $5 \mathrm{U}$ \\
\hline Chlorodibromomethi ne & 100 & 100 & 200 & 100 & su & $5 \mathbf{v}$ & $5 \mathbf{v}$ & $5 U$ \\
\hline Chloroethane & 200 & 200 & 400 & 200 & 100 & 100 & 100 & 100 \\
\hline Chloroform & 100 & 3 & 200 & 100 & 50 & 5v & $5 \mathbf{v}$ & 50 \\
\hline Chloromethane & 200 & 200 & 400 & 200 & 100 & 100 & 100 & 100 \\
\hline 1,1-Dichloroethane & 100 & 100 & 200 & 100 & su & 50 & 50 & 50 \\
\hline 1,2-Dlchloroethane & 100 & 100 & 200 & 100 & 50 & $5 v$ & 50 & 50 \\
\hline 1,1-Dichloroethene & 100 & 100 & 200 & 100 & 50 & $5 \mathbf{u}$ & $5 v$ & 5u \\
\hline 1,2-Dichloroethene & 72 & 71 & 73 & 84 & $5 \mathbf{v}$ & 50 & 50 & 7 \\
\hline 1,2-Dichloropropane & 100 & 100 & 200 & 100 & $5 \mathbf{v}$ & 50 & 50 & 5u \\
\hline cis-1,3-Dichloropropene & 100 & 100 & 200 & 100 & 50 & $5 \mathbf{u}$ & $5 \mathbf{v}$ & 5u \\
\hline trans-1,3-Dichloropropene & 100 & 100 & 200 & 100 & 5u & $5 \mathbf{v}$ & $5 \mathbf{v}$ & 5u \\
\hline Bthylbenzene & 100 & 100 & 200 & 100 & 50 & $5 \mathbf{v}$ & 50 & 5u \\
\hline 2-Hexanone & 200 & 200 & 400 & 200 & 100 & 100 & $10 \mathrm{U}$ & 100 \\
\hline 4-Methyl-2-pentanone & 3 & 200 & 400 & 200 & 100 & 2 & 100 & 100 \\
\hline Methylene chloride & 100 & 100 & 200 & 100 & su & 1 & $5 \mathbf{U}$ & su \\
\hline Styrene & 100 & 100 & $20 U$ & 100 & $5 \mathbf{v}$ & $5 \mathrm{u}$ & 50 & 50 \\
\hline $1,1,2,2$-Tetrachloroethane & $10 v$ & 200 & 200 & 100 & su & 5u & $5 \mathbf{v}$ & $5 U$ \\
\hline Tetrachloroethene & 300 & 400 & 120 & 190 & 50 & $5 \mathrm{u}$ & 50 & 50 \\
\hline Toluene & 100 & 100 & 200 & 100 & $5 \mathbf{u}$ & 50 & 50 & 50 \\
\hline 1,1,1-Trichloroethane & $10 \mathrm{v}$ & 100 & 200 & 100 & $5 \mathbf{v}$ & $5 u$ & $5 U$ & 50 \\
\hline $1,1,2$-Trichloroethane & 100 & 100 & 200 & 100 & 50 & $5 v$ & 50 & 50 \\
\hline Trichloroethene & 120 & 130 & 48 & 77 & $5 \mathbf{U}$ & 50 & 50 & 50 \\
\hline Vinyl acetate & 200 & 200 & 400 & 200 & 100 & 100 & 100 & 100 \\
\hline Vinyl chloride & 200 & 200 & 400 & 200 & 100 & 100 & 100 & 100 \\
\hline Xylenes & 100 & 100 & 200 & 100 & su & $5 U$ & $5 \mathbf{U}$ & $5 \mathrm{v}$ \\
\hline
\end{tabular}

(CONTINUED) 
APPENDIX E. 2

Groundwater Quality Data, 1993

\begin{tabular}{|c|c|c|c|c|c|c|c|c|}
\hline \multirow{3}{*}{$\begin{array}{l}\text { Sampling Point } \\
\text { Location } \\
\text { Date Sampled }\end{array}$} & \multicolumn{4}{|c|}{$G W-385$} & \multicolumn{4}{|c|}{ GW-505 } \\
\hline & \multicolumn{4}{|c|}{ NHP } & \multicolumn{4}{|c|}{ RG } \\
\hline & $01 / 12 / 93$ & $04 / 19 / 93$ & $08 / 03 / 93$ & $10 / 27 / 93$ & $03 / 01 / 93$ & $06 / 18 / 93$ & $09 / 15 / 93$ & $11 / 16 / 93$ \\
\hline VOLATILE ORGANICS (ug/L) & $\cdot$ & - & $\cdot$ & $\cdot$ & $\cdot$ & - & $\cdot$ & - \\
\hline Acetone & 100 & 100 & 100 & 100 & 100 & 100 & 100 & 100 \\
\hline Benzene & $5 \mathbf{u}$ & $5 \mathbf{u}$ & $\mathbf{5 u}$ & 50 & $5 \mathbf{u}$ & 50 & 50 & 50 \\
\hline Bromodichloromethane & 50 & 50 & 5u & 50 & 50 & 50 & 50 & 5u \\
\hline Bromoform & $5 v$ & $5 \mathbf{u}$ & 50 & 50 & 50 & 50 & 50 & 50 \\
\hline Bromomethane & 100 & 100 & 100 & 100 & 100 & 100 & 100 & 100 \\
\hline 2-Butanone & 100 & 100 & 100 & 100 & 100 & 100 & 100 & 100 \\
\hline Carbon disulfide & 50 & $5 \mathbf{v}$ & 50 & so & su & $5 \mathrm{U}$ & 50 & 5u \\
\hline Carbon tetrachloride & 5u & 5u & su & 50 & $5 U$ & 5u & 50 & 50 \\
\hline Chlorobenzene & 5u & 5u & 5u & su & su & su & 5u & 50 \\
\hline Chlorodibromomethane & 50 & 50 & $5 v$ & 50 & 5u & 5u & $5 U$ & 50 \\
\hline Chloroethane & 100 & 100 & 100 & 100 & 100 & 100 & 100 & 100 \\
\hline Chloroform & su & 5u & 50 & 50 & su & su & su & 50 \\
\hline Chloromethane & 100 & 100 & 100 & 100 & 100 & 100 & 100 & 100 \\
\hline 1,1-Dichloroethane & $5 \mathbf{v}$ & $5 \mathbf{u}$ & 50 & su & su & 50 & 5u & 5U \\
\hline 1,2-Dichloroethane & 5u & 5u & $5 \mathrm{v}$ & 5u & su & 50 & 5u & su \\
\hline 1,1-Dichloroethene & 5u & 50 & 50 & su & 50 & 1 & 50 & 50 \\
\hline 1,2-Dichloroethene & 5u & 50 & 50 & 50 & 5u & 5u & su & su \\
\hline 1,2-Dichloropropane & 50 & 50 & su & su & 5u & so & 5v & 5u \\
\hline cis-1,3-Dichloropropene & $5 u$ & $5 \mathrm{v}$ & 50 & 50 & 50 & 50 & 50 & 5u \\
\hline trans-1, 3-Dichloropropene & 50 & 50 & 50 & su & 50 & 50 & 50 & 50 \\
\hline Ethylbenzene & 5u & 50 & 50 & 5u & 50 & 50 & 5U & $5 \mathrm{U}$ \\
\hline 2-Hexanone & 100 & 100 & 100 & 100 & 100 & 100 & 100 & 100 \\
\hline 4-Methy1-2-pentanone & 100 & 100 & 100 & 100 & 100 & 100 & 100 & 100 \\
\hline Methylene chloride & 2 & 50 & 50 & 50 & 50 & 50 & $5 U$ & 1 \\
\hline styrene & 50 & 5u & 5u & 5u & 50 & 5u & 50 & $5 \mathrm{U}$ \\
\hline 1,1,2,2-Tetrachloroethane & 5u & 50 & su & so & 50 & 50 & 50 & 50 \\
\hline Tetrachloroethene & 50 & 50 & 50 & 50 & 50 & 0.8 & 50 & 50 \\
\hline Toluene & 50 & 50 & 5u & 5u & 50 & 5u & 50 & 50 \\
\hline 1,1,1-Trichloroethane & 50 & 50 & su & 5u & 50 & 50 & 5U & 50 \\
\hline 1,1,2-rrichloroethane & $5 u$ & 50 & 50 & 50 & 50 & 5u & $5 U$ & 50 \\
\hline Trichloroethene & 5u & 50 & 50 & 50 & $5 \mathbf{v}$ & 50 & 50 & 50 \\
\hline Vinyl acetate & 100 & 100 & 100 & 100 & 100 & 100 & 100 & 100 \\
\hline vinyl chloride & 100 & 100 & 100 & 100 & 100 & 100 & 100 & 100 \\
\hline xylenes & 50 & 50 & su & 50 & 50 & $5 \mathbf{U}$ & $5 U$ & 50 \\
\hline
\end{tabular}

(CONTINUED) 
APPENDIX E. 2

Groundwater Quality Data, 1993

\begin{tabular}{|c|c|c|c|c|c|c|c|c|}
\hline \multirow{3}{*}{$\begin{array}{l}\text { Sampling Point } \\
\text { Location } \\
\text { - } \\
\text { Date Sampled }\end{array}$} & \multicolumn{4}{|c|}{$G W-508$} & \multicolumn{4}{|c|}{$G W-603$} \\
\hline & \multicolumn{4}{|c|}{ RG } & \multicolumn{4}{|c|}{$\mathbf{E X P}$} \\
\hline & $03 / 05 / 93$ & $06 / 23 / 93$ & $09 / 21 / 93$ & $11 / 19 / 93$ & $01 / 29 / 93$ & $05 / 04 / 93$ & $08 / 16 / 93$ & $10 / 28 / 93$ \\
\hline VOLAIILE ORGANICS (ug/L) & - & - & - & - & - & - & - & . \\
\hline Acetone & 25000 & 25000 & $2500 \mathrm{U}$ & 25000 & 100 & 100 & 100 & 100 \\
\hline Benzene & 9500 & 8800 & 7600 & 7700 & $5 U$ & $\mathbf{s u}$ & su & su \\
\hline Bromodichloromethane & $1300 \mathrm{U}$ & $1300 \mathrm{U}$ & 13000 & $1300 \mathrm{U}$ & 50 & 5u & su & 5u \\
\hline Bromoform & 13000 & 13000 & 13000 & 13000 & $5 \mathrm{U}$ & $\mathbf{5 u}$ & 50 & 50 \\
\hline Bromomethane & 25000 & 25000 & 25000 & 25000 & 100 & $10 \mathrm{U}$ & 100 & 100 \\
\hline 2-Eutanone & 25000 & $2500 \mathrm{U}$ & 25000 & 25000 & 100 & 100 & 100 & 100 \\
\hline Carbon diaulfito & $1300 \mathrm{v}$ & 13000 & $1300 \mathrm{U}$ & $1300 \mathrm{v}$ & $\mathbf{5 u}$ & 5U & $5 \mathbf{u}$ & $5 \mathbf{v}$ \\
\hline Carbon tetrachloride & 13000 & 13000 & 13000 & 13000 & 50 & 50 & 50 & 50 \\
\hline Chlorobenzene & $1300 \mathrm{U}$ & $1300 \mathrm{U}$ & $1300 \mathrm{U}$ & $1300 \mathrm{U}$ & 50 & $5 v$ & $5 \mathbf{U}$ & 50 \\
\hline Chlorodibromomethane & 13000 & 13000 & $1300 \mathrm{u}$ & 13000 & su & $5 \mathbf{u}$ & $5 \mathbf{u}$ & 5u \\
\hline Chloroethane & 25000 & 25000 & 25000 & 25000 & 100 & 100 & 100 & 100 \\
\hline Chloroform & 13000 & 13000 & $1300 \mathrm{U}$ & $1300 \mathrm{v}$ & 50 & $5 \mathbf{v}$ & 50 & 5u \\
\hline Chloromethane & 25000 & 25000 & $2500 u$ & 25000 & 100 & 100 & 100 & 100 \\
\hline 1,1-Dichloroethane & 13000 & $1300 \mathrm{U}$ & $1300 \mathrm{U}$ & 13000 & $\mathbf{5 0}$ & 50 & $5 \mathbf{v}$ & $\mathbf{5 u}$ \\
\hline 1,2-Dichloroethane & 13000 & $1300 \mathrm{v}$ & 13000 & $1300 \mathrm{U}$ & 50 & 50 & 5u & 50 \\
\hline 1,1-Dichloroethene & 13000 & $1300 \mathrm{v}$ & $1300 \mathrm{U}$ & $1300 \mathrm{U}$ & 50 & 50 & 50 & $\mathbf{5 u}$ \\
\hline 1,2-Dichloroethene & 13000 & 13000 & 13000 & 13000 & 50 & $5 \mathbf{v}$ & 50 & 50 \\
\hline 1,2-Dichloropropane & 13000 & 13000 & 13000 & $1300 \mathrm{U}$ & 50 & $5 v$ & 50 & 50 \\
\hline cis-1,3-Dichloropropene & 13000 & 13000 & $1300 \mathrm{U}$ & 13000 & $5 \mathbf{v}$ & $5 u$ & $5 v$ & $5 v$ \\
\hline trans-1,3-Dichloropropene & 13000 & 13000 & 13000 & 13000 & 50 & $5 \mathbf{v}$ & $5 \mathbf{v}$ & 50 \\
\hline Ethylbenzene & 3100 & 2900 & 2700 & 2500 & 50 & $5 \mathbf{v}$ & 5u & $5 \mathbf{u}$ \\
\hline 2-Hexanone & 25000 & 25000 & 25000 & 25000 & 100 & 100 & 100 & 100 \\
\hline 4-Nethyl-2-pentanone & 25000 & 25000 & 25000 & 25000 & 100 & 100 & 100 & 100 \\
\hline Methylene chloride & $1300 \mathrm{U}$ & 310 & 350 & 13000 & $\mathbf{5 u}$ & 50 & 1 & $5 \mathbf{U}$ \\
\hline styrene & $1300 \mathrm{U}$ & 13000 & 13000 & 13000 & 50 & su & 5u & su \\
\hline $1,1,2,2$-Tetrachloroethane & 13000 & 13000 & 13000 & $1300 \mathrm{U}$ & $5 \mathbf{v}$ & su & $5 \mathbf{v}$ & 50 \\
\hline Tetrachloroethene & $1300 \mathrm{U}$ & 13000 & 13000 & 13000 & 50 & 50 & su & 50 \\
\hline Toluene & 29000 & 29000 & 32000 & 28000 & 50 & 50 & 5u & 5U \\
\hline $1,1,1$-Tr1chloroethane & 13000 & 13000 & 13000 & 13000 & 50 & 50 & 5u & 50 \\
\hline $1,1,2$-Trichloroethane & $1300 \mathrm{U}$ & $1300 \mathrm{U}$ & $1300 \mathrm{U}$ & $1300 \mathrm{U}$ & $5 \mathbf{v}$ & 50 & 50 & 50 \\
\hline Trichloroethene & $1300 \mathrm{u}$ & 13000 & 13000 & 13000 & 50 & 5u & su & $5 \mathrm{U}$ \\
\hline Vinyl acetate & 25000 & 25000 & 25000 & 25000 & 100 & 100 & 100 & 100 \\
\hline Vinyl chloride & 25000 & 25000 & 25000 & 25000 & 100 & 100 & 100 & 100 \\
\hline Xylenes & 18000 & 17000 & 16000 & 17000 & $5 \mathrm{U}$ & 50 & 50 & 50 \\
\hline
\end{tabular}

(CONTINUED) 
APPENDIX E. 2

\begin{tabular}{|c|c|c|c|c|c|c|c|c|}
\hline \multirow{3}{*}{$\begin{array}{l}\text { Sampling Point } \\
\text { Iocation } \\
\text { - } \\
\text { Date Sampled }\end{array}$} & \multicolumn{4}{|c|}{$G W-604$} & \multicolumn{4}{|c|}{ GW- 605} \\
\hline & \multicolumn{4}{|c|}{$\mathbf{E X P}$} & \multicolumn{4}{|c|}{$\mathbf{E X P}$} \\
\hline & $01 / 30 / 93$ & $05 / 04 / 93$ & $08 / 16 / 93$ & $10 / 28 / 93$ & $02 / 02 / 93$ & $05 / 10 / 93$ & $08 / 19 / 93$ & $11 / 10 / 93$ \\
\hline VOLATILE ORGANICS (ug/L) & - & - & - & . & • & - & - & - \\
\hline Acetone & 3 & 100 & 100 & 100 & 100 & 100 & 100 & 100 \\
\hline Benzene & $5 U$ & 50 & $5 \mathrm{v}$ & su & $5 \mathbf{u}$ & 50 & $5 v$ & su \\
\hline Bromodichloromethane & 50 & 50 & 50 & $5 U$ & 50 & $5 U$ & 50 & $5 \mathbf{v}$ \\
\hline Bromoform & $5 \mathrm{U}$ & 50 & 50 & su & $5 U$ & 50 & 50 & 50 \\
\hline Bromomethane & 100 & 100 & 100 & 100 & 100 & 100 & 100 & 100 \\
\hline 2-Butanone & 100 & 100 & 100 & 100 & 100 & 100 & 100 & 100 \\
\hline Carbon disulfide & $5 \mathbf{5}$ & 50 & $5 \mathrm{U}$ & $5 \mathrm{U}$ & $\mathbf{5 u}$ & $5 \mathbf{U}$ & 50 & $\mathbf{5 u}$ \\
\hline Carbon tetrachloride & $5 \mathbf{U}$ & 50 & $5 \mathrm{U}$ & $5 U$ & 170 & 50 & 180 & 160 \\
\hline Chlorobenzene & 50 & 50 & 50 & $5 \mathrm{U}$ & $5 \mathrm{U}$ & 50 & 50 & $\mathbf{5 u}$ \\
\hline Chlorodibromomethane & $5 \mathbf{U}$ & 50 & $5 U$ & $5 \mathrm{u}$ & $5 \mathbf{v}$ & $5 \mathbf{U}$ & 50 & $5 \mathbf{v}$ \\
\hline Chloroethane & 100 & 100 & 100 & 100 & 100 & 100 & 100 & 100 \\
\hline Chloroform & $5 U$ & 1 & 1 & 1 & 23 & 20 & 28 & 29 \\
\hline Chloromethane & 100 & 100 & 100 & 100 & 100 & 100 & 100 & 100 \\
\hline 1,1-Dichloroethane & $5 U$ & $5 U$ & 50 & $5 \mathrm{U}$ & 3 & $5 U$ & 3 & 3 \\
\hline 1,2-Dichloroethane & $5 U$ & 50 & 50 & 5u & 50 & $5 v$ & 50 & $5 \mathbf{v}$ \\
\hline 1,1-Dichloroethene & $5 \mathrm{u}$ & 50 & $5 \mathrm{U}$ & 50 & 2 & $5 u$ & 1 & 1 \\
\hline 1,2-Dichloroethene & $5 u$ & 50 & 50 & $5 \mathrm{U}$ & 22 & 10 & 30 & 30 \\
\hline 1,2-Dichloropropane & 50 & 50 & 50 & 5u & 50 & 50 & 50 & 50 \\
\hline cis-1,3-Dichloropropene & $5 U$ & 50 & $5 \mathbf{v}$ & 5u & 50 & 50 & 50 & 50 \\
\hline trans-1,3-Dichloropropene & $5 \mathbf{u}$ & 50 & 50 & 50 & 50 & 50 & $5 U$ & $5 \mathbf{U}$ \\
\hline Ethylbenzene & $5 v$ & 50 & 50 & 50 & $5 \mathbf{v}$ & 50 & 50 & $5 \mathbf{v}$ \\
\hline 2-Hexanone & 100 & 100 & 100 & 100 & 100 & 100 & 100 & 100 \\
\hline 4-Methyl-2-pentanone & 2 & 100 & 100 & 100 & 100 & 100 & 100 & 100 \\
\hline Methylene chloride & $5 \mathrm{U}$ & $5 \mathrm{U}$ & $5 \mathrm{U}$ & $5 U$ & $5 U$ & su & 1 & 5u \\
\hline Styrene & 50 & 50 & $5 \mathbf{v}$ & 50 & 50 & 50 & $5 \mathbf{U}$ & $5 \mathbf{v}$ \\
\hline $1,1,2,2$-Tetrachloroethane & 50 & 50 & 50 & $5 v$ & $5 v$ & 50 & 50 & $5 \mathbf{U}$ \\
\hline Tetrachloroethene & 50 & 50 & $5 \mathbf{v}$ & 50 & 25 & 7 & 32 & 30 \\
\hline Toluene & $5 U$ & 50 & $5 \mathbf{v}$ & $5 \mathrm{U}$ & $5 \mathbf{v}$ & 50 & 50 & $5 U$ \\
\hline $1,1,1$-Trichloroethane & 50 & $5 U$ & 50 & $5 U$ & $5 \mathbf{v}$ & 50 & $5 \mathbf{v}$ & $5 U$ \\
\hline $1,1,2$-Trichloroethane & 50 & $5 \mathrm{U}$ & $5 U$ & 50 & 50 & 50 & $5 \mathbf{U}$ & 50 \\
\hline Trichloroethene & $5 v$ & 50 & $5 \mathbf{5}$ & $5 v$ & 8 & 3 & 10 & 9 \\
\hline Vinyl acetate & 100 & 100 & 100 & 100 & 100 & 100 & 100 & 100 \\
\hline vinyl chloride & 100 & 100 & 100 & $10 \mathrm{~J}$ & 100 & 100 & 2 & $10 U$ \\
\hline Xylenes & 50 & $5 U$ & 50 & $5 U$ & 50 & 50 & 50 & $5 U$ \\
\hline
\end{tabular}

( CONTINUED) 
APPENDIX E.2

Groundwater Quality Data, 1993

\begin{tabular}{|c|c|c|c|c|c|c|c|c|}
\hline \multirow{3}{*}{$\begin{array}{l}\text { Sampling Point } \\
\text { - } \\
\text { - } \\
\text { Date Sampled }\end{array}$} & \multicolumn{4}{|c|}{ GW-606 } & \multicolumn{4}{|c|}{ GW-617 } \\
\hline & \multicolumn{4}{|c|}{$\mathbf{E X P}$} & \multicolumn{4}{|c|}{ EXP } \\
\hline & $02 / 03 / 93$ & $05 / 10 / 93$ & $08 / 20 / 93$ & $11 / 11 / 93$ & $01 / 12 / 93$ & $05 / 04 / 93$ & $09 / 16 / 93$ & $12 / 07 / 93$ \\
\hline VOLATILE ORGANICS (ug/I) & $\cdot$ & - & $\cdot$ & $\cdot$ & $\cdot$ & - & $\cdot$ & - \\
\hline Acetone & 29 & 500 & 500 & 500 & 2 & 100 & 7 & 4 \\
\hline Benzene & $25 \mathrm{u}$ & $25 U$ & 250 & $25 v$ & $5 \mathbf{v}$ & $5 \mathrm{U}$ & $5 v$ & $5 v$ \\
\hline Bromodichloromethane & 250 & 250 & 250 & $25 U$ & 50 & $5 U$ & $5 U$ & 50 \\
\hline Bromoform & $25 U$ & 250 & $25 \mathrm{U}$ & $25 U$ & $5 U$ & 50 & 50 & $5 U$ \\
\hline Bromomethane & 500 & 500 & 500 & 500 & 100 & 100 & 100 & 100 \\
\hline 2-Butanone & $50 \mathrm{U}$ & 500 & 500 & 500 & 100 & 100 & 100 & 7 \\
\hline Carbon disulfide & $25 U$ & 250 & 25U & 250 & 50 & 5U & $5 U$ & 50 \\
\hline Carbon tetrachloride & 1100 & 580 & 770 & 500 & 50 & $5 U$ & 50 & 50 \\
\hline Chlorobenzene & 250 & $25 U$ & 250 & 250 & $5 \mathbf{U}$ & 50 & $5 \mathbf{U}$ & $5 \mathrm{~J}$ \\
\hline Chlorodibromomethane & $25 \mathrm{U}$ & $25 v$ & $25 v$ & 250 & $5 \mathbf{v}$ & $5 v$ & $5 \mathbf{u}$ & 50 \\
\hline Chloroethane & 500 & 500 & 500 & 500 & $10 \mathrm{U}$ & 100 & 100 & 100 \\
\hline Chloroform & 310 & 250 & 250 & 250 & $5 U$ & $5 \mathbf{5}$ & $5 \mathbf{U}$ & 50 \\
\hline Chloromethane & 500 & 500 & 500 & 500 & 100 & 100 & 100 & 100 \\
\hline 1,1-Dichloroethane & $25 \mathrm{U}$ & $25 \mathrm{U}$ & 250 & 250 & $5 \mathbf{v}$ & $5 U$ & $5 \mathbf{v}$ & 50 \\
\hline 1,2-Dichloroethane & $25 U$ & $25 U$ & 250 & 250 & 50 & 50 & 50 & 50 \\
\hline 1,1-Dichloroethene & $25 U$ & 250 & $25 U$ & $25 U$ & 50 & 50 & 50 & 50 \\
\hline 1,2-Dichloroethene & $25 \mathrm{U}$ & 250 & $25 U$ & 250 & 4 & 8 & 22 & 18 \\
\hline 1,2-Dichloropropane & $25 \mathrm{U}$ & $25 \mathrm{U}$ & 250 & 250 & 50 & 50 & 50 & 50 \\
\hline cis-1,3-Dichloropropene & $25 \mathrm{U}$ & $25 \mathrm{U}$ & $25 U$ & $25 \mathrm{U}$ & $5 \mathbf{U}$ & 50 & 50 & 50 \\
\hline trans-1,3-Dichloropropene & $25 \mathrm{U}$ & 250 & 250 & 250 & 50 & 50 & 50 & $5 U$ \\
\hline Ethylbenzene & 250 & 250 & 250 & 250 & 50 & 50 & $5 \mathrm{U}$ & $5 \mathbf{5}$ \\
\hline 2-Hexanone & 500 & 500 & 500 & 500 & 100 & 100 & $10 \mathrm{U}$ & 100 \\
\hline 4-Methyl-2-pentanone & 11 & 500 & $50 \mathrm{v}$ & $50 \mathrm{U}$ & 2 & 100 & 100 & 1 \\
\hline Methylene chloride & 17 & 250 & 10 & 12 & $5 \mathrm{U}$ & 50 & 50 & 2 \\
\hline Styrene & 250 & $25 \mathrm{U}$ & $25 \mathrm{U}$ & $25 \mathrm{U}$ & $5 \mathbf{U}$ & 50 & $5 \mathbf{v}$ & 50 \\
\hline $1,1,2,2$-Tetrachloroethane & 250 & $25 \mathrm{U}$ & 250 & 250 & $5 \mathrm{U}$ & 50 & 50 & 50 \\
\hline Tetrachloroethene & 6 & 250 & 6 & 5 & 2 & $5 U$ & 14 & 14 \\
\hline Toluene & 250 & 250 & 250 & 250 & $5 u$ & 50 & 50 & 50 \\
\hline 1,1,1-Trichloroethane & 250 & $25 U$ & $25 U$ & $25 \mathrm{u}$ & $5 U$ & $5 \mathbf{0}$ & 50 & $5 \mathbf{U}$ \\
\hline 1,1,2-Trichloroethane & $25 \mathrm{U}$ & 250 & 250 & $25 \mathrm{U}$ & 50 & 50 & $5 \mathbf{v}$ & $5 U$ \\
\hline Trichloroethene & 250 & $25 \mathrm{U}$ & $25 U$ & $25 \mathrm{U}$ & 2 & 50 & 16 & 14 \\
\hline Vinyl acetate & $50 \mathrm{U}$ & $50 \mathrm{U}$ & 500 & 500 & $10 \mathrm{U}$ & 100 & 10 & 100 \\
\hline Vinyl chloride & $50 \mathrm{U}$ & 500 & 500 & 500 & $10 u$ & $10 \mathrm{v}$ & 100 & 100 \\
\hline xylenes & $25 U$ & $25 U$ & $25 \mathrm{U}$ & 250 & 5u & $5 U$ & 50 & 50 \\
\hline
\end{tabular}

(CONTINUED) 
APPENDIX E. 2

\begin{tabular}{|c|c|c|c|c|c|c|c|c|}
\hline \multirow{3}{*}{$\begin{array}{l}\text { Sampling Point } \\
\text { Location } \\
\text { - } \\
\text { Date Sampled }\end{array}$} & \multicolumn{4}{|c|}{$G W-618$} & \multicolumn{4}{|c|}{ GW-619 } \\
\hline & \multicolumn{4}{|c|}{ EXP } & \multicolumn{4}{|c|}{ PWF } \\
\hline & $01 / 12 / 93$ & $05 / 05 / 93$ & $09 / 16 / 93$ & $12 / 08 / 93$ & $01 / 20 / 93$ & $05 / 05 / 93$ & $09 / 17 / 93$ & $12 / 08 / 93$ \\
\hline VOLATILE ORGANICS (ug/L) & - & . & - & - & - & - & - & - \\
\hline Acetone & 1 & 100 & 100 & 100 & 100 & 100 & 100 & 100 \\
\hline Benzene & $5 \mathbf{U}$ & 50 & $5 \mathbf{v}$ & $5 U$ & $5 \mathrm{U}$ & $5 U$ & $5 \mathbf{U}$ & 50 \\
\hline Bromodichloromethane & 50 & 50 & 50 & $5 \mathrm{U}$ & 50 & $5 v$ & $5 u$ & 50 \\
\hline Bromoform & 50 & $5 \mathbf{u}$ & 5v & 50 & 50 & 50 & $5 U$ & su \\
\hline Bromomethane & 100 & 100 & 100 & 100 & 100 & 100 & $10 \mathrm{v}$ & 100 \\
\hline 2-Butanone & 100 & 100 & 100 & 100 & 100 & 100 & 100 & 100 \\
\hline Carbon disulfide & $5 \mathbf{S}$ & 50 & 50 & $5 \mathbf{v}$ & $5 \mathbf{U}$ & 50 & 50 & 50 \\
\hline Carbon tetrachloride & 50 & 50 & 50 & 50 & $5 U$ & $5 \mathbf{v}$ & 50 & $5 U$ \\
\hline Chlorobenzene & $5 \mathrm{U}$ & $5 v$ & 50 & $5 \mathbf{s u}$ & $5 v$ & 50 & $5 \mathrm{U}$ & $5 \mathbf{U}$ \\
\hline Chlorodibromomethane & $5 \mathbf{U}$ & $5 \mathbf{U}$ & 50 & $5 \mathbf{U}$ & 50 & $5 \mathbf{v}$ & $5 \mathbf{U}$ & 5U \\
\hline Chloroethane & 100 & 100 & 100 & 100 & 100 & 100 & 100 & 100 \\
\hline Chloroform & $\mathbf{5 u}$ & 50 & $5 v$ & $5 \mathbf{U}$ & $5 \mathbf{U}$ & $5 \mathbf{U}$ & 50 & 50 \\
\hline Chloromethane & 100 & 100 & 100 & 100 & 100 & 100 & 100 & 100 \\
\hline 1,1-Dichloroethane & 50 & 50 & 50 & $5 \mathbf{U}$ & 50 & $5 \mathbf{v}$ & $5 \mathbf{U}$ & $5 \mathbf{v}$ \\
\hline 1,2-Dichloroethane & 50 & $5 v$ & $5 v$ & su & 50 & 50 & $5 \mathbf{v}$ & $5 \mathbf{U}$ \\
\hline 1,1-Dichloroethene & 50 & $5 U$ & 50 & 50 & 50 & 50 & 50 & 50 \\
\hline 1,2-Dichloroethene & 41 & 34 & 39 & 36 & 61 & 170 & 95 & 79 \\
\hline 1,2-Dichloropropane & $5 \mathbf{U}$ & 50 & su & su & 50 & 5U & 50 & $5 \mathbf{v}$ \\
\hline Cis-1,3-Dichloropropene & 50 & 50 & 50 & $5 \mathrm{U}$ & 50 & $5 \mathbf{U}$ & 50 & $\mathbf{5 u}$ \\
\hline trans-1,3-Dichloropropene & 50 & 5u & 50 & $5 v$ & $5 U$ & $5 v$ & 50 & 50 \\
\hline Ethylbenzene & 50 & 50 & $5 \mathrm{v}$ & 50 & 50 & 50 & 50 & 50 \\
\hline 2-Hexanone & $10 U$ & 100 & 100 & $10 \mathrm{U}$ & 100 & 100 & 100 & 100 \\
\hline 4-Methyl-2-pentanone & 2 & 100 & 100 & 100 & 100 & 200 & 100 & 100 \\
\hline Methylene chloride & $5 v$ & 50 & $\mathbf{5 U}$ & $5 \mathbf{U}$ & 50 & 50 & $5 \mathbf{v}$ & 5u \\
\hline Styrene & 50 & 50 & 50 & 50 & 50 & $5 \mathrm{U}$ & 50 & 50 \\
\hline $1,1,2,2$-Tetrachloroethane & $5 \mathbf{u}$ & $5 v$ & 50 & $5 \mathbf{U}$ & 50 & $5 \mathbf{U}$ & $5 \mathbf{v}$ & $\mathbf{5 v}$ \\
\hline Tetrachloroethene & 23 & 27 & 10 & 43 & 140 & 230 & 95 & 150 \\
\hline Toluene & $5 U$ & 50 & 50 & 50 & $5 \mathbf{U}$ & 50 & $5 \mathbf{u}$ & 5u \\
\hline 1,1,1-Trichloroethane & $5 U$ & 50 & $5 v$ & 50 & $5 \mathbf{v}$ & $5 \mathrm{U}$ & $5 \mathbf{U}$ & 5u \\
\hline 1,1,2-Trichloroethane & $5 \mathbf{v}$ & $5 v$ & $5 \mathrm{U}$ & $5 U$ & $5 v$ & 50 & 50 & 50 \\
\hline Trichloroethene & 27 & 30 & 19 & 41 & 35 & 93 & 43 & 42 \\
\hline Vinyl acetate & 100 & 100 & 100 & 100 & 100 & 100 & 100 & 100 \\
\hline vinyl chloride & 100 & 100 & 100 & 100 & 100 & 100 & $10 \mathrm{U}$ & 100 \\
\hline Xylenes & $5 U$ & $5 \mathbf{U}$ & 50 & 50 & 50 & $5 \mathbf{v}$ & $5 U$ & 50 \\
\hline
\end{tabular}

( CONTINUED) 
APPENDIX $\mathrm{E} .2$

Groundwater Quality Data, 1993

\begin{tabular}{|c|c|c|c|c|c|c|c|c|}
\hline \multirow{3}{*}{$\begin{array}{l}\text { Sampling Point } \\
\text { Location } \\
\text { - } \\
\text { Date Sampled }\end{array}$} & \multicolumn{4}{|c|}{$G W-620$} & \multicolumn{4}{|c|}{ GW-631 } \\
\hline & \multicolumn{4}{|c|}{ FTF } & \multicolumn{4}{|c|}{ RG } \\
\hline & $01 / 20 / 93$ & $05 / 06 / 93$ & $09 / 17: 93$ & $12 / 09 / 93$ & $03 / 02 / 93$ & $06 / 18 / 93$ & $09 / 16 / 93$ & $11 / 17 / 93$ \\
\hline VOLATILE ORGANICS (ug/I) & - & $\cdot$ & - & - & $\cdot$ & - & - & - \\
\hline Acetone & 400 & 60 & 400 & 20 & 100 & 100 & 100 & 100 \\
\hline Benzene & 200 & $25 v$ & 200 & $20 \mathrm{u}$ & 2 & 2 & 1 & 2 \\
\hline Bromodichloromethane & 200 & 250 & 200 & 200 & $5 \mathrm{v}$ & 5U & 50 & 50 \\
\hline Bromoform & 200 & 250 & 200 & 200 & $5 \mathrm{U}$ & 50 & $5 U$ & $5 \mathbf{u}$ \\
\hline Bromomethane & 400 & 500 & 400 & 400 & $10 \mathrm{U}$ & 100 & 100 & 100 \\
\hline 2-Butanone & 400 & 500 & $40 U$ & 41 & 100 & 100 & 7 & 100 \\
\hline Carbon disulfide & 200 & 250 & 200 & 200 & $5 U$ & $5 \mathbf{U}$ & 50 & 5v \\
\hline Carbon tetrachloride & 200 & $25 \mathrm{U}$ & 200 & 200 & 50 & $5 v$ & su & 50 \\
\hline Chlorobenzene & 200 & $25 \mathrm{U}$ & 200 & 200 & $5 U$ & 50 & $5 \mathbf{v}$ & $5 \mathbf{U}$ \\
\hline Chlorodibromomethane & 200 & $25 U$ & 200 & 200 & 50 & $5 U$ & 50 & $5 \mathbf{v}$ \\
\hline Chloroethane & 400 & $50 \mathrm{U}$ & 400 & 400 & 100 & $10 \mathrm{U}$ & 100 & 100 \\
\hline Chloroform & 200 & 250 & 200 & 200 & 50 & $5 \mathbf{v}$ & 50 & $5 \mathbf{U}$ \\
\hline Chloromethane & 400 & 500 & 400 & $40 \mathrm{U}$ & 100 & 100 & 100 & 100 \\
\hline 1,1-Dichloroethane & 200 & $25 U$ & 200 & 200 & 50 & 50 & $5 \mathbf{U}$ & 50 \\
\hline 1,2-Dichloroethane & 200 & $25 U$ & $20 \mathrm{U}$ & 200 & $5 v$ & 5u & 5u & $5 \mathbf{v}$ \\
\hline 1-Dichloroethene & 200 & 250 & 200 & 200 & 50 & 50 & 50 & $5 \mathbf{U}$ \\
\hline 1,2-Dichloroethene & 560 & 400 & 540 & 620 & 1 & 1 & $5 \mathbf{v}$ & $5 U$ \\
\hline 1,2-Dichloropropane & 200 & $25 U$ & 200 & 200 & 50 & 50 & $5 \mathbf{v}$ & $5 \mathbf{U}$ \\
\hline cis-1,3-Dichloropropene & 200 & $25 U$ & 200 & 200 & 50 & 50 & $5 \mathbf{v}$ & $5 \mathbf{v}$ \\
\hline trans-1,3-Dichloropropene & 200 & 250 & 200 & 200 & 50 & 50 & $5 \mathbf{v}$ & $5 \mathbf{U}$ \\
\hline Ethylbenzene & 200 & $25 \mathrm{U}$ & 200 & 200 & 50 & 5u & $5 \mathbf{U}$ & 50 \\
\hline 2-Hexanone & 400 & 500 & 400 & 400 & 100 & 100 & 100 & 100 \\
\hline 4-Methyl-2-pentanone & 400 & 500 & 400 & 4 & 100 & 100 & 2 & $10 \mathrm{U}$ \\
\hline Methylene chloride & 9 & 250 & 200 & 8 & $5 \mathbf{U}$ & 50 & $5 \mathbf{U}$ & $\mathbf{5 u}$ \\
\hline Styrene & 200 & 250 & 200 & 200 & $5 v$ & $5 \mathrm{U}$ & $5 \mathrm{U}$ & $5 U$ \\
\hline $1,1,2,2$-Tetrachloroethane & 200 & $25 \mathrm{U}$ & 200 & 200 & $5 u$ & 50 & $5 \mathbf{v}$ & $5 v$ \\
\hline Tetrachloroethene & 540 & 430 & 530 & 780 & 4 & 5 & 4 & 5 \\
\hline Toluene & $20 U$ & $25 \mathrm{U}$ & 200 & 200 & 50 & $5 \mathbf{u}$ & 0.8 & $5 U$ \\
\hline 1,1,1-Trichloroethane & 200 & $25 U$ & 200 & 200 & 5u & 50 & 50 & 50 \\
\hline 1,1,2-Trichloroethane & 200 & $25 U$ & 200 & 200 & $5 U$ & $5 \mathbf{u}$ & 50 & $5 U$ \\
\hline Trichloroethene & 170 & 150 & 190 & 230 & 50 & $5 \mathrm{U}$ & $5 \mathbf{U}$ & 50 \\
\hline Vinyl acetate & $40 u$ & 500 & 400 & 400 & 100 & $10 \mathrm{U}$ & 100 & 100 \\
\hline vinyl chloride & $40 \mathrm{U}$ & 500 & 400 & 400 & 100 & 100 & 100 & 100 \\
\hline xylenes & 200 & $25 U$ & 200 & 200 & $5 U$ & $5 \mathrm{U}$ & 6 & $5 \mathbf{U}$ \\
\hline
\end{tabular}

(CONTINOED) 
APPENDIX E. 2

Groundwater Quality Data, 1993

\begin{tabular}{|c|c|c|c|c|c|c|c|c|}
\hline \multirow{3}{*}{$\begin{array}{l}\text { Sampling Point } \\
\text { - } \\
\text { Location } \\
\text { Date Sampled }\end{array}$} & \multicolumn{4}{|c|}{$G W-632$} & \multicolumn{4}{|c|}{$G W-633$} \\
\hline & \multicolumn{4}{|c|}{ RG } & \multicolumn{4}{|c|}{ RG } \\
\hline & $03 / 03 / 93$ & $06 / 21 / 93$ & $09 / 16 / 93$ & $11 / 18 / 93$ & $03 / 03 / 93$ & $06 / 21 / 93$ & $09 / 20 / 93$ & $11 / 19 / 93$ \\
\hline VOLATILE ORGANICS (ug/L) & $\cdot$ & $\cdot$ & • & - & $\cdot$ & - & - & - \\
\hline Acetone & 5000 & 10000 & 100 & 2000 & 100 & 100 & $10 \mathrm{U}$ & 100 \\
\hline Benzene & 9900 & 9300 & 2300 & 11000 & 6 & 8 & 10 & 4 \\
\hline Bromodichloromethane & 2500 & 5000 & 50 & 1000 & 5u & 50 & 50 & 5u \\
\hline Bromoform & 2500 & 5000 & su & 1000 & 2 & 50 & 1 & $5 \mathbf{u}$ \\
\hline Bromomethane & 5000 & $1000 \mathrm{U}$ & 100 & 2000 & 100 & 100 & 100 & 100 \\
\hline 2-Butanone & 5000 & 10000 & 100 & 2000 & $10 \mathrm{U}$ & $10 \mathrm{v}$ & $10 \mathrm{v}$ & 100 \\
\hline Carbon disulfide & 2500 & 5000 & 50 & 1000 & 50 & 50 & $5 \mathbf{v}$ & $\mathbf{5 u}$ \\
\hline Carbon tetrachloride & 2500 & 5000 & $5 \mathrm{U}$ & 1000 & 50 & 50 & $5 v$ & $5 \mathbf{5}$ \\
\hline Chlorobenzene & 2500 & 5000 & 50 & 1000 & $5 \mathrm{v}$ & 50 & 50 & $5 \mathbf{v}$ \\
\hline Chlorodibromomethane & 2500 & 5000 & $5 u$ & 1000 & 50 & $5 v$ & $5 U$ & $5 \mathbf{v}$ \\
\hline Chloroethane & 5000 & 10000 & 100 & 2000 & 100 & 100 & 100 & 100 \\
\hline Chloroform & 2500 & 5000 & 0.5 & 1000 & 24 & 28 & 27 & 6 \\
\hline Chloromethane & 160 & 10000 & 100 & 2000 & 100 & 100 & 100 & 100 \\
\hline 1,1-Dichloroethane & 2500 & 5000 & 2 & 1000 & $5 \mathbf{U}$ & 50 & 50 & $\mathbf{s u}$ \\
\hline 1,2-Dichloroethane & 2500 & 5000 & 50 & 1000 & 5u & 50 & 50 & $5 \mathbf{u}$ \\
\hline 1,1-Dichloroethene & 2500 & 5000 & 50 & 1000 & 50 & 2 & 1 & 0.8 \\
\hline 1,2-Dichloroethene & 2500 & 5000 & 15 & 1000 & 3 & 4 & 3 & $\mathbf{5 u}$ \\
\hline 1,2-Dichloropropane & 2500 & 5000 & 50 & 1000 & 50 & su & 50 & $5 \mathbf{v}$ \\
\hline cis-1,3-Dichloropropene & 2500 & 5000 & 50 & 1000 & 50 & 50 & 50 & $5 \mathbf{U}$ \\
\hline trans-1,3-Dichloropropene & 2500 & 5000 & 50 & 1000 & $5 u$ & 50 & 5u & $5 \mathbf{U}$ \\
\hline Ethylbenzene & 260 & 360 & 170 & 400 & $5 \mathbf{U}$ & $5 \mathbf{v}$ & 1 & 50 \\
\hline 2-Hexanone & 5000 & 10000 & $10 \mathrm{U}$ & 2000 & $10 \mathrm{U}$ & $10 \mathrm{U}$ & 100 & $10 U$ \\
\hline 4-Methy1-2-pentanone & 71 & 10000 & 100 & 2000 & 100 & 100 & 100 & 100 \\
\hline Methylene chloride & 2500 & 5000 & $5 \mathrm{U}$ & 1000 & 18 & 29 & 24 & 7 \\
\hline Styrene & 2500 & 5000 & $5 U$ & 1000 & 50 & 5u & 50 & $5 \mathbf{u}$ \\
\hline $1,1,2,2$-Tetrachloroethane & 2500 & 5000 & $5 U$ & 1000 & 50 & 5u & 50 & $5 \mathbf{v}$ \\
\hline Tetrachloroethene & 2500 & 5000 & 2 & 1000 & 60 & 74 & 78 & 30 \\
\hline Toluene & 3600 & 4100 & 690 & 4100 & 3 & 6 & 8 & 4 \\
\hline 1,1,1-Trichloroethane & 2500 & 5000 & 50 & 1000 & 50 & 50 & 50 & $5 \mathbf{v}$ \\
\hline $1,1,2$-Trichloroethane & 2500 & 5000 & 50 & 1000 & 50 & 50 & 5U & 50 \\
\hline Trichloroethene & 2500 & 5000 & 1 & 1000 & 7 & 8 & 8 & 2 \\
\hline Vinyl acetate & 1000 & $1000 \mathrm{U}$ & 100 & 2000 & 100 & 100 & $10 \mathrm{U}$ & 100 \\
\hline Vinyl chloride & 5000 & 10000 & 100 & 2000 & $10 \mathrm{U}$ & 100 & 100 & 100 \\
\hline Xylenes & 2600 & 3000 & 1200 & 4100 & 1 & 4 & 6 & 3 \\
\hline
\end{tabular}

( CONTINUED) 
APPBNDIX E. 2

Groundwater Quality Data, 1993

\begin{tabular}{|c|c|c|c|c|c|c|c|c|}
\hline \multirow{3}{*}{$\begin{array}{l}\text { Sampling Point } \\
\text { Location } \\
\text { - } \\
\text { Date sampled }\end{array}$} & \multicolumn{4}{|c|}{$G W-634$} & \multicolumn{4}{|c|}{$G W-656$} \\
\hline & \multicolumn{4}{|c|}{ RG } & \multicolumn{4}{|c|}{ T0134 } \\
\hline & $03 / 02 / 93$ & $06 / 18 / 93$ & $09 / 16 / 93$ & $11 / 18 / 93$ & $03 / 11 / 93$ & $06 / 22 / 93$ & $09 / 23 / 93$ & $11 / 17 / 93$ \\
\hline VOLATILE ORGANICS (ug/I) & - & - & - & - & $\cdot$ & - & - & - \\
\hline Acetone & 2500 & 2500 & 100 & 2000 & 10000 & 10000 & $1000 \mathrm{v}$ & 10000 \\
\hline Benzene & 430 & 380 & 270 & 310 & 5000 & 5000 & 5000 & 5000 \\
\hline Bromodichloromethane & 1300 & 1300 & $5 \mathbf{u}$ & 1000 & 5000 & 5000 & 5000 & 5000 \\
\hline Bromoform & $130 \mathrm{u}$ & 1300 & 5u & $100 \mathrm{U}$ & 5000 & 5000 & 5000 & 5000 \\
\hline Bromomethane & 2500 & 2500 & 100 & 2000 & $1000 \mathrm{U}$ & 10000 & 10000 & 10000 \\
\hline 2-Butanone & $250 u$ & 2500 & 100 & 2000 & $1000 \mathrm{U}$ & $1000 \mathrm{U}$ & $1000 u$ & 10000 \\
\hline Carbon disulfide & 1300 & 1300 & 50 & 1000 & 5000 & 5000 & 5000 & 5000 \\
\hline Carbon tetrachloride & 1300 & 1300 & 5u & 1000 & $500 u$ & 5000 & $500 \mathrm{U}$ & 5000 \\
\hline Chlorobenzene & $130 \mathrm{u}$ & 1300 & 50 & 1000 & 5000 & 5000 & 5000 & 5000 \\
\hline Chlorodibromomethane & 1300 & 1300 & 5u & 1000 & $500 u$ & 5000 & 5000 & 5000 \\
\hline Chloroethane & 2500 & 2500 & 100 & 2000 & 10000 & 10000 & 10000 & 10000 \\
\hline Chloroform & $130 \mathrm{U}$ & 1300 & 50 & 1000 & 5000 & 5000 & 5000 & 5000 \\
\hline Chloromethane & 130 & 2500 & 100 & 2000 & 10000 & 10000 & 10000 & 10000 \\
\hline 1,1-Dichloroethane & 1300 & $130 \mathrm{U}$ & 50 & 1000 & $500 \mathrm{U}$ & 5000 & 5000 & 5000 \\
\hline 1,2-Dichloroethane & 1300 & 1300 & 50 & 1000 & 5000 & 5000 & 5000 & 5000 \\
\hline 1,1-Dichloroethene & 1300 & 1300 & $5 \mathrm{U}$ & 1000 & 1700 & 1700 & 1500 & 1500 \\
\hline 1,2-Dichloroethene & 1300 & 1300 & 2 & $100 \mathrm{U}$ & 300 & 340 & 230 & 250 \\
\hline 1,2-Dichloropropane & 1300 & 1300 & $5 \mathbf{v}$ & 1000 & 5000 & 5000 & 5000 & 5000 \\
\hline cis-1,3-Dichloropropene & 1300 & 1300 & 50 & 1000 & 5000 & 5000 & 5000 & 5000 \\
\hline trans-1,3-Dichloropropene & 1300 & 1300 & $\mathbf{5 u}$ & $100 \mathrm{u}$ & 5000 & 5000 & 5000 & 5000 \\
\hline Ethylbenzene & 790 & 710 & 550 & 680 & 5000 & 5000 & 5000 & 5000 \\
\hline 2-Hexanone & 2500 & 2500 & 100 & 2000 & 10000 & 10000 & 10000 & 10000 \\
\hline 4-Methyl-2-pentanone & 2500 & 2500 & 100 & 2000 & 10000 & 10000 & 10000 & 10000 \\
\hline Methylene chloride & 62 & 1300 & su & 1000 & 5000 & 5000 & 5000 & 5000 \\
\hline styrene & 53 & 1300 & 50 & 1000 & 5000 & 5000 & 5000 & 5000 \\
\hline $1,1,2,2$-Tetrachloroethane & 1300 & 1300 & 5u & 1000 & 5000 & $500 \mathrm{U}$ & 5000 & 5000 \\
\hline Tetrachloroethene & 1300 & 1300 & 50 & 1000 & 5000 & 99 & 81 & 5000 \\
\hline Toluene & 680 & 540 & 300 & 350 & 5000 & 5000 & 5000 & 5000 \\
\hline $1,1,1$-Trichloroethane & 1300 & 1300 & 50 & 1000 & 5000 & 5000 & 5000 & 5000 \\
\hline $1,1,2$-Trichloroethane & 1300 & 1300 & 50 & 1000 & $500 u$ & 5000 & $500 \mathrm{U}$ & 5000 \\
\hline Trichloroethene & 1300 & 1300 & 50 & 1000 & 11000 & 11000 & 10000 & 8600 \\
\hline Vinyl acetate & 2500 & 2500 & 100 & 2000 & $1000 \mathrm{U}$ & $1000 \mathrm{U}$ & $1000 \mathrm{U}$ & 10000 \\
\hline vinyl chlor 1 de & 2500 & 2500 & 100 & 2000 & $1000 \mathrm{U}$ & $1000 \mathrm{U}$ & $1000 \mathrm{U}$ & 10000 \\
\hline Xylenes & 3100 & 2800 & 1800 & 2200 & 5000 & 5000 & 5000 & 5000 \\
\hline
\end{tabular}

(CONTINUED) 
APPENDIX $\mathrm{E} .2$

\begin{tabular}{|c|c|c|c|c|c|c|c|c|}
\hline \multirow{3}{*}{$\begin{array}{l}\text { Sampling Polnt } \\
\text { Location } \\
\text { - } \\
\text { Date sampled }\end{array}$} & \multicolumn{4}{|c|}{$G w-657$} & \multicolumn{4}{|c|}{$G W-658$} \\
\hline & \multicolumn{4}{|c|}{ T2331 } & \multicolumn{4}{|c|}{$\mathbf{F F}$} \\
\hline & $03 / 10 / 93$ & $06 / 18 / 93$ & $09 / 21 / 93$ & $11 / 15 / 93$ & $03 / 11 / 93$ & $06 / 23 / 93$ & $11 / 19 / 93$ & $09 / 27 / 04$ \\
\hline VOLATILE ORCANICS (ug/L) & - & - & $\cdot$ & • & - & $\cdot$ & $\cdot$ & - \\
\hline Acetone & 100 & 100 & $10 \mathrm{v}$ & $10 \mathrm{U}$ & $1000 \mathrm{v}$ & 10000 & 5000 & 10000 \\
\hline Benzene & 50 & 50 & su & $5 \mathbf{u}$ & 10000 & 13000 & 4800 & 14000 \\
\hline Bromodichloromethane & 5u & 5u & 50 & 5v & 5000 & 5000 & 2500 & 5000 \\
\hline Bromotorm & 50 & 5u & su & $5 v$ & 5000 & 5000 & 2500 & 5000 \\
\hline Bromomethane & $10 v$ & 100 & 100 & 100 & 10000 & $1000 \mathrm{U}$ & 5000 & 10000 \\
\hline 2-Butanone & 100 & 100 & 100 & 100 & 10000 & 10000 & 5000 & 10000 \\
\hline Carbon disulfide & su & 50 & 50 & 50 & 5000 & 5000 & 2500 & 5000 \\
\hline Carbon tetrachloride & 50 & 5u & su & 50 & 5000 & 5000 & 2500 & 5000 \\
\hline Chlorobenzene & 50 & 5u & su & su & 5000 & 5000 & 2500 & $500 u$ \\
\hline Chlorodibroxomethane & su & 5u & 50 & 50 & 5000 & 5000 & 2500 & 5000 \\
\hline Chloroethane & 100 & 100 & 100 & 100 & 10000 & 10000 & 5000 & 10000 \\
\hline Chloroform & 5u & $5 \mathbf{v}$ & 5v & su & 5000 & 5000 & 2500 & 5000 \\
\hline Chloromethane & 100 & 100 & 100 & 100 & 10000 & $1000 \mathrm{u}$ & 5000 & 10000 \\
\hline 1,1-Dichloroethane & 50 & $5 U$ & $5 \mathbf{v}$ & 5u & 5000 & 5000 & 2500 & 5000 \\
\hline 1,2-Dichloroethane & 5u & 50 & $5 v$ & su & 560 & 790 & 370 & 1000 \\
\hline 1,1-D1chloroethene & 50 & 50 & 50 & $5 \mathbf{v}$ & 5000 & 5000 & 2500 & 5000 \\
\hline 1,2-Dichloroethene & 5u & su & su & su & 5000 & 5000 & 2500 & 5000 \\
\hline 1,2-Dichloropropane & 50 & 5u & 5u & 50 & $500 \mathrm{U}$ & 5000 & 2500 & 5000 \\
\hline cie-2,3-Dichloropropene & 50 & 50 & 50 & 50 & 5000 & 5000 & 2500 & 5000 \\
\hline trans-1,3-Dichloropropene & $5 v$ & su & 5u & su & 5000 & 5000 & 2500 & 5000 \\
\hline Bthylbenzene & su & 50 & 50 & 5u & 1100 & 1400 & 760 & 1500 \\
\hline 2-Hexanone & 100 & 100 & 100 & 100 & $1000 \mathrm{U}$ & 10000 & 5000 & 10000 \\
\hline 4-Methyl-2-pentanone & 2 & 100 & 100 & 100 & 10000 & 10000 & 5000 & 10000 \\
\hline Methylene chloride & 2 & 50 & su & su & 120 & 5000 & 2500 & $500 v$ \\
\hline Styrane & 50 & su & 50 & 5u & 5000 & 5000 & 2500 & 5000 \\
\hline $1,1,2,2$-Tetrachloroethane & 5U & su & $5 \mathbf{v}$ & 50 & 5000 & 5000 & 2500 & 5000 \\
\hline Setrachloroethene & $5 u$ & 50 & 50 & 50 & 5000 & 5000 & 2500 & 5000 \\
\hline Toluene & 50 & 50 & 5u & su & 5700 & 8400 & 3400 & 9300 \\
\hline 1,1,1-Trichloroethane & 50 & 50 & $5 U$ & 5u & 5000 & 5000 & 2500 & 5000 \\
\hline 1,1,2-Trichloroethane & 50 & 50 & su & 50 & 5000 & 5000 & 2500 & 5000 \\
\hline Trichloroethene & 50 & 50 & 50 & 5u & 5000 & 5000 & 2500 & $500 u$ \\
\hline Vinyl acetate & 2 & 100 & 100 & 100 & $1000 \mathrm{U}$ & 10000 & 5000 & 10000 \\
\hline vinyl chloride & 100 & 100 & $10 \mathrm{U}$ & 100 & $1000 \mathrm{u}$ & $1000 \mathrm{v}$ & 5000 & 10000 \\
\hline Xylenes & $5 U$ & su & 5u & 50 & 6800 & 6400 & 4000 & 7500 \\
\hline
\end{tabular}

(CONT INUED) 
APPENDIX E. 2

\begin{tabular}{|c|c|c|c|c|c|c|c|c|}
\hline \multirow{3}{*}{$\begin{array}{l}\text { Sampling Polnt } \\
\text { Locetion } \\
\text { Date sampled }\end{array}$} & \multicolumn{4}{|c|}{$6 w-659$} & \multicolumn{4}{|c|}{$0 w-707$} \\
\hline & \multicolumn{4}{|c|}{$\mathbf{r}$} & \multicolumn{4}{|c|}{ T2331 } \\
\hline & $03 / 10 / 93$ & $06 / 18 / 93$ & $09 / 22 / 93$ & $11 / 15 / 93$ & $03 / 11 / 93$ & $06 / 23 / 93$ & $09 / 27 / 93$ & $11 / 18 / 93$ \\
\hline VOLATILE ORGNNICS (ug/I) & - & - & • & • & - & - & - & - \\
\hline Acetone & 1 & 200 & 200 & 100 & 100 & 200 & 100 & 100 \\
\hline Benzene & 11 & 27 & 42 & 2 & $5 v$ & $\mathbf{5 u}$ & $5 \mathbf{v}$ & 5u \\
\hline Bromodichloromethane & 50 & su & $5 \mathbf{v}$ & $\mathbf{5 u}$ & $5 v$ & $\mathbf{s u}$ & $5 \mathbf{v}$ & $\mathbf{s u}$ \\
\hline Bromotorm & $\mathbf{5 u}$ & sv & 50 & sv & $5 v$ & su & 50 & 50 \\
\hline Bromomethane & 100 & 100 & 100 & 100 & 100 & 100 & 100 & 100 \\
\hline 2-Butanone & 100 & 100 & 100 & 100 & 100 & 100 & 100 & 100 \\
\hline Carbon disulfide & 50 & 5u & su & $5 \mathbf{v}$ & $5 \mathbf{v}$ & Su & 50 & su \\
\hline Carbon tetrachlorlde & su & $5 v$ & su & 50 & 50 & su & su & su \\
\hline Chlorobanzene & 50 & sv & 50 & su & $5 u$ & su & su & $\mathbf{5 u}$ \\
\hline Chlorodibromomethane & $\mathbf{s u}$ & su & su & $5 \mathbf{v}$ & $5 \mathbf{v}$ & su & su & $\mathbf{s u}$ \\
\hline Chloroethane & 100 & 100 & 100 & 100 & 100 & 100 & 100 & 100 \\
\hline Chloroform & 50 & 5u & su & $\mathbf{s u}$ & 5u & 50 & $\mathbf{5 u}$ & 5u \\
\hline Chloromethane & 100 & 100 & 100 & 100 & 100 & 100 & 100 & 100 \\
\hline 1,1-Dichloroethane & su & $5 \mathbf{v}$ & su & 50 & $5 \mathbf{v}$ & $5 \mathbf{v}$ & 50 & 50 \\
\hline 1,2-Dichloroethane & 5u & 5u & so & su & $5 \mathbf{v}$ & $5 \mathbf{v}$ & $5 \mathbf{u}$ & 50 \\
\hline 1,1-Dichloroethene & $5 \mathbf{v}$ & 5v & su & su & 50 & 50 & su & 50 \\
\hline 1,2-Dichloroethene & su & sv & su & su & su & su & $5 \mathbf{v}$ & 5u \\
\hline 1,2-Dichloropropane & su & 50 & so & so & su & 50 & 50 & $\mathbf{5 u}$ \\
\hline cis-1,3-Dichloropropene & $5 \mathbf{v}$ & 5u & su & $\mathbf{s u}$ & $5 \mathbf{v}$ & $5 \mathbf{v}$ & su & 50 \\
\hline trane-1,3-Dichloropropene & 50 & su & su & $\mathbf{5 u}$ & 5v & $\mathbf{s u}$ & su & $\mathbf{s u}$ \\
\hline Ethylbenzene & 2 & so & 4 & su & 5u & 50 & $\mathbf{5 u}$ & 50 \\
\hline 2-Hexanone & 100 & 100 & 200 & 100 & 100 & 100 & 100 & 100 \\
\hline 1-Mathyl-2-pentanone & 2 & 100 & 100 & 100 & 100 & 100 & 100 & 100 \\
\hline Methylene chlorlde & 2 & $5 \mathbf{v}$ & 50 & su & $\mathbf{5 u}$ & $\mathbf{5 u}$ & su & 50 \\
\hline styrene & $5 \mathbf{v}$ & su & $\mathbf{s u}$ & $\mathbf{5 u}$ & $5 \mathbf{v}$ & 5u & 5u & 50 \\
\hline $1,1,2,2$-Tetrachloroethane & 50 & su & $\mathbf{5 0}$ & su & 50 & su & su & 50 \\
\hline Tetrachloroethene & su & 50 & 50 & 5u & su & su & su & 50 \\
\hline Toluene & 50 & 50 & su & 5u & $5 \mathbf{U}$ & $5 u$ & su & 50 \\
\hline $1,1,1$-Trichloroethane & $5 \mathbf{v}$ & su & 5u & so & $5 v$ & su & $\mathbf{5 u}$ & 50 \\
\hline $1,1,2$-Trichloroethane & 50 & 5v & su & su & $5 v$ & $5 v$ & $5 \mathbf{v}$ & 50 \\
\hline Trichloroethene & $5 \mathbf{v}$ & su & su & $5 \mathbf{v}$ & 1 & $\mathbf{5 u}$ & $\mathbf{5 u}$ & 50 \\
\hline Vinyl acetate & 100 & 100 & 100 & 100 & 100 & 100 & 100 & 100 \\
\hline vinyl chloride & 100 & 100 & 100 & 100 & 100 & 100 & 100 & 100 \\
\hline xylenes & 1 & 9 & 17 & 6 & $5 v$ & 50 & $5 u$ & su \\
\hline
\end{tabular}

(CONT INUED) 
APPENDTX E.2

\begin{tabular}{|c|c|c|c|c|c|c|c|c|}
\hline \multirow{3}{*}{$\begin{array}{l}\text { Sampling point } \\
\text { Location } \\
\text { Date sampled }\end{array}$} & \multicolumn{4}{|c|}{$6 W-708$} & \multicolumn{4}{|c|}{ GW-733 } \\
\hline & \multicolumn{4}{|c|}{ T2331 } & \multicolumn{4}{|c|}{$\mathbf{E X P}$} \\
\hline & $03 / 10 / 93$ & $06 / 18 / 93$ & $09 / 21 / 93$ & $11 / 12 / 93$ & $02 / 02 / 93$ & $05 / 06 / 93$ & $08 / 18 / 93$ & $11 / 04 / 93$ \\
\hline VOLATILE ORENSICS (Ug/L) & $\cdot$ & $\cdot$ & $\cdot$ & $\cdot$ & $\cdot$ & - & $\cdot$ & $\cdot$ \\
\hline Acetone & 2000 & 2000 & 2000 & 2000 & 100 & 100 & 100 & 100 \\
\hline Benzene & 870 & 1200 & 1100 & 780 & su & su & su & sv \\
\hline Bromodichloromethane & 1000 & 1000 & 1000 & 1000 & su & so & su & su \\
\hline Bromotorm & 1000 & 1000 & 1000 & 1000 & su & $\mathbf{s u}$ & su & su \\
\hline Bromomethane & 2000 & 2000 & 2000 & 2000 & 200 & 100 & 200 & 100 \\
\hline 2-Butanone & $200 \mathrm{w}$ & 2000 & 2000 & 2000 & 100 & 100 & 100 & 100 \\
\hline Carbon dluulelde & 1000 & 2000 & 1000 & 1000 & su & so & sv & 50 \\
\hline Carbon tetrachloride & 1000 & 1000 & 1000 & 1000 & 60 & 61 & 72 & 87 \\
\hline Chlorobenzene & 1000 & 1000 & 1000 & 1000 & 5u & so & su & 50 \\
\hline Chlorodibromomethane & 1000 & 1000 & 1000 & 1000 & su & so & 50 & su \\
\hline Chloroethane & 2000 & 2000 & 2000 & 2000 & 100 & 100 & 100 & 100 \\
\hline Chlorotorm & 1000 & 1000 & 1000 & 1000 & 8 & 5 & 6 & 7 \\
\hline Chloromethane & 2000 & 2000 & 2000 & 2000 & 100 & 100 & 100 & 100 \\
\hline 1,1-Dichloroethane & 1000 & 1000 & 1000 & 1000 & su & 50 & sv & su \\
\hline 1,2-Dichloroethane & 1000 & 1000 & 1000 & 1000 & su & so & su & su \\
\hline 1,1-Dichloroathene & 1000 & 1000 & 1000 & 1000 & so & 50 & 50 & su \\
\hline 1,2-Dichloroethene & 1000 & 1000 & 1000 & 1000 & su & so & so & su \\
\hline 1,2-Dichloropropane & 1000 & 1000 & 1000 & 1000 & so & so & so & 50 \\
\hline cie-1,3-D1chloropropene & 1000 & 1000 & 1000 & 1000 & 50 & so & su & su \\
\hline trans-1, 3-Dichloropropane & 1000 & 1000 & 2000 & 1000 & 50 & so & su & su \\
\hline sthylbenzene & 300 & 620 & 720 & 670 & su & su & su & so \\
\hline 2-Hexanone & 2000 & 2000 & 2000 & 2000 & 100 & 100 & 100 & 100 \\
\hline 4-Methyl-2-pentanone & 57 & 2000 & 2000 & 2000 & 100 & 1 & 100 & 100 \\
\hline Methylene chloride & 63 & 1000 & 1000 & 32 & su & 2 & 1 & 1 \\
\hline styrene & 1000 & 1000 & 1000 & 1000 & 50 & 5u & so & su \\
\hline 1,1,2,2-Tetrachloroethane & 1000 & 1000 & 1000 & 1000 & su & su & su & su \\
\hline Tetrachloroethene & 1000 & 1000 & 1000 & 1000 & 2 & 2 & 2 & 3 \\
\hline Toluene & 150 & 120 & 120 & 98 & 50 & su & su & su \\
\hline 1,1,1-Trichloroethane & 1000 & 1000 & 1000 & 1000 & 50 & 5u & so & su \\
\hline 1,1,2-Trichloroethane & 1000 & 1000 & 1000 & 1000 & su & 5u & su & 5u \\
\hline Trichloroethene & 1000 & 1000 & 2000 & 1000 & 0.9 & su & 1 & 1 \\
\hline Vinyl acetate & 2000 & 2000 & 2000 & 2000 & 100 & 100 & 100 & 100 \\
\hline Vinyl chlorida & $200 \mathrm{U}$ & 2000 & 2000 & 2000 & 100 & 100 & 100 & 100 \\
\hline xylenes & 2500 & 2600 & 2400 & 1800 & 50 & su & so & su \\
\hline
\end{tabular}

(CONTINURD) 
APPENDIX $\mathrm{E} .2$

\begin{tabular}{|c|c|c|c|c|c|c|c|c|}
\hline \multirow{3}{*}{$\begin{array}{l}\text { Sampling point } \\
\text { Looation } \\
\text { - } \\
\text { Date sampled }\end{array}$} & \multicolumn{4}{|c|}{ ow-73s } & \multicolumn{4}{|c|}{ CW-744 } \\
\hline & \multicolumn{4}{|c|}{ NHP } & \multicolumn{4}{|c|}{ ORIDK1 } \\
\hline & $02 / 02 / 93$ & $05 / 07 / 93$ & $08 / 28 / 93$ & $10 / 29 / 93$ & $01 / 18 / 93$ & $04 / 12 / 93$ & $08 / 04 / 93$ & $10 / 22 / 93$ \\
\hline VOLATIL ORCNAICs (ug/L) & - & - & - & - & - & - & - & - \\
\hline Acetone & 100 & 100 & 100 & 100 & 100 & 100 & 100 & 5 \\
\hline Bensene & su & $\mathbf{5 u}$ & su & su & su & $\mathbf{s u}$ & su & su \\
\hline Bromodichloromethane & $5 \mathbf{v}$ & su & su & su & $\mathbf{s u}$ & $\mathbf{s u}$ & $\mathbf{5 u}$ & $\mathbf{5 u}$ \\
\hline Bromoform & $\mathbf{5 u}$ & $\mathbf{s u}$ & su & su & su & su & su & su \\
\hline Eromomethane & 100 & 100 & 100 & 100 & 100 & 200 & 100 & 100 \\
\hline 2-Butanone & 100 & 100 & 100 & 100 & 100 & 100 & 100 & 10 \\
\hline Carbon disulfide & su & su & su & $5 \mathbf{v}$ & su & su & su & su \\
\hline Carbon tetrachlortde & su & su & su & su & su & su & su & so \\
\hline Chlorobanzene & su & su & $\mathbf{5 u}$ & su & su & su & su & $\mathbf{s u}$ \\
\hline Chlorod1bromomethene & su & so & su & su & $\mathbf{5 u}$ & so & su & su \\
\hline Chloroethane & 100 & 100 & 100 & 100 & 100 & 100 & 100 & 100 \\
\hline Chlorotorm & so & so & su & 50 & su & so & su & su \\
\hline Chloromethane & 100 & 100 & 100 & 100 & 100 & 100 & 100 & 100 \\
\hline 1,1-Dlehloroethane & 50 & $\mathbf{5 u}$ & su & $\mathbf{s u}$ & $\mathbf{5 0}$ & 50 & $\mathbf{s u}$ & $\mathbf{5 u}$ \\
\hline 1,2-Dlehloroethane & so & 50 & $\mathbf{s u}$ & su & $\mathbf{5 u}$ & su & su & $\mathbf{5 u}$ \\
\hline 1,1-Dichloroethene & sv & so & su & su & su & su & su & $\mathbf{s u}$ \\
\hline 1,2-Dlehloroethene & su & $\mathbf{5 0}$ & su & su & su & so & su & $\mathbf{5 v}$ \\
\hline 1,2-D1chloropropane & so & 50 & su & so & 50 & su & $\mathbf{s u}$ & $\mathbf{5 0}$ \\
\hline c1s-1,3-DLchloropropene & so & $\mathbf{5 0}$ & su & su & $\mathbf{5 0}$ & sv & so & $\mathbf{5 u}$ \\
\hline trens-1,3-DLchloropropene & so & $\mathbf{5 0}$ & su & $\mathbf{s u}$ & $\mathbf{5 0}$ & $5 v$ & $\mathbf{s u}$ & su \\
\hline ethylbenzene & su & su & su & $\mathbf{s u}$ & $\mathbf{s 0}$ & su & so & $\mathbf{s u}$ \\
\hline 2-Hexanone & 100 & 100 & 100 & 100 & 100 & $10 \mathrm{v}$ & 100 & 100 \\
\hline 4-Methyl-2-pentanone & 100 & 100 & 100 & 100 & 100 & 100 & 100 & 100 \\
\hline methylene chloride & so & 0.8 & su & 1 & 0.6 & su & $\mathbf{s u}$ & 3 \\
\hline styrene & 50 & $\mathbf{5 0}$ & su & $5 \mathbf{v}$ & $5 \mathbf{v}$ & $\mathbf{s u}$ & $\mathbf{s u}$ & 50 \\
\hline $1,1,2,2$-Tetrechloroethane & su & $\mathbf{5 U}$ & su & $5 \mathbf{v}$ & $\mathbf{5 u}$ & $5 \mathbf{v}$ & su & $\mathbf{5 u}$ \\
\hline Tetrachloroethene & so & 50 & su & $5 \mathbf{v}$ & $\mathbf{s u}$ & su & 50 & su \\
\hline Toluene & su & 50 & $5 \mathbf{u}$ & 50 & 50 & 5u & so & su \\
\hline $1,1,1-$ Ir 1ehloroethane & $\mathbf{s u}$ & $\mathbf{5 u}$ & su & 50 & sv & $\mathbf{5 u}$ & 5u & su \\
\hline $1,1,2$-Trichloroethane & su & su & $\mathbf{s u}$ & $\mathbf{s u}$ & $\mathbf{5 u}$ & su & so & $\mathbf{s u}$ \\
\hline Irichloroethene & 50 & 50 & $\mathbf{5 u}$ & su & su & $\mathbf{s u}$ & so & $5 U$ \\
\hline VInyl seetate & 100 & 100 & 100 & 100 & 100 & 100 & 100 & 100 \\
\hline Vinyl chloride & 100 & 100 & 100 & 100 & 100 & 100 & 100 & 100 \\
\hline xylenes & so & su & $5 u$ & 50 & 50 & 50 & 50 & $\mathbf{S U}$ \\
\hline
\end{tabular}

(CONTINUED) 
APPENDIX 8.2

\begin{tabular}{|c|c|c|c|c|c|c|c|c|}
\hline \multirow{3}{*}{$\begin{array}{l}\text { 8ampling Point } \\
\text { Location } \\
\text { Date sampled }\end{array}$} & \multicolumn{4}{|c|}{$O W-745$} & \multicolumn{4}{|c|}{ OW-746 } \\
\hline & \multicolumn{4}{|c|}{ GRIDK1 } & \multicolumn{4}{|c|}{ ORIDKI } \\
\hline & $01 / 19 / 93$ & $04 / 12 / 93$ & $08 / 04 / 93$ & $10 / 23 / 93$ & $01 / 19 / 93$ & $04 / 12 / 93 \mid$ & $08 / 05 / 93$ & $10 / 23 / 93$ \\
\hline VOLATILE ORONHICS (ug/L) & $\cdot$ & $\cdot$ & $\cdot$ & - & $\cdot$ & $\cdot$ & $\cdot$ & - \\
\hline Acetone & 100 & 100 & 100 & 100 & 100 & 100 & 100 & 100 \\
\hline Bonzene & su & su & su & so & su & so & 50 & su \\
\hline Bronodichloromethane & su & 50 & su & 50 & su & su & su & su \\
\hline Bromotorm & so & so & su & 50 & su & su & su & su \\
\hline Bromomethane & 100 & 100 & 100 & 100 & 100 & 100 & 100 & 100 \\
\hline 2-Butanone & 100 & 100 & 100 & $10 u$ & 100 & 100 & 100 & 100 \\
\hline Carbon dlsuleide & su & su & sv & 50 & su & $\mathbf{s v}$ & su & su \\
\hline Carbon tetrachloride & so & su & so & so & so & $5 u$ & su & su \\
\hline Chlorobenzone & su & so & su & so & so & su & su & 50 \\
\hline Chlorodlbromomothane & 50 & su & so & so & su & su & su & sv \\
\hline Chloroethane & 100 & 100 & 100 & 100 & 100 & 100 & 100 & 100 \\
\hline Chloroform & so & su & so & so & su & so & su & so \\
\hline Chloromethane & 100 & 100 & 100 & 100 & 100 & 100 & 100 & 100 \\
\hline 1,1-Dichloronthane & so & so & su & so & su & so & su & su \\
\hline 1,2-Dichloroethane & su & su & so & so & so & so & su & so \\
\hline 1,1-Dlchloroethene & su & su & so & so & so & 50 & su & 50 \\
\hline 1,2-Dichloroethene & so & so & so & so & su & so & su & So \\
\hline 1,2-Dichloropropane & so| & so & so & so & so & so & su & so \\
\hline cle-1,3-Dichloropropene & su & su & su & so & 5u & so & 50 & so \\
\hline trane-1, 3-Dichloropropene & so & su & su & so & su & so & su & su \\
\hline Ethylbenzone & so & su & so & so & su & 50 & su & so \\
\hline 2-Hexanone & 100 & 100 & 100 & 100 & 100 & 100 & 100 & 100 \\
\hline 1-Mothyl-2-pentanone & 2 & 100 & 100 & 100 & 100 & 100 & 100 & 100 \\
\hline Methylene chloride & 3 & 0.9 & su & 1 & su & 1 & su & 0.9 \\
\hline 8tyrene & su & su & su & so & su & so & su & su \\
\hline 1,1,2,2-Tetrachloroethane & 50 & su & so & so & 50 & su & su & so \\
\hline Tetrachloroethene & su & su & 50 & su & 5v & su & so & so \\
\hline Toluene & su & 5u & su & so & 5u & su & su & su \\
\hline $1,1,1$-rrichloroethane & su & su & so & su & so & so & su & su \\
\hline 1,1,2-Trichloroethane & su & su & so & 50 & su & so & so & su \\
\hline Trlehlorouthene & so & su & 50 & su & 5U & $\mathbf{s u}$ & su & su \\
\hline Vinyl acetate & 100 & 100 & 100 & 100 & 100 & 100 & 100 & 100 \\
\hline Vinyl chlorlde & 100 & 100 & 100 & 100 & 100 & 100 & 100 & 100 \\
\hline xylenes & 50 & su & 5u & su & su & su & su & su \\
\hline
\end{tabular}

(CONTINURD) 
APPENDIX 8.2

Groundwater Quallty Data, 1993

\begin{tabular}{|c|c|c|c|c|c|c|c|c|}
\hline \multirow{3}{*}{$\begin{array}{l}\text { Sampling Point } \\
\text { Location } \\
\text { Date sampled }\end{array}$} & \multicolumn{4}{|c|}{ ON-747 } & \multicolumn{4}{|c|}{ OW-748 } \\
\hline & \multicolumn{4}{|c|}{ GRIDK2 } & \multicolumn{4}{|c|}{ GRIDK2 } \\
\hline & $|01 / 19 / 93|$ & $|04 / 13 / 93|$ & $08 / 03 / 93$ & $10 / 23 / 93$ & $01 / 20 / 93$ & $04 / 13 / 93$ & $08 / 06 / 93$ & $10 / 24 / 93$ \\
\hline VOLATILE ORCAMICS (ug/L) & . & - & - & - & $\cdot$ & . & - & • \\
\hline Acetone & 100 & 100 & 100 & 100 & 1 & 100 & 100 & 100 \\
\hline Benzene & su & su & so & so & su & sv & su & su \\
\hline Bromodichloromethane & su & su & so & so & su & so & so & su \\
\hline Eromolorm & su & su & so & so & so & su & su & su \\
\hline Bromomethane & 100 & 100 & 100 & 100 & 100 & 100 & 100 & 100 \\
\hline 2-Butanone & 100 & 100 & 100 & 100 & 100 & 100 & 100 & 100 \\
\hline Carbon dieuleide & su & su & so & su & su & su & su & su \\
\hline Carbon tetrachloride & su & su & so & so & so & su & su & 5u \\
\hline Chlorobenzene & su & su & so & so & 50 & su & su & su \\
\hline Chlorod Ibromomethane & su & su & so & so & su & su & su & su \\
\hline Chloroethane & 100 & 100 & 100 & 100 & 100 & 100 & 100 & 100 \\
\hline Chlorotorm & su & su & so & 50 & 50 & su & su & su \\
\hline Chloromethane & 100 & 100 & 100 & 100 & 100 & 100 & 100 & 100 \\
\hline 1,1-Dlchloroethane & su & su & so & su & su & so & su & 50 \\
\hline 1,2-Dichloroothane & su & so & so & so & su & su & su & su \\
\hline 1,1-Dichloroethene & su & su & so & so & su & su & so & su \\
\hline 1,2-Dichloroethene & su & su & so & so & so & su & su & su \\
\hline 1,2-Dichloropropane & su & so & so & 50 & su & su & so & 50 \\
\hline cil-1, 3-Dichloropropene & so & su & so & so & su & su & so & su \\
\hline trans-1,3-Dichloropropene & su & 50 & 50 & 50 & su & 5u & su & su \\
\hline Ethylbenzene & su & su & 50 & su & su & su & su & su \\
\hline 2-Lexanone & 100 & 100 & 100 & 100 & 100 & 100 & 100 & 100 \\
\hline 1-Mathyl-2-pentanone & 2 & 100 & 100 & 100 & 2 & 100 & 100 & 100 \\
\hline Methylene chloride & su & so & so & 1 & su & su & su & 1 \\
\hline styrane & su & sv & so & 50 & su & so & so & su \\
\hline $1,1,2,2$-Tutrachloroethane & su & 5u & 50 & 50 & 5u & su & su & 5u \\
\hline Tetrachloroethene & su & so & 5u & so & su & su & so & su \\
\hline Toluene & 50 & su & su & so & su & su & su & su \\
\hline 1,1,1-Trichloroethane & su & 5u & so & so & su & 5u & su & so \\
\hline 1,1,2-Trienloroethane & so & su & 50 & 50 & so & 50 & su & so \\
\hline Trichloroethene & su & so & 50 & so & 50 & sv & su & 50 \\
\hline Vinyl acetate & 200 & 100 & 100 & 100 & 100 & 100 & 100 & 100 \\
\hline VInyl chlorlde & 100 & 100 & 100 & 100 & 100 & 100 & 100 & 100 \\
\hline xylenes & su & su & so & 50 & su & su & 50 & 5u \\
\hline
\end{tabular}

(CONTINUED) 
APPENDIX E. 2

\begin{tabular}{|c|c|c|c|c|c|c|c|c|}
\hline \multirow{3}{*}{$\begin{array}{l}\text { Sampling Point } \\
\text { Location } \\
\text { Date sampled }\end{array}$} & \multicolumn{4}{|c|}{ GW-749 } & \multicolumn{4}{|c|}{ GW-750 } \\
\hline & \multicolumn{4}{|c|}{ GRIDK2 } & \multicolumn{4}{|c|}{ GRIDR2 } \\
\hline & $01 / 21 / 93$ & $04 / 14 / 93$ & $08 / 06 / 93$ & $10 / 24 / 93$ & $01 / 21 / 93$ & $04 / 15 / 93$ & $08 / 06 / 93$ & $10 / 25 / 93$ \\
\hline VOLATILE ORGANICS (ug/L) & $\cdot$ & . & $\cdot$ & $\cdot$ & $\cdot$ & $\cdot$ & $\cdot$ & - \\
\hline Acetone & 100 & 100 & 100 & 100 & 100 & 100 & 100 & 100 \\
\hline Benzene & su & 5u & su & $5 \mathbf{5 u}$ & su & su & su & su \\
\hline Bromodichloromathane & su & su & so & 5u & 5u & su & 5u & su \\
\hline Bromoform & su & 50 & 5u & su & su & su & 5u & 50 \\
\hline Bromomethane & 100 & 100 & 100 & 100 & 100 & 100 & 100 & 100 \\
\hline 2-Butanone & 100 & 100 & 100 & 100 & 100 & 100 & 100 & 100 \\
\hline Carbon disulfide & su & so & $\mathbf{s u}$ & 5u & 50 & su & $5 \mathbf{v}$ & su \\
\hline Carbon tetrachloride & su & 50 & so & 5u & 50 & su & 50 & 50 \\
\hline Chlorobenzene & $5 u$ & 50 & su & su & 50 & su & 50 & 50 \\
\hline Chlorodibromomethane & su & 50 & so & 50 & 50 & su & 5u & su \\
\hline Chloroethane & 100 & 100 & 100 & 100 & 100 & 100 & 100 & 100 \\
\hline Chlorotorm & 50 & $5 \mathbf{v}$ & su & 5u & 50 & 5u & 50 & 50 \\
\hline Chloromethane & 100 & 100 & 100 & 100 & 100 & 100 & 100 & 100 \\
\hline 1,1-Dichloroethane & 50 & su & $5 \mathbf{v}$ & 5u & 50 & su & su & su \\
\hline 1,2-Dichloroethane & 5u & su & su & 5u & 50 & su & 50 & 5u \\
\hline 1,1-Dichloroethane & su & su & 5u & 5u & su & 5u & su & su \\
\hline 1,2-Dichloroethene & su & 5u & 50 & 50 & 50 & 50 & 50 & 5u \\
\hline 1,2-Dichloropropane & 50 & 50 & 5u & 50 & su & 5u & 50 & su \\
\hline C1s-1,3-Dichloropropene & 50 & 5u & 50 & su & 5u & su & 50 & 5u \\
\hline trans-1, 3-Dichloropropene & 50 & 50 & su & su & 50 & 5u & 50 & 50 \\
\hline zthylbenzene & 5u & 50 & $5 \mathbf{u}$ & 5u & 5u & su & 5u & su \\
\hline 2-Hexanone & 100 & 100 & 100 & 100 & 100 & 100 & 100 & 100 \\
\hline 1-Methyl-2-pentanone & 100 & 100 & 100 & 100 & 100 & 100 & 100 & 100 \\
\hline Hethylene chloride & 5u & $5 \mathbf{v}$ & 5u & 1 & 50 & 50 & 50 & $5 \mathrm{U}$ \\
\hline Styrene & $5 u$ & su & 50 & 5u & 5u & 5u & $5 \mathbf{v}$ & 50 \\
\hline $1,1,2,2$-Tetrachloroethane & 50 & 50 & 5u & 5u & su & su & 50 & su \\
\hline Tetrachloroethene & 50 & su & 5u & 50 & 50 & 5u & 5u & su \\
\hline Toluene & 50 & su & so & 5u & 50 & 50 & 50 & 50 \\
\hline $1,1,1-\operatorname{Tr} 1 \mathrm{ch}$ loroethane & $5 U$ & 50 & 5u & 5u & $5 v$ & 5u & 5u & 50 \\
\hline $1,1,2$-Trichloroethane & 50 & 50 & 5u & su & 50 & $\mathbf{5 u}$ & 50 & 5u \\
\hline Trlchloroethene & 50 & su & 5u & 5u & 50 & $5 \mathbf{u}$ & 50 & 50 \\
\hline Vinyl acetate & 100 & 100 & 100 & 100 & 100 & 100 & 100 & 100 \\
\hline vinyl chloride & 100 & 100 & 100 & 100 & 100 & 100 & 100 & 100 \\
\hline Xylenes & 50 & 5u & 50 & su & 50 & su & $5 U$ & 50 \\
\hline
\end{tabular}

(CONTINUED) 
APPENDIX R.2

Groundwater Quality Data, 1993

\begin{tabular}{|c|c|c|c|c|c|c|c|c|}
\hline \multirow{3}{*}{$\begin{array}{l}\text { Sampling Point } \\
\text { Location } \\
\text { Date Sampled }\end{array}$} & \multicolumn{4}{|c|}{$6 W-751$} & \multicolumn{4}{|c|}{ GW-752 } \\
\hline & \multicolumn{4}{|c|}{ GRIDJ3 } & \multicolumn{4}{|c|}{ GRIDJ3 } \\
\hline & $01 / 11 / 93$ & $04 / 16 / 93$ & $08 / 02 / 93$ & $10 / 26 / 93$ & $01 / 11 / 93$ & $04 / 16 / 93$ & $08 / 02 / 93$ & $10 / 26 / 93$ \\
\hline VOLATILE ORGANICS (ug/L) & . & . & $\cdot$ & $\cdot$ & $\cdot$ & - & . & - \\
\hline Acetone & 100 & 100 & 100 & 100 & 42 & 100 & 100 & 100 \\
\hline Benzene & $\mathbf{s u}$ & su & su & 50 & su & su & su & su \\
\hline Bromodichloromethane & 50 & so & su & so & su & su & su & 50 \\
\hline Bromoform & su & 50 & 5u & su & su & 5u & su & 5u \\
\hline Bromomethane & 100 & 100 & 100 & 100 & 100 & 100 & 100 & 100 \\
\hline 2-Butanone & 100 & 100 & 100 & 100 & 100 & 100 & 100 & 100 \\
\hline Carbon disulfide & 50 & 50 & $\mathbf{s u}$ & 50 & su & 50 & su & su \\
\hline Carbon tetrachloride & su & so & 50 & sv & 50 & 5u & 5u & 50 \\
\hline Chlorobenzene & 50 & so & su & $5 \mathbf{v}$ & su & so & 5u & 5v \\
\hline Chlorodibromomethane & so & 50 & 50 & 50 & 5u & 5v & su & su \\
\hline Chloroethane & 100 & 100 & 100 & 100 & 100 & 100 & 100 & 100 \\
\hline$\therefore$ loroform & so & su & su & $5 \mathbf{v}$ & 50 & so & su & so \\
\hline cnloromethane & 100 & 100 & 100 & 100 & 100 & 100 & 100 & 100 \\
\hline 1,1-Dichloroethane & su & su & 50 & so & su & su & so & so \\
\hline 1,2-Dichloroethane & sv & 5u & 50 & 50 & 50 & su & su & so \\
\hline 1,1-Dichloroethene & 50 & su & 50 & so & 5u & $5 v$ & so & so \\
\hline 1,2-Dichloroethene & su & 5u & 5v & 50 & 50 & 50 & so & so \\
\hline 1,2-Dichloropropane & su & 50 & 50 & 50 & 50 & 5u & 5u & 50 \\
\hline Cis-1,3-Dichloropropene & 50 & 50 & 5v & so & so & 5u & su & 50 \\
\hline trans-1, 3-Dichloropropene & 50 & so & so & 5u & 5u & su & su & 50 \\
\hline Ethylbenzene & su & 5u & 5u & 5u & 50 & su & su & 50 \\
\hline 2-Hexanone & 100 & 100 & 100 & 100 & 100 & 100 & 100 & 100 \\
\hline 4-Methyl-2-pentanone & 100 & 100 & 100 & 100 & 100 & 100 & 100 & 100 \\
\hline Methylene chloride & $\mathbf{s u}$ & 1 & 50 & $5 \mathbf{v}$ & 50 & 50 & 5u & su \\
\hline styrene & 5u & so & 50 & su & su & su & 5u & 50 \\
\hline $1,1,2,2$-Tetrachloroethane & 50 & 50 & 50 & 50 & su & 50 & 5u & 50 \\
\hline Tetrachloroethene & su & 50 & 50 & 5u & su & so & 50 & 50 \\
\hline Toluene & 5u & 50 & 50 & 50 & 5v & 50 & 5u & 50 \\
\hline $1,1,1$-Trichloroethane & 50 & 5u & 5u & 50 & 50 & 5u & 50 & su \\
\hline 1,1,2-Trichloroethane & 50 & 50 & 50 & 50 & 50 & 50 & su & 5u \\
\hline Trichloroethene & 5u & 50 & 5u & 50 & 50 & 50 & su & 50 \\
\hline vinyl acetate & 100 & 100 & 100 & 100 & 100 & 100 & 100 & 100 \\
\hline vinyl chloride & 200 & 100 & 100 & 100 & 100 & 100 & 100 & 100 \\
\hline xylenes & 50 & 50 & su & su & su & 50 & 5u & 50 \\
\hline
\end{tabular}

(CONTINUED) 
APPENDIX E. 2

Groundwater Quality Data, 1993

\begin{tabular}{|c|c|c|c|c|c|c|c|c|}
\hline \multirow{3}{*}{$\begin{array}{l}\text { Sampling Point } \\
\text { - } \\
\text { Location } \\
\text { Date Sampled }\end{array}$} & \multicolumn{4}{|c|}{ GW-753 } & \multicolumn{4}{|c|}{ GW-754 } \\
\hline & \multicolumn{4}{|c|}{ GRIDJ 2} & \multicolumn{4}{|c|}{ GRIDJ2 } \\
\hline & $01 / 15 / 93$ & $04 / 08 / 93$ & $08 / 03 / 93$ & $10 / 21 / 93$ & $01 / 18 / 93$ & $04 / 08 / 93$ & $08 / 04 / 93$ & $10 / 22 / 93$ \\
\hline VOLATILE ORGANICS (ug/L) & $\cdot$ & - & - & - & $\cdot$ & - & $\cdot$ & - \\
\hline Acetone & 100 & $10 \mathrm{U}$ & $10 \mathrm{U}$ & 100 & 100 & 100 & 100 & 3 \\
\hline Benzene & 50 & $5 \mathrm{U}$ & 50 & 50 & 50 & 5u & 5u & $5 \mathbf{v}$ \\
\hline Bromodichloromethane & 50 & $5 \mathrm{U}$ & 50 & 50 & 50 & 50 & $5 v$ & 50 \\
\hline Bromoform & 50 & 50 & 50 & $5 \mathbf{U}$ & 50 & 5u & 5u & 50 \\
\hline Bromomethane & 100 & 100 & 100 & 100 & 100 & 100 & 100 & 100 \\
\hline 2-Butanone & 100 & $10 \mathrm{U}$ & 100 & 100 & 100 & 100 & 100 & 10 \\
\hline Carbon disulfide & 50 & 50 & 50 & $5 \mathbf{U}$ & 50 & 50 & 50 & 50 \\
\hline Carbon tetrachloride & 50 & 50 & 50 & 50 & 50 & 50 & 50 & $5 \mathbf{U}$ \\
\hline Chlorobenzene & 50 & $5 U$ & 50 & 50 & 50 & 50 & $5 U$ & 50 \\
\hline Chlorodibromomethane & $5 \mathbf{U}$ & 5u & 50 & $5 U$ & 5u & 5U & 50 & $5 \mathbf{5}$ \\
\hline Chloroethane & 100 & 100 & 100 & 100 & 100 & 100 & 100 & 100 \\
\hline Chloroform & 50 & $5 U$ & $5 \mathbf{v}$ & $5 \mathbf{U}$ & $5 \mathbf{U}$ & $5 v$ & $5 \mathbf{v}$ & 5U \\
\hline Chloromethane & 100 & $10 \mathrm{U}$ & 100 & 100 & $10 \mathrm{U}$ & 100 & 100 & 100 \\
\hline 1,1-Dichloroethane & 50 & $5 U$ & $5 v$ & 50 & $5 \mathbf{U}$ & 50 & $5 \mathrm{U}$ & $\mathbf{5 u}$ \\
\hline 1,2-Dichloroethane & 50 & $5 U$ & 50 & 50 & 5u & 50 & 50 & 50 \\
\hline 1,1-Dichloroethene & 50 & $5 U$ & $5 U$ & 50 & $5 u$ & 50 & 50 & 50 \\
\hline 1,2-Dichloroethene & 50 & $5 U$ & 50 & 50 & $5 U$ & 50 & 50 & $5 \mathbf{v}$ \\
\hline 1,2-Dichloropropane & 50 & $5 U$ & 50 & 50 & 50 & 50 & 5v & 50 \\
\hline cis-1,3-Dichloropropene & 50 & 50 & 50 & 50 & 50 & $5 U$ & $5 \mathrm{U}$ & $5 \mathbf{0}$ \\
\hline trans-1,3-Dichloropropene & 50 & $5 U$ & 50 & 50 & 5u & 50 & 5U & $5 \mathbf{U}$ \\
\hline Ethylbenzene & $\mathbf{5 0}$ & 5u & $5 v$ & 50 & 5u & 50 & $5 u$ & 50 \\
\hline 2-Hexanone & $10 \mathrm{U}$ & 100 & 100 & 100 & 100 & 100 & 100 & 100 \\
\hline 4-Methy1-2-pentanone & 100 & 100 & 100 & 100 & 100 & 100 & 100 & 100 \\
\hline Methylene chloride & $\mathbf{5 u}$ & 1 & 50 & $5 U$ & $\mathbf{5 u}$ & $5 \mathbf{v}$ & 50 & 3 \\
\hline Styrene & 50 & 50 & 5u & 5U & 50 & 50 & 50 & 50 \\
\hline $1,1,2,2$-Tetrachloroethane & 50 & $5 \mathrm{v}$ & 50 & $5 \mathbf{v}$ & 50 & $5 U$ & 50 & 50 \\
\hline Tetrachloroethene & 50 & $5 v$ & 50 & 50 & 50 & 50 & 50 & $5 \mathbf{U}$ \\
\hline Toluene & 50 & $5 U$ & 50 & $5 U$ & 50 & 50 & 50 & 50 \\
\hline $1,1,1$-Trichloroethane & 50 & $5 U$ & 50 & 50 & 50 & 50 & su & 50 \\
\hline 1,1,2-Trichloroethane & 50 & $5 U$ & 50 & 50 & 50 & 50 & 50 & 50 \\
\hline Trichloroethene & $5 \mathbf{U}$ & 50 & 50 & 50 & $5 \mathrm{U}$ & 50 & $5 \mathbf{5}$ & $5 \mathbf{5}$ \\
\hline Vinyl acetate & 100 & 100 & 100 & 100 & 100 & 100 & 100 & 100 \\
\hline Vinyl chloride & 100 & 100 & 100 & 100 & 100 & 100 & 100 & 100 \\
\hline Xylenes & 50 & 50 & 50 & 50 & 50 & 5v & $5 \mathrm{U}$ & 50 \\
\hline
\end{tabular}

(CONTINUED) 
APPENDIX E. 2

\begin{tabular}{|c|c|c|c|c|c|c|c|c|}
\hline \multirow{3}{*}{$\begin{array}{l}\text { Sampling Point } \\
\text { scation } \\
\text { Date Sampled }\end{array}$} & \multicolumn{4}{|c|}{$G w-755$} & \multicolumn{4}{|c|}{ GW-756 } \\
\hline & \multicolumn{4}{|c|}{ GRIDJ 1} & \multicolumn{4}{|c|}{ GRIDJ 1} \\
\hline & $01 / 14 / 93$ & $04 / 07 / 93$ & $08 / 03 / 93$ & $10 / 21 / 93$ & $01 / 15 / 93$ & $04 / 08 / 93$ & $08 / 03 / 93$ & $10 / 21 / 93$ \\
\hline vOLATILE ORGANICS (ug/L) & - & - & - & - & - & - & - & - \\
\hline setone & 100 & 100 & 100 & $10 \mathrm{U}$ & 100 & 100 & 100 & 2 \\
\hline Benzene & 50 & $\mathbf{5 u}$ & $5 U$ & 50 & $5 U$ & $5 \mathbf{v}$ & so & $5 U$ \\
\hline Bromodichloromethane & 50 & 50 & 50 & $5 U$ & $5 U$ & 50 & 50 & 50 \\
\hline Bromoform & $5 v$ & $5 \mathbf{v}$ & $5 U$ & 50 & $\mathbf{5 U}$ & $5 \mathbf{v}$ & $5 U$ & 50 \\
\hline Aromomethane & 100 & 100 & 100 & 100 & 100 & 100 & 100 & $10 \mathrm{U}$ \\
\hline -Butanone & 100 & 100 & 100 & 100 & 100 & 100 & 100 & 8 \\
\hline Carbon disulfide & $5 \mathrm{U}$ & $5 v$ & $5 U$ & $5 \mathbf{u}$ & $5 U$ & $5 \mathbf{v}$ & $5 \mathbf{u}$ & 50 \\
\hline Carbon tetrachloride & 50 & $5 \mathbf{v}$ & $5 U$ & $5 \mathbf{v}$ & 50 & $5 \mathbf{u}$ & $5 \mathbf{v}$ & 50 \\
\hline Chlorobenzene & 50 & 5u & $5 \mathbf{v}$ & 50 & 50 & 50 & $5 \mathbf{U}$ & 50 \\
\hline Chlorodibromomethane & $5 v^{1}$ & 50 & 50 & $5 \mathbf{v}$ & $5 U$ & $5 v$ & 50 & 50 \\
\hline Chloroethane & 100 & 100 & 100 & 100 & $10 \mathrm{U}$ & 100 & 100 & 100 \\
\hline Chloroform & $5 u$ & 50 & $5 U$ & $5 \mathbf{v}$ & $5 \mathbf{U}$ & $5 \mathbf{U}$ & $5 U$ & $5 U$ \\
\hline Chloromethane & 100 & 100 & 100 & 100 & $10 \mathrm{U}$ & 100 & 100 & 100 \\
\hline 1,1-Dichloroethane & $5 v$ & $5 \mathrm{U}$ & 50 & $5 \mathbf{U}$ & $\mathbf{5 U}$ & $\mathbf{5 0}$ & $5 \mathbf{5}$ & $5 \mathbf{U}$ \\
\hline 1,2-Dichloroethane & 50 & $5 \mathrm{U}$ & 50 & 50 & $5 \mathbf{5}$ & 50 & $5 \mathbf{v}$ & $5 \mathbf{U}$ \\
\hline 1,1-Dichloroethene & 50 & 50 & 50 & $5 \mathbf{U}$ & $5 U$ & 50 & $5 \mathbf{U}$ & 50 \\
\hline 1,2-Dichloroethene & $5 \mathbf{v}$ & $5 U$ & 50 & $5 \mathbf{u}$ & $5 \mathbf{5 u}$ & $5 U$ & $5 \mathbf{v}$ & 50 \\
\hline 1,2-Dichloropropane & $5 \mathbf{v}$ & $5 \mathrm{v}$ & 50 & 50 & 50 & 50 & 50 & 50 \\
\hline Cis-1, 3-Dichloropropene & 50 & 5U & 50 & $5 \mathbf{u}$ & $5 v$ & 50 & 50 & $5 U$ \\
\hline trans-1,3-Dichloropropene & su & su & 5U & 50 & 5u & 5u & 50 & 50 \\
\hline Ethylbenzene & 50 & 50 & 50 & $5 \mathbf{v u}$ & $5 \mathbf{v}$ & 50 & 50 & 50 \\
\hline 2-Hexanone & 100 & $10 \mathrm{U}$ & 100 & 100 & 100 & $10 \mathrm{U}$ & 100 & 100 \\
\hline 4-Methy1-2-pentanone & 1 & 100 & 100 & 100 & 100 & 100 & 100 & 100 \\
\hline Methylene chloride & $5 \mathbf{v}$ & su & $5 U$ & 50 & 50 & $5 \mathbf{u}$ & 50 & 3 \\
\hline Styrene & 50 & 50 & 50 & $5 \mathbf{U}$ & 50 & $5 U$ & 50 & 50 \\
\hline $1,1,2,2$-Tetrachloroethane & $5 \mathbf{v}$ & $5 v$ & 50 & $5 \mathbf{U}$ & 50 & $5 \mathbf{u}$ & 50 & $5 \mathrm{U}$ \\
\hline Tetrachloroethene & $5 \mathbf{v}$ & $5 \mathbf{v}$ & 50 & $5 \mathbf{v}$ & $5 \mathbf{v}$ & $5 \mathrm{U}$ & $5 \mathrm{U}$ & $5 U$ \\
\hline Toluene & $5 \mathbf{U}$ & 50 & 50 & $5 U$ & 50 & 50 & 5v & 50 \\
\hline $1,1,1$-Trichloroethane & $5 \mathrm{U}$ & 50 & 50 & $5 U$ & $5 \mathbf{v}$ & $5 \mathrm{U}$ & $5 U$ & 50 \\
\hline $1,1,2$-Trichloroethane & $5 u$ & 50 & 50 & 50 & 50 & $5 U$ & 50 & 50 \\
\hline Trichloroethene & $5 U$ & $5 \mathrm{U}$ & $5 \mathrm{U}$ & 50 & 50 & $5 U$ & $5 \mathrm{U}$ & 50 \\
\hline VinyI acetate & $10 \mathrm{U}$ & 100 & 100 & 100 & 100 & 100 & 100 & 100 \\
\hline vinyl chloride & 100 & 100 & 100 & 100 & 100 & 100 & 100 & $10 U$ \\
\hline xylenes & $5 U$ & $5 \mathrm{U}$ & 50 & su & $5 U$ & 50 & $5 U$ & $5 U$ \\
\hline
\end{tabular}

(CONTINUED) 
APPENDIX E. 2

Groundwater Quality Data, 1993

\begin{tabular}{|c|c|c|c|c|c|c|c|c|}
\hline \multirow{3}{*}{$\begin{array}{l}\text { Sampling Point } \\
\text { - } \\
\text { Location } \\
\text { Date Sampled }\end{array}$} & \multicolumn{4}{|c|}{ GW-758 } & \multicolumn{4}{|c|}{ GW-759 } \\
\hline & \multicolumn{4}{|c|}{ GRIDG1 } & \multicolumn{4}{|c|}{ GRIDG1 } \\
\hline & $01 / 25 / 93$ & $04 / 13 / 93$ & $09 / 12 / 93$ & $11 / 19 / 93$ & $01 / 26 / 93$ & $04 / 15 / 93$ & $09 / 13 / 93$ & $11 / 19 / 93$ \\
\hline VOLATILB ORGANICS $(\mathrm{ug} / \mathrm{L})$ & - & - & - & - & $\cdot$ & - & - & • \\
\hline Acetone & 15 & 100 & 100 & 100 & 100 & 100 & 100 & 100 \\
\hline Benzene & $5 \mathrm{u}$ & 5u & $5 \mathrm{v}$ & $5 U$ & 50 & $\mathbf{5 u}$ & 50 & $5 U$ \\
\hline Bromodichloromethane & $5 v$ & $5 v$ & $5 v$ & $5 v$ & 50 & 50 & $5 v$ & $5 \mathrm{U}$ \\
\hline Bromoform & 50 & $5 \mathrm{U}$ & 5u & 50 & $5 \mathbf{u}$ & 50 & 50 & 50 \\
\hline Bromomethane & 100 & 100 & 100 & 100 & 100 & 100 & 100 & 100 \\
\hline 2-Butanone & 100 & 100 & 100 & 100 & 100 & 100 & 100 & 100 \\
\hline Carbon disulfide & $5 \mathbf{U}$ & $5 \mathrm{U}$ & $5 U$ & 50 & 50 & $5 v$ & 50 & 50 \\
\hline Carbon tetrachloride & 50 & 50 & 50 & 50 & 50 & 50 & 50 & 50 \\
\hline Chlorobenzene & 50 & 50 & $5 u$ & 50 & $5 \mathrm{U}$ & 50 & 50 & 50 \\
\hline Chlorodibromomethane & 50 & 50 & 50 & su & $5 \mathrm{U}$ & 50 & 50 & 5u \\
\hline Chloroethane & 100 & 100 & 100 & 100 & 100 & 100 & 100 & 100 \\
\hline Chloroform & 50 & 50 & 50 & 50 & 50 & 50 & 50 & 50 \\
\hline Chloromethane & 100 & 100 & 100 & 100 & 100 & 100 & 100 & 100 \\
\hline 1,1-Dichloroethane & $5 u$ & su & 50 & 50 & 50 & $5 v$ & $5 \mathrm{U}$ & su \\
\hline 1,2-Dichloroethane & 50 & 50 & so & 50 & 50 & 50 & 5u & 50 \\
\hline 1,1-Dichloroethene & $5 \mathbf{v}$ & 50 & 50 & 5v & 50 & 50 & $5 U$ & 50 \\
\hline 1,2-Dichloroethene & su & 50 & 50 & 5u & 50 & 50 & 5U & 50 \\
\hline 1,2-Dichloropropane & 50 & 50 & 50 & 50 & 50 & 5u & $5 U$ & 50 \\
\hline Cis-1, 3-Dichloropropene & 5u & 50 & 50 & 50 & 50 & 50 & $5 U$ & 50 \\
\hline trans-1,3-Dichloropropene & 5u & 50 & 50 & 50 & 50 & 50 & $5 \mathbf{U}$ & 50 \\
\hline Ethylbenzene & 50 & 50 & 50 & 50 & 50 & $5 U$ & $5 U$ & 50 \\
\hline 2-Hexanone & 100 & 100 & 100 & 100 & 100 & 100 & 100 & 100 \\
\hline 4-Methy1-2-pentanone & 100 & 100 & 100 & 100 & 100 & 100 & 100 & 100 \\
\hline Methylene chloride & su & 50 & 1 & 5u & 50 & 50 & 50 & 50 \\
\hline Styrene & 50 & $5 \mathbf{v}$ & 50 & 50 & 50 & 50 & $5 \mathrm{U}$ & $5 \mathbf{v}$ \\
\hline $1,1,2,2$-Tetrachloroethane & $5 u$ & 50 & 50 & 50 & 50 & 50 & $5 U$ & 50 \\
\hline Tetrachloroethene & $5 \mathbf{v}$ & 50 & 50 & 50 & 50 & 50 & 50 & $5 v$ \\
\hline Toluene & 50 & 50 & 50 & 5u & 50 & 50 & 50 & 50 \\
\hline 1,1,1-Trichloroethane & 50 & 5u & 50 & $5 U$ & 50 & 50 & 50 & 5u \\
\hline 1,1,2-Trichloroethane & $5 v$ & 50 & 50 & $5 U$ & 50 & 50 & 50 & 50 \\
\hline Prichloroethene & $5 v$ & 50 & $5 \mathrm{v}$ & $5 U$ & $5 u$ & $5 u$ & su & $5 \mathbf{u}$ \\
\hline Vinyl acetate & 100 & 100 & 100 & 100 & 100 & 100 & 100 & 100 \\
\hline vinyl chloride & 100 & 100 & 100 & 100 & 100 & 100 & 100 & 100 \\
\hline Xylenes & $5 \mathrm{U}$ & 50 & 50 & $5 U$ & $5 \mathrm{U}$ & 50 & $5 \mathrm{U}$ & $5 \mathbf{u}$ \\
\hline
\end{tabular}

(CONTINUED) 
APPENDIX E.2

\begin{tabular}{|c|c|c|c|c|c|c|c|c|}
\hline \multirow{3}{*}{$\begin{array}{l}\text { Sampling Point } \\
\text { Location } \\
\text { - } \\
\text { Date Sampled }\end{array}$} & \multicolumn{4}{|c|}{$G W-760$} & \multicolumn{4}{|c|}{ GW-761 } \\
\hline & \multicolumn{4}{|c|}{ GRIDG2 } & \multicolumn{4}{|c|}{ GRIDG2 } \\
\hline & $01 / 27 / 93$ & $04 / 16 / 93$ & $09 / 14 / 93$ & $11 / 30 / 93$ & $01 / 26 / 93$ & $04 / 16 / 93$ & $09 / 13 / 93$ & $11 / 29 / 93$ \\
\hline VOLATIIE ORGANICS ( $\mathrm{ug} / \mathrm{L}$ ) & . & $\cdot$ & - & - & . & - & - & - \\
\hline Acetone & 100 & 100 & 100 & 100 & 100 & 100 & $10 \mathrm{U}$ & 100 \\
\hline Benzene & $5 \mathbf{v}$ & 50 & 50 & 5U & su & 50 & 50 & 5u \\
\hline Bromodichloromethane & $5 \mathbf{v}$ & so & 5u & 5u & 5u & 50 & 5u & 50 \\
\hline Bromoform & 5u & 50 & 50 & 50 & 50 & 50 & $5 \mathrm{u}$ & 50 \\
\hline Bromomethane & 100 & 100 & 100 & 100 & 100 & 100 & 100 & 100 \\
\hline 2-Butanone & 100 & 100 & 100 & 10U & 100 & 100 & 100 & 100 \\
\hline Carbon disulfide & $\mathbf{5 u}$ & 50 & 50 & 5U & su & 50 & $5 U$ & 50 \\
\hline Carbon tetrachloride & 5u & 5u & 50 & 50 & 50 & 50 & 50 & 50 \\
\hline Chlorobenzene & $5 v$ & 50 & su & 5U & 5U & 50 & 50 & 50 \\
\hline Chlorodibromomethane & 50 & 50 & 50 & 50 & 50 & $5 \mathbf{v}$ & 50 & 50 \\
\hline Chloroethane & 100 & 100 & 100 & 100 & 100 & 100 & 100 & 100 \\
\hline Chloroform & $5 \mathbf{v}$ & so & $5 u$ & 5u & 50 & 50 & $5 U$ & 5U \\
\hline Chloromethane & $10 \mathrm{U}$ & 100 & 100 & 100 & 100 & 100 & 100 & 100 \\
\hline 1,1-Dichloroethane & 5u & 50 & 50 & 5u & 5U & 50 & 50 & $5 U$ \\
\hline 1,2-Dichloroethane & 50 & 50 & 50 & 50 & $5 u$ & su & $5 U$ & 5u \\
\hline 1,1-Dichloroethene & 50 & 50 & 50 & 5U & 5v & 50 & 50 & 5u \\
\hline 1,2-Dichloroethene & 50 & 50 & $5 v$ & 5u & 50 & 50 & $5 v$ & 50 \\
\hline 1,2-Dichloropropane & 50 & 50 & 50 & 5u & 50 & 50 & 50 & 50 \\
\hline cis-1,3-Dichloropropene & 50 & 50 & 50 & 5U & 5u & 50 & $5 u$ & 50 \\
\hline trans-1,3-Dichloropropene & 50 & $5 \mathrm{U}$ & 50 & 50 & 50 & 50 & 50 & 50 \\
\hline Ethylbenzene & so & 50 & 5u & 50 & su & $5 \mathbf{v}$ & $5 \mathbf{u}$ & 50 \\
\hline 2-Hexanone & 100 & 100 & 100 & 100 & 100 & 100 & 100 & 100 \\
\hline 4-Methy1-2-pentanone & 100 & 100 & 100 & 100 & 100 & 100 & 100 & 100 \\
\hline Methylene chloride & 5u & $5 v$ & 50 & 5u & 50 & 5u & 50 & $5 \mathbf{u}$ \\
\hline Styrene & 50 & 50 & su & 5u & 50 & 50 & 50 & 5u \\
\hline $1,1,2,2$-Tetrachloroethane & 50 & $5 v$ & 5u & 50 & 50 & 50 & 50 & 50 \\
\hline Tetrachloroethene & 5u & 50 & 5u & 50 & 5u & 50 & 50 & 50 \\
\hline Toluene & 50 & 5u & 50 & $5 U$ & 5U & 50 & 50 & 50 \\
\hline 1,1,1-Trichloroethane & 50 & $5 \mathrm{v}$ & 50 & $5 U$ & 50 & 50 & 50 & 50 \\
\hline 1,1,2-Trichloroethane & 50 & 50 & $5 u$ & $5 U$ & $5 U$ & 50 & 50 & 50 \\
\hline Trichloroethene & $5 U$ & 50 & 5u & 50 & su & 50 & 50 & 50 \\
\hline Vinyl acetate & 100 & 100 & 100 & 100 & 100 & 100 & 100 & 100 \\
\hline Vingl chloride & 100 & 100 & 100 & 100 & 100 & 100 & 100 & 100 \\
\hline xylenes & $5 \mathrm{U}$ & 50 & 50 & $5 \mathrm{U}$ & 50 & 50 & 50 & su \\
\hline
\end{tabular}

(CONTINUED) 
APPENDIX R.2

\begin{tabular}{|c|c|c|c|c|c|c|c|c|}
\hline \multirow{3}{*}{$\begin{array}{l}\text { Sampling Point } \\
\text { L } \\
\text { Location } \\
\text { Date Sampled }\end{array}$} & \multicolumn{4}{|c|}{ GW-762 } & \multicolumn{4}{|c|}{ GW-763 } \\
\hline & \multicolumn{4}{|c|}{ GRIDJ 3} & \multicolumn{4}{|c|}{ GRIDJ3 } \\
\hline & $02 / 22 / 93$ & $04 / 20 / 93$ & $08 / 04 / 93$ & $10 / 28 / 93$ & $01 / 25 / 93$ & $04 / 21 / 93$ & $08 / 05 / 93$ & $10 / 29 / 93$ \\
\hline VOLATILE ORGANICS (Ug/L) & $\cdot$ & $\cdot$ & $\cdot$ & $\cdot$ & $\cdot$ & $\cdot$ & $\cdot$ & - \\
\hline Acetone & 1000 & 1000 & 1000 & 1000 & 100 & 100 & 100 & 100 \\
\hline Benzene & 500 & 500 & 500 & 500 & 50 & $5 v$ & 50 & 5u \\
\hline Bromodichloromethane & 500 & 500 & 500 & 500 & 50 & so & su & 50 \\
\hline Bromoform & 500 & 500 & 500 & 500 & $5 v$ & 50 & $5 v$ & 50 \\
\hline Bromomethane & 1000 & 1000 & 1000 & 1000 & 100 & 100 & 100 & 100 \\
\hline 2-Butanone & 1000 & 1000 & 1000 & 1000 & 100 & 100 & 100 & 100 \\
\hline Carbon disulfide & 500 & 500 & 500 & 500 & 50 & 50 & 50 & su \\
\hline Carbon tetrachloride & 500 & 500 & 500 & 500 & 5u & 50 & 5u & 50 \\
\hline Chlorobenzene & 500 & 500 & 500 & 500 & 50 & 50 & 50 & su \\
\hline Chlorodibromomethane & 500 & 500 & 500 & 500 & 50 & 50 & 50 & su \\
\hline Chloroethane & 1000 & 1000 & 1000 & 1000 & 100 & 100 & 100 & 100 \\
\hline Chloroform & 500 & 500 & 500 & 500 & 50 & 50 & $5 \mathrm{u}$ & $5 \mathbf{u}$ \\
\hline Chloromethane & 1000 & 1000 & 1000 & 1000 & 100 & 100 & 100 & 100 \\
\hline 1,1-Dichloroethane & 500 & 500 & 500 & 500 & su & $5 \mathrm{U}$ & $5 \mathbf{u}$ & 50 \\
\hline 1,2-Dichloroethane & 500 & 500 & 500 & 500 & 50 & 50 & 50 & 5U \\
\hline 1,1-Dichloroethene & 12 & 500 & 500 & 500 & 5u & 5u & 50 & 2 \\
\hline 1,2-Dichloroethene & 85 & 500 & 71 & 78 & 59 & 42 & 98 & 120 \\
\hline 1,2-Dichloropropane & 500 & 500 & 500 & 500 & 50 & 50 & su & 5u \\
\hline Cis-1,3-Dichloropropene & 500 & 500 & 500 & 500 & 50 & 5u & su & su \\
\hline trans-1, 3-Dichloropropene & 500 & 500 & 500 & 500 & 50 & 50 & 5u & su \\
\hline Ethylbenzene & 500 & 500 & 500 & 500 & $5 U$ & su & 50 & $5 v$ \\
\hline 2-Hexanone & 1000 & 1000 & 1000 & 1000 & 100 & 100 & 100 & 100 \\
\hline 4-Methyl-2-pentanone & 1000 & 24 & 1000 & 1000 & 100 & 100 & 100 & 100 \\
\hline Methylene chloride & 500 & 11 & 500 & 500 & 50 & 50 & 5u & 1 \\
\hline Styrene & 500 & 500 & 500 & 500 & 50 & 5u & 5u & 50 \\
\hline $1,1,2,2$-Tetrachloroethane & 500 & 500 & 500 & 500 & 50 & 5u & 5u & 5u \\
\hline Tetrachloroethene & 900 & 1000 & 920 & 1000 & 8 & 5 & 27 & 41 \\
\hline Toluene & 500 & 500 & 500 & 500 & $5 \mathrm{U}$ & 50 & 5u & su \\
\hline 1,1,1-Trichloroethane & 500 & 500 & 500 & 500 & 50 & 50 & 5u & 50 \\
\hline 1,1,2-Trichloroethane & 500 & 500 & 500 & 500 & 5u & 5u & 5u & 50 \\
\hline Trichloroethene & 38 & 46 & 500 & 44 & 2 & 1 & 4 & 7 \\
\hline vingl acetate & 1000 & 1000 & 1000 & 1000 & 100 & 100 & 100 & 100 \\
\hline Vinyl chloride & 1000 & 1000 & 1000 & 1000 & 45 & 35 & 48 & 59 \\
\hline xylenes & 500 & 500 & 500 & 500 & 50 & $5 \mathrm{u}$ & 50 & $5 \mathrm{U}$ \\
\hline
\end{tabular}

(CONTINUED) 
APPENDIX R. 2

\begin{tabular}{|c|c|c|c|c|c|c|c|c|}
\hline \multirow{3}{*}{$\begin{array}{l}\text { Sampling point } \\
\text { Location } \\
\text { - } \\
\text { Date Sampled }\end{array}$} & \multicolumn{4}{|c|}{ GW-764 } & \multicolumn{4}{|c|}{ GW-765 } \\
\hline & \multicolumn{4}{|c|}{ GRIDE I } & \multicolumn{4}{|c|}{ GRIDEI } \\
\hline & $01 / 23 / 93$ & $04 / 13 / 93$ & $09 / 12 / 93$ & $11 / 12 / 93$ & $01 / 25 / 93$ & $04 / 13 / 93$ & $09 / 12 / 93$ & $11 / 19 / 93$ \\
\hline VOLATILE ORGANICS (ug/L) & - & . & - & - & - & - & - & - \\
\hline Acetone & $10 \dot{0}$ & $20 \dot{u}$ & $10 \dot{u}$ & $10 \dot{U}$ & $10 \dot{U}$ & 100 & $10 \dot{U}$ & $10 \dot{0}$ \\
\hline Benzene & 5u & 50 & 5u & 5u & 5u & su & 5u & 50 \\
\hline Bromodichloromethane & 5u & 50 & 50 & 5U & 5u & 5u & 50 & 50 \\
\hline Bromoform & 50 & 50 & 50 & 5u & 50 & 50 & 5u & 5u \\
\hline Bromomethane & 100 & 100 & 100 & 100 & 100 & 100 & 100 & 100 \\
\hline 2-Butanone & 100 & 100 & 100 & 100 & 100 & 100 & 100 & 100 \\
\hline Carbon disulfide & 5u & su & su & 5u & 5u & 50 & su & 4 \\
\hline Carbon tetrachloride & 50 & 5u & 50 & 50 & 50 & 50 & 5U & su \\
\hline Chlorobenzene & 50 & 50 & su & 50 & su & 50 & su & su \\
\hline Chlorodibromomethane & 5u & 5u & $5 u$ & su & 5u & 5u & 5u & 50 \\
\hline Chloroethane & 100 & 100 & 100 & 100 & 100 & 100 & 100 & 100 \\
\hline Chloroform & 5u & 5u & 50 & 50 & 50 & 50 & 5u & 50 \\
\hline Chloromethane & 100 & 100 & 100 & 100 & 100 & 100 & 100 & 100 \\
\hline 1,1-Dichloroethane & so & 5v & $5 u$ & su & su & so & 5u & 50 \\
\hline 1,2-Dichloroethane & 50 & 50 & $5 u$ & 5u & 5v & 5u & 5u & 5U \\
\hline 1,1-Dichloroethene & 5u & $5 \mathrm{v}$ & $5 u$ & su & 50 & 5u & su & 50 \\
\hline$\therefore$ 2-Dichloroethene & 5u & 5v & 50 & 5u & 5u & 50 & 5u & so \\
\hline .,2-Dichloropropane & 5u & 5u & su & su & 5u & 50 & 5u & 50 \\
\hline cis-1,3-Dichloropropene & 50 & $5 \mathrm{v}$ & 50 & su & su & 5u & 5u & su \\
\hline trans-1,3-Dichloropropene & su & 5u & 5u & 5u & 50 & $5 v$ & 5u & 50 \\
\hline Bthylbenzene & 5u & $5 \mathrm{v}$ & 50 & 5u & 50 & 5u & 5u & 50 \\
\hline 2-Hexanone & 100 & 100 & 100 & 100 & 100 & 100 & 100 & 100 \\
\hline 4-Methy1-2-pentanone & 100 & 100 & 100 & 100 & 100 & 100 & 100 & 100 \\
\hline Methylene chloride & 50 & 1 & 50 & 50 & $5 \mathrm{U}$ & 50 & su & 50 \\
\hline Styrene & 50 & $5 \mathrm{v}$ & 50 & $5 \mathbf{u}$ & su & 50 & su & 50 \\
\hline $1,1,2,2$-Tetrachloroethane & 5u & 50 & 50 & 5u & 5u & $5 v$ & 5u & 50 \\
\hline Tetrachloroethene & 5u & 50 & 5u & 50 & 5u & $5 v$ & 5u & 50 \\
\hline Toluene & 50 & $5 \mathrm{U}$ & 5u & 5u & 50 & 5u & 5u & 50 \\
\hline $1,1,1$-Trichloroethane & 50 & 50 & 50 & 5U & 5u & 50 & 5u & 50 \\
\hline $1,1,2$-Trichloroethane & 5u & 50 & 50 & 50 & 5u & $5 v$ & 50 & 50 \\
\hline Trichloroethene & su & 50 & 50 & su & su & 50 & 50 & 50 \\
\hline vinyl acetate & 100 & 100 & 100 & 100 & 100 & 100 & 100 & 100 \\
\hline vinyl chloride & 100 & 100 & 100 & 100 & 100 & 100 & 100 & 100 \\
\hline xylenes & 5u & 50 & $5 v$ & 5u & 5U & $5 \mathbf{u}$ & 50 & $5 \mathbf{U}$ \\
\hline
\end{tabular}

(CONTINUED) 
APPENDIX E.2

\begin{tabular}{|c|c|c|c|c|c|c|c|c|}
\hline \multirow{3}{*}{$\begin{array}{l}\text { Sampling Polnt } \\
\text { Location } \\
\text { Date Sampled }\end{array}$} & \multicolumn{4}{|c|}{ GW-766 } & \multicolumn{4}{|c|}{$G W-767$} \\
\hline & \multicolumn{4}{|c|}{ GRIDI2 } & \multicolumn{4}{|c|}{ GRIDI2 } \\
\hline & $02 / 01 / 93$ & $04 / 15 / 93$ & $08 / 03 / 93$ & $10 / 13 / 93$ & $02 / 02 / 93$ & $04 / 15 / 93$ & $08 / 04 / 93$ & $10 / 13 / 93$ \\
\hline VOLATILS ORGANICS (ug/L) & - & . & . & . & . & . & . & • \\
\hline Acetone & 2 & 100 & 100 & 100 & 1 & 100 & 100 & 100 \\
\hline Benzene & 5u & su & 5u & 50 & 5u & 50 & su & su \\
\hline Bromodichloromethane & 50 & 5u & 50 & 50 & su & so & su & su \\
\hline Bromoform & su & su & 50 & su & su & 50 & 50 & 5u \\
\hline Bromomethane & 100 & 100 & 100 & 100 & 100 & 100 & 100 & 100 \\
\hline 2-Butanone & 100 & 100 & 100 & 100 & 4 & 100 & 100 & 100 \\
\hline Carbon dieulfide & su & 50 & 50 & $5 v$ & so & 5u & 50 & su \\
\hline Carbon tetrachlorido & su & 5u & 5u & su & su & su & su & su \\
\hline Chlorobenzene & su & su & su & su & su & su & su & su \\
\hline Chlorodibromomethane & su & su & su & 5u & su & 50 & 5u & 50 \\
\hline Chloroethane & 100 & 100 & 100 & 100 & 100 & 100 & 100 & 100 \\
\hline Chloroform & 5u & su & su & su & su & 50 & so & 50 \\
\hline Chloromethane & 100 & 100 & 100 & 100 & 100 & 100 & 100 & 100 \\
\hline 1, 1-Dichloroethane & 5u & su & 50 & 5u & 5u & 50 & su & so \\
\hline 1,2-Dlchloroethane & 5u & 5u & 5u & 50 & 50 & 5u & 50 & su \\
\hline 1,1-Dichloroethene & 50 & 5u & 50 & su & 50 & so & su & su \\
\hline 1,2-Dichloroethene & 50 & su & 5u & su & 50 & 5u & 5u & su \\
\hline 1,2-Dichloropropane & 50 & su & 5u & su & 5u & 5u & 5u & su \\
\hline cis-1,3-Dichloropropene & 50 & 5u & 50 & 5u & su & 50 & 50 & su \\
\hline trans-1,3-Dichloropropane & su & 5u & 50 & 5u & 5u & 5u & 5u & su \\
\hline Rthylbenzene & su & su & su & su & su & 50 & 50 & su \\
\hline 2-Hexanone & 100 & 100 & 100 & 100 & 100 & 100 & 100 & 100 \\
\hline 4-Methyl-2-pentanone & 2 & 100 & 100 & 100 & 2 & 100 & 100 & 100 \\
\hline Methylene chloride & su & 5u & 5u & su & 5u & 1 & 50 & 5u \\
\hline styrene & su & 5u & 5u & 50 & su & 5u & 5u & su \\
\hline 1,1,2,2-Tetrachloroethane & 50 & 50 & 50 & 50 & 50 & 5u & su & 5u \\
\hline Tetrachloroethene & su & 50 & 50 & 5v & $5 \mathrm{U}$ & 50 & 5u & 50 \\
\hline Tolvene & su & 50 & 50 & $5 \mathrm{v}$ & su & 50 & 50 & 50 \\
\hline 1,1,1-Trichloroethane & 50 & 5u & $5 u$ & 50 & su & su & 5u & so \\
\hline 1,1,2-Trichloroethane & 50 & 5u & 50 & 50 & su & 50 & 50 & 50 \\
\hline Trichloroethene & $5 \mathrm{v}$ & su & su & $5 \mathrm{v}$ & 5u & 50 & su & so \\
\hline Vinyl acetate & 100 & 100 & 100 & 100 & 100 & 100 & 100 & 100 \\
\hline Vinyl chloride & 100 & 100 & 100 & 100 & 100 & 100 & 100 & 100 \\
\hline xylenes & 5u & su & $5 u$ & su & $5 \mathrm{U}$ & 50 & 5u & 5u \\
\hline
\end{tabular}

(CONTINUED) 
APPENDIX 2.2

Groundwater Quallty Data, 1993

\begin{tabular}{|c|c|c|c|c|c|c|c|c|}
\hline \multirow{3}{*}{$\begin{array}{l}\text { Sampling Polnt } \\
\text { Location } \\
\text { - } \\
\text { Date sampled }\end{array}$} & \multicolumn{4}{|c|}{ OW-768 } & \multicolumn{4}{|c|}{ OW-769 } \\
\hline & \multicolumn{4}{|c|}{ ORIDII } & \multicolumn{4}{|c|}{ GRIDO3 } \\
\hline & $|01 / 29 / 93|$ & $04 / 07 / 93$ & $08 / 04 / 93$ & $10 / 13 / 93$ & $01 / 27 / 93$ & $04 / 19 / 93$ & $09 / 14 / 93$ & $11 / 30 / 93$ \\
\hline VOLATILS ORGANICS (Ug/L) & . &. &. & . & • &. & . & . \\
\hline Acetone & 100 & 100 & 100 & 100 & 100 & 200 & 100 & 100 \\
\hline Benzene & su & su & su & su & su & su & su & su \\
\hline Bromodichloromethane & 50 & su & su & 50 & su & su & su & su \\
\hline Bromotorm & su & so & su & su & 5u & 5u & su & su \\
\hline Bromomethane & 100 & 100 & 100 & 100 & 100 & 100 & 100 & 100 \\
\hline 2-Butanone & 100 & 100 & 100 & 100 & 100 & 100 & 100 & 100 \\
\hline Carbon diauletide & su & so & su & su & so & su & su & so \\
\hline Carbon tetrachloride & sv & so & so & so & 9 & 5 & 5 & 6 \\
\hline Chlorobenzene & 50 & 50 & so & sv & 50 & so & su & su \\
\hline Chlorodibromomethane & so & su & su & su & 50 & so & su & su \\
\hline Chloroethane & 100 & 200 & 100 & 100 & 100 & 100 & 100 & 100 \\
\hline Chlorotorm & su & su & su & su & 1 & 1 & 0.9 & 0.7 \\
\hline Chloromethane & 100 & 100 & 100 & 100 & 100 & 100 & 200 & 100 \\
\hline 1,1-Dichloroethane & su & su & su & su & 1 & 1 & su & su \\
\hline 1,2-Dichloroethane & su & su & so & 5u & su & 50 & su & 50 \\
\hline 1,1-Dichloroethene & su & 50 & su & su & 1 & 1 & su & so \\
\hline 1,2-Dlohloroethene & so & sv & su & su & 1 & su & su & su \\
\hline 1,2-D1chloropropane & su & so & su & so & 50 & $\mathbf{5 u}$ & su & 50 \\
\hline cis-1,3-Dichloropropene & 50 & 5u & su & su & 50 & $\mathbf{5 u}$ & su & su \\
\hline trans-1,3-Dichloropropene & so & su & su & 5u & su & 50 & su & su \\
\hline Ethylbenzene & su & so & 5u & 5u & 5u & so & su & su \\
\hline 2-Hexanone & 100 & 100 & 100 & 100 & 100 & 100 & 100 & 100 \\
\hline 4-Methy 1-2-pentanone & 100 & 100 & 100 & 100 & 2 & 100 & 200 & 100 \\
\hline Mathylene chloride & 5u & so & 5u & su & su & sv & su & su \\
\hline styrene & 5u & su & su & 5u & 5u & so & su & su \\
\hline 1,1,2,2-Tetrachloroethane & su & su & 50 & 5u & so & $5 v$ & 5u & 50 \\
\hline Tetrachloroethene & so & 50 & 5u & su & 4 & 4 & 3 & 2 \\
\hline Toluene & su & su & su & su & su & 5u & su & 5u \\
\hline 1,1,1-Trichloroethane & su & 50 & 50 & 5u & so & 0.8 & 0.4 & 50 \\
\hline 1,1,2-Trichloroethane & su & 50 & su & 5u & 50 & 50 & su & su \\
\hline Trichloroethene & 50 & 50 & su & 50 & 1 & 1 & 1 & 0.7 \\
\hline Vinyl acetate & 100 & 100 & 100 & 100 & 100 & 100 & 100 & 100 \\
\hline Vinyl chloride & 100 & 100 & 100 & 100 & 100 & 100 & 100 & 200 \\
\hline xylenes & $5 u$ & 50 & 5u & 5u & 5u & 50 & 5u & su \\
\hline
\end{tabular}

(CONT INUED) 
APPENDIX E.2

\begin{tabular}{|c|c|c|c|c|c|c|c|c|}
\hline \multirow{3}{*}{$\begin{array}{l}\text { sampling point } \\
\text { Looation } \\
\text { Date sampled }\end{array}$} & \multicolumn{4}{|c|}{$0 w-770$} & \multicolumn{4}{|c|}{ ON-771 } \\
\hline & \multicolumn{4}{|c|}{ GRIDO3 } & \multicolumn{4}{|c|}{ ORIDCL } \\
\hline & $01 / 27 / 93$ & $04 / 19 / 93$ & $09 / 14 / 93$ & $11 / 29 / 93$ & $01 / 23 / 93$ & $|04 / 12 / 93|$ & $09 / 03 / 93$ & $11 / 11 / 93$ \\
\hline VOLATILE OREAHICs (ug/L) & $\cdot$ & - & - & - & - & $\cdot$ & . & • \\
\hline Acetone & 100 & 100 & 100 & 100 & 100 & 100 & 20 & 100 \\
\hline Bensene & su & su & so & so & 50 & so & 1 & so \\
\hline Bromodichloromethane & su & su & so & su & su & su & so & so \\
\hline Bromotorm & su & su & so & su & su & su & $\mathbf{s u}$ & su \\
\hline Bromomethane & 100 & 100 & 100 & 100 & 100 & 100 & 100 & 100 \\
\hline 2-Butanone & 100 & 100 & 100 & 100 & 100 & luv & 100 & 100 \\
\hline Carbon disulfide & su & so & su & su & so & su & 2 & su \\
\hline Carbon tetrachloride & su & su & so & su & so & so & su & so \\
\hline Chlorobenzene & so & so & su & su & su & su & so & so \\
\hline Chlorodibromomathane & so & sv & su & su & so & su & so & so \\
\hline Chloroethane & 100 & 100 & 200 & 100 & 100 & 100 & 100 & 100 \\
\hline Chlorotorm & 4 & 4 & s & 9 & so & su & sv & so \\
\hline Chloromethane & 100 & 100 & 100 & 100 & 100 & 100 & 100 & 100 \\
\hline 1,1-Dichloroethane & su & su & su & su & su & su & su & su \\
\hline 1,2-Dlchloroethane & 5u & so & su & su & su & su & su & so \\
\hline 1,1-Dlchloroethene & su & so & su & 50 & so & so & so & su \\
\hline 1,2-DLChloroethene & so & 50 & su & su & su & su & so & su \\
\hline 1,2-Dichloropropane & su & so & su & so & su & su & 50 & su \\
\hline Cle-1,3-Dlchloropropene & su & sv & so & so & su & su & su & 5u \\
\hline trana-1, 3-Dichloropropane & 50 & so & su & su & su & su & so & su \\
\hline ethylbenzene & 5u & su & so & su & 5u & 5u & so & su \\
\hline 2-Hexanone & 100 & 100 & 100 & 100 & 100 & 100 & 100 & 100 \\
\hline 4-Methyl-2-pantanone & 2 & 100 & 100 & 100 & 200 & 100 & 100 & 100 \\
\hline Mothylene chloride & su & so & 0.9 & so & su & 50 & su & 1 \\
\hline Styrane & su & su & 5u & 50 & 5u & sv & su & su \\
\hline 1,1,2,2-Tetrachloroethane & su & so & su & su & 50 & su & su & 5u \\
\hline Tetrachloroethene & so & 5u & so & su & su & su & so & su \\
\hline Toluene & su & su & su & so & so & 50 & 1 & su \\
\hline 1,1,1-Trichloroethane & su & su & su & su & su & so & 5u & su \\
\hline 1,1,2-Trichloroethane & su & 50 & su & su & su & su & su & su \\
\hline Trichloroethene & su & 50 & su & su & su & 5u & 4 & 5u \\
\hline vinyl acetate & 100 & 100 & 100 & 100 & 100 & 100 & 200 & 100 \\
\hline Vinyl chloride & 100 & 100 & 100 & 100 & 100 & 100 & 100 & 100 \\
\hline xylenes & 5u & su & su & su & su & su & su & su \\
\hline
\end{tabular}

(CONTINOED) 
APPINDIX E.2

Oroundwater Quallty Date, 1993

\begin{tabular}{|c|c|c|c|c|c|c|c|c|}
\hline \multirow{3}{*}{$\begin{array}{l}\text { Sampling polnt } \\
\text { Location } \\
\text { Date sempled }\end{array}$} & \multicolumn{4}{|c|}{ ON-772 } & \multicolumn{4}{|c|}{ 6w-773 } \\
\hline & \multicolumn{4}{|c|}{ ORIDCL } & \multicolumn{4}{|c|}{ ORIDH2 } \\
\hline & $01 / 23 / 93$ & $04 / 12 / 93$ & $09 / 12 / 93$ & $11 / 12 / 93$ & $02 / 03 / 93$ & $04 / 20 / 93 \mid$ & $|00 / 06 / 93|$ & $10 / 15 / 93$ \\
\hline vorartis ORensics (ug/h) & - & $\cdot$ & - & - & $\cdot$ & - & - & - \\
\hline Acetone & 100 & 100 & 100 & 100 & 100 & 100 & 100 & $100^{\circ}$ \\
\hline Benzene & so & so & su & su & su & su & su & so \\
\hline Eromodichloromethane & su & so & su & so & su & su & su & so \\
\hline Bromotorm & su & so & su & su & su & su & su & so \\
\hline Dromomathane & 100 & 100 & 100 & 100 & 100 & 100 & 100 & 100 \\
\hline 2-8utanone & 100 & 100 & 100 & 100 & 100 & 100 & 100 & 100 \\
\hline Cerbon dieulelde & su & so & su & so & su & su & so & so \\
\hline Carbon tetrachloride & so & so & su & so & su & su & su & so \\
\hline Chlorobanzene & so & so & so & so & su & su & su & so \\
\hline Chlorodibromomethane & su & so & so & so & su & su & su & su \\
\hline Chloroethane & 100 & 100 & 100 & 100 & 200 & 100 & 100 & 100 \\
\hline Chlorotorm & so & 30 & su & so & su & su & su & so \\
\hline Chloromethane & 200 & 100 & 100 & 200 & 200 & 100 & 100 & 100 \\
\hline 1,1-Dlohloroethane & 50 & su & su & su & so & su & so & su \\
\hline 1,2-Dichloroethane & so & su & su & su & so & su & su & so \\
\hline 1,1-Dichloroethene & su & su & su & su & so & su & $\mathbf{s u}$ & su \\
\hline 1,2-Dichloroethene & so & su & su & so & so & su & so & su \\
\hline 1,2-D1Ch Loropropane & su & su & so & su & so & so & so & su \\
\hline CLE-1, 3-DLChloropropene & so & su & su & su & so & so & so & so \\
\hline trans-1,3-Dich loropropene & so & so & su & so & so & so & so & so \\
\hline Ethylbonzone & so & su & su & so & su & su & so & su \\
\hline 2-Hexanone & 100 & 100 & 100 & 100 & 100 & 200 & 100 & 100 \\
\hline 1-Mothyl-2-pentanone & 100 & 100 & 100 & 100 & 2 & 2 & 100 & 100 \\
\hline Mathylene chloride & so & su & su & 1 & 2 & su & 50 & su \\
\hline Styrane & su & 50 & 5u & so & so & su & so & so \\
\hline $1,1,2,2$-Tetrachloroethane & so & su & so & so & su & $\mathbf{s u}$ & su & su \\
\hline Tetrachloroethene & su & su & so & so & su & su & su & su \\
\hline Toluene & 5u & so & so & 50 & su & su & so & su \\
\hline $1,1,1-$ Trichloroothane & su & su & 5u & so & su & su & su & su \\
\hline 1,1,2-Trichloroethane & so & su & so & 50 & su & su & su & so \\
\hline Trichloroethene & su & su & 5u & so & su & so & su & su \\
\hline vinyl acetate & 200 & 100 & 200 & 100 & 100 & 100 & 100 & 100 \\
\hline vinyl chloride & 200 & 100 & 100 & 100 & 100 & 100 & 100 & 100 \\
\hline xylenos & su & 50 & 5u & su & $\mathbf{5 u}$ & su & su & su \\
\hline
\end{tabular}

(CONTIRURE) 
APPENDIX 8.2

Oroundwater Quadlty Data, 1993

\begin{tabular}{|c|c|c|c|c|c|c|c|c|}
\hline \multirow{3}{*}{$\begin{array}{l}\text { Eapling Point } \\
\text { Location } \\
\text { Date sampled }\end{array}$} & \multicolumn{4}{|c|}{$a N-774$} & \multicolumn{4}{|c|}{ an-773 } \\
\hline & \multicolumn{4}{|c|}{ ORIDH2 } & \multicolumn{4}{|c|}{ ORIDH3 } \\
\hline & $02 / 04 / 93$ & $04 / 20 / 93$ & $08 / 09 / 93$ & $20 / 19 / 93$ & $02 / 04 / 83$ & $04 / 20 / 93$ & $08 / 09 / 93$ & $10 / 15 / 93$ \\
\hline VOLATILS OACNAICS (Ug/L) & $\cdot$ & $\cdot$ & $\cdot$ & • & - & - & - & \\
\hline Acetone & 100 & 100 & 100 & 100 & 100 & 100 & 100 & 100 \\
\hline Denzene & su & so & su & su & so & so & su & su \\
\hline Bromodlehloromethane & su & su & su & su & su & su & su & su \\
\hline Bromotorm & so & so & sv & sv & su & su & so & su \\
\hline Bromomethane & 100 & 100 & 100 & 100 & 100 & 100 & 100 & 100 \\
\hline 2-Dutanone & 100 & 100 & 100 & 100 & 100 & 200 & 100 & 100 \\
\hline Carbon dieulelde & so & su & su & sv & so & $\mathbf{s u}$ & su & su \\
\hline Carbon tetrachloride & so & so & su & su & so & so & so & 1 \\
\hline Chlorobenzene & su & so & su & so & so & su & su & su \\
\hline Chlorodibromowethane & so & so & su & su & so & su & su & su \\
\hline Chloroethane & 100 & 100 & 100 & 100 & 100 & 200 & 100 & 100 \\
\hline Chlorotorm & su & so & su & so & 1 & su & su & 0.8 \\
\hline Chloromethane & 100 & 100 & 100 & 100 & 100 & 100 & 100 & 100 \\
\hline 1,1-Diohloroethane & so & su & su & 50 & so & su & so & su \\
\hline 1,2-DLohloroethane & su & so & su & so & su & su & su & su \\
\hline 1,1-Dichloroethene & so & su & so & su & so & su & so & su \\
\hline 1,2-Dlehloroethene & so & so & su & so & so & su & so & su \\
\hline 1,2-Dichloropropane & su & so & so & so & su & so & su & su \\
\hline cle-1,3-Dichloropropene & su & so & so & so & su & su & so & su \\
\hline trane-1, 3-DLehLoropropene & so & so & so & 50 & su & su & su & su \\
\hline Ethylbenzene & so & so & so & so & so & su & 50 & su \\
\hline 2-Hexanone & 100 & 100 & 100 & 100 & 100 & 100 & 100 & 100 \\
\hline 4-Mathy 1-2-pantanone & 100 & 2 & 100 & 100 & 100 & 2 & 100 & 100 \\
\hline Methylene ohloride & so & 50 & so & so & su & 3 & so & so \\
\hline styrene & 5u & 50 & so & so & su & su & so & su \\
\hline $1,1,2,2$-Tetrachloroethane & su & so & so & so & so & so & so & su \\
\hline Tets echloroethene & su & su & su & su & 0.6 & su & su & su \\
\hline Toluene & su & su & su & so & so & su & su & su \\
\hline $1,1,1-\operatorname{Tr}$ lehloroethane & so & 50 & su & so & su & su & 50 & su \\
\hline 1,1,2-Trichloroethane & su & su & su & su & so & su & su & su \\
\hline Trlehloroethene & 5u & su & so & su & $s$ & 3 & 4 & 5 \\
\hline vinyl acetate & 100 & 100 & 100 & 100 & 100 & 100 & 100 & 100 \\
\hline Vinyl chloride & 100 & 100 & 100 & 100 & 100 & 100 & 100 & 100 \\
\hline xylenes & su & su & su & su & su & su & 50 & su \\
\hline
\end{tabular}

(CONTINURD) 
APPENDIX 8.2

Groundwater Quellty Data, 1993

\begin{tabular}{|c|c|c|c|c|}
\hline \multirow{3}{*}{$\begin{array}{l}\text { Sampling Point } \\
\text { Looation } \\
\text { Date sampled }\end{array}$} & \multicolumn{4}{|c|}{ OW-776 } \\
\hline & \multicolumn{4}{|c|}{ ORIDH3 } \\
\hline & $02 / 04 / 93$ & $\mid 04 / 20 / 93$ & $08 / 10 / 93$ & $10 / 18 / 93$ \\
\hline VOLATIL ORCNAICS (ug/L) & $\cdot$ & $\cdot$ & $\cdot$ & - \\
\hline Aoetone & 100 & 100 & 100 & 100 \\
\hline Bensene & su & su & sv & su \\
\hline Bromodlehloromethane & so & su & su & sv \\
\hline Bromotorm & su & su & so & su \\
\hline Bromomothane & 100 & 100 & 100 & 100 \\
\hline 2-Butanona & 100 & 100 & 100 & 100 \\
\hline Carbon disuletde & su & su & su & su \\
\hline Carbon tetrachloride & so & su & su & su \\
\hline Chlorobensene & so & sv & so & su \\
\hline Chlorodibromomethane & so & su & so & su \\
\hline Chloroethane & 100 & 100 & 100 & 100 \\
\hline Chloroform & 1 & 1 & 2 & 2 \\
\hline Chloromethane & 100 & 100 & 100 & 100 \\
\hline 1,1-Dichloroethane & su & $\mathbf{s u}$ & $\mathbf{3 0}$ & su \\
\hline 1,2-DLchloroothane & su & so & su & so \\
\hline 1,1-Dichloroethene & so & so & $\mathbf{s u}$ & so \\
\hline 1,2-DLchloroethene & su & so & so & 5u \\
\hline 1,2-Dichloropropane & su & so & so & su \\
\hline ola-1, 3-Dichloropropene & so & so & su & 5v \\
\hline trane-1, 3-Dichloropropane & so & 50 & so & su \\
\hline Ethylbenzene & $\mathbf{5 u}$ & so & so & so \\
\hline 2-Hexanone & 100 & 100 & 100 & 100 \\
\hline 4-Methyl-2-pentanone & 100 & 1 & 100 & 100 \\
\hline Methylene ohloride & so & so & su & so \\
\hline styrone & su & 50 & so & su \\
\hline 1,1,2,2-Tatrachloroethane & so & so & su & su \\
\hline Tetrachloroethene & 3 & 2 & 2 & 2 \\
\hline Tolvene & 50 & so & so & su \\
\hline 1,1,1-Trichloroethane & so & su & so & so \\
\hline 1,1,2-Trichloroethane & su & so & su & so \\
\hline Trichloroethene & 4 & 3 & 4 & 4 \\
\hline Vinyl acetate & 100 & 100 & 100 & 100 \\
\hline Vinyl chloride & 100 & 100 & 100 & 100 \\
\hline xylenes & su & 5u & su & so \\
\hline
\end{tabular}




\section{APPENDIX E.3}

FIELD MEASUREMENTS, MISCLLANEOUS PARAMETERS, AND GROSS ALPHA AND GROSS BETA ACTIVITIES 
APPENDIX $\mathrm{E} \cdot 3$

Groundwater Quality Data, 1993

\begin{tabular}{|c|c|c|c|c|c|c|c|c|}
\hline \multirow{3}{*}{$\begin{array}{l}\text { Sampling Polnt } \\
\text { - } \\
\text { Location } \\
\text { Date Sampled }\end{array}$} & \multicolumn{4}{|c|}{$6 w-151$} & \multicolumn{4}{|c|}{$G W-168$} \\
\hline & \multicolumn{4}{|c|}{ NHP } & \multicolumn{4}{|c|}{ NHP } \\
\hline & $01 / 26 / 93$ & $04 / 22 / 93$ & $08 / 06 / 93$ & $11 / 02 / 93$ & $01 / 22 / 93$ & $05 / 14 / 93$ & $08 / 19 / 93$ & $11 / 02 / 93$ \\
\hline FIELD MEASUREMENTS & - & - & - & - & - & - & - & - \\
\hline Depth to Water $(f t)$ & 14.3 & 14.58 & 15.1 & 15.24 & 28.1 & 30.21 & 30.89 & 31.21 \\
\hline Water Temp (degrees C) & 13.4 & 15.6 & 17.2 & 14.8 & 13.9 & 15.7 & 16.9 & 13.8 \\
\hline pH (pH units) & 7.5 & 7.5 & 7.3 & 7.2 & 6.9 & 7.1 & 7.2 & $7 \cdot 4$ \\
\hline Sp. Cond. (umho/cm) & 428 & 411 & 443 & 466 & 524 & 476 & 907 & 502 \\
\hline Dissolved Oxygen (ppm) & 0.7 & 3 & 0.9 & 1 & 3.4 & 5.4 & $1 \cdot 4$ & 5.7 \\
\hline Oxidation/Reduction (mV) & 177 & 78 & 167 & 210 & 15 & -47 & -318 & 14 \\
\hline MISCEILANEOUS PARAMETERS & $\cdot$ & $\cdot$ & $\cdot$ & • & $\cdot$ & $\cdot$ & • & • \\
\hline pH (pH unite) & 7.7 & 7.8 & 7.6 & 7.7 & 7.3 & 7.8 & 8 & 7.5 \\
\hline Sp. Cond. (umho/cm) & 515 & 494 & 495 & 484 & 587 & 558 & 553 & 566 \\
\hline TPH $(\mathrm{mg} / \mathrm{L})$ & $\cdot$ & - & • & + & - & - & • & - \\
\hline $\operatorname{TDS}(m g / L)$ & 278 & 276 & 300 & 302 & 324 & 322 & 334 & 348 \\
\hline TSS (mg/L) & $<1$ & 1 & 1 & $<1$ & 3 & 1 & 1 & $<1$ \\
\hline Turbidity (NTO) & 2.3 & 0.5 & 3.7 & 0.8 & 2 & 2.2 & 1.2 & 3 \\
\hline RADIOCIMMICAI PARAMETERS & - & - & - & - & - & - & - & - \\
\hline Grose Alpha (pCl/I) & 1.74 & -1.00 & 0.90 & 1.10 & 2.66 & -5.13 & 4.60 & 1.44 \\
\hline Grose Alpha $(\mathrm{CB}+/-)$ & $2 \cdot 30$ & 2.20 & 3.60 & 1.70 & 2.10 & 3.80 & 2.90 & 1.80 \\
\hline Gross Beta (pCl/L) & $-5 \cdot 22$ & 4.27 & 2.97 & 1.38 & 1.89 & -1.68 & 5.57 & 2.16 \\
\hline Gross Beta $(\mathrm{CB}+/-)$ & 3.40 & 2.80 & 5.10 & 2.90 & 2.80 & 5.60 & 3.00 & 3.00 \\
\hline
\end{tabular}

(CONTINOED) 
APPENDIX E.3

Groundwater Quality Data, 1993

\begin{tabular}{|c|c|c|c|c|c|c|c|c|}
\hline \multirow{3}{*}{$\begin{array}{l}\text { Sampling Point } \\
\text { Location } \\
\text { - } \\
\text { Date Sampled }\end{array}$} & \multicolumn{4}{|c|}{ GW-169 } & \multicolumn{4}{|c|}{ GW-170 } \\
\hline & \multicolumn{4}{|c|}{ EXP } & \multicolumn{4}{|c|}{$\mathbf{E X P}$} \\
\hline & $01 / 23 / 93$ & $05 / 21 / 93$ & $08 / 19 / 93$ & $11 / 01 / 93$ & $01 / 23 / 93$ & $05 / 24 / 93$ & $08 / 21 / 93$ & $11 / 04 / 93$ \\
\hline FIBID MEASUREMENTS & - & - & & & - & - & - & - \\
\hline Depth to Water (ft) & 27 & 30.21 & 31.43 & 32 & 31.8 & 32.4 & 33.15 & 32.85 \\
\hline Water Temp (degrees C) & 13.1 & 13.6 & 15.6 & 12.3 & 12.7 & 16.6 & 15.8 & 13.2 \\
\hline pH (pH units) & 7.2 & 6.6 & 7.3 & 7.6 & 7.6 & 7.8 & 7.6 & 7.7 \\
\hline Sp. Cond. (umho/cm) & 304 & 281 & 619 & 356 & 444 & 377 & 453 & 462 \\
\hline Dissolved Oxygen (ppm) & 5.8 & 5.9 & 5.4 & 7.3 & 1.5 & 0.8 & 1.5 & 2.9 \\
\hline Oxidation/Reduction (mv) & 165 & 236 & 125 & 101 & 190 & -46 & 135 & 176 \\
\hline MISCEILANEOUS PARAMETERS & . & . & . & . & . & . & . & • \\
\hline pH (pH units) & 7.2 & 7.4 & 8 & 7.6 & 7.7 & 7.9 & 7.9 & 7.7 \\
\hline Sp. Cond. (umho/cm) & 320 & 350 & 377 & 342 & 485 & 461 & 471 & 514 \\
\hline TPH $(\mathrm{mg} / \mathrm{I})$ & - & - & • & - & • & • & • & • \\
\hline TDS (mg/L) & 228 & 240 & 312 & 274 & 266 & 278 & 286 & 294 \\
\hline TSS $(m g / I)$ & 2100 & 404 & 1388 & 1960 & $<1$ & $<1$ & $<1$ & $<1$ \\
\hline Turbidity (NTU) & 2250 & 20 & 1200 & 46 & 1.2 & 1.2 & 0.8 & 0.7 \\
\hline RADIOCFEMICAI PARAMBTERS & - & - & - & - & - & - & - & • \\
\hline Gross Alpha (pCi/L) & 25.60 & 4.76 & 24.30 & 72.00 & 1.63 & -0.90 & -1.76 & 2.04 \\
\hline Gross Alphe $(\mathrm{CR}+/-)$ & 6.70 & 2.80 & 7.30 & 26.00 & 1.70 & 3.10 & 2.10 & 1.90 \\
\hline Gross Beta (pci/L) & 29.60 & 6.16 & 32.40 & 65.50 & 1.19 & $-2 \cdot 14$ & 2.25 & 2.93 \\
\hline Gross Beta $(\mathrm{CB}+/-)$ & 7.00 & 3.20 & 7.10 & 32.00 & 2.80 & 5.40 & 2.80 & 3.00 \\
\hline
\end{tabular}

(CONTINUED) 
APPENDIX E.3

Groundwater Quality Data, 1993

\begin{tabular}{|c|c|c|c|c|c|c|c|c|}
\hline \multirow{3}{*}{$\begin{array}{l}\text { Sampling Point } \\
\text { Location } \\
\text { - } \\
\text { Date Sampled }\end{array}$} & \multicolumn{5}{|c|}{ GW-183 } & \multicolumn{3}{|c|}{ GW-190 } \\
\hline & \multicolumn{5}{|c|}{$\mathbf{F F}$} & \multicolumn{3}{|c|}{ RG } \\
\hline & $03 / 10 / 93$ & $06 / 21 / 93$ & $06 / 29 / 93$ & $09 / 22 / 93$ & $11 / 16 / 93$ & $03 / 01 / 93$ & $06 / 17 / 93$ & $07 / 07 / 93$ \\
\hline FIELD MEASUREMENTS & - & • & - & - & - & • & - & - \\
\hline Depth to Water $(f t)$ & 10.2 & 11 & 11.31 & 11.79 & 10.91 & 12.75 & 13.44 & 13.57 \\
\hline Water Temp (degrees C) & 17.5 & 17.6 & 17.8 & 19 & 18.5 & 16.8 & 19.4 & 23.6 \\
\hline pH (ph units) & 6.9 & 6.8 & 6.6 & 7 & 6.8 & 6.9 & 6.7 & 6.8 \\
\hline Sp. Cond. (umho/cm) & 878 & 760 & 784 & 837 & 924 & 235 & 236 & 245 \\
\hline Dissolved Oxygen (ppm) & 0.8 & 1.2 & 1.9 & 4.6 & 1.6 & 1.5 & 4.2 & 7.3 \\
\hline Oxidation/Reduction (mv) & -63 & -51 & 19 & 35 & 61 & 172 & 202 & 118 \\
\hline MISCELIAANBOUS PARAMETERS & • & $\cdot$ & $\cdot$ & • & • & $\cdot$ & $\bullet$ & • \\
\hline pH (pH units) & 6.9 & 7.6 & $\cdot$ & $7 . \dot{1}$ & $7 . \dot{1}$ & $6 . \dot{7}$ & 7.5 & $\cdot$ \\
\hline Sp. Cond. (umho/cm) & 954 & 915 & - & 959 & 934 & 225 & 259 & - \\
\hline$T P H(m g / L)$ & 0.034 & $\cdot$ & 0.014 & $<0.1$ & $<0.1$ & $<0.1$ & $\cdot$ & $<0.1$ \\
\hline TDS (mg/L) & 554 & 584 & $\cdot$ & 572 & 540 & 168 & 178 & • \\
\hline TSS (mg/L) & 13 & 8 & - & 18 & 3 & 7 & 5 & - \\
\hline Turbidity (NTU) & 5.9 & 6.6 & . & 10 & 2.5 & 4.7 & 12 & - \\
\hline RADIOCHEMICAI PARAMETERS & . & . & . & . & . & $\cdot$ &. & • \\
\hline Gross Alpha (pCi/L) & -1.84 & 0.00 & $\cdot$ & 3.51 & 2.09 & 0.30 & -2.54 & • \\
\hline Gross Alphe $(\mathrm{CE}+/-)$ & 5.00 & 3.70 & - & 4.40 & 2.40 & 1.30 & 1300.00 & • \\
\hline Gross Beta $(\mathrm{pCi} / \mathrm{L})$ & 4.88 & 4.01 & - & 3.97 & 0.51 & 0.66 & -4.67 & . \\
\hline Gross Beta $(\mathrm{CE}+/-)$ & 5.70 & 5.40 & - & 5.60 & 2.70 & 3.10 & 2500.00 & - \\
\hline
\end{tabular}

(CONTINUED) 
APPENDIX E. 3

Groundwater Quality Data, 1993

\begin{tabular}{|c|c|c|c|c|c|c|c|c|}
\hline \multirow{3}{*}{$\begin{array}{l}\text { Sampling Point } \\
\text { Location } \\
\text { - } \\
\text { Date Sampled }\end{array}$} & \multicolumn{2}{|c|}{ GW-190 } & \multicolumn{4}{|c|}{ GW-191 } & \multicolumn{2}{|c|}{$G W-192$} \\
\hline & \multicolumn{2}{|c|}{$\mathbf{R G}$} & \multicolumn{4}{|c|}{ B4 } & \multicolumn{2}{|c|}{ B4 } \\
\hline & $09 / 15 / 93$ & $11 / 16 / 93$ & $01 / 12 / 93$ & $04 / 12 / 93$ & $07 / 08 / 93$ & $12 / 09 / 93$ & $01 / 14 / 93$ & $04 / 13 / 93$ \\
\hline FIELD MBASUREMENTS & - & - & - & - & - & - & - & - \\
\hline Depth to Water (ft) & 13.87 & 12.93 & 4.1 & 4.2 & 6.68 & 3.29 & 4.2 & 4.81 \\
\hline Water Temp (degrees C) & 18.1 & $17 \cdot 1$ & 15.7 & 21.1 & 23.1 & 16 & 11.8 & 19.7 \\
\hline pH (pH units) & 6.4 & 6.3 & 7.4 & 7.6 & 7.1 & 7.4 & 6.4 & 6.5 \\
\hline Sp. Cond. (umho/cm) & 236 & 259 & 453 & 462 & 541 & 479 & 723 & 562 \\
\hline Dissolved Oxygen (ppm) & 4.4 & 0.9 & 8.4 & 5.4 & 5.5 & 7 & 4.7 & 5.6 \\
\hline Oxidation/Reduction (mv) & 341 & 198 & 88 & 21 & 169 & 186 & 24 & -12 \\
\hline MISCELIAANEOUS PARAMETERS & - & - & - & - & - & - & - & • \\
\hline pH (pH units) & 7 & 7.2 & 7.6 & 8 & 8 & 7.6 & 6.8 & 6.7 \\
\hline Sp. Cond. (umho/cm) & 261 & 279 & 493 & 430 & 498 & 524 & 630 & 648 \\
\hline $\operatorname{TPH}(\mathrm{mg} / \mathrm{L})$ & $<0.1$ & $<0.1$ & $\cdot$ & - & • & • & • & • \\
\hline $\operatorname{TDS}(\mathrm{mg} / \mathrm{L})$ & 180 & 144 & 310 & 334 & 322 & 352 & 392 & 402 \\
\hline TSS $(\mathrm{mg} / \mathrm{L})$ & 1 & 9 & 21 & 2162 & 107 & 143 & 61 & 6 \\
\hline Turbidity (NTU) & 4 & 15 & 20 & 560 & 230 & 1650 & 66 & 5.8 \\
\hline RADIOCHEMICAL PARAMETERS & - & - & - & - & - & - & - & - \\
\hline Gross Alpha (pci/L) & 2.33 & 2.51 & 0.00 & 28.00 & -0.00 & 3.32 & -0.21 & 0.26 \\
\hline Gross Alpha $(\mathrm{CE}+/-)$ & 1.90 & 1.40 & 2.20 & 13.00 & 2.60 & 1.80 & 2.10 & 2.10 \\
\hline Gross Beta (pCi/L) & -0.34 & 3.86 & 1.89 & 80.60 & -5.31 & 3.42 & 2.58 & 5.56 \\
\hline Gross Beta $(\mathrm{CE}+/-)$ & 2.60 & 2.60 & 2.90 & 15.00 & 5.60 & 2.60 & 2.90 & 2.90 \\
\hline
\end{tabular}

(CONTINUED) 
APPENDIX E. 3

Groundwater Quality Data, 1993

\begin{tabular}{|c|c|c|c|c|c|c|c|c|}
\hline \multirow{3}{*}{$\begin{array}{l}\text { Sampling Point } \\
\text { Location } \\
\text { - } \\
\text { Date Sampled }\end{array}$} & \multicolumn{2}{|c|}{ GW-192 } & \multicolumn{4}{|c|}{ GW-193 } & \multicolumn{2}{|c|}{ GW-194 } \\
\hline & \multicolumn{2}{|c|}{ B4 } & \multicolumn{4}{|c|}{ T2331 } & \multicolumn{2}{|c|}{ B4 } \\
\hline & $07 / 08 / 93$ & $12 / 09 / 93$ & $03 / 11 / 93$ & $06 / 22 / 93$ & $09 / 23 / 93$ & $11 / 18 / 93$ & $01 / 13 / 93$ & $04 / 13 / 93$ \\
\hline FIELD MEASUREMENTS & - & - & - & - & - & - & - & - \\
\hline Depth to Water (ft) & 6.7 & 4.11 & 8.45 & 8.85 & 9.54 & 8.65 & 5.68 & 6.24 \\
\hline Water Temp (degrees $C$ ) & 20.8 & 16.6 & 17.1 & 23 & 19.1 & 19.9 & 12.4 & 15.2 \\
\hline pH (pH units) & 6.4 & 6.7 & 7.4 & 7.2 & 7.2 & 7.2 & 7 & 7 \\
\hline Sp. Cond. (umho/cm) & 619 & 608 & 634 & 754 & 793 & 816 & 424 & 377 \\
\hline Dissolved Oxygen (ppm) & 2.3 & 6.4 & 1.4 & 2.2 & 4.1 & 3.1 & 4.3 & 6.8 \\
\hline Oxidation/Reduction (mv) & -36 & 91 & -192 & -84 & -16 & -14 & 219 & 107 \\
\hline MISCELLANEOUS PARAMETERS & - & - & - & - & - & • & - & - \\
\hline pH (pH units) & 7.3 & 6.8 & 7.7 & 7.7 & 7.4 & 7.4 & 7.2 & 7.2 \\
\hline Sp. Cond. (umho/cm) & 657 & 652 & 719 & 780 & 808 & 866 & 460 & 435 \\
\hline TPH (mg/L) & - & - & 3.478 & 3.64 & 2.18 & 2.29 & - & - \\
\hline $\operatorname{TDS}(\mathrm{mg} / \mathrm{L})$ & 418 & 432 & 398 & 500 & 484 & 462 & 278 & 294 \\
\hline TSS (mg/L) & 11 & 10 & 35 & 830 & 23 & 98 & 102 & 65 \\
\hline Turbidity (NTU) & 7.5 & 22 & 52 & 440 & 22 & 29 & 34 & 150 \\
\hline RADIOCHEMICAL PARAMETERS & - & - & - & - & - & - & - & - \\
\hline Gross Alpha (pci/L) & -1.09 & 0.37 & 7.40 & 15.20 & 19.00 & 42.10 & 27.20 & 2.29 \\
\hline Gross Alpha $(\mathrm{CB}+/-)$ & $2 \cdot 40$ & 1.10 & 3.80 & 3.80 & 5.10 & 5.80 & 5.10 & 2.30 \\
\hline Gross Beta (pCi/L) & -8.53 & 2.95 & 11.80 & 15.20 & 24.90 & 41.60 & $30 \cdot 30$ & 14.00 \\
\hline Gross Beta $(\mathrm{CE}+/-)$ & 5.40 & 2.50 & 3.70 & 3.50 & 4.30 & 4.60 & 4.20 & 3.30 \\
\hline
\end{tabular}

(CONTINUED) 
APPENDIX E. 3

Groundwater Qual1ty Data, 1993

\begin{tabular}{|c|c|c|c|c|c|c|c|c|}
\hline \multirow{3}{*}{$\begin{array}{l}\text { Sampling Point } \\
\text { - } \\
\text { Location } \\
\text { - } \\
\text { Date Sampled }\end{array}$} & \multicolumn{2}{|c|}{ GW-194 } & \multicolumn{4}{|c|}{ GW-195 } & \multicolumn{2}{|c|}{ GW-199 } \\
\hline & \multicolumn{2}{|c|}{ B4 } & \multicolumn{4}{|c|}{ B4 } & \multicolumn{2}{|c|}{ GRIDI 1} \\
\hline & $07 / 08 / 93$ & $12 / 09 / 93$ & $01 / 13 / 93$ & $04 / 13 / 93$ & $07 / 08 / 93$ & $12 / 09 / 93$ & $01 / 29 / 93$ & $04 / 07 / 93$ \\
\hline VIELD MEASUREMENTS & • & - & - & - & $\cdot$ & - & - & - \\
\hline Depth to Water (ft) & 6.83 & 6.12 & 5.85 & 6.37 & 8.1 & 6.79 & 16.62 & 16.6 \\
\hline Water Temp (degrees C) & 23.1 & 16.7 & 15.9 & 18.1 & 22.1 & $15 \cdot 3$ & 15.2 & 16 \\
\hline pH (pH units) & 6.6 & 7.5 & 6.7 & 6.7 & 6.3 & 6.9 & 6.6 & $6 \cdot 4$ \\
\hline Sp. Cond. (umho/cm) & 419 & 439 & 364 & 335 & 338 & 338 & 506 & 319 \\
\hline Dissolved Oxygen (ppm) & 3.9 & 7.2 & 1.5 & 3 & 3.5 & 6.7 & 1.9 & 2.4 \\
\hline Oxidation/Reduction (mV) & 187 & 188 & 159 & 160 & 200 & 217 & 200 & 116 \\
\hline MISCELLAKEOUS PARAKETERS & - & - & - & - & $\cdot$ & - & - & - \\
\hline pH (pH units) & 7.7 & 7.3 & 6.9 & 6.8 & 7.6 & 7.1 & 6.8 & 6.9 \\
\hline Sp. Cond. (umho/cm) & 454 & 460 & 390 & 375 & 372 & 372 & 387 & 378 \\
\hline TPH (mg/L) & & & - & - & - & - & - & - \\
\hline $\operatorname{TDS}(\mathrm{mg} / \mathrm{L})$ & 290 & 312 & 254 & 310 & 300 & 282 & 280 & 222 \\
\hline TSS (mg/L) & 90 & 30 & 524 & 527 & 510 & 292 & 628 & 40 \\
\hline Turbidity (NTU) & 64 & 120 & 240 & 220 & 240 & 360 & 3900 & 15 \\
\hline RADIOCHEMICAI PARAMETERS & • & - & - & - & - & - & - & - \\
\hline Gross Alpha (pCi/L) & -1.66 & 6.06 & 2.50 & 9.68 & 2.18 & 5.05 & 4.23 & $-1 \cdot 14$ \\
\hline Gross Alpha $(\mathrm{CE}+/-)$ & 2.00 & 2.30 & 2.10 & 4.60 & 2.90 & 2.30 & 2.80 & 6.60 \\
\hline Gross Beta (pCi/L) & -9.04 & 11.00 & 7.89 & 44.10 & 6.78 & 25.40 & 5.45 & 14.70 \\
\hline Gross Beta $(\mathrm{CE}+/-)$ & 5.50 & 3.20 & 3.20 & 7.20 & 6.40 & 4.50 & 5.40 & 10.00 \\
\hline
\end{tabular}

(CONTINUED) 
APPENDIX R.3

Groundwater Quality Data, 1993

\begin{tabular}{|c|c|c|c|c|c|c|c|c|}
\hline \multirow{3}{*}{$\begin{array}{l}\text { Sampling Point } \\
\text { - } \\
\text { Location } \\
\text { Date Sampled }\end{array}$} & \multicolumn{2}{|c|}{ GW-199 } & \multicolumn{4}{|c|}{ GW-204 } & \multicolumn{2}{|c|}{ GW-206 } \\
\hline & \multicolumn{2}{|c|}{ GRIDI 1} & \multicolumn{4}{|c|}{ T0134 } & \multicolumn{2}{|c|}{$\mathbf{E X P}$} \\
\hline & $08 / 02 / 93$ & $10 / 12 / 93$ & $03 / 11 / 93$ & $06 / 22 / 93$ & $09 / 23 / 93$ & $11 / 16 / 93$ & $01 / 19 / 93$ & $05 / 06 / 93$ \\
\hline FIELD MEASUREMBNTS & - & - & $\cdot$ & . & $\cdot$ & . & - & - \\
\hline Depth to Water (ft) & 16 & 17.55 & 11.65 & 10.55 & 10.9 & 10.4 & 9 & 8.68 \\
\hline Water Temp (degrees C) & 19.4 & 12.2 & 11.1 & 22.6 & 19.8 & 20.7 & 12.9 & 15.1 \\
\hline $\mathrm{PH}$ (pH unite) & 6.1 & 6.4 & 7 & 7.2 & 7.6 & 7.4 & 6.6 & 6.9 \\
\hline Sp. Cond. (umho/cm) & 366 & 451 & 422 & 368 & 453 & 399 & 335 & 394 \\
\hline Dissolved Oxygen (ppm) & 1.3 & 2.3 & 4.5 & 2.9 & 8.5 & 4.2 & 6.7 & 5.3 \\
\hline Oxidation/Reduction (mv) & 183 & 16 & -29 & 80 & 212 & 140 & -54 & 59 \\
\hline MISCELIANEOUS PARAMETERS & & • & $\cdot \vec{\cdot}$ & $\cdot$ & $\cdot$ & $\bullet$ & • & $\cdot$ \\
\hline $\mathrm{pH}$ (pH units) & 6.5 & 6.6 & 7.6 & 7.6 & 7.6 & $7 . \dot{7}$ & $6 . \dot{7}$ & 6.9 \\
\hline Sp. Cond. (umho/cm) & 432 & 446 & 447 & 413 & 470 & 391 & 355 & 439 \\
\hline TPH $(\mathrm{mg} / \mathrm{L})$ & - & $\cdot$ & 0.44 & 0.003 & $<0.1$ & 0.113 & . & • \\
\hline $\operatorname{TDS}(\mathrm{mg} / \mathrm{L})$ & 250 & 332 & 294 & 268 & 312 & 192 & 220 & 248 \\
\hline TSS (mg/L) & 14 & 16 & 25 & 3 & 26 & 250 & 2750 & 1210 \\
\hline Turbidity (NTU) & 25 & 6.5 & 24 & 3.3 & 6.5 & 110 & 1900 & 750 \\
\hline RADIOCHBMICAL PARARETERS & . & . & . & . & . & . & . & . \\
\hline Gross Alphe $(\mathrm{pCl} / \mathrm{L})$ & -2.38 & 6.93 & 30.70 & 102.00 & 50.10 & 67.10 & 44.00 & 10.90 \\
\hline Gross Alpha $(\mathrm{CE}+/-)$ & 4.50 & 6.70 & 5.20 & 8.50 & 8.60 & 6.70 & 13.00 & 4.40 \\
\hline Gross Beta (pCi/L) & -7.41 & 7.54 & 20.80 & 36.80 & 32.80 & 39.10 & 133.00 & 38.80 \\
\hline Gross Beta $(\mathrm{CE}+/-)$ & 5.80 & 10.00 & 4.00 & 4.40 & 7.10 & 4.50 & 11.00 & 5.80 \\
\hline
\end{tabular}

( CONTINURD) 
APPENDIX E.3

Groundwater Quality Data, 1993

\begin{tabular}{|c|c|c|c|c|c|c|c|c|}
\hline \multirow{3}{*}{$\begin{array}{l}\text { Sampling Point } \\
\text { Location } \\
\text { Date Sampled }\end{array}$} & \multicolumn{2}{|c|}{ GW-206 } & \multicolumn{4}{|c|}{ GW-207 } & \multicolumn{2}{|c|}{$G W-208$} \\
\hline & \multicolumn{2}{|c|}{$\mathbf{E x P}$} & \multicolumn{4}{|c|}{$\mathbf{E X P}$} & \multicolumn{2}{|c|}{$\mathbf{E X P}$} \\
\hline & $08 / 12 / 93$ & $10 / 27 / 93$ & $01 / 14 / 93$ & $05 / 04 / 93$ & $08 / 11 / 93$ & $10 / 26 / 93$ & $01 / 19 / 93$ & $05 / 06 / 93$ \\
\hline YIBLL MEASUREKBNTS & $\cdot$ & • & $\cdot$ & - & - & - & - & - \\
\hline Dapth to Water $(f t)$ & 9.27 & 9.81 & 0 & 0 & 0 & 0 & 0 & 0 \\
\hline Water Temp (degrees C) & 18.2 & 14.8 & 14.5 & 16.6 & 16.8 & 15.8 & 12.6 & 16.8 \\
\hline pH (ph unita) & 6.9 & 6.7 & 7.3 & 7.3 & 7.4 & 7.4 & 7.1 & 7.2 \\
\hline Sp. Cond. (umho/cm) & 506 & 552 & 580 & 500 & 526 & 543 & 542 & 516 \\
\hline Dissolved Oxygen (ppm) & 1.5 & 6.2 & 1.3 & 0.2 & 0.3 & 6.2 & 4.2 & 2.7 \\
\hline Oxidation/Reduction (mV) & -63 & -39 & -22 & -4 & -22 & -2 & -54 & 56 \\
\hline MISCELLANEOUS PARAMETERS & $\cdot$ & $\cdot$ & . & . & . & . & . & . \\
\hline pH (pH units) & 7 & 6.6 & 7.4 & 7.5 & 7.6 & 7.8 & 7.5 & 7.5 \\
\hline Sp. Cond. (umho/cm) & 460 & 498 & 590 & 568 & 568 & 592 & 595 & 613 \\
\hline TPH $(\mathrm{mg} / \mathrm{L})$ & $\cdot$ & . & . & - & - & • & • & • \\
\hline TDS $(\mathrm{mg} / \mathrm{L})$ & 296 & 300 & 348 & 338 & 366 & 362 & 406 & 388 \\
\hline TSS (mg/L) & 67 & 1187 & $<1$ & $<1$ & 1 & $<1$ & 2 & $<1$ \\
\hline Turbidity (NTU) & 1050 & 13 & 3.1 & 1.4 & 1.4 & 0.7 & 4.5 & 2.2 \\
\hline RADIOCHRMICAL PARAMETERS & & . & . & - & - & . & . & . \\
\hline Gross Alpha (pCi/L) & 6.74 & 75.20 & -0.19 & $-0.7 \dot{1}$ & -0.84 & $2.7 \dot{3}$ & -0.36 & -0.89 \\
\hline Gross Alphe $(\mathrm{CE}+/-)$ & 8.90 & 40.00 & 1.90 & 0.85 & 2.00 & 1.80 & 1.80 & 0.94 \\
\hline Gross Beta (PC1/L) & 24.70 & 204.00 & 4.93 & -1.31 & 2.09 & 3.17 & 4.49 & 5.01 \\
\hline Gross Beta $(\mathrm{Cz}+/-)$ & 11.00 & 64.00 & 3.00 & 1.40 & 2.50 & 2.80 & 2.90 & 1.30 \\
\hline
\end{tabular}

(CONT INUED) 
APPENDIX $\mathbf{Z} \cdot 3$

Groundwater Quality Data, 1993

\begin{tabular}{|c|c|c|c|c|c|c|c|c|}
\hline \multirow{3}{*}{$\begin{array}{l}\text { Sampling Point } \\
\text { - } \\
\text { Location } \\
\text { - } \\
\text { Date sampled }\end{array}$} & \multicolumn{2}{|c|}{$G W-208$} & \multicolumn{4}{|c|}{$G w-220$} & \multicolumn{2}{|c|}{ GW-232 } \\
\hline & \multicolumn{2}{|c|}{$\mathbf{E X P}$} & \multicolumn{4}{|c|}{ NHP } & \multicolumn{2}{|c|}{$\mathbf{E X P}$} \\
\hline & $08 / 13 / 93$ & $10 / 28 / 93$ & $01 / 25 / 93$ & $04 / 22 / 93$ & $08 / 06 / 93$ & $11 / 01 / 93$ & $01 / 26 / 93$ & $05 / 21 / 93$ \\
\hline FIELD MRASUREMENTS & - & & - & - & - & - & . & - \\
\hline Depth to Water $(t t)$ & 0 & 2.86 & 15.75 & $15 \cdot 7$ & 15.85 & 16.22 & 30.15 & 30.82 \\
\hline Water Tamp (degrees C) & 17.3 & 14 & 14.8 & 14.5 & 17.1 & 14.7 & 12 & 14 \\
\hline ph (pH units) & 7.2 & 6.9 & 7.6 & 7 & 6.7 & 7.4 & 9 & 8.1 \\
\hline Sp. Cond. (umho/cm) & 567 & 545 & 395 & 423 & 410 & 453 & 811 & 701 \\
\hline Dissolved Oxygen (ppm) & 0.2 & 0.8 & 1.6 & 2.9 & 1.4 & 0.9 & 1.3 & 0.7 \\
\hline Oxidetion/Reduction (mV) & 39 & 42 & 99 & 190 & 201 & 144 & -12 & 198 \\
\hline MISCRLIAYEOUS PARAYTIRRS & - & - & - & $\cdot$ & - & - & - & - \\
\hline ph (pH units) & 7.6 & 7.6 & 7.6 & 7.8 & 7.6 & 7.6 & 9.2 & 9.2 \\
\hline sp. Cond. (umho/cm) & 592 & 602 & 482 & 516 & 478 & 524 & 877 & 923 \\
\hline TPH $(m g / L)$ & - & $\cdot$ & - & $\cdot$ & - & - & - & • \\
\hline $\operatorname{TDS}\left(\mathrm{mg} / I_{n}\right)$ & 428 & 424 & 256 & 332 & 292 & 320 & 580 & 562 \\
\hline $\operatorname{Tss}(\mathrm{mg} / \mathrm{L})$ & 3 & 2 & 7 & 414 & 107 & 11 & 4 & 1 \\
\hline Turbldity (NTU) & 8.1 & 7 & 6 & 280 & 74 & 8.2 & 2.6 & $1 \cdot 2$ \\
\hline RADIOCHEMICAL PARAMETERS & - & - & . & $\cdot$ & - & - & $\cdot$ & - \\
\hline Grose Alpha (pCi/L) & -0.99 & 2.05 & 0.00 & 2.57 & 1.51 & 2.80 & -3.53 & $-1 \cdot 18$ \\
\hline Grose Alpha $(\mathrm{CE}+/-)$ & 1.30 & 1.80 & 1.90 & 5.60 & 3.70 & 2.30 & 2.60 & 3.20 \\
\hline Grose Beta (pCL/L) & -1.19 & 4.12 & -5.47 & 28.80 & 5.45 & 4.15 & -2.29 & 1.49 \\
\hline Gross Beta $(\mathrm{CR}+/-)$ & 3.10 & 2.90 & 3.40 & 6.30 & 5.30 & 2.80 & 2.90 & 3.10 \\
\hline
\end{tabular}

(CONTINUED) 
APPENDIX R.3

Groundwater Quality Data, 1993

\begin{tabular}{|c|c|c|c|c|c|c|c|c|}
\hline \multirow{3}{*}{$\begin{array}{l}\text { Sampling Point } \\
\text { Location } \\
\text { Date sampled }\end{array}$} & \multicolumn{2}{|c|}{ OW-232 } & \multicolumn{4}{|c|}{ Gw-239 } & \multicolumn{2}{|c|}{$O W-240$} \\
\hline & \multicolumn{2}{|c|}{$\mathbf{E x p}$} & \multicolumn{4}{|c|}{ MHP } & \multicolumn{2}{|c|}{ MHP } \\
\hline & $08 / 21 / 93$ & $11 / 01 / 93$ & $01 / 23 / 93$ & $05 / 13 / 93$ & $08 / 18 / 93$ & $10 / 30 / 93$ & $01 / 27 / 93$ & $04 / 21 / 93$ \\
\hline TIELD MEASUREMENTS & - & - & - & . & - & - & - & . \\
\hline Dopth to Water $(f t)$ & 31.4 & 31.61 & 28.95 & 29.62 & 30.43 & 30.71 & 20.3 & 20.65 \\
\hline Water Tump (degrees C) & 16.8 & 13 & 13.5 & 14.9 & 19.2 & 11.7 & 14.8 & 13.5 \\
\hline ph (ph unite) & 8.9 & 9 & 8.9 & 8.8 & 8.8 & 7.9 & 7.1 & 7.4 \\
\hline 8p. Cond. (umho/cm) & 814 & 746 & 1779 & 1367 & 1736 & 1721 & 705 & 451 \\
\hline DLusolved Oxygen (ppm) & 0.9 & 0.7 & 9 & 4.2 & 1.3 & 1.2 & 5.4 & 10.8 \\
\hline Oxidation/Reduction (mV) & 93 & 135 & -101 & 141 & 118 & 122 & 93 & 150 \\
\hline MISCELLANEOUS PARARTIRRS & . & • & . & . & . & . & . & . \\
\hline pH (pH unite) & $9 . \dot{3}$ & $9 . \dot{2}$ & 0.8 & $8 . \dot{9}$ & $8 . \dot{9}$ & $0 . \dot{9}$ & 7.5 & 7.6 \\
\hline 8p. Cond. (unho/cm) & 876 & 955 & 2920 & 1800 & 1800 & 1890 & 733 & 551 \\
\hline TPH $(\mathrm{mg} / \mathrm{L})$ & - & • & • & • & • & • & • & 。 \\
\hline $\operatorname{TDS}(\mathrm{mg} / \mathrm{L})$ & 576 & 584 & 1102 & 1114 & 1108 & 1120 & 396 & 30 \\
\hline Tss (mg/L) & 5 & 2 & 4 & 3 & $<1$ & 21 & $<1$ & $<1$ \\
\hline Turbidity (NTU) & 4.3 & 1.8 & 5.6 & 3.9 & 7.9 & 10 & 2.1 & 1.4 \\
\hline RADIOCHEMICAL PARAMETERS & . & • & . & . & . & • & . & . \\
\hline Gros: Alpha $(\mathrm{pCi} / L)$ & -2.53 & -0.21 & 3.11 & -1.61 & -10.80 & 0.00 & 1.32 & -0.3 \\
\hline Grose Alpha $(\mathrm{CB}+/-)$ & 3.70 & 2.00 & 3.30 & 7.00 & 10.00 & 5.10 & 2.30 & 2.00 \\
\hline Gross Beta $(\mathrm{PC} 1 / \mathrm{L})$ & -0.53 & -1.25 & 2.90 & 1.77 & 5.52 & 6.22 & 4.34 & 5.7 \\
\hline Orose Beta $(\mathrm{CE}+1-)$ & 2.80 & 2.80 & 3.00 & 6.10 & 11.00 & 5.80 & 3.10 & 2.80 \\
\hline
\end{tabular}

(CONTINUSD) 
APPENDIX $\mathbf{E}, 3$

Oroundwater Quality Data, 1993

\begin{tabular}{|c|c|c|c|c|c|c|c|c|}
\hline \multirow{3}{*}{$\begin{array}{l}\text { sampling Point } \\
\text { Location } \\
\text { Date sampled }\end{array}$} & \multicolumn{2}{|c|}{ ON-240 } & \multicolumn{4}{|c|}{ ov-251 } & \multicolumn{2}{|c|}{$G W-252$} \\
\hline & \multicolumn{2}{|c|}{ NHP } & \multicolumn{4}{|c|}{$\mathbf{s 2}$} & \multicolumn{2}{|c|}{$\mathbf{8 2}$} \\
\hline & $08 / 05 / 93$ & $11 / 01 / 93$ & $01 / 20 / 93$ & $05 / 05 / 93$ & $09 / 17 / 93$ & $12 / 08 / 93$ & $01 / 08 / 93$ & $05 / 03 / 93$ \\
\hline TIELD MASUREMETS & - & - & - & $\cdot$ & - & - & - & - \\
\hline Depth to Water $(t t)$ & 21.25 & 21.37 & 13.95 & 26.48 & 20.78 & 11.25 & 19 & 23.64 \\
\hline Water Temp (degrees $C$ ) & 16.8 & 15.1 & 13.9 & 16.6 & 16.3 & 14.6 & 12.3 & 16.8 \\
\hline pH (pH unito) & 6.5 & 7.5 & 6 & 6.2 & 6.4 & 6.6 & 6.6 & 6.9 \\
\hline sp. Cond, (umho/cm) & 471 & 581 & 976 & 961 & 744 & 683 & 136 & 246 \\
\hline Dlesolved Oxygen (ppm) & 4.2 & 4.3 & s & 0.6 & 0.5 & 0.5 & 12.3 & $\theta$ \\
\hline Oxidation/Raduction (mv) & 43 & 176 & 225 & 222 & 177 & 200 & 218 & 209 \\
\hline MISCELTMNEOUS PARARTTERS & $\dot{.}$ & $\bullet$ &. & $\dot{.}$ & $\cdot$ & . & $\cdot$ & • \\
\hline ph (pH unita) & $7 . \dot{6}$ & 7.6 & $6 . \dot{9}$ & $7 . \dot{2}$ & $6 . \dot{9}$ & $6 . \dot{7}$ & $7 . \dot{2}$ & $7 . \dot{3}$ \\
\hline sp. Cond, (umho/cm) & 536 & 665 & 1040 & 1080 & 792 & 706 & 125 & 134 \\
\hline TPH $(\mathrm{mg} / \mathrm{L})$ & $\cdot$ & $\cdot$ & $\cdot$ & $\cdot$ & $\cdot$ & - & $\cdot$ & • \\
\hline $\operatorname{TDS}(\mathrm{mg} / \mathrm{L})$ & 312 & 100 & 670 & 722 & 520 & 472 & 60 & 102 \\
\hline$T 88(m g / L)$ & s & 1 & 119 & 104 & 434 & 150 & 4 & 8 \\
\hline Turbldity (nTU) & 1.7 & 1.8 & 120 & 105 & 220 & 81 & 12 & 8.5 \\
\hline RADIOCHEICAL PARAMETERS & . & • & $\cdot$ & $\cdot$ & . & - & - & - \\
\hline Grose Alpha $(\mathrm{pC} 1 / L)$ & 3.36 & 3.94 & 19.20 & 5.94 & 22.20 & 9.11 & 0.00 & 5.58 \\
\hline Grose Alpha $(\mathrm{CX}+1-)$ & 4.00 & 2.60 & 6.00 & 2.40 & 4.30 & 2.80 & 1.60 & 1.40 \\
\hline Grose Beta $(\mathrm{PC} i / L)$ & 5.13 & 7.15 & 19.40 & 12.60 & 11.50 & 4.19 & -1.10 & -0.34 \\
\hline Grose Beta $(\mathrm{Cz}+/-1$ & 5.30 & 3.00 & 6.50 & 2.70 & 3.30 & 2.70 & 2.60 & 1.40 \\
\hline
\end{tabular}

(CONTINURD) 
APPENDIX 2.3

Groundwater Quality Data, 1993

\begin{tabular}{|c|c|c|c|c|c|c|c|c|}
\hline \multirow{3}{*}{$\begin{array}{l}\text { Sampling Point } \\
\text { Location } \\
\text { Date sampled }\end{array}$} & \multicolumn{2}{|c|}{ GW-2s2 } & \multicolumn{4}{|c|}{ ON-25s } & \multicolumn{2}{|c|}{$O W-261$} \\
\hline & \multicolumn{2}{|c|}{82} & \multicolumn{4}{|c|}{82} & \multicolumn{2}{|c|}{$\mathbf{8 y}$} \\
\hline & $|09 / 15 / 93|$ & $12 / 07 / 93$ & $01 / 08 / 93$ & $05 / 03 / 93$ & $09 / 15 / 93$ & $12 / 07 / 93$ & $|01 / 08 / 93|$ & $04 / 07 / 93$ \\
\hline TIELD MasuREMATS & - & - & - & $\cdot$ & . & - & - & . \\
\hline Dopth to Water $(f t)$ & 32.65 & 14.3 & 21.7 & 26.4 & 36.97 & 17.36 & 17.5 & 27.5 \\
\hline Water Tenp (degrens C) & 22.7 & 13.3 & 14 & 14.9 & 23.6 & 14.2 & 13 & 17.9 \\
\hline pH (PH unite) & 6.1 & 7 & 7.3 & 6.9 & 7 & 7.3 & 0.1 & 7 \\
\hline 8p. Cond. (umho/cm) & 345 & 141 & 448 & 391 & 478 & 174 & 339 & 300 \\
\hline DLeselved Oxygen (ppm) & 7.8 & 9.4 & 7.5 & 4.3 & 4.4 & 4.4 & 6.3 & 6.7 \\
\hline Oxidation/Raduction (mV) & 238 & 102 & 220 & 226 & 271 & 138 & 143 & 189 \\
\hline MISCELLANROUS PARAETERS & - & . & . & . & . & . & • & • \\
\hline pH (pH unite) & 7.2 & i & 7.7 & $7 . \dot{6}$ & 7.4 & 7.5 & $7 . \dot{9}$ & 7.3 \\
\hline 8p. Cond. (umho/cm) & 396 & 172 & 508 & 503 & 490 & 488 & 379 & 358 \\
\hline TPH $(m g / 2)$ & $\cdot$ & - & . & . & . & . & • & • \\
\hline TD8 (mg/L) & 264 & 150 & 300 & 310 & 352 & 360 & 230 & 220 \\
\hline TS8 (mg/L) & 29 & 5 & 57 & 95 & 187 & 1260 & $<1$ & 2 \\
\hline Turbidity (Nru) & 250 & 11 & 44 & 113 & 140 & 560 & 15 & 20 \\
\hline RADIOCHEMICAL PARALTIZRS & . & . & - & - & - & - & . & • \\
\hline Grose Npha $(\mathrm{PCl} / \mathrm{L})$ & 0.63 & -0.31 & 0.58 & 4.72 & $3.99^{\circ}$ & 2.60 & -0.84 & -0.31 \\
\hline Grose Alpha $(\mathrm{Cz}+/-)$ & 1.70 & 0.60 & 2.20 & 2.10 & 2.20 & 2.10 & 1.70 & 3.60 \\
\hline Orose Beta $(\mathrm{pCl} / \mathrm{L})$ & 2.41 & 0.08 & 3.46 & 0.46 & 3.54 & 6.91 & 1.22 & 7.12 \\
\hline Grose Beta $(C 2+1-1$ & 2.80 & 2.40 & 3.00 & 2.90 & 2.80 & 2.90 & 2.80 & 5.10 \\
\hline
\end{tabular}

(CONTINORD) 
APPENDIX 8.3

Groundwater Quadity Data, 1993

\begin{tabular}{|c|c|c|c|c|c|c|c|c|}
\hline \multirow{3}{*}{$\begin{array}{l}\text { Sampling Point } \\
\text { Lootion } \\
\text { Date sumpled }\end{array}$} & \multicolumn{2}{|c|}{$O W-261$} & \multicolumn{4}{|c|}{$G W-262$} & \multicolumn{2}{|c|}{ GW-263 } \\
\hline & \multicolumn{2}{|c|}{$\mathbf{s y}$} & \multicolumn{4}{|c|}{ sy } & \multicolumn{2}{|c|}{ 8Y } \\
\hline & $|07 / 01 / 93|$ & $12 / 07 / 93$ & $01 / 11 / 93$ & $04 / 08 / 93$ & $07 / 01 / 93$ & $12 / 07 / 93$ & $|01 / 11 / 93|$ & $04 / 08 / 93$ \\
\hline FIILD masurarants & - & - & - & - & - & - & - & • \\
\hline Depth to Water (ft) & 19.2 & 17.7 & 17.38 & 17.2 & 18.53 & 17.65 & 26.5 & 24.78 \\
\hline Water Tanp (degreas C) & 20.1 & 14.7 & 12.6 & 16.8 & 28.7 & 14.1 & 15.5 & 28.4 \\
\hline pH (pH unIte) & 6.8 & 7.1 & 7.6 & 7.6 & 7.6 & 7.9 & 7.3 & 7.3 \\
\hline 8p. Cond. (umho/cm) & 363 & 350 & 383 & 362 & 354 & 375 & 539 & 455 \\
\hline Dissolvad oxygen (ppm) & 5.7 & 3.3 & 3.1 & 5.7 & 5.7 & 6.2 & 6.6 & 7.4 \\
\hline Oxidation/Reduction (mV) & 186 & 220 & 138 & 69 & 146 & 99 & 173 & 197 \\
\hline MISCELLAREOUS PARNETERS & . & . & . & . & . & . & $\cdot$ & . \\
\hline pH (pH unite) & $7 . \dot{2}$ & 7.2 & $8 . \dot{3}$ & 7.7 & $8 . \dot{2}$ & 7.9 & $\dot{8}$ & 7.6 \\
\hline sp. Cond, (umho/cm) & 364 & 365 & 399 & 405 & 395 & 391 & 590 & 532 \\
\hline TPH $(\mathrm{mg} / \mathrm{L})$ & $\cdot$ & - & • & - & • & • & $\cdot$ & • \\
\hline $\operatorname{TDS}(m g / L)$ & 224 & 272 & 252 & 262 & 272 & 296 & 332 & 314 \\
\hline Ts8 (mg/L) & 8 & 1 & 3 & 8 & 48 & 19 & 152 & 7 \\
\hline Turbldity (WTO) & 5.5 & 3.8 & 1.9 & 3.5 & 29 & 18 & 230 & 6 \\
\hline RADTOCIINICAL PARAMETERS & . & . & • & . & . & . & . & . \\
\hline Orose Alpha $(\mathrm{pCl} / \mathrm{L})$ & -2.96 & -0.71 & 2.99 & 1.17 & -2.17 & 1.62 & 0.00 & 0.00 \\
\hline Grose Npha $(\mathrm{Cr}+1-)$ & 3.50 & 1.30 & 2.50 & 2.10 & 3.80 & 1.90 & 3.00 & 2.00 \\
\hline Grose Beta $(\mathrm{pCl} / \mathrm{L})$ & -2.47 & 2.45 & 2.38 & 13.90 & -3.15 & 4.64 & 13.90 & 39.10 \\
\hline Orose Bota $(\mathrm{Cz}+1-)$ & 5.50 & 2.60 & 2.90 & 3.40 & 5.50 & 2.70 & 3.50 & 4.50 \\
\hline
\end{tabular}

(CONTIMUED) 
APPENDIX E. 3

Groundwater Quality Data, 1993

\begin{tabular}{|c|c|c|c|c|c|c|c|c|}
\hline \multirow{3}{*}{$\begin{array}{l}\text { Sampling polnt } \\
\text { L } \\
\text { Location } \\
\text { Date sampled }\end{array}$} & \multicolumn{2}{|c|}{$G W-263$} & \multicolumn{4}{|c|}{$G W-264$} & \multicolumn{2}{|c|}{$G W-281$} \\
\hline & \multicolumn{2}{|c|}{$\mathbf{S Y}$} & \multicolumn{4}{|c|}{ sy } & \multicolumn{2}{|c|}{$\mathbf{F F}$} \\
\hline & $07 / 01 / 93$ & $12 / 07 / 93$ & $01 / 12 / 93$ & $04 / 12 / 93$ & $07 / 02 / 93$ & $12 / 08 / 93$ & $02 / 02 / 93$ & $04 / 16 / 93$ \\
\hline FIELD MEASUREMEHTS & - & - & • & . & - & . & . & • \\
\hline Depth to Water $(t t)$ & 31.1 & 29.97 & 27.18 & 25.78 & 31.85 & 29.91 & 6.22 & 5.12 \\
\hline Water Temp (degrees C) & 24.5 & 13 & 13.6 & 18.8 & 21.1 & 16.5 & 12.1 & 11.1 \\
\hline pH (pH units) & 7.2 & 7.6 & 7.1 & 7.1 & 6.9 & 7.3 & 6.9 & 7.1 \\
\hline Sp. Cond. (umho/cm) & 447 & 538 & 996 & 913 & 895 & 1049 & 346 & 301 \\
\hline Dissolved Oxygen (ppm) & 5.3 & 5.9 & 1.2 & 3.8 & 7.4 & 7.5 & 5.4 & 1.3 \\
\hline Oxidation/Reduction (mV) & 162 & 219 & -162 & -66 & -100 & 42 & 174 & 138 \\
\hline MISCEILAMEOUS PARAMETERS & - & - & • & . & - & . & . & . \\
\hline pH (pH units) & 7.9 & 7.6 & 7.5 & 7.7 & 7.4 & 7.4 & 7.2 & 7.4 \\
\hline Sp. Cond. (umho/cm) & 461 & 559 & 1050 & 945 & 968 & 1080 & 402 & 313 \\
\hline $\operatorname{TPH}(m g / L)$ & - & - & • & • & • & • & 0.0125 & 0.012 \\
\hline TDS (mg/L) & 286 & 394 & 658 & 624 & 658 & 740 & 246 & 214 \\
\hline Tss (mg/L) & 158 & 7 & 2 & 22 & 8 & 34 & 59 & 129 \\
\hline Turbidity (NIO) & 110 & 20 & 6.5 & 1 & 22 & 160 & 23 & 190 \\
\hline RADIOCEMYCAL PARAMETERS & . & . & . & . & . & . & . & . \\
\hline Grose Alpha $(\mathrm{pC} i / \mathrm{L})$ & -4.25 & -0.01 & -1.03 & -2.18 & -0.39 & 1.35 & 1.74 & 6.20 \\
\hline Grose Alpha $(\mathrm{CE}+/-)$ & 3.30 & 1.60 & 3.60 & 3.10 & 2.80 & 1.60 & 2.10 & 2.00 \\
\hline Grose Beta $(\mathrm{pC} 1 / \mathrm{L})$ & -3.47 & 3.22 & 2.26 & 3.54 & -4.87 & 1.80 & 2.70 & 9.88 \\
\hline Gross Beta (Cs+/-) & 5.50 & 2.60 & 3.10 & 3.00 & 5.50 & 2.50 & 3.00 & 2.70 \\
\hline
\end{tabular}

(CONTINURD) 


\begin{tabular}{|c|c|c|c|c|c|c|c|c|}
\hline \multirow{3}{*}{$\begin{array}{l}\text { Sampling Point } \\
\text { Location } \\
\text { Date sampled }\end{array}$} & \multicolumn{2}{|c|}{$G W-281$} & \multicolumn{4}{|c|}{$G W-283$} & \multicolumn{2}{|c|}{ GW-284 } \\
\hline & \multicolumn{2}{|c|}{$\mathbf{F}$} & \multicolumn{4}{|c|}{$\mathbf{r r}$} & \multicolumn{2}{|c|}{$\mathbf{F}$} \\
\hline & $08 / 04 / 93$ & $10 / 24 / 93$ & $02 / 03 / 93$ & $04 / 19 / 93$ & $08 / 06 / 93$ & $10 / 14 / 93$ & $02 / 02 / 93$ & $04 / 16 / 93$ \\
\hline FIRLD MEASUREMENTS & - & - & - & - & - & • & - & \\
\hline Depth to Water (ft) & 9.2 & 7.25 & 6.15 & 5.8 & 6.73 & 6.85 & 8.73 & 8.45 \\
\hline Water Temp (degreen C) & 20.1 & 16 & 15.8 & 16.8 & 18.3 & 19.2 & 14.4 & 14.3 \\
\hline pH (pH units) & 6.6 & 6.5 & 6.7 & 6.6 & 6.3 & 6.6 & 7 & 6.6 \\
\hline Sp. Cond. (unho/cm) & 396 & 325 & 992 & 816 & 864 & 828 & 1115 & 1198 \\
\hline Dlesolved Oxygen (ppm) & 2.1 & 1.7 & 4 & 2.2 & 1.5 & 1.2 & 6.2 & 1 \\
\hline Oxidation/Reduction (mv) & 126 & 197 & 85 & 74 & 62 & -15 & 114 & -8 \\
\hline MISCRLIANEOUS PARAMETERS & $\dot{\bullet}$ & $\dot{\bullet}$ & $\dot{\bullet}$ & $\dot{\bullet}$ & $\cdot$ & . & • & • \\
\hline pH (pH unite) & $\dot{7}$ & $\dot{7}$ & 6.8 & $\dot{7}$ & 6.7 & 6.7 & 7.1 & $\dot{7}$ \\
\hline sp. Cond. (umho/cm) & 420 & 357 & 920 & 950 & 1020 & 987 & 1310 & 1430 \\
\hline TPH $(m g / L)$ & 0.017 & $<0.1$ & 0.0091 & $<0.1$ & $<0.1$ & $<0.1$ & $<0.1$ & $<0.1$ \\
\hline TDS $(\mathrm{mg} / \mathrm{L})$ & 280 & 230 & 630 & 650 & 692 & 680 & 788 & 908 \\
\hline T8s (mg/L) & 120 & 21 & 1077 & 348 & 32 & 50 & 18 & 22 \\
\hline Turbidity (NTO) & 40 & 24 & 300 & 37 & 15 & 25 & 8.6 & 15 \\
\hline RADIOCHEMICAC PARAMETERS & $\cdot$ & . & $\dot{\bullet}$ & $\dot{\bullet}$ & $\cdot$ & • & • & $\bullet$ \\
\hline Gross Mlpha (PCi/L) & 1.75 & 3.11 & 1.13 & 6.65 & -1.01 & 0.37 & 3.43 & 1.37 \\
\hline Gross Alpha $(\mathrm{CE}+/-)$ & 9.30 & 2.00 & 2.00 & 2.00 & 3.60 & 1.80 & 4.20 & 0.86 \\
\hline Gross Beta $(\mathrm{PCl} / \mathrm{L})$ & 5.12 & 4.89 & 3.74 & 3.97 & 4.20 & 4.16 & 4.15 & 2.98 \\
\hline Gross Beta $(\mathrm{CE}+/-)$ & 12.00 & 2.80 & 4.30 & 1.50 & 5.30 & 2.80 & 3.20 & 1.30 \\
\hline
\end{tabular}

(CONTINOED) 
APPENDIX E. 3

Groundwater Quality Data, 1993

\begin{tabular}{|c|c|c|c|c|c|c|c|c|}
\hline \multirow{3}{*}{$\begin{array}{l}\text { Sampling Point } \\
\text { Location } \\
\text { - } \\
\text { Date Sampled }\end{array}$} & \multicolumn{2}{|c|}{ GW-284 } & \multicolumn{4}{|c|}{$G W-285$} & \multicolumn{2}{|c|}{ GW-337 } \\
\hline & \multicolumn{2}{|c|}{ FF } & \multicolumn{4}{|c|}{ FF } & \multicolumn{2}{|c|}{ WC } \\
\hline & $08 / 05 / 93$ & $10 / 14 / 93$ & $02 / 02 / 93$ & $04 / 19 / 93$ & $08 / 05 / 93$ & $10 / 14 / 93$ & $01 / 21 / 93$ & $05 / 07 / 93$ \\
\hline FIELD MEASUREMENTS & • & $\cdot$ & $\cdot$ & • & $\cdot$ & - & $\cdot$ & - \\
\hline Depth to Water ( $f t$ ) & 10.34 & 9.75 & 9.61 & 9.4 & 10.7 & 10.55 & 10.8 & 10.7 \\
\hline Water Temp (degrees C) & $21 \cdot 5$ & 18.6 & 14.3 & 16 & 21.1 & 18.5 & 16.2 & 22.9 \\
\hline pH (pH units) & 6.5 & 6.7 & 6.9 & 6.8 & 6.7 & 6.6 & $6 \cdot 3$ & 6.8 \\
\hline Sp. Cond. (umho/cm) & 1208 & 1280 & 439 & 393 & 432 & 450 & 484 & 504 \\
\hline Dissolved Oxygen (ppm) & 2.2 & 1.3 & 6.3 & 3.6 & $3 \cdot 3$ & 3.1 & 6 & 2.2 \\
\hline Oxidation/Reduction (mV) & 122 & -11 & 182 & 162 & 96 & 20 & 236 & 208 \\
\hline MISCBLLANEOUS PARAMETERS & • & - & . & . & - & $\cdot$ & • & - \\
\hline pH (pH units) & 6.9 & 6.8 & 7.2 & 7.3 & $\dot{7}$ & 6.8 & $7 . \dot{1}$ & 7.3 \\
\hline Sp. Cond. (umho/cm) & 1340 & 1320 & 478 & 488 & 453 & 453 & 545 & 538 \\
\hline TPH (mg/L) & $<0.1$ & $<0.1$ & 0.0075 & $<0.1$ & 0.015 & $<0.1$ & $\cdot$ & • \\
\hline TDS $(m g / I)$ & 1048 & 856 & 304 & 330 & 284 & 300 & 320 & 332 \\
\hline TSS $(m g / L)$ & 48 & 20 & 15 & 201 & 26 & 6 & 26 & 11 \\
\hline Turbidity (NTU) & 25 & 15 & 25 & 280 & 18 & 6.1 & 14 & 11 \\
\hline RADIOCERMICAI PARAYETERS & - & - & - & . & $\cdot$ & - & $\cdot$ & - \\
\hline Gross Alpha (pCi/I) & 3.81 & 3.20 & 3.89 & 3.02 & 0.88 & 3.85 & 13.50 & -0.17 \\
\hline Gross Alpha $(\mathrm{CE}+/-)$ & 5.80 & 2.50 & 2.60 & 1.30 & 4.70 & 2.20 & 5.20 & 0.76 \\
\hline Gross Beta (pci/I) & 2.27 & 2.66 & 7.05 & 2.11 & -0.34 & 4.50 & 19.30 & 1.49 \\
\hline Gross Beta $(\mathrm{CE}+/-)$ & 5.90 & 2.80 & 3.20 & 1.40 & 5.70 & 2.80 & 6.50 & 1.20 \\
\hline
\end{tabular}

(CONTINUED) 
APPENDIX E.3

Groundwater Quality Data, 1993

\begin{tabular}{|c|c|c|c|c|c|c|c|c|}
\hline \multirow{3}{*}{$\begin{array}{l}\text { Sampling Point } \\
\text { Iocation } \\
\text { Date Sampled }\end{array}$} & \multicolumn{2}{|c|}{ GW-337 } & \multicolumn{4}{|c|}{$G W-338$} & \multicolumn{2}{|c|}{$G W-380$} \\
\hline & \multicolumn{2}{|c|}{ WC } & \multicolumn{4}{|c|}{ WC } & \multicolumn{2}{|c|}{ NHP } \\
\hline & $09 / 17 / 93$ & $12 / 09 / 93$ & $01 / 11 / 93$ & $05 / 04 / 93$ & $09 / 23 / 93$ & $12 / 07 / 93$ & $01 / 25 / 93$ & $04 / 21 / 93$ \\
\hline FIELD MEASUREMENTS & . & . & . & . & . & - & . & . \\
\hline Depth to Water (ft) & 10.17 & 10.54 & 8.55 & 8.82 & 8.85 & 8.24 & 9.4 & 9.52 \\
\hline Water Temp (degrees C) & 20.7 & 17.4 & 13.1 & 20.1 & 21 & 15.5 & 12.5 & 12.5 \\
\hline pH (pH units) & 6.6 & 6.6 & 6.6 & 7.1 & 6.9 & 7 & 6.9 & 6.9 \\
\hline Sp. Cond. (umho/cm) & 514 & 484 & 460 & 422 & 491 & 450 & 940 & 632 \\
\hline Dissolved Oxygen (ppm) & 1.1 & 1.4 & 6.5 & 3 & 3.3 & 3 & 5.2 & 4.8 \\
\hline Oxidation/Reduction (mV) & 184 & 176 & 203 & 183 & 195 & 161 & 97 & 109 \\
\hline MISCELLANEOUS PARAMETERS & . & - & $\cdot$ & - & - & $\cdot$ & • & • \\
\hline pH (pH units) & $7 . \dot{5}$ & $6 . \dot{9}$ & $7 . \dot{7}$ & $7 . \dot{2}$ & $7 . \dot{4}$ & 7.4 & $\dot{7}$ & 7.4 \\
\hline Sp. Cond. (umho/cm) & 542 & 535 & 394 & 478 & 501 & 488 & 1160 & 809 \\
\hline TPH $(m g / L)$ & $\cdot$ & . & $\cdot$ & • & - & • & • & • \\
\hline TDS $(m g / I)$ & 336 & 350 & 300 & 304 & 312 & 368 & 590 & 452 \\
\hline TSS $(m g / I)$ & 578 & 6 & 3 & 5 & 7 & 21 & 15 & 10 \\
\hline Turbidity (NTU) & 335 & 3.8 & 4.4 & 8 & 110 & 4.1 & 16 & 20 \\
\hline RADIOCHEMICAL PARAMETRRS & . & • & $\cdot$ & - & - & - & • & • \\
\hline Gross Alpha $(\mathrm{pCi} / \mathrm{L})$ & -0.16 & 1.28 & -0.21 & -0.55 & -1.18 & -0.56 & 1.79 & -0.25 \\
\hline Gross Alpho $(\mathrm{CB}+/-)$ & 1.50 & 1.50 & 2.20 & 1.00 & 3.40 & 1.40 & 3.70 & 3.00 \\
\hline Gross Beta $(\mathrm{pCi} / \mathrm{L})$ & 1.21 & 2.57 & 0.54 & -1.87 & 0.51 & 1.88 & -6.25 & 3.01 \\
\hline Gross Beta $(\mathrm{CE}+/-)$ & 2.70 & 2.50 & 2.80 & 1.50 & 5.30 & 2.50 & 3.60 & 2.80 \\
\hline
\end{tabular}

(CONTINUED) 
APPENDIX E. 3

Groundwater Quality Data, 1993

\begin{tabular}{|c|c|c|c|c|c|c|c|c|}
\hline \multirow{3}{*}{$\begin{array}{l}\text { Sampling Point } \\
\text { - } \\
\text { Location } \\
\text { - } \\
\text { Date Sampled }\end{array}$} & \multicolumn{2}{|c|}{ GW-380 } & \multicolumn{4}{|c|}{ GW-381 } & \multicolumn{2}{|c|}{$G W-382$} \\
\hline & \multicolumn{2}{|c|}{ NHP } & \multicolumn{4}{|c|}{ NHP } & \multicolumn{2}{|c|}{ NHP } \\
\hline & $08 / 05 / 93$ & $10 / 29 / 93$ & $01 / 27 / 93$ & $04 / 26 / 93$ & $08 / 08 / 93$ & $11 / 08 / 93$ & $01 / 28 / 93$ & $04 / 26 / 93$ \\
\hline FIELD MRASUREMENTS & $\cdot$ & & - & $\cdot$ & - & - & $\cdot$ & - \\
\hline Depth to Water $(f t)$ & 11.52 & 11.82 & 10 & 10.04 & 11.32 & 11.5 & 10.85 & 10.85 \\
\hline Water Temp (degrees $C$ ) & 22.7 & 17 & 15.1 & 17.3 & 18.5 & 16 & $15 \cdot 3$ & 15.8 \\
\hline pH (pH units) & 5.6 & 6.7 & 7.2 & 7.6 & 7.5 & 7.2 & 7.6 & 7.4 \\
\hline Sp. Cond. (umho/cm) & 560 & 578 & 636 & 609 & 646 & 614 & 445 & 425 \\
\hline Dissolved Oxygen (ppm) & 2.2 & 6.9 & 1.4 & 3 & 1 & 0.9 & 0.9 & 2.8 \\
\hline Oxidation/Reduction (mv) & 30 & 65 & -26 & 58 & -93 & -64 & 71 & 35 \\
\hline MISCBLTANEOUS PARAMETERS & • & • & • & • & • & • & $\cdot$ & - \\
\hline pH (pH units) & 6.7 & 6.8 & 7.4 & 7.4 & 7.8 & 7.6 & 8 & 7.5 \\
\hline 5p. Cond. (umho/cm) & 640 & 661 & 691 & 654 & 667 & 656 & 488 & 475 \\
\hline TPH $(\mathrm{mg} / \mathrm{L})$ & • & $\cdot$ & • & • & $\bullet$ & - & $\cdot$ & - \\
\hline TDS (mg/I) & 378 & 388 & 390 & 392 & 398 & 420 & 272 & 284 \\
\hline TSS $(m g / I)$ & 124 & 11 & 10 & 9 & 4 & 4 & 1 & 3 \\
\hline Turbidity (NTU) & 21 & 50 & 21 & 23 & 5.5 & 4.6 & 5.4 & 13 \\
\hline RADIOCFEMICAI PARAMETERS & - & - & - & - & - & - & $\cdot$ & - \\
\hline Gross Alpha $(\mathrm{pCi} / \mathrm{I})$ & -3.32 & 0.51 & 1.32 & 1.17 & 1.52 & $1.80^{\circ}$ & 0.87 & 0.31 \\
\hline Gross Alpha $(\mathrm{CE}+/-1$ & 2.80 & 2.10 & 2.30 & 2.20 & 3.50 & 1.90 & 1.80 & 1.90 \\
\hline Gross Beta (pci/L) & 2.31 & 5.13 & 3.56 & 4.00 & 3.47 & 0.00 & 3.12 & 2.06 \\
\hline Gross Beta $(\mathrm{CB}+/-)$ & 5.10 & 2.90 & 3.00 & 2.70 & 5.50 & 2.80 & 2.90 & 2.60 \\
\hline
\end{tabular}

(CONTINUED) 
APPENDIX E. 3

Groundwater Quality Data, 1993

\begin{tabular}{|c|c|c|c|c|c|c|c|c|}
\hline \multirow{3}{*}{$\begin{array}{l}\text { Sampling Point } \\
\text { - } \\
\text { Location } \\
\text { - } \\
\text { Date Sampled }\end{array}$} & \multicolumn{2}{|c|}{$G W-382$} & \multicolumn{4}{|c|}{ GW-383 } & \multicolumn{2}{|c|}{ GW-384 } \\
\hline & \multicolumn{2}{|c|}{ NHP } & \multicolumn{4}{|c|}{ NHP } & \multicolumn{2}{|c|}{ NHP } \\
\hline & $08 / 08 / 93$ & $11 / 04 / 93$ & $01 / 26 / 93$ & $04 / 22 / 93$ & $08 / 07 / 93$ & $11 / 03 / 93$ & $01 / 13 / 93$ & $04 / 20 / 93$ \\
\hline FIELD MEASURBMENTS & • & - & $\cdot$ & - & - & - & - & - \\
\hline Depth to water (ft) & 11.4 & 11.6 & 9.5 & 9.59 & 9.62 & 9.68 & 12.5 & 10.94 \\
\hline Water Temp (degrees C) & 17 & 14.2 & 14.9 & 15.2 & 17 & 17 & 15.8 & 16.6 \\
\hline $\mathrm{pH}$ (pH units) & 7.9 & 7.5 & 7.3 & 7 & 7.2 & 7.1 & 7.2 & 6.9 \\
\hline Sp. Cond. (umho/cm) & 481 & 470 & 489 & 444 & 573 & 514 & 544 & 623 \\
\hline Dissolved Oxygen (ppm) & 1.1 & 1.2 & 1.2 & 2.3 & 0.6 & 0.6 & 2.5 & 1.9 \\
\hline Oxidation/Reduction (mv) & -57 & -92 & 32 & 64 & -105 & -51 & 24 & 47 \\
\hline MISCELLANEOUS PARAMETERS & - & - & - & - & - & - & - & • \\
\hline pH (pH units) & 7.8 & 7.7 & 7.5 & 7.8 & 7.8 & 7.5 & 7.2 & 7.3 \\
\hline Sp. Cond. (umho/cm) & 482 & 517 & 572 & 542 & 521 & 550 & 620 & 637 \\
\hline TPH (mg/I) & • & • & • & • & $\cdot$ & - & - & - \\
\hline TDS (mg/L) & 288 & 298 & 334 & 320 & 324 & 354 & 370 & 380 \\
\hline TSS (mg/L) & 2 & $<1$ & $<1$ & 2 & 2 & 3 & 2 & 4 \\
\hline Turbidity (NTU) & 2.5 & 1.4 & 0.8 & 1.8 & 10 & 12 & 5 & 1.8 \\
\hline RADIOCHEMICAL PARAMETERS & - & - & - & - & - & - & - & - \\
\hline Gross Alpha (pci/L) & 0.30 & 0.46 & 0.88 & -0.65 & -1.52 & 0.16 & 0.00 & 1.96 \\
\hline Gross Alpha $(\mathrm{CB}+/-)$ & 3.20 & 1.60 & 2.10 & 1.80 & 2.90 & 1.60 & 2.00 & 1.40 \\
\hline Gross Beta (pci/L) & -0.33 & -2.58 & -8.53 & 4.85 & -2.48 & 3.54 & 2.40 & 2.88 \\
\hline Gross Beta $(\mathrm{CE}+/-)$ & 5.20 & 2.60 & 3.30 & 2.80 & 5.10 & 3.00 & 2.80 & 1.40 \\
\hline
\end{tabular}

(CONTINUED) 
APPENDIX E. 3

Groundwater Quality Data, 1993

\begin{tabular}{|c|c|c|c|c|c|c|c|c|}
\hline \multirow{3}{*}{$\begin{array}{l}\text { Sampling Point } \\
\text { Location } \\
\text { - } \\
\text { Date Sampled }\end{array}$} & \multicolumn{2}{|c|}{ GW-384 } & \multicolumn{4}{|c|}{$G W-385$} & \multicolumn{2}{|c|}{$G W-505$} \\
\hline & \multicolumn{2}{|c|}{ NHP } & \multicolumn{4}{|c|}{ NHP } & \multicolumn{2}{|c|}{ RG } \\
\hline & $08 / 04 / 93$ & $10 / 28 / 93$ & $01 / 12 / 93$ & $04 / 19 / 93$ & $08 / 03 / 93$ & $10 / 27 / 93$ & $03 / 01 / 93$ & $06 / 18 / 93$ \\
\hline FIELD MEASUREMENTS & - & - & - & - & - & - & - & - \\
\hline Depth to Water $(f t)$ & 12.85 & 12.98 & 3 & 2.5 & 4.9 & 4.7 & 4.62 & 3.36 \\
\hline Water Temp (degrees $C$ ) & 18.7 & 15.7 & 14.8 & 16.5 & 17.6 & 16 & 12.6 & 19.6 \\
\hline pH (pH units) & 6.8 & 7 & 9.1 & 9 & 8.7 & 9.1 & 5.7 & 5.3 \\
\hline Sp. Cond. (umho/cm) & 581 & 543 & 675 & 619 & 663 & 655 & 234 & 183 \\
\hline Dissolved Oxygen (ppm) & 0.4 & 0.2 & 8.5 & 5.2 & 7.2 & 6.9 & 4.9 & 6.3 \\
\hline Oxidation/Reduction (mV) & -54 & -96 & 112 & 148 & 152 & 56 & 251 & 254 \\
\hline MISCELLANEOUS PARAMETRRS & . & . & . & . & . & . & . & • \\
\hline pH (pH units) & 7.3 & 7.4 & 9.1 & 9 & 9.2 & 9.1 & 6 & 5.9 \\
\hline Sp. Cond. (umho/cm) & 611 & 616 & 760 & 702 & 736 & 743 & 247 & 213 \\
\hline TPH $(\mathrm{mg} / \mathrm{L})$ & • & • & • & • & • & . & 0.0166 & • \\
\hline $\operatorname{TDS}(\mathrm{mg} / \mathrm{L})$ & 382 & 390 & 452 & 466 & 474 & 476 & 186 & 142 \\
\hline TSS $(\mathrm{mg} / \mathrm{L})$ & 3 & 2 & 3 & 5 & 20 & 3 & 87 & 133 \\
\hline Turbidity (NTU) & 2.5 & 3.9 & 5 & 17 & 9.8 & 3 & 50 & 16 \\
\hline RADIOCHEMICAL PARAMETERS & . & . & . & . & . & . & . & . \\
\hline Gross Alpha (pCi/L) & -4.58 & 2.60 & $0.2 \dot{2}$ & 0.74 & -4.90 & 1.94 & 16.40 & $16.20^{\circ}$ \\
\hline Gross Alpha $(\mathrm{CE}+/-)$ & 4.10 & 2.30 & 2.40 & 1.10 & 4.70 & 2.00 & 3.50 & 9.00 \\
\hline Gross Beta $(\mathrm{pCi} / \mathrm{L})$ & -1.55 & 6.84 & 1.04 & -0.17 & -4.18 & 2.56 & 12.30 & 9.84 \\
\hline Gross Beta $(\mathrm{CE}+/-)$ & 5.60 & 3.00 & 2.90 & 1.30 & 6.10 & 2.80 & 3.70 & 11.00 \\
\hline
\end{tabular}

(CONTINUED) 
APPENDIX E.3

Groundwater Quality Data, 1993

\begin{tabular}{|c|c|c|c|c|c|c|c|c|}
\hline \multirow{3}{*}{$\begin{array}{l}\text { Sampling Point } \\
\text { - } \\
\text { Location } \\
\text { Date Sampled }\end{array}$} & \multicolumn{3}{|c|}{ GW-505 } & \multicolumn{5}{|c|}{$G W-508$} \\
\hline & \multicolumn{3}{|c|}{ RG } & \multicolumn{5}{|c|}{ RG } \\
\hline & $07 / 07 / 93$ & $09 / 15 / 93$ & $11 / 16 / 93$ & $03 / 05 / 93$ & $06 / 23 / 93$ & $06 / 29 / 93$ & $09 / 21 / 93$ & $09 / 22 / 93$ \\
\hline FIELD MRASUREMENTS & - & - & - & - & - & - & - & - \\
\hline Depth to Water (ft) & 5.1 & 6.15 & 4.9 & 10.83 & 11.83 & 12.6 & 11.35 & $11 \cdot 35$ \\
\hline Water Temp (degrees C) & 24.5 & $15 \cdot 3$ & $18 \cdot 3$ & 14.3 & $18 \cdot 5$ & 18.4 & 20.8 & 20.9 \\
\hline pH (pH units) & 5.3 & 5.2 & 5.7 & 5.4 & 4.7 & 5 & 5.4 & 4.8 \\
\hline Sp. Cond. (umho/cm) & 198 & 237 & 305 & 419 & 298 & 320 & 256 & 369 \\
\hline Dissolved Oxygen (ppm) & 5.6 & 5.6 & 3.3 & 5.5 & 7.1 & 3 & 1.7 & 5.8 \\
\hline Oxidation/Reduction (mv) & 182 & 373 & 214 & 253 & 237 & 245 & 252 & 195 \\
\hline MISCEILANEOUS PARAMETERS & - & - & . & . & . & - & • & - \\
\hline pH (pH units) & - & 5.6 & 6.3 & 5.4 & 5 & - & 4.5 & - \\
\hline Sp. Cond. (umho/cm) & - & 188 & 273 & 320 & 272 & - & 254 & - \\
\hline TPH (mg/L) & 0.017 & 0.017 & 0.017 & 24.06 & - & 1422.9 & 88.08 & - \\
\hline $\operatorname{TDS}(\mathrm{mg} / \mathrm{I})$ & - & 148 & 148 & 196 & 140 & • & 158 & - \\
\hline TSS $(m g / L)$ & - & 42 & 103 & 11 & 7 & - & 32 & - \\
\hline Turbidity (NTO) & - & 34 & 26 & 1 & 5.5 & - & 4.5 & - \\
\hline RADIOCHEMICAI PARAMETERS & - & - & - & - & - & - & - & - \\
\hline Gross Alpha (pCi/L) & - & 10.80 & 18.90 & 0.29 & 0.62 & - & - & 2.08 \\
\hline Gross Alpha $(\mathrm{CE}+/-)$ & - & 2.90 & 4.30 & 2.90 & 1.70 & - & - & 3.90 \\
\hline Gross Beta (pci/L) & - & 4.71 & 16.90 & 4.27 & 4.13 & - & - & 5.30 \\
\hline Gross Beta $(\mathrm{CB}+/-)$ & - & 2.90 & 3.60 & 5.40 & 2.80 & . & - & 5.60 \\
\hline
\end{tabular}

(CONTINUED) 
APPENDIX R. 3

Groundwater Quality Data, 1993

\begin{tabular}{|c|c|c|c|c|c|c|c|c|}
\hline \multirow{3}{*}{$\begin{array}{l}\text { Sampling Point } \\
\text { Location } \\
\text { Date sampled }\end{array}$} & \multirow{3}{*}{\begin{tabular}{|c|} 
GW-508 \\
RG \\
$11 / 19 / 93$ \\
\end{tabular}} & \multicolumn{4}{|c|}{ GW-603 } & \multicolumn{3}{|c|}{$G W-604$} \\
\hline & & \multicolumn{4}{|c|}{ EXP } & \multicolumn{3}{|c|}{ EXP } \\
\hline & & $01 / 29 / 93$ & $05 / 04 / 93$ & $08 / 16 / 93$ & $10 / 28 / 93$ & $01 / 30 / 93$ & $05 / 04 / 93$ & $08 / 16 / 93$ \\
\hline FIEID MBASUREMENTS & - & - & - & - & - & - & - & - \\
\hline Depth to Water (ft) & 11.35 & 57.88 & 58.56 & 59.41 & 59.85 & 58.12 & 58.71 & 59.4 \\
\hline Water Temp (degrees C) & 19.2 & 14 & 17.8 & 16.1 & 13.3 & 14.1 & 16.6 & 16.6 \\
\hline pH (pH units) & 9.8 & 7.3 & 7.2 & 7.6 & 6.9 & 7.4 & 7.6 & 8 \\
\hline Sp. Cond. (unho/cm) & 590 & 494 & 515 & 529 & 503 & 378 & 327 & 308 \\
\hline Dissolved Oxygen (ppm) & 3.2 & 3 & 3.1 & 3.9 & 2.1 & 4.6 & 4.5 & 5.8 \\
\hline Oxidation/Reduction (mv) & 230 & 229 & 92 & 143 & 226 & 206 & 52 & 122 \\
\hline MISCELIAARBOUS PARAMETERS & - & - & . & . & - & . & • & • \\
\hline pH (pH units) & 4.6 & $7 . \dot{4}$ & $7 . \dot{2}$ & 7.6 & 8.1 & $7 . \dot{4}$ & 7.5 & 7.9 \\
\hline Sp. Cond. (umho/cm) & 272 & 540 & 578 & 592 & 562 & 418 & 352 & 339 \\
\hline TPH $(m g / L)$ & 21.49 & • & • & - & • & . & • & • \\
\hline $\operatorname{TDS}(\mathrm{mg} / \mathrm{L})$ & 160 & 346 & 346 & 366 & 360 & 262 & 210 & 210 \\
\hline TsS $(m g / L)$ & 9 & 9 & 78 & 19 & 16 & 8 & $<1$ & 4 \\
\hline Turbidity (NTU) & 1.6 & 10 & 62 & 19 & 14 & 16.5 & 4.5 & 10 \\
\hline RADIOCHEMICAL PARAMETERS & - & - & - & . & . & . & . & - \\
\hline Gross Alpha (pCi/L) & 1.78 & 2.37 & 1.77 & 0.00 & 4.94 & 0.18 & -0.00 & -1.42 \\
\hline Gross Alpha $(\mathrm{CE}+/-)$ & 1.20 & 1.90 & 2.00 & 2.20 & 2.20 & 1.90 & 0.87 & 1.80 \\
\hline Gross Beta $(\mathrm{pC} i / \mathrm{L})$ & 4.28 & 7.61 & 4.85 & 8.43 & 7.16 & 5.16 & -2.92 & 4.05 \\
\hline Gross Beta $(\mathrm{CB}+/-)$ & 2.60 & 3.10 & 3.00 & 2.90 & 3.00 & 2.80 & 1.40 & 2.60 \\
\hline
\end{tabular}

(CONTINUED) 
APPENDIX E. 3

Groundwater Quallty Data, 1993

\begin{tabular}{|c|c|c|c|c|c|c|c|c|}
\hline \multirow{3}{*}{$\begin{array}{l}\text { Sampling Point } \\
\text { Location } \\
\text { Date Sampled }\end{array}$} & \multirow{3}{*}{\begin{tabular}{|c|} 
GW-604 \\
$\operatorname{Exp}$ \\
$10 / 28 / 93$ \\
\end{tabular}} & \multicolumn{4}{|c|}{ GW-605 } & \multicolumn{3}{|c|}{ GW-606 } \\
\hline & & \multicolumn{4}{|c|}{$\exp$} & \multicolumn{3}{|c|}{$\mathbf{E X P}$} \\
\hline & & $02 / 02 / 93$ & $05 / 10 / 93$ & $08 / 19 / 93$ & $11 / 10 / 93$ & $02 / 03 / 93$ & $05 / 10 / 93$ & $08 / 20 / 93$ \\
\hline FIELD MEASUREMENTS & - & . & . & . & . & - & - & • \\
\hline Depth to Water (ft) & 59.64 & 11.15 & 10.9 & 11 & 11.1 & 13.85 & 13.9 & 14.4 \\
\hline Water Temp (degrees C) & 13.8 & 13.6 & 17.6 & 22 & 16 & 13.3 & 17.5 & 19.5 \\
\hline pH (pH units) & 7.5 & 7 & 7.1 & 7.5 & 6.8 & 7.5 & 7.5 & 7.7 \\
\hline Sp. Cond. (unho/cm) & 294 & 501 & 488 & 678 & 555 & 582 & 501 & 593 \\
\hline Dissolved Oxygen (ppm) & 4.6 & 0.9 & 1.1 & 3 & 1 & 1.1 & 1.4 & 2.4 \\
\hline Oxidation/Reduction (mv) & 200 & 139 & 36 & 53 & 106 & 115 & 133 & 78 \\
\hline MISCELLANEOUS PARAMETERS & $\dot{\bullet}$ & $\cdot$ & $\cdot$ & $\cdot$ & $\cdot$ & • & • & • \\
\hline$\overline{p H}$ (pH units) & $7 . \dot{9}$ & 7.3 & $7 . \dot{9}$ & $\dot{8}$ & 7.3 & 7.6 & $8 . \dot{3}$ & 7.6 \\
\hline sp. Cond. (umho/cm) & 317 & 613 & 603 & 571 & 591 & 594 & 600 & 608 \\
\hline TPH $(\mathrm{mg} / \mathrm{L})$ & $\cdot$ & $\cdot$ & $\cdot$ & - & $\cdot$ & • & • & - \\
\hline $\operatorname{TDS}(\mathrm{mg} / \mathrm{L})$ & 202 & 354 & 354 & 370 & 394 & 366 & 360 & 358 \\
\hline TSS $(\mathrm{mg} / \mathrm{L})$ & 2 & $<1$ & 1 & 7 & $<1$ & 2 & $<1$ & $<1$ \\
\hline Turbidity (NTU) & 6 & 1.8 & 2.4 & 3.2 & 1.9 & 1.4 & 1.5 & 0.7 \\
\hline RADIOCHEMICAL PARAKGTERS & . &. & . & . & . & - & - & • \\
\hline Gross Alpha $(\mathrm{pCi} / \mathrm{L})$ & $0.7 \dot{5}$ & 53.40 & 61.40 & 29.00 & 83.00 & 4.22 & 4.93 & 1.13 \\
\hline Gross Alphe $(\mathrm{CE}+/-)$ & 1.90 & 6.70 & 10.00 & 5.00 & 12.00 & 1.90 & 5.10 & 2.50 \\
\hline Gross Beta $(\mathrm{pC} 1 / \mathrm{L})$ & 0.91 & 43.40 & 51.40 & 27.40 & 58.10 & 6.37 & 1.84 & 5.09 \\
\hline Gross Beta $(\mathrm{CB}+/-)$ & 2.60 & 4.80 & 8.20 & 4.00 & 7.70 & 4.10 & 5.80 & 3.00 \\
\hline
\end{tabular}

(CONTINUED) 
APPENDIX B. 3

Groundwater Quadity Data, 1993

\begin{tabular}{|c|c|c|c|c|c|c|c|c|}
\hline \multirow{3}{*}{$\begin{array}{l}\text { Sampling Point } \\
\text { Location } \\
\text { Date sampled }\end{array}$} & \multirow{3}{*}{\begin{tabular}{|c|} 
GW-606 \\
$\operatorname{Exp}$ \\
$11 / 11 / 93$ \\
\end{tabular}} & \multicolumn{4}{|c|}{ GW-617 } & \multicolumn{3}{|c|}{$G W-618$} \\
\hline & & \multicolumn{4}{|c|}{$\mathbf{E x p}$} & \multicolumn{3}{|c|}{$\mathbf{E x p}$} \\
\hline & & $01 / 12 / 93$ & $05 / 04 / 93$ & $09 / 16 / 93$ & $12 / 07 / 93$ & $01 / 12 / 93$ & $05 / 05 / 93$ & $09 / 16 / 93$ \\
\hline FIELd masurRMENTS & - & . & - & . & . & . & . & • \\
\hline Depth to Water (ft) & 14.25 & 12.8 & 13.56 & 15 & 13.6 & 12.6 & 13.29 & 14.51 \\
\hline Water Temp (degrees C) & 13.6 & 25.1 & 18.9 & 19.1 & 15.2 & 17 & 16.9 & 17.8 \\
\hline pH (pH units) & 7.1 & 5.5 & 5.7 & 4.8 & 5.7 & 6.6 & 6.4 & 5.9 \\
\hline sp. Cond. (unho/cm) & 577 & 181 & 151 & 216 & 254 & 623 & 549 & 647 \\
\hline Diesolved Oxygen (ppm) & 0.9 & 9.3 & 4.4 & 2.3 & 3.7 & 2 & 0.6 & 0.4 \\
\hline Oxidation/Reduction (mV) & 191 & 243 & 61 & 158 & 40 & -38 & 18 & 148 \\
\hline MISCELLANBOUS PARANETERS & • & $\dot{\bullet}$ & • & $\dot{\bullet}$ & $\dot{\bullet}$ & $\cdot$ & • & • \\
\hline pH (pH units) & $\dot{8}$ & $6 . \dot{3}$ & $5 . \dot{7}$ & $5 . \dot{9}$ & $6 . \dot{3}$ & $6 . \dot{7}$ & $7 . \dot{5}$ & $6 . \dot{6}$ \\
\hline Sp. Cond. (umho/cm) & 617 & 189 & 169 & 230 & 270 & 664 & 624 & 672 \\
\hline TPH $(\mathrm{mg} / \mathrm{L})$ & - & • & • & • & • & . & • & • \\
\hline TDS (mg/L) & 382 & 116 & 110 & 136 & 204 & 350 & 350 & 380 \\
\hline TsS $(m g / L)$ & 2 & 9 & 19 & 25 & 9 & $<1$ & $<1$ & 1 \\
\hline Turbidity (NTU) & 2 & 10 & 16 & 52 & 8 & 1.6 & 3.3 & 1.5 \\
\hline RADIOCHEHICAL PARAMETERS & - & - & - & • & - & - & - & • \\
\hline Gross Alpha (PCi/L) & 6.31 & 0.45 & 0.61 & 1.02 & $0.80^{\circ}$ & -1.11 & -1.40 & 0.83 \\
\hline Gross Alpha $(\mathrm{CE}+/-)$ & 2.80 & 1.70 & 0.69 & 1.60 & 1.10 & 2.20 & 0.93 & 1.80 \\
\hline Gross Beta $(\mathrm{pCl} / \mathrm{L})$ & 0.26 & 3.79 & -4.01 & 4.52 & 1.27 & 4.60 & -1.19 & 2.17 \\
\hline Gross Beta $(\mathrm{CE}+/-)$ & 3.10 & 2.90 & 1.40 & 2.90 & 2.50 & 3.10 & 1.50 & 2.80 \\
\hline
\end{tabular}

(CONTINURD) 
APPENDIX $\mathbf{E} .3$

Groundwater Quallty Data, 1993

\begin{tabular}{|c|c|c|c|c|c|c|c|c|}
\hline \multirow{3}{*}{$\begin{array}{l}\text { 8anpling Polnt } \\
\text { Location } \\
\text { - } \\
\text { Date sampled }\end{array}$} & $0 w-618$ & \multicolumn{4}{|c|}{ OW-619 } & \multicolumn{3}{|c|}{$Q W-620$} \\
\hline & $\mathbf{B X P}$ & \multicolumn{4}{|c|}{ PTF } & \multicolumn{3}{|c|}{ MTF } \\
\hline & $12 / 08 / 93$ & $01 / 20 / 93$ & $05 / 05 / 93$ & $09 / 17 / 93$ & $12 / 08 / 93$ & $01 / 20 / 93$ & $05 / 06 / 93$ & $09 / 17 / 93$ \\
\hline FIELD MASUREMLNTS & - & - & - & - & - & - & - & - \\
\hline Depth to Water (ft) & 13.37 & 20.15 & 23.3 & 24.58 & 17.65 & 21.9 & 25 & 27.65 \\
\hline Water Temp (degrees C) & 16.5 & 14.6 & 17.5 & 16.1 & 15.2 & 10.5 & 19 & 19.7 \\
\hline ph (ph unfts) & 6.3 & 20.6 & 11 & 10.1 & 10.2 & 11 & 21.8 & 9.1 \\
\hline sp. Cond. (umho/cm) & 626 & 287 & 528 & 277 & 242 & 618 & 2140 & 292 \\
\hline Dienolved Oxygen (ppm) & 0.3 & 6.3 & 2.2 & 1.9 & 2.5 & 6.2 & 4 & 5.2 \\
\hline Oxidation/Reduction (mV) & 48 & 11 & -37 & 43 & 15 & 20 & -34 & 84 \\
\hline MISCELINALOUS PARANETERS & - & - & - & - & - & - & - & - \\
\hline pH (pH units) & 6.8 & 9.4 & 10.7 & 9.1 & 9.1 & 8.9 & 12 & $11 \cdot 4$ \\
\hline Sp, Cond. (umho/cm) & 659 & 124 & 270 & 160 & 142 & 156 & 2220 & 510 \\
\hline TPH $(m g / L)$ & & - & - & - & - & . & - & - \\
\hline $\operatorname{TDS}(\mathrm{mg} / \mathrm{L})$ & 402 & 72 & 158 & 106 & 118 & 148 & 558 & 158 \\
\hline T88 (mg/L) & $<1$ & $<1$ & 42 & 41 & 30 & 78 & 65 & 60 \\
\hline Turbldity (NFU) & 1 & 6 & 2.9 & 21 & 20 & 34 & 36 & 20 \\
\hline RADIOCLEMICAI PARAMETERS & - & - & - & - & - & - & - & - \\
\hline Gross Alpha (PCI/I) & 0.70 & 2.61 & -0.67 & 3.06 & 0.63 & 1.01 & 0.16 & -0.45 \\
\hline Grose Alpha $(\mathrm{Cs}+/-)$ & 1.10 & 1.90 & 0.80 & 2.00 & 1.00 & 1.50 & 0.82 & 1.40 \\
\hline Gross Beta (pC1/L) & 3.21 & 14.80 & 14.30 & 15.00 & 14.80 & 10.20 & 8.51 & 7.28 \\
\hline Gross Beta $(\mathrm{CE}+/-1$ & 2.60 & 3.50 & 1.80 & 3.50 & 3.60 & 3.20 & 1.40 & 3.00 \\
\hline
\end{tabular}

(CONTINUED) 
APPENDIX E.3

Groundwater Quality Data, 1993

\begin{tabular}{|c|c|c|c|c|c|c|c|c|}
\hline \multirow{3}{*}{$\begin{array}{l}\text { 8ampling Point } \\
\text { Location } \\
\text { Date sampled }\end{array}$} & \multirow{3}{*}{\begin{tabular}{|c|}
$O W-620$ \\
$M T$ \\
$12 / 09 / 93$ \\
\end{tabular}} & \multicolumn{5}{|c|}{ OW-631 } & \multirow{2}{*}{\multicolumn{2}{|c|}{$\frac{0 w-632}{R G}$}} \\
\hline & & \multicolumn{5}{|c|}{ RO } & & \\
\hline & & $03 / 02 / 93$ & $06 / 18 / 93$ & $07 / 07 / 93$ & $09 / 16 / 93$ & $11 / 17 / 93$ & $03 / 03 / 93$ & $06 / 21 / 93$ \\
\hline TISLD MEASURAMHTS & - & - & $\cdot$ & $\cdot$ & $\cdot$ & $\cdot$ & - & • \\
\hline Dopth to Water $(f t)$ & 20.96 & 6.8 & 7.1 & 7.45 & 7.85 & 6.85 & 7.5 & 8.34 \\
\hline Water Temp (degrees C) & 13.2 & 13 & 23.1 & 24.2 & 20.1 & 20.6 & 14 & 19.5 \\
\hline pH (pH unite) & 9.9 & 4.7 & 4.5 & 4.5 & 4.2 & 3.9 & 5.2 & 5.7 \\
\hline 8p. Cond. (unho/cm) & 348 & 242 & 248 & 400 & 202 & 240 & 279 & 320 \\
\hline Diesulved oxygen (ppa) & 3.8 & 5.9 & 5.7 & 2.9 & 4 & 2.9 & 3.5 & 3.5 \\
\hline Oxidation/Reduction (mV) & 78 & 277 & 278 & 240 & 383 & 318 & 145 & 140 \\
\hline MISCELIAMOUS PARAMTIRRS & $\cdot$ & . & . & . &. & . & . & . \\
\hline pH (pH unite) & 8.2 & 4.9 & $\dot{s}$ & . & 4.8 & $4 . \dot{9}$ & $5 . \dot{9}$ & $6 . \dot{8}$ \\
\hline 8p. Cond. (unho/cm) & 328 & 232 & 313 & $\cdot$ & 188 & 212 & 290 & 467 \\
\hline TPH $(m g / L)$ & $\cdot$ & 0.003 & $\cdot$ & $<0.1$ & o & 0.002 & 2.098 & • \\
\hline $\operatorname{TDS}(m g / L)$ & 228 & 166 & 238 & $\cdot$ & 142 & 152 & 198 & 272 \\
\hline T8s $(m g / L)$ & 55 & 2 & 4 & - & 2 & 10 & 27 & 19 \\
\hline Turbidity (NTU) & 9.5 & 2.6 & 5.5 & . & 1.5 & so & 22 & 56 \\
\hline RADIOCHEMICAX PARAKETERS & . & . & . & . & . & . & . & . \\
\hline Grose Alpha (pCi/L) & 0.16 & 1.78 & 4.56 & . & 0.90 & 0.79 & 0.57 & 0.88 \\
\hline Gross Alpha $(\mathrm{Cz}+/-)$ & 0.94 & 1.60 & 3.90 & • & 1.60 & 0.85 & 2.90 & 3.50 \\
\hline Grose Beta $(\mathrm{PC} 1 / \mathrm{L})$ & 20.10 & 8.65 & 3.27 & . & 3.08 & 2.47 & -0.98 & 2.63 \\
\hline Grose Buta $(\mathrm{CR}+/-)$ & 4.00 & 3.50 & 5.30 & - & 2.80 & 2.50 & 5.00 & 5.20 \\
\hline
\end{tabular}

(CONTINUED) 
Groundwater Quallty Data, 1993

\begin{tabular}{|c|c|c|c|c|c|c|c|c|}
\hline \multirow{3}{*}{$\begin{array}{l}\text { sumpling polnt } \\
\text { Looation } \\
\text { Dute sampled }\end{array}$} & \multicolumn{3}{|c|}{ ON-632 } & \multicolumn{5}{|c|}{$6 w-633$} \\
\hline & \multicolumn{3}{|c|}{ RO } & \multicolumn{5}{|c|}{ no } \\
\hline & $06 / 29 / 93$ & $09 / 26 / 93$ & $11 / 18 / 93$ & $03 / 03 / 93$ & $06 / 21 / 93$ & $06 / 29 / 93$ & $09 / 20 / 93$ & $11 / 19 / 93$ \\
\hline FIELD MABUREMGATS & • & $\cdot$ & - & - & - & - & - & $\bullet^{\bullet}$ \\
\hline Dopth to Water (ft) & 0.35 & $8 . \dot{9}$ & $8 . \dot{1}$ & 3.54 & 2.61 & 2.05 & 3.7 & 2.55 \\
\hline Water Tamp (degrees C) & 17.8 & 19.8 & 15.1 & 14 & 17.6 & 19.1 & 21.1 & 16.7 \\
\hline pH (pH unite) & 6.1 & 3.3 & 3 & 5.3 & 5.4 & 5.1 & 5.3 & 9 \\
\hline 8p. Cond. (unho/cm) & 792 & 396 & 273 & 2590 & 1860 & 23200 & 26500 & 1005 \\
\hline Dlesolved Oxygen (ppm) & 4.1 & 2.1 & 3.4 & 3.1 & 3.9 & 1.9 & 1.8 & 3.3 \\
\hline Oxldation/Raduction (mV) & 138 & 177 & 205 & 250 & 98 & 190 & 258 & 215 \\
\hline MISCELWMLOOS PARARETERS & - & . & . & . & . & . & . & \\
\hline pH (pH unite) & . & $5 . \dot{7}$ & 6 & 5.7 & 6 & . & 5.5 & $5 . \dot{8}$ \\
\hline 8p. Cond. (unho/cm) & • & 312 & 423 & 19870 & 23800 & . & 2650 & 2820 \\
\hline TPH $(m g / L)$ & 3.3 & 2.61 & 4.2 & 0.0221 & $\cdot$ & 0.05 & 0.044 & $<0.1$ \\
\hline $208(\mathrm{mg} / \mathrm{L})$ & • & 234 & 188 & 21966 & 23196 & • & 25532 & 1326 \\
\hline $788(\mathrm{mg} / \mathrm{L})$ & - & 19 & 8 & 219 & 169 & - & 133 & 16 \\
\hline Turbidity (RTO) & - & 66 & 5 & 220 & 95 & - & 70 & 12 \\
\hline RADIOCERICAL PARALTERS & . & . & . & . &. & . & . & \\
\hline Grose Alpha (pCi/L) & - & 4.31 & 1.30 & 13.50 & 14.30 & $\dot{\bullet}$ & -44.40 & 2.85 \\
\hline Grose Npha $(\mathrm{Cz}+/-)$ & . & 2.30 & 1.00 & 9.70 & 43.00 & . & 170.00 & 1.80 \\
\hline Gross Beta $(\mathrm{pcl} / \mathrm{L})$ & - & 5.62 & 3.97 & 75.60 & 364.00 & - & 581.00 & 43.80 \\
\hline Grose Beta $(\mathrm{CB}+/-)$ & - & 3.00 & 2.60 & 9.30 & 72.00 & - & 300.00 & 6.20 \\
\hline
\end{tabular}

(CONTrNURD) 
APPENDIX 8.3

Groundwater Quallty Data, 1993

\begin{tabular}{|c|c|c|c|c|c|c|c|c|}
\hline \multirow{3}{*}{$\begin{array}{l}\text { 8umpling Point } \\
\text { Location } \\
\text { Date sampled }\end{array}$} & \multicolumn{5}{|c|}{ GW-634 } & \multicolumn{3}{|c|}{$O W-656$} \\
\hline & \multicolumn{5}{|c|}{ RO } & \multicolumn{3}{|c|}{ T0134 } \\
\hline & $03 / 02 / 93$ & $06 / 18 / 93$ & $07 / 07 / 93$ & $09 / 16 / 93$ & $11 / 18 / 93$ & $03 / 11 / 93$ & $06 / 22 / 93$ & $09 / 23 / 93$ \\
\hline FIELD MAsUREMETS & , & • & - & . & . & . & • & • \\
\hline Depth to Water (ft) & 8.42 & 9.74 & 9.5 & 9.65 & 8.81 & 10.12 & 9.9 & 9.19 \\
\hline Water Tamp (degrees C) & 13.8 & 21.6 & 24.5 & 20.1 & 15.8 & 16.1 & 22.2 & 22.2 \\
\hline pH (pH unite) & 5.4 & 4.8 & 4.8 & 4.6 & 4.2 & 6.7 & 6.5 & 7.1 \\
\hline Sp. Cond. (wwho/on) & 75 & 69 & 68 & 75 & 70 & 608 & 562 & 599 \\
\hline Dlesolved Oxygen (ppm) & 2.3 & 5.4 & 1.8 & 3.2 & 2.5 & 1.1 & 1.2 & 4.7 \\
\hline Oxidation/Raduction (mV) & 63 & 41 & 70 & 199 & 233 & 2 & 9 & 241 \\
\hline MISCELLANLOUS PARNATERS & - & - & - & - & • & $\cdot$ & $\cdot$ & • \\
\hline pH (pH unite) & 5.3 & 5.6 & $\cdot$ & $5 . \dot{3}$ & $5 . \dot{3}$ & 7.3 & $6 . \dot{9}$ & $7 . \dot{1}$ \\
\hline 8p. Cond. (umho/cm) & 73 & 74 & - & 71 & 71 & 648 & 624 & 672 \\
\hline TPH $(m g / c)$ & 6.032 & - & 4.48 & 2.48 & 5.16 & 0.002 & $<0.1$ & 0.003 \\
\hline $\operatorname{TDS}(\mathrm{mg} / \mathrm{L})$ & 58 & 46 & • & 58 & 52 & 448 & 454 & 476 \\
\hline$T 88$ (mg/L) & $<1$ & $<1$ & - & 2 & 1 & 92 & 83 & 48 \\
\hline Turbidity (NTO) & 1.2 & 1.4 & . & 3.6 & 1.4 & 66 & 130 & 100 \\
\hline RDIOCKMICAI PARARETERS & • & - & - & . & - & • & $\cdot$ & • \\
\hline Grose Alpha (pCL/L) & 2.11 & 2.26 & - & 0.71 & 0.44 & 0.33 & 0.93 & -1.25 \\
\hline Orose Alphe $(\mathrm{Cs}+/-)$ & 1.60 & 3.60 & . & 1.50 & 0.67 & 2.10 & 3.20 & 3.00 \\
\hline Grose Beta (pCi/L) & 11.40 & 5.40 &. & 1.36 & 3.08 & 2.59 & 4.30 & 4.30 \\
\hline Gros: Beta $(\mathrm{CB}+/-)$ & 3.60 & 5.40 & $\cdot$ & 2.70 & 2.60 & 3.10 & 5.30 & 3.30 \\
\hline
\end{tabular}

(CONTIMURD) 
APPENDIX $\mathrm{E}, 3$

Groundwater Quallty Data, 1993

\begin{tabular}{|c|c|c|c|c|c|c|c|c|}
\hline \multirow{3}{*}{$\begin{array}{l}\text { 8aupling Point } \\
\text { Location } \\
\text { Date Sampled }\end{array}$} & \multirow{3}{*}{\begin{tabular}{|c|}
$G W-656$ \\
$T 0134$ \\
$11 / 17 / 93$ \\
\end{tabular}} & \multicolumn{4}{|c|}{$6 W-657$} & \multicolumn{3}{|c|}{$G W-658$} \\
\hline & & \multicolumn{4}{|c|}{ T2331 } & \multicolumn{3}{|c|}{$\mathbf{r r}$} \\
\hline & & $03 / 10 / 93$ & $06 / 18 / 93$ & $09 / 21 / 93$ & $11 / 15 / 93$ & $03 / 11 / 93$ & $06 / 23 / 93$ & $11 / 19 / 93$ \\
\hline FIRLD meAsUREMENTS & - & - & - & • & . & . & • & • \\
\hline Depth to Water (ft) & 9.25 & 6.01 & 6.17 & 7.4 & 5.9 & 10.95 & 11.8 & 11 \\
\hline Water Tamp (degrees C) & 21 & 16.3 & 22 & 25.2 & 21 & 15.3 & 22.6 & 18.2 \\
\hline pH (pH units) & 6.6 & 7.3 & 6.8 & 6.8 & 6.9 & 6.4 & 6.2 & 6.4 \\
\hline Sp. Cond. (umho/cm) & 654 & 498 & 452 & 538 & 498 & 477 & 546 & 486 \\
\hline Diesolved Oxygen (ppm) & 2.6 & 0.7 & 1.3 & 0.7 & 1.2 & 0.9 & 1.7 & 1.3 \\
\hline Oxidation/Rer'sction (mV) & 55 & -14 & -49 & -59 & 166 & -65 & -43 & -25 \\
\hline MISCELLANEOUS PARAMETERS & • & • & $\dot{\bullet}$ & $\cdot$ & $\cdot$ & $\dot{\cdot}$ & $\cdot$ & $\cdot$ \\
\hline pH (pH units) & 7.1 & 7.1 & $7 . \dot{3}$ & $7 . \dot{2}$ & $7 . \dot{1}$ & 6.9 & $6 . \overline{7}$ & ; \\
\hline Sp. Cond. (unho/cm) & 635 & 535 & 520 & 530 & 526 & 462 & 482 & 590 \\
\hline TPH $(\mathrm{mg} / \mathrm{L})$ & $<0.1$ & 0.002 & • & 0.003 & 0.02 & 8.155 & 9.81 & 64.65 \\
\hline $\operatorname{TDS}(m g / L)$ & 422 & 292 & 286 & 310 & 302 & 286 & 290 & 332 \\
\hline $\mathrm{Ts8}(\mathrm{mg} / \mathrm{L})$ & 746 & $<1$ & 7 & 4 & 3 & 29 & 29 & 91 \\
\hline Turbidity (NTU) & 380 & 1.2 & 4 & 5.8 & 1.6 & 28 & 36 & 18 \\
\hline RADTOCHEMICAL PARARETZRS & - & - & . & $\dot{.}$ & $\dot{.}$ & $\dot{\bullet}$ & . & • \\
\hline Grose Alpha $(\mathrm{pCl} / \mathrm{L})$ & 5.63 & 0.94 & 0.61 & -1.83 & 2.39 & 1.36 & 1.00 & 4.04 \\
\hline Grose Alpha $(\mathrm{CB}+/-)$ & 2.90 & 3.80 & 3.60 & 3.30 & 2.20 & 4.40 & 1.80 & 2.50 \\
\hline Grose Bata $(\mathrm{pCi} / \mathrm{I})$ & 8.02 & 6.64 & 4.80 & 4.46 & 4.99 & 1.01 & 2.43 & 4.42 \\
\hline Gross Beta $(\mathrm{Cr}+/-)$ & 3.20 & 5.60 & 5.40 & 5.60 & 2.90 & 6.00 & 2.70 & 2.90 \\
\hline
\end{tabular}

(CONTINOED) 
APPENDIX E. 3

Groundwater Quality Data, 1993

\begin{tabular}{|c|c|c|c|c|c|c|c|c|}
\hline \multirow{3}{*}{$\begin{array}{l}\text { Sampling Point } \\
\text { Iocation } \\
\text { - } \\
\text { Date Sampled }\end{array}$} & \multirow{3}{*}{$\begin{array}{c}\text { GW-658 } \\
\text { FF } \\
09 / 27 / 04\end{array}$} & \multicolumn{5}{|c|}{ GW-659 } & \multicolumn{2}{|c|}{ GW-707 } \\
\hline & & \multicolumn{5}{|c|}{$\mathbf{F F}$} & \multicolumn{2}{|c|}{ T2331 } \\
\hline & & $03 / 10 / 93$ & $06 / 18 / 93$ & $07 / 07 / 93$ & $09 / 22 / 93$ & $11 / 15 / 93$ & $03 / 11 / 93$ & $06 / 23 / 93$ \\
\hline FIEID VEASUREMENTS & - & - & - & - & $\cdot$ & - & - & • \\
\hline Depth to Water (ft) & 11.85 & 7.4 & 8.12 & 8.5 & 8.69 & 1.9 & 5.95 & 5.8 \\
\hline Water Temp (degrees C) & 21.6 & 18.1 & 25.7 & 21.8 & 22.7 & 20 & 15.1 & 21.8 \\
\hline pH (pH units) & 6.4 & 6.2 & 6.2 & 6.3 & $6 \cdot 3$ & 6.7 & 7.9 & 7.8 \\
\hline Sp. Cond. (umho/cm) & 493 & 1791 & 741 & 1014 & 308 & 109 & 332 & 431 \\
\hline Dissolved oxygen (ppm) & 5.4 & 3.5 & 3 & 1.5 & $4 \cdot 4$ & 6.3 & 5.6 & $5 \cdot 5$ \\
\hline Oxidation/Reduction (mV) & -26 & 147 & 67 & -54 & 57 & 175 & -27 & 188 \\
\hline MISCELLANEOUS PARAMETERS & - & - & - & - & - & - & - & - \\
\hline pH (pk units) & 6.7 & 6.2 & 6.6 & - & 6.4 & 6.9 & 8.2 & 7.9 \\
\hline Sp. Cond. (umho/cm) & 538 & 1740 & 905 & - & 355 & 101 & 349 & 701 \\
\hline TPH $(m g / L)$ & 1006.5 & 0.01 & - & 0.046 & 0.015 & $<0.1$ & 0.01 & 0.003 \\
\hline TDS $(\mathrm{mg} / \mathrm{L})$ & 294 & 946 & 640 & - & 224 & 306 & 216 & 514 \\
\hline TSS (mg/I) & 31 & 391 & 234 & - & 253 & 743 & 11 & 1 \\
\hline Turbidity (NTO) & 220 & 460 & 340 & - & 680 & 750 & 6.2 & 2.9 \\
\hline RADIOCHOMICAL PARAYETERS & - & - & - & - & - & - & - & - \\
\hline Gross Alpha (pCi/L) & -1.00 & 3.74 & 10.70 & - & -1.47 & 4.55 & 2.76 & 3.66 \\
\hline Gross Alpha $(\mathrm{CB}+/-)$ & 2.80 & 6.10 & 8.80 & - & 3.30 & 4.00 & 2.40 & 2.50 \\
\hline Gross Beta (pCi/L) & -1.56 & $16 \cdot 40$ & $25 \cdot 30$ & - & 2.90 & $12 \cdot 40$ & 3.92 & 8.15 \\
\hline Gross Beta $(\mathrm{CB}+/-)$ & 3.00 & 6.40 & 12.00 & - & 5.40 & 5.90 & 3.20 & 3.10 \\
\hline
\end{tabular}

(CONTINUED) 
APPENDIX E. 3

Groundwater Quality Data, 1993

\begin{tabular}{|c|c|c|c|c|c|c|c|c|}
\hline \multirow{3}{*}{$\begin{array}{l}\text { Sampling Point } \\
\text { Location } \\
\text { Date Sampled }\end{array}$} & \multicolumn{2}{|c|}{ GW-707 } & \multicolumn{5}{|c|}{ GW-708 } & GW-733 \\
\hline & \multicolumn{2}{|c|}{ T2331 } & \multicolumn{5}{|c|}{ T2331 } & EXP \\
\hline & $09 / 27 / 93$ & $11 / 18 / 93$ & $03 / 10 / 93$ & $06 / 18 / 93$ & $07 / 07 / 93$ & $09 / 21 / 93$ & $11 / 12 / 93$ & $02 / 02 / 93$ \\
\hline FIELD MEASUREMENTS & $\cdot$ & $\cdot$ & $\cdot$ & $\cdot$ & • & - & • & - \\
\hline Depth to Water $(f t)$ & 5.95 & 6 & 4.5 & 4.98 & 5.55 & 5.15 & 4.8 & 55.85 \\
\hline Water Temp (degrees C) & 22.8 & 21.5 & 13.7 & 23.9 & 25.7 & 25.9 & 15.9 & 14 \\
\hline $\mathrm{pH}$ (pH units) & 7.3 & 7.2 & 7 & 6.8 & 6.6 & 6.5 & 6.9 & 8 \\
\hline Sp. Cond. (umho/cm) & 759 & 485 & 481 & 597 & 646 & 784 & 788 & 330 \\
\hline Dissolved Oxygen (ppm) & 6 & 7 & 0.5 & 0.8 & 0.5 & 0.6 & 1.6 & 1.4 \\
\hline Oxidation/Reduction (mV) & 8 & 16 & -92 & -80 & -104 & -85 & -54 & 22 \\
\hline MISCELLANEOUS PARAMETERS & $\cdot$ & $\cdot$ & $\bullet$ & $\cdot$ & • & • & $\dot{\bullet}$ & • \\
\hline pH (pH units) & $7 . \dot{7}$ & 7.6 & $\dot{7}$ & $7 . \dot{1}$ & • & $6 . \dot{9}$ & $6 . \dot{9}$ & 8 \\
\hline Sp. Cond. (umho/cm) & 661 & 447 & 554 & 688 & • & 792 & 799 & 367 \\
\hline TPH $(m g / L)$ & $<0.1$ & $<0.1$ & 4.88 & • & 5.22 & 4.58 & 4.89 & • \\
\hline TDS (mg/L) & 310 & 242 & 324 & 426 & • & 522 & 496 & 194 \\
\hline TSS $(m g / L)$ & 2 & 3 & 31 & 10 & - & 9 & 12 & 9 \\
\hline Turbidity (NTO) & 3.6 & 2.4 & 15 & 22 & - & 50 & 36 & 15 \\
\hline RADIOCHEMICAL PARAMETERS & . & . & . & . & - & • & . & . \\
\hline Gross Alpha (pCi/L) & -0.69 & 1.89 & -1.96 & 0.32 & $\bullet$ & -2.15 & 0.34 & 0.64 \\
\hline Gross Alphe $(\mathrm{CE}+/-)$ & 3.00 & 2.10 & 3.50 & 3.70 & - & $\mathbf{3 . 3 0}$ & 2.10 & 1.80 \\
\hline Gross Beta $(\mathrm{pCi} / \mathrm{L})$ & 6.81 & 5.82 & 4.01 & 10.70 & - & 6.19 & 7.75 & 4.49 \\
\hline Gross Beta $(\mathrm{CE}+/-)$ & 3.40 & 3.00 & 5.40 & 5.80 & - & 5.70 & 3.10 & 3.00 \\
\hline
\end{tabular}

(CONTINUED) 
APPENDIX E.3

Groundwater Quality Data, 1993

\begin{tabular}{|c|c|c|c|c|c|c|c|c|}
\hline \multirow{3}{*}{$\begin{array}{l}\text { Sampling Point } \\
\text { Location } \\
\text { Date Sampled }\end{array}$} & \multicolumn{3}{|c|}{$G W-733$} & \multicolumn{4}{|c|}{ GW-735 } & \multirow{2}{*}{$\begin{array}{l}\text { GW-744 } \\
\text { GRIDK1 }\end{array}$} \\
\hline & \multicolumn{3}{|c|}{$\operatorname{EXP}$} & \multicolumn{4}{|c|}{ NHP } & \\
\hline & $05 / 06 / 93$ & $08 / 18 / 93$ & $11 / 04 / 93$ & $02 / 02 / 93$ & $05 / 07 / 93$ & $08 / 18 / 93$ & $10 / 29 / 93$ & $01 / 18 / 93$ \\
\hline FIELD MRASUREMENTS & - & - & - & - & • & - & • & - \\
\hline$n$th to Water $(f t)$ & 56.5 & 57.65 & 57.92 & 22.52 & 22.61 & 23.22 & 23.7 & 6.01 \\
\hline ter Temp (degrees $C$ ) & 15.9 & 16.5 & 13.8 & 12.7 & 16.6 & 19.8 & 14 & 15.6 \\
\hline $\mathrm{pH}$ (pH units) & 7.8 & 7.9 & 7.9 & 7.2 & 7.1 & 7 & 6.5 & 7.4 \\
\hline Sp. Cond. (umho/cm) & 314 & 356 & 348 & 558 & 520 & 542 & 570 & 393 \\
\hline Dissolved Oxygen (ppm) & 1.5 & 3.1 & 1.4 & 0.9 & 0.7 & 3.1 & 0.4 & 0.7 \\
\hline Oxidation/Reduction (mv) & 65 & 95 & 62 & 91 & 18 & 51 & 95 & -74 \\
\hline MISCELIANEOUS PARAMETERS & - & . & . & . & . & . & . & - \\
\hline $\mathrm{pH}$ (pH units) & 7.7 & 7.9 & 7.9 & 7.3 & 7.7 & 7.4 & $7 . \dot{3}$ & 7.7 \\
\hline Sp. Cond. (umho/cm) & 357 & 355 & 383 & 638 & 614 & 576 & 618 & 435 \\
\hline TPH $(\mathrm{mg} / \mathrm{L})$ & • & - & & - & - & . & . & • \\
\hline $\operatorname{TDS}(\mathrm{mg} / \mathrm{L})$ & 186 & 228 & 222 & 384 & 374 & 386 & 398 & 258 \\
\hline TSS $(m g / I)$ & 37 & 20 & 2 & 7 & 7 & $<1$ & 14 & $<1$ \\
\hline Turbidity (NTU) & 38 & 20 & 4.8 & 14 & 14 & 2.4 & 18 & 1.8 \\
\hline RADIOCHEMICAI PARAMETERS & . & . & . & . & . & . & . & . \\
\hline Gross Alpha (pCi/L) & -0.96 & 1.22 & 1.06 & 0.20 & -1.03 & -0.83 & 1.98 & 2.98 \\
\hline Gross Alpha $(\mathrm{CE}+/-)$ & 0.64 & 2.40 & 1.70 & 2.20 & 0.68 & 2.30 & 2.30 & 2.30 \\
\hline Gross Beta (pCi/L) & 0.23 & 4.96 & -0.69 & 6.35 & 3.15 & 2.51 & 4.10 & 6.41 \\
\hline Gross Beta $(\mathrm{CE}+/-)$ & 1.20 & 3.00 & 2.80 & 3.20 & 1.30 & 2.80 & 2.90 & 3.00 \\
\hline
\end{tabular}

(CONTIRUED) 
APPENDIX E.3

Groundwater Quality Data, 1993

\begin{tabular}{|c|c|c|c|c|c|c|c|c|}
\hline \multirow{3}{*}{$\begin{array}{l}\text { Sampling Point } \\
\text { Location } \\
\text { - } \\
\text { Date Sampled }\end{array}$} & \multicolumn{3}{|c|}{ GW-744 } & \multicolumn{4}{|c|}{$G W-745$} & GW-746 \\
\hline & \multicolumn{3}{|c|}{ GRIDK1 } & \multicolumn{4}{|c|}{ GRIDK1 } & GRIDK1 \\
\hline & $04 / 12 / 93$ & $08 / 04 / 93$ & $10 / 22 / 93$ & $01 / 19 / 93$ & $04 / 12 / 93$ & $08 / 04 / 93$ & $10 / 23 / 93$ & $01 / 19 / 93$ \\
\hline FIEID MEASUREMENTS & - & - & - & - & • & - & - & - \\
\hline Depth to Water (ft) & 5.8 & 7.9 & 6.65 & 8.63 & 8.25 & 10.05 & 6.35 & 5 \\
\hline Water Temp (degrees C) & 16.5 & 17.7 & 15.8 & 12.2 & 16.3 & 19.9 & 15.5 & 13.1 \\
\hline pH (pH units) & 7.5 & 7.6 & 7.4 & 7.1 & 7.1 & 7.3 & 7 & 5.8 \\
\hline Sp. Cond. (umho/cm) & 343 & 386 & 407 & 385 & 345 & 414 & 364 & 137 \\
\hline Dissolved Oxygen (ppm) & 1.2 & 0.3 & 0.5 & 6.6 & 6.6 & 3.2 & 2.7 & 6.2 \\
\hline Oxidation/Reduction (mV) & 54 & -97 & -95 & 158 & 168 & 28 & 93 & 206 \\
\hline MISCELIANEOUS PARAMETERS & - & - & - & - & - & - & - & - \\
\hline ph (pH units) & 8.1 & 7.6 & 7.6 & 7.3 & 7.9 & 7.4 & 7.3 & 6 \\
\hline Sp. Cond. (umho/cm) & 414 & 423 & 438 & 428 & 419 & 442 & 446 & 152 \\
\hline TPH $(m g / L)$ & - & - & - & - & - & - & - & - \\
\hline TDS (mg/r) & 266 & 242 & 286 & 268 & 270 & 284 & 298 & 102 \\
\hline TSS (mg/L) & $<1$ & $<1$ & $<1$ & 3 & 2 & $<1$ & $<1$ & 12 \\
\hline Turbidity (NTU) & 2.5 & 1.4 & 1 & 1.8 & 6.2 & 1.5 & 2.6 & 120 \\
\hline RADIOCHEMICAL PARAMETERS & - & - & • & - & - & - & - & - \\
\hline Gross Alpha (pCi/L) & 1.29 & -3.64 & 0.48 & -0.17 & 0.18 & -2.48 & 0.32 & 0.29 \\
\hline Gross Alpha $(\mathrm{CE}+/-)$ & 1.50 & 4.20 & 2.00 & 1.80 & 1.50 & 4.50 & 1.90 & 1.60 \\
\hline Gross Beta (pCi/L) & 3.74 & 1.20 & 0.50 & 2.02 & 3.30 & $-1 \cdot 38$ & 1.75 & 0.82 \\
\hline Gross Beta $(\mathrm{CE}+/-)$ & 2.70 & 5.80 & 2.80 & 2.80 & 2.60 & 5.60 & 2.90 & 2.60 \\
\hline
\end{tabular}

(CONTINUED) 
APPENDIX E. 3

Groundwater Quality Data, 1993

\begin{tabular}{|c|c|c|c|c|c|c|c|c|}
\hline \multirow{3}{*}{$\begin{array}{l}\text { Sampling Poin } \\
\text { Location } \\
\text { Date Sampled }\end{array}$} & \multicolumn{3}{|c|}{ GW-746 } & \multicolumn{4}{|c|}{$G W-747$} & GW-748 \\
\hline & \multicolumn{3}{|c|}{ GRIDK1 } & \multicolumn{4}{|c|}{ GRIDK2 } & GRIDK2 \\
\hline & $04 / 12 / 93$ & $08 / 05 / 93$ & $10 / 23 / 93$ & $01 / 19 / 93$ & $04 / 13 / 93$ & $08 / 05 / 93$ & $10 / 23 / 93$ & $01 / 20 / 93$ \\
\hline FIELD MEASUREMENTS & - & - & $\cdot$ & $\cdot$ & $\cdot$ & $\cdot$ & $\cdot$ & • \\
\hline Depth to Water (ft) & 4.83 & 5.78 & 5.55 & 4.46 & 4.52 & 9.01 & 5.67 & 6.16 \\
\hline Water Temp (degrees $C$ ) & 16 & 21.1 & 17.8 & 13.8 & 16.1 & 17.8 & 16 & 13.6 \\
\hline H (pH units) & 5.5 & 6.9 & 5.6 & 7.5 & 7.6 & 7.7 & 7.6 & 7.6 \\
\hline sp. Cond. (umho/cm) & 115 & 220 & 176 & 426 & 383 & 456 & 411 & 462 \\
\hline Dissolved Oxygen (ppm) & 8.7 & 4.7 & 2.7 & 1.3 & 1.7 & 0.5 & 0.5 & 5 \\
\hline Oxidation/Reduction (mV) & 232 & 59 & 154 & 23 & 133 & -71 & -64 & 169 \\
\hline MISCELLANEOUS PARAMETERS & . & $\cdot$ & • & . & $\dot{.}$ & $\cdot$ & $\cdot$ & • \\
\hline pH (pH units) & $6 . \dot{6}$ & $6 . \dot{2}$ & 5.9 & 7.7 & 7.5 & 7.7 & 7.6 & 8 \\
\hline Sp. Cond. (umho/cm) & 140 & 158 & 168 & 455 & 440 & 446 & 448 & 480 \\
\hline $\mathrm{TPH}(\mathrm{mg} / \mathrm{L})$ & • & • & - & • & - & $\cdot$ & $\cdot$ & - \\
\hline $\operatorname{TDS}(\mathrm{mg} / L)$ & 104 & 104 & 142 & 276 & 272 & 264 & 286 & 326 \\
\hline TsS (mg/L) & 13 & 414 & 6 & $<1$ & 1 & 2 & $<1$ & 79 \\
\hline Turbidity (NTU) & 210 & 230 & 4.3 & 1.3 & 0.6 & 1.1 & 1.6 & 160 \\
\hline RADIOCHEMICAL PARAMETERS & $\cdot$ & - & - & - & - & . & . & - \\
\hline Gross Alphe (pCi/L) & 0.72 & 4.67 & 0.00 & -1.25 & 0.20 & -0.90 & 0.48 & 1.40 \\
\hline Gross Alpha $(\mathrm{CB}+/-)$ & 1.30 & 4.00 & 1.80 & 1.60 & 1.70 & 3.20 & 2.00 & 2.40 \\
\hline Gross Beta $(\mathrm{pC} i / \mathrm{L})$ & 2.87 & 2.79 & 1.40 & 1.18 & 4.46 & 9.74 & -0.33 & 4.29 \\
\hline Gross Beta $(\mathrm{CE}+/-)$ & 2.50 & 5.10 & 2.80 & 2.70 & 2.70 & 5.50 & 2.80 & 3.00 \\
\hline
\end{tabular}

(CONTINUED) 
APPENDIX E. 3

Groundwater Quality Data, 1993

\begin{tabular}{|c|c|c|c|c|c|c|c|c|}
\hline \multirow{3}{*}{$\begin{array}{l}\text { Sampling Point } \\
\text { Location } \\
\text { - } \\
\text { Date Sampled }\end{array}$} & \multicolumn{3}{|c|}{$G W-748$} & \multicolumn{4}{|c|}{ Gw-749 } & GW-750 \\
\hline & \multicolumn{3}{|c|}{ GRIDK2 } & \multicolumn{4}{|c|}{ GRIDK2 } & GRIDK2 \\
\hline & $04 / 13 / 93$ & $08 / 06 / 93$ & $10 / 24 / 93$ & $01 / 21 / 93$ & $04 / 14 / 93$ & $08 / 06 / 93$ & $10 / 24 / 93$ & $01 / 21 / 93$ \\
\hline FIELD MEASUREMENTS & - & - & - & - & - & $\cdot$ & - & - \\
\hline Depth to Water (ft) & 6.38 & 9.42 & 7 & 6.17 & 5.18 & 5.61 & 7.7 & 10.47 \\
\hline Water Temp (degrees C) & 16.4 & 16.9 & 17.1 & 12.2 & 15 & 19.5 & 20.2 & 13.5 \\
\hline pH (pH units) & 7.8 & 7.7 & 7.5 & 6.7 & 7 & 7.1 & 7.3 & 6.9 \\
\hline sp. Cond. (umho/cm) & 444 & 529 & 494 & 516 & 460 & 593 & 666 & 448 \\
\hline Dissolved Oxygen (ppm) & 4.6 & 1.4 & 4 & 4.7 & 4.2 & 5.9 & 5.1 & 6.5 \\
\hline Oxidation/Reduction (mv) & 171 & 176 & 165 & 21 & 138 & 15 & -44 & 84 \\
\hline MISCELLANEOUS PARAMETERS & - & - & - & - & - & - & - & - \\
\hline $\mathrm{pH}$ (pH units) & 7.7 & 7.5 & 7.7 & 7.3 & 7.4 & 7.4 & 7.5 & 7.6 \\
\hline Sp. Cond. (umho/cm) & 457 & 533 & 524 & 550 & 512 & 644 & 704 & 475 \\
\hline TPH $(\mathrm{mg} / \mathrm{L})$ & • & • & • & • & • & • & $\cdot$ & • \\
\hline $\operatorname{TDS}(\mathrm{mg} / \mathrm{L})$ & 316 & 348 & 350 & 540 & 316 & 436 & 470 & 274 \\
\hline TSS (mg/L) & 142 & 12 & 17 & 2 & 20 & 13 & $<1$ & $<1$ \\
\hline Turbidity (NTU) & 1800 & 16 & 17 & 4.3 & 18 & 14 & 3.3 & 0.9 \\
\hline RADIOCHEMICAI PARAMETERS & - & • & • & - & - & • & • & • \\
\hline Gross Alpha (pCi/I) & 5.69 & -1.20 & 0.32 & 0.19 & 1.57 & 1.61 & 0.54 & -1.31 \\
\hline Gross Alpha $(\mathrm{CE}+/-)$ & 3.80 & 2.90 & 2.00 & 2.10 & 0.94 & 3.70 & 2.50 & 1.70 \\
\hline Gross Beta (pCi/L) & 13.70 & 0.33 & 0.58 & 5.12 & 3.76 & 1.50 & -0.88 & 4.42 \\
\hline Gross Beta $(\mathrm{CB}+/-)$ & 5.60 & 5.30 & 2.80 & 3.00 & 1.40 & 5.40 & 3.10 & 2.90 \\
\hline
\end{tabular}

(CONTINUED) 
APRENDIX R. 3

Groundwater Quality Data, 1993

\begin{tabular}{|c|c|c|c|c|c|c|c|c|}
\hline \multirow{3}{*}{$\begin{array}{l}\text { Sampling Point } \\
\text { Location } \\
\text { - } \\
\text { Date Sampled }\end{array}$} & \multicolumn{3}{|c|}{ GW-750 } & \multicolumn{4}{|c|}{ GW-751 } & \multirow{2}{*}{$\frac{G W-752}{\text { GRIDJ3 }}$} \\
\hline & \multicolumn{3}{|c|}{ GRIDK2 } & \multicolumn{4}{|c|}{ GRIDJ 3} & \\
\hline & $04 / 15 / 93$ & $08 / 06 / 93$ & $10 / 25 / 93$ & $01 / 11 / 93$ & $04 / 16 / 93$ & $08 / 02 / 93$ & $10 / 26 / 93$ & $01 / 11 / 93$ \\
\hline FIELD MBASUREMENTS & - & • & - & $\cdot$ & $\cdot$ & - & - & - \\
\hline Depth to Water (ft) & 10.2 & 13.25 & 13.5 & 4.45 & 4.14 & $6 \cdot 3$ & 6.15 & 3.95 \\
\hline Water Temp (degrees c) & 15.2 & 16.8 & $15 \cdot 4$ & 14.2 & 15.4 & 17.3 & 16.4 & 12.8 \\
\hline pH (pH units) & 7.1 & 7.7 & 7.2 & 7.4 & 7.1 & 7.2 & 7.5 & 5.5 \\
\hline Sp. Cond. (umho/cm) & 399 & 531 & 465 & 361 & 361 & 355 & 365 & 249 \\
\hline Dissolved Oxygen (ppm) & 2.2 & 0.7 & 0.4 & 3 & $2 \cdot 1$ & 0.4 & 0.3 & 5 \\
\hline Oxidation/Reduction (mV) & 51 & -57 & -85 & 38 & 110 & 72 & -76 & 125 \\
\hline MISCBLLANBOUS PARAMETERS & . & . & • & . & - & . & . & . \\
\hline pH (pH units) & 7.6 & 7.5 & 7.8 & 8 & 7.7 & 7.8 & 7.9 & 6.3 \\
\hline Sp. Cond. (umho/cm) & 478 & 498 & 488 & 389 & 392 & 372 & 390 & 276 \\
\hline $\operatorname{TPH}(\mathrm{mg} / \mathrm{L})$ & $\cdot$ & $\cdot$ & $\cdot$ & $\cdot$ & $\cdot$ & $\cdot$ & $\cdot$ & • \\
\hline $\operatorname{TDS}(\mathrm{mg} / L)$ & 290 & 308 & 312 & 226 & 218 & 228 & 2.52 & 160 \\
\hline TsS (mg/L) & $<1$ & 7 & $<1$ & 7 & 4 & 2 & 9 & $<1$ \\
\hline Turbidity (NTU) & 0.9 & 7.9 & 1 & 2.1 & 1.7 & 1.6 & 2.2 & 7.6 \\
\hline RADIOCHEMICAI RARAMBTERS & - & - & $\cdot$ & - & $\cdot$ & $\cdot$ & • & - \\
\hline Gross Alpha (pCi/L) & -0.80 & -0.60 & 1.25 & 0.71 & -0.61 & -1.47 & 2.44 & -0.72 \\
\hline Gross Alpha $(\mathrm{CB}+/-)$ & 0.79 & 3.10 & 1.50 & 2.10 & 0.60 & 4.60 & 1.70 & 1.40 \\
\hline Gross Beta (pCi/L) & 0.70 & 5.44 & 1.99 & 1.49 & 1.82 & -6.08 & 1.98 & 3.44 \\
\hline Gross Beta $(\mathrm{CB}+/-)$ & 1.30 & 5.60 & 2.70 & 2.80 & 1.30 & 5.90 & 2.70 & 2.90 \\
\hline
\end{tabular}

(CONTINOED) 
APPENDIX $\mathrm{E} .3$

\begin{tabular}{|c|c|c|c|c|c|c|c|c|}
\hline \multirow{3}{*}{$\begin{array}{l}\text { Sampling Point } \\
\text { Location } \\
\text { Date Sampled }\end{array}$} & \multicolumn{3}{|c|}{ GW-752 } & \multicolumn{4}{|c|}{ GW-753 } & \multirow{2}{*}{$\begin{array}{l}\text { GW-754 } \\
\text { GRIDJ } 2\end{array}$} \\
\hline & \multicolumn{3}{|c|}{ GRIDJ3 } & \multicolumn{4}{|c|}{ GRIDJ2 } & \\
\hline & $04 / 16 / 93$ & $08 / 02 / 93$ & $10 / 26 / 93$ & $01 / 15 / 93$ & $04 / 08 / 93$ & $08 / 03 / 93$ & $10 / 21 / 93$ & $01 / 18 / 93$ \\
\hline FIELD MBASUREMENTS & $\cdot$ & $\cdot$ & $\cdot$ & $\cdot$ & • & . & $\cdot$ & - \\
\hline Depth to Water (ft) & 3.64 & 6.1 & 5.95 & 4 & 4.62 & 10.68 & 9.6 & 8.15 \\
\hline Water Temp (degrees $C$ ) & 12.6 & 21.1 & 19.7 & 14.3 & 15.8 & 18.3 & 15.7 & 12.8 \\
\hline pH (pH units) & 5.1 & 4.7 & 5.6 & 7.4 & 7.4 & 7.8 & 7.3 & 5.8 \\
\hline Sp. Cond. (umho/cm) & 251 & 233 & 348 & 399 & 331 & 381 & 335 & 214 \\
\hline Dissolved Oxygen (ppm) & 2.1 & 0.5 & 0.4 & 4.2 & 2.3 & 0.4 & 0.6 & 3.6 \\
\hline Oxidation/Reduction (mv) & 142 & 129 & 96 & -85 & 73 & -77 & -66 & -4 \\
\hline MISCELIANEOUS PARAMETERS & • & $\cdot$ & $\cdot$ & $\cdot$ & $\cdot \dot{ }$ & • & $\cdot$ & • \\
\hline pH (pH units) & $5 . \dot{9}$ & $5 . \dot{8}$ & 6 & $7 . \dot{5}$ & $7 . \dot{5}$ & $7 . \dot{8}$ & $7 . \dot{7}$ & 7.2 \\
\hline Sp. Cond. (umho/cm) & 269 & 262 & 254 & 383 & 394 & 398 & 402 & 297 \\
\hline TPH $(m g / L)$ & • & $\cdot$ & $\cdot$ & . & $\cdot$ & • & $\cdot$ & • \\
\hline $\operatorname{TDS}(\mathrm{mg} / \mathrm{I})$ & 160 & 184 & 180 & 242 & 238 & 258 & 256 & 178 \\
\hline TSS $(m g / L)$ & 19 & 18 & 5 & $<1$ & 5 & 2 & 2 & 70 \\
\hline Turbidity (NTO) & 13 & 14 & 3.7 & 1.3 & 1 & 1.4 & 3 & 35 \\
\hline RADIOCHRMICAL PARAMETRRS & - & $\cdot$ & $\cdot$ & $\cdot$ & - & • & - & • \\
\hline Gross Alpha $(\mathrm{pC} i / L)$ & -0.18 & 1.72 & 0.88 & 0.85 & -0.78 & -4.46 & 1.69 & 2.38 \\
\hline Gross Alpha $(\mathrm{CB}+/-)$ & 0.57 & 4.90 & 1.40 & 1.90 & 1.80 & 4.20 & 2.10 & 2.00 \\
\hline Gross Beta $(\mathrm{pCl} / \mathrm{L})$ & 0.31 & -0.49 & 0.58 & 2.77 & 3.34 & -8.57 & 0.41 & 6.90 \\
\hline Gross Beta $(\mathrm{CE}+/-)$ & 1.20 & 6.20 & 2.60 & 2.80 & 2.90 & 5.80 & 2.80 & 3.00 \\
\hline
\end{tabular}

(CONTINUED) 
APPENDIX $\mathrm{B} .3$

Groundwater Quality Data, 1993

\begin{tabular}{|c|c|c|c|c|c|c|c|c|}
\hline \multirow{3}{*}{$\begin{array}{l}\text { Sampling Point } \\
\text { Location } \\
\text { - } \\
\text { Date Sampled }\end{array}$} & \multicolumn{3}{|c|}{$G W-754$} & \multicolumn{4}{|c|}{$G W-755$} & \multirow{2}{*}{$\frac{\text { GW-756 }}{\text { GRIDJ1 }}$} \\
\hline & \multicolumn{3}{|c|}{ GRIDJ 2} & \multicolumn{4}{|c|}{ GRIDJ1 } & \\
\hline & $04 / 08 / 93$ & $08 / 04 / 93$ & $10 / 22 / 93$ & $01 / 14 / 93$ & $04 / 07 / 93$ & $08 / 03 / 93$ & $10 / 21 / 93$ & $0.1 / 15 / 93$ \\
\hline FIELD MRASUREMENTS & - & - & - & $\cdot$ & - & - & - & - \\
\hline Depth to Water ( $f t$ ) & 8.15 & 13.75 & $12 \cdot 4$ & 6.35 & 7.15 & 9.2 & 8.92 & 5.42 \\
\hline Water Temp (degrees C) & 16.5 & 20 & 13.8 & 13.5 & 16.2 & 18.3 & 16 & 13.2 \\
\hline $\mathrm{pH}$ (pH unitz) & 6.9 & 7.5 & 6.9 & 7.9 & 8.2 & 8.1 & 7.7 & 5.4 \\
\hline Sp. Cond. (umho/cm) & 237 & 369 & 294 & 371 & 385 & 428 & 416 & 299 \\
\hline Dissolved Oxygen (ppm) & 7.3 & 2.4 & 2 & $5 \cdot 1$ & 7.9 & 3.5 & 2 & 6.4 \\
\hline Oxidation/Reduction (mv) & 64 & -76 & -19 & 128 & 203 & 92 & 120 & 135 \\
\hline MISCELLANBOUS PARAMETERS & • & . & . & - & • & - & . & - \\
\hline pH (pH units) & 6.3 & 7.2 & 7.1 & 8.2 & 8.4 & 8.3 & 8 & 6 \\
\hline sp. Cond. (umho/cm) & 187 & 277 & 298 & 460 & 452 & 460 & 479 & 289 \\
\hline MPH $(\mathrm{mg} / \mathrm{L})$ & • & $\cdot$ & $\cdot$ & $\cdot$ & $\cdot$ & - & $\cdot$ & • \\
\hline TDS (mg/L) & 136 & 182 & 202 & 282 & 290 & 304 & 308 & 172 \\
\hline $\operatorname{TsS}(\mathrm{mg} / \mathrm{L})$ & 110 & 22 & 7 & 12 & 118 & 12 & $<1$ & 1729 \\
\hline Turb1dity (NTU) & 24 & 20 & 5.2 & 10 & 16 & 12 & 2.7 & 680 \\
\hline RADIOCKEMICAL PARAMETERS & - & - & - & - & - & - & - & • \\
\hline Gross Alpha (pCi/L) & 3.14 & $-2 \cdot 30$ & 0.75 & -1.00 & -1.34 & -3.68 & 1.51 & $18 \cdot 30$ \\
\hline Gross Alphe $(\mathrm{CE}+/-)$ & 2.10 & 4.10 & 1.90 & 1.50 & 3.80 & 4.50 & 2.20 & 6.70 \\
\hline Gross Beta (pci/L) & 6.15 & $-2 \cdot 38$ & 0.91 & 0.33 & 5.53 & -8.94 & 1.34 & 38.60 \\
\hline Gross Beta $(\mathrm{CB}+/-)$ & 3.00 & 5.50 & 2.80 & 2.70 & 5.00 & 5.80 & 2.90 & 7.50 \\
\hline
\end{tabular}

(CONTINUED) 
APPENDIX E.3

Groundwater Quality Data, 1993

\begin{tabular}{|c|c|c|c|c|c|c|c|c|}
\hline \multirow{3}{*}{$\begin{array}{l}\text { Sampling Point } \\
\text { Location } \\
\text { - } \\
\text { Date Sampled }\end{array}$} & \multicolumn{3}{|c|}{ GW-756 } & \multicolumn{4}{|c|}{$6 w-758$} & \multirow{2}{*}{$\begin{array}{c}\text { GW-759 } \\
\text { GRIDG1 }\end{array}$} \\
\hline & \multicolumn{3}{|c|}{ GRIDJ1 } & \multicolumn{4}{|c|}{ GRIDG1 } & \\
\hline & $04 / 08 / 93$ & $08 / 03 / 93$ & $10 / 21 / 93$ & $01 / 25 / 93$ & $04 / 13 / 93$ & $09 / 12 / 93$ & $11 / 19 / 93$ & $01 / 26 / 93$ \\
\hline FIELD MEASUREMENTS & $\cdot$ & $\cdot$ & $\cdot$ & $\cdot$ & - & $\cdot$ & $\cdot$ & . \\
\hline Dapth to Water $(f t)$ & 5.85 & 7.45 & 7.25 & 13.55 & 15 & 17.25 & 14.9 & 15.85 \\
\hline Water Temp (degrees C) & 13.2 & 19.6 & 18.6 & 15.6 & 17.3 & 17.4 & 15.6 & 9.5 \\
\hline pH (pH units) & 5.4 & 5.9 & 5.7 & 7.6 & 7.6 & 6.9 & 7.4 & 7.3 \\
\hline Sp. Cond. (wmho/cm) & 245 & 304 & 314 & 373 & 367 & 421 & 422 & 399 \\
\hline Diseolved Oxygen (ppm) & 5.1 & 0.9 & 1 & 3.1 & 6 & 0.5 & 4.4 & 8.1 \\
\hline Oxidation/Reduction (mV) & 168 & 50 & 23 & -24 & -11 & 92 & 65 & 106 \\
\hline MISCELIANEOUS PARAMETERS & - & • & - & $\cdot$ & - & $\cdot$ & - & - \\
\hline $\mathrm{pH}$ (pH units) & 5.9 & $6 . \dot{3}$ & i & 7.5 & 7.6 & $7 . \dot{8}$ & 7.6 & 7.6 \\
\hline Sp. Cond. (umho/cm) & 283 & 330 & 350 & 475 & 444 & 447 & 465 & 475 \\
\hline TPH $(\mathrm{mg} / \mathrm{L})$ & • & • & & $\cdot$ & • & . & $\cdot$ & • \\
\hline TDS (mg/L) & 194 & 226 & 228 & 276 & 278 & 292 & 284 & 300 \\
\hline Tss (mg/L) & 1233 & 482 & 114 & 4 & 18 & 3 & 1 & 53 \\
\hline Turbidity (NTU) & 1500 & 340 & 105 & 1.7 & 3 & 2.5 & 3.6 & 52 \\
\hline RADIOCHEMICAI PARAMETERS & • & $\cdot \dot{1}$ & $\cdot$ & $\cdot \dot{ }$ & • & $\cdot \dot{ }$ & $\cdot$ & • \\
\hline Gross Alpha (pCi/L) & 2.40 & -1.17 & 1.73 & $0.5 \dot{4}$ & $0.8 \dot{1}$ & -1.70 & -0.53 & 2.94 \\
\hline Gross Alpha $(\mathrm{CE}+/-)$ & 3.40 & 4.60 & 2.20 & 2.10 & 1.00 & 3.80 & 1.40 & 2.40 \\
\hline Gross Beta $(\mathrm{pCi} / \mathrm{L})$ & 7.92 & -3.78 & 6.32 & -8.12 & 1.31 & 1.74 & -5.33 & 5.99 \\
\hline Gross Beta $(\mathrm{CE}+/-)$ & 5.60 & 6.00 & 3.10 & 3.30 & 1.30 & 5.80 & 2.90 & 3.10 \\
\hline
\end{tabular}

( CONTINOED) 
APPENDIX E. 3

Groundwater Quality Data, 1993

\begin{tabular}{|c|c|c|c|c|c|c|c|c|}
\hline \multirow{3}{*}{$\begin{array}{l}\text { Sampling Point } \\
\text { Location } \\
\text { - } \\
\text { Date Sampled }\end{array}$} & \multicolumn{3}{|c|}{ Gw-759 } & \multicolumn{4}{|c|}{ GW-760 } & Gw-761 \\
\hline & \multicolumn{3}{|c|}{ GRIDG1 } & \multicolumn{4}{|c|}{ GRIDG2 } & \multirow{2}{*}{\begin{tabular}{|c|} 
GRIDE2 \\
$01 / 26 / 93$
\end{tabular}} \\
\hline & $04 / 15 / 93$ & $09 / 13 / 93$ & $11 / 19 / 93$ & $01 / 27 / 93$ & $04 / 16 / 93$ & $09 / 14 / 93$ & $11 / 30 / 93$ & \\
\hline FIELD MBASUREMBNTS & - & $\cdot$ & - & $\cdot$ & $\cdot$ & - & $\cdot$ & - \\
\hline Depth to Water (ft) & 16.8 & 18.9 & 20.15 & 9.22 & 9.8 & 13.6 & 10.75 & 8.95 \\
\hline Water Termp (degrees C) & 19.7 & 16.8 & 14.5 & 17.2 & 16.5 & 23.3 & 14.6 & 12.3 \\
\hline pH (pH units) & 7.4 & 7.5 & 7.5 & 7 & 7 & 7.3 & 8 & 6.9 \\
\hline Sp. Cond. (umho/cm) & 383 & 462 & 424 & 609 & 571 & 823 & 593 & 191 \\
\hline Diesolved Oxygen (ppm) & 2.2 & 4.4 & 7.9 & 7.7 & 6.8 & 4.4 & 8.1 & 7.2 \\
\hline Oxidation/Reduction (mv) & 215 & 285 & 164 & 172 & 231 & 361 & 124 & 158 \\
\hline MISCELIANEOUS PARAMRTERS & - & - & • & - & • & - & . & - \\
\hline pH (ph units) & 7.8 & 7.8 & 7.7 & 7.7 & 7.8 & 7.9 & 0.1 & 7.2 \\
\hline sp. Cond. (umho/om) & 458 & 470 & 477 & 700 & 755 & 826 & 734 & 222 \\
\hline $\operatorname{TPH}(\mathrm{mg} / \mathrm{I})$ & • & & $\cdot$ & • & - & • & • & • \\
\hline $\operatorname{TDS}(\mathrm{mg} / \mathrm{I})$ & 270 & 270 & 284 & 464 & 468 & 566 & 440 & 162 \\
\hline TSS (mg/L) & 2 & 592 & 13 & 6 & 2 & 7 & $<1$ & 41 \\
\hline Turbidity (NTU) & 3.5 & 80 & 12 & 3.5 & 6 & 24 & 2.5 & 14 \\
\hline RADIOCHEMICAC PARAMTIERS & - & - & - & - & - & - & - & - \\
\hline Grose Alpha (pc1/I) & -1.82 & 8.50 & -1.23 & -0.20 & 4.53 & -2.60 & -1.15 & 3.34 \\
\hline Grose Alpha $(\mathrm{CB}+/-)$ & 0.67 & 9.20 & 1.30 & 1.80 & 1.40 & 4.00 & 1.30 & 2.10 \\
\hline Gross Beta (pci/L) & -2.19 & 15.30 & -5.17 & -1.97 & 1.30 & -1.23 & 1.18 & 2.83 \\
\hline Gross Beta $(\mathrm{CB}+/-)$ & 1.20 & 12.00 & 2.90 & 2.70 & 1.30 & 5.70 & 2.50 & 2.90 \\
\hline
\end{tabular}

(CONTINUED) 
APPENDIX $\mathrm{R} \cdot 3$

Groundwater Quality Data, 1993

\begin{tabular}{|c|c|c|c|c|c|c|c|c|}
\hline \multirow{3}{*}{$\begin{array}{l}\text { Sampling Point } \\
\text { Location } \\
\text { Date Sampled }\end{array}$} & \multicolumn{3}{|c|}{ OW-761 } & \multicolumn{5}{|c|}{ Gw-762 } \\
\hline & \multicolumn{3}{|c|}{ GRIDE2 } & \multicolumn{5}{|c|}{ ERIDJ3 } \\
\hline & $04 / 16 / 93 \mid$ & $09 / 13 / 93$ & $11 / 29 / 93$ & $01 / 23 / 93$ & $02 / 22 / 93$ & $04 / 20 / 93$ & $08 / 04 / 93$ & $10 / 28 / 93$ \\
\hline FIELD MAASUREMENTS & - & - & $\cdot$ & - & $\cdot$ & $\cdot$ & $\cdot$ & $\cdot$ \\
\hline Depth to Water (ft) & 9.15 & 13.25 & 10.22 & 13.25 & . & 13 & 13.9 & 13.75 \\
\hline Water Temp (dagrees C) & 14 & 21.3 & 15.3 & 17.4 & $\cdot$ & 17.9 & 19.2 & 17.3 \\
\hline pH (pH units) & 6.6 & 6.6 & 7 & 7.2 & - & 7.3 & 6.8 & 7.3 \\
\hline 8p. Cond. (umho/cm) & 166 & 201 & 169 & 597 & $\cdot$ & 502 & 595 & 560 \\
\hline Diesolved Oxygen (ppm) & 4 & 4.2 & 7.5 & 1 & - & 1.2 & 1.4 & 0.4 \\
\hline Oxidation/Reduction (mv) & 298 & 427 & 189 & 63 & . & 106 & 27 & -45 \\
\hline MISCELLANEOUS PARAKETERS & $\cdot$ & $\dot{.}$ & $\cdot$ & $\dot{.}$ & $\cdot$ & $\cdot$ &. & • \\
\hline pH (pH unites) & $7 . \dot{1}$ & 7.3 & 7.2 & 7.4 & $\cdot$ & 7.5 & 7.6 & 7.5 \\
\hline sp. Cond. (umho/cm) & 212 & 215 & 200 & 604 & - & 592 & 611 & 608 \\
\hline TPH $(m g / L)$ & $\cdot$ & $\cdot$ & $\cdot$ & $\cdot$ & $\cdot$ & $\cdot$ & $\cdot$ & • \\
\hline $\operatorname{TDS}(\mathrm{mg} / \mathrm{L})$ & 122 & 132 & 138 & 332 & $\cdot$ & 344 & 358 & 368 \\
\hline Ts8 (mg/I) & 6 & 12 & 27 & 13 & $\cdot$ & 15 & 21 & 8 \\
\hline Turbidity (NTU) & 5.5 & 520 & 22 & 9.9 & - & 16 & 35 & 7.5 \\
\hline RADTOCHEMICAI PARARERER & $\cdot$ & $\cdot$ & - & - & $\cdot$ & $\cdot$ & . & $\cdot$ \\
\hline Gross Alpha (pCi/L) & 1.41 & 0.59 & 0.33 & -0.60 & $\cdot$ & -0.48 & -3.28 & -1.13 \\
\hline Grose Alpha $(\mathrm{CE}+/-)$ & 0.79 & 3.60 & 1.50 & 1.90 & $\cdot$ & 0.96 & 4.20 & 1.70 \\
\hline Gross Beta $(\mathrm{pCl} / \mathrm{L})$ & 1.27 & -0.51 & 5.26 & 6.09 & . & 4.68 & 1.54 & 2.42 \\
\hline Gross Beta $(\mathrm{CE}+/-)$ & 1.30 & 5.50 & 2.80 & 2.90 & - & 1.40 & 5.80 & 2.70 \\
\hline
\end{tabular}

(CONTINUED) 
APPENDIX 4.3

Groundwater Quallty Data, 1993

\begin{tabular}{|c|c|c|c|c|c|c|c|c|}
\hline \multirow{3}{*}{$\begin{array}{l}\text { sampling point } \\
\text { Location } \\
\text { Date sampled }\end{array}$} & \multicolumn{4}{|c|}{$6 w-763$} & \multicolumn{4}{|c|}{ CW-764 } \\
\hline & \multicolumn{4}{|c|}{ ERIDJ3 } & \multicolumn{4}{|c|}{ GRIDII } \\
\hline & $01 / 25 / 93$ & $04 / 21 / 93$ & $08 / 05 / 93$ & $10 / 29 / 93$ & $01 / 23 / 93$ & $04 / 13 / 93$ & $09 / 12 / 93$ & $11 / 12 / 93$ \\
\hline FIELd masurEments & - & - & . & . & - & . & - & - \\
\hline Depth to Water (ft) & 10.2 & 9.95 & 10.65 & 10.35 & 20.68 & 20.25 & 21 & 20.87 \\
\hline Water Temp (degrees C) & 7.9 & 12.7 & 22.3 & 19.1 & 16.7 & 27.7 & 18.6 & 16.5 \\
\hline pH (pB units) & 6.9 & 6.5 & 6.3 & 6.9 & 7.6 & 7.9 & 7.2 & 7.7 \\
\hline sp. Cond. (umho/cm) & 628 & 593 & 665 & 668 & 360 & 303 & 350 & 355 \\
\hline Diseolved Oxygen (ppm) & 1.5 & 2.9 & 1.7 & 2.9 & 2.8 & 9 & 3.2 & 7.3 \\
\hline Oxidation/Reduction (mV) & -58 & -27 & -34 & -70 & 22 & 137 & 165 & 55 \\
\hline MI8CELLANEOOS PARAMETERS & $\cdot$ & . & $\cdot$ & $\cdot$ & $\cdot$ & $\cdot$ & $\cdot$ & • \\
\hline pH (pH undte) & $\dot{7}$ & $7 . \dot{1}$ & 6.9 & 7.1 & 7.7 & $\dot{8}$ & $7 . \dot{9}$ & 7.7 \\
\hline 8p. Cond. (unho/cm) & 718 & 695 & 716 & 717 & 380 & 327 & 356 & 391 \\
\hline TPH $(\mathrm{mg} / \mathrm{I})$ & & $\cdot$ & $\cdot$ & $\cdot$ & - & $\cdot$ & $\cdot$ & • \\
\hline TDS (mg/L) & 388 & 412 & 422 & 434 & 238 & 240 & 246 & 254 \\
\hline Ts8 $(m g / L)$ & 53 & 30 & 52 & 35 & 2 & 3 & 5 & 113 \\
\hline Turbidity (RTO) & 35 & 30 & 46 & 30 & 2.1 & 1.1 & 7.1 & 320 \\
\hline RADIOCHEMICAL PARAKETERS & - & - & $\cdot$ & • & . & . & • & • \\
\hline Grose Alpha $(\mathrm{pCl} / \mathrm{L})$ & 1.51 & -3.42 & 2.81 & 3.94 & -0.35 & 2.34 & 2.55 & -0.89 \\
\hline Grose Alpha $(\mathrm{CB}+/-)$ & 2.70 & 3.50 & 4.00 & 2.50 & 1.90 & 2.10 & 4.20 & 1.40 \\
\hline Grose Beta $(\mathrm{pC} 1 / \mathrm{L})$ & -4.15 & 1.40 & 3.32 & 4.10 & -5.49 & 8.72 & 2.07 & -4.76 \\
\hline Grose Beta $(\mathrm{Cs}+/-)$ & 3.60 & 2.70 & 5.20 & 2.90 & 3.40 & 3.00 & 5.70 & 2.90 \\
\hline
\end{tabular}

(CONTINUED) 
APPENDIX 8.3

Groundwater Qual1ty Data, 1993

\begin{tabular}{|c|c|c|c|c|c|c|c|c|}
\hline \multirow{3}{*}{$\begin{array}{l}\text { Sampling Point } \\
\text { - } \\
\text { Location } \\
\text { - } \\
\text { Date sampled }\end{array}$} & \multicolumn{4}{|c|}{$G W-765$} & \multicolumn{4}{|c|}{ GW-766 } \\
\hline & \multicolumn{4}{|c|}{ ORIDE 1} & \multicolumn{4}{|c|}{ GRIDI2 } \\
\hline & $01 / 25 / 93$ & $04 / 13 / 93$ & $09 / 12 / 93$ & $11 / 19 / 93$ & $02 / 01 / 93$ & $04 / 15 / 93$ & $08 / 03 / 93$ & $10 / 13 / 93$ \\
\hline FIELD MASURENQHTS & - & - & - & - & $\cdot$ & $\cdot$ & - & - \\
\hline Depth to Water (ft) & 19.54 & 20.33 & 21.45 & 19.55 & 11.73 & 11.55 & 12.25 & 22.33 \\
\hline Water Tamp (degrees C) & 13.2 & 17.6 & 19.1 & 15.7 & 17.1 & 16.1 & 21.8 & 17.1 \\
\hline pH (pH undte) & 7.1 & 7.2 & $6 \cdot 5$ & 7.1 & 10.1 & 8.5 & 9.1 & 8.7 \\
\hline 8p. Cond. (umho/cm) & 419 & 448 & 515 & 490 & 271 & 237 & 261 & 260 \\
\hline Diseolved Oxygen (ppm) & 6.7 & $7 \cdot 3$ & 1.9 & 6.9 & 1.5 & 2.5 & 5.3 & 4 \\
\hline Oxidation/Reduction (mV) & 171 & 139 & 340 & 220 & 138 & 112 & 11 & 133 \\
\hline HISCELWATEOUS PARARTERS & - & - & $\cdot$ & $\cdot$ & - & $\cdot$ & - & - \\
\hline pH (pH unite) & 7.3 & 7.3 & 7.5 & 7.5 & 9.4 & 8.8 & 8.5 & 8.6 \\
\hline 8p. Cond. (umho/cm) & 576 & 548 & 553 & 568 & 166 & 257 & 269 & 275 \\
\hline $\operatorname{TPH}(\mathrm{mg} / \mathrm{L})$ & & $\cdot$ & $\cdot$ & $\cdot$ & $<0.1$ & $<0.1$ & 0.007 & $<0.1$ \\
\hline $\operatorname{TDs}(\mathrm{mg} / \mathrm{L})$ & 434 & 364 & 362 & 380 & 128 & 166 & 182 & 202 \\
\hline $288(\mathrm{mg} / \mathrm{L})$ & 349 & 97 & 264 & 158 & 64 & 1 & 2 & 3 \\
\hline Turbidity (WMU) & 620 & 80 & 250 & 130 & 7.4 & 2 & 1.6 & $2 \cdot 4$ \\
\hline RADIOCHAICAI PARANTYRS & - & - & . & - & - & - & - & - \\
\hline Grose Alpha (pCl/I) & 4.93 & -0.19 & 17.50 & -0.36 & -0.69 & -0.53 & $-2 \cdot 30$ & 3.97 \\
\hline Grose Alpha $(\mathrm{CB}+/-)$ & 4.80 & 0.80 & 10.00 & 1.50 & 1.60 & 0.72 & 4.40 & 2.10 \\
\hline Grove Beta $(p C I / L)$ & 4.90 & 0.22 & 6.93 & $-5 \cdot 15$ & 6.32 & 3.45 & -6.88 & 5.21 \\
\hline Grose Beta $(\mathrm{CB}+/-)$ & 7.60 & 1.20 & 12.00 & 2.90 & 2.90 & 1.30 & 5.80 & 2.80 \\
\hline
\end{tabular}

(CONTINUSD) 
APPENDIX E.3

Groundwater Quality Data, 1993

\begin{tabular}{|c|c|c|c|c|c|c|c|c|}
\hline \multirow{3}{*}{$\begin{array}{l}\text { Sampling Point } \\
\text { Iocation } \\
\text { - } \\
\text { Date Sampled }\end{array}$} & \multicolumn{4}{|c|}{$G W-767$} & \multicolumn{4}{|c|}{$G W-768$} \\
\hline & \multicolumn{4}{|c|}{ GRIDI2 } & \multicolumn{4}{|c|}{ GRIDI1 } \\
\hline & $02 / 02 / 93$ & $04 / 15 / 93$ & $08 / 04 / 93$ & $10 / 13 / 93$ & $01 / 29 / 93$ & $04 / 07 / 93$ & $08 / 04 / 93$ & $10 / 13 / 93$ \\
\hline FIELD MEASUREMENTS & $\cdot$ & $\cdot$ & $\cdot$ & - & $\cdot$ & - & $\cdot$ & - \\
\hline Depth to Water ( $f t)$ & 12.98 & 12.65 & 12.6 & 13.67 & 18.1 & 18.2 & 19.4 & 19.1 \\
\hline Water Temp (degrees C) & 12 & 16.3 & 19.7 & 20 & 15.2 & 16 & 16.4 & 13.4 \\
\hline $\mathrm{pH}$ (pH units) & 5.8 & 5.2 & 4.8 & 5.5 & 7.6 & $7 \cdot 4$ & 8.3 & 7 \\
\hline Sp. Cond. (umho/cm) & 66 & 33 & 46 & 63 & 518 & 388 & 599 & 498 \\
\hline Dissolved Oxygen (ppm) & 6.6 & 6.7 & 3.5 & 2.7 & 8.9 & 4.6 & 5.1 & 3.9 \\
\hline Oxidation/Reduction (mv) & 245 & 292 & 244 & 213 & 148 & 71 & 94 & 198 \\
\hline MISCELIANEOUS PARAMETERS & . & . & . & • & . & . &. & - \\
\hline $\mathrm{pH}$ (pH units) & 5.8 & 5.7 & 5.5 & 5.9 & 7.9 & 8 & 7.8 & 7.8 \\
\hline Sp. Cond. (unho/cm) & 45 & 39 & 52 & 42 & 472 & 490 & 512 & 505 \\
\hline $\operatorname{TPH}(m g / L)$ & 0.0038 & $<0.1$ & $<0.1$ & $<0.1$ & • & • & - & • \\
\hline TDS (mg/L) & 54 & 32 & 56 & 64 & 340 & 304 & 322 & 342 \\
\hline TsS (mg/I) & 33 & 7 & $<1$ & 3 & 5 & $<1$ & 2 & $<1$ \\
\hline Turbidity (NTU) & 2.9 & 0.5 & 1.6 & 1.5 & 3 & 1.2 & 1.9 & 1 \\
\hline RADIOCHEMICAL PARAMETERS & - & - & - & - & - & - & - & - \\
\hline Gross Alpha (pCi/L) & 1.56 & 1.82 & -2.23 & 2.56 & 2.57 & -2.54 & -3.38 & 2.22 \\
\hline Gross Alpha $(\mathrm{CB}+/-)$ & 1.80 & 0.70 & 4.00 & 1.80 & 1.80 & 3.40 & 4.30 & 2.00 \\
\hline Gross Beta (pCi/I) & 3.11 & 0.65 & -2.37 & 4.34 & 5.57 & 6.82 & 0.52 & 5.63 \\
\hline Gross Beta $(\mathrm{CE}+/-)$ & 2.90 & 1.30 & 5.50 & 2.80 & 3.00 & 5.10 & 5.70 & 2.90 \\
\hline
\end{tabular}

(CONTINUED) 
APPENDIX E. 3

Groundwater Quality Data, 1993

\begin{tabular}{|c|c|c|c|c|c|c|c|c|}
\hline \multirow{3}{*}{$\begin{array}{l}\text { Sampling Point } \\
\text { - } \\
\text { Location } \\
\text { Date Sampled }\end{array}$} & \multicolumn{4}{|c|}{$G W-769$} & \multicolumn{4}{|c|}{$\mathrm{GW}-770$} \\
\hline & \multicolumn{4}{|c|}{ GRIDG3 } & \multicolumn{4}{|c|}{ GRIDG3 } \\
\hline & $01 / 27 / 93$ & $04 / 19 / 93$ & $09 / 14 / 93$ & $11 / 30 / 93$ & $01 / 27 / 93$ & $04 / 19 / 93$ & $09 / 14 / 93$ & $11 / 29 / 93$ \\
\hline FIELD MEASUREMENTS & - & - & $\cdot$ & - & $\cdot$ & $\cdot$ & - & • \\
\hline Depth to Water ( $f t$ ) & 10.36 & 11.5 & 12.57 & 10 & 12.89 & 13.39 & 14.75 & 11.83 \\
\hline Water Temp (degrees C) & 17.5 & 19.2 & 20.5 & 18.2 & 18.3 & 17.2 & 20.8 & 19.3 \\
\hline pH (pH units) & 7.2 & 7.5 & 7 & 7.5 & 6 & 7.2 & 6.7 & 7.1 \\
\hline Sp. Cond. (umho/cm) & 422 & 378 & 469 & 424 & 287 & 228 & 316 & 298 \\
\hline Dissolved Oxygen (ppm) & 2.1 & 0.9 & 0.5 & 5 & 7.7 & 5.4 & 3.6 & 7.7 \\
\hline Oxidation/Reduction (mV) & 36 & 62 & 123 & 107 & 139 & 237 & 277 & 210 \\
\hline MISCELIANEOUS PARAMETERS & • & $\dot{.}$ & • & $\cdot$ & . & $\cdot$ & • & • \\
\hline pH (pH units) & 7.5 & $7 . \dot{7}$ & 7.7 & 7.9 & 7.4 & 7.4 & 7.4 & 7.3 \\
\hline Sp. Cond. (umho/cm) & 460 & 455 & 477 & 480 & 321 & 288 & 325 & 320 \\
\hline TPH $(\mathrm{mg} / \mathrm{L})$ & • & . & • & • & $\cdot$ & . & • & • \\
\hline $\operatorname{TDS}(\mathrm{mg} / \mathrm{L})$ & 282 & 278 & 294 & 298 & 188 & 190 & 212 & 212 \\
\hline $\operatorname{TSS}(\mathrm{mg} / \mathrm{L})$ & $<1$ & 1 & $<1$ & $<1$ & 8 & 41 & 2 & 10 \\
\hline Turbidity (NTU) & 1.1 & 0.9 & 2 & 1 & 7.6 & 8.5 & 11 & 13 \\
\hline RADIOCHEMICAL PARAMETERS & - & $\cdot$ & $\cdot$ & $\cdot$ & $\cdot$ & $\cdot$ & • & • \\
\hline Gross Alpha (pCi/L) & 0.59 & -0.46 & -0.96 & -0.90 & 2.28 & 3.96 & 1.23 & -0.52 \\
\hline Gross Alpha $(\mathrm{CE}+/-)$ & 2.00 & 0.91 & 3.70 & 1.30 & 2.10 & 1.30 & 1.70 & 1.30 \\
\hline Gross Beta (pCi/L) & 2.40 & 1.05 & -2.77 & 1.89 & 3.54 & 3.54 & 3.35 & 3.26 \\
\hline Gross Beta $(\mathrm{CE}+/-)$ & 2.90 & 1.30 & 5.40 & 2.50 & 3.00 & 1.40 & 2.80 & 2.60 \\
\hline
\end{tabular}

(CONTINUED) 
APPEHDIX E. 3

Groundwater Quality Data, 1993

\begin{tabular}{|c|c|c|c|c|c|c|c|c|}
\hline \multirow{3}{*}{$\begin{array}{l}\text { Sampling Point } \\
\text { Location } \\
\text { - } \\
\text { Date Sampled }\end{array}$} & \multicolumn{4}{|c|}{ GW-771 } & \multicolumn{4}{|c|}{ GW-772 } \\
\hline & \multicolumn{4}{|c|}{ GRIDC1 } & \multicolumn{4}{|c|}{ GRIDC1 } \\
\hline & $01 / 23 / 93$ & $04 / 12 / 93$ & $09 / 03 / 93$ & $11 / 11 / 93$ & $01 / 23 / 93$ & $04 / 12 / 93$ & $09 / 12 / 93$ & $11 / 12 / 93$ \\
\hline FIELD MEASUREMENTS & - & - & - & - & $\cdot$ & $\cdot$ & $\cdot$ & $\cdot$ \\
\hline Depth to Water (ft) & 9.62 & 8.65 & 13.28 & 11.68 & 11.23 & 10.1 & 14.8 & 13.48 \\
\hline Water Temp (degrees $C$ ) & 17.1 & 19.6 & 19.1 & 19 & 16.5 & 18.1 & 18 & 16.1 \\
\hline $\mathrm{pH}$ (pH units) & 7.3 & 7.3 & 7.5 & 7.6 & 6 & 6 & 5.6 & 6.1 \\
\hline Sp. Cond. (umho/cm) & 374 & 357 & 392 & 375 & 362 & 323 & 306 & 304 \\
\hline Dissolved Oxygen (ppm) & 3.6 & 5.3 & 4.8 & 6.7 & 2 & 4.4 & 1.1 & 5.7 \\
\hline Oxidation/Reduction (mv) & 52 & 53 & 218 & 160 & -13 & -26 & 30 & 22 \\
\hline MISCELLANEOUS PARAMETERS & - & - & - & - & . & $\cdot$ & - & • \\
\hline pH (pH units) & 7.5 & $\dot{8}$ & 7.5 & 7.8 & $6 . \dot{3}$ & $6 . \dot{4}$ & $6 . \dot{4}$ & $6 . \dot{2}$ \\
\hline Sp. Cond. (umho/cm) & 421 & 409 & 417 & 415 & 364 & 360 & 316 & 334 \\
\hline TPH $(m g / L)$ & • & • & • & • & - & • & • & • \\
\hline $\operatorname{TDS}(\mathrm{mg} / \mathrm{L})$ & 264 & 268 & 266 & 306 & 190 & 214 & 194 & 204 \\
\hline TSS (mg/I) & 3 & $<1$ & 6 & 31 & 7 & 1 & 11 & 4 \\
\hline Turbidity (NTU) & 1.9 & 1.9 & 4 & 4.1 & 27 & 8.8 & 6 & 20 \\
\hline RADIOCHEMICAI PARAMBTERS & • & • & • & • & • & $\cdot$ & • & $\cdot$ \\
\hline Gross Alpha (pCi/L) & -0.19 & 0.57 & 1.59 & -0.86 & 0.31 & -0.32 & -0.61 & -0.86 \\
\hline Gross Alpha $(\mathrm{CE}+/-)$ & 2.10 & 2.00 & 4.10 & 1.30 & 1.80 & 1.50 & 3.60 & 1.30 \\
\hline Gross Beta $(\mathrm{pC} i / L)$ & -6.80 & 3.92 & -0.86 & -5.91 & -9.29 & -0.58 & -0.69 & -2.17 \\
\hline Gross Beta $(\mathrm{CB}+/-)$ & 3.40 & 2.90 & 5.60 & 2.90 & 3.20 & 2.60 & 5.50 & 3.00 \\
\hline
\end{tabular}

(CONTINUED) 
APPENDIX E. 3

Groundwater Quality Data, 1993

\begin{tabular}{|c|c|c|c|c|c|c|c|c|}
\hline \multirow{3}{*}{$\begin{array}{l}\text { Sampling Point } \\
\text { Location } \\
\text { - } \\
\text { Date Sampled }\end{array}$} & \multicolumn{4}{|c|}{$\mathrm{GW}-773$} & \multicolumn{4}{|c|}{$G W-774$} \\
\hline & \multicolumn{4}{|c|}{ GRIDH 2} & \multicolumn{4}{|c|}{ GRIDH2 } \\
\hline & $02 / 03 / 93$ & $04 / 20 / 93$ & $08 / 06 / 93$ & $10 / 15 / 93$ & $02 / 04 / 93$ & $04 / 20 / 93$ & $08 / 09 / 93$ & $10 / 15 / 93$ \\
\hline FIELD MEASUREMENTS & - & - & $\cdot$ & - & - & $\cdot$ & - & \\
\hline Depth to Water $(f t)$ & 12.8 & 12.92 & 15.23 & 15.95 & 14.7 & 14.8 & 16.77 & 17.3 \\
\hline Water Temp (degrees C) & 15.2 & 15.7 & 17 & 15.7 & 14.7 & 15.8 & 22.6 & 17.6 \\
\hline $\mathrm{pH}$ (pH units) & 7.9 & 7.5 & 7.4 & 7.7 & 7.3 & 7.1 & 7.4 & 7.2 \\
\hline Sp. Cond. (umho/cm) & 335 & 291 & 312 & 310 & 384 & 336 & 369 & 379 \\
\hline Dissolved Oxygen (ppm) & 5.3 & 0.5 & 1 & 0.4 & 3 & 0.9 & 2.3 & 1.5 \\
\hline Oxidation/Reduction (mv) & 97 & 146 & 73 & 12 & 221 & 190 & 53 & 95 \\
\hline MISCELLANEOUS PARAMETERS & . & . & . & . & . & . & . & . \\
\hline $\mathrm{pH}$ (pH units) & 7.7 & 7.8 & 7.7 & 7.7 & $8 . \dot{2}$ & 7.4 & $7 . \dot{5}$ & $7 . \dot{3}$ \\
\hline Sp. Cond. (umho/cm) & 318 & 333 & 339 & 335 & 421 & 391 & 386 & 394 \\
\hline TPH $(\mathrm{mg} / \mathrm{L})$ & $\cdot$ & . &. & $\cdot$ &. &. & . & • \\
\hline TDS $(m g / L)$ & 210 & 204 & 218 & 234 & 316 & 222 & 256 & 260 \\
\hline TSS (mg/L) & 2 & 2 & $<1$ & $<1$ & 22 & 10 & 20 & 5 \\
\hline Turbidity (NTU) & 1.4 & 0.9 & 1.7 & 1.5 & 150 & 150 & 14 & 4 \\
\hline RADIOCHEMICAL PARAMETERS & . &. & $\cdot$ &. & $\cdot$ & $\cdot$ & $\cdot$ & • \\
\hline Gross Alpha $(\mathrm{pC} i / I)$ & 0.47 & -0.83 & -2.03 & 0.00 & 1.22 & -0.21 & $0.0 \dot{0}$ & -0.79 \\
\hline Gross Alpha $(\mathrm{CE}+/-)$ & 1.30 & 0.77 & 2.90 & 2.10 & 1.60 & 0.86 & 3.10 & 2.00 \\
\hline Gross Beta (pCi/L) & 2.74 & 0.80 & 1.15 & -1.21 & -2.95 & -0.41 & -0.99 & -1.21 \\
\hline Gross Beta $(\mathrm{CE}+1-)$ & 4.10 & 1.30 & 5.00 & 3.00 & 3.90 & 1.30 & 5.20 & 3.00 \\
\hline
\end{tabular}

(CONTINUED) 
APPENDIX E. 3

Groundwater Quality Data, 1993

\begin{tabular}{|c|c|c|c|c|c|c|c|c|}
\hline \multirow{3}{*}{$\begin{array}{l}\text { Sampling Point } \\
\text { - } \\
\text { Location } \\
\text { - } \\
\text { Date Sampled }\end{array}$} & \multicolumn{4}{|c|}{ GW -775} & \multicolumn{4}{|c|}{$G W-776$} \\
\hline & \multicolumn{4}{|c|}{ GRIDH3 } & \multicolumn{4}{|c|}{ GRIDH3 } \\
\hline & $02 / 04 / 93$ & $04 / 20 / 93$ & $08 / 09 / 93$ & $10 / 15 / 93$ & $02 / 04 / 93$ & $04 / 20 / 93$ & $08 / 10 / 93$ & $10 / 18 / 93$ \\
\hline FIELD MEASURBMENTS & - & - & - & - & - & - & - & - \\
\hline Depth to Water (ft) & 15.15 & 14.9 & 12.47 & 16.1 & 16 & 15.7 & 16 & 16.55 \\
\hline Water Temp (degrees C) & 18.2 & 17.8 & 19.8 & 18.7 & 18.4 & 18 & 18.8 & 19.3 \\
\hline pH (pH units) & 7.6 & 7.6 & 7.6 & 7.6 & 7.5 & 7.6 & 7.1 & 7.1 \\
\hline Sp. Cond. (umho/cm) & 467 & 385 & 396 & 403 & 573 & 486 & 522 & 478 \\
\hline Dissolved Oxygen (ppm) & 3.2 & 1.6 & 1.2 & 0.8 & 8.9 & $4 \cdot 3$ & 9.5 & 4.4 \\
\hline Oxidation/Reduction (mv) & 168 & 186 & 12 & 117 & 165 & 192 & 174 & 73 \\
\hline MISCELIAANEOUS PARAMETERS & - & - & - & - & - & - & - & - \\
\hline $\mathrm{pH}$ (pH units) & 8.2 & 7.7 & 7.7 & 7.6 & 8 & 7.5 & 7.5 & 7.5 \\
\hline Sp. Cond. (umho/cm) & 444 & 441 & 430 & 441 & 571 & 550 & 563 & 530 \\
\hline $\operatorname{TPH}(\mathrm{mg} / \mathrm{L})$ & - & - & - & • & • & . & - & • \\
\hline $\operatorname{TDS}(\mathrm{mg} / \mathrm{L})$ & 274 & 254 & 272 & 302 & 350 & 338 & 356 & 356 \\
\hline TSS (mg/L) & 4 & 3 & $<1$ & $<1$ & 13 & 45 & 135 & 13 \\
\hline Turbidity (NTU) & 2.8 & 1.8 & 1.4 & 0.7 & 12 & 22 & 28 & 18 \\
\hline RADIOCHEMICAL PARAMETERS & - & - & - & - & - & - & - & - \\
\hline Gross Alpha (pCi/L) & 1.10 & 0.22 & -0.30 & 0.32 & 0.37 & -0.83 & 1.81 & -0.17 \\
\hline Gross Alpha $(\mathrm{CE}+/-)$ & 1.40 & 0.96 & 3.10 & 2.20 & 1.50 & 3.20 & 3.50 & 2.20 \\
\hline Gross Beta (pCi/L) & -1.66 & 3.32 & 0.49 & 0.26 & -2.89 & 5.88 & -1.98 & 1.30 \\
\hline Gross Beta $(\mathrm{CB}+/-)$ & 3.90 & 1.40 & 5.30 & 3.10 & 3.90 & 3.00 & 5.20 & 3.20 \\
\hline
\end{tabular}


APPENDIX F

FIELD DUPLICATE DATA 


\section{EXPLANATION}

\section{LOCATION:}

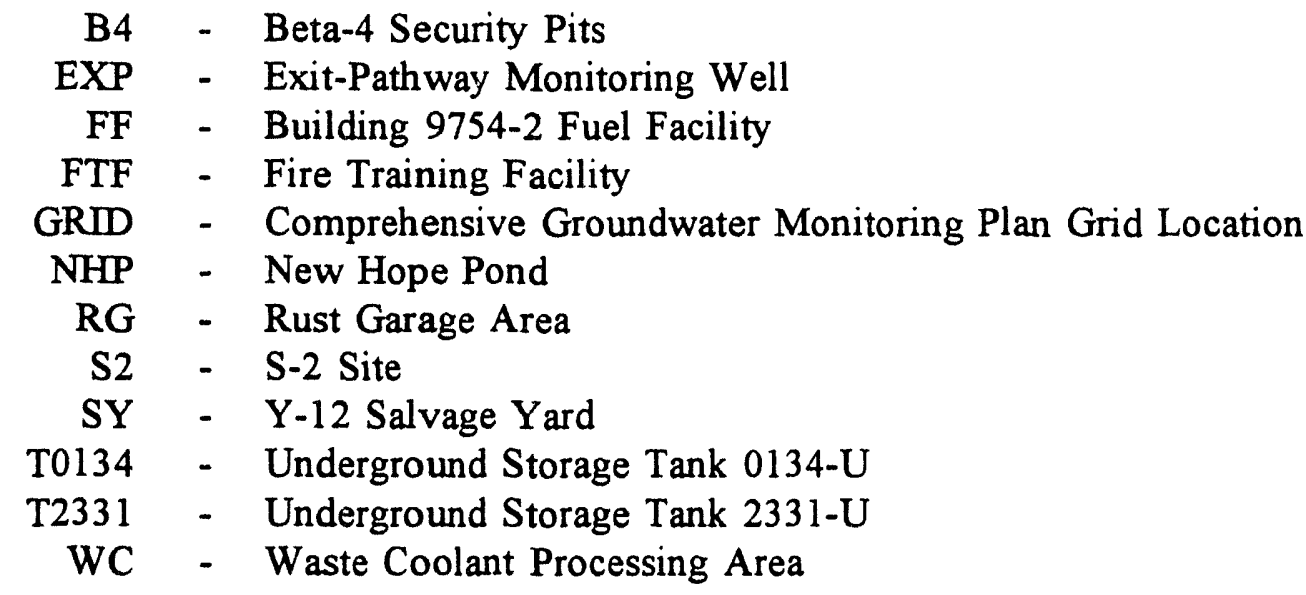

ALL DATA:

- Not Analyzed

VOLATILE ORGANIC COMPOUNDS:

All results in micrograms per Liter (ug/L).

$\mathrm{U}$ - Compound not detected at the reported minimum attainable detection limit.

METALS, MAJOR IONS, MISCELLANEOUS, AND RADIOCHEMICAL PARAMETERS:

All analyses are by Inductively Coupled Plasma (ICP) Spectroscopy unless otherwise noted.

Bicarbonate and carbonate alkalinity reported as $\mathrm{CaCO}_{3}$.

$<\quad$ - Compound not detected at the reported minimum attainable detection limit.

$\mathrm{mg} / \mathrm{L} \quad-\quad$ milligrams per Liter

AAS - Atomic Absorption Spectroscopy

CVAA - Cold Vapor Atomic Absorption

Fluor - Fluorometric

DIS - Dissolved Concentration (Filtered Sample)

TOT - Total Concentration (Unfiltered Sample) 


\section{EXPLANATION (cont'd)}

TPH - Total Petroleum Hydrocarbons

TDS - Total Dissolved Solids

TSS - Total Suspended Solids

Sp. Cond. - Specific Conductance

ppm - parts per million

umho/cm - micromhos per centimeter

NTU - Nephelometric Turbidity Unit

$\mathrm{mV}$ - millivolts

$\mathrm{pCi} / \mathrm{L}$ - picoCuries per liter

$\mathrm{Bq} / \mathrm{L}$ - Becquerels per liter

$\mathrm{CE}+/$ - - Counting Error (two standard deviations) 


\section{APPENDIX F.1}

TRACE METALS AND MAJOR IONS 
APPENDIX F.1

Field Duplicate Groundwater Quality Data, 1993

\begin{tabular}{|c|c|c|c|c|c|c|c|c|}
\hline \multirow{4}{*}{$\begin{array}{l}\text { Sampling Point } \\
\text { Location } \\
\text { Date Sampled }\end{array}$} & \multicolumn{2}{|c|}{$G W-151$} & \multicolumn{2}{|c|}{ GW-170 } & \multicolumn{4}{|c|}{ GW- 183} \\
\hline & \multicolumn{2}{|c|}{ NHP } & \multicolumn{2}{|c|}{ EXP } & \multicolumn{4}{|c|}{$\mathbf{F F}$} \\
\hline & \multicolumn{2}{|c|}{$08 / 06 / 93$} & \multicolumn{2}{|c|}{$05 / 24 / 93$} & \multicolumn{2}{|c|}{$03 / 10 / 93$} & \multicolumn{2}{|c|}{$06 / 21 / 93$} \\
\hline & TOT & DIS & TOT & DIS & TOT & DIS & TOT & DIS \\
\hline METALS (mg/L) & - & • & - & . & - & . & . & . \\
\hline Aluminum & $<0.02$ & $<0.02$ & $<0.02$ & $<0.02$ & 0.26 & 0.037 & 0.21 & $<0.02$ \\
\hline Antimony & $<0.05$ & $<0.05$ & $<0.05$ & $<0.05$ & $<0.05$ & $<0.05$ & $<0.05$ & $<0.05$ \\
\hline Arsenic & $<0.05$ & $<0.05$ & $<0.05$ & $<0.05$ & $<0.05$ & $<0.05$ & $<0.05$ & $<0.05$ \\
\hline Barium & 0.19 & 0.19 & 0.086 & 0.084 & 0.22 & 0.22 & 0.21 & 0.22 \\
\hline Beryllium & $<0.0003$ & $<0.0003$ & $<0.0003$ & $<0.0003$ & $<0.0003$ & $<0.0003$ & $<0.0003$ & $<0.0003$ \\
\hline Boron & 0.074 & 0.087 & 0.079 & 0.071 & 0.037 & 0.028 & 0.049 & 0.06 \\
\hline Cadmium (AAS) & $<0.002$ & $<0.002$ & $<0.002$ & $<0.002$ & $<0.002$ & $<0.002$ & $<0.002$ & $<0.002$ \\
\hline Cadmium & $<0.003$ & $<0.003$ & $<0.003$ & $<0.003$ & $<0.003$ & $<0.003$ & $<0.003$ & $<0.003$ \\
\hline Chromium (AAS) & $<0.01$ & $<0.01$ & $<0.01$ & $<0.01$ & $<0.01$ & $<0.01$ & $<0.01$ & $<0.01$ \\
\hline Chromium & $<0.01$ & $<0.01$ & $<0.01$ & $<0.01$ & $<0.01$ & $<0.01$ & $<0.01$ & $<0.01$ \\
\hline Cobalt & $<0.005$ & $<0.005$ & $<0.005$ & $<0.005$ & $<0.005$ & $<0.005$ & $<0.005$ & $<0.005$ \\
\hline Copper & 0.0044 & $<0.004$ & $<0.004$ & 0.066 & $<0.004$ & $<0.004$ & $<0.004$ & $<0.004$ \\
\hline Iron & 0.038 & $<0.005$ & 0.051 & $<0.005$ & 2.1 & 0.86 & 1.1 & 0.86 \\
\hline Lead (AAS) & $<0.004$ & $<0.004$ & $<0.004$ & $<0.004$ & $<0.004$ & $<0.004$ & $<0.004$ & $<0.004$ \\
\hline Mercury (CVAA) & $<0.0002$ & $<0.0002$ & $<0.0002$ & $<0.0002$ & $<0.0002$ & $<0.0002$ & $<0.0002$ & $<0.0002$ \\
\hline Mol ybdenum & $<0.01$ & $<0.01$ & $<0.01$ & $<0.01$ & $<0.01$ & $<0.01$ & $<0.01$ & $<0.01$ \\
\hline Nickel & $<0.01$ & $<0.01$ & $<0.01$ & $<0.01$ & $<0.01$ & $<0.01$ & $<0.01$ & $<0.01$ \\
\hline Selenium & $<0.05$ & $<0.05$ & $<0.05$ & $<0.05$ & $<0.05$ & $<0.05$ & $<0.05$ & $<0.05$ \\
\hline Silver & $<0.006$ & $<0.006$ & $<0.006$ & $<0.006$ & $<0.006$ & $<0.006$ & $<0.006$ & $<0.006$ \\
\hline Strontium & 0.52 & 0.53 & 0.51 & 0.5 & 0.65 & 0.65 & 0.64 & 0.64 \\
\hline Thorium & $<0.2$ & $<0.2$ & $<0.2$ & $<0.2$ & $<0.2$ & $<0.2$ & $<0.2$ & $<0.2$ \\
\hline Uranium (Fluor) & $<0.001$ & $<0.001$ & 0.001 & 0.001 & 0.001 & 0.002 & 0.002 & 0.002 \\
\hline Vanadium & $<0.005$ & $<0.005$ & $<0.005$ & $<0.005$ & $<0.005$ & $<0.005$ & $<0.005$ & $<0.005$ \\
\hline Zinc & 0.0093 & 0.0065 & 0.0088 & 0.025 & $<0.002$ & 0.0042 & 0.0026 & 0.0067 \\
\hline MAJOR IONS (mg/L) & $\cdot$ & . & $\cdot$ & . & $\cdot$ & $\cdot$ & $\cdot$ & • \\
\hline Alkalinity-HCO3 & 217 & $\cdot$ & $21 \dot{2}$ & $\cdot$ & 437 & $\cdot$ & $45 \dot{3}$ & • \\
\hline Alkalinity-CO3 & $<1$ & $\cdot$ & $<1$ & • & $<1$ & $\cdot$ & $<1$ & - \\
\hline Calcium & 54 & 53 & 45 & 44 & 140 & 140 & 140 & 140 \\
\hline Chloride & 13 & $\cdot$ & 11.9 & $\cdot$ & 33.6 & $\cdot$ & 31 & • \\
\hline Fluoride & 0.1 & $\cdot$ & 0.2 & $\cdot$ & $<0.1$ & $\cdot$ & $<0.1$ & • \\
\hline Magnesium & 24 & 24 & 23 & 23 & 23 & 23 & 24 & 24 \\
\hline Manganese & 0.0015 & 0.0014 & $<0.001$ & 0.0017 & 0.74 & 0.5 & 0.3 & 0.34 \\
\hline Nitrate-N & 0.92 & $\cdot$ & 0.71 & $\cdot$ & $<0.2$ & $\cdot$ & $<0.2$ & • \\
\hline Potassium & 2.7 & 2.5 & 2.1 & 2.3 & 2.4 & 1.8 & 1.8 & 1.7 \\
\hline Sodium & 7 & 7 & 14 & 14 & 25 & 26 & 24 & 24 \\
\hline Sulfate & 22 & . & 17.6 & . & 26.3 & . & 29 & • \\
\hline
\end{tabular}

(CONTINUED) 
APPENDIX F.1

Field Duplicate Groundwater Quality Data, 1993

\begin{tabular}{|c|c|c|c|c|c|c|c|c|}
\hline \multirow{4}{*}{$\begin{array}{l}\text { Sampling Point } \\
\text { Location } \\
\text { Date Sampled }\end{array}$} & \multicolumn{4}{|c|}{$G W-183$} & \multicolumn{4}{|c|}{$G W-190$} \\
\hline & \multicolumn{4}{|c|}{$\mathbf{F F}$} & \multicolumn{4}{|c|}{ RG } \\
\hline & \multicolumn{2}{|c|}{$09 / 22 / 93$} & \multicolumn{2}{|c|}{$21 / 16 / 93$} & \multicolumn{2}{|c|}{$06 / 17 / 93$} & \multicolumn{2}{|c|}{$09 / 15 / 93$} \\
\hline & TOT & DIS & TOT & DIS & TOT & DIS & TOT & DIS \\
\hline METALS (mg/L) & - & - & . & - & - & - & - & - \\
\hline Aluminum & 1.9 & 0.035 & 0.12 & $<0.02$ & 0.26 & $<0.02$ & 0.076 & 0.021 \\
\hline Antimony & $<0.05$ & $<0.05$ & $<0.05$ & $<0.05$ & $<0.05$ & $<0.05$ & $<0.05$ & $<0.05$ \\
\hline Arsenic & $<0.05$ & $<0.05$ & $<0.05$ & $<0.05$ & $<0.05$ & $<0.05$ & $<0.05$ & $<0.05$ \\
\hline Barium & 0.25 & 0.24 & 0.23 & 0.21 & 0.13 & 0.11 & 0.13 & 0.12 \\
\hline Beryllium & $<0.0003$ & $<0.0003$ & $<0.0003$ & $<0.0003$ & $<0.0003$ & $<0.0003$ & 0.00044 & $<0.0003$ \\
\hline Boron & 0.073 & 0.055 & 0.046 & 0.076 & 0.013 & 0.015 & 0.019 & 0.02 \\
\hline Cadmium (AAS) & $<0.002$ & $<0.002$ & $<0.002$ & $<0.002$ & $<0.002$ & $<0.002$ & $<0.002$ & $<0.002$ \\
\hline Cadmium & $<0.003$ & $<0.003$ & $<0.003$ & $<0.003$ & $<0.003$ & $<0.003$ & $<0.003$ & $<0.003$ \\
\hline Chromium (AAS) & $<0.01$ & $<0.01$ & $<0.01$ & $<0.01$ & $<0.01$ & $<0.01$ & $<0.01$ & $<0.01$ \\
\hline Chromium & $<0.01$ & $<0.01$ & $<0.01$ & $<0.01$ & $<0.01$ & $<0.01$ & $<0.01$ & $<0.01$ \\
\hline Cobalt & $<0.005$ & $<0.005$ & $<0.005$ & $<0.005$ & $<0.005$ & $<0.005$ & $<0.005$ & $<0.005$ \\
\hline Copper & $<0.004$ & $<0.004$ & $<0.004$ & $<0.004$ & $<0.004$ & 0.0046 & 0.011 & $<0.004$ \\
\hline Iron & 2.7 & 0.51 & 0.36 & 0.024 & 0.7 & 0.1 & 0.33 & 0.027 \\
\hline Lead (AAS) & $<0.004$ & $<0.004$ & $<0.004$ & $<0.004$ & $<0.004$ & $<0.004$ & $<0.004$ & $<0.004$ \\
\hline Mercury (CVAA) & $<0.0002$ & $<0.0002$ & $<0.0002$ & $<0.0002$ & $<0.0002$ & $<0.0002$ & $<0.0002$ & $<0.0002$ \\
\hline Molybdenum & $<0.01$ & $<0.01$ & $<0.01$ & $<0.01$ & $<0.01$ & $<0.01$ & $<0.01$ & $<0.01$ \\
\hline Nickel & $<0.01$ & $<0.01$ & $<0.01$ & $<0.01$ & $<0.01$ & 0.011 & $<0.01$ & $<0.01$ \\
\hline Selenium & $<0.05$ & $<0.05$ & $<0.05$ & $<0.05$ & $<0.05$ & $<0.05$ & $<0.05$ & $<0.05$ \\
\hline silver & $<0.006$ & $<0.006$ & $<0.006$ & $<0.006$ & $<0.006$ & $<0.006$ & $<0.006$ & $<0.006$ \\
\hline Strontium & 0.74 & 0.73 & 0.72 & 0.7 & 0.11 & 0.11 & 0.14 & 0.12 \\
\hline Thorium & $<0.2$ & $<0.2$ & $<0.2$ & $<0.2$ & $<0.2$ & $<0.2$ & $<0.2$ & $<0.2$ \\
\hline Uranium (Fluor) & 0.003 & 0.002 & 0.002 & 0.003 & $<0.001$ & $<0.001$ & $<0.001$ & $<0.001$ \\
\hline Vanadium & $<0.005$ & $<0.005$ & $<0.005$ & $<0.005$ & $<0.005$ & $<0.005$ & $<0.005$ & $<0.005$ \\
\hline Zinc & 0.012 & 0.0056 & 0.028 & 0.035 & 0.021 & 0.024 & 0.024 & 0.017 \\
\hline MAJOR IONS (mg/L) & . & . & . & . & . & . & . & . \\
\hline Alkalinity-HCO3 & ${ }_{453}$ & $\cdot$ & $\begin{array}{r}467 \\
\end{array}$ & $\cdot$ & 110 & $\cdot$ & 116 & • \\
\hline Alkalinity- $\mathrm{CO}_{3}$ & $<1$ &. & $<1$ &. & $<1$ &. & $<1$ & - \\
\hline Calcium & 150 & 150 & 160 & 150 & 35 & 36 & 43 & 35 \\
\hline Chloride & 31 & . & 32 & $\cdot$ & 6.4 & $\cdot$ & 5.7 & $\cdot$ \\
\hline Fluoride & $<0.1$ & . & $<0.1$ & $\cdot$ & 0.2 & $\cdot$ & 0.1 & • \\
\hline Magnesium & 25 & 25 & 28 & 26 & 6.1 & 6.1 & 6.3 & 6.2 \\
\hline Manganese & 0.21 & 0.18 & 0.21 & 0.23 & 0.35 & 0.19 & 0.28 & 0.34 \\
\hline Nitrate-N & $<0.2$ &. & $<0.2$ & $\cdot$ & $<0.2$ &. & $<0.2$ & \\
\hline Potassium & 2.5 & 1.9 & 2.1 & 1.8 & 0.67 & 1.3 & 0.99 & 1.2 \\
\hline Sodium & 28 & 27 & 25 & 27 & 8.2 & 8.3 & 8.7 & 8.1 \\
\hline Sulfate & 30 & . & 24 & $\cdot$ & 16 & . & 20 & 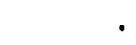 \\
\hline
\end{tabular}

(CONTINUED) 
APPENDIX F.1

Field Duplicate Groundwater Quality Data, 1993

\begin{tabular}{|c|c|c|c|c|c|c|c|c|}
\hline \multirow{4}{*}{$\begin{array}{l}\text { Sampling Point } \\
\text { Location } \\
\text { Date Sampled }\end{array}$} & \multicolumn{4}{|c|}{$G W-207$} & \multicolumn{4}{|c|}{ GW-208 } \\
\hline & \multicolumn{4}{|c|}{ EXP } & \multicolumn{4}{|c|}{ EXP } \\
\hline & \multicolumn{2}{|c|}{$08 / 11 / 93$} & \multicolumn{2}{|c|}{$10 / 26 / 93$} & \multicolumn{2}{|c|}{$01 / 19 / 93$} & \multicolumn{2}{|c|}{$05 / 06 / 93$} \\
\hline & TOT & DIS & TOT & DIS & TOT & DIS & TOT & DIS \\
\hline METALS (mg/L) & $\cdot$ & $\cdot$ & $\cdot$ & $\cdot$ & $\cdot$ & $\cdot$ & $\cdot$ & - \\
\hline Aluminum & $<0.02$ & $<0.32$ & $<0.02$ & $<0.02$ & $<0.02$ & $<0.02$ & $<0.02$ & 0.027 \\
\hline Antimony & $<0.05$ & $<0.05$ & $<0.05$ & $<0.05$ & $<0.05$ & $<0.05$ & $<0.05$ & $<0.05$ \\
\hline Arsenic & $<0.05$ & $<0.05$ & $<0.05$ & $<0.05$ & $<0.05$ & $<0.05$ & $<0.05$ & $<0.05$ \\
\hline Barium & 0.05 & 0.049 & 0.047 & 0.047 & 0.039 & 0.04 & 0.037 & 0.039 \\
\hline Beryllium & $<0.0003$ & $<0.0003$ & $<0.0003$ & $<0.0003$ & $<0.0003$ & $<0.0003$ & $<0.0003$ & $<0.0003$ \\
\hline Boron & 0.1 & 0.11 & 0.11 & 0.11 & 0.22 & 0.21 & 0.23 & 0.34 \\
\hline Cadmium (AAS) & $<0.002$ & $<0.002$ & $<0.002$ & $<0.002$ & $<0.002$ & $<0.002$ & $<0.002$ & $<0.002$ \\
\hline Cadmium & $<0.003$ & $<0.003$ & $<0.003$ & $<0.003$ & $<0.003$ & $<0.003$ & $<0.003$ & $<0.003$ \\
\hline Chromium (AAS) & $<0.01$ & $<0.01$ & $<0.01$ & $<0.01$ & $<0.01$ & $<0.01$ & $<0.01$ & $<0.01$ \\
\hline Chromium & $<0.01$ & $<0.01$ & $<0.01$ & $<0.01$ & $<0.01$ & $<0.01$ & $<0.01$ & 0.014 \\
\hline Cobalt & $<0.005$ & $<0.005$ & $<0.005$ & $<0.005$ & $<0.005$ & $<0.005$ & $<0.005$ & $<0.005$ \\
\hline Copper & $<0.004$ & $<0.004$ & $<0.004$ & $<0.004$ & $<0.004$ & $<0.004$ & $<0.004$ & 0.0074 \\
\hline Iron & 0.22 & 0.2 & 0.2 & 0.2 & 0.63 & 0.47 & 0.49 & 0.54 \\
\hline Lead (AAS) & $<0.004$ & $<0.004$ & $<0.004$ & $<0.004$ & $<0.004$ & $<0.004$ & $<0.004$ & $<0.004$ \\
\hline Mercury (CVAA) & $<0.0002$ & $<0.0002$ & $<0.0002$ & $<0.0002$ & $<0.0002$ & $<0.0002$ & $<0.0002$ & $<0.0002$ \\
\hline Molybdenum & $<0.01$ & $<0.01$ & $<0.01$ & $<0.01$ & $<0.01$ & $<0.01$ & $<0.01$ & $<0.01$ \\
\hline Nickel & $<0.01$ & $<0.01$ & $<0.01$ & $<0.01$ & $<0.01$ & $<0.01$ & $<0.01$ & 0.015 \\
\hline Selenium & $<0.05$ & $<0.05$ & $<0.05$ & $<0.05$ & $<0.05$ & $<0.05$ & $<0.05$ & $<0.05$ \\
\hline silver & $<0.006$ & $<0.006$ & $<0.006$ & $<0.006$ & $<0.006$ & $<0.006$ & $<0.006$ & $<0.006$ \\
\hline Strontium & 0.53 & 0.52 & 0.48 & 0.48 & 1.3 & 1.3 & 1.3 & 1.3 \\
\hline Thorium & $<0.2$ & $<0.2$ & $<0.2$ & $<0.2$ & $<0.2$ & $<0.2$ & $<0.2$ & $<0.2$ \\
\hline Uranium (Fluor) & $<0.001$ & 0.002 & $<0.001$ & 0.001 & $<0.001$ & $<0.001$ & $<0.001$ & $<0.001$ \\
\hline Vanadium & $<0.005$ & $<0.005$ & $<0.005$ & $<0.005$ & $<0.005$ & $<0.005$ & $<0.005$ & $<0.005$ \\
\hline zine & 0.0041 & 0.011 & $<0.002$ & 0.0078 & 1.8 & 1.4 & 1.2 & 1 \\
\hline MAJOR IONS $(\mathrm{mg} / \mathrm{L})$ & . & $\cdot$ & . & $\cdot$ & . & . &. & . \\
\hline Alkalinity- $\mathrm{HCO} 3$ & 265 & $\cdot$ & 272 & $\cdot$ & $22 \dot{2}$ & $\cdot$ & $22 \dot{0}$ & . \\
\hline Alkalinity-CO3 & $<1$ & $\cdot$ & $<1$ & $\cdot$ & $<1$ & $\cdot$ & $<1$ & • \\
\hline Calcium & 59 & 59 & 57 & 58 & 72 & 71 & 75 & 75 \\
\hline Chloride & 1.9 & $\cdot$ & 1 & $\cdot$ & 1.3 & $\cdot$ & 1.6 & • \\
\hline Fluoride & 0.2 & $\cdot$ & 0.2 & $\cdot$ & 0.3 & . & 0.2 & - \\
\hline Magnesium & 39 & 39 & 37 & 37 & 23 & 23 & 24 & 24 \\
\hline Manganese & 0.01 & 0.0098 & 0.0099 & 0.01 & 0.015 & 0.014 & 0.014 & 0.018 \\
\hline Nitrate-N & $<0.2$ &. & $<0.2$ & $\cdot$ & $<0.2$ & . & $<0.2$ & • \\
\hline Potassium & 2.5 & 2.9 & 3.1 & 3 & 2.8 & 2.9 & 2.9 & 7.8 \\
\hline Sodium & 11 & 11 & 10 & 9.7 & 15 & 15 & 15 & 16 \\
\hline sulfate & 51 & $\cdot$ & 55 & $\cdot$ & 116 & $\cdot$ & 100 & • \\
\hline
\end{tabular}

(CONTINUED) 
APPENDIX F.1

Field Duplicate Groundwater Quality Data, 1993

\begin{tabular}{|c|c|c|c|c|c|c|c|c|}
\hline \multirow{4}{*}{$\begin{array}{l}\text { Sampling Point } \\
\text { Iocation } \\
\text { - } \\
\text { Date Sarmled }\end{array}$} & \multicolumn{6}{|c|}{ GW-255 } & \multicolumn{2}{|c|}{$G W-262$} \\
\hline & \multicolumn{6}{|c|}{$\mathbf{s 2}$} & \multicolumn{2}{|c|}{ SY } \\
\hline & \multicolumn{2}{|c|}{$01 / 08 / 93$} & \multicolumn{2}{|c|}{$05 / 03 / 93$} & \multicolumn{2}{|c|}{$12 / 07 / 93$} & \multicolumn{2}{|c|}{$01 / 11 / 93$} \\
\hline & TOT & DIS & TOT & DIS & TOT & DIS & TOT & DIS \\
\hline METALS (mg/I) & - & $\cdot$ & $\cdot$ & • & - & - & - & - \\
\hline Aluminum & 2 & $<0.02$ & 3.7 & $<0.02$ & 29 & 0.025 & 0.039 & $<0.02$ \\
\hline Antimony & $<0.05$ & $<0.05$ & $<0.05$ & $<0.05$ & $<0.05$ & 0.071 & $<0.05$ & $<0.05$ \\
\hline Arsenic & $<0.05$ & $<0.05$ & $<0.05$ & $<0.05$ & $<0.05$ & $<0.05$ & $<0.05$ & $<0.05$ \\
\hline Barium & 0.04 & 0.031 & 0.046 & 0.029 & 0.21 & 0.04 & 0.28 & 0.27 \\
\hline Beryllium & $<0.0003$ & $<0.0003$ & 0.00035 & $<0.0003$ & 0.0034 & $<0.0003$ & $<0.0003$ & $<0.0003$ \\
\hline Boron & 0.025 & 0.0096 & 0.023 & 0.019 & 0.065 & 0.034 & 0.012 & 0.013 \\
\hline Cacmium (AAS) & $<0.002$ & $<0.002$ & $<0.002$ & $<0.002$ & 0.0025 & $<0.002$ & $<0.002$ & $<0.002$ \\
\hline Cadmium & $<0.003$ & $<0.003$ & $<0.003$ & $<0.003$ & 0.0044 & $<0.003$ & $<0.003$ & $<0.003$ \\
\hline Chromiun (AAS) & $<0.01$ & $<0.01$ & 0.012 & $<0.01$ & 0.05 & $<0.01$ & 0.01 & $<0.01$ \\
\hline Chromium & $<0.01$ & $<0.01$ & $<0.01$ & $<0.01$ & 0.028 & $<0.01$ & $<0.01$ & $<0.01$ \\
\hline Cobalt & $<0.005$ & $<0.005$ & $<0.005$ & $<0.005$ & 0.031 & $<0.005$ & $<0.005$ & $<0.005$ \\
\hline Copper & 0.011 & $<0.004$ & 0.0079 & $<0.004$ & 0.099 & $<0.004$ & $<0.004$ & $<0.004$ \\
\hline Iron & 3 & $<0.005$ & 4.8 & $<0.005$ & 38 & $<0.005$ & 0.17 & 0.035 \\
\hline Lead (AAS) & 0.0053 & $<0.004$ & 0.0087 & $<0.004$ & 0.076 & $<0.004$ & $<0.004$ & $<0.004$ \\
\hline Mercury (CVAA) & $<0.0002$ & $<0.0002$ & $<0.0002$ & $<0.0002$ & 0.00039 & $<0.0002$ & $<0.0002$ & $<0.0002$ \\
\hline Molybdenum & $<0.01$ & $<0.01$ & $<0.01$ & $<0.01$ & $<0.01$ & $<0.01$ & $<0.01$ & $<0.01$ \\
\hline Nickel & $<0.01$ & $<0.01$ & 0.012 & $<0.01$ & 0.044 & $<0.01$ & $<0.01$ & 0.011 \\
\hline Selenium & $<0.05$ & $<0.05$ & $<0.05$ & $<0.05$ & $<0.05$ & $<0.05$ & $<0.05$ & $<0.05$ \\
\hline Silver & $<0.006$ & $<0.006$ & $<0.006$ & $<0.006$ & $<0.006$ & $<0.006$ & $<0.006$ & $<0.006$ \\
\hline Strontium & 0.028 & 0.027 & 0.026 & 0.025 & 0.039 & 0.034 & 0.15 & 0.15 \\
\hline Thorium & $<0.2$ & $<0.2$ & $<0.2$ & $<0.2$ & $<0.2$ & $<0.2$ & $<0.2$ & $<0.2$ \\
\hline Uranium (Fluor) & 0.001 & 0.001 & 0.001 & 0.001 & 0.001 & 0.001 & 0.001 & 0.001 \\
\hline Vanadium & 0.0065 & $<0.005$ & $<0.005$ & $<0.005$ & 0.053 & $<0.005$ & $<0.005$ & $<0.005$ \\
\hline Zinc & 0.019 & $<0.002$ & 0.04 & 0.012 & 0.19 & $<0.002$ & 0.0099 & 0.0059 \\
\hline MAJOR IONS $(\mathrm{mg} / \mathrm{L})$ & $\cdot$ & $\cdot$ & - & - & - & - & - & - \\
\hline Alkalinity- $\mathrm{HCO} 3$ & 238 &. & 239 &. & 245 &. & 195 & • \\
\hline Alkalinity-CO3 & $<1$ & $\cdot$ & $<1$ & • & $<1$ & • & $<1$ & - \\
\hline Calcium & 56 & 58 & 50 & 49 & 66 & 74 & 61 & 63 \\
\hline Chloride & 5.5 & $\cdot$ & 4.8 & - & 6 & - & $<1$ & - \\
\hline Fluoride & $<0.1$ & - & $<0.1$ & - & $<0.1$ & - & 0.1 & - \\
\hline Magnesium & 31 & 30 & 32 & 30 & 42 & 37 & 5.9 & 6 \\
\hline Manganese & 0.1 & $<0.001$ & 0.23 & $<0.001$ & 2.7 & $<0.001$ & 0.066 & 0.062 \\
\hline Nitrate-N & 1.7 & $\cdot$ & 2.2 & $\cdot$ & 3 & • & $<0.2$ & • \\
\hline Potassium & 1.6 & 0.91 & 1.6 & 1.1 & 6.9 & 1.4 & 1.4 & 1.3 \\
\hline Sodium & 3.4 & 3.3 & 3.3 & 3.2 & 3.8 & 3.4 & 14 & 14 \\
\hline Sulfate & 11 & . & 12 & • & 9 & $\cdot$ & 14 & • \\
\hline
\end{tabular}

(CONTINOED) 
APPENDIX F.1

Field Duplicate Groundwater Quality Data, 1993

\begin{tabular}{|c|c|c|c|c|c|c|c|c|}
\hline \multirow{4}{*}{$\begin{array}{l}\text { Sampling point } \\
\text { L } \\
\text { Location } \\
\text { Date Sampled }\end{array}$} & \multicolumn{4}{|c|}{ GW-262 } & \multicolumn{2}{|c|}{ GW-264 } & \multicolumn{2}{|c|}{ GW-382 } \\
\hline & \multicolumn{4}{|c|}{ SY } & \multirow{2}{*}{\multicolumn{2}{|c|}{$\frac{S Y}{12 / 08 / 93}$}} & \multirow{2}{*}{\multicolumn{2}{|c|}{$\begin{array}{c}\text { NHP } \\
01 / 28 / 93\end{array}$}} \\
\hline & \multicolumn{2}{|c|}{$04 / 08 / 93$} & \multicolumn{2}{|c|}{$07 / 01 / 93$} & & & & \\
\hline & TOT & DIS & TOT & DIS & TOT & DIS & TOT & DIS \\
\hline METALS (mg/L) & $\cdot$ & - & - & - & - & - & - & • \\
\hline Aluminum & 0.18 & 0.07 & 1.1 & $<0.02$ & 0.64 & $<0.02$ & $<0.02$ & $<0.02$ \\
\hline Antimony & $<0.05$ & $<0.05$ & $<0.05$ & $<0.05$ & $<0.05$ & $<0.05$ & $<0.05$ & $<0.05$ \\
\hline Arsenic & $<0.05$ & $<0.05$ & $<0.05$ & $<0.05$ & $<0.05$ & $<0.05$ & $<0.05$ & $<0.05$ \\
\hline Barium & 0.27 & 0.27 & 0.28 & 0.27 & 0.039 & 0.029 & 0.11 & 0.11 \\
\hline Beryllium & $<0.0003$ & $<0.0003$ & $<0.0003$ & $<0.0003$ & $<0.0003$ & $<0.0003$ & $<0.0003$ & $<0.0003$ \\
\hline Boron & 0.012 & 0.013 & 0.011 & 0.0099 & 0.072 & 0.14 & 0.068 & 0.062 \\
\hline Cadmium (AAS) & $<0.002$ & $<0.002$ & $<0.002$ & $<0.002$ & $<0.002$ & $<0.002$ & $<0.002$ & $<0.002$ \\
\hline Cadmium & $<0.003$ & $<0.003$ & $<0.003$ & $<0.003$ & $<0.003$ & $<0.003$ & $<0.003$ & $<0.003$ \\
\hline Chromium (AAS) & $<0.01$ & $<0.01$ & $<0.01$ & $<0.01$ & $<0.01$ & $<0.01$ & $<0.01$ & $<0.01$ \\
\hline Chromium & $<0.01$ & $<0.01$ & $<0.01$ & $<0.01$ & $<0.01$ & $<0.01$ & $<0.01$ & $<0.01$ \\
\hline Cobalt & $<0.005$ & $<0.005$ & $<0.005$ & $<0.005$ & $<0.005$ & $<0.005$ & $<0.005$ & $<0.005$ \\
\hline Copper & $<0.004$ & $<0.004$ & $<0.004$ & 0.005 & $<0.004$ & $<0.004$ & $<0.004$ & 0.0043 \\
\hline Iron & 0.71 & 0.022 & 1.3 & $<0.005$ & 0.92 & 0.044 & 0.91 & 0.17 \\
\hline Lead (AAS) & $<0.004$ & $<0.004$ & $<0.004$ & $<0.004$ & $<0.004$ & $<0.004$ & $<0.004$ & $<0.004$ \\
\hline Mercury (CVAA) & $<0.0002$ & $<0.0002$ & $<0.0002$ & $<0.0002$ & $<0.0002$ & $<0.0002$ & $<0.0002$ & $<0.0002$ \\
\hline Molybdenum & $<0.01$ & $<0.01$ & $<0.01$ & $<0.01$ & $<0.01$ & $<0.01$ & $<0.01$ & $<0.01$ \\
\hline Nickel & $<0.01$ & $<0.01$ & $<0.01$ & $<0.01$ & $<0.01$ & $<0.01$ & $<0.01$ & 0.011 \\
\hline Selenium & $<0.05$ & $<0.05$ & $<0.05$ & $<0.05$ & $<0.05$ & $<0.05$ & $<0.05$ & $<0.05$ \\
\hline Silver & $<0.006$ & $<0.006$ & $<0.006$ & $<0.006$ & $<0.006$ & $<0.006$ & $<0.006$ & $<0.006$ \\
\hline Strontium & 0.15 & 0.15 & 0.15 & 0.15 & 0.33 & 0.33 & 0.4 & 0.4 \\
\hline Thorium & $<0.2$ & $<0.2$ & $<0.2$ & $<0.2$ & $<0.2$ & $<0.2$ & $<0.2$ & $<0.2$ \\
\hline Oranium (Fluor) & 0.001 & 0.001 & 0.001 & 0.002 & 0.001 & 0.001 & 0.001 & 0.001 \\
\hline Vanadium & $<0.005$ & $<0.005$ & $<0.005$ & $<0.005$ & $<0.005$ & $<0.005$ & $<0.005$ & $<0.005$ \\
\hline zinc & 0.021 & 0.013 & 0.011 & 0.01 & 0.0066 & 0.0039 & $<0.002$ & $<0.002$ \\
\hline MAJOR IONS (mg/L) & • & $\cdot$ & $\dot{\bullet}$ & $\dot{.}$ & • & $\cdot$ & $\dot{\bullet}$ & • \\
\hline Alkalinity-HCO3 & 201 & • & 208 & $\cdot$ & ${ }_{431}^{\circ}$ & $\cdot$ & 205 & • \\
\hline Alkalinity $-\mathrm{CO} 3$ & $<1$ & • & $<1$ & $\cdot$ & $<1$ & $\cdot$ & $<1$ & . \\
\hline Calcium & 65 & 64 & 66 & 63 & 150 & 150 & 51 & 51 \\
\hline Chloride & $<1$ & . & $<1$ & • & 11.6 & . & 20 & • \\
\hline Fluoride & 0.2 & - & 0.1 & - & $<0.1$ & . & 0.2 & • \\
\hline Magnesium & 6.2 & 6 & 6.4 & 6 & 42 & 39 & 25 & 25 \\
\hline Manganese & 0.1 & 0.097 & 0.094 & 0.057 & 0.05 & 0.04 & 0.006 & 0.006 \\
\hline Nitrate-N & $<0.2$ & • & $<0.2$ & • & $<0.2$ & • & 2.8 & • \\
\hline Potassium & 1.7 & 1.6 & 2.2 & 1.6 & 3.9 & 3.4 & 1.1 & 1.3 \\
\hline Sodium & 14 & 14 & 14 & 15 & 29 & 28 & 6.2 & 6.3 \\
\hline Sulfate & 13 & • & 12 & $\cdot$ & 155 & $\cdot$ & 16 & • \\
\hline
\end{tabular}

(CONTINUED) 
APPENDIX F.1

Field Duplicate Groundwater Quality Data, 1993

\begin{tabular}{|c|c|c|c|c|c|c|c|c|}
\hline \multirow{4}{*}{$\begin{array}{l}\text { Sampling point } \\
\text { Location } \\
\text { Date Sampled }\end{array}$} & \multicolumn{2}{|c|}{ GW-382 } & \multicolumn{2}{|c|}{ GW-606 } & \multicolumn{2}{|c|}{ GW- 618} & \multicolumn{2}{|c|}{$G W-631$} \\
\hline & \multicolumn{2}{|c|}{ NHP } & \multicolumn{2}{|c|}{ EXP } & \multicolumn{2}{|c|}{ EXP } & \multicolumn{2}{|c|}{ RG } \\
\hline & \multicolumn{2}{|c|}{$04 / 26 / 93$} & \multicolumn{2}{|c|}{$08 / 20 / 93$} & \multicolumn{2}{|c|}{$09 / 16 / 93$} & \multicolumn{2}{|c|}{$03 / 02 / 93$} \\
\hline & TOT & DIS & $T r J T$ & DIS & TOT & DIs & TOT & DIS \\
\hline METALS (mg/I) & - & . & . & - & . & - & $\cdot$ & • \\
\hline Aluminum & $<0.02$ & $<0.02$ & $<0.02$ & $<0.02$ & $<0.02$ & $<0.02$ & 0.33 & 0.19 \\
\hline Antimony & $<0.05$ & $<0.05$ & $<0.05$ & $<0.05$ & $<0.05$ & $<0.05$ & $<0.05$ & $<0.05$ \\
\hline Arsenic & $<0.05$ & $<0.05$ & $<0.05$ & $<0.05$ & $<0.05$ & $<0.05$ & $<0.05$ & $<0.05$ \\
\hline Barium & 0.15 & 0.15 & 0.094 & 0.095 & 0.061 & 0.061 & 0.33 & 0.37 \\
\hline Beryllium & $<0.0003$ & $<0.0003$ & $<0.0003$ & $<0.0003$ & $<0.0003$ & $<0.0003$ & 0.00082 & 0.00082 \\
\hline Boron & 0.035 & 0.038 & 0.03 & 0.033 & 0.15 & 0.15 & 0.037 & 0.047 \\
\hline Cadmium (AAS) & $<0.002$ & $<0.002$ & $<0.002$ & $<0.002$ & 0.029 & $<0.002$ & $<0.002$ & $<0.002$ \\
\hline Cadmium & $<0.003$ & $<0.003$ & $<0.003$ & $<0.003$ & 0.026 & $<0.003$ & $<0.003$ & $<0.003$ \\
\hline Chromium (AAS) & $<0.01$ & $<0.01$ & $<0.01$ & $<0.01$ & $<0.01$ & $<0.01$ & $<0.01$ & $<0.01$ \\
\hline Chromium & $<0.01$ & $<0.2:$ & $<0.01$ & $<0.6 i$ & $<0.01$ & $<0.01$ & $<0.01$ & $<0.01$ \\
\hline Cobalt & $<0.005$ & $<0.005$ & $<0.005$ & $<0.005$ & $<0.005$ & $<0.005$ & 0.028 & 0.026 \\
\hline Copper & 0.0055 & $<0.004$ & $<0.004$ & $<0.004$ & $<0.004$ & $<0.004$ & $<0.004$ & $<0.004$ \\
\hline Iron & 1.5 & 0.36 & $<0.005$ & $<0.005$ & 0.14 & 0.14 & 0.14 & 0.012 \\
\hline Lead (AAS) & $<0.004$ & $<0.004$ & $<0.004$ & $<0.004$ & $<0.004$ & $<0.004$ & $<0.004$ & $<0.004$ \\
\hline Mercury (CVAA) & $<0.0002$ & $<0.0002$ & $<0.0002$ & $<0.0002$ & $<0.0002$ & $<0.0002$ & $<0.0002$ & $<0.0002$ \\
\hline Molybdenum & $<0.01$ & $<0.01$ & $<0.01$ & $<0.01$ & $<0.01$ & $<0.01$ & $<0.01$ & $<0.01$ \\
\hline Nickel & $<0.01$ & $<0.01$ & $<0.01$ & $<0.01$ & $<0.01$ & $<0.01$ & 0.051 & 0.042 \\
\hline Selenium & $<0.05$ & $<0.05$ & $<0.05$ & $<0.05$ & $<0.05$ & $<0.05$ & $<0.05$ & $<0.05$ \\
\hline Silver & $<0.006$ & $<0.006$ & $<0.006$ & $<0.006$ & $<0.006$ & $<0.006$ & $<0.006$ & $<0.006$ \\
\hline Strontium & 0.36 & 0.36 & 0.5 & 0.5 & 0.18 & 0.18 & 0.095 & 0.11 \\
\hline Thorium & $<0.2$ & $<0.2$ & $<0.2$ & $<0.2$ & $<0.2$ & $<0.2$ & $<0.2$ & $<0.2$ \\
\hline Uranium (Fluor) & $<0.001$ & $<0.001$ & 0.008 & 0.01 & $<0.001$ & $<0.001$ & $<0.001$ & $<0.001$ \\
\hline Vanadium & $<0.005$ & $<0.005$ & $<0.005$ & $<0.005$ & $<0.005$ & $<0.005$ & $<0.005$ & $<0.005$ \\
\hline Zinc & 0.024 & 0.002 & 0.0034 & 0.015 & 0.0069 & 0.0049 & 0.11 & 0.097 \\
\hline MAJOR IONS (mg/L) & . &. & . & . &. & . &. & . \\
\hline Alkalinity-HCO3 & 203 &. & 227 & . & 279 & . & $\dot{8}$ & • \\
\hline Alkalinity- $\mathrm{CO} 3$ & $<1$ &. & $<1$ & . & $<1$ & . & $<1$ & . \\
\hline Calcium & 52 & 52 & 75 & 74 & 100 & 100 & 13 & 16 \\
\hline Chloride & 19.5 &. & 46 & • & 28 & . & 48 & . \\
\hline Fluoride & 0.2 &. & $<0.1$ & • & 0.2 & . & 0.1 & • \\
\hline Magnesium & 25 & 25 & 36 & 36 & 9.5 & 9.6 & 9.1 & 10 \\
\hline Manganese & 0.014 & 0.011 & 0.02 & 0.021 & 2.9 & 2 & 0.95 & 0.86 \\
\hline aitrate-N & 2.08 &. & 0.58 & • & 2.1 & . & 2.8 & • \\
\hline Potassium & 1.5 & 0.73 & 3.4 & 3.5 & 3.5 & 3.6 & 2.2 & 2.5 \\
\hline Sodium & 6.1 & 6.1 & 5 & 5 & 18 & 18 & 17 & 17 \\
\hline sulfate & 13.3 &. & 26 & . & 68 & . & 3 & • \\
\hline
\end{tabular}

(CONTINUED) 
A.PPENDIX F. 1

Field Duplicate Groundwater Quality Data, 1993

\begin{tabular}{|c|c|c|c|c|c|c|c|c|}
\hline \multirow{4}{*}{$\begin{array}{l}\text { Sampling Point } \\
\text { Location } \\
\text { Date Sampled }\end{array}$} & \multicolumn{2}{|c|}{ GW-631 } & \multicolumn{6}{|c|}{$G W-735$} \\
\hline & \multicolumn{2}{|c|}{ RG } & \multicolumn{6}{|c|}{ NHP } \\
\hline & \multicolumn{2}{|c|}{$11 / 17 / 93$} & \multicolumn{2}{|c|}{$02 / 02 / 93$} & \multicolumn{2}{|c|}{$05 / 07 / 93$} & \multicolumn{2}{|c|}{$10 / 29 / 93$} \\
\hline & TOT & DIS & TOT & DIS & TOT & DIS & TOT & DIS \\
\hline METALS (mg/L) & $\cdot$ & - & • & - & - & - & • & • \\
\hline Aluminum & 2.3 & 0.18 & 0.52 & $<0.02$ & 0.45 & 0.036 & 0.25 & $<0.02$ \\
\hline Antimony & $<0.05$ & $<0.05$ & $<0.05$ & $<0.05$ & $<0.05$ & $<0.05$ & $<0.05$ & $<0.05$ \\
\hline Arsenic & $<0.05$ & $<0.05$ & $<0.05$ & $<0.05$ & $<0.05$ & $<0.05$ & $<0.05$ & $<0.05$ \\
\hline Barium & 0.29 & 0.19 & 0.28 & 0.27 & 0.29 & 0.28 & 0.31 & 0.3 \\
\hline Beryllium & 0.00079 & 0.00088 & $<0.0003$ & $<0.0003$ & $<0.0003$ & $<0.0003$ & $<0.0003$ & $<0.0003$ \\
\hline Boron & 0.063 & 0.052 & 0.031 & 0.017 & 0.038 & 0.045 & 0.025 & 0.027 \\
\hline Cadmium (AAS) & $<0.002$ & $<0.002$ & $<0.002$ & $<0.002$ & $<0.002$ & $<0.002$ & $<0.002$ & $<0.002$ \\
\hline Cadmium & $<0.003$ & $<0.003$ & $<0.003$ & $<0.003$ & $<0.003$ & $<0.003$ & $<0.003$ & $<0.003$ \\
\hline Chromium (AAS) & $<0.01$ & $<0.01$ & $<0.01$ & $<0.01$ & $<0.01$ & $<0.01$ & $<0.01$ & $<0.01$ \\
\hline Chromium & $<0.01$ & $<0.01$ & $<0.01$ & $<0.01$ & $<0.01$ & $<0.01$ & $<0.01$ & $<0.01$ \\
\hline Cobalt & 0.022 & 0.032 & $<0.005$ & $<0.005$ & $<0.005$ & $<0.005$ & $<0.005$ & $<0.005$ \\
\hline Copper & 0.0051 & 0.0066 & 0.0045 & $<0.004$ & $<0.004$ & $<0.004$ & $<0.004$ & $<0.004$ \\
\hline Iron & 2.3 & 0.047 & 0.6 & 0.043 & 0.63 & 0.04 & 0.45 & 0.03 \\
\hline Lead (AAS) & $<0.004$ & $<0.004$ & $<0.004$ & $<0.004$ & $<0.004$ & $<0.004$ & $<0.004$ & $<0.004$ \\
\hline Mercury (CVAA) & $<0.0002$ & $<0.0002$ & $<0.0002$ & $<0.0002$ & $<0.0002$ & $<0.0002$ & $<0.0002$ & $<0.0002$ \\
\hline Hol ybdenum & $<0.01$ & $<0.01$ & $<0.01$ & $<0.01$ & $<0.01$ & $<0.01$ & $<0.01$ & $<0.01$ \\
\hline Nickel & 0.033 & 0.029 & $<0.01$ & $<0.01$ & $<0.01$ & $<0.01$ & $<0.01$ & $<0.01$ \\
\hline Selenium & $<0.05$ & $<0.05$ & $<0.05$ & $<0.05$ & $<0.05$ & $<0.05$ & $<0.05$ & $<0.05$ \\
\hline Silver & $<0.006$ & $<0.006$ & $<0.006$ & $<0.006$ & $<0.006$ & $<0.006$ & $<0.006$ & $<0.006$ \\
\hline Strontium & 0.078 & 0.047 & 0.24 & 0.24 & 0.27 & 0.26 & 0.26 & 0.26 \\
\hline Thorium & $<0.2$ & $<0.2$ & $<0.2$ & $<0.2$ & $<0.2$ & $<0.2$ & $<0.2$ & $<0.2$ \\
\hline Uranium (Fluor) & 0.001 & 0.001 & $<0.001$ & $<0.001$ & $<0.001$ & $<0.001$ & $<0.001$ & $<0.001$ \\
\hline Vanadium & $<0.005$ & $<0.005$ & $<0.005$ & $<0.005$ & $<0.005$ & $<0.005$ & $<0.005$ & $<0.005$ \\
\hline zinc & 0.21 & 0.062 & 0.0044 & 0.0024 & 0.0046 & 0.0036 & 0.0044 & 0.011 \\
\hline MAJOR IONS (mg/L) & $\cdot$ & • & • & $\cdot$ & . & • & . & . \\
\hline Alkalinity-HCO3 & $\dot{9}$ & • & $289^{\circ}$ & • & 280 & • & $292^{\circ}$ & . \\
\hline Alkalinity- $\mathrm{CO} 3$ & $<1$ & • & $<1$ & • & $<1$ & - & $<1$ & . \\
\hline Calcium & 11 & 6.1 & 110 & 110 & 110 & 110 & 120 & 110 \\
\hline Chloride & 44 & • & 13 & • & 15 & . & 11 & . \\
\hline Fluoride & $<0.1$ & - & $<0.1$ & - & $<0.1$ & . & $<0.1$ & . \\
\hline Magnesium & 7.1 & 5 & 8.2 & 8.1 & 8.2 & 8 & 8.7 & 8.5 \\
\hline Manganese & 0.85 & 0.89 & 0.23 & 0.22 & 0.25 & 0.24 & 0.27 & 0.25 \\
\hline Nitrate-N & 3.9 & . & $<0.2$ & • & $<0.2$ & • & $<0.2$ & . \\
\hline Potassium & 2.9 & 2.2 & 2.5 & 2.1 & 2.4 & 2.2 & 2.2 & 2.3 \\
\hline Sodium & 17 & 16 & 3.5 & 3.5 & 3.4 & 3.4 & 3.8 & 3.8 \\
\hline Sulfate & 3 & • & 23 & • & 19 & . & 20 & . \\
\hline
\end{tabular}

(CONTINUED) 
APPENDIX F.1

Field Duplicate Groundwater Quality Data, 1993

\begin{tabular}{|c|c|c|c|c|c|c|c|c|}
\hline \multirow{4}{*}{$\begin{array}{l}\text { Sampling Point } \\
\text { - } \\
\text { Location } \\
\text { Date Sampled }\end{array}$} & \multicolumn{4}{|c|}{ GW-747 } & \multicolumn{2}{|c|}{ GW-751 } & \multicolumn{2}{|c|}{$G W-753$} \\
\hline & \multicolumn{4}{|c|}{ GRIDK2 } & \multirow{2}{*}{\multicolumn{2}{|c|}{$\frac{\text { GRIDJ3 }}{10 / 26 / 93}$}} & \multirow{2}{*}{\multicolumn{2}{|c|}{$\frac{\text { GRIDJ2 }}{08 / 03 / 93}$}} \\
\hline & \multicolumn{2}{|c|}{$01 / 19 / 93$} & \multicolumn{2}{|c|}{$04 / 13 / 93$} & & & & \\
\hline & TOT & DIS & TOT & DIS & TOT & DIS & TOT & DIS \\
\hline METALS (mg/L) & - & - & - & - &. & . & . & • \\
\hline Aluminum & 0.021 & $<0.02$ & 0.024 & $<0.02$ & 0.12 & $<0.02$ & $<0.02$ & $<0.02$ \\
\hline Antimony & $<0.05$ & $<0.05$ & $<0.05$ & $<0.05$ & $<0.05$ & $<0.05$ & $<0.05$ & $<0.05$ \\
\hline Arsenic & $<0.05$ & $<0.05$ & $<0.05$ & $<0.05$ & $<0.05$ & $<0.05$ & $<0.05$ & $<0.05$ \\
\hline Barium & 0.13 & 0.12 & 0.13 & 0.13 & 0.57 & 0.57 & 0.22 & 0.21 \\
\hline Beryllium & $<0.0003$ & $<0.0003$ & $<0.0003$ & $<0.0003$ & $<0.0003$ & $<0.0003$ & $<0.0003$ & $<0.0003$ \\
\hline Boron & 0.064 & 0.088 & 0.086 & 0.071 & 0.042 & 0.037 & 0.04 & 0.053 \\
\hline Cadmium (AAS) & $<0.002$ & $<0.002$ & $<0.002$ & $<0.002$ & $<0.002$ & $<0.002$ & $<0.002$ & $<0.002$ \\
\hline Cadmium & $<0.003$ & $<0.003$ & $<0.003$ & $<0.003$ & $<0.003$ & $<0.003$ & $<0.003$ & $<0.003$ \\
\hline Chromium (AAS) & $<0.01$ & $<0.01$ & $<0.01$ & $<0.01$ & $<0.01$ & $<0.01$ & $<0.01$ & $<0.01$ \\
\hline Chromium & $<0.01$ & $<0.01$ & $<0.01$ & $<0.01$ & $<0.01$ & $<0.01$ & $<0.01$ & $<0.01$ \\
\hline Cobalt & $<0.005$ & $<0.005$ & $<0.005$ & $<0.005$ & $<0.005$ & $<0.005$ & $<0.005$ & $<0.005$ \\
\hline Copper & $<0.004$ & $<0.004$ & $<0.004$ & $<0.004$ & $<0.004$ & $<0.004$ & $<0.004$ & $<0.004$ \\
\hline Iron & 0.13 & 0.11 & 0.1 & 0.088 & 0.2 & 0.12 & 0.23 & 0.15 \\
\hline Lead (AAS) & $<0.004$ & $<0.004$ & $<0.004$ & $<0.004$ & $<0.004$ & $<0.004$ & $<0.004$ & $<0.004$ \\
\hline Mercury (CVAA) & $<0.0002$ & $<0.0002$ & $<0.0002$ & $<0.0002$ & $<0.0002$ & $<0.0002$ & $<0.0002$ & $<0.0002$ \\
\hline Mol ybdenum & $<0.01$ & $<0.01$ & $<0.01$ & $<0.01$ & $<0.01$ & $<0.01$ & $<0.01$ & $<0.01$ \\
\hline Nickel & $<0.01$ & $<0.01$ & $<0.01$ & $<0.01$ & $<0.01$ & $<0.01$ & $<0.01$ & $<0.01$ \\
\hline Selenium & $<0.05$ & $<0.05$ & $<0.05$ & $<0.05$ & $<0.05$ & $<0.05$ & $<0.05$ & $<0.05$ \\
\hline Silver & $<0.006$ & $<0.006$ & $<0.006$ & $<0.006$ & $<0.006$ & $<0.006$ & $<0.006$ & $<0.006$ \\
\hline Strontium & 0.58 & 0.57 & 0.58 & 0.58 & 0.46 & 0.46 & 0.37 & 0.36 \\
\hline Thorium & $<0.2$ & $<0.2$ & $<0.2$ & $<0.2$ & $<0.2$ & $<0.2$ & $<0.2$ & $<0.2$ \\
\hline Uranium (Fluor) & $<0.001$ & $<0.001$ & $<0.001$ & $<0.001$ & 0.001 & $<0.001$ & $<0.001$ & $<0.001$ \\
\hline Vanadium & $<0.005$ & $<0.005$ & $<0.005$ & $<0.005$ & $<0.005$ & $<0.005$ & $<0.005$ & $<0.005$ \\
\hline zinc & 0.0067 & 0.0063 & $<0.002$ & $<0.002$ & 0.0026 & $<0.002$ & 0.011 & 0.0064 \\
\hline MAJOR IONS (mg/L) & • & - & . & - & . & - & . & . \\
\hline Alkalinity-HCO3 & 206 & $\dot{\bullet}$ & 203 & • & $18 \dot{4}$ & $\cdot$ & $19 \dot{9}$ & . \\
\hline Alkalinity- $\mathrm{CO} 3$ & $<1$ & . & $<1$ & . & $<1$ & . & $<1$ & . \\
\hline Calcium & 51 & 50 & 53 & 53 & 57 & 56 & 61 & 58 \\
\hline Chloride & 6 & . & 5.5 & • & 10 &. & 3.1 & . \\
\hline Fluoride & $<0.1$ & - & 0.2 & • & $<0.1$ & . & $<0.1$ & . \\
\hline Magnesium & 11 & 10 & 10 & 10 & 9.9 & 9.7 & 9.4 & 9 \\
\hline Manganese & 0.014 & 0.014 & 0.013 & 0.013 & 0.014 & 0.013 & 0.036 & 0.034 \\
\hline Nitrate-N & $<0.2$ & . & $<0.2$ & • & $<0.2$ & . & 0.83 & . \\
\hline Potassium & 1.9 & 1.8 & 2 & 1.7 & 3.1 & 3.2 & 2.3 & 2.1 \\
\hline Sodium & 23 & 23 & 24 & 24 & 8.7 & 8.7 & 12 & 11 \\
\hline Sulfate & 26 & . & 23.9 & . & 4 &. & 15 & • \\
\hline
\end{tabular}

(CONTINUED) 
Field Duplicate Groundwater Quality Data, 1993

\begin{tabular}{|c|c|c|c|c|c|c|c|c|}
\hline \multirow{4}{*}{$\begin{array}{l}\text { Sampling Point } \\
\text { Location } \\
\text { Date Sampled }\end{array}$} & \multicolumn{2}{|c|}{ GW-755 } & \multicolumn{6}{|c|}{ GW-758 } \\
\hline & \multicolumn{2}{|c|}{ GRIDJI } & \multicolumn{6}{|c|}{ GRIDG1 } \\
\hline & \multicolumn{2}{|c|}{$10 / 21 / 93$} & \multicolumn{2}{|c|}{$04 / 13 / 93$} & \multicolumn{2}{|c|}{$09 / 12 / 93$} & \multicolumn{2}{|c|}{$11 / 19 / 93$} \\
\hline & TOT & DIS & TOT & DIS & TOT & DIS & TOT & DIS \\
\hline METALS (mg/L) & - & - & . & • & - & . & . & - \\
\hline Aluminum & 0.024 & 0.024 & 0.082 & $<0.02$ & 0.033 & $<0.02$ & 0.043 & 0.029 \\
\hline Antimony & $<0.05$ & $<0.05$ & $<0.05$ & $<0.05$ & $<0.05$ & $<0.05$ & $<0.05$ & $<0.05$ \\
\hline Arsenic & $<0.05$ & $<0.05$ & $<0.05$ & $<0.05$ & $<0.05$ & $<0.05$ & $<0.05$ & $<0.05$ \\
\hline Barium & 0.19 & 0.19 & 0.11 & 0.11 & 0.12 & 0.12 & 0.11 & 0.12 \\
\hline Beryllium & $<0.0003$ & 0.00034 & $<0.0003$ & $<0.0003$ & $<0.0003$ & $<0.0003$ & $<0.0003$ & $<0.0003$ \\
\hline Boron & 0.13 & 0.11 & 0.061 & 0.06 & 0.072 & 0.05 & 0.094 & 0.11 \\
\hline Cadmium (AAS) & $<0.002$ & $<0.002$ & $<0.002$ & $<0.002$ & $<0.002$ & $<0.002$ & $<0.002$ & $<0.002$ \\
\hline Cadmium & $<0.003$ & $<0.003$ & $<0.003$ & $<0.003$ & $<0.003$ & $<0.003$ & $<0.003$ & $<0.003$ \\
\hline Chromium (AAS) & $<0.01$ & $<0.01$ & $<0.01$ & $<0.01$ & $<0.01$ & $<0.01$ & $<0.01$ & $<0.01$ \\
\hline Chromium & $<0.01$ & $<0.01$ & $<0.01$ & $<0.01$ & $<0.01$ & $<0.01$ & 0.014 & 0.012 \\
\hline Cobalt & $<0.005$ & $<0.005$ & $<0.005$ & $<0.005$ & $<0.005$ & $<0.005$ & $<0.005$ & $<0.005$ \\
\hline Copper & 0.0084 & 0.0063 & $<0.004$ & $<0.004$ & $<0.004$ & $<0.004$ & $<0.004$ & $<0.004$ \\
\hline Iron & 0.054 & 0.024 & 0.28 & 0.19 & 0.43 & 0.19 & 0.46 & 0.28 \\
\hline Lead (AAS) & $<0.004$ & $<0.004$ & $<0.004$ & $<0.004$ & $<0.004$ & $<0.004$ & $<0.004$ & $<0.004$ \\
\hline Mercury (CVAA) & $<0.0002$ & $<0.0002$ & $<0.0002$ & $<0.0002$ & $<0.0002$ & $<0.0002$ & $<0.0002$ & $<0.0002$ \\
\hline Molybdenum & $<0.01$ & $<0.01$ & $<0.01$ & $<0.01$ & $<0.01$ & $<0.01$ & $<0.01$ & $<0.01$ \\
\hline Nickel & $<0.01$ & $<0.01$ & $<0.01$ & $<0.01$ & $<0.01$ & $<0.01$ & $<0.01$ & 0.013 \\
\hline Selenium & $<0.05$ & $<0.05$ & $<0.05$ & $<0.05$ & 0.053 & $<0.05$ & $<0.05$ & $<0.05$ \\
\hline silver & $<0.006$ & $<0.006$ & $<0.006$ & $<0.006$ & $<0.006$ & $<0.006$ & $<0.006$ & $<0.006$ \\
\hline Strontium & 0.86 & 0.89 & 0.43 & 0.43 & 0.46 & 0.47 & 0.48 & 0.5 \\
\hline Thorium & $<0.2$ & $<0.2$ & $<0.2$ & $<0.2$ & $<0.2$ & $<0.2$ & $<0.2$ & $<0.2$ \\
\hline Oranium (Fluor) & $<0.001$ & $<0.001$ & $<0.001$ & $<0.001$ & $<0.001$ & $<0.001$ & $<0.001$ & $<0.001$ \\
\hline Vanadium & $<0.005$ & $<0.005$ & $<0.005$ & $<0.005$ & $<0.005$ & $<0.005$ & $<0.005$ & $<0.005$ \\
\hline zinc & 0.0097 & 0.0097 & 0.0032 & 0.0032 & 0.0036 & 0.0072 & $<0.002$ & $<0.002$ \\
\hline MAJOR IONS $(\mathrm{mg} / \mathrm{L})$ & $\cdot$ & $\cdot$ & . & . & $\cdot$ & $\cdot$ & $\cdot$ & • \\
\hline Alkalinity-HCO3 & $2 \dot{7}$ & $\cdot$ & $18 \dot{8}$ & $\cdot$ & $19 \dot{9}$ & $\cdot$ & $19 \dot{8}$ & • \\
\hline Alkalinity-CO3 & $<1$ & $\cdot$ & $<1$ & . & $<1$ & . & $<1$ & . \\
\hline Calcium & 23 & 25 & 62 & 62 & 72 & 73 & 66 & 70 \\
\hline Chloride & 15 & - & 14 & . & 14.7 & . & 20 & • \\
\hline Fluoride & 0.5 &. & 0.1 & . & 0.1 & . & 0.2 & • \\
\hline Magnesium & 5.8 & 6.1 & 8 & 8 & 9.1 & 9.2 & 8.7 & 9.1 \\
\hline Manganese & 0.013 & 0.018 & 0.19 & 0.19 & 0.25 & 0.25 & 0.21 & 0.23 \\
\hline Nitrate-N & $<0.2$ &. & $<0.2$ & . & $<0.2$ &. & $<0.2$ & • \\
\hline Potassium & 3.1 & 3 & 2.6 & 2.6 & 1.9 & 2 & 2.5 & 2.7 \\
\hline Sodium & 83 & 75 & 14 & 14 & 14 & 14 & 14 & 15 \\
\hline Sulfate & 16 & . & 21 &. & 19.5 & . & 17 & - \\
\hline
\end{tabular}

(CONTINURD) 
Field Duplicate Groundwater Quality Data, 1993

\begin{tabular}{|c|c|c|c|c|c|c|c|c|}
\hline \multirow{4}{*}{$\begin{array}{l}\text { Sampling Point } \\
\text { Location } \\
\text { - } \\
\text { Date Sampled }\end{array}$} & \multicolumn{2}{|c|}{ GW-760 } & \multicolumn{6}{|c|}{$G W-766$} \\
\hline & \multicolumn{2}{|c|}{ GRIDG2 } & \multicolumn{6}{|c|}{ GRIDI 2} \\
\hline & \multicolumn{2}{|c|}{$01 / 27 / 93$} & \multicolumn{2}{|c|}{$04 / 15 / 93$} & \multicolumn{2}{|c|}{$08 / 03 / 93$} & \multicolumn{2}{|c|}{$10 / 13 / 93$} \\
\hline & TOT & DIS & TOT & DIS & TOT & DIS & TOT & DIS \\
\hline METALS (mg/L) & • & $\cdot$ & $\cdot$ & - & $\cdot$ & • & $\cdot$ & • \\
\hline Aluminum & 0.16 & 0.15 & 0.056 & $<0.02$ & 0.03 & 0.026 & 0.049 & 0.045 \\
\hline Antimony & $<0.05$ & $<0.05$ & $<0.05$ & $<0.05$ & $<0.05$ & $<0.05$ & $<0.05$ & $<0.05$ \\
\hline Arsenic & $<0.05$ & $<0.05$ & $<0.05$ & $<0.05$ & $<0.05$ & $<0.05$ & $<0.05$ & $<0.05$ \\
\hline Barium & 0.43 & 0.44 & 0.22 & 0.23 & 0.23 & 0.23 & 0.26 & 0.28 \\
\hline Beryllium & $<0.0003$ & $<0.0003$ & $<0.0003$ & $<0.0003$ & $<0.0003$ & $<0.0003$ & $<0.0003$ & $<0.0003$ \\
\hline Boron & 0.029 & 0.037 & 0.039 & 0.027 & 0.056 & 0.04 & 0.03 & 0.034 \\
\hline Cadmium (AAS) & $<0.002$ & $<0.002$ & $<0.002$ & $<0.002$ & $<0.002$ & $<0.002$ & $<0.002$ & $<0.002$ \\
\hline Cadmium & $<0.003$ & $<0.003$ & $<0.003$ & $<0.003$ & $<0.003$ & $<0.003$ & $<0.003$ & $<0.003$ \\
\hline Chromium (AAS) & 0.18 & $<0.01$ & $<0.01$ & $<0.01$ & $<0.01$ & $<0.01$ & $<0.01$ & $<0.01$ \\
\hline Chromium & 0.043 & $<0.01$ & $<0.01$ & $<0.01$ & $<0.01$ & $<0.01$ & $<0.01$ & $<0.01$ \\
\hline Cobalt & $<0.005$ & $<0.005$ & $<0.005$ & $<0.005$ & $<0.005$ & $<0.005$ & $<0.005$ & $<0.005$ \\
\hline Copper & $<0.004$ & $<0.004$ & $<0.004$ & $<0.004$ & $<0.004$ & $<0.004$ & $<0.004$ & $<0.004$ \\
\hline Iron & 0.34 & 0.0069 & 0.025 & 0.015 & 0.058 & 0.011 & 0.056 & $<0.005$ \\
\hline Lead (AAS) & $<0.004$ & $<0.004$ & $<0.004$ & $<0.004$ & $<0.004$ & $<0.004$ & $<0.004$ & $<0.004$ \\
\hline Mercury (CVAA) & $<0.0002$ & $<0.0002$ & $<0.0002$ & $<0.0002$ & $<0.0002$ & $<0.0002$ & $<0.0002$ & $<0.0002$ \\
\hline Molybdenum & $<0.01$ & $<0.01$ & $<0.01$ & $<0.01$ & $<0.01$ & $<0.01$ & $<0.01$ & $<0.01$ \\
\hline Nickel & 0.082 & 0.064 & $<0.01$ & $<0.01$ & $<0.01$ & $<0.01$ & $<0.01$ & $<0.01$ \\
\hline Selenium & $<0.05$ & $<0.05$ & 0.059 & $<0.05$ & $<0.05$ & $<0.05$ & $<0.05$ & $<0.05$ \\
\hline silver & $<0.006$ & $<0.006$ & $<0.006$ & $<0.006$ & $<0.006$ & $<0.006$ & $<0.006$ & $<0.006$ \\
\hline Strontium & 0.23 & 0.24 & 0.37 & 0.38 & 0.43 & 0.42 & 0.52 & 0.72 \\
\hline Thorium & $<0.2$ & $<0.2$ & $<0.2$ & $<0.2$ & $<0.2$ & $<0.2$ & $<0.2$ & $<0.2$ \\
\hline Urantum (Fluor) & $<0.001$ & $<0.001$ & $<0.001$ & $<0.001$ & $<0.001$ & $<0.001$ & $<0.001$ & $<0.001$ \\
\hline Vanadium & $<0.005$ & $<0.005$ & $<0.005$ & $<0.005$ & $<0.005$ & $<0.005$ & $<0.005$ & $<0.005$ \\
\hline zinc & 0.0043 & 0.011 & 0.0046 & 0.0058 & 0.0048 & 0.0089 & $<0.002$ & 0.0045 \\
\hline MAJOR IONS $(\mathrm{mg} / \mathrm{L})$ & & - & . & . & $\cdot$ & $\cdot$ & $\cdot$ & $\cdot$ \\
\hline Alkalinity-HCO3 & 154 & $\cdot$ & 109 & $\dot{\bullet}$ & $12 \dot{4}$ & $\cdot$ & $12 \dot{2}$ & • \\
\hline Alkalinity-CO3 & $<1$ & • & 16 & - & 4 & $\cdot$ & 12 & • \\
\hline Calcium & 110 & 110 & 33 & 35 & 35 & 33 & 35 & 39 \\
\hline Chloride & 182 & . & 2.7 & • & 3.9 & . & 4 & • \\
\hline Fluoride & $<0.1$ & $\cdot$ & 0.1 & . & $<0.1$ & . & 0.1 & . \\
\hline Magnesium & 12 & 12 & 6.1 & 6.4 & 7.2 & 7 & 7.3 & 8.9 \\
\hline Manganese & 0.042 & 0.038 & 0.0041 & 0.0033 & 0.006 & 0.0052 & 0.0076 & 0.0067 \\
\hline Nitrate-N & 0.29 & $\cdot$ & $<0.2$ & . & $<0.2$ & $\cdot$ & $<0.2$ & • \\
\hline Potassium & 2 & 2.2 & 3.7 & 3.7 & 3.6 & 4.1 & 4 & 4.6 \\
\hline Sodium & 8.9 & 9.1 & 12 & 12 & 12 & 12 & 13 & 12 \\
\hline Sulfate & 17 & . & 10 & • & 14 & $\cdot$ & 9 & • \\
\hline
\end{tabular}

(CONTINUED) 
APPENDIX F.1

Field Duplicate Groundwater Quality Data, 1993

\begin{tabular}{|c|c|c|}
\hline \multirow{4}{*}{$\begin{array}{l}\text { Sampling Point } \\
\text { Location } \\
\text { - } \\
\text { Date Sampled }\end{array}$} & \multicolumn{2}{|c|}{$G W-773$} \\
\hline & \multicolumn{2}{|c|}{ GRIDH2 } \\
\hline & \multicolumn{2}{|c|}{$02 / 03 / 93$} \\
\hline & TOT & DIS \\
\hline METALS (mg/L) & - & • \\
\hline Aluminum & 0.051 & 0.034 \\
\hline Antimony & $<0.05$ & $<0.05$ \\
\hline Arsentc & $<0.05$ & $<0.05$ \\
\hline Barlum & 0.35 & 0.35 \\
\hline Beryl11um & $<0.0003$ & $<0.0003$ \\
\hline Boron & 0.034 & 0.035 \\
\hline Cadmium (AAS) & $<0.002$ & $<0.002$ \\
\hline Cadmium & $<0.003$ & $<0.003$ \\
\hline Chromium (AAS) & $<0.01$ & $<0.01$ \\
\hline Chromi um & $<0.01$ & $<0.01$ \\
\hline Cobalt & $<0.005$ & $<0.005$ \\
\hline Copper & $<0.004$ & $<0.004$ \\
\hline Iron & 0.098 & 0.14 \\
\hline Lead (AAS) & $<0.004$ & $<0.004$ \\
\hline Mercury (CVAA) & $<0.0002$ & $<0.0002$ \\
\hline Molybdenum & $<0.01$ & $<0.01$ \\
\hline Nickel & $<0.01$ & $<0.01$ \\
\hline Selenium & $<0.05$ & $<0.05$ \\
\hline Silver & $<0.006$ & $<0.006$ \\
\hline Strontium & 0.16 & 0.15 \\
\hline Thorium & $<0.2$ & $<0.2$ \\
\hline Uranium (Fluor) & $<0.001$ & $<0.001$ \\
\hline Vanadium & $<0.005$ & $<0.005$ \\
\hline zinc & 0.0089 & 0.0074 \\
\hline MAJOR IONS (mg/L) & - & - \\
\hline Alkalinity-hCO3 & 163 & - \\
\hline Alkalinity-co3 & $<1$ & • \\
\hline Calcium & 52 & 52 \\
\hline Chloride & 2.7 & • \\
\hline Fluoride & $<0.1$ & • \\
\hline Magnesium & 6.9 & 6.8 \\
\hline Manganese & 0.037 & 0.032 \\
\hline Nitrate-N & $<0.2$ & • \\
\hline Potaseium & 1.2 & 1.2 \\
\hline Sodium & 4.8 & 4.6 \\
\hline Sulfate & 7.6 & • \\
\hline
\end{tabular}




\section{APPENDIX F.2}

\section{VOLATILE ORGANIC COMPOUNDS}


APPENDIX F. 2

Field Duplicate Data, 1993

\begin{tabular}{|c|c|c|c|c|c|c|c|c|}
\hline \multirow{3}{*}{$\begin{array}{l}\text { Sampling Point } \\
\text { Location } \\
\text { - } \\
\text { Date Sampled }\end{array}$} & \multirow{3}{*}{\begin{tabular}{|c|} 
(iW-151 \\
NHP \\
$08 / 06 / 93$ \\
\end{tabular}} & \multirow{3}{*}{\begin{tabular}{|c|} 
GW-170 \\
EXP \\
$05 / 24 / 93$ \\
\end{tabular}} & \multicolumn{5}{|c|}{$G W-183$} & \multirow{3}{*}{$\begin{array}{c}\text { GW-190 } \\
\text { RG } \\
06 / 17 / 93\end{array}$} \\
\hline & & & \multicolumn{5}{|c|}{$\mathbf{F F}$} & \\
\hline & & & $03 / 10 / 93$ & $06 / 21 / 93$ & $06 / 29 / 93$ & $09 / 22 / 93$ & $11 / 16 / 93$ & \\
\hline VOLATILE ORGANICS (ug/L) & - & - & - & - & - & - & - & - \\
\hline Acetone & 200 & 100 & 10 & 100 & . & 100 & 100 & 200 \\
\hline Benzene & 100 & $5 \mathbf{U}$ & 310 & 32 & . & 71 & 7 & 100 \\
\hline Bromodichloromethane & 200 & 50 & $5 \mathrm{U}$ & $5 \mathbf{U}$ & . & $5 U$ & $5 u$ & 100 \\
\hline Bromoform & 100 & 5u & $5 \mathbf{u}$ & 5u & - & $5 \mathbf{U}$ & su & 100 \\
\hline Bromomethane & 200 & 100 & 100 & 100 & - & 100 & 100 & 200 \\
\hline 2-Butanone & 200 & 4 & 100 & 100 & - & 100 & $10 \mathrm{U}$ & 200 \\
\hline Carbon disulfide & 100 & $5 \mathbf{u}$ & 50 & $\mathbf{5 u}$ & • & $\mathbf{5 u}$ & 50 & 100 \\
\hline Carbon tetrachloride & 520 & $5 u$ & $5 \mathbf{v}$ & 50 & - & 50 & $5 \mathbf{v}$ & 100 \\
\hline Chlorobenzene & 100 & $5 \mathbf{u}$ & $\mathbf{5 u}$ & 50 & $\cdot$ & 50 & $5 u$ & 100 \\
\hline Chlorodlbromomethane & 100 & $5 \mathbf{u}$ & $\mathbf{5 u}$ & $\mathbf{5 v}$ & - & $\mathbf{5 u}$ & $5 \mathbf{u}$ & 100 \\
\hline Chloroethane & 200 & 100 & 100 & 100 & - & 100 & $10 \mathrm{U}$ & 200 \\
\hline Chloroform & 20 & 63 & 50 & 5u & - & 50 & 50 & 100 \\
\hline Chloromethane & 200 & 100 & 100 & 100 & - & 100 & 100 & 200 \\
\hline 1,1-Dichloroethane & 100 & $\mathbf{5 u}$ & $\mathbf{5 u}$ & $\mathbf{s u}$ & - & su & su & 7 \\
\hline 1,2-Dichloroethane & 100 & $5 \mathrm{U}$ & 6 & $5 v$ & - & 50 & 50 & 100 \\
\hline 1,1-Dichloroethene & 100 & $5 \mathrm{U}$ & 50 & $5 \mathbf{v}$ & - & 50 & $5 U$ & 100 \\
\hline 1,2-Dichloroethene & 100 & $5 v$ & $\mathbf{5 u}$ & $5 \mathbf{v}$ & . & 50 & $5 \mathrm{U}$ & 250 \\
\hline 1,2-Dichloropropane & 100 & 50 & 5U & $5 v$ & - & su & 50 & 100 \\
\hline c1s-1,3-Dichloropropene & 100 & $5 \mathbf{u}$ & 50 & su & $\cdot$ & 50 & 50 & 100 \\
\hline trans-1,3-Dichloropropene & 100 & $5 \mathrm{U}$ & $\mathbf{5 u}$ & $5 \mathbf{v}$ & - & 50 & $5 v$ & 100 \\
\hline Ethylbenzene & 100 & 5u & 19 & 3 & - & 4 & 1 & 100 \\
\hline 2-Hexanone & 200 & 100 & 100 & 100 & - & 100 & 100 & 200 \\
\hline 4-Methy 1-2-pentanone & 200 & 1 & 100 & 100 & - & 100 & 100 & 200 \\
\hline Methylene chloride & 100 & 2 & 2 & $\mathbf{5 u}$ & $\cdot$ & 50 & 0.9 & 100 \\
\hline styrene & 100 & $5 U$ & 1 & $\mathbf{5 u}$ & $\cdot$ & $5 v$ & $5 v$ & 100 \\
\hline $1,1,2,2$-Tetrachloroethane & 100 & $5 \mathrm{U}$ & 50 & 50 & $\cdot$ & 50 & $5 u$ & 100 \\
\hline Tetrachloroethene & 21 & 5 & su & $5 \mathbf{v}$ & - & 5u & $5 v$ & 72 \\
\hline Toluene & 100 & $5 \mathbf{u}$ & 140 & 26 & - & 35 & 5 & 100 \\
\hline $1,1,1$-Trichloroethane & 100 & $5 \mathrm{U}$ & $5 \mathrm{U}$ & $5 \mathbf{v}$ & - & $5 \mathbf{U}$ & $5 \mathbf{v}$ & 100 \\
\hline $1,1,2$-Trichloroethane & 100 & 50 & $5 v$ & 50 & - & 50 & $5 \mathbf{s}$ & 100 \\
\hline Trichloroethene & 100 & 2 & $5 v$ & 50 & - & $5 \mathbf{v}$ & su & 79 \\
\hline Vinyl acetate & 200 & 100 & 66 & 100 & - & 100 & 100 & 200 \\
\hline Vinyl chloride & 200 & 100 & 100 & 100 & . & 100 & 100 & 59 \\
\hline Xylenes & 100 & 50 & 71 & 11 & . & 20 & 4 & 100 \\
\hline
\end{tabular}

(CONTINUED) 
APPENDIX F.2

Field Duplicate Data, 1993

\begin{tabular}{|c|c|c|c|c|c|c|c|c|}
\hline \multirow{3}{*}{$\begin{array}{l}\text { Sampling Point } \\
\text { Location } \\
\text { Date Sampled }\end{array}$} & \multicolumn{2}{|c|}{ GW- 190} & \multicolumn{2}{|c|}{ GW-207 } & \multicolumn{2}{|c|}{$G W-208$} & \multicolumn{2}{|c|}{$G W-255$} \\
\hline & \multicolumn{2}{|c|}{ RG } & \multicolumn{2}{|c|}{ EXP } & \multicolumn{2}{|c|}{ EXP } & \multicolumn{2}{|c|}{$\mathbf{s 2}$} \\
\hline & $07 / 07 / 93$ & $09 / 15 / 93$ & $08 / 11 / 93$ & $10 / 26 / 93$ & $01 / 19 / 93$ & $05 / 06 / 93$ & $01 / 08 / 93$ & $05 / 03 / 93$ \\
\hline VOLATILE ORGANICS (Ug/L) & - & $\cdot$ & $\cdot$ & $\cdot$ & $\cdot$ & - & - & - \\
\hline Acetone & . & 200 & 100 & 3 & 100 & 100 & 100 & 100 \\
\hline Benzene & . & 100 & 50 & 50 & su & 50 & su & su \\
\hline Bromodichloromethane & . & 100 & su & 5u & 50 & 50 & su & so \\
\hline Bromoform & . & 100 & su & 5u & su & su & su & 5v \\
\hline Bromomethane & . & 200 & 100 & 100 & 100 & $10 u$ & 100 & 100 \\
\hline 2-Butanone & . & 200 & 100 & 8 & 100 & 100 & 100 & 100 \\
\hline Carbon disulfide & - & 100 & $5 u$ & so & 50 & 5u & su & 5u \\
\hline Carbon tetrachloride & . & 100 & su & 50 & su & 5u & 50 & su \\
\hline Chlorobenzene & . & 100 & 5u & 5u & su & su & 50 & su \\
\hline Chlorodibromomethane & - & 100 & 5u & su & 50 & 50 & 50 & 50 \\
\hline Chloroethane & . & 200 & 100 & 100 & 100 & 100 & 100 & 100 \\
\hline Chloroform & - & 100 & su & su & su & 50 & 1 & 1 \\
\hline Chloromethane & . & 200 & 100 & 100 & 100 & 100 & 100 & 100 \\
\hline 1,1-Dichloroethane & . & 5 & 50 & su & 50 & su & $5 \mathbf{v}$ & su \\
\hline 1,2-Dichloroethane & . & 100 & 5u & 5u & 50 & 50 & su & 50 \\
\hline 1,1-Dichloroethene & . & 100 & 50 & 5u & 5u & 5u & 50 & 5u \\
\hline 1,2-Dichloroethene & . & 170 & sv & 50 & 5u & 50 & su & 50 \\
\hline 1,2-Dichloropropane & . & 100 & 50 & 50 & su & su & su & 5u \\
\hline Cis-1,3-Dichloropropene & - & 100 & 50 & 50 & 50 & su & $5 v$ & 5u \\
\hline trans-1,3-Dichloropropene & . & 100 & 50 & 5u & 50 & 5u & $5 v$ & 5u \\
\hline Ethylbenzene & - & 100 & 50 & 50 & 50 & 50 & $5 \mathbf{v}$ & su \\
\hline 2-Hexanone & . & 200 & 100 & 100 & 100 & 100 & 100 & 100 \\
\hline 4-Methyl-2-pentanone & - & 200 & 100 & $10 \mathrm{U}$ & 100 & 100 & 1 & 100 \\
\hline Methylene chloride & . & 100 & 5u & 2 & 5u & su & su & 5u \\
\hline Styrene & . & 100 & 50 & 50 & 50 & so & 50 & 5u \\
\hline $1,1,2,2$-Tetrachloroethane & - & 100 & $5 \mathbf{u}$ & so & 5u & 5u & 50 & 5u \\
\hline Tetrachloroethene & . & 94 & 50 & 50 & su & 50 & su & 5u \\
\hline roluene & . & 100 & 50 & 50 & 50 & 5u & 5u & 50 \\
\hline $1,1,1-\operatorname{Tr} 1 \mathrm{chl}$ loroethane & - & 100 & su & 50 & 50 & 5v & 50 & 50 \\
\hline $1,1,2$-Trichloroethane & . & 100 & 50 & 50 & su & su & 50 & 50 \\
\hline Trichloroethene & . & 46 & $5 \mathrm{u}$ & su & su & $5 \mathrm{u}$ & 50 & 50 \\
\hline vinyl acetate & $\cdot$ & 200 & 100 & 100 & 100 & 100 & 100 & 100 \\
\hline vinyl chloride & - & 49 & 100 & 100 & 100 & 100 & 100 & 100 \\
\hline xylenes & . & 100 & 50 & 50 & su & su & $5 u$ & su \\
\hline
\end{tabular}

(CONTINUED) 
APPENDIX F.2

Field Duplicate Data, 1993

\begin{tabular}{|c|c|c|c|c|c|c|c|c|}
\hline \multirow{3}{*}{$\begin{array}{l}\text { Sampling Point } \\
\text { Location } \\
\text { Date Sampled }\end{array}$} & \multirow{3}{*}{\begin{tabular}{|c|}
$G W-255$ \\
$S 2$ \\
$12 / 07 / 93$ \\
\end{tabular}} & \multicolumn{3}{|c|}{$G W-262$} & \multirow{3}{*}{\begin{tabular}{|c|}
$G W-264$ \\
$S Y$ \\
$12 / 08 / 93$ \\
\end{tabular}} & \multirow{2}{*}{\multicolumn{2}{|c|}{$\frac{G W-382}{\text { NHP }}$}} & \multirow{3}{*}{\begin{tabular}{|c|} 
GW-606 \\
EXP \\
$08 / 20 / 93$
\end{tabular}} \\
\hline & & \multicolumn{3}{|c|}{ sy } & & & & \\
\hline & & $01 / 11 / 93$ & $04 / 08 / 93$ & $07 / 01 / 93$ & & $01 / 28 / 93$ & $04 / 26 / 93$ & \\
\hline VOLATILE ORCANICS $(\mathrm{ug} / \mathrm{L})$ & - & - & - & - & . & . & . & • \\
\hline Acetone & 100 & 100 & 100 & 100 & 100 & $80 u$ & 1000 & 500 \\
\hline Benzene & 5u & su & $5 U$ & su & 5u & 400 & 500 & 250 \\
\hline Bromodichloromethane & su & 5u & 5v & su & 50 & $40 v$ & 500 & 250 \\
\hline Bromoform & 5u & 5u & 5u & $5 U$ & 50 & 400 & 500 & 250 \\
\hline Bromomethane & 100 & 100 & 100 & 100 & 100 & 800 & 1000 & 500 \\
\hline 2-Butanone & 100 & 100 & 100 & 100 & 100 & 800 & 1000 & 500 \\
\hline Carbon diaulfide & 5u & 50 & 5u & su & 5u & 400 & 500 & 250 \\
\hline Carbon tetrachloride & 5u & 50 & 50 & su & 5u & 1200 & 5400 & 730 \\
\hline Chlorobenzene & 50 & 5u & 5u & 5u & $5 U$ & 400 & $50 u$ & 250 \\
\hline Chlorodibrimomethane & 5u & su & 50 & su & 50 & 400 & 500 & 250 \\
\hline Chloroethane & 100 & 100 & 100 & 100 & 100 & 800 & 1000 & 500 \\
\hline Chloroform & 1 & su & su & su & su & 890 & 380 & 250 \\
\hline Chloromethane & 100 & 100 & 100 & 100 & 100 & 800 & 1000 & 500 \\
\hline 1,1-Dichloroethane & 5U & so & 50 & 5u & 5u & $40 \mathrm{v}$ & 500 & 250 \\
\hline 1,2-Dichloroethane & 50 & 5u & su & su & 5u & 400 & 500 & 250 \\
\hline 1,1-Dichloroethene & 5u & su & 5u & $5 U$ & $5 \mathbf{U}$ & 400 & 500 & $25 \mathrm{U}$ \\
\hline 1,2-Dichloroethene & 50 & su & 50 & 5u & su & 400 & 500 & 250 \\
\hline 1,2-Dichloropropane & 5u & 50 & 5u & su & 50 & 400 & 500 & 250 \\
\hline cis-1,3-Dichloropropene & 50 & 5u & 5u & 50 & 50 & 400 & 500 & 250 \\
\hline trana-1,3-Dichloropropene & 5u & 50 & su & 50 & su & 400 & 500 & 250 \\
\hline Rthylbenzene & 5u & su & 5u & su & 50 & 400 & 500 & 250 \\
\hline 2-Hexanone & 200 & 100 & 100 & 100 & 100 & 800 & 1000 & 500 \\
\hline 4-Methy1-2-pentanone & 100 & 2 & 100 & 100 & 100 & 800 & 1000 & 500 \\
\hline Methylene chloride & 5u & 5u & 50 & $5 U$ & 50 & 24 & 500 & 250 \\
\hline styrene & 50 & 50 & 50 & 50 & 5u & 400 & 500 & 250 \\
\hline $1,1,2,2$-Tetrachloroethane & su & SU & 5U & su & 50 & 400 & 500 & 250 \\
\hline Tetrachloroethene & 5u & 50 & 50 & su & 5u & 94 & 250 & 6 \\
\hline Toluene & 50 & 5u & 5u & $5 U$ & 50 & $40 U$ & 500 & 250 \\
\hline $1,1,1-$ Trichloroethane & $5 u$ & su & 50 & su & 50 & 400 & 500 & 250 \\
\hline 1,1,2-Trichloroethane & 50 & 50 & 5u & su & $5 U$ & 400 & 500 & 250 \\
\hline Trichloroethene & 5U & 50 & 50 & so & 5u & 19 & 34 & 250 \\
\hline Vinyl acetate & 100 & 100 & 100 & 100 & 100 & 800 & 10010 & 500 \\
\hline Vinyl chloride & 200 & 100 & 100 & 100 & 100 & 800 & 1000 & 500 \\
\hline Xylenes & su & 50 & su & su & su & $40 u$ & 500 & 250 \\
\hline
\end{tabular}

(CONTINUED) 
APPENDIX F.2

Field Duplicate Data, 1993

\begin{tabular}{|c|c|c|c|c|c|c|c|c|}
\hline \multirow{3}{*}{$\begin{array}{l}\text { Sampling point } \\
\text { Location } \\
\text { Date Sampled }\end{array}$} & \multirow{3}{*}{\begin{tabular}{|c|} 
GW-618 \\
EXP \\
$09 / 16 / 93$ \\
\end{tabular}} & \multicolumn{2}{|c|}{$G W-631$} & \multicolumn{3}{|c|}{ GW-735 } & \multicolumn{2}{|c|}{$G w-747$} \\
\hline & & \multicolumn{2}{|c|}{ RG } & \multicolumn{3}{|c|}{ NHP } & \multicolumn{2}{|c|}{ GRIDK2 } \\
\hline & & $03 / 02 / 93$ & $11 / 17 / 93$ & $02 / 02 / 93$ & $05 / 07 / 93$ & $10 / 29 / 93$ & $01 / 19 / 93$ & $04 / 13 / 93$ \\
\hline VOLATILE ORGANICS (ug/L) & $\cdot$ & - & . & - & - & $\cdot$ & $\cdot$ & $\cdot$ \\
\hline Acetone & 100 & 100 & 100 & 100 & 100 & 100 & 1 & 100 \\
\hline Benzene & su & 2 & 2 & $5 v$ & su & $5 u$ & su & su \\
\hline Bromodichloromethane & 50 & su & 50 & 50 & $5 \mathrm{U}$ & $5 \mathrm{u}$ & 50 & 5u \\
\hline Bromoform & 50 & su & 50 & su & $5 \mathrm{U}$ & 5u & 50 & 50 \\
\hline Bromomethane & 100 & 100 & 100 & 100 & 100 & 100 & 100 & 100 \\
\hline 2-Butanone & 9 & 100 & 100 & 100 & 100 & 100 & 100 & 100 \\
\hline Carbon disulfide & 50 & 5u & $5 v$ & 50 & 50 & 50 & $5 \mathbf{5 u}$ & 50 \\
\hline Carbon tetrachloride & 50 & su & 50 & $5 \mathrm{v}$ & 50 & 50 & 50 & 5u \\
\hline Chlorobenzene & 50 & su & 5u & 50 & 5u & su & 50 & $5 v$ \\
\hline Chlorodibromomethane & su & su & su & 50 & 5u & $5 v$ & 5u & 50 \\
\hline Chloroethane & 100 & 200 & 100 & 100 & 100 & 200 & 100 & 100 \\
\hline Chloroform & 50 & su & 50 & 50 & 5u & $5 \mathrm{U}$ & $5 v$ & $5 \mathrm{U}$ \\
\hline Chloromethane & 100 & 100 & 100 & 100 & 100 & 100 & 100 & 100 \\
\hline 1,1-Dichloroethane & 50 & $\mathbf{5 u}$ & su & 50 & 50 & 5u & $5 u$ & 5v \\
\hline 1,2-Dichloroethane & su & su & 50 & 50 & 50 & 50 & 50 & 50 \\
\hline 1,1-Dichloroethene & su & 50 & 50 & 50 & $5 \mathrm{U}$ & $5 v$ & 50 & su \\
\hline 1,2-Dichloroethene & 39 & 1 & 5u & 50 & su & 50 & 5u & 5U \\
\hline 1,2-Dichloropropane & 50 & 50 & 50 & 5u & 50 & 50 & 50 & 50 \\
\hline cis-1,3-Dichloropropene & $5 v$ & 50 & 50 & 50 & 50 & 50 & 50 & 50 \\
\hline trans-1, 3-Dichloropropene & su & $5 \mathbf{u}$ & $5 v$ & 5u & 50 & 5u & 50 & 50 \\
\hline Ethylbenzene & su & 5u & 50 & 50 & su & 50 & 50 & 50 \\
\hline 2-Hexanone & 100 & 100 & 100 & 100 & 100 & 100 & 100 & 100 \\
\hline 4-Methyl-2-pentanone & 100 & 100 & 100 & 100 & 100 & 100 & 2 & 100 \\
\hline Methylene chloride & 50 & su & 50 & 50 & 0.7 & 1 & 5u & 50 \\
\hline Styrene & 50 & 50 & 50 & 50 & 50 & 50 & 50 & 50 \\
\hline 1,1,2,2-Tetrachloroethane & so & 5u & 50 & 50 & 50 & 5u & 50 & 50 \\
\hline Tetrachloroethene & 10 & 4 & 5 & 50 & $5 U$ & $5 \mathrm{u}$ & 5u & 50 \\
\hline Toluene & su & 50 & 5u & 50 & 5u & 50 & $5 \mathrm{u}$ & 5U \\
\hline 1,1,1-Trichloroethane & $5 v$ & 50 & 50 & $5 u$ & 50 & 50 & $5 \mathrm{u}$ & 5u \\
\hline 1,1,2-Trichloroethane & $5 U$ & $5 u$ & su & 50 & 5u & 50 & 50 & su \\
\hline Trichloroethene & 17 & 50 & 50 & $5 u$ & 50 & $5 \mathrm{v}$ & 50 & 50 \\
\hline Vinyl acetate & 100 & 100 & 100 & 100 & 100 & 100 & 100 & 100 \\
\hline vinyl chloride & 5 & 100 & 100 & 100 & 100 & 100 & 100 & 100 \\
\hline xylenes & 50 & su & $5 U$ & 50 & $5 v$ & 50 & $5 u$ & $5 U$ \\
\hline
\end{tabular}

(CONT INUED) 
APPENDIX F. 2

Field Duplicate Data, 1993

\begin{tabular}{|c|c|c|c|c|c|c|c|c|}
\hline \multirow{3}{*}{$\begin{array}{l}\text { Sampling Point } \\
\text { Location } \\
\text { Date Sampled }\end{array}$} & \multirow{3}{*}{\begin{tabular}{c|} 
GW-751 \\
GRIDJ3 \\
$10 / 26 / 93$
\end{tabular}} & \multirow{3}{*}{\begin{tabular}{|c|} 
GW-753 \\
GRIDJ2 \\
$08 / 03 / 93$ \\
\end{tabular}} & \multirow{3}{*}{\begin{tabular}{|c|} 
GW-755 \\
GRIDJ 1 \\
$10 / 21 / 93$ \\
\end{tabular}} & \multicolumn{3}{|c|}{ GW-758 } & \multirow{3}{*}{\begin{tabular}{|c|} 
GW-760 \\
GRIDG2 \\
$01 / 27 / 93$ \\
\end{tabular}} & \multirow{3}{*}{$\begin{array}{c}\text { GW-766 } \\
\text { GRIDI2 } \\
04 / 15 / 93\end{array}$} \\
\hline & & & & \multicolumn{3}{|c|}{ GRIDGI } & & \\
\hline & & & & $04 / 13 / 93$ & $09 / 12 / 93$ & $11 / 19 / 93$ & & \\
\hline VOLATILE ORGANICS (ug/L) & • & - & - & - & $\cdot$ & $\cdot$ & - & - \\
\hline Acetone & 100 & 100 & 100 & 100 & 100 & 100 & 100 & 100 \\
\hline Benzene & $5 \mathbf{u}$ & 5u & $5 U$ & su & 50 & 50 & 50 & su \\
\hline Bromodichloromethane & 50 & su & 50 & 50 & $5 v$ & $5 v$ & 50 & su \\
\hline Bromoform & 5u & 5u & 50 & su & 5u & 50 & su & su \\
\hline Bromomethane & 100 & 100 & 100 & 100 & 100 & 100 & 100 & 100 \\
\hline 2-Butanone & 100 & 100 & 100 & 100 & 100 & 100 & 100 & 100 \\
\hline Carbon disulfide & 50 & su & 50 & $5 U$ & 5u & su & su & 50 \\
\hline Carbon tetrachloride & 50 & 50 & 50 & 50 & 50 & $5 v$ & 5u & su \\
\hline Chlorobenzene & 50 & 50 & 50 & 50 & 50 & $5 v$ & 50 & 50 \\
\hline Chlorodibromomethane & 50 & $5 v$ & 50 & 50 & 50 & 50 & 5u & 50 \\
\hline Chloroethane & 100 & 100 & 100 & 100 & 100 & 100 & 100 & 100 \\
\hline chloroform & 50 & 50 & 50 & $5 U$ & 50 & 50 & 50 & 50 \\
\hline Chloromethane & 100 & 100 & 100 & 100 & 100 & 100 & 100 & 100 \\
\hline 1,1-Dichloroethane & 50 & 50 & 50 & 50 & su & $5 \mathbf{v}$ & 50 & 5u \\
\hline 1,2-Dichloroethane & 50 & so & 50 & 5u & 50 & $5 v$ & su & 5u \\
\hline 1,1-Dichloroethene & 50 & 50 & 50 & 50 & 50 & 5u & 50 & su \\
\hline 1,2-Dichloroethene & 50 & 50 & 50 & so & 50 & 5u & 5u & 50 \\
\hline 1,2-Dichloropropane & 5u & su & 50 & 50 & su & $5 u$ & $5 v$ & 511 \\
\hline cis-1,3-Dichloropropene & 50 & 50 & 50 & su & 50 & $5 \mathbf{v}$ & 5u & 50 \\
\hline trans-1,3-Dichloropropene & su & 5u & 50 & 50 & 50 & $5 \mathbf{v}$ & 5u & su \\
\hline Ethylbenzene & 50 & 50 & 50 & 50 & 5u & $5 \mathbf{u}$ & su & 5u \\
\hline 2-Hexanone & 100 & 100 & 100 & 100 & 100 & 100 & 100 & iou \\
\hline 4-Methyl-2-pentanone & 100 & 100 & 100 & 100 & 100 & 100 & 100 & 100 \\
\hline Methylene chloride & su & 50 & 50 & 50 & 1 & 1 & 5u & 50 \\
\hline Styrene & 5v & 50 & 50 & 50 & 5u & $5 \mathbf{u}$ & 5u & 5u \\
\hline $1,1,2,2$-Tetrachloroethane & 50 & $5 v$ & 50 & su & 50 & 5u & 50 & 50 \\
\hline Tetrachloroethene & 50 & 50 & 50 & 50 & 50 & 50 & 5u & su \\
\hline Toluene & 50 & 50 & 50 & $5 v$ & 50 & 50 & 50 & 50 \\
\hline 1,1,1-Trichloroethane & 5u & 50 & 50 & su & so & 50 & $5 U$ & 50 \\
\hline 1,1,2-Trichloroethane & 50 & $5 u$ & 50 & 5u & 50 & 5u & 5u & 50 \\
\hline Trichloroethene & su & $5 U$ & 50 & 50 & 50 & 50 & $5 u$ & 50 \\
\hline Vinyl acetate & 100 & 100 & 100 & 100 & 100 & 100 & 100 & 100 \\
\hline vinyl chloride & 100 & 100 & 100 & 100 & 100 & 100 & 100 & 100 \\
\hline Xylenes & 50 & 50 & 5v & $5 U$ & $5 U$ & 50 & 5u & $5 U$ \\
\hline
\end{tabular}

(CONTINURD) 
Field Duplicate Data, 1993

\begin{tabular}{|c|c|c|c|}
\hline \multirow{3}{*}{$\begin{array}{l}\text { Sampling Point } \\
\text { Location } \\
\text { - } \\
\text { Date Sampled }\end{array}$} & \multicolumn{2}{|c|}{ GW-766 } & GW-773 \\
\hline & \multicolumn{2}{|c|}{ GRIDI2 } & GRIDH2 \\
\hline & $08 / 03 / 93$ & $10 / 13 / 93$ & $02 / 03 / 93$ \\
\hline VOLATIIE ORGANICS (ug/I) & • & $\cdot$ & - \\
\hline Acetone & 100 & 100 & 100 \\
\hline Benzene & 50 & 50 & $5 \mathbf{u}$ \\
\hline Bromodichloromethane & 50 & 50 & 50 \\
\hline Bromoform & $5 \mathbf{v}$ & $5 U$ & $5 \mathbf{U}$ \\
\hline Bromomethane & 100 & 100 & 100 \\
\hline 2-Butanone & 100 & 100 & 100 \\
\hline Carbon disulfide & 50 & $5 U$ & $5 \mathbf{U}$ \\
\hline Carbon tetrachloride & 50 & 50 & 50 \\
\hline Chlorobenzene & 50 & $5 U$ & 50 \\
\hline Chlorodibromomethane & 50 & 50 & 50 \\
\hline Chloroethane & 100 & 100 & 100 \\
\hline Chloroform & $5 \mathbf{U}$ & $5 \mathbf{U}$ & $5 \mathbf{U}$ \\
\hline Chloromethane & 100 & 100 & 100 \\
\hline 1,1-Dichloroethane & 50 & $5 \mathbf{v}$ & $5 \mathbf{U}$ \\
\hline 1,2-Dichloroethane & 50 & 50 & $5 \mathbf{u}$ \\
\hline 1,1-Dichloroethene & 50 & 50 & $5 \mathbf{U}$ \\
\hline 1,2-Dichloroethene & 50 & 50 & $5 \mathbf{U}$ \\
\hline 1,2-Dichloropropane & 50 & 50 & $5 \mathbf{U}$ \\
\hline cis-1, 3-Dichloropropene & 50 & $5 v$ & $5 \mathbf{U}$ \\
\hline trans-1,3-Dichloropropene & $5 \mathbf{v}$ & 50 & $5 \mathbf{U}$ \\
\hline Ethylbenzene & $5 U$ & 50 & $\mathbf{5 U}$ \\
\hline 2-Hexanone & 100 & 100 & 100 \\
\hline 4-Methyl-2-pentanone & 100 & 100 & 2 \\
\hline Methylene chloride & $\mathbf{5 0}$ & $5 \mathbf{U}$ & 2 \\
\hline styrene & 50 & 50 & 50 \\
\hline $1,1,2,2$-Tetrachloroethane & 50 & $5 \mathbf{v}$ & 50 \\
\hline Tetrachloroethene & 50 & $5 \mathbf{v}$ & $5 \mathbf{U}$ \\
\hline Toluene & $5 U$ & 50 & $5 U$ \\
\hline $1,1,1$-Trichloroethane & $5 U$ & 50 & $5 U$ \\
\hline 1,1,2-Trichloroethane & $5 \mathbf{v}$ & 5U & 50 \\
\hline Trichloroethene & 50 & 50 & 50 \\
\hline Vinyl acetate & 100 & 100 & 100 \\
\hline vinyl chloride & 100 & 100 & 100 \\
\hline xylenes & 50 & 50 & $5 U$ \\
\hline
\end{tabular}




\section{APPENDIX F.3}

FIELD MEASUREMENTS, MISCELLANEOUS PARAMETERS, AND GROSS ALPHA AND GROSS BETA ACTIVITIES 
APPENDIX F. 3

Field Duplicate Groundwater Data, 1993

\begin{tabular}{|c|c|c|c|c|c|c|c|c|}
\hline \multirow{3}{*}{$\begin{array}{l}\text { Sampling Point } \\
\text { Iocation } \\
\text { - } \\
\text { Date Sampled }\end{array}$} & GW-151 & $G W-170$ & \multicolumn{5}{|c|}{ GW- 183} & \multirow{3}{*}{$\begin{array}{c}\text { GW- } 190 \\
\text { RG } \\
06 / 17 / 93\end{array}$} \\
\hline & NHP & EXP & \multicolumn{5}{|c|}{ FF } & \\
\hline & $08 / 06 / 93$ & $05 / 24 / 93$ & $03 / 10 / 93$ & $06 / 21 / 93$ & $06 / 29 / 93$ & $09 / 22 / 93$ & $11 / 16 / 93$ & \\
\hline FIELD MEASUREMENTS & - & - & - & - & - & - & - & • \\
\hline Depth to Water (ft) & . & - & - & - & - & . & - & • \\
\hline Water Temp (degrees C) & 17.2 & 16.5 & 17.5 & 17.5 & 17.9 & 18.5 & 18.4 & 19.5 \\
\hline $\mathrm{pH}$ (pH units) & 7.3 & 7.8 & 6.9 & 6.8 & 6.6 & 6.9 & 6.8 & 6.8 \\
\hline Sp. Cond. (umho/cm) & 437 & 363 & 863 & 764 & 779 & 825 & 921 & 238 \\
\hline Dissolved Oxygen (ppm) & 0.5 & 0.9 & 1.1 & 1.3 & 1.9 & 3.4 & 1.3 & 4.8 \\
\hline Oxidation/Reduction (mV) & 169 & 26 & -55 & -30 & 25 & 41 & 59 & 192 \\
\hline MISCELLANEOUS PARAMETERS & • & • & $\bullet$ & $\bullet$ & $\cdot$ & • & $\begin{array}{l}\cdot \\
.\end{array}$ & • \\
\hline pH (pH units) & 7.6 & 7.9 & 6.9 & 7.7 & - & 7.1 & 7.1 & 7.5 \\
\hline Sp. Cond. (umho/cm) & 495 & 464 & 951 & 914 & • & 948 & 938 & 259 \\
\hline TPH (mg/I) & $\cdot$ & - & 0.045 & $\cdot$ & - & $<0.1$ & 0.005 & - \\
\hline TDS (mg/L) & 262 & 262 & 560 & 566 & - & 574 & 554 & 178 \\
\hline TSS (mg/L) & $<1$ & $<1$ & 11 & 10 & - & 27 & 3 & 5 \\
\hline Turbidity (NTU) & 3.8 & 1.4 & 5.8 & 7.2 & - & 15 & 2.5 & 11 \\
\hline RADIOCHEMICAL PARAMETERS & - & • & - & • & • & - & . & - \\
\hline Gross Alpha $(\mathrm{pCi} / \mathrm{I})$ & -0.60 & -2.42 & 0.00 & 3.59 & - & $1.6 \dot{ }$ & 1.62 & -2.59 \\
\hline Gross Alpha $(\mathrm{CE}+/-)$ & 3.30 & 2.80 & 4.90 & 4.70 & • & 4.20 & 2.40 & 1300.00 \\
\hline Gross Beta (pCi/I) & 3.79 & -2.48 & 1.55 & 7.62 & - & 3.64 & 2.03 & -4.28 \\
\hline Gross Beta $(\mathrm{CE}+/-)$ & 5.20 & 5.40 & 5.40 & 5.70 & - & 5.60 & 2.80 & 2500.00 \\
\hline
\end{tabular}

(CONTINUED) 
APPENDIX F. 3

Field Duplicate Groundwater Data, 1993

\begin{tabular}{|c|c|c|c|c|c|c|c|c|}
\hline \multirow{3}{*}{$\begin{array}{l}\text { Sampling Point } \\
\text { - } \\
\text { - } \\
\text { Dacation Sampled }\end{array}$} & \multicolumn{2}{|c|}{$6 W-190$} & \multicolumn{2}{|c|}{ GW-207 } & \multicolumn{2}{|c|}{$G W-208$} & \multicolumn{2}{|c|}{$G W-255$} \\
\hline & \multicolumn{2}{|c|}{ RG } & \multicolumn{2}{|c|}{$\mathbf{E X P}$} & \multicolumn{2}{|c|}{ EXP } & \multicolumn{2}{|c|}{$\mathbf{s 2}$} \\
\hline & $07 / 07 / 93$ & $09 / 15 / 93$ & $08 / 11 / 93$ & $10 / 26 / 93$ & $01 / 19 / 93$ & $05 / 06 / 93$ & $01 / 08 / 93$ & $05 / 03 / 93$ \\
\hline FIELD MRASUREMENTS & - & - & - & - & - & - & - & - \\
\hline Depth to water (ft) & . & . & - & . & . & - & - & . \\
\hline Water Temp (degrees C) & 19.4 & 18 & 16.7 & 15.8 & 12.8 & 16.7 & 13.9 & 14.9 \\
\hline pH (pH units) & 6.8 & 6.5 & 7.4 & 7.4 & 7.2 & 7.1 & 7.5 & 7 \\
\hline Sp. Cond. (umho/cm) & 221 & 248 & 515 & 538 & 563 & 497 & 453 & 388 \\
\hline Dissolved Oxygen (ppm) & 7.4 & 4.2 & 0.5 & 5.9 & 4.3 & 2.9 & 7.3 & 4.4 \\
\hline Oxidation/Reduction (mv) & 128 & 334 & -8 & -2 & -50 & 70 & 214 & 226 \\
\hline MISCEILANEOUS PARAMETERS & - & - & - & - & - & - & - & - \\
\hline pH (pH units) & - & 7 & 7.6 & 7.9 & 7.5 & 7.5 & 7.7 & 7.7 \\
\hline Sp. Cond. (umho/cm) & $\cdot$ & 258 & 576 & 590 & 598 & 614 & 508 & 503 \\
\hline TPH $(m g / L)$ & $<0.1$ & $<0.1$ & - & • & - & • & • & • \\
\hline TDS (mg/L) & - & 192 & 368 & 360 & 410 & 392 & 304 & 310 \\
\hline TsS (mg/L) & - & 1 & 1 & $<1$ & 2 & $<1$ & 57 & 93 \\
\hline Turbidity (NTU) & - & 3 & 1.3 & 0.7 & 3.6 & 1.8 & 42 & 120 \\
\hline RADIOCHEMICAL PARAYETERS & - & - & - & - & - & - & - & • \\
\hline Gross Alpha (pCi/I) & - & 0.47 & -1.69 & 2.60 & -0.18 & -1.50 & 1.31 & 1.69 \\
\hline Gross Alpha $(\mathrm{CE}+/-)$ & - & 1.60 & 1.90 & 1.80 & 1.90 & 0.82 & 2.30 & 0.94 \\
\hline Gross Beta (pCi/L) & - & 0.86 & 4.97 & 1.42 & 1.27 & 3.83 & 1.76 & 0.48 \\
\hline Gross Beta $(\mathrm{CB}+/-)$ & - & 2.70 & 2.70 & 2.70 & 2.80 & 2.30 & 2.80 & 1.50 \\
\hline
\end{tabular}

(CONTINUED) 
APPENDIX F.3

Field Duplicate Groundwater Data, 1993

\begin{tabular}{|c|c|c|c|c|c|c|c|c|}
\hline \multirow{3}{*}{$\begin{array}{l}\text { Sampling Point } \\
\text { Iocation } \\
\text { - } \\
\text { Date Sampled }\end{array}$} & \multirow{3}{*}{\begin{tabular}{c|} 
GW-255 \\
$S 2$ \\
$12 / 07 / 93$ \\
\end{tabular}} & \multicolumn{3}{|c|}{$G W-262$} & $G W-264$ & \multicolumn{2}{|c|}{ GW-382 } & \multirow{2}{*}{$\begin{array}{c}\text { GW-606 } \\
\text { BXP }\end{array}$} \\
\hline & & \multicolumn{3}{|c|}{ SY } & SY & \multicolumn{2}{|c|}{ NHP } & \\
\hline & & $01 / 11 / 93$ & $04 / 08 / 93$ & $07 / 01 / 93$ & $12 / 08 / 93$ & $01 / 28 / 93$ & $04 / 26 / 93$ & $08 / 20 / 93$ \\
\hline FIBLD MEASUREMENTS & • & - & - & - & - & - & - & • \\
\hline Depth to water (ft) & - & - & - & . & - & . & . & • \\
\hline Water Temp (degrees C) & 14.1 & 14 & 16.4 & 20 & 16 & 15.2 & 15.9 & 18.8 \\
\hline pH (pH units) & 7.2 & 7.6 & 7.6 & 7.6 & 7.2 & 7.6 & 7.4 & 7.6 \\
\hline Sp. Cond. (umho/cm) & 464 & 384 & 353 & 414 & 1010 & 444 & 424 & 595 \\
\hline Dissolved Oxygen (ppm) & 4.5 & 1.5 & 7.4 & 5.8 & 7.2 & 1.8 & 2.4 & 2.5 \\
\hline Oxidation/Reduction (mv) & 140 & 104 & 76 & 126 & 54 & 66 & 32 & 83 \\
\hline MISCELLANEOUS PARAMETERS & - & - & • & • & - & - & - & - \\
\hline pH (pH units) & 7.5 & 8.3 & 7.7 & 8.1 & 7.3 & 8 & 7.6 & 7.6 \\
\hline Sp. Cond. (umho/cm) & 489 & 400 & 405 & 400 & 1090 & 490 & 476 & 609 \\
\hline TPH $(\mathrm{mg} / \mathrm{L})$ & - & - & $\cdot$ & - & - & - & - & • \\
\hline TDS (mg/I) & 340 & 252 & 254 & 272 & 752 & 268 & 282 & 354 \\
\hline TSS $(\mathrm{mg} / \mathrm{L})$ & 1064 & 1 & 12 & 40 & 44 & $<1$ & 2 & $<1$ \\
\hline Turbidity (NTU) & 580 & 1.8 & 9 & 60 & 200 & 5 & 13 & 0.9 \\
\hline RADIOCHEMICAL PARAMETERS & - & - & - & - & - & - & - & - \\
\hline Gross Alpha (pCi/L) & 2.77 & 1.85 & 0.87 & -1.84 & 1.07 & 2.10 & -0.83 & 4.06 \\
\hline Gross Alpha $(\mathrm{CB}+/-)$ & 2.10 & 2.30 & 2.30 & 3.80 & 1.40 & 1.60 & 2.30 & 3.00 \\
\hline Gross Beta (pci/L) & 5.17 & 2.12 & 1.99 & $-1 \cdot 16$ & 2.03 & 5.15 & 2.68 & 3.11 \\
\hline Gross Beta $(\mathrm{CE}+/-)$ & 2.70 & 2.90 & 2.80 & 5.60 & 2.50 & 2.90 & 2.70 & 2.90 \\
\hline
\end{tabular}

(CONTINUED) 
APPENDIX $\mathbf{F} .3$

Field Duplicate Groundwater Data, 1993

\begin{tabular}{|c|c|c|c|c|c|c|c|c|}
\hline \multirow{3}{*}{$\begin{array}{l}\text { Sampling point } \\
\text { Location } \\
\text { Date Sampled }\end{array}$} & \multirow{3}{*}{\begin{tabular}{c|} 
GW-618 \\
EXP \\
$09 / 16 / 93$
\end{tabular}} & \multicolumn{2}{|c|}{$G W-631$} & \multicolumn{3}{|c|}{ OW-735 } & \multicolumn{2}{|c|}{$6 w-747$} \\
\hline & & \multicolumn{2}{|c|}{ RG } & \multicolumn{3}{|c|}{ NHP } & \multicolumn{2}{|c|}{ GRIDK2 } \\
\hline & & $03 / 02 / 93$ & $11 / 17 / 93$ & $02 / 02 / 93$ & $05 / 07 / 93$ & $10 / 29 / 93$ & $01 / 19 / 93$ & $04 / 13 / 93$ \\
\hline FIELD MEASURBGRNTS & . & - & - & . & . & . & - & . \\
\hline Depth to Water (tt) & & . & . & . & . & . &. & • \\
\hline Water Tamp (degrees C) & 17.7 & 13.1 & 20.6 & 13.3 & 16.1 & 14.1 & 13.6 & 16.1 \\
\hline pH (pH units) & 6 & 4.3 & 3.8 & 7.1 & 7.1 & 6.5 & 7.4 & 7.6 \\
\hline Sp. Cond, (umho/cm) & 638 & 235 & 189 & 572 & 505 & 578 & 428 & 381 \\
\hline Dissolved Oxygen (ppm) & 0.4 & 6.4 & 3.1 & 0.9 & 0.6 & 0.4 & 1.2 & 1.5 \\
\hline Oxidation/Reduction (mV) & 145 & 276 & 318 & 88 & 19 & 102 & 27 & 129 \\
\hline MISCELLANEOUS PARANETERS & - & . & . & . & . & . & . & . \\
\hline $\mathrm{pH}$ (pH units) & $6 . \dot{6}$ & $4 . \dot{9}$ & 4.8 & $7 . \dot{3}$ & 7.6 & 7.3 & $7 . \overline{7}$ & 7.5 \\
\hline Sp. Cond. (umho/cm) & 675 & 231 & 222 & 635 & 617 & 618 & 456 & 438 \\
\hline TPH $(m g / L)$ & . & 0.0031 & 0.003 & . & • & . & . & • \\
\hline TDS (mg/L) & 376 & 152 & 150 & 384 & 382 & 402 & 276 & 272 \\
\hline TSS (mg/L) & 1 & 3 & 3 & 7 & 6 & 13 & $<1$ & 2 \\
\hline Turbidity (NTO) & 1.2 & 2.5 & 5 & 15 & 14 & 20 & 1.2 & 0.6 \\
\hline RADIOCHEMICAL PARAMETERS & . & . & . & . & . & . &. & . \\
\hline Gross Alphe $(\mathrm{PCi} / L)$ & 1.52 & 2.07 & 0.63 & 1.26 & -1.04 & 1.97 & -1.16 & 1.18 \\
\hline Gross Alpha $(\mathrm{CE}+/-)$ & 1.90 & 1.60 & 0.78 & 2.50 & 0.69 & 2.30 & 1.50 & 1.90 \\
\hline Gross Beta $(\mathrm{pCl} / \mathrm{L})$ & 5.40 & 9.39 & 2.55 & 4.32 & 4.57 & 4.01 & 1.17 & 4.97 \\
\hline Gross Beta $(\mathrm{CB}+/-)$ & 3.00 & 3.50 & 2.50 & 3.10 & 1.30 & 2.80 & 2.70 & 2.80 \\
\hline
\end{tabular}

(CONTINUED) 
APPENDIX $\mathrm{F} .3$

Field Duplicate Groundwater Data, 1993

\begin{tabular}{|c|c|c|c|c|c|c|c|c|}
\hline \multirow{3}{*}{$\begin{array}{l}\text { Sampling Point } \\
\text { Iocation } \\
\text { - } \\
\text { Date Sampled }\end{array}$} & OW-751 & $G W-753$ & GW-755 & \multicolumn{3}{|c|}{ GW-758 } & GW-760 & \multirow{2}{*}{$\frac{G W-766}{\text { GRIDI2 }}$} \\
\hline & GRIDJ 3 & GRIDJ 2 & GRIDJ1 & \multicolumn{3}{|c|}{ GRIDG1 } & GRIDE2 & \\
\hline & $10 / 26 / 93$ & $08 / 03 / 93$ & $10 / 21 / 93$ & $04 / 13 / 93$ & $09 / 12 / 93$ & $11 / 19 / 93$ & $01 / 27 / 93$ & $04 / 15 / 93$ \\
\hline FIELD MEASURERENTS & • & - & $\cdot$ & - & - & - & - & - \\
\hline $\begin{array}{l}\text { Depth to Water ( } f t \text { ) } \\
\text { Water Temp (degrees } C \text { ) }\end{array}$ & 16.3 & 17.9 & 15.8 & 17.3 & 17.2 & 15.8 & 16.9 & $\dot{16}$ \\
\hline pH (pH units) & 7.5 & 7.8 & 7.6 & 7.6 & 6.9 & 7.4 & 6.9 & 8.5 \\
\hline sp. Cond. (umho/cm) & 365 & 362 & 409 & 360 & 418 & 427 & 619 & 241 \\
\hline DLesolved Oxygen (ppta) & 0.3 & 0.4 & 2.2 & 6 & 0.5 & 5.6 & 7.8 & 2.5 \\
\hline Oxidation/Reduction (mv) & -90 & -80 & 110 & -10 & 101 & 65 & 175 & 116 \\
\hline MISCELIANROUS PARARETERS & - & - & $\cdot$ & - & - & $\cdot$ & - & - \\
\hline pH (pH undts) & 7.9 & 7.9 & 8 & 7.6 & 7.8 & 7.6 & 7.7 & 8.9 \\
\hline Sp. Cond. (umho/cm) & 387 & 397 & 475 & 443 & 446 & 464 & 698 & 195 \\
\hline $\operatorname{TPA}(\mathrm{mg} / \mathrm{L})$ & & $\cdot$ & $\cdot$ & $\cdot$ & • & $\cdot$ & $\cdot$ & $<0.1$ \\
\hline $\operatorname{TDS}(m g / L)$ & 246 & 262 & 304 & 282 & 290 & 280 & 436 & 166 \\
\hline Tss $(m g / L)$ & 10 & $<1$ & $<1$ & 9 & 2 & 2 & 6 & $<1$ \\
\hline Turbidity (NIU) & 2.8 & $1 \cdot 3$ & 2.4 & 3.8 & 2.3 & 3.7 & 3.3 & 2.9 \\
\hline RADIOCERMICAX PARARTERS & - & - & . & - & - & - & - & - \\
\hline Grose Alpha (pci/L) & 1.53 & -6.18 & 0.50 & 0.81 & 0.00 & -1.05 & 2.05 & -1.08 \\
\hline Grose Alpha $(\mathrm{Cz}+/-)$ & 1.60 & 4.00 & 2.10 & 1.00 & 3.90 & $1 \cdot 30$ & 2.50 & 0.67 \\
\hline Gross Beta (pCi/L) & 2.15 & -6.42 & 0.42 & 0.38 & 4.85 & -2.79 & -0.78 & 3.45 \\
\hline Grove Beta $(\mathrm{CB}+/-)$ & 2.70 & 5.90 & 2.80 & 1.20 & 5.90 & 3.00 & 2.80 & 1.30 \\
\hline
\end{tabular}

(CONTINUED) 
APPENDIX Y.3

Field Duplloate Groundwater Data, 1993

\begin{tabular}{|c|c|c|c|}
\hline \multirow{3}{*}{$\begin{array}{l}\text { sampling point } \\
\text { Location } \\
\text { Date sampled }\end{array}$} & \multicolumn{2}{|c|}{ OW-766 } & ON-773 \\
\hline & \multicolumn{2}{|c|}{ ORIDI2 } & GRIDH2 \\
\hline & $08 / 03 / 93$ & $10 / 13 / 93$ & $02 / 03 / 93$ \\
\hline FIELD MRASUREMETS & - & - & \\
\hline Dopth to Water $(t t)$ & . & . & • \\
\hline Mater Temp (degrees C) & 20.7 & 17.2 & 15.3 \\
\hline pH (pH unite) & 9 & 8.6 & 7.9 \\
\hline sp. Cond. (umho/cm) & 255 & 265 & 365 \\
\hline Dlesolved Oxygen (Ppm) & 8 & 5.4 & $\mathbf{s}$ \\
\hline OxLdation/Raduction (mV) & 26 & 131 & 85 \\
\hline MISCELLAROUS PARAMTERS & $\cdot$ & $\cdot$ & • \\
\hline pH (pH unite) & 8.5 & 8.6 & 7.7 \\
\hline 8p. Cond. (umho/cm) & 270 & 275 & 318 \\
\hline $\operatorname{TPH}(m g / L)$ & $<0.1$ & $<0.1$ & - \\
\hline $\operatorname{TDS}(m g / L)$ & 186 & 204 & 204 \\
\hline TsS (mg/c) & 1 & 3 & 34 \\
\hline Turbldity (NTU) & 1.9 & 1.4 & 1.2 \\
\hline RADIOCHEMICAL PARAMTERS & - & . & • \\
\hline Grose Alphe (pC1/I) & -3.74 & 2.61 & 0.47 \\
\hline Orose Alphe $(\mathrm{Cz}+/-)$ & 4.20 & 1.90 & 1.30 \\
\hline Orose Bata $(p C 1 / L)$ & -6.89 & 3.39 & -2.32 \\
\hline Oroses Beta $(\mathrm{Cs}+1-)$ & 3.80 & 2.70 & 3.80 \\
\hline
\end{tabular}


APPENDIX G

ION-CHARGE BALANCE CALCULATIONS 


\section{EXPLANATION}

\section{LOCATION:}

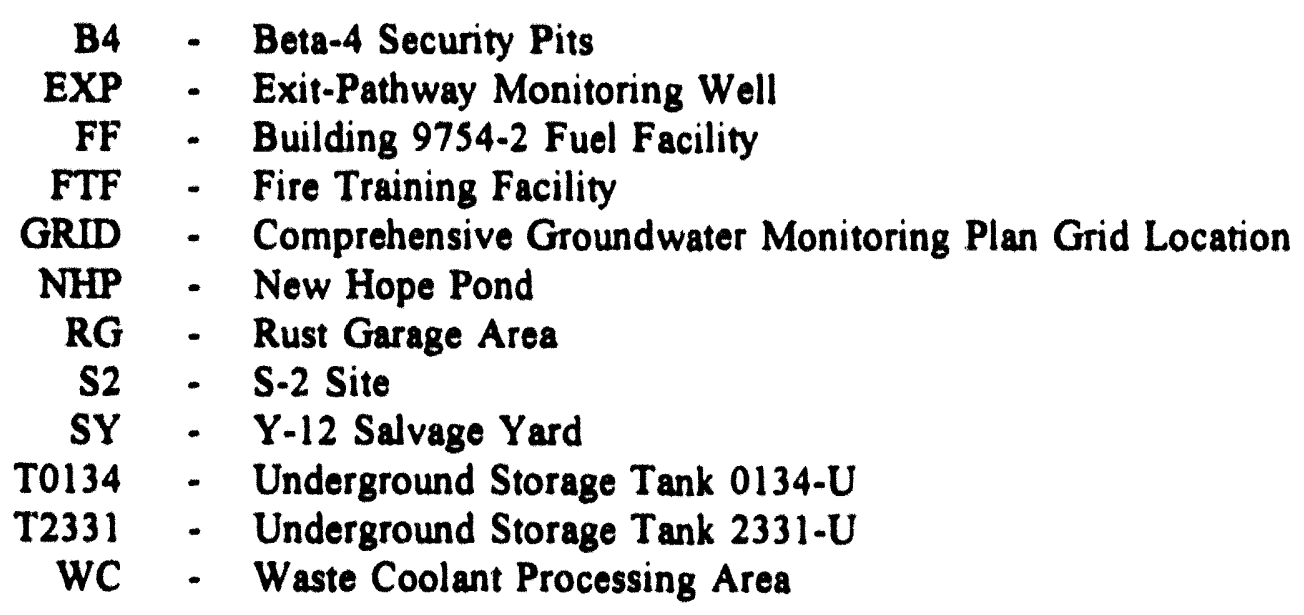

NOTES:

$$
\begin{array}{rll}
\mathrm{mg} / \mathrm{L} & - \text { milligrams per Liter } \\
\mathrm{meq} / \mathrm{L} & - & \text { milliequivalents per Liter } \\
\mathrm{TDS} & - & \text { Total Dissolved Solids } \\
\text { TSS } & - & \text { Total Suspended Solids }
\end{array}
$$

Bicarbonate and carbonate alkalinities (reported as $\mathrm{CaCO}_{3}$ ) were converted to $\mathrm{HCO}_{3}$ and $\mathrm{CO}_{3}$ as described in Mercier and Turner (1990).

Charge balances for each sample were evaluated by computing a relative percent difference (RPD) in the summed milliequivalent concentrations of the principal cations and anions using the following formula:

$$
\text { RPD }(\%)=\left(\frac{\sum \text { cattons }-\sum \text { anions }}{\sum \text { cations }+\sum \text { anions }}\right) \times 100
$$




\section{EXPLANATION}

Using this formula, the RPD calculated for the following samples exceeded $10 \%$ :

\begin{tabular}{|c|c|c|c|}
\hline Monitoring Well & Location & Sampling Date & RPD \\
\hline$G W-151$ & NHP & $4 / 22 / 93$ & 39.6 \\
\hline GW-190 & $\mathbf{R G}$ & $3 / 1 / 93$ & 48.5 \\
\hline GW-193 & $\mathrm{T} 2331$ & $9 / 23 / 93$ & 40.1 \\
\hline GW-206 & EXP & $8 / 12 / 93$ & 37.1 \\
\hline GW-206 & EXP & $10 / 27 / 93$ & -11.2 \\
\hline$G W-232$ & EXP & $8 / 21 / 93$ & 11.1 \\
\hline GW-232 & EXP & $11 / 1 / 93$ & 11.2 \\
\hline GW-251 & S2 & $01 / 20 / 93$ & -11.2 \\
\hline GW-251 & S2 & $09 / 17 / 93$ & -51.8 \\
\hline GW-252 & $\mathbf{S 2}$ & $01 / 08 / 93$ & -24.7 \\
\hline GW-252 & $\mathbf{S} 2$ & $5 / 3 / 93$ & 43.4 \\
\hline GW-252 & $\mathbf{S} 2$ & $12 / 07 / 93$ & -54.3 \\
\hline GW-281 & FF & $10 / 14 / 93$ & -14.2 \\
\hline$G W-283$ & FF & $10 / 14 / 93$ & -36.2 \\
\hline GW-337 & WC & $12 / 09 / 93$ & -11.2 \\
\hline GW-380 & NHP & $08 / 05 / 93$ & -36.6 \\
\hline GW-380 & NHP & $10 / 29 / 93$ & 12.1 \\
\hline GW-508 & $\mathbf{R G}$ & $03 / 05 / 93$ & -28.3 \\
\hline GW-508 & RG & $11 / 19 / 93$ & -28.9 \\
\hline GW-617 & EXP & $09 / 16 / 93$ & -16.1 \\
\hline GW-617 & EXP & $12 / 07 / 93$ & -11.4 \\
\hline GW-619 & FTF & $1 / 20 / 93$ & 48.1 \\
\hline GW-619 & FTF & $5 / 5 / 93$ & 49.6 \\
\hline GW-619 & FTF & $9 / 17 / 93$ & 30.2 \\
\hline GW-619 & FTF & $12 / 8 / 93$ & 30 \\
\hline GW-620 & FTF & $1 / 20 / 93$ & 64.4 \\
\hline GW-620 & FTF & $09 / 17 / 93$ & -38.6 \\
\hline
\end{tabular}


APFEADIX $G$

Ion-Charge Balance Calculations, 1993

\begin{tabular}{|c|c|c|c|c|c|c|c|c|c|c|c|c|c|c|}
\hline \multirow{3}{*}{$\begin{array}{l}\text { Sampling Point } \\
\text { Location } \\
\text { Date Sampled }\end{array}$} & \multicolumn{4}{|c|}{$G u-151$} & \multicolumn{4}{|c|}{$G w-168$} & \multicolumn{4}{|c|}{ Gu-169 } & \multicolumn{2}{|c|}{$6:-170$} \\
\hline & \multicolumn{4}{|c|}{ MHP } & \multicolumn{4}{|c|}{$\operatorname{maP}$} & \multicolumn{4}{|c|}{$\mathbf{E X P}$} & \multicolumn{2}{|c|}{$\mathbf{E x P}$} \\
\hline & $01 / 26 / 93$ & $04 / 22 / 93$ & $08 / 06 / 93$ & $11 / 02 / 93$ & $01 / 22 / 93$ & $05 / 14 / 93$ & $08 / 19 / 93$ & $11 / 02 / 93$ & $01 / 23 / 93$ & $05 / 21 / 93$ & $08 / 19 / 93$ & $11 / 01 / 93$ & $01 / 23 / 93$ & $05 / 24 / 93$ \\
\hline CATIONS (meg/L) & - & $\cdot$ & $\cdot$ & $\cdot$ & $\cdot$ & $\cdot$ & - & $\cdot$ & $\cdot$ & - & $\cdot$ & - & - & - \\
\hline Calcium & 2.69 & 0.04 & 2.45 & 2.79 & 3.84 & 3.74 & 4.04 & 3.99 & 2.50 & 3.59 & 3.34 & 3.14 & 2.25 & 2.20 \\
\hline Magnesium & 1.97 & 0.02 & 1.81 & 2.06 & 1.73 & 1.65 & 1.81 & 1.73 & 0.22 & 0.30 & 0.33 & 0.26 & 1.89 & 1.89 \\
\hline Potassium & 0.04 & 0.04 & 0.05 & 0.05 & 0.06 & 0.04 & 0.05 & 0.06 & 0.06 & 0.07 & 0.05 & 0.06 & 0.05 & 0.05 \\
\hline Sodium & 0.30 & 11.75 & 0.29 & 0.30 & 0.28 & 0.20 & 0.26 & 0.25 & 0.06 & 0.07 & 0.06 & 0.05 & 0.70 & 0.61 \\
\hline Hydrogen Ion & 0.00 & 0.00 & 0.00 & 0.00 & 0.00 & 0.00 & 0.00 & 0.00 & 0.00 & 0.00 & 0.00 & 0.00 & 0.00 & 0.00 \\
\hline Total Cations & 5.01 & 11.85 & 4.59 & 5.20 & 5.91 & 5.62 & 6.16 & 6.03 & 2.84 & 4.03 & 3.78 & 3.51 & 4.89 & 4.75 \\
\hline ANIONS (meq $/ L$ ) & & $\cdot$ & & $\bullet$ & & $\cdot$ & $\cdot$ & $\cdot$ & & $\cdot$ & $\cdot$ & $\dot{\bullet}$ & $\cdot$ & $\cdot$ \\
\hline Alkalinity- $\mathrm{CO} 3$ & 0.00 & 0.00 & 0.00 & 0.00 & 0.00 & 0.00 & 0.00 & 0.00 & 0.00 & 0.00 & 0.00 & 0.00 & 0.00 & 0.00 \\
\hline Alkalinity-HCO3 & 4.36 & 4.26 & 4.30 & 4.26 & 5.50 & 5.30 & 5.50 & 5.62 & 2.90 & 3.36 & 3.80 & 3.50 & 4.22 & 4.18 \\
\hline Chloride & 0.54 & 0.37 & 0.37 & 0.45 & 0.59 & 0.37 & 0.34 & 0.37 & 0.05 & 0.05 & 0.05 & 0.04 & 0.46 & 0.34 \\
\hline Fluoride & 0.01 & 0.01 & 0.01 & 0.01 & 0.00 & 0.00 & 0.01 & 0.01 & 0.00 & 0.00 & 0.00 & 0.00 & 0.01 & 0.01 \\
\hline Nitrate Mitrogen & 0.05 & 0.06 & 0.07 & 0.07 & 0.04 & 0.03 & 0.03 & 0.04 & 0.08 & 0.06 & 0.05 & 0.06 & 0.06 & 0.05 \\
\hline Sulfate & 0.42 & 0.44 & 0.46 & 0.35 & 0.25 & 0.23 & 0.17 & 0.21 & 0.10 & 0.15 & 0.12 & 0.12 & 0.41 & 0.37 \\
\hline Hydroxyl Ion & 0.00 & 0.00 & 0.00 & 0.00 & 0.00 & 0.00 & 0.00 & 0.00 & 0.00 & 0.00 & 0.00 & 0.00 & 0.00 & 0.00 \\
\hline$\cdot$ & & & & & & & & & & & $\cdot$ & • & & • \\
\hline Total Anions & 5.36 & 5.13 & 5.19 & 5.14 & 6.37 & 5.92 & 6.04 & 6.23 & 3.13 & 3.62 & 4.02 & 3.73 & 5.15 & 4.94 \\
\hline CHARGE BALANCE (8) & -3 & 40 & -6 & 1 & -4 & -3 & 1 & -2 & -5 & 5 & -3 & -3 & -3 & -2 \\
\hline $\operatorname{TDS}(m g / L)$ & 278 & 276 & 300 & 302 & 324 & 322 & 334 & 348 & 228 & 240 & 312 & 274 & 266 & $278^{\circ}$ \\
\hline TSS (mg/L) & $<1$ & 1 & 1 & $<1$ & 3 & 1 & 1 & $<1$ & 2100 & 404 & 1388 & 1960 & $<1$ & $<1$ \\
\hline Turbidity (NTU) & 2.3 & 0.5 & 3.7 & 0.8 & 2 & 2.2 & 1.2 & 3 & 2250 & 20 & 1200 & 46 & 1.2 & 1.2 \\
\hline
\end{tabular}


APPEADIX G

Ion-Charge Balance Calculations, 1993

\begin{tabular}{|c|c|c|c|c|c|c|c|c|c|c|c|c|c|c|}
\hline \multirow{3}{*}{$\begin{array}{l}\text { Sampling Point } \\
\text { Location } \\
\text { Date Sampled }\end{array}$} & \multicolumn{2}{|c|}{ GW-170 } & \multicolumn{4}{|c|}{$6 w-183$} & \multicolumn{4}{|c|}{ GW-190 } & \multicolumn{4}{|c|}{ GW-191 } \\
\hline & \multicolumn{2}{|c|}{$\mathbf{E X P}$} & \multicolumn{4}{|c|}{$\mathbf{F F}$} & \multicolumn{4}{|c|}{ RG } & \multicolumn{4}{|c|}{ B4 } \\
\hline & $08 / 21 / 93$ & $11 / 04 / 93$ & $03 / 10 / 93$ & $06 / 21 / 93$ & $09 / 22 / 93$ & $11 / 16 / 93$ & $03 / 01 / 93$ & $06 / 17 / 93$ & $09 / 15 / 93$ & $11 / 16 / 93$ & $01 / 12 / 93$ & $04 / 12 / 93$ & $07 / 08 / 93$ & $12 / 09 / 93$ \\
\hline CATIONS (meq/L) & - & - & - & - & - & - & • & - & • & - & - & - & $\cdot$ & - \\
\hline Calcium & 2.40 & 2.40 & 6.99 & 6.99 & 7.49 & 7.49 & 1.40 & 1.80 & 2.80 & 1.65 & 4.14 & 3.64 & 3.44 & 4.19 \\
\hline Magnesium & 2.06 & 1.97 & 1.89 & 1.97 & 1.97 & 2.22 & 0.47 & 0.50 & 0.52 & 0.49 & 0.90 & 0.80 & 0.74 & 0.90 \\
\hline Potassium & 0.04 & 0.04 & 0.07 & 0.04 & 0.05 & 0.06 & 0.03 & 0.02 & 0.03 & 0.02 & 0.08 & 0.05 & 0.07 & 0.07 \\
\hline Sodium & 0.74 & 0.70 & 1.09 & 1.04 & 1.17 & 1.13 & 0.29 & 0.37 & 0.36 & 0.37 & 0.38 & 0.52 & 0.61 & 0.48 \\
\hline Hydrogen Ion & 0.00 & 0.00 & 0.00 & 0.00 & 0.00 & 0.00 & 0.00 & 0.00 & 0.00 & 0.00 & 0.00 & 0.00 & 0.00 & 0.00 \\
\hline . & & & & & & & & & & & • & & & • \\
\hline Total Cations & 5.23 & 5.11 & 10.04 & 10.05 & 10.68 & 10.89 & 2.19 & 2.69 & 2.70 & 2.54 & 5.51 & 5.01 & 4.86 & 5.64 \\
\hline ANIONS (meg/ll) & & & & . & & & • & $\bullet$ & & • & & • & . & - \\
\hline & 0.00 & & & 0.00 & 0.00 & 0.00 & 0.00 & 0.00 & 0.00 & 0.00 & 0.00 & 0.00 & 0.00 & 0.00 \\
\hline $\begin{array}{l}\text { Alkalinity- } \mathrm{CO} 3 \\
\text { Alkalinity- } \mathrm{HCO} 3\end{array}$ & $\begin{array}{l}0.00 \\
4.32\end{array}$ & $\begin{array}{l}0.00 \\
4.26\end{array}$ & $\begin{array}{l}0.00 \\
8.69\end{array}$ & $\begin{array}{l}0.00 \\
8.97\end{array}$ & $\begin{array}{l}0.00 \\
9.19\end{array}$ & $\begin{array}{l}0.00 \\
9.21\end{array}$ & $\begin{array}{l}0.00 \\
0.28\end{array}$ & $\begin{array}{l}0.00 \\
2.24\end{array}$ & 2.24 & 2.42 & 4.78 & 4.66 & 5.10 & 5.14 \\
\hline $\begin{array}{l}\text { Alkaldnity-HCOS } \\
\text { Chloride }\end{array}$ & $\begin{array}{l}4.32 \\
0.41\end{array}$ & $\begin{array}{l}4.20 \\
0.48\end{array}$ & 1.00 & 0.85 & 0.85 & 0.93 & 0.15 & 0.17 & 0.17 & 0.17 & 0.10 & 0.11 & 0.12 & 0.17 \\
\hline Fluoride & 0.01 & 0.01 & 0.00 & 0.00 & 0.00 & 0.00 & 0.01 & 0.01 & 0.01 & 0.01 & 0.00 & 0.01 & 0.01 & 0.00 \\
\hline Nitrate Nitrogen & 0.05 & 0.08 & 0.00 & 0.00 & 0.00 & 0.00 & 0.00 & 0.00 & 0.02 & 0.00 & 0.00 & 0.00 & 0.00 & 0.00 \\
\hline Sulfate & 0.38 & 0.42 & 0.55 & 0.60 & 0.60 & 0.52 & 0.32 & 0.35 & 0.40 & 0.34 & 0.25 & 0.24 & 0.42 & 0.27 \\
\hline Hydroxy 1 Ion & 0.00 & 0.00 & 0.00 & 0.00 & 0.00 & 0.00 & 0.00 & 0.00 & 0.00 & 0.00 & 0.00 & 0.00 & 0.00 & 0.00 \\
\hline & • & & • & • & • & $\bullet$ & • & & $\cdot$ & • & • & • & $\bullet$ & $\bullet$ \\
\hline Total Anions & 5.17 & 5.24 & 10.25 & 10.42 & 10.64 & 10.66 & 0.76 & 2.78 & 2.83 & 2.93 & 5.12 & 5.01 & 5.64 & 5.58 \\
\hline$\overline{\text { CHARGE BALANCE (8) }}$ & $\dot{1}$ & -1 & -1 & -2 & $\dot{0}$ & $\dot{1}$ & 49 & -2 & -2 & -7 & 4 & $\dot{0}$ & -7 & $\dot{1}$ \\
\hline & . & & & . &. & - & • & & - & - & $\cdot$ & - & $\cdot$ & - \\
\hline $\operatorname{TDS}(\mathrm{mg} / \mathrm{L})$ & 286 & 294 & 554 & 584 & 572 & 540 & 168 & 178 & 180 & 144 & 310 & 334 & 322 & 352 \\
\hline TSS $(\mathrm{mg} / \mathrm{L})$ & $<1$ & $<1$ & 13 & 8 & 18 & 3 & 7 & 5 & 1 & 9 & 21 & 2162 & 107 & 143 \\
\hline Turbidity (NTU) & 0.8 & 0.7 & 5.9 & 6.6 & 10 & 2.5 & 4.7 & 12 & 4 & 15 & 20 & 560 & 230 & 1650 \\
\hline
\end{tabular}

(CONTINUED) 
APPENDIX G

Ion-Charge Balance Calculations, 1993

\begin{tabular}{|c|c|c|c|c|c|c|c|c|c|c|c|c|c|c|}
\hline \multirow{3}{*}{$\begin{array}{l}\text { Sampling Point } \\
\text { Location } \\
\text { Date Sampled }\end{array}$} & \multicolumn{4}{|c|}{$G w-192$} & \multicolumn{4}{|c|}{ Gw-193 } & \multicolumn{4}{|c|}{ Gw-194 } & \multicolumn{2}{|c|}{ GW-195 } \\
\hline & \multicolumn{4}{|c|}{$\mathbf{B 4}$} & \multicolumn{4}{|c|}{ T2331 } & \multicolumn{4}{|c|}{ B4 } & \multicolumn{2}{|c|}{ B4 } \\
\hline & $01 / 14 / 93 \mid$ & $104 / 13 / 93$ & $07 / 08 / 9 \Xi$ & $=2 / 09 / 93$ & $03 / 11 / 93$ & $06 / 22 / 93$ & $09 / 23 / 93$ & $11 / 18 / 93$ & $01 / 13 / 93$ & $04 / 13 / 93$ & $07 / 08 / 93$ & $12 / 09 / 93$ & $01 / 13 / 93$ & $04 / 13 / 93$ \\
\hline CATIONS (meq/L) & - & - & $\cdot$ & $\cdot$ & $\cdot$ & $\cdot$ & $\cdot$ & $\cdot$ & $\cdot$ & $\cdot$ & - & - & - & - \\
\hline Calcium & 5.49 & 5.49 & 5.49 & 5.49 & 4.59 & 5.99 & 6.99 & 6.99 & 3.69 & 3.79 & 3.84 & 3.99 & 3.14 & 3.24 \\
\hline Magnesium & 0.90 & 0.99 & 1.07 & 0.99 & 1.23 & 1.56 & 1.73 & 1.89 & 0.59 & 0.49 & 0.49 & 0.53 & 0.47 & 0.47 \\
\hline Potassium & 0.02 & 0.03 & 0.04 & 0.03 & 0.19 & 0.19 & 0.26 & 0.31 & 0.03 & 0.02 & 0.02 & 0.02 & 0.02 & 0.00 \\
\hline Sodium & 0.30 & 0.31 & 0.31 & 0.34 & 0.33 & 0.48 & 0.41 & 0.70 & 0.22 & 0.29 & 0.30 & 0.30 & 0.24 & 0.26 \\
\hline Hydrogen Ion & 0.00 & 0.00 & 0.00 & 0.00 & 0.00 & 0.00 & 0.00 & 0.00 & 0.00 & 0.00 & 0.00 & 0.00 & 0.00 & 0.00 \\
\hline . & & & & & & & & & & & & & & \\
\hline Total Cations & 6.71 & 6.82 & 6.91 & 6.85 & 6.35 & 8.22 & 9.38 & 9.88 & 4.53 & 4.59 & 4.66 & 4.84 & 3.87 & 3.97 \\
\hline ANIONS (meq/L) & $\cdot$ & $\cdot$ & $\cdot$ & $\cdot$ & $\cdot$ & • & $\cdot$ & $\cdot$ & $\cdot$ & $\cdot$ & $\cdot$ & . & $\cdot$ & - \\
\hline Alkalinity-CO3 & 0.00 & 0.00 & 0.00 & 0.00 & 0.00 & 0.00 & 0.00 & 0.00 & 0.00 & 0.00 & 0.00 & 0.00 & 0.00 & 0.00 \\
\hline Alkalinity-HCO3 & 6.43 & 6.61 & 6.99 & 6.51 & 6.35 & 8.35 & 3.48 & 9.13 & 4.50 & 4.34 & 4.56 & 4.58 & 3.66 & 3.58 \\
\hline Chloride & 0.31 & 0.28 & 0.37 & 0.42 & 0.19 & 0.42 & 0.18 & 0.45 & 0.06 & 0.09 & 0.10 & 0.11 & 0.10 & 0.10 \\
\hline Fluoride & 0.01 & 0.01 & 0.01 & 0.01 & 0.03 & 0.03 & 0.03 & 0.03 & 0.00 & 0.00 & 0.00 & 0.00 & 0.00 & 0.00 \\
\hline Nitrate Nitrogen & 0.00 & 0.00 & 0.00 & 0.00 & 0.00 & 0.00 & 0.09 & 0.00 & 0.00 & 0.00 & 0.00 & 0.00 & 0.00 & 0.00 \\
\hline Sulfate & 0.09 & 0.14 & 0.04 & 0.04 & 0.23 & 0.17 & 0.23 & 0.08 & 0.29 & 0.29 & 0.25 & 0.23 & 0.29 & 0.31 \\
\hline Hydroxyl Ion & 0.00 & 0.00 & 0.00 & 0.00 & 0.00 & 0.00 & 0.00 & 0.00 & 0.00 & 0.00 & 0.00 & 0.00 & 0.00 & 0.00 \\
\hline & & & & & & & & & & & & & & \\
\hline Total Anions & 6.85 & 7.04 & 7.41 & 6.98 & 6.80 & 8.97 & 4.01 & 9.69 & 4.85 & 4.72 & 4.91 & 4.92 & 4.05 & 3.99 \\
\hline CHARGE BALANCE (8) & -1 & -2 & -4 & -1 & -3 & -4 & 40 & 1 & -3 & -1 & -3 & -1 & -2 & -0 \\
\hline$\overline{T D S}(\mathrm{mg} / \mathrm{L})$ & 392 & $\begin{array}{r}\cdot \\
402\end{array}$ & $\begin{array}{r}0 \\
418\end{array}$ & ${ }_{432}$ & 398 & ${ }_{500}$ & 484 & $\begin{array}{r}\cdot \\
462\end{array}$ & 278 & 294 & 290 & 312 & 254 & 310 \\
\hline TSS (mg/L) & 61 & 6 & 11 & 10 & 35 & 830 & 23 & 98 & 102 & 65 & 90 & 30 & 524 & 527 \\
\hline Turbidity (NTU) & 66 & 5.8 & 7.5 & 22 & 52 & 440 & 22 & 29 & 34 & 150 & 64 & 120 & 240 & 220 \\
\hline
\end{tabular}

(CONTINUED) 
APPENDIX G

Ion-Charge Balance Calculations, 1993

\begin{tabular}{|c|c|c|c|c|c|c|c|c|c|c|c|c|c|c|}
\hline \multirow{3}{*}{$\begin{array}{l}\text { Sampling Point } \\
\text { Location } \\
\text { Date Sampled }\end{array}$} & \multicolumn{2}{|c|}{ GW-195 } & \multicolumn{4}{|c|}{ GW-199 } & \multicolumn{4}{|c|}{$G W-204$} & \multicolumn{4}{|c|}{ GW-206 } \\
\hline & \multicolumn{2}{|c|}{ B4 } & \multicolumn{4}{|c|}{ GRIDI1 } & \multicolumn{4}{|c|}{ T0134 } & \multicolumn{4}{|c|}{ EXP } \\
\hline & $07 / 08 / 93$ & $12 / 09 / 93$ & $01 / 29 / 93$ & $04 / 07 / 93$ & $08 / 02 / 93$ & $10 / 12 / 93$ & $03 / 11 / 93$ & $06 / 22 / 93$ & $09 / 23 / 93$ & $11 / 16 / 93$ & $01 / 19 / 93$ & $05 / 06 / 93$ & $08 / 12 / 93$ & $10 / 27 / 93$ \\
\hline CATIONS (meg/L) & - & - & - & - & - & • & - & - & $\cdot$ & - & - & - & - & 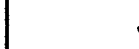 \\
\hline Calcium & 3.09 & 3.24 & $2.74^{\circ}$ & 2.59 & 2.94 & 2.74 & 1.95 & 2.64 & 3.19 & 2.79 & 1.95 & 2.54 & 5.99 & 2.74 \\
\hline Magnesium & 0.47 & 0.47 & 0.99 & 0.99 & 1.23 & 1.15 & 0.53 & 0.58 & 0.77 & 0.70 & 2.07 & 1.56 & 4.36 & 1.48 \\
\hline Potassium & 0.02 & 0.02 & 0.06 & 0.06 & 0.07 & 0.08 & 0.05 & 0.07 & 0.09 & 0.08 & 0.11 & 0.19 & 0.16 & 0.17 \\
\hline Sodium & 0.27 & 0.27 & 0.27 & 0.32 & 0.29 & 0.29 & 2.13 & 0.87 & 1.13 & 0.70 & 0.20 & 0.25 & 0.21 & 0.24 \\
\hline Hydrogen Ion & 0.00 & 0.00 & 0.00 & 0.00 & 0.00 & 0.00 & 0.00 & 0.00 & 0.00 & 0.00 & 0.00 & 0.00 & 0.00 & 0.00 \\
\hline - & & & & & & & & & & & & • & & 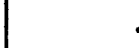 \\
\hline Total Cations & 3.85 & 4.00 & 4.06 & 3.96 & 4.54 & 4.26 & 4.66 & 4.16 & 5.18 & 4.27 & 3.33 & 4.54 & 10.71 & 4.63 \\
\hline ANIONS (meq/L) & & & $\cdot$ & & & • & $\dot{.}$ &. & & . & & . &. & . \\
\hline Alkalinity-CO3 & 0.00 & 0.00 & 0.00 & 0.00 & $\begin{array}{r}. \\
0.00\end{array}$ & 0.00 & 0.00 & 0.00 & 0.00 & 0.00 & 0.00 & 0.00 & 0.00 & 0.00 \\
\hline Alkalinity-HCO3 & 3.62 & 3.60 & 3.52 & 2.96 & 3.62 & 3.42 & 4.78 & 3.42 & 3.72 & 3.50 & 3.50 & 4.26 & 4.32 & 5.02 \\
\hline Chloride & 0.10 & 0.11 & 0.08 & 0.59 & 0.21 & 0.59 & 0.12 & 0.11 & 0.10 & 0.10 & 0.15 & 0.11 & 0.16 & 0.59 \\
\hline Fluoride & 0.00 & 0.00 & 0.01 & 0.01 & 0.01 & 0.01 & 0.10 & 0.05 & 0.07 & 0.07 & 0.00 & 0.00 & 0.00 & 0.00 \\
\hline Nitrate Nitrogen & 0.00 & 0.02 & 0.02 & 0.02 & 0.02 & 0.03 & 0.00 & 0.00 & 0.02 & 0.02 & 0.00 & 0.00 & 0.00 & 0.00 \\
\hline Sulfate & 0.29 & 0.25 & 0.56 & 0.52 & 0.62 & 0.65 & 0.09 & 1.04 & 0.96 & 0.66 & 0.22 & 0.29 & 0.44 & 0.19 \\
\hline Hydroxyl Ion & 0.00 & 0.00 & 0.00 & 0.00 & 0.00 & 0.00 & 0.00 & 0.00 & 0.00 & 0.00 & 0.00 & 0.00 & 0.00 & 0.00 \\
\hline - & & & & & & & & & & & & & & \\
\hline Total Anions & 4.01 & 3.98 & 4.20 & 4.10 & 4.48 & 4.69 & 5.09 & 4.62 & 4.86 & 4.35 & 3.86 & 4.66 & 4.91 & 5.80 \\
\hline CHARGE BALANCE (8) & $-\dot{2}$ & $\dot{0}$ & $\begin{array}{r}-2 \\
-2\end{array}$ & $\begin{array}{r}\cdot \\
-2\end{array}$ & $\dot{1}$ & $\begin{array}{r}-5 \\
-\end{array}$ & -4 & $\begin{array}{r}-5 \\
-5\end{array}$ & $\dot{3}$ & $\begin{array}{r}0 \\
-1\end{array}$ & $\dot{-7}$ & $\begin{array}{r}\dot{-1} \\
\text { - }\end{array}$ & 37 & -11 \\
\hline TDS $(\mathrm{mo} / \mathrm{I})$ & ${ }_{300}$ & $\begin{array}{r}\cdot \\
282\end{array}$ & $28 \dot{0}$ & 222 & 250 & . & 294 & 268 & . & • & 220 & 248 & $\begin{array}{r}\cdot \\
296\end{array}$ & 300 \\
\hline $\begin{array}{l}\text { TDS }(m g / L) \\
\text { TSS }(m g / L)\end{array}$ & $\begin{array}{l}300 \\
510\end{array}$ & $\begin{array}{l}282 \\
292\end{array}$ & $\begin{array}{l}280 \\
628\end{array}$ & $\begin{array}{r}222 \\
40\end{array}$ & $\begin{array}{r}250 \\
14\end{array}$ & $\begin{array}{r}332 \\
16\end{array}$ & $\begin{array}{r}294 \\
25\end{array}$ & $\begin{array}{r}268 \\
3\end{array}$ & $\begin{array}{r}312 \\
26\end{array}$ & $\begin{array}{l}192 \\
250\end{array}$ & $\begin{array}{r}220 \\
2750\end{array}$ & $\begin{array}{r}248 \\
1210\end{array}$ & $\begin{array}{r}296 \\
67\end{array}$ & 1187 \\
\hline Turbidity (NTU) & 240 & 360 & 3900 & 15 & 25 & 6.5 & 24 & 3.3 & 6.5 & 110 & 1900 & 750 & 1050 & 13 \\
\hline
\end{tabular}

(CONTINUED) 
APPENDIX G

Ion-Charge Balance Calculations, 1993

\begin{tabular}{|c|c|c|c|c|c|c|c|c|c|c|c|c|c|c|}
\hline \multirow{3}{*}{$\begin{array}{l}\text { Sampling Point } \\
\text { Location } \\
\text { - } \\
\text { Date Sampled }\end{array}$} & \multicolumn{4}{|c|}{ GW-207 } & \multicolumn{4}{|c|}{ GW-208 } & \multicolumn{4}{|c|}{$G W-220$} & \multicolumn{2}{|c|}{ GW-232 } \\
\hline & \multicolumn{4}{|c|}{ EXP } & \multicolumn{4}{|c|}{ EXP } & \multicolumn{4}{|c|}{ NHP } & \multicolumn{2}{|c|}{ EXP } \\
\hline & $01 / 14 / 93$ & $05 / 04 / 93$ & $08 / 11 / 93$ & $10 / 26 / 93$ & $01 / 19 / 93$ & $05 / 06 / 93$ & $08 / 13 / 93$ & $10 / 28 / 93$ & $01 / 25 / 93$ & $04 / 22 / 93$ & $08 / 06 / 93$ & $11 / 01 / 93$ & $01 / 26 / 93$ & $05 / 21 / 93$ \\
\hline CATIONS (meq/L) & - & - & $\cdot$ & - & - & - & - & - & - & - & - & - & - & . \\
\hline Calcium & 2.74 & 2.74 & 2.84 & 2.89 & 3.44 & 3.69 & 3.79 & 3.69 & 2.69 & 3.14 & 2.84 & 3.14 & 0.05 & 0.06 \\
\hline Magnesium & 2.96 & 2.96 & 3.13 & 3.04 & 1.81 & 1.97 & 2.06 & 1.97 & 1.81 & 1.89 & 1.81 & 1.89 & 0.07 & 0.07 \\
\hline Potassium & 0.07 & 0.07 & 0.06 & 0.07 & 0.07 & 0.09 & 0.07 & 0.07 & 0.06 & 0.06 & 0.05 & 0.07 & 0.07 & 0.07 \\
\hline Sodium & 0.43 & 0.41 & 0.48 & 0.43 & 0.65 & 0.65 & 0.70 & 0.61 & 0.16 & 0.15 & 0.20 & 0.18 & 9.14 & 10.00 \\
\hline Hydrogen Ion & 0.00 & 0.00 & 0.00 & 0.00 & 0.00 & 0.00 & 0.00 & 0.00 & 0.00 & 0.00 & 0.00 & 0.00 & 0.00 & 0.00 \\
\hline Total Cations & 6.20 & 6.19 & 6.51 & 6.44 & 5.97 & 6.41 & 6.61 & 6.34 & 4.72 & 5.25 & 4.91 & 5.29 & 9.33 & 10.21 \\
\hline ANIONS (meq $/ L$ ) & & & & & & $\dot{\bullet}$ & & . & $\dot{.}$ & . & $\begin{array}{l}\cdot \\
\cdot\end{array}$ & $\dot{\cdot}$ & $\dot{.}$ & • \\
\hline Alkalinity-CO3 & 0.00 & 0.00 & 0.00 & 0.00 & 0.00 & 0.00 & 0.00 & 0.00 & 0.00 & 0.00 & 0.00 & 0.00 & 1.44 & 1.64 \\
\hline Alkalinity- $\mathrm{HCO} 3$ & 5.32 & 5.32 & 5.26 & 5.34 & 4.46 & 4.44 & 4.54 & 4.52 & 4.44 & 4.68 & 4.40 & 4.78 & 7.35 & 7.03 \\
\hline Chloride & 0.04 & 0.03 & 0.05 & 0.03 & 0.04 & 0.04 & 0.06 & 0.06 & 0.23 & 0.19 & 0.24 & 0.26 & 0.16 & 0.16 \\
\hline Fluoride & 0.01 & 0.01 & 0.01 & 0.01 & 0.02 & 0.01 & 0.02 & 0.02 & 0.00 & 0.00 & 0.00 & 0.01 & 0.11 & 0.10 \\
\hline Nitrate Nitrogen & 0.00 & 0.00 & 0.00 & 0.00 & 0.00 & 0.00 & 0.00 & 0.00 & 0.03 & 0.06 & 0.06 & 0.05 & 0.00 & 0.00 \\
\hline Sulfate & 1.06 & 1.08 & 1.06 & 1.10 & 2.21 & 2.00 & 2.15 & 1.83 & 0.21 & 0.35 & 0.40 & 0.26 & 0.65 & 0.62 \\
\hline Hydroxyl Ion & 0.00 & 0.00 & 0.00 & 0.00 & 0.00 & 0.00 & 0.00 & 0.00 & 0.00 & 0.00 & 0.00 & 0.00 & 0.02 & 0.02 \\
\hline Total Anions & 6.43 & 6.44 & 6.38 & 6.47 & 6.72 & 6.49 & 6.76 & 6.43 & 4.91 & 5.28 & 5.09 & 5.36 & & 9.57 \\
\hline Total Anions & $\begin{array}{r}6.43 \\
.\end{array}$ & $\begin{array}{r}6.44 \\
.\end{array}$ & 6.38 & $\begin{array}{r}6.47 \\
.\end{array}$ & $\begin{array}{r}6.72 \\
.\end{array}$ & 6.49 & $\begin{array}{r}6.76 \\
.\end{array}$ & $\begin{array}{r}6.43 \\
.\end{array}$ & $\begin{array}{r}4.91 \\
.\end{array}$ & $\begin{array}{r}5.28 \\
.\end{array}$ & 5.09 & 5.36 & 9.73 & 9.57 \\
\hline CHARGE BALANCE (8) & -2 & -2 & 1 & -0 & -6 & -1 & -1 & -1 & -2 & -0 & -2 & -1 & -2 & 3 \\
\hline $\operatorname{TDS}(m g / L)$ & 348 & ri & $\begin{array}{r}\cdot \\
366\end{array}$ & $\begin{array}{r}\cdot \\
362\end{array}$ & ${ }_{406}$ & • & 428 & 424 & . & 332 & $\dot{2}$ & $\dot{3}$ & 5 & ; \\
\hline $\begin{array}{l}\text { TDS (mg/L) } \\
\text { TSS (mg/L) }\end{array}$ & $\begin{array}{r}348 \\
<1\end{array}$ & $\begin{array}{r}338 \\
<1\end{array}$ & $\begin{array}{r}366 \\
1\end{array}$ & $\begin{array}{r}362 \\
<1\end{array}$ & $\begin{array}{r}406 \\
2\end{array}$ & $\begin{array}{r}388 \\
<1\end{array}$ & $\begin{array}{r}428 \\
3\end{array}$ & $\begin{array}{r}424 \\
2\end{array}$ & $\begin{array}{r}256 \\
7\end{array}$ & $\begin{array}{l}332 \\
414\end{array}$ & $\begin{array}{l}292 \\
107\end{array}$ & $\begin{array}{r}310 \\
11\end{array}$ & $\begin{array}{r}580 \\
4\end{array}$ & $\begin{array}{r}562 \\
1\end{array}$ \\
\hline Turbidity (NTU) & 3.1 & 1.4 & 1.4 & 0.7 & 4.5 & 2.2 & 8.1 & 7 & 6 & 280 & 74 & 8.2 & 2.6 & 1.2 \\
\hline
\end{tabular}

(CONTINUED) 
APPENDIX G

Ion-Charge Balance Calculations, 1993

\begin{tabular}{|c|c|c|c|c|c|c|c|c|c|c|c|c|c|c|}
\hline \multirow{3}{*}{$\begin{array}{l}\text { Sampling Point } \\
\text { Location } \\
\text { - } \\
\text { Date Sampled }\end{array}$} & \multicolumn{2}{|c|}{ GW-232 } & \multicolumn{4}{|c|}{ GW-239 } & \multicolumn{4}{|c|}{ GW-240 } & \multicolumn{4}{|c|}{ GW-251 } \\
\hline & \multicolumn{2}{|c|}{$\mathbf{E X P}$} & \multicolumn{4}{|c|}{ NHP } & \multicolumn{4}{|c|}{ NHP } & \multicolumn{4}{|c|}{ s2 } \\
\hline & $08 / 21 / 93$ & $11 / 01 / 93$ & $01 / 23 / 93$ & $05 / 13 / 93$ & $08 / 18 / 93$ & $10 / 30 / 93$ & $01 / 27 / 93$ & $04 / 21 / 93$ & $08 / 05 / 93$ & $11 / 01 / 93$ & $01 / 20 / 93$ & $05 / 05 / 93$ & $09 / 17 / 93$ & $12 / 08 / 93$ \\
\hline CATIONS (meq/L) & - & - & - & - & $\cdot$ & - & - & - & $\cdot$ & - & • & - & • & • \\
\hline Calcium & 0.11 & 0.05 & 0.08 & 0.08 & 0.07 & 0.08 & 2.40 & 1.80 & 2.25 & 2.59 & 5.49 & 6.49 & 4.74 & 5.49 \\
\hline Magnesium & 0.08 & 0.07 & 0.04 & 0.04 & 0.04 & 0.04 & 1.40 & 1.23 & 1.32 & 1.40 & 1.48 & 1.56 & 1.40 & 1.48 \\
\hline Potassium & 0.08 & 0.06 & 0.09 & 0.10 & 0.10 & 0.08 & 0.10 & 0.28 & 0.06 & 0.05 & 0.09 & 0.09 & 0.06 & 0.06 \\
\hline Sodium & 9.57 & 10.00 & 17.83 & 16.96 & 17.83 & 18.70 & 3.09 & 1.83 & 1.52 & 2.31 & 0.78 & 0.87 & 0.52 & 0.43 \\
\hline Hydrogen Ion & 0.00 & 0.00 & 0.00 & 0.00 & 0.00 & 0.00 & 0.00 & 0.00 & 0.00 & 0.00 & 0.00 & 0.00 & 0.00 & 0.00 \\
\hline$\cdot$ & & & & & & & $\cdot$ & $\cdot$ & $\cdot$ & $\cdot$ & & $\cdot$ & $\cdot$ & • \\
\hline Total Cations & 9.85 & 10.19 & 18.05 & 17.19 & 18.05 & 18.91 & 6.98 & 5.14 & 5.14 & 6.35 & 7.85 & 9.01 & 6.72 & 7.47 \\
\hline ANIONS (meq/L) & & & & & & & & & & . & & • & $\cdot$ & • \\
\hline Alkalinity- $\mathrm{CO} 3$ & 0.00 & 0.00 & 0.88 & 1.12 & $0.8 \dot{0}$ & 1.00 & 0.00 & 0.00 & 0.00 & 0.00 & 0.00 & 0.00 & 0.00 & 0.00 \\
\hline Alkalinity-HCO3 & 7.03 & 7.23 & 9.87 & 9.91 & 10.21 & 10.09 & 3.82 & 3.64 & 3.22 & 3.12 & 3.18 & 3.20 & 3.28 & 3.16 \\
\hline Chloride & 0.16 & 0.19 & 7.90 & 7.93 & 7.22 & 6.46 & 3.92 & 1.61 & 1.10 & 2.25 & 0.34 & 0.31 & 0.17 & 0.20 \\
\hline Fluoride & 0.12 & 0.11 & 0.16 & 0.16 & 0.15 & 0.19 & 0.02 & 0.01 & 0.02 & 0.03 & 0.07 & 0.06 & 0.08 & 0.08 \\
\hline Nitrate Nitrogen & 0.00 & 0.00 & 0.00 & 0.00 & 0.00 & 0.00 & 0.08 & 0.06 & 0.18 & 0.29 & 5.86 & 5.93 & 17.42 & 3.06 \\
\hline Sulfate & 0.55 & 0.60 & 0.98 & 0.92 & 0.90 & 0.94 & 0.29 & 0.29 & 0.75 & 1.21 & 0.40 & 0.46 & 0.23 & 0.19 \\
\hline Hydroxyl Ion & 0.02 & 0.02 & 0.01 & 0.01 & 0.01 & 0.01 & 0.00 & 0.00 & 0.00 & 0.00 & 0.00 & 0.00 & 0.00 & 0.00 \\
\hline$\cdot$ & & & & & & & & & $\cdot$ & & $\cdot$ & & $\cdot$ & • \\
\hline Total Anions & 7.88 & 8.14 & 19.79 & 20.04 & 19.29 & 18.69 & 8.12 & 5.61 & 5.26 & 6.89 & 9.84 & 9.96 & 21.18 & 6.69 \\
\hline$\overline{\text { CHARGE BALANCE }(8)}$ & 11 & 11 & -5 & -8 & -3 & $\dot{1}$ & -8 & -4 & -1 & -4 & -11 & -5 & -52 & $\dot{5}$ \\
\hline $\operatorname{TDS}(\mathrm{mg} / \mathrm{L})$ & $\begin{array}{r}\cdot \\
576\end{array}$ & $\begin{array}{r}\cdot \\
584\end{array}$ & $\begin{array}{r}. \\
1102\end{array}$ & 1114 & 1108 & 1120 & 396 & $\begin{array}{r}\cdot \\
304\end{array}$ & 312 & $\begin{array}{r}\cdot \\
400\end{array}$ & 670 & $\begin{array}{r}\cdot \\
722\end{array}$ & 520 & $\begin{array}{r}. \\
472\end{array}$ \\
\hline TSS (mg/L) & 5 & 2 & 4 & 3 & $<1$ & 21 & $<1$ & $<1$ & 5 & 1 & 119 & 104 & 434 & 150 \\
\hline Turbidity (NTU) & 4.3 & 1.8 & 5.6 & 3.9 & 7.9 & 10 & 2.1 & 1.4 & 1.7 & 1.8 & 120 & 105 & 220 & 81 \\
\hline
\end{tabular}

(CONTINUED) 
APPENDIX G

Ion-Charge Balance Calculations, 1993

\begin{tabular}{|c|c|c|c|c|c|c|c|c|c|c|c|c|c|c|}
\hline \multirow{3}{*}{$\begin{array}{l}\text { Sampling Point } \\
\text { Location } \\
\text { Date Sampled }\end{array}$} & \multicolumn{4}{|c|}{ GW-252 } & \multicolumn{4}{|c|}{ GW-255 } & \multicolumn{4}{|c|}{$G W-261$} & \multicolumn{2}{|c|}{$G W-262$} \\
\hline & \multicolumn{4}{|c|}{ s2 } & \multicolumn{4}{|c|}{ s2 } & \multicolumn{4}{|c|}{ SY } & \multicolumn{2}{|c|}{ SY } \\
\hline & $01 / 08 / 93$ & $05 / 03 / 93$ & $09 / 15 / 93$ & $12 / 07 / 93$ & $01 / 08 / 93$ & $05 / 03 / 93$ & $09 / 15 / 93$ & $12 / 07 / 93$ & $01 / 08 / 93$ & $04 / 07 / 93$ & $07 / 01 / 93$ & $12 / 07 / 93$ & $01 / 11 / 93$ & $04 / 08 / 93$ \\
\hline CATIONS (meq/L) & - & $\cdot$ & $\cdot$ & - & $\cdot$ & $\cdot$ & - & $\cdot$ & $\cdot$ & - & $\cdot$ & $\cdot$ & - & - \\
\hline Calcium & 0.26 & 1.55 & 1.95 & 0.25 & 2.84 & 2.40 & 2.94 & 2.89 & 2.30 & 2.40 & 2.40 & 2.45 & 3.04 & 3.19 \\
\hline Magnesium & 0.22 & 1.81 & 1.97 & 0.23 & 2.47 & 2.47 & 2.47 & 2.55 & 0.90 & 0.99 & 0.99 & 0.99 & 0.49 & 0.50 \\
\hline Potassium & 0.00 & 0.04 & 0.04 & 0.02 & 0.02 & 0.03 & 0.03 & 0.03 & 0.03 & 0.04 & 0.05 & 0.04 & 0.05 & 0.05 \\
\hline Sodium & 0.15 & 0.43 & 0.57 & 0.09 & 0.15 & 0.14 & 0.18 & 0.17 & 0.42 & 0.41 & 0.41 & 0.43 & 0.61 & 0.61 \\
\hline Hydrogen Ion & 0.00 & 0.00 & 0.00 & 0.00 & 0.00 & 0.00 & 0.00 & 0.00 & 0.00 & 0.00 & 0.00 & 0.00 & 0.00 & 0.00 \\
\hline Total Cations & 0.63 & 3.84 & 4.52 & $0.5 \dot{9}$ & 5.48 & 5.04 & 5.63 & 5.64 & 3.66 & 3.83 & 3.84 & 3.91 & 4.19 & 4.35 \\
\hline ANIONS (meq/I) & & $\cdot$ & &. & $\cdot$ & $\cdot$ & $\cdot$ & $\cdot$ & $\cdot$ & $\cdot$ & $\cdot \dot{1}$ & $\cdot \dot{ }$ & $\cdot$ & • \\
\hline Alkalinity-CO3 & 0.00 & 0.00 & 0.00 & 0.00 & 0.00 & 0.00 & 0.00 & 0.00 & 0.00 & 0.00 & 0.00 & 0.00 & 0.00 & $0.0 \dot{0}$ \\
\hline Alkalinity-HCO3 & 0.92 & 1.18 & 3.94 & 1.90 & 4.72 & 4.74 & 4.78 & 4.88 & 2.98 & 3.06 & 3.00 & 3.06 & 3.96 & 4.00 \\
\hline Chloride & 0.04 & 0.06 & 0.10 & 0.03 & 0.16 & 0.14 & 0.17 & 0.17 & 0.10 & 0.11 & 0.12 & 0.14 & 0.03 & 0.03 \\
\hline Fluoride & 0.00 & 0.01 & 0.01 & 0.00 & 0.00 & 0.00 & 0.00 & 0.00 & 0.01 & 0.01 & 0.02 & 0.01 & 0.01 & 0.01 \\
\hline Nitrate Nitrogen & 0.03 & 0.06 & 0.18 & 0.04 & 0.11 & 0.16 & 0.13 & 0.22 & 0.00 & 0.00 & 0.00 & 0.00 & 0.00 & 0.00 \\
\hline Sulfate & 0.06 & 0.20 & 0.23 & 0.02 & 0.25 & 0.25 & 0.33 & 0.19 & 0.69 & 0.69 & 0.75 & 0.65 & 0.29 & 0.31 \\
\hline Hydroxy I Ion & 0.00 & 0.00 & 0.00 & 0.00 & 0.00 & 0.00 & 0.00 & 0.00 & 0.00 & 0.00 & 0.00 & 0.00 & 0.00 & 0.00 \\
\hline - & & & & & & $\cdot$ & & & & & $\cdot$ & . & $\cdot$ & - \\
\hline Total Anions & 1.05 & 1.51 & 4.45 & 1.98 & 5.24 & 5.28 & 5.41 & 5.45 & 3.78 & 3.86 & 3.88 & 3.85 & 4.29 & 4.35 \\
\hline CHARGE BALANCE (8) & -25 & 43 & $\dot{1}$ & -54 & $\dot{2}$ & $-\dot{2}$ & $\dot{2}$ & $\dot{2}$ & $\dot{-2}$ & $\dot{-0}$ & $\begin{array}{r}\cdot \\
-1\end{array}$ & $\dot{1}$ & $\dot{-1}$ & ; \\
\hline $\operatorname{TDS}(m g / L)$ & $\dot{60}$ & 102 & 264 & 150 & 300 & 310 & $35 \dot{2}$ & 360 & 230 & 220 & 224 & $27 \dot{2}$ & $\begin{array}{r}\cdot \\
252\end{array}$ & $26 \dot{\circ}$ \\
\hline TSS (mg/L) & 4 & 8 & 29 & 5 & 57 & 95 & 187 & 1260 & $<1$ & 2 & 8 & 1 & 3 & 8 \\
\hline Turbidity (NTU) & 12 & 8.5 & 250 & 11 & 44 & 115 & 140 & 560 & 15 & 20 & 5.5 & 3.8 & 1.9 & 3.5 \\
\hline
\end{tabular}

(CONTINUED) 
APPENDIX G

Ion-Charge Balance Calculations, 1993

\begin{tabular}{|c|c|c|c|c|c|c|c|c|c|c|c|c|c|c|}
\hline \multirow{3}{*}{$\begin{array}{l}\text { Sampling Point } \\
\text { Location } \\
\text { Date Sampled }\end{array}$} & \multicolumn{2}{|c|}{ GW-262 } & \multicolumn{4}{|c|}{ GW-263 } & \multicolumn{4}{|c|}{$G W-264$} & \multicolumn{4}{|c|}{$G w-281$} \\
\hline & \multicolumn{2}{|c|}{ SY } & \multicolumn{4}{|c|}{ SY } & \multicolumn{4}{|c|}{ sY } & \multicolumn{4}{|c|}{$\mathbf{F F}$} \\
\hline & $07 / 01 / 93$ & $12 / 07 / 93$ & $01 / 11 / 93$ & $04 / 08 / 93$ & $07 / 01 / 93$ & $12 / 07 / 93$ & $01 / 12 / 93$ & $04 / 12 / 93$ & $07 / 02 / 93 \mid$ & $12 / 08 / 93$ & $02 / 02 / 93$ & $04 / 16 / 93$ & $08 / 04 / 93$ & $10 / 14 / 93$ \\
\hline CATIONS (meg/L) & - & - & - & - & $\cdot$ & $\cdot$ & $\cdot$ & - & $\cdot$ & $\cdot$ & $\cdot$ & $\cdot$ & $\cdot$ & - \\
\hline Calcium & 3.09 & 3.19 & 3.24 & 3.04 & 2.94 & 3.54 & 6.99 & 7.49 & 5.99 & 7.49 & 2.64 & 2.15 & 2.99 & 1.90 \\
\hline Magnesium & 0.49 & 0.50 & 1.23 & 1.23 & 1.15 & 1.40 & 3.21 & 3.37 & 2.80 & 3.21 & 0.73 & 0.54 & 0.90 & 0.62 \\
\hline Potassium & 0.04 & 0.03 & 0.05 & 0.04 & 0.05 & 0.04 & 0.09 & 0.10 & 0.09 & 0.09 & 0.03 & 0.05 & 0.05 & 0.06 \\
\hline Sodium & 0.65 & 0.61 & 0.87 & 0.86 & 0.87 & 0.91 & 1.13 & 1.30 & 1.17 & 1.22 & 0.31 & 0.37 & 0.37 & 0.24 \\
\hline Hydrogen Ion & 0.00 & 0.00 & 0.00 & 0.00 & 0.00 & 0.00 & 0.00 & 0.00 & 0.00 & 0.00 & 0.00 & 0.00 & 0.00 & 0.00 \\
\hline - & & & & & & & & & & & & & $\cdot$ & \\
\hline Total Cations & 4.27 & 4.34 & 5.40 & 5.18 & 5.01 & 5.89 & 11.42 & 12.26 & 10.05 & 12.01 & 3.71 & 3.11 & 4.32 & 2.82 \\
\hline ANIONS (meg/L) & & $\cdot$ & $\cdot$ & $\cdot$ & & $\bullet$ & & $\cdot$ & & $\cdot$ & & $\cdot$ & & • \\
\hline & & & & & & 0.00 & 0.00 & 0.00 & 0.00 & & 0.00 & 0.00 & 0.00 & 0.00 \\
\hline $\begin{array}{l}\text { Alkalinity- } \mathrm{CO} 3 \\
\text { Alkalinity- } \mathrm{HCO} 3\end{array}$ & $\begin{array}{l}0.00 \\
4.14\end{array}$ & $\begin{array}{l}0.00 \\
4.08\end{array}$ & $\begin{array}{l}0.00 \\
3.80\end{array}$ & $\begin{array}{l}0.00 \\
3.26\end{array}$ & $\begin{array}{l}0.00 \\
3.14\end{array}$ & $\begin{array}{l}0.00 \\
3.96\end{array}$ & $\begin{array}{l}0.00 \\
9.05\end{array}$ & $\begin{array}{l}0.00 \\
7.95\end{array}$ & $\begin{array}{l}0.00 \\
8.13\end{array}$ & $\begin{array}{l}0.00 \\
8.47\end{array}$ & $\begin{array}{l}0.00 \\
3.52\end{array}$ & $\begin{array}{l}0.00 \\
2.46\end{array}$ & $\begin{array}{l}0.00 \\
3.80\end{array}$ & $\begin{array}{l}0.00 \\
3.24\end{array}$ \\
\hline Chloride & 0.00 & 0.00 & 1.66 & 1.64 & 1.21 & 1.50 & 0.45 & 0.44 & 0.56 & 0.33 & 0.15 & 0.27 & 0.21 & 0.17 \\
\hline Fluoride & 0.01 & 0.01 & 0.01 & 0.02 & 0.01 & 0.01 & 0.00 & 0.01 & 0.01 & 0.00 & 0.01 & 0.01 & 0.01 & 0.01 \\
\hline Nitrate Nitrogen & 0.00 & 0.00 & 0.04 & 0.03 & 0.02 & 0.04 & 0.00 & 0.00 & 0.00 & 0.00 & 0.00 & 0.03 & 0.03 & 0.00 \\
\hline Sulfate & 0.27 & 0.21 & 0.33 & 0.40 & 0.33 & 0.33 & 2.94 & 2.29 & 2.35 & 2.94 & 0.31 & 0.44 & 0.37 & 0.33 \\
\hline Hydroxyl Ion & 0.00 & 0.00 & 0.00 & 0.00 & 0.00 & 0.00 & 0.00 & 0.00 & 0.00 & 0.00 & 0.00 & 0.00 & 0.00 & 0.00 \\
\hline - & & & & & & & & & & $\cdot$ & & & $\cdot$ & - \\
\hline Total Anions & 4.41 & 4.29 & 5.84 & 5.33 & 4.71 & 5.83 & 12.44 & 10.69 & 11.06 & 11.74 & 3.99 & 3.20 & 4.42 & 3.75 \\
\hline CHARGE BALANCE (z) & -2 & $\dot{1}$ & -4 & -1 & 3 & 1 & -4 & 7 & -5 & 1 & -4 & -1 & -1 & -14 \\
\hline $\operatorname{TDS}(\mathrm{mg} / \mathrm{I})$ & $\begin{array}{r}\cdot \\
272\end{array}$ & $\begin{array}{r}\cdot \\
296\end{array}$ & 332 & 314 & 286 & 394 & 65B & 624 & 658 & 740 & $24 \dot{ }$ & 214 & $280^{\circ}$ & 230 \\
\hline $\begin{array}{l}\text { TDS }(m g / L) \\
\operatorname{TSS}(m g / L)\end{array}$ & 48 & 19 & 152 & 7 & 158 & 7 & 2 & 22 & 8 & 34 & 59 & 129 & 120 & 21 \\
\hline Turbidity (NTU) & 29 & 18 & 230 & 6 & 110 & 20 & 6.5 & 1 & 22 & 160 & 23 & 190 & 40 & 24 \\
\hline
\end{tabular}

(CONTINUED) 
PPENDIX G

Ion-Charge Balance Calculetions, 1993

\begin{tabular}{|c|c|c|c|c|c|c|c|c|c|c|c|c|c|c|}
\hline \multirow{3}{*}{$\begin{array}{l}\text { Sampling Point } \\
\text { Location } \\
\text { Date Sampled }\end{array}$} & \multicolumn{4}{|c|}{ GW-283 } & \multicolumn{4}{|c|}{$G W-284$} & \multicolumn{4}{|c|}{ GW-285 } & \multicolumn{2}{|c|}{$G w-337$} \\
\hline & \multicolumn{4}{|c|}{$\mathbf{F F}$} & \multicolumn{4}{|c|}{$\mathbf{F F}$} & \multicolumn{4}{|c|}{$\mathbf{F F}$} & \multicolumn{2}{|c|}{$w c$} \\
\hline & $02 / 03 / 93$ & $04 / 19 / 93$ & $08 / 06 / 93$ & $10 / 14 / 93$ & $02 / 02 / 93$ & $04 / 16 / 93$ & $08 / 05 / 93$ & $10 / 14 / 93$ & $02 / 02 / 93$ & $04 / 19 / 93$ & $08 / 05 / 93$ & $10 / 14 / 93$ & $05 / 07 / 93$ & $09 / 17 / 93$ \\
\hline CATIONS (meq/L) & - & . & - & . & - & - & . & - & - & - & - & - & - & - \\
\hline Calcium & 7.98 & 7.49 & 8.48 & 7.49 & 9.48 & 8.98 & 9.98 & 9.48 & 3.64 & 3.54 & 3.69 & 3.24 & 4.19 & 4.84 \\
\hline Magnesium & 1.40 & 1.23 & 1.48 & 1.40 & 1.56 & 2.14 & 1.81 & 1.81 & 0.56 & 0.50 & 0.56 & 0.58 & 0.67 & 0.79 \\
\hline Potassium & 0.07 & 0.07 & 0.07 & 0.07 & 0.05 & 0.04 & 0.04 & 0.04 & 0.05 & 0.03 & 0.05 & 0.04 & 0.04 & 0.05 \\
\hline Sodium & 0.52 & 0.48 & 0.41 & 0.48 & 1.13 & 1.39 & 1.22 & 1.39 & 0.40 & 0.33 & 0.35 & 0.52 & 0.17 & 0.18 \\
\hline Hydrogen Ion & 0.00 & 0.00 & 0.00 & 0.00 & 0.00 & 0.00 & 0.00 & 0.00 & 0.00 & 0.00 & C.00 & 0.00 & 0.00 & 0.00 \\
\hline . & & & & & & & & & • & & $\cdot$ & • & • & • \\
\hline Total Cations & 9.98 & 9.27 & 10.45 & 9.43 & 12.22 & 12.55 & 13.05 & 12.72 & 4.65 & 4.41 & 4.65 & 4.39 & 5.06 & 5.86 \\
\hline ANIONS (meq/L) & & - & & & • & &. & & • & $\cdot$ & $\dot{.}$ & $\cdot$ & • & . \\
\hline Alkalinity-co3 & 0.00 & 0.00 & 0.00 & 0.00 & 0.00 & 0.00 & 0.00 & 0.00 & 0.00 & 0.00 & 0.00 & 0.00 & 0.00 & 0.00 \\
\hline Alkalinity-HCO3 & 6.77 & 6.99 & 7.65 & 7.59 & 5.70 & 5.26 & 6.04 & 6.21 & 3.62 & 3.82 & 3.70 & 3.56 & 4.72 & 5.02 \\
\hline Chloride & 3.44 & 2.54 & 2.65 & 12.38 & 5.98 & 6.71 & 6.21 & 7.02 & 0.56 & 0.65 & 0.62 & 1.33 & 0.68 & 0.62 \\
\hline Fluoride & 0.00 & 0.00 & 0.00 & 0.00 & 0.00 & 0.00 & 0.00 & 0.00 & 0.01 & 0.01 & 0.00 & 0.01 & 0.00 & 0.00 \\
\hline Nitrate Nitrogen & 0.00 & 0.00 & 0.00 & 0.00 & 0.00 & 0.00 & 0.00 & 0.00 & 0.04 & 0.02 & 0.00 & 0.00 & 0.00 & 0.00 \\
\hline Sulfate & 0.25 & 0.22 & 0.27 & 0.17 & 0.67 & 0.48 & 0.58 & 0.62 & 0.94 & 0.40 & 0.15 & 0.29 & 0.15 & 0.17 \\
\hline Hydroxyl Ion & 0.00 & 0.00 & 0.00 & 0.00 & 0.00 & 0.00 & 0.00 & 0.00 & 0.00 & 0.00 & 0.00 & 0.00 & 0.00 & 0.00 \\
\hline - & & & & & & & & & & & & & • & \\
\hline Total Anions & 10.47 & 9.75 & 10.58 & 20.14 & 12.34 & 12.45 & 12.82 & 13.86 & 5.17 & 4.89 & 4.47 & 5.18 & 5.54 & 5.80 \\
\hline CHARGE BALANCE (8) & -2 & -3 & $-\dot{1}$ & -36 & $-\dot{0}$ & 0 & 1 & -4 & -5 & $-\dot{5}$ & $\dot{2}$ & -8 & -5 & 0 \\
\hline $\operatorname{TDS}(\mathrm{mg} / \mathrm{L})$ & 630 & 650 & 692 & $68 \dot{0}$ & 788 & 908 & 1048 & ${ }_{856}$ & 304 & 330 & 284 & 300 & 332 & 336 \\
\hline TSS $(m g / L)$ & 1077 & 348 & 32 & 50 & 18 & 22 & 48 & 20 & 15 & 201 & 26 & 6 & 11 & 578 \\
\hline Turbidity (NTU) & 300 & 37 & 15 & 25 & 8.6 & is & 25 & 15 & 25 & 280 & 18 & 6.1 & 11 & 335 \\
\hline
\end{tabular}

(CONTINUED) 
APPEADIX 6

Inm-Charge Balance Calcuıu_s . 6, 1993

\begin{tabular}{|c|c|c|c|c|c|c|c|c|c|c|c|c|c|c|}
\hline \multirow{3}{*}{$\begin{array}{l}\text { Sampling Point } \\
\text { Location } \\
\text { Date Sampled }\end{array}$} & \multicolumn{2}{|c|}{ Gw-337 } & \multicolumn{4}{|c|}{ GN-338 } & \multirow{2}{*}{\multicolumn{4}{|c|}{$\frac{G x-380}{\text { nnP }}$}} & \multirow{2}{*}{\multicolumn{4}{|c|}{$\frac{\text { Gu-381 }}{\text { map }}$}} \\
\hline & \multirow{2}{*}{\begin{tabular}{|c|} 
wc \\
$12 / 09 / 93$
\end{tabular}} & \multirow{2}{*}{\begin{tabular}{|c|} 
WCPA \\
$01 / 21 / 93$
\end{tabular}} & \multicolumn{3}{|c|}{$w c$} & \multirow{2}{*}{$\begin{array}{c}\text { WIPA } \\
01 / 11 / 93\end{array}$} & & & & & & & & \\
\hline & & & $05 / 04 / 93$ & $09 / 23 / 93$ & $12 / 07 / 93$ & & $01 / 25 / 93$ & $04 / 21 / 93$ & $08 / 05 / 93$ & $10 / 29 / 93$ & $01 / 27 / 93$ & $04 / 26 / 93$ & $08 / 08 / 93$ & $11 / 08 / 93$ \\
\hline CATIONS (meq/L) & & - & • & - & - & . & . & - & - & $\cdot$ & - & - & - & • \\
\hline Calcium & 3.39 & 4.39 & 4.14 & 4.44 & 4.44 & 4.24 & 2.54 & 1.35 & 1.45 & 2.45 & 3.74 & 3.49 & 3.54 & 4.14 \\
\hline Magnesium & 0.71 & 0.70 & 0.72 & 0.79 & 0.81 & 0.77 & 1.73 & 0.90 & 0.99 & 1.23 & 1.56 & 1.65 & 1.65 & 1.89 \\
\hline Potassium & 0.08 & 0.07 & 0.05 & 0.05 & 0.06 & 0.03 & 0.03 & 0.02 & 0.02 & 0.04 & 0.05 & 0.04 & 0.03 & 0.06 \\
\hline Sodium & 0.18 & 0.16 & 0.22 & 0.27 & 0.27 & 0.23 & 5.65 & 4.26 & 3.39 & 4.05 & 1.09 & 1.22 & 0.91 & 0.87 \\
\hline Hydrogen Ion & 0.00 & 0.00 & 0.00 & 0.00 & 0.00 & 0.00 & 0.00 & 0.00 & 0.00 & 0.00 & 0.00 & 0.00 & 0.00 & 0.00 \\
\hline$\cdot$ & & & & & & & & & & & & & & \\
\hline Total Cations & 4.36 & 5.32 & 5.14 & 5.54 & 5.57 & 5.28 & 9.95 & 6.54 & 5.85 & 7.76 & 6.44 & 6.40 & 6.13 & 6.96 \\
\hline ANrons (meq/L) & &. & & & $\cdot$ & . & $\cdot$ & & & . & & $\cdot$ & & • \\
\hline & & & & & & & & & & & & & & 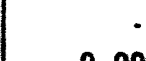 \\
\hline Alkalinity-CO3 & 0.00 & 0.00 & 0.00 & 0.00 & 0.00 & 0.00 & 0.00 & 0.00 & 0.00 & 0.00 & 0.00 & 0.00 & 0.00 & 0.00 \\
\hline Alkalinity-HCO3 & 4.80 & 5.02 & 4.68 & 5.04 & 4.90 & 4.66 & 3.28 & 3.54 & 1.56 & 3.58 & 4.64 & 4.30 & 4.90 & 5.12 \\
\hline Chloride & 0.53 & 0.99 & 0.18 & 0.16 & 0.20 & 0.17 & 7.05 & 3.95 & 10.27 & 1.33 & 2.14 & 2.34 & 1.86 & 1.92 \\
\hline Fluoride & 0.00 & 0.00 & 0.00 & 0.01 & 0.00 & 0.00 & 0.01 & 0.01 & 0.00 & 0.01 & 0.00 & 0.01 & 0.00 & 0.01 \\
\hline Nitrate Nitrogen & 0.00 & 0.00 & 0.03 & 0.02 & 0.04 & 0.04 & 0.10 & 0.08 & 0.13 & 0.26 & 0.00 & 0.02 & 0.00 & 0.00 \\
\hline Sulfate & 0.13 & 0.16 & 0.29 & 0.37 & 0.25 & 0.31 & 0.43 & 0.27 & 0.65 & 0.92 & 0.15 & 0.11 & 0.14 & 0.13 \\
\hline Hydroxyl Ion & 0.00 & 0.00 & 0.00 & 0.00 & 0.00 & 0.00 & 0.00 & 0.00 & 0.00 & 0.00 & 0.00 & 0.00 & 0.00 & 0.00 \\
\hline - & & & & $\cdot$ & & & • & & & & & & & • \\
\hline Total Anions & 5.46 & 6.16 & 5.18 & 5.60 & 5.39 & 5.18 & 10.87 & 7.85 & 12.60 & 6.09 & 6.93 & 6.78 & 6.89 & 7.17 \\
\hline CHARGE BALANCE (8) & -11 & $\begin{array}{r}-1 \\
-7\end{array}$ & $\begin{array}{r}0 \\
-0\end{array}$ & $\begin{array}{r}\cdot \\
-1\end{array}$ & $\dot{2}$ & $\dot{1}$ & -4 & $-\dot{9}$ & -37 & 12 & -4 & $\begin{array}{r}-3 \\
-3\end{array}$ & $\begin{array}{r}\cdot \\
-6\end{array}$ & -1 \\
\hline & • & • & $\cdot$ & $\cdot$ & $\bullet$ & $\cdot$ & $\cdot$ & $\cdot$ & $\cdot$ & $\cdot$ & $\cdot$ & $\cdot$ & $\cdot$ & • \\
\hline $\operatorname{TDS}(\mathrm{mg} / \mathrm{L})$ & 350 & 320 & 304 & 312 & 368 & 300 & 590 & 452 & 378 & 388 & 390 & 392 & 398 & 420 \\
\hline TSS (mg/L) & 6 & 26 & 5 & 7 & 21 & 3 & 15 & 10 & 124 & 11 & 10 & 9 & 4 & 4 \\
\hline Turbidity (NTU) & 3.8 & 14 & 8 & 110 & 4.1 & 4.4 & 16 & 20 & 21 & 50 & 21 & 23 & 5.5 & 4.6 \\
\hline
\end{tabular}

(CONTINUED) 
APPEADIX G

Ion-Charge Balance Calculations, 1993

\begin{tabular}{|c|c|c|c|c|c|c|c|c|c|c|c|c|c|c|}
\hline \multirow{3}{*}{$\begin{array}{l}\text { Sampling Point } \\
\text { Location } \\
\text { Date Sampled }\end{array}$} & \multicolumn{4}{|c|}{$G w-382$} & \multicolumn{4}{|c|}{ Gw-383 } & \multicolumn{4}{|c|}{$a-384$} & \multicolumn{2}{|c|}{ Gx-385 } \\
\hline & \multicolumn{4}{|c|}{ NHP } & \multicolumn{4}{|c|}{ MHP } & \multicolumn{4}{|c|}{ MEP } & \multicolumn{2}{|c|}{ MaP } \\
\hline & $01 / 28 / 93$ & $04 / 26 / 93$ & $08 / 08 / 93$ & $11 / 04 / 93$ & $|01 / 26 / 93|$ & $04 / 22 / 93$ & $08 / 07 / 93$ & $11 / 03 / 93$ & $01 / 13 / 93$ & $04 / 20 / 93$ & $\mid 08 / 04 / 93$ & $10 / 28 / 93$ & $01 / 12 / 93$ & $04 / 19 / 93$ \\
\hline CATIOAS (meq/L) & - & $\cdot$ & - & $\cdot$ & $\cdot$ & - & - & - & - & - & $\cdot$ & - & - & - \\
\hline Calcium & 2.50 & 2.54 & 2.50 & 2.64 & 3.79 & 3.74 & 3.64 & 4.09 & 4.84 & 4.84 & 4.69 & 4.84 & 0.05 & 0.05 \\
\hline Magnesium & 2.06 & 2.06 & 2.22 & 2.30 & 0.81 & 0.82 & 0.82 & 0.90 & 0.99 & 0.99 & 0.99 & 0.99 & 0.02 & 0.02 \\
\hline Potassium & 0.03 & 0.02 & 0.03 & 0.03 & 0.06 & 0.07 & 0.07 & 0.07 & 0.07 & 0.06 & 0.07 & 0.07 & 0.05 & 0.05 \\
\hline Sodium & 0.27 & 0.26 & 0.29 & 0.30 & 0.70 & 0.70 & 0.78 & 0.70 & 0.61 & 0.57 & 0.65 & 0.70 & 7.83 & 6.96 \\
\hline Hydrogen Ion & 0.00 & 0.00 & 0.00 & 0.00 & 0.00 & 0.00 & 0.00 & 0.00 & 0.00 & 0.00 & 0.00 & 0.00 & 0.00 & 0.00 \\
\hline & & & & & & $\cdot$ & & & & & & & & \\
\hline Total Cations & 4.85 & 4.88 & 5.04 & 5.28 & 5.37 & 5.33 & 5.32 & 5.76 & 6.50 & 6.45 & 6.40 & 6.60 & 7.95 & 7.07 \\
\hline ANIONS (meq/L) &. &. &. & $\cdot$ &. &. & $\cdot$ & $\cdot$ & $\cdot$ & $\bullet$ & $\cdot$ & 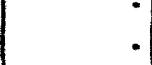 & $\cdot$ & • \\
\hline Alkalinity-CO3 & 0.00 & 0.00 & 0.00 & 0.00 & 0.00 & 0.00 & 0.00 & 0.00 & 0.00 & 0.00 & 0.00 & 0.00 & 0.92 & 0.96 \\
\hline Alkalinity- $\mathrm{HCO}_{3}$ & 4.12 & 4.08 & 4.14 & 4.12 & 4.38 & 4.26 & 4.44 & 4.40 & 5.40 & 5.54 & 5.58 & 5.62 & 6.35 & 5.90 \\
\hline Chloride & 0.62 & 0.55 & 0.55 & 0.56 & 0.96 & 0.76 & 0.68 & 0.85 & 0.85 & 0.78 & 0.73 & 0.85 & 0.06 & 0.07 \\
\hline Fluoride & 0.01 & 0.01 & 0.01 & 0.01 & 0.00 & 0.00 & 0.00 & 0.01 & 0.00 & 0.01 & 0.01 & 0.00 & 0.12 & 0.16 \\
\hline Nitrate Nitrogen & 0.20 & 0.15 & 0.25 & 0.26 & 0.00 & 0.00 & 0.00 & 0.00 & 0.00 & 0.00 & 0.00 & 0.00 & 0.00 & 0.00 \\
\hline Sulfate & 0.35 & 0.31 & 0.29 & 0.33 & 0.40 & 0.44 & 0.46 & 0.35 & 0.29 & 0.32 & 0.37 & 0.25 & 0.37 & 0.39 \\
\hline Hydroxyl Ion & 3.00 & 0.00 & 0.00 & 0.00 & 0.00 & 0.00 & 0.00 & 0.00 & 0.00 & 0.00 & 0.00 & 0.00 & 0.01 & 0.01 \\
\hline & & & & & & & & & & & & & & \\
\hline Total Anions & 5.30 & 5.10 & 5.23 & 5.28 & 5.73 & 5.46 & 5.57 & 5.60 & 6.53 & 6.64 & 6.69 & 6.71 & 7.84 & 7.48 \\
\hline CHARGE BAIANCE (8) & -4 & -2 & -2 & -0 & -3 & -1 & -2 & $i$ & -0 & -1 & -2 & -1 & $i$ & -3 \\
\hline $\operatorname{Tos}(\mathrm{mo} / \mathrm{L})$ & $27 \dot{2}$ & 284 & $28 \dot{ }$ & 298 & 334. & 320 & 324. & 354. & $37 \dot{0}$ & 380 & $\begin{array}{r}\cdot \\
392\end{array}$ & 390 & 452 & 466 \\
\hline $\begin{array}{l}\text { TDS (mg/L) } \\
\text { TSS (mg/L) }\end{array}$ & $\begin{array}{r}272 \\
1\end{array}$ & 200 & 2 & $<1$ & $<1$ & 2 & 2 & 3 & 2 & 4 & 3 & 2 & 3 & 5 \\
\hline Turbidity (NTU) & 5.4 & 13 & 2.5 & 1.4 & 0.8 & 1.8 & 10 & 12 & 5 & 1.8 & 2.5 & 3.9 & 5 & 17 \\
\hline
\end{tabular}

(CONTIMUED) 
APPEndIX 6

Ion-Charge Balance Calculations, 1993

\begin{tabular}{|c|c|c|c|c|c|c|c|c|c|c|c|c|c|c|}
\hline \multirow{3}{*}{$\begin{array}{l}\text { Sampling point } \\
\text { Locetion } \\
\text { - } \\
\text { Date Sampled }\end{array}$} & \multicolumn{2}{|c|}{$G w-385$} & \multicolumn{4}{|c|}{ Gu-505 } & \multicolumn{4}{|c|}{$60-500$} & \multicolumn{4}{|c|}{$6 n-603$} \\
\hline & \multicolumn{2}{|c|}{ MHP } & \multicolumn{4}{|c|}{ RE } & \multicolumn{4}{|c|}{ Ro } & \multicolumn{4}{|c|}{$\exp$} \\
\hline & $|08 / 03 / 93|$ & $10 / 27 / 93$ & $03 / 01 / 93$ & $06 / 18 / 93$ & $09 / 15 / 93$ & $11 / 16 / 93$ & $03 / 05 / 93$ & $\mid 06 / 23 / 93$ & $09 / 22 / 93$ & $11 / 19 / 93$ & $01 / 29 / 93$ & $05 / 04 / 93$ & $|08 / 16 / 93|$ & $10 / 28 / 93$ \\
\hline CArrons $(\mathrm{meq} / \mathrm{I})$ & $\cdot$ & - & $\cdot 1$ & $\cdot$ & - & - & $\cdot$ & - & - & - & $\cdot$ & - & & - \\
\hline Calcium & 0.05 & 0.05 & 1.35 & 1.10 & 0.85 & 1.95 & 0.42 & 0.36 & 0.30 & 0.28 & 3.54 & 3.94 & 4.24 & 3.99 \\
\hline Magnesi un & 0.02 & 0.02 & 0.45 & 0.46 & 0.38 & 0.64 & 0.77 & 1.23 & 1.48 & 0.82 & 1.89 & 2.14 & 2.22 & 2.06 \\
\hline Potassium & 0.07 & 0.05 & 0.03 & 0.04 & 0.04 & 0.05 & 0.03 & 0.03 & 0.03 & 0.02 & 0.14 & 0.15 & 0.19 & 0.17 \\
\hline Sodium & 7.39 & 7.83 & 0.29 & 0.32 & 0.32 & 0.31 & 1.09 & 1.13 & 1.39 & 1.00 & 0.16 & 0.16 & 0.17 & 0.17 \\
\hline Hydrogen Ion & 0.00 & 0.00 & 0.00 & 0.00 & 0.00 & 0.00 & 0.00 & 0.01 & 0.02 & 0.03 & 0.00 & 0.00 & 0.00 & 0.00 \\
\hline$\cdot$ & & & & & & & & & & & $\cdot$ & & $\cdot$ & \\
\hline Total Cations & 7.53 & 7.95 & 2.13 & 1.92 & 1.59 & 2.95 & 2.31 & 2.76 & 3.22 & 2.15 & 5.73 & 6.38 & 6.83 & 6.38 \\
\hline ANIOUS (meq/L) & $\cdot$ & - & $\cdot$ & $\cdot$ & . & . & - & - & $\cdot$ & - & $\cdot$ & - & $\cdot$ & . \\
\hline Alkalinity-co3 & 1.00 & 1.08 & 0.00 & 0.00 & 0.00 & 0.00 & 0.00 & 0.00 & $\cdot$ & 0.00 & 0.00 & 0.00 & 0.00 & 0.00 \\
\hline Alkalinity-nCO3 & 6.33 & 6.59 & 1.04 & 0.74 & 0.60 & 1.52 & 0.34 & 0.10 &. & 0.04 & 5.66 & 5.82 & 5.76 & 6.06 \\
\hline Chloride & 0.08 & 0.08 & 0.78 & 0.68 & 0.68 & 0.60 & 3.58 & 1.92 &. & 3.78 & 0.15 & 0.19 & 0.18 & 0.14 \\
\hline Fluoride & 0.16 & 0.17 & 0.02 & 0.01 & 0.01 & 0.02 & 0.01 & 0.01 & $\cdot$ & 0.01 & 0.01 & 0.01 & 0.01 & 0.01 \\
\hline witrate Nitrogen & 0.00 & 0.00 & 0.09 & 0.10 & 0.07 & 0.07 & 0.00 & 0.00 &. & 0.00 & 0.05 & 0.05 & 0.06 & 0.06 \\
\hline Sulfate & 0.48 & 0.35 & 0.42 & 0.31 & 0.44 & 0.61 & 0.21 & 0.27 & $\cdot 1$ & 0.06 & 0.29 & 0.37 & 0.26 & 0.25 \\
\hline Hydroxyl Ion & 0.02 & 0.01 & 0.00 & 0.00 & 0.00 & 0.00 & 0.00 & 0.00 & 0.00 & 0.00 & 0.00 & 0.00 & 0.00 & 0.00 \\
\hline$\cdot$ & & & & $\cdot$ & & & & & $\cdot$ & & & & & \\
\hline Total Anions & 8.08 & 8.29 & 2.34 & 1.84 & 1.80 & 2.82 & 4.14 & 2.30 & $\cdot$ & 3.89 & 6.16 & 6.44 & 6.27 & 6.52 \\
\hline CHARGE BALAHCE (*) & -3 & -2 & -5 & 2 & -6 & 2 & -28 & 9 & . & -29 & -4 & -0 & 4 & -1 \\
\hline $\operatorname{TDS}(m g / L)$ & 474 & $\begin{array}{r}- \\
476\end{array}$ & 186 & 142 & 148 & 148 & 196 & 140 & . & 160 & $\begin{array}{r}\cdot \\
346\end{array}$ & 346 & 366 & 360 \\
\hline ISS $(m g / L)$ & 20 & 3 & 87 & 133 & 42 & 103 & 11 & 7 &. & 9 & 9 & 78 & 19 & 16 \\
\hline Turbidity (HrY) & 9.8 & 3 & 50 & 16 & 34 & 26 & 1 & 5.5 &. & 1.6 & 10 & 62 & 19 & 14 \\
\hline
\end{tabular}

(COArrmued) 
APPESDIX 6

Ion-Charge Balance Calculations, 1993

\begin{tabular}{|c|c|c|c|c|c|c|c|c|c|c|c|c|c|c|}
\hline \multirow{3}{*}{$\begin{array}{l}\text { Sampling Point } \\
\text { Location } \\
\text { Date Sampled }\end{array}$} & \multicolumn{4}{|c|}{ GW-604 } & \multicolumn{4}{|c|}{ Gai-605 } & \multicolumn{4}{|c|}{$0-606$} & \multicolumn{2}{|c|}{$0 x-617$} \\
\hline & \multicolumn{4}{|c|}{$\operatorname{Exp}$} & \multicolumn{4}{|c|}{$\operatorname{ExP}$} & \multicolumn{4}{|c|}{$\mathbf{E x P}$} & \multicolumn{2}{|c|}{$\mathbf{E x P}$} \\
\hline & $01 / 30 / 93 \mid$ & $05 / 04 / 93$ & $08 / 16 / 93$ & $10 / 28 / 93$ & $02 / 02 / 93$ & $05 / 10 / 93$ & $|08 / 19 / 93|$ & $11 / 10 / 93$ & $02 / 03 / 93$ & $05 / 10 / 93$ & $08 / 20 / 93$ & $11 / 11 / 93$ & $01 / 12 / 93$ & $05 / 04 / 93$ \\
\hline CATrons (meq/L) & - & - & - & - & - & - & - & - & - & - & - & - & - & \\
\hline Calcium & 2.69 & 2.30 & 2.40 & 2.05 & 4.09 & 4.19 & 4.89 & 4.39 & 2.99 & 3.19 & 3.84 & 3.44 & 1.15 & 0.85 \\
\hline Magnes i um & 1.48 & 1.40 & 1.40 & 1.32 & 1.23 & 1.23 & 1.32 & 1.40 & 2.55 & 2.55 & 3.04 & 2.88 & 0.42 & 0.36 \\
\hline Potassium & 0.03 & 0.04 & 0.03 & 0.03 & 0.08 & 0.06 & 0.13 & 0.09 & 0.10 & 0.08 & 0.08 & 0.09 & 0.00 & 0.02 \\
\hline Sodium & 0.09 & 0.07 & 0.06 & 0.08 & 0.52 & 0.57 & 0.37 & 0.65 & 0.20 & 0.19 & 0.22 & 0.21 & 0.17 & 0.14 \\
\hline Hydrogen Ion & 0.00 & 0.00 & 0.00 & 0.00 & 0.00 & 0.00 & 0.00 & 0.00 & 0.00 & 0.00 & 0.00 & 0.00 & 0.00 & 0.00 \\
\hline Total Cations & 4.30 & 3.80 & 3.89 & 3.48 & 5.93 & 6.05 & 6.70 & 6.53 & 5.84 & 6.01 & 7.19 & 6.63 & 1.74 & 1.38 \\
\hline & & & & & & & & & & & & & & \\
\hline AMIOMS (meq/L) & • & - & - & - & - & - & - & . & - & - & $\cdot$ & - & - & . \\
\hline Alkalinity-CO3 & 0.00 & 0.00 & 0.00 & 0.00 & 0.00 & 0.00 & 0.00 & 0.00 & 0.00 & 0.00 & 0.00 & 0.00 & 0.00 & 0.00 \\
\hline Alkalinity-uCO3 & 4.16 & 3.66 & 3.34 & 3.20 & 5.12 & 5.04 & 5.10 & 5.20 & 4.14 & 4.36 & 4.48 & 4.86 & 0.68 & 0.72 \\
\hline Chloride & 0.08 & 0.07 & 0.06 & 0.06 & 0.62 & 0.73 & 0.65 & 0.75 & 1.27 & 1.21 & 1.30 & 1.44 & 0.09 & 0.13 \\
\hline Fluoride & 0.01 & 0.01 & 0.01 & 0.01 & 0.00 & 0.00 & 0.00 & 0.01 & 0.01 & 0.01 & 0.01 & 0.01 & 0.00 & 0.00 \\
\hline Nitrate Nitrogen & 0.04 & 0.03 & 0.04 & 0.04 & 0.00 & 0.03 & 0.00 & 0.02 & 0.05 & 0.06 & 0.05 & 0.07 & 0.17 & 0.11 \\
\hline Sulfate & 0.14 & 0.12 & 0.09 & 0.10 & 0.50 & 0.54 & 0.33 & 0.53 & 0.71 & 0.65 & 0.58 & 0.70 & 0.77 & 0.65 \\
\hline Hydroxyl Ion & 0.00 & 0.00 & 0.00 & 0.00 & 0.00 & 0.00 & 0.00 & 0.00 & 0.00 & 0.00 & 0.00 & 0.00 & 0.00 & 0.00 \\
\hline & & & & & & & & & & & & & $\cdot$ & • \\
\hline Total Anions & 4.43 & 3.88 & 3.54 & 3.40 & 6.24 & 6.34 & 6.08 & 6.50 & 6.17 & 6.28 & 6.41 & 7.08 & 1.71 & 1.60 \\
\hline CHARGE BALAMCE (ह) & -2 & -1 & 5 & 1 & -3 & -2 & $\dot{5}$ & o & -3 & -2 & $\dot{6}$ & -3 & $\dot{2}$ & -8 \\
\hline TOS $-0 / 11$ & 262 & 210 & 210 & $20 \dot{2}$ & $\bullet$ & 25e & 27. & & $26 \%$ & & . & & 116 & 110 \\
\hline $\begin{array}{l}\operatorname{TDS}(\mathrm{mg} / \mathrm{L}) \\
\operatorname{TSS}(\mathrm{mg} / \mathrm{L})\end{array}$ & $\begin{array}{r}262 \\
8\end{array}$ & $\begin{array}{r}210 \\
<1\end{array}$ & $\begin{array}{r}210 \\
4\end{array}$ & $\begin{array}{r}202 \\
2\end{array}$ & $\begin{array}{r}354 \\
<1\end{array}$ & $\begin{array}{r}354 \\
1\end{array}$ & $\begin{array}{r}370 \\
7\end{array}$ & $\begin{array}{r}394 \\
<1\end{array}$ & $\begin{array}{r}366 \\
2\end{array}$ & $\begin{array}{r}360 \\
<1\end{array}$ & $\begin{array}{r}358 \\
<1\end{array}$ & $\begin{array}{r}382 \\
2\end{array}$ & $\begin{array}{r}116 \\
9\end{array}$ & $\begin{array}{r}110 \\
19\end{array}$ \\
\hline Turbidity (mrv) & 16.5 & 4.5 & 10 & 6 & 1.8 & 2.4 & 3.2 & 1.9 & 1.4 & 1.5 & 0.7 & 2 & 10 & 16 \\
\hline
\end{tabular}

(CONTIMUED) 
APPEODIX 6

Ion-Charge Balence Calculatione, 1993

\begin{tabular}{|c|c|c|c|c|c|c|c|c|c|c|c|c|c|c|}
\hline \multirow{3}{*}{$\begin{array}{l}\text { Sampling Point } \\
\text { Location } \\
\text { Date Sampled }\end{array}$} & \multicolumn{2}{|c|}{ Gin-617 } & \multicolumn{4}{|c|}{$6 w-618$} & \multicolumn{4}{|c|}{$6-619$} & \multicolumn{4}{|c|}{$6-620$} \\
\hline & \multicolumn{2}{|c|}{$\mathbf{E x P}$} & \multicolumn{4}{|c|}{$\mathbf{E x P}$} & \multicolumn{4}{|c|}{$m$} & \multicolumn{4}{|c|}{$m$} \\
\hline & $09 / 16 / 93$ & $12 / 07 / 93$ & $01 / 12 / 93$ & $05 / 05 / 93$ & $09 / 16 / 93$ & $12 / 08 / 93$ & $01 / 20 / 93$ & $05 / 05 / 93$ & $09 / 17 / 93$ & $12 / 08 / 93$ & $01 / 20 / 93$ & $05 / 06 / 93$ & $09 / 17 / 93$ & $12 / 09 / 93$ \\
\hline Cations (meq/L) & - & - & - & - & - & - & - & - & - & - & - & - & 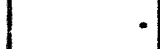 & - \\
\hline Calcium & 0.95 & 1.25 & 4.94 & 4.74 & 4.99 & 4.99 & 2.45 & 2.64 & 2.64 & 2.20 & 1.00 & 8.98 & 2.40 & 4.39 \\
\hline Magnesi ius & 0.41 & 0.49 & 0.75 & 0.72 & 0.77 & 0.77 & 0.11 & 0.06 & 0.14 & 0.12 & 0.49 & 0.00 & 0.70 & 0.01 \\
\hline Potassium & 0.03 & 0.04 & 0.08 & 0.09 & 0.09 & 0.09 & 0.43 & 0.69 & 0.49 & 0.41 & 0.24 & 0.82 & 0.13 & 0.69 \\
\hline Sodium & 0.17 & 0.26 & 0.78 & 0.74 & 0.78 & 0.78 & 0.14 & 0.20 & 0.17 & 0.12 & 0.13 & 0.26 & 0.11 & 0.23 \\
\hline Hydrogen Ion & 0.00 & 0.00 & 0.00 & 0.00 & 0.00 & 0.00 & 0.00 & 0.00 & 0.00 & 0.00 & 0.00 & 0.00 & 0.00 & 0.00 \\
\hline Total Cations & 1.56 & 2.05 & 6.55 & 6.29 & 6.64 & 6.63 & 3.13 & 3.60 & 3.44 & 2.84 & 2.65 & 10.06 & 3.33 & 5.32 \\
\hline MIÖuS (neq/L) & & $\cdot$ & • & & $\cdot$ & $\cdot$ & $\cdot$ & $\cdot$ & $\cdot$ & $\cdot$ & $\cdot$ & $\cdot$ & $\cdot$ & - \\
\hline Alkalinity- $\mathrm{CO} 3$ & 0.00 & 0.00 & 0.00 & 0.00 & 0.00 & 0.00 & 0.16 & 0.56 & 0.24 & 0.16 & 0.20 & 0.80 & 4.74 & 0.00 \\
\hline Alkalinity-HCO3 & 1.34 & 1.52 & 5.42 & 5.32 & 5.58 & 5.64 & 0.74 & 0.00 & 1.24 & 1.18 & 0.00 & 0.00 & 0.00 & 2.76 \\
\hline Chloride & 0.31 & 0.39 & 0.85 & 0.65 & 0.76 & 0.66 & 0.06 & 0.05 & 0.08 & 0.05 & 0.09 & 0.00 & 0.08 & 0.09 \\
\hline Fluoride & 0.00 & 0.00 & 0.01 & 0.01 & 0.01 & 0.02 & 0.00 & 0.01 & 0.00 & 0.01 & 0.00 & 0.01 & 0.01 & 0.00 \\
\hline witrate Mitrogen & 0.04 & 0.06 & 0.09 & 0.11 & 0.13 & 0.08 & 0.05 & 0.03 & 0.14 & 0.05 & 0.14 & 0.09 & 0.08 & 0.16 \\
\hline Sulfate & 0.48 & 0.60 & 0.50 & 0.44 & 0.46 & 0.43 & 0.06 & 0.06 & 0.12 & 0.06 & 0.14 & 0.12 & 0.08 & 0.19 \\
\hline Hydroxyl Ion & 0.00 & 0.00 & 0.00 & 0.00 & 0.00 & 0.00 & 0.03 & 0.50 & 0.01 & 0.01 & 0.01 & 10.00 & 2.51 & 0.00 \\
\hline - & & & & & & & $\cdot$ & & & & & & & • \\
\hline Total Anions & 2.16 & 2.57 & 6.86 & 6.53 & 6.93 & 6.83 & 1.10 & 1.21 & 2.84 & 1.53 & 0.57 & 11.09 & 7.50 & 3.20 \\
\hline CHARGE BALAMCE (3) & -16 & -11 & -2 & -2 & -2 & -1 & 48 & so & 30 & 30 & 64 & -5 & -39 & 25 \\
\hline mong &. & - & - & $\therefore$ & $\cdot$ & $\cdot$ & 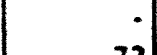 & 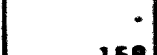 & $\cdot$ & $\dot{0}$ & 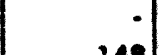 & $\dot{\theta}$ & - & \\
\hline $\operatorname{TDS}(\mathrm{mg} / \mathrm{L})$ & 136 & 204 & 350 & 350 & 380 & 402 & 72 & 158 & 106 & 118 & 148 & 358 & 158 & 228 \\
\hline ISS (mg/L) & 25 & 9 & $<1$ & $<1$ & 1 & $<1$ & $<1$ & 42 & 41 & 30 & 78 & 65 & 60 & 55 \\
\hline Turbidity (MrU) & 52 & 8 & 1.6 & 3.3 & 1.5 & 1 & 6 & 2.9 & 21 & 20 & 34 & 36 & 20 & 9.5 \\
\hline
\end{tabular}

(COArTrued) 
Appendix $G$

Ion-Charge Balance Calculations, 1993

\begin{tabular}{|c|c|c|c|c|c|c|c|c|c|c|c|c|c|c|}
\hline \multirow{3}{*}{$\begin{array}{l}\text { Sampling Point } \\
\text { Location } \\
\text { Date Sampled }\end{array}$} & \multicolumn{4}{|c|}{$G+631$} & \multicolumn{4}{|c|}{ Gw-632 } & \multicolumn{4}{|c|}{ Cun-633 } & \multicolumn{2}{|c|}{$G \mathrm{G}-634$} \\
\hline & \multicolumn{4}{|c|}{ RG } & \multicolumn{4}{|c|}{ RG } & \multicolumn{4}{|c|}{ IG } & \multicolumn{2}{|c|}{ RE } \\
\hline & $03 / 02 / 93$ & $06 / 18 / 93$ & $09 / 16 / 93$ & $11 / 17 / 93$ & $03 / 03 / 93$ & $06 / 21 / 93$ & $|09 / 16 / 93|$ & $11 / 18 / 93$ & $03 / 03 / 93$ & $06 / 21 / 93$ & $09 / 20 / 93$ & $11 / 19 / 93$ & $\mid 03 / 02 / 93$ & $06 / 18 / 93$ \\
\hline Cations (meq/L) & - & - & - & - & - & - & - & - & - & - & $\cdot$ & - & - & - \\
\hline Calcium & 0.80 & 0.35 & 0.36 & 0.36 & 3.89 & 1.85 & 2.69 & 1.40 & 354.29 & 234.53 & 244.51 & 11.98 & 0.12 & 0.09 \\
\hline Magnesium & 0.79 & 0.53 & 0.48 & 0.44 & 0.90 & 0.68 & 0.71 & 0.65 & 37.84 & 27.15 & 27.15 & 1.81 & 0.16 & 0.18 \\
\hline Potessium & 0.06 & 0.05 & 0.06 & 0.06 & 0.05 & 0.03 & 0.04 & 0.05 & 0.00 & 0.00 & 0.36 & 0.13 & 0.02 & 0.02 \\
\hline Sodium & 0.70 & 0.70 & 0.74 & 0.74 & 0.20 & 0.17 & 0.22 & 0.19 & 3.13 & 2.04 & 2.65 & 0.38 & 0.15 & 0.16 \\
\hline Hydrogen Ion & 0.01 & 0.01 & 0.02 & 0.01 & 0.00 & 0.00 & 0.00 & 0.00 & 0.00 & 0.00 & 0.00 & 0.00 & 0.01 & 0.00 \\
\hline Total Cations & 2.36 & .63 & .6 & 1.61 & 5.04 & 2.74 & 3.67 & 2.29 & 395.26 & 263.72 & 274.67 & 14.29 & 0.45 & 0.45 \\
\hline & & & & - & & & & & - & & & & - & \\
\hline ANIONS (meq/L) & & - & $\cdot$ & $\cdot$ & $\cdot$ & $\cdot$ & $\cdot$ & $\cdot$ & $\cdot$ & - & - & $\cdot$ &. & ' \\
\hline Alkalinity- $\mathrm{CO} 3$ & 0.00 & 0.00 & 0.00 & 0.00 & 0.00 & 0.00 & 0.00 & 0.00 & 0.00 & 0.00 & 0.00 & 0.00 & 0.00 & 0.00 \\
\hline Alkalinity-uCO3 & 0.22 & 0.22 & 0.14 & 0.20 & 1.02 & 2.76 & 2.40 & 2.94 & 5.30 & 5.46 & 5.22 & 1.94 & 0.42 & 0.24 \\
\hline Chloride & 1.35 & 1.33 & 1.66 & 1.27 & 0.42 & 0.51 & 0.45 & 0.59 & 0.00 & 0.00 & 0.00 & 0.23 & 0.22 & 0.26 \\
\hline Fluoride & 0.00 & 0.00 & 0.00 & 0.00 & 0.00 & 0.01 & 0.00 & 0.00 & 0.00 & 0.00 & 0.00 & 0.00 & 0.00 & 0.00 \\
\hline Witrate Nitrogen & 0.20 & 1.00 & 0.08 & 0.31 & 0.79 & 0.79 & 0.99 & 0.88 & 276.62 & 307.04 & 392.80 & 60.05 & 0.00 & 0.00 \\
\hline Sulfate & 0.09 & 0.04 & 0.08 & 0.08 & 0.00 & 0.00 & 0.15 & 0.04 & 0.00 & 0.00 & 6.35 & 0.60 & 0.00 & 0.0 \\
\hline Hydroxyl Ion & 0.00 & 0.00 & 0.00 & 0.00 & 0.00 & 0.00 & 0.00 & 0.00 & 0.00 & 0.00 & 0.00 & 0.00 & 0.00 & 0.00 \\
\hline - & 1.87 & 2.59 & i) & .8 & 2.23 & 4.06 & 3.99 & 4.45 & $281.92^{\circ}$ & 312.49 & 404.36 & 62.82 & 0.64 & 0.53 \\
\hline Total AnLons & 1.81 & 2.39 & 1.91 & 2.00 & 200 & 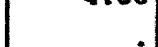 & & & & & & - & -1 & \\
\hline CHARGE BALANCE (8) & 12 & -23 & -9 & -7 & 39 & -19 & -4 & -32 & 17 & -8 & -19 & -63 & -17 & -9 \\
\hline $\operatorname{TDS}(\mathrm{mg} / \mathrm{L})$ & 166 & 238 & 142 & 152 & 198 & 272 & 234 & 188 & 21966 & 23196 & 25552 & 1326 & 58 & 46 \\
\hline TSS (mg/L) & 2 & 4 & 2 & 10 & 27 & 19 & 19 & 8 & 219 & 169 & 133 & 16 & $<1$ & $<$ \\
\hline Turbidity (NTU) & 2.6 & 5.5 & 1.5 & 50 & 22 & 56 & 66 & 5 & 220 & 95 & 70 & 12 & 1.2 & 1.4 \\
\hline
\end{tabular}

(CONTIMUED) 
APPENDIX G

Ion-Charge Balance Calnulations, 1993

\begin{tabular}{|c|c|c|c|c|c|c|c|c|c|c|c|c|c|c|}
\hline \multirow{3}{*}{$\begin{array}{l}\text { Sampling Point } \\
\text { Location } \\
\text { Date Sampled }\end{array}$} & \multicolumn{2}{|c|}{$G W-634$} & \multicolumn{4}{|c|}{ Gw-656 } & \multicolumn{4}{|c|}{$G W-657$} & \multicolumn{4}{|c|}{$G w-658$} \\
\hline & \multicolumn{2}{|c|}{ RG } & \multicolumn{4}{|c|}{20134} & \multicolumn{4}{|c|}{$\mathbf{2 2 3 3 1}$} & \multicolumn{4}{|c|}{$\boldsymbol{F}$} \\
\hline & $09 / 16 / 93$ & $11 / 18 / 93$ & $03 / 11 / 93$ & $06 / 22 / 93$ & $09 / 23 / 93$ & $11 / 17 / 93$ & $03 / 10 / 93$ & $06 / 18 / 93$ & $09 / 21 / 93$ & $11 / 15 / 93$ & $03 / 11 / 93$ & $06 / 23 / 93$ & $11 / 19 / 93$ & $09 / 27 / 04$ \\
\hline CATIONS (meq/L) & $\cdot$ & - & - & - & - & - & $\cdot$ & - & - & - & - & - & - & . \\
\hline Calcium & 0.08 & 0.09 & 4.99 & 4.99 & 5.99 & 5.49 & 3.49 & 3.49 & 3.89 & 4.04 & 2.30 & 2.35 & 3.74 & 2.79 \\
\hline Magnesium & 0.16 & 0.15 & 0.49 & 0.49 & 0.51 & 0.50 & 0.67 & 0.73 & 0.74 & 0.79 & 0.99 & 1.15 & 1.15 & 1.07 \\
\hline Potessium & 0.03 & 0.03 & 0.07 & 0.05 & 0.06 & 0.06 & 0.14 & 0.15 & 0.15 & 0.14 & 0.00 & 0.00 & 0.03 & 0.02 \\
\hline Sodium & 0.16 & 0.17 & 0.33 & 0.30 & 0.35 & 0.35 & 0.57 & 0.65 & 0.52 & 0.52 & 0.70 & 0.57 & 0.70 & 0.70 \\
\hline Hydrogen Ion & 0.01 & 0.01 & 0.00 & 0.00 & 0.00 & 0.00 & 0.00 & 0.00 & 0.00 & 0.00 & 0.00 & 0.00 & 0.00 & 0.00 \\
\hline - & & & & & & & 8. & & & & & 4.06 & 5.62 & 58 \\
\hline Total Cations & 0.43 & 0.44 & 5.87 & 5.83 & 6.91 & 6.40 & 4.86 & 5.03 & 5.30 & 5.49 & 3.98 & 4.06 & 5.62 & 4.58 \\
\hline ANIONS (meg/L) & $\cdot$ & - & - & $\cdot$ & $\cdot$ & - & $\cdot$ & - & $\cdot$ & - & $\cdot$ & - & - & $\cdot$ \\
\hline Alkalinity-CO3 & 0.00 & 0.00 & 0.00 & 0.00 & 0.00 & 0.00 & 0.00 & 0.00 & 0.00 & 0.00 & 0.00 & 0.00 & 0.00 & 0.00 \\
\hline Alkal inity-HCO3 & 0.36 & 0.38 & 3.18 & 3.10 & 3.56 & 3.50 & 3.90 & 4.42 & 4.40 & 4.22 & 4.38 & 4.82 & 5.40 & 5.18 \\
\hline Chloride & 0.25 & 0.23 & 1.96 & 1.86 & 1.92 & 1.81 & 0.71 & 0.62 & 0.42 & 0.52 & 0.54 & 0.39 & 0.76 & 0.73 \\
\hline Fluoride & 0.00 & 0.00 & 0.00 & 0.01 & 0.00 & 0.00 & 0.02 & 0.03 & 0.03 & 0.02 & 0.01 & 0.00 & 0.01 & 0.00 \\
\hline Nitrate Nitrogen & 0.00 & 0.00 & 0.00 & 0.02 & 0.00 & 0.00 & 0.00 & 0.00 & 0.00 & 0.02 & 0.00 & 0.00 & 0.00 & 0.00 \\
\hline Sulfate & 0.02 & 0.00 & 1.30 & 1.27 & 1.35 & 1.40 & 0.64 & 0.46 & 0.62 & 0.78 & 0.34 & 0.16 & 0.04 & 0.00 \\
\hline Hydroxyl Ion & 0.00 & 0.00 & 0.00 & 0.00 & 0.00 & 0.00 & 0.00 & 0.00 & 0.00 & 0.00 & 0.00 & 0.00 & 0.00 & 0.00 \\
\hline & & & & & & & & & & & $\cdot$ & $\cdot$ & $\bullet$ & $\bullet$ \\
\hline Total Anions & 0.63 & 0.61 & 6.44 & 6.26 & 6.83 & 6.70 & 5.27 & 5.52 & 5.47 & 5.56 & 5.26 & 5.37 & 6.20 & 5.91 \\
\hline$\overline{\text { CHARGE BALANCE (8) }}$ & -19 & -16 & -5 & -4 & $\dot{1}$ & -2 & -4 & -5 & -2 & -1 & -14 & -14 & -5 & -13 \\
\hline $\operatorname{TDS}(\mathrm{mg} / \mathrm{L})$ & 58 & 52 & 448 & 454 & 476 & $42 \dot{2}$ & 292 & 286 & $31 \dot{0}$ & 302 & 286 & 290 & 332 & 294 \\
\hline TSS (mg/L) & 2 & 1 & 92 & 83 & 48 & 746 & $<1$ & 7 & 4 & 3 & 29 & 29 & 91 & 31 \\
\hline Turbidity (NTU) & 3.6 & 1.4 & 66 & 130 & 100 & 380 & 1.2 & 4 & 5.8 & 1.6 & 28 & 36 & 18 & 220 \\
\hline
\end{tabular}

(CONTINUED) 
APPENDIX G

Ion-Charge Balance Calculations, 1993

\begin{tabular}{|c|c|c|c|c|c|c|c|c|c|c|c|c|c|c|}
\hline \multirow{3}{*}{$\begin{array}{l}\text { Sampling Point } \\
\text { Location } \\
\text { - } \\
\text { Date Sampled }\end{array}$} & \multicolumn{4}{|c|}{ GW-659 } & \multicolumn{4}{|c|}{$6 w-707$} & \multicolumn{4}{|c|}{ Gw-708 } & \multicolumn{2}{|c|}{ Gw-733 } \\
\hline & \multicolumn{4}{|c|}{$\mathbf{F F}$} & \multicolumn{4}{|c|}{ T2331 } & \multicolumn{4}{|c|}{ T2331 } & \multicolumn{2}{|c|}{ EXP } \\
\hline & $03 / 10 / 93$ & $06 / 18 / 93$ & $09 / 22 / 93$ & $11 / 15 / 93$ & $03 / 11 / 93$ & $06 / 23 / 93$ & |09/27/93 & $11 / 18 / 93$ & $03 / 10 / 93$ & $06 / 18 / 93$ & $09 / 21 / 93 \mid$ & $11 / 12 / 93$ & $02 / 02 / 93$ & $05 / 06 / 93$ \\
\hline CATIONS (meq/L) & - & - & - & - & $\cdot$ & - & - & $\cdot$ & - & $\cdot$ & $\cdot$ & - & - & - \\
\hline Calcium & 2.45 & 0.55 & 0.39 & 0.55 & 1.30 & 2.40 & 3.64 & 2.79 & 4.79 & 5.99 & 6.99 & 6.99 & 1.95 & 1.90 \\
\hline Magnesium & 0.47 & 0.11 & 0.11 & 0.06 & 0.18 & 0.52 & 0.82 & 0.59 & 0.79 & 1.07 & 1.32 & 1.48 & 1.23 & 1.23 \\
\hline Potassium & 0.12 & 0.06 & 0.05 & 0.03 & 0.12 & 0.23 & 0.20 & 0.13 & 0.15 & 0.22 & 0.28 & 0.18 & 0.04 & 0.04 \\
\hline Sodium & 13.05 & 5.65 & 3.57 & 0.28 & 0.17 & 1.13 & 0.83 & 0.52 & 0.10 & 0.16 & 0.19 & 0.18 & 0.20 & 0.18 \\
\hline Hydrogen Ion & 0.00 & 0.00 & 0.00 & 0.00 & 0.00 & 0.00 & 0.00 & 0.00 & 0.00 & 0.00 & 0.00 & 0.00 & 0.00 & 0.00 \\
\hline Total Cations & 16.08 & 6. & 4 & 0.92 & 1.77 & 4.27 & 5.49 & 4.04 & 5.84 & 7.43 & 8.77 & 8.83 & 3.41 & 3.35 \\
\hline Holas Latsomb & 10.00 & . & .1 & 0.52 & 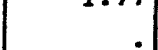 & 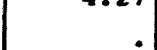 & .0. & 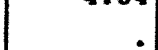 & 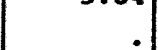 & & & . & 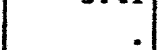 & 2.00 \\
\hline ANIONS (meq/L) & • & $\cdot$ & $\cdot$ & $\cdot$ & $\cdot$ & $\cdot$ & $\cdot$ & $\cdot$ & $\cdot$ & $\cdot$ & - & $\cdot$ & $\cdot$ & • \\
\hline Alkalinity-CO3 & 0.00 & 0.00 & 0.00 & 0.00 & 0.00 & 0.00 & 0.00 & 0.00 & 0.00 & 0.00 & 0.00 & 0.00 & 0.00 & 0.00 \\
\hline Alkalinity- $\mathrm{HCO} 3$ & 0.66 & 1.44 & 1.26 & 0.74 & 2.62 & 3.02 & 4.00 & 3.10 & 5.28 & 7.43 & 7.85 & 8.43 & 2.98 & 3.02 \\
\hline Chloride & 16.31 & 6.71 & 1.41 & 0.09 & 0.48 & 5.70 & 2.31 & 0.65 & 0.09 & 0.17 & 0.15 & 0.17 & 0.48 & 0.37 \\
\hline Fluoride & 0.00 & 0.00 & 0.01 & 0.01 & 0.02 & 0.02 & 0.03 & 0.03 & 0.01 & 0.02 & 0.03 & 0.03 & 0.02 & 0.01 \\
\hline Nitrate Nitrogen & 0.00 & 0.00 & 0.00 & 0.02 & 0.02 & 0.00 & 0.02 & 0.02 & 0.00 & 0.00 & 0.00 & 0.00 & 0.00 & 0.00 \\
\hline Sulfate & 0.41 & 0.21 & 0.23 & 0.11 & 0.44 & 0.67 & 0.94 & 0.79 & 0.08 & 0.00 & 0.12 & 0.08 & 0.25 & 0.25 \\
\hline Hydroxyl Ion & 0.00 & 0.00 & 0.00 & 0.00 & 0.00 & 0.00 & 0.00 & 0.00 & 0.00 & 0.00 & 0.00 & 0.00 & 0.00 & 0.00 \\
\hline$\cdot$ & & & & & & & & & & & & & & \\
\hline Total Anions & 17.37 & 8.36 & 2.90 & 0.97 & 3.58 & 9.40 & 7.29 & 4.59 & 5.46 & 7.63 & 8.15 & 8.71 & 3.72 & 3.65 \\
\hline CHARGE BALANCE (8) & -4 & -14 & 17 & -2 & -34 & -38 & -14 & -6 & 3 & -1 & 4 & 1 & -4 & -4 \\
\hline$\overline{\operatorname{TDS}(\mathrm{mg} / \mathrm{L})}$ & 946 & 640 & 224 & 306 & 216 & 514 & 310 & 242 & 324 & 426 & 522 & 496 & 194 & 186 \\
\hline ISS (mg/L) & 391 & 234 & 253 & 743 & 11 & 1 & 2 & 3 & 31 & 10 & 9 & 12 & 9 & 37 \\
\hline Turbidity (NTU) & 460 & 340 & 680 & 750 & 6.2 & 2.9 & 3.6 & 2.4 & 15 & 22 & so & 36 & 15 & 38 \\
\hline
\end{tabular}

(CONTINUED) 
APPENDIX G

Ion-Charge Balance Calculations, 1993

\begin{tabular}{|c|c|c|c|c|c|c|c|c|c|c|c|c|c|c|}
\hline \multirow{3}{*}{$\begin{array}{l}\text { Sampling Point } \\
\text { Location } \\
\text { Date Sampled }\end{array}$} & \multicolumn{2}{|c|}{ GW-733 } & \multicolumn{4}{|c|}{ Gw-735 } & \multicolumn{4}{|c|}{ Gw-744 } & \multicolumn{4}{|c|}{ GW-745 } \\
\hline & \multicolumn{2}{|c|}{ BXP } & \multicolumn{4}{|c|}{ NHP } & \multicolumn{4}{|c|}{ GRIDK1 } & \multicolumn{4}{|c|}{ GRIDK1 } \\
\hline & $08 / 18 / 93$ & $11 / 04 / 93$ & $02 / 02 / 93$ & $05 / 07 / 93$ & $08 / 18 / 93$ & $10 / 29 / 93$ & $01 / 18 / 93$ & $04 / 12 / 93$ & $08 / 04 / 93$ & $10 / 22 / 93$ & $01 / 19 / 93$ & $04 / 12 / 93$ & $08 / 04 / 93$ & $10 / 23 / 93$ \\
\hline CATIONS (meq/I) & $\cdot$ & - & - & $\cdot$ & - & - & $\cdot$ & - & $\cdot$ & - & $\cdot$ & - & - & - \\
\hline Calcium & 2.50 & 2.05 & 5.49 & 5.49 & 5.99 & 5.49 & 2.54 & 2.40 & 2.50 & 2.69 & 2.45 & 2.64 & 2.94 & 2.94 \\
\hline Magnesium & 1.48 & 1.32 & 0.67 & 0.65 & 0.75 & 0.70 & 0.67 & 0.65 & 0.69 & 0.75 & 0.69 & 0.72 & 0.81 & 0.82 \\
\hline Potassium & 0.05 & 0.05 & 0.07 & 0.06 & 0.06 & 0.07 & 0.06 & 0.08 & 0.08 & 0.08 & 0.06 & 0.08 & 0.09 & 0.08 \\
\hline Sodium & 0.23 & 0.08 & 0.16 & 0.14 & 0.16 & 0.17 & 1.26 & 1.26 & 1.26 & 1.30 & 0.78 & 0.83 & 0.91 & 0.91 \\
\hline Hydrogen Ion & 0.00 & 0.00 & 0.00 & 0.00 & 0.00 & 0.00 & 0.00 & 0.00 & 0.00 & 0.00 & 0.00 & 0.00 & 0.00 & 0.00 \\
\hline Total Cations & $\begin{array}{r}. \\
4.25\end{array}$ & 3.49 & 6.39 & 6.34 & 6.96 & 6.43 & 4.54 & 4.38 & 4.53 & 4.82 & 3.98 & 4.28 & 4.76 & 4.76 \\
\hline ANIONS (meq/L) & & $\cdot$ & $\dot{\bullet}$ & & $\bullet$ & $\dot{\bullet}$ & & $\cdot$ & $\dot{.}$ & $\cdot$ & & $\stackrel{\bullet}{.}$ & $\cdot$ & • \\
\hline Alkalinity-CO3 & 0.00 & 0.00 & 0.00 & 0.00 & 0.00 & 0.00 & 0.00 & 0.00 & 0.00 & 0.00 & 0.00 & 0.00 & 0.00 & 0.00 \\
\hline Alkalinity-HCO3 & 3.16 & 3.16 & 5.80 & 5.62 & 5.72 & 5.80 & 3.98 & 3.84 & 3.90 & 3.98 & 3.56 & 3.44 & 3.72 & 3.66 \\
\hline Chloride & 1.10 & 0.37 & 0.34 & 0.42 & 0.27 & 0.31 & 0.09 & 0.09 & 0.10 & 0.11 & 0.28 & 0.27 & 0.27 & 0.45 \\
\hline Fluoride & 0.01 & 0.02 & 0.00 & 0.00 & 0.00 & 0.00 & 0.01 & 0.01 & 0.01 & 0.01 & 0.00 & 0.01 & 0.01 & 0.00 \\
\hline Nitrate Nitrogen & 0.00 & 0.01 & 0.00 & 0.00 & 0.00 & 0.00 & 0.00 & 0.00 & 0.00 & 0.00 & 0.00 & 0.00 & 0.00 & 0.00 \\
\hline Sulfate & 0.23 & 0.21 & 0.44 & 0.40 & 0.37 & 0.44 & 0.47 & 0.45 & 0.52 & 0.42 & 0.62 & 0.62 & 0.60 & 0.54 \\
\hline Hydroxyl Ion & 0.00 & 0.00 & 0.00 & 0.00 & 0.00 & 0.00 & 0.00 & 0.00 & 0.00 & 0.00 & 0.00 & 0.00 & 0.00 & 0.00 \\
\hline - & & & & & & & & & & & & & $\cdot$ & \\
\hline Total Anions & 4.50 & 3.76 & 6.57 & 6.43 & 6.36 & 6.54 & 4.55 & 4.39 & 4.53 & 4.51 & 4.46 & 4.34 & 4.60 & 4.65 \\
\hline CHARGE BALANCE (8) & -3 & -4 & -1 & -1 & $\dot{5}$ & -1 & -0 & -0 & $\dot{0}$ & 3 & -6 & -1 & $\dot{2}$ & $\dot{1}$ \\
\hline Imas & $\cdot$ & $\cdot$ & $\cdot$ & $\cdot$ & 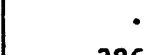 & $\cdot$ & 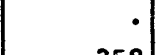 & 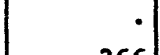 & $\bullet$ & $\cdot$ & $\cdot$ & 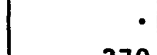 & 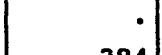 & 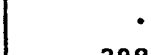 \\
\hline $\operatorname{TDS}$ (mg/L) & 228 & 222 & 384 & 374 & 386 & 398 & 258 & 266 & 242 & 286 & 268 & 270 & 284 & 298 \\
\hline TSS (mg/L) & 20 & 2 & 7 & 7 & $<1$ & 14 & $<1$ & $<1$ & $<1$ & $<1$ & 3 & 2 & $<1$ & $<1$ \\
\hline Turbidity (NTU) & 20 & 4.8 & 14 & 14 & 2.4 & 18 & 1.8 & 2.5 & 1.4 & 1 & 1.8 & 6.2 & 1.5 & 2.6 \\
\hline
\end{tabular}

(CONTINUED) 


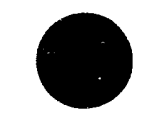

APPENDIX G

Ion-Charge Balance Calculations, 1993

\begin{tabular}{|c|c|c|c|c|c|c|c|c|c|c|c|c|c|c|}
\hline \multirow{3}{*}{$\begin{array}{l}\text { Sampling Point } \\
\text { Location } \\
\text { Date Sampled }\end{array}$} & \multicolumn{4}{|c|}{ GW-746 } & \multicolumn{4}{|c|}{ GW-747 } & \multicolumn{4}{|c|}{ GW-748 } & \multicolumn{2}{|c|}{ GH-749 } \\
\hline & \multicolumn{4}{|c|}{ GRIDKI } & \multicolumn{4}{|c|}{ GRIDK2 } & \multicolumn{4}{|c|}{ GRIDK2 } & \multicolumn{2}{|c|}{ GRIDK2 } \\
\hline & $01 / 19 / 93$ & $04 / 12 / 93$ & $08 / 05 / 93$ & $10 / 23 / 93$ & $01 / 19 / 93$ & $04 / 13 / 93$ & $08 / 05 / 93$ & $10 / 23 / 93$ & $01 / 20 / 93$ & $04 / 13 / 93$ & $08 / 06 / 93$ & $10 / 24 / 93$ & $01 / 21 / 93$ & $04 / 14 / 93$ \\
\hline CATIONS (meq/L) & - & $\cdot$ & $\cdot$ & - & $\cdot$ & - & $\cdot$ & - & - & - & - & $\cdot$ & $\cdot$ & - \\
\hline Calcium & 0.65 & 0.60 & 0.90 & 0.65 & 2.64 & 2.64 & 2.69 & 2.74 & 3.69 & 3.84 & 4.24 & 4.54 & 4.39 & 3.99 \\
\hline Magnesium & 0.35 & 0.34 & 0.45 & 0.39 & 0.90 & 0.82 & 0.90 & 0.90 & 0.67 & 0.67 & 0.79 & 0.82 & 0.73 & 0.63 \\
\hline Potassium & 0.03 & 0.03 & 0.05 & 0.04 & 0.05 & 0.05 & 0.06 & 0.05 & 0.02 & 0.03 & 0.02 & 0.02 & 0.10 & 0.08 \\
\hline Sodium & 0.25 & 0.26 & 0.35 & 0.30 & 1.00 & 1.09 & 1.17 & 1.30 & 0.40 & 0.42 & 0.42 & 0.48 & 0.27 & 0.31 \\
\hline Hydrogen Ion & 0.00 & 0.00 & 0.00 & 0.00 & 0.00 & 0.00 & 0.00 & 0.00 & 0.00 & 0.00 & 0.00 & 0.00 & 0.00 & 0.00 \\
\hline - & & & & & & & & & & & & $\therefore$ & - & \\
\hline Total Cations & 1.29 & 1.22 & 1.75 & 1.38 & 4.60 & 4.60 & 4.84 & 5.01 & 4.78 & 4.97 & 5.47 & 5.87 & 5.50 & 5.01 \\
\hline ANIONS (meg/L) & & • & . & - &. & - & . & $\cdot$ &. & 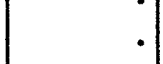 & $\cdot$ & $\cdot$ & $\cdot$ & $\cdot$ \\
\hline Alkalinity- $\mathrm{CO} 3$ & 0.00 & 0.00 & 0.00 & 0.00 & 0.00 & $0 . \dot{00}$ & 0.00 & 0.00 & 0.00 & 0.00 & 0.00 & 0.00 & 0.00 & 0.00 \\
\hline Alkalinity- & 0.64 & 0.66 & 0.76 & 0.80 & 4.14 & 4.00 & 4.14 & 4.06 & 4.06 & 3.94 & 4.38 & 4.44 & 4.18 & 4.04 \\
\hline Chloride & 0.19 & 0.29 & 0.21 & 0.23 & 0.18 & 0.16 & 0.13 & 0.11 & 0.65 & 0.27 & 0.42 & 0.52 & 0.56 & 0.39 \\
\hline Fluoride & 0.00 & 0.00 & 0.00 & 0.00 & 0.00 & 0.01 & 0.01 & 0.01 & 0.00 & 0.01 & 0.01 & 0.00 & 0.00 & 0.01 \\
\hline Nitrate Nitrogen & 0.04 & 0.03 & 0.04 & 0.03 & 0.00 & 0.00 & 0.00 & 0.00 & 0.00 & 0.00 & 0.00 & 0.00 & 0.03 & 0.00 \\
\hline Sulfate & 0.52 & 0.38 & 0.50 & 0.54 & 0.58 & 0.50 & 0.50 & 0.46 & 0.81 & 0.56 & 0.60 & 0.67 & 1.12 & 0.79 \\
\hline Hydroxyl Ion & 0.00 & 0.00 & 0.00 & 0.00 & 0.00 & 0.00 & 0.00 & 0.00 & 0.00 & 0.00 & 0.00 & 0.00 & 0.00 & 0.00 \\
\hline & & & & & & & & & $\cdot$ & & & & $\cdot$ & \\
\hline Total Anions & 1.40 & 1.37 & 1.50 & 1.60 & 4.90 & 4.67 & 4.77 & 4.64 & 5.52 & 4.78 & 5.41 & 5.63 & 5.90 & 5.23 \\
\hline$\overline{\text { CHARGE BAIANCE ( }(8)}$ & -4 & $-\dot{6}$ & $\dot{8}$ & $\dot{-7}$ & -3 & $-\dot{1}$ & $\dot{1}$ & $\dot{4}$ & -7 & $\dot{2}$ & $\dot{1}$ & $\dot{2}$ & -3 & -2 \\
\hline $\operatorname{TDS}(\mathrm{mg} / \mathrm{L})$ & $\begin{array}{r}\cdot \\
102\end{array}$ & 104 & 104 & $14 \dot{2}$ & $27 \dot{6}$ & $27 \dot{2}$ & 264 & $28 \dot{ }$ & $32 \dot{0}$ & $31 \dot{6}$ & $34 \dot{8}$ & $35 \dot{0}$ & 540 & 316 \\
\hline TSS (mg/L) & 12 & 13 & 414 & 6 & $<1$ & 1 & 2 & $<1$ & 79 & 142 & 12 & 17 & 2 & 20 \\
\hline Turbidity (NTU) & 120 & 210 & 230 & 4.3 & 1.3 & 0.6 & 1.1 & 1.6 & 160 & 1800 & 16 & 17 & 4.3 & 18 \\
\hline
\end{tabular}

(CONTINUED) 
APPENDIX $G$

Ion-Charge Balance Calculations, 1993

\begin{tabular}{|c|c|c|c|c|c|c|c|c|c|c|c|c|c|c|}
\hline \multirow{3}{*}{$\begin{array}{l}\text { Sampling Point } \\
\text { Location } \\
\text { Date Sampled }\end{array}$} & \multicolumn{2}{|c|}{ GW-749 } & \multicolumn{4}{|c|}{ GW-750 } & \multicolumn{4}{|c|}{ GW-751 } & \multicolumn{4}{|c|}{ GW-752 } \\
\hline & \multicolumn{2}{|c|}{ GRIDK2 } & \multicolumn{4}{|c|}{ GRIDK2 } & \multicolumn{4}{|c|}{ GRIDJ3 } & \multicolumn{4}{|c|}{ GRIDJ3 } \\
\hline & $08 / 06 / 93$ & $10 / 24 / 93$ & $01 / 21 / 93$ & $04 / 15 / 93$ & $08 / 06 / 93$ & $10 / 25 / 93$ & $01 / 11 / 93$ & $|04 / 16 / 93|$ & $08 / 02 / 93$ & $10 / 26 / 93$ & $\mid 01 / 11 / 93$ & $04 / 16 / 93$ & $08 / 02 / 93$ & $10 / 26 / 93$ \\
\hline CATIONS (meg/L) & - & - & - & • & - & - & - & - & - & - & - & $\cdot$ & $\cdot$ & - \\
\hline Calcium & 5.49 & 5.49 & 3.59 & 3.79 & 3.74 & 3.69 & 2.84 & 2.59 & 2.50 & 2.74 & 1.50 & 1.45 & 1.45 & 1.40 \\
\hline Magnesium & 0.80 & 0.77 & 0.90 & 0.82 & 1.07 & 0.99 & 0.80 & 0.72 & 0.75 & 0.79 & 0.29 & 0.28 & 0.30 & 0.28 \\
\hline Potassium & 0.03 & 0.04 & 0.12 & 0.11 & 0.12 & 0.15 & 0.08 & 0.08 & 0.08 & 0.08 & 0.04 & 0.04 & 0.06 & 0.06 \\
\hline Sodium & 0.52 & 0.57 & 0.22 & 0.20 & 0.27 & 0.26 & 0.37 & 0.34 & 0.37 & 0.39 & 0.35 & 0.35 & 0.39 & 0.39 \\
\hline Hydrogen Ion & 0.00 & 0.00 & 0.00 & 0.00 & 0.00 & 0.00 & 0.00 & 0.00 & 0.00 & 0.00 & 0.00 & 0.00 & 0.00 & 0.00 \\
\hline - & & & & & & & & & & • & & $\cdot$ & & \\
\hline Total Cations & 6.84 & 6.86 & 4.84 & 4.93 & 5.21 & 5.09 & 4.08 & 3.73 & 3.69 & 4.01 & 2.17 & 2.12 & 2.20 & 2.12 \\
\hline ANIONS (meq/L) & & . & & & $\bullet$ & & & $\cdot$ & & $\bullet$ & & • & $\cdot$ & • \\
\hline Alkalinity-CO3 & 0.00 & 0.00 & 0.00 & 0.00 & 0.00 & 0.00 & 0.00 & 0.00 & 0.00 & 0.00 & 0.00 & 0.00 & 0.00 & 0.00 \\
\hline $\begin{array}{l}\text { Alkalinity- } \mathrm{CO} 3 \\
\text { Alkalinity-HCO3 }\end{array}$ & 4.12 & 5.42 & 4.68 & 4.58 & 4.82 & 4.82 & 3.62 & 3.54 & 3.62 & 3.64 & 1.16 & 1.08 & 1.14 & 0.90 \\
\hline Chloride & 0.45 & 1.08 & 0.12 & 0.10 & 0.12 & 0.12 & 0.26 & 0.25 & 0.23 & 0.28 & 1.47 & 1.13 & 1.52 & 1.10 \\
\hline Fluoride & 0.01 & 0.01 & 0.00 & 0.00 & 0.00 & 0.00 & 0.00 & 0.00 & 0.00 & 0.00 & 0.00 & 0.00 & 0.00 & 0.00 \\
\hline Nitrate Nitrogen & 0.51 & 0.00 & 0.00 & 0.00 & 0.00 & 0.00 & 0.00 & 0.00 & 0.00 & 0.00 & 0.00 & 0.00 & 0.00 & 0.00 \\
\hline Sulfate & 1.69 & 1.00 & 0.40 & 0.40 & 0.44 & 0.34 & 0.13 & 0.19 & 0.08 & 0.08 & 0.10 & 0.17 & 0.10 & 0.10 \\
\hline Hydroxyl Ion & 0.00 & 0.00 & 0.00 & 0.00 & 0.00 & 0.00 & 0.00 & 0.00 & 0.00 & 0.00 & 0.00 & 0.00 & 0.00 & 0.00 \\
\hline & & & & & - & & & & & & & . & & \\
\hline Total Anions & 6.77 & 7.50 & 5.19 & 5.07 & 5.37 & 5.28 & 4.00 & 3.98 & 3.92 & 4.00 & 2.72 & 2.38 & 2.76 & 2.10 \\
\hline CHARGE BALANCE ( $(8)$ & $\dot{1}$ & -4 & -4 & $\begin{array}{r}\cdot \\
-1\end{array}$ & -2 & $-\dot{-2}$ & $\dot{1}$ & -3 & -3 & $\dot{0}$ & $-1 i$ & $-\dot{6}$ & -11 & $\dot{0}$ \\
\hline & • & • & $\cdot$ & • & $\cdot$ & • & $\cdot$ & $\cdot$ & $\cdot$ & $\cdot$ & $\cdot$ & $\cdot$ & $\cdot$ & • \\
\hline $\operatorname{TDS}(\mathrm{mg} / \mathrm{L}$ ) & 436 & 470 & 274 & 290 & 308 & 312 & 226 & 218 & 228 & 252 & 160 & 160 & 184 & 180 \\
\hline TSS (mg/L) & 13 & $<1$ & $<1$ & $<1$ & 7 & $<1$ & 7 & 4 & 2 & 9 & $<1$ & 19 & 18 & 5 \\
\hline Turbidity (NTU) & 14 & 3.3 & 0.9 & 0.9 & 7.9 & 1 & 2.1 & 1.7 & 1.6 & 2.2 & 7.6 & 13 & 14 & 3.7 \\
\hline
\end{tabular}

(CONTINUED) 
APPENDIX G

Ion-Charge Balance Calculations, 1993

\begin{tabular}{|c|c|c|c|c|c|c|c|c|c|c|c|c|c|c|}
\hline \multirow{3}{*}{$\begin{array}{l}\text { Sampling Point } \\
\text { Location } \\
\text { Date Sampled }\end{array}$} & \multicolumn{4}{|c|}{$G W-753$} & \multicolumn{4}{|c|}{$G W-754$} & \multicolumn{4}{|c|}{$G W-755$} & \multicolumn{2}{|c|}{ GW-756 } \\
\hline & \multicolumn{4}{|c|}{ GRIDJ2 } & \multicolumn{4}{|c|}{ GRIDJ2 } & \multicolumn{4}{|c|}{ GRIDJ1 } & \multicolumn{2}{|c|}{ GRIDJ1 } \\
\hline & $01 / 15 / 93$ & $04 / 08 / 93$ & $08 / 03 / 93$ & $10 / 21 / 93$ & $01 / 18 / 93$ & $04 / 08 / 93$ & $08 / 04 / 93$ & $10 / 22 / 93$ & $01 / 14 / 93$ & 04/07/93 & $08 / 03 / 93$ & $10 / 21 / 93$ & $01 / 15 / 93$ & $04 / 08 / 93$ \\
\hline CATIONS (meq/L) & $\cdot$ & $\cdot$ & $\cdot$ & $\cdot$ & $\cdot$ & $\cdot$ & $\cdot$ & - & - & - & $\cdot$ & $\cdot$ & $\cdot$ & • \\
\hline Calcium & 3.04 & 2.89 & 2.89 & 2.99 & 1.90 & 2.54 & 2.64 & 2.69 & 1.05 & 0.95 & 1.20 & 1.35 & 1.45 & 1.35 \\
\hline Magnesium & 0.74 & 0.72 & 0.74 & 0.79 & 0.67 & 0.73 & 0.78 & 0.81 & 0.39 & 0.33 & 0.44 & 0.52 & 0.42 & 0.44 \\
\hline Potessium & 0.05 & 0.04 & 0.05 & 0.05 & 0.05 & 0.04 & 0.04 & 0.04 & 0.06 & 0.06 & 0.09 & 0.08 & 0.03 & 0.03 \\
\hline Sodium & 0.43 & 0.48 & 0.48 & 0.52 & 0.21 & 0.20 & 0.20 & 0.22 & 2.96 & 3.31 & 3.22 & 3.44 & 0.70 & 0.78 \\
\hline Hydrogen Ion & 0.00 & 0.00 & 0.00 & 0.00 & 0.00 & 0.00 & 0.00 & 0.00 & 0.00 & 0.00 & 0.00 & 0.00 & 0.00 & 0.00 \\
\hline Total Cations & 4.27 & 4.14 & 4.16 & 4.36 & 2.82 & 3.52 & 3.67 & 3.77 & 4.45 & 4.64 & 4.95 & 5.38 & 2.59 & 2.60 \\
\hline ANIONS (meq/L) & & & & . & $\cdot$ & $\cdot$ & $\cdot \dot{.}$ & $\dot{\bullet}$ & $\cdot$ & $\cdot$ & $\cdot$ & $\cdot$ & $\cdot$ & $\cdot$ \\
\hline Alkalinity-CO3 & 0.00 & 0.00 & 0.00 & 0.00 & 0.00 & 0.00 & 0.00 & 0.00 & 0.00 & 0.04 & 0.00 & 0.00 & 0.00 & 0.00 \\
\hline Alkalinity- $-\mathrm{HCO} 3$ & 3.98 & 3.86 & 4.00 & 3.92 & 2.60 & 1.82 & 2.88 & 2.84 & 4.24 & 4.34 & 4.30 & 4.32 & 1.24 & 1.12 \\
\hline Chloride & 0.07 & 0.07 & 0.08 & 0.08 & 0.12 & 0.09 & 0.13 & 0.11 & 0.17 & 0.27 & 0.23 & 0.39 & 1.10 & 0.82 \\
\hline Fluoride & 0.00 & 0.01 & 0.00 & 0.00 & 0.00 & 0.00 & 0.00 & 0.00 & 0.03 & 0.02 & 0.04 & 0.03 & 0.00 & 0.01 \\
\hline Nitrate Nitrogen & 0.00 & 0.00 & 0.00 & 0.00 & 0.00 & 0.00 & 0.00 & 0.00 & 0.00 & 0.00 & 0.00 & 0.00 & 0.00 & 0.00 \\
\hline Sulfate & 0.25 & 0.23 & 0.31 & 0.19 & 0.27 & 0.35 & 0.29 & 0.23 & 0.40 & 0.42 & 0.44 & 0.33 & 0.67 & 0.75 \\
\hline Hydroxyl Ion & 0.00 & 0.00 & 0.00 & 0.00 & 0.00 & 0.00 & 0.00 & 0.00 & 0.00 & 0.00 & 0.00 & 0.00 & 0.00 & 0.00 \\
\hline & & & & & & & & &. & & $\cdot$ & - & & \\
\hline Total Anions & 4.30 & 4.16 & 4.39 & 4.19 & 2.98 & 2.26 & 3.30 & 3.18 & 4.83 & 5.08 & 5.00 & 5.07 & 3.01 & 2.69 \\
\hline CHARGE BALANCE (8) & $\begin{array}{r}\dot{0} \\
-\dot{0}\end{array}$ & $\dot{-0}$ & -3 & $\dot{2}$ & $\dot{-3}$ & $\dot{22}$ & $\dot{5}$ & $\dot{9}$ & -4 & $\begin{array}{r}\cdot \\
-5\end{array}$ & $\begin{array}{r}\cdot \\
-1\end{array}$ & $\dot{3}$ & -7 & -2 \\
\hline $\operatorname{TDS}(m q / L)$ & $24 \dot{ }$ & 238 & 258 & 256 & 178 & 136 & $18 \dot{8}$ & 202 & 282 & 290 & $\begin{array}{r}\cdot \\
304\end{array}$ & 308 & 172 & 194 \\
\hline TSS (mg/L) & $\begin{array}{r}242 \\
<1\end{array}$ & $\begin{array}{r}230 \\
5\end{array}$ & $\begin{array}{r}250 \\
2\end{array}$ & $\begin{array}{r}230 \\
2\end{array}$ & 70 & $\begin{array}{l}130 \\
110\end{array}$ & $\begin{array}{r}102 \\
22\end{array}$ & $\begin{array}{r}202 \\
7\end{array}$ & $\begin{array}{r}202 \\
12\end{array}$ & 118 & $\begin{array}{r}304 \\
12\end{array}$ & $\begin{array}{r}<00 \\
<1\end{array}$ & $\begin{array}{r}172 \\
1729\end{array}$ & 1233 \\
\hline Turbidity (NTU) & 1.3 & 1 & 1.4 & 3 & 35 & 24 & 20 & 5.2 & 10 & 16 & 12 & 2.7 & 680 & 1500 \\
\hline
\end{tabular}

(CONTINUED) 
APPENDIX $G$

Ion-Charge Balance Colculations, 1993

\begin{tabular}{|c|c|c|c|c|c|c|c|c|c|c|c|c|c|c|}
\hline \multirow{3}{*}{$\begin{array}{l}\text { Sampling Point } \\
\text { Location } \\
\text { Date Sampled }\end{array}$} & \multicolumn{2}{|c|}{ GW-756 } & \multicolumn{4}{|c|}{ GW-758 } & \multicolumn{4}{|c|}{ GW-759 } & \multicolumn{4}{|c|}{ GW-760 } \\
\hline & \multicolumn{2}{|c|}{ GRIDJ 1} & \multicolumn{4}{|c|}{ GRIDGI } & \multicolumn{4}{|c|}{ GRIDG1 } & \multicolumn{4}{|c|}{ GRIDG2 } \\
\hline & $08 / 03 / 93$ & $10 / 21 / 93$ & $01 / 25 / 93$ & $04 / 13 / 93$ & $09 / 12 / 93$ & $11 / 19 / 93$ & $\mid 01 / 26 / 93$ & 04/15/93 & $09 / 13 / 93$ & $11 / 19 / 93$ & $\mid 01 / 27 / 93$ & $04 / 16 / 93$ & $09 / 14 / 93$ & $11 / 30 / 93$ \\
\hline CATIONS (meg/L) & - & - & - & - & • & - & - & - & - & - & - & - & - & $\cdot$ \\
\hline Calcium & 1.40 & 1.45 & 3.39 & 3.14 & 3.59 & 3.64 & 1.60 & 3.29 & 3.74 & 3.44 & 5.49 & 4.99 & 6.49 & 5.49 \\
\hline Magnesium & 0.50 & 0.52 & 0.72 & 0.66 & 0.75 & 0.77 & 0.24 & 0.72 & 0.82 & 0.78 & 0.90 & 0.90 & 1.15 & 1.07 \\
\hline Potassium & 0.05 & 0.04 & 0.06 & 0.07 & 0.06 & 0.07 & 0.00 & 0.04 & 0.04 & 0.05 & 0.05 & 0.04 & 0.03 & 0.04 \\
\hline Sodium & 0.91 & 0.96 & 0.65 & 0.61 & 0.61 & 0.61 & 0.28 & 0.57 & 0.52 & 0.48 & 0.38 & 0.43 & 0.48 & 0.48 \\
\hline Hydrogen Ion & 0.00 & 0.00 & 0.00 & 0.00 & 0.00 & 0.00 & 0.00 & 0.00 & 0.00 & 0.00 & 0.00 & 0.00 & 0.00 & 0.00 \\
\hline - & & & & & & & & & & $\cdot$ & & $\cdot$ & - & - \\
\hline Total Cations & 2.86 & 2.96 & 4.83 & 4.48 & 5.01 & 5.09 & 2.12 & 4.62 & 5.12 & 4.75 & 6.83 & 6.37 & 8.15 & 7.07 \\
\hline ANIONS (meq/L) & • & & • & $\cdot$ & & $\bullet$ & $\cdot$ & $\dot{\bullet}$ & $\bullet$ & $\bullet$ & $\cdot$ & $\cdot$ & $\cdot$ & • \\
\hline Alkelinity-CO3 & 0.00 & 0.00 & 0.00 & 0.00 & 0.00 & 0.00 & 0.00 & 0.00 & 0.00 & 0.00 & 0.00 & 0.00 & 0.00 & 0.00 \\
\hline Alkalinity-HCO3 & 1.08 & 1.08 & 3.84 & 3.74 & 4.00 & 4.02 & 3.82 & 3.72 & 3.90 & 3.96 & 3.08 & 3.04 & 3.32 & 3.24 \\
\hline Chloride & 1.18 & 1.27 & 0.47 & 0.39 & 0.43 & 0.54 & 1.89 & 0.59 & 0.50 & 0.59 & 4.88 & 3.44 & 2.14 & 3.44 \\
\hline Fluoride & 0.00 & 0.00 & 0.01 & 0.01 & 0.01 & 0.01 & 0.01 & 0.01 & 0.00 & 0.01 & 0.00 & 0.00 & 0.00 & 0.00 \\
\hline Nitrate Nitrogen & 0.00 & 0.00 & 0.00 & 0.00 & 0.00 & 0.00 & 0.00 & 0.00 & 0.00 & 0.00 & 0.03 & 0.04 & 0.00 & 0.03 \\
\hline Sulfate & 0.75 & 0.67 & 0.41 & 0.42 & 0.42 & 0.33 & 0.42 & 0.35 & 0.44 & 0.37 & 0.44 & 0.44 & 0.44 & 0.37 \\
\hline Hydroxyl Ion & 0.00 & 0.00 & 0.00 & 0.00 & 0.00 & 0.00 & 0.00 & 0.00 & 0.00 & 0.00 & 0.00 & 0.00 & 0.00 & 0.00 \\
\hline - & & & • & & & & & $\cdot$ & & & & . & . & \\
\hline Total Anions & 3.01 & 3.02 & 4.72 & 4.55 & 4.86 & 4.90 & 6.13 & 4.67 & 4.84 & 4.93 & 8.42 & 6.96 & 5.90 & 7.08 \\
\hline CHARGE BALANCE (8) & -3 & -1 & 1 & -1 & $\dot{2}$ & $\dot{2}$ & -49 & -1 & 3 & -2 & -10 & -4 & 16 & -0 \\
\hline & . &. & $\dot{0}$ &. & $\cdot$ & $\therefore$ & & $\cdot$ & $\cdot$ & $\cdot$ & $\bullet$ &. & $\cdot$ & • \\
\hline TDS (mg/L) & 226 & 228 & 276 & 278 & 292 & 284 & 300 & 270 & 270 & 284 & $4 \epsilon 4$ & 468 & 566 & $\mathbf{4 4 0}$ \\
\hline TSS $(\mathrm{mg} / \mathrm{I})$ & 482 & 114 & 4 & 18 & 3 & 1 & 53 & 2 & 592 & 13 & 6 & 2 & 7 & $<1$ \\
\hline Turbidity (NTU) & 340 & 105 & 1.7 & 3 & 2.5 & 3.6 & 52 & 3.5 & 80 & 12 & 3.5 & 6 & 24 & 2.5 \\
\hline
\end{tabular}

(CONTINUED)

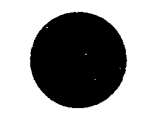


APPENDIX $G$

Ion-Charge Balance Calculations, 1993

\begin{tabular}{|c|c|c|c|c|c|c|c|c|c|c|c|c|c|c|}
\hline \multirow{3}{*}{$\begin{array}{l}\text { Sampling Point } \\
\text { Location } \\
\text { Date Sampled }\end{array}$} & \multicolumn{4}{|c|}{ Gw-761 } & \multicolumn{4}{|c|}{ GW-762 } & \multicolumn{4}{|c|}{ Gw-763 } & \multicolumn{2}{|c|}{ Gw-764 } \\
\hline & \multicolumn{4}{|c|}{ GRIDG2 } & \multicolumn{4}{|c|}{ GRIDJ3 } & \multicolumn{4}{|c|}{ GRIDJ3 } & \multicolumn{2}{|c|}{ GRIDB 1} \\
\hline & $01 / 26 / 93$ & $04 / 16 / 93$ & $09 / 13 / 93$ & $11 / 29 / 93$ & $01 / 23 / 93$ & $04 / 20 / 93$ & $08 / 04 / 93$ & $10 / 28 / 93$ & $01 / 25 / 93$ & $04 / 21 / 93$ & $08 / 05 / 93$ & $10 / 29 / 93$ & $01 / 23 / 93 \mid$ & $04 / 13 / 93$ \\
\hline CATIONS (meg/L) & - & - & $\cdot$ & - & - & - & - & - & - & $\cdot$ & - & - & $\cdot$ & - \\
\hline Calcium & 3.39 & 1.40 & 1.65 & 1.45 & 3.64 & 3.54 & 3.44 & 4.09 & 5.49 & 4.99 & 5.49 & 5.99 & 2.40 & 2.25 \\
\hline Magnesium & 0.72 & 0.21 & 0.24 & 0.21 & 1.89 & 1.81 & 1.89 & 2.14 & 1.07 & 0.99 & 0.99 & 1.07 & 0.49 & 0.45 \\
\hline Potassium & 0.06 & 0.02 & 0.00 & 0.00 & 0.09 & 0.08 & 0.12 & 0.10 & 0.02 & 0.02 & 0.05 & 0.03 & 0.05 & 0.06 \\
\hline Sodium & 0.65 & 0.25 & 0.27 & 0.27 & 0.42 & 0.35 & 0.35 & 0.39 & 0.48 & 0.41 & 0.37 & 0.41 & 1.13 & 1.04 \\
\hline Hydrogen Ion & 0.00 & 0.00 & 0.00 & 0.00 & 0.00 & 0.00 & 0.00 & 0.00 & 0.00 & 0.00 & 0.00 & 0.00 & 0.00 & 0.00 \\
\hline . & & & & & & & & & & & & & & \\
\hline Total Cations & 4.83 & 1.87 & 2.15 & 1.93 & 6.04 & 5.78 & 5.81 & 6.72 & 7.06 & 6.41 & 6.89 & 7.50 & 4.07 & 3.80 \\
\hline ANIONS (meq/L) & & &. & . &. &. & . & $\cdot$ &. &. & . &. &. & . \\
\hline Alkalinity-CO3 & 0.00 & 0.00 & 0.00 & 0.00 & 0.00 & 0.00 & 0.00 & 0.00 & 0.00 & 0.00 & 0.00 & 0.00 & 0.00 & 0.00 \\
\hline Alkalinity-HCO3 & 1.44 & 1.48 & 1.68 & 1.56 & 5.28 & 5.20 & 5.62 & 5.62 & 6.19 & 6.35 & 6.45 & 6.45 & 3.72 & 3.58 \\
\hline Chloride & 0.28 & 0.20 & 0.21 & 0.20 & 0.71 & 0.72 & 0.68 & 0.79 & 1.32 & 1.16 & 1.02 & 1.10 & 0.08 & 0.08 \\
\hline Fluoride & 0.01 & 0.01 & 0.00 & 0.01 & 0.01 & 0.00 & 0.00 & 0.00 & 0.01 & 0.02 & 0.01 & 0.01 & 0.01 & 0.01 \\
\hline Nitrate Nitrogen & 0.00 & 0.02 & 0.00 & 0.01 & 0.00 & 0.00 & 0.00 & 0.00 & 0.00 & 0.00 & 0.00 & 0.00 & 0.00 & 0.00 \\
\hline Sulfate & 0.27 & 0.33 & 0.25 & 0.23 & 0.33 & 0.25 & 0.37 & 0.27 & 0.00 & 0.15 & 0.17 & 0.04 & 0.21 & 0.27 \\
\hline Hydroxyl Ion & 0.00 & 0.00 & 0.00 & 0.00 & 0.00 & 0.00 & 0.00 & 0.00 & 0.00 & 0.00 & 0.00 & 0.00 & 0.00 & 0.00 \\
\hline - & & & & & & & & & & & & & & \\
\hline Total Anions & 1.99 & 2.04 & 2.13 & 2.01 & 6.31 & 6.17 & 6.67 & 6.68 & 7.53 & 7.67 & 7.65 & 7.61 & 4.01 & 3.93 \\
\hline CHARGE BALANCE (8) & 42 & -4 & $\dot{0}$ & -2 & -2 & -3 & -7 & 0 & -3 & -9 & -5 & -1 & $i$ & -2 \\
\hline & $\cdot$ & 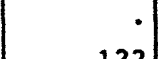 & $\dot{1}$ & $\dot{1}$ & $\cdot$ & $\cdot$ & 250 & 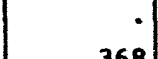 & $\dot{1}$ & $\cdot$ & $\cdot$ & $\cdot$ & 230 & \\
\hline $\operatorname{TDS}(m g / L)$ & 162 & 122 & 132 & 138 & 332 & 344 & 358 & 368 & 388 & 412 & 422 & 434 & 238 & 240 \\
\hline TSS $(m g / L)$ & 41 & 6 & 12 & 27 & 13 & 15 & 21 & 8 & 53 & 30 & 52 & 35 & 2 & 3 \\
\hline Turbidity (NTU) & 14 & 5.5 & 520 & 22 & 9.9 & 16 & 35 & 7.5 & 35 & 30 & 46 & 30 & 2.1 & 1.1 \\
\hline
\end{tabular}

(CONTINUED) 
APPBndIX 6

Ion-Charge Balance Calculations, 1993

\begin{tabular}{|c|c|c|c|c|c|c|c|c|c|c|c|c|c|c|}
\hline \multirow{3}{*}{$\begin{array}{l}\text { Sampling Foint } \\
\text { Location } \\
\text { Dete Sompled }\end{array}$} & \multicolumn{2}{|c|}{ Gw-764 } & \multicolumn{4}{|c|}{ Gw-765 } & \multicolumn{4}{|c|}{ Gw-766 } & \multicolumn{4}{|c|}{$G w-767$} \\
\hline & \multicolumn{2}{|c|}{ GRIDE1 } & \multicolumn{4}{|c|}{ GRIDE1 } & \multicolumn{4}{|c|}{ GRIDI2 } & \multicolumn{4}{|c|}{ GRIDI2 } \\
\hline & $09 / 12 / 93$ & $11 / 12 / 93$ & $01 / 25 / 93$ & $04 / 13 / 93$ & $09 / 12 / 93$ & $11 / 19 / 93$ & $02 / 01 / 93$ & $04 / 15 / 93$ & $08 / 03 / 93$ & $10 / 13 / 93$ & $02 / 02 / 93$ & $04 / 15 / 93$ & $08 / 04 / 93$ & $10 / 13 / 93$ \\
\hline CATIONS (meq/L) & $\cdot$ & - & - & $\cdot$ & - & $\cdot$ & $\cdot$ & $\cdot$ & - & $\cdot$ & - & $\cdot$ & $\cdot$ & - \\
\hline Calcium & 2.54 & 2.30 & 2.69 & 4.44 & 4.99 & 4.94 & 2.10 & 1.75 & 1.65 & 1.10 & 0.28 & 0.12 & 0.14 & 0.18 \\
\hline Magnesium & 0.52 & 0.49 & 0.29 & 0.72 & 0.82 & 0.81 & 0.53 & 0.53 & 0.58 & 0.39 & 0.12 & 0.09 & 0.10 & 0.09 \\
\hline Potassium & 0.04 & 0.05 & 0.06 & 0.00 & 0.00 & 0.03 & 0.13 & 0.10 & 0.09 & 0.14 & 0.02 & 0.00 & 0.03 & 0.02 \\
\hline Sodium & 1.17 & 1.17 & 0.22 & 0.35 & 0.39 & 0.42 & 0.43 & 0.52 & 0.52 & 0.57 & 0.13 & 0.10 & 0.12 & 0.13 \\
\hline Hydrogen Ion & 0.00 & 0.00 & 0.00 & 0.00 & 0.00 & 0.00 & 0.00 & 0.00 & 0.00 & 0.00 & 0.00 & 0.00 & 0.00 & 0.00 \\
\hline Total Cations & 4.28 & 4.01 & 3.26 & 5.51 & 6.20 & 6.20 & 3.19 & 2.89 & 2.84 & 2.19 & 0.55 & 0.31 & 0.40 & 0.42 \\
\hline ANIONS (meq/L) & - & - & $\cdot$ & $\cdot$ & $\dot{.}$ &. & $\cdot$ & 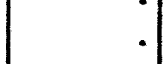 & - & $\cdot$ & & $\dot{.}$ & $\cdot$ & . \\
\hline Alkalinity-CO3 & 0.00 & 0.00 & 0.00 & 0.00 & 0.00 & 0.00 & 0.52 & 0.32 & 0.08 & 0.20 & 0.00 & 0.00 & 0.00 & 0.00 \\
\hline Alkalinity-HCO3 & 3.78 & 3.90 & 5.36 & 5.36 & 5.70 & 5.68 & 1.02 & 2.18 & 2.52 & 2.46 & 0.16 & 0.14 & 0.26 & 0.26 \\
\hline Chloride & 0.08 & 0.08 & 0.23 & 0.19 & 0.26 & 0.25 & 0.06 & 0.07 & 0.11 & 0.11 & 0.09 & 0.07 & 0.15 & 0.11 \\
\hline Fluoride & 0.01 & 0.01 & 0.01 & 0.01 & 0.01 & 0.01 & 0.00 & 0.01 & 0.00 & 0.01 & 0.00 & 0.00 & 0.00 & 0.00 \\
\hline Nitrate Nitrogen & 0.00 & 0.00 & 0.00 & 0.00 & 0.00 & 0.00 & 0.00 & 0.00 & 0.00 & 0.00 & 0.00 & 0.00 & 0.02 & 0.00 \\
\hline Sulfate & 0.20 & 0.21 & 0.25 & 0.27 & 0.21 & 0.21 & 0.22 & 0.18 & 0.29 & 0.19 & 0.09 & 0.07 & 0.19 & 0.06 \\
\hline Hydroxyl Ion & 0.00 & 0.00 & 0.00 & 0.00 & 0.00 & 0.00 & 0.03 & 0.01 & 0.00 & 0.00 & 0.00 & 0.00 & 0.00 & 0.00 \\
\hline - & & & & & & & & & & & & & & • \\
\hline Total Anions & 4.07 & 4.20 & 5.84 & 5.83 & 6.17 & 6.14 & 1.85 & 2.76 & 3.00 & 2.97 & 0.34 & 0.28 & 0.62 & 0.44 \\
\hline CHARGE BALANCE $(8)$ & $\dot{2}$ & -2 & -28 & -3 & $\dot{0}$ & $\dot{0}$ & 27 & $\dot{2}$ & -3 & -15 & 24 & $\dot{5}$ & -21 & -1 \\
\hline mos $1 \mathrm{mo} / \mathrm{T}$ & 246 & 254 & 434 & 364 & $\dot{362}$ & 380 & 128 & $2 \dot{166}$ & 182 & 202 & $\dot{54}$ & $\dot{32}$ & 56 & 64 \\
\hline $\begin{array}{l}\text { TDS }(m g / L) \\
\text { TSS }(m g / L)\end{array}$ & $\begin{array}{r}246 \\
5\end{array}$ & $\begin{array}{l}254 \\
113\end{array}$ & $\begin{array}{l}434 \\
349\end{array}$ & $\begin{array}{r}364 \\
97\end{array}$ & $\begin{array}{l}362 \\
264\end{array}$ & $\begin{array}{l}380 \\
158\end{array}$ & $\begin{array}{r}128 \\
64\end{array}$ & $\begin{array}{r}166 \\
1\end{array}$ & $\begin{array}{r}182 \\
2\end{array}$ & $\begin{array}{r}202 \\
3\end{array}$ & $\begin{array}{l}54 \\
33\end{array}$ & $\begin{array}{r}32 \\
7\end{array}$ & $\begin{array}{l}56 \\
<1\end{array}$ & $\begin{array}{r}64 \\
3\end{array}$ \\
\hline Turbidity (NTU) & 7.1 & 320 & 620 & 80 & 250 & 130 & 7.4 & 2 & 1.6 & 1.4 & 2.9 & 0.5 & 1.6 & 1.5 \\
\hline
\end{tabular}

(CONTINUED) 
APPENDIX G

Ion-Charge Balance Calculations, 1993

\begin{tabular}{|c|c|c|c|c|c|c|c|c|c|c|c|c|c|c|}
\hline \multirow{3}{*}{$\begin{array}{l}\text { Sampling Point } \\
\text { Location } \\
\text { Date Sampled }\end{array}$} & \multicolumn{4}{|c|}{ GW-768 } & \multicolumn{4}{|c|}{ GW-769 } & \multicolumn{4}{|c|}{ Gi-770 } & \multicolumn{2}{|c|}{$64-771$} \\
\hline & \multicolumn{4}{|c|}{ GRIDII } & \multicolumn{4}{|c|}{ GRIDG3 } & \multicolumn{4}{|c|}{ GRIDG3 } & \multicolumn{2}{|c|}{ GRIDCI } \\
\hline & $01 / 29 / 93$ & $04 / 07 / 93$ & $08 / 04 / 93$ & $10 / 13 / 93$ & $01 / 27 / 93$ & $04 / 19 / 93$ & $09 / 14 / 93$ & $11 / 30 / 93$ & $01 / 27 / 93$ & $04 / 19 / 93$ & $09 / 14 / 93$ & $11 / 29 / 93$ & $01 / 23 / 93$ & $04 / 12 / 93$ \\
\hline CATIONS (meq/L) & $\cdot$ & $\cdot$ & $\cdot$ & $\cdot$ & $\cdot$ & - & $\cdot$ & $\cdot$ & - & - & - & - & - & - \\
\hline Calcium & 1.80 & 1.80 & 1.95 & 1.80 & 3.69 & 3.39 & 3.94 & 3.79 & 3.49 & 2.15 & 2.99 & 2.69 & 3.64 & 3.49 \\
\hline Magnesium & 0.54 & 0.55 & 0.62 & 0.54 & 0.82 & 0.76 & 0.90 & 0.90 & 0.78 & 0.26 & 0.33 & 0.30 & 0.59 & 0.56 \\
\hline Potassium & 0.06 & 0.05 & 0.07 & 0.07 & 0.08 & 0.08 & 0.07 & 0.07 & 0.05 & 0.05 & 0.06 & 0.05 & 0.03 & 0.03 \\
\hline Sodi un & 2.87 & 2.91 & 3.00 & 3.00 & 0.28 & 0.26 & 0.29 & 0.30 & 0.52 & 0.20 & 0.23 & 0.25 & 0.24 & 0.22 \\
\hline Hydrogen Ion & 0.00 & 0.00 & 0.00 & 0.00 & 0.00 & 0.00 & 0.00 & 0.00 & 0.00 & 0.00 & 0.00 & 0.00 & 0.00 & 0.00 \\
\hline$\cdot$ & & & & $\cdot$ & & & & & & & $\cdot$ & $\cdot$ & $\cdot$ & \\
\hline Total Cations & 5.27 & 5.32 & 5.64 & 5.41 & 4.87 & 4.49 & 5.21 & 5.08 & 4.84 & 2.66 & 3.62 & 3.30 & 4.50 & 4.30 \\
\hline ANIONS (meq/L) & - & . & . & . & - & - & - & $\cdot$ & . & $\cdot$ & - & . & . & - \\
\hline Alkalinity-CO3 & 0.00 & 0.00 & 0.00 & 0.00 & 0.00 & 0.00 & 0.00 & 0.00 & 0.00 & 0.00 & 0.00 & 0.00 & 0.00 & 0.00 \\
\hline Alkal inity- $\mathrm{HCO} 3$ & 5.00 & 5.06 & 5.14 & 5.10 & 3.98 & 3.82 & 4.10 & 4.14 & 2.66 & 2.24 & 2.68 & 2.82 & 3.74 & 3.76 \\
\hline Chloride & 0.09 & 0.08 & 0.10 & 0.08 & 0.59 & 0.44 & 0.51 & 0.59 & 0.12 & 0.12 & 0.15 & 0.17 & 0.28 & 0.27 \\
\hline Fluoride & 0.02 & 0.02 & 0.02 & 0.02 & 0.00 & 0.00 & 0.00 & 0.00 & 0.01 & 0.01 & 0.01 & 0.01 & 0.01 & 0.01 \\
\hline Nitrate Nitrogen & 0.00 & 0.00 & 0.00 & 0.00 & 0.00 & 0.00 & 0.02 & 0.00 & 0.03 & 0.04 & 0.05 & 0.04 & 0.00 & 0.00 \\
\hline Sulfate & 0.27 & 0.27 & 0.35 & 0.25 & 0.40 & 0.38 & 0.44 & 0.35 & 0.46 & 0.45 & 0.52 & 0.42 & 0.34 & 0.28 \\
\hline Hydroxyl Ion & 0.00 & 0.00 & 0.00 & 0.00 & 0.00 & 0.00 & 0.00 & 0.00 & 0.00 & 0.00 & 0.00 & 0.00 & 0.00 & 0.00 \\
\hline - & & & & & & & & & & & & & & \\
\hline Total Anions & 5.38 & 5.43 & 5.61 & 5.45 & 4.97 & 4.63 & 5.06 & 5.08 & 3.28 & 2.86 & 3.41 & 3.45 & 4.36 & 4.31 \\
\hline$\overline{\text { CHARGE BALANCE (8) }}$ & -1 & -1 & 0 & -0 & -1 & -2 & $i$ & -0 & 19 & -4 & $\dot{3}$ & -2 & $\dot{2}$ & -0 \\
\hline & $\cdot$ & $\cdot$ & $\cdot$ & $\cdot$ & $\cdot$ & $\cdot$ & $\cdot$ & $\cdot$ & $\cdot$ & $\bullet$ & $\cdot$ & $\cdot$ & $\cdot$ & • \\
\hline TDS (mg/L) & 340 & 304 & 322 & 342 & 282 & 278 & 294 & 298 & 188 & 190 & 212 & 212 & 264 & 268 \\
\hline TSS (mg/L) & 5 & $<1$ & 2 & $<1$ & $<1$ & 1 & $<1$ & $<1$ & 8 & 41 & 2 & 10 & 3 & $<1$ \\
\hline Turbidity (NTU) & 3 & 1.2 & 1.9 & 1 & 1.1 & 0.9 & 2 & 1 & 7.6 & 8.5 & 11 & 13 & 1.9 & 1.9 \\
\hline
\end{tabular}

(CONTINUED) 
APPEADIX 6

Ion-Charge Balance Calculatione, 1993

\begin{tabular}{|c|c|c|c|c|c|c|c|c|c|c|c|c|c|c|}
\hline \multirow{3}{*}{$\begin{array}{l}\text { Sampling Point } \\
\text { Location } \\
\text { Date Sampled }\end{array}$} & \multicolumn{2}{|c|}{ Gw-771 } & \multicolumn{4}{|c|}{ Gw-772 } & \multicolumn{4}{|c|}{$6 n-773$} & \multicolumn{4}{|c|}{$60-774$} \\
\hline & \multicolumn{2}{|c|}{ GRIDC1 } & \multicolumn{4}{|c|}{ GRIDC1 } & \multicolumn{4}{|c|}{ Grinu2 } & \multicolumn{4}{|c|}{ EnIDIR } \\
\hline & $09 / 03 / 93$ & $11 / 11 / 93$ & $01 / 23 / 93 \mid$ & $04 / 12 / 93$ & $09 / 12 / 93$ & $11 / 12 / 93$ & $02 / 03 / 93$ & $04 / 20 / 93$ & $\mid 08 / 06 / 93$ & $10 / 15 / 93$ & $02 / 04 / 93$ & $04 / 20 / 93$ & $08 / 09 / 93$ & $10 / 15 / 93$ \\
\hline Carrons (meg/L) & - & - & - & - & - & - & - & $\cdot$ & - & - & - & - & - & • \\
\hline Calcium & 3.84 & 3.49 & 0.60 & 0.60 & 0.55 & 0.65 & 2.59 & 2.59 & 2.69 & 2.64 & 3.39 & 3.19 & 3.34 & 3.44 \\
\hline Magnesium & 0.62 & 0.58 & 0.44 & 0.50 & 0.41 & 0.42 & 0.56 & 0.53 & 0.59 & 0.53 & 0.50 & 0.47 & 0.52 & 0.51 \\
\hline Potassium & 0.03 & 0.03 & 0.03 & 0.00 & 0.04 & 0.03 & 0.03 & 0.00 & 0.03 & 0.03 & 0.05 & 0.05 & 0.08 & 0.05 \\
\hline Sodium & 0.23 & 0.23 & 0.91 & 0.96 & 0.87 & 0.91 & 0.20 & 0.18 & 0.20 & 0.19 & 0.19 & 0.19 & 0.20 & 0.19 \\
\hline Hydrogen Ion & 0.00 & 0.00 & 0.00 & 0.00 & 0.00 & 0.00 & 0.00 & 0.00 & 0.00 & 0.00 & 0.00 & 0.00 & 0.00 & 0.00 \\
\hline Total Cations & 4.72 & 4.35 & 1.99 & 2.06 & $\begin{array}{r}. \\
1.87\end{array}$ & 2.02 & 3.38 & 3.30 & 3.52 & 3.40 & 4.14 & 3.90 & 4.13 & 4.19 \\
\hline ANIONS (meq/L) & & & & & & $\cdot$ & $\cdot$ & $\cdot$ & $\cdot$ & $\cdot$ & $\cdot$ & & $\bullet$ & $\cdot$ \\
\hline Alkalinity-CO3 & 0.00 & 0.00 & 0.00 & 0.00 & 0.00 & 0.00 & 0.00 & 0.00 & 0.00 & 0.00 & 0.00 & 0.00 & 0.00 & 0.00 \\
\hline Alkalinity-HCO3 & 3.88 & 4.04 & 2.16 & 2.32 & 2.56 & 2.38 & 3.24 & 3.30 & 3.36 & 3.38 & 3.88 & 3.98 & 3.92 & 3.90 \\
\hline Chloride & 0.25 & 0.28 & 1.57 & 1.26 & 0.75 & 0.82 & 0.08 & 0.09 & 0.09 & 0.08 & 0.11 & 0.12 & 0.11 & 0.11 \\
\hline Fluoride & 0.01 & 0.00 & 0.01 & 0.01 & 0.01 & 0.01 & 0.00 & 0.01 & 0.00 & 0.01 & 0.00 & 0.01 & 0.00 & 0.01 \\
\hline Nitrate Mitrogen & 0.00 & 0.00 & 0.00 & 0.00 & 0.00 & 0.00 & 0.00 & 0.00 & 0.00 & 0.00 & 0.00 & 0.00 & 0.00 & 0.00 \\
\hline Sulfate & 0.31 & 0.24 & 0.10 & 0.15 & 0.05 & 0.04 & 0.11 & 0.18 & 0.23 & 0.10 & 0.21 & 0.21 & 0.27 & 0.12 \\
\hline Hydroxyl Ion & 0.00 & 0.00 & 0.00 & 0.00 & 0.00 & 0.00 & 0.00 & 0.00 & 0.00 & 0.00 & 0.00 & 0.00 & 0.00 & 0.00 \\
\hline - & & & & & & & & & & & $\cdot$ & & $\cdot$ & • \\
\hline Total Anions & 4.45 & 4.57 & 3.83 & 3.74 & 3.37 & 3.24 & 3.43 & 3.57 & 3.67 & 3.57 & 4.19 & 4.30 & 4.30 & 4.14 \\
\hline CHARGE BALAMCE (*) & 3 & -2 & -32 & -29 & -29 & -23 & -1 & -4 & -2 & -2 & -1 & -5 & -2 & $i$ \\
\hline $\operatorname{TDS}(\operatorname{mog}(u)$ & 266 & $\begin{array}{r}\cdot \\
306\end{array}$ & 190 & 214 & 194 & 204 & 210 & 204 & $21 \dot{0}$ & 234 & 316 & 222 & 256 & 260 \\
\hline $\begin{array}{l}\text { TDS }(m g / L) \\
\text { TSS }(m g / L)\end{array}$ & $\begin{array}{r}266 \\
6\end{array}$ & $\begin{array}{r}300 \\
31\end{array}$ & $\begin{array}{r}190 \\
7\end{array}$ & $\begin{array}{r}218 \\
1\end{array}$ & $\begin{array}{r}198 \\
11\end{array}$ & 4 & 2 & 2 & $<1$ & $<1$ & 22 & $\begin{array}{r}222 \\
10\end{array}$ & 20 & 5 \\
\hline Turbidity (NTU) & 4 & 4.1 & 17 & 8.8 & 6 & 20 & 1.4 & 0.9 & 2.7 & 1.5 & 150 & 150 & 14 & 4 \\
\hline
\end{tabular}

(CONTIMUED) 
APPEADIX G

Ion-Charge Balance Calculations, 1993

\begin{tabular}{|c|c|c|c|c|c|c|c|c|}
\hline \multirow{3}{*}{$\begin{array}{l}\text { Sampling Point } \\
\text { Location } \\
\text { Date Sampled }\end{array}$} & \multicolumn{4}{|c|}{ ow-775 } & \multicolumn{4}{|c|}{$0 w-776$} \\
\hline & \multicolumn{4}{|c|}{ GRIDA3 } & \multicolumn{4}{|c|}{ GRIDA3 } \\
\hline & $02 / 04 / 93$ & $04 / 20 / 93$ & $08 / 09 / 93$ & $10 / 15 / 93$ & $02 / 04 / 93$ & $04 / 20 / 93$ & $08 / 10 / 93$ & $10 / 18 / 93$ \\
\hline CATrons (meq/L) & - & - & - & - & - & - & - & - \\
\hline Calcium & 3.39 & 3.54 & 3.59 & 3.44 & 4.44 & 4.39 & 4.59 & 4.54 \\
\hline Magnesium & 0.48 & 0.49 & 0.54 & 0.54 & 0.41 & 0.36 & 0.42 & 0.39 \\
\hline Potassium & 0.10 & 0.05 & 0.06 & 0.06 & 0.08 & 0.07 & 0.08 & 0.07 \\
\hline Sodium & 0.17 & 0.16 & 0.17 & 0.18 & 0.48 & 0.48 & 0.48 & 0.43 \\
\hline Hydrogen Ion & 0.00 & 0.00 & 0.00 & 0.00 & 0.00 & 0.00 & 0.00 & 0.00 \\
\hline Total Cations & 4.14 & 4.24 & 4.37 & 4.23 & & $5.30^{\circ}$ & 5.57 & 5.43 \\
\hline Awrons (meq/L) & . & & 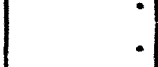 & & $\dot{\bullet}$ & & . & $\stackrel{.}{.}$ \\
\hline Alkalinity- $\mathrm{CO} 3$ & 0.00 & 0.00 & 0.00 & 0.00 & 0.00 & 0.00 & 0.00 & 0.00 \\
\hline Alkalinity-aCO3 & 3.14 & 3.18 & 3.28 & 3.36 & 3.96 & 3.82 & 3.94 & 3.96 \\
\hline Chloride & 0.62 & 0.76 & 0.62 & 2.16 & 0.76 & 1.17 & 0.82 & 1.06 \\
\hline Fluoride & 0.00 & 0.00 & 0.00 & 0.00 & 0.00 & 0.00 & 0.00 & 0.00 \\
\hline Nitrate Mitrogen & 0.02 & 0.03 & 0.03 & 0.03 & 0.08 & 0.08 & 0.09 & 0.10 \\
\hline Sulfate & 0.60 & 0.56 & 0.56 & 0.52 & 0.83 & 0.84 & 0.79 & 0.72 \\
\hline Hydroxyl Ion & 0.00 & 0.00 & 0.00 & 0.00 & 0.00 & 0.00 & 0.00 & 0.00 \\
\hline rotal Anions & 4.39 & 4.53 & 4.49 & 5.06 & 5.63 & 5.90 & 5.64 & 5.83 \\
\hline Chares Batance (8) & -3 & -3 & -1 & -9 & -2 & -5 & -1 & -4 \\
\hline $\operatorname{mos}(\operatorname{mg} / L)$ & $274^{\circ}$ & $254^{\circ}$ & 272 & 302 & 350 & 338 & 356 & 356 \\
\hline TSS $(m g / L)$ & 4 & 3 & $<1$ & $<1$ & 13 & 45 & 135 & 23 \\
\hline Turbidity (mo) & 2.8 & 1.8 & 1.4 & 0.7 & 12 & 22 & 28 & 18 \\
\hline
\end{tabular}


APPENDIX $\mathrm{H}$

TRACE METAL DATA SUMMARY 


\section{EXPLANATION}

\section{LOCATION:}

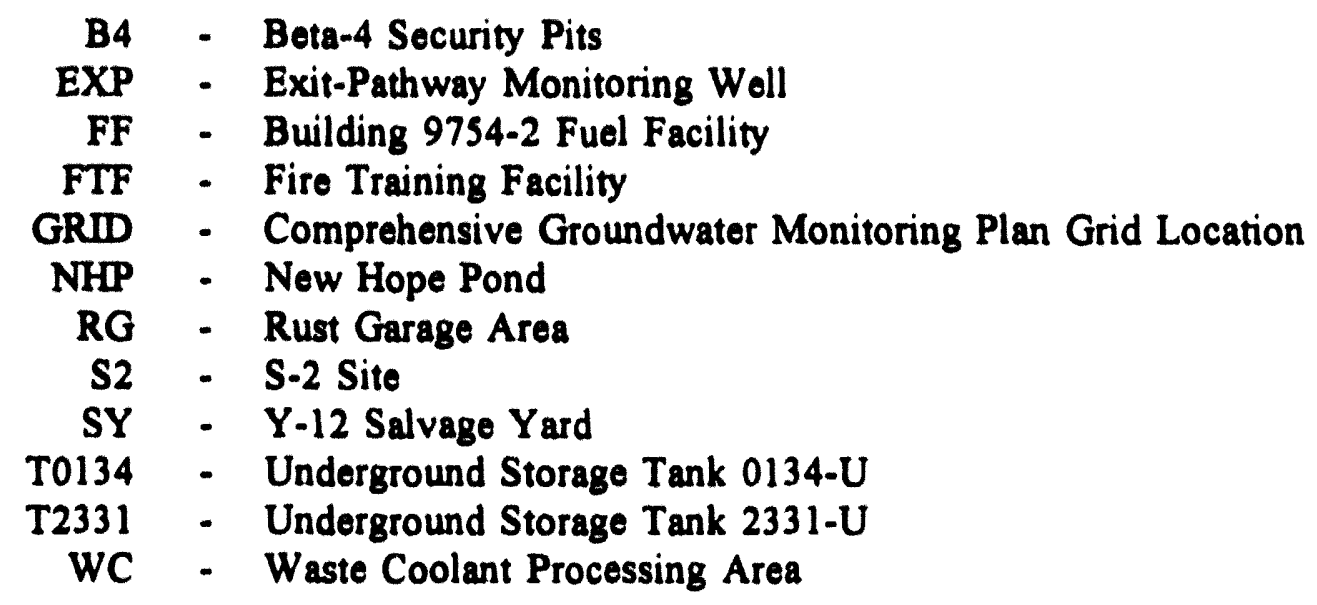

NOTES:

$\mathrm{mg} / \mathrm{L}$ - milligrams per Liter

- Concentration less than the Maximum Contaminant Level (MCL) or Screening Level

TOT - Total Concentration (Unfiltered Sample)

DIS - Dissolved Concentration (Filtered Sample)

TSS - Total Suspended Solids

NTU - Nephelometric Turbidity Unit

MCLs:

\section{Metal}

Barium (ICP)

Cadmium (AAS)

Chromium (AAS)

Lead (AAS)

Mercury (CVAA)
Maximum Contaminant Level (mg/L)

2.0

0.005

0.10

0.05

0.002

Because of inherent analytical interferences, ICP results for arsenic, selenium, and silver were not evaluated with respect to MCLs. Historical AAS results for these metals have shown only isolated and sporadic occurrences where concentrations exceeded MCLs. 


\section{EXPLANATION (cont'd)}

\section{SCREENING LEVELS:}

Metal (ICP)

Aluminum

Beryllium

Boron

Cobalt

Copper

Iron

Manganese

Molybdenum

Nickel

Strontium

Thorium

Uranium

Vanadium

Zinc

\section{Screening Level $(\mathrm{mg} / \mathrm{L})$}

8.3

0.0066

1.1

0.012

0.44

13.91

2.4

0.023

0.18

0.88

$<0.2$

0.01

0.064

0.14

Values represent the maximum total concentration (in $\mathrm{mg} / \mathrm{L}$ ) that would be expected in uncontaminated, unfiltered groundwater samples collected from monitoring wells located upgradient from waste-management sites at the Y-12 Plant. 


\section{APPENDIX H.1}

TRACE METAL CONCENTRATIONS THAT EXCEED MAXIMUM CONTAMINANT LEVELS 
Trace Metal Concentrations That Exceed Maximum Contaminant Levels, 1993

\begin{tabular}{|c|c|c|c|c|c|c|c|c|c|c|c|c|c|c|}
\hline \multirow{4}{*}{$\begin{array}{l}\text { Sampling Point } \\
\text { Location } \\
\text { Date Sampled }\end{array}$} & \multicolumn{6}{|c|}{ GW-169 } & \multicolumn{6}{|c|}{ GW-206 } & \multicolumn{2}{|c|}{$G W-251$} \\
\hline & \multicolumn{6}{|c|}{ EXP } & \multicolumn{6}{|c|}{$\mathbf{E X P}$} & \multirow{2}{*}{\multicolumn{2}{|c|}{$\frac{s 2}{01 / 20 / 93}$}} \\
\hline & \multicolumn{2}{|c|}{$01 / 23 / 93$} & \multicolumn{2}{|c|}{$08 / 19 / 93$} & \multicolumn{2}{|c|}{$11 / 01 / 93$} & \multicolumn{2}{|c|}{$05 / 06 / 93$} & \multicolumn{2}{|c|}{$08 / 12 / 93$} & \multicolumn{2}{|c|}{$10 / 27 / 93$} & & \\
\hline & TOT & DIS & TOT & DIS & TOT & DIS & TOT & DIS & TOT & DIS & TOT & DIS & TOT & DIS \\
\hline Barium & $\cdot$ & $\cdot$ & $\cdot$ & $\cdot$ & - & $\cdot$ & $\cdot$ & $\cdot$ & $\cdot$ & - & • & - & . & \\
\hline Cadmium (AAS) & $\cdot$ & $\cdot$ & - &. & $\cdot$ & $\cdot$ & $\cdot$ & - & $\cdot$ & $\cdot$ & $\cdot$ & . & 0.16 & 0.15 \\
\hline Chromium (AAS) & $\cdot$ & $\cdot$ & $\cdot$ & $\cdot$ & $\cdot$ & $\cdot$ & $\cdot$ & $\cdot$ & 0.11 & $<0.01$ & 0.2 & $<0.01$ & . & • \\
\hline Lead (AAS) & 0.15 & $<0.004$ & 0.18 & $<0.004$ & 0.091 & $<0.004$ & 0.14 & $<0.004$ & 0.13 & 0.064 & 0.12 & $<0.004$ & $\cdot$ & - \\
\hline Mercury (CVAA) & $\cdot$ & $\bullet$ & & $\cdot$ & $\cdot$ & $\cdot$ & $\cdot$ & $\cdot$ & $\cdot$ & $\cdot$ & $\cdot$ & . & $\cdot$ & - \\
\hline - & & $\cdot$ & & $\cdot$ & $\cdot$ & $\cdot$ & $\cdot$ & $\cdot$ & $\cdot$ & $\cdot$ & $\cdot$ & • & $\cdot$ & - \\
\hline TSS $(\mathrm{mg} / \mathrm{L})$ & 2100 & $\cdot$ & 1388 & $\cdot$ & 1960 & $\cdot$ & 1210 & $\cdot$ & 67 & $\cdot$ & 1187 & - & 119 & - \\
\hline Turbidity (NTU) & 2250 & $\cdot$ & 1200 & $\cdot$ & 46 & $\cdot$ & 750 & $\cdot$ & 1050 & $\cdot$ & 13 & $\cdot$ & 120 & - \\
\hline
\end{tabular}

\section{(CONTINUED)}

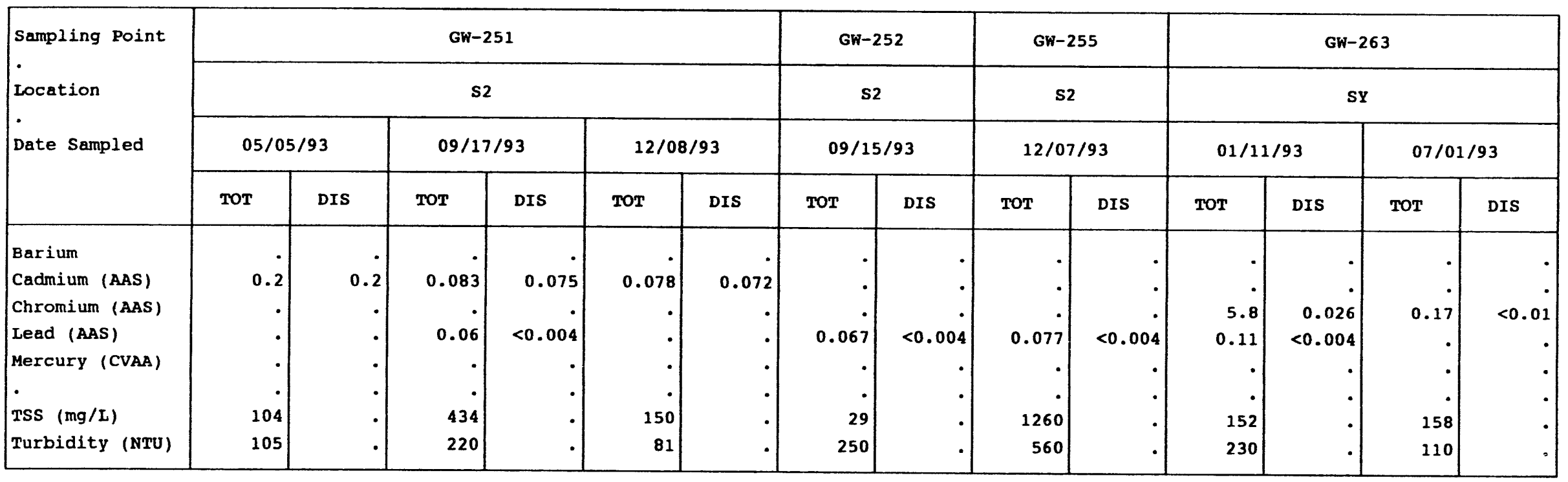

(CONTINUED) 
APPENDIX H.1

Trace Metal Concentrations That Exceed Maximum Contaminant Levels, 1993

\begin{tabular}{|c|c|c|c|c|c|c|c|c|c|c|c|c|c|c|}
\hline \multirow{4}{*}{$\begin{array}{l}\text { Sampling Point } \\
\text { Location } \\
\text { Date Sampled }\end{array}$} & \multicolumn{2}{|c|}{ GW-263 } & \multicolumn{8}{|c|}{$G W-284$} & \multicolumn{2}{|c|}{ GW-285 } & \multicolumn{2}{|c|}{ GW-337 } \\
\hline & \multicolumn{2}{|c|}{ sy } & \multicolumn{8}{|c|}{$\mathbf{F F}$} & \multirow{2}{*}{\multicolumn{2}{|c|}{$\frac{F F}{04 / 19 / 93}$}} & \multirow{2}{*}{\multicolumn{2}{|c|}{$\frac{\text { wC }}{01 / 21 / 93}$}} \\
\hline & \multicolumn{2}{|c|}{$12 / 07 / 93$} & \multicolumn{2}{|c|}{$02 / 02 / 93$} & \multicolumn{2}{|c|}{$04 / 16 / 93$} & \multicolumn{2}{|c|}{$08 / 05 / 93$} & \multicolumn{2}{|c|}{$10 / 14 / 93$} & & & & \\
\hline & TOT & DIS & TOT & DIS & TOT & DIS & TOT & DIS & TOT & DIS & TOT & DIS & TOT & DIS \\
\hline Barium & & • & $\cdot$ & $\cdot$ & $\cdot$ & $\cdot$ & $\cdot$ & $\cdot$ & $\cdot$ & $\cdot$ & $\cdot$ & $\cdot$ & $\cdot$ & \\
\hline Cadmium (AAS) & 0.0067 & 0.0025 & . & $\cdot$ & - & $\cdot$ & $\cdot$ & $\cdot$ & $\cdot$ & $\cdot$ & $\cdot$ & . & 0.0069 & 0.0051 \\
\hline Chromium (AAS) & $\cdot$ & • & 0.64 & $<0.01$ & 0.16 & $<0.01$ & 1.9 & $<0.01$ & 0.12 & $<0.01$ & 0.76 & $<0.01$ & $\cdot$ & • \\
\hline Lead (AAS) & $\cdot$ & - & $\cdot$ & $\cdot$ & $\cdot$ & $\cdot$ & $\cdot$ & $\cdot$ & $\cdot$ & $\cdot$ & $\cdot$ & $\cdot$ & $\cdot$ & • \\
\hline Mercury (CVAA) & • & - & $\cdot$ & $\cdot$ & $\cdot$ & $\cdot$ & $\cdot$ & $\cdot$ & $\cdot$ & $\cdot$ & $\cdot$ & $\cdot$ & $\cdot$ & • \\
\hline - & $\cdot$ & - & $\cdot$ & $\cdot$ & $\cdot$ & $\cdot$ & $\cdot$ & $\cdot$ & $\cdot$ & $\cdot$ & $\cdot$ & $\cdot$ & -1 & • \\
\hline TSS (mg/L) & 7 & - & 18 & - & 22 & $\cdot$ & 48 & $\cdot$ & 20 & • & 201 & $\cdot$ & 26 & • \\
\hline Turbidity (NTU) & 20 & - & 8.6 & $\cdot$ & 15 & $\cdot$ & 25 & $\cdot$ & 15 & • & 280 & • & 14 & - \\
\hline
\end{tabular}

(CONTINUED)

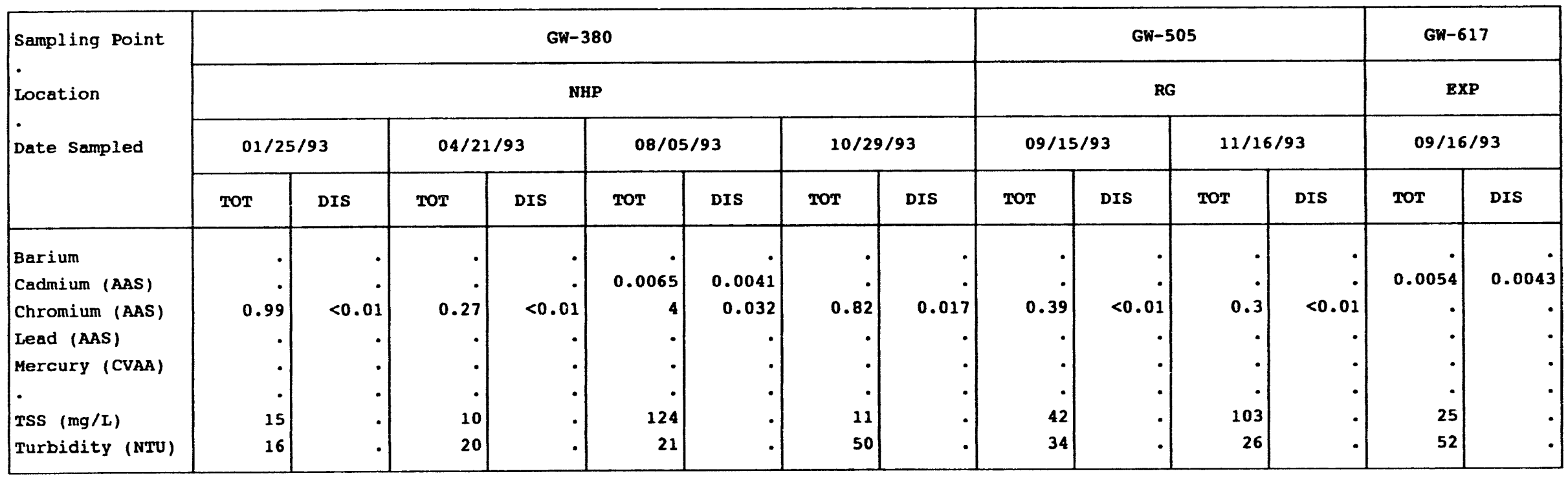

(CONTI 
Trace Metal Concentrations That Exceed Maximum Contaminant Levels, 1993

\begin{tabular}{|c|c|c|c|c|c|c|c|c|c|c|c|c|c|c|}
\hline \multirow{4}{*}{$\begin{array}{l}\text { Sampling Point } \\
\text { Location } \\
\text { Date Sampled }\end{array}$} & \multicolumn{8}{|c|}{$G W-618$} & \multicolumn{6}{|c|}{ GW-633 } \\
\hline & \multicolumn{8}{|c|}{$\operatorname{EXP}$} & \multicolumn{6}{|c|}{$\mathbf{R G}$} \\
\hline & \multicolumn{2}{|c|}{$01 / 12 / 93$} & \multicolumn{2}{|c|}{$05 / 05 / 93$} & \multicolumn{2}{|c|}{$09 / 16 / 93$} & \multicolumn{2}{|c|}{$12 / 08 / 93$} & \multicolumn{2}{|c|}{$03 / 03 / 93$} & \multicolumn{2}{|c|}{$06 / 21 / 93$} & \multicolumn{2}{|c|}{$09 / 20 / 93$} \\
\hline & TOT & DIS & TOT & DIS & TOT & DIS & TOT & DIS & TOT & DIS & TOT & DIS & TOT & DIS \\
\hline Barium & - & - & - & - & - & - & - & - & 33 & 37 & 22 & 21 & 22 & 24 \\
\hline Cadmium (AAS) & 0.014 & $<0.002$ & 0.02 & $<0.002$ & 0.03 & $<0.002$ & 0.02 & 0.0053 & . &. & . & . & . & • \\
\hline Chromium (AAS) & . & • & • & • & • & • & • & • & $\cdot$ &. & . & . & . & . \\
\hline Lead (AAS) & . & - & . & - & - & - & . & - & . &. & . & - & . & . \\
\hline Mercury (CVAA) & - & - & - & - & - & - & - & - & . &. & . & - & . & • \\
\hline & $\cdot$ & - & $\cdot$ & - & - & - & $\cdot$ & $\cdot$ & $\cdot$ & $\cdot$ & $\cdot$ & - & . & • \\
\hline TSS (mg/L) & $<1$ & - & $<1$ & - & 1 & - & $<1$ & - & 219 &. & 169 & - & 133 & - \\
\hline Turbidity (NTU) & 1.6 & - & 3.3 & - & 1.5 & - & 1 & . & 220 & $\cdot$ & 95 & . & 70 & • \\
\hline
\end{tabular}

(CONTINUED)

\begin{tabular}{|c|c|c|c|c|c|c|c|c|c|c|c|c|c|c|}
\hline \multirow{4}{*}{$\begin{array}{l}\text { Sampling Point } \\
\text { Location } \\
\text { Date Sampled }\end{array}$} & \multicolumn{6}{|c|}{ GW-659 } & \multicolumn{2}{|c|}{ GW-707 } & \multicolumn{2}{|c|}{$G W-746$} & \multicolumn{2}{|c|}{ GW-756 } & \multicolumn{2}{|c|}{ GW-760 } \\
\hline & \multicolumn{6}{|c|}{$\mathbf{F F}$} & \multicolumn{2}{|c|}{ T2331 } & \multicolumn{2}{|c|}{ GRIDK1 } & \multicolumn{2}{|c|}{ GRIDJ1 } & \multicolumn{2}{|c|}{ GRIDG2 } \\
\hline & \multicolumn{2}{|c|}{$06 / 18 / 93$} & \multicolumn{2}{|c|}{$09 / 22 / 93$} & \multicolumn{2}{|c|}{$11 / 15 / 93$} & \multicolumn{2}{|c|}{$06 / 23 / 93$} & \multicolumn{2}{|c|}{$08 / 05 / 93$} & \multicolumn{2}{|c|}{$01 / 15 / 93$} & \multicolumn{2}{|c|}{$01 / 27 / 93$} \\
\hline & TOT & DIS & TOT & DIS & TOT & DIS & TOT & DIS & TOT & DIS & TOT & DIS & TOT & DIS \\
\hline Barium & & & . & - & . & - &. &. &. & . &. &. & . & . \\
\hline Cadmium (AAS) & 0.0067 & $<0.002$ & $\cdot$ & $\cdot$ & • & • & 0.0061 & $<0.002$ &. & $\cdot$ & . &. &. & . \\
\hline Chromium (AAS) & 0.18 & $<0.01$ & 0.13 & 0.011 & 0.24 & $<0.01$ & $\cdot$ & $\cdot$ & 0.2 & $<0.01$ & 0.12 & $<0.01$ & 0.15 & $<0.01$ \\
\hline Lead (AAS) & • & • & . & • & . & • & .1 &. &. &. &. &. &. & . \\
\hline Mercury (CVAA) & $\cdot$ & - & $\cdot$ & $\cdot$ & . & - & $\cdot$ &. &. &. &. &. &. & . \\
\hline • & $\cdot$ & • & $\cdot$ & $\cdot$ & $\cdot$ & - & $\cdot$ & $\cdot$ & $\cdot$ & $\cdot$ & $\cdot$ & $\cdot$ &. & - \\
\hline TSS (mg/L) & 234 & - & 253 & $\cdot$ & 743 & • & 1 & $\cdot$ & 414 & $\cdot$ & 1729 &. & 6 & . \\
\hline Turbidity (NTU) & 340 & . & 680 & $\cdot$ & 750 & - & 2.9 &. & 230 & . & 680 &. & 3.5 & . \\
\hline
\end{tabular}

(CONTINUED) 
APPENDIX H.1

Trace Metal Concentrations That Exceed Maximum Contaminant Levels, 1993

\begin{tabular}{|c|c|c|c|c|c|c|c|c|}
\hline \multirow{4}{*}{$\begin{array}{l}\text { Sampling Point } \\
\text { Location } \\
\text { Date Sampled }\end{array}$} & \multicolumn{4}{|c|}{$G W-760$} & \multicolumn{4}{|c|}{ GW-776 } \\
\hline & \multicolumn{4}{|c|}{ GRIDG2 } & \multicolumn{4}{|c|}{ GRIDH3 } \\
\hline & \multicolumn{2}{|c|}{$04 / 16 / 93$} & \multicolumn{2}{|c|}{$09 / 14 / 93$} & \multicolumn{2}{|c|}{$04 / 20 / 93$} & \multicolumn{2}{|c|}{$10 / 18 / 93$} \\
\hline & TOT & DIS & TOT & DIS & TOT & DIS & TOT & DIS \\
\hline Barium & & & • & . & . & . & . & • \\
\hline Cadmium (AAS) & 0.0087 & 0.014 & 0.02 & 0.013 & - & - & - & • \\
\hline Chromium (AAS) & $\cdot$ & • & $\cdot$ & • & 0.16 & $<0.01$ & 0.31 & $<0.01$ \\
\hline Lead (AAS) & $\cdot$ & - & . & . & • & • & . & • \\
\hline Mercury (CVAA) & $\cdot$ & - & $\cdot$ & $\cdot$ & $\cdot$ & $\cdot$ & $\cdot$ & • \\
\hline - & • & - & - & . & . & . & - & . \\
\hline TSS (mg/L) & 2 & $\cdot$ & 7 & $\cdot$ & 45 & . & 13 & . \\
\hline Turbidity (NTU) & 6 &. & 24 & $\cdot$ & 22 & . & 18 & . \\
\hline
\end{tabular}




\section{APPENDIX H.2 \\ TRACE METAL CONCENTRATIONS THAT EXCEED SCREENING LEVELS}


APPENDIX H. 2

Trace Metal Concentrations That Exceed

Screening Level6, 1993

\begin{tabular}{|c|c|c|c|c|c|c|c|c|c|c|c|c|c|c|}
\hline \multirow{4}{*}{$\begin{array}{l}\text { Sampling Point } \\
\text { Location } \\
\text { Date Sampled }\end{array}$} & \multicolumn{8}{|c|}{$G W-169$} & \multicolumn{2}{|c|}{ GW-191 } & \multicolumn{2}{|c|}{ GW-192 } & \multicolumn{2}{|c|}{ Gw-193 } \\
\hline & \multicolumn{8}{|c|}{ EXP } & \multirow{2}{*}{\multicolumn{2}{|c|}{$\frac{B 4}{04 / 12 / 93}$}} & \multirow{2}{*}{\multicolumn{2}{|c|}{$\frac{B 4}{01 / 14 / 93}$}} & \multirow{2}{*}{\multicolumn{2}{|c|}{$\frac{T 2331}{03 / 11 / 93}$}} \\
\hline & \multicolumn{2}{|c|}{$01 / 23 / 93$} & \multicolumn{2}{|c|}{$05 / 21 / 93$} & \multicolumn{2}{|c|}{$08 / 19 / 93$} & \multicolumn{2}{|c|}{$11 / 01 / 93$} & & & & & & \\
\hline & TOT & DIS & TOT & DIS & TOT & DIS & TOT & DIS & TOT & DIS & TOT & Drs & TOT & DIS \\
\hline Aluminum & 75 & 0.023 & 14 & 0.041 & 71 & $<0.02$ & 29 & 0.045 & 42 & 0.051 & . & - & . & . \\
\hline Beryllium & 0.0092 & $<0.0003$ & $\cdot$ & • & 0.0092 & $<0.0003$ & $\cdot$ & • & $\cdot$ & & . & - & . & - \\
\hline Boron & & & $\cdot$ & $\cdot$ & $\cdot$ & & $\cdot$ & $\cdot$ & . & $\cdot$ & $\cdot$ & - & - & - \\
\hline Cobalt & 0.052 & $<0 . \operatorname{cus}$ & 0.015 & $<0.005$ & 0.054 & $<0.005$ & 0.031 & $<0.005$ & 0.03 & $<0.005$ & - & - & . & - \\
\hline Copper & - & $\cdot$ & • & & $\cdot$ & & . & . & . &. &. & . & . & . \\
\hline Iron & 79 & 0.014 & 24 & $<0.005$ & 83 & $<0.005$ & 45 & 0.11 & 56 & 0.084 & 18 & 2.7 & . & • \\
\hline Manganese & 3.1 & 0.0026 & $\cdot$ & $\cdot$ & 3.3 & 0.0012 & $\cdot$ & $\cdot$ & $\cdot$ & • & $\cdot$ & $\cdot$ & . & . \\
\hline Mol ybdenum & . & . & . & . & $\cdot$ & &. &. & . & - & . & . & . & . \\
\hline Nickel & - & $\cdot$ & $\cdot$ & $\cdot$ & $\cdot$ & $\cdot$ & - & . & $\cdot$ & . & . & . & . & . \\
\hline Strontium & - & $\cdot$ & $\cdot$ & - & $\cdot$ & - & - & . & 0.96 & 0.48 & . & . & . & . \\
\hline Thorium & - & $\cdot$ & $\cdot$ & $\cdot$ & $\cdot$ &. & • & - & • & • & - & - & $\cdot$ & \\
\hline Uranium & & $\cdot$ & $\cdot$ & $\cdot$ & & $\cdot$ & - & - & - &. & - & . & 0.017 & 0.018 \\
\hline Vanadium & 0.13 & $<0.005$ & • & $\cdot$ & 0.13 & $<0.005$ & • & • & $\cdot$ & $\cdot$ & - & . & . & \\
\hline zinc & 0.36 & 0.0056 & . &. & 0.36 & 0.0082 & 0.21 & 0.0046 & 0.2 & 0.0066 & - & $\cdot$ &. & • \\
\hline isen & $\cdot$ & $\cdot$ & $\cdot$ & $\cdot$ & & $\cdot$ & & $\cdot$ & & $\cdot$ & $\cdot$ & $\cdot$ & $\cdot$ & $\cdot$ \\
\hline TSS (mg/L) & 2100 & $\cdot$ & 404 & $\cdot$ & 1388 & $\cdot$ & 1960 & $\cdot$ & 2162 & - & 61 & - & 35 & • \\
\hline Turbidity (NTU) & 2250 & $\cdot$ & 20 & $\cdot$ & 1200 & $\cdot$ & 46 & $\cdot$ & 560 & $\cdot$ & 66 & - & 52 & - \\
\hline
\end{tabular}

(CONTINUED) 
APPENDIX H.2

Trace Metal Concentrations That Exceed

Screening Levels, 1993

\begin{tabular}{|c|c|c|c|c|c|c|c|c|c|c|c|c|c|c|}
\hline \multirow{4}{*}{$\begin{array}{l}\text { Sampling Point } \\
\text { Location } \\
\text { Date Sampled }\end{array}$} & \multicolumn{6}{|c|}{ GW-193 } & \multicolumn{2}{|c|}{ GW-194 } & \multicolumn{6}{|c|}{ GW-195 } \\
\hline & \multicolumn{6}{|c|}{ T2331 } & \multirow{2}{*}{\multicolumn{2}{|c|}{$\frac{B 4}{01 / 13 / 93}$}} & \multicolumn{6}{|c|}{ B4 } \\
\hline & \multicolumn{2}{|c|}{$06 / 22 / 93$} & \multicolumn{2}{|c|}{$09 / 23 / 93$} & \multicolumn{2}{|c|}{$11 / 18 / 93$} & & & \multicolumn{2}{|c|}{$01 / 13 / 93$} & \multicolumn{2}{|c|}{$04 / 13 / 93$} & \multicolumn{2}{|c|}{$07 / 08 / 93$} \\
\hline & тот & DIS & TOT & DIS & TOT & DIS & TOT & DIS & TOT & DIS & TOT & DIS & TOT & DIS \\
\hline Aluminum & 9.3 & $<0.02$ & - & $\cdot 1$ & $\cdot$ & $\cdot$ & $\cdot$ & - & 14 & 0.097 & 13) & 0.055 & 12 & $<0.02$ \\
\hline Beryllium & $\cdot$ &. & $\cdot$ & $\cdot 1$ & $\cdot$ & $\cdot$ & $\cdot$ & $\cdot$ & $\cdot$ & $\cdot$ & $\cdot$ & $\cdot$ & $\cdot$ & • \\
\hline Boron &. &. &. &. & $\cdot$ & $\cdot$ & $\cdot$ & $\cdot$ & $\cdot 1$ & $\cdot$ & $\cdot$ & $\cdot$ & $\cdot$ & • \\
\hline Cobalt &. &. &. & $\cdot$ &. & $\cdot$ & $\cdot 1$ & $\cdot$ & 0.02 & $<0.005$ & 0.013 & 0.0053 & 0.015 & $<0.005$ \\
\hline Copper & $\cdot$ & $\cdot$ & $\cdot$ & $\cdot$ & $\cdot$ & $\cdot$ & $\cdot$ & $\cdot$ & $\cdot$ & $\cdot$ & $\cdot$ & $\cdot$ & $\cdot$ & • \\
\hline Iron & $\cdot$ &. & $\cdot$ & . & $\cdot$ & $\cdot$ &. & $\cdot$ & 24 & 0.12 & 17 & 0.04 & 18 & 0.014 \\
\hline Manganese & $\cdot$ & $\cdot$ & $\cdot$ & $\cdot$ & $\cdot$ & $\cdot$ & $\cdot$ & $\cdot$ & $\cdot$ & $\cdot$ & $\cdot$ & $\cdot$ & $\cdot$ & • \\
\hline Molybdenum & $\cdot$ & $\cdot$ & $\cdot$ & $\cdot$ & $\cdot$ & $\cdot$ & $\cdot$ & $\cdot$ & $\cdot$ & $\cdot$ & $\cdot$ & $\cdot$ & $\cdot$ & • \\
\hline Nickel & $\cdot$ & $\cdot$ & $\cdot$ & $\cdot$ & $\cdot$ & $\cdot$ & $\cdot$ & $\cdot$ & $\cdot$ & $\cdot$ & $\cdot$ & $\cdot$ & $\cdot$ & • \\
\hline Strontium & $\cdot 1$ & $\cdot$ & $\cdot$ & $\cdot$ & $\cdot$ & $\cdot$ & $\cdot$ & $\cdot$ & $\cdot$ & $\cdot$ & $\cdot$ & $\cdot$ & $\cdot$ & • \\
\hline Thorium & $\cdot$ &. & $\cdot$ & $\cdot$ & $\cdot$ & $\cdot$ & $\cdot$ &. & $\cdot$ & $\cdot$ & $\cdot$ & $\cdot$ & $\cdot 1$ & • \\
\hline Uranium & $\cdot$ & $\cdot 1$ & 0.086 & 0.077 & 0.08 & 0.08 & 0.09 & 0.09 & $\cdot$ & $\cdot$ &. & $\cdot$ &. & • \\
\hline Vanadium & $\cdot$ & $\cdot$ & $\cdot$ & $\cdot$ & $\cdot$ & $\cdot$ & $\cdot$ & $\cdot$ & $\cdot$ & $\cdot$ & $\cdot 1$ & $\cdot$ & $\cdot$ & • \\
\hline zinc & $\cdot$ & $\cdot$ & $\cdot$ & $\cdot$ & $\cdot$ & $\cdot$ & $\cdot$ & $\cdot$ & $\cdot$ & $\cdot$ & $\cdot$ & $\cdot$ & $\cdot$ & • \\
\hline - & $\cdot$ & $\cdot$ & $\cdot$ & $\cdot$ & $\cdot$ & $\cdot$ & $\cdot$ & $\cdot$ &. & $\cdot$ & $\cdot$ & $\cdot$ & $\cdot$ & • \\
\hline TSS (mg/L) & 830 & $\cdot$ & 23 & $\cdot$ & 98 & $\cdot$ & 102 & $\cdot$ & 524 & - & 527 & $\cdot$ & 510 & • \\
\hline Turbidity (NTU) & 440 & $\cdot$ & 22 & $\cdot$ & 29 & $\cdot$ & 34 & $\cdot$ & 240 & $\cdot$ & 220 & $\cdot$ & 240 & . \\
\hline
\end{tabular}

(CONTINUED) 
Trace Metal Concentrations That Exceed

Screening Levels, 1993

\begin{tabular}{|c|c|c|c|c|c|c|c|c|c|c|c|c|c|c|}
\hline \multirow{4}{*}{$\begin{array}{l}\text { Sampling Point } \\
\text { Location } \\
\text { - } \\
\text { Date Sampled }\end{array}$} & \multicolumn{2}{|c|}{ GW-195 } & \multicolumn{6}{|c|}{ GW-199 } & \multicolumn{6}{|c|}{$G w-204$} \\
\hline & \multirow{2}{*}{\multicolumn{2}{|c|}{$\frac{B 4}{12 / 09 / 93}$}} & \multicolumn{6}{|c|}{ GRIDI 1} & \multicolumn{6}{|c|}{20134} \\
\hline & & & \multicolumn{2}{|c|}{$01 / 29 / 93$} & \multicolumn{2}{|c|}{$08 / 02 / 93$} & \multicolumn{2}{|c|}{$10 / 12 / 93$} & \multicolumn{2}{|c|}{$03 / 11 / 93$} & \multicolumn{2}{|c|}{$06 / 22 / 93$} & \multicolumn{2}{|c|}{$09 / 23 / 93$} \\
\hline & TOT & DIS & TOT & DIS & TOT & DIS & Tor & Drs & TOT & DIS & TOT & DIS & Tor & DIS \\
\hline Aluminum & 9.3 & $<0.02$ & . & . &. & . & . & . & . & $\cdot$ &. &. &. & . \\
\hline Beryllium & . & . & - & - & $\cdot$ & . & - & . &. &. & . & . & . & - \\
\hline Boron &. & $\cdot$ & - & - &. &. & - & $\cdot$ & $\cdot$ & $\cdot$ & . & . & . & - \\
\hline Cobalt & .1 & $\cdot$ & . & - & $\cdot$ &. & - & . & $\cdot$ & $\cdot$ & $\cdot$ & $\cdot$ & $\cdot$ & - \\
\hline Copper & . & & . & - & $\cdot$ & . & - & . & $\cdot$ &. & . & . & . & - \\
\hline Iron & 14 & 0.0095 & . & • &. &. & . & . & $\cdot$ & $\cdot$ & . & . & . & - \\
\hline Manganese & . & $\cdot$ & 4 & 1.5 & 3.4 & 1.8 & - & $\cdot$ &. &. & $\cdot$ & . & . & - \\
\hline Molybdenum & $\cdot$ & $\cdot$ & - & • & $\cdot$ & $\cdot$ & - & $\cdot$ & $\cdot$ & $\cdot$ &. & - & - & - \\
\hline Nickel &. &. & - & . &. &. & . & . & $\cdot$ &. &. & . & - & . \\
\hline strontium & $\cdot$ & - & . & - & $\cdot$ &. & - & $\cdot$ &. & $\cdot$ &. & - & $\cdot$ & - \\
\hline Thorium &. & . & - & - & $\cdot 1$ & $\cdot 1$ & - & . & $\cdot$ & $\cdot$ & $\cdot$ & • & . & • \\
\hline Uranium & . & . & . & . & $\cdot$ & $\cdot$ & . & $\cdot$ & 0.075 & 0.068 & 0.21 & 0.185 & 0.126 & 0.12 \\
\hline Vanedium & - & . & . & - & $\cdot$ & $\cdot 1$ & . & $\cdot$ & $\cdot 1$ & $\cdot$ & $\cdot$ & $\cdot$ &. & • \\
\hline Zinc & • & - & . & . & $\cdot$ & $\cdot$ & 0.17 & 0.18 & $\cdot$ & $\cdot$ &. & $\cdot$ & $\cdot$ & - \\
\hline - & • & . & • & . & $\cdot$ &. & . & $\cdot$ & . & $\cdot$ &. & . & $\cdot$ & - \\
\hline TSS (mg/L) & 292 & . & 628 & - & 14 & $\cdot$ & 16 & $\cdot$ & 25 &. & 3 & - & 26 & - \\
\hline Turbidity (NTU) & 360 & . & 3900 & - & 25 & - & 6.5 & $\cdot 1$ & 24 & $\cdot$ & 3.3 & . & 6.5 & - \\
\hline
\end{tabular}

(CONTINUED) 
APPENDIX H.2

Trace Metal Concentrations That Exceed

Screening Levels, 1993

\begin{tabular}{|c|c|c|c|c|c|c|c|c|c|c|c|c|c|c|}
\hline \multirow{4}{*}{$\begin{array}{l}\text { Sampling Point } \\
\text { Location } \\
\text { Date Sampled }\end{array}$} & \multicolumn{2}{|c|}{ GW-204 } & \multicolumn{8}{|c|}{ GW-206 } & \multicolumn{4}{|c|}{ Gn-208 } \\
\hline & \multicolumn{2}{|c|}{ T0134 } & \multicolumn{8}{|c|}{$\operatorname{EXP}$} & \multicolumn{4}{|c|}{$\exp$} \\
\hline & \multicolumn{2}{|c|}{$11 / 16 / 93$} & \multicolumn{2}{|c|}{$01 / 19 / 93$} & \multicolumn{2}{|c|}{$05 / 06 / 93$} & \multicolumn{2}{|c|}{$08 / 12 / 93$} & \multicolumn{2}{|c|}{$10 / 27 / 93$} & \multicolumn{2}{|c|}{$01 / 19 / 93$} & \multicolumn{2}{|c|}{$05 / 06 / 93$} \\
\hline & TOT & DIS & TOT & DIS & TOT & DIS & TOT & DIS & TOT & DIS & TOT & DIS & TOT & DIS \\
\hline Aluminum & - & - & - & - & 22 & 0.29 & 49 & 2.3 & 42 & 1 & - & $\cdot$ & $\cdot$ & - \\
\hline Beryllium & - & - & $\cdot$ & - & - & $\cdot$ & • & $\cdot 1$ & $\cdot$ & $\cdot$ & - & . & $\cdot$ & • \\
\hline Boron & - & - & $\cdot$ & - & • & • & • & $\cdot$ & • & . & . & - & . & . \\
\hline Cobalt & - & - & $\cdot 1$ & - & 0.058 & 0.0069 & 0.074 & 0.025 & 0.089 & $<0.005$ & - & $\cdot$ & - & - \\
\hline Copper & - & . &. & • & • & . & • &. & • & $\cdot$ & . & . & . & - \\
\hline Iron & - & - & 23 & 5.9 & 78 & 3.6 & 120 & 47 & 130 & 19 & - & $\cdot$ & . & - \\
\hline Manganese & - & - & $\cdot$ & • & 4.8 & 1.1 & 7.5 & 5.7 & 7.8 & 1.3 & $\cdot$ & $\cdot$ & • & - \\
\hline Mol ybdenum & - & - & - & - & $<0.05$ & $<0.01$ & $\cdot$ & $\cdot$ & $<0.1$ & $<0.01$ & - & $\cdot$ & - & - \\
\hline Nickel & - & - & $\cdot 1$ & - & • & $\cdot$ & $\cdot$ &. & $\cdot$ & - & $\cdot$ & $\cdot$ & $\cdot$ & • \\
\hline Strontium & - & - & $\cdot$ & - & • & $\cdot$ & - & $\cdot$ & .1 & $\cdot$ & 1.3 & 1.3 & 1.3 & 1.3 \\
\hline Thorium & • & - & $\cdot$ & - & $<1$ & $<0.2$ & . &. & $<2$ & $<0.2$ & $\cdot$ & $\cdot$ & $\cdot$ & • \\
\hline Uranium & 0.094 & 0.108 &. & . & • & . & . & $\cdot$ & & & - & . & - & . \\
\hline Vanadium & • & • & $\cdot$ & - & • & $\cdot$ & 0.1 & 0.021 & 0.087 & $<0.005$ & • & • & • & • \\
\hline zinc & - & - & $\cdot$ & - & 0.15 & 0.053 & 0.15 & 0.027 & 0.21 & 0.0067 & 1.9 & 1.4 & 1.2 & 0.91 \\
\hline - & . & - &. & - & & $\cdot$ & $\cdot$ & $\cdot$ & $\cdot$ & $\bullet$ & $\cdot$ & $\cdot$ & $\cdot$ & • \\
\hline TSS (mg/L) & 250 & - & 2750 & - & 1210 &. & 67 & $\cdot 1$ & 1187 & • & 2 & $\cdot$ & $<1$ & - \\
\hline Turbidity (NTU) & 110 & - & 1900 & . & 750 &. & 1050 & . & 13 & . & 4.5 & - & 2.2 & • \\
\hline
\end{tabular}

(CONTINUED) 
APPENDIX $\mathrm{H.2}$

Trace Metel Concentrations That Exceed

Screening Levels, 1993

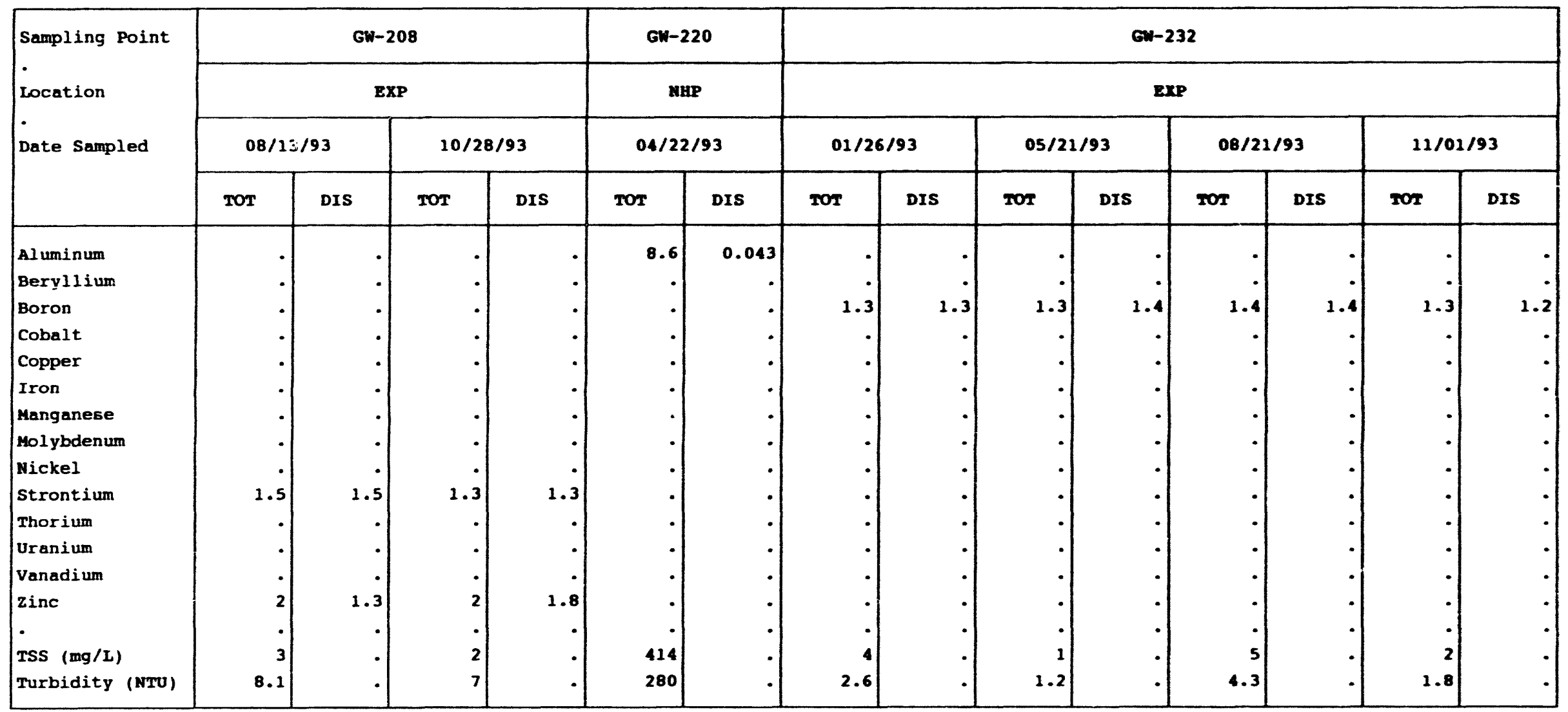

(CONTINUED) 
APPEXDIX $\mathrm{A.2}$

Trace Metal Concentrations that Exceed

Screening Levels, 1993

\begin{tabular}{|c|c|c|c|c|c|c|c|c|c|c|c|c|c|c|}
\hline \multirow{4}{*}{$\begin{array}{l}\text { Sampling Point } \\
\text { Location } \\
\text { Date Sampled }\end{array}$} & \multicolumn{8}{|c|}{ Gw-239 } & \multicolumn{6}{|c|}{$c u-251$} \\
\hline & \multicolumn{8}{|c|}{ MHP } & \multicolumn{6}{|c|}{ s2 } \\
\hline & \multicolumn{2}{|c|}{$01 / 23 / 93$} & \multicolumn{2}{|c|}{$05 / 13 / 93$} & \multicolumn{2}{|c|}{$08 / 18 / 93$} & \multicolumn{2}{|c|}{$10 / 30 / 93$} & \multicolumn{2}{|c|}{$01 / 20 / 93$} & \multicolumn{2}{|c|}{$05 / 05 / 93$} & \multicolumn{2}{|c|}{$09 / 17 / 93$} \\
\hline & TOT & DIS & TOT & DIS & TOT & DIs & TOT & DIs & TOT & DIS & Tor & DIs & $\operatorname{sot}$ & DIS \\
\hline Aluminum & $\cdot$ & - & - & - & - & - & - & - & - & - & • & - & 17 & 0.033 \\
\hline Beryllium &. & . & . & • & • & . & • & • & - & - & - & - & . & • \\
\hline Boron & 1.5 & 1.5 & 1.5 & 1.5 & 1.6 & 1.6 & 1.5 & 1.5 & • & • & • & • & . & • \\
\hline Cobalt & . & • & . & . & • & • & • & • & 0.035 & 0.032 & 0.039 & 0.036 & 0.023 & 0.0063 \\
\hline Copper & . & . & . &. & . & . & . & . & 0.58 & 0.42 & 0.65 & 0.46 & 0.95 & 0.17 \\
\hline Iron & . &. & . & - & - & . & 16 & 0.37 & • & • & • & • & 23 & $<0.005$ \\
\hline Manganese & . & - & - & - & - & . & • & • & 4.7 & 4.6 & 5.4 & 5.2 & 2.9 & 1.8 \\
\hline Mol ybdenum & . &. & - & - & • & $\cdot$ & - & - & • & • & - & • & - & • \\
\hline wickel & . & - & - & . & - & - & • & - & $\cdot$ & - & - & - & - & . \\
\hline Strontium & . & $\cdot$ & - & - & - & $\cdot$ & - & - & . & • & • & - & - & • \\
\hline Thorium & . & . & . & . & - & . & - & - & - & - & . & - & - & - \\
\hline Uranium & . & . & . & - & - & . & - & - & . & - & . & - &. & • \\
\hline Vanadiun & . & . & . & . & . & . & - & - & - & - & - & - & - & . \\
\hline zinc & - & $\cdot$ & - & $\cdot$ & - & $\cdot$ & - & - & - & - & - & - & • & • \\
\hline . & - & . & - & . & • & - & - & - & • & - & • & - & • & • \\
\hline TSS (mg/L) & 4 & . & 3 & . & $<1$ & . & 21 & - & 119 & - & 104 & - & 434 & • \\
\hline Turbidity (NTU) & 5.6 & . & 3.9 & - & 7.9 & - & 10 & - & 120 & - & 105 & - & 220 & . \\
\hline
\end{tabular}

(CONTINUED) 
Irace hetal concentrations That exceed

Screening Levels, 1993

\begin{tabular}{|c|c|c|c|c|c|c|c|c|c|c|c|c|c|c|}
\hline \multirow{4}{*}{$\begin{array}{l}\text { Sampling Point } \\
\text { Location } \\
\text { Date Sampled }\end{array}$} & \multicolumn{2}{|c|}{$G+251$} & \multicolumn{2}{|c|}{$G w-252$} & \multicolumn{2}{|c|}{ GW-255 } & \multicolumn{8}{|c|}{ GW-263 } \\
\hline & \multicolumn{2}{|c|}{ s2 } & \multicolumn{2}{|c|}{ s2 } & \multirow{2}{*}{\multicolumn{2}{|c|}{$12 / 07 / 93$}} & \multicolumn{8}{|c|}{$\mathbf{s y}$} \\
\hline & \multicolumn{2}{|c|}{$12 / 08 / 93$} & \multicolumn{2}{|c|}{$09 / 15 / 93$} & & & \multicolumn{2}{|c|}{$01 / 11 / 93$} & \multicolumn{2}{|c|}{$04 / 08 / 93$} & \multicolumn{2}{|c|}{$07 / 01 / 93$} & \multicolumn{2}{|c|}{$12 / 07 / 93$} \\
\hline & Tor & DIS & TOT & DIS & Tor & DIS & Tor & DIS & TOT & DIS & TOT & DIS & TOT & DIS \\
\hline Aluminum & $\cdot$ & - & 16 & $<0.02$ & 29 & $<0.02$ & 13 & 0.063 & - & - & $\cdot$ & - & $\cdot$ & - \\
\hline Beryllium & - & - & $\cdot$ & • & - & • & $\cdot$ & • & $\cdot$ & - & - & - & $\cdot$ & - \\
\hline Boron & & • & $\cdot$ & - & $\cdot$ & $\cdot$ & $\cdot 1$ & $\bullet$ & $\cdot$ & $\cdot$ & $\cdot$ & $\cdot$ & $\cdot$ & • \\
\hline Cobalt & 0.015 & 0.013 & $\cdot$ & - & 0.03 & $<0.005$ & 0.048 & 0.0091 &. & - & - & - &. & - \\
\hline Copper & $\cdot$ & • & $\cdot$ & • & $\cdot$ & • & $\cdot$ & $\bullet$ & $\cdot$ & - & • & - & $\cdot$ & - \\
\hline Iron & . & - & 20 & $<0.005$ & 39 & $<0.005$ & 37 & 0.15 &. & - & $\cdot$ & - & $\cdot$ & - \\
\hline Manganese & - & - & $\cdot$ & • & 2.8 & $<0.001$ & & & $\cdot$ & - & $\cdot$ & - &. & - \\
\hline Mol ybdenum & - & - & $\cdot$ & - & $\cdot$ & $\cdot$ & 0.051 & $<0.01$ & $\cdot$ & $\cdot$ & $\cdot$ & $\cdot$ & $\cdot$ & • \\
\hline Hickel & - & - & $\cdot$ & - & - & . & 2.1 & 0.96 & 0.33 & 0.33 & 0.36 & 0.15 & 0.28 & 0.26 \\
\hline Strontium & - & - & $\cdot$ & - & • & $\cdot$ & $\cdot$ & $\cdot$ & $\cdot$ & $\cdot$ & $\cdot$ & $\cdot$ & $\cdot$ & • \\
\hline Thorium & . & - & $\cdot$ & - & $\cdot$ & $\cdot$ & $\cdot$ & $\cdot$ & $\cdot$ & $\cdot$ & $\cdot$ & - & $\cdot$ & - \\
\hline Oranium & $\cdot$ & - & $\cdot$ & - & $\cdot$ & $\cdot$ & $\cdot$ & - & $\cdot$ & $\cdot$ & $\cdot$ & $\cdot$ & $\cdot$ & - \\
\hline Vanadium & $\cdot$ & - &. & • & & $\cdot$ &. & . &. & - & $\cdot$ & - &. & - \\
\hline zinc & - & - & 0.15 & 0.037 & 0.19 & $<0.002$ & $\cdot$ & • & $\cdot$ & $\cdot$ & $\cdot$ & - & $\cdot 1$ & - \\
\hline . & • & - & $\cdot$ & . & &. & $\cdot 1$ & • &. & $\cdot$ & $\cdot$ & - &. & - \\
\hline TSS $(\mathrm{mg} / \mathrm{L})$ & 150 & - & 29 & - & 1260 & . & 152 & $\cdot$ & 7 & - & 258 & - & 7 & - \\
\hline Turbidity (NTU) & 81 & - & 250 & - & 560 & $\cdot$ & 230 & - & 6 & $\cdot$ & 110 & - & 20 & - \\
\hline
\end{tabular}

(CONTINUED) 
APPEMDIX $\mathrm{H} .2$

Trace Metal Concentrations that Exceed

Screenlng Levels, 1993

\begin{tabular}{|c|c|c|c|c|c|c|c|c|c|c|c|c|c|c|}
\hline \multirow{4}{*}{$\begin{array}{l}\text { Sampling Point } \\
\text { Location } \\
\text { Date Sampled }\end{array}$} & \multicolumn{2}{|c|}{$G W-281$} & \multicolumn{8}{|c|}{ Gw-284 } & \multicolumn{4}{|c|}{$60-285$} \\
\hline & \multicolumn{2}{|c|}{$\mathbf{F F}$} & \multicolumn{8}{|c|}{$\mathbf{F}$} & \multicolumn{4}{|c|}{$\boldsymbol{F}$} \\
\hline & \multicolumn{2}{|c|}{$08 / 04 / 93$} & \multicolumn{2}{|c|}{$02 / 02 / 93$} & \multicolumn{2}{|c|}{$04 / 16 / 93$} & \multicolumn{2}{|c|}{$08 / 05 / 93$} & \multicolumn{2}{|c|}{$10 / 14 / 93$} & \multicolumn{2}{|c|}{$02 / 02 / 93$} & \multicolumn{2}{|c|}{$04 / 19 / 93$} \\
\hline & TOT & DIS & TOT & DIS & TOT & DIS & TOT & DIS & TOT & Drs & TOT & DIs & Tor & Drs \\
\hline Aluminum & 18 & $<0.02$ & $\cdot$ & - & $\cdot$ & - & 14 & $<0.02$ & - & $\cdot$ & $\cdot$ & $\cdot$ & $\cdot$ & $\cdot$ \\
\hline Beryllium & $\cdot$ & $\cdot$ & $\cdot$ & - & - & - & $\cdot$ & $\cdot$ & $\cdot$ & - & $\cdot$ & $\cdot$ & $\cdot$ & - \\
\hline Boron & & & $\cdot$ & - & - & . & $\cdot$ & $\cdot$ & $\cdot$ & $\cdot$ & - & $\cdot$ & $\cdot$ & - \\
\hline Cobalt & 0.014 & $<0.005$ & $\cdot 1$ & - & • & - & 0.064 & 0.016 & $\cdot$ & - & $\cdot$ & $\cdot$ & $\cdot$ & • \\
\hline Copper & $\cdot$ & $\cdot$ & $\cdot$ & $\cdot$ & $\cdot$ & - & $\cdot$ & $\cdot$ & $\cdot$ & $\cdot$ & $\cdot$ & $\cdot$ & $\cdot$ & - \\
\hline Iron & 23 & 1.8 & $\cdot$ & - & - & • & 35 & 0.0063 & $\cdot$ & - & $\cdot$ & $\cdot$ & $\cdot$ & - \\
\hline Hanganese & $\cdot$ & • & $\cdot$ & - & - & - & $\cdot$ & $\cdot$ & $\cdot$ & - & $\cdot$ & $\cdot 1$ & $\cdot$ & • \\
\hline Mol ybdenum & $\cdot$ & • &. & • & $\cdot$ & $\cdot$ & $\cdot$ & $\cdot$ & $\cdot$ & $\cdot$ & • & $\cdot$ & $\cdot$ & • \\
\hline Nickel &. & . & 0.96 & 0.92 & 0.8 & 1.4 & 1.8 & 0.98 & 0.59 & 0.96 & 0.58 & 0.58 & 0.91 & 0.58 \\
\hline Strontium & $\cdot$ & • & $\cdot$ & $\cdot$ & $\cdot$ & $\cdot$ & $\cdot$ & $\cdot$ & $\cdot$ & $\cdot$ & $\cdot$ & $\cdot$ & $\cdot$ & • \\
\hline Thorium & $\cdot 1$ & - & $\cdot 1$ & - & - & - & $\cdot 1$ & $\cdot$ & $\cdot$ & $\cdot$ & - & $\cdot$ & $\cdot$ & • \\
\hline Uranium & $\cdot$ & - & $\cdot$ & . & . & - & $\cdot$ & $\cdot$ & $\cdot$ & $\cdot$ & $\cdot$ & $\cdot$ & $\cdot$ & $\cdot$ \\
\hline Vanadiun & & & $\cdot$ & - & - & $\cdot$ & $\cdot$ & $\cdot$ & $\cdot$ & $\cdot$ & - & $\cdot$ & $\cdot$ & • \\
\hline zinc & 0.63 & 0.009 & $\cdot$ & - & $\cdot$ & - & $\cdot$ & $\cdot$ & • & - & $\cdot$ & $\cdot$ & $\cdot$ & - \\
\hline - & $\cdot$ & $\cdot$ & $\cdot$ & - & • & - & $\cdot$ & $\cdot$ & $\cdot$ & $\cdot$ & $\cdot$ & $\cdot$ & $\cdot$ & $\cdot$ \\
\hline TSS $(\mathrm{mg} / L)$ & 120 & • & 18 & - & 22 & - & 48 & $\cdot$ & 20 & $\cdot$ & 15 &. & 201 & • \\
\hline Turbidity (NTU) & 40 & - & 8.6 & - & 15 & - & 25 & $\cdot$ & 15 & $\cdot$ & 25 & $\cdot$ & 280 & • \\
\hline
\end{tabular}

(CONTINUED) 
APPEADIX 日. 2

Irace Metal Concentrations That Exceed

Screening Levels, 1993

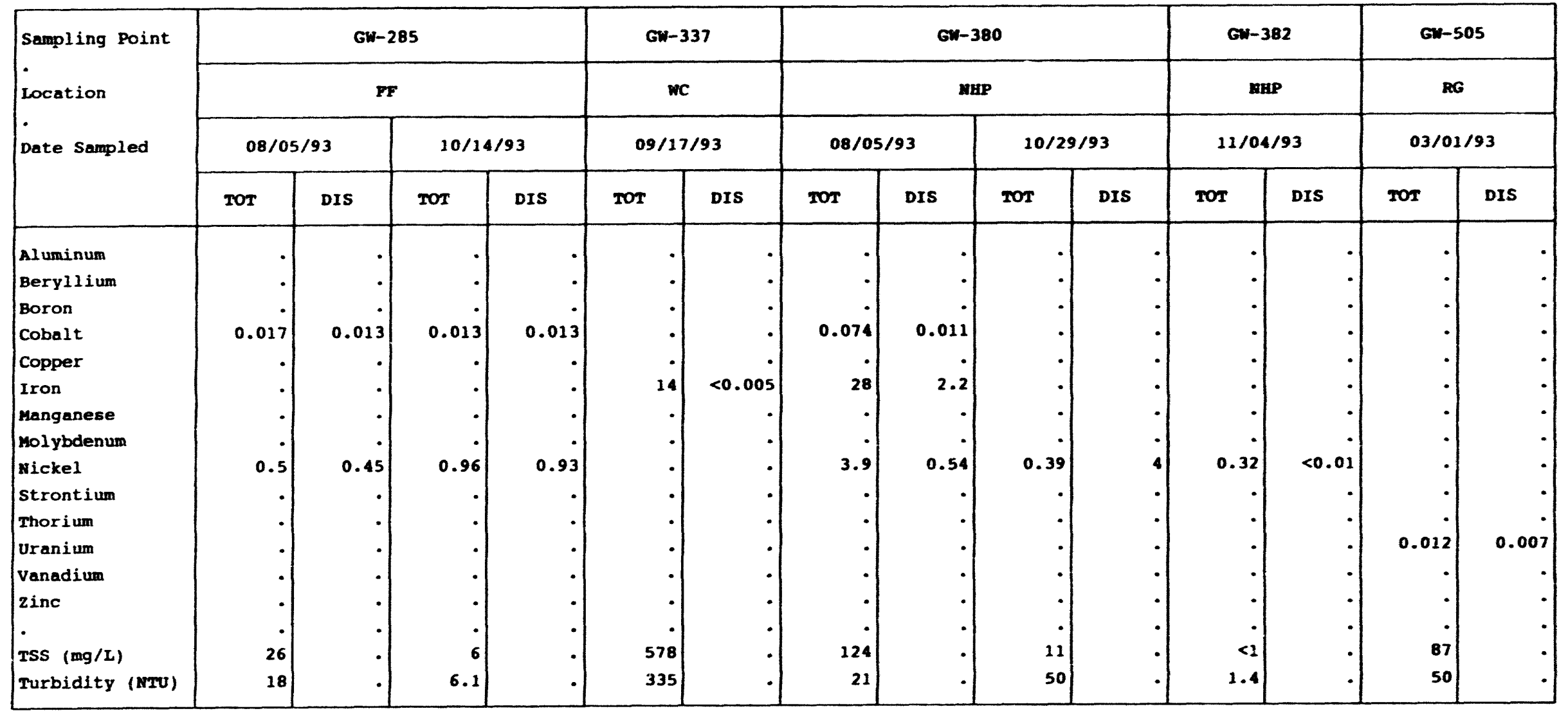

(CONTINUED) 
APPENDIX H. 2

Trace Metal Concentrations That Exceed

Screening Levels, 1993

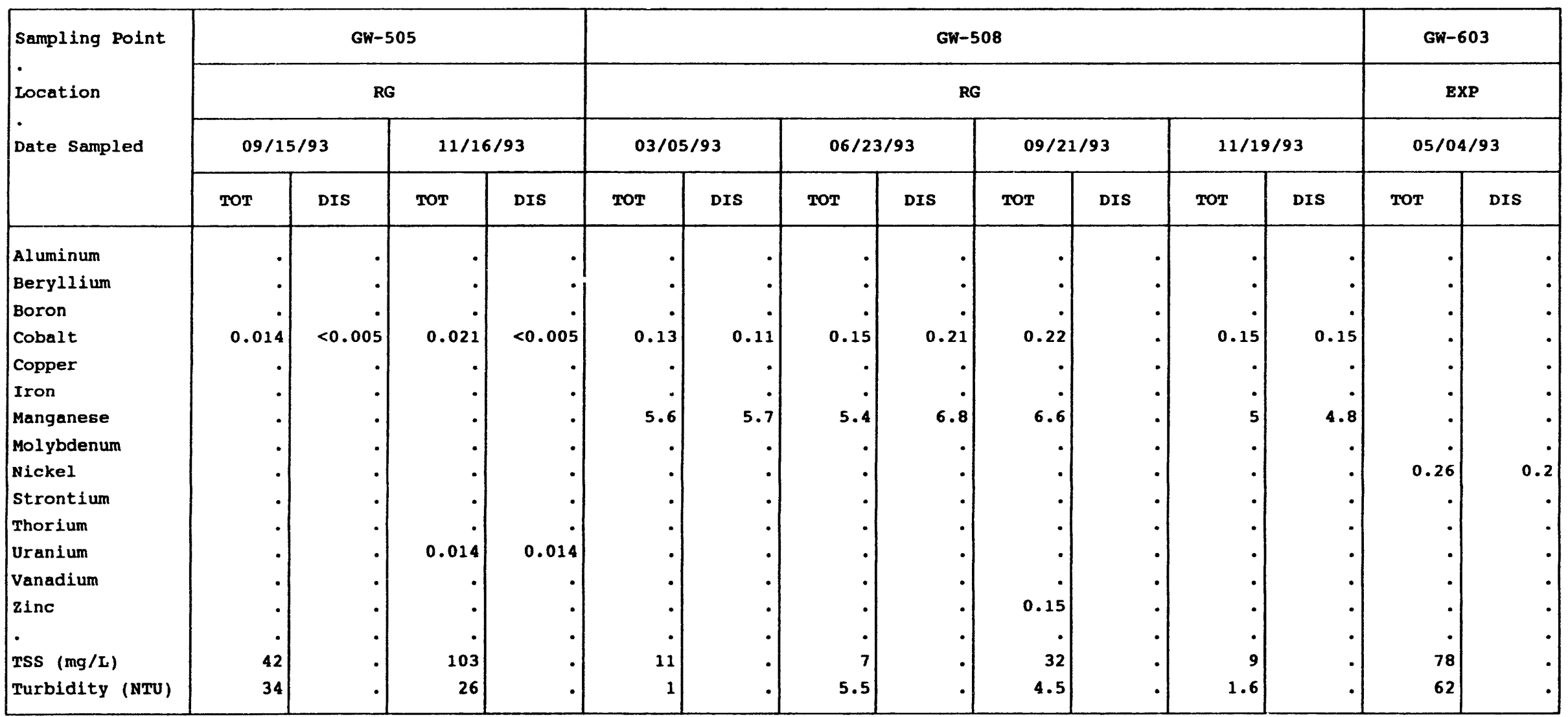

(CONTINUED) 
APPENDIX H.2

Trace Metal Concentrations That Exceed

Screening Levels, 1993

\begin{tabular}{|c|c|c|c|c|c|c|c|c|c|c|c|c|c|c|}
\hline \multirow{4}{*}{$\begin{array}{l}\text { Sampling Point } \\
\text { - } \\
\text { Location } \\
\text { Date Sampled }\end{array}$} & \multicolumn{6}{|c|}{$G W-631$} & \multicolumn{8}{|c|}{$G W-632$} \\
\hline & \multicolumn{6}{|c|}{ RG } & \multicolumn{8}{|c|}{ RG } \\
\hline & \multicolumn{2}{|c|}{$06 / 18 / 93$} & \multicolumn{2}{|c|}{$09 / 16 / 93$} & \multicolumn{2}{|c|}{$11 / 17 / 93$} & \multicolumn{2}{|c|}{$03 / 03 / 93$} & \multicolumn{2}{|c|}{$06 / 21 / 93$} & \multicolumn{2}{|c|}{$09 / 16 / 93$} & \multicolumn{2}{|c|}{$11 / 18 / 93$} \\
\hline & TOT & DIS & TOT & Drs & TOT & DIS & TOT & DIS & TOT & DIS & TOT & DIS & TOT & Drs \\
\hline Aluminum & - & $\cdot$ & $\cdot$ & - & - & . & . & . & . & . &. & . & . & . \\
\hline Beryllium & $\cdot$ & $\cdot$ & $\cdot$ & . & $\cdot$ & - &. & $\cdot$ & $\cdot$ &. & $\cdot$ & . &. & . \\
\hline Boron & $\cdot$ & $\cdot$ & $\cdot$ & $\cdot$ & $\cdot$ & $\bullet$ & $\cdot$ & $\cdot$ & $\cdot$ &. &. & $\cdot$ & $\cdot$ & - \\
\hline Cobalt & 0.028 & 0.032 & 0.034 & 0.034 & 0.025 & 0.028 & 0.071 & 0.044 & 0.08 & 0.08 & 0.11 & 0.055 & 0.084 & 0.11 \\
\hline Copper & $\cdot$ & $\cdot$ & $\cdot$ & $\cdot$ & $\cdot$ & . & $\cdot$ & $\cdot$ & . &. & $\cdot$ & $\cdot$ & $\cdot$ & • \\
\hline Iron & $\cdot$ & $\cdot$ & $\cdot$ & $\cdot$ & $\cdot$ & $\cdot$ &. & $\cdot$ & $\cdot$ & . &. & . &. & - \\
\hline Manganese & $\cdot$ & $\cdot$ & $\cdot$ & $\cdot$ & $\cdot$ & $\cdot$ & 6.1 & 5.4 & 6.5 & 6.2 & 5.8 & 4.4 & 5.2 & 4.9 \\
\hline Mol ybdenum & $\cdot$ & $\cdot$ & $\cdot$ & $\cdot$ & $\cdot$ & $\cdot$ & $\cdot$ & $\cdot$ & $\cdot$ & $\cdot$ & $\cdot$ &. & $\cdot$ & • \\
\hline Nickel & $\cdot$ & $\cdot$ & $\cdot$ & $\cdot$ & $\cdot$ & $\cdot$ & $\cdot$ & $\cdot$ & $\cdot$ & . & . &. &. & . \\
\hline Strontium & $\cdot$ & $\cdot$ & $\cdot$ & $\cdot$ & $\cdot$ & $\cdot$ & $\cdot$ & $\cdot$ & $\cdot$ &. & $\cdot$ &. & $\cdot$ & . \\
\hline Thorium & $\cdot$ & $\cdot$ & $\cdot$ & $\cdot$ & $\cdot$ & $\cdot$ & $\cdot$ & $\cdot$ & $\cdot$ & $\cdot$ & $\cdot$ &. & $\cdot$ & - \\
\hline Uranium & $\cdot$ & $\cdot$ & $\cdot$ & $\cdot$ & $\cdot$ & $\cdot$ & $\cdot$ & $\cdot$ & $\cdot$ & $\cdot$ &. &. &. & - \\
\hline Vanadium & $\cdot$ & $\cdot$ & $\cdot$ & $\cdot$ & $\cdot$ & $\cdot$ & $\cdot$ & $\cdot$ & $\cdot$ & $\cdot$ & $\cdot$ & $\cdot$ & $\cdot$ & - \\
\hline zinc & $\cdot$ & • & $\cdot$ & $\cdot$ & 0.23 & 0.066 & $\cdot$ & $\cdot$ & $\cdot$ &. & $\cdot$ &. & $\cdot$ & - \\
\hline$\cdot$ & $\cdot$ & $\cdot$ & $\cdot$ & $\cdot$ & $\cdot$ & $\cdot$ & $\cdot$ & $\cdot$ & $\cdot$ & $\cdot$ & $\cdot$ & $\cdot$ & $\cdot$ & - \\
\hline TSS $(m g / L)$ & 4 & $\cdot$ & 2 & $\cdot$ & 10 & $\cdot$ & 27 & $\cdot$ & 19 & $\cdot$ & 19 & $\cdot$ & 8 & - \\
\hline Turbidity (NTU) & 5.5 & $\cdot$ & 1.5 & $\cdot$ & 50 & $\cdot$ & 22 & $\cdot$ & 56 & $\cdot$ & 66 & $\cdot$ & 5 & - \\
\hline
\end{tabular}

(CONTINUED) 
APPENDIX H.2

Trace Metal Concentrations That Exceed

Screening LevelE, 1993

\begin{tabular}{|c|c|c|c|c|c|c|c|c|c|c|c|c|c|c|}
\hline \multirow{4}{*}{$\begin{array}{l}\text { Sampling Point } \\
\text { L } \\
\text { Location } \\
\text { Date Sampled }\end{array}$} & \multicolumn{2}{|c|}{ GW-603 } & \multicolumn{6}{|c|}{$G W-605$} & \multicolumn{4}{|c|}{ GW-617 } & \multicolumn{2}{|c|}{$G W-631$} \\
\hline & E) & & \multicolumn{6}{|c|}{ EXP } & \multicolumn{4}{|c|}{ EXP } & \multirow{2}{*}{\multicolumn{2}{|c|}{$\frac{\text { RG }}{03 / 02 / 93}$}} \\
\hline & \multicolumn{2}{|c|}{$08 / 16 / 93$} & \multicolumn{2}{|c|}{$02 / 02 / 93$} & \multicolumn{2}{|c|}{$05 / 10 / 93$} & \multicolumn{2}{|c|}{$11 / 10 / 93$} & \multicolumn{2}{|c|}{$09 / 16 / 93$} & \multicolumn{2}{|c|}{$12 / 07 / 93$} & & \\
\hline & TOT & DIS & TOT & DIS & TOT & DIS & TOT & DIS & TOT & DIS & TOT & DIS & TOT & DIS \\
\hline Aluminum & - & $=$ & $\cdot$ & $\cdot$ & $\cdot 1$ & $\cdot$ & $\cdot$ & $\cdot$ & $\cdot$ & • & . & . & $\cdot$ & • \\
\hline Beryllium & - & - & $\cdot$ & $\cdot$ & $\cdot$ & $\cdot$ &. & $\cdot$ &. & . & - & . &. & • \\
\hline Boron & . & - & . & $\cdot$ & . & . & . & $\cdot$ & . & . & - & . &. & • \\
\hline Cobalt & . & . & $\cdot$ &. & $\cdot$ & $\cdot$ &. & $\cdot$ &. & . & . & . & 0.035 & 0.027 \\
\hline Copper & . & . & $\cdot$ & $\cdot$ & $\cdot$ & . & $\cdot$ & . &. & . & . & - &. & • \\
\hline Iron & . & . & $\cdot 1$ & $\cdot$ & $\cdot$ &. & $\cdot$ &. &. & . & . & • &. & - \\
\hline Manganese & . & . &. & $\cdot 1$ &. &. &. &. & 4.2 & 3.9 & 3.2 & 2.7 &. & . \\
\hline Molybdenum & $\cdot$ & . & $\cdot$ & $\cdot$ & $\cdot$ & $\cdot$ & $\cdot$ & $\cdot$ & $\cdot$ &. & . & . &. & . \\
\hline Nickel & 0.37 & 0.3 & $\cdot$ & $\cdot$ & $\cdot$ & $\cdot$ & $\cdot$ & $\cdot$ & $\cdot$ &. & . & - &. & - \\
\hline Strontium & $\cdot$ & • & $\cdot$ & $\cdot$ & $\cdot$ & $\cdot$ & $\cdot$ & $\cdot$ & $\cdot 1$ &. & $\cdot$ & - &. & • \\
\hline Thorium & $\cdot$ & - & $\cdot$ & $\cdot$ & $\cdot$ & . & . &. &. &. & . & . &. & . \\
\hline Uranium & $\cdot$ & $\cdot$ & 0.149 & 0.152 & 0.189 & 0.194 & 0.19 & 0.16 & $\cdot$ & $\cdot$ & $\cdot$ & . & $\cdot$ & - \\
\hline Vanadium & $\cdot$ & - & . & $\cdot$ & $\cdot$ & $\cdot$ & . &. & $\cdot$ &. &. & . &. & . \\
\hline Zinc & $\cdot$ & . & $\cdot$ & $\cdot$ & $\cdot$ & $\cdot$ & $\cdot$ & $\cdot$ & $\cdot$ & $\cdot$ & 0.15 & 0.19 & $\cdot$ & - \\
\hline - & $\cdot$ & - & $\cdot$ & $\cdot$ & . & $\cdot$ & . &. &. &. &. & . & . & - \\
\hline TSS (mg/L) & 19 & $\cdot$ & $<1$ & $\cdot$ & 1 & $\cdot$ & $<1$ & $\cdot$ & 25 & $\cdot$ & 9 & $\cdot$ & 2 & - \\
\hline Turbidity (NTU) & 19 & $\cdot$ & 1.8 & $\cdot$ & 2.4 & $\cdot$ & 1.9 & $\cdot$ & 52 & . & 8 & . & 2.6 & - \\
\hline
\end{tabular}

(CONTINUED) 
APPENDIX H.2

Trace Metal Concentrations That Exceed

Screening Levels, 1993

\begin{tabular}{|c|c|c|c|c|c|c|c|c|c|c|c|c|c|c|}
\hline \multirow{4}{*}{$\begin{array}{l}\text { Sampling Point } \\
\text { Location } \\
\text { Date Sampled }\end{array}$} & \multicolumn{6}{|c|}{ GW-633 } & \multicolumn{8}{|c|}{$G W-634$} \\
\hline & \multicolumn{6}{|c|}{$\mathbf{R G}$} & \multicolumn{8}{|c|}{ RG } \\
\hline & \multicolumn{2}{|c|}{$03 / 03 / 93$} & \multicolumn{2}{|c|}{$06 / 21 / 93$} & \multicolumn{2}{|c|}{$09 / 20 / 93$} & \multicolumn{2}{|c|}{$03 / 02 / 93$} & \multicolumn{2}{|c|}{$06 / 18 / 93$} & \multicolumn{2}{|c|}{$09 / 16 / 93$} & \multicolumn{2}{|c|}{$11 / 18 / 93$} \\
\hline & TOT & DIS & TOT & DIS & TOT & DIS & TOT & DIS & TOT & DIS & TOT & DIS & TOT & DIS \\
\hline Aluminum & 11 & $<2$ & & $\cdot$ & - & - & . & $\cdot$ & $\cdot$ & - & . & . & . & \\
\hline Beryllium & $<0.03$ & $<0.03$ & $<0.015$ & $<0.015$ & - &. &. & . & .1 & . &. &. &. & • \\
\hline Boron & & & & & . & $\cdot$ &. & . &. & . &. &. &. & • \\
\hline Cobalt & $<0.5$ & $<0.5$ & $<0.25$ & $<0.25$ & $<0.1$ & 0.086 & 0.046 & 0.047 & 0.052 & 0.052 & 0.051 & 0.05 & 0.051 & 0.048 \\
\hline Copper & . & & $\cdot$ & $\cdot$ & $\cdot$ & $\cdot$ & $\cdot$ & $\cdot$ & . & . &. & . & • & • \\
\hline Iron & 14 & $<0.5$ & $\cdot$ & $\cdot$ & $\cdot$ & $\cdot$ & $\cdot$ & $\cdot$ & $\cdot$ & $\cdot$ & $\cdot$ & $\cdot$ & • & • \\
\hline Manganese &. & $\cdot$ & 5.5 & 7.2 & 11 & 13 & 3.6 & 3.7 & 4.3 & 4.2 & 3.9 & 3.8 & 3.9 & 3.7 \\
\hline Molybdenum & $<1$ & $<1$ & $<0.5$ & $<0.5$ & $<0.2$ & $<0.1$ &. &. &. &. & . &. &. & • \\
\hline Nickel & $<1$ & $<1$ & $<0.5$ & $<0.5$ & $<0.2$ & 0.28 &. &. & . & . & . & . &. & . \\
\hline strontium & 15 & 17 & 11 & 10 & 11 & 12 & $\cdot$ & $\cdot$ & . & . & . & . & . & . \\
\hline Thorium & $<20$ & $<20$ & $<10$ & $<10$ & $<4$ & $<2$ &. & $\cdot$ & . & . & . & . &. & . \\
\hline Uranium & & . & $\cdot$ & $\cdot$ & &. &. &. & . & . & . & . &. & . \\
\hline Vanadium & $<0.5$ & $<0.5$ & $<0.25$ & $<0.25$ & $<0.1$ & $<0.05$ &. &. & . & . &. & . &. & . \\
\hline zinc & $<0.2$ & $<0.2$ & $\cdot$ & $\cdot$ & $\cdot$ & $\cdot$ & - & $\cdot$ & . & $\cdot$ & . & . & . & • \\
\hline - & $\cdot$ & $\cdot$ & $\cdot$ & $\cdot$ & $\cdot$ & $\cdot$ & $\cdot$ & $\cdot$ & $\cdot$ & $\cdot$ & $\cdot$ & $\cdot$ & $\cdot$ & • \\
\hline TSS (mg/L) & 219 & $\cdot$ & 169 & $\cdot$ & 133 & . & $<1$ &. & $<1$ & $\cdot$ & 2 & . & 1 & • \\
\hline Turbidity (NTU) & 220 & $\cdot$ & 95 & $\cdot$ & 70 & - & 1.2 & $\cdot$ & 1.4 & $\cdot$ & 3.6 & . & 1.4 & • \\
\hline
\end{tabular}

(CONTINUED) 
APPENDIX H.2

Trace Metal Concentrations That Exceed

Screening Levels, 1993

\begin{tabular}{|c|c|c|c|c|c|c|c|c|c|c|c|c|c|c|}
\hline \multirow{4}{*}{$\begin{array}{l}\text { Sampling Point } \\
\text { Location } \\
\text { - } \\
\text { Date Sampled }\end{array}$} & \multicolumn{4}{|c|}{$\cdots-656$} & \multicolumn{8}{|c|}{$G W-658$} & \multicolumn{2}{|c|}{ GW-659 } \\
\hline & \multicolumn{4}{|c|}{ T0134 } & \multicolumn{8}{|c|}{$\mathbf{F F}$} & \multirow{2}{*}{\multicolumn{2}{|c|}{$\frac{F F}{03 / 10 / 93}$}} \\
\hline & \multicolumn{2}{|c|}{$06 / 22 / 93$} & \multicolumn{2}{|c|}{$11 / 17 / 93$} & \multicolumn{2}{|c|}{$03 / 11 / 93$} & \multicolumn{2}{|c|}{$06 / 23 / 93$} & \multicolumn{2}{|c|}{$11 / 19 / 93$} & \multicolumn{2}{|c|}{$09 / 27 / 04$} & & \\
\hline & тот & DIS & TOT & DIS & TOT & DIS & TOT & DIS & TOT & DIS & TOT & DIS & TOT & DIS \\
\hline Aluminum & . & $\cdot$ & 14 & 0.022 & $\cdot$ & - & - & - & . & . & . & . &. & . \\
\hline Beryllium & $\cdot$ & $\cdot$ & • & $\cdot$ & $\cdot 1$ & - & $\cdot$ & - & . & . &. & - &. & • \\
\hline Boron &. &. & . & $\cdot$ & $\cdot$ & . &. & . & . & . &. & . & . & • \\
\hline Cobalt & $\cdot$ & $\cdot$ & 0.022 & $<0.005$ & $\cdot 1$ & . &. & - & • & . & . & . & 0.015 & 0.011 \\
\hline Copper & . & $\cdot$ & • & & . & . & $\cdot$ & $\cdot$ & . & . & . & . & $\cdot$ & • \\
\hline Iron & . & $\cdot$ & 41 & 0.12 & 19 & 18 & $\cdot$ & . &. & . & 16 & 14 &. & • \\
\hline Manganese & . &. & . &. & 22 & 22 & 29 & 28 & 8.4 & 8.5 & 24 & 23 & 3.6 & 3.6 \\
\hline Molybdenum & $\cdot$ & $\cdot$ & . & $\cdot$ & $\cdot$ & $\cdot$ & $\cdot$ & $\cdot$ & . &. & $\cdot$ & . & $\cdot$ & • \\
\hline Nickel &. &. & . & . & $\cdot$ & . &. & $\cdot$ & . & . &. &. &. & . \\
\hline Strontium & $\cdot$ & $\cdot$ & $\cdot$ & $\cdot$ & $\cdot$ &. &. & $\cdot$ &. & . &. & . &. & . \\
\hline Thorium & $\cdot$ & $\cdot$ & $\cdot$ &. & $<0.4$ & $<0.4$ & $<0.4$ & $<0.4$ & $\cdot$ &. &. & . & . & . \\
\hline Uranium & $\cdot$ & $\cdot$ & $\cdot$ & $\cdot$ &. & . & . & $\cdot$ & . &. & . &. & . & • \\
\hline Vanadium & . & $\cdot$ & $\cdot$ & $\cdot$ &. & $\cdot$ & $\cdot$ &. &. & $\cdot$ &. & $\cdot$ &. & • \\
\hline Zinc & 0.25 & 0.2 & 0.19 & 0.078 & $\cdot$ &. & $\cdot 1$ &. &. &. &. & $\cdot$ & . & • \\
\hline - & $\cdot$ & $\cdot$ & $\cdot$ & $\cdot$ & $\cdot$ & $\cdot$ & $\cdot$ & $\cdot$ & $\cdot$ &. &. &. &. & . \\
\hline TSS (mg/L) & 83 & $\cdot$ & 746 &. & 29 & $\cdot$ & 29 &. & 91 & $\cdot$ & 31 &. & 391 & - \\
\hline Turbidity (NTU) & & $\cdot$ & 380 & $\cdot$ & 28 & $\cdot$ & 36 & $\cdot$ & 18 &. & 220 & . & 460 & • \\
\hline
\end{tabular}

(CONTINUED) 
APPENDIX H. 2

Trace Metal Concentrations That Exceed

Screening Levels, 1993

\begin{tabular}{|c|c|c|c|c|c|c|c|c|c|c|c|c|c|c|}
\hline \multirow{4}{*}{$\begin{array}{l}\text { Sampling Point } \\
\text { Location } \\
\text { Date Sampled }\end{array}$} & \multicolumn{6}{|c|}{$G W-659$} & \multicolumn{2}{|c|}{ GW-707 } & \multicolumn{2}{|c|}{ GW-733 } & \multicolumn{4}{|c|}{$G W-744$} \\
\hline & \multicolumn{6}{|c|}{$\mathbf{F F}$} & \multirow{2}{*}{\multicolumn{2}{|c|}{$\frac{T 2331}{11 / 18 / 93}$}} & \multirow{2}{*}{\multicolumn{2}{|c|}{$\frac{\text { EXP }}{08 / 18 / 93}$}} & \multicolumn{4}{|c|}{ GRIDK1 } \\
\hline & \multicolumn{2}{|c|}{$06 / 18 / 93$} & \multicolumn{2}{|c|}{$09 / 22 / 93$} & \multicolumn{2}{|c|}{$11 / 15 / 93$} & & & & & \multicolumn{2}{|c|}{$08 / 04 / 93$} & \multicolumn{2}{|c|}{$10 / 22 / 93$} \\
\hline & TOT & DIS & TOT & DIS & тот & DIS & TOT & DIS & TOT & DIS & TOT & DIS & TOT & DIS \\
\hline Aluminum & 23 & 0.03 & - & - & 14 & 0.17 & - & - & - & - & - & $\cdot$ & - & $\cdot$ \\
\hline Beryllium & $\cdot$ & • & - & - & • & • & - & - & - & - & - & - & - & • \\
\hline Boron & • & • & • & • & - & • & - & - & - & . & - & . & - & . \\
\hline Cobalt & 0.025 & 0.0063 & 0.013 & 0.029 & 0.019 & $<0.005$ & - & - & - & . & - & . & - & • \\
\hline Copper & • & & . & • & • & • & . & - & - & . & - & . & . & • \\
\hline Iron & 27 & 0.45 & - & - & 18 & 0.27 & . & - & 16 & 0.034 & - & . & - & . \\
\hline Manganese & $\cdot$ & • & - & - & • & . & . & . & $\cdot$ & • & . & . & - & • \\
\hline Molybdenum & - & • & - & - & - & . & 0.028 & 0.025 & . & . & . & . & - & • \\
\hline Nickel & 0.23 & 0.13 & - & - & - & . & • & • & - & - & • & • & . & • \\
\hline strontium & $\cdot$ & • & . & - & - & . & . & - & . & . & 0.94 & 0.94 & 1.1 & 1.1 \\
\hline Thorium & $\cdot$ & - & - & - & - & . & . & . & . & . & • & . & . & • \\
\hline uranium & . & . & - & - & . & $\cdot$ & - & - & . & - & - & . & - & - \\
\hline Vanadium & & & - & - & . &. & • & . & - & . & . & . & - & - \\
\hline zinc & 0.15 & 0.04 & $\cdot$ & $\cdot$ & 0.19 & 0.012 & - & -. & . & - & - & • & . & - \\
\hline . & & • & $\cdot$ & $\cdot$ & & $\cdot$ & . & - & . & . & . & - & - & • \\
\hline TSS (mg/L) & 234 & - & 253 & $\cdot$ & 743 & . & 3 & - & 20 & . & $<1$ & . & $<1$ & • \\
\hline Turbidity (NTU) & 340 & - & 680 & - & 750 & - & 2.4 & • & 20 & - & 1.4 & - & 1 & - \\
\hline
\end{tabular}

(CONTINUED) 
APPENDIX H.2

Trace Metal Concentrations That Exceed

Screening Levels, 1993

\begin{tabular}{|c|c|c|c|c|c|c|c|c|c|c|c|c|c|c|}
\hline \multirow{4}{*}{$\begin{array}{l}\text { Sampling Point } \\
\text { - } \\
\text { Location } \\
\text { Date Sampled }\end{array}$} & \multicolumn{2}{|c|}{ GW-745 } & \multicolumn{2}{|c|}{ GW-746 } & \multicolumn{4}{|c|}{ GW-755 } & \multicolumn{6}{|c|}{$G W-756$} \\
\hline & \multicolumn{2}{|c|}{ GRIDK1 } & \multicolumn{2}{|c|}{ GRIDK1 } & \multicolumn{4}{|c|}{ GRIDJI } & \multicolumn{6}{|c|}{ GRIDJ 1} \\
\hline & \multicolumn{2}{|c|}{$10 / 23 / 93$} & \multicolumn{2}{|c|}{$08 / 05 / 93$} & \multicolumn{2}{|c|}{$04 / 07 / 93$} & \multicolumn{2}{|c|}{$10 / 21 / 93$} & \multicolumn{2}{|c|}{$01 / 15 / 93$} & \multicolumn{2}{|c|}{$08 / 03 / 93$} & \multicolumn{2}{|c|}{$10 / 21 / 93$} \\
\hline & TOT & DIS & TOT & DIS & TOT & DIS & TOT & DIS & TOT & DIS & TOT & DIS & TOT & DIS \\
\hline Aluminum & $\cdot$ & $\cdot$ & 20 & 1.3 & $\cdot$ & $\cdot 1$ & $\cdot$ & $\cdot$ & 47 & 0.028 & 16 & $<0.02$ & $\cdot$ & . \\
\hline Beryllium & $\cdot$ & $\cdot$ & $\cdot$ & $\cdot$ & $\cdot$ & $\cdot$ & $\cdot$ &. & $\cdot$ &. & $\cdot$ &. &. & - \\
\hline Boron &. & $\cdot$ & $\cdot$ &. & $\cdot$ & $\cdot$ &. &. &. &. &. &. &. & . \\
\hline Cobalt & 0.019 & $<0.005$ & 0.014 & $<0.005$ & $\cdot$ & $\cdot$ & $\cdot$ &. & 0.048 & $<0.005$ & 0.025 & 0.015 & 0.013 & 0.0093 \\
\hline Copper & $\cdot$ & $\cdot$ & $\cdot$ & $\cdot$ & $\cdot$ & $\cdot$ & $\cdot$ & $\cdot$ &. & & $\cdot$ & & $\cdot$ & . \\
\hline Iron & $\cdot$ &. & 18 & 0.86 & . &. & $\cdot$ & $\cdot$ & 110 & 0.12 & 30 & 1.3 & $\cdot$ & . \\
\hline Manganese & $\cdot$ & $\cdot$ &. & . &. &. &. &. &. &. & 3 & 3 & 2.5 & 2.4 \\
\hline Mol ybdenum & $\cdot$ & $\cdot$ & $\cdot$ & $\cdot$ & $\cdot$ &. &. &. &. & .1 &. &. &. & . \\
\hline Nickel & $\cdot$ &. & .1 &. &. &. &. &. &. &. &. &. &. & . \\
\hline Strontium & $\cdot$ & $\cdot$ &. &. & 0.91 & 0.55 & 0.9 & 0.93 &. &. &. &. &. & . \\
\hline Thorium & $\cdot$ &. & $\cdot$ &. &. &. &. &. &. &. &. &. &. & . \\
\hline Urenium & $\cdot 1$ & $\cdot$ &. &. & $\cdot$ & $\cdot$ & $\cdot$ &. & $\cdot$ &. &. &. &. & . \\
\hline Vanadium & $\cdot$ & $\cdot$ & $\cdot$ &. &. & $\cdot$ & $\cdot$ &. & 0.087 & $<0.005$ &. &. &. &. \\
\hline zinc & $\cdot$ & $\cdot$ & $\cdot 1$ & $\cdot$ & $\cdot$ & $\cdot$ &. &. & 0.15 & 0.016 & .1 &. &. & . \\
\hline - & $\cdot$ & $\cdot$ & $\cdot$ & $\cdot$ & $\cdot$ &. & $\cdot$ &. & & $\cdot$ &. &. &. &. \\
\hline TsS (mg/L) & $<1$ & $\cdot$ & 414 & $\cdot$ & 118 & $\cdot$ & $<1$ &. & 1729 & $\cdot$ & 482 & $\cdot$ & 114 & . \\
\hline Turbidity (NTU) & 2.6 & $\cdot$ & 230 & $\cdot$ & 16 & $\cdot$ & 2.7 &. & 680 & $\cdot$ & 340 &. & 105 & . \\
\hline
\end{tabular}

(CONTINUED) 
Trace Hetal Concentrations That Exceed

Screening Levels, 1993

\begin{tabular}{|c|c|c|c|c|c|c|c|c|c|c|c|c|c|c|}
\hline \multirow{4}{*}{$\begin{array}{l}\text { Sampling Point } \\
\text { Location } \\
\text { Date Sampled }\end{array}$} & \multicolumn{2}{|c|}{ GW-759 } & \multicolumn{2}{|c|}{ GW-761 } & \multicolumn{8}{|c|}{ GW-763 } & \multicolumn{2}{|c|}{$G w-765$} \\
\hline & \multicolumn{2}{|c|}{ GRIDG1 } & \multicolumn{2}{|c|}{ GRIDG2 } & \multicolumn{8}{|c|}{ GRIDJ3 } & \multirow{2}{*}{\multicolumn{2}{|c|}{$\frac{\text { GRIDE } 1}{01 / 25 / 93}$}} \\
\hline & \multicolumn{2}{|c|}{$09 / 13 / 93$} & \multicolumn{2}{|c|}{$11 / 29 / 93$} & \multicolumn{2}{|c|}{$01 / 25 / 93$} & \multicolumn{2}{|c|}{$04 / 21 / 93$} & \multicolumn{2}{|c|}{$08 / 05 / 93$} & \multicolumn{2}{|c|}{$10 / 29 / 93$} & & \\
\hline & TOT & DIS & TOT & DIS & TOT & DIS & TOT & DIS & TOT & DIS & TOT & DIS & Tor & DIS \\
\hline Aluminum & 12 & 0.03 & $\cdot$ & - & - & - & $\cdot$ & $\cdot$ & - & $\cdot$ & - & - & 21 & 0.19 \\
\hline Beryllium & . & . & - & - & - & . & $\cdot$ & $\cdot$ & . & $\cdot$ & $\cdot$ & $\cdot 1$ & $\cdot$ & • \\
\hline Boron & . & . &. & . & - &. &. &. &. & $\cdot 1$ &. & $\cdot$ &. & • \\
\hline Cobalt & . & $\cdot$ & 0.015 & $<0.005$ & $\cdot$ &. & $\cdot$ & $\cdot$ & $\cdot$ & $\cdot$ & $\cdot$ & $\cdot$ & $\cdot 1$ & • \\
\hline Copper & . & . &. & . & . &. & .1 &. & $\cdot 1$ &. & . &. & .1 & • \\
\hline Iron & 24 & 0.055 &. & • & 20 & 21 & 22 & 23 & 18 & 12 & 16 & 16 & 27 & 0.22 \\
\hline Monganese & $\cdot$ & . & $\cdot 1$ & - & $\cdot$ &. & $\cdot$ & $\cdot$ & $\cdot$ & $\cdot$ & $\cdot$ & $\cdot$ & $\cdot$ & • \\
\hline Molybdenum & . & . & $\cdot$ & . & . & $\cdot$ & $\cdot$ & $\cdot$ & $\cdot$ & $\cdot$ & $\cdot$ & $\cdot$ & $\cdot 1$ & • \\
\hline Nickel & . & $\cdot$ & $\cdot 1$ & . & $\cdot$ & $\cdot$ & $\cdot$ & $\cdot$ & $\cdot$ & $\cdot$ & $\cdot$ & $\cdot$ & $\cdot$ & • \\
\hline Strontium & . & $\cdot$ & - & - & $\cdot$ & $\cdot$ & $\cdot$ & $\cdot$ &. & $\cdot$ & $\cdot$ & $\cdot$ & $\cdot$ & • \\
\hline Thorium &. & $\cdot$ & . & - & . &. & . & $\cdot$ &. &. & $\cdot$ & $\cdot 1$ & $\cdot$ & • \\
\hline Uranium & $\cdot$ & $\cdot$ & $\cdot 1$ & • & $\cdot$ & $\cdot 1$ & $\cdot$ & $\cdot$ & $\cdot$ & $\cdot$ & $\cdot$ & $\cdot$ & $\cdot$ & • \\
\hline Vanadium & . & . &. & . & $\cdot$ & $\cdot$ &. & $\cdot$ & $\cdot 1$ & $\cdot$ & $\cdot$ & . & $\cdot$ & - \\
\hline zinc & . & $\cdot$ & $\cdot$ & $\cdot$ & $\cdot$ & • & $\cdot$ & $\cdot$ & $\cdot$ & $\cdot$ & $\cdot$ & $\cdot$ & $\cdot$ & • \\
\hline - &. & . & . & . & $\cdot$ & - & $\cdot$ & $\cdot$ & $\cdot$ & $\cdot$ & . & $\cdot$ & $\cdot$ & • \\
\hline TSS (mg/L) & 592 & $\cdot$ & 27 & - & 53 & $\cdot$ & 30 & $\cdot$ & 52 & $\cdot$ & 35 & $\cdot$ & 349 & - \\
\hline Turbidity (NTU) & 80 & $\cdot$ & 22 & $\cdot$ & 35 & $\cdot$ & 30 & $\cdot$ & 46 & $\cdot 1$ & 30 & • & 620 & • \\
\hline
\end{tabular}

(CONTINUED) 
AYPENDIX H. 2

Trace Metal Concentrations That Exceed

Screening Levels, 1993

\begin{tabular}{|c|c|c|c|c|c|c|c|c|c|c|c|c|c|c|}
\hline \multirow{4}{*}{$\begin{array}{l}\text { Sampling Point } \\
\text { Location } \\
\text { Date Sampled }\end{array}$} & \multicolumn{2}{|c|}{ GW-765 } & \multicolumn{2}{|c|}{$G W-767$} & \multicolumn{8}{|c|}{ GW-772 } & \multicolumn{2}{|c|}{ Gw-773 } \\
\hline & \multicolumn{2}{|c|}{ GRIDE 1} & \multicolumn{2}{|c|}{ GRIDI2 } & \multicolumn{8}{|c|}{ GRIDCI } & \multirow{2}{*}{\multicolumn{2}{|c|}{$\begin{array}{c}\text { GRIDH2 } \\
08 / 06 / 93\end{array}$}} \\
\hline & \multicolumn{2}{|c|}{$09 / 12 / 93$} & \multicolumn{2}{|c|}{$08 / 04 / 93$} & \multicolumn{2}{|c|}{$01 / 23 / 93$} & \multicolumn{2}{|c|}{$04 / 12 / 93$} & \multicolumn{2}{|c|}{$09 / 12 / 93$} & \multicolumn{2}{|c|}{$11 / 12 / 93$} & & \\
\hline & TOT & DIS & TOT & DIS & TOT & DIS & TOT & DIS & TOT & DIS & TOT & DrS & TOT & DIS \\
\hline Aluminum & 14 & $<0.02$ & $\cdot$ & - & $\cdot$ & & $\bullet$ & $\cdot$ & - & $\cdot$ & $\cdot$ & $\cdot$ & $\cdot$ & • \\
\hline Beryllium & $\cdot$ & • & $\cdot$ & $\cdot$ & 0.0079 & $<0.0003$ & 0.0074 & $<0.003$ & - & - & $\cdot$ & $\cdot$ & $\cdot$ & • \\
\hline Boron & . & $\cdot$ & $\cdot$ & $\cdot$ & & & $\cdot$ & & • & $\cdot$ & $\cdot$ & $\cdot$ & $\cdot$ & • \\
\hline Cobalt & . & $\cdot$ & $\cdot$ & $\cdot$ & 0.069 & 0.063 & 0.056 & 0.051 & 0.056 & 0.06 & 0.056 & 0.055 & $\cdot$ & • \\
\hline Copper & $\cdot$ & $\cdot$ & $\cdot$ & $\cdot$ & $\cdot$ & $\cdot$ & $\cdot$ & $\cdot$ & • & $\cdot$ & $\cdot$ & • & - & • \\
\hline Iron & 24 & $<0.005$ & $\cdot$ & $\cdot$ & 36 & 37 & 37 & 36 & 34 & 32 & 34 & 34 & $\cdot$ & • \\
\hline Manganese & $\cdot$ & $\cdot$ & $\cdot$ & $\cdot$ & 13 & 13 & 13 & 12 & 12 & 12 & 12 & 13 & $\cdot$ & • \\
\hline Molybdenum & . &. & $\cdot$ & $\cdot$ &. & $\cdot$ & $<0.1$ & $<0.1$ & • & . & $\cdot$ & $\cdot$ & $\cdot$ & • \\
\hline Nickel & $\cdot$ & $\cdot$ & $\cdot$ & $\cdot$ & $\cdot$ & $\cdot 1$ & - & $\cdot$ & $\cdot$ & $\cdot$ & $\cdot$ & - & $\cdot$ & • \\
\hline Strontium & . & $\cdot$ & $\cdot$ & $\cdot$ & $\cdot$ & $\cdot$ & $\cdot$ & $\cdot$ & $\cdot$ & $\cdot$ & $\cdot$ & $\cdot$ & $\cdot$ & • \\
\hline Thorium & . &. &. & $\cdot$ &. & $\cdot$ & $<2$ & $<2$ & $\cdot$ & - & $\cdot$ & $\cdot$ &. & . \\
\hline Uranium & $\cdot$ & $\cdot$ & $\cdot$ & $\cdot$ & $\cdot$ & $\cdot$ & $\cdot$ & $\cdot$ & $\cdot$ & $\cdot$ & $\cdot$ & $\cdot$ & $\cdot$ & $\cdot$ \\
\hline Vanadium & . & . &. &. & . & $\cdot$ & - & $\cdot$ & $\cdot$ & - & $\cdot$ & $\cdot$ & $\cdot$ & • \\
\hline zinc & $\cdot$ & $\cdot$ & 0.16 & 0.18 & $\cdot$ & $\cdot$ & • & $\cdot$ & $\cdot$ & $\cdot$ & $\cdot$ & $\cdot$ & 0.23 & 0.0098 \\
\hline - &. & - & $\cdot$ & $\cdot$ & $\cdot$ & $\cdot$ & - & $\cdot$ & - & - & $\cdot$ & $\cdot$ & $\cdot$ & • \\
\hline TSS $(m g / L)$ & 264 & - & $<1$ & $\cdot$ & 7 & $\cdot$ & 1 & $\cdot$ & 11 & - & 4 & - & $<1$ & $\cdot$ \\
\hline Turbidity (NTU) & 250 & - & 1.6 & $\cdot$ & 17 & $\cdot$ & 8.8 & $\cdot$ & 6 & - & 20 & • & 1.7 & • \\
\hline
\end{tabular}

(CONTINUED) 
Trace Metal Concentrations That Bxceed

Screening Levels, 1993

\begin{tabular}{|c|c|c|c|c|c|c|c|c|}
\hline \multirow{4}{*}{$\begin{array}{l}\text { Sampling Point } \\
\text { Location } \\
\text { Date Sampled }\end{array}$} & \multicolumn{2}{|c|}{ GW-774 } & \multicolumn{6}{|c|}{$G W-776$} \\
\hline & \multicolumn{2}{|c|}{ GRIDH2 } & \multicolumn{6}{|c|}{ GRIDH3 } \\
\hline & \multicolumn{2}{|c|}{$02 / 04 / 93$} & \multicolumn{2}{|c|}{$04 / 20 / 93$} & \multicolumn{2}{|c|}{$08 / 10 / 93$} & \multicolumn{2}{|c|}{$10 / 18 / 93$} \\
\hline & TOT & DIS & TOT & DIS & TOT & DIS & TOT & DIS \\
\hline Aluminum & 24 & 0.27 & $\cdot$ & • & • & $\cdot$ & $\cdot$ & - \\
\hline Beryllium & $\cdot$ & $\cdot$ & $\cdot$ & - & • & $\cdot$ & - & - \\
\hline Boron & $\cdot$ & & $\cdot$ & - & - & $\cdot$ & - & - \\
\hline Cobalt & 0.023 & $<0.005$ & $\cdot$ & - & - & $\cdot$ & - & - \\
\hline Copper & $\cdot$ & & $\cdot$ & - & • & $\cdot 1$ & - & - \\
\hline Iron & 32 & 0.34 & $\cdot$ & - & • & $\cdot$ & $\cdot$ & - \\
\hline Manganese & $\cdot$ & . & $\cdot$ & - & - & $\cdot$ & $\cdot$ & - \\
\hline Mol ybdenum &. & $\cdot$ & $\cdot$ & • & • & $\cdot$ & $\cdot$ & • \\
\hline Nickel & $\cdot$ & . & 0.19 & 0.15 & 0.33 & 0.32 & 0.24 & 0.22 \\
\hline Strontium &. & $\cdot$ & $\cdot$ & $\cdot$ & • & $\cdot$ & $\cdot$ & • \\
\hline Thorium & $\cdot$ &. &. & - & • & $\cdot$ & $\cdot$ & - \\
\hline Uranium & - & $\cdot$ &. & - & - & $\cdot$ & $\cdot$ & - \\
\hline Vanadium & $\cdot$ & $\cdot$ & $\cdot$ & - & $\cdot$ & $\cdot$ & $\cdot$ & - \\
\hline zinc & . & . &. & - & - & $\cdot$ & $\cdot$ & • \\
\hline - &. & $\cdot$ & $\cdot$ & - & • & $\cdot$ & $\cdot$ & - \\
\hline TsS (mg/I) & 22 &. & 45 & - & 135 & $\cdot$ & 13 & - \\
\hline Turbidity (NTU) & 150 & . & 22 & - & 28 & $\cdot 1$ & 18 & - \\
\hline
\end{tabular}




\section{APPENDIX I}

QA/QC DATA SUMMARIES 


\section{EXPLANATION}

\section{LOCATION:}

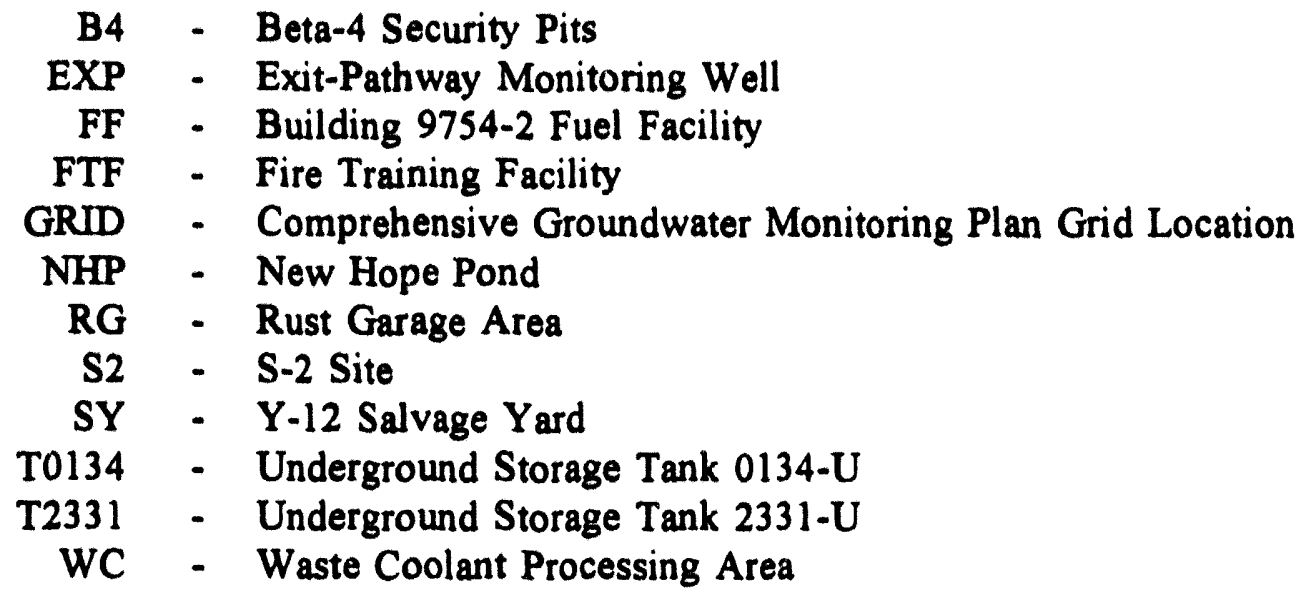

NOTES:

- Not analyzed

$\mathrm{U}$ - Compound not detected at the reported minimum attainable detection limit.

B - Well was bailed

W - Well Wizard (Dedicated sampling equipment)

\section{UNITS:}

$\mu \mathrm{g} / \mathrm{L} \quad$ - micrograms per liter

$\mathrm{mg} / \mathrm{L} \quad-\quad$ milligrams per liter

$\mathrm{pCi} / \mathrm{L}$ - picoCuries per liter

$\mathrm{Bq} / \mathrm{L} \quad-\quad$ Becquerels per liter

CE +/- - Counting Error (two standard deviations) 
APPENDIX 1.1

CORRELATION OF GROUNDWATER SAMPLES TO ASSOCIATED BLANKS 
APPENDIX I.1

Correlation of samples to Associated Blanks

\begin{tabular}{ccccc}
\hline & & Pump \\
$\begin{array}{c}\text { 8ampling } \\
\text { Pt. }\end{array}$ & Location No. & Sample & Trip Blank & Lab Blank \\
\hline
\end{tabular}

Firat Quarter 1993

\begin{tabular}{|c|c|c|c|c|c|c|}
\hline GW-151 & NBP & 7 & $01 / 26 / 93$ & $930126-094$ & $930126-096$ & $930127-001$ \\
\hline$O W-168$ & NHP & 8 & $01 / 22 / 93$ & $930122-070$ & $930122-072$ & $930125-004$ \\
\hline OW-169 & EXP & B & $01 / 23 / 93$ & $930123-021$ & $930123-025$ & $930125-004$ \\
\hline GW-170 & EXP & 8 & $01 / 23 / 93$ & $930123-022$ & $930123-025$ & $930125-004$ \\
\hline$G w-183$ & $\mathbf{F F}$ & 8 & $03 / 10 / 93$ & $930311-011$ & $930311-021$ & $930311-091$ \\
\hline GW-190 & RG & 3 & $03 / 01 / 93$ & $930302-001$ & $930302-007$ & $930304-076$ \\
\hline$G W-191$ & B4 & 6 & $01 / 12 / 93$ & $930112-083$ & $930112-087$ & $930113-147$ \\
\hline$a w-192$ & B4 & 6 & $01 / 14 / 93$ & $930114-028$ & $930114-030$ & $930115-001$ \\
\hline GW-193 & T2331 & 8 & $03 / 11 / 93$ & $930312-006$ & $930312-012$ & $930316-001$ \\
\hline GW-194 & B4 & 7 & $01 / 13 / 93$ & $930113-115$ & $930113-117$ & $930113-147$ \\
\hline GW-195 & 84 & 7 & $01 / 13 / 93$ & $930113-113$ & $930113-117$ & $930113-147$ \\
\hline GW-199 & GRIDI 1 & 6 & $01 / 29 / 93$ & $930129-094$ & $930129-096$ & $930202-095$ \\
\hline$G W-204$ & T0134 & 8 & $03 / 11 / 93$ & $930312-010$ & $930312-012$ & $930312-001$ \\
\hline GW-206 & ExP & $\mathbf{B}$ & $01 / 19 / 93$ & $930119-064$ & $930119-070$ & $930119-088$ \\
\hline$O W-207$ & EXP & 8 & $01 / 14 / 93$ & $930114-223$ & $930114-225$ & $930115-001$ \\
\hline$G W-208$ & $\mathbf{E X P}$ & 8 & $01 / 19 / 93$ & $930119-066$ & $930119-070$ & $930120-001$ \\
\hline GW-220 & NBP & 7 & $01 / 25 / 93$ & $930125-256$ & $930125-262$ & $930127-005$ \\
\hline GW-232 & $\mathbf{E X P}$ & 2 & $01 / 26 / 93$ & $930126-074$ & $930126-076$ & $930127-005$ \\
\hline GW-239 & NEP & 2 & $01 / 23 / 93$ & $930123-016$ & $930123-018$ & $930125-004$ \\
\hline$G K-240$ & NAP & 3 & $01 / 27 / 93$ & $930127-088$ & $930127-090$ & $930128-009$ \\
\hline GH-251 & $\mathbf{s 2}$ & 4 & $01 / 20 / 93$ & $930121-016$ & $930121-018$ & $930121-066$ \\
\hline $6 w-252$ & 82 & 4 & $01 / 08 / 93$ & $930108-135$ & $930108-137$ & $930111-139$ \\
\hline GW-255 & $\mathbf{8 2}$ & 4 & $01 / 08 / 93$ & $930108-131$ & $930108-137$ & $930111-008$ \\
\hline$G W-261$ & SY & 6 & $01 / 08 / 93$ & $930108-138$ & $930108-140$ & $930111-139$ \\
\hline$G W-262$ & $\mathbf{S Y}$ & 6 & $01 / 11 / 93$ & $930111-144$ & $930111-148$ & $930112-042$ \\
\hline$G \%-263$ & SY & 6 & $01 / 11 / 93$ & $930111-142$ & $930111-148$ & $930112-042$ \\
\hline$G W-264$ & $\mathbf{S Y}$ & 6 & $01 / 12 / 93$ & $930112-085$ & $930112-087$ & $930113-147$ \\
\hline$G W-281$ & $\mathbf{F F}$ & 6 & $02 / 02 / 93$ & $930203-009$ & $930203-017$ & $930203-057$ \\
\hline$G W-283$ & $\mathbf{F F}$ & 6 & $02 / 03 / 93$ & $930203-124$ & $930203-128$ & $930205-035$ \\
\hline GW-284 & $\mathbf{F F}$ & 6 & $02 / 02 / 93$ & $930203-011$ & $930203-017$ & $930203-057$ \\
\hline$G W-285$ & $\mathbf{F F}$ & 6 & $02 / 02 / 93$ & $930203-013$ & $930203-017$ & $930203-057$ \\
\hline GW-337 & พc & 4 & $01 / 21 / 93$ & $930121-060$ & $930121-062$ & $930122-001$ \\
\hline$G W-338$ & พc & 4 & $01 / 11 / 93$ & $930111-089$ & $930111-091$ & $930111-139$ \\
\hline$G W-380$ & NEP & 7 & $01 / 25 / 93$ & $930125-258$ & $930125-262$ & $930126-030$ \\
\hline$G W-381$ & NAP & 3 & $01 / 27 / 93$ & $930127-086$ & $930127-090$ & $930128-009$ \\
\hline GW-382 & NaP & 7 & $01 / 28 / 93$ & $930128-199$ & $930128-203$ & $930202-095$ \\
\hline$G W-383$ & NAP & 7 & $01 / 26 / 93$ & $930126-092$ & $930126-096$ & $930127-005$ \\
\hline$G W-384$ & NAP & 5 & $01 / 13 / 93$ & $930113-118$ & $930113-120$ & $930113-147$ \\
\hline GW-385 & NEP & 5 & $01 / 12 / 93$ & $930112-100$ & $930112-102$ & $930113-147$ \\
\hline GW -505 & RG & $\mathbf{B}$ & $03 / 01 / 93$ & $930302-002$ & $930302-007$ & $930302-060$ \\
\hline$G W-508$ & $\mathbf{R G}$ & B & $03 / 05 / 93$ & $930305-040$ & $930305-042$ & $930308-083$ \\
\hline $34-603$ & $\mathbf{E X P}$ & 4 & $01 / 29 / 93$ & $930129-114$ & $930129-116$ & $930201-001$ \\
\hline$G W-604$ & EXP & 4 & $01 / 30 / 93$ & $930201-089$ & $930201-091$ & $930202-004$ \\
\hline OW-605 & $\mathbf{E X P}$ & 8 & $02 / 02 / 93$ & $930203-031$ & $930203-033$ & $930203-001$ \\
\hline GW -606 & EXP & 8 & $02 / 03 / 93$ & $930203-091$ & $930203-093$ & $930204-034$ \\
\hline
\end{tabular}


APPENDIX I. 1

Correlation of Samples to Associated Blanks

\begin{tabular}{|c|c|c|c|c|c|c|}
\hline $\begin{array}{l}\text { Sampling } \\
\text { pt. }\end{array}$ & Location & $\begin{array}{l}\text { Pump } \\
\text { No. }\end{array}$ & Date & $\begin{array}{c}\text { sample } \\
\text { No. }\end{array}$ & $\begin{array}{c}\text { Trip Blank } \\
\text { No. }\end{array}$ & $\begin{array}{c}\text { Lab Blank } \\
\text { No. }\end{array}$ \\
\hline GW-617 & EXP & 4 & $01 / 12 / 93$ & $930112-057$ & $930112-061$ & $930113-002$ \\
\hline GW-618 & EXP & 4 & $01 / 12 / 93$ & $930112-059$ & $930112-061$ & $930113-002$ \\
\hline$G W-619$ & FTF & 4 & $01 / 20 / 93$ & $930121-012$ & $930121-018$ & $930125-263$ \\
\hline$G W-620$ & FTF & 4 & $01 / 20 / 93$ & $930121-014$ & $930121-018$ & $930125-263$ \\
\hline$G W-631$ & $\mathbf{R G}$ & 3 & $03 / 02 / 93$ & $930303-007$ & $930303-013$ & $930303-002$ \\
\hline$G W-632$ & RG & 3 & $03 / 03 / 93$ & $930303-110$ & $930303-114$ & $930304-069$ \\
\hline GW -633 & RG & 3 & $03 / 03 / 93$ & $930303-112$ & $930303-114$ & $930304-069$ \\
\hline$G W-634$ & RG & 3 & $03 / 02 / 93$ & $930303-009$ & $930303-013$ & $930303-002$ \\
\hline$G W-656$ & T0134 & 8 & $03 / 11 / 93$ & $930312-002$ & $930312-012$ & $930312-001$ \\
\hline GW -657 & T2331 & 8 & $03 / 10 / 93$ & $930311-015$ & $930311-021$ & $930311-091$ \\
\hline$G W-658$ & $\mathbf{F F}$ & 8 & $03 / 11 / 93$ & $930312-004$ & $930312-012$ & $930316-001$ \\
\hline GW-659 & FF & 8 & $03 / 10 / 93$ & $930311-017$ & $930311-021$ & $930312-066$ \\
\hline GW -707 & T2331 & 8 & $03 / 11 / 93$ & $930312-008$ & $930312-012$ & $930312-001$ \\
\hline GW-708 & T2331 & 8 & $03 / 10 / 93$ & $930311-019$ & $930311-021$ & $930311-091$ \\
\hline$G W-733$ & $\operatorname{EXP}$ & 4 & $02 / 02 / 93$ & $930203-024$ & $930203-030$ & $930203-001$ \\
\hline$G W-735$ & NEP & 4 & $02 / 02 / 93$ & $930203-026$ & $930203-030$ & $930203-601$ \\
\hline GW-744 & GRIDK 1 & 7 & $01 / 18 / 93$ & $930118-103$ & $930118-105$ & $930119-012$ \\
\hline GW -745 & GRIDK1 & 7 & $01 / 19 / 93$ & $930120-009$ & $930120-011$ & $930121-029$ \\
\hline$G W-746$ & GRIDR1 & 7 & $01 / 19 / 93$ & $930120-007$ & $930120-011$ & $930120-001$ \\
\hline GW -747 & GRIDK2 & 7 & $01 / 19 / 93$ & $930120-012$ & $930120-011$ & $930120-037$ \\
\hline GW-748 & GRIDK2 & 7 & $01 / 20 / 93$ & $930120-098$ & $930120-100$ & $930121-029$ \\
\hline$G W-749$ & GRIDK2 & 7 & $01 / 21 / 93$ & $930121-078$ & $930121-082$ & $930122-001$ \\
\hline$G W-750$ & GRIDK2 & 7 & $01 / 21 / 93$ & $930121-080$ & $930121-082$ & $930122-001$ \\
\hline$G W-751$ & GRIDJ3 & 5 & $01 / 11 / 93$ & $930111-127$ & $930111-131$ & $930111-139$ \\
\hline GW-752 & GRIDJ 3 & 5 & $01 / 11 / 93$ & $930111-129$ & $930111-131$ & $930114-221$ \\
\hline$G W-753$ & GRIDJ2 & 7 & $01 / 15 / 93$ & $930115-095$ & $930115-099$ & $930118-002$ \\
\hline GW-754 & GRIDJ2 & 7 & $01 / 18 / 93$ & $930118-101$ & $930118-105$ & $930119-012$ \\
\hline$G W-755$ & GRIDJ 1 & 7 & $01 / 14 / 93$ & $930114-218$ & $930114-220$ & $930115-001$ \\
\hline$G W-756$ & GRIDJ 1 & 7 & $01 / 15 / 93$ & $930115-097$ & $930115-099$ & $930118-002$ \\
\hline$G W-758$ & GRIDG 1 & 6 & $01 / 25 / 93$ & $930125-236$ & $930125-238$ & $930126-001$ \\
\hline$G W-759$ & GRIDG 1 & 6 & $01 / 26 / 93$ & $930126-110$ & $930126-112$ & $930128-002$ \\
\hline$G W-760$ & GRIDG2 & 6 & $01 / 27 / 93$ & $930128-012$ & $930128-018$ & $930128-002$ \\
\hline GW-761 & GRIDG2 & 6 & $01 / 26 / 93$ & $930126-108$ & $930126-112$ & $930128-002$ \\
\hline$G W-762$ & GRIDJ 3 & 7 & $01 / 23 / 93$ & $930123-019$ & $930123-025$ & \\
\hline$G W-762$ & GRIDJ 3 & $\mathbf{B}$ & $02 / 22 / 93$ & $930222-102$ & $930222-103$ & $930223-001$ \\
\hline$G W-763$ & GRIDJ 3 & 7 & $01 / 25 / 93$ & $930125-260$ & $930125-262$ & $930126-001$ \\
\hline$G W-764$ & GRIDE 1 & 6 & $01 / 23 / 93$ & $930123-029$ & $930123-033$ & $930125-263$ \\
\hline$G W-765$ & GRIDE 1 & 6 & $01 / 25 / 93$ & $930125-234$ & $930125-238$ & $930126-001$ \\
\hline$G W-766$ & GRIDI2 & 6 & $02 / 01 / 93$ & $930201-114$ & $930201-116$ & $930202-004$ \\
\hline$G W-767$ & GRIDI2 & 6 & $02 / 02 / 93$ & $930203-015$ & $930203-017$ & $930203-057$ \\
\hline GW-768 & GRIDI 1 & 6 & $01 / 29 / 93$ & $930129-092$ & $930129-096$ & $930129-124$ \\
\hline GW -769 & GRIDG 3 & 6 & $01 / 27 / 93$ & $930128-016$ & $930128-018$ & $930128-009$ \\
\hline$G W-770$ & GRIDG 3 & 6 & $01 / 27 / 93$ & $930128-010$ & $930128-018$ & $930128-009$ \\
\hline GW-771 & GRIDC 1 & 6 & $01 / 23 / 93$ & $930123-027$ & $930123-033$ & $930125-263$ \\
\hline GW-772 & GRIDC1 & 6 & $01 / 23 / 93$ & $930123-028$ & $930123-033$ & $930125-263$ \\
\hline GW -773 & GRIDH2 & 6 & $02 / 03 / 93$ & $930203-122$ & $930203-128$ & $930205-035$ \\
\hline GW-774 & GRIDH2 & 6 & $02 / 04 / 93$ & $930205-016$ & $930205-018$ & $930209-001$ \\
\hline
\end{tabular}


APPENDIX I. 1

Correlation of samples to Associated Blanks

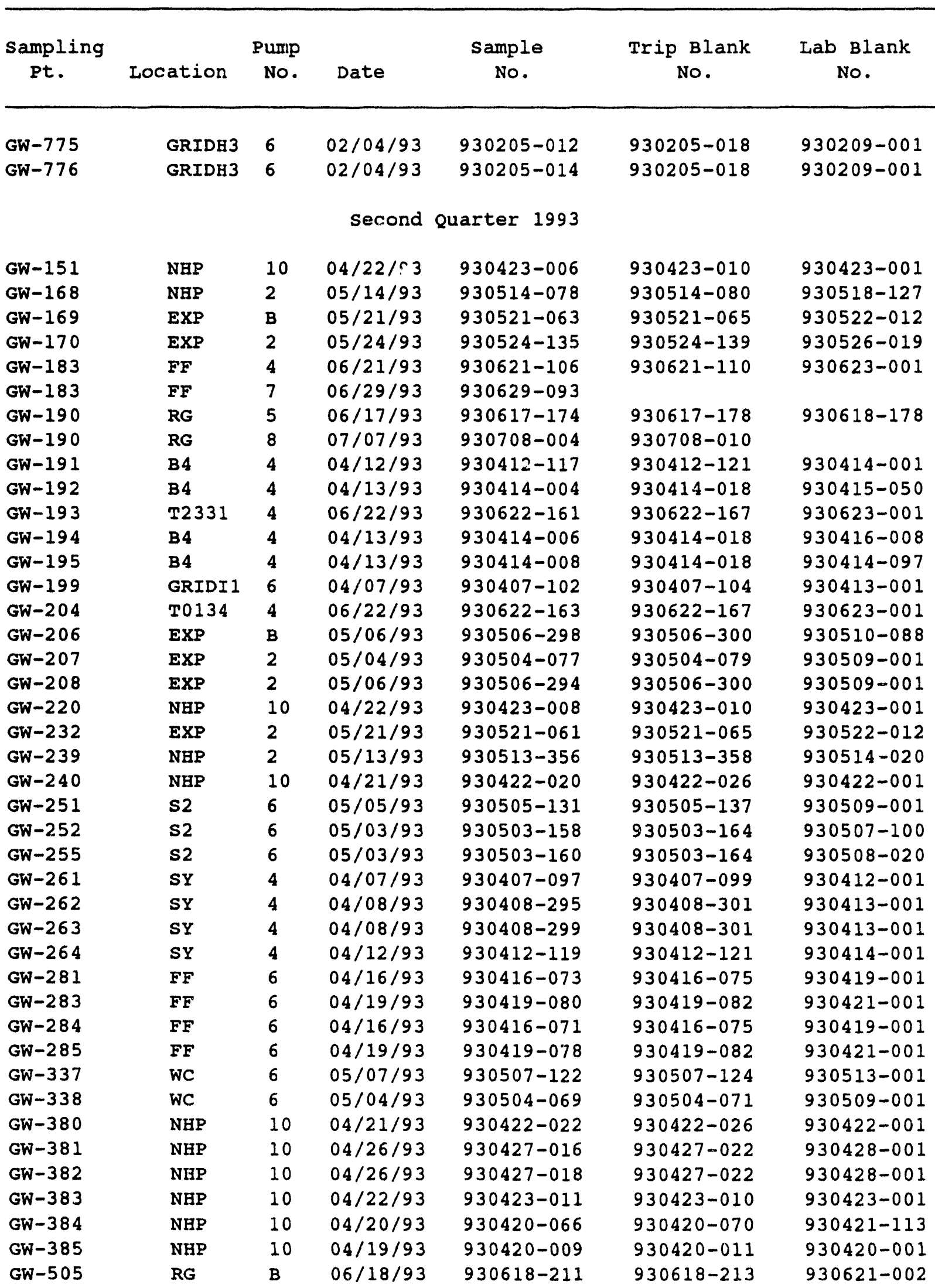


APPENDIX I.I

Correlation of Samples to Associated Blanks

\begin{tabular}{|c|c|c|c|c|c|c|}
\hline $\begin{array}{l}\text { sampling } \\
\text { pt. }\end{array}$ & Location & $\begin{array}{l}\text { Pump } \\
\text { No. }\end{array}$ & Date & $\begin{array}{c}\text { sample } \\
\text { No. }\end{array}$ & $\begin{array}{c}\text { Trip Blank } \\
\text { No. }\end{array}$ & $\begin{array}{c}\text { Lab Blank } \\
\text { No. }\end{array}$ \\
\hline$G W-505$ & RG & B & $07 / 07 / 93$ & $930708-008$ & $930708-010$ & \\
\hline$G W-508$ & RG & B & $06 / 23 / 93$ & $930624-010$ & $930624-012$ & $930625-001$ \\
\hline GW-508 & $\mathbf{R G}$ & 1 & $06 / 29 / 93$ & $930630-007$ & $930630-010$ & \\
\hline GW-603 & EXP & 10 & $05 / 04 / 93$ & $930504-074$ & $930504-076$ & $930508-020$ \\
\hline$G W-604$ & EXP & 10 & $05 / 04 / 93$ & $930504-072$ & $930504-076$ & $930508-020$ \\
\hline$G W-605$ & EXP & 10 & $05 / 10 / 93$ & $930511-020$ & $930511-022$ & $930513-045$ \\
\hline$G W-606$ & EXP & 10 & $05 / 10 / 93$ & $930511-023$ & $930511-022$ & $930513-045$ \\
\hline$G W-617$ & EXP & 6 & $05 / 04 / 93$ & $930504-067$ & $930504-071$ & $930508-020$ \\
\hline$G W-618$ & EXP & 6 & $05 / 05 / 93$ & $930505-133$ & $930505-137$ & $930509-001$ \\
\hline$G W-619$ & FTF & 6 & $05 / 05 / 93$ & $930505-135$ & $930505-137$ & $930509-001$ \\
\hline GW -620 & FTF & 6 & $05 / 06 / 93$ & $930506-053$ & $930506-055$ & $930511-065$ \\
\hline$G W-631$ & RG & 5 & $06 / 18 / 93$ & $930618-209$ & $930618-213$ & $930621-002$ \\
\hline$G W-631$ & RG & 8 & $07 / 07 / 93$ & $930708-006$ & $930708-310$ & \\
\hline$G W-632$ & RG & 5 & $06 / 21 / 93$ & $930622-012$ & $930622-016$ & $930623-001$ \\
\hline$G W-632$ & RG & 1 & $06 / 29 / 93$ & $930630-005$ & $930630-010$ & \\
\hline GW -633 & RG & 5 & $06 / 21 / 93$ & $930622-014$ & $930622-016$ & $930623-001$ \\
\hline$G W-633$ & RG & 1 & $06 / 29 / 93$ & $930630-006$ & $930630-010$ & \\
\hline$G W-634$ & RG & 5 & $06 / 18 / 93$ & $930618-207$ & $930618-213$ & $930619-007$ \\
\hline$G W-634$ & RG & 8 & $07 / 07 / 93$ & $930708-007$ & $930708-010$ & \\
\hline$G W-656$ & T0134 & 4 & $06 / 22 / 93$ & $930622-165$ & $930622-167$ & $930623-001$ \\
\hline GW -657 & T2331 & 4 & $06 / 18 / 93$ & $930618-247$ & $930618-249$ & $930621-002$ \\
\hline$G W-658$ & $\mathbf{F F}$ & 4 & $06 / 23 / 93$ & $930624-018$ & $930624-022$ & $930625-001$ \\
\hline$G W-659$ & $\mathbf{F F}$ & 4 & $06 / 18 / 93$ & $930618-245$ & $930618-249$ & $930619-007$ \\
\hline GW-659 & FF & 3 & $07 / 07 / 93$ & $930707-079$ & $930707-081$ & \\
\hline GW -707 & T2331 & 4 & $06 / 23 / 93$ & $930624-020$ & $930624-022$ & $930625-001$ \\
\hline GW-708 & T2331 & 4 & $06 / 18 / 93$ & $930618-243$ & $930618-249$ & $930619-007$ \\
\hline GW-708 & T2331 & 3 & $07 / 07 / 93$ & $930707-078$ & $930707-081$ & \\
\hline GW -733 & EXP & 10 & $05 / 06 / 93$ & $930506-301$ & $930506-303$ & $930511-041$ \\
\hline GW -735 & NHP & 10 & $05 / 07 / 93$ & $930507-126$ & $930507-130$ & $930511-065$ \\
\hline$G W-744$ & GRIDK1 & 3 & $04 / 12 / 93$ & $930412-126$ & $930412-128$ & $930414-001$ \\
\hline GW-745 & GRIDK 1 & 3 & $04 / 12 / 93$ & $930412-124$ & $930412-128$ & $930414-001$ \\
\hline GW -746 & GRIDK 1 & 3 & $04 / 12 / 93$ & $930412-122$ & $930412-128$ & $930414-001$ \\
\hline GW -747 & GRIDK2 & 3 & $04 / 13 / 93$ & $930413-077$ & $930413-083$ & $930414-097$ \\
\hline GW -748 & GRIDK2 & 3 & $04 / 13 / 93$ & $930413-081$ & $930413-083$ & $930415-050$ \\
\hline GW-749 & GRIDK2 & 3 & $04 / 14 / 93$ & $930414-126$ & $930414-128$ & $930416-008$ \\
\hline GW -750 & GRIDK2 & 3 & $04 / 15 / 93$ & $930415-035$ & $930415-037$ & $930416-061$ \\
\hline GW-751 & GRIDJ 3 & 10 & $04 / 16 / 93$ & $930416-121$ & $930416-125$ & $930419-001$ \\
\hline GW-752 & GRIDJ 3 & 10 & $04 / 16 / 93$ & $930416-123$ & $930416-125$ & $930419-001$ \\
\hline GW -753 & GRIDJ2 & 3 & $04 / 08 / 93$ & $930408-317$ & $930408-319$ & $930413-001$ \\
\hline GW-754 & GRIDJ2 & 3 & $04 / 08 / 93$ & $930408-315$ & $930408-319$ & $930413-001$ \\
\hline GW -755 & GRIDJ 1 & 8 & $04 / 07 / 93$ & $930407-105$ & $930407-107$ & $930412-001$ \\
\hline GW-756 & GRIDJ 1 & 3 & $04 / 08 / 93$ & $930408-313$ & $930408-319$ & $930413-001$ \\
\hline GW-758 & GRIDG 1 & 5 & $04 / 13 / 93$ & $930414-010$ & $930414-019$ & $930415-001$ \\
\hline GW-759 & GRIDG 1 & 5 & $04 / 15 / 93$ & $930415-213$ & $930415-215$ & $930416-061$ \\
\hline GW -760 & GRIDG2 & 5 & $04 / 16 / 93$ & $930416-098$ & $930416-102$ & $930419-001$ \\
\hline$G W-761$ & GRIDG2 & 5 & $04 / 16 / 93$ & $930416-100$ & $930416-102$ & $930419-001$ \\
\hline$G W-762$ & GRIDJ3 & 10 & $04 / 20 / 93$ & $930420-068$ & $930420-070$ & $930421-113$ \\
\hline
\end{tabular}


APPENDIX I. 1

Correlation of samples to Associated Blanks

\begin{tabular}{lllllll}
\hline $\begin{array}{c}\text { Sampling } \\
\text { Pt. }\end{array}$ & Location & $\begin{array}{c}\text { Pump } \\
\text { No. }\end{array}$ & Date & $\begin{array}{c}\text { Sample } \\
\text { No. }\end{array}$ & Trip Blank & Lab Blank \\
\hline & & & & & No. \\
GW-763 & GRIDJ3 & 10 & $04 / 21 / 93$ & $930422-024$ & $930422-026$ & $930422-001$ \\
GW-764 & GRIDE1 & 5 & $04 / 13 / 93$ & $930414-014$ & $930414-019$ & $930415-001$ \\
GW-765 & GRIDE1 & 5 & $04 / 13 / 93$ & $930414-016$ & $930414-019$ & $930415-001$ \\
GW-766 & GRIDI2 & 6 & $04 / 15 / 93$ & $930415-267$ & $930415-273$ & $930416-008$ \\
GW-767 & GRIDI2 & 6 & $04 / 15 / 93$ & $930415-271$ & $930415-273$ & $930416-008$ \\
GW-768 & GRIDI1 & 6 & $04 / 07 / 93$ & $930407-100$ & $930407-104$ & $930413-001$ \\
GW-769 & GRIDG3 & 5 & $04 / 19 / 93$ & $930419-065$ & $930419-067$ & $930420-001$ \\
GW-770 & GRIDG3 & 5 & $04 / 19 / 93$ & $930419-063$ & $930419-067$ & $930420-001$ \\
GW-771 & GRIDC1 & 5 & $04 / 12 / 93$ & $930412-111$ & $930412-113$ & $930414-001$ \\
GW-772 & GRIDC1 & 5 & $04 / 12 / 93$ & $930412-109$ & $930412-113$ & $930414-001$ \\
GW-773 & GRIDH2 & 6 & $04 / 20 / 93$ & $930421-005$ & $930421-007$ & $930421-113$ \\
GW-774 & GRIDH2 & 6 & $04 / 20 / 93$ & $930421-008$ & $930421-007$ & $930421-113$ \\
GW-775 & GRIDH3 & 6 & $04 / 20 / 93$ & $930421-010$ & $930421-007$ & $930421-113$ \\
GW-776 & GRIDH3 & 6 & $04 / 20 / 93$ & $930421-012$ & $930421-007$ & $930421-113$
\end{tabular}

Third Quarter 1993

\begin{tabular}{|c|c|c|c|c|c|c|}
\hline GW-151 & NEP & 4 & $08 / 06 / 93$ & $930806-118$ & $930806-124$ & $930809-070$ \\
\hline$G W-168$ & NHP & 2 & $08 / 19 / 93$ & $930819-301$ & $930819-305$ & $930820-001$ \\
\hline GW-169 & EXP & B & $08 / 19 / 93$ & $930819-303$ & $930819-305$ & $930820-001$ \\
\hline GW- 170 & EXP & 2 & $08 / 21 / 93$ & $930823-107$ & $930823-112$ & $930825-035$ \\
\hline$G W-183$ & $\mathbf{F F}$ & 1 & $09 / 22 / 93$ & $930922-071$ & $9300922-68$ & $930923-093$ \\
\hline GW-190 & RG & 1 & $09 / 15 / 93$ & $930915-146$ & $930915-152$ & $930917-070$ \\
\hline GW-191 & B4 & 10 & $07 / 08 / 93$ & $930709-001$ & $930709-008$ & $930709-031$ \\
\hline$G W-192$ & B4 & 10 & $07 / 08 / 93$ & $930709-003$ & $930709-008$ & $930709-031$ \\
\hline GW-193 & T2331 & 1 & $09 / 23 / 93$ & $930924-014$ & $930924-013$ & $930927-018$ \\
\hline$G W-194$ & B4 & 10 & $07 / 08 / 93$ & $930709-005$ & $930709-008$ & $930709-031$ \\
\hline$G W-195$ & B4 & 10 & $07 / 08 / 93$ & $930709-009$ & $930709-008$ & $930709-031$ \\
\hline GW-199 & GRIDI 1 & 7 & $08 / 02 / 93$ & $9.30802-137$ & $930802-139$ & $930803-001$ \\
\hline$G W-204$ & T0134 & 1 & $09 / 23 / 93$ & $930924-011$ & $930924-013$ & $930927-018$ \\
\hline$G W-206$ & EXP & B & $08 / 12 / 93$ & $930813-007$ & $930813-009$ & $930816-088$ \\
\hline$G W-207$ & EXP & 2 & $08 / 11 / 93$ & $930812-005$ & $930812-009$ & $930812-271$ \\
\hline$G W-208$ & EXP & 2 & $08 / 13 / 93$ & $930813-164$ & $930813-166$ & $930816-126$ \\
\hline$G W-220$ & NEP & 4 & $08 / 06 / 93$ & $930806-122$ & $930806-124$ & $930809-070$ \\
\hline$G W-232$ & EXP & 2 & $08 / 21 / 93$ & $930823-105$ & $930823-112$ & $930825-035$ \\
\hline$G W-239$ & NAP & 2 & $08 / 18 / 93$ & $930818-114$ & $930818-119$ & $930820-001$ \\
\hline$G W-240$ & NHP & 4 & $08 / 05 / 93$ & $930805-421$ & $930805-427$ & $930807-045$ \\
\hline$G W-251$ & s2 & 4 & $09 / 17 / 93$ & $930918-004$ & $930918-011$ & $930921-039$ \\
\hline$G W-252$ & s2 & 4 & $09 / 15 / 93$ & $930915-133$ & $930915-135$ & $930916-031$ \\
\hline$G W-255$ & s2 & 4 & $09 / 15 / 93$ & $930915-136$ & $930915-135$ & $930916-031$ \\
\hline$G W-2 \downarrow 1$ & SY & 10 & $07 / 01 / 93$ & $930702-006$ & $930702-019$ & $930707-032$ \\
\hline$G W-262$ & SY & 10 & $07 / 01 / 93$ & $930702-008$ & $930702-019$ & $930707-032$ \\
\hline$G W-263$ & SY & 10 & $07 / 01 / 93$ & $930702-012$ & $930702-019$ & $930707-032$ \\
\hline$G W-264$ & SY & 10 & $07 / 02 / 93$ & $930702-100$ & $930702-102$ & $930707-090$ \\
\hline$G W-281$ & $\mathrm{FF}$ & 7 & $08 / 04 / 93$ & $930805-409$ & $930805-415$ & $930806-047$ \\
\hline$G W-283$ & FF & 7 & $08 / 06 / 93$ & $930806-081$ & $930806-083$ & $930809-070$ \\
\hline$G W-284$ & FF & 7 & $08 / 05 / 93$ & $930805-413$ & $930805-415$ & $930806-047$ \\
\hline
\end{tabular}


APPENDIX I. 1

Correlation of samples to Associated Blanks

\begin{tabular}{|c|c|c|c|c|c|c|}
\hline $\begin{array}{l}\text { sampling } \\
\text { pt. }\end{array}$ & Location & $\begin{array}{l}\text { Pump } \\
\text { No. }\end{array}$ & Date & $\begin{array}{c}\text { sample } \\
\text { No. }\end{array}$ & $\begin{array}{c}\text { Trip Blank } \\
\text { No. }\end{array}$ & $\begin{array}{c}\text { Lab Blank } \\
\text { No. }\end{array}$ \\
\hline GW -285 & $\mathbf{F F}$ & 7 & $08 / 05 / 93$ & $930805-411$ & $930805-415$ & $930806-047$ \\
\hline GW -337 & WC & 4 & $09 / 17 / 93$ & $930918-009$ & $930918-011$ & $930920-116$ \\
\hline$G W-338$ & พC & 4 & $09 / 23 / 93$ & $930923-129$ & $930923-131$ & $930927-018$ \\
\hline$G W-380$ & NEP & 4 & $08 / 05 / 93$ & $930805-423$ & $930805-427$ & $930807-045$ \\
\hline GW -381 & NHP & 4 & $08 / 08 / 93$ & $930809-019$ & $930809-021$ & $930811-031$ \\
\hline$G W-382$ & NHP & 4 & $08 / 08 / 93$ & $930809-017$ & $930809-021$ & $930811-031$ \\
\hline GW -383 & NHP & 4 & $08 / 07 / 93$ & $930809-014$ & $930809-016$ & $930810-039$ \\
\hline$G W-384$ & NHP & 4 & $08 / 04 / 93$ & $930804-086$ & $930804-090$ & $930805-116$ \\
\hline$G W-385$ & NHP & 1 & $08 / 03 / 93$ & $930804-005$ & $930804-016$ & $930804-035$ \\
\hline$G W-505$ & $\mathbf{R G}$ & B & $09 / 15 / 93$ & $930915-150$ & $930915-152$ & $930917-070$ \\
\hline$G W-508$ & RG & B & $09 / 21 / 93$ & $930921-168$ & $930921-169$ & $930922-010$ \\
\hline$G W-508$ & $\mathbf{R G}$ & B & $09 / 22 / 93$ & $930922-065$ & & \\
\hline GW -603 & EXP & 7 & $08 / 16 / 93$ & $930816-200$ & $930816-204$ & $930818-001$ \\
\hline$G W-604$ & EXP & 7 & $08 / 16 / 93$ & $930816-202$ & $930816-204$ & $930818-001$ \\
\hline$G W-605$ & EXP & 7 & $08 / 19 / 93$ & $930819-254$ & $930819-256$ & $930820-001$ \\
\hline$G W-606$ & EXP & 7 & $08 / 20 / 93$ & $930820-084$ & $930820-088$ & $930823-002$ \\
\hline GW -617 & EXP & 4 & $09 / 16 / 93$ & $930916-126$ & $930916-132$ & $930918-029$ \\
\hline$G W-618$ & EXP & 4 & $09 / 16 / 93$ & $930916-128$ & $930916-132$ & $930918-029$ \\
\hline GW-619 & FTF & 4 & $09 / 17 / 93$ & $930918-002$ & $930918-011$ & $930921-039$ \\
\hline$G W-620$ & FTF & 4 & $09 / 17 / 93$ & $930918-007$ & $930918-011$ & $930921-039$ \\
\hline$G W-631$ & $\mathbf{R G}$ & 1 & $09 / 16 / 93$ & $930917-005$ & $930917-007$ & $930918-029$ \\
\hline$G W-632$ & RG & 1 & $09 / 16 / 93$ & $930917-001$ & $930917-007$ & $930918-029$ \\
\hline$G W-633$ & RG & 1 & $09 / 20 / 93$ & $930920-164$ & $930920-166$ & $930921-025$ \\
\hline$G W-634$ & $\mathbf{R G}$ & 1 & $09 / 16 / 93$ & $930917-003$ & $930917-007$ & $930918-029$ \\
\hline$G W-656$ & T0134 & 1 & $09 / 23 / 93$ & $930924-016$ & $930924-013$ & $930927-018$ \\
\hline GW -657 & T2331 & 1 & $09 / 21 / 93$ & $930921-164$ & $930921-169$ & $930922-033$ \\
\hline$G W-658$ & FF & 1 & $09 / 27 / 04$ & $930927-229$ & $930927-228$ & $930928-089$ \\
\hline$G W-659$ & $\mathbf{F F}$ & 1 & $09 / 22 / 93$ & $930922-066$ & $930922-068$ & $930923-093$ \\
\hline GW -707 & T2331 & 1 & $09 / 27 / 93$ & $930927-226$ & $930927-228$ & $930928-089$ \\
\hline$G W-708$ & T2331 & 1 & $09 / 21 / 93$ & $930921-166$ & $930921-169$ & $930922-033$ \\
\hline$G W-733$ & $\operatorname{EXP}$ & 7 & $08 / 18 / 93$ & $930818-112$ & $930818-118$ & $930820-001$ \\
\hline GW -735 & NHP & 7 & $08 / 18 / 93$ & $930818-116$ & $930818-118$ & $930820-001$ \\
\hline$G W-744$ & GRIDK 1 & 10 & $08 / 04 / 93$ & $930804-108$ & $930804-110$ & $930806-047$ \\
\hline GW -745 & GRIDK 1 & 10 & $08 / 04 / 93$ & $930804-106$ & $930804-110$ & $930806-047$ \\
\hline GW-746 & GRIDK 1 & 10 & $08 / 05 / 93$ & $930805-418$ & $930805-420$ & $930806-047$ \\
\hline GW -747 & GRIDK2 & 10 & $08 / 05 / 93$ & $930805-416$ & $930805-420$ & $930806-047$ \\
\hline GW-748 & GRIDK2 & 10 & $08 / 06 / 93$ & $930806-160$ & $930806-164$ & $930810-039$ \\
\hline GW -749 & GRIDK2 & 10 & $08 / 06 / 93$ & $930806-162$ & $930806-164$ & $930810-039$ \\
\hline$G W-750$ & GRIDK2 & 10 & $08 / 06 / 93$ & $930806-158$ & $930806-164$ & $930810-039$ \\
\hline$G W-751$ & GRIDJ 3 & 1 & $08 / 02 / 93$ & $930802-126$ & $930802-130$ & $930803-001$ \\
\hline GW -752 & GRIDJ 3 & 1 & $08 / 02 / 93$ & $930802-128$ & $930802-130$ & $930804-035$ \\
\hline GW -753 & GRIDJ2 & 10 & $08 / 03 / 93$ & $930804-007$ & $930804-017$ & $930804-035$ \\
\hline$G W-754$ & GRIDJ2 & 10 & $08 / 04 / 93$ & $930804-104$ & $930804-110$ & $930806-047$ \\
\hline GW-755 & GRIDJ 1 & 10 & $08 / 03 / 93$ & $930804-011$ & $930804-017$ & $930804-035$ \\
\hline$G W-756$ & GRIDJ 1 & 10 & $08 / 03 / 93$ & $930804-013$ & $930804-017$ & $930804-035$ \\
\hline$G W-758$ & GRIDG 1 & 4 & $09 / 12 / 93$ & $930913-080$ & $930913-075$ & $930915-110$ \\
\hline$G W-759$ & GRIDG 1 & 4 & $09 / 13 / 93$ & $930914-006$ & $930914-008$ & $930916-020$ \\
\hline
\end{tabular}


APPENDIX I. 1

Correlation of Samples to Associated Blanks

\begin{tabular}{|c|c|c|c|c|c|c|}
\hline $\begin{array}{l}\text { sampling } \\
\text { pt. }\end{array}$ & Location & $\begin{array}{l}\text { Pump } \\
\text { No. }\end{array}$ & Date & $\begin{array}{c}\text { Sample } \\
\text { No. }\end{array}$ & $\begin{array}{c}\text { Trip Blank } \\
\text { No. }\end{array}$ & $\begin{array}{c}\text { Lab Blank } \\
\text { No. }\end{array}$ \\
\hline GW-760 & GRIDG2 & 4 & $09 / 14 / 93$ & $930915-001$ & $930915-007$ & $930916-020$ \\
\hline GW-761 & GRIDG2 & 4 & $09 / 13 / 93$ & $930914-004$ & $930914-008$ & $930915-107$ \\
\hline GW-762 & GRIDJ 3 & 4 & $08 / 04 / 93$ & $930804-088$ & $930804-090$ & $930805-116$ \\
\hline$G W-763$ & GRIDJ 3 & 4 & $08 / 05 / 93$ & $930805-425$ & $930805-427$ & $930807-045$ \\
\hline$G W-764$ & GRIDE 1 & 4 & $09 / 12 / 93$ & $930913-076$ & $930913-075$ & $930915-110$ \\
\hline GW -765 & GRIDEI & 4 & $09 / 12 / 93$ & $930913-078$ & $930913-075$ & $930915-110$ \\
\hline GW-766 & GRIDI2 & 7 & $08 / 03 / 93$ & $930804-001$ & $930804-015$ & $930804-035$ \\
\hline$G W-767$ & GRIDI2 & 7 & $08 / 04 / 93$ & $930805-060$ & $930805-064$ & $930805-116$ \\
\hline GW-768 & GRIDI1 & 7 & $08 / 04 / 93$ & $930805-062$ & $930805-064$ & $930805-116$ \\
\hline GW-769 & GRIDG3 & 4 & $09 / 14 / 93$ & $930915-003$ & $930915-007$ & $930916-020$ \\
\hline GW -770 & GRIDG 3 & 4 & $09 / 14 / 93$ & $930915-005$ & $930915-007$ & $930916-020$ \\
\hline GW -771 & GRIDCl & 4 & $09 / 03 / 93$ & $930903-143$ & $930903-145$ & $930908-076$ \\
\hline GW-772 & GRIDC 1 & 4 & $09 / 12 / 93$ & $930913-073$ & $930913-075$ & $930915-107$ \\
\hline GW -773 & GRIDH2 & 7 & $08 / 06 / 93$ & $930806-079$ & $930806-083$ & $930809-070$ \\
\hline$G W-774$ & GRIDH2 & 7 & $08 / 09 / 93$ & $930810-003$ & $930810-007$ & $930811-031$ \\
\hline GW-775 & GRIDH 3 & 7 & $08 / 09 / 93$ & $930810-005$ & $930810-007$ & $930811-031$ \\
\hline GW-776 & GRIDH 3 & 7 & $08 / 10 / 93$ & $930810-117$ & $930810-119$ & $930811-031$ \\
\hline
\end{tabular}

Fourth Quarter 1993

\begin{tabular}{|c|c|c|c|c|c|c|}
\hline$G W-151$ & NEP & 5 & $11 / 02 / 93$ & $931102-047$ & $931102-050$ & $931109-111$ \\
\hline GW-168 & NEP & 8 & $11 / 02 / 93$ & $931102-045$ & $931102-049$ & $931119-123$ \\
\hline GW-169 & EXP & B & $11 / 01 / 93$ & $931101-146$ & $931101-150$ & $931102-001$ \\
\hline$G W-170$ & EXP & 8 & $11 / 04 / 93$ & $931104-062$ & $931104-064$ & $931109-111$ \\
\hline GW-183 & $\mathbf{F F}$ & 10 & $11 / 16 / 93$ & $931116-109$ & $931116-111$ & $931118-001$ \\
\hline GW-190 & RG & 6 & $11 / 16 / 93$ & $931116-096$ & $931116-100$ & $931118-001$ \\
\hline GW-191 & B4 & 5 & $12 / 09 / 93$ & $931210-007$ & $931210-016$ & $931214-001$ \\
\hline GW-192 & B4 & 5 & $12 / 09 / 93$ & $931210-009$ & $931210-016$ & $931214-001$ \\
\hline GW-193 & T2331 & 10 & $11 / 18 / 93$ & $931118-161$ & $931118-163$ & $931119-102$ \\
\hline$G W-1.94$ & B4 & 5 & $12 / 09 / 93$ & $931210-011$ & $931210-016$ & $931214-001$ \\
\hline GW-195 & B4 & 5 & $12 / 09 / 93$ & $931210-013$ & $931210-016$ & $931214-001$ \\
\hline GW-199 & GRIDI 1 & 7 & $10 / 12 / 93$ & $931012-154$ & $931012-156$ & $931015-141$ \\
\hline$G W-204$ & T0134 & 10 & $11 / 16 / 93$ & $931116-114$ & $931116-111$ & $931118-001$ \\
\hline$G W-206$ & $\operatorname{EXP}$ & B & $10 / 27 / 93$ & $931027-120$ & $931027-122$ & $931028-022$ \\
\hline$G W-207$ & EXP & 8 & $10 / 26 / 93$ & $931026-031$ & $931026-035$ & $931028-007$ \\
\hline$G W-208$ & EXP & 8 & $10 / 28 / 93$ & $931028-032$ & $931028-034$ & $931028-022$ \\
\hline$G W-220$ & NHP & 5 & $11 / 01 / 93$ & $931101-133$ & $931101-135$ & $931102-001$ \\
\hline$G W-232$ & FXP & 2 & $11 / 01 / 93$ & $931101-148$ & $931101-150$ & $931102-001$ \\
\hline$G W-239$ & NHP & 2 & $10 / 30 / 93$ & $931101-045$ & $931101-047$ & $931102-001$ \\
\hline$G W-240$ & NHP & 5 & $11 / 01 / 93$ & $931101-131$ & $931101-135$ & $931102-001$ \\
\hline$G W-251$ & $\mathbf{s} 2$ & 10 & $12 / 08 / 93$ & $931208-086$ & $331208-092$ & $931209-029$ \\
\hline$G W-252$ & $s 2$ & 10 & $12 / 07 / 93$ & $931208-021$ & $931208-023$ & $931209-025$ \\
\hline$G W-255$ & s2 & 10 & $12 / 07 / 93$ & $931208-017$ & $931208-023$ & $931208-005$ \\
\hline$G W-261$ & SY & 5 & $12 / 07 / 93$ & $931208-010$ & $931208-012$ & $931208-005$ \\
\hline GW-262 & SY & 5 & $12 / 07 / 93$ & $931208-006$ & $931208-012$ & $931208-005$ \\
\hline GW-263 & SY & 5 & $12 / 07 / 93$ & $931208-008$ & $931208-012$ & $931208-005$ \\
\hline$G W-264$ & SY & 5 & $12 / 08 / 93$ & $931208-093$ & $931208-097$ & $931209-029$ \\
\hline
\end{tabular}


APPENDIX I. 1

Correlation of samples to Associated Blanks

\begin{tabular}{|c|c|c|c|c|c|c|}
\hline $\begin{array}{l}\text { Sampling } \\
\text { pt. }\end{array}$ & Location & $\begin{array}{l}\text { Pump } \\
\text { No. }\end{array}$ & Date & $\begin{array}{c}\text { Sample } \\
\text { No. }\end{array}$ & $\begin{array}{c}\text { Trip Blank } \\
\text { No. }\end{array}$ & $\begin{array}{c}\text { Lab Blank } \\
\text { No. }\end{array}$ \\
\hline$G W-281$ & FF & 7 & $10 / 14 / 93$ & $931014-059$ & $931014-056$ & $931018-003$ \\
\hline$G W-283$ & $F F$ & 7 & $10 / 14 / 93$ & $931014-057$ & $931014-056$ & $931018-003$ \\
\hline$G W-284$ & FF & 7 & $10 / 14 / 93$ & $931014-054$ & $931014-056$ & $931014-010$ \\
\hline GW -285 & $\mathbf{F F}$ & 7 & $10 / 14 / 93$ & $931014-061$ & $931014-056$ & $931018-003$ \\
\hline GW-337 & WC & 10 & $12 / 09 / 93$ & $931209-098$ & $931209-102$ & $931210-049$ \\
\hline$G W-338$ & wc & 10 & $12 / 07 / 93$ & $931208-015$ & $931208-023$ & $931208-005$ \\
\hline GW -380 & NHP & 5 & $10 / 29 / 93$ & $931029-067$ & $931029-071$ & $931101-042$ \\
\hline$G W-381$ & NHP & 5 & $11 / 08 / 93$ & $931108-192$ & $931108-194$ & $931110-011$ \\
\hline$G W-382$ & NHP & 5 & $11 / 04 / 93$ & $931104-066$ & $931104-068$ & $931109-029$ \\
\hline GW -383 & NGP & 5 & $11 / 03 / 93$ & $931103-013$ & $931103-015$ & $931109-111$ \\
\hline$G W-384$ & NHP & 5 & $10 / 28 / 93$ & $931028-078$ & $931028-080$ & $931029-026$ \\
\hline$G W-385$ & NHP & 5 & $10 / 27 / 93$ & $931027-118$ & $931027-123$ & $931028-022$ \\
\hline GW -505 & RG & $\mathbf{B}$ & $11 / 16 / 93$ & $931116-098$ & $931116-100$ & $931118-001$ \\
\hline GW-508 & $\mathbf{R G}$ & $\mathbf{B}$ & $11 / 19 / 93$ & $931119-064$ & $931119-066$ & $931124-240$ \\
\hline$G W-603$ & EXP & 6 & $10 / 28 / 93$ & $931028-071$ & $931028-075$ & $931029-026$ \\
\hline$G W-604$ & EXP & 6 & $10 / 28 / 93$ & $931028-073$ & $931028-075$ & $931029-026$ \\
\hline GW-605 & EXP & 6 & $11 / 10 / 93$ & $931110-069$ & $931110-071$ & $931111-014$ \\
\hline GW-606 & EXP & 6 & $11 / 11 / 93$ & $931111-105$ & $931111-107$ & $931117-001$ \\
\hline GW-617 & EXP & 10 & $12 / 07 / 93$ & $931208-019$ & $931208-023$ & $931206-102$ \\
\hline$G W-618$ & EXP & 10 & $12 / 08 / 93$ & $931208-088$ & $931208-092$ & $931209-029$ \\
\hline GW-619 & FTF & 10 & $12 / 08 / 93$ & $931208-090$ & $931208-092$ & $931209-029$ \\
\hline GW -620 & FTF & 10 & $12 / 09 / 93$ & $931209-100$ & $931209-102$ & $931210-049$ \\
\hline$G W-631$ & RG & 6 & $11 / 17 / 93$ & $931117-045$ & $931117-047$ & $931118-146$ \\
\hline GW-632 & RG & 6 & $11 / 18 / 93$ & $931118-154$ & $931118-158$ & $931119-102$ \\
\hline$G W-633$ & RG & 6 & $11 / 19 / 93$ & $931119-062$ & $931119-066$ & $931123-001$ \\
\hline GW-634 & RG & 6 & $11 / 18 / 93$ & $931118-156$ & $931118-158$ & $931119-102$ \\
\hline$G W-656$ & T0134 & 10 & $11 / 17 / 93$ & $931117-042$ & $931117-044$ & $931118-146$ \\
\hline GW -657 & T2331 & 10 & $11 / 15 / 93$ & $931115-205$ & $931115-207$ & $931116-035$ \\
\hline$G W-658$ & $\mathbf{F F}$ & 10 & $11 / 19 / 93$ & $931119-058$ & $931119-060$ & $931124-240$ \\
\hline$G W-659$ & FF & 10 & $11 / 15 / 93$ & $931115-203$ & $931115-207$ & $931116-035$ \\
\hline GW-707 & T2331 & 10 & $11 / 18 / 93$ & $931118-159$ & $931118-163$ & $931119-102$ \\
\hline GW-708 & T2331 & 10 & $11 / 12 / 93$ & $931112-063$ & $931112-065$ & $931117-001$ \\
\hline GW-733 & EXP & 6 & $11 / 04 / 93$ & $931104-072$ & $931104-074$ & $931109-029$ \\
\hline GW -735 & NHP & 6 & $10 / 29 / 93$ & $931029-053$ & $931029-057$ & $931101-042$ \\
\hline GW-744 & GRIDK 1 & 5 & $10 / 22 / 93$ & $931022-044$ & $931022-048$ & $931025-031$ \\
\hline GW -745 & GRIDK 1 & 5 & $10 / 23 / 93$ & $931025-006$ & $931025-008$ & $931027-001$ \\
\hline GW-746 & GRIDK 1 & 5 & $10 / 23 / 93$ & $931025-004$ & $931025-008$ & $931027-001$ \\
\hline$G W-747$ & GRIDK2 & 5 & $10 / 23 / 93$ & $931025-002$ & $931025-008$ & $931027-001$ \\
\hline$G W-748$ & GRIDK2 & 5 & $10 / 24 / 93$ & $931025-017$ & $931025-021$ & $931027-001$ \\
\hline GW -749 & GRIDK2 & 5 & $10 / 24 / 93$ & $931025-019$ & $931025-021$ & $931027-001$ \\
\hline GW -750 & GRIDK2 & 5 & $10 / 25 / 93$ & $931025-076$ & $931025-078$ & $931027-002$ \\
\hline GW-751 & GRIDJ 3 & 5 & $10 / 26 / 93$ & $931026-019$ & $931026-023$ & $931027-002$ \\
\hline GW-752 & GRIDJ 3 & 5 & $10 / 26 / 93$ & $931026-017$ & $931026-023$ & $931027-002$ \\
\hline GW -753 & GRIDJ 2 & 5 & $10 / 21 / 93$ & $931021-108$ & $931021-114$ & $931023-006$ \\
\hline GW-754 & GRIDJ2 & 5 & $10 / 22 / 93$ & $931022-046$ & $931022-048$ & $931025-031$ \\
\hline GW -755 & GRIDJ 1 & 5 & $10 / 21 / 93$ & $931021-110$ & $931021-114$ & $931023-006$ \\
\hline$G W-756$ & GRIDJ 1 & 5 & $10 / 21 / 93$ & $931021-106$ & $931021-114$ & $931025-031$ \\
\hline
\end{tabular}


APPENDIX I. 1

Correlation of Samples to Associated Blanks

\begin{tabular}{|c|c|c|c|c|c|c|}
\hline $\begin{array}{l}\text { sampling } \\
\text { pt. }\end{array}$ & Location & $\begin{array}{l}\text { Pump } \\
\text { No. }\end{array}$ & Date & $\begin{array}{c}\text { Sample } \\
\text { No. }\end{array}$ & $\begin{array}{c}\text { Trip Blank } \\
\text { No. }\end{array}$ & $\begin{array}{c}\text { Lab Blank } \\
\text { No. }\end{array}$ \\
\hline$G W-758$ & GRIDGI & 8 & $11 / 19 / 93$ & $931119-108$ & $931119-114$ & $931123-001$ \\
\hline$G W-759$ & GRIDG 1 & 8 & $11 / 19 / 93$ & $931119-110$ & $931119-114$ & $931123-053$ \\
\hline GW -760 & GRIDG2 & 8 & $11 / 30 / 93$ & $931130-063$ & $931130-065$ & $931201-064$ \\
\hline GW -761 & GRIDG2 & 8 & $11 / 29 / 93$ & $931129-127$ & $931129-129$ & $931130-055$ \\
\hline GW -762 & GRIDJ 3 & 5 & $10 / 28 / 93$ & $931028-076$ & $931028-080$ & $931029-026$ \\
\hline GW -763 & GRIDJ3 & 5 & $18 / 29 / 93$ & $931029-069$ & $931029-071$ & $931101-042$ \\
\hline$G W-764$ & GRIDE 1 & 8 & $11 / 12 / 93$ & $931112-080$ & $931112-079$ & $931116-035$ \\
\hline$G W-765$ & GRIDE 1 & 8 & $11 / 19 / 93$ & $931119-112$ & $931119-114$ & $931124-240$ \\
\hline$G W-766$ & GRIDI2 & 7 & $10 / 13 / 93$ & $931013-068$ & $931013-072$ & $931014-010$ \\
\hline GW-767 & GRIDI2 & 7 & $10 / 13 / 93$ & $931013-064$ & $931013-072$ & $931014-010$ \\
\hline GW-768 & GRIDI 1 & 7 & $10 / 13 / 93$ & $931013-066$ & $931013-072$ & $931014-010$ \\
\hline GW-769 & GRIDG 3 & 8 & $11 / 30 / 93$ & $931130-061$ & $931130-065$ & $931201-064$ \\
\hline$G W-770$ & GRIDG 3 & 8 & $11 / 29 / 93$ & $931129-125$ & $931129-129$ & $931130-055$ \\
\hline GW-771 & GRIDC 1 & 8 & $11 / 11 / 93$ & $931111-109$ & $931111-111$ & $931116-002$ \\
\hline GW -772 & GRIDC 1 & 8 & $11 / 12 / 93$ & $931112-077$ & $931112-079$ & $931117-001$ \\
\hline GW -773 & GRIDH2 & 7 & $10 / 15 / 93$ & $931015-226$ & $931015-228$ & $931018-196$ \\
\hline GW -774 & GRIDH2 & 7 & $10 / 15 / 93$ & $931015-224$ & $931015-228$ & $931018-196$ \\
\hline$G W-775$ & GRIDH 3 & 7 & $10 / 15 / 93$ & $931015-222$ & $931015-228$ & $931018-196$ \\
\hline GW -776 & GRIDH 3 & 7 & $10 / 18 / 93$ & $931018-202$ & $931018-204$ & $931019-038$ \\
\hline
\end{tabular}


APPENDIX L.2

TRIP BLANK DATA SUMMARY 
APPENDIX 1.2

1993 Trip Blank Data Summary

\begin{tabular}{|c|c|c|c|c|c|c|c|c|c|c|}
\hline \multirow{2}{*}{ Compound } & \multicolumn{2}{|c|}{ Finst } & \multicolumn{2}{|c|}{ Second } & \multicolumn{2}{|c|}{ Thind } & \multicolumn{2}{|c|}{ Fourth } & \multicolumn{2}{|c|}{ Total } \\
\hline & No. & $\%$ & No. & $\%$ & No. & $\%$ & No. & $\%$ & No. & $\%$ \\
\hline Total Samples & 50 & & 50 & & 46 & & 51 & & 197 & \\
\hline Not Detected & 22 & 44 & 34 & 68 & 36 & 78 & 21 & 41 & 113 & 57 \\
\hline VOCs Detected & 28 & 56 & 16 & 32 & 10 & 22 & 30 & 59 & 84 & 43 \\
\hline Methylene chloride & 11 & 22 & 14 & 28 & 6 & 13 & 21 & 41 & 52 & 26 \\
\hline Acetone & 15 & 30 & 1 & 2 & 4 & 9 & 20 & 39 & 40 & 20 \\
\hline 4-Methyl-2-pentanone & 19 & 38 & 1 & 2 & & & 2 & 4 & 22 & 11 \\
\hline 2-Butanone & 1 & 2 & 1 & 2 & & & 5 & 10 & 7 & 4 \\
\hline Benzene & & & & & 2 & 4 & & & 2 & 1 \\
\hline Trichloroethene & & & 1 & 2 & 1 & 2 & & & 2 & 1 \\
\hline Carbon disulfide & & & & & 1 & 2 & & & 1 & 1 \\
\hline Chloroform & & & 1 & 2 & & & & & 1 & 1 \\
\hline Xylenes & & & & & 1 & 2 & & & 1 & 1 \\
\hline
\end{tabular}

PC12.TAB/2E200125 
APPENDIX 1.2

VoCs Detected in Trip Blank Samples, First Quarter 1993

\begin{tabular}{|c|c|c|c|c|c|c|c|c|}
\hline Sample No. & $\begin{array}{c}930108- \\
137\end{array}$ & $\begin{array}{c}930108- \\
140\end{array}$ & $\begin{array}{c}930111- \\
091\end{array}$ & $\begin{array}{c}930111- \\
131\end{array}$ & $\begin{array}{c}930111- \\
148\end{array}$ & $\begin{array}{c}930112- \\
061\end{array}$ & $\begin{array}{c}930112- \\
087\end{array}$ & $\begin{array}{c}930112- \\
102\end{array}$ \\
\hline Acetone & 100 & 100 & 100 & 2 & 100 & 2 & 100 & 100 \\
\hline 2-Butanone & 100 & 100 & 100 & 100 & 100 & 100 & 100 & 100 \\
\hline 4-Methyl-2-pentanone & 100 & 100 & 100 & 2 & 2 & 2 & 100 & 100 \\
\hline Methylene chloride & 2 & 3 & 3 & 1 & $5 U$ & $5 U$ & 50 & 2 \\
\hline
\end{tabular}

(CONTINUED)

\begin{tabular}{|c|c|c|c|c|c|c|c|c|}
\hline Sample No. & $\begin{array}{c}930113- \\
117\end{array}$ & $\begin{array}{c}930113- \\
120\end{array}$ & $\begin{array}{c}930114- \\
030\end{array}$ & $\begin{array}{c}930114- \\
220\end{array}$ & $\begin{array}{c}930114- \\
225\end{array}$ & $\begin{array}{c}930115- \\
099\end{array}$ & $\begin{array}{c}930118- \\
105\end{array}$ & $\begin{array}{c}930119- \\
070\end{array}$ \\
\hline Acetone & 100 & 100 & 2 & 100 & 1 & 100 & 100 & 100 \\
\hline 2-Butanone & 100 & 100 & 100 & 100 & 100 & 100 & 100 & 100 \\
\hline 4-Methyl-2-pentanone & 100 & 100 & 2 & 2 & 1 & 100 & 100 & 100 \\
\hline Methylene chloride & 50 & $5 U$ & $5 U$ & 50 & 50 & 50 & 50 & $5 \mathrm{U}$ \\
\hline
\end{tabular}

(CONTINUED)

\begin{tabular}{|c|c|c|c|c|c|c|c|c|}
\hline Sample No. & $\begin{array}{c}930120- \\
011\end{array}$ & $\begin{array}{c}930120- \\
100\end{array}$ & $\begin{array}{c}930121- \\
018\end{array}$ & $\begin{array}{c}930121- \\
062\end{array}$ & $\begin{array}{c}930121- \\
082\end{array}$ & $\begin{array}{c}930122- \\
072\end{array}$ & $\begin{array}{c}930123- \\
018\end{array}$ & $\begin{array}{c}930123- \\
025\end{array}$ \\
\hline Acetone & 3 & 1 & 100 & 100 & 100 & 2 & 2 & 100 \\
\hline 2-Butanone & 100 & 100 & 100 & 100 & 100 & 100 & 100 & 100 \\
\hline 4-Methy1-2-pentanone & 2 & 2 & 100 & 100 & 100 & 2 & 1 & 100 \\
\hline Methylene chloride & 50 & 50 & $5 U$ & 50 & 50 & 50 & 50 & 50 \\
\hline
\end{tabular}

(CONTINUED)

\begin{tabular}{|c|c|c|c|c|c|c|c|c|}
\hline Sample No. & $\begin{array}{c}930123- \\
033\end{array}$ & $\begin{array}{c}930125- \\
238\end{array}$ & $\begin{array}{c}930125- \\
262\end{array}$ & $\begin{array}{c}930126- \\
076\end{array}$ & $\begin{array}{c}930126- \\
096\end{array}$ & $\begin{array}{c}930126- \\
112\end{array}$ & $\begin{array}{c}930127- \\
090\end{array}$ & $\begin{array}{c}930128- \\
018\end{array}$ \\
\hline Acetone & 100 & 220 & 31 & 33 & 100 & 100 & 2 & 100 \\
\hline 2-Butanone & 100 & 100 & 100 & 100 & 100 & 100 & 100 & 100 \\
\hline 4-Methy1-2-pentanone & 100 & 100 & 2 & 1 & 100 & 100 & 2 & 2 \\
\hline Methylene chloride & 50 & 50 & $5 \mathbf{S}$ & $5 u$ & 15 & 1 & 5U & 50 \\
\hline
\end{tabular}

(CONTINUED) 
APPENDIX I. 2

vocs Detected in Trip Blank Samples, First Quarter 1993

\begin{tabular}{|c|c|c|c|c|c|c|c|c|}
\hline Sample No. & $\begin{array}{c}930128- \\
203\end{array}$ & $\begin{array}{c}930129- \\
096\end{array}$ & $\begin{array}{c}930129- \\
116\end{array}$ & $\begin{array}{c}930201- \\
091\end{array}$ & $\begin{array}{c}930201- \\
1.16\end{array}$ & $\begin{array}{c}930203- \\
017\end{array}$ & $\begin{array}{c}930203- \\
030\end{array}$ & $\begin{array}{c}930203- \\
033\end{array}$ \\
\hline Acetone & 100 & 100 & 100 & 1 & 2 & $10 \mathrm{U}$ & 100 & 100 \\
\hline 2-Butanone & 100 & 100 & 100 & 100 & 100 & 3 & 100 & 100 \\
\hline 4-Methyl-2-pentanone & 100 & 100 & 100 & 2 & 2 & 2 & 100 & 100 \\
\hline Methylene chloride & $5 \mathbf{u}$ & $5 u$ & $5 v$ & 50 & $5 \mathbf{U}$ & $5 U$ & 0.7 & 50 \\
\hline
\end{tabular}

(CONTINUED)

\begin{tabular}{|c|c|c|c|c|c|c|c|c|}
\hline Sample No. & $\begin{array}{c}930203- \\
093\end{array}$ & $\begin{array}{c}930203- \\
128\end{array}$ & $\begin{array}{c}930205- \\
018\end{array}$ & $\begin{array}{c}930222- \\
103\end{array}$ & $\begin{array}{c}930302- \\
007\end{array}$ & $\begin{array}{c}930303- \\
013\end{array}$ & $\begin{array}{c}930303- \\
114\end{array}$ & $\begin{array}{c}930305- \\
042\end{array}$ \\
\hline Acetone & 6 & 100 & $10 u$ & 100 & 100 & 100 & 100 & 100 \\
\hline 2-Butanone & 100 & 100 & 100 & 100 & $10 \mathrm{u}$ & 100 & 100 & 100 \\
\hline 4-Methyl-2-pentanone & 2 & 2 & $10 u$ & 100 & 100 & 100 & 100 & 100 \\
\hline Methylene chloride & 2 & 2 & $5 u$ & 1 & $5 u$ & 50 & $5 u$ & 50 \\
\hline
\end{tabular}

(CONTINUED)

\begin{tabular}{|l|r|r|}
\hline Sample No. & $\begin{array}{r}930311- \\
021\end{array}$ & $\begin{array}{c}930312- \\
012\end{array}$ \\
\hline Acetone & $10 \mathrm{U}$ & $10 \mathrm{U}$ \\
2-Butanone & $10 \mathrm{U}$ & $10 \mathrm{U}$ \\
4-Methyl-2-pentanone & $10 \mathrm{U}$ & $10 \mathrm{U}$ \\
Methylene chloride & $5 \mathrm{U}$ & $5 \mathrm{U}$ \\
\hline
\end{tabular}


APPENDIX I.2

Trip Blank Data Summary, Second Quarter 1993

\begin{tabular}{|c|c|c|c|c|c|c|c|c|}
\hline Sample No. & $\begin{array}{c}930407- \\
099\end{array}$ & $\begin{array}{c}930407- \\
104\end{array}$ & $\begin{array}{c}930407- \\
107\end{array}$ & $\begin{array}{l}930408- \\
301\end{array}$ & $\begin{array}{c}930408- \\
319\end{array}$ & $\begin{array}{c}930412- \\
113\end{array}$ & $\begin{array}{c}930412- \\
121\end{array}$ & $\begin{array}{c}930412- \\
128\end{array}$ \\
\hline Acetone & $10 \mathrm{U}$ & 100 & 100 & 100 & 100 & 100 & 100 & 100 \\
\hline 2-Butanone & 100 & 100 & 100 & 100 & 100 & 100 & $10 \mathrm{U}$ & 100 \\
\hline Cisloroform & 50 & $5 U$ & $5 \mathrm{U}$ & $5 U$ & $5 U$ & 50 & 50 & 50 \\
\hline 4-Methyl-2-pentanone & 100 & $10 \mathrm{U}$ & 100 & 100 & 100 & 100 & $10 \mathrm{U}$ & 100 \\
\hline Methylene chloride & 2 & $5 U$ & 6 & $5 U$ & $5 \mathrm{U}$ & 1 & $5 U$ & $5 U$ \\
\hline Trichloroethene & 50 & $5 U$ & $5 U$ & $5 \mathrm{U}$ & 50 & 50 & 50 & $5 U$ \\
\hline
\end{tabular}

(CONTINUED)

\begin{tabular}{|c|c|c|c|c|c|c|c|c|}
\hline Sample No. & $\begin{array}{c}930413- \\
083\end{array}$ & $\begin{array}{c}930414- \\
018\end{array}$ & $\begin{array}{c}930414- \\
019\end{array}$ & $\begin{array}{c}930414- \\
128\end{array}$ & $\begin{array}{c}930415- \\
037\end{array}$ & $\begin{array}{c}930415- \\
215\end{array}$ & $\begin{array}{c}930415- \\
273\end{array}$ & $\begin{array}{c}930416- \\
075\end{array}$ \\
\hline Acetone & 100 & 100 & $10 \mathrm{U}$ & $10 \mathrm{U}$ & 100 & 100 & 100 & 100 \\
\hline 2-Butanone & $10 \mathrm{U}$ & 100 & 100 & 100 & 100 & 100 & $10 \mathrm{U}$ & 100 \\
\hline Chloroform & 50 & 50 & 50 & $5 U$ & 50 & 50 & $5 U$ & 50 \\
\hline 4-Methyl-2-pentanone & 100 & 100 & 100 & 100 & 100 & 100 & 100 & 100 \\
\hline Methylene chloride & $5 U$ & 50 & $5 U$ & $5 U$ & $5 \mathbf{v}$ & 50 & $5 U$ & $5 U$ \\
\hline Trichloroethene & 50 & 50 & 50 & 50 & 50 & 50 & $5 v$ & 50 \\
\hline
\end{tabular}

(CONTINUED)

\begin{tabular}{|c|c|c|c|c|c|c|c|c|}
\hline Sample No. & $\begin{array}{l}930416- \\
102\end{array}$ & $\begin{array}{c}930416- \\
125\end{array}$ & $\begin{array}{c}930419- \\
067\end{array}$ & $\begin{array}{c}930419- \\
082\end{array}$ & $\begin{array}{c}930420- \\
011\end{array}$ & $\begin{array}{c}930420- \\
070\end{array}$ & $\begin{array}{c}930421- \\
007\end{array}$ & $\begin{array}{c}930422- \\
026\end{array}$ \\
\hline Acetone & 100 & 100 & 100 & 100 & 100 & 100 & 100 & 200 \\
\hline 2-Butanone & 100 & 100 & 100 & 100 & 100 & 100 & 100 & 100 \\
\hline Chloroform & $5 U$ & 50 & 50 & $5 \mathrm{U}$ & $5 U$ & 50 & 50 & $5 U$ \\
\hline 4-Methyl-2-pentanone & 100 & $10 U$ & 100 & 100 & $10 \mathrm{U}$ & 2 & 100 & 100 \\
\hline Methylene chloride & $5 U$ & $5 U$ & 2 & 1 & $5 U$ & 1 & 50 & 50 \\
\hline Trichloroethene & 50 & 50 & 50 & 0.8 & $5 u$ & 50 & 50 & $5 U$ \\
\hline
\end{tabular}

(CONTINUED)

\begin{tabular}{|c|c|c|c|c|c|c|c|c|}
\hline Sample No. & $\begin{array}{c}930423- \\
010\end{array}$ & $\begin{array}{c}930427- \\
022\end{array}$ & $\begin{array}{c}930503- \\
164\end{array}$ & $\begin{array}{c}930504- \\
0: 1\end{array}$ & $\begin{array}{c}930504- \\
076\end{array}$ & $\begin{array}{c}930504- \\
079\end{array}$ & $\begin{array}{c}930505- \\
137\end{array}$ & $\begin{array}{c}930506- \\
055\end{array}$ \\
\hline retone & 100 & 100 & 100 & 100 & 100 & 100 & 100 & 100 \\
\hline -Butanone & 100 & 100 & 100 & 100 & 100 & 100 & 100 & 100 \\
\hline Chloroform & $5 U$ & 50 & 50 & $5 \mathrm{U}$ & $5 U$ & 50 & $5 U$ & $5 U$ \\
\hline 4-Methyl-2-pentanone & 100 & 100 & 100 & 100 & 100 & 100 & 100 & 100 \\
\hline Methylene chloride & $5 U$ & $5 U$ & 50 & $5 \mathrm{U}$ & $5 \mathrm{U}$ & 50 & $5 U$ & $5 U$ \\
\hline Trichloroethene & $5 U$ & $5 \mathbf{U}$ & 50 & 50 & $5 \mathbf{u}$ & $5 \mathrm{U}$ & $5 U$ & $5 U$ \\
\hline
\end{tabular}


APPENDIX I. 2

Trip Blank Data Summary, Second Quarter 1993

\begin{tabular}{|c|c|c|c|c|c|c|c|c|}
\hline Sample No. & $\begin{array}{c}930506- \\
300\end{array}$ & $\begin{array}{c}930506- \\
303\end{array}$ & $\begin{array}{c}930507- \\
124\end{array}$ & $\begin{array}{c}930507- \\
130\end{array}$ & $\begin{array}{c}930511- \\
022\end{array}$ & $\begin{array}{c}930513- \\
358\end{array}$ & $\begin{array}{c}930514- \\
080\end{array}$ & $\begin{array}{c}930521- \\
065\end{array}$ \\
\hline Acetone & 9 & 100 & 100 & 100 & 100 & 100 & 100 & 100 \\
\hline 2-Butanone & 100 & 100 & 100 & 100 & 100 & 100 & 100 & 100 \\
\hline Chloroform & $5 \mathbf{U}$ & 50 & $5 \mathbf{U}$ & 50 & 50 & $5 U$ & 50 & $5 U$ \\
\hline 4-Methyl-2-pentanone & 100 & 100 & 100 & $10 \mathrm{U}$ & 100 & 100 & 100 & 100 \\
\hline Methylene chloride & 0.8 & 50 & 0.8 & $5 U$ & $5 v$ & 2 & 2 & $5 U$ \\
\hline Trichloroethene & $5 U$ & 50 & 50 & 50 & 50 & 50 & $5 \mathbf{U}$ & su \\
\hline
\end{tabular}

( CONTINUED)

\begin{tabular}{|c|c|c|c|c|c|c|c|c|}
\hline Sample No. & $\begin{array}{c}930524- \\
139\end{array}$ & $\begin{array}{c}930617- \\
178\end{array}$ & $\begin{array}{c}930618- \\
213\end{array}$ & $\begin{array}{c}930618- \\
249\end{array}$ & $\begin{array}{c}930621- \\
110\end{array}$ & $\begin{array}{c}930622- \\
016\end{array}$ & $\begin{array}{c}930622- \\
167\end{array}$ & $\begin{array}{c}930624- \\
012\end{array}$ \\
\hline Acetone & 100 & 100 & 100 & 100 & 100 & 100 & 100 & 100 \\
\hline 2-Butanone & 4 & 100 & 100 & 100 & 100 & 100 & 100 & 100 \\
\hline Chloroform & 50 & 50 & 50 & 50 & 50 & 50 & 50 & 0.4 \\
\hline 4-Methy:-2-pentanone & 100 & 100 & 100 & 100 & $10 U$ & 100 & 100 & 100 \\
\hline Methylene chloride & $5 \mathbf{S}$ & $5 \mathrm{U}$ & 2 & 2 & 2 & $5 U$ & $5 \mathrm{U}$ & 50 \\
\hline Trichloroethene & $5 U$ & 50 & 50 & $5 U$ & 50 & $5 U$ & $5 U$ & 50 \\
\hline
\end{tabular}

(CONTINUED)

\begin{tabular}{|l|r|r|}
\hline Sample No. & $\begin{array}{r}930624- \\
022\end{array}$ & $\begin{array}{c}930630- \\
010\end{array}$ \\
\hline Acetone & $10 \mathrm{U}$ & $10 \mathrm{U}$ \\
2-Butanone & $10 \mathrm{U}$ & $10 \mathrm{U}$ \\
Chloroform & $5 \mathrm{U}$ & $5 \mathrm{U}$ \\
4-Methyl-2-pentanone & $10 \mathrm{U}$ & $10 \mathrm{U}$ \\
Methylene chloride & $5 \mathrm{U}$ & 1 \\
Trichloroethene & $5 \mathrm{U}$ & $5 \mathrm{U}$ \\
\hline
\end{tabular}


APPENDIX I.2

Trip Blank Data Sumnary, Third Quarter 1993

\begin{tabular}{|c|c|c|c|c|c|c|c|c|}
\hline Sample No. & $\begin{array}{c}930702- \\
019\end{array}$ & $\begin{array}{c}930702- \\
102\end{array}$ & $\begin{array}{c}930709- \\
008\end{array}$ & $\begin{array}{c}930802- \\
130\end{array}$ & $\begin{array}{c}930802- \\
139\end{array}$ & $\begin{array}{c}930804- \\
015\end{array}$ & $\begin{array}{c}930804- \\
016\end{array}$ & $\begin{array}{c}930804- \\
017\end{array}$ \\
\hline Acetone & $10 U$ & $10 U$ & 100 & $10 U$ & $10 U$ & 20 & 18 & 100 \\
\hline Benzene & $5 U$ & $5 U$ & $5 v$ & $5 U$ & $5 U$ & $5 \mathbf{U}$ & $5 U$ & 50 \\
\hline Carbon disulfide & 50 & $5 U$ & $5 v$ & $5 U$ & $5 U$ & $5 U$ & 50 & 50 \\
\hline Methylene chloride & $5 v$ & $5 \%$ & 50 & 50 & $5 U$ & $5 \mathrm{U}$ & 50 & $5 U$ \\
\hline Trichloroethene & 50 & घU & 5 's & 50 & 50 & $5 \mathbf{U}$ & $5 U$ & 50 \\
\hline XYlenes & 50 & $5 \mathbf{U}$ & $5 U$ & 50 & $5 U$ & $5 U$ & $5 \mathrm{U}$ & $5 U$ \\
\hline
\end{tabular}

( CONTINUED)

\begin{tabular}{|c|c|c|c|c|c|c|c|c|}
\hline Sample No. & $\begin{array}{l}930804- \\
090\end{array}$ & $\begin{array}{c}930804- \\
110\end{array}$ & $\begin{array}{c}930805- \\
064\end{array}$ & $\begin{array}{c}930805- \\
415\end{array}$ & $\begin{array}{c}930805- \\
420\end{array}$ & $\begin{array}{c}930805- \\
427\end{array}$ & $\begin{array}{c}930806- \\
083\end{array}$ & $\begin{array}{c}930806- \\
124\end{array}$ \\
\hline Acetone & 100 & 100 & 100 & 100 & 100 & 100 & 100 & $10 \mathrm{U}$ \\
\hline Benzene & $5 U$ & $5 U$ & $5 \mathrm{U}$ & 50 & $5 \mathrm{U}$ & $5 \mathrm{U}$ & 50 & $5 U$ \\
\hline Carbon disulfide & 50 & 50 & 50 & $5 U$ & 50 & 50 & $5 \mathbf{U}$ & $5 U$ \\
\hline Methylene chloride & 50 & $5 U$ & $5 \mathbf{U}$ & $5 U$ & 50 & $5 U$ & 50 & $5 U$ \\
\hline Trichloroethene & $5 \mathrm{U}$ & $5 U$ & 50 & 50 & 50 & 50 & $5 U$ & 50 \\
\hline Xylenes & 50 & $5 \mathbf{v}$ & $5 U$ & $5 \mathbf{v}$ & 50 & $5 U$ & 50 & 50 \\
\hline
\end{tabular}

(CONTINUED)

\begin{tabular}{|c|c|c|c|c|c|c|c|c|}
\hline Sample No. & $\begin{array}{c}930806- \\
164\end{array}$ & $\begin{array}{c}930809- \\
016\end{array}$ & $\begin{array}{c}930809- \\
021\end{array}$ & $\begin{array}{c}930810- \\
007\end{array}$ & $\begin{array}{c}930810- \\
119\end{array}$ & $\begin{array}{c}930812- \\
009\end{array}$ & $\begin{array}{c}930813- \\
009\end{array}$ & $\begin{array}{c}930813- \\
166\end{array}$ \\
\hline Acetone & 100 & 100 & 100 & 100 & 100 & $10 \mathrm{U}$ & 100 & 100 \\
\hline Benzene & 50 & $5 v$ & $5 \mathbf{v}$ & 50 & 50 & $5 U$ & $5 v$ & $5 U$ \\
\hline Carbon disulfide & 50 & $5 \mathrm{~J}$ & $5 \mathbf{U}$ & 50 & 50 & 50 & $5 U$ & $5 \mathbf{U}$ \\
\hline Methylene chloride & 50 & 50 & $5 U$ & 50 & $5 \mathbf{u}$ & $5 U$ & 50 & $5 U$ \\
\hline Trichloroethene & $5 U$ & 50 & $5 U$ & 50 & 50 & $5 U$ & $5 U$ & 50 \\
\hline Xylenes & $5 U$ & 50 & $5 U$ & 50 & 50 & $5 U$ & $5 \mathbf{U}$ & $5 \mathrm{~J}$ \\
\hline
\end{tabular}

(CONTINUED) 
APPENDIX I. 2

Trip Blank Data Summary, Third Quarter 1993

\begin{tabular}{|c|c|c|c|c|c|c|c|c|}
\hline Sample No. & $\begin{array}{c}930816- \\
204\end{array}$ & $\begin{array}{c}930818- \\
118\end{array}$ & $\begin{array}{c}930818- \\
119\end{array}$ & $\begin{array}{c}930819- \\
256\end{array}$ & $\begin{array}{c}930819- \\
305\end{array}$ & $\begin{array}{c}930820- \\
088\end{array}$ & $\begin{array}{c}930823- \\
112\end{array}$ & $\begin{array}{c}930903- \\
145\end{array}$ \\
\hline Acetone & 100 & 100 & 100 & 100 & 100 & 10 & 100 & 26 \\
\hline Benzene & su & 50 & 50 & $5 u$ & 50 & 50 & su & 1 \\
\hline Carbon disulfide & $5 U$ & 50 & 50 & 50 & 50 & 50 & 50 & 2 \\
\hline Methylene chloride & 1 & 1 & 50 & 1 & 1 & 1 & 2 & 50 \\
\hline Trichloroethene & $5 v$ & 50 & $5 u$ & 50 & $5 \mathrm{u}$ & 50 & $5 \mathrm{U}$ & 1 \\
\hline xylenes & 50 & 50 & 50 & 50 & $5 v$ & $5 \mathrm{v}$ & 50 & 50 \\
\hline
\end{tabular}

(CONT INUED)

\begin{tabular}{|c|c|c|c|c|c|c|c|c|}
\hline Sample No. & $\begin{array}{c}930913- \\
075\end{array}$ & $\begin{array}{l}930914- \\
008\end{array}$ & $\begin{array}{c}930915- \\
007\end{array}$ & $\begin{array}{c}930915- \\
135\end{array}$ & $\begin{array}{c}930915- \\
152\end{array}$ & $\begin{array}{c}930916- \\
132\end{array}$ & $\begin{array}{l}930917- \\
007\end{array}$ & $\begin{array}{c}930918- \\
011\end{array}$ \\
\hline Acetone & 100 & 100 & 100 & 100 & 100 & 100 & 100 & 100 \\
\hline Benzene & 50 & $5 \mathrm{U}$ & su & 50 & $5 U$ & 50 & 1 & 50 \\
\hline Carbon disulfide & 50 & 50 & 50 & 50 & 50 & 50 & $5 U$ & 50 \\
\hline Methylene chloride & 50 & 50 & 50 & $5 \mathrm{v}$ & 50 & $5 U$ & $5 U$ & 50 \\
\hline Trichloroethene & $5 u$ & 50 & 50 & $5 U$ & $5 U$ & $5 U$ & 50 & 50 \\
\hline xylenes & 50 & 50 & 50 & 50 & $5 U$ & 50 & 3 & 50 \\
\hline
\end{tabular}

(CONTINUED)

\begin{tabular}{|l|r|r|r|r|r|r|}
\hline Sample No. & $\begin{array}{l}930920- \\
166\end{array}$ & $\begin{array}{l}930921- \\
169\end{array}$ & $\begin{array}{c}930922- \\
068\end{array}$ & $\begin{array}{c}930923- \\
131\end{array}$ & $\begin{array}{c}930924- \\
013\end{array}$ & $\begin{array}{c}930927- \\
228\end{array}$ \\
\hline Acetone & $10 \mathrm{U}$ & $10 \mathrm{U}$ & $10 \mathrm{U}$ & $10 \mathrm{U}$ & $10 \mathrm{U}$ & $10 \mathrm{~V}$ \\
Benzene & $5 \mathrm{U}$ & $5 \mathrm{U}$ & $5 \mathrm{U}$ & $5 \mathrm{U}$ & $5 \mathrm{U}$ & $5 \mathrm{U}$ \\
Carbon disulfide & $5 \mathrm{U}$ & $5 \mathrm{U}$ & $5 \mathrm{U}$ & $5 \mathrm{U}$ & $5 \mathrm{U}$ & $5 \mathrm{U}$ \\
Methylene chloride & $5 \mathrm{U}$ & $5 \mathrm{U}$ & $5 \mathrm{U}$ & $5 \mathrm{U}$ & $5 \mathrm{U}$ & $5 \mathrm{U}$ \\
Trichloroethene & $5 \mathrm{U}$ & $5 \mathrm{U}$ & $5 \mathrm{U}$ & $5 \mathrm{U}$ & $5 \mathrm{U}$ & $5 \mathrm{U}$ \\
Xylenes & $5 \mathrm{U}$ & $5 \mathrm{U}$ & $5 \mathrm{U}$ & $5 \mathrm{U}$ & $5 \mathrm{U}$ & $5 \mathrm{U}$ \\
\hline
\end{tabular}


APPENDIX I.2

Trip Blank Data Summary, Fourth Quarter 1993

\begin{tabular}{|c|c|c|c|c|c|c|c|c|}
\hline Sample No. & $\begin{array}{c}931012- \\
156\end{array}$ & $\begin{array}{c}931013- \\
072\end{array}$ & $\begin{array}{c}931014- \\
056\end{array}$ & $\begin{array}{c}931015- \\
228\end{array}$ & $\begin{array}{c}931021- \\
114\end{array}$ & $\begin{array}{c}931022- \\
048\end{array}$ & $\begin{array}{c}931025- \\
008\end{array}$ & $\begin{array}{c}931025- \\
021\end{array}$ \\
\hline Acetone & 100 & 100 & 4 & 100 & 100 & 100 & 8 & 6 \\
\hline 2-Butanone & 10 & 100 & 10 & 100 & 100 & 100 & 100 & 100 \\
\hline 4-Methyl-2-pentanone & 100 & 100 & 100 & 100 & $10 U$ & 100 & 100 & 100 \\
\hline Methylene chloride & 50 & 50 & 2 & 50 & 50 & $5 U$ & 1 & 1 \\
\hline
\end{tabular}

( CONTINUED)

\begin{tabular}{|c|c|c|c|c|c|c|c|c|}
\hline Sample No. & $\begin{array}{c}931025- \\
078\end{array}$ & $\begin{array}{c}931026- \\
023\end{array}$ & $\begin{array}{c}931026- \\
035\end{array}$ & $\begin{array}{c}931027- \\
122\end{array}$ & $\begin{array}{c}931027- \\
123\end{array}$ & $\begin{array}{c}931028- \\
034\end{array}$ & $\begin{array}{c}931028- \\
075\end{array}$ & $\begin{array}{c}931028- \\
080\end{array}$ \\
\hline Acetone & 100 & 100 & 100 & 21 & 16 & 29 & 30 & 29 \\
\hline 2-Butanone & 100 & 100 & 9 & $10 \mathrm{U}$ & 100 & 100 & 100 & 100 \\
\hline 4-Methyl-2-pentanone & 100 & 100 & 100 & 100 & 100 & 100 & 100 & 100 \\
\hline Methylene chloride & $5 \mathrm{u}$ & $5 \mathbf{U}$ & 2 & $5 U$ & 50 & $5 \mathbf{U}$ & $5 U$ & $5 \mathbf{U}$ \\
\hline
\end{tabular}

(CONTINUED)

\begin{tabular}{|c|c|c|c|c|c|c|c|c|}
\hline Sample No. & $\begin{array}{c}931029- \\
057\end{array}$ & $\begin{array}{c}931029- \\
071\end{array}$ & $\begin{array}{c}931101- \\
047\end{array}$ & $\begin{array}{c}931101- \\
135\end{array}$ & $\begin{array}{c}931101- \\
150\end{array}$ & $\begin{array}{c}931102- \\
049\end{array}$ & $\begin{array}{c}931102- \\
050\end{array}$ & $\begin{array}{c}931103- \\
015\end{array}$ \\
\hline Acetone & 23 & 22 & 19 & 22 & 20 & $10 \mathrm{v}$ & 100 & 37 \\
\hline 2-Butanone & 100 & 100 & 100 & 100 & 100 & 100 & 100 & 100 \\
\hline 4-Methy1-2-pentanone & 100 & 100 & $10 \mathrm{U}$ & 100 & 100 & 100 & 100 & 100 \\
\hline Methylene chloride & 1 & 1 & 5u & 1 & 1 & 50 & 50 & 50 \\
\hline
\end{tabular}

(CONTINUED)

\begin{tabular}{|c|c|c|c|c|c|c|c|c|}
\hline Sample No. & $\begin{array}{c}931104- \\
064\end{array}$ & $\begin{array}{c}931104- \\
068\end{array}$ & $\begin{array}{c}931104- \\
074\end{array}$ & $\begin{array}{c}931108- \\
194\end{array}$ & $\begin{array}{c}931110- \\
071\end{array}$ & $\begin{array}{c}931111- \\
107\end{array}$ & $\begin{array}{c}931111- \\
111\end{array}$ & $\begin{array}{l}931112- \\
065\end{array}$ \\
\hline Acetone & 41 & 39 & 35 & 100 & 100 & 100 & 100 & 100 \\
\hline 2-Butanone & 100 & 100 & 100 & 100 & $10 \mathrm{U}$ & 100 & 100 & 100 \\
\hline 4-Methyl-2-pentanone & 100 & 100 & 100 & 100 & 100 & 100 & 100 & 100 \\
\hline Methylene chloride & $5 U$ & 0.7 & 1 & 50 & $5 \mathrm{U}$ & 2 & 50 & 1 \\
\hline
\end{tabular}

(CONTINUED) 
APPENDIX I, 2

Trip Blank Data Summary, Fourth Quarter 1993

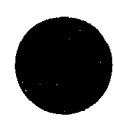

\begin{tabular}{|c|c|c|c|c|c|c|c|c|}
\hline Sample No. & $\begin{array}{c}931112- \\
079\end{array}$ & $\begin{array}{c}931115- \\
207\end{array}$ & $\begin{array}{c}931116- \\
100\end{array}$ & $\begin{array}{c}931116- \\
111\end{array}$ & $\begin{array}{c}931117- \\
044\end{array}$ & $\begin{array}{c}931117- \\
047\end{array}$ & $\begin{array}{c}931118- \\
158\end{array}$ & $\begin{array}{c}931118- \\
163\end{array}$ \\
\hline Acetone & 15 & $10 U$ & $10 U$ & 100 & $10 \mathrm{U}$ & 100 & 100 & 100 \\
\hline 2-Butanone & 100 & 100 & 100 & 100 & 100 & 100 & 100 & 100 \\
\hline 4-Methyl-2-pentanone & 100 & 100 & 100 & 100 & $10 \mathrm{U}$ & 100 & 100 & 100 \\
\hline Methylene chloride & 2 & su & 1 & 1 & $5 \mathbf{U}$ & $5 v$ & 50 & $5 \mathrm{u}$ \\
\hline
\end{tabular}

(CONTINUED)

\begin{tabular}{|c|c|c|c|c|c|c|c|c|}
\hline Sample No. & $\begin{array}{c}931119- \\
060\end{array}$ & $\begin{array}{c}931119- \\
066\end{array}$ & $\begin{array}{c}931119- \\
114\end{array}$ & $\begin{array}{c}931129- \\
129\end{array}$ & $\begin{array}{c}931130- \\
065\end{array}$ & $\begin{array}{c}931208- \\
012\end{array}$ & $\begin{array}{c}931208- \\
023\end{array}$ & $\begin{array}{c}931208- \\
092\end{array}$ \\
\hline Acetone & 100 & 100 & $10 U$ & 100 & 100 & 100 & 100 & 100 \\
\hline 2-Butar sne & 100 & 100 & 100 & 100 & 100 & 100 & 100 & 100 \\
\hline 4-Mechyl-2-pentanone & 100 & 100 & 100 & 100 & $10 U$ & 100 & 100 & 100 \\
\hline Methylene chloride & 1 & 1 & 50 & 50 & 50 & 1 & 1 & $5 U$ \\
\hline
\end{tabular}

(CONTINUED)

\begin{tabular}{|l|r|r|r|}
\hline Sample No. & $\begin{array}{c}931208- \\
097\end{array}$ & $\begin{array}{c}931209- \\
102\end{array}$ & $\begin{array}{c}931210- \\
016\end{array}$ \\
\hline Acetone & 100 & 5 & 3 \\
2-Butanone & 100 & 9 & 8 \\
4-Methyl-2-pentanone & 100 & 1 & 1 \\
Methylene chloride & $5 \mathrm{U}$ & 2 & 2 \\
\hline
\end{tabular}


APPENDIX 1.3

LABORATORY BLANK DATA SUMMARY 
APPENDIX L3

1993 Laboratory Blank Data Summary

\begin{tabular}{|c|c|c|c|c|c|c|c|c|c|c|}
\hline \multirow{2}{*}{ Compound } & \multicolumn{2}{|c|}{ First } & \multicolumn{2}{|c|}{ Second } & \multicolumn{2}{|c|}{ Thind } & \multicolumn{2}{|c|}{ Fourth } & \multicolumn{2}{|c|}{ Total } \\
\hline & No. & $\%$ & No. & $\%$ & No. & $\%$ & No. & $\%$ & No. & $\%$ \\
\hline Total Samples & 42 & & 32 & & 33 & & 36 & & 143 & \\
\hline Not Detected & 13 & 31 & 17 & 53 & 23 & 70 & 18 & 50 & 71 & 50 \\
\hline VOCs Detected & 29 & 69 & 15 & 47 & 10 & 30 & 18 & 50 & 72 & 50 \\
\hline Methylene chloride & 17 & 41 & 11 & 34 & 8 & 24 & 17 & 47 & 53 & 37 \\
\hline 4-Methyl-2-pentanone & 17 & 41 & 7 & 22 & 1 & 3 & 5 & 14 & 30 & 21 \\
\hline Acetone & 11 & 26 & & & & & 8 & 22 & 19 & 13 \\
\hline 2-Butanone & 1 & 2 & 1 & 3 & 1 & 3 & 7 & 19 & 10 & 7 \\
\hline 2-Hexanone & & & 7 & 22 & 1 & 3 & 1 & 3 & 9 & 6 \\
\hline Vinyl Acetate & & & 2 & 6 & & & & & 2 & 1 \\
\hline Benzene & & & & & 1 & 3 & & & 1 & 1 \\
\hline Styrene & & & & & & & 1 & 3 & 1 & 1 \\
\hline Toluene & & & & & 1 & 3 & & & 1 & 1 \\
\hline 1,1,2-Trichloroethane & & & 1 & 3 & & & & & 1 & 1 \\
\hline
\end{tabular}

PCU.TAB/2E20012S 
APPENDIX 1.3

VOCs Detected in Laboratory Blank Samples, First Quarter 1993

\begin{tabular}{|c|c|c|c|c|c|c|c|c|}
\hline Sample No. & $\begin{array}{c}930111- \\
008\end{array}$ & $\begin{array}{c}930111- \\
139\end{array}$ & $\begin{array}{c}930112- \\
042\end{array}$ & $\begin{array}{c}930113- \\
002\end{array}$ & $\begin{array}{c}930113- \\
147\end{array}$ & $\begin{array}{c}930114- \\
221\end{array}$ & $\begin{array}{c}930115- \\
001\end{array}$ & $\begin{array}{c}930118- \\
002\end{array}$ \\
\hline Acetone & 1 & 100 & 1 & 2 & 100 & 100 & 1 & 100 \\
\hline 2-Butanone & 100 & 100 & 100 & $10 u$ & 100 & 100 & 100 & 100 \\
\hline 4-Methyl-2-pentanone & 2 & 100 & 2 & 2 & 100 & 100 & 2 & 100 \\
\hline Methylene chloride & 50 & 2 & $5 \mathbf{U}$ & $5 \mathbf{v}$ & 2 & 3 & $5 \mathbf{u}$ & 50 \\
\hline
\end{tabular}

(CONTINUBD)

\begin{tabular}{|c|c|c|c|c|c|c|c|c|}
\hline Sample No. & $\begin{array}{c}930119- \\
012\end{array}$ & $\begin{array}{c}930119- \\
088\end{array}$ & $\begin{array}{c}930120- \\
001\end{array}$ & $\begin{array}{c}930120- \\
037\end{array}$ & $\begin{array}{c}930121- \\
029\end{array}$ & $\begin{array}{c}930121- \\
066\end{array}$ & $\begin{array}{c}930122- \\
001\end{array}$ & $\begin{array}{c}930125- \\
004\end{array}$ \\
\hline Acetono & 100 & 100 & 100 & 2 & 2 & 100 & 100 & 1 \\
\hline 2-Butanone & 100 & 100 & 100 & 100 & 100 & 100 & 100 & 100 \\
\hline 4-Methyl-2-pentanone & 100 & 100 & 100 & 2 & 3 & 100 & 100 & 3 \\
\hline Methylene chloride & 50 & 2 & 50 & 50 & 50 & 2 & 0.7 & 50 \\
\hline
\end{tabular}

(CONTINUED)

\begin{tabular}{|c|c|c|c|c|c|c|c|c|}
\hline Sample No. & $\begin{array}{c}930125- \\
263\end{array}$ & $\begin{array}{c}930126- \\
001\end{array}$ & $\begin{array}{c}930126- \\
030\end{array}$ & $\begin{array}{c}930127- \\
001\end{array}$ & $\begin{array}{c}930127- \\
005\end{array}$ & $\begin{array}{c}930128- \\
002\end{array}$ & $\begin{array}{c}930128- \\
009\end{array}$ & $\begin{array}{c}930129- \\
124\end{array}$ \\
\hline Acetone & 100 & 100 & 3 & 100 & 100 & 100 & 100 & 100 \\
\hline 2-Butanone & 100 & 100 & 100 & 100 & 100 & 100 & 100 & 100 \\
\hline 1-Methyl-2-pentanone & 100 & 100 & 2 & 100 & 1 & 100 & 2 & 100 \\
\hline thylene chloride & 2 & 50 & 50 & 50 & 50 & su & 50 & 2 \\
\hline
\end{tabular}

( CONTINUED)

\begin{tabular}{|c|c|c|c|c|c|c|c|c|}
\hline Sample No. & $\begin{array}{c}930201- \\
001\end{array}$ & $\begin{array}{c}930202- \\
004\end{array}$ & $\begin{array}{c}930202- \\
095\end{array}$ & $\begin{array}{c}930203- \\
001\end{array}$ & $\begin{array}{c}930203- \\
057\end{array}$ & $\begin{array}{c}930204- \\
034\end{array}$ & $\begin{array}{c}930205- \\
035\end{array}$ & $\begin{array}{c}930209- \\
001\end{array}$ \\
\hline Acetone & 100 & 1 & 100 & 100 & 2 & 100 & 100 & 100 \\
\hline 2-Butanone & 100 & 100 & 100 & 100 & 5 & 100 & 100 & 100 \\
\hline 4-Methy 1-2-pentanone & 100 & 2 & 100 & 100 & 2 & 2 & 2 & 100 \\
\hline Methylene chlorlde & $5 v$ & 50 & 2 & $5 U$ & 50 & 1 & 1 & su \\
\hline
\end{tabular}

(CONTINUED) 
APPENDIX 1.3

VOCs Detected in Laboratory Blank Samples, First Quarter 1993

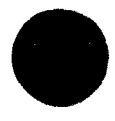

\begin{tabular}{|c|c|c|c|c|c|c|c|c|}
\hline Sample No. & $\begin{array}{c}930223- \\
001\end{array}$ & $\begin{array}{c}930302- \\
060\end{array}$ & $\begin{array}{c}930303- \\
002\end{array}$ & $\begin{array}{c}930304- \\
069\end{array}$ & $\begin{array}{c}930304- \\
076\end{array}$ & $\begin{array}{c}930308- \\
083\end{array}$ & $\begin{array}{c}930311- \\
091\end{array}$ & $\begin{array}{c}930312- \\
001\end{array}$ \\
\hline Acetone & 100 & 100 & 100 & 100 & 100 & 100 & 3 & 100 \\
\hline 2-Butanone & 100 & 100 & 100 & 100 & 100 & 100 & 100 & 100 \\
\hline 4-Methyl-2-pentanone & 100 & 100 & 2 & 100 & 100 & 100 & 3 & 100 \\
\hline Methylene chloride & 1 & su & 2 & 1 & 5u & 50 & 2 & Su \\
\hline
\end{tabular}

( CONTINURD)

\begin{tabular}{|l|r|r|}
\hline Sample No. & $\begin{array}{c}930312- \\
066\end{array}$ & $\begin{array}{c}930316- \\
001\end{array}$ \\
\hline Acetone & 100 & 100 \\
2-Butanone & 100 & 100 \\
4-Methyl-2-pentanone & 1 & 100 \\
Methylene chloride & 2 & 1 \\
\hline
\end{tabular}


APPBNDIX I. 3

voce Detected in Laboratory Blank Samples, Second Quarter 1993

\begin{tabular}{|c|c|c|c|c|c|c|c|c|}
\hline Sample No. & $\begin{array}{c}930412- \\
001\end{array}$ & $\begin{array}{c}930413- \\
001\end{array}$ & $\begin{array}{c}930414- \\
001\end{array}$ & $\begin{array}{c}930414- \\
097\end{array}$ & $\begin{array}{c}930415- \\
001\end{array}$ & $\begin{array}{c}930415- \\
050\end{array}$ & $\begin{array}{c}930416- \\
008\end{array}$ & $\begin{array}{c}930416- \\
061\end{array}$ \\
\hline 2-Butanone & 100 & 100 & 100 & 100 & 100 & 100 & 100 & 100 \\
\hline 2-Hexanone & 100 & 100 & 100 & 100 & 100 & $10 \mathrm{U}$ & 100 & 100 \\
\hline 4-Methyl-2-pentanone & 100 & 100 & 100 & $10 \mathrm{u}$ & 100 & 100 & 100 & 100 \\
\hline Methylene chlorlde & $5 v$ & $\mathbf{5 u}$ & $5 \mathbf{U}$ & $5 \mathbf{u}$ & su & su & 1 & $\mathbf{5 u}$ \\
\hline $1,1,2-\operatorname{Tr} 1 \mathrm{chl}$ oroethane & 50 & su & $5 \mathbf{u}$ & $5 U$ & 50 & SU & $5 \mathbf{u}$ & 50 \\
\hline VInyl acetate & 100 & 100 & 100 & 100 & 100 & 100 & 100 & 100 \\
\hline
\end{tabular}

( CONTINURD)

\begin{tabular}{|c|c|c|c|c|c|c|c|c|}
\hline Sample No. & $\begin{array}{c}930419- \\
001\end{array}$ & $\begin{array}{c}930420- \\
001\end{array}$ & $\begin{array}{c}930421- \\
001\end{array}$ & $\begin{array}{c}930421- \\
113\end{array}$ & $\begin{array}{c}930422- \\
001\end{array}$ & $\begin{array}{c}930423- \\
001\end{array}$ & $\begin{array}{l}930428- \\
001\end{array}$ & $\begin{array}{c}930507- \\
100\end{array}$ \\
\hline 2-Butanone & 100 & 100 & 100 & 100 & 100 & $10 \mathrm{U}$ & 100 & 100 \\
\hline 2-Hexanone & 100 & 100 & 100 & 5 & 100 & 100 & 100 & 1 \\
\hline 4-Methyl-2-pentanone & 100 & 100 & 100 & 3 & 100 & $10 \mathrm{U}$ & 100 & 2 \\
\hline Methylene chloride & su & 1 & 1 & $5 \mathrm{v}$ & $\mathbf{s u}$ & $5 \mathbf{u}$ & 1 & 2 \\
\hline 1,1,2-Trichloroethane & su & $\mathbf{5 u}$ & $\mathbf{5 u}$ & $5 \mathrm{U}$ & $\mathbf{5 u}$ & $5 \mathbf{u}$ & $5 \mathbf{u}$ & $\mathbf{5 u}$ \\
\hline Vingl acetate & 100 & 100 & 100 & 1 & 100 & 100 & 100 & 100 \\
\hline
\end{tabular}

(CONTINUED)

\begin{tabular}{|c|c|c|c|c|c|c|c|c|}
\hline Sample Ho. & $\begin{array}{c}930508- \\
020\end{array}$ & $\begin{array}{c}930509- \\
001\end{array}$ & $\begin{array}{c}930510- \\
088\end{array}$ & $\begin{array}{c}930511- \\
041\end{array}$ & $\begin{array}{c}930511- \\
065\end{array}$ & $\begin{array}{c}930513- \\
001\end{array}$ & $\begin{array}{c}930513- \\
045\end{array}$ & $\begin{array}{l}930514- \\
020\end{array}$ \\
\hline 2-Butanone & 100 & 100 & 100 & 100 & 100 & $10 u$ & 100 & 100 \\
\hline 2-Hexanone & 100 & 100 & 3 & 3 & 100 & 100 & 5 & 2 \\
\hline 4-Methy 1-2-pentanone & 100 & 100 & 3 & 2 & 200 & 100 & 5 & 3 \\
\hline Methylene chloride & 1 & $\mathbf{5 u}$ & 2 & 2 & su & 1 & 50 & 2 \\
\hline 1,1,2-Trichloroethane & su & 5u & $\mathbf{5 u}$ & so & so & $5 u$ & $5 v$ & $\mathbf{5 u}$ \\
\hline VInyl acetate & 100 & 100 & 100 & 100 & 100 & 100 & 100 & 200 \\
\hline
\end{tabular}

(CONTINUED)

\begin{tabular}{|c|c|c|c|c|c|c|c|c|}
\hline Sample No. & $\begin{array}{c}930518- \\
127\end{array}$ & $\begin{array}{c}930522- \\
012\end{array}$ & $\begin{array}{c}930526- \\
019\end{array}$ & $\begin{array}{c}930618- \\
178\end{array}$ & $\begin{array}{c}930619- \\
007\end{array}$ & $\begin{array}{c}930621- \\
002\end{array}$ & $\begin{array}{c}930623 \ldots \\
001\end{array}$ & $\begin{array}{l}930625- \\
001\end{array}$ \\
\hline 2-Butanone & 100 & 100 & 5 & 100 & $10 u$ & 100 & 100 & $10 U$ \\
\hline 2-Hexanone & 100 & 100 & 1 & 100 & 100 & 100 & ou & 100 \\
\hline 4-Methy 1-2-pentanone & 100 & 100 & 2 & 100 & 100 & 100 & 100 & 100 \\
\hline Methylene chloride & 50 & $5 U$ & $5 v$ & su & $\mathbf{s u}$ & 1 & $5 U$ & $5 \mathrm{u}$ \\
\hline 1,2,2-Trichloroethane & 1 & $5 U$ & $5 U$ & su & so & $5 U$ & $5 U$ & $5 u$ \\
\hline Vinyl acetate & 5 & 100 & 100 & 100 & 100 & 100 & 100 & 100 \\
\hline
\end{tabular}


APPENDIX I.3

vocs Detected in Laboratory Blank Samples, Third Quarter 1993

\begin{tabular}{|c|c|c|c|c|c|c|c|c|}
\hline Sample No. & $\begin{array}{c}930707- \\
032\end{array}$ & $\begin{array}{c}930707- \\
090\end{array}$ & $\begin{array}{c}930709- \\
031\end{array}$ & $\begin{array}{c}930803- \\
001\end{array}$ & $\begin{array}{c}930804- \\
035\end{array}$ & $\begin{array}{c}930805- \\
116\end{array}$ & $\begin{array}{c}930806- \\
047\end{array}$ & $\begin{array}{c}930807- \\
045\end{array}$ \\
\hline Benzene & $5 v$ & 50 & 50 & $5 U$ & 50 & $5 \mathrm{U}$ & $5 U$ & $5 U$ \\
\hline 2-Butanone & 100 & 100 & 100 & 100 & 100 & 100 & 100 & 100 \\
\hline 2-Hexanone & 100 & 3 & 100 & 100 & 100 & 100 & 100 & 100 \\
\hline 4-Methy 1-2-pentanone & 100 & 3 & 100 & 100 & 100 & 100 & 100 & 100 \\
\hline Methylene chloride & $5 u$ & 3 & su & 1 & $\mathbf{s u}$ & $5 \mathbf{5 u}$ & 5u & 50 \\
\hline Toluene & su & $5 \mathbf{v}$ & 50 & 5u & $5 v$ & 5u & 50 & 50 \\
\hline
\end{tabular}

(CONTINUED)

\begin{tabular}{|c|c|c|c|c|c|c|c|c|}
\hline Sample No. & $\begin{array}{c}930809- \\
070\end{array}$ & $\begin{array}{c}930810- \\
039\end{array}$ & $\begin{array}{c}930811- \\
031\end{array}$ & $\begin{array}{c}930812- \\
271\end{array}$ & $\begin{array}{c}930816- \\
088\end{array}$ & $\begin{array}{c}930816- \\
126\end{array}$ & $\begin{array}{c}930818- \\
001\end{array}$ & $\begin{array}{c}930820- \\
001\end{array}$ \\
\hline Benzene & 5u & 50 & so & $5 \mathbf{v}$ & 50 & $5 \mathbf{u}$ & $5 \mathbf{u}$ & 50 \\
\hline 2-Butanone & 100 & 100 & 100 & 100 & $10 U$ & 100 & 100 & 100 \\
\hline 2-Hexanone & $10 v$ & 100 & 100 & $10 U$ & 100 & $10 U$ & 100 & 100 \\
\hline 4-Methy 1-2-pentanone & 100 & 100 & 100 & 100 & 100 & 100 & 200 & 100 \\
\hline Methylane chloride & $5 \mathbf{v}$ & su & $5 \mathbf{U}$ & $5 \mathbf{U}$ & $\mathbf{5 v}$ & 1 & 1 & 2 \\
\hline Toluene & 50 & su & 50 & $5 U$ & 50 & $5 \mathbf{s}$ & $5 u$ & 50 \\
\hline
\end{tabular}

(CONTINUED)

\begin{tabular}{|c|c|c|c|c|c|c|c|c|}
\hline Sample No. & $\begin{array}{c}930823- \\
002\end{array}$ & $\begin{array}{c}930825- \\
035\end{array}$ & $\begin{array}{c}930908- \\
076\end{array}$ & $\begin{array}{l}930915- \\
107\end{array}$ & $\begin{array}{c}930915- \\
110\end{array}$ & $\begin{array}{c}930916- \\
020\end{array}$ & $\begin{array}{c}930916- \\
031\end{array}$ & $\begin{array}{c}930917- \\
070\end{array}$ \\
\hline Benzene & $5 \mathbf{u}$ & 50 & 1 & 50 & $5 \mathrm{U}$ & 50 & $5 \mathbf{v}$ & 50 \\
\hline 2-Butanone & $10 \mathrm{v}$ & 100 & 100 & 100 & 100 & 100 & 100 & 100 \\
\hline 2-Hexanone & 100 & 100 & 100 & 100 & 100 & 100 & 100 & 100 \\
\hline 4-Methyl-2-pentanone & 100 & 100 & 100 & 100 & 100 & 100 & 100 & 100 \\
\hline Hethylene chloride & 1 & 50 & 50 & 50 & $5 u$ & 2 & $5 U$ & 50 \\
\hline Toluene & $5 u$ & $5 U$ & 1 & 50 & 5U & su & 50 & 50 \\
\hline
\end{tabular}

(CONTINUED) 
APPENDIX I.3

VOCs Detected in Iaboratory Blank Samples, Third Quarter 1993

\begin{tabular}{|c|c|c|c|c|c|c|c|c|}
\hline Sample No. & $\begin{array}{c}930918- \\
029\end{array}$ & $\begin{array}{c}930920- \\
116\end{array}$ & $\begin{array}{c}930921- \\
025\end{array}$ & $\begin{array}{c}930921- \\
039\end{array}$ & $\begin{array}{c}930922- \\
010\end{array}$ & $\begin{array}{c}930922- \\
033\end{array}$ & $\begin{array}{c}930923- \\
093\end{array}$ & $\begin{array}{c}930927- \\
018\end{array}$ \\
\hline Benzene & $5 \mathrm{U}$ & $5 U$ & $5 U$ & $5 U$ & $5 U$ & $5 U$ & 50 & 50 \\
\hline 2-Butanone & 8 & 100 & 100 & 100 & 100 & 100 & 100 & 100 \\
\hline 2-Hexanone & 100 & 100 & 100 & 100 & 100 & 100 & 100 & 100 \\
\hline 4-Methy 1-2-pentanone & 100 & 100 & 100 & 100 & $10 \mathrm{U}$ & 100 & 100 & 100 \\
\hline Methylene chloride & su & su & 50 & $\mathbf{5 U}$ & 1 & $5 \mathbf{U}$ & 50 & $\mathbf{5 U}$ \\
\hline Toluene & 50 & $5 U$ & 50 & 50 & $5 U$ & 50 & 50 & $5 U$ \\
\hline
\end{tabular}

(CONTINUED)

\begin{tabular}{|l|r|}
\hline Sample No. & $\begin{array}{c}930928- \\
089\end{array}$ \\
\hline Benzene & 50 \\
2-Butanone & $10 U$ \\
2-Hexanone & 100 \\
4-Methyl-2-pentanone & 100 \\
Methylene chloride & 50 \\
Toluene & 50 \\
\hline
\end{tabular}


APPENDIX I. 3

vOCs Detected in Laboratory Blank Samples, Fourth Quarter 1993

\begin{tabular}{|c|c|c|c|c|c|c|c|c|}
\hline Sample No. & $\begin{array}{c}931014- \\
010\end{array}$ & $\begin{array}{c}931015- \\
141\end{array}$ & $\begin{array}{c}931018- \\
003\end{array}$ & $\begin{array}{c}931018- \\
196\end{array}$ & $\begin{array}{c}931019- \\
038\end{array}$ & $\begin{array}{c}931023- \\
006\end{array}$ & $\begin{array}{c}931025- \\
031\end{array}$ & $\begin{array}{c}931027- \\
001\end{array}$ \\
\hline Acetone & 100 & 100 & 4 & 100 & 100 & 100 & 3 & 8 \\
\hline 2-Butanone & 100 & 100 & 10 & $10 U$ & $10 \mathrm{U}$ & 100 & 9 & 100 \\
\hline 2-Hexanone & 100 & 100 & 100 & 100 & 100 & 100 & 100 & 100 \\
\hline 4-Methy 1-2-pentanone & 100 & 100 & 1 & $10 \mathrm{U}$ & 100 & 100 & 100 & 100 \\
\hline Methylene chloride & 50 & $5 \mathbf{U}$ & 3 & $5 \mathbf{S}$ & 50 & 0.6 & 2 & 1 \\
\hline styrene & $5 u$ & 50 & $5 v$ & 0.6 & $5 U$ & 50 & 50 & su \\
\hline
\end{tabular}

(CONTINUED)

\begin{tabular}{|c|c|c|c|c|c|c|c|c|}
\hline Sample No. & $\begin{array}{c}931027- \\
002\end{array}$ & $\begin{array}{c}931028- \\
007\end{array}$ & $\begin{array}{c}931028- \\
022\end{array}$ & $\begin{array}{c}931029- \\
026\end{array}$ & $\begin{array}{c}931101- \\
042\end{array}$ & $\begin{array}{c}931102- \\
001\end{array}$ & $\begin{array}{c}931109- \\
029\end{array}$ & $\begin{array}{c}931109- \\
111\end{array}$ \\
\hline Acetone & 100 & 3 & 100 & 100 & 100 & $10 U$ & 100 & 100 \\
\hline 2-Butanone & 100 & 15 & $10 u$ & 100 & 100 & 100 & 100 & 100 \\
\hline 2-Hexanone & 100 & 100 & 100 & 100 & 100 & 100 & 100 & 100 \\
\hline 4-Methyl-2-pentanorie & $10 \mathrm{U}$ & $10 \mathrm{U}$ & 100 & 100 & 100 & 100 & 100 & 100 \\
\hline Methylene chloride & $5 u$ & 3 & $5 v$ & $5 \mathbf{u}$ & 2 & 2 & 2 & $\mathbf{s u}$ \\
\hline Styrene & $5 \mathrm{U}$ & 50 & $5 v$ & 50 & $5 \mathbf{u}$ & 5u & su & 50 \\
\hline
\end{tabular}

(CONTINUED)

\begin{tabular}{|c|c|c|c|c|c|c|c|c|}
\hline Sample No. & $\begin{array}{c}931110- \\
011\end{array}$ & $\begin{array}{c}931111- \\
014\end{array}$ & $\begin{array}{c}931116- \\
002\end{array}$ & $\begin{array}{c}931116- \\
035\end{array}$ & $\begin{array}{c}931117- \\
001\end{array}$ & $\begin{array}{c}931118- \\
001\end{array}$ & $\begin{array}{c}931118- \\
146\end{array}$ & $\begin{array}{c}931119- \\
102\end{array}$ \\
\hline Acetone & 100 & 100 & 100 & $10 \mathrm{U}$ & 100 & 100 & 100 & 100 \\
\hline 2-Butanone & 100 & 100 & 100 & 100 & 100 & 100 & 100 & 100 \\
\hline 2-Hexanone & $10 \mathrm{U}$ & 100 & 100 & 100 & 100 & 100 & 100 & 100 \\
\hline 4-Methyl-2-pentanone & 100 & 100 & 100 & $10 \mathrm{U}$ & 100 & 100 & 100 & 100 \\
\hline Methylene chloride & $5 \mathbf{u}$ & $5 \mathbf{5}$ & 1 & su & 2 & 1 & $5 U$ & $5 \mathbf{U}$ \\
\hline Styrene & 50 & 50 & 50 & $5 U$ & 50 & $5 U$ & $5 U$ & 50 \\
\hline
\end{tabular}

(CONTINUED) 
APPENDIX I.3

VOCs Detected in Laboratory Blank Samples, Fourth Quarter 1993

\begin{tabular}{|c|c|c|c|c|c|c|c|c|}
\hline Sample No. & $\begin{array}{c}931119- \\
123\end{array}$ & $\begin{array}{c}931123- \\
001\end{array}$ & $\begin{array}{c}931123- \\
053\end{array}$ & $\begin{array}{c}931124- \\
240\end{array}$ & $\begin{array}{c}931130- \\
055\end{array}$ & $\begin{array}{c}931201- \\
064\end{array}$ & $\begin{array}{c}931206- \\
102\end{array}$ & $\begin{array}{l}931208- \\
005\end{array}$ \\
\hline Acetone & 100 & 100 & $10 \mathrm{U}$ & 100 & $10 \mathrm{U}$ & 100 & 7 & 100 \\
\hline 2-Butanone & 100 & 100 & 100 & 100 & 100 & 100 & 15 & 100 \\
\hline 2-Hexanone & 100 & 100 & 100 & 100 & 100 & 100 & 100 & 100 \\
\hline 4-Methyl-2-pentanone & 100 & 100 & 100 & 100 & 100 & 100 & 1 & 100 \\
\hline Methylene chloride & 50 & 2 & $5 \mathbf{v}$ & 5u & 5u & 50 & 2 & 1 \\
\hline Styrene & 50 & $5 \mathbf{U}$ & $5 \mathbf{u}$ & 50 & $5 \mathbf{v}$ & 50 & $5 \mathbf{u}$ & 50 \\
\hline
\end{tabular}

( CONTINUED)

\begin{tabular}{|l|r|r|r|r|}
\hline Sample No. & $\begin{array}{c}931209- \\
025\end{array}$ & $\begin{array}{c}931209- \\
029\end{array}$ & $\begin{array}{c}931210- \\
049\end{array}$ & $\begin{array}{c}931214- \\
001\end{array}$ \\
\hline Acetone & 5 & $10 \mathrm{U}$ & 4 & 3 \\
2-Butanone & 10 & $10 \mathrm{U}$ & 8 & 8 \\
2-Hexanone & $10 \mathrm{U}$ & $10 \mathrm{U}$ & 2 & $10 \mathrm{U}$ \\
4-Methyl-2-pentanone & 2 & $10 \mathrm{U}$ & 3 & 1 \\
Methylene chloride & 2 & $5 \mathrm{U}$ & 2 & 2 \\
Styrene & $5 \mathrm{U}$ & $5 \mathrm{U}$ & $5 \mathrm{U}$ & $5 \mathrm{~V}$ \\
\hline
\end{tabular}


APPENDIX $\mathbf{L} .4$

EQUIPMENT RINSATE DATA SUMMARY 
APPENDIX I. 4

Equipment Rinsate Data Summary, First Quarter 1993

\begin{tabular}{|c|c|c|c|c|c|c|c|c|}
\hline Sample No. & $\begin{array}{c}930114- \\
031\end{array}$ & $\begin{array}{c}930121- \\
063\end{array}$ & $\begin{array}{c}930121- \\
083\end{array}$ & $\begin{array}{c}930123- \\
026\end{array}$ & $\begin{array}{c}930127- \\
091\end{array}$ & $\begin{array}{c}930128- \\
019\end{array}$ & $\begin{array}{c}930128- \\
204\end{array}$ & $\begin{array}{c}930201- \\
060\end{array}$ \\
\hline VOLATILE ORGANICS (ug/I) & - & - & - & - & - & - & - & - \\
\hline Acetone & 2 & 100 & 100 & 100 & 1 & 2 & 100 & - \\
\hline 4-Methyl-2-pentanone & 2 & 100 & 100 & 100 & 2 & 1 & 100 & - \\
\hline Methylene chloride & $5 U$ & 2 & 1 & $5 \mathbf{U}$ & $5 U$ & 50 & $5 U$ & - \\
\hline OTHER PARAMETERS (mg/L) & - & - & - & - & - & - & - & - \\
\hline Nitrate (as $N)$ & $\bullet$ & $\cdot$ & $\cdot$ & $\cdot$ & $\bullet$ & $\cdot$ & $\cdot$ & $\cdot$ \\
\hline Aluminum & • & - & $\cdot$ & - & 0.051 & $\cdot$ & $0.037^{\circ}$ & 0.023 \\
\hline Antimony & - & - & - & - & $<0.05$ & - & $<0.05$ & $<0.05$ \\
\hline Arsenic & - & - & - & - & $<0.05$ & - & $<0.05$ & $<0.05$ \\
\hline Barium & - & - & - & - & 0.014 & - & 0.023 & 0.0037 \\
\hline Beryllium & - & - & - & - & $<0.0003$ & - & $<0.0003$ & $<0.0003$ \\
\hline Boron & - & - & - & - & 0.018 & - & 0.016 & 0.0061 \\
\hline Cadmium & - & - & - & - & $<0.003$ & - & $<0.003$ & $<0.003$ \\
\hline Calcium & - & - & - & - & 34 & - & 27 & 0.28 \\
\hline Chromium & - & - & - & - & $<0.01$ & - & $<0.01$ & $<0.01$ \\
\hline Cobalt & - & - & - & - & $<0.005$ & - & $<0.005$ & $<0.005$ \\
\hline Copper & - & - & - & - & $<0.004$ & - & 0.0044 & $<0.004$ \\
\hline Iron & - & - & - & - & 0.016 & - & 1.5 & 0.065 \\
\hline Magnesium & - & - & - & - & 9.6 & - & 7.6 & 0.04 \\
\hline Manganese & - & - & - & - & $<0.001$ & - & 0.0071 & 0.0036 \\
\hline Mol ybdenum & - & - & - & - & $<0.01$ & - & $<0.01$ & $<0.01$ \\
\hline Nickel & - & - & - & - & $<0.01$ & - & $<0.01$ & $<0.01$ \\
\hline Potassium & • & - & - & - & 1.5 & - & 1.2 & $<0.6$ \\
\hline Selenium & - & - & - & - & $<0.05$ & - & 0.059 & $<0.05$ \\
\hline silver & - & - & - & - & $<0.006$ & - & $<0.006$ & $<0.006$ \\
\hline Sodium & - & - & - & - & 6.3 & - & 4.5 & $<0.02$ \\
\hline strontium & - & - & - & - & 0.079 & - & 0.067 & 0.0022 \\
\hline Thorium & - & - & - & - & $<0.2$ & - & $<0.2$ & $<0.2$ \\
\hline Vanadium & - & - & - & - & $<0.005$ & - & $<0.005$ & $<0.005$ \\
\hline Zinc & - & - & - & - & 1.3 & - & 0.85 & 0.0061 \\
\hline Gross Alpha (pci/L) & • & - & - & • & - & - & - & - \\
\hline Gross Alpha $(\mathrm{CE}+/-)$ & - & - & - & - & - & • & - & - \\
\hline Gross Beta (pCi/I) & - & - & - & - & - & - & . & - \\
\hline Gross Beta $(\mathrm{CE}+/-)$ & - & - & - & - & - & - & . & - \\
\hline
\end{tabular}

(CONTINUED) 
APPENDIX I.4

Equipment Rinsate Data Summary, First Quarter 1993

\begin{tabular}{|c|c|c|c|c|}
\hline Sample No. & $\begin{array}{c}930203- \\
094\end{array}$ & $\begin{array}{c}930224- \\
003\end{array}$ & $\begin{array}{c}930303- \\
115\end{array}$ & $\begin{array}{c}930312- \\
013\end{array}$ \\
\hline VOLATILE ORGANICS (ug/L) & - & - & - & - \\
\hline Acetone & 3 & 100 & 100 & 100 \\
\hline 4-Methyl-2-pentanone & 2 & 100 & 100 & $10 U$ \\
\hline Methylene chloride & 2 & 1 & $5 U$ & $5 U$ \\
\hline OTHER PARAMETBRS (mg/L) & - & • & . & • \\
\hline Nitrate (as N) & - & $\cdot$ & $<0.2$ & • \\
\hline Aluminum & - & - & 0.043 & - \\
\hline Antimony & - & - & $<0.05$ & - \\
\hline Arsenic & - & - & $<0.05$ & - \\
\hline Barium & - & - & 0.034 & - \\
\hline Beryllium & - & - & 0.0021 & - \\
\hline Boron & - & - & 0.022 & - \\
\hline Cadmium & - & - & $<0.003$ & - \\
\hline Calcium & - & - & 33 & - \\
\hline Chromium & - & - & $<0.01$ & - \\
\hline Cobalt & - & - & $<0.005$ & - \\
\hline Copper & - & - & $<0.004$ & - \\
\hline Iron & - & - & 0.43 & - \\
\hline Magnesium & - & - & 8.9 & - \\
\hline Manganese & - & - & 0.1 & - \\
\hline Molybdenum & - & - & $<0.01$ & - \\
\hline Nickel & - & - & 0.01 & - \\
\hline Potassium & - & - & 1.5 & - \\
\hline Selentum & - & - & $<0.05$ & - \\
\hline Silver & - & - & $<0.006$ & - \\
\hline Sodium & - & - & 5.6 & - \\
\hline strontium & - & - & 0.086 & - \\
\hline Thorium & - & - & $<0.2$ & - \\
\hline Vanadium & - & - & $<0.005$ & - \\
\hline zinc & - & - & 1.2 & - \\
\hline Gross Alpha (pCi/L) & - & - & 0 & - \\
\hline Gross Alpha $(\mathrm{CE}+/-)$ & - & - & 2.9 & - \\
\hline Gross Beta $(p c i / I)$ & - & - & 3.96 & - \\
\hline Gross Beta $(\mathrm{CE}+/-)$ & - & . & 5.4 & - \\
\hline
\end{tabular}


APPENDIX I. 4

Equipment Rinsate Data Summary, Second Quarter 1993

\begin{tabular}{|c|c|c|c|c|c|c|c|c|}
\hline Sample No. & $\begin{array}{c}930414- \\
020\end{array}$ & $\begin{array}{c}930415- \\
038\end{array}$ & $\begin{array}{c}930419- \\
068\end{array}$ & $\begin{array}{c}930421- \\
004\end{array}$ & $\begin{array}{c}930427- \\
023\end{array}$ & $\begin{array}{c}930507- \\
125\end{array}$ & $\begin{array}{c}930511- \\
025\end{array}$ & $\begin{array}{c}930524- \\
140\end{array}$ \\
\hline VOLATILE ORGANICS (ug/L) & $\cdot$ & $\cdot$ & $\cdot$ & - & • & - & $\cdot$ & - \\
\hline Acetone & 100 & 100 & 100 & 100 & 100 & 100 & 100 & 100 \\
\hline Bromodichloromethane & $5 \mathbf{U}$ & $5 U$ & $5 \mathbf{U}$ & 3 & $5 \mathbf{u}$ & 50 & $5 U$ & 50 \\
\hline 2-Butanone & 100 & 100 & 100 & 100 & 100 & 100 & 100 & 4 \\
\hline Chloroform & 50 & 50 & su & 23 & $5 u$ & 50 & 50 & 50 \\
\hline 1,2-Dichloropropane & $5 \mathbf{v}$ & $5 \mathrm{U}$ & $5 \mathbf{v}$ & 9 & $5 \mathbf{u}$ & 50 & $5 u$ & 50 \\
\hline 4-Methy1-2-pentanone & 100 & 100 & 100 & 2 & $10 u$ & 100 & 100 & 1 \\
\hline Methylene chloride & $5 U$ & su & $5 U$ & $5 v$ & $5 \mathbf{U}$ & 1 & $5 U$ & 50 \\
\hline Trichloroethene & 5u & 50 & $5 U$ & 50 & $5 \mathrm{u}$ & 50 & $5 U$ & $5 \mathbf{U}$ \\
\hline OTHER PARAMETERS (mg/L) &. & - & . & • & - & - & - & - \\
\hline Nitrate (as $N)$ & $\cdot$ & $\cdot$ & $\cdot$ & $\cdot$ & $\cdot$ & $\cdot$ & $\cdot$ & - \\
\hline TPH $(\mathrm{mg} / \mathrm{L})$ & $\cdot$ & - & & $<0.1$ & - & - & - & - \\
\hline Aluminum & . & . & - & $\cdot$ & $<0.02$ & - & - & - \\
\hline Antimony & - & . & - & . & $<0.05$ & - & - & - \\
\hline Arsenic & . & . & $\cdot$ & $\cdot$ & $<0.05$ & - & - & - \\
\hline Barium & - & . & - & - & 0.021 & - & - & - \\
\hline Beryllium & - & $\cdot$ & - & - & $<0.0003$ & - & • & - \\
\hline Boron & $\cdot$ & $\cdot$ & - & $\cdot$ & 0.0047 & . & - & - \\
\hline Cadmium & - & - & - & . & $<0.003$ & . & - & - \\
\hline Calcium & - & - & - & - & 31 & $\cdot$ & - & - \\
\hline Chromium & - & - & - & . & $<0.01$ & - & - & - \\
\hline Cobalt & - & - & - & $\cdot$ & $<0.005$ & - & - & - \\
\hline Copper & - & - & - & - & $<0.004$ & - & - & - \\
\hline Iron & $\cdot$ & - & - & - & 0.3 & - & - & - \\
\hline Magnesium & $\cdot$ & - & • & • & 9.2 & - & - & - \\
\hline Manganese & - & - & . & - & 0.0076 & $\cdot$ & - & - \\
\hline Molybdenum & - & - & - & - & $<0.01$ & - & - & - \\
\hline Nickel & - & - & - & - & $<0.01$ & . & - & - \\
\hline Potassium & - & - & - & - & 1.5 & - & - & - \\
\hline Selenium & $\cdot$ & - & . & . & $<0.05$ & - & - & - \\
\hline silver & - & - & - & - & $<0.006$ & - & - & - \\
\hline Sodium & - & - & - & - & 4.9 & - & - & - \\
\hline Strontiun & - & - & - & - & 0.08 & - & - & - \\
\hline Thorium & - & - & - & - & $<0.2$ & - & - & - \\
\hline Vanadium & - & - & - & . & $<0.005$ & - & - & - \\
\hline Zinc & - & - & - & $\cdot$ & 0.74 & - & - & - \\
\hline Gross Alpha (pCi/L) & - & - & $\cdot$ & $\cdot$ & • & - & - & - \\
\hline Gross Alpha $(\mathrm{CE}+/-)$ & - & - & - & - & - & - & - & - \\
\hline Gross Beta (pCi/L) & $\cdot$ & - & $\cdot$ & - & - & - & - & - \\
\hline Gross Beta $(\mathrm{CE}+/-)$ & $\cdot$ & $\cdot$ & $\cdot$ & - & - & - & • & • \\
\hline
\end{tabular}

(CONTINUED) 
APPENDIX I.4

Equipment Rinsate Data Summary, Second Quarter 1993

\begin{tabular}{|c|c|c|c|c|c|c|}
\hline Sample No. & $\begin{array}{c}930622- \\
017\end{array}$ & $\begin{array}{c}930624- \\
023\end{array}$ & $\begin{array}{c}930629- \\
095\end{array}$ & $\begin{array}{c}930630- \\
008\end{array}$ & $\begin{array}{c}930707- \\
080\end{array}$ & $\begin{array}{c}930708- \\
009\end{array}$ \\
\hline VOLATILB ORGANICS (ug/I) & - & - & - & - & - & - \\
\hline Acetone & 6 & 100 & 100 & 100 & 100 & 100 \\
\hline Bromodichloromethane & $\mathbf{5 u}$ & $5 \mathbf{v}$ & 5u & 5u & $\mathbf{5 u}$ & 50 \\
\hline 2-Butanone & 100 & 100 & 100 & 100 & 100 & 100 \\
\hline chloroform & $\mathbf{5 u}$ & su & 50 & 50 & $\mathbf{s u}$ & $5 \mathbf{v}$ \\
\hline 1,2-Dichloropropane & su & su & $\mathbf{5 u}$ & $\mathbf{5 u}$ & $\mathbf{5 u}$ & 5u \\
\hline 4-Methyl-2-pentanone & 100 & 100 & 100 & 100 & 100 & 100 \\
\hline Methylene chlorlde & $\mathbf{5 u}$ & $5 \mathbf{s}$ & SU & 1 & $\mathbf{5 u}$ & $\mathbf{5 u}$ \\
\hline Trichloroethene & 1 & $\mathbf{5 0}$ & 50 & $5 \mathbf{v}$ & 50 & su \\
\hline OTHER PARAYKTERS (mg/L) & - & - & - & . & . & • \\
\hline Nitrate (as N) & 0.28 & • & • & $<0.2$ & • & 0.55 \\
\hline TPH (mg/L) & • & 0.006 & 0.003 & 0.079 & $<0.1$ & 0.028 \\
\hline Aluminum & $<0.02$ & • & • & $<0.02$ & $\cdot$ & $<0.02$ \\
\hline Antimony & $<0.05$ & - & - & $<0.05$ & - & $<0.05$ \\
\hline Arsentc & $<0.05$ & - & - & $<0.05$ & - & $<0.05$ \\
\hline Barlum & 0.03 & - & - & 0.0063 & - & 0.016 \\
\hline Bery 111 um & $<0.0003$ & - & - & $<0.0003$ & - & $<0.0003$ \\
\hline Boron & 0.035 & - & - & 0.024 & - & 0.035 \\
\hline Cadmium & $<0.003$ & - & . & $<0.003$ & - & $<0.003$ \\
\hline Calcium & 33 & - & - & $<0.008$ & - & 25 \\
\hline Chromium & $<0.01$ & - & - & $<0.01$ & • & $<0.01$ \\
\hline Cobalt & $<0.005$ & - & - & $<0.005$ & - & $<0.005$ \\
\hline Copper & $<0.004$ & - & - & 0.12 & - & 0.012 \\
\hline Iron & $<0.005$ & - & - & 0.022 & - & 0.019 \\
\hline Magnesium & 9.4 & - & - & 0.0046 & - & 6.3 \\
\hline Manganese & 0.006 & - & - & 0.0021 & - & 0.014 \\
\hline Molybdenum & $<0.01$ & - & - & $<0.01$ & • & $<0.01$ \\
\hline Nickel & $<0.01$ & - & - & 0.012 & • & $<0.01$ \\
\hline Potass Ium & 1.4 & - & - & $<0.6$ & - & 0.89 \\
\hline Selenium & $<0.05$ & - & - & $<0.05$ & - & $<0.05$ \\
\hline silver & $<0.006$ & - & - & $<0.006$ & - & $<0.006$ \\
\hline sodium & 5.2 & - & - & 0.13 & - & 3.4 \\
\hline Strontium & 0.086 & - & - & $<0.0004$ & - & 0.054 \\
\hline Thorium & $<0.2$ & - & - & $<0.2$ & - & $<0.2$ \\
\hline Vanadium & $<0.005$ & - & . & $<0.005$ & - & $<0.005$ \\
\hline Zinc & 0.71 & - & - & 0.015 & - & 0.3 \\
\hline Gross Alpha (pci/L) & 0.45 & - & - & 0 & - & $-1 \cdot 22$ \\
\hline Gross Alpha $(\mathrm{CE}+/-)$ & 1.6 & - & - & 1.4 & - & 1.9 \\
\hline Grose Beta $(p C 1 / L)$ & 2.91 & - & - & 0.762 & - & -7 \\
\hline Gross Beta $(\mathrm{CE}+/-)$ & 2.7 & - & - & 2.5 & - & 5.7 \\
\hline
\end{tabular}


APPENDIX 1.4

Equipment Rinsate Data summary, Third Quarter 1993

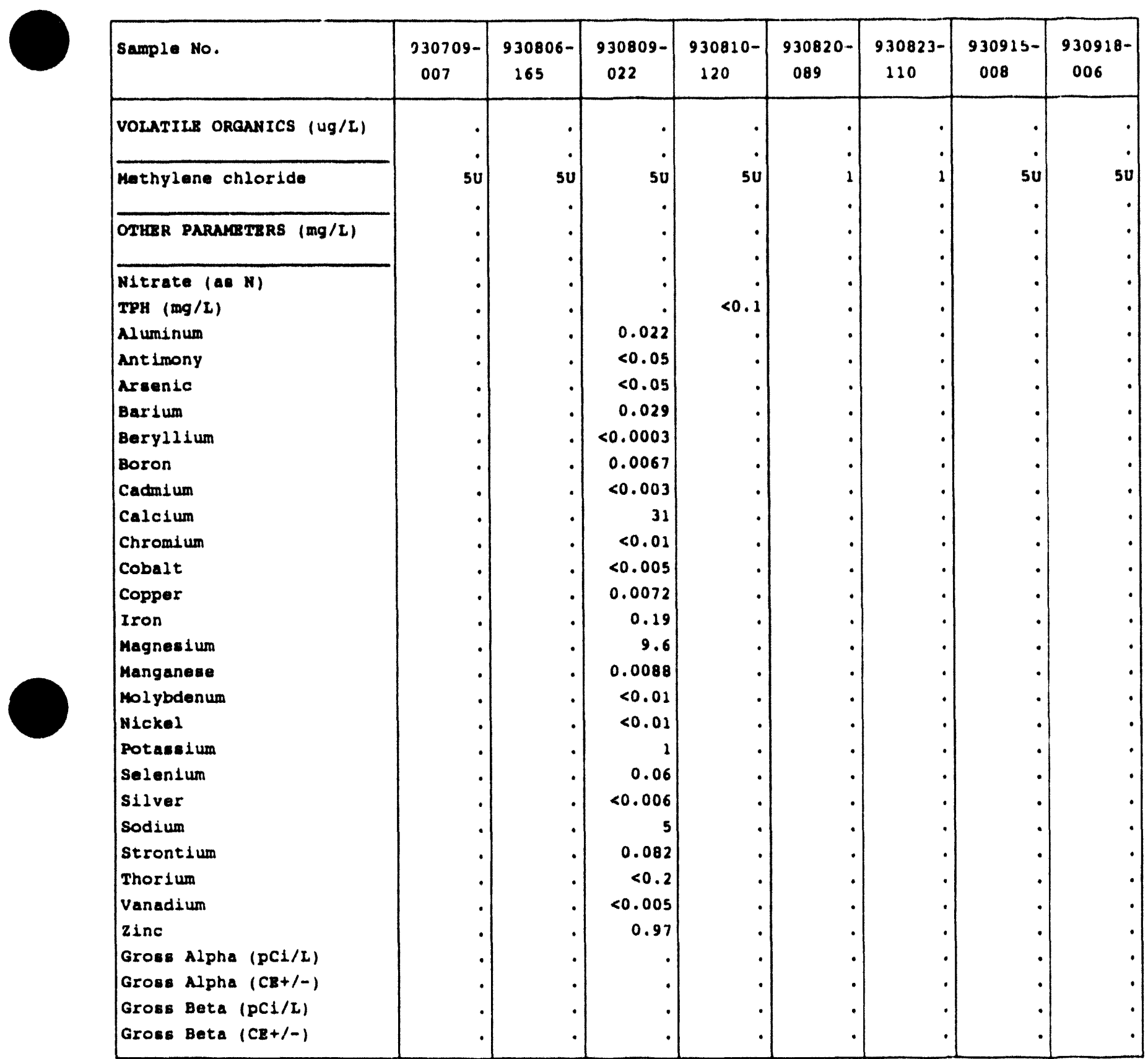

(CONTINUED) 
Bquipment Rineate Data Summary, Thlrd Quarter 1993

\begin{tabular}{|c|c|c|}
\hline samplo No. & $\begin{array}{c}930920- \\
167\end{array}$ & $\begin{array}{c}930927- \\
231\end{array}$ \\
\hline VOLATILE ORCANICS (ug/L) & - & - \\
\hline Mathylene chloride & 1 & su \\
\hline OTHER PARAMTERS (mg/L) & - & - \\
\hline Nitrate (ae N) & 0.55 & - \\
\hline $\operatorname{TPH}(\mathrm{mg} / \mathrm{L})$ & 0 & 0.285 \\
\hline Aluminum & $<0.02$ & • \\
\hline Antimony & $<0.05$ & - \\
\hline Areen 10 & $<0.0 s$ & - \\
\hline Barium & 0.026 & - \\
\hline Bery 11 ium & $<0.0003$ & - \\
\hline Boron & 0.018 & - \\
\hline Cadini um & $<0.003$ & - \\
\hline Calclum & 30 & - \\
\hline Chromium & $<0.01$ & - \\
\hline Cobalt & $<0.005$ & - \\
\hline Copper & $<0.004$ & - \\
\hline Iron & $<0.005$ & - \\
\hline Magnes 1 um & 8 & - \\
\hline Manganese & 0.0033 & - \\
\hline Molybdenum & $<0.01$ & - \\
\hline Nickel & $<0.01$ & - \\
\hline Potanesum & 1.1 & - \\
\hline Selc ium & $<0.05$ & - \\
\hline sllver & $<0.006$ & - \\
\hline Sodium & 4 & - \\
\hline strontium & 0.078 & - \\
\hline Thorium & $<0.2$ & - \\
\hline Vanadium & $<0.005$ & - \\
\hline Zinc & 0.99 & - \\
\hline Gross Alpha, $p(1 / L)$ & -2.03 & - \\
\hline Groes Alpha $(C E+/-)$ & 3.1 & - \\
\hline Gross Beta (pCl/L) & 2.9 & - \\
\hline Grose Beta $(\mathrm{CE}+/-)$ & 5.4 & - \\
\hline
\end{tabular}


APPENDIX 1.4

Equipment Rineate Data Summary, Fourth Quarter 1993

\begin{tabular}{|c|c|c|c|c|c|c|c|c|}
\hline sample No. & $\begin{array}{c}931018- \\
209\end{array}$ & $\begin{array}{c}931025- \\
079\end{array}$ & $\begin{array}{c}931101- \\
151\end{array}$ & $\begin{array}{c}931104- \\
065\end{array}$ & $\begin{array}{c}931108- \\
195\end{array}$ & $\begin{array}{c}931111- \\
108\end{array}$ & $\begin{array}{c}931119- \\
061\end{array}$ & $\begin{array}{c}931129- \\
063\end{array}$ \\
\hline VOLATIL ORCAyICs (ug/L) & $\cdot$ & - & $\cdot$ & - & - & - & - & - \\
\hline Acetone & 3 & 100 & 31 & 39 & 100 & 100 & 200 & 100 \\
\hline 2-Butanone & 8 & 100 & 100 & 100 & 100 & 100 & 100 & 100 \\
\hline 1,2-Dlehloropropano & 5 & su & su & su & su & su & su & su \\
\hline 4-Methyl-2-pentanone & 100 & 100 & 100 & 100 & 100 & 100 & 100 & 100 \\
\hline Methylene chlorlde & 2 & su & 1 & su & su & 2 & su & su \\
\hline OTHER PARANETERS $(\mathrm{mg} / \mathrm{L})$ & . & - & . & . & . & - & . & - \\
\hline Mitrate (as $\left.N_{1}\right)$ & & $\cdot$ & . & . &. & . &. & $<0 . \dot{2}$ \\
\hline XPH $(m g / L)$ & $<0.1$ & . & . & . & . & . &. & \\
\hline Aluminum & . & . & • & - & 0.021 & - & $\cdot$ & $<0.02$ \\
\hline Antimony & - & - & . & - & $<0.05$ & . & . & $<0.03$ \\
\hline Areenice & - & - & . & - & $<0.05$ & - & $\cdot$ & $<0.05$ \\
\hline Barium & $\cdot$ & . & . & . & 0.042 & . & $\cdot$ & $<0.001$ \\
\hline Bery111um & - & - & . & . & $<0.0003$ & - & . & $<0.0003$ \\
\hline Boron & - & - & . & - & 0.016 & $\cdots$ & . & 0.0041 \\
\hline Cadmi um & - & . & . & - & $<0.003$ & - & $\cdot$ & $<0.003$ \\
\hline calctum & - & - & . & - & 36 & - & . & 0.21 \\
\hline Chromiun & - & - & - & - & $<0.01$ & - & $\cdot$ & $<0.01$ \\
\hline Cobalt & • & - & . & - & $<0.005$ & - & $\cdot$ & $<0.005$ \\
\hline Copper & - & • & • & - & 0.01 & - & $\cdot$ & 0.0053 \\
\hline Iron & . & - & • & - & 0.23 & - & $\cdot$ & 0.01 \\
\hline Magnee i um & - & - & . & - & 20 & . & . & 0.043 \\
\hline Manganose & - & - & . & - & 0.014 & - & $\cdot$ & $<0.001$ \\
\hline Molybdenum & . & . & . & . & $<0.01$ & - &. & $<0.01$ \\
\hline Mlckel & - & - & - & - & $<0.01$ & - & $\cdot$ & $<0.01$ \\
\hline Potaseium & - & - & - & - & 1.4 & - & $\cdot$ & $<0.6$ \\
\hline selenium & - & - & . & - & $<0.05$ & - & $\cdot$ & $<0.05$ \\
\hline silver & . & - & - & - & $<0.006$ & - & . & $<0.006$ \\
\hline Sodium & . & - & - & - & 4.9 & - & . & 0.094 \\
\hline strontium & . & - & $\cdot$ & - & 0.095 & - & . & 0.00088 \\
\hline Thorium & - & $\cdot$ & - & - & $<0.2$ & - & $\cdot$ & $<0.2$ \\
\hline Vanadium & . & - & . & - & $<0.005$ & - &. & $<0.005$ \\
\hline zinc & - & - & - & - & 0.83 & . & . & 0.014 \\
\hline Grose Alpha (PCi/L) & - & - & - & - & - & - & . & 0.134 \\
\hline Gross Alpha $(\mathrm{CB}+/-)$ & . & - & . & . & . & . & . & 0.48 \\
\hline Grose Beta $(p \mathrm{Cl} / \mathrm{L})$ & - & - & . & - & - & - & . & -1.83 \\
\hline Grose Beta $(\mathrm{Cz}+1-1$ & - & - & . & - & - & - & $\cdot$ & 2.3 \\
\hline
\end{tabular}

(CONTINUED) 
APPEHDTX 1.4

Iquipment Rineate Data summary, rourth Quarter 1993

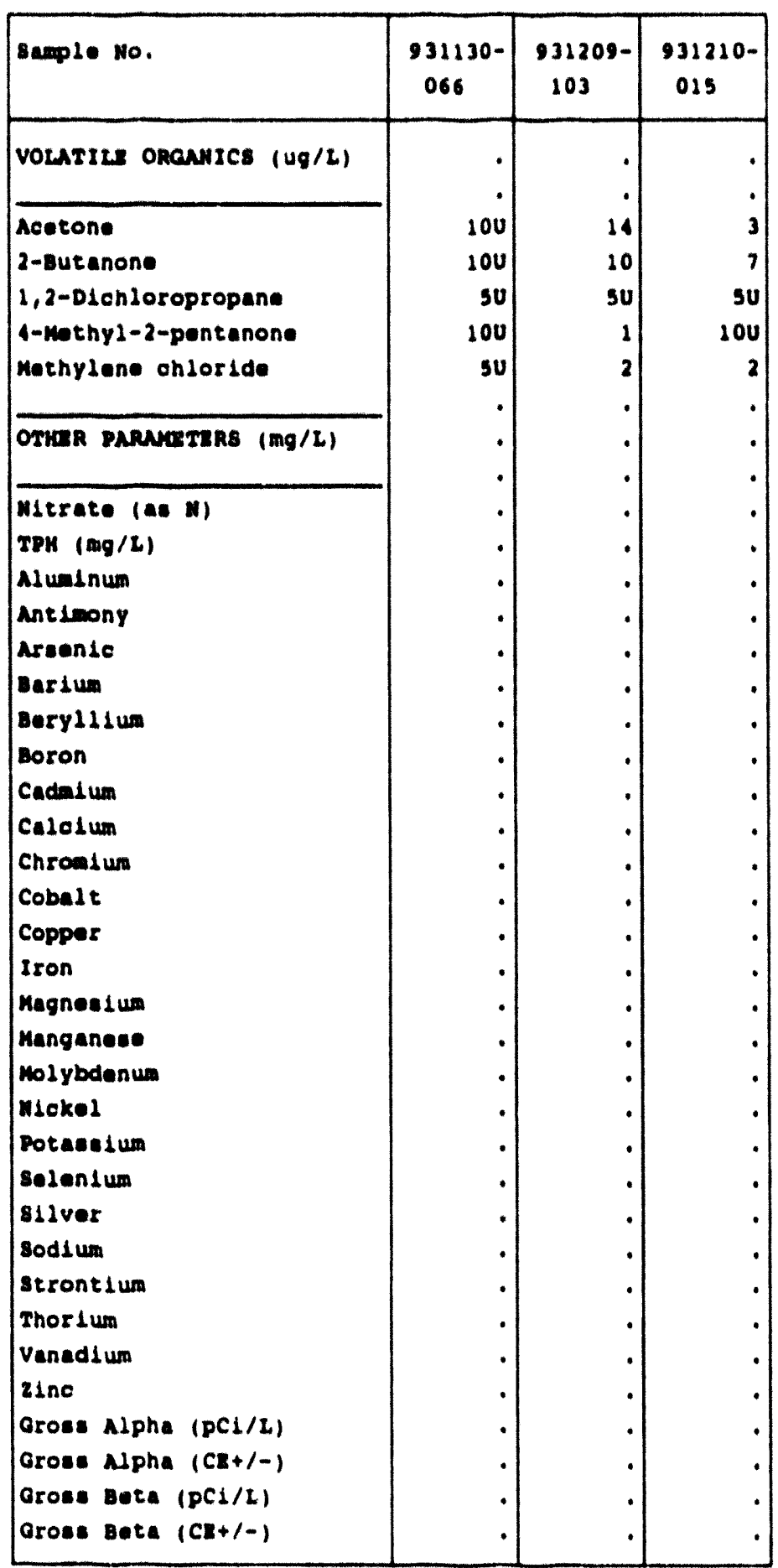


APPENDIX J

VALIDATED RESULTS OF VOLATIL ORGANIC COMPOUND ANALYSES 


\section{EXPLANATION}

\section{LOCATION:}

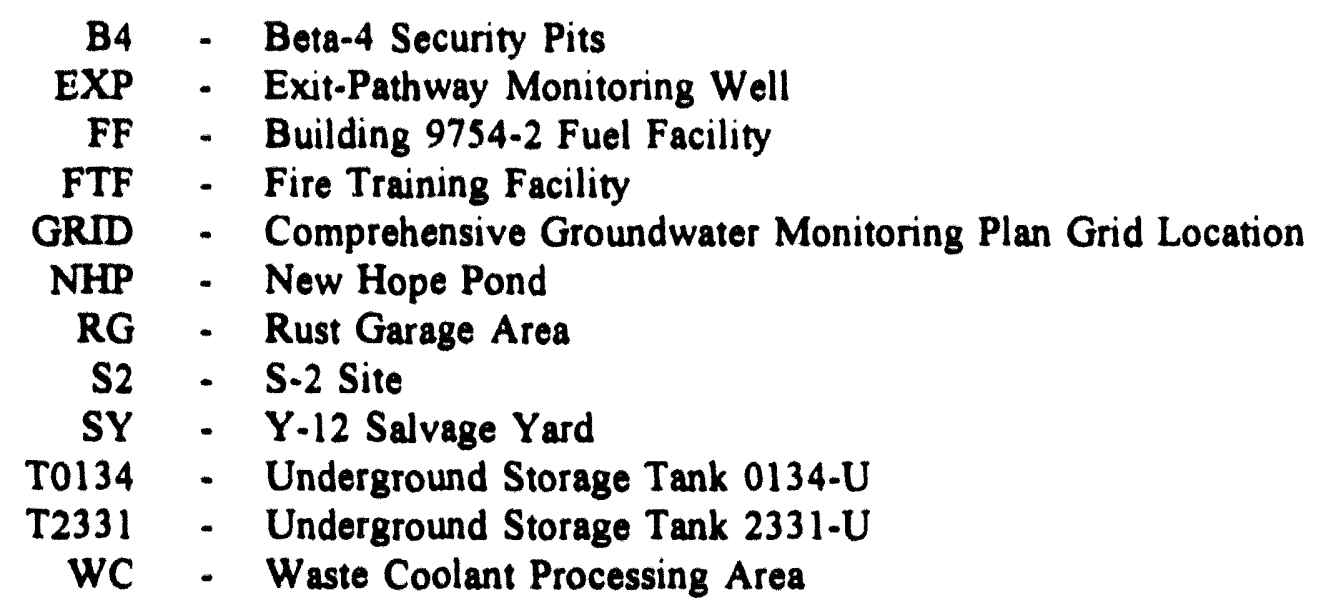

\section{NOTES:}

All results in micrograms per Liter (ug/L).

FP1 - False Positive result, screened by laboratory blank

FP2 - False Positive result, screened by trip blank

FP3 - Anomalous Result

$U$ - Compound not detected at the reported mininum attainable detection limit.

Results of VOC analyses reported for each groundwater sample were evaluated using results for each associated laboratory and trip blank sample to identify false positives (i.e., results that are probable artifacts of the groundwater sampling or analysis procedures). To identify the false positives, the maximum concentration of each VOC detected in either blank sample (Appendix I) is multiplied by a qualification factor to determine the blank qualification result (BQR) for the compound. A factor of 5 is used for most compounds and a factor of 10 is used for the common laboratory reagents methylene chloride, acetone, 2-butanone, and toluene (U.S. Environmental Protection Agency 1988b). If a compound detected in the blank samples is also detected in an associated groundwater sample, the concentration reported for the groundwater sample (Appendix E) is compared to the corresponding $B Q R$. If the concentration of the compound in the groundwater sample is at or below the $B Q R$, the result is considered a false positive and is excluded from the summed VOC concentration determined for the sample.

If a compound was detected in only one of at least four consecutive quarterly samples collected at a sampling point, the result was assumed to be anomalous. Anomalous VOC results were not identified in samples for which elevated analytical detection limits were reported for the compound during any other consecutive sampling event. 
APPEADIX J

Valldated voc Results, 1993

\begin{tabular}{|c|c|c|c|c|c|c|c|c|c|c|c|c|}
\hline \multirow{4}{*}{$\begin{array}{l}\text { Sampling Point } \\
\text { Location } \\
\text { - } \\
\text { Date Sampled }\end{array}$} & \multicolumn{8}{|c|}{ GN-151 } & \multicolumn{4}{|c|}{$6 w-168$} \\
\hline & \multicolumn{8}{|c|}{ map } & \multicolumn{4}{|c|}{$\operatorname{MiP}$} \\
\hline & \multicolumn{2}{|c|}{$01 / 26 / 93$} & \multicolumn{2}{|c|}{$04 / 22 / 93$} & \multicolumn{2}{|c|}{$08 / 06 / 93$} & \multicolumn{2}{|c|}{$11 / 02 / 93$} & \multicolumn{2}{|c|}{$01 / 22 / 93$} & \multicolumn{2}{|c|}{$05 / 14 / 93$} \\
\hline & ug/x & sum & ug/x & \& sum & $u g / L$ & 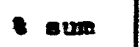 & ug/L & sum & $u g / L$ & Q =um & ug/L & sum \\
\hline Acetone & 200 & - & 200 & . & 200 &. & 400 & - & FP1 & - & 200 & \\
\hline Benzene & 100 &. & 100 &. & 100 & $\cdot$ & 200 & $\cdot$ & 50 & . & 5u & . \\
\hline Bromoform & 100 & $\cdot$ & 100 & $\cdot$ & 100 & $\cdot 1$ & 200 & $\cdot$ & so & - & 5u & . \\
\hline 2-Butanone & 200 & $\cdot$ & 200 & $\cdot$ & 200 & .1 & 400 & $\cdot$ & 100 & - & 100 & . \\
\hline Carbon disulfide & 100 &. & 100 &. & 100 & $\cdot 1$ & 200 & $\cdot$ & so & - & 5u & . \\
\hline Carbon tetrachloride & 220 & 90.5 & 330 & 89.9 & 520 & 92.9 & 610 & 92.1 & 50 & - & so & . \\
\hline Chloroform & 9) & 3.7 & 15 & 4.1 & 19 & 3.4 & 21 & 3.2 & so & - & 50 & . \\
\hline Chloromethane & 200 & $\cdot$ & 200 & $\cdot$ & 200 & $\cdot$ & 400 & $\cdot$ & 100 & $\cdot$ & 100 & . \\
\hline 1,1-Dichloroethane & 100 & $\cdot$ & 100 & $\cdot$ & 100 & $\cdot$ & 200 & $\cdot$ & 50 & - & 50 & . \\
\hline 1,2-Dichloroethane & 100 & $\cdot$ & 100 & $\cdot$ & 100 & $\cdot$ & 200 & $\cdot$ & 50 & - & so & . \\
\hline 1,1-Dichloroethene & 100 & $\cdot$ & 100 & $\cdot$ & 100 & $\cdot$ & 200 & $\cdot$ & 50 & - & 50| & \\
\hline 1,2-Dichloroethene & 100 & $\cdot$ & 100 & $\cdot$ & 100 & $\cdot$ & 200 & $\cdot$ & 50 & $\cdot$ & 5u & . \\
\hline Ethylbenzene & 100 & $\cdot$ & 100 & $\cdot$ & 100 & $\cdot$ & 200 & $\cdot$ & 50 & - & 50 & - \\
\hline 4-Methy1-2-pentanone & 200 & $\cdot$ & 200 & $\cdot 1$ & 200 & $\cdot$ & 400 & $\cdot$ & $\mathbf{F P 1}$ & - & 100 & . \\
\hline Nethylene chlorlde & 100 & $\cdot$ & 100 & $\cdot$ & 100 & $\cdot$ & 200 & $\cdot$ & 5v & - & FP2 & \\
\hline Styrene & 100 &. & 100 & $\cdot 1$ & 100 & $\cdot$ & 200 & $\cdot$ & 50 & - & 50 & . \\
\hline Tetrachloroethene & 12 & 4.9 & 19 & 5.2 & 21 & 3.8 & 27 & 4.1 & 50 & - & 50 & . \\
\hline Tolvene & 100 & $\cdot$ & 100 & $\cdot$ & 100 & $\cdot$ & 200 & $\cdot$ & 50 & $\cdot$ & 50 & - \\
\hline 1,1,1-Trichloroethane & 100 & $\cdot$ & 100 & $\cdot$ & 100 & $\cdot$ & 200 & $\cdot$ & 50 & - & 50 & . \\
\hline Irichloroethene & 2 & 0.8 & 3 & 0.8 & 100 & $\cdot$ & 4 & 0.6 & su & $\cdot$ & so & \\
\hline Vinyl acetate & 200 & $\cdot$ & 200 & $\cdot$ & 200 & $\cdot$ & 200 & $\cdot$ & 100 & $\cdot$ & 100 & . \\
\hline vinyl chloride & 200 & $\cdot$ & 200 &. & 200 & $\cdot$ & 400 & $\cdot$ & 100 & - & 100 & . \\
\hline xylenes & 100 & $\cdot$ & 100 &. & 100 & $\cdot$ & 200 & $\cdot 1$ & 50 & - & 50 & . \\
\hline Summed vOCs & 243 & 99.9 & 367 & 100 & 560 & 100.1 & 662 & 100 & 0 & - & o & . \\
\hline
\end{tabular}

(CONTINUED) 
APPENDIX J

Valldated voC Results, 1993

\begin{tabular}{|c|c|c|c|c|c|c|c|c|c|c|c|c|}
\hline \multirow{4}{*}{$\begin{array}{l}\text { Sempling Point } \\
\text { Location } \\
\text { Date Sampled }\end{array}$} & \multicolumn{4}{|c|}{ GN-168 } & \multicolumn{8}{|c|}{ Gn-169 } \\
\hline & \multicolumn{4}{|c|}{ MHP } & \multicolumn{8}{|c|}{$\mathbf{E x P}$} \\
\hline & \multicolumn{2}{|c|}{$08 / 19 / 93$} & \multicolumn{2}{|c|}{$11 / 02 / 93$} & \multicolumn{2}{|c|}{$01 / 23 / 93$} & \multicolumn{2}{|c|}{$05 / 21 / 93$} & \multicolumn{2}{|c|}{$08 / 19 / 93$} & \multicolumn{2}{|c|}{$11 / 01 / 93$} \\
\hline & $\mathrm{ug} / \mathrm{L}$ & sum & ug/L & sum & $u g / L$ & s sum & $\mathbf{u g} / \mathrm{t}$ & s sum & $\mathbf{u g} / \mathbf{L}$ & sum & ug/L & s sum \\
\hline Acetone & 100 &. & 100 & . & 100 &. & 100 & . & 100 & . & 100 & . \\
\hline Benzene & 50 & . & su & - & 50 &. & so & - & 50 & - & 5u & - \\
\hline Bromoform & so & . & 50 & - & 50 & $\cdot$ & 50 & - & so & . & so & • \\
\hline 2-Butanone & 100 &. & 100 & - & 100 & $\cdot$ & 100 & - & 100 & - & 100 & - \\
\hline Carbon disulfide & so & $\cdot 1$ & 50 & - & 50 & $\cdot 1$ & 50 & $\cdot$ & 50 & $\cdot$ & 5u & • \\
\hline Carbon tetrachloride & 50 & $\cdot$ & 50 & - & 50 & $\cdot$ & so & $\cdot$ & 50 & • & so & • \\
\hline chloroform & 50 & $\cdot$ & 50 & - & 50 & $\cdot$ & 50 & - & 5v & - & 50 & - \\
\hline Chloromethane & 100 & . & 100 & - & 100 &. & 100 & • & 100 & - & 100 & • \\
\hline 1,1-Dichloroethane & so &. & 50 & - & 50 & $\cdot$ & so & - & so & . & 50 & - \\
\hline 1,2-Dichloroethane & 50 & . & 50 & - & so & $\cdot$ & 50 & - & 50 & - & 50 & • \\
\hline 1,1-Dichloroethene & 50 & $\cdot$ & 50 & - & 50 & $\cdot$ & 50 & - & 50 & - & 50 & • \\
\hline 1,2-Dichloroethene & 5u &. & 50 & - & 50 & $\cdot$ & so & - & 5v & $\cdot$ & so & • \\
\hline Ethylbenzene & 50 & . & sv & . & 50 & $\cdot$ & 50 & - & so & $\cdot$ & 50 & • \\
\hline 4-Methyl-2-pentanone & 100 &. & 100 & - & FP1 & $\cdot$ & 100 & - & 100 & $\cdot$ & 100 & $\cdot$ \\
\hline Methylene chloride & 5u & $\cdot$ & 50 & • & 50 & $\cdot$ & so & - & 50 & • & 50 & • \\
\hline Styrene & 50 & $\cdot 1$ & so & $\cdot$ & 50 & $\cdot$ & 50 & $\cdot$ & 50 & $\cdot$ & 5v & • \\
\hline Tetrachloroethene & so &. & 50 & - & 3 & 50 & 3 & 50 & 3 & 60 & 3 & 60 \\
\hline Toluene & so & $\cdot$ & 50 & $\cdot$ & 50 & $\cdot$ & 50 & $\cdot$ & 5u & $\cdot$ & 50 & - \\
\hline 1,1,1-Trichloroethane & 50 &. & 50 & . & 50 & $\cdot$ & so & $\cdot$ & 50 & $\cdot$ & 50 & • \\
\hline Trichloroethene & 5v & $\cdot$ & so & • & 3 & 50 & 3 & 50 & 2 & 40 & 2 & 40 \\
\hline vinyl acetate & 100 &. & 100 & - & 100 & $\cdot$ & 100 & $\cdot$ & 100 & $\cdot$ & 100 & • \\
\hline vinyl chloride & 100 &. & 100 & . & 100 & $\cdot$ & 100 &. & 100 &. & 100 & - \\
\hline xylenes & 50 & $\cdot$ & 50 & • & 50 & $\cdot$ & 50 & - & so & - & 50 & - \\
\hline Summed voCs & o &. & $\dot{0}$ & $\bullet$ & $\dot{6}$ & 100 & 6 & 100 & 5 & 100 & 5 & 100 \\
\hline
\end{tabular}

(CONTINUED) 
APPENDIX $J$

Valldated vOC Results, 1993

\begin{tabular}{|c|c|c|c|c|c|c|c|c|c|c|c|c|}
\hline \multirow{4}{*}{$\begin{array}{l}\text { Sarmpling Point } \\
\text { Location } \\
\text { - } \\
\text { Date Sampled }\end{array}$} & \multicolumn{8}{|c|}{$G W-170$} & \multicolumn{4}{|c|}{$G W-183$} \\
\hline & \multicolumn{8}{|c|}{$\mathbf{E X P}$} & \multicolumn{4}{|c|}{$\mathbf{F}$} \\
\hline & \multicolumn{2}{|c|}{$01 / 23 / 93$} & \multicolumn{2}{|c|}{$05 / 24 / 93$} & \multicolumn{2}{|c|}{$08 / 21 / 93$} & \multicolumn{2}{|c|}{$11 / 04 / 93$} & \multicolumn{2}{|c|}{$03 / 10 / 93$} & \multicolumn{2}{|c|}{$06 / 21 / 93$} \\
\hline & $u g / L$ & sum & ug/L & 8 sum & ug/L & $8 \mathrm{sum}$ & $u g / L$ & s sum & ug/L & s sum & $u g / L$ & sum \\
\hline Acetone & 100 & - & 100 & . & 100 & - & 100 & . & FP1 & . & 100 & \\
\hline Benzene & 5v & - & so & .1 & 5u &. & 5v &. & 350 & 54.3 & 32 & 45.7 \\
\hline Bromoform & so & - & so & $\cdot$ & $5 v$ & $\cdot$ & 50 & $\cdot$ & 50 & $\cdot$ & 50 & • \\
\hline 2-Butanone & 100 & - & FP1 & . & 100 &. & FP3 &. & 100 &. & 100 & • \\
\hline Carbon disulfide & 50 & - & 50 &. & sv &. & 5u &. & so & . & 50 & . \\
\hline Carbon tetrachloride & 1 & 1.4 & 50 &. & 16 & 23.5 & 39 & 43.8 & 50 & . & 5u & . \\
\hline Chloroform & 57 & 82.6 & 59 & 85.5 & 43 & 63.2 & 41 & 46.1 & 50 & - & 50 & • \\
\hline Chloromethane & 100 & . & 100 & . & 100 & $\cdot$ & 100 &. & 100 & . & 100 & . \\
\hline 1,1-D1chloroethane & 50 & - & 5v & $\cdot$ & 50 & $\cdot$ & 50 & $\cdot$ & 5u & - & 5u & • \\
\hline 1,2-Dichloroethane & 50 & - & 50 & $\cdot 1$ & su &. & 50 & . & FP3 & . & 5u & . \\
\hline 1,1-Dichloroethene & 50 & - & su & $\cdot$ & 50 & $\cdot$ & su & $\cdot$ & 50 & $\cdot$ & 50 & • \\
\hline 1,2-Dichloroethene & 50 & - & 50 & $\cdot$ & 50 & $\cdot$ & FP3 & $\cdot$ & 50 & $\cdot$ & 5u & • \\
\hline Ethylbenzene & 50 & . & 50 & . & 5u &. & 50 & . & 22 & 3.4 & 2 & 2.9 \\
\hline 4-Methy1-2-pentanone & FP1 & . & 100 & $\cdot$ & 100 & - & 100 & $\cdot$ & 100 & $\cdot$ & 100 & • \\
\hline Methylene chloride & 2 & 2.9 & 2 & 2.9 & FP2 & $\cdot$ & 1 & 1.1 & FP1 & . & FP2 & . \\
\hline Styrene & 5u & • & 50 &. & 5u & $\cdot$ & 50 &. & FP3 & - & 50 & - \\
\hline Tetrachloroethene & 7 & 10.1 & 6 & 8.7 & 6 & 8.8 & 6 & 6.7 & 50 & - & 5u & . \\
\hline Toluene & 50 & $\cdot$ & 50 & $\cdot$ & 50 & $\cdot$ & 50 &. & 180 & 28 & 25 & 35.7 \\
\hline 1,1,1-Trichloroethane & 50 & $\cdot$ & 50 &. & 50 & $\cdot$ & 50 & $\cdot$ & 5u & $\cdot$ & 50 & • \\
\hline Trichloroethene & 2 & 2.9 & 2 & 2.9 & 3 & 4.4 & 2 & 2.2 & 5v & $\cdot$ & 50 & - \\
\hline vinyl acetate & 100 & $\cdot$ & 100 & $\cdot 1$ & 100 & $\cdot$ & 100 & $\cdot$ & FP3 & $\cdot$ & 100 & • \\
\hline vinyl chloride & 100 & $\cdot$ & 100 & $\cdot$ & 100 & $\cdot$ & 100 & $\cdot$ & 100 &. & 100 & - \\
\hline Xylenes & $5 u$ & . & 5u & . & 50 &. & 50 &. & 92 & 14.3 & 11 & 15.7 \\
\hline Summed VOCs & 69 & 99.9 & 69 & 100 & 68 & 99.9 & 89 & 99.9 & 644 & 100 & 70 & 100 \\
\hline
\end{tabular}

(CONTINUED) 
APPENDIX $J$

Valldated vOC Results, 1993

\begin{tabular}{|c|c|c|c|c|c|c|c|c|c|c|c|c|}
\hline \multirow{4}{*}{$\begin{array}{l}\text { Sampling Point } \\
\text { Location } \\
\text { Date Sampled }\end{array}$} & \multicolumn{4}{|c|}{$G W-183$} & \multicolumn{8}{|c|}{ GW-190 } \\
\hline & \multicolumn{4}{|c|}{$\mathbf{F F}$} & \multicolumn{8}{|c|}{ RG } \\
\hline & \multicolumn{2}{|c|}{$09 / 22 / 93$} & \multicolumn{2}{|c|}{$11 / 16 / 93$} & \multicolumn{2}{|c|}{$03 / 01 / 93$} & \multicolumn{2}{|c|}{$06 / 17 / 93$} & \multicolumn{2}{|c|}{$09 / 15 / 93$} & \multicolumn{2}{|c|}{$11 / 16 / 93$} \\
\hline & ug/L & sum & ug/L & sum & ug/L & $8 \mathrm{sum}$ & $u g / L$ & s sum & $u g / L$ & s sum & $u g / L$ & 8 sum \\
\hline Acetone & 100 & • & 100 & - & 100 & - & 200 & . & 200 & - & 100 & . \\
\hline Benzene & 57 & 47.1 & 7 & 36.8 & 50 & • & 50 & - & 100 & - & 50 & • \\
\hline Bromoform & 5u & $\cdot$ & 50 & $\cdot$ & 50 & • & 50 & $\cdot$ & 100 & - & 50 & • \\
\hline 2-Butanone & 100 & • & 100 & • & 100 & • & 100 & - & 200 & - & 100 & • \\
\hline Carbon disulfide & 50 & - & 50 & . & $5 v$ & $\cdot$ & 50 & . & 100 & - & 50 & • \\
\hline Carbon tetrachloride & 50 & • & 50 & $\cdot$ & 50 & $\cdot$ & 50 & - & 100 & - & 50 & . \\
\hline Chloroform & $5 v$ & - & 5u & . & 50 & . & 50 & $\cdot$ & 100 & - & 50 & • \\
\hline Chloromethane & 100 & . & 100 & . & 100 & $\cdot$ & 100 & - & 200 & - & 100 & • \\
\hline 1,1-Dichloroethane & 50 & • & 50 & $\cdot$ & 50 & • & 6 & 1.5 & 170 & 48.9 & 50 & • \\
\hline 1,2-Dichloroethane & 50 & $\cdot$ & 5u & $\cdot 1$ & 50 &. & 50 & $\cdot$ & 100 & $\cdot$ & 50 & - \\
\hline 1,1-Dichloroethene & 50 & $\cdot$ & 50 & $\cdot$ & 50 & $\cdot$ & 50 & • & FP3 & - & 50 & • \\
\hline 1,2-Dichloroethene & 50 & $\cdot$ & 5v & $\cdot$ & 5u & $\cdot$ & 220 & 53.5 & 100 & - & 7 & 10.8 \\
\hline Ethylbenzene & 5 & 4.1 & 1 & 5.3 & 5u & $\cdot$ & 50 & $\cdot$ & 100 & - & 50 & • \\
\hline 4-Methy1-2-pentanone & 100 & $\cdot$ & 100 & $\cdot$ & 100 & $\cdot$ & 100 & • & 200 & - & 100 & • \\
\hline Methylene chloride & $5 \mathbf{v}$ & $\cdot$ & FP1 & $\cdot 1$ & 50 & $\cdot$ & 50 & $\cdot$ & 100 & - & FP1 & • \\
\hline styrene & $\mathbf{5 u}$ & $\cdot$ & 50 &. & 50 &. & 50 & . & 100 & - & 50 & • \\
\hline Tetrachloroethene & 50 & $\cdot$ & 50 & $\cdot$ & 110 & 90.9 & 61 & 14.8 & 89 & 25.6 & 49 & 75.4 \\
\hline Toluene & 38 & 31.4 & 6 & 31.6 & 50 & $\cdot$ & 50 & $\cdot$ & 100 & $\cdot$ & 50 & • \\
\hline 1,1,1-Trichloroethane & 5u & $\cdot$ & 50 & $\cdot$ & 50 & $\cdot$ & 50 & $\cdot$ & 100 & $\cdot$ & 50 & • \\
\hline Trichloroethene & su & $\cdot 1$ & 5u & - & 11 & 9.1 & 67 & 16.3 & 39 & 11.2 & 7 & 10.8 \\
\hline vinyl acetate & 100 & $\cdot$ & 100 & • & 100 & $\cdot$ & 100 & $\cdot$ & 200 & $\cdot$ & 100 & • \\
\hline vinyl chloride & 100 & $\cdot$ & 100 & $\cdot$ & 100 & - & 57 & 13.9 & 50 & 14.4 & 2 & 3.1 \\
\hline Xylenes & 21 & 17.4 & 5 & 26.3 & 5u & - & 5u & . & 100 & $\cdot$ & 50 & • \\
\hline Summed VOCs & 121 & 100 & 19 & 100 & 121 & 100 & 411 & 100 & 348 & 100.1 & 65 & 100.1 \\
\hline
\end{tabular}

(CONTINUED) 
APPENDIX $J$

Valldated vOC Results, 1993

\begin{tabular}{|c|c|c|c|c|c|c|c|c|c|c|c|c|}
\hline \multirow{4}{*}{$\begin{array}{l}\text { Sampling Point } \\
\text { Location } \\
\text { Date Sampled }\end{array}$} & \multicolumn{8}{|c|}{$G W-191$} & \multicolumn{4}{|c|}{ GW-192 } \\
\hline & \multicolumn{8}{|c|}{ B4 } & \multicolumn{4}{|c|}{ B4 } \\
\hline & \multicolumn{2}{|c|}{$01 / 12 / 93$} & \multicolumn{2}{|c|}{$04 / 12 / 93$} & \multicolumn{2}{|c|}{$07 / 08 / 93$} & \multicolumn{2}{|c|}{$12 / 09 / 93$} & \multicolumn{2}{|c|}{$01 / 14 / 93$} & \multicolumn{2}{|c|}{$04 / 13 / 93$} \\
\hline & $\mathrm{ug} / \mathrm{L}$ & sum & ug/L & sum & ug/L & 8 sum & $u g / L$ & 8 sum & $u g / L$ & s sum & ug $/ \mathbf{L}$ & 8 sum \\
\hline Acetone & 100 & . & 100 & - & 100 & $\cdot$ & FP1 & . & 100 & . & 100 & . \\
\hline Benzene & 50 & $\cdot$ & 5u & - & su &. & 50 & . & 50 & - & $5 v$ & • \\
\hline Bromoform & 50 & - & 50 & - & su &. & 50 & . & 50 &. & 50 & . \\
\hline 2-Butanone & 100 &. & 100 & . & 100 &. & FP1 & . & 100 & $\cdot$ & 100 & • \\
\hline Carbon disulfide & 50 & $\cdot$ & 5u & $\cdot$ & 5u & $\cdot$ & 5u & $\cdot$ & 50 &. & 50 & • \\
\hline Carbon tetrachloride & 5v & . & $5 v$ & $\cdot$ & 5u & $\cdot$ & 5u & . & 50 &. & $5 v$ & - \\
\hline Chloroform & 50 & $\cdot$ & $5 \mathbf{v}$ & $\cdot$ & 50 & $\cdot$ & 5u & . & $5 v$ &. & 50 & . \\
\hline Chloromethane & 100 & $\cdot$ & 100 & . & 100 & $\cdot$ & 100 & . & 100 &. & 100 & . \\
\hline 1,1-Dichloroethane & 50 & $\cdot$ & 50 & - & 5u & $\cdot$ & 50 & . & 1 & 4.2 & 50 & . \\
\hline 1,2-D1chloroethane & $5 v$ & . & 50 & . & 5u & $\cdot$ & 5u &. & $5 u$ & $\cdot$ & 50 & . \\
\hline 1,1-Dichloroetherie & 5v & $\cdot$ & 50 & $\cdot$ & 5v &. & 50 & - & 50 & . & 50 & • \\
\hline 1,2-Dichloroethene & $5 v$ & . & 50 & . & 50 &. & 50 & • & 19 & 79.2 & 18 & 81.8 \\
\hline Ethylbenzene & 50 & $\cdot$ & 50 & $\cdot$ & 5v & $\cdot$ & 50 & $\cdot$ & 5v & $\cdot$ & 50 & • \\
\hline 4-Methy1-2-pentanone & 100 & - & 100 & - & 100 &. & FP1 & • & FP1 & . & 100 & • \\
\hline Methylene chloride & 50 & . & 50 & - & 5u & $\cdot$ & FP1 & $\cdot$ & 5u & $\cdot$ & 50 & - \\
\hline styrene & $5 \mathbf{v}$ & - & 50 & - & 50 & $\cdot$ & $5 v$ & $\cdot$ & 50 & . & 50 & • \\
\hline Tetrachloroethene & 50 & - & 50 & $\cdot$ & 50 & $\cdot$ & 5u & $\cdot$ & 1 & 4.2 & 1 & 4.5 \\
\hline Toluene & 50 & - & 5u & - & 50 & . & 50 &. & $5 u$ &. & so & • \\
\hline $1,1,1$-Trichloroethane & 50 & • & 50 & - & 50 & . & 5v & . & 50 & • & su & • \\
\hline Trichloroethene & 50 & $\cdot$ & 5u & - & 5u & $\cdot$ & 50 &. & 3 & 12.5 & 3 & 13.6 \\
\hline vinyl acetate & 100 & - & 100 & - & 100 &. & 100 &. & 100 &. & 100 & • \\
\hline vinyl chloride & 100 & $\cdot$ & 100 & - & 100 & - & 100 & $\cdot$ & 100 & - & 100 & • \\
\hline xylenes & 50 & $\cdot$ & 50 & . & 50 & . & 50 &. & 50 & . & 50 & . \\
\hline Summed vOCs & 0 &. & 0 & . & 0 & . & 0 & . & 24 & 100.1 & 22 & 99.9 \\
\hline
\end{tabular}

(CONTINUED) 
APPENDIX $J$

Validated VOC Results, 1993

\begin{tabular}{|c|c|c|c|c|c|c|c|c|c|c|c|c|}
\hline \multirow{4}{*}{$\begin{array}{l}\text { Sarmiling Point } \\
\text { - } \\
\text { Location } \\
\text { - } \\
\text { Date Sampled }\end{array}$} & \multicolumn{4}{|c|}{ Gw-192 } & \multicolumn{8}{|c|}{ Gw-193 } \\
\hline & \multicolumn{4}{|c|}{ B4 } & \multicolumn{8}{|c|}{ T2331 } \\
\hline & \multicolumn{2}{|c|}{$07 / 08 / 93$} & \multicolumn{2}{|c|}{$12 / 09 / 93$} & \multicolumn{2}{|c|}{$03 / 11 / 93$} & \multicolumn{2}{|c|}{$06 / 22 / 93$} & \multicolumn{2}{|c|}{$09 / 23 / 93$} & \multicolumn{2}{|c|}{$11 / 18 / 93$} \\
\hline & ug/L & 8 sum & ug/L & sum & $u g / L$ & sum & ug $/ \mathrm{I}$ & sum & ug/L & sum & ug/L & sum \\
\hline Acetone & 100 & $\cdot$ & FP1 & $\cdot$ & 2500 & $\cdot$ & 2500 & $\cdot$ & 1000 & • & 1000 & \\
\hline Benzene & 50 & $\cdot$ & 50 & $\cdot$ & 2200 & 54 & 1400 & 55.2 & 1200 & 56.6 & 2800 & 61.8 \\
\hline Bromoform & 50 & $\cdot$ & 50 & . & 1300 &. & 1300 & $\cdot$ & 500 & $\cdot$ & 500 & • \\
\hline 2-Butanone & 100 & $\cdot$ & FP1 &. & 2500 &. & 2500 & . & 1000 &. & 1000 & - \\
\hline Carbon disulfide & 50 & $\cdot$ & 50 & $\cdot$ & 1300 &. & 1300 & $\cdot$ & 500 &. & 500 & - \\
\hline Carbon tetrachloride & 50 & $\cdot$ & 5u & . & 1300 & $\cdot$ & 1300 & . & 500 & . & 500 & - \\
\hline Chloroform & 50 &. & su & $\cdot$ & 1300 & $\cdot$ & 1300 &. & 500 & . & 500 & • \\
\hline Chloromethane & 100 & $\cdot$ & 100 &. & 2500 & $\cdot$ & 2500 & . & 1000 & - & 1000 & - \\
\hline 1,1-Dichloroethane & su & $\cdot$ & 1 & 4.8 & 1300 &. & 1300 &. & 500 & . & 500 & . \\
\hline 1,2-Dichloroethane & 50 & $\cdot$ & 50 &. & 1300 & $\cdot$ & 1300 & $\cdot$ & 500 & - & 500 & - \\
\hline 1,1-Dichloroethene & 50 &. & 50 & $\cdot$ & 1300 & $\cdot 1$ & 1300 &. & 500 & . & 500 & • \\
\hline 1,2-Dichloroethene & 9 & 100 & 16 & 76.2 & 1300 &. & 1300 & $\cdot$ & 500 & $\cdot$ & 500 & • \\
\hline Ethylbenzene & 50 & $\cdot$ & 50 & $\cdot$ & 790 & 19.4 & 740 & 29.2 & 520 & 24.5 & 450 & 9.9 \\
\hline 4-Methy1-2-pentanone & 100 & $\cdot$ & FPI &. & 2500 & . & 2500 & $\cdot$ & 1000 &. & 1000 & • \\
\hline Methylene chloride & 50 & $\cdot$ & FP1 & $\cdot$ & 34 & 0.8 & 1300 &. & 500 &. & 500 & - \\
\hline Styrene & 50 & $\cdot$ & 50 & $\cdot$ & 1300 &. & 1300 &. & 500 &. & 500 & . \\
\hline Tetrachloroethene & 50 & $\cdot$ & 1 & 4.8 & 1300 &. & 1300 & $\cdot$ & 500 & $\cdot$ & 500 & • \\
\hline Toluene & 50 &. & 50 &. & 370 & 9.1 & 75 & 3 & 100 & 4.7 & 700 & 15.5 \\
\hline $1,1,1-\operatorname{Tr} 1$ chloroethane & 50 & $\cdot$ & 50 & $\cdot$ & 1300 & $\cdot$ & 1300 & $\cdot$ & 500 & $\cdot$ & 500 & • \\
\hline Trichloroethene & 50 &. & 3 & 14.3 & 1300 &. & 1300 &. & 500 &. & 500 & - \\
\hline vinyl acetate & 100 & $\cdot$ & 100 & $\cdot$ & 2500 & $\cdot$ & 2500 & . & 1000 & $\cdot$ & 1000 & - \\
\hline vinyl chloride & 100 & $\cdot$ & 100 &. & 2500 &. & 2500 &. & 1000 &. & 1000 & . \\
\hline Xylenes & 50 &. & 5u & $\cdot$ & 680 & 16.7 & 320 & 12.6 & 300 & 14.2 & 580 & 12.8 \\
\hline Summed vocs & 9 & 100 & 21 & 100.1 & 4074 & 100 & 2535 & 100 & 2120 & 100 & 4530 & 100 \\
\hline
\end{tabular}

(CONTINUED) 
APPERDIX $J$

validated voC Results, 1993

\begin{tabular}{|c|c|c|c|c|c|c|c|c|c|c|c|c|}
\hline \multirow{4}{*}{$\begin{array}{l}\text { Sampling Point } \\
\text { Location } \\
\text { - } \\
\text { Date Sampled }\end{array}$} & \multicolumn{8}{|c|}{ GW-194 } & \multicolumn{4}{|c|}{ GW-195 } \\
\hline & \multicolumn{8}{|c|}{ B4 } & \multicolumn{4}{|c|}{ B4 } \\
\hline & \multicolumn{2}{|c|}{$01 / 13 / 93$} & \multicolumn{2}{|c|}{$04 / 13 / 93$} & \multicolumn{2}{|c|}{$07 / 08 / 93$} & \multicolumn{2}{|c|}{$12 / 09 / 93$} & \multicolumn{2}{|c|}{$01 / 13 / 93$} & \multicolumn{2}{|c|}{$04 / 13 / 93$} \\
\hline & ug/L & sum & $\mathrm{ug} / \mathrm{I}$ & sum & $u g / L$ & sum & $\mathrm{ug} / \mathrm{L}$ & sum & ug/L & sum & $u g / L$ & sum \\
\hline Acetone & 100 & - & 100 & - & 100 & - & FP1 & - & 100 & . & 100 & . \\
\hline Benzene & 50 & - & 5v & - & 50 & - & so & - & 50 & - & 5v & • \\
\hline Bromoform & 50 & $\cdot$ & 5v & - & so & - & 50 & • & 50 & - & 5u & • \\
\hline 2-Butanone & 100 & • & 100 & • & 100 & - & FP1 & - & 100 & - & 100 & . \\
\hline Carbon disulfide & $5 \mathbf{v}$ & $\cdot$ & 50 & $\cdot$ & 50 & - & $5 \mathbf{v}$ & - & su & - & su & $\cdot$ \\
\hline Carbon tetrachloride & 5u & $\cdot$ & 50 & • & 50 & - & $5 v$ & - & $5 v$ & - & 50 & $\cdot$ \\
\hline Chloroform & 5u & $\cdot$ & 5u & $\cdot$ & 50 & - & 5v & - & 50 & - & 5u & • \\
\hline Chloromethane & 100 & $\cdot$ & 100 & $\cdot$ & 100 & - & 100 & - & 100 & - & 100 & • \\
\hline 1,1-Dichloroethane & 50 & $\cdot$ & $\mathbf{5 u}$ & $\cdot$ & 50 & - & 50 & - & 5u & - & 50 & $\cdot$ \\
\hline 1,2-Dichloroethane & 50 & $\cdot$ & 50 & $\cdot$ & 50 & - & 50 & $\cdot$ & 50 & - & 50 & $\cdot$ \\
\hline 1,1-Dichloroethene & 50 & $\cdot$ & 5u & $\cdot$ & 50 & - & 50 & - & 50 & - & 50 & • \\
\hline 1,2-Dichloroethene & 50 & $\cdot$ & $5 v$ & $\cdot$ & 5u & - & 50 & - & 50 & - & 5u & $\cdot$ \\
\hline Ethylbenzene & 50 & $\cdot$ & $5 v$ & $\cdot$ & 5u & - & 50 & • & 5u & - & 50 & • \\
\hline 4-Methy 1-2-pentanone & 100 & $\cdot$ & 100 & $\cdot$ & 100 & - & FP1 & • & 100 & - & 100 & • \\
\hline Methylene chloride & 50 & $\cdot$ & 50 & $\cdot$ & 5u & - & FP1 & - & 50 & - & 5v & • \\
\hline Styrene & 50 & $\cdot$ & 50 & $\cdot$ & 50 & - & 5u & - & 50 & - & 5u & $\cdot$ \\
\hline Tetrachloroethene & 50 & $\cdot$ & 50 & $\cdot$ & 50 & $\cdot$ & 50 & - & 50 & - & 50 & $\cdot$ \\
\hline Toluene & 50 & - & 50 & - & 50 & - & 50 & - & 50 & • & 5u & • \\
\hline $1,1,1$-Trichloroethane & 50 & $\cdot$ & $5 \mathbf{v}$ & $\cdot$ & 50 & $\cdot$ & 50 & - & 50 & - & $5 u$ & • \\
\hline Trichloroethene & 5v & $\cdot$ & 50 & $\cdot$ & 5v & $\cdot$ & 5v & - & 5u & • & 50 & • \\
\hline vinyl acetate & 100 & $\cdot$ & 100 & $\cdot$ & 100 & - & 100 & - & 100 & - & 100 & • \\
\hline vinyl chloride & 100 & $\cdot$ & 100 & $\cdot$ & 100 & $\cdot$ & 100 & - & 100 & - & 100 & • \\
\hline Xylenes & 50 & $\cdot$ & 50 & $\cdot$ & 50 & $\cdot$ & 50 & - & 5u & - & 50 & • \\
\hline Summed VOCs & 0 & . & 0 &. & 0 & . & 0 & . & 0 & . & 0 & . \\
\hline
\end{tabular}

(CONTINUED) 
APPENDIX $J$

Valldated voc Results, 1993

\begin{tabular}{|c|c|c|c|c|c|c|c|c|c|c|c|c|}
\hline \multirow{4}{*}{$\begin{array}{l}\text { Sampling Point } \\
\text { Location } \\
\text { Date Sampled }\end{array}$} & \multicolumn{4}{|c|}{ GW-195 } & \multicolumn{8}{|c|}{$G w-199$} \\
\hline & \multicolumn{4}{|c|}{ B4 } & \multicolumn{8}{|c|}{ GRIDI1 } \\
\hline & \multicolumn{2}{|c|}{$07 / 08 / 93$} & \multicolumn{2}{|c|}{$12 / 09 / 93$} & \multicolumn{2}{|c|}{$01 / 29 / 93$} & \multicolumn{2}{|c|}{$04 / 07 / 93$} & \multicolumn{2}{|c|}{$08 / 02 / 93$} & \multicolumn{2}{|c|}{$10 / 12 / 93$} \\
\hline & ug/L & s sum & $u g / L$ & $8 \mathrm{sum}$ & $u g / L$ & s sum & ug/L & s sum & ug/I & $\operatorname{sum}$ & $u g / L$ & s sum \\
\hline Acetone & 100 & $\cdot$ & FP1 & $\cdot$ & 100 & $\cdot$ & 100 & $\cdot$ & 100 & $\cdot$ & 100 & \\
\hline Benzene & 50 & $\cdot$ & 50 & $\bullet$ & 50 & - & so & $\cdot$ & 50 & $\cdot$ & 50 & \\
\hline Bromoform & 50 &. & so & - & su & $\cdot$ & 50 & $\cdot$ & 50 & - & su & \\
\hline 2-Butanone & 100 & $\cdot$ & $\mathbf{F P 1}$ & - & 100 & - & 100 & $\cdot$ & 100 & $\cdot$ & 100 & \\
\hline Carbon disulfide & su & $\cdot$ & su &. & so & - & su & $\cdot$ & 50 & $\cdot$ & 50 & \\
\hline Carbon tetrachloride & 50 & $\cdot$ & 50 & $\cdot$ & 50 & - & 50 & $\cdot$ & 50 & $\cdot$ & 50 & \\
\hline Chloroform & 50 & $\cdot$ & so & $\cdot$ & 50 & - & 50 & $\cdot$ & 50 & $\cdot$ & 50 & \\
\hline Chloromethane & 100 & $\cdot$ & 100 & $\cdot$ & 100 & $\cdot$ & 100 & $\cdot$ & 100 & $\cdot$ & 100 & \\
\hline 1,1-Dichloroethane & so & $\cdot$ & 50 & • & 50 & $\cdot$ & 50 & $\cdot$ & 50 & $\cdot$ & 5v & \\
\hline 1,2-Dichloroethane & su & $\cdot$ & 5v & $\cdot$ & 5v & $\cdot$ & so & $\cdot$ & 50 & - & 50 & \\
\hline 1,1-Dichloroethene & 50 & $\cdot$ & 50 & $\cdot$ & su & - & 50 & $\cdot$ & 50 & - & 50 & \\
\hline 1,2-Dichloroethene & 50 & $\cdot$ & 50 & $\cdot$ & su & $\cdot$ & 50 & $\cdot$ & 50 & $\cdot$ & 50 & \\
\hline Ethylbenzene & 50 & $\cdot$ & 5u & $\cdot$ & 5u & - & 50 & $\cdot$ & 50 & $\cdot$ & 50 & \\
\hline 4-Methyl-2-pentanone & 100 &. & 100 & $\cdot$ & 100 & • & 100 & $\cdot$ & 100 & $\cdot$ & 100 & \\
\hline Methylene chloride & 5u & $\cdot$ & $\mathbf{F P 1}$ & - & so & $\cdot$ & 50 & $\cdot$ & FP1 & - & 50 & \\
\hline Styrene & 50 & $\cdot$ & 50 & $\cdot$ & sv & • & 5v & $\cdot$ & $\mathbf{5 0}$ & $\cdot$ & 50 & \\
\hline Tetrachloroethene & su & • & so & $\cdot$ & su & - & 50 & $\cdot$ & 50 & $\cdot$ & so & \\
\hline Toluene & su & $\cdot$ & 50 & $\cdot$ & 5u & - & su & $\cdot$ & 50 & $\cdot$ & 50 & \\
\hline $1,1,1$-Trichloroethene & 5v & $\cdot$ & 5v & . & 50 & • & 5v & $\cdot$ & 50 & $\cdot$ & 5v & \\
\hline Trichloroethene & 50 & $\cdot$ & 50 & $\cdot$ & 5v & $\cdot$ & 50 & $\cdot$ & 50 & $\cdot$ & sv & \\
\hline Vinyl acetate & 100 & $\cdot$ & 100 & - & 100 & - & 100 & $\cdot$ & 100 & $\cdot$ & 100 & \\
\hline vingl chloride & 100 & $\cdot$ & 100 & $\cdot$ & 100 & $\cdot$ & 100 & $\cdot$ & 100 & • & 100 & \\
\hline & 50 & $\cdot$ & 5v & $\cdot$ & 5v & $\cdot$ & 5o & $\cdot$ & 50 & - & 50 & \\
\hline Summed VOCs & 0 &. & 0 & $\cdot$ & 0 & . & 0 & . & 0 & . & 0 & \\
\hline
\end{tabular}

(CONTINUED) 
APPEIDIX J

validated voc Results, 1993

\begin{tabular}{|c|c|c|c|c|c|c|c|c|c|c|c|c|}
\hline \multirow{4}{*}{$\begin{array}{l}\text { Sampling Point } \\
\text { Location } \\
\text { Date Sampled }\end{array}$} & \multicolumn{8}{|c|}{ GW-204 } & \multicolumn{4}{|c|}{ GN-206 } \\
\hline & \multicolumn{8}{|c|}{ T0134 } & \multicolumn{4}{|c|}{$\exp$} \\
\hline & \multicolumn{2}{|c|}{$03 / 11 / 93$} & \multicolumn{2}{|c|}{$06 / 22 / 93$} & \multicolumn{2}{|c|}{$09 / 23 / 93$} & \multicolumn{2}{|c|}{$11 / 16 / 93$} & \multicolumn{2}{|c|}{$01 / 19 / 93$} & \multicolumn{2}{|c|}{$05 / 06 / 93$} \\
\hline & ug/L & - sum & $\operatorname{ug} / 2$ & $8 \operatorname{sum}$ & $u g / L$ & - sum & $\mathbf{u g} / \mathbf{L}$ & I sum & ug/L & > sum & $u g / L$ & e sum \\
\hline Acetone & 100 & - & 100 & - & 100 & $\cdot$ & 100 & - & 100 & . & 100 & . \\
\hline Benzene & 50 & - & 50 & - & 5u & - & so & - & 50 & - & su & . \\
\hline Bromoform & 50 & - & 5u & - & su & . & 50 & - & 50 & - & so & . \\
\hline 2-Butanone & 100 & - & 100 & - & 100 & . & 100 & $\cdot$ & 100 & - & 100 & . \\
\hline Carbon disulfide & 5v & - & so & - & 50 & . & 5v & $\cdot$ & 50 & . & 5v & . \\
\hline Carbon tetrachloride & 50 & - & su & - & 50 & . & sv & . & 5v & - & 50 & . \\
\hline Chloroform & 50 & - & 50 & - & 50 & $\cdot$ & 50 & - & 50 & - & 50 & . \\
\hline Chloromethane & 100 & - & 100 & - & 100 & . & 100 & - & 100 & - & 100 & . \\
\hline 1,1-D1chloroethane & 5v & - & su & - & 50 & $\cdot$ & so & $\cdot$ & 50 & - & 50 & . \\
\hline 1,2-Dichloroethene & 5v & - & 50 & - & 50 & $\cdot$ & 5v & $\cdot$ & 50 & - & 50 & . \\
\hline 1,1-Dichloroethene & 5v & - & so & - & so & - & su & . & 50 & - & 50 & . \\
\hline 1.2-Dichloroethene & so & $\cdot$ & 50 & - & 5v & - & so & $\cdot$ & su & - & 50 & . \\
\hline Ethylbenzene & 2 & 100 & 50 & - & 50 & - & 2 & 100 & 50 & - & so & . \\
\hline 4-Methy1-2-pentanone & 100 & $\cdot$ & 100 & - & 100 & • & 100 & $\cdot$ & 100 & $\cdot$ & FP1 & . \\
\hline Methylene chloride & FP3 & • & 50 & - & so & - & $\mathbf{F P 1}$ & - & 50 & . & FP1 & . \\
\hline Styrene & 50 & $\cdot$ & 50 & - & 5v & $\cdot$ & 50 & - & 50 & - & so & . \\
\hline Tetrachloroethene & 50 & • & 50 & - & 5v &. & so & - & 50 & $\cdot$ & 50 & . \\
\hline Toluene & 50 & . & so & - & 50 & . & 5v & - & 50 & - & so & . \\
\hline $1,1,1-\operatorname{Tr} 1 \mathrm{chl}$ oroethane & 50 & . & 50 & - & so & $\cdot$ & 5u & $\cdot$ & so & - & so & . \\
\hline Trichloroethene & so & - & so & . & so & - & so & - & 5v & - & 50 & . \\
\hline Vinyl acetate & 100 & $\cdot$ & 100 & - & 100 & $\cdot$ & 100 & $\cdot$ & 100 & $\cdot$ & 100 & . \\
\hline vinyl chloride & 100 & $\cdot$ & 100 & - & 100 & . & 100 & - & 100 & - & 100 & . \\
\hline xylenes & 50 & $\cdot$ & su & - & 50 & $\cdot$ & FP3 & - & 50 & $\cdot$ & 50 & • \\
\hline Summed vocs & 2 & 100 & 0 & . & 0 &. & 2 & 100 & 0 & - & 0 & . \\
\hline
\end{tabular}

(CONTINURD) 
APPENDIX J

valldated voc Reeulte, 1993

\begin{tabular}{|c|c|c|c|c|c|c|c|c|c|c|c|c|}
\hline \multirow{4}{*}{$\begin{array}{l}\text { Sampling Point } \\
\text { Location } \\
\text { Date Sampled }\end{array}$} & \multicolumn{4}{|c|}{ GW-206 } & \multicolumn{8}{|c|}{$6 w-207$} \\
\hline & \multicolumn{4}{|c|}{$\mathbf{E x P}$} & \multicolumn{8}{|c|}{$\mathbf{E x P}$} \\
\hline & \multicolumn{2}{|c|}{$08 / 12 / 93$} & \multicolumn{2}{|c|}{$10 / 27 / 93$} & \multicolumn{2}{|c|}{$01 / 14 / 93$} & \multicolumn{2}{|c|}{$05 / 04 / 93$} & \multicolumn{2}{|c|}{$08 / 11 / 93$} & \multicolumn{2}{|c|}{$10 / 26 / 93$} \\
\hline & $u g / L$ & c sum & $u g / 2$ & - an & $u g / L$ & $\because=$ & $u g / L$ & - & ug/L & เ cum & $u g / L$ & 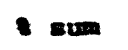 \\
\hline Acetone & 100 & - & 100 & - & 100 & . & 100 & - & 100 & - & FPI & . \\
\hline Benzene & 50 & . & 50 & - & so & - & 50 & $\cdot$ & so & - & so & . \\
\hline Bromoform & 50 & - & so & - & so & - & 50 & $\cdot$ & sv & $\cdot$ & 50 & · \\
\hline 2-Butanone & 100 & $\cdot$ & 100 & - & 100 & - & 100 & - & 100 & $\cdot$ & $\mathbf{P P 1}$ & • \\
\hline Carbon disulfide & 50 & . & 50 & - & su & - & 50 & . & 50 & - & 50 & • \\
\hline Carbon tetrachloride & 50 & $\cdot$ & so & - & so & $\cdot$ & 50 & $\cdot$ & su & - & 50 & . \\
\hline Chloroform & so & . & 50 & - & so & . & so & - & sv & - & 50 & • \\
\hline Chloromethane & 100 & - & 100 & - & 100 & $\cdot$ & 100 & $\cdot$ & 100 & $\cdot$ & 100 & • \\
\hline 1,1-D1chloroethane & so & $\cdot$ & so & - & so & $\cdot$ & so & $\cdot$ & so & $\cdot$ & 50 & • \\
\hline 1,2-Dichloroethane & 50 & $\cdot$ & 50 & - & 50 & $\cdot$ & so & $\cdot$ & sv & - & 50 & • \\
\hline 1,1-Dichloroethene & 50 & . & 50 & - & so & . & 50 & $\cdot$ & 50 & - & 50) & . \\
\hline 1,2-Dichloroethene & 50 & $\cdot$ & 50 & - & 50 & $\cdot$ & so & $\cdot$ & 5v & $\cdot$ & 50 & • \\
\hline Ethylbenzene & so & - & 50 & - & so & $\cdot$ & so & $\cdot$ & 50 & $\cdot$ & 50 & • \\
\hline 1-Methy1-2-pentanone & 100 & . & 100 & - & FP1 & - & 100 & $\cdot$ & 100 & - & 100 & . \\
\hline Nethylene chloride & so & $\cdot$ & 50 & - & 5v & $\cdot$ & so & $\cdot$ & so & $\cdot$ & $\mathbf{P P 1}$ & $\cdot$ \\
\hline styrene & 5v & - & 5v & - & 50 & $\cdot$ & 50 & $\cdot$ & so & - & 50 & • \\
\hline Tetrachloroethene & sv &. & 50 & - & 50 & $\cdot$ & 50 & $\cdot$ & 50 & $\cdot$ & 50 & • \\
\hline Toluene & 50 & $\cdot$ & so & - & so & $\cdot$ & 50 & $\cdot$ & so & - & su & . \\
\hline $1,1,1$-Trichloroethane & 50 & $\cdot$ & so & - & 50 & $\cdot$ & so & $\cdot$ & 50 & $\cdot$ & 50 & $\cdot$ \\
\hline Irichloroethene & so & $\cdot$ & so & - & sv & $\cdot$ & 50 & $\cdot$ & 50 & - & su & . \\
\hline Vinyl acetate & 100 & - & 100 & - & 100 &. & 100 & $\cdot$ & 200 & - & 100 & . \\
\hline vinyl chloride & 100 & - & 100 & - & 100 & $\cdot$ & 100 & $\cdot$ & 100 & $\cdot$ & 100 & . \\
\hline xylenes & su & $\cdot$ & 50 & - & 50 & - & 50 & - & so & - & so & . \\
\hline Summed vOCB & 0 & . & o) & . & 0 & . & -) & . & 0 & - & o) & . \\
\hline
\end{tabular}

(COArTINUED) 
valldated voc nosulte, 1993

\begin{tabular}{|c|c|c|c|c|c|c|c|c|c|c|c|c|}
\hline \multirow{4}{*}{$\begin{array}{l}\text { Sarpling Point } \\
\text { Location } \\
\text { Dete Sampled }\end{array}$} & \multicolumn{8}{|c|}{$\sigma w-220$} & \multicolumn{4}{|c|}{$6-232$} \\
\hline & \multicolumn{8}{|c|}{$\max$} & \multicolumn{4}{|c|}{ exp } \\
\hline & \multicolumn{2}{|c|}{$01 / 25 / 93$} & \multicolumn{2}{|c|}{$04 / 22 / 93$} & \multicolumn{2}{|c|}{$08 / 06 / 93$} & \multicolumn{2}{|c|}{$11 / 01 / 93$} & \multicolumn{2}{|c|}{$01 / 26 / 93$} & \multicolumn{2}{|c|}{$05 / 21 / 93$} \\
\hline & $\operatorname{ug} / 2$ & - แm & $\mathrm{ug} / \mathrm{L}$ & sen & $\operatorname{ug} / 2$ & an & $\operatorname{ug} / 2$ & sen & $\log / \mathrm{L}$ & $\because=$ & $u g / 2$ & $1=$ \\
\hline Acetone & $\mathbf{F P 2}$ & - & 200 & - & 200 & - & 500 & - & 100 & $\cdot$ & 100 & \\
\hline Benzene & 100 & $\cdot$ & 100 & $\cdot$ & 100 & - & 250 & - & $\mathbf{s 0}$ & $\cdot$ & so & rat \\
\hline Bromoform & 100 & . & 100 & - & 100 & - & 250 & - & so & $\cdot$ & so & . \\
\hline 2-Butanone & 200 & $\cdot$ & 200 & $\cdot$ & 200 & $\cdot$ & 500 & - & 100 & $\cdot$ & 100 & sina \\
\hline Carbon disulfide & 100 & $\cdot$ & 100 & $\cdot$ & 100 & $\cdot$ & 250 & • & so & $\cdot$ & so & ra \\
\hline Carbon tetrachloride & 320 & อ8.9 & 200 & 87.2 & 490 & $\mathbf{8 0 . 9}$ & 400 & 05.7 & so & $\cdot$ & 50 & r \\
\hline Chloroform & 17 & 4.7 & 14 & 4.4 & 23 & 4.2 & 21 & 4.5 & so & - & so & . \\
\hline Chloromethane & 200 & $\cdot$ & 200 & $\cdot$ & 200 & $\cdot$ & 500 & • & 100 & $\cdot$ & 100 & ra \\
\hline 1,1-Dichloroethene & 100 &. & 100 &. & 100 & - & 258 & - & 50 & $\cdot$ & 50 & . \\
\hline 1,2-Dichloroethane & 100 & $\cdot 1$ & 100 & $\cdot$ & 100 & $\cdot$ & 250 & - & 50 & - & so & ra \\
\hline 1,1-Dichloroethene & 100 & $\cdot 1$ & 100 & $\cdot$ & 100 & $\cdot$ & 250 & - & 50 & $\cdot$ & so & sina \\
\hline 1.2-Dichloroethene & 100 &. & 100 & $\cdot$ & 100 & $\cdot$ & 250 & - & 50 & $\cdot$ & 50 & . \\
\hline zthylbenzene & 100 & $\cdot$ & 100 & $\cdot$ & 100 & $\cdot$ & 250 & - & so & $\cdot$ & 50 & . \\
\hline 1-methy1-2-pentanone & FP1 & $\cdot$ & 200 & $\cdot$ & 200 & $\cdot$ & 500 & - & $\boldsymbol{F} 1$ & - & 100 & r \\
\hline Wethylene chloride & 100 & $\cdot$ & 100 & $\cdot$ & 200 & $\cdot$ & $\mathbf{r P 1}$ & • & so & $\cdot$ & so & . \\
\hline Styrene & 100 & $\cdot$ & 100 & $\cdot$ & 100 & $\cdot$ & 250 & • & 50 & $\cdot$ & so & . \\
\hline Tetrachloroethene & 19 & 5.3 & 23 & 7.2 & 32 & 5.0 & 39 & 3.4 & so & $\cdot$ & so & ra \\
\hline Toluene & 100 & $\cdot$ & 100 & $\cdot$ & 200 & $\cdot$ & 250 & $\cdot$ & so & $\cdot$ & so & r \\
\hline 1,1,1-Trichloroethane & 100 & $\cdot$ & 100 & $\cdot$ & 100 & $\cdot$ & 250 & - & 50 & . & so & . \\
\hline Irichloroethene & 4 & 1.1 & 4 & 1.2 & 6 & 1.1 & 7 & 1.5 & so &. & 50 & . \\
\hline Vinyl acetate & 200 & $\cdot$ & 200 & $\cdot$ & 200 & $\cdot$ & sou & $\cdot$ & 100 & - & 100 & ra \\
\hline vinyl chloride & 200 &. & 200 & $\cdot$ & 200 & - & 500 & - & 100 & - & 100 & . \\
\hline xylenes & 100 & $\cdot$ & 100 & $\cdot$ & 100 &. & 250 & - & so & - & 50 & 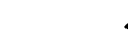 \\
\hline Summed vOCs & 360 & 100 & 321 & 100 & 551 & 100 & 467 & 100.1 & 0 & . & 0 & . \\
\hline
\end{tabular}

(Contrimued) 
APrewdix $J$

valldated voc mesulte, 1993

\begin{tabular}{|c|c|c|c|c|c|c|c|c|c|c|c|c|}
\hline \multirow{4}{*}{$\begin{array}{l}\text { Sampling Point } \\
\text { Location } \\
\text { Date Sompled }\end{array}$} & \multicolumn{4}{|c|}{$6 w-232$} & \multicolumn{8}{|c|}{$6-239$} \\
\hline & \multicolumn{4}{|c|}{$\mathbf{E x P}$} & \multicolumn{8}{|c|}{$\max$} \\
\hline & \multicolumn{2}{|c|}{$08 / 21 / 93$} & \multicolumn{2}{|c|}{$11 / 01 / 93$} & \multicolumn{2}{|c|}{$01 / 23 / 93$} & \multicolumn{2}{|c|}{$05 / 13 / 93$} & \multicolumn{2}{|c|}{$00 / 18 / 93$} & \multicolumn{2}{|c|}{$10 / 30 / 93$} \\
\hline & $\operatorname{ug} / 2$ & - sum & $\operatorname{ug} / 2$ & - cuen & $\log / 2$ & 10 & $\operatorname{ug} / 2$ & - sen & $\operatorname{ug} / 2$ & - cu & $\operatorname{ug} / 2$ & - en \\
\hline Acetone & 100 & . & 100 & $\cdot$ & 200 & $\cdot$ & 100 & - & 100 & - & 100 & • \\
\hline Benzene & 5v & - & so & $\cdot$ & so & $\cdot$ & so & $\cdot$ & so & $\cdot$ & 50 & • \\
\hline Bromoform & so & - & so & $\cdot$ & so & $\cdot$ & so & $\cdot$ & 50 & $\cdot$ & 50 & • \\
\hline 2-Butanone & 100 & - & 100 & - & 100 & $\cdot$ & 100 & $\cdot$ & 100 & $\cdot$ & 100 & $\cdot$ \\
\hline Carbon disulfide & so & - & so & $\cdot$ & so & $\cdot$ & so & $\cdot$ & so & $\cdot$ & 50 & - \\
\hline Carbon tetrachloride & so & - & so & $\cdot$ & so & $\cdot$ & so & $\cdot$ & so & $\cdot$ & so & • \\
\hline chloroform & so & - & so & $\cdot$ & 50 & $\cdot$ & 50 & $\cdot$ & so & $\cdot$ & so & • \\
\hline Chloromethane & 100 & • & 100 & $\cdot$ & 100 & $\cdot$ & 100 & $\cdot$ & 100 & $\cdot$ & 100 & $\cdot$ \\
\hline 1,1-Dlchloroethane & 50 & • & 50 & $\cdot$ & so & $\cdot$ & sol & $\cdot$ & so & - & so & $\cdot$ \\
\hline 1,2-Dichloroethane & so & $\cdot$ & so & $\cdot 1$ & 50 & $\cdot$ & so) & $\cdot$ & 50 & $\cdot$ & so & $\cdot$ \\
\hline 1,1-Dlchloroethene & so & . & so & $\cdot$ & so & $\cdot$ & so & $\cdot$ & sv & - & 50 & • \\
\hline 1,2-Dichloroethene & so & $\cdot$ & 50 & $\cdot$ & 50 & $\cdot$ & 50 & $\cdot$ & 50 & $\cdot$ & so & $\cdot$ \\
\hline Ethylbenzene & 5v & $\cdot$ & so & $\cdot$ & so &. & so & $\cdot$ & so & - & 50 & • \\
\hline 4-methy1-2-pentanone & 200 & $\cdot$ & 100 & $\cdot$ & $\boldsymbol{m}$ & $\cdot$ & 100 & $\cdot$ & 100 & • & 200 & • \\
\hline wethylene chloride & so & $\cdot$ & $\mathbf{P P 1}$ & $\cdot$ & 50 & $\cdot$ & $\mathbf{P P 2}$ & $\cdot$ & 50 & $\cdot$ & $\mathbf{m 1}$ & $\cdot$ \\
\hline Styrene & so & - & su & $\cdot$ & so & $\cdot$ & $\mathbf{5 0}$ & $\cdot$ & so & - & $\mathbf{s o}$ & • \\
\hline Tetrachloroethene & sv & $\cdot$ & 50 & $\cdot$ & 50 & $\cdot$ & so & $\cdot$ & so & - & 50 & $\cdot$ \\
\hline Toluene & so & $\cdot$ & so & $\cdot$ & 50 & $\cdot$ & 50 & $\cdot$ & sol & $\cdot$ & so & • \\
\hline 1,1,1-Irichloroethane & so! & - & so & $\cdot$ & so & $\cdot$ & so & $\cdot$ & 50 & $\cdot$ & 50 & $\cdot$ \\
\hline Irichloroethene & sv & - & so & $\cdot$ & 50 & $\cdot$ & 30 & $\cdot$ & so & $\cdot$ & so & $\cdot$ \\
\hline Vinyl acetate & 100 & $\cdot$ & 100 & $\cdot$ & 100 & $\cdot$ & 100 & $\cdot$ & 100 & $\cdot$ & 100 & $\cdot$ \\
\hline vinyl chloride & 100 & $\cdot$ & 100 &. & 100 & $\cdot$ & 100 & $\cdot$ & 100 & $\cdot$ & 100 & $\cdot$ \\
\hline xylene: & 50 & $\cdot$ & so & $\cdot$ & so & $\cdot$ & sol & $\cdot$ & 50 & $\cdot$ & So & - \\
\hline Sumped vocs & 0 & - & 0 &. & 0 & - & . & - & - & - & - & - \\
\hline
\end{tabular}

(COArrimued) 
APFEADIX $J$

valldated voc meevite, 1993

\begin{tabular}{|c|c|c|c|c|c|c|c|c|c|c|c|c|}
\hline \multirow{4}{*}{$\begin{array}{l}\text { Sempling point } \\
\text { Locetion } \\
\text { Dete Sempled }\end{array}$} & \multicolumn{8}{|c|}{$0 n-240$} & \multicolumn{4}{|c|}{ can-251 } \\
\hline & \multicolumn{8}{|c|}{$\max$} & \multicolumn{4}{|c|}{$\mathbf{8 2}$} \\
\hline & \multicolumn{2}{|c|}{$01 / 27 / 93$} & \multicolumn{2}{|c|}{$04 / 21 / 93$} & \multicolumn{2}{|c|}{$00 / 05 / 93$} & \multicolumn{2}{|c|}{$11 / 01 / 93$} & \multicolumn{2}{|c|}{$01 / 20 / 93$} & \multicolumn{2}{|c|}{$05 / 05 / 93$} \\
\hline & $u g / L$ & - ะm & $\operatorname{ug} / 2$ & c sen & $\operatorname{ug} / 2$ & $0=$ & $\log / 2$ & - von & $\log / 2$ & - & $0 g / 2$ & - sx \\
\hline Acetone & FP2 & - & 100 & - & 100 & - & 100 & - & 100 & - & 100 & - \\
\hline Benzene & so &. & so & $\cdot 1$ & 50 & $\bullet$ & 50 & - & so & $\cdot$ & so & - \\
\hline Bromoform & so &. & so &. & so & - & so & - & 50 & $\cdot$ & 50 & - \\
\hline 2-Butanone & 100 &. & 100 & $\cdot 1$ & 100 & - & 100 & . & 100 &. & 100 & - \\
\hline Carbon disulfide & so & & so & $\cdot$ & so & $\cdot$ & so & - & 50 & $\cdot$ & so & $\cdot$ \\
\hline Carbon tetrachloride & 10 & 90.9 & $\bullet$ & 85.1 & 10 & $\mathbf{8 3 . 3}$ & 15 & 03.3 & so & $\cdot$ & 11 & 3.5 \\
\hline Chloroform & 1 & 9.1 & 0.8 & 8.5 & 2 & 16.7 & 2 & 11.1 & 2 & 6.1 & $\bullet$ & 2.6 \\
\hline Chlororethane & 100 &. & 100 & $\cdot$ & 100 & $\cdot$ & 100 & $\cdot$ & 100 & $\cdot$ & 200 & - \\
\hline 1,1-Dlchloroethane & 50 & $\cdot$ & so & $\cdot$ & so & $\cdot$ & so & $\cdot$ & so & $\cdot$ & sol & • \\
\hline 1,2-Dichloroethane & 50 & $\cdot 1$ & 50 & $\cdot$ & 50 & $\cdot$ & so & $\cdot$ & so & $\cdot$ & sol & - \\
\hline 1,1-Dichloroethene & so & $\cdot$ & 50 & $\cdot$ & so & - & so & $\cdot$ & so & $\cdot$ & so & - \\
\hline 1,2-Dichloroethene & 50 & $\cdot$ & 50 & $\cdot$ & so & $\cdot$ & so & • & so & $\cdot$ & $\mathbf{P 3}$ & $\cdot$ \\
\hline zthylbenzene & so &. & $\mathbf{s o}$ & $\cdot$ & 50 & - & 50 & $\cdot$ & 50 & $\cdot$ & so & - \\
\hline 4-Methy 1-2-pentanone & $\mathbf{P P 1}$ & .1 & 100 &. & 200 & - & 100 & $\cdot$ & 100 & $\cdot$ & 100 & - \\
\hline methylene chloride & so & $\cdot$ & so & $\cdot$ & so & - & $\mathbf{r P 1}$ & $\cdot$ & 50 & $\cdot$ & so & - \\
\hline Styrene & 50 & $\cdot$ & so & $\cdot$ & so & $\cdot$ & 50 & $\cdot$ & 50 & $\cdot$ & so & • \\
\hline Tetrachloroethene & so & $\cdot$ & 0.6 & 6.4 & so & $\cdot$ & 1 & 5.6 & 24) & 72.7 & 210 & 67.5 \\
\hline Toluene & so & $\cdot$ & so & $\cdot$ & so & $\cdot$ & so & $\cdot$ & so & $\cdot$ & so & $\cdot$ \\
\hline 1,1,1-Trichloroethane & 50 &. & so & $\cdot$ & 50 & $\cdot$ & so & $\cdot$ & 50 & $\cdot$ & 50 & $\cdot$ \\
\hline Irlchloroethene & so & $\cdot$ & so &. & so & - & sol & $\cdot$ & 7 & 21.2 & 62 & 26.4 \\
\hline Vinyl acetate & 100 & $\cdot$ & 100 & $\cdot$ & 100 & $\cdot$ & 100 & $\cdot$ & 100 & $\cdot$ & 100 & • \\
\hline Vinyl shloride & 100 &. & 100 &. & 100 & $\cdot$ & 100 & $\cdot$ & 200 & $\cdot$ & 200 & - \\
\hline xylenea & so &. & so & $\cdot$ & 50 & - & sv & $\cdot$ & so & - & so & - \\
\hline Sumed vocs & & 100 & 9.4 & 100 & 12 & 100 & 18) & 200 & 33 & 100 & 311 & 100 \\
\hline
\end{tabular}

(CONTIMUED) 
Appandx 3

Valldated voC neavite, 1993

\begin{tabular}{|c|c|c|c|c|c|c|c|c|c|c|c|c|}
\hline \multirow{4}{*}{$\begin{array}{l}\text { Sempling Polnt } \\
\text { Location } \\
\text { Date Sempled }\end{array}$} & \multicolumn{4}{|c|}{$6-251$} & \multicolumn{8}{|c|}{$c 0-252$} \\
\hline & \multicolumn{4}{|c|}{$\mathbf{s 2}$} & \multicolumn{8}{|c|}{$\mathbf{8 2}$} \\
\hline & \multicolumn{2}{|c|}{$09 / 17 / 93$} & \multicolumn{2}{|c|}{$12 / 08 / 93$} & \multicolumn{2}{|c|}{$01 / 08 / 93$} & \multicolumn{2}{|c|}{$05 / 03 / 93$} & \multicolumn{2}{|c|}{$09 / 15 / 93$} & \multicolumn{2}{|c|}{$22 / 07 / 93$} \\
\hline & $\operatorname{ug} / 2$ & - sum & ug/L & - อบ & $\operatorname{ug} / 2$ & an & $\log / 2$ & $=0$ & $\lg / 2$ & oum & $\operatorname{ug} / 2$ & 1 ex \\
\hline Acetone & 100 & $\cdot 1$ & 100 &. & 100 & $\cdot$ & 100 & - & 100 & - & FP1 & . \\
\hline Benzene & so & $\cdot$ & so & $\cdot 1$ & so &. & sol & - & so & - & so & . \\
\hline Bromoform & so & $\cdot$ & so & $\cdot$ & so & $\cdot$ & so & - & so & - & so & . \\
\hline 2-Butanone & 100 & $\cdot$ & 100 & $\cdot$ & 100 & $\cdot$ & 100 & - & 200 & - & $\mathbf{m P 1}$ & . \\
\hline Carbon disulfide & so & $\cdot$ & so & $\cdot$ & so & $\cdot$ & so & - & se & - & so & . \\
\hline Carbon tetrachloride & 2 & 2.7 & 1 & 2.2 & so & .1 & 50 & - & so & - & so & . \\
\hline chloroform & 6 & 8.2 & 4 & 8.7 & 50 & $\cdot 1$ & so & . & 50 & $\cdot$ & 50 & • \\
\hline Chloronethane & 100 & $\cdot$ & 100 & $\cdot$ & 100 & $\cdot 1$ & 100 & - & 100 & - & 100 & • \\
\hline 1,1-Dichloroethane & 50 &. & so & $\cdot$ & 50 & .1 & so & - & so & $\cdot$ & 50 & • \\
\hline 1,2-Dichloroethane & 50 & $\cdot 1$ & 50 & $\cdot$ & so & $\cdot$ & 50 & - & so & $\cdot$ & so & • \\
\hline 1,1-Dichloroethene & 50 & $\cdot$ & 50 & $\cdot$ & so & $\cdot$ & so & $\cdot$ & so & $\cdot$ & 50 & • \\
\hline 1.2-Dichloroethene & 50 & $\cdot$ & so & $\cdot$ & so & $\cdot$ & so & - & so & - & 50 & • \\
\hline Ethylbenzene & 50 & $\cdot$ & 50 & $\cdot$ & 50 &. & so & $\cdot$ & 50 & $\cdot$ & so & • \\
\hline 1-Methy1-2-pentanone & 100 & $\cdot$ & 100 & $\cdot$ & 100 & $\cdot 1$ & $\mathbf{r P 1}$ & - & 200 & $\cdot$ & FP1 & - \\
\hline Nethylene chloride & 50 & $\cdot$ & so & $\cdot$ & $\mathbf{r P 1}$ & $\cdot$ & $\boldsymbol{F P}$ & - & so & - & $\mathbf{P} \mathbf{1}$ & • \\
\hline Styrene & 50 & $\cdot$ & so & $\cdot$ & 50 & $\cdot$ & 50 & - & so & $\cdot$ & so & • \\
\hline Tetrachloroethene & 47 & 64.4 & 31 & 67.4 & 50 & $\cdot$ & so & - & so &. & so & • \\
\hline Toluene & so & $\cdot$ & so & $\cdot$ & so & $\cdot$ & 50 & • & 50 & $\cdot$ & so & • \\
\hline 1,1,1-Irichloroethane & 50 & $\cdot$ & 50 & & 50 & $\cdot$ & 50 & $\cdot$ & so & $\cdot$ & 50 & • \\
\hline Irichloroethene & 18 & 24.7 & 10 & 21.7 & 50 & $\cdot$ & 50 & - & 50 & $\cdot$ & 50 & - \\
\hline Vinyl acetate & 100 & $\cdot$ & 100 & $\cdot$ & 100 & $\cdot$ & 100 & • & 100 & $\cdot$ & 100 & • \\
\hline Vinyl chloride & 100 & $\cdot$ & 100 & $\cdot$ & 100 & $\cdot$ & 100 & $\cdot$ & 100 &. & 100 & - \\
\hline xylenes & 50 & $\cdot$ & 50 & $\cdot$ & 50 & $\cdot$ & 50 & • & so & $\cdot$ & so & • \\
\hline Sumned vocs & 73 & 100 & 46 & 100 & o) &. & 0 & - & - &. & 0 & . \\
\hline
\end{tabular}

(CONTImUED) 
APPETDIX J

Valldated voC Results, 1993

\begin{tabular}{|c|c|c|c|c|c|c|c|c|c|c|c|c|}
\hline \multirow{4}{*}{$\begin{array}{l}\text { Sampling Point } \\
\text { Location } \\
\text { Date Sampled }\end{array}$} & \multicolumn{8}{|c|}{ Gw-255 } & \multicolumn{4}{|c|}{$G 2-261$} \\
\hline & \multicolumn{8}{|c|}{$\mathbf{s 2}$} & \multicolumn{4}{|c|}{ sy } \\
\hline & \multicolumn{2}{|c|}{$01 / 08 / 93$} & \multicolumn{2}{|c|}{$05 / 03 / 93$} & \multicolumn{2}{|c|}{$09 / 15 / 93$} & \multicolumn{2}{|c|}{$12 / 07 / 93$} & \multicolumn{2}{|c|}{$01 / 08 / 93$} & \multicolumn{2}{|c|}{$04 / 07 / 93$} \\
\hline & $\mathbf{u g} / \mathbf{L}$ & isum & $u g / L$ & เ $\operatorname{sum}$ & $\mathbf{u g} / \mathrm{L}$ & เ sm & $\operatorname{ug} / \mathrm{I}$ & - 8и & $u g / L$ & : sum & $\mathbf{v g} / \mathrm{L}$ & $8 \mathrm{xum}$ \\
\hline Acetone & 100 & - & $\mathbf{F P 3}$ & - & 100 & - & 100 & - & 100 & - & 100 & . \\
\hline Benzene & 5v & - & so & • & 50 & $\cdot$ & so & - & so & $\cdot$ & sv & rata \\
\hline Bromoform & so & - & so & - & 50 & $\cdot$ & 50 & $\cdot$ & 50 & $\cdot$ & so & . \\
\hline 2-Butanone & 100 & - & 100 & . & 100 & . & 100 & - & 100 & - & 100 & ra \\
\hline Carbon disulfide & su & - & 50 & $\cdot$ & 50 & $\cdot$ & so & $\cdot$ & so & $\cdot$ & 50 & r \\
\hline Carbon tetrachloricie & 50 & $\cdot$ & so & - & so & $\cdot$ & so & $\cdot$ & 50 & - & so & . \\
\hline Chloroform & 1 & 100 & 1 & 100 & 1 & 100 & 1 & 100 & so & $\cdot$ & 50 & rata \\
\hline Chloromethane & 100 & $\cdot$ & 100 & $\cdot$ & 100 & $\cdot$ & 100 & $\cdot$ & 100 & - & 100 & . \\
\hline 1,1-Dichloroethane & 50 & - & so & $\cdot$ & 50 & - & 50 & - & 50 & • & 50 & r \\
\hline 1,2-Dichloroethane & so & - & sv & $\cdot$ & 50 &. & 50 & $\cdot$ & so & - & 50 & ra \\
\hline 1,1-Dichloroethene & su & - & 50 & $\cdot$ & 50 &. & 50 & - & so & $\cdot$ & 50 & . \\
\hline 1,2-Dichloroethene & 50 & - & 50 & $\cdot$ & 50 & - & sv & $\cdot$ & 50 & $\cdot$ & 5v & r \\
\hline Ethylbenzene & so & - & 50 & $\cdot$ & 50 & - & 50 & - & so & $\cdot$ & so & ra \\
\hline 4-Hethy1-2-pentanone & FP1 & - & 100 & $\cdot$ & 100 & - & 100 & - & 100 & - & 100 & r \\
\hline Methylene chloride & so & - & su & $\cdot$ & so & - & FP1 & - & FP1 & $\cdot$ & FP2 & . \\
\hline Styrene & 5v & $\cdot$ & so & $\cdot$ & 50 & - & so & $\cdot$ & 50 & • & 50 & 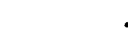 \\
\hline Tetrachloroethene & 5u & - & 50 & $\cdot$ & so & $\cdot$ & 5v & $\cdot$ & 50 & $\cdot$ & 50 & rata \\
\hline Toluene & 50 & - & 50 & $\cdot$ & 50 & - & so & - & so & - & 50 & . \\
\hline $1,1,1-\mathrm{Tr}$ ichloroethane & 50 & - & 5u & $\cdot$ & 50 & - & 50 & $\cdot$ & 50 & $\cdot$ & 50 & r \\
\hline Trichloroethene & 50 & $\cdot$ & 50 & $\cdot$ & 50 & - & 50 & - & so & - & so & . \\
\hline vinyl ecetate & 100 & - & 100 & $\cdot$ & 100 & - & 100 & $\cdot$ & 100 & - & 100 & . \\
\hline vingl chloride & 100 & - & 100 & $\cdot$ & 100 & $\cdot$ & 100 & $\cdot$ & 100 & - & 100 & rata \\
\hline xylenes & so & - & 50 & $\cdot$ & so & . & 50 & - & 50 & - & so & . \\
\hline Summed vocs & $\dot{1}$ & 100 & $\dot{1}$ & 100 & $i$ & 100 & 1 & 100 & 0 & . & 0 & . \\
\hline
\end{tabular}

(CONTINUED) 
APPEADIX J

Valldated voc Results, 1993

\begin{tabular}{|c|c|c|c|c|c|c|c|c|c|c|c|c|}
\hline \multirow{4}{*}{$\begin{array}{l}\text { Sampling Point } \\
\text { Location } \\
\text { - } \\
\text { Date Sampled }\end{array}$} & \multicolumn{4}{|c|}{ GW-261 } & \multicolumn{8}{|c|}{$6 w-262$} \\
\hline & \multicolumn{4}{|c|}{$\mathbf{S Y}$} & \multicolumn{8}{|c|}{$\mathbf{s y}$} \\
\hline & \multicolumn{2}{|c|}{$07 / 01 / 93$} & \multicolumn{2}{|c|}{$12 / 07 / 93$} & \multicolumn{2}{|c|}{$01 / 11 / 93$} & \multicolumn{2}{|c|}{$04 / 08 / 93$} & \multicolumn{2}{|c|}{$07 / 01 / 93$} & \multicolumn{2}{|c|}{$12 / 07 / 93$} \\
\hline & ug/L & sum & $u g / L$ & s sum & $u g / L$ & sum & $\mathrm{ug} / \mathrm{x}$ & s $\mathrm{sum}$ & $u g / L$ & sum & $u g / L$ & t sum \\
\hline Acetone & 100 & • & 100 & $\cdot$ & 100 & $\cdot$ & 100 & $\cdot$ & 100 & - & 100 & • \\
\hline Benzene & su & - & 50 & - & 50 & $\cdot$ & 50 & $\cdot$ & 50 & $\cdot$ & 50 & • \\
\hline Bromoform & sv & - & so & $\cdot$ & 50 & $\cdot$ & so & $\cdot$ & so & $\cdot$ & so & • \\
\hline 2-Butanone & 100 & - & 100 & $\cdot$ & 100 & $\cdot$ & 100 & • & 100 & - & 100 & $\cdot$ \\
\hline Carbon disulfide & 50 & $\cdot$ & 50 & $\cdot$ & 50 & - & so & • & 50 & - & so & • \\
\hline Carbon tetrachloride & 50 & . & 50 & - & so & - & so & - & so & - & 50 & • \\
\hline Chloroform & so & $\cdot$ & 50 & $\cdot$ & 50 & - & so & $\cdot$ & 50 & - & so & • \\
\hline Chloromethane & 100 & $\cdot$ & 100 & $\cdot$ & 100 & - & 100 & - & 100 & . & 100 & . \\
\hline 1.1-Dichloroethane & 50 & $\cdot$ & sv & $\cdot$ & 50 & $\cdot$ & 5u & $\cdot$ & so & - & 50 & . \\
\hline 1,2-Dichloroethane & 50 & - & 50 & $\cdot$ & 50 & $\cdot$ & 50 & $\cdot$ & so & $\cdot$ & 50 & $\cdot$ \\
\hline 1,1-Dichloroethene & 5u &. & 50 & $\cdot$ & 50 &. & 50 & $\cdot$ & 5v & . & 50 & - \\
\hline 1.2-Dichloroethene & 5v & $\cdot$ & 50 &. & 5o & $\cdot$ & su & $\cdot$ & 5v & $\cdot$ & 5v & $\cdot$ \\
\hline Ethylbenzene & 50 & $\cdot$ & so & $\cdot$ & 50 & $\cdot$ & so & $\cdot$ & 50 & $\cdot$ & so & • \\
\hline 4-Methy1-2-pentanone & 100 & $\cdot$ & 100 & $\cdot 1$ & $\mathbf{F P 1}$ & . & 100 & $\cdot$ & 100 & $\cdot$ & 100 & $\cdot$ \\
\hline Methylene chloride & so &. & FP1 & $\cdot$ & 50 & $\cdot$ & 50 & $\cdot$ & 50 & $\cdot$ & FP1 & • \\
\hline Styrene & so & $\cdot$ & sv & $\cdot$ & 50 & $\cdot$ & 50 & $\cdot$ & 50 & $\cdot$ & so & • \\
\hline Tetrachloroethene & 50 & $\cdot$ & 5v & $\cdot$ & so & $\cdot$ & 50 & $\cdot$ & 50 & - & so & • \\
\hline Toluene & 50 & $\cdot$ & 50 & $\cdot$ & 50 & $\cdot$ & 50 & $\cdot$ & 50 & - & so & • \\
\hline 1,1,1-Trichloroethane & 5v & $\cdot$ & 50 & $\cdot$ & 50 & $\cdot$ & 5v & $\cdot$ & so & - & so & • \\
\hline Irichloroethene & 50 &. & 50 & $\cdot$ & 50 & $\cdot$ & 50 & $\cdot 1$ & so & - & 50 & • \\
\hline vingl acetate & 100 & $\cdot$ & 100 &. & 100 & $\cdot$ & 100 & $\cdot$ & 100 & - & 100 & • \\
\hline vinyl chloride & 100 & $\cdot$ & 100 & $\cdot$ & 100 & $\cdot$ & 100 & $\cdot$ & 100 & - & 200 & • \\
\hline xylenes & 50 & $\cdot$ & 50 & $\cdot 1$ & 50 & $\cdot$ & so & $\cdot$ & 50 & $\cdot$ & 50 & • \\
\hline Summed vOCs & 0 &. & 0 &. & 0 & . & o & . & 0 & - & 0 & - \\
\hline
\end{tabular}

(CONTINUED) 
APPENDIX $J$

Valldated voC Results, 1993

\begin{tabular}{|c|c|c|c|c|c|c|c|c|c|c|c|c|}
\hline \multirow{4}{*}{$\begin{array}{l}\text { Sampling Point } \\
\text { Location } \\
\text { Date Sampled }\end{array}$} & \multicolumn{8}{|c|}{$G N-263$} & \multicolumn{4}{|c|}{ GN-264 } \\
\hline & \multicolumn{8}{|c|}{ sY } & \multicolumn{4}{|c|}{$\mathbf{S Y}$} \\
\hline & \multicolumn{2}{|c|}{$01 / 11 / 93$} & \multicolumn{2}{|c|}{$04 / 08 / 93$} & \multicolumn{2}{|c|}{$07 / 01 / 93$} & \multicolumn{2}{|c|}{$12 / 07 / 93$} & \multicolumn{2}{|c|}{$01 / 12 / 93$} & \multicolumn{2}{|c|}{$04 / 12 / 93$} \\
\hline & ug/I & sum & ug/L & sum & ug $/ \mathrm{L}$ & sum & $u g / L$ & sum & ug/L & sum & $u g / L$ & sum \\
\hline Acetone & 100 & $\cdot$ & $10 \mathrm{r}$ & - & FP3 & $\cdot$ & 100 & - & 100 & - & 100 & \\
\hline Benzene & 50 & $\cdot$ & 50 & $\cdot$ & 50 & $\cdot$ & 50 & - & 5u & • & 50 & \\
\hline Bromoform & sv & $\cdot$ & 50 & • & 50 & $\cdot$ & 50 & - & 50 & - & 50 & \\
\hline 2-Butanone & 100 & $\cdot$ & 100 & $\cdot$ & 100 & $\cdot$ & 100 & - & 100 & - & 100 & \\
\hline Carbon disulfide & 50 & $\cdot$ & 5u & $\cdot$ & 50 & $\cdot 1$ & 50 & - & 50 & $\cdot$ & 50 & \\
\hline Carbon tetrachloride & 50 & $\cdot$ & 5u & $\cdot$ & 5u & $\cdot$ & 5u & - & 50 & $\cdot$ & 50 & \\
\hline Chloroform & 50 & $\cdot$ & 5u & $\cdot$ & 5u & $\cdot$ & 50 & - & 50 & - & 50 & \\
\hline Chloromethane & 100 & $\cdot$ & 100 & $\cdot$ & 100 & $\cdot$ & 100 & - & 100 & - & 100 & \\
\hline 1,1-Dichloroethane & 50 & $\cdot 1$ & 50 & $\cdot$ & 50 & $\cdot$ & 50 & - & 50 & - & 50 & \\
\hline 1,2-Dichloroethane & 50 & $\cdot$ & 50 & $\cdot$ & 50 & $\cdot$ & 50 & - & 50 & $\cdot$ & 50 & \\
\hline 1,1-Dichloroethene & 50 & $\cdot$ & 50 & $\cdot$ & 50 & $\cdot$ & 50 & - & 50 & $\cdot$ & 5u & \\
\hline 1,2-Dichloroethene & $5 \mathbf{U}$ & $\cdot$ & 50 & $\cdot$ & 50 & $\cdot$ & 50 & - & 50 & $\cdot$ & 50 & \\
\hline Ethylbenzene & 50 & $\cdot$ & 50 & $\cdot$ & 5u & $\cdot$ & 5v & - & 50 & $\cdot$ & 50 & \\
\hline 4-Methy1-2-pentanone & FP1 & $\cdot$ & 100 & $\cdot$ & 100 & $\cdot$ & 100 & - & 100 & $\cdot$ & 100 & \\
\hline Methylene chloride & 50 & $\cdot$ & 5u & $\cdot$ & 50 & $\cdot$ & FP1 & - & 50 & $\cdot$ & FP3 & \\
\hline Styrene & 5u & $\cdot$ & 50 & $\cdot$ & 5u & $\cdot$ & 5u & - & 50 & $\cdot$ & 50 & \\
\hline Tetrachloroethene & 50 & $\cdot$ & 50 & $\cdot$ & 5u & $\cdot$ & 50 & - & 50 & $\cdot$ & 5u & \\
\hline Toluene & 50 & $\cdot$ & 50 & $\cdot$ & 50 & $\cdot$ & 50 & - & 50 & $\cdot$ & $5 \mathrm{v}$ & \\
\hline $1,1,1$-Trichloroethane & su & $\cdot$ & 50 & $\cdot$ & 50 & $\cdot$ & 50 & - & 50 & $\cdot$ & 50 & \\
\hline Trichloroethene & su & $\cdot$ & 50 & $\cdot$ & 50 & $\cdot$ & 50 & - & 50 & $\cdot 1$ & 50 & \\
\hline vinyl acetate & 100 & $\cdot$ & 100 & $\cdot$ & 100 & $\cdot$ & 100 & - & 100 & $\cdot$ & 100 & \\
\hline vinyl chloride & 100 & $\cdot$ & 100 & $\cdot$ & 100 & $\cdot$ & 100 & - & 100 & $\cdot$ & 100 & \\
\hline xylenes & 5u & $\cdot$ & 50 & $\cdot$ & 50 & $\cdot$ & 50 & - & 50 & $\cdot$ & 50 & \\
\hline Sumned vocs & &. & 0 & $\cdot$ & 0 &. & o & - & 0 & . & 0 & \\
\hline
\end{tabular}

(CONTINUED) 
APPENDIX $J$

Valldated VOC Results, 1993

\begin{tabular}{|c|c|c|c|c|c|c|c|c|c|c|c|c|}
\hline \multirow{4}{*}{$\begin{array}{l}\text { Sampling Point } \\
\text { Location } \\
\text { Date Sampled }\end{array}$} & \multicolumn{4}{|c|}{ GW-264 } & \multicolumn{8}{|c|}{$G W-281$} \\
\hline & \multicolumn{4}{|c|}{$\mathbf{s Y}$} & \multicolumn{8}{|c|}{$\mathbf{F r}$} \\
\hline & \multicolumn{2}{|c|}{$07 / 02 / 93$} & \multicolumn{2}{|c|}{$12 / 08 / 93$} & \multicolumn{2}{|c|}{$02 / 02 / 93$} & \multicolumn{2}{|c|}{$04 / 16 / 93$} & \multicolumn{2}{|c|}{$08 / 04 / 93$} & \multicolumn{2}{|c|}{$10 / 14 / 93$} \\
\hline & $u g / L$ & s sum & ug/I & 8 sum & $\mathbf{u g} / \mathrm{L}$ & sum & $\mathrm{ug} / \mathrm{L}$ & s sum & $u g / L$ & s sum & ug/L & 8 sum \\
\hline Acetone & 100 & . & 100 & - & FP1 & - & 100 & - & 100 & $\cdot$ & FP1 & \\
\hline Benzene & 50 & $\cdot$ & su & - & 50 & - & 50 & - & 50 & - & 50 & \\
\hline Bromoform & 50 & $\cdot$ & 50 & - & 5v & - & 5v & - & 50 & $\cdot$ & 50 & \\
\hline 2-Butanone & 100 & . & 100 & - & FP1 & - & 100 & - & 100 & - & FP1 & \\
\hline Carbon disulfide & 5u & $\cdot$ & 5u & - & 50 & - & 50 & $\cdot$ & 50 & - & 50 & \\
\hline Carbon tetrachloride & 50 &. & 5v & - & 50 & • & 5v & $\cdot$ & 50 & - & 50 & \\
\hline Chloroform & 50 & $\cdot$ & 50 & - & 5v & - & 50 & $\cdot$ & 50 & - & $5 v$ & \\
\hline Chloromethane & 100 & - & 100 & - & 100 & - & 100 & - & 100 & $\cdot$ & 100 & \\
\hline 1,1-Dichloroethane & 50 & - & 5u & - & 50 & - & 5v & $\cdot$ & 50 & - & 5u & \\
\hline 1,2-Dichloroethane & 50 & - & 50 & - & 5u & - & 50 & - & 50 & - & 50 & \\
\hline 1,1-Dichloroethene & 50 & - & 50 & - & $5 v$ & - & 50 & $\cdot$ & 50 & - & 50 & \\
\hline 1,2-Dichloroethene & 50 & . & 50 & - & 50 & - & 5v & $\cdot$ & 50 & - & 50 & \\
\hline Ethylbenzene & 5u & • & 5u & - & 50 & • & 5v & $\cdot$ & 50 & - & 50 & \\
\hline 4-Methyl-2-pentanone & 100 & - & 100 & - & FP1 & - & 100 & $\cdot$ & 100 & - & 100 & \\
\hline Methylene chloride & 50 & • & 5u & - & 5v & - & 50 & $\cdot$ & 50 & - & FP1 & \\
\hline Styrene & 5u & - & 5v & - & 50 & - & 5v & $\cdot$ & 50 & - & 5v & \\
\hline Tetrachloroethene & su & - & 50 & - & 50 & - & 5u & $\cdot$ & 50 & $\cdot$ & 50 & \\
\hline Toluene & 5u & • & 50 & - & 50 & - & 5u & $\cdot$ & 50 & - & 50 & \\
\hline 1,1,1-Trichloroethane & $5 \mathbf{5 u}$ & • & 50 & - & 50 & - & 50 & $\cdot$ & 50 & $\cdot$ & 50 & \\
\hline Trichloroethene & 50 & . & so & - & 5v & - & 5u & . & 50 & - & 50 & \\
\hline vinyl acetate & 100 & - & 100 & - & 100 & - & 100 & $\cdot$ & 100 & . & 100 & \\
\hline Vinyl chloride & 100 & - & 100 & - & 100 & - & 100 & $\cdot$ & 100 & $\cdot$ & 100 & \\
\hline xylenes & 50 & $\cdot$ & 5u & - & 50 & - & 50 & $\cdot$ & su & $\cdot$ & $5 v$ & \\
\hline Summed Vocs & 0 & . & 0 & - & 0 & • & 0 & . & 0 & . & 0 & \\
\hline
\end{tabular}

(CONTINUED) 
APPENDIX J

Valldated VOC Results, 1993

\begin{tabular}{|c|c|c|c|c|c|c|c|c|c|c|c|c|c|}
\hline \multirow{4}{*}{$\begin{array}{l}\text { Sampling Point } \\
\text { Location } \\
\text { Date Sampled }\end{array}$} & \multicolumn{8}{|c|}{ GW-283 } & \multicolumn{5}{|c|}{$G w-284$} \\
\hline & \multicolumn{8}{|c|}{$\mathbf{F F}$} & \multicolumn{5}{|c|}{$\mathbf{F F}$} \\
\hline & \multicolumn{2}{|c|}{$02 / 03 / 93$} & \multicolumn{2}{|c|}{$04 / 19 / 93$} & \multicolumn{2}{|c|}{$08 / 06 / 93$} & \multicolumn{2}{|c|}{$10 / 14 / 93$} & \multicolumn{2}{|c|}{$02 / 02 / 93$} & \multicolumn{3}{|c|}{$04 / 16 / 93$} \\
\hline & ug/L & sum & $\mathbf{u g} / \mathrm{x}$ & 8 sum & $u g / L$ & s sum & $u g / L$ & sum & ug/L & sum & $u g / L$ & 8 sum & \\
\hline Acetone & 100 & . & 100 & . & 100 & . & FP1 & - & 100 & . & 100 & & - \\
\hline Benzene & 50 & . & 50 & - & 5v & . & su & . & 50 & - & 50 & & - \\
\hline Bromoform & 50 & . & 50 & - & $5 v$ & $\cdot$ & 50 & . & 50 & - & 50 & & - \\
\hline 2-Butanone & 100 & $\cdot$ & 100 & - & 100 & $\cdot$ & FP1 & $\cdot$ & FP1 & $\cdot$ & 100 & & - \\
\hline Carbon disulfide & $5 \mathbf{v}$ & - & 5u & - & 50 & $\cdot$ & 50 & - & 5u & - & 5u & & - \\
\hline Carbon tetrachloride & 5u &. & 50 & - & 50 & . & 50 & - & 50 & - & 50 & & - \\
\hline Chloroform & 50 & • & 50 & - & 50 & $\cdot$ & 5u & $\cdot$ & 50 & - & 50 & & - \\
\hline Chloromethane & 100 & $\cdot 1$ & 100 & - & 100 & $\cdot$ & 100 & . & 100 & - & 100 & & - \\
\hline 1,1-Dichloroethane & su & $\cdot$ & $5 v$ & • & 5v & $\cdot$ & 50 & $\cdot$ & 50 & $\cdot$ & 50 & & - \\
\hline 1,2-Dichloroethane & 50 & $\cdot$ & 50 & . & 50 & $\cdot$ & 50 & . & 50 & . & so & & - \\
\hline 1,1-Dichloroethene & 50 & - & 50 & - & 50 & $\cdot$ & 50 & - & 50 & $\cdot$ & 50 & & - \\
\hline 1,2-Dichloroethene & 50 & - & 50 & - & 50 &. & 50 & - & 50 & . & 50 & & - \\
\hline Ethylbenzene & sv & $\cdot$ & 50 & - & 50 & - & 5u & - & sv & . & 50 & & - \\
\hline 4-Methy1-2-pentanone & FP1 & - & 100 & - & 100 & $\cdot$ & 100 & $\cdot$ & FP1 & - & 100 & & - \\
\hline Methylene chloride & FP1 & $\cdot$ & 50 & - & 50 & $\cdot$ & FP1 & $\cdot$ & 50 & - & 50 & & - \\
\hline Styrene & 50 & $\cdot$ & 50 & - & 50 & $\cdot$ & 50 & $\cdot$ & 50 & - & 50 & & - \\
\hline Tetrachloroethene & 5v & $\cdot$ & 50 & $\cdot$ & 50 & $\cdot$ & 50 & $\cdot$ & 50 & $\cdot$ & 50 & & - \\
\hline Toluene & 50 & $\cdot$ & 50 & $\cdot$ & $5 v$ & $\cdot$ & 50 & • & 50 & $\cdot$ & 50 & & - \\
\hline 1,1,1-Trichloroethane & $5 \mathbf{v}$ & $\cdot$ & 50 & - & $5 \mathrm{v}$ & $\cdot$ & 50 & $\cdot$ & 50 & . & 50 & & - \\
\hline Trichloroethene & 50 & $\cdot$ & FP2 & - & 50 & $\cdot$ & 50 & $\cdot$ & su & . & 50 & & - \\
\hline vinyl acetate & 100 &. & 100 & - & 100 & $\cdot$ & 100 & $\cdot$ & 100 & - & 100 & & - \\
\hline vinyl chloride & 100 &. & 100 & - & 100 & $\cdot$ & 100 & . & 100 & - & 100 & & - \\
\hline xylenes & 5v &. & 50 & - & 5u & $\cdot$ & 50 & . & SU & • & 50 & & . \\
\hline Summed VOCs & 0 &. & 0 & . & 0 & . & 0 & . & 0 & . & 0 & & . \\
\hline
\end{tabular}

(CONTINUED) 
APPENDIX $J$

Valldated VOC Results, 1993

\begin{tabular}{|c|c|c|c|c|c|c|c|c|c|c|c|c|}
\hline \multirow{4}{*}{$\begin{array}{l}\text { Sampling Point } \\
\text { Location } \\
\text { Date Sampled }\end{array}$} & \multicolumn{4}{|c|}{$G W-284$} & \multicolumn{8}{|c|}{ GW-285 } \\
\hline & \multicolumn{4}{|c|}{$\mathbf{F F}$} & \multicolumn{8}{|c|}{$\mathbf{F r}$} \\
\hline & \multicolumn{2}{|c|}{$08 / 05 / 93$} & \multicolumn{2}{|c|}{$10 / 14 / 93$} & \multicolumn{2}{|c|}{$02 / 02 / 93$} & \multicolumn{2}{|c|}{$04 / 19 / 93$} & \multicolumn{2}{|c|}{$08 / 05 / 93$} & \multicolumn{2}{|c|}{$10 / 14 / 93$} \\
\hline & ug $/ \mathrm{L}$ & 8 sum & $\mathrm{ug} / \mathrm{L}$ & 8 sum & $u g / x$ & s sum & $u g / L$ & sum & $u g / L$ & sum & $\mathrm{ug} / \mathrm{I}$ & sun \\
\hline Acetone & 100 & - & 100 & - & 100 & - & 100 & - & 100 & . & FP1 & . \\
\hline Benzene & 50 & - & 50 & - & 5v & - & 50 & - & 50 & . & 50 & ra \\
\hline Bromoform & 50 & - & 50 & - & 50 & - & 50 & - & 50 & . & $5 v$ & . \\
\hline 2-Butanone & 100 & - & 100 & - & FP1 & - & 100 & - & 100 & - & FP1 & . \\
\hline Carbon disulfide & 50 & - & 50 & - & su & - & 50 & - & 5u & - & 50 & . \\
\hline Carbon tetrachloride & 50 & • & 50 & - & 50 & - & $5 \mathbf{v}$ & - & 50 & . & 50 & . \\
\hline Chloroform & 5u & - & 50 & - & 50 & - & 5v & - & 50 & $\cdot$ & 50 & ra \\
\hline Chloromethane & 100 & • & 100 & - & 100 & - & 100 & - & 100 & $\cdot$ & 100 & . \\
\hline 1,1-Dichloroethane & 5u & - & 50 & - & 50 & - & 5u & - & 50 & $\cdot$ & 50 & r \\
\hline 1,2-Dichloroethane & 50 & - & 50 & - & 50 & - & 50 & - & 50 & . & 50 & ra \\
\hline 1,1-Dichloroethene & 50 & - & 50 & - & 50 & - & 5v & - & 50 & $\cdot$ & 50 & r \\
\hline 1,2-Dichloroethene & 50 & - & 50 & - & 50 & - & 50 & - & 50 & - & FP3 & . \\
\hline Bthylbenzene & 5v & - & 50 & - & 50 & • & 50 & - & 5u & $\cdot$ & 50 & . \\
\hline 4-Methy1-2-pentanone & 100 & - & 100 & - & FP1 & - & 100 & - & 100 & $\cdot$ & 100 & r \\
\hline Methylene chloride & 5v & - & 5u & - & 50 & - & 50 & - & 5U & $\cdot$ & FP1 & . \\
\hline styrene & 5v & - & 5v & - & 50 & - & su & - & 5u & $\cdot$ & 50 & . \\
\hline Tetrachloroethene & su & • & 50 & - & 5v & - & 5u & - & 50 & $\cdot$ & 50 & . \\
\hline Toluene & $\mathbf{5 0}$ & - & 50 & - & 50 & - & 50 & - & 50 & . & 50 & ra \\
\hline $1,1,1-\operatorname{Tr} 1 \mathrm{chl}$ oroethane & $5 \mathbf{v}$ & - & 50 & - & 50 & - & $5 \mathbf{v}$ & - & 50 & $\cdot$ & 50 & . \\
\hline Trichloroethene & so & - & 50 & - & 5u & - & FP2 & - & su & $\cdot$ & 50 & ra \\
\hline vinyl acetate & 100 & - & 100 & - & 100 & - & 100 & - & 100 & $\cdot$ & 100 & . \\
\hline vinyl chloride & 100 & - & 100 & - & 100 & - & 100 & - & 100 & $\cdot$ & 100 & . \\
\hline xylenes & 5u & - & 50 & - & 50 & - & 5o & - & 50 & $\cdot$ & 50 & ra \\
\hline Summed VOCs & $\mathbf{0}$ & - & 0 & . & 0 & . & 0 & . & 0 & . & 0 & . \\
\hline
\end{tabular}

(CONTINUED) 
APPEHDIX J

Validated voC Results, 1993

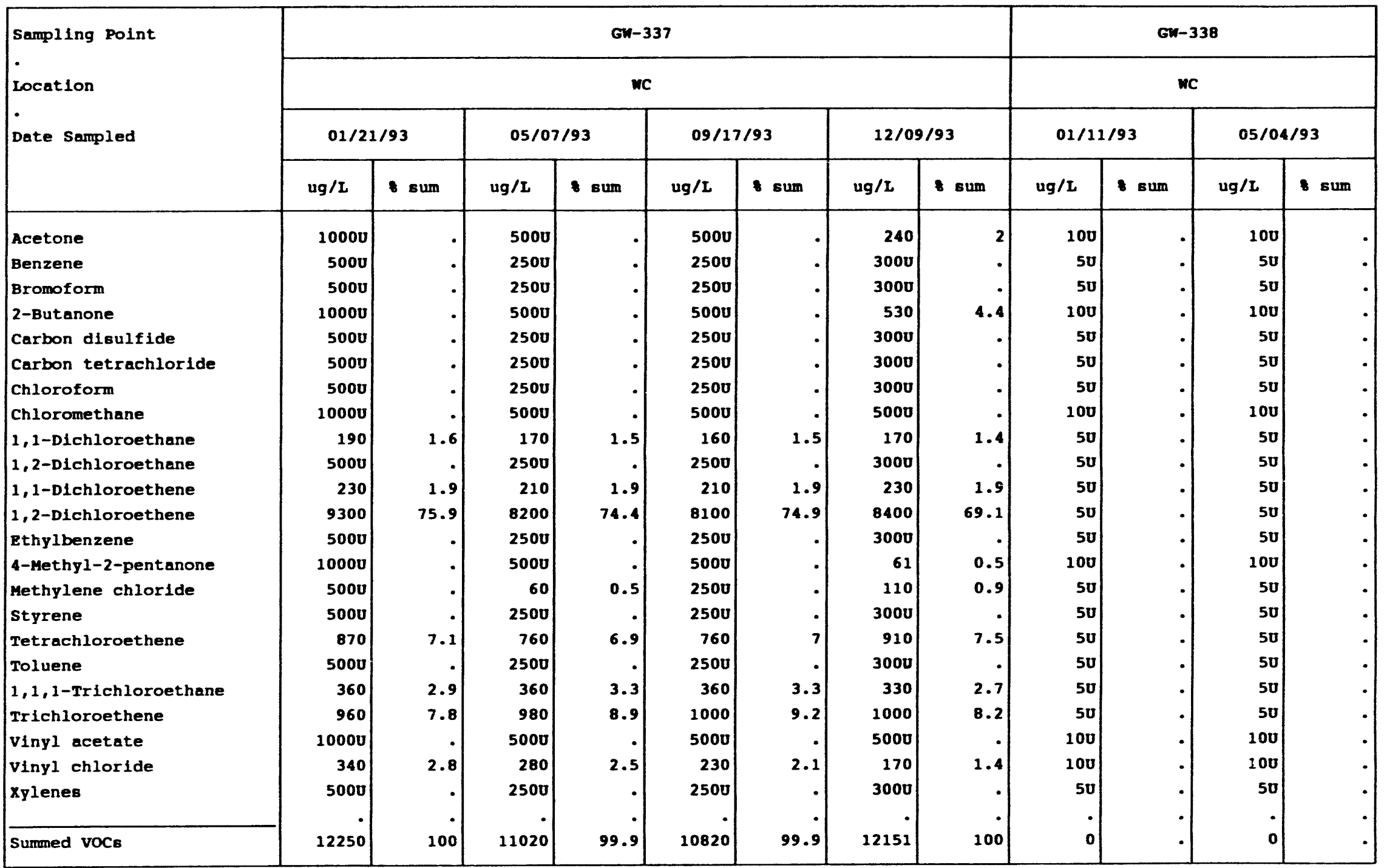

(CONTINUED) 
APPENDIX J

Validated voC Results, 1993

\begin{tabular}{|c|c|c|c|c|c|c|c|c|c|c|c|c|}
\hline \multirow{4}{*}{$\begin{array}{l}\text { Sampling Point } \\
\text { Location } \\
\text { Date Sampled }\end{array}$} & \multicolumn{4}{|c|}{$G x-338$} & \multicolumn{8}{|c|}{$G W-380$} \\
\hline & \multicolumn{4}{|c|}{ wC } & \multicolumn{8}{|c|}{ MHP } \\
\hline & \multicolumn{2}{|c|}{$09 / 23 / 93$} & \multicolumn{2}{|c|}{$12 / 07 / 93$} & \multicolumn{2}{|c|}{$01 / 25 / 93$} & \multicolumn{2}{|c|}{$04 / 21 / 93$} & \multicolumn{2}{|c|}{$08 / 05 / 93$} & \multicolumn{2}{|c|}{$10 / 29 / 93$} \\
\hline & $u g / L$ & sum & ug/L & 8 sum & $\mathbf{u g} / \mathrm{L}$ & sum & ug/L & s sum & $u g / I$ & sum & $u g / L$ & sum \\
\hline Acetone & 100 & $\cdot$ & 100 & - & FP1 & $\cdot$ & 100 & - & 100 & - & 100 & \\
\hline Benzene & 50 & - & $\mathbf{5 v}$ & - & 50 & . & 50 & - & 50 & - & 5v & \\
\hline Bromoform & so & $\cdot$ & 50 & - & 50 & . & 50 & $\cdot$ & 50 & . & 50 & \\
\hline 2-Butanone & 100 & $\cdot$ & 100 & - & 100 & . & 100 &. & 100 & - & 100 & \\
\hline Carbon disulfide & 50 & $\cdot$ & 50 & - & 50 & - & sv & $\cdot$ & 50 & - & 50 & 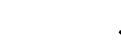 \\
\hline Carbon tetrachloride & 50 & $\cdot$ & 50 & . & 50 & - & 5v &. & 50 & . & 50 & \\
\hline Chloroform & 50 & $\cdot$ & 50 & - & 50 & - & 5v & $\cdot$ & 50 & . & 5u & \\
\hline Chloromethane & 100 & $\cdot$ & 100 & $\cdot$ & 100 & . & 100 & $\cdot$ & 100 &. & 100 & \\
\hline 1,1-Dichloroethane & 50 &. & 50 & . & 50 & . & 50 &. & 50 & . & 50 & \\
\hline 1,2-Dichloroethane & 5v & $\cdot 1$ & 5v &. & 5v & $\cdot$ & 50 &. & su &. & 50 & 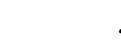 \\
\hline 1,1-Dichloroethene & 50 & . & 50 &. & 50 & - & 50 & - & 50 &. & 50 & ta \\
\hline 1,2-Dichloroethene & 5v &. & 50 &. & 50 & $\cdot$ & 50 & - & 5v & - & 50 & 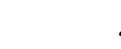 \\
\hline Ethylbenzene & 50 &. & 50 & $\cdot$ & 50 & $\cdot$ & sv & - & su &. & 50 & 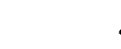 \\
\hline 4-Methy1-2-pentanone & 100 & $\cdot$ & 100 & $\cdot$ & FP1 & • & 100 & $\cdot$ & 100 & $\cdot$ & 100 & r \\
\hline Methylene chloride & 50 & $\cdot$ & FP1 & $\cdot$ & 50 & $\cdot$ & 50 & $\cdot$ & 5v &. & FP1 & 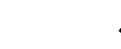 \\
\hline Styrene & 50 & $\cdot$ & $\mathbf{s u}$ & .1 & so & $\cdot$ & 50 & - & 5v & - & 50 & \\
\hline Tetrachloroethene & 50 &. & 50 &. & 50 & - & 50 & - & 5v & - & 50 & 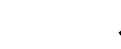 \\
\hline Toluene & 50 & $\cdot$ & 50 & - & $5 \mathbf{v}$ & . & 50 & $\cdot$ & so & . & 50 & rat \\
\hline 1,1,1-Tr1chloroethane & 50 & $\cdot$ & 5v & - & 5u & $\cdot$ & 5v & - & 50 & - & 50 & rata \\
\hline Trichloroethene & 50 & - & 5v & $\cdot$ & 50 &. & 50 & . & 50 &. & so & . \\
\hline Vinyl acetate & 100 & - & 100 &. & 100 & $\cdot$ & 100 & - & 100 & . & 100 & ra \\
\hline Vinyl chloride & 100 & $\cdot$ & 100 &. & 100 &. & 100 & $\cdot$ & 100 &. & 100 & ra \\
\hline xylenes & su & . & 50 & . & 50 &. & 50 & . & 50 & $\cdot$ & 50 & . \\
\hline Summed vocs & $\dot{0}$ & $\cdot$ & $\dot{0}$ & $\cdot$ & $\dot{0}$ & $\cdot$. & $\dot{0}$ & $\cdot$ & . & $\cdot$. & . & . \\
\hline
\end{tabular}

(CONTINUED) 
APPEADIX $J$

Valldated voc Resulta, 1993

\begin{tabular}{|c|c|c|c|c|c|c|c|c|c|c|c|c|}
\hline \multirow{4}{*}{$\begin{array}{l}\text { Sampling Point } \\
\text { Location } \\
\text { Date Sampled }\end{array}$} & \multicolumn{8}{|c|}{ Gn-381 } & \multicolumn{4}{|c|}{$6 n-382$} \\
\hline & \multicolumn{8}{|c|}{ NAP } & \multicolumn{4}{|c|}{ MAP } \\
\hline & \multicolumn{2}{|c|}{$01 / 27 / 93$} & \multicolumn{2}{|c|}{$04 / 26 / 93$} & \multicolumn{2}{|c|}{$08 / 08 / 93$} & \multicolumn{2}{|c|}{$11 / 08 / 93$} & \multicolumn{2}{|c|}{$01 / 28 / 93$} & \multicolumn{2}{|c|}{$04 / 26 / 93$} \\
\hline & $u g / L$ & sum & $\operatorname{ug} / \mathbf{L}$ & - sum & ug $/ \mathrm{L}$ & sum & ug $/ \mathrm{L}$ & Eum & $\operatorname{ug} / 2$ & e sum & ug/L & $5 \mathrm{~cm}$ \\
\hline Acetone & 52 & 0.7 & 5000 & - & 5000 & - & 5000 & - & 800 & - & 1000 & - \\
\hline Benzene & 2500 & $\cdot$ & 2500 & $\cdot$ & 2500 & - & 2500 & - & 400 & - & 500 & • \\
\hline Bromoform & 2500 & - & 2500 & $\cdot$ & 2500 & - & 2500 & - & 400 & - & 500 & - \\
\hline 2-Butanone & 5000 & $\cdot$ & 5000 & $\cdot$ & 5000 & - & 5000 & $\cdot$ & 800 & - & 1000 & - \\
\hline Carbon disulfide & 2500 & $\cdot$ & 2500 & $\cdot$ & 2500 & $\cdot$ & 2500 & $\cdot$ & 400 & - & 500 & • \\
\hline Carbon tetrachloride & 6400 & 90.8 & 4800 & 92.7 & 6600 & 92.1 & 5500 & 92.6 & 1500 & 57.2 & 5500 & 89.2 \\
\hline chloroform & 520 & 7.4 & 380 & 7.3 & 570 & 7.9 & 440 & 7.4 & 950 & 36.2 & 380 & 6.2 \\
\hline Chloromethane & 5000 & $\cdot$ & 5000 & $\cdot$ & 5000 & $\cdot$ & 5000 & $\cdot$ & 800 & • & 1000 & • \\
\hline 1,1-Dichloroethane & 2500 & $\cdot$ & 2500 & $\cdot$ & 2500 & $\cdot$ & 2500 & $\cdot$ & 400 & - & 500 & • \\
\hline 1,2-Dichloroethane & 2500 & - & 2500 & $\cdot$ & 2500 & $\cdot$ & 2500 & $\cdot$ & 400 & - & 500 & - \\
\hline 1,1-Dichloroethene & 2500 & - & 2500 &. & 2500 & . & 2500 &. & 400 & - & 500 & • \\
\hline 1,2-Dichloroethene & 2500 & . & 2500 & - & 2500 & - & 2500 & $\cdot$ & 400 & - & 500 & • \\
\hline Ethylbenzene & 2500 & $\cdot$ & 2500 & • & 2500 & $\cdot$ & 2500 & $\cdot$ & 400 & - & 500 & • \\
\hline 4-Methy1-2-pentanone & 78 & 1.1 & 5000 & • & 5000 & $\cdot$ & 5000 & $\cdot$ & 800 & - & 1000 & - \\
\hline Methylene chloride & 2500 & $\cdot$ & 2500 & . & 2500 & $\cdot$ & 2500 & $\cdot$ & 24 & 0.9 & 500 & • \\
\hline Stprene & 2500 & $\cdot$ & 2500 & $\cdot$ & 2500 & $\cdot$ & 2500 & - & 400 & • & 500 & • \\
\hline Tetrachloroethene & 2500 & $\cdot$ & 2500 & $\cdot$ & 2500 & $\cdot$ & 2500 & - & 130 & 5 & 250 & 4.1 \\
\hline Toluene & 2500 & $\cdot$ & 2500 & • & 2500 & $\cdot$ & 2500 & $\cdot$ & 400 & - & 500 & • \\
\hline $1,1,1$-Trichloroethane & 2500 & $\cdot$ & 2500 & $\cdot$ & 2500 & $\cdot$ & 2500 & $\cdot$ & 400 & - & 500 & • \\
\hline Trichlcroethene & 2500 & $\cdot$ & 2500 & - & 2500 & $\cdot$ & 2500 & $\cdot$ & 20 & 0.8 & 34 & 0.6 \\
\hline vinyl acetate & 5000 & $\cdot$ & 5000 & $\cdot$ & 5000 & $\cdot$ & 5000 & $\cdot$ & 800 & - & 1000 & • \\
\hline vinyl chloride & 5000 & . & 5000 & - & 5000 & $\cdot$ & 5000 & $\cdot$ & 800 & - & 1000 & • \\
\hline xylenes & 2500 & $\cdot$ & 2500 & . & 2500 & $\cdot$ & 2500 & . & 400 & - & 500 & • \\
\hline Summed VOCB & 7050 & 100 & 5180 & 100 & 7170 & 100 & 5940 & 100 & 2624 & 100.1 & 6164 & 100.1 \\
\hline
\end{tabular}

(CONTINUED) 
APPEMDIX $J$

valldated voc Resulta, 1993

\begin{tabular}{|c|c|c|c|c|c|c|c|c|c|c|c|c|}
\hline \multirow{4}{*}{$\begin{array}{l}\text { Sampling Point } \\
\text { Location } \\
\text { Date Sampled }\end{array}$} & \multicolumn{4}{|c|}{$G W-382$} & \multicolumn{8}{|c|}{$6 m-383$} \\
\hline & \multicolumn{4}{|c|}{ MHP } & \multicolumn{8}{|c|}{$\operatorname{MaP}$} \\
\hline & \multicolumn{2}{|c|}{$08 / 08 / 93$} & \multicolumn{2}{|c|}{$11 / 04 / 93$} & \multicolumn{2}{|c|}{$01 / 26 / 93$} & \multicolumn{2}{|c|}{$04 / 22 / 93$} & \multicolumn{2}{|c|}{$08 / 07 / 93$} & \multicolumn{2}{|c|}{$11 / 03 / 93$} \\
\hline & ug/t & sum & $\operatorname{ug} / 2$ & จ sum & $\mathrm{ug} / \mathrm{I}$ & t sum & ug/L & - sum & $\operatorname{ug} / \mathrm{L}$ & $2 \sin$ & $u g / L$ & c sum \\
\hline Acetone & 5000 & . & 5000 & . & 200 & - & 200 & . & 400 & . & 200 & . \\
\hline Benzene & 2500 & - & 2500 & .1 & 100 & . & 100 & . & 200 & . & 100 & - \\
\hline Bromoform & 2500 & - & 2500 & $\cdot$ & 100 & - & 100 & $\cdot$ & 200 & - & 100 & - \\
\hline 2-Butanone & 5000 & $\cdot$ & 5000 & $\cdot 1$ & 200 & - & 200 & $\cdot$ & 400 & $\cdot$ & 200 & • \\
\hline Carbon disulfide & 2500 & . & 2500 &. & 100 & . & 100 &. & 200 & . & 100 & • \\
\hline Carbon tetrachloride & 6500 & 92.1 & 7000 & 92.6 & 3 & 0.6 & 4 & 0.7 & 200 & $\cdot$ & 100 & • \\
\hline chloroform & 330 & 4.7 & 220 & 2.9 & 100 & • & 3 & 0.5 & 200 & $\cdot$ & 100 & • \\
\hline Chloromethane & 5000 & $\cdot$ & 5000 & $\cdot$ & 200 & $\cdot$ & 200 & $\cdot$ & 400 & - & 200 & • \\
\hline 1,1-Dichloroethane & 2500 & . & 2500 & - & 100 & - & 100 & $\cdot$ & 200 & $\cdot$ & 100 & • \\
\hline 1,2-Dichloroethane & 2500 & $\cdot$ & 2500 & - & 100 & - & 100 & - & 200 & - & 100 & • \\
\hline 1,1-Dichloroethene & 2500 & . & 2500 & . & 200 & $\cdot$ & 100 & - & 200 & $\cdot$ & 100 & • \\
\hline 1,2-Dichloroethene & 2500 & . & 2500 & . & 72 & 14.5 & 71 & 11.7 & 73 & 30.3 & 84 & 23.9 \\
\hline Ethylberzene & 2500 & . & 2500 & - & 100 & $\cdot$ & 100 & $\cdot$ & 200 & $\cdot$ & 100 & • \\
\hline 4-Methy1-2-pentanone & 5000 & $\cdot$ & 5000 &. & $\mathbf{P 1}$ & $\cdot$ & 200 &. & 400 & - & 200 & • \\
\hline Methylene chloride & 2500 & $\cdot$ & 80 & 1.1 & 100 & $\cdot$ & 100 & $\cdot$ & 200 & $\cdot$ & 100 & • \\
\hline styrene & 2500 &. & 2500 & $\cdot$ & 100 & • & 100 & - & 200 & - & 100 & - \\
\hline Tetrachloroethene & 230 & 3.3 & 260 & 3.4 & 300 & 60.6 & 400 & 65.8 & 120 & 49.8 & 190 & 54.1 \\
\hline Toluene & 2500 & $\cdot$ & 2500 & $\cdot$ & 100 & $\cdot$ & 100 & $\cdot$ & 200 & $\cdot$ & 100 & • \\
\hline $1,1,1$-Trichloroethane & 2500 & $\cdot$ & 2500 & $\cdot$ & 100 & $\cdot$ & 100 & $\cdot$ & 200 & • & 100 & • \\
\hline Trichloroethene & 2500 &. & 2500 &. & 120 & 24.2 & 130 & 21.4 & 48 & 19.9 & 77 & 21.9 \\
\hline vinyl acetate & 5000 & - & 5000 & $\cdot$ & 200 & • & 200 & $\cdot$ & 400 & $\cdot$ & 200 & • \\
\hline vinyl chloride & 5000 & - & 5000 &. & 200 & - & 200 & $\cdot$ & 400 & - & 200 & • \\
\hline xylenes & 2500 & $\cdot$ & 2500 & $\cdot$ & 100 & - & 100 & - & 200 & $\cdot$ & 100 & - \\
\hline Summed vocs & 7060 & 100.1 & 7560 & 100 & 495 & 99.9 & 608 & 100.1 & 241 & 100 & 351 & 99.9 \\
\hline
\end{tabular}

(COnTIRUED) 
APPENDIX $J$

valldated voc Renults, 1993

\begin{tabular}{|c|c|c|c|c|c|c|c|c|c|c|c|c|}
\hline \multirow{4}{*}{$\begin{array}{l}\text { Sampling Polnt } \\
\text { Location } \\
\text { Date Sampled }\end{array}$} & \multicolumn{8}{|c|}{$6 w-384$} & \multicolumn{4}{|c|}{ Ger-3es } \\
\hline & \multicolumn{8}{|c|}{ MuP } & \multicolumn{4}{|c|}{$\operatorname{mip}$} \\
\hline & \multicolumn{2}{|c|}{$01 / 13 / 93$} & \multicolumn{2}{|c|}{$04 / 20 / 93$} & \multicolumn{2}{|c|}{$08 / 04 / 93$} & \multicolumn{2}{|c|}{$10 / 28 / 93$} & \multicolumn{2}{|c|}{$01 / 12 / 93$} & \multicolumn{2}{|c|}{$04 / 19 / 93$} \\
\hline & ug/L & - ะหน & $u g / L$ & sum & $\operatorname{ug} / 2$ & - sun & $\operatorname{ug} / \mathrm{L}$ & san & $\operatorname{ug} / 2$ & 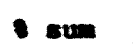 & $\operatorname{ug} / x$ & - sum \\
\hline Acetone & 100 & . & 100 & • & 100 & - & 100 & - & 100 & - & 100 & \\
\hline Benzene & so & $\cdot$ & sv & - & 50 & . & 5v & - & 50 & - & so & \\
\hline Bromoform & 50 & . & 50 & - & so & . & 5v & - & 50 & - & sv & \\
\hline 2-Butanone & 100 &. & 100 & - & 100 &. & 100 & - & 100 & - & 100 & \\
\hline Carbon disulfide & so &. & 5v & . & so &. & so & - & 50 & . & 50 & \\
\hline Carbon tetrachloride & 50 &. & so & - & so & $\cdot$ & so & - & so & - & 50 & \\
\hline Chloroform & so &. & 50 & - & 50 &. & so & - & so & - & so & \\
\hline Chloromethane & 100 &. & 100 & - & 100 &. & 100 & - & 100 & - & 100 & \\
\hline 1,1-Dichloroethane & 5v &. & so & - & so &. & so & - & sv & - & so & \\
\hline 1,2-Dichloroethane & 50 &. & so & - & 50 &. & so & - & 50 & - & so & \\
\hline 1,1-Dichloroethene & 50 &. & so & - & 50 &. & 50 & - & so & . & so & \\
\hline 1,2-Dichloroethene & so & $\cdot$ & 50 & - & so & . & FP3 & - & so & - & 50 & \\
\hline Ethylbenzene & sv & $\cdot$ & 50 & - & so &. & 50 & - & 50 & - & 50 & \\
\hline 4-Methy1-2-pentanone & 100 &. & $\mathbf{F P 1}$ & . & 100 &. & 100 & - & 200 & - & 100 & \\
\hline Methylene chloride & 50 & $\cdot 1$ & $\mathbf{F P 2}$ & - & sv & $\cdot$ & so & - & $\mathbf{F P 1}$ & - & so & \\
\hline styrene & so & $\cdot$ & 50 & - & 50 & $\cdot$ & so & - & 50 & - & 5v & \\
\hline Tetrachloroethene & 50 &. & 5v & - & 50 & $\cdot$ & so & - & 50 & - & 50 & 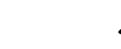 \\
\hline Toluene & 50 & $\cdot 1$ & 50 & - & so & $\cdot$ & 50 & - & so & - & so & \\
\hline 1,1,1-Trichloroethane & so & .1 & so & - & so &. & 50 & - & so & - & so & 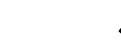 \\
\hline Trichloroethene & so & .1 & 50 & - & 50 & . & 50 & - & so & - & so & \\
\hline vinyl acetote & 100 & $\cdot$ & 100 & - & 100 &. & 100 & - & 100 & - & 100 & 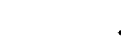 \\
\hline Vinyl chloride & 100 &. & 100 & . & 100 &. & 100 & - & 100 & - & 100 & rat \\
\hline xylenes & so &. & so & - & 50 &. & 5v & - & so & - & so & 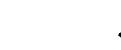 \\
\hline Summed vocs & &. & o) & . & 0 &. & 0 & . & o & . & o & . \\
\hline
\end{tabular}

(CONTImUEd) 
APPEDIXIX $J$

Valldated voc mosulte, 1993

\begin{tabular}{|c|c|c|c|c|c|c|c|c|c|c|c|c|}
\hline \multirow{4}{*}{$\begin{array}{l}\text { Sampling Point } \\
\text { Location } \\
\text { - } \\
\text { Date Sampled }\end{array}$} & \multicolumn{4}{|c|}{ Gm-3es } & \multicolumn{8}{|c|}{$0 m-505$} \\
\hline & \multicolumn{4}{|c|}{ map } & \multicolumn{8}{|c|}{ mo } \\
\hline & \multicolumn{2}{|c|}{$08 / 03 / 93$} & \multicolumn{2}{|c|}{$10 / 27 / 93$} & \multicolumn{2}{|c|}{$03 / 01 / 93$} & \multicolumn{2}{|c|}{$06 / 18 / 93$} & \multicolumn{2}{|c|}{$09 / 15 / 93$} & \multicolumn{2}{|c|}{$11 / 16 / 93$} \\
\hline & $\mathrm{ug} / \mathrm{L}$ & exim & $\operatorname{ug} / \mathrm{L}$ & เ & $\operatorname{ug} / \mathrm{x}$ & ous & $\operatorname{ng} / 2$ & com & $\operatorname{ng} / \mathbf{L}$ & $-\infty$ & $\operatorname{ug} / 2$ & evm \\
\hline Acetone & 100 & $\cdot$ & 100 & - & 100 & - & 100 & - & 100 & - & 100 & - \\
\hline Benzene & 50 & $\cdot 1$ & 50 & - & 5v & - & so & - & so & - & 50 & - \\
\hline Bromoform & 50 &. & 50 & - & 50 & - & 50 & $\cdot$ & 50 & - & 50 & - \\
\hline 2-Butanone & 100 & $\cdot$ & 100 & - & 100 & - & 100 & $\cdot 1$ & 100 & - & 100 & - \\
\hline Carbon disulfide & 50 & $\cdot$ & so & - & so & - & 50 & $\cdot$ & so & - & 50 & - \\
\hline Carbon tetrachloride & so & $\cdot$ & 50 & - & 50) & - & so & $\cdot 1$ & so) & - & 50| & - \\
\hline Chloroform & so & $\cdot$ & 50 & - & so & - & so & $\cdot$ & 50 & - & 50 & - \\
\hline Chloromethane & 100 & $\cdot$ & 100 & • & 100 & - & 100 & $\cdot$ & 100 & - & 100 & - \\
\hline 1.1-Dichloroethane & 50 & $\cdot$ & so & - & so & - & so & $\cdot$ & 50 & - & so & - \\
\hline 1,2-Dichloroethane & so & $\cdot$ & 50 & - & 50 & - & so & $\cdot$ & 50 & - & sol & - \\
\hline 1,1-Dlchloroethene & 50 & $\cdot 1$ & 50 & - & so & - & $\mathbf{r e 3}$ & $\cdot$ & 50 & - & 5o| & - \\
\hline 1,2-D1chloroethene & 50 &. & so & - & so & - & 50 & $\cdot$ & so & - & so & - \\
\hline Ethylbenzene & so & $\cdot$ & 50 & - & 50 & - & so & $\cdot$ & 50 & - & so & * \\
\hline 4-Methyl-2-pentanone & 100 & $\cdot$ & 100 & - & 100 & - & 100 & $\cdot$ & 100 & - & 100 & - \\
\hline Methylene chloride & so &. & 50 & - & 50 & - & 50 & $\cdot$ & so & - & $\boldsymbol{F P 1}$ & - \\
\hline styrene & so & $\cdot 1$ & so & - & so & - & 50 & $\cdot 1$ & so & - & 50 & $\cdot$ \\
\hline Tetrachloroethene & so &. & 50 & - & so & - & $\mathbf{P P 3}$ & $\cdot$ & 50 & - & so & - \\
\hline Toluene & so & $\cdot$ & 50 & - & so & - & so & $\cdot$ & 50 & - & 50 & - \\
\hline 1,1,1-Trichloroethane & so &. & so & - & 50 & - & so & $\cdot$ & 50 & - & 50 & - \\
\hline Trichloroethene & 50 & $\cdot 1$ & so & - & so & - & 50 & $\cdot$ & so & - & so & - \\
\hline Vinyl acetate & 100 & $\cdot$ & 100 & - & 100 & - & 100 & $\cdot$ & 100 & - & 100 & - \\
\hline vingl chloride & 100 &. & 100 & - & 100 & - & 100 & $\cdot$ & 200 & - & 100 & - \\
\hline xylenes & 50 & $\cdot$ & 50 & - & 50 & - & 50 & $\cdot$ & so & - & sol & • \\
\hline Sumed vocs & o &. & o) & - & 0) & - & - & - & 0 & - & - & - \\
\hline
\end{tabular}

(COATIMUED) 
Apredor $J$

Valldated voc Reeulte, 1993

\begin{tabular}{|c|c|c|c|c|c|c|c|c|c|c|c|c|c|}
\hline \multirow{4}{*}{$\begin{array}{l}\text { Saupling Point } \\
\text { Location } \\
\text { Date Sampled }\end{array}$} & \multicolumn{8}{|c|}{$G w-500$} & \multicolumn{5}{|c|}{$\cos -603$} \\
\hline & \multicolumn{8}{|c|}{ R.G } & \multicolumn{5}{|c|}{$\operatorname{sex}$} \\
\hline & \multicolumn{2}{|c|}{$03 / 05 / 93$} & \multicolumn{2}{|c|}{$06 / 23 / 93$} & \multicolumn{2}{|c|}{$09 / 21 / 93$} & \multicolumn{2}{|c|}{$11 / 19 / 93$} & \multicolumn{2}{|c|}{$01 / 29 / 93$} & \multicolumn{3}{|c|}{$05 / 04 / 93$} \\
\hline & $\operatorname{ug} / 2$ & \& & $\log / 2$ & in & $\log / 2$ & - con & $\operatorname{ug} / \mathrm{L}$ & - an & $\log / x$ & - & $\operatorname{lng} / 2$ & com & \\
\hline Acetone & 25000 & & 25000 & $\cdot$ & 25000 & $\cdot$ & 25000 & • & 100 & - & 100 & & - \\
\hline Benzene & 9500 & 15.9 & 8800 & 15.2 & 7600 & 13 & 7700 & 13.9 & so & - & 50 & & - \\
\hline Bromofor & 13000 & $\cdot$ & 13000 & . & 13000 & - & 13000 & • & $\mathbf{5 0}$ & - & 50 & & - \\
\hline 2-Butanone & 25000 & . & 25000 & - & 25000 & - & 25000 & - & 100 & - & 100 & & - \\
\hline Carbon disulfide & 13000 & . & 13000 & - & 13000 & - & 13000 & - & so & - & so & & - \\
\hline Carbon tetrachloride & 13000 & . & 13000 & - & 13000 & - & 13000 & - & so & - & so & & - \\
\hline Chloroform & 13000 & $\cdot$ & 13000 & - & 13000 & - & 13000 & - & 50 & - & so & & - \\
\hline Chloromethane & 25000 & . & 25000 & - & 25000 & - & 25000 & - & 100 & • & 100 & & - \\
\hline 1,1-Dichloroethane & 13000 & . & 13000 &. & 13000 & . & 13000 & - & 50 & - & so & & - \\
\hline 1,2-Dichloroethane & 13000 & - & 13000 & $\cdot$ & 13000 & - & 13000 & - & so & - & 50 & & - \\
\hline 1,1-Dichloroethene & 13000 &. & 13000 &. & 23000 & - & 13000 & - & so & - & 50 & & - \\
\hline 1,2-Dichloroethene & 13000 & . & 13000 &. & 13000 & $\cdot$ & 13000 & • & 50 & - & so & & - \\
\hline Ethylbenzene & 3100 & 5.2 & 2900 & 5 & 2700 & 4.6 & 2500 & 4.5 & so & - & 50 & & - \\
\hline 1-Hethyl-2-pentanone & 25000 & $\cdot$ & 25000 & $\cdot$ & 25000 & $\cdot$ & 25000 & • & 100 & - & 100 & & - \\
\hline methylene chloride & 13000 & - & 310 & 0.5 & 350 & 0.6 & 13000 & - & so & - & 50 & & - \\
\hline styrene & 13000 &. & 13000 & $\cdot$ & 13000 & $\cdot$ & 13000 & - & so & - & 5v & & - \\
\hline Tetrachloroethene & 13000 &. & 13000 &. & 13000 & & 13000 & & so & - & so & & - \\
\hline Toluene & 29000 & 48.7 & 29000 & so & 32000 & 54.6 & 28000 & 50.7 & 50 & - & so & & - \\
\hline 1,1,1-Trichloroethane & 13000 & $\cdot$ & 13000 & $\cdot$ & 13000 & $\cdot$ & 13000 & - & 50 & - & 50 & & - \\
\hline Irichloroethene & 13000 &. & 13000 & . & 13000 & - & 13000 & - & so & - & so & & - \\
\hline Vingl acetate & 25000 & . & 25000 & $\cdot$ & 25000 & • & 25000 & - & 100 & - & 100 & & - \\
\hline vinyl chloride & 25000 &. & 25000 & $\cdot$ & 25000 & & 25000 & & 100 & - & 100 & & - \\
\hline xylenes & 18000 & 30.2 & 17000 & 29.3 & 16000 & 27.3 & 17000 & 30.8 & 50 & • & so & & - \\
\hline Sunmed vocs & 59600 & 100 & 58010 & 100 & 58650 & 100.1 & 55200 & 99.9 & $\mathbf{0}$ & - & - & & - \\
\hline
\end{tabular}

(CONTINUED) 
APPSEDIX J

velldated voc neavite, 1993

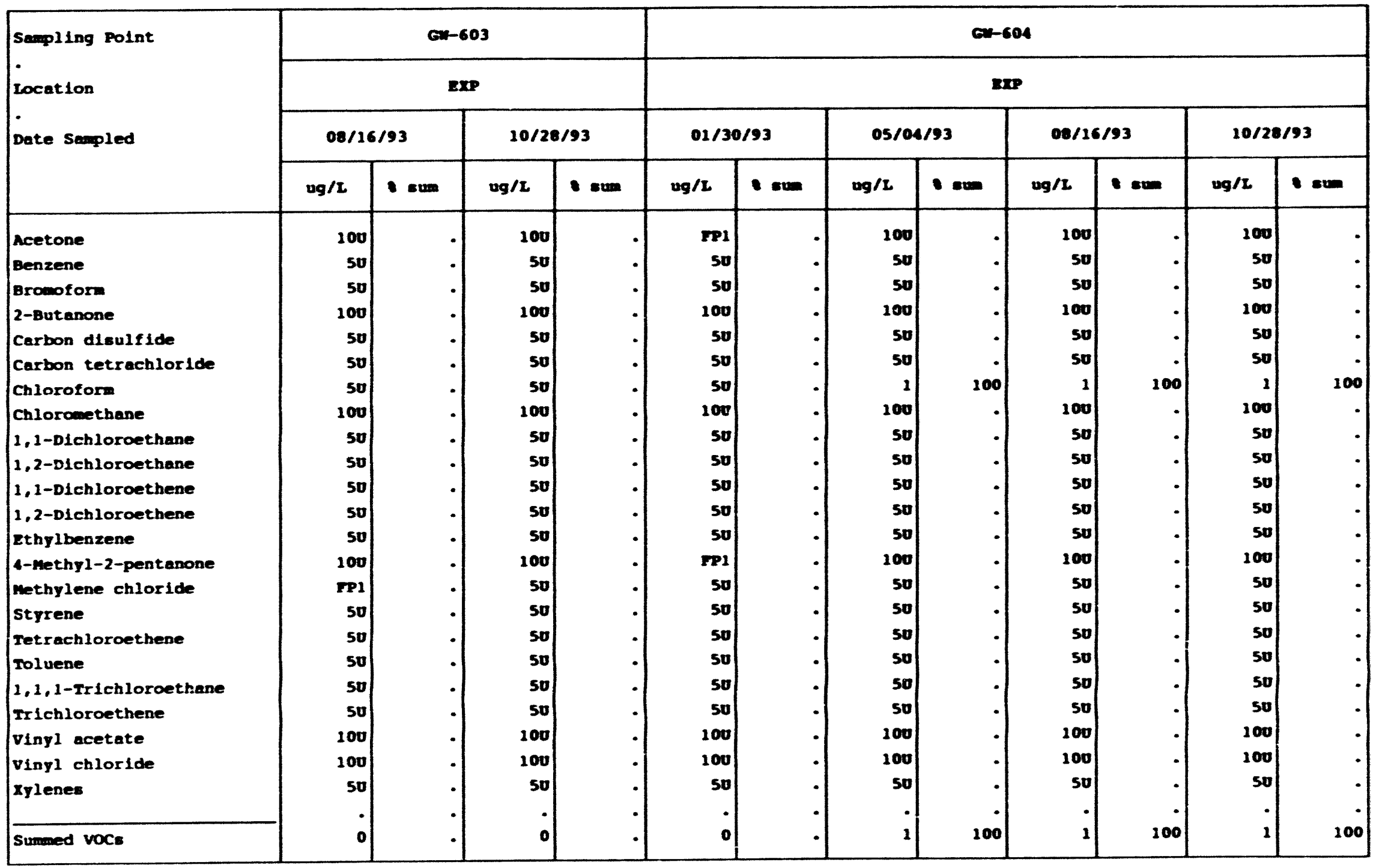

(contrinued) 
valldated voc Resulte, 1993

\begin{tabular}{|c|c|c|c|c|c|c|c|c|c|c|c|c|}
\hline \multirow{4}{*}{$\begin{array}{l}\text { Sampling Point } \\
\text { Location } \\
\text { Date Sampled }\end{array}$} & \multicolumn{8}{|c|}{$6 w-605$} & \multicolumn{4}{|c|}{$0 w-606$} \\
\hline & \multicolumn{8}{|c|}{$\mathbf{E x P}$} & \multicolumn{4}{|c|}{$\mathbf{E x P}$} \\
\hline & \multicolumn{2}{|c|}{$02 / 02 / 93$} & \multicolumn{2}{|c|}{$05 / 10 / 93$} & \multicolumn{2}{|c|}{$08 / 19 / 93$} & \multicolumn{2}{|c|}{$11 / 10 / 93$} & \multicolumn{2}{|c|}{$02 / 03 / 93$} & \multicolumn{2}{|c|}{$05 / 10 / 93$} \\
\hline & $u g / t$ & t $\operatorname{sum}$ & $0 g / L$ & : & $u g / L$ & > $=\pi$ & $u g / L$ & cum & ug/I & $=\sin$ & ug/x & $8 \mathrm{sun}$ \\
\hline Acetone & 100 & - & 100 & - & 100 & $\cdot$ & 100 & $\cdot$ & EP2 & - & 500 & - \\
\hline Benzene & 50 & $\cdot$ & 50 & $\cdot$ & 50 & $\cdot$ & $\mathbf{5 0}$ & $\cdot$ & 250 & - & 250 & • \\
\hline Bromoform & 50 & $\cdot$ & 50 & $\cdot$ & so & $\cdot$ & 50 & $\cdot$ & 250 & $\cdot$ & 250 & • \\
\hline 2-Butanone & 100 & $\cdot$ & 100 & $\cdot$ & 100 & $\cdot$ & 100 & $\cdot$ & 500 & $\cdot$ & 500 & - \\
\hline Carbon disulifide & so & $\cdot$ & so & $\cdot$ & $\mathbf{5 0}$ & $\cdot$ & 50 & $\cdot$ & 250 & $\cdot$ & 250 & • \\
\hline Carbon tetrachloride & 170 & 67.2 & so & 55.6 & 180 & 63.4 & 160 & 61.1 & 1100 & 77.1 & 580 & 69.9 \\
\hline Chloroform & 23 & 9.1 & 20 & 22.2 & 28 & 9.9 & 29 & 11.1 & 310 & 21.7 & 250 & 30.1 \\
\hline Chloromethane & 100 & $\cdot$ & 100 & $\cdot$ & 100 & $\cdot$ & 100 & $\cdot$ & 500 & $\cdot$ & 500 & - \\
\hline 1,1-Dichloroethane & 3 & 1.2 & 5v & $\cdot$ & 3 & 2.1 & 3 & 1.1 & 250 & $\cdot$ & 250 & $\cdot$ \\
\hline 1,2-Dichloroethane & 50 & $\cdot$ & 50 & $\cdot$ & so & $\cdot$ & so & $\cdot$ & 250 & - & 250 & • \\
\hline 1,1-Dlchloroethene & 2 & 0.8 & 50 & $\cdot$ & 1 & 0.4 & 1 & 0.4 & 250 & $\cdot$ & 250 & - \\
\hline 1,2-Dichloroethene & 22 & 8.7 & 10 & 11.1 & 30 & 10.6 & 30 & 11.5 & 250 & $\cdot$ & 250 & - \\
\hline Ethylbenzene & so & $\cdot$ & so & $\cdot$ & so & $\cdot$ & 5v & $\cdot$ & 250 & $\cdot$ & 250 & • \\
\hline 4-methy1-2-pentanone & 100 & $\cdot$ & 100 & - & 100 & $\cdot$ & 100 & $\cdot$ & 11 & 0.8 & 500 & • \\
\hline Methylene chloride & so & $\cdot 1$ & 50 & $\cdot$ & FP1 & $\cdot$ & 50 & $\cdot$ & FP2 & $\cdot$ & 250 & - \\
\hline Styrene & 50 & $\cdot$ & 50 & $\cdot$ & 50 & $\cdot$ & 50 & $\cdot$ & 250 & $\cdot$ & 250 & $\cdot$ \\
\hline Tetrachloroethene & 25 & 9.9 & 7 & 7.8 & 32 & 11.3 & 30 & 11.5 & 6 & 0.4 & 250 & • \\
\hline Toluene & 50 & $\cdot$ & 50 & $\cdot$ & 50 & $\cdot$ & 50 & $\cdot$ & 250 & $\cdot$ & 250 & • \\
\hline 1,1,1-Trichloroethane & 50 & $\cdot$ & 50 & $\cdot$ & 50 & $\cdot$ & 50 & $\cdot$ & 250 & $\cdot$ & 250 & • \\
\hline Trlchloroethene & 8 & 3.2 & 3 & 3.3 & 10 & 3.5 & 9 & 3.4 & 250 & . & 250 & • \\
\hline vinyl acetate & 100 & $\cdot 1$ & 100 &. & 100 & $\cdot 1$ & 100 & $\cdot$ & 500 & $\cdot$ & 500 & • \\
\hline vinyl chloride & 100 & $\cdot$ & 100 & $\cdot$ & FP3 & $\cdot$ & 100 & - & 500 & - & 500 & $\cdot$ \\
\hline xylenes & 50 & $\cdot 1$ & so & $\cdot$ & 50 & $\cdot$ & 5v & $\cdot$ & 250 & $\cdot$ & 250 & • \\
\hline Summed $\mathrm{VOC}_{\mathrm{B}}$ & 253 & & 90 & 200 & 284 & 100.2 & 262 & 100.1 & 1427 & 100 & 830 & 100 \\
\hline
\end{tabular}

(CONTIRUED) 
APPEEDIX $J$

Valldated voc Reeults, 1993

\begin{tabular}{|c|c|c|c|c|c|c|c|c|c|c|c|c|}
\hline \multirow{4}{*}{$\begin{array}{l}\text { Sampling Point } \\
\text { Location } \\
\text { Date Sempled }\end{array}$} & \multicolumn{4}{|c|}{ Gu-606 } & \multicolumn{8}{|c|}{$G n-617$} \\
\hline & \multicolumn{4}{|c|}{$\mathbf{E x P}$} & \multicolumn{8}{|c|}{$\mathbf{E x P}$} \\
\hline & \multicolumn{2}{|c|}{$08 / 20 / 93$} & \multicolumn{2}{|c|}{$11 / 11 / 93$} & \multicolumn{2}{|c|}{$01 / 12 / 93$} & \multicolumn{2}{|c|}{$05 / 04 / 93$} & \multicolumn{2}{|c|}{$09 / 16 / 93$} & \multicolumn{2}{|c|}{$12 / 07 / 93$} \\
\hline & ug/L & sum & ug/L & sum & $u g / x$ & - sum & $u g / L$ & - $=\operatorname{com}$ & $u g / 2$ & s $8 x$ & $\operatorname{ug} / \mathrm{L}$ & s sum \\
\hline Acetone & 500 & - & 500 & • & $\mathbf{F P 1}$ & . & 100 & . & FP3 & $\cdot$ & $\mathbf{F P 1}$ & - \\
\hline Benzene & 250 &. & 250 & $\cdot$ & 50 & $\cdot$ & 5v & $\cdot$ & so & $\cdot$ & 50 & • \\
\hline Bromoform & 250 &. & 250 & $\cdot$ & so & $\cdot$ & so & $\cdot$ & 50 & $\cdot$ & so & - \\
\hline 2-Butanone & 500 &. & 500 & $\cdot$ & 100 & $\cdot$ & 100 & $\cdot$ & 100 & $\cdot$ & $\mathbf{F P 1}$ & - \\
\hline Carbon disulfide & 250 & $\cdot$ & 250 & $\cdot$ & 50 & $\cdot$ & 5v & $\cdot$ & 50 & $\cdot$ & 50 & • \\
\hline Carbon tetrachloride & 770 & 74.3 & 500 & 66.2 & 50 & $\cdot$ & so & $\cdot$ & 50 &. & so & • \\
\hline Chloroform & 250 & 24.1 & 250 & 33.1 & 50 & $\cdot$ & so & $\cdot$ & 50 & $\cdot$ & so & • \\
\hline Chloromethane & 500 & $\cdot$ & 500 & . & 100 & $\cdot$ & 100 & $\cdot$ & 100 &. & 100 & • \\
\hline 1,1-Dichloroethane & 250 & $\cdot$ & 250 & $\cdot$ & 50 & $\cdot$ & 50 & $\cdot$ & 5o &. & so & - \\
\hline 1,2-Dichloroethane & 250 &. & 250 & . & 50 &. & 50 & $\cdot$ & 50 &. & 50 & • \\
\hline 1,1-Dichloroethene & 250 & $\cdot$ & 250 & $\cdot$ & 50 & $\cdot$ & 50 & $\cdot$ & 50 & $\cdot$ & so & • \\
\hline 1,2-Dichloroethene & 250 & $\cdot$ & 250 & $\cdot$ & 4 & so & 8 & 100 & 22 & 42.3 & 18 & 39.1 \\
\hline Ethylbenzene & 250 &. & 250 & $\cdot$ & 50 & $\cdot$ & 50 & $\cdot$ & so & $\cdot$ & 50 & • \\
\hline 4-Methy1-2-pentanone & 500 &. & 500 & $\cdot$ & $\boldsymbol{F P 1}$ & $\cdot$ & 100 &. & 100 &. & $\boldsymbol{F P 1}$ & • \\
\hline Methylene chloride & 10 & 1 & $\mathbf{F P 1}$ &. & 50 & $\cdot$ & 50 & $\cdot$ & so & $\cdot$ & $\mathbf{F P 1}$ & • \\
\hline styrene & 250 & $\cdot$ & 250 &. & so &. & so & $\cdot 1$ & so &. & so & • \\
\hline Tetrachloroethene & 6 & 0.6 & 5 & 0.7 & 2 & 25 & 50 & $\cdot$ & 14 & 26.9 & 14 & 30.4 \\
\hline Toluene & 250 & $\cdot$ & 250 & $\cdot$ & 50 & $\cdot$ & 5v & $\cdot$ & 50 & $\cdot$ & so & • \\
\hline 1,1,1-Irichloroethane & 250 & $\cdot 1$ & 250 & $\cdot$ & 50) & $\cdot$ & 50 & $\cdot$ & so &. & 50 & - \\
\hline Trichloroethene & 250 & $\cdot$ & 250 & $\cdot$ & 2 & 25 & so & $\cdot$ & 16 & 30.8 & 14 & 30.4 \\
\hline vinyl acetate & 500 & $\cdot$ & 500 & $\cdot$ & 100 & $\cdot$ & 100 &. & $\mathbf{F P 3}$ &. & 100 & • \\
\hline vinyl chloride & 500 &. & 500 & $\cdot$ & 100 & $\cdot 1$ & 100 &. & 100 & $\cdot$ & 100 & - \\
\hline xylenes & 250 & $\cdot$ & 250 & $\cdot$ & 50 &. & 50 & $\cdot$ & so &. & 50 & - \\
\hline Sumened VOCs & 1036 & 100 & 755 & 100 & 8 & 100 & 8 & 200 & 52 & 100 & 46 & 99.9 \\
\hline
\end{tabular}

(COMTINUED) 
APPERDIX J

Valldated voC Results, 1993

\begin{tabular}{|c|c|c|c|c|c|c|c|c|c|c|c|c|}
\hline \multirow{4}{*}{$\begin{array}{l}\text { Sampling Point } \\
\text { Location } \\
\text { Date Sampled }\end{array}$} & \multicolumn{8}{|c|}{$G W-618$} & \multicolumn{4}{|c|}{ GW-619 } \\
\hline & \multicolumn{8}{|c|}{$\mathbf{E x P}$} & \multicolumn{4}{|c|}{ FTF } \\
\hline & \multicolumn{2}{|c|}{$01 / 12 / 93$} & \multicolumn{2}{|c|}{$05 / 05 / 93$} & \multicolumn{2}{|c|}{$09 / 16 / 93$} & \multicolumn{2}{|c|}{$12 / 08 / 93$} & \multicolumn{2}{|c|}{$01 / 20 / 93$} & \multicolumn{2}{|c|}{$05 / 05 / 93$} \\
\hline & $u g / L$ & sum & $u g / L$ & sum & $u g / L$ & sum & $u g / L$ & s sum & ug/L & s sum & ug/L & sum \\
\hline Acetone & FP1 & - & 100 & $\cdot$ & 100 & . & 100 & $\cdot 1$ & 100 & - & 100 & - \\
\hline Benzene & 50 & $\cdot$ & 50 & $\cdot$ & 50 & $\cdot$ & su & $\cdot$ & 50 & $\cdot$ & 50 & • \\
\hline Bromoform & 50 & $\cdot$ & 50 & $\cdot 1$ & 50 & $\cdot$ & 50 & $\cdot$ & 50 & $\cdot$ & 50 & • \\
\hline 2-Butanone & 100 & $\cdot$ & 100 & $\cdot$ & 100 & $\cdot$ & 100 & $\cdot$ & 100 & $\cdot$ & 100 & • \\
\hline Carbon disulfide & 5u & $\cdot$ & su & $\cdot$ & 5u & $\cdot$ & 50 & $\cdot$ & 50 & $\cdot$ & 50 & • \\
\hline Carbon tetrachloride & 50 & $\cdot$ & 50 & $\cdot$ & 50 & $\cdot$ & 50 & $\cdot$ & 50 & $\cdot$ & 50 & • \\
\hline Chloroform & 5u & $\cdot$ & 5v & $\cdot$ & 50 & $\cdot$ & 50 & $\cdot$ & 50 & $\cdot$ & 50 & • \\
\hline Chloromethane & 100 & $\cdot$ & 100 & $\cdot$ & 100 & $\cdot$ & 100 &. & 100 & $\cdot$ & 100 & • \\
\hline 1,1-Dichloroethane & 5u & $\cdot$ & su & $\cdot$ & su & $\cdot$ & 50 & $\cdot$ & 50 & $\cdot$ & 50 & - \\
\hline 1,2-Dichloroethane & so & $\cdot$ & 5u & $\cdot 1$ & 50 & $\cdot$ & 50 & $\cdot$ & 50 & $\cdot$ & 50 & • \\
\hline 1,1-Dichloroethene & 50 & $\cdot$ & 5v & $\cdot$ & 50 & $\cdot$ & 50 & $\cdot$ & 50 & $\cdot$ & 50 & • \\
\hline 1,2-Dichloroethene & 41 & 45.1 & 34 & 37.4 & 39 & 57.4 & 36 & 30 & 61 & 25.8 & 170 & 34.5 \\
\hline Ethylbenzene & 5u &. & 50 & $\cdot$ & 50 & $\cdot$ & 5u & $\cdot$ & 50 & $\cdot$ & 50 & • \\
\hline 4-Methy1-2-pentanone & FPI & $\cdot$ & 100 & $\cdot$ & 100 & $\cdot$ & 100 & $\cdot$ & 100 & $\cdot$ & 100 & • \\
\hline Methylene chloride & 50 & $\cdot$ & 50 & $\cdot$ & 50 & $\cdot$ & 50 & $\cdot$ & 50 & $\cdot$ & su & • \\
\hline Styrene & 5v & $\cdot$ & 50 & $\cdot$ & 50 & $\cdot$ & 50 & $\cdot$ & 50 & $\cdot$ & 50 & • \\
\hline Tetrachloroethene & 23 & 25.3 & 27 & 29.7 & 10 & 14.7 & 43 & 35.8 & 140 & 59.3 & 230 & 46.7 \\
\hline Toluene & 5u & $\cdot$ & 50 & $\cdot$ & 50 & $\cdot$ & 50 & $\cdot$ & 50 & $\cdot$ & 50 & • \\
\hline 1,1,1-Trichloroethane & 50 & $\cdot$ & 50 & $\cdot$ & 50 & $\cdot$ & 5u & $\cdot$ & 50 & $\cdot$ & 50 & • \\
\hline Trichloroethene & 27 & 29.7 & 30 & 33 & 19 & 27.9 & 41 & 34.2 & 35 & 14.8 & 93 & 18.9 \\
\hline Vinyl acetate & 100 & $\cdot$ & 100 & $\cdot$ & 100 & $\cdot$ & 100 & $\cdot$ & 100 & $\cdot$ & 100 & • \\
\hline vinyl chloride & 100 & $\cdot$ & 100 & $\cdot$ & 100 & $\cdot$ & 100 & $\cdot$ & 100 & $\cdot$ & 100 & • \\
\hline xylenes & 50 & $\cdot$ & 50 & $\cdot$ & 50 & $\cdot$ & 50 & $\cdot$ & 50 & $\cdot$ & $5 \mathrm{U}$ & • \\
\hline Summed vOCB & 91 & 100.1 & 91 & 100.1 & 68 & 100 & 120 & 100 & 236 & 99.9 & 493 & 100.1 \\
\hline
\end{tabular}

(CONTINUED) 
APPEHDIX $J$

Validated voc Results, 1993

\begin{tabular}{|c|c|c|c|c|c|c|c|c|c|c|c|c|}
\hline \multirow{4}{*}{$\begin{array}{l}\text { Sampling Point } \\
\text { Location } \\
\text { Date Sampled }\end{array}$} & \multicolumn{4}{|c|}{ GW-619 } & \multicolumn{8}{|c|}{$G W-620$} \\
\hline & \multicolumn{4}{|c|}{ FTF } & \multicolumn{8}{|c|}{$\mathbf{F T}$} \\
\hline & \multicolumn{2}{|c|}{$09 / 17 / 93$} & \multicolumn{2}{|c|}{$12 / 08 / 93$} & \multicolumn{2}{|c|}{$01 / 20 / 93$} & \multicolumn{2}{|c|}{$05 / 06 / 93$} & \multicolumn{2}{|c|}{$09 / 17 / 93$} & \multicolumn{2}{|c|}{$12 / 09 / 93$} \\
\hline & ug/L & 8 sum & $u g / x$ & sum & $u g / L$ & sum & ug/L & sum & $\operatorname{ug} / \mathrm{L}$ & sum & ug/L & sum \\
\hline Acetone & 100 & . & 100 & . & 400 & - & 60 & 5.8 & 400 & . & FP1 & - \\
\hline Benzene & sv & $\cdot$ & 5u & - & 200 & - & 250 & $\cdot$ & 200 & - & 200 & • \\
\hline Bromoform & 5v & $\cdot$ & 50 & - & 200 & - & 250 & $\cdot$ & 200 & - & 200 & • \\
\hline 2-Butanone & 100 & $\cdot 1$ & 100 & - & 400 & - & 500 & $\cdot$ & 400 & - & FP1 & • \\
\hline Carbon disulfide & 50 & $\cdot$ & su & • & 200 & - & 250 & - & 200 & - & 200 & • \\
\hline Carbon tetrachloride & so & $\cdot$ & 50 & - & 200 & - & 250 & - & 200 & - & 200 & • \\
\hline Chloroform & 50 & . & 50 & - & 200 & • & 250 & - & 200 & - & 200 & • \\
\hline Chloromethane & 100 & $\cdot$ & 100 & - & 400 & - & 500 & - & 400 & - & 400 & • \\
\hline 1,1-Dichloroethane & 5u & $\cdot$ & 50 & - & 200 & - & 250 & - & 200 & - & 200 & • \\
\hline 1,2-Dichloroethane & 5v & . & 50 & • & 200 & - & 250 & - & 200 & - & 200 & • \\
\hline 1,1-Dichloroethene & so & $\cdot$ & 50 & $\cdot$ & 200 & • & 250 & $\cdot$ & 200 & $\cdot$ & 200 & • \\
\hline 1,2-Dichloroethene & 95 & 40.8 & 79 & 29.2 & 560 & 44.1 & 400 & 38.5 & 540 & 42.9 & 620 & 38 \\
\hline Ethylbenzene & 50 & $\cdot$ & 50 & • & 200 & • & 250 & $\cdot$ & 200 & • & 200 & • \\
\hline 4-Methy1-2-pentanone & 100 & - & 100 & • & 400 & - & 500 & $\cdot$ & 400 & - & FP1 & • \\
\hline Methylene chloride & 5u & $\cdot$ & 50 & • & FP1 & - & 250 & $\cdot$ & 200 & $\cdot$ & FP1 & • \\
\hline styrene & 5u & $\cdot$ & 50 & • & 200 & • & 250 & $\cdot$ & 200 & • & 200 & • \\
\hline Tetrachloroethene & 95 & 40.8 & 150 & 55.4 & 540 & 42.5 & 430 & 41.3 & 530 & 42.1 & 780 & 47.9 \\
\hline Toluene & 50 & $\cdot$ & 50 & • & 200 & - & 250 & $\cdot$ & 200 & $\cdot$ & 200 & • \\
\hline 1,1,1-Trichloroethane & 50 & $\cdot$ & 50 & $\cdot$ & 200 & • & 250 & $\cdot$ & 200 & - & 200 & - \\
\hline Trichloroethene & 43 & 18.5 & 42 & 15.5 & 170 & 13.4 & 150 & 14.4 & 190 & 15.1 & 230 & 14.1 \\
\hline Vinyl acetate & 100 & $\cdot$ & 100 & . & 400 & • & 500 & $\cdot$ & 400 & $\cdot$ & 400 & • \\
\hline vinyl chloride & 100 & $\cdot$ & 100 & . & 400 & - & 500 & - & 400 & - & 400 & - \\
\hline xylenes & 50 &. & 5u & . & 200 & - & 250 & - & 200 & - & 200 & - \\
\hline Summed vocs & 233 & 100.1 & 271 & 100.1 & 1270 & 100 & 1040 & 100 & 1260 & 100.1 & 1630 & 100 \\
\hline
\end{tabular}

(CONT INUED) 
APPENDIX J

Validated voC Results, 1993

\begin{tabular}{|c|c|c|c|c|c|c|c|c|c|c|c|c|}
\hline \multirow{4}{*}{$\begin{array}{l}\text { Sampling Point } \\
\text { Location } \\
\text { Date Sampled }\end{array}$} & \multicolumn{8}{|c|}{ Gx-631 } & \multicolumn{4}{|c|}{$G W-632$} \\
\hline & \multicolumn{8}{|c|}{$\mathbf{R G}$} & \multicolumn{4}{|c|}{$\mathbf{R G}$} \\
\hline & \multicolumn{2}{|c|}{$03 / 02 / 93$} & \multicolumn{2}{|c|}{$06 / 18 / 93$} & \multicolumn{2}{|c|}{$09 / 16 / 93$} & \multicolumn{2}{|c|}{$11 / 17 / 93$} & \multicolumn{2}{|c|}{$03 / 03 / 93$} & \multicolumn{2}{|c|}{$06 / 21 / 93$} \\
\hline & $u g / L$ & sum & $u g / L$ & sum & ug/L & sum & $\mathrm{ug} / \mathrm{L}$ & 8 sum & ug/L & sum & ug $/ \mathrm{L}$ & s sum \\
\hline Acetone & 100 & • & 100 & . & 100 & . & 100 & . & 5000 & . & 10000 & . \\
\hline Benzene & 2 & 28.6 & 2 & 25 & FP2 & . & 2 & 28.6 & 9900 & 56.3 & 9300 & 55.5 \\
\hline Bromoform & 50 & • & so &. & so &. & 50 & . & 2500 & • & 5000 & • \\
\hline 2-Butanone & 100 & - & 100 & $\cdot$ & FP1 & $\cdot$ & 100 & - & 5000 & - & 10000 & • \\
\hline Carbon disulfide & 50 & - & su &. & 50 &. & 50 &. & 2500 & - & 5000 & . \\
\hline Carbon tetrachloride & 50 & - & 50 & - & so & . & 50 & . & 2500 & - & 5000 & • \\
\hline Chloroform & 50 & . & 50 & - & 50 & - & 50 &. & 2500 & . & 5000 & • \\
\hline Chloromethane & 100 & . & 100 & - & 100 & - & 100 & . & 160 & 0.9 & 10000 & • \\
\hline 1,1-Dichloroethane & $5 \mathbf{v}$ & - & so & . & 50 & . & 50 &. & 2500 & $\cdot$ & 5000 & • \\
\hline 1,2-Dichloroethane & $5 v$ & . & 50 & . & 50 &. & 50 & - & 2500 & - & 5000 & • \\
\hline 1,1-Dichloroethene & 5u & • & 5u & • & 50 & . & 50 & $\cdot$ & 2500 & . & 5000 & • \\
\hline 1,2-Dichloroethene & 1 & 14.3 & 1 & 12.5 & 50 & . & su & - & 2500 & - & 5000 & • \\
\hline Ethylbenzene & 5u & $\cdot$ & 50 & $\cdot$ & 50 & $\cdot$ & 50 & $\cdot$ & 260 & 1.5 & 360 & 2.1 \\
\hline 4-Hethyl-2-pentanone & 100 & • & 100 & $\cdot$ & FP3 & $\cdot$ & 100 & - & 71 & 0.4 & 10000 & • \\
\hline Methylene chloride & 50 & - & 50 & $\cdot$ & 50 & . & 50 & - & 2500 &. & 5000 & - \\
\hline Styrene & 5u & $\cdot$ & 50 & $\cdot$ & su &. & 5v & - & 2500 & . & 5000 & • \\
\hline Tetrachloroethene & 4 & 57.1 & 5 & 62.5 & 4 & 100 & 5 & 71.4 & 2500 & • & 5000 & • \\
\hline Toluene & 50 & $\cdot$ & 50 & $\cdot$ & FP3 &. & 50 &. & 3600 & 20.5 & 4100 & 24.5 \\
\hline $1,1,1-\mathrm{Tr}$ ichloroethane & 5u & $\cdot$ & su & $\cdot$ & 50 & $\cdot$ & 50 & $\cdot$ & 2500 & $\cdot$ & 5000 & • \\
\hline Trichloroethene & 50 &. & 5u & . & 50 & - & 5u & . & 2500 & . & 5000 & • \\
\hline vinyl acetate & 100 & - & 100 & $\cdot$ & 100 & $\cdot$ & 100 & $\cdot$ & 1000 & 5.7 & 10000 & • \\
\hline vinyl chloride & 100 & - & 100 & $\cdot$ & 100 & $\cdot$ & 100 & . & 5000 & $\cdot$ & 20000 & • \\
\hline xylenes & 50 & - & 50 &. & FP2 & . & 50 & . & 2600 & 14.8 & 3000 & 17.9 \\
\hline Summed vOCs & $\dot{7}$ & 100 & $\dot{8}$ & 100 & $\dot{4}$ & 100 & $\dot{7}$ & 100 & 17591 & $100 . \dot{1}$ & 16760 & $100^{\circ}$ \\
\hline
\end{tabular}

(CONTINUED) 
APPENDIX J

Valldated voc Results, 1993

\begin{tabular}{|c|c|c|c|c|c|c|c|c|c|c|c|c|}
\hline \multirow{4}{*}{$\begin{array}{l}\text { Sampling Point } \\
\text { Location } \\
\text { Date Sampled }\end{array}$} & \multicolumn{4}{|c|}{ GW-632 } & \multicolumn{8}{|c|}{$G w-633$} \\
\hline & \multicolumn{4}{|c|}{$\mathbf{R G}$} & \multicolumn{8}{|c|}{ RG } \\
\hline & \multicolumn{2}{|c|}{$09 / 16 / 93$} & \multicolumn{2}{|c|}{$11 / 18 / 93$} & \multicolumn{2}{|c|}{$03 / 03 / 93$} & \multicolumn{2}{|c|}{$06 / 21 / 93$} & \multicolumn{2}{|c|}{$09 / 20 / 93$} & \multicolumn{2}{|c|}{$11 / 19 / 93$} \\
\hline & ug/L & sum & ug/L & s sum & ug/L & s sum & $u g / L$ & sum & ug/L & 8 sum & $u g / L$ & sum \\
\hline Acetone & 100 & $\cdot$ & 2000 & $\cdot$ & 100 & $\cdot$ & 100 & $\cdot$ & 100 & $\cdot$ & 100 & - \\
\hline Benzene & 2300 & 52.5 & 11000 & 56.1 & 6 & 4.8 & 8 & 4.9 & 10 & 6 & 4 & 8 \\
\hline Bromoform & 5u & $\cdot$ & 1000 & $\cdot$ & 2 & 1.6 & 50 &. & 1 & 0.6 & 50 & • \\
\hline 2-Butanone & 100 & . & 2000 &. & 100 & $\cdot$ & 100 & $\cdot$ & 100 & $\cdot$ & 100 & • \\
\hline Carbon disulfide & 50 & . & 1000 & • & 50 & $\cdot$ & 50 & $\cdot$ & 5u & - & 50 & • \\
\hline Carbon tetrachloride & 50 & . & 1000 &. & 50 & $\cdot$ & 50 & $\cdot$ & 50 & $\cdot$ & 50 & • \\
\hline chloroform & 0.5 & 0 & 1000 & - & 24 & 19.4 & 28 & 17.2 & 27 & 16.3 & 6 & 12 \\
\hline Chloromethane & 100 & $\cdot$ & 2000 & $\cdot$ & 100 & $\bullet$ & 100 & $\cdot$ & 100 & $\cdot$ & 100 & • \\
\hline 1,1-Dichloroethane & 2 & 0 & 1000 & • & 50 & $\cdot$ & 50 & $\cdot$ & 50 & - & 5u & • \\
\hline 1,2-Dichloroethane & 5u & $\cdot$ & 1000 & $\cdot$ & 50 & $\cdot$ & 50 & $\cdot$ & 50 & $\cdot$ & 50 & • \\
\hline 1,1-Dichloroethene & 50 & . & 1000 &. & 5u & . & 2 & 1.2 & 1 & 0.6 & 0.8 & 1.6 \\
\hline 1,2-Dichloroethene & 15 & 0.3 & 1000 & $\cdot$ & 3 & 2.4 & 4 & 2.5 & 3 & 1.8 & su & • \\
\hline Ethylbenzene & 170 & 3.9 & 400 & 2 & 50 & • & 5v & $\cdot$ & FP3 & $\cdot$ & 50 & • \\
\hline 4-Methy1-2-pentanone & 100 & $\cdot$ & 2000 & $\cdot$ & 100 & $\cdot$ & 100 & $\cdot$ & 100 & $\cdot$ & 100 & • \\
\hline Methylene chloride & 50 & . & 1000 & $\cdot$ & 18 & 14.5 & 29 & 17.8 & 24 & 14.5 & FP1 & • \\
\hline styrene & 50 & $\cdot$ & 1000 & $\cdot 1$ & 50 & $\cdot$ & 50 & $\cdot$ & 50 & $\cdot$ & 50 & • \\
\hline Tetrachloroethene & 2 & 0 & 1000 & $\cdot$ & 60 & 48.4 & 74 & 45.4 & 78 & 47 & 30 & 60.2 \\
\hline Toluene & 690 & 15.8 & 4100 & 20.9 & 3 & 2.4 & 6 & 3.7 & 8 & 4.8 & 4 & 8 \\
\hline $1,1,1-\operatorname{Tr} 1$ chloroethane & 50 & $\cdot$ & 1000 & $\cdot$ & 5u & $\cdot$ & 5u & $\cdot$ & 5u & $\cdot$ & 50 & $\cdot$ \\
\hline Trichloroethene & 1 & o) & 1000 & $\cdot$ & 7 & 5.6 & 8 & 4.9 & 8 & 4.8 & 2 & 4 \\
\hline Vinyl acetate & 100 & $\cdot$ & 2000 & $\cdot$ & 100 & $\cdot$ & 100 & $\cdot$ & 100 & $\cdot$ & 100 & $\cdot$ \\
\hline vinyl chloride & 100 & $\cdot$ & 2000 & $\cdot$ & 100 & $\cdot$ & 100 & $\cdot$ & 100 & $\cdot$ & 100 & • \\
\hline Xylenes & 1200 & 27.4 & 4100 & 20.9 & 1 & 0.8 & 4 & 2.5 & 6 & 3.6 & 3 & 6 \\
\hline Summed Vocs & 4380.5 & 99.9 & 19600 & 99.9 & 124 & 99.9 & 163 & 100.1 & 166 & 100 & 49.8 & 99.8 \\
\hline
\end{tabular}

(CONTINUED) 
APPENDIX J

Valldated voc Resulte, 1993

\begin{tabular}{|c|c|c|c|c|c|c|c|c|c|c|c|c|}
\hline \multirow{4}{*}{$\begin{array}{l}\text { Sampling Point } \\
\text { Location } \\
\text { - Date Sampled }\end{array}$} & \multicolumn{8}{|c|}{ GW-634 } & \multicolumn{4}{|c|}{$G W-656$} \\
\hline & \multicolumn{8}{|c|}{$\mathbf{R G}$} & \multicolumn{4}{|c|}{ T0134 } \\
\hline & \multicolumn{2}{|c|}{$03 / 02 / 93$} & \multicolumn{2}{|c|}{$06 / 18 / 93$} & \multicolumn{2}{|c|}{$09 / 16 / 93$} & \multicolumn{2}{|c|}{$11 / 18 / 93$} & \multicolumn{2}{|c|}{$03 / 11 / 93$} & \multicolumn{2}{|c|}{$06 / 22 / 93$} \\
\hline & $u g / L$ & sum & $u g / L$ & 8 sum & ug/L & sum & ug/L & 8 sum & ug/L & s sum & ug/L & s sum \\
\hline Acetone & 2500 & - & 2500 & - & 100 & . & 2000 & - & 10000 & - & 10000 & - \\
\hline Benzene & 430 & 8.2 & 380 & 8.6 & 270 & 9.2 & 310 & 8.8 & 5000 & - & 5000 & - \\
\hline Bromoform & 1300 & • & 1300 & $\cdot$ & 5v & $\cdot$ & 1000 & $\cdot$ & $500 U$ & - & 5000 & $\cdot$ \\
\hline 2-Butanone & 2500 & - & 2500 & $\cdot$ & 100 & - & 2000 & $\cdot$ & 10000 & . & 10000 & • \\
\hline Carbon disulfide & 1300 & - & 1300 & $\cdot$ & 50 & - & 1000 & - & 5000 & • & 5000 & • \\
\hline Carbon tetrachloride & 1300 & - & 1300 & $\cdot$ & 50 & $\cdot$ & 1000 & $\cdot$ & 5000 & $\cdot$ & 5000 & • \\
\hline Chloroform & 1300 & • & 1300 & $\cdot$ & su & - & 1000 & - & 5000 & $\cdot$ & 5000 & $\cdot$ \\
\hline Chloromethane & 130 & 2.5 & 2500 & . & 100 & - & 2000 & - & 10000 & - & 10000 & • \\
\hline 1,1-Dichloroethane & 1300 & • & 1300 & $\cdot$ & 5v & - & 1000 & - & 5000 & $\cdot$ & 5000 & • \\
\hline 1,2-Dichloroethane & 1300 & • & 1300 & $\cdot$ & 5u & - & 1000 & $\cdot$ & 5000 & $\cdot$ & 5000 & • \\
\hline 1,1-Dichloroethene & 1300 & • & 1300 & $\cdot$ & 5u & $\cdot$ & $100 \mathrm{U}$ & $\cdot$ & 1700 & 13.1 & 1700 & 12.9 \\
\hline 1,2-Dichloroethene & 1300 & - & 1300 & $\cdot$ & 2 & 0.1 & 1000 & • & 300 & 2.3 & 340 & 2.6 \\
\hline Ethylbenzene & 790 & 15.1 & 710 & 16 & 550 & 18.8 & 680 & 19.2 & 5000 & $\cdot$ & 5000 & • \\
\hline 4-Methyl-2-pentanone & 2500 & • & 2500 & $\cdot$ & 100 & $\cdot$ & 2000 & $\bullet$ & 10000 & $\cdot$ & 10000 & • \\
\hline Methylene chioride & 62 & 1.2 & 1300 & $\cdot$ & su & - & 1000 & • & 5000 & • & 5000 & • \\
\hline styrene & 53 & 1 & 1300 & $\cdot$ & 50 & $\cdot$ & 1000 & $\cdot$ & 5000 & $\cdot$ & 5000 & $\bullet$ \\
\hline Tetrachloroethene & 1300 & - & 1300 & $\cdot$ & 50 & . & 1000 & $\cdot$ & 5000 & - & 99 & 0.8 \\
\hline Toluene & 680 & 13 & 540 & 12.2 & 300 & 10.3 & 350 & 9.9 & $500 \mathrm{U}$ & $\cdot$ & 5000 & • \\
\hline 1,1,1-Trichloroethene & 1300 & • & 1300 & $\cdot$ & su & $\cdot$ & 1000 & $\cdot$ & 5000 & $\cdot$ & 5000 & • \\
\hline Trichloroethene & 1300 & - & 1300 & $\cdot$ & 50 & $\cdot$ & 1000 & $\cdot$ & 11000 & 84.6 & 11000 & 83.7 \\
\hline vinyl acetate & 2500 & - & 2500 & . & 100 & - & 2000 & $\cdot$ & 10000 & - & 10000 & • \\
\hline vinyl chloride & 2500 & • & 2500 & $\cdot$ & 100 & • & $\mathbf{2 0 0 0}$ & $\cdot$ & 10000 & - & 10000 & - \\
\hline xylenes & 3100 & 59.1 & 2800 & 63.2 & 1800 & 61.6 & 2200 & 62.1 & 5000 & - & 5000 & • \\
\hline Summed Vocs & 5245 & 100.1 & 4430 & 100 & $2922^{\circ}$ & 100 & $3540^{\circ}$ & 100 & 13000 & 100 & 13139 & 100 \\
\hline
\end{tabular}

(CONTINUED) 
APPENDIX J

Validated voC Results, 1993

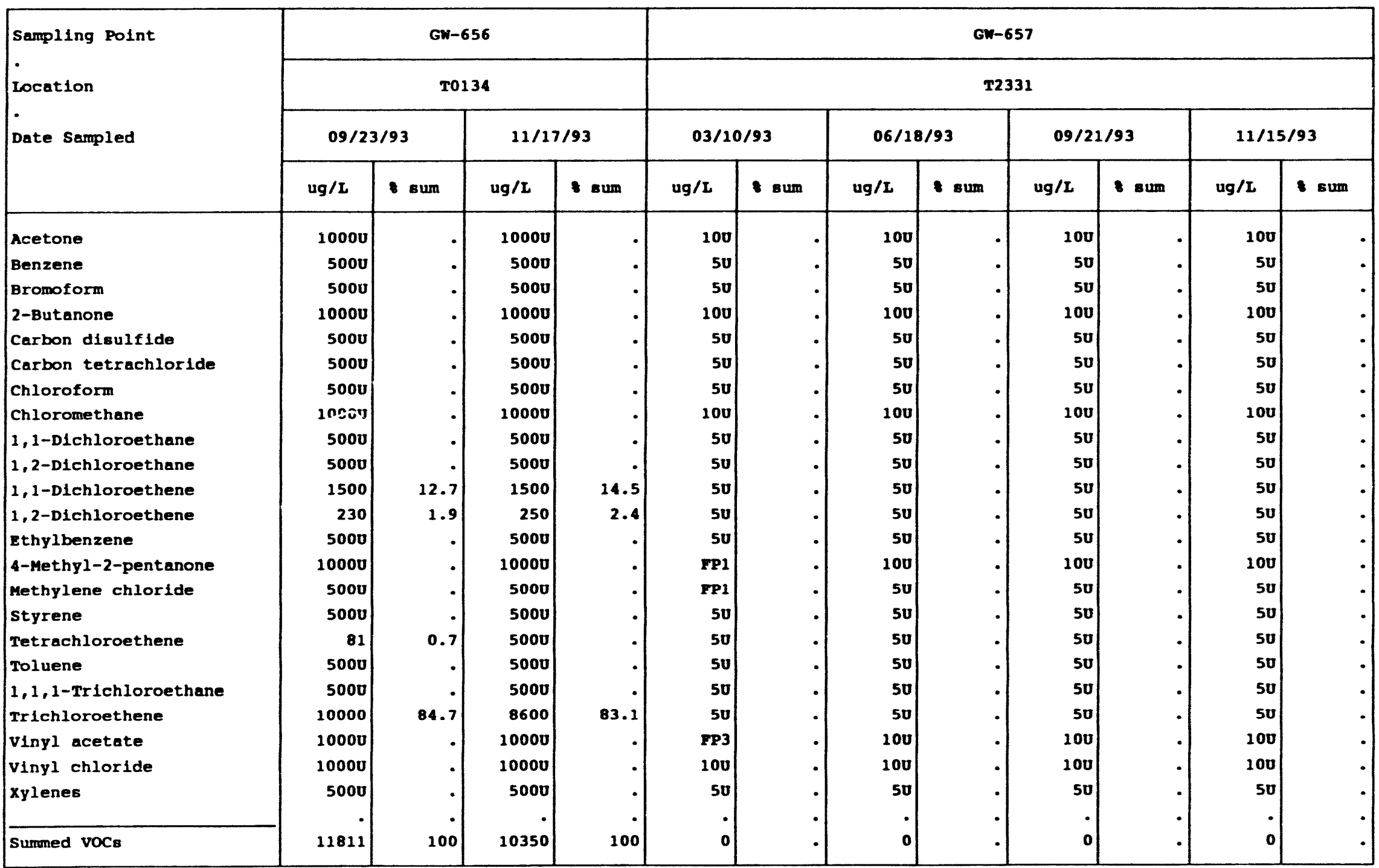

(CONTINUED) 
APPERDIX $J$

Valldated VOC Results, 1993

\begin{tabular}{|c|c|c|c|c|c|c|c|c|c|c|c|c|}
\hline \multirow{4}{*}{$\begin{array}{l}\text { Sampling Polnt } \\
\text { Location } \\
\text { Date Sampled }\end{array}$} & \multicolumn{8}{|c|}{$G W-658$} & \multicolumn{4}{|c|}{ GW-659 } \\
\hline & \multicolumn{8}{|c|}{$\mathbf{F F}$} & \multicolumn{4}{|c|}{$\mathbf{F F}$} \\
\hline & \multicolumn{2}{|c|}{$03 / 11 / 93$} & \multicolumn{2}{|c|}{$06 / 23 / 93$} & \multicolumn{2}{|c|}{$11 / 19 / 93$} & \multicolumn{2}{|c|}{$09 / 27 / 04$} & \multicolumn{2}{|c|}{$03 / 10 / 93$} & \multicolumn{2}{|c|}{$06 / 18 / 93$} \\
\hline & $u g / t$ & s sum & ug/L & sum & ug/L & 8 sum & ug/L & sum & ug/L & sum & ug/L & ร $64 \mathrm{~mm}$ \\
\hline Acetone & 10000 & - & 10000 & $\cdot$ & 5000 & - & 10000 & - & FP3 & - & 100 & - \\
\hline Benzene & 10000 & 41.2 & 13000 & 43.3 & 4800 & 36 & 14000 & 42 & 11 & 78.6 & 27 & 75 \\
\hline Bromoform & 5000 & - & 5000 & - & 2500 & - & 5000 & - & 50 & - & 5u & - \\
\hline 2-Butanone & 10000 & . & 10000 & - & 5000 & - & 10000 & - & 100 & - & 100 & - \\
\hline Carbon disulfide & 5000 & $\cdot$ & 5000 & $\cdot$ & 2500 & - & 5000 & - & 50 & $\cdot$ & so & - \\
\hline Carbon tetrachloride & 5000 & $\cdot$ & 5000 & $\cdot$ & $250 u$ & - & 5000 & - & 50 & $\cdot$ & 50 & • \\
\hline Chloroform & 5000 & $\cdot$ & 5000 & $\cdot$ & 2500 & $\cdot$ & 5000 & - & 50 & $\cdot$ & so & • \\
\hline Chloromethane & 10000 & $\cdot$ & 10000 & $\cdot$ & 5000 & - & 10000 & - & 100 & $\cdot$ & 100 & • \\
\hline 1,1-Dichloroethane & 5000 & $\cdot$ & 5000 & $\cdot$ & 2500 & - & 5000 & - & 50 & $\cdot$ & so & - \\
\hline 1,2-Dichloroethane & 560 & 2.3 & 790 & 2.6 & 370 & 2.8 & 1000 & 3 & $5 v$ & $\cdot$ & 50 & $\cdot$ \\
\hline 1,1-Dichloroethene & 5000 & - & 5000 & - & 2500 & $\cdot$ & 5000 & - & 50 & - & 50 & - \\
\hline 1,2-Dichloroethene & 5000 & $\cdot$ & 5000 & $\cdot$ & 2500 & $\cdot$ & 5000 & • & 50 & $\cdot$ & 50 & - \\
\hline Ethylbenzene & 1100 & 4.5 & 1400 & 4.7 & 760 & 5.7 & 1500 & 4.5 & 2 & 14.3 & 5u & • \\
\hline 4-Methy1-2-pentanone & 10000 & $\cdot$ & 10000 & $\cdot$ & 5000 & - & 10000 & - & FP1 & - & 100 & - \\
\hline Methylene chloride & 120 & 0.5 & 5000 & $\cdot$ & 2500 & - & 5000 & - & FP1 & - & 50 & - \\
\hline Styrene & 5000 & $\cdot$ & 5000 & $\cdot$ & 2500 & - & 5000 & - & 50 & $\cdot$ & 50 & - \\
\hline Tetrachloroethene & 5000 & $\cdot$ & 5000 & $\cdot$ & 2500 & $\cdot$ & 5000 & $\cdot$ & 50 & $\cdot$ & 5u & • \\
\hline Toluene & 5700 & 23.5 & 8400 & 28 & 3400 & 25.5 & 9300 & 27.9 & 50 & - & 50 & • \\
\hline $1,1,1$-Trichloroethane & 5000 & - & 5000 & $\cdot$ & 2500 & - & 5000 & - & 50 & $\cdot$ & 50 & $\cdot$ \\
\hline Trichloroethene & 5000 & - & 5000 & $\cdot$ & 2500 & - & 5000 & - & 50 & - & so & • \\
\hline vinyl acetate & 10000 & $\cdot$ & 10000 & $\cdot$ & 5000 & - & 10000 & - & 100 & - & 100 & • \\
\hline Vinyl chloride & 10000 & $\cdot$ & 10000 &. & 5000 & - & 10000 & - & 100 & - & 100 & - \\
\hline xylenes & 6800 & 28 & 6400 & 21.3 & 4000 & 30 & 7500 & 22.5 & 1 & 7.1 & 9 & 25 \\
\hline Summed VOCB & 24280 & 100 & 29990 & 99.9 & 13330 & 100 & 33300 & 99.9 & 14 & 100 & 36 & 100 \\
\hline
\end{tabular}

(CONTINUED) 
APPENDIX $J$

Valldated voC Results, 1993

\begin{tabular}{|c|c|c|c|c|c|c|c|c|c|c|c|c|}
\hline \multirow{4}{*}{$\begin{array}{l}\text { Sampling Point } \\
\text { Location } \\
\text { Date Sampled }\end{array}$} & \multicolumn{4}{|c|}{ GW-659 } & \multicolumn{8}{|c|}{ Gw-707 } \\
\hline & \multicolumn{4}{|c|}{$\mathbf{F r}$} & \multicolumn{8}{|c|}{$\mathbf{T 2 3 3 1}$} \\
\hline & \multicolumn{2}{|c|}{$09 / 22 / 93$} & \multicolumn{2}{|c|}{$11 / 15 / 93$} & \multicolumn{2}{|c|}{$03 / 11 / 93$} & \multicolumn{2}{|c|}{$06 / 23 / 93$} & \multicolumn{2}{|c|}{$09 / 27 / 93$} & \multicolumn{2}{|c|}{$11 / 18 / 93$} \\
\hline & ug/L & sum & ug/I & * eum & $u g / L$ & - sum & ug/L & sum & $\operatorname{ug} / \mathrm{L}$ & t sum & $\mathbf{u g} / \mathbf{L}$ & $5 \operatorname{sun}$ \\
\hline Acetone & 100 & - & 100 & $\cdot$ & 100 & - & 100 & - & 100 & - & 100 & - \\
\hline Benzene & 42 & 66.7 & 2 & 25 & 50 & - & 50 & - & 50 & - & 50 & • \\
\hline Bromoform & 50 & $\cdot$ & 50 & $\cdot$ & 50 & - & 50 & $\cdot$ & 5v & - & 50 & - \\
\hline 2-Butanone & 100 & . & 100 & $\cdot$ & 100 & - & 100 & $\cdot$ & 100 & . & 100 & • \\
\hline Carbon disulfide & 50 & $\cdot$ & 5u & $\cdot$ & 50 & - & su & $\cdot$ & 5v & - & 50 & • \\
\hline Carbon tetrachloride & 50 & - & 50 & $\cdot$ & 50 & - & 5v & $\cdot$ & 5v & $\cdot$ & 50 & • \\
\hline Chloroform & 50 & $\cdot$ & 50 & $\cdot$ & 50 & - & 50 & $\cdot$ & 5v & - & 50 & • \\
\hline Chloromethane & 100 & • & 100 & $\cdot$ & 100 & - & 100 & $\cdot$ & 100 & $\cdot$ & 100 & • \\
\hline 1,1-Dichloroethane & 5v & $\cdot$ & 50 & $\cdot$ & 50 & • & 50 & $\cdot$ & su & $\cdot$ & 50 & $\cdot$ \\
\hline 1,2-Dichloroethane & 5u & $\cdot$ & 50 & $\cdot$ & 50 & - & 50 & $\cdot$ & $5 v$ & $\cdot$ & 50 & $\cdot$ \\
\hline 1,1-Dichloroethene & 5v & $\cdot$ & 50 & $\cdot$ & 50 & - & 50 & $\cdot$ & 5v & $\cdot$ & 50 & - \\
\hline 1,2-Dichloroethene & 50 & $\cdot$ & 50 & - & 5v & - & 5v & $\cdot$ & $5 v$ & $\cdot$ & 50 & - \\
\hline Ethylbenzene & 4 & 6.3 & $5 v$ & $\cdot$ & 5v & - & 50 & $\cdot$ & 50 & . & 50 & - \\
\hline 4-Methyl-2-pentanone & 100 & • & 100 & $\cdot$ & 100 & - & 100 & $\cdot$ & 100 & - & 200 & • \\
\hline Methylene chloride & 5v & • & 50 & $\cdot$ & 50 & - & 50 & $\cdot$ & 50 & • & $\mathbf{5 v}$ & • \\
\hline styrene & 50 & $\cdot$ & 50 & • & 50 & - & 50 & $\cdot$ & 50 & $\cdot$ & so & • \\
\hline Tetrachloroethene & 50 & $\cdot$ & 50 & $\cdot$ & 50 & - & $5 \mathbf{v}$ & $\cdot$ & 50 & $\cdot$ & 50 & - \\
\hline Toluene & 50 & $\cdot$ & 5u & $\cdot$ & 50 & - & 50 & $\cdot$ & 50 & $\cdot$ & 50 & $\cdot$ \\
\hline 1,1,1-Trichloroethane & 50 & $\cdot$ & 5v & $\cdot$ & 50 & - & so & $\cdot$ & su & - & 50 & • \\
\hline Trichloroethene & 50 & $\cdot$ & 50 & $\cdot$ & FP3 & - & sv & $\cdot$ & 50 & $\cdot$ & 50 & • \\
\hline vinyl acetate & 100 & $\cdot$ & 100 & $\cdot$ & 100 & - & 100 & $\cdot$ & 100 & - & 100 & $\cdot$ \\
\hline vinyl chloride & 100 & $\cdot$ & 100 & $\cdot$ & 100 & - & 100 & $\cdot$ & 100 & $\cdot$ & 100 & . \\
\hline xylenes & 17 & 27 & 6 & 75 & 50 & - & 50 & $\cdot$ & 50 & - & 50 & • \\
\hline Summed vocs & 63 & 100 & 8 & 100 & 0 & - & 0 & . & 0 & . & 0 & . \\
\hline
\end{tabular}

(CONTIMUED) 
APPEADIX $J$

Valldated voc Resulta, 1993

\begin{tabular}{|c|c|c|c|c|c|c|c|c|c|c|c|c|}
\hline \multirow{4}{*}{$\begin{array}{l}\text { Sampling Point } \\
\text { Location } \\
\text { Date Sampled }\end{array}$} & \multicolumn{8}{|c|}{ GW-708 } & \multicolumn{4}{|c|}{$64-733$} \\
\hline & \multicolumn{8}{|c|}{$\mathbf{T 2 3 3 1}$} & \multicolumn{4}{|c|}{$\mathbf{2 x P}$} \\
\hline & \multicolumn{2}{|c|}{$03 / 10 / 93$} & \multicolumn{2}{|c|}{$06 / 18 / 93$} & \multicolumn{2}{|c|}{$09 / 21 / 93$} & \multicolumn{2}{|c|}{$11 / 12 / 93$} & \multicolumn{2}{|c|}{$02 / 02 / 93$} & \multicolumn{2}{|c|}{$05 / 06 / 93$} \\
\hline & ug/L & s sum & $u g / L$ & sum & $u g / L$ & - cum & $u g / L$ & t sum & ug/L & $1 \sin$ & $\operatorname{ug} / \mathrm{x}$ & $\operatorname{sen}$ \\
\hline Acetone & 2000 & • & 2000 & & 2000 & - & 2000 & - & 100 & - & 100 & - \\
\hline Benzene & 870 & 22.1 & 1200 & 26.4 & 1100 & 25.3 & 780 & 23.1 & 50 & - & 50 & - \\
\hline Bromoform & 1000 & $\cdot$ & 1000 & $\cdot$ & 1000 & $\cdot$ & 1000 & $\cdot$ & 50 & - & so & - \\
\hline 2-Butanone & 2000 & • & 2000 & $\cdot$ & 2000 & $\cdot$ & 2000 & - & 100 & - & 100 & - \\
\hline Carbon disulfide & 1000 & $\cdot$ & 1000 & $\cdot$ & 1000 & $\cdot 1$ & 1000 & - & so & • & 50 & • \\
\hline Carbon tetrachloride & 1000 & $\cdot$ & 1000 & $\cdot$ & 1000 & $\cdot$ & 1000 & $\cdot$ & 60 & 84.6 & 61 & 89.7 \\
\hline Chloroform & 1000 & $\cdot$ & 1000 & $\cdot$ & 1000 & $\cdot$ & 1000 & $\cdot$ & e & 11.3 & 5 & 7.4 \\
\hline Chloromethane & 2000 & $\cdot$ & 2000 & $\cdot$ & 2000 & - & 2000 & $\cdot$ & 100 & • & 100 & • \\
\hline 1,1-Dichloroethane & 1000 & - & 1000 & $\cdot$ & 1000 & $\cdot$ & 1000 & $\cdot$ & 50 & - & so & - \\
\hline 1,2-Dichloroethane & 1000 & $\cdot$ & 1000 & $\cdot$ & 1000 & - & 1000 & - & 50 & - & 50 & - \\
\hline 1,1-Dichloroethene & 2000 & $\cdot$ & 1000 &. & 1000 & $\cdot$ & 1000 & • & so & - & 50 & - \\
\hline 1.2-Dichloroethene & 1000 & $\cdot$ & 1000 & $\cdot 1$ & 1000 &. & 1000 & $\cdot$ & 50 & - & 50 & - \\
\hline Ethylbenzene & 300 & 7.6 & 620 & 13.7 & 720 & 16.6 & 670 & 19.8 & su & - & 50 & - \\
\hline 4-Methy1-2-pentanone & 57 & 1.4 & 2000 & $\cdot$ & 2000 & $\cdot$ & 2000 & • & 100 & - & $\mathbf{F P 1}$ & - \\
\hline Methylene chloride & 63 & 1.6 & 1000 & $\cdot$ & 1000 & $\cdot$ & 32 & 0.9 & 50 & - & FP1 & - \\
\hline Styrene & 1000 & $\cdot$ & 1000 & $\cdot$ & 1000 & $\cdot$ & 1000 & • & so & - & 50 & • \\
\hline Tetrachloroethene & 1000 & $\cdot$ & 1000 & $\cdot$ & 1000 & $\cdot$ & 1000 & $\cdot$ & 2 & 2.8 & 2 & 2.9 \\
\hline Toluene & 150 & 3.8 & 120 & 2.6 & 120 & 2.8 & 98 & 2.9 & 50 & $\cdot$ & 50 & - \\
\hline 1,1,1-Trichloroethane & 1000 & $\cdot$ & 1000 & $\cdot$ & 1000 & $\cdot$ & 1000 & $\cdot$ & 50 & $\cdot$ & 50 & - \\
\hline Trichloroethene & 1000 & $\cdot$ & 1000 & $\cdot$ & 2000 & $\cdot$ & 1000 & $\cdot$ & 0.9 & 1.3 & 50 & - \\
\hline vingl acetate & 2000 &. & 2000 & $\cdot 1$ & 2000 & $\cdot$ & 2000 & - & 100 & $\cdot$ & 100 & - \\
\hline vinyl chloride & 2000 & . & 2000 &. & 2000 & $\cdot$ & 2000 & $\cdot$ & 100 & $\cdot$ & 100 & - \\
\hline Xylenes & 2500 & 63.5 & 2600 & 57.3 & 2400 & 55.3 & 1800 & 53.3 & 50 & - & so & - \\
\hline Summed vocs & 3940 & 200 & 4540 & 100 & 4340 & 100 & 3380 & 100 & 70.9 & 100 & 68 & 100 \\
\hline
\end{tabular}

(CONTIMUED) 
APPEDDIX J

Valldated voc Reaulte, 1993

\begin{tabular}{|c|c|c|c|c|c|c|c|c|c|c|c|c|}
\hline \multirow{4}{*}{$\begin{array}{l}\text { Sampling Point } \\
\text { Location } \\
\text { Date Sampled }\end{array}$} & \multicolumn{4}{|c|}{ GW-733 } & \multicolumn{8}{|c|}{ GN-735 } \\
\hline & \multicolumn{4}{|c|}{$\exp$} & \multicolumn{8}{|c|}{$\mathbf{m P}$} \\
\hline & \multicolumn{2}{|c|}{$08 / 18 / 93$} & \multicolumn{2}{|c|}{$11 / 04 / 93$} & \multicolumn{2}{|c|}{$02 / 02 / 93$} & \multicolumn{2}{|c|}{$05 / 07 / 93$} & \multicolumn{2}{|c|}{$08 / 18 / 93$} & \multicolumn{2}{|c|}{$10 / 29 / 93$} \\
\hline & $\mathbf{u g} / \mathrm{L}$ & sum & ug/L & - sum & $u g / L$ & -1 & $u g / L$ & c sum & ug/L & sen & $\operatorname{ug} / 2$ & cos \\
\hline Acetone & 100 & - & 100 & - & 100 & - & 100 & .1 & 100 & - & 100 & - \\
\hline Benzene & so & - & 50 & - & $\mathbf{5 0}$ & - & 50 & $\cdot$ & 50 & - & 50 & - \\
\hline Bromoform & so & - & 50 & - & 50 & - & so &. & sv & . & 50 & - \\
\hline 2-Butanone & 100 & - & 100 & - & 100 & - & 100 & $\cdot$ & 100 & - & 100 & • \\
\hline Carbon disulfide & 50 & - & so & • & so & - & so & $\cdot 1$ & 50 & - & so & • \\
\hline Carbon tetrachloride & 72 & 88.9 & 87 & 88.8 & 50 & - & 50 & $\cdot$ & so & $\cdot$ & 50 & • \\
\hline Chloroform & 6 & 7.4 & 7 & 7.1 & 50 & - & so &. & so & - & 50 & • \\
\hline Chloromethane & 100 & • & 100 & • & 100 & - & 100 &. & 100 & $\cdot$ & 100 & - \\
\hline 1,1-Dichloroethane & 50 & . & 50 & . & 50 & - & 50 &. & so & . & 50 & • \\
\hline 1,2-Dichloroethane & 50 & - & 50 & - & 50 & - & 50 & $\cdot$ & so & $\cdot$ & 50 & - \\
\hline 1,1-Dichloroethene & 50 & $\cdot$ & 50 & . & 50 & - & 50 &. & 5v &. & 50 & • \\
\hline 1,2-Dichloroethene & 50 & $\cdot$ & 50 & $\cdot$ & 50 & - & so & $\cdot$ & 50 & . & so & . \\
\hline Ethylbenzene & sv &. & 5v & $\cdot$ & 5v & . & so & $\cdot$ & 50 & $\cdot$ & 50 & • \\
\hline 4-Methy1-2-pentanone & 100 & $\cdot$ & 100 & $\cdot$ & 100 & - & 100 & $\cdot 1$ & 100 & $\cdot$ & 100 & • \\
\hline Methylene chloride & FP1 & $\cdot$ & $\mathbf{F P 1}$ & $\cdot$ & 5v & - & FP3 &. & so & $\cdot$ & FP1 & - \\
\hline Styrene & 50 &. & 5v &. & 50 & - & so & $\cdot$ & so & . & 50 & - \\
\hline Tetrachloroethene & 2 & 2.5 & 3 & 3.1 & 5v & - & 50 & $\cdot 1$ & so & $\cdot$ & so & • \\
\hline Toluene & $\mathbf{s u}$ &. & 50 & $\cdot$ & 50 & - & 50 & $\cdot$ & 50 & . & 50 & - \\
\hline 1,1,1-Trichloroethane & so & $\cdot$ & so &. & 50 & - & so &. & 50 & - & so & • \\
\hline Trichloroethene & 1 & 1.2 & 1 & 1 & 50 & - & 50 &. & 50 & $\cdot$ & 50 & • \\
\hline Vinyl acetate & 100 & $\cdot$ & 100 & $\cdot$ & 100 & - & 100 &. & 100 & $\cdot$ & 100 & . \\
\hline Vinyl chloride & 100 &. & 100 & $\cdot$ & 100 & - & 100 & - & 100 & $\cdot$ & 100 & . \\
\hline xylenes & 50 & $\cdot$ & 5v & $\cdot$ & so & - & so & - & so & $\cdot$ & so & - \\
\hline Sunmed vocs & 81 & 100 & 98 & 100 & 0 & - & 0 & - & 0 & - & o) & . \\
\hline
\end{tabular}

(CONTIMUED) 
Valldated voc Reeulte, 1993

\begin{tabular}{|c|c|c|c|c|c|c|c|c|c|c|c|c|}
\hline \multirow{4}{*}{$\begin{array}{l}\text { Sampling Point } \\
\text { Location } \\
\text { Date Sampled }\end{array}$} & \multicolumn{8}{|c|}{ 6n-744 } & \multicolumn{4}{|c|}{$G x-745$} \\
\hline & \multicolumn{8}{|c|}{ GRIDx1 } & \multicolumn{4}{|c|}{ GRIDRI } \\
\hline & \multicolumn{2}{|c|}{$01 / 18 / 93$} & \multicolumn{2}{|c|}{$04 / 12 / 93$} & \multicolumn{2}{|c|}{$08 / 04 / 93$} & \multicolumn{2}{|c|}{$10 / 22 / 93$} & \multicolumn{2}{|c|}{$01 / 19 / 93$} & \multicolumn{2}{|c|}{$04 / 12 / 93$} \\
\hline & $\mathbf{u g} / \mathbf{L}$ & E sum & $u g / L$ & $2 \sin$ & ug/l & - en & ng/L & - sum & $u g / L$ & > & $\operatorname{ug} / \mathrm{x}$ & sem \\
\hline Acetone & 100 & - & 100 & - & 100 & . & IPI & - & 100 & . & 100 & - \\
\hline Benzene & 50 & - & so & - & so & - & so & - & 50 & - & 50 & - \\
\hline Bromoform & 50 & - & so & - & 5v & $\cdot$ & 50 & - & so & - & 50 & - \\
\hline 2-Butanone & 100 & - & 100 & - & 100 & .1 & $\mathbf{R P 1}$ & - & 100 & $\cdot$ & 100 & - \\
\hline Carbon disulfide & so & - & so & - & sv &. & $\mathbf{5 0}$ & - & so & $\cdot$ & so & - \\
\hline Carbon tetrachloride & 50 & - & 50 & - & so & . & so & - & so & . & so & - \\
\hline Chloroform & 50 & - & so & - & 50 & $\cdot$ & 50 & - & so & $\cdot$ & so & - \\
\hline Chloromethane & 100 & - & 100 & - & 100 &. & 100 & - & 100 & - & 100 & - \\
\hline 1,1-Dichloroethane & 5v & - & 50 & - & 50 & $\cdot$ & so & - & 50 & $\cdot$ & 50 & - \\
\hline 1,2-Dichloroethane & so & - & So & - & 5v &. & so & - & so & $\cdot$ & 50 & - \\
\hline 1,1-Dichloroethene & 50 & - & 50 & - & so & $\cdot$ & 50 & - & so & . & 50 & - \\
\hline 1,2-Dichloroethene & su & - & 50 & - & 50 & . & so & - & so & - & so & - \\
\hline Ethylbenzene & so & - & sv & - & so & $\cdot$ & 50 & - & so & - & so & - \\
\hline 4-methy1-2-pentanone & 100 & - & 100 & - & 100 & $\cdot$ & 100 & - & $\mathbf{P P 1}$ & $\cdot$ & 100 & • \\
\hline Methylene chloride & $\mathbf{r p 3}$ & - & 50 & - & 50 & $\cdot$ & $\mathbf{F P 1}$ & - & 3 & 100 & 0.9 & 100 \\
\hline Styrene & so & - & 50 & - & so & $\cdot$ & 50 & - & so & $\cdot$ & so & • \\
\hline Tetrachloroethene & 50 & - & 50 & - & so & $\cdot$ & so & - & so & $\cdot$ & so & - \\
\hline Toluene & 5v & - & 50 & - & 50 & $\cdot$ & 50 & $\cdot$ & 50 & $\cdot$ & so & - \\
\hline 1,1,1-Trichloroethane & 50 & - & 50 & - & so & $\cdot$ & 50 & - & 50 & $\cdot$ & so & - \\
\hline Irichloroethene & 50 & - & so & - & so & $\cdot$ & 50 & • & so & $\cdot$ & 50 & - \\
\hline Vinyl acetate & 100 & - & 100 & - & 100 &. & 100 & - & 100 & $\cdot$ & 100 & - \\
\hline vingl chloride & 100 & - & 100 & - & 100 & $\cdot$ & 100 & - & 100 &. & 100 & - \\
\hline xylenes & 50 & - & so & - & 50 & . & 50 & . & 50 &. & 50 & - \\
\hline Summed VOCB & 0 & . & 0 & . & 0 & . & 0 & . & 3 & 100 & 0.9 & 100 \\
\hline
\end{tabular}

(CONTIMUED) 
APPADDIX $J$

valldeted voc meeulte, 1993

\begin{tabular}{|c|c|c|c|c|c|c|c|c|c|c|c|c|}
\hline \multirow{4}{*}{$\begin{array}{l}\text { Sampling Polnt } \\
\text { Location } \\
\text { Date Sampled }\end{array}$} & \multicolumn{4}{|c|}{ Gw-745 } & \multicolumn{8}{|c|}{$6-746$} \\
\hline & \multicolumn{4}{|c|}{ GRIDK1 } & \multicolumn{8}{|c|}{ enrox } \\
\hline & \multicolumn{2}{|c|}{$08 / 04 / 93$} & \multicolumn{2}{|c|}{$10 / 23 / 93$} & \multicolumn{2}{|c|}{$01 / 19 / 93$} & \multicolumn{2}{|c|}{$04 / 12 / 93$} & \multicolumn{2}{|c|}{$08 / 05 / 93$} & \multicolumn{2}{|c|}{ 10/23/93 } \\
\hline & $\operatorname{ug} / 2$ & - sum & $\operatorname{ug} / 2$ & 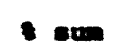 & $\mathrm{ug} / \mathrm{z}$ & com & $\operatorname{ug} / 2$ & - sum & $\operatorname{ug} / 2$ & 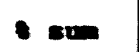 & $\operatorname{ug} / 2$ & 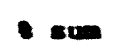 \\
\hline Acetone & 100 & . & 100 & - & 100 & - & 200 & - & 100 & - & 100 & • \\
\hline Benzene & so & - & 50 & - & so & - & 50 & $\cdot$ & so & - & so & - \\
\hline Bromoform & 50 & $\cdot$ & so & - & 5o & - & so & - & so & - & so & - \\
\hline 2-Butanone & 100 &. & 100 & - & 100 & $\cdot$ & 100 & - & 100 & - & 100 & • \\
\hline Carbon disulfide & so & $\cdot$ & 50 & - & 50 & - & so & - & so & - & 50 & - \\
\hline Carbon tetrachloride & so & $\cdot$ & 50 & - & so & $\cdot$ & so & - & so & - & so & • \\
\hline Chloroform & 50 & $\cdot$ & 50 & - & 50 & . & 50 & - & 50 & - & so & • \\
\hline Chloromethane & 100 &. & 100 & - & 100 & $\cdot$ & 100 & - & 100 & $\cdot$ & 100 & • \\
\hline 1,1-Dichloroethane & so & $\cdot$ & so & - & 50 &. & so & - & 50 & - & so & • \\
\hline 1,2-Dichloroethane & so &. & so & - & so & - & so & - & 50 & - & so & • \\
\hline 1,1-Dichloroethene & 50 &. & so & - & 50 & . & so & - & 50 & - & $5 \mathbf{0}$ & • \\
\hline 1,2-Dichloroethene & so &. & so & - & 50 & . & so & - & 50 & - & so & • \\
\hline Ethylbenzene & so &. & so & - & 50 &. & so & - & so & - & so & - \\
\hline 4-methyl-2-pentanone & 100 &. & 100 & - & 100 & $\cdot$ & 100 & • & 100 & - & 100 & • \\
\hline Methylene chloride & 50 &. & FP1 & - & so &. & $\mathbf{F P 3}$ & - & so & - & FP1 & • \\
\hline styrene & 50 &. & 50 & $\cdot$ & 50 &. & 50 & - & so & - & so & • \\
\hline Tetrechloroethene & 50 &. & 50 & - & 50 &. & so & - & 50 & - & so & • \\
\hline Toluene & so & .1 & 50 & - & 50 & $\cdot$ & so & $\cdot$ & so & - & so & • \\
\hline $1,1,1$-Trichloroethane & 50 & $\cdot$ & so & - & so & $\cdot$ & so & - & so & - & 50 & • \\
\hline Trichloroethene & 50 &. & 50 & - & 50 & $\cdot$ & so & $\cdot$ & so & . & so & - \\
\hline Vinyl acetate & 100 & $\cdot$ & 100 & - & 100 & $\cdot$ & 100 & - & 100 & $\cdot$ & 100 & - \\
\hline vinyl chloride & 100 &. & 100 & - & 100 & $\cdot 1$ & 100 & - & 100 & - & 100 & - \\
\hline xylenes & 50 & .1 & so & • & so & $\cdot$ & 50 & - & 50 & $\cdot$ & so & - \\
\hline Surmed vocs & &. & o) & . & 0 & . & . & . & 0 & . & 0 & - \\
\hline
\end{tabular}

(CONTIMUED) 
APPADDIX J

Valldated voc nesulte, 1993

\begin{tabular}{|c|c|c|c|c|c|c|c|c|c|c|c|c|}
\hline \multirow{4}{*}{$\begin{array}{l}\text { Sampling Point } \\
\text { Location } \\
\text { Date Sampled }\end{array}$} & \multicolumn{8}{|c|}{$6 w-747$} & \multicolumn{4}{|c|}{$60-748$} \\
\hline & \multicolumn{8}{|c|}{ GRIDI2 } & \multicolumn{4}{|c|}{ GRIDIR } \\
\hline & \multicolumn{2}{|c|}{$01 / 19 / 93$} & \multicolumn{2}{|c|}{$04 / 13 / 93$} & \multicolumn{2}{|c|}{$08 / 05 / 93$} & \multicolumn{2}{|c|}{$10 / 23 / 93$} & \multicolumn{2}{|c|}{$01 / 20 / 93$} & \multicolumn{2}{|c|}{$04 / 13 / 93$} \\
\hline & $u g / 2$ & $\rightarrow \sin$ & $u g / L$ & : & $\operatorname{ug} / 2$ & - sem & $\operatorname{ug} / 2$ & $-\infty$ & $\operatorname{ug} / \mathrm{L}$ & s. & $\operatorname{ug} / \mathrm{L}$ & som \\
\hline Acetone & 100 & - & 100 & - & 100 & - & 100 & • & PP1 & - & 100 & . \\
\hline Benzene & 50 & $\cdot 1$ & 50 & $\cdot$ & 50 & - & so & - & 50 & . & so & . \\
\hline Bronoform & 50 & - & 50 & - & 50 & - & 50 & - & 50 & - & so & ra \\
\hline 2-Butanone & 100 & - & 100 & - & 100 & - & 100 & - & 100 & - & 100 & ra \\
\hline Carbon disulfide & su &. & 50 & - & 50 & - & 50 & - & 50 & - & so & rata \\
\hline Carbon tetrachloride & 50 & - & so & - & 50 & - & 50 & - & so & - & so & ra \\
\hline Chloroform & so & - & 50 & $\cdot$ & 50 & - & so & - & 50 & $\cdot$ & so & r \\
\hline Chloromethane & 100 & . & 100 & $\cdot$ & 100 & & 100 & - & 100 & - & 100 & ra \\
\hline 1,1-Dichloroethane & so & $\cdot$ & 50 & $\cdot$ & so & - & so & - & so & $\cdot$ & sv & . \\
\hline 1,2-Dichloroethane & 50 & . & so & $\cdot$ & so & - & so & - & so & - & so & . \\
\hline 1,1-Dichloroethene & so &. & so &. & so & - & so & - & so & - & so & r \\
\hline 1,2-Dichloroethene & so & $\cdot$ & so & $\cdot$ & so & - & so & - & su & - & so & 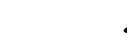 \\
\hline Ethylbenzene & 50 &. & so & . & 50 & - & so & - & so & - & so & 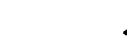 \\
\hline 4-Hethy1-2-pentanone & FP1 & $\cdot$ & 100 & $\cdot$ & 100 & - & 100 & - & $\mathbf{F} \mathbf{1}$ & - & 100 & . \\
\hline Methylene chloride & 50 &. & 50 & . & so & - & FP1 & - & so & . & so & . \\
\hline Styrene & so & $\cdot 1$ & 50 & $\cdot$ & so & - & 50 & - & 50 & $\cdot$ & so & 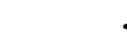 \\
\hline Tetrachloroethene & 5v &. & 50 & - & 50 & - & 50 & - & so & $\cdot$ & so & . \\
\hline Toluene & so & $\cdot 1$ & 50 &. & 50 & - & so & - & 50 & $\cdot$ & 50 & . \\
\hline $1,1,1$-Trichloroethane & so &. & 50 &. & 50 & - & so & - & so & $\cdot$ & 50 & . \\
\hline Trlchloroethene & 50 & $\cdot 1$ & 50 & $\cdot$ & 50 & - & 50 & - & so & $\cdot$ & 50 & . \\
\hline Vinyl acetate & 100 &. & 100 & $\cdot$ & 100 & - & 100 & - & 100 & - & 100 & . \\
\hline vinyl chloride & 100 &. & 100 & - & 100 & - & 100 & - & 100 & - & 100 & . \\
\hline xylenes & 50 & - & so & - & 50 & - & 50 & - & so & - & 50 & . \\
\hline Summed VOCs & 0 & . & 0 & - & 0 & . & o) & - & 0 & . & 0 & . \\
\hline
\end{tabular}

(COArInUED) 
APPEADIX $J$

valldated voc Reaulta, 1993

\begin{tabular}{|c|c|c|c|c|c|c|c|c|c|c|c|c|}
\hline \multirow{4}{*}{$\begin{array}{l}\text { Sampling Point } \\
\text { Location } \\
\text { Date Sampled }\end{array}$} & \multicolumn{4}{|c|}{ Gw-748 } & \multicolumn{8}{|c|}{$6 w-749$} \\
\hline & \multicolumn{4}{|c|}{ GRIDK2 } & \multicolumn{8}{|c|}{ GRIDI2 } \\
\hline & \multicolumn{2}{|c|}{$08 / 06 / 93$} & \multicolumn{2}{|c|}{$10 / 24 / 93$} & \multicolumn{2}{|c|}{$01 / 21 / 93$} & \multicolumn{2}{|c|}{$04 / 14 / 93$} & \multicolumn{2}{|c|}{$08 / 06 / 93$} & \multicolumn{2}{|c|}{$19 / 24 / 93$} \\
\hline & $u g / x$ & sum & ug/L & sum & $u g / L$ & s. & $u g / t$ & - & $u g / L$ & sam & $\mathrm{ug} / \mathrm{L}$ & s sw \\
\hline Acetone & 100 & $\cdot$ & 100 & • & 100 & - & 100 & - & 100 & - & 100 & • \\
\hline Benzene & so & - & so & - & so & - & so & - & so & - & 50 & . \\
\hline Bromoform & 50 & $\cdot$ & 50 & $\cdot$ & 50 & - & 50 & . & so & $\cdot$ & so & . \\
\hline 2-Butanone & 100 & $\cdot$ & 100 & - & 100 & - & 100 & $\cdot$ & 100 & $\cdot$ & 100 & • \\
\hline Carbon disulfide & 50 & - & 50 & - & so & - & so & $\cdot$ & so & $\cdot$ & 50 & • \\
\hline Carbon tetrachloride & 50 & $\cdot$ & 50 & - & 50 & - & 50 & - & 50 & $\cdot$ & 50 & . \\
\hline chloroform & sv & $\cdot$ & 50 & - & so & $\cdot$ & so & $\cdot$ & 5v & $\cdot$ & so & • \\
\hline Chloromethane & 100 & . & 100 & - & 100 & - & 100 & - & 100 & - & 100 & • \\
\hline 1,1-Dichloroethane & so & $\cdot$ & so & - & so & $\cdot$ & so & $\cdot$ & 50 & $\cdot$ & 50 & • \\
\hline 1,2-Dichloroethane & so & $\cdot$ & su & $\cdot$ & su & $\cdot$ & so & - & su & $\cdot$ & so & • \\
\hline 1,1-Dichloroethene & 5v & $\cdot$ & 50 & • & so & - & 50 & $\cdot$ & 50 & - & so & - \\
\hline 1,2-Dichloroethene & sol & $\cdot$ & su & $\cdot$ & so & $\cdot$ & so & $\cdot$ & sv & $\cdot$ & so & • \\
\hline Ethylbenzene & su & $\cdot$ & 50 & • & 50 & $\cdot$ & 50 & $\cdot$ & 5v & $\cdot$ & 50 & • \\
\hline 4-Methyl-2-pentanone & 100 & $\cdot$ & 100 & - & 1001 & $\cdot$ & 100 & $\cdot$ & 100 & - & 100 & • \\
\hline Hethylene chloride & so & $\cdot$ & $\boldsymbol{F P 1}$ & $\cdot$ & so & $\cdot$ & so & $\cdot$ & 50 & $\cdot$ & $\mathbf{F P 1}$ & • \\
\hline Styrene & 50 & $\cdot$ & sv & $\cdot$ & 50 & $\cdot$ & so & $\cdot$ & 50 & $\cdot$ & 50 & • \\
\hline Tetrachloroethene & so & $\cdot$ & su & $\cdot$ & so & $\cdot$ & so & $\cdot$ & 50 & $\cdot$ & so & • \\
\hline Toluene & 50) & $\cdot 1$ & 50 &. & 50) & $\cdot$ & 50 & - & 50 & $\cdot$ & 50 & • \\
\hline 1,1,1-Trichloroethane & su & $\cdot$ & 50 & $\cdot$ & so & $\cdot$ & so & - & so & $\cdot$ & 50 & • \\
\hline Trichloroethene & so & $\cdot$ & 50 & $\cdot$ & 50 & $\cdot$ & so & - & so & $\cdot$ & so & • \\
\hline vinyl acetote & 100 & $\cdot$ & 100 & $\cdot$ & 100 & $\cdot$ & 100 & $\cdot$ & 100 & $\cdot$ & 100 & • \\
\hline vinyl chloride & 100 & . & 100 & $\cdot$ & 100 &. & 100 & - & 100 & $\cdot$ & 100 & - \\
\hline xylenes & 50 & $\cdot$ & 50 & $\cdot$ & so & $\cdot$ & 50 & $\cdot$ & sv & $\cdot$ & 50 & • \\
\hline Sumed VOCs & 0 & . & 0 & . & 0 & . & 0 & - & 0 & $\cdot$ & 0 & - \\
\hline
\end{tabular}

(CONTIMUED) 
APPENDIX J

Valldated Voc Results, 1993

\begin{tabular}{|c|c|c|c|c|c|c|c|c|c|c|c|c|}
\hline \multirow{4}{*}{$\begin{array}{l}\text { Sampling Polnt } \\
\text { Location } \\
\text { Date Sampled }\end{array}$} & \multicolumn{8}{|c|}{$G N-751$} & \multicolumn{4}{|c|}{ GW-752 } \\
\hline & \multicolumn{8}{|c|}{ GRIDJ3 } & \multicolumn{4}{|c|}{ GRIDJ3 } \\
\hline & \multicolumn{2}{|c|}{$01 / 11 / 93$} & \multicolumn{2}{|c|}{$04 / 16 / 93$} & \multicolumn{2}{|c|}{$08 / 02 / 93$} & \multicolumn{2}{|c|}{$10 / 26 / 93$} & \multicolumn{2}{|c|}{$01 / 11 / 93$} & \multicolumn{2}{|c|}{$04 / 16 / 93$} \\
\hline & ug/L & sum & ug/L & sum & ug/l & sum & ug/L & \& $\operatorname{sum}$ & $\mathbf{u g} / \mathbf{L}$ & c sum & $\mathrm{ug} / \mathrm{L}$ & sum \\
\hline Acetone & 100 & . & 100 & . & 100 & . & 100 & - & FP3 & . & 100 & . \\
\hline Benzene & 5u &. & 50 & $\cdot$ & su & $\cdot$ & 50 & • & 50 & $\cdot$ & 50 & . \\
\hline Bromoform & 50 & - & 50 & $\cdot 1$ & 5u &. & 50 & • & 50 & $\cdot$ & 50 & . \\
\hline 2-Butanone & 100 & - & 100 & $\cdot$ & 100 &. & 100 & - & 100 & $\cdot$ & 100 & . \\
\hline Carbon disulfide & 5u &. & 50 & $\cdot 1$ & 50 & $\cdot$ & su & - & 50 & $\cdot$ & su & . \\
\hline Carbon tetrachloride & 5u & $\cdot$ & 50 & $\cdot 1$ & 50 & $\cdot$ & 50 & - & 50 & • & 50 & . \\
\hline Chloroform & 50 & $\cdot$ & 50 & $\cdot$ & su & $\cdot$ & 50 & - & 50 & $\cdot$ & 50 & . \\
\hline Chloromethane & 100 & - & 100 & $\cdot$ & 100 & $\cdot 1$ & 100 & - & 100 & $\cdot$ & 100 & . \\
\hline 1,1-Dichloroethane & 50 &. & su & $\cdot$ & 50 & $\cdot$ & 5u & - & 50 & $\cdot$ & 50 & . \\
\hline 1,2-Dichloroethane & 5u & - & 50 & $\cdot$ & 5v &. & 50 & • & 50 & • & so & . \\
\hline 1,1-Dichloroethene & 50 & $\cdot$ & 50 & $\cdot$ & 5u & $\cdot$ & 50 & - & 50 & $\cdot$ & 50 & . \\
\hline 1,2-Dichloroethene & 50 &. & 5u & $\cdot$ & 50 & $\cdot$ & 50 & • & so & $\cdot$ & 5u & . \\
\hline zthylbenzene & 50 &. & 50 & $\cdot$ & 50 & $\cdot$ & 50 & • & 50 & . & 50 & . \\
\hline 4-Methy1-2-pentanone & 100 & - & 100 & $\cdot$ & 100 & $\cdot$ & 100 & - & 100 & $\cdot$ & 100 & . \\
\hline Methylene chloride & 50 & $\cdot$ & FP3 & $\cdot$ & 5v & $\cdot$ & 50 & - & 50 & $\cdot$ & 50 & . \\
\hline Styrene & 50 & $\cdot$ & 50 & $\cdot$ & 50 & $\cdot$ & 50 & - & 50 & $\cdot$ & 50 & . \\
\hline Tetrachloroethene & 50 & $\cdot$ & 50 & $\cdot$ & 50 & $\cdot$ & 50 & - & 50 & $\cdot$ & 50 & . \\
\hline Toluene & 50 & $\cdot$ & 50 & $\cdot 1$ & 50 & $\cdot$ & 50 & - & 50 & $\cdot$ & 50 & . \\
\hline $1,1,1$-Trichloroethane & 50 & . & 50 & $\cdot$ & 50 & $\cdot$ & 50 & - & 50 & - & 50 & . \\
\hline Trichloroethene & 50 & $\cdot$ & 50 & $\cdot$ & so & $\cdot$ & 50 & - & 50 & $\cdot$ & so & . \\
\hline vinyl acetate & 100 & $\cdot$ & 100 & $\cdot$ & 100 & $\cdot$ & 100 & - & 100 & - & 100 & 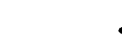 \\
\hline vinyl chloride & 100 &. & 100 & $\cdot 1$ & 100 & $\cdot$ & 100 & • & 100 & $\cdot$ & 100 & . \\
\hline xylenes & 50 &. & 50 &. & 5v & $\cdot 1$ & 5u & - & 50 & $\cdot$ & 5u & ra \\
\hline Sumned vocs & 0 &. & 0 &. & 0 & . & 0 & - & 0 & . & 0 & . \\
\hline
\end{tabular}

(CONTINUED) 
APPENDIX $J$

Valldated voc Results, 1993

\begin{tabular}{|c|c|c|c|c|c|c|c|c|c|c|c|c|}
\hline \multirow{4}{*}{$\begin{array}{l}\text { Sampling Polnt } \\
\text { Location } \\
\text { - } \\
\text { Date Sampled }\end{array}$} & \multicolumn{4}{|c|}{ GW-752 } & \multicolumn{8}{|c|}{ GW-753 } \\
\hline & \multicolumn{4}{|c|}{ GRIDJ3 } & \multicolumn{8}{|c|}{ GRIDJ2 } \\
\hline & \multicolumn{2}{|c|}{$08 / 02 / 93$} & \multicolumn{2}{|c|}{$10 / 26 / 93$} & \multicolumn{2}{|c|}{$01 / 15 / 93$} & \multicolumn{2}{|c|}{$04 / 08 / 93$} & \multicolumn{2}{|c|}{$08 / 03 / 93$} & \multicolumn{2}{|c|}{$10 / 21 / 93$} \\
\hline & ug/L & sum & ug/L & - sum & $u g / L$ & sum & $u g / L$ & sum & $\mathrm{ug} / \mathrm{t}$ & sum & $\operatorname{ug} / \mathrm{L}$ & s sum \\
\hline Acetone & 100 & $\cdot$ & 100 & $\cdot$ & 100 & $\cdot$ & 100 & $\cdot$ & 100 & $\cdot$ & 100 & - \\
\hline Benzene & 5v & $\cdot$ & 50 & $\cdot$ & su & $\cdot$ & 50 & $\cdot$ & 50 & $\cdot$ & 5u & . \\
\hline Bromoform & 5u & $\cdot$ & su & $\cdot$ & 50 & $\cdot$ & su & $\cdot$ & 50 & $\cdot$ & 50 & • \\
\hline 2-Butanone & 100 & $\cdot$ & 100 & $\cdot$ & 100 & $\cdot$ & 100 & $\cdot$ & 100 &. & 100 & • \\
\hline Carbon disulfide & 50 & $\cdot$ & 50 & $\cdot$ & 50 & $\cdot$ & 50 & $\cdot$ & 50 & $\cdot$ & 50 & • \\
\hline Carbon tetrachloride & 50 &. & 50 & $\cdot$ & so & $\cdot$ & 50 & $\cdot$ & 50 & $\cdot$ & su & • \\
\hline Chloroform & 50 & $\cdot$ & 50 & $\cdot$ & 50 & $\cdot$ & su & $\cdot$ & 5v & . & 50 & • \\
\hline Chloromethane & 100 & $\cdot$ & 100 & $\cdot$ & 100 & $\cdot$ & 100 & $\cdot$ & 100 & $\cdot$ & 100 & • \\
\hline 1,1-Dichloroethane & 5v & $\cdot$ & 50 & $\cdot$ & 50 & $\cdot$ & 50 & $\cdot$ & 50 & $\cdot$ & 50 & - \\
\hline 1,2-Dichloroethane & 50 & $\cdot$ & 50 & $\cdot$ & 50 & $\cdot$ & 50 & $\cdot$ & 50 & $\cdot$ & 50 & • \\
\hline 1,1-D1chloroethene & 50 & $\cdot$ & 50 & $\cdot$ & 50 & $\cdot$ & 50 & $\cdot$ & 50 & $\cdot$ & 50 & • \\
\hline 1,2-Dichloroethene & 50 & $\cdot$ & 50 & $\cdot$ & 50 &. & 50 & $\cdot$ & 50 & $\cdot 1$ & 50 & • \\
\hline Ethylbenzene & 50 & $\cdot$ & 50 & $\cdot$ & su & $\cdot$ & 50 & $\cdot$ & 50 & $\cdot$ & 50 & • \\
\hline 4-Methyl-2-pentanone & 100 & $\cdot$ & 100 & $\cdot$ & 100 & $\cdot$ & 100 & $\cdot$ & 100 & $\cdot$ & 100 & • \\
\hline Methylene chloride & 5u & $\cdot$ & 50 & $\cdot$ & sv & $\cdot$ & FP3 & $\cdot$ & 50 & $\cdot$ & so & . \\
\hline Styrene & 50 & $\cdot$ & 50 &. & 5u & $\cdot$ & 5u & $\cdot$ & 50 & $\cdot$ & so & - \\
\hline Tetrachloroethene & 5u & $\cdot$ & 5v & $\cdot$ & 50 & $\cdot$ & 50 & $\cdot$ & 50 & $\cdot$ & 50 & - \\
\hline Toluene & 50 & $\cdot$ & 50 & $\cdot$ & 5u & $\cdot$ & 5u & $\cdot$ & 50 & $\cdot$ & 50 & - \\
\hline 1,1,1-Trichloroethane & 5v & $\cdot$ & 50 & $\cdot$ & 50 & $\cdot$ & 50 & $\cdot$ & 50 & $\cdot$ & 50 & • \\
\hline Trichloroethene & 50 & $\cdot$ & 50 & $\cdot$ & su & $\cdot$ & 5u & $\cdot$ & 5u & $\cdot$ & 5u & - \\
\hline vinyl acetate & 100 & $\cdot$ & 100 & $\cdot$ & 100 & $\cdot$ & 100 & $\cdot$ & 100 & $\cdot$ & 100 & - \\
\hline vinyl chloride & 100 & $\cdot$ & 100 & $\cdot$ & 100 & $\cdot$ & 100 & $\cdot$ & 100 & $\cdot$ & 100 & - \\
\hline xylenes & 5v & $\cdot$ & 50 & $\cdot$ & 50 & $\cdot$ & 50 & $\cdot$ & 5u & $\cdot$ & 50 & • \\
\hline Summed vOCs & 0 & . & 0 & $\cdot$ & 0 &. & 0 &. & 0 &. & 0 & . \\
\hline
\end{tabular}

(CONTINUED) 
APPENDIX $J$

Valldated VOC Results, 1993

\begin{tabular}{|c|c|c|c|c|c|c|c|c|c|c|c|c|}
\hline \multirow{4}{*}{$\begin{array}{l}\text { Sampling Polnt } \\
\text { Location } \\
\text { - } \\
\text { Date Sampled }\end{array}$} & \multicolumn{8}{|c|}{ GW-754 } & \multicolumn{4}{|c|}{ GW-755 } \\
\hline & \multicolumn{8}{|c|}{ GRIDJ2 } & \multicolumn{4}{|c|}{ GRIDJI } \\
\hline & \multicolumn{2}{|c|}{$01 / 18 / 93$} & \multicolumn{2}{|c|}{$04 / 08 / 93$} & \multicolumn{2}{|c|}{$08 / 04 / 93$} & \multicolumn{2}{|c|}{$10 / 22 / 93$} & \multicolumn{2}{|c|}{$01 / 14 / 93$} & \multicolumn{2}{|c|}{$04 / 07 / 93$} \\
\hline & ug/L & Eum & $u g / L$ & sum & $u g / L$ & sum & ug/L & sum & ug/L & $8 \mathrm{sum}$ & ug/L & 8 sum \\
\hline Acetone & 100 & - & 100 & - & 100 & - & FP1 & - & 100 & - & 100 & ( \\
\hline Benzene & 5u & - & 50 & - & 5u & - & 50 & - & 50 & . & 5u & 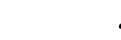 \\
\hline Bromoform & 50 & - & 50 & - & 50 & - & 5u & - & 5u & - & 50 & se \\
\hline 2-Butanone & 100 & - & 100 & - & 100 & - & FP1 & - & 100 & - & 100 & ra \\
\hline Carbon disulfide & $5 v$ & $\cdot$ & 50 & - & su & - & 5v & - & 50 & - & 5u & rat \\
\hline Carbon tetrachloride & 50 & $\cdot$ & 50 & - & 50 & - & 50 & - & 5v & - & 50 & ris \\
\hline Chloroform & $5 \mathbf{v}$ & - & 50 & - & 50 & - & 50 & - & 50 & - & 50 & . \\
\hline Chloromethane & 100 & $\cdot$ & 100 & - & 100 & - & 100 & - & 100 & • & 100 & ra \\
\hline 1,1-Dichloroethane & 5u & • & 50 & - & 50 & - & 50 & - & 50 & - & 50 & ra \\
\hline 1,2-Dichloroethane & 50 & • & 50 & - & 50 & - & 50 & - & 5u & - & 50 & r \\
\hline 1,1-Dichloroethene & 50 & • & 50 & - & 50 & - & 50 & - & 5u & - & 50 & sina \\
\hline 1,2-Dichloroethene & 50 & $\cdot$ & 5v & - & 50 & - & 50 & - & 50 & - & 50 & rata \\
\hline zthylbenzene & 50 & $\cdot$ & 50 & - & 50 & - & 5u & - & 50 & - & 50 & sina \\
\hline 4-Methyl-2-pentanone & 100 & $\cdot$ & 100 & - & 100 & - & 100 & - & FP1 & - & 100 & sina \\
\hline Methylene chloride & 50 & - & 50 & - & 5u & - & FP1 & - & 50 & • & 50 & ra \\
\hline Styrene & 50 & $\cdot$ & 5u & - & 50 & $\cdot$ & 5u & - & 50 & - & 50 & rat \\
\hline Tetrachloroethene & 5u & • & 50 & - & 50 & - & 50 & - & 50 & - & 50 & ra \\
\hline Toluene & 5u & $\cdot$ & 50 & - & 5u & $\cdot$ & 50 & - & 50 & - & 50 & r \\
\hline 1,1,1-Trichloroethane & 50 & $\cdot$ & so & - & 50 & - & 50 & $\cdot$ & 5u & - & 50 & r \\
\hline Trichloroethene & 50 & $\cdot$ & 5u & - & 50 & - & 50 & - & 50 & - & 50 & r \\
\hline vinyl acetate & 100 & $\cdot$ & 100 & - & 100 & • & 100 & - & 100 & - & 100 & ra \\
\hline vinyl chloride & 100 & $\cdot$ & 100 & - & 100 & $\cdot$ & 100 & - & 100 & - & 100 & rata \\
\hline xylenes & 5u & $\cdot$ & 5u & - & 50 & • & 50 & - & 5u & - & 50 & rat \\
\hline Summed VOCs & 0 & . & $\mathbf{0}$ & - & 0 & - & 0 & - & 0 & - & 0 & ra \\
\hline
\end{tabular}

(CONTINUED) 
APPENDIX $J$

Validated VOC Results, 1993

\begin{tabular}{|c|c|c|c|c|c|c|c|c|c|c|c|c|c|}
\hline \multirow{4}{*}{$\begin{array}{l}\text { Sampling Point } \\
\text { Location } \\
\text { Date Sampled }\end{array}$} & \multicolumn{4}{|c|}{ GW-755 } & \multicolumn{9}{|c|}{ GW-756 } \\
\hline & \multicolumn{4}{|c|}{ GRIDJI } & \multicolumn{9}{|c|}{ GRIDJ1 } \\
\hline & \multicolumn{2}{|c|}{$08 / 03 / 93$} & \multicolumn{2}{|c|}{$10 / 21 / 93$} & \multicolumn{2}{|c|}{$01 / 15 / 93$} & \multicolumn{2}{|c|}{$04 / 08 / 93$} & \multicolumn{2}{|c|}{$08 / 03 / 93$} & \multicolumn{3}{|c|}{$10 / 21 / 93$} \\
\hline & $u g / L$ & sum & ug/L & sum & $\mathrm{ug} / \mathrm{L}$ & 8 sum & $u g / L$ & sum & $\mathrm{ug} / \mathrm{L}$ & 8 sum & $u g / L$ & 8 sum & \\
\hline Acetone & 100 & . & 100 & - & 100 & . & 100 & - & 100 & . & FP1 & & - \\
\hline Benzene & 50 & - & 5v & • & 50 & $\cdot$ & 50 & - & 50 & $\cdot$ & 50 & & - \\
\hline Bromoform & 5v & - & so & • & su & $\cdot$ & su & - & su & - & 50 & & - \\
\hline 2-Butanone & 100 & . & 100 & - & 100 & - & 100 & - & 100 & - & FP1 & & - \\
\hline Carbon disulfide & so & - & 5v & - & so & • & 50 & - & 50 & - & 50 & & - \\
\hline Carbon tetrachloride & 5u & - & 5v & • & 5u & - & 50 & - & 50 & - & 50 & & - \\
\hline Chloroform & so & $\cdot$ & 5v & - & 50 & $\cdot$ & 50 & - & $\mathbf{5 u}$ & - & 50 & & - \\
\hline Chloromethane & 100 & - & 100 & - & 100 & $\cdot$ & 100 & $\cdot$ & 100 & - & 100 & & - \\
\hline 1,1-Dichloroethane & 50 & - & 5u & - & 5u & • & 50 & - & 5u & - & 50 & & - \\
\hline 1,2-Dichloroethane & 50 & - & 50 & $\cdot$ & 5v & $\cdot$ & 50 & $\cdot$ & 50 & $\cdot$ & su & & - \\
\hline 1,1-Dichloroethene & 50 & - & 5v & $\cdot$ & 50 & - & 50 & . & 50 & - & 50 & & • \\
\hline 1,2-Dichloroethene & 5u & $\cdot$ & sv & - & 5u & $\cdot$ & 50 & $\cdot$ & 50 & $\cdot$ & 5u & & - \\
\hline Ethyibenzene & su & - & 50 & - & 50 & $\cdot$ & $5 \mathbf{v}$ & - & $5 v$ & $\cdot$ & 50 & & - \\
\hline 4-Methy1-2-pentanone & 100 & - & 100 & $\cdot$ & 100 & $\cdot$ & 100 & • & 100 & $\cdot$ & 100 & & - \\
\hline Methylene chloride & 50 & - & 50 & $\cdot$ & 50 & - & 50 & - & 50 & $\cdot$ & FP1 & & • \\
\hline Styrene & su & - & 5v & $\cdot$ & so & $\cdot$ & $5 \mathbf{v}$ & - & 50 & $\cdot$ & 50 & & - \\
\hline Tetrachloroethene & 50 & - & 50 & - & 50 & • & 5u & - & 50 & $\cdot$ & 50 & & - \\
\hline Toluene & 50 & . & 50 & $\cdot$ & $5 v$ & $\cdot$ & 50 & $\cdot$ & 50 & $\cdot$ & 50 & & - \\
\hline 1,1,1-Trichloroethane & $5 \mathbf{v}$ & - & $5 \mathbf{v}$ & - & $5 \mathbf{u}$ & - & 5u & $\cdot$ & 50 & $\cdot$ & su & & - \\
\hline Trichloroethene & 5u & $\cdot$ & 50 & - & 50 & $\cdot$ & 50 & $\cdot$ & 50 & $\cdot$ & 50 & & - \\
\hline vinyl acetate & 100 & - & 100 & - & 100 & $\cdot$ & 100 & $\cdot$ & 100 & $\cdot$ & 100 & & - \\
\hline vingl chloride & 100 & $\cdot$ & 100 & - & 100 & $\cdot$ & 100 & - & 100 & - & 100 & & - \\
\hline xylenes & 50 & - & 5v & . & $5 v$ & • & 50 & • & 50 & . & 50 & & - \\
\hline Surmed vocs & $\dot{0}$ & $\cdot$ & 0 & • & 0 & - & 0 & . & $\dot{0}$ & $\cdot$ & $\dot{0}$ & & • \\
\hline
\end{tabular}

(CONTINURD) 
APPEXTDIX $J$

Validated voc Results, 1993

\begin{tabular}{|c|c|c|c|c|c|c|c|c|c|c|c|c|}
\hline \multirow{4}{*}{$\begin{array}{l}\text { Sampling Point } \\
\text { Location } \\
\text { - } \\
\text { Date Sampled }\end{array}$} & \multicolumn{8}{|c|}{ GW-758 } & \multicolumn{4}{|c|}{ GW-762 } \\
\hline & \multicolumn{8}{|c|}{ GRIDGI } & \multicolumn{4}{|c|}{ GRIDJ3 } \\
\hline & \multicolumn{2}{|c|}{$01 / 25 / 93$} & \multicolumn{2}{|c|}{$04 / 13 / 93$} & \multicolumn{2}{|c|}{$09 / 12 / 93$} & \multicolumn{2}{|c|}{$11 / 19 / 93$} & \multicolumn{2}{|c|}{$02 / 22 / 93$} & \multicolumn{2}{|c|}{$04 / 20 / 93$} \\
\hline & ug/L & s sum & ug/L & sum & $u g / L$ & s sum & ug/L & 8 sum & ug/L & s sum & ug/L & 8 sum \\
\hline Acetone & FP? & $\cdot$ & 100 & $\cdot 1$ & 100 & $\cdot$ & 100 & - & 1000 & - & 1000 & - \\
\hline Benzene & 5u| & • & 50 & $\cdot$ & 5u & $\cdot 1$ & 50 & $\cdot$ & 500 & $\cdot$ & 500 & • \\
\hline Bromoform & 50 & $\cdot$ & 50 & $\cdot$ & 50 & $\cdot$ & 5u & • & $50 \mathrm{v}$ & $\cdot$ & 500 & • \\
\hline 2-Butanone & 100 & - & 100 & $\cdot$ & 100 & $\cdot$ & 100 & - & 1000 & $\cdot$ & 1000 & • \\
\hline Carbon disulfide & 50 & $\cdot$ & so & $\cdot$ & 50 & $\cdot$ & 50 & $\cdot$ & 500 & $\cdot$ & 500 & • \\
\hline Carbon tetrachloride & 50 & $\cdot$ & 50 & $\cdot$ & 5u & $\cdot$ & 50 & $\cdot$ & 500 & $\cdot$ & 500 & • \\
\hline Chloroform & 50 & $\cdot$ & so & $\cdot$ & $5 \mathbf{5 u}$ & $\cdot$ & 50 & $\cdot$ & 500 & $\cdot$ & 500 & • \\
\hline Chloromethane & 100 & $\cdot$ & 100 & $\cdot$ & 100 & $\cdot 1$ & 100 & $\cdot$ & 1000 & $\cdot$ & 1000 & - \\
\hline 1,1-Dichloroethane & 5u & $\cdot$ & 5u & $\cdot$ & 50 & $\cdot$ & 50 & $\cdot$ & 500 & $\cdot$ & 500 & • \\
\hline 1,2-Dichloroethane & su & $\cdot$ & 50 & $\cdot$ & su & $\cdot$ & 50 & $\cdot$ & 500 & $\cdot$ & 500 & • \\
\hline 1,1-Dichloroethene & 5u & $\cdot$ & 50 & $\cdot$ & 50 & $\cdot$ & 50 & $\cdot$ & 12 & 1.2 & 500 & • \\
\hline 1,2-Dichloroethene & su & $\cdot$ & 5u & $\cdot$ & 50 & $\cdot$ & 50 & $\cdot$ & 85 & 8.2 & 500 & • \\
\hline Ethylbenzene & 50 & $\cdot$ & 5v & $\cdot$ & 50 & $\cdot$ & 50 & $\cdot$ & 500 & $\cdot$ & 500 & • \\
\hline 4-Methy1-2-pentanone & 100 & $\cdot$ & 100 & $\cdot$ & 100 & $\cdot$ & 100 & $\cdot$ & 1000 & $\cdot$ & 24 & 2.2 \\
\hline Methylene chloride & 50 & $\cdot$ & 50 & $\cdot$ & FP3 & $\cdot$ & 50 & $\cdot$ & 500 & $\cdot$ & 11 & 1 \\
\hline Styrene & 5v & $\cdot$ & su & $\cdot$ & 50 & $\cdot$ & 50 & $\cdot$ & 500 & $\cdot$ & 500 & • \\
\hline Tetrachloroethene & 50 & $\cdot$ & 50 & $\cdot$ & 50 & $\cdot$ & 50 & $\cdot$ & 900 & 87 & 1000 & 92.5 \\
\hline Toluene & 50 & $\cdot$ & 50 & $\cdot$ & 5u & $\cdot$ & 50 & $\cdot$ & 500 & $\cdot$ & 500 & • \\
\hline $1,1,1$-Trichloroethane & 50 & $\cdot$ & 50 & $\cdot$ & su & $\cdot$ & 50 & $\cdot$ & 500 & $\cdot$ & 500 & • \\
\hline Trichloroethene & 50 & $\cdot$ & 50 & $\cdot$ & 50 & $\cdot$ & 50 & $\cdot$ & 38 & 3.7 & 46 & 4.3 \\
\hline vinyl acetate & 100 & $\cdot$ & 100 & $\cdot$ & 100 & $\cdot$ & 100 & $\cdot$ & 1000 & $\cdot$ & 1000 & • \\
\hline vinyl chloride & 100 & $\cdot$ & 100 & $\cdot$ & 100 & $\cdot$ & 100 & $\cdot$ & 1000 & $\cdot$ & 1000 & • \\
\hline Xylenes & 50 & $\cdot$ & 50 & $\cdot$ & 50 & $\cdot$ & 50 & $\cdot$ & 500 & $\cdot$ & 500 & • \\
\hline Summed VOCB & 0 & . & 0 & . & 0 & . & 0 & . & 1035 & 100.1 & 1081 & 100 \\
\hline
\end{tabular}

(CONTINUED) 
APPENDIX J

Validated vOC Results, 1993

\begin{tabular}{|c|c|c|c|c|c|c|c|c|c|c|c|c|}
\hline \multirow{4}{*}{$\begin{array}{l}\text { Sampling Point } \\
\text { Location } \\
\text { Date Sampled }\end{array}$} & \multicolumn{4}{|c|}{ GW-762 } & \multicolumn{8}{|c|}{ GW-763 } \\
\hline & \multicolumn{4}{|c|}{ GRIDJ3 } & \multicolumn{8}{|c|}{ GRIDJ3 } \\
\hline & \multicolumn{2}{|c|}{$08 / 04 / 93$} & \multicolumn{2}{|c|}{$10 / 28 / 93$} & \multicolumn{2}{|c|}{$01 / 25 / 93$} & \multicolumn{2}{|c|}{$04 / 21 / 93$} & \multicolumn{2}{|c|}{$08 / 05 / 93$} & \multicolumn{2}{|c|}{$10 / 29 / 93$} \\
\hline & ug/L. & sum & ug/L & sum & $u g / x$ & sum & $\operatorname{ug} / \mathrm{L}$ & 8 sum & $\operatorname{ug} / \mathrm{L}$ & 8 sum & $\mathrm{ug} / \mathrm{L}$ & sum \\
\hline Acetone & 1000 & $\cdot$ & 1000 & - & 100 & - & 100 & • & 100 & - & 100 & 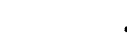 \\
\hline Benzene & 500 & - & 500 & - & 50 & - & 50 & - & 50 & - & 50 & . \\
\hline Bromoform & 500 & - & 500 & $\cdot$ & 50 & • & 5u & $\cdot$ & $5 \mathbf{v}$ & - & 50 & r \\
\hline 2-Butanone & 1000 & - & 1000 & - & 100 & - & 100 & $\cdot$ & 100 & - & 100 & . \\
\hline Cerbon disulfide & 500 & $\cdot$ & 500 & $\cdot$ & 50 & $\cdot$ & 50 & $\cdot$ & 50 & - & 50 & r \\
\hline Carbon tetrachloride & 500 & $\cdot$ & 500 & $\cdot$ & so & $\cdot$ & $5 v$ & $\cdot$ & 5v & - & 50 & r \\
\hline Chloroform & 500 & $\cdot$ & 500 & $\cdot$ & 50 & $\cdot$ & 50 & $\cdot$ & 50 & - & 50 & . \\
\hline Chloromethane & 1000 & $\cdot$ & 1000 & $\cdot$ & 100 & $\cdot$ & 100 & - & 100 & - & 100 & . \\
\hline 1,1-Dichloroethane & 500 & $\cdot$ & 500 & $\cdot$ & 50 & $\cdot$ & 50 & $\cdot$ & 50 & - & 50 & . \\
\hline 1,2-Dichloroethane & 500 & $\cdot$ & 500 & $\cdot$ & 50 & $\cdot$ & 50 & $\cdot$ & so & - & 50 & . \\
\hline 1,1-Dichloroethene & 500 & $\cdot$ & 500 & $\cdot$ & 50 & $\cdot$ & 50 & • & 50 & • & FP3 & . \\
\hline 1,2-Dichloroethene & 71 & 7.2 & 78 & 7 & 59 & 51.8 & 42 & 50.6 & 98 & 55.4 & 120 & 52.5 \\
\hline Ethylbenzene & 500 & $\cdot$ & 500 & $\cdot$ & 5u & $\cdot$ & 50 & • & 50 & • & 50 & r \\
\hline 4-Methy1-2-pentanone & 1000 & . & 1000 & $\cdot$ & 100 & - & 100 & $\cdot$ & 100 & • & 100 & . \\
\hline Methylene chloride & 500 & - & 500 & $\cdot$ & $5 \mathbf{5}$ & $\cdot$ & 5u & • & 50 & - & FP1 & . \\
\hline Styrene & 500 & $\cdot$ & 500 & • & 50 & $\cdot$ & 5v & . & 50 & • & 5v & r \\
\hline Tetrachloroethene & 920 & 92.8 & 1000 & 89.1 & 8 & 7 & 5 & 6 & 27 & 15.3 & 41 & 18.1 \\
\hline Toluene & 500 & $\cdot$ & 500 & $\cdot$ & 50 & $\cdot$ & 50 & - & 50 & • & 50 & r \\
\hline $1,1,1-\operatorname{Tr}$ ichloroethane & 500 & - & 500 & $\cdot$ & 50 & $\cdot$ & 5u & $\cdot$ & 50 & • & su & . \\
\hline Trichloroethene & 500 & $\cdot$ & 44 & 3.9 & 2 & 1.8 & 1 & 1.2 & 4 & 2.3 & 7 & 3.1 \\
\hline vinyl acetate & 1000 & $\cdot$ & 1000 & $\bullet$ & 100 & $\cdot$ & 100 & $\cdot$ & 100 & $\cdot$ & 100 & . \\
\hline vinyl chloride & 1000 & $\cdot$ & 1000 & $\cdot$ & 45 & 39.5 & 35 & 42.2 & 48 & 27.1 & 59 & 26 \\
\hline xylenes & 500 & $\cdot$ & 500 & . & 50 & $\cdot$ & 50 & • & 50 & • & 50 & . \\
\hline Summed voCs & 991 & 100 & 1122 & 100 & 114 & 100.1 & 83 & 100 & 177 & 100.1 & 227 & 100.1 \\
\hline
\end{tabular}

(CONTINUED) 
APPERDIX $J$

Validated voC Results, 1993

\begin{tabular}{|c|c|c|c|c|c|c|c|c|c|c|c|c|}
\hline \multirow{4}{*}{$\begin{array}{l}\text { Sampling Point } \\
\text { Location } \\
\text { Date Sampled }\end{array}$} & \multicolumn{8}{|c|}{ GN-764 } & \multicolumn{4}{|c|}{ GW-765 } \\
\hline & \multicolumn{8}{|c|}{ GRIDE 1} & \multicolumn{4}{|c|}{ GRIDE 1} \\
\hline & \multicolumn{2}{|c|}{$01 / 23 / 93$} & \multicolumn{2}{|c|}{$04 / 13 / 93$} & \multicolumn{2}{|c|}{$09 / 12 / 93$} & \multicolumn{2}{|c|}{$11 / 12 / 93$} & \multicolumn{2}{|c|}{$01 / 25 / 93$} & \multicolumn{2}{|c|}{$04 / 13 / 93$} \\
\hline & ug/L & sum & $\mathrm{ug} / \mathrm{L}$ & sum & ug/L & sum & ug/L & sum & ug/L & sum & ug/L & 8 sum \\
\hline Acetone & 100 & $\cdot$ & 100 & - & 100 & - & 100 & - & 100 & - & 100 & - \\
\hline Benzene & 5u & $\cdot$ & 50 & • & 5v & $\cdot$ & $\mathbf{5 0}$ & - & 50 & $\cdot$ & 50 & • \\
\hline Bromoform & 50 & $\cdot$ & su & $\cdot$ & 50 & $\cdot$ & 50 & - & so & $\cdot$ & 50 & • \\
\hline 2-Butanone & 100 & $\cdot 1$ & 100 & $\cdot$ & 100 & $\cdot$ & 100 & - & 100 & $\cdot$ & 100 & • \\
\hline Carbon disulfide & 50 & $\cdot$ & 5u & $\cdot$ & so & $\cdot$ & 50 & - & 50 & - & 50 & • \\
\hline Carbon tetrachloride & 50 & $\cdot$ & 50 & $\cdot$ & 50 & $\cdot$ & 50 & - & 5v & $\cdot$ & 50 & • \\
\hline Chloroform & 50 & $\cdot$ & 50 & $\cdot$ & 50 &. & 50 & - & 50 & - & 50 & • \\
\hline Chloromethane & 100 & $\cdot$ & 100 & $\cdot$ & 100 & $\cdot$ & 100 & - & 100 & $\cdot$ & 100 & • \\
\hline 1,1-Dichloroethane & 50 & $\cdot$ & 5v & $\cdot$ & 5v & $\cdot$ & $5 \mathbf{5}$ & - & 5v & - & 50 & • \\
\hline 1,2-Dichloroethane & 50 & $\cdot$ & 5v & $\cdot$ & su & $\cdot$ & 50 & - & sv & - & 50 & • \\
\hline 1,1-Dichloroethene & 50 & $\cdot$ & 5v & $\cdot$ & 50 & • & 50 & $\cdot$ & su & $\cdot$ & 50 & - \\
\hline 1,2-Dichloroethene & 5u & $\cdot$ & 5u & $\cdot$ & 50 & - & 50 & - & 50 & - & 5u & • \\
\hline Ethylbenzene & 50 & $\cdot$ & so & $\cdot$ & 50 & $\cdot$ & su & $\cdot$ & 50 & $\cdot$ & 50 & - \\
\hline 4-Methy1-2-pentanone & 100 & $\cdot$ & 100 & $\cdot$ & 100 & $\cdot$ & 100 & . & 100 & - & 100 & • \\
\hline Methylene chloride & 5u & $\cdot$ & FP3 & $\cdot$ & 50 & • & sv & $\cdot$ & 50 & • & 50 & • \\
\hline Styrene & 50 & $\cdot$ & 50 & $\cdot$ & 50 & $\cdot$ & 50 & - & 50 & $\cdot$ & 50 & - \\
\hline Tetrachloroethene & 50 & $\cdot$ & 50 & • & 50 & $\cdot$ & $5 v$ & - & so & $\cdot$ & 50 & • \\
\hline Toluene & 50 & $\cdot$ & 50 & $\cdot$ & 50 & $\cdot$ & su & • & so & • & 50 & • \\
\hline 1,1,1-Trichloroethane & 50 & $\cdot$ & 5v & $\cdot$ & 50 & $\cdot$ & 50 & $\cdot$ & 50 & $\cdot$ & 50 & • \\
\hline Trichloroethene & 50 & $\cdot$ & 50 & $\cdot$ & 50 & $\cdot$ & 50 & • & 50 & $\cdot$ & 50 & - \\
\hline Vinyl acetate & 100 & $\cdot$ & 100 & $\cdot$ & 100 & $\cdot$ & 100 & $\cdot$ & 100 & $\cdot$ & 100 & * \\
\hline vinyl chloride & 100 & $\cdot$ & 100 & $\cdot$ & 100 & $\cdot$ & 100 & $\cdot$ & 100 & $\cdot$ & 100 & • \\
\hline Xylenes & so & $\cdot$ & 50 & . & 50 & . & 50 & . & 50 & . & 50 & . \\
\hline Summed vocs & 0 & . & 0 & . & 0 & $\cdot$ & 0 & . & 0 & . & 0 & . \\
\hline
\end{tabular}

(CONTINUED) 
APPENDIX $J$

Validated VoC Results, 2993

\begin{tabular}{|c|c|c|c|c|c|c|c|c|c|c|c|c|}
\hline \multirow{4}{*}{$\begin{array}{l}\text { Sampling Point } \\
\text { Location } \\
\text { Date Sampled }\end{array}$} & \multicolumn{4}{|c|}{ GW-765 } & \multicolumn{8}{|c|}{ GW-766 } \\
\hline & \multicolumn{4}{|c|}{ GRIDEI } & \multicolumn{8}{|c|}{ GRIDI2 } \\
\hline & \multicolumn{2}{|c|}{$0 y / 12 / 93$} & \multicolumn{2}{|c|}{$11 / 19 / 93$} & \multicolumn{2}{|c|}{$02 / 01 / 93$} & \multicolumn{2}{|c|}{$04 / 15 / 93$} & \multicolumn{2}{|c|}{$08 / 03 / 93$} & \multicolumn{2}{|c|}{$10 / 13 / 93$} \\
\hline & ug/L & sum & ug/L & sum & ug/t & sum & ug/L & sum & ug/L & sum & ug/L & $\operatorname{sum}$ \\
\hline Acetone & 100 & $\cdot$ & 100 & - & EP1 & - & 100 & - & 100 & $\cdot$ & 100 & . \\
\hline Benzene & 50 & $\cdot$ & 50 & $\cdot$ & 5v & $\cdot$ & $\mathbf{5 u}$ & - & 5v & $\cdot$ & so & r \\
\hline Bromoform & 5u & $\cdot$ & 50 & $\cdot$ & 5v & $\cdot 1$ & 50 & - & 50 &. & 50 & . \\
\hline 2-Butanone & 100 & $\cdot$ & 100 & $\cdot$ & 100 & - & 100 & - & 100 & $\cdot$ & 100 & . \\
\hline Carbon disulfide & su & $\cdot$ & FP3 & $\cdot$ & 5v & - & 5u & - & 5v &. & 5v & . \\
\hline Carbon tetrachloride & 50 & $\cdot$ & 5u & $\cdot$ & 5u & $\cdot$ & 50 & $\cdot$ & 50 & $\cdot$ & 5u & ra \\
\hline Chloroform & 50 & $\cdot$ & 50 & - & sv & • & 50 & - & 50 & $\cdot$ & 50 & . \\
\hline Chloromethane & 100 & $\cdot 1$ & 100 & $\cdot$ & 100 & $\cdot$ & 100 & $\cdot$ & 100 & - & 100 & . \\
\hline 1,1-Dichloroethane & 50 & $\cdot$ & 50 & $\cdot$ & 5v & $\cdot$ & 5u & $\cdot$ & sv & $\cdot$ & 50 & . \\
\hline 1,2-Dichloroethane & 5u & $\cdot$ & 5u & $\cdot$ & 5u & $\cdot$ & 50 & $\cdot$ & 50 & $\cdot$ & 50 & . \\
\hline 1,1-vichloroethene & 50 & $\cdot$ & so & $\cdot$ & 50 & $\cdot$ & 50 & $\cdot$ & 50 & $\cdot$ & 5u & 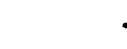 \\
\hline 1,2-Dichloroethene & 5u & $\cdot$ & 50 & • & 50 & $\cdot$ & $\equiv \mathbf{0}$ & $\cdot$ & 50 & $\cdot$ & 50 & r \\
\hline Ethylbenzene & 5u & $\cdot$ & su & $\cdot$ & 50 & $\cdot$ & 50 & $\cdot$ & 50 & - & 5u & . \\
\hline 4-Methy1-2-pentanone & 100 & $\cdot$ & 100 & . & FP1 & $\cdot$ & 100 & $\cdot$ & 100 & - & 100 & . \\
\hline Methylene chloride & 50 & $\cdot$ & 50 & $\cdot$ & 5v & $\cdot$ & 5v & $\cdot$ & 50 & $\cdot$ & 50 & . \\
\hline Styrene & 50 & $\cdot$ & 50 & $\cdot$ & 50 & $\cdot$ & 50 & $\cdot$ & 5v & . & 50 & . \\
\hline Tetrachloroethene & so & $\cdot$ & 50 &. & su & $\cdot$ & 5v & $\cdot$ & 50 & - & 50 & . \\
\hline Toluene & $5 v$ & $\cdot$ & so & $\cdot$ & 5v &. & 5v & $\cdot$ & 50 & $\cdot$ & 50 & . \\
\hline $1,1,1-\operatorname{Tr}$ ichloroethane & 50 & $\cdot$ & 5u & $\cdot$ & so & $\cdot 1$ & 5u & $\cdot$ & 5u & $\cdot$ & su & . \\
\hline Trichloroethene & 50 & $\cdot$ & 50 & $\cdot$ & 50 & $\cdot$ & 50 & $\cdot$ & 50 & - & 50 & . \\
\hline vinyl acetate & 100 & $\cdot$ & 100 & $\cdot$ & 100 & $\cdot$ & 100 & $\cdot$ & 100 & - & 100 & . \\
\hline vinyl chloride & 100 & $\cdot$ & 100 & $\cdot$ & 100 & $\cdot$ & 100 & $\cdot$ & 100 & $\cdot$ & 100 & . \\
\hline xylenes & 5v & $\cdot$ & 50 & $\cdot$ & so & $\cdot$ & 5v & $\cdot$ & 50 & $\cdot$ & 5v & . \\
\hline Sumed vocs & 0 & . & 0 &. & 0 & - & 0 & $\cdot$ & 0 & - & 0 & . \\
\hline
\end{tabular}

(CONTINUED) 
Valldated voc Results, 1993

\begin{tabular}{|c|c|c|c|c|c|c|c|c|c|c|c|c|}
\hline \multirow{4}{*}{$\begin{array}{l}\text { Sampling Point } \\
\text { Location } \\
\text { Date Sampled }\end{array}$} & \multicolumn{8}{|c|}{ GW-767 } & \multicolumn{4}{|c|}{$G w-769$} \\
\hline & \multicolumn{8}{|c|}{ GRIDI2 } & \multicolumn{4}{|c|}{ GRIDG3 } \\
\hline & \multicolumn{2}{|c|}{$02 / 02 / 93$} & \multicolumn{2}{|c|}{$04 / 15 / 93$} & \multicolumn{2}{|c|}{$08 / 04 / 93$} & \multicolumn{2}{|c|}{$10 / 13 / 93$} & \multicolumn{2}{|c|}{$01 / 27 / 93$} & \multicolumn{2}{|c|}{$04 / 19 / 93$} \\
\hline & ug/L & s sum & ug/L & - sum & $u g / L$ & - sum & $u g / L$ & s sum & $u g / L$ & - sum & $u g / L$ & sum \\
\hline Acetone & FP1 & - & 100 & . & 100 & - & 100 & - & 100 & $\cdot$ & 100 & - \\
\hline Benzene & 50 & $\cdot$ & 50 & - & 50 & $\cdot$ & 50 & $\cdot$ & 50 & $\cdot$ & so & - \\
\hline Bromoform & 5u & $\cdot$ & su & - & $5 \mathbf{v}$ & $\cdot$ & 5v & - & 50 & $\cdot 1$ & 50) & $\cdot$ \\
\hline 2-Butanone & $\mathbf{F P 1}$ & $\cdot$ & 100 & $\cdot$ & 100 & $\cdot$ & 100 & - & 100 & $\cdot$ & 100 & - \\
\hline Carbon disulfide & 5v & $\cdot$ & 5u & - & 50 & $\cdot$ & su & $\cdot$ & 50 & $\cdot$ & so & • \\
\hline Carbon tetrachloride & 50 & $\cdot$ & 5u & • & 50 & $\cdot$ & 50 & $\cdot$ & 9 & 52.9 & 5 & 36.2 \\
\hline Chloroform & 5v & $\cdot 1$ & 50 & - & 50 & $\cdot$ & 5v & • & 1 & 5.9 & 1 & 7.2 \\
\hline Chloromethane & 100 & $\cdot$ & 100 & • & 100 & $\cdot$ & 100 & $\cdot$ & 100 & $\cdot 1$ & 100 & - \\
\hline 1,1-Dichloroethane & 50 & $\cdot 1$ & 50 & $\cdot$ & 5v & $\cdot$ & 50 & • & 1 & 5.9 & 1 & 7.2 \\
\hline 1,2-Dichloroethane & so & $\cdot$ & su & $\cdot$ & 50 & $\cdot$ & 50 & $\cdot$ & so & $\cdot$ & 5u & - \\
\hline 1,1-Dichloroethene & 50 & $\cdot$ & 50 & - & 50 & $\cdot$ & 50 & $\cdot$ & 1 & 5.9 & 1 & 7.2 \\
\hline 1,2-Dichloroethene & 5v & $\cdot$ & 5u & $\cdot$ & 50 & $\cdot$ & 5v & $\cdot$ & FP3 & $\cdot$ & 50 & • \\
\hline Ethylbenzene & so & $\cdot$ & 5u & - & so & $\cdot$ & 50 & - & 50 & $\cdot$ & 50 & - \\
\hline 4-Methy1-2-pentanone & FP1 & $\cdot$ & 100 & - & 100 & $\cdot$ & 100 & $\cdot$ & FP1 & $\cdot$ & 100 & - \\
\hline Methylene chloride & 5u & $\cdot$ & FP1 & - & 50 & $\cdot$ & sv & $\cdot$ & 50 & $\cdot$ & 50 & $\cdot$ \\
\hline styrene & su &. & so & - & 50 &. & 50 & $\cdot$ & 50 & $\cdot$ & 50 & - \\
\hline Tetrachloroethene & so & $\cdot$ & 50 & $\cdot$ & 50 & $\cdot$ & 5v & $\cdot$ & 4 & 23.5 & 4 & 29 \\
\hline Toluene & su & $\cdot$ & 50 & . & 50 & $\cdot$ & 5u & $\cdot$ & 50 & $\cdot$ & so & • \\
\hline $1,1,1-\operatorname{Tr} 1 \mathrm{chl}$ oroethane & 50 & $\cdot$ & 5u & $\cdot$ & 50 & $\cdot$ & 50 & - & 50 & $\cdot$ & 0.8 & 5.8 \\
\hline Trichloroethene & 5u & $\cdot$ & su & $\cdot$ & 5v & $\cdot$ & so & $\cdot$ & 1 & 5.9 & 1 & 7.2 \\
\hline Vinyl acetate & 100 & $\cdot$ & 100 & • & 100 & $\cdot$ & 100 & $\cdot$ & 100 & $\cdot$ & 100 & • \\
\hline vinyl chloride & 100 & $\cdot$ & 100 & $\cdot$ & 100 & $\cdot$ & 100 & - & 100 & $\cdot$ & 100 & - \\
\hline xylenes & so &. & 5u & - & 50 & $\cdot$ & 50 & . & 50 & $\cdot$ & 50 & - \\
\hline Summed VOCB & 0 &. & 0 & . & 0 &. & 0 & . & 17 & 100 & 13.8 & 99.8 \\
\hline
\end{tabular}

(CONTINUED) 
APPENDIX $J$

Valldated VOC Results, 1993

\begin{tabular}{|c|c|c|c|c|c|c|c|c|c|c|c|c|}
\hline \multirow{4}{*}{$\begin{array}{l}\text { Sampling Point } \\
\text { Location } \\
\text { Date Sampled }\end{array}$} & \multicolumn{4}{|c|}{ GW-769 } & \multicolumn{8}{|c|}{$G w-770$} \\
\hline & \multicolumn{4}{|c|}{ GRIDG3 } & \multicolumn{8}{|c|}{ GRIDG3 } \\
\hline & \multicolumn{2}{|c|}{$09 / 14 / 93$} & \multicolumn{2}{|c|}{$11 / 30 / 93$} & \multicolumn{2}{|c|}{$01 / 27 / 93$} & \multicolumn{2}{|c|}{$04 / 19 / 93$} & \multicolumn{2}{|c|}{$09 / 14 / 93$} & \multicolumn{2}{|c|}{$11 / 29 / 93$} \\
\hline & ug/L & sum & ug/L & sum & $\mathrm{ug} / \mathrm{t}$ & sum & ug/I & เ anm & $u g / x$ & sum & ug/L & s sum \\
\hline Acetone & 100 & $\cdot$ & 100 & - & 100 & $\cdot$ & 100 & . & 100 & - & 100 & - \\
\hline Benzene & 5u & $\cdot$ & 50 & $\cdot$ & 5v & $\cdot$ & 5v & $\cdot$ & 50 & $\cdot$ & 50 & • \\
\hline Bromoform & 5v & $\cdot$ & so & $\cdot$ & 50 &. & 50 & $\cdot$ & 5v & $\cdot$ & 5v & - \\
\hline 2-Butanone & 100 & $\cdot$ & 100 & $\cdot$ & 100 & $\cdot$ & 100 & $\cdot$ & 100 & $\cdot$ & 100 & • \\
\hline Carbon disulfide & 50 & $\cdot$ & 5v & $\cdot$ & 50 & $\cdot$ & 50 & $\cdot$ & 50 & $\cdot$ & so & - \\
\hline Carbon tetrachloride & 5 & 48.5 & 6 & 63.8 & 50 & $\cdot$ & 50 & $\cdot$ & 50 & $\cdot$ & 50. & • \\
\hline Chloroform & 0.9 & 8.7 & 0.7 & 7.4 & 4 & 100 & 4 & 100 & 5 & 100 & 9 & 100 \\
\hline Chloromethane & 100 & $\cdot$ & 100 & $\cdot$ & 100 & $\cdot$ & 100 & $\cdot$ & 100 & $\cdot$ & 100 & • \\
\hline 1,1-Dichloroethane & 50 &. & 50 & $\cdot$ & so & $\cdot$ & so & $\cdot$ & 50 &. & 5u & • \\
\hline 1,2-Dichloroethane & 50 & $\cdot$ & 50 &. & 50 & $\cdot 1$ & 50 & $\cdot$ & 50 & $\cdot$ & 50 & - \\
\hline 1,1-Dichioroethene & sol & $\cdot$ & 50 & $\cdot$ & 50 & $\cdot$ & 50 & $\cdot$ & so & $\cdot$ & 50 & • \\
\hline 1,2-Dichloroethene & 50i & $\cdot$ & 50 & $\cdot 1$ & 50) & $\cdot$ & 50) & $\cdot$ & 50 & $\cdot$ & 50 & - \\
\hline Ethylbenzene & 5u &. & 50 & $\cdot$ & 50 & $\cdot$ & 50 &. & 50 & $\cdot$ & 50 & - \\
\hline 4-Methy1-2-pentanone & 100 & $\cdot$ & 100 & $\cdot$ & FP1 & $\cdot$ & 100 & $\cdot$ & 100 & $\cdot$ & 100 & • \\
\hline Methylene chloride & so & $\cdot$ & 5v & $\cdot$ & 5u & $\cdot$ & 50 & $\cdot 1$ & FT: & $\cdot$ & 50 & • \\
\hline styrene & so & $\cdot$ & 50 & $\cdot$ & 50 & $\cdot$ & 50 & $\cdot$ & 50 & $\cdot$ & 50 & - \\
\hline Tetrachloroethene & 3 & 29.1 & 2 & 21.3 & su & $\cdot$ & 50 &. & 50 &. & 50 & - \\
\hline Toluene & 50 & $\cdot$ & su & $\cdot$ & 50 & $\cdot$ & 50 & $\cdot$ & 50 &. & 50 & - \\
\hline 1,1,1-Trichloroethane & 0.4 & 3.9 & 5v & $\cdot$ & 50 & $\cdot$ & 50 & $\cdot$ & 50 & $\cdot$ & 5u & - \\
\hline Trichloroethene & 1 & 9.7 & 0.7 & 7.4 & 50 & $\cdot$ & so & $\cdot$ & so & $\cdot$ & 50 & • \\
\hline vinyl acetate & 100 & $\cdot$ & 100 & $\cdot$ & 100 & $\cdot$ & 100 & $\cdot$ & 100 & $\cdot$ & 100 & - \\
\hline vingl chloride & 100 & $\cdot$ & 100 & $\cdot$ & 100 & $\cdot$ & 100 & - & 100 & $\cdot$ & 100 & • \\
\hline xylenes & 50 & $\cdot$ & 50 &. & 50 & $\cdot$ & 50 &. & 50 & $\cdot$ & 50 & . \\
\hline Summed vOCs & 10.3 & 99.9 & 9.4 & 99.9 & 4 & 100 & 4 & 100 & 5 & 100 & 9 & 100 \\
\hline
\end{tabular}

(CONTIRUED) 
Valldated voC Results, 1993

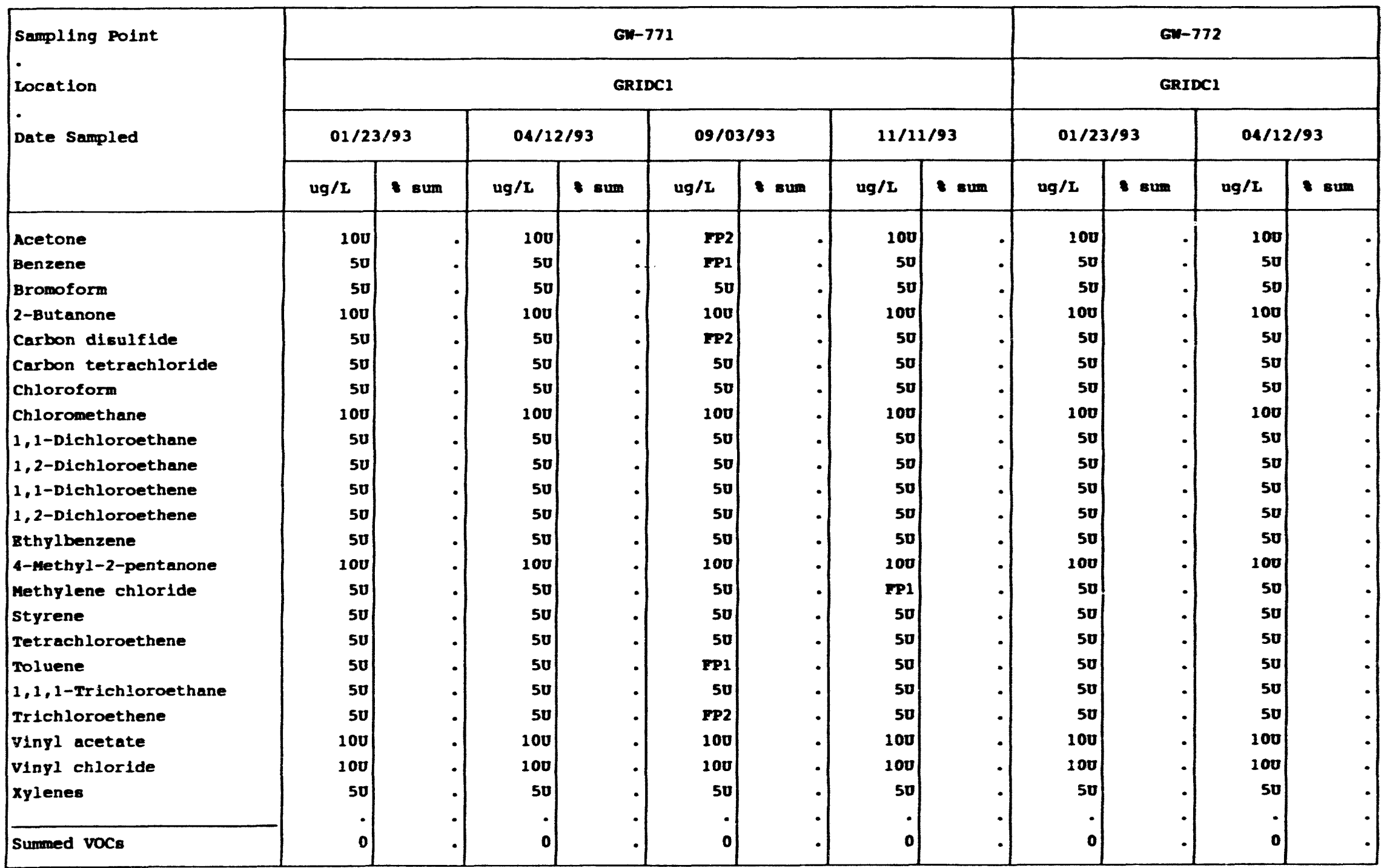

(CONTINUED) 
APPSADIX $J$

Validated voc Results, 1993

\begin{tabular}{|c|c|c|c|c|c|c|c|c|c|c|c|c|}
\hline \multirow{4}{*}{$\begin{array}{l}\text { Sampling Point } \\
\text { Location } \\
\text { Date Sampled }\end{array}$} & \multicolumn{4}{|c|}{ Gw-772 } & \multicolumn{8}{|c|}{$64-773$} \\
\hline & \multicolumn{4}{|c|}{ GRIDCI } & \multicolumn{8}{|c|}{ GRIDA2 } \\
\hline & \multicolumn{2}{|c|}{$09 / 12 / 93$} & \multicolumn{2}{|c|}{$11 / 12 / 93$} & \multicolumn{2}{|c|}{$02 / 03 / 93$} & \multicolumn{2}{|c|}{$04 / 20 / 93$} & \multicolumn{2}{|c|}{$08 / 06 / 93$} & \multicolumn{2}{|c|}{$10 / 15 / 93$} \\
\hline & ug/L & sum & $u g / L$ & sum & $\operatorname{ug} / \mathrm{L}$ & \& sum & $u g / L$ & s.m & $\mathbf{n g} / \mathbf{L}$ & $5 \sin$ & $\operatorname{ng} / \mathbf{L}$ & c sum \\
\hline Acetone & 100 & - & 100 & - & 100 & - & 100 & - & 100 & - & 100 & 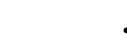 \\
\hline Benzene & su & • & 50 & - & 50 & - & 50 & - & so & - & 50 & . \\
\hline Bromoform & 5u & • & 50 & - & so & - & 50 & - & so & - & 50 & . \\
\hline 2-Butanone & 100 & • & 100 & - & 100 & - & 100 & - & 100 & - & 100 & 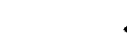 \\
\hline Carbon disulfide & 50 & - & 5u & - & so & - & 50 & - & 50 & - & so & rat \\
\hline Carben tetrachloride & 50 & • & 50 & - & 50 & . & 50 & - & 5v & - & so & ra \\
\hline Chloroform & 50 & • & 5v & - & 50 & - & 5v & - & 5v & - & 50 & ratan \\
\hline Chloromethane & 100 & - & 100 & - & 100 & - & 100 & - & 100 & - & 100 & rata \\
\hline 1,1-Dichloroethane & 50 & • & 50 & - & 50 & - & 50 & - & $\mathbf{5 u}$ & - & 50 & 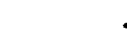 \\
\hline 1,2-Dichloroethane & 5v & . & 5v & - & 50 & - & 50 & - & 50 & - & so & r \\
\hline 1,1-Dichloroethene & 50 & • & 50 & - & 50 & - & 50 & - & 50 & - & so & . \\
\hline 1,2-Dichloroethene & so & • & 50 & - & 50 & - & 50 & - & so & - & so & r \\
\hline zthylbenzene & 50 & • & 50 & - & 50 & - & 50 & - & 5v & - & 50 & rat \\
\hline 4-methy1-2-pentanone & 100 & • & 100 & - & $\mathbf{F P 1}$ & • & FP1 & - & 100 & - & 100 & . \\
\hline Methylene chloride & 50 & • & FP1 & - & FP1 & • & 50 & - & so & - & 50 & rat \\
\hline Styrene & 50 & - & 50 & - & so & - & 50 & - & 50 & - & 50 & 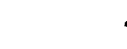 \\
\hline Tetrachloroethene & 50 & • & so & - & so & - & 50 & - & so & - & 50 & r \\
\hline Toluene & 50 & • & 50 & - & 50 & - & su & - & so & - & 50 & rat \\
\hline 1,1,1-Trichloroethane & 50 & • & 50 & - & 50 & • & 50 & - & so & - & so & r \\
\hline Trichloroethene & 50 & - & 50 & - & so & - & 50 & - & so & - & 50 & ratan \\
\hline Vinyl acetate & 100 & - & 100 & - & 100 & - & 100 & - & 100 & - & 100 & ra \\
\hline Vinyl chloride & 100 & - & 100 & - & 100 & • & 100 & - & 100 & - & 100 & ratan \\
\hline xylenes & so & • & 50 & - & 50 & - & 50 & - & 50 & - & 50 & r \\
\hline Summed vocs & 0 & - & 0 & - & o) & - & o) & - & o) & - & 0 & r \\
\hline
\end{tabular}

(CONTINUED) 
APPEMDIX J

Valldated voc Resulte, 1993

\begin{tabular}{|c|c|c|c|c|c|c|c|c|c|c|c|c|}
\hline \multirow{4}{*}{$\begin{array}{l}\text { Sampling Point } \\
\text { Location } \\
\text { Date Sampled }\end{array}$} & \multicolumn{8}{|c|}{ Gw-774 } & \multicolumn{4}{|c|}{$6 n-775$} \\
\hline & \multicolumn{8}{|c|}{ GRIDH2 } & \multicolumn{4}{|c|}{ GRIDA3 } \\
\hline & \multicolumn{2}{|c|}{$02 / 04 / 93$} & \multicolumn{2}{|c|}{$04 / 20 / 93$} & \multicolumn{2}{|c|}{$08 / 09 / 93$} & \multicolumn{2}{|c|}{$10 / 15 / 93$} & \multicolumn{2}{|c|}{$02 / 04 / 93$} & \multicolumn{2}{|c|}{$04 / 20 / 93$} \\
\hline & ug/L & s $\operatorname{sum}$ & $u g / x$ & sum & $u g / L$ & เ & $u g / L$ & > an & $\mathrm{ng} / \mathrm{x}$ & - smin & $\operatorname{ug} / \mathrm{L}$ & s.um \\
\hline Acetone & 100 & - & 100 & - & 100 & - & 100 & - & 100 & . & 100 & - \\
\hline Benzene & 5v & $\cdot$ & 50 & - & so & - & 50 & - & so & $\cdot$ & so & - \\
\hline Bromoform & 50 & - & 50 & - & so & - & 50 & - & sv & . & so & . \\
\hline 2-Butanone & 100 & - & 100 & - & 100 & - & 100 & - & 100 & - & 100 & • \\
\hline Carbon disulfide & 50 & - & 50 & - & su & - & so & - & so & $\cdot$ & so & • \\
\hline Carbon tetrachloride & 50 & $\cdot$ & 50 & - & 50 & - & so & - & 50 & $\cdot$ & so & • \\
\hline Chloroform & 50 & $\cdot$ & 50 & - & 50 & - & so & - & 1 & 16.7 & so & • \\
\hline Chloromethane & 100 & $\cdot$ & 100 & - & 100 & - & 100 & - & 100 & $\cdot$ & 100 & • \\
\hline 1,1-Dichloroethane & 50 & - & 50 & - & so & - & 50 & - & so & - & 50 & • \\
\hline 1,2-Dichloroethane & so & - & 50 & - & 50 & - & so & - & so & - & so & • \\
\hline 1,1-Dichloroethene & 50 & - & so & - & 50 & - & sv & - & 50 & - & 50 & • \\
\hline 1,2-Dichloroethene & su & - & 5v & - & 50 & - & 50 & • & 50 & $\cdot$ & 50 & - \\
\hline Ethylbenzene & 50 & - & 5v & - & 50 & - & 50 & • & so & - & 50 & • \\
\hline 4-Methy1-2-pentanone & 100 & - & FP1 & - & 100 & - & 100 & $\cdot$ & 100 & - & $\mathbf{F P 1}$ & • \\
\hline Methylene chloride & 50 & - & so & - & 50 & - & 50 & - & su & $\cdot$ & FP3 & • \\
\hline styrene & 50 & - & 50 & - & 50 & - & 50 & $\cdot$ & $\mathbf{5 0}$ & - & 50 & $\cdot$ \\
\hline Tetrachloroethene & 5u & - & 5v & - & 50 & • & 50 & $\cdot$ & $\mathbf{F P 3}$ & - & so & • \\
\hline Toluene & 50 & - & so & - & 50 & - & so & - & so & $\cdot$ & so & • \\
\hline 1,1,1-Trichloroethane & 50 & - & so & - & 50 & $\cdot$ & 50 & $\cdot$ & 50 & $\cdot$ & so & • \\
\hline Trlchloroethene & 50 & • & so & - & 50 & - & so & $\cdot$ & 5 & 83.3 & 3 & 100 \\
\hline vinyl acetate & 100 & - & 100 & - & 100 & - & 100 & - & 100 & $\cdot$ & 100 & • \\
\hline VInyl chloride & 100 & - & 100 & • & 100 & $\cdot$ & 100 & $\cdot$ & 100 & $\cdot$ & 100 & • \\
\hline xylenes & so & - & 50 & . & 50 & . & 50 & . & so & . & 50 & • \\
\hline Summed VOCE & 0 & . & 0 & . & 0 & . & 0 & . & 6 & 100 & 3 & 100 \\
\hline
\end{tabular}

(COMTINUED) 
APPEDDIX J

validated voc neaulte, 1993

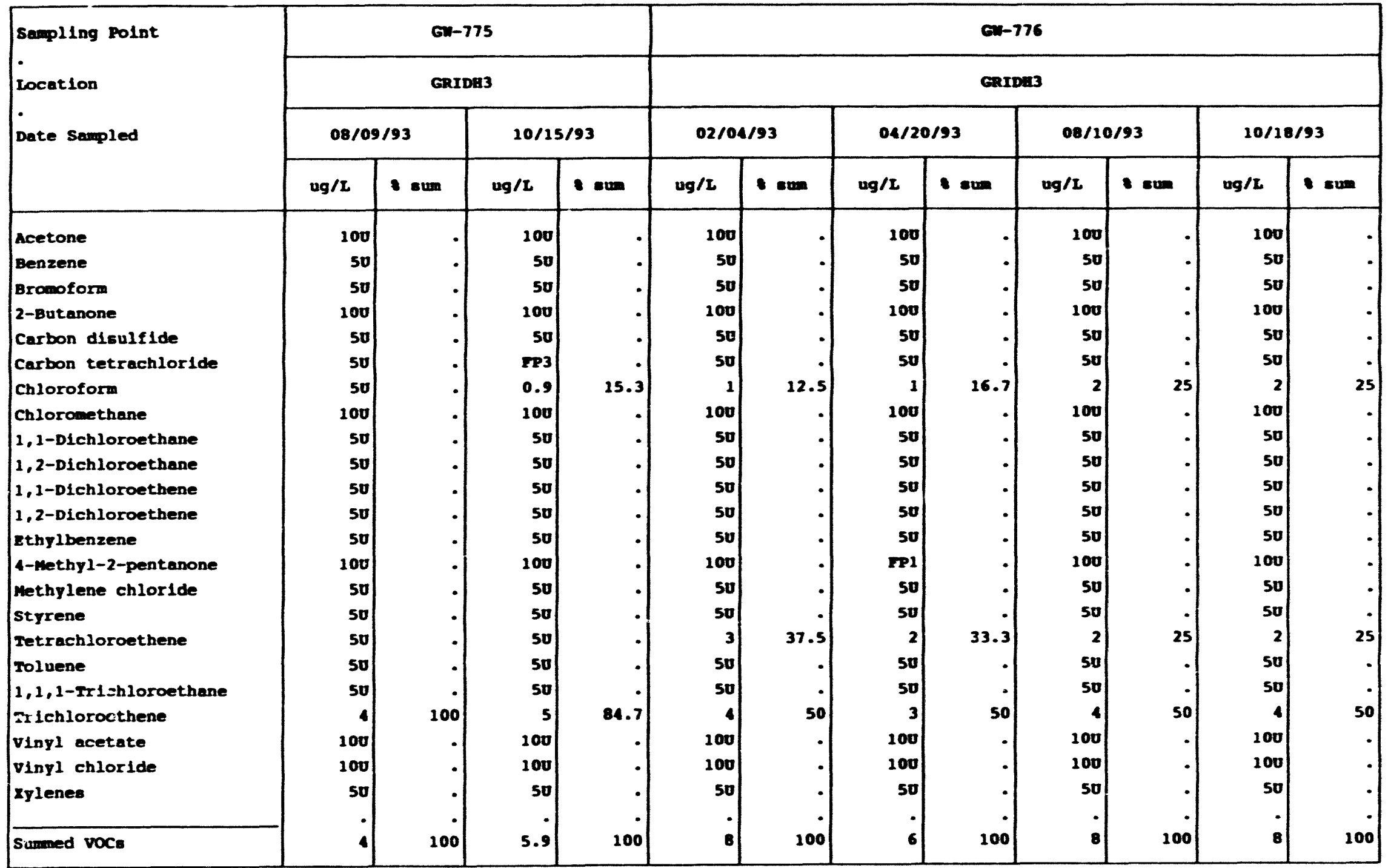


APPENDIX $K$

RADIOCHEMICAL DATA SUMMARY 


\section{EXPLANATION}

\section{LOCATION:}

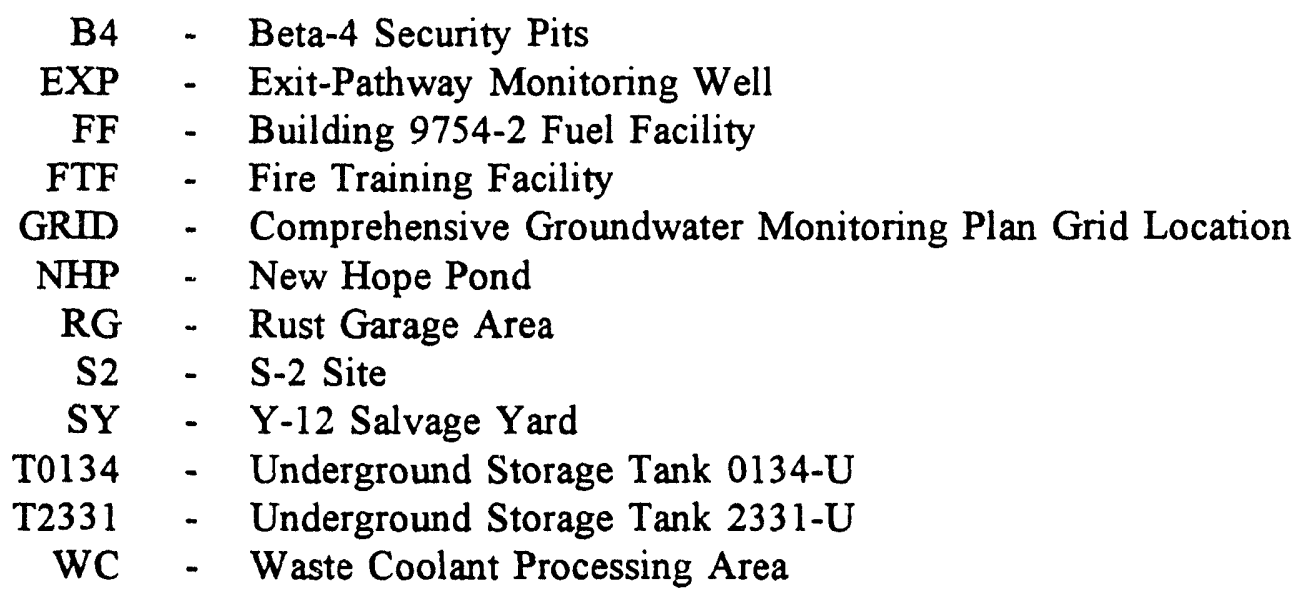

NOTES:
$\mathrm{pCi} / \mathrm{L}$ - picoCuries per Liter
$\pm \quad$ - Counting Error (two standard deviations)
- Not sampled

Mean counting error (e) calculated using the following formula from Evans (1955):

$$
e=\sqrt{\frac{E_{1}^{2}}{n^{2}}+\frac{E_{2}^{2}}{n^{2}}+\frac{E_{3}^{2}}{n^{2}}+\ldots}
$$

where $\mathbf{E}_{1}, \mathbf{E}_{2}, \ldots \mathbf{E}_{\mathbf{x}}$ are the individual errors, and $\mathbf{n}$ is the number of observations. 
Gross Alpha and Gross Beta Activity Data Summary, 1993

\begin{tabular}{|c|c|c|c|c|c|c|c|c|c|c|c|c|c|c|}
\hline \multirow{3}{*}{$\begin{array}{l}\text { Sampling Point } \\
\text { Location }\end{array}$} & \multicolumn{3}{|c|}{ GW-151 } & \multicolumn{3}{|c|}{$G W-168$} & \multicolumn{2}{|c|}{ GW-169 } & \multicolumn{3}{|c|}{$G W-170$} & \multicolumn{3}{|c|}{$G W-183$} \\
\hline & \multicolumn{3}{|c|}{ NHP } & \multicolumn{3}{|c|}{ NHP } & \multicolumn{2}{|c|}{$\operatorname{EXP}$} & \multicolumn{3}{|c|}{ EXP } & \multicolumn{3}{|c|}{ FF } \\
\hline & $\mathrm{pCi} / \mathrm{L}$ & $\mathrm{CE}$ & $+1-$ & $\mathrm{pCi} / \mathrm{L}$ & $\mathrm{CE}$ & $+1-$ & $\mathrm{pCi} / \mathrm{L}$ & $\mathrm{CE}+1-$ & $\mathrm{pCi} / \mathrm{L}$ & $\mathrm{CE}$ & $+1-$ & $\mathrm{pCi} / \mathrm{L}$ & $\mathrm{CE}$ & $+1-$ \\
\hline GROSS ALPHA & - & & - & - & & . & - & - & . & & . & - & & - \\
\hline First Quarter & 1.74 & & 2.30 & 2.66 & & 2.10 & 25.60 & 6.70 & 1.63 & & 1.70 & -1.84 & & 5.00 \\
\hline Second Quarter & -1.00 & & 2.20 & -5.13 & & 3.80 & 4.76 & 2.80 & -0.90 & & 3.10 & 0.00 & & 3.70 \\
\hline Third Quarter & 0.90 & & 3.60 & 4.60 & & 2.90 & 24.30 & 7.30 & -1.76 & & 2.10 & 3.51 & & 4.40 \\
\hline Fourth Quarter & 1.10 & & 1.70 & 1.44 & & 1.80 & 72.00 & 26.00 & 2.04 & & 1.90 & 2.09 & & 2.40 \\
\hline - & & & & & & & & & - & & - & - & & - \\
\hline Mean & 0.69 & & 1.30 & 0.89 & & 1.40 & 31.66 & 7.00 & 0.25 & & 1.10 & 0.94 & & 2.00 \\
\hline GROSS BETA & - & & - & - & & . & - & - & - & & - & - & & - \\
\hline First Quarter & -5.22 & & 3.40 & 1.89 & & 2.80 & 29.60 & 7.00 & 1.19 & & 2.80 & 4.88 & & 5.70 \\
\hline Second Quarter & 4.27 & & 2.80 & -1.68 & & 5.60 & 6.16 & 3.20 & -2.14 & & 5.40 & 4.01 & & 5.40 \\
\hline Third Quarter & 2.97 & & 5.10 & 5.57 & & 3.00 & 32.40 & 7.10 & 2.25 & & 2.80 & 3.97 & & 5.60 \\
\hline Fourth Quarter & 1.38 & & 2.90 & 2.16 & & 3.00 & 65.50 & 32.00 & 2.93 & & 3.00 & 0.51 & & 2.70 \\
\hline - & & & & • & & • & $\cdot$ & - & • & & $\cdot$ & • & & • \\
\hline Mean & 0.85 & & 1.80 & 1.98 & & 1.90 & 33.42 & 8.40 & 1.06 & & 1.80 & 3.34 & & 2.50 \\
\hline
\end{tabular}

(CONTINUED)

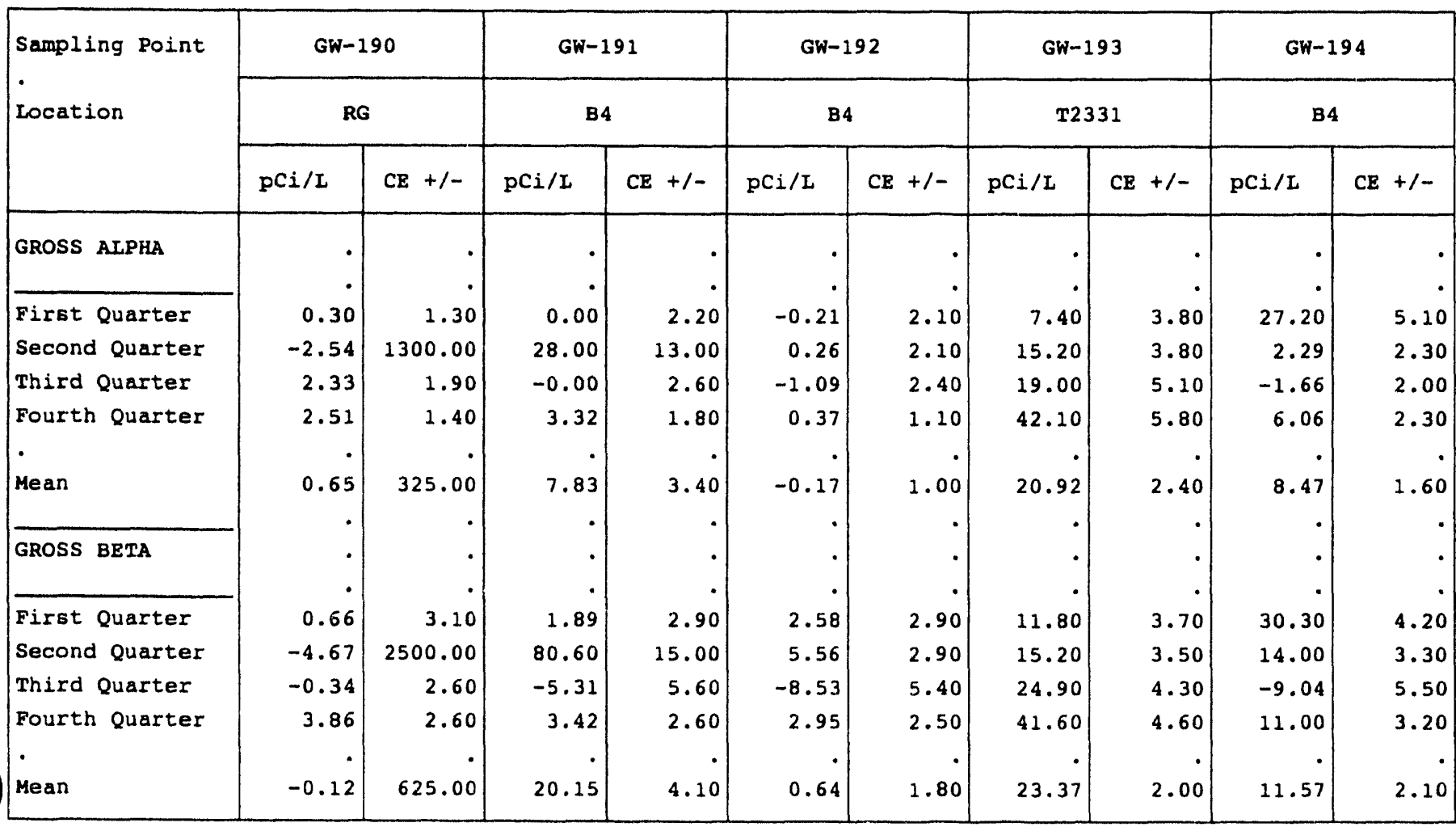

(CONT INUED) 
APPENDIX $\mathrm{K}$

Gross Alpha and Gross Beta Activity Data Summary, 1993

\begin{tabular}{|c|c|c|c|c|c|c|c|c|c|c|}
\hline \multirow{3}{*}{$\begin{array}{l}\text { Sampling Point } \\
\text { Location }\end{array}$} & \multicolumn{2}{|c|}{$G W-251$} & \multicolumn{2}{|c|}{ GW-252 } & \multicolumn{2}{|c|}{$G W-255$} & \multicolumn{2}{|c|}{$G W-261$} & \multicolumn{2}{|c|}{$G W-262$} \\
\hline & \multicolumn{2}{|c|}{ s2 } & \multicolumn{2}{|c|}{ s2 } & \multicolumn{2}{|c|}{ s2 } & \multicolumn{2}{|c|}{ SY } & \multicolumn{2}{|c|}{ SY } \\
\hline & $\mathrm{pCi} / \mathrm{L}$ & $\mathrm{CE}+1-$ & $\mathrm{pCi} / \mathrm{L}$ & $\mathrm{CE}+/-$ & $\mathrm{pCi} / \mathrm{L}$ & $\mathrm{CE}+/-$ & $\mathrm{pCi} / \mathrm{L}$ & $\mathrm{CE}+/-$ & $\mathrm{pCi} / \mathrm{L}$ & $\mathrm{CE}+/-$ \\
\hline GROSS ALPHA & - & - & - & - & - & - & - & • & . & • \\
\hline First Quarter & 19.20 & 6.00 & 0.00 & 1.60 & 0.58 & 2.20 & $-0.8 \dot{4}$ & 1.70 & 2.99 & $2.50^{\circ}$ \\
\hline Second Quarter & 5.94 & 2.40 & 5.58 & 1.40 & 4.72 & 2.10 & -0.31 & 3.60 & 1.17 & 2.10 \\
\hline Third Quarter & 22.20 & 4.30 & 0.63 & 1.70 & 3.99 & 2.20 & -2.96 & 3.50 & -2.17 & 3.80 \\
\hline Fourth Quarter & 9.11 & 2.80 & -0.31 & 0.60 & 2.60 & 2.10 & -0.71 & 1.30 & 1.62 & 1.90 \\
\hline & & & & & & & $\cdot$ & • & & \\
\hline Mean & 14.11 & 2.10 & 1.48 & 0.70 & 2.97 & 1.10 & -1.20 & 1.40 & 0.90 & 1.30 \\
\hline GROSS BETA & & . & . & . & . & . & . & $\cdot$ & $\cdot$ & " \\
\hline First Quarter & 19.40 & 6.50 & -1.10 & 2.60 & 3.46 & 3.00 & 1.22 & $2.80^{\circ}$ & 2.38 & $2.90^{\circ}$ \\
\hline Second Quarter & 12.60 & 2.70 & -0.34 & 1.40 & 0.46 & 2.90 & 7.12 & 5.10 & 13.90 & 3.40 \\
\hline Third Quarter & 11.50 & 3.30 & 2.41 & 2.80 & 3.54 & 2.80 & -2.47 & 5.50 & -3.15 & 5.50 \\
\hline Fourth Quarter & 4.19 & 2.70 & 0.08 & 2.40 & 6.91 & 2.90 & 2.45 & 2.60 & 4.64 & 2.70 \\
\hline & & & & & & & & & $\cdot$ & • \\
\hline Mean & 11.92 & 2.10 & 0.26 & 1.20 & 3.59 & 1.50 & 2.08 & 2.10 & 4.44 & 1.90 \\
\hline
\end{tabular}

(CONTINUED)

\begin{tabular}{|c|c|c|c|c|c|c|c|c|c|c|}
\hline \multirow{3}{*}{$\begin{array}{l}\text { Sampling Point } \\
\text { Location }\end{array}$} & \multicolumn{2}{|c|}{$G W-263$} & \multicolumn{2}{|c|}{ GW-264 } & \multicolumn{2}{|c|}{ GW-281 } & \multicolumn{2}{|c|}{$G W-283$} & \multicolumn{2}{|c|}{ GW-284 } \\
\hline & \multicolumn{2}{|c|}{ SY } & \multicolumn{2}{|c|}{ SY } & \multicolumn{2}{|c|}{ FF } & \multicolumn{2}{|c|}{$\mathrm{FF}$} & \multicolumn{2}{|c|}{$\mathbf{F F}$} \\
\hline & $\mathrm{pCi} / \mathrm{L}$ & $C E+/-$ & $\mathrm{pCi} / \mathrm{L}$ & $\mathrm{CE}+/-$ & $\mathrm{pCi} / \mathrm{L}$ & $\mathrm{CE}+/-$ & $\mathrm{pCi} / \mathrm{L}$ & $\mathrm{CE}+/-$ & $\mathrm{pCi} / \mathrm{L}$ & $\mathrm{CE}+1-$ \\
\hline GROSS ALPHA & • & - & - & . & - & - & - & • & • & \\
\hline First Quarter & 8.00 & 3.00 & -1.03 & 3.60 & 1.74 & 2.10 & 1.13 & 2.00 & 3.43 & $4.20^{\circ}$ \\
\hline Second Quarter & 0.00 & 2.00 & -2.18 & 3.10 & 6.20 & 2.00 & 6.65 & 2.00 & 1.37 & 0.86 \\
\hline Third Quarter & -4.25 & 3.30 & -0.39 & 2.80 & 1.75 & 9.30 & -1.01 & 3.60 & 3.81 & 5.80 \\
\hline Fourth Quarter & -0.01 & 1.60 & 1.35 & 1.60 & 3.11 & 2.00 & 0.37 & 1.80 & 3.20 & 2.50 \\
\hline Mean & 0.94 & & -0.56 & $\cdot$ & $\dot{3}$ & & & $\cdot$ & & \\
\hline & & 1.30 & -0.56 & 1.40 & 3.20 & 2.50 & 1.78 & 1.20 & 2.95 & 1.90 \\
\hline GROSS BETA & - & - & . & . & . & . & . & . & ${ }^{\circ}$ & \\
\hline First Quarter & 13.90 & 3.50 & 2.26 & 3. $\dot{0}$ & 2.70 & 3.00 & 3.74 & 4.30 & - & 30 \\
\hline Second Qurter & 39.10 & 4.50 & 3.54 & 3.00 & 9.88 & 2.70 & 3.97 & $\begin{array}{l}4.30 \\
1.50\end{array}$ & $\begin{array}{l}4.15 \\
2.98\end{array}$ & $\begin{array}{l}3.20 \\
1.30\end{array}$ \\
\hline Third Qua ter & -3.47 & 5.50 & -4.87 & 5.50 & 5.12 & 12.00 & 4.20 & 5.30 & 2.27 & 5.90 \\
\hline Fourth Quarter & 3.22 & 2.60 & 1.80 & 2.50 & 4.89 & 2.80 & 4.16 & 2.80 & 2.66 & 2.80 \\
\hline - & & & & & & • & & & & \\
\hline Mean & 13.19 & 2.10 & 0.68 & 1.90 & 5.65 & 3.20 & 4.02 & 1.90 & 3.01 & 1.80 \\
\hline
\end{tabular}

(CONTINUED) 
Gross Alpha and Gross Beta Activity Data Summary, 1993

\begin{tabular}{|c|c|c|c|c|c|c|c|c|c|c|c|}
\hline \multirow{3}{*}{$\begin{array}{l}\text { Sampling Point } \\
\text { Location }\end{array}$} & \multicolumn{2}{|c|}{$G W-285$} & \multicolumn{2}{|c|}{ GW-337 } & \multicolumn{2}{|c|}{$G W-338$} & \multicolumn{2}{|c|}{$G W-380$} & \multicolumn{3}{|c|}{$G W-381$} \\
\hline & \multicolumn{2}{|c|}{$\mathbf{F F}$} & \multicolumn{2}{|c|}{ WC } & \multicolumn{2}{|c|}{ พc } & \multicolumn{2}{|c|}{ NHP } & \multicolumn{3}{|c|}{ NHP } \\
\hline & $\mathrm{pCi} / \mathrm{I}$ & $\mathrm{CE}+1-$ & $\mathrm{pCi} / \mathrm{L}$ & $\mathrm{CE}+/-$ & $\mathrm{pCi} / \mathrm{L}$ & $\mathrm{CE}+/-$ & $\mathrm{pCi} / \mathrm{L}$ & $\mathrm{CE}+1-$ & $\mathrm{pCi} / \mathrm{L}$ & $\mathrm{CE}$ & $+1-$ \\
\hline GROSS ALPHA & • & - & - & - & - & - & $\cdot$ & $\cdot$ & $\cdot$ & & - \\
\hline First Quarter & 3.89 & 2.60 & 13.50 & 5.20 & -0.21 & 2.20 & 1.79 & 3.70 & 1.32 & & 2.30 \\
\hline Second Quarter & 3.02 & 2.30 & -0.17 & 0.76 & -0.55 & 1.00 & -0.25 & 3.00 & 1.17 & & 2.20 \\
\hline Third Quarter & 0.88 & 4.70 & -0.16 & 1.50 & $-1 \cdot 18$ & 3.40 & -3.32 & 2.80 & 1.52 & & 3.50 \\
\hline Fourth Quarter & 3.85 & 2.20 & 1.28 & 1.50 & $-0,56$ & 1.40 & 0.51 & 2.10 & 1.80 & & 1.90 \\
\hline - & • & & - & & $\cdot$ & $\cdot$ & $\cdot$ & $\cdot$ & $\cdot$ & & • \\
\hline Mean & 2.91 & 1.50 & 3.61 & 1.40 & -0.62 & 1.10 & -0.32 & 1.50 & 1.45 & & 1.30 \\
\hline GROSS BETA & . & $\cdot$ & $\cdot$ & $\cdot$ & . & . & $\cdot$ & . & . & & - \\
\hline irst Quarter & 7.05 & 3.20 & $19 \cdot 30$ & 6.50 & 0.54 & 2.80 & -6.25 & 3.60 & 3.56 & & 3.00 \\
\hline recond Quarter & 2.11 & 1.40 & 1.49 & 1.20 & -1.87 & 1.50 & 3.01 & 2.80 & 4.00 & & 2.70 \\
\hline Third Quarter & -0.34 & 5.70 & 1.21 & 2.70 & 0.51 & 5.30 & 2.31 & 5.10 & 3.47 & & 5.50 \\
\hline Fourth Quarter & 4.50 & 2.80 & 2.57 & 2.50 & 1.88 & 2.50 & 5.13 & 2.90 & 0.00 & & 2.80 \\
\hline & $\cdot$ & • & $\bullet$ & $\cdot$ & • & • & $\cdot$ & $\cdot$ & • & & • \\
\hline Mean & 3.33 & 1.80 & 6.14 & 1.90 & 0.26 & 1.70 & 1.05 & 1.90 & 2.76 & & 1.80 \\
\hline
\end{tabular}

(CONTINUED)

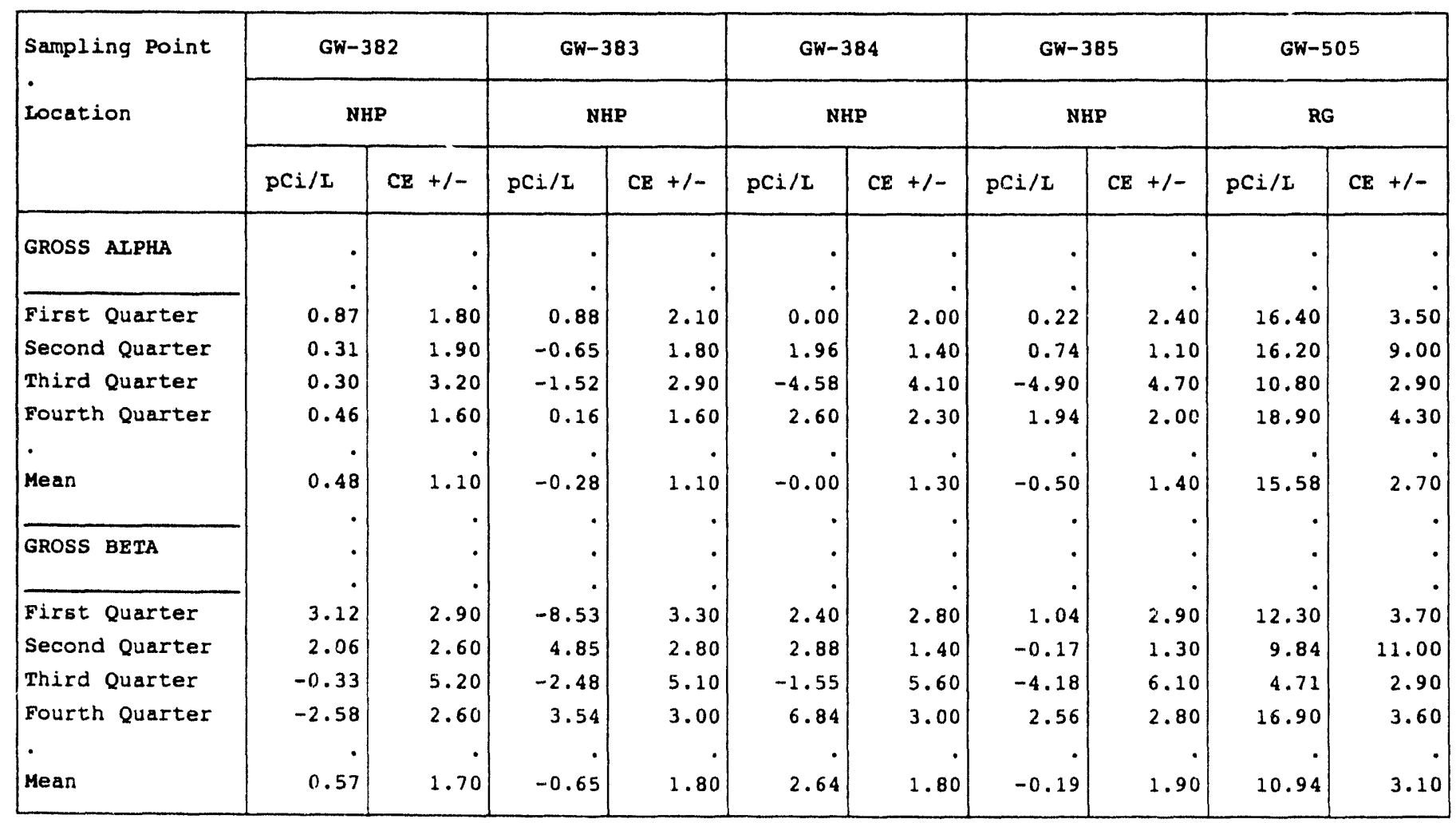

(CONTINUED) 
Gross Alpha and Gross Beta Activity Data Sumary, 1993

\begin{tabular}{|c|c|c|c|c|c|c|c|c|c|c|}
\hline \multirow{3}{*}{$\begin{array}{l}\text { Sampling Point } \\
\text { Location }\end{array}$} & \multicolumn{2}{|c|}{ GW-508 } & \multicolumn{2}{|c|}{ GW-603 } & \multicolumn{2}{|c|}{$G W-604$} & \multicolumn{2}{|c|}{$G W-605$} & \multicolumn{2}{|c|}{ GW-606 } \\
\hline & \multicolumn{2}{|c|}{ RG } & \multicolumn{2}{|c|}{$\operatorname{EXP}$} & \multicolumn{2}{|c|}{$\operatorname{EXP}$} & \multicolumn{2}{|c|}{ Exp } & \multicolumn{2}{|c|}{ EXP } \\
\hline & $\mathrm{pCi} / \mathrm{L}$ & $\mathrm{CE}+/-$ & $\mathrm{pCi} / \mathrm{L}$ & $\mathrm{CE}+/-$ & $\mathrm{pCi} / \mathrm{L}$ & $\mathrm{CE}+1-$ & $\mathrm{pCi} / \mathrm{L}$ & $C E+1-$ & $\mathrm{pCi} / \mathrm{L}$ & $\mathrm{CE}+/-$ \\
\hline GROSS ALPHA & - & - & - & . & - & - & - & - & . & - \\
\hline First Quarter & 0.29 & 2.90 & 2.37 & 1.90 & 0.18 & 1.90 & 53.40 & 6.70 & 4.22 & 1.90 \\
\hline Second Quarter & 0.62 & 1.70 & 1.77 & 2.00 & -0.00 & 0.87 & 61.40 & 10.00 & 4.93 & 5.10 \\
\hline Third Quarter & 2.08 & 3.90 & 0.00 & 2.20 & -1.42 & 1.80 & 29.00 & 5.00 & 1.13 & 2.50 \\
\hline Fourth Quarter & 1.78 & 1.20 & 4.94 & 2.20 & 0.75 & 1.90 & 83.00 & 12.00 & 6.31 & 2.80 \\
\hline & & & . & . & . & - & . & . & - & • \\
\hline Mean & 1.19 & 1.30 & 2.27 & 1.00 & -0.12 & 0.80 & 56.70 & 4.40 & 4.15 & 1.70 \\
\hline GROSS BETA &. & . & . & . & . & . & . & . & . & • \\
\hline First Quarter & 4.27 & 5.40 & 7.61 & 3.10 & 5.16 & 2.80 & 43.40 & 4.80 & 6.37 & 4.10 \\
\hline Second Quarter & 4.13 & 2.80 & 4.85 & 3.00 & -2.92 & 1.40 & 51.40 & 8.20 & 1.84 & 5.80 \\
\hline Third Quarter & 5.30 & 5.60 & 8.43 & 2.90 & 4.05 & 2.60 & 27.40 & 4.00 & 5.09 & 3.00 \\
\hline Fourth Quarter & 4.28 & 2.60 & 7.16 & 3.00 & 0.91 & 2.60 & 58.10 & 7.70 & 0.26 & 3.10 \\
\hline & & & & - & - & . & . & - & - & . \\
\hline Mean & 4.49 & 2.20 & 7.01 & 1.50 & 1.80 & 1.20 & 45.07 & 3.20 & 3.39 & 2.10 \\
\hline
\end{tabular}

(CONTINUED)

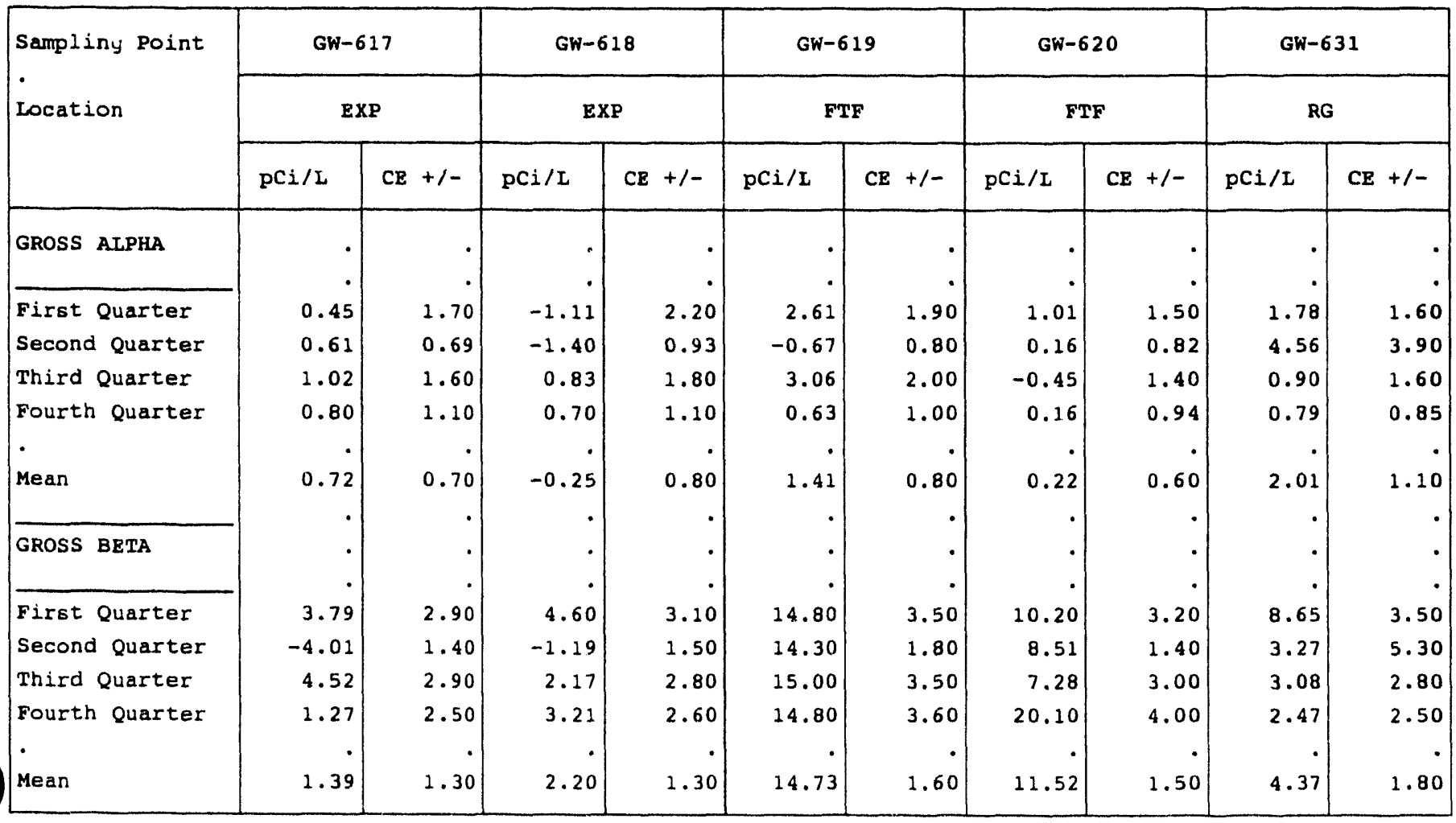

(CONTINUED) 
Gross Alpha and Gross Beta Activity Data Sumnary, 1993

\begin{tabular}{|c|c|c|c|c|c|c|c|c|c|c|}
\hline \multirow{3}{*}{$\begin{array}{l}\text { Sampling Point } \\
\text { Location }\end{array}$} & \multicolumn{2}{|c|}{$G W-632$} & \multicolumn{2}{|c|}{ GW-633 } & \multicolumn{2}{|c|}{$G W-634$} & \multicolumn{2}{|c|}{ GW- 656} & \multicolumn{2}{|c|}{$G W-657$} \\
\hline & \multicolumn{2}{|c|}{ RG } & \multicolumn{2}{|c|}{ RG } & \multicolumn{2}{|c|}{ RG } & \multicolumn{2}{|c|}{ T0134 } & \multicolumn{2}{|c|}{ T2331 } \\
\hline & $\mathrm{pCi} / \mathrm{L}$ & $\mathrm{CE}+/-$ & $\mathrm{pCi} / \mathrm{L}$ & $\mathrm{CE}+1-$ & $\mathrm{pCi} / \mathrm{L}$ & $\mathrm{CE}+/-$ & $\mathrm{pCi} / \mathrm{L}$ & $C E+/-$ & $\mathrm{pCi} / \mathrm{L}$ & $\mathrm{CE}+1-$ \\
\hline GROSS ALPHA & - & - & . & - & $\cdot$ & - & - & - & $\cdot$ & \\
\hline First Quarter & 0.57 & 2.90 & 13.50 & 9.70 & 2.11 & 1.60 & 0.33 & 2.10 & 0.94 & 3.80 \\
\hline Second Quarter & 0.88 & 3.50 & 14.30 & 43.00 & 2.26 & 3.60 & 0.93 & 3.20 & 0.61 & 3.60 \\
\hline Third Quarter & 4.31 & 2.30 & -44.40 & 170.00 & 0.71 & 1.50 & -1.25 & 3.00 & -2.83 & 3.30 \\
\hline Fourth Quarter & 1.30 & 1.00 & 2.85 & 1.80 & 0.44 & 0.67 & 5.63 & 2.90 & 2.39 & 2.20 \\
\hline & & & & $\cdot$ & & & $\cdot$ & $\cdot$ & & • \\
\hline Mean & 1.77 & 1.30 & -3.44 & 43.90 & 1.38 & 1.10 & 1.41 & 1.40 & 0.53 & 1.60 \\
\hline GROSS BETA & . & &. & . &. & & . & . &. & . \\
\hline First Quarter & -0.98 & 5.00 & 75.60 & 9.30 & $11.40^{\circ}$ & 3.60 & 2.59 & 3.10 & 6.64 & 5.60 \\
\hline Second Quarter & 2.63 & 5.20 & 364.00 & 72.00 & 5.40 & 5.40 & 4.30 & 5.30 & 4.80 & 5.40 \\
\hline Third Quarter & 5.62 & 3.00 & 581.00 & 300.00 & 1.36 & 2.70 & 4.30 & 3.30 & 4.46 & 5.60 \\
\hline Fourth Quarter & 3.97 & 2.60 & 43.80 & 6.20 & 3.08 & 2.60 & 8.02 & 3.20 & 4.99 & 2.90 \\
\hline & & & & . & . & & . & - & . & \\
\hline Mean & 2.81 & 2.10 & 266.10 & 77.20 & 5.31 & 1.90 & 4.80 & 1.90 & 5.22 & 2.50 \\
\hline
\end{tabular}

(CONTINUED)

\begin{tabular}{|c|c|c|c|c|c|c|c|c|c|c|}
\hline \multirow{3}{*}{$\begin{array}{l}\text { Sampling Point } \\
\text { Location }\end{array}$} & \multicolumn{2}{|c|}{$G W-658$} & \multicolumn{2}{|c|}{ GW-659 } & \multicolumn{2}{|c|}{ GW-707 } & \multicolumn{2}{|c|}{$G W-708$} & \multicolumn{2}{|c|}{ GW-733 } \\
\hline & \multicolumn{2}{|c|}{ FF } & \multicolumn{2}{|c|}{$\mathbf{F F}$} & \multicolumn{2}{|c|}{$T 2331$} & \multicolumn{2}{|c|}{ T2331 } & \multicolumn{2}{|c|}{ EXP } \\
\hline & $\mathrm{pCi} / \mathrm{L}$ & $\mathrm{CE}+1-$ & $\mathrm{pCi} / \mathrm{L}$ & $\mathrm{CE}+1-$ & $\mathrm{pCi} / \mathrm{L}$ & $\mathrm{CE}+1-$ & $\mathrm{pCi} / \mathrm{L}$ & $C E+/-$ & $\mathrm{pCi} / \mathrm{L}$ & $\mathrm{CE}+/-$ \\
\hline GROSS ALPHA & - & . & - & - & . & - & - & - & . & 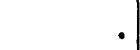 \\
\hline First Quarter & 1.36 & 4.40 & 3.74 & 6.10 & 2.76 & 2.40 & -1.96 & 3.50 & 0.64 & 1.80 \\
\hline Second Quarter & 3.00 & 1.80 & 10.70 & 8.80 & 3.66 & 2.50 & 0.32 & 3.70 & -0.96 & 0.64 \\
\hline Third Quarter & -1.00 & 2.80 & -1.47 & 3.30 & -0.69 & 3.00 & -2.15 & 3.30 & 1.22 & 2.40 \\
\hline Fourth Quarter & 4.04 & 2.50 & 4.55 & 4.00 & 1.89 & 2.10 & 0.34 & 2.10 & 1.06 & 1.70 \\
\hline - & & & & & & & . & • & & \\
\hline Mean & 1.35 & 1.50 & 4.38 & 3.00 & 1.91 & 1.30 & -0.86 & 1.60 & 0.49 & 0.90 \\
\hline GROSS BETA & - & & & $\cdot$ &. & $\cdot$ &. & . & $\cdot$ & . \\
\hline First Quarter & 1.01 & 6.00 & 16.40 & 6.40 & $3.9 \dot{2}$ & 3.20 & 4.01 & 5.40 & 4.49 & 3.00 \\
\hline Second Quarter & 2.43 & 2.70 & 25.30 & 12.00 & 8.15 & 3.10 & 10.70 & 5.80 & 0.23 & 1.20 \\
\hline Third Quarter & -1.56 & 3.00 & 2.90 & 5.40 & 6.81 & 3.40 & 6.19 & 5.70 & 4.96 & 3.00 \\
\hline Fourth Quarter & 4.42 & 2.90 & 12.40 & 5.90 & 5.82 & 3.00 & 7.75 & 3.10 & -0.69 & 2.80 \\
\hline$\cdot$ & & & & & & & & & • & \\
\hline Mean & 1.58 & 1.90 & 14.25 & 3.90 & 6.17 & 1.60 & 7.16 & 2.60 & 2.25 & 1.30 \\
\hline
\end{tabular}

(CONTINUED) 
APPENDIX $\mathrm{K}$

Gross Alpha and Gross Beta Activity Data Summary, 1993

\begin{tabular}{|c|c|c|c|c|c|c|c|c|c|c|c|c|}
\hline \multirow{3}{*}{$\begin{array}{l}\text { Sampling Point } \\
\text { Location }\end{array}$} & \multicolumn{2}{|c|}{$G W-735$} & \multicolumn{2}{|c|}{$G W-744$} & \multicolumn{2}{|c|}{$G W-745$} & \multicolumn{3}{|c|}{ GW-746 } & \multicolumn{3}{|c|}{ GW-747 } \\
\hline & \multicolumn{2}{|c|}{ NHP } & \multicolumn{2}{|c|}{ GRIDKI } & \multicolumn{2}{|c|}{ GRIDKI } & \multicolumn{3}{|c|}{ GRIDK1 } & \multicolumn{3}{|c|}{ GRIDK2 } \\
\hline & $\mathrm{pCi} / \mathrm{L}$ & $\mathrm{CE}+/-$ & $\mathrm{pCi} / \mathrm{L}$ & $\mathrm{CE}+/-$ & $\mathrm{pCi} / \mathrm{L}$ & $\mathrm{CE}+1-$ & $\mathrm{pCi} / \mathrm{L}$ & $\mathrm{CE}$ & $+1-$ & $\mathrm{pCi} / \mathrm{L}$ & $\mathrm{CE}$ & $+1-$ \\
\hline GROSS ALPHA & - & - & - & - & - & - & - & & - & - & & - \\
\hline First Quarter & 0.20 & 2.20 & 2.98 & 2.30 & -0.17 & 1.80 & 0.29 & & 1.60 & -1.25 & & 1.60 \\
\hline Second Quarter & -1.03 & 0.68 & 1.29 & 1.80 & 0.18 & 1.50 & 0.72 & & 1.30 & 0.20 & & 1.70 \\
\hline Third Quarter & -0.83 & 2.30 & -3.64 & 4.20 & -2.48 & 4.50 & 4.67 & & 4.00 & -0.90 & & 3.20 \\
\hline Fourth Quarter & 1.98 & 2.30 & 0.48 & 2.00 & 0.32 & 1.90 & 0.00 & & 1.80 & 0.48 & & 2.00 \\
\hline - & & & & & & & & & • & $\bullet$ & & • \\
\hline Mean & 0.08 & 1.00 & 0.28 & 1.40 & -0.54 & 1.40 & 1.42 & & 1.20 & -0.37 & & 1.10 \\
\hline GROSS BETA & - & - & - & - & - & - & - & & - & - & & • \\
\hline First Quarter & 6.35 & 3.20 & 6.41 & 3.00 & 2.02 & 2.80 & 0.82 & & 2.60 & 1.18 & & 2.70 \\
\hline Second Quarter & 3.15 & 1.30 & 3.74 & 2.70 & 3.30 & 2.60 & 2.87 & & 2.50 & 4.46 & & 2.70 \\
\hline Third Quarter & 2.51 & 2.80 & 1.20 & 5.80 & -1.38 & 5.60 & 2.79 & & 5.10 & 9.74 & & 5.50 \\
\hline Fourth Quarter & 4.10 & 2.90 & 0.50 & 2.80 & 1.75 & 2.90 & 1.40 & & 2.80 & -0.33 & & 2.80 \\
\hline - & & & & & & & & & $\bullet$ & • & & • \\
\hline Mean & 4.03 & 1.30 & 2.96 & 1.90 & 1.42 & 1.80 & 1.97 & & 1.70 & 3.76 & & 1.80 \\
\hline
\end{tabular}

(CONTINUED)

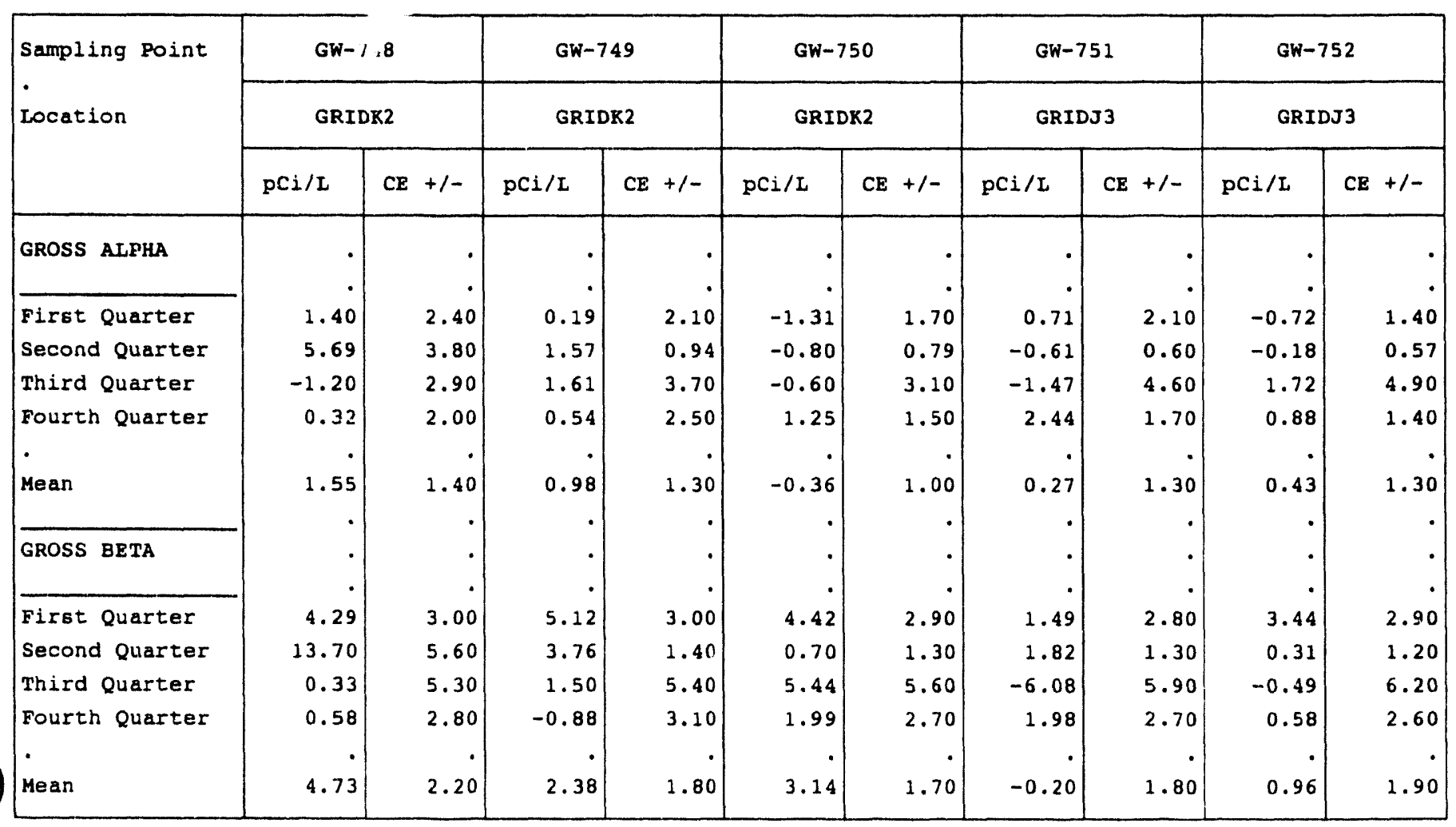

(CONTINUED) 
APPENDIX $\mathrm{K}$

Gross Alpha and Gross Beta Activity Data Summary, 1993

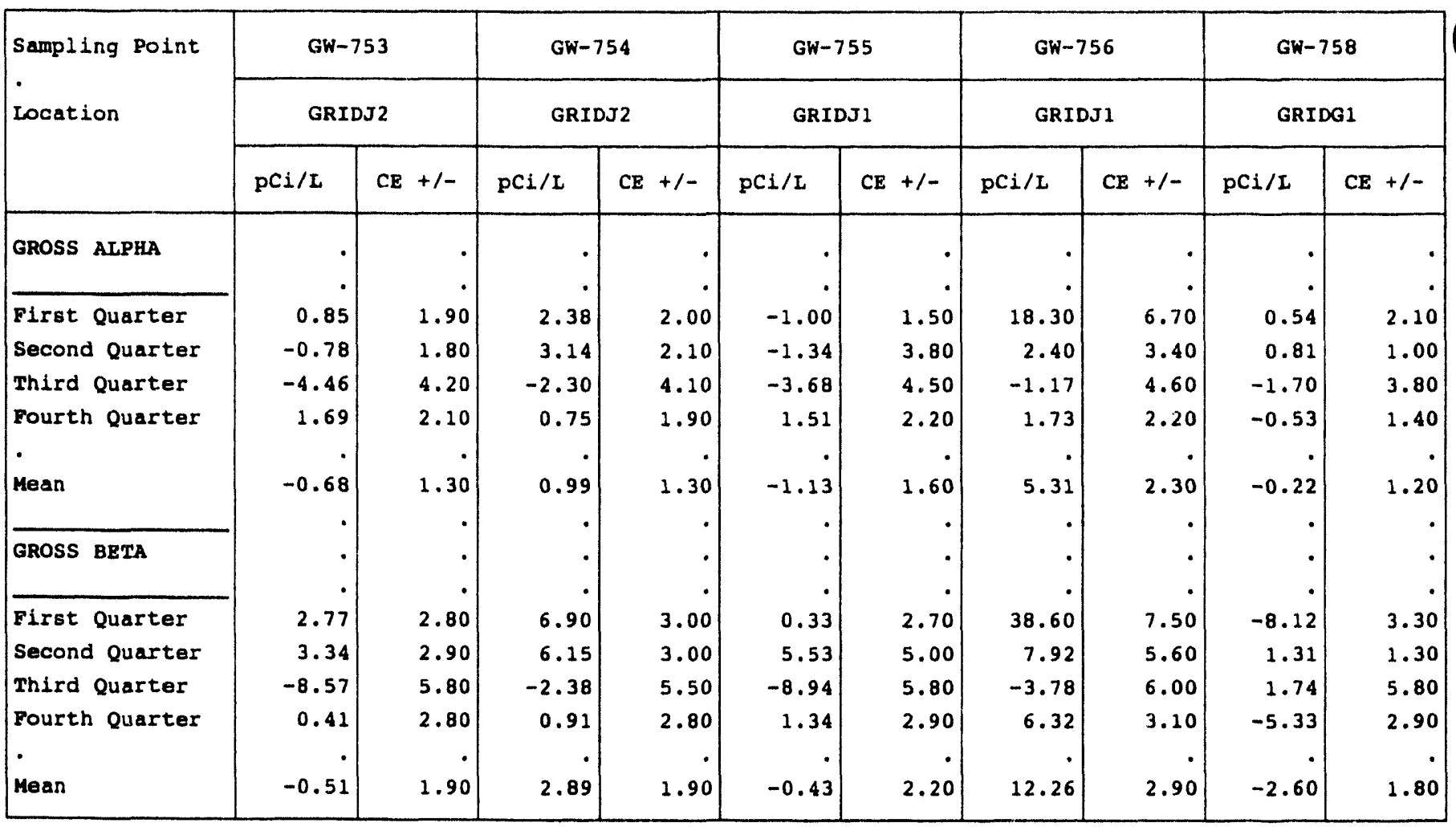

(CONTINUED)

\begin{tabular}{|c|c|c|c|c|c|c|c|c|c|c|c|c|c|}
\hline \multirow{3}{*}{$\begin{array}{l}\text { Sampling Point } \\
\text { L } \\
\text { Location }\end{array}$} & \multicolumn{2}{|c|}{ GW-759 } & \multicolumn{3}{|c|}{ GW-760 } & \multicolumn{2}{|c|}{ GW-761 } & \multicolumn{3}{|c|}{ GW-762 } & \multicolumn{3}{|c|}{ GW-763 } \\
\hline & \multicolumn{2}{|c|}{ GRIDGI } & \multicolumn{3}{|c|}{ GRIDG 2} & \multicolumn{2}{|c|}{ GRIDG2 } & \multicolumn{3}{|c|}{ GRIDJ3 } & \multicolumn{3}{|c|}{ GRIDJ3 } \\
\hline & $\mathrm{pCi} / \mathrm{L}$ & $\mathrm{CE}+1-$ & $\mathrm{pCi} / \mathrm{L}$ & $\mathrm{CE}$ & $+1-$ & $\mathrm{pCi} / \mathrm{L}$ & $\mathrm{CE}+1-$ & $\mathrm{pCi} / \mathrm{L}$ & $\mathrm{CE}$ & $+1-$ & $\mathrm{pCi} / \mathrm{L}$ & CE & $+1-$ \\
\hline GROSS ALPHA & $\cdot$ & • & - & & - & • & • & $\cdot$ & & - & • & & - \\
\hline First Quarter & 2.94 & 2.40 & -0.20 & & 1.80 & 3.34 & 2.10 & -0.60 & & 1.90 & 1.51 & & 2.70 \\
\hline Second Quarter & -1.82 & 0.67 & 4.53 & & 1.40 & 1.41 & 0.79 & -0.48 & & 0.96 & -3.42 & & 3.50 \\
\hline Third Quarter & 8.50 & 9.20 & -2.60 & & 4.00 & 0.59 & 3.60 & -3.28 & & 4.20 & 2.81 & & 4.00 \\
\hline Fourth Quarter & -1.23 & 1.30 & -1.15 & & 1.30 & 0.33 & 1.50 & -1.13 & & 1.70 & 3.94 & & 2.50 \\
\hline & & $\cdot$ & & & - & $\cdot$ & $\cdot$ & - & & • & $\cdot$ & & - \\
\hline Mean & 2.10 & 2.40 & 0.15 & & 1.20 & 1.42 & 1.10 & -1.37 & & 1.30 & 1.21 & & 1.60 \\
\hline GROSS BETA & - & $\cdot$ & - & & - & - & - & - & & - & - & & - \\
\hline First Quarter & 5.99 & 3.10 & -1.97 & & 2.70 & 2.83 & 2.90 & 6.09 & & 2.90 & $-4 \cdot 15$ & & 3.60 \\
\hline Second Quarter & -2.19 & 1.20 & 1.30 & & 1.30 & 1.17 & 1.30 & 4.68 & & 1.40 & 1.40 & & 2.70 \\
\hline Third Quarter & 15.30 & 12.00 & -1.23 & & 5.70 & -0.51 & 5.50 & 1.54 & & 5.80 & 3.32 & & 5.20 \\
\hline Fourth Quarter & -5.17 & 2.90 & 1.18 & & 2.50 & 5.26 & 2.80 & 2.42 & & 2.70 & 4.10 & & 2.90 \\
\hline & & $\cdot$ & • & & & • & $\cdot$ & . & & • & . & & • \\
\hline Mean & 3.48 & 3.20 & -0.18 & & 1.70 & 2.19 & 1.70 & 3.68 & & 1.80 & 1.17 & & 1.90 \\
\hline
\end{tabular}


APPENDIX $R$

Gross Alpha and Gross Beta Activity Data Sumnary, 1993

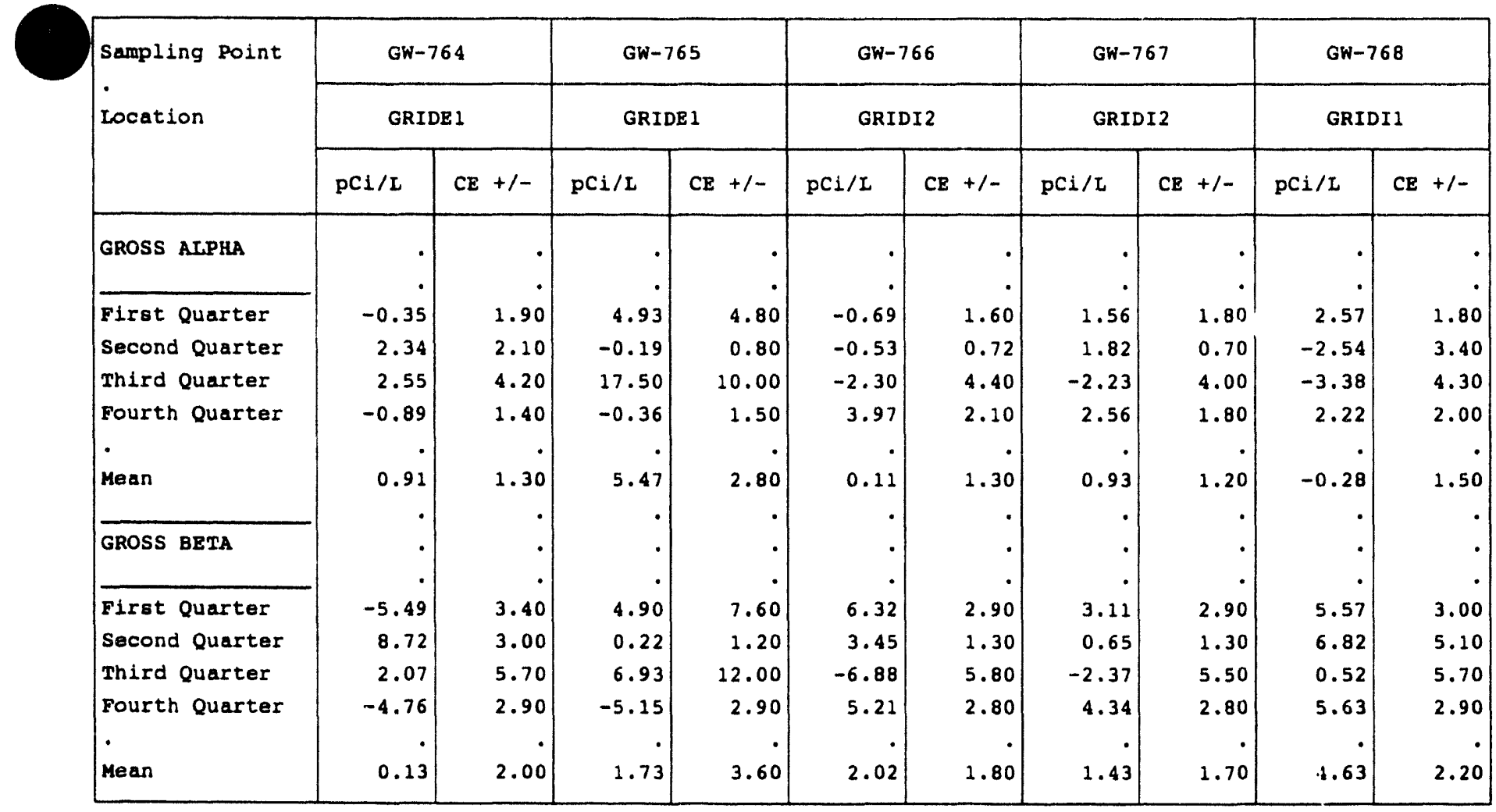

(CONTINUED)

\begin{tabular}{|c|c|c|c|c|c|c|c|c|c|c|c|c|c|}
\hline \multirow{3}{*}{$\begin{array}{l}\text { Sampling Point } \\
\text { Location }\end{array}$} & \multicolumn{3}{|c|}{ GW-769 } & \multicolumn{2}{|c|}{ GW-770 } & \multicolumn{2}{|c|}{ GW-771 } & \multicolumn{3}{|c|}{ GW-772 } & \multicolumn{3}{|c|}{ GW-773 } \\
\hline & \multicolumn{3}{|c|}{ GRIDG3 } & \multicolumn{2}{|c|}{ GRIDG 3} & \multicolumn{2}{|c|}{ GRIDC1 } & \multicolumn{3}{|c|}{ GRIDC1 } & \multicolumn{3}{|c|}{ GRIDH2 } \\
\hline & $\mathrm{pCi} / \mathrm{L}$ & $\mathrm{CE}$ & $+1-$ & $\mathrm{pCi} / \mathrm{L}$ & $\mathrm{CE}+1-$ & $\mathrm{pCi} / \mathrm{L}$ & $\mathrm{CE}+1-$ & $\mathrm{pCi} / \mathrm{L}$ & CE & $+1-$ & $\mathrm{pCi} / \mathrm{L}$ & $\mathrm{CE}$ & $+1-$ \\
\hline GROSS ALPHA & • & & - & - & - & • & - & • & & - & - & & - \\
\hline First Quarter & 0.59 & & 2.00 & 2.28 & 2.10 & -0.19 & 2.10 & 0.31 & & 1.80 & 0.47 & & 1.30 \\
\hline Second Quarter & -0.46 & & 0.91 & 3.96 & 1.30 & 0.57 & 2.00 & -0.32 & & 1.50 & -0.83 & & 0.77 \\
\hline Third Quarter & -0.96 & & 3.70 & 1.23 & 1.70 & 1.59 & 4.10 & -0.61 & & 3.60 & -2.03 & & 2.90 \\
\hline Fourth Quarter & -0.90 & & 1.30 & -0.52 & 1.30 & -0.86 & 1.30 & -0.86 & & 1.30 & 0.00 & & 2.10 \\
\hline & & & $\cdot$ & • & - & $\cdot$ & $\cdot$ & - & & • & - & & - \\
\hline Mean & -0.43 & & 1.10 & 1.74 & 0.80 & 0.28 & 1.30 & -0.37 & & 1.10 & -0.60 & & 1.00 \\
\hline GROSS BETA & - & & - & - & . & $\cdot$ & - & - & & - & - & & - \\
\hline First Quarter & 2.40 & & 2.90 & 3.54 & 3.00 & -6.80 & 3.40 & -9.29 & & 3.20 & 2.74 & & 4.10 \\
\hline Second Quarter & 1.05 & & 1.30 & 3.54 & 1.40 & 3.92 & 2.90 & -0.58 & & 2.60 & 0.80 & & 1.30 \\
\hline Third Quarter & -2.77 & & 5.40 & 3.35 & 2.80 & -0.86 & 5.60 & -0.69 & & 5.50 & 1.15 & & 5.00 \\
\hline Fourth Quarter & 1.89 & & 2.50 & 3.26 & 2.60 & -5.91 & 2.90 & -2.17 & & 3.00 & -1.21 & & 3.00 \\
\hline$\cdot$ & $\bullet$ & & $\cdot$ & - & $\cdot$ & $\cdot$ & $\cdot$ & • & & - & • & & - \\
\hline Mean & 0.64 & & 1.70 & 3.42 & 1.30 & -2.41 & 1.90 & -3.18 & & 1.90 & 0.87 & & 1.80 \\
\hline
\end{tabular}


Gross Alpha and Gross Beta Activity Data Summary, 1993

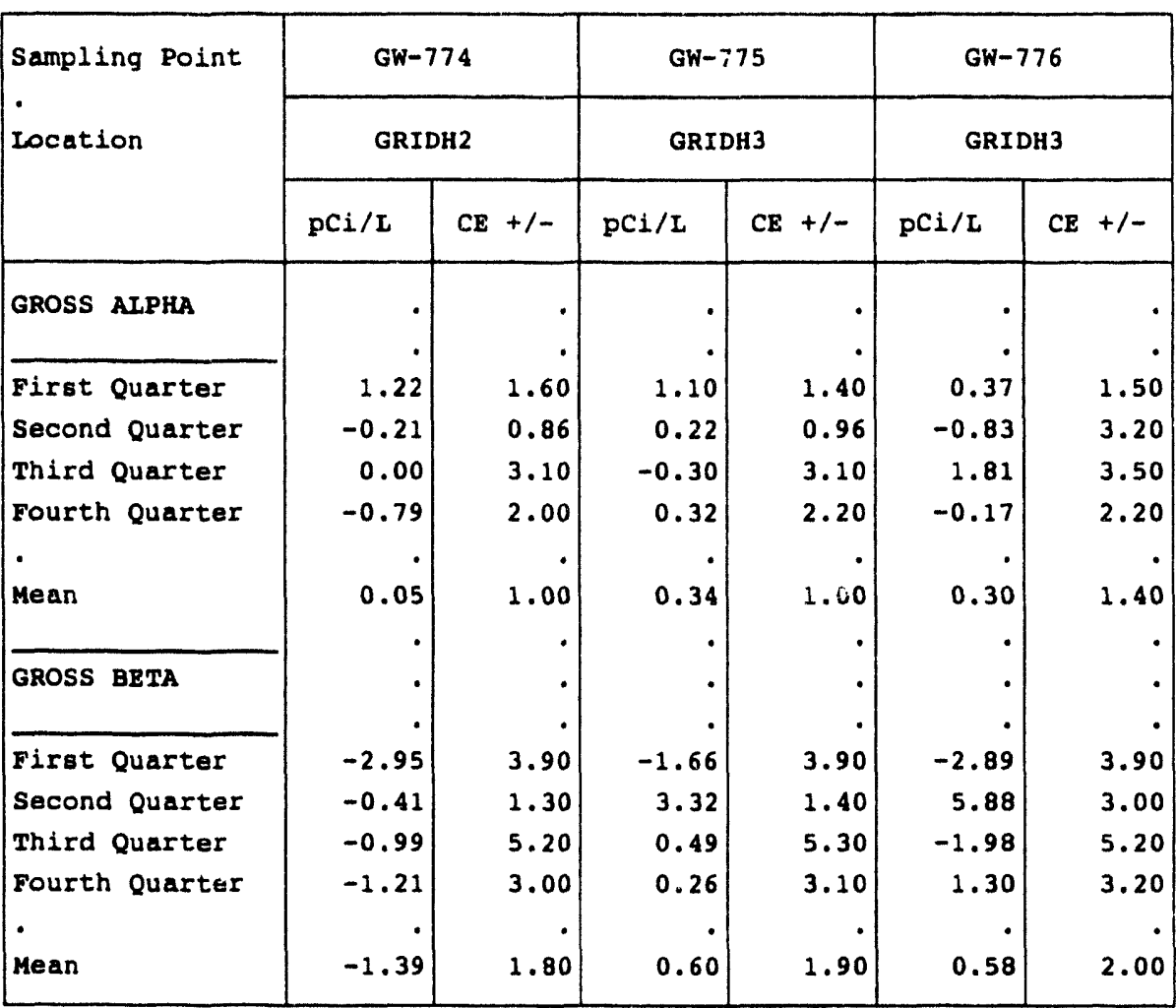


APPENDIX L

ANNUAL A VERAGE NITRATE (as N) CONCENTRATIONS 


\section{EXPLANATION}

\section{LOCATION:}

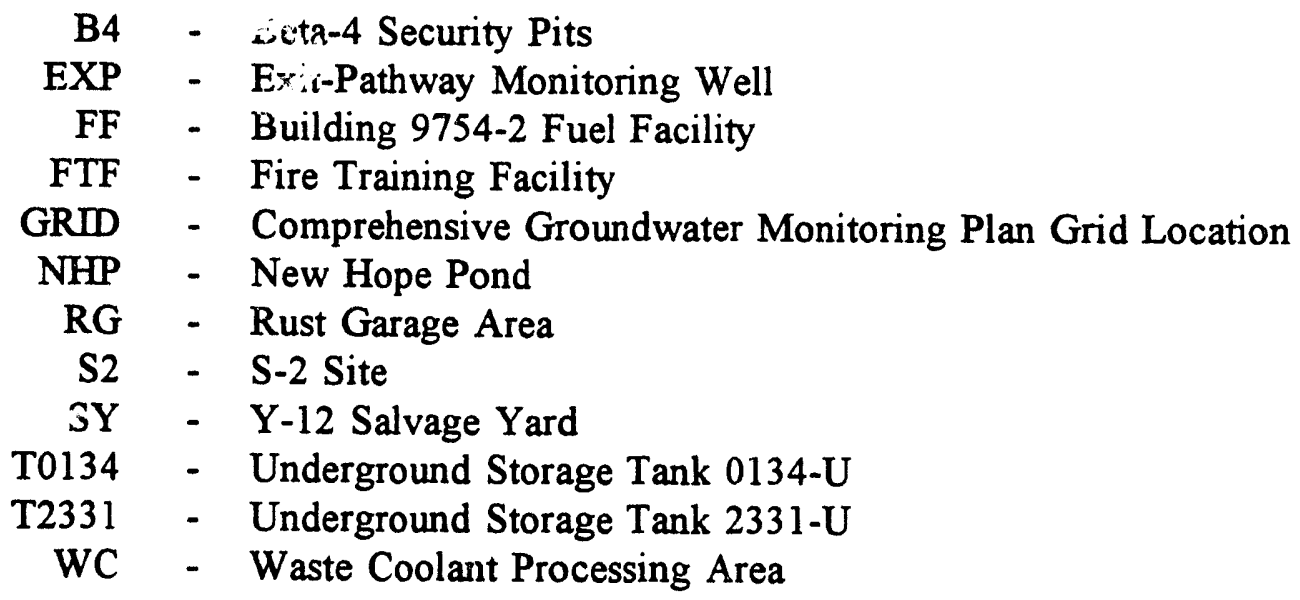

NOTES:

All concentrations reported as nitrate (as $\mathrm{N}$ ) in milligrams per Liter $(\mathrm{mg} / \mathrm{L})$.

Results reported as less than the detection limit were not used to calculate mean concentrations.

ND - Not detected, annual average

. - Not sampled

$<\quad$ - Not detected at the minimum attainable detection limit. 
APPENDIX I

Annual Average Nitrate Concentrations, 1993

\begin{tabular}{|c|c|c|c|c|c|c|}
\hline $\begin{array}{l}\text { sampling } \\
\text { Point }\end{array}$ & Location & $\begin{array}{c}1993 \\
\text { Average }\end{array}$ & First & $\begin{array}{l}\text { Eerly co } \\
\text { second }\end{array}$ & $\begin{array}{l}\text { atration } \\
\text { Third }\end{array}$ & $\begin{array}{l}\text { (mg/L) } \\
\text { Fourth }\end{array}$ \\
\hline GW-151 & NHP & 0.87 & 0.7 & 0.86 & 0.92 & 1 \\
\hline GW-168 & NHP & 0.46 & 0.51 & 0.42 & 0.41 & 0.5 \\
\hline GW-169 & EXP & 0.88 & 1.1 & 0.79 & 0.71 & 0.91 \\
\hline GW-170 & EXP & 0.83 & 0.79 & 0.66 & 0.76 & 1.1 \\
\hline GW-183 & FF & ND & $<0.2$ & $<0.2$ & $<0.2$ & $<0.2$ \\
\hline GW-190 & RG & 0.26 & $<0.2$ & $<0.2$ & 0.26 & $<0.2$ \\
\hline GW-191 & B4 & ND & $<0.2$ & $<0.2$ & $<0.2$ & $<0.2$ \\
\hline GW-192 & B4 & ND & $<0.2$ & $<0.2$ & $<0.2$ & $<0.2$ \\
\hline GW-193 & T2331 & 1.3 & $<0.2$ & $<0.2$ & 1.3 & $<0.2$ \\
\hline GW-194 & B4 & ND & $<0.2$ & $<0.2$ & $<0.2$ & $<0.2$ \\
\hline GW-195 & B4 & 0.3 & $<0.2$ & $<0.2$ & $<0.2$ & 0.3 \\
\hline GW-199 & GRIDI 1 & 0.3 & 0.3 & 0.23 & 0.25 & 0.4 \\
\hline GW-204 & T0134 & 0.27 & $<0.2$ & $<0.2$ & 0.26 & 0.28 \\
\hline GW-206 & $\operatorname{EXP}$ & ND & $<0.2$ & $<0.2$ & $<0.2$ & $<0.2$ \\
\hline GW-207 & EXP & ND & $<0.2$ & $<0.2$ & $<0.2$ & $<0.2$ \\
\hline GW-208 & EXP & ND & $<0.2$ & $<0.2$ & $<0.2$ & $<0.2$ \\
\hline GW-220 & NHP & 0.7 & 0.43 & 0.78 & 0.85 & 0.72 \\
\hline$G W-232$ & EXP & ND & $<0.2$ & $<0.2$ & $<0.2$ & $<0.2$ \\
\hline$G W-239$ & NEP & ND & $<0.2$ & $<0.2$ & $<0.2$ & $<0.2$ \\
\hline$G W-240$ & NHP & 2.11 & 1.1 & 0.85 & 2.5 & 4 \\
\hline GW -251 & s2 & 112.98 & 82 & 83 & 244 & 42.9 \\
\hline$G W-252$ & s2 & 1.09 & 0.47 & 0.87 & 2.5 & 0.5 \\
\hline GW-255 & s2 & 2.18 & 1.6 & 2.2 & 1.8 & 3.1 \\
\hline GW-261 & SY & ND & $<0.2$ & $<0.2$ & $<0.2$ & $<0.2$ \\
\hline GW-262 & SY & ND & $<0.2$ & $<0.2$ & $<0.2$ & $<0.2$ \\
\hline$G W-263$ & SY & 0.45 & 0.52 & 0.39 & 0.27 & 0.6 \\
\hline$G W-264$ & SY & ND & $<0.2$ & $<0.2$ & $<0.2$ & $<0.2$ \\
\hline$G W-281$ & FF & 0.45 & $<0.2$ & 0.44 & 0.45 & $<0.2$ \\
\hline$G W-283$ & FF & ND & $<0.2$ & $<0.2$ & $<0.2$ & $<0.2$ \\
\hline$G W-284$ & $\mathbf{F F}$ & ND & $<0.2$ & $<0.2$ & $<0.2$ & $<0.2$ \\
\hline$G W-285$ & FF & 0.44 & 0.58 & 0.3 & $<0.2$ & $<0.2$ \\
\hline$G W-337$ & WC & ND & $<0.2$ & $<0.2$ & $<0.2$ & $<0.2$ \\
\hline$G W-338$ & WC & 0.46 & 0.51 & 0.41 & 0.31 & 0.6 \\
\hline$G W-380$ & NHP & 1.98 & 1.4 & 1.1 & 1.8 & 3.6 \\
\hline$G W-381$ & NHP & 0.34 & $<0.2$ & 0.34 & $<0.2$ & $<0.2$ \\
\hline$G W-382$ & NEP & 2.98 & 2.8 & 2.08 & 3.45 & 3.6 \\
\hline$G W-383$ & NHP & ND & $<0.2$ & $<0.2$ & $<0.2$ & $<0.2$ \\
\hline$G W-384$ & NEP & ND & $<0.2$ & $<0.2$ & $<0.2$ & $<0.2$ \\
\hline$G W-385$ & NHP & ND & $<0.2$ & $<0.2$ & $<0.2$ & $<0.2$ \\
\hline GW-505 & RG & 1.13 & 1.2 & 1.4 & 1 & 0.93 \\
\hline$G W-508$ & RG & ND & $<0.2$ & $<0.2$ & $<0.2$ & $<0.2$ \\
\hline$G W-603$ & EXP & 0.78 & 0.73 & 0.69 & 0.8 & 0.9 \\
\hline GW- 604 & EXP & 0.52 & 0.62 & 0.45 & 0.5 & 0.5 \\
\hline$G W-605$ & EXP & 0.32 & $<0.2$ & 0.38 & $<0.2$ & 0.25 \\
\hline$G W-606$ & EXP & 0.8 & 0.73 & 0.87 & 0.64 & 0.95 \\
\hline
\end{tabular}


APPENDIX I

Annual Average Nitrate Concentrations, 1993

\begin{tabular}{|c|c|c|c|c|c|c|}
\hline \multirow{2}{*}{$\begin{array}{l}\text { Sampling } \\
\text { Point }\end{array}$} & \multirow[b]{2}{*}{ Location } & \multirow{2}{*}{$\begin{array}{c}1993 \\
\text { Average }\end{array}$} & \multicolumn{2}{|c|}{ Quarterly c } & Concentration & \multirow{2}{*}{$\begin{array}{l}\text { (mg/L) } \\
\text { Fourth }\end{array}$} \\
\hline & & & First & second & Third & \\
\hline$G W-617$ & EXP & 1.3 & 2.4 & 1.5 & 0.51 & 0.8 \\
\hline$G W-618$ & EXP & 1.43 & 1.2 & 1.6 & 1.8 & 1.11 \\
\hline GW-619 & FTF & 0.99 & 0.71 & 0.49 & 2 & 0.77 \\
\hline$G W-620$ & FTF & 1.65 & 1.9 & 1.3 & 1.1 & 2.31 \\
\hline$G W-631$ & RG & 5.58 & 2.8 & 14 & 1.1 & 4.4 \\
\hline GW -632 & RG & 12.05 & 11 & 11 & 13.9 & 12.3 \\
\hline$G W-633$ & RG & 3629 & 3874 & 4300 & 5501 & 841 \\
\hline$G W-634$ & RG & ND & $<0.2$ & $<0.2$ & $<0.2$ & $<0.2$ \\
\hline$G W-656$ & T0134 & 0.33 & $<0.2$ & 0.33 & $<0.2$ & $<0.2$ \\
\hline$G W-657$ & T2331 & 0.26 & $<0.2$ & $<0.2$ & $<0.2$ & 0.26 \\
\hline$G W-658$ & $\mathbf{F F}$ & ND & $<0.2$ & $<0.2$ & $<0.2$ & $<0.2$ \\
\hline GW-659 & $\mathbf{F F}$ & 0.33 & $<2.26$ & $<0.2$ & $<0.2$ & 0.33 \\
\hline GW -707 & T2331 & 0.28 & 0.31 & $<0.2$ & 0.23 & 0.3 \\
\hline GW -708 & T2331 & ND & $<0.2$ & $<0.2$ & $<0.2$ & $<0.2$ \\
\hline$G W-733$ & $\operatorname{EXP}$ & 0.2 & $<0.2$ & $<0.2$ & $<0.2$ & 0.2 \\
\hline GW-735 & NBP & ND & $<0.2$ & $<0.2$ & $<0.2$ & $<0.2$ \\
\hline$G W-744$ & GRIDK 1 & ND & $<0.2$ & $<0.2$ & $<0.2$ & $<0.2$ \\
\hline$G W-745$ & GRIDK1 & ND & $<0.2$ & $<0.2$ & $<0.2$ & $<0.2$ \\
\hline$G W-746$ & GRIDK 1 & 0.49 & 0.61 & 0.46 & 0.5 & 0.4 \\
\hline GW -747 & GRIDK2 & ND & $<0.2$ & $<0.2$ & $<0.2$ & $<0.2$ \\
\hline$G W-748$ & GRIDK2 & ND & $<0.2$ & $<0.2$ & $<0.2$ & $<0.2$ \\
\hline$G W-749$ & GRIDK2 & 3.78 & 0.45 & $<0.2$ & 7.1 & $<0.2$ \\
\hline$G W-750$ & GRIDK2 & ND & $<0.2$ & $<0.2$ & $<0.2$ & $<0.2$ \\
\hline$G W-751$ & GRIDJ3 & ND & $<0.2$ & $<0.2$ & $<0.2$ & $<0.2$ \\
\hline GW-752 & GRIDJ 3 & ND & $<0.2$ & $<0.2$ & $<0.2$ & $<0.2$ \\
\hline$G W-753$ & GRIDJ2 & ND & $<0.2$ & $<0.2$ & $<0.2$ & $<0.2$ \\
\hline GW-754 & GRIDJ2 & ND & $<0.2$ & $<0.2$ & $<0.2$ & $<0.2$ \\
\hline$G W-755$ & GRIDJ 1 & ND & $<0.2$ & $<0.2$ & $<0.2$ & $<0.2$ \\
\hline GW-756 & GRIDJ 1 & ND & $<0.2$ & $<0.2$ & $<0.2$ & $<0.2$ \\
\hline$G W-758$ & GRIDGI & ND & $<0.2$ & $<0.2$ & $<0.2$ & $<0.2$ \\
\hline$G W-759$ & GRIDG 1 & ND & $<0.2$ & $<0.2$ & $<0.2$ & $<0.2$ \\
\hline$G W-760$ & GRIDG2 & 0.44 & 0.38 & 0.54 & $<0.2$ & 0.4 \\
\hline$G W-761$ & GRIDG2 & 0.23 & $<0.2$ & 0.26 & $<0.2$ & 0.2 \\
\hline GW-762 & GRIDJ 3 & ND & $<0.2$ & $<0.2$ & $<0.2$ & $<0.2$ \\
\hline GW -763 & GRIDJ3 & ND & $<0.2$ & $<0.2$ & $<0.2$ & $<0.2$ \\
\hline$G W-764$ & GRIDE 1 & ND & $<0.2$ & $<0.2$ & $<0.2$ & $<0.2$ \\
\hline$G W-765$ & GRIDE 1 & ND & $<0.2$ & $<0.2$ & $<0.2$ & $<0.2$ \\
\hline GW-766 & GRIDI2 & ND & $<0.2$ & $<0.2$ & $<0.2$ & $<0.2$ \\
\hline$G W-767$ & GRIDI2 & 0.25 & $<0.2$ & $<0.2$ & 0.25 & $<0.2$ \\
\hline$G W-768$ & GRIDI 1 & ND & $<0.2$ & $<0.2$ & $<0.2$ & $<0.2$ \\
\hline GW-769 & GRIDG 3 & 0.25 & $<0.2$ & $<0.2$ & 0.25 & $<0.2$ \\
\hline GW-770 & GRIDG 3 & 0.59 & 0.46 & 0.51 & 0.77 & 0.6 \\
\hline$G W-771$ & GRIDC 1 & ND & $<0.2$ & $<0.2$ & $<0.2$ & $<0.2$ \\
\hline GW-772 & GRIDC 1 & ND & $<0.2$ & $<0.2$ & $<0.2$ & $<0.2$ \\
\hline$G W-773$ & GRIDH2 & ND & $<0.2$ & $<0.2$ & $<0.2$ & $<0.2$ \\
\hline
\end{tabular}


APPENDIX L

Annual Average Nitrate Concentrations, 1993

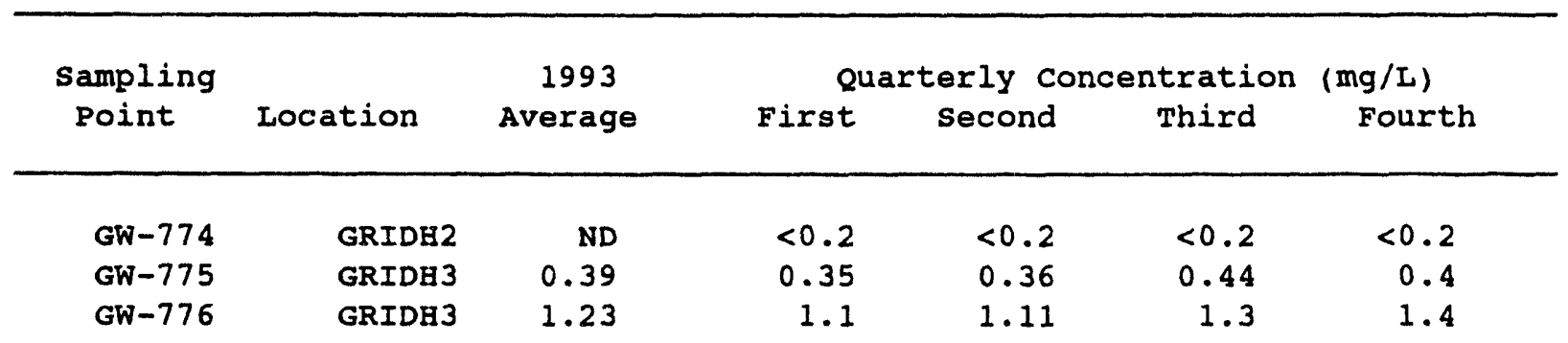




\section{APPENDIX M}

\section{GROUNDWATER ELEVATIONS}




\section{EXPLANATION}

\section{LOCATION:}

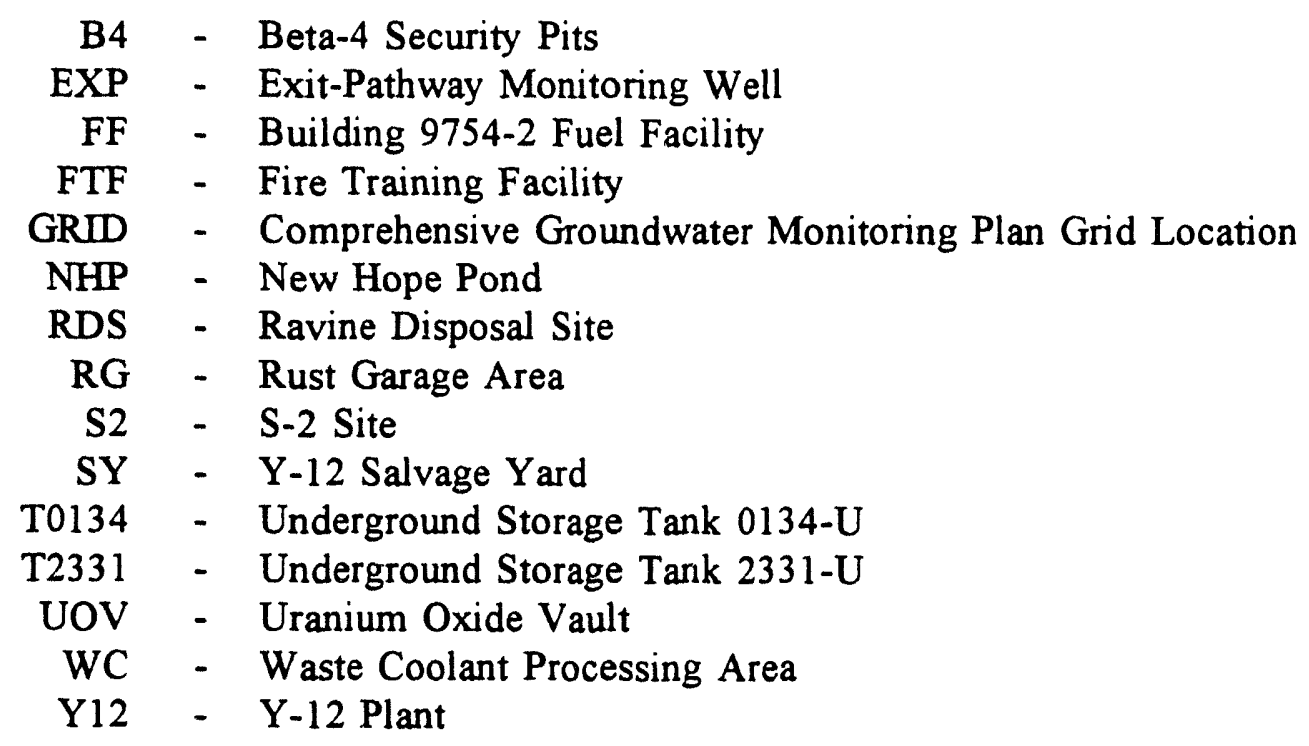

\section{SAMPLED IN 93:}

Y - Wells included in the 1993 groundwater monitoring program.

\section{NOTES:}

CTP Elev. - The elevation of the centerpoint of the monitored interval.

January - Measuring event from January 10 to 14, 1993.

August - Measuring event from August 31 to September 3, 1993.

Depth - The distance in $\mathrm{ft}$ from the top-of-casing measuring point to the static water level.

Elev. - The groundwater elevation in $\mathrm{ft}$ above mean sea level.

+/ Ft - The calculated drop in water level from the January to the August measuring events. Negative results show a rise in water level.

- No information available. 
APPENDIX $M$

Groundwater Elevations, 1993

$\begin{array}{lccccc}\text { Sampling } & & & & & \text { August } \\ \text { Pt. } & \text { Lampled } & \text { CTP } & \text { January } & +/- \\ \end{array}$

Water Table Interval

\begin{tabular}{|c|c|c|c|c|c|c|c|c|}
\hline $55-1 A$ & Y12 & & 970.05 & 10.7 & 975.97 & 10.9 & 975.77 & 0.2 \\
\hline $55-1 B$ & Y12 & & 950.25 & 10.5 & 975.76 & 10.8 & 975.46 & 0.3 \\
\hline $55-1 c$ & Y12 & & 913.45 & 11.2 & 975.53 & 11.9 & 974.83 & 0.7 \\
\hline $55-2 A$ & Y 12 & & 965.12 & 8.2 & 968.54 & 8.6 & 968.14 & 0.4 \\
\hline $55-2 B$ & Y12 & & 951.87 & 7.7 & 969.55 & 8.6 & 968.65 & 0.9 \\
\hline $55-3 A$ & Y 12 & & 960.44 & 11.3 & 961.16 & 11.7 & 960.76 & 0.4 \\
\hline $55-3 B$ & $\times 12$ & & 936.71 & 12.1 & 961.22 & 12.5 & 960.82 & 0.4 \\
\hline $55-3 c$ & Y12 & & 897.51 & 12.5 & 961.84 & 12.9 & 961.44 & 0.4 \\
\hline $55-6 A$ & Y12 & & 977.37 & 7.9 & 981.14 & 9.8 & 979.24 & 1.9 \\
\hline $56-1 A$ & Y12 & & 952.72 & 8.2 & 961.05 & 8.1 & 961.15 & -0.1 \\
\hline $56-2 A$ & $Y 12$ & & 950.47 & 7.5 & 955.80 & 8.4 & 954.90 & 0.9 \\
\hline $56-2 B$ & Y12 & & 926.81 & 7.0 & 955.28 & 7.9 & 954.38 & 0.9 \\
\hline $56-3 A$ & Y 12 & & 947.95 & 10.6 & 952.43 & 10.7 & 952.33 & 0.1 \\
\hline $56-3 B$ & Y 12 & & 932.16 & 10.2 & 953.20 & 10.5 & 952.90 & 0.3 \\
\hline $56-3 c$ & Y12 & & 910.11 & 10.3 & 952.56 & 10.6 & 952.26 & 0.3 \\
\hline $56-4 A$ & Y 12 & & 951.05 & 10.3 & 951.77 & 10.3 & 951.77 & 0.0 \\
\hline $56-6 A$ & Y12 & & 940.12 & • & • & 12.1 & 948.18 & • \\
\hline $56-7 A$ & Y12 & & 938.57 & • & • & 19.3 & 939.15 & • \\
\hline $56-8 A$ & Y 12 & & 936.85 & 19.3 & 943.20 & 20.3 & 942.16 & 1.0 \\
\hline $59-1 A$ & Y 12 & & 934.26 & 3.0 & 942.75 & 4.9 & 940.85 & 1.9 \\
\hline $59-1 B$ & $Y 12$ & & 911.62 & 3.8 & 941.89 & 5.0 & 940.69 & 1.2 \\
\hline $60-1 A$ & Y 12 & & 909.19 & 12.6 & 917.06 & 13.5 & 916.16 & 0.9 \\
\hline $60-1 B$ & Y 12 & & 903.06 & 12.8 & 917.00 & 13.5 & 916.30 & 0.7 \\
\hline $60-2 A$ & Y 12 & & 917.52 & 10.1 & 919.59 & 10.7 & 918.99 & 0.6 \\
\hline$G W-105$ & s3 & & 1000.81 & 8.1 & 1009.25 & 9.3 & 1008.05 & 1.2 \\
\hline$G W-106$ & s3 & & 950.15 & 4.1 & 1011.91 & 5.4 & 1010.61 & 1.3 \\
\hline$G W-107$ & s3 & & 985.43 & 7.2 & 990.99 & 7.4 & 990.79 & 0.2 \\
\hline$G W-108$ & s3 & & 945.81 & 8.4 & 989.78 & 8.7 & 989.48 & 0.3 \\
\hline GW-132 & s3 & & 622.76 & 150.3 & 903.62 & 150.0 & 903.92 & -0.3 \\
\hline GW-135 & s3 & & 497.92 & 181.6 & 996.18 & 177.0 & 1000.78 & -4.6 \\
\hline$G W-148$ & NHP & & 896.68 & 8.3 & 899.06 & 9.1 & 898.26 & 0.8 \\
\hline$G W-150$ & NHP & & 904.53 & 14.2 & 901.36 & 14.1 & 901.46 & -0.1 \\
\hline GW-152 & NHP & $\mathbf{Y}$ & 904.31 & 19.2 & 901.98 & 20.0 & 901.18 & 0.8 \\
\hline$G W-154$ & NEP & & 900.65 & 8.5 & 903.00 & 9.8 & 901.70 & 1.3 \\
\hline$G W-167$ & NEP & $\mathbf{Y}$ & 902.12 & 23.7 & 907.68 & 32.0 & 899.38 & 8.3 \\
\hline$G W-169$ & EXP & $Y$ & 898.25 & 23.9 & 907.97 & 33.4 & 898.47 & 9.5 \\
\hline GW-171 & EXP & & 889.95 & 8.9 & 911.20 & 14.6 & 905.50 & 5.7 \\
\hline$G W-183$ & $\mathbf{F F}$ & $\mathbf{Y}$ & 925.21 & 9.8 & 936.22 & 11.9 & 934.12 & 2.1 \\
\hline GW-191 & B4 & $\mathbf{Y}$ & 954.35 & 4.2 & 1006.94 & 6.8 & 1004.34 & 2.6 \\
\hline GW-192 & B4 & $\mathbf{Y}$ & 994.04 & 4.8 & 1003.83 & 6.8 & 1001.83 & 2.0 \\
\hline GW-193 & T2331 & $\mathbf{Y}$ & 919.16 & 8.5 & 925.50 & 9.7 & 924.30 & 1.2 \\
\hline$G W-194$ & B4 & $Y$ & 982.92 & 6.4 & 988.96 & • & • & • \\
\hline GW-196 & B4 & & 1010.44 & 13.0 & 1024.23 & 19.3 & 1017.93 & 6.3 \\
\hline$G W-198$ & RDS & & 940.54 & 19.7 & 944.82 & 22.4 & 942.12 & 2.7 \\
\hline GW-199 & GRIDI 1 & $\mathbf{Y}$ & 938.56 & 16.6 & 944.48 & 18.2 & 942.88 & 1.6 \\
\hline
\end{tabular}


APPENDIX $M$

Groundwater Elevations, 1993

\begin{tabular}{|c|c|c|c|c|c|c|c|c|}
\hline \multirow{2}{*}{$\begin{array}{l}\text { sampling } \\
\text { pt. }\end{array}$} & \multirow{2}{*}{ Location } & \multirow{2}{*}{$\begin{array}{l}\text { sampled } \\
\text { in } 93\end{array}$} & \multirow{2}{*}{$\begin{array}{c}\text { CTP } \\
\text { Elev. }\end{array}$} & \multicolumn{2}{|c|}{ January } & \multicolumn{2}{|c|}{ August } & \multirow{2}{*}{$\begin{array}{r}+/- \\
\text { Ft }\end{array}$} \\
\hline & & & & Depth & Elev. & Depth & Elev. & \\
\hline$G W-201$ & RDS & & 947.87 & 19.2 & 949.69 & 19.8 & 949.09 & 0.6 \\
\hline GW-202 & RDS & & 947.12 & 10.1 & 957.92 & 11.5 & 956.52 & 1.4 \\
\hline$G W-204$ & T0134 & $\mathbf{Y}$ & 943.57 & 11.6 & 946.97 & 10.9 & 947.67 & -0.7 \\
\hline GW-206 & EXP & $\mathbf{Y}$ & 881.20 & 9.1 & 885.40 & 9.7 & 884.80 & 0.6 \\
\hline$G W-218$ & vov & & 911.82 & 13.2 & 922.61 & 15.9 & 919.91 & 2.7 \\
\hline GW-219 & uov & & 923.47 & 11.5 & 924.14 & 14.2 & 921.44 & 2.7 \\
\hline GW-252 & $\mathbf{s} 2$ & $Y$ & 978.06 & 17.5 & 1006.71 & 32.8 & 991.41 & 15.3 \\
\hline$G W-253$ & $\mathbf{s} 2$ & & 958.50 & 2.3 & 1001.69 & 15.7 & 988.29 & 13.4 \\
\hline GW-255 & s2 & $Y$ & 950.37 & 21.1 & 1006.03 & 37.4 & 989.73 & 16.3 \\
\hline$G W-261$ & SY & $\mathbf{Y}$ & 1027.37 & 17.9 & 1032.09 & 19.9 & 1030.09 & 2.0 \\
\hline$G W-263$ & $\mathbf{s Y}$ & $\mathbf{Y}$ & 1028.23 & 26.3 & 1031.43 & 33.4 & 1024.33 & 7.1 \\
\hline GW-269 & SY & & 999.43 & 18.9 & 1008.91 & • & • & • \\
\hline$G W-272$ & SY & & 994.12 & 7.2 & 1001.96 & . & . & - \\
\hline$G W-273$ & $\mathbf{S Y}$ & & 973.34 & 6.1 & 997.42 & - & - & - \\
\hline$G W-274$ & $\mathbf{S Y}$ & & 962.54 & 5.0 & 990.43 & . & • & - \\
\hline$G W-281$ & $\mathbf{F F}$ & $\mathbf{Y}$ & 937.01 & 5.3 & 940.78 & 7.3 & 938.78 & 2.0 \\
\hline$G W-282$ & $F F$ & & 936.19 & 0.4 & 943.54 & 6.3 & 937.64 & 5.9 \\
\hline$G W-283$ & FE & $\mathbf{Y}$ & 927.22 & 4.5 & 934.12 & 7.2 & 931.42 & 2.7 \\
\hline$G W-284$ & $\mathbf{F F}$ & $\mathbf{Y}$ & 942.30 & 7.9 & 933.71 & 10.2 & 931.41 & 2.3 \\
\hline GW-285 & $\mathbf{F F}$ & $Y$ & 930.63 & 8.7 & 933.76 & 10.9 & 931.56 & 2.2 \\
\hline$G W-332$ & พC & & 959.10 & 10.1 & 970.97 & 11.6 & 969.47 & 1.5 \\
\hline$G W-333$ & WC & & 960.72 & 11.0 & 972.83 & 12.4 & 971.43 & 1.4 \\
\hline$G W-334$ & WC & & 956.73 & 11.3 & 972.43 & 12.3 & 971.43 & 1.0 \\
\hline$G W-335$ & WC & & 969.45 & 9.9 & 971.98 & 11.7 & 970.18 & 1.8 \\
\hline$G W-337$ & wc & $\mathbf{Y}$ & 965.57 & 10.6 & 976.71 & 11.6 & 975.71 & 1.0 \\
\hline$G W-338$ & WC & $\mathbf{Y}$ & 969.62 & 9.0 & 978.27 & 9.1 & 978.17 & 0.1 \\
\hline$G W-349$ & s2 & & 976.33 & 3.7 & 989.80 & 6.7 & 986.80 & 3.0 \\
\hline GW-350 & s2 & & 955.15 & 3.6 & 989.91 & 6.5 & 987.01 & 2.9 \\
\hline$G W-380$ & NEP & $\mathbf{Y}$ & 904.51 & 8.9 & 904.65 & 11.6 & 901.95 & 2.7 \\
\hline$G W-383$ & NHP & $Y$ & 885.90 & 9.3 & 899.20 & 9.8 & 898.70 & 0.5 \\
\hline$G W-384$ & NHP & $Y$ & 859.78 & 12.3 & 896.08 & 12.9 & 895.48 & 0.6 \\
\hline$G W-617$ & EXP & $\mathbf{Y}$ & 970.21 & 13.0 & 972.11 & 15.1 & 970.01 & 2.1 \\
\hline$G W-618$ & EXP & $Y$ & 951.14 & 12.6 & 972.34 & 14.8 & 970.14 & 2.2 \\
\hline$G W-619$ & FTE & $Y$ & 978.94 & 17.4 & 997.84 & 29.5 & 985.74 & 12.1 \\
\hline$G W-620$ & FTF & $\mathbf{Y}$ & 944.49 & 19.8 & 995.54 & 30.4 & 984.94 & 10.6 \\
\hline$G W-633$ & $R G$ & $\mathbf{Y}$ & 987.27 & 6.2 & 990.30 & 4.0 & 992.50 & $-2 \cdot 2$ \\
\hline$G W-634$ & RG & $\mathbf{Y}$ & 998.06 & 7.9 & 999.25 & 10.1 & 997.05 & 2.2 \\
\hline$G W-656$ & T0134 & $\mathbf{Y}$ & 940.00 & 10.0 & 944.79 & 9.9 & 944.89 & -0.1 \\
\hline$G W-657$ & T2331 & $Y$ & 920.25 & 6.5 & 924.00 & • & • & • \\
\hline$G W-658$ & FF & $Y$ & 929.04 & 10.2 & 934.61 & 12.7 & 932.11 & 2.5 \\
\hline GW-659 & $F F$ & $Y$ & 931.14 & 7.0 & 934.16 & 8.9 & 932.26 & 1.9 \\
\hline GW-707 & T2331 & $\mathbf{Y}$ & 915.41 & 5.9 & 925.00 & • & • & • \\
\hline GW-708 & T2331 & $\mathbf{Y}$ & 922.83 & 4.6 & 926.30 & • & • & - \\
\hline GW-735 & NHP & $\mathbf{Y}$ & 847.99 & 19.8 & 904.48 & 23.7 & 900.58 & 3.9 \\
\hline$G W-746$ & GRIDK 1 & $Y$ & 895.06 & 3.8 & 903.08 & 6.6 & 900.28 & 2.8 \\
\hline$G W-749$ & GRIDK2 & $\mathbf{Y}$ & 908.09 & 4.0 & 917.19 & 9.3 & 911.89 & 5.3 \\
\hline$G W-752$ & GRIDJ 3 & $Y$ & 899.41 & 3.7 & 909.08 & 6.4 & 906.38 & 2.7 \\
\hline
\end{tabular}


APPENDIX $M$

Groundwater Elevations, 1993

\begin{tabular}{|c|c|c|c|c|c|c|c|c|}
\hline \multirow{2}{*}{$\begin{array}{c}\text { sampling } \\
\text { pt. }\end{array}$} & \multirow[b]{2}{*}{ Location } & \multirow{2}{*}{$\begin{array}{l}\text { Sampled } \\
\text { in } 93\end{array}$} & \multirow{2}{*}{$\begin{array}{c}\text { CTP } \\
\text { Elev. }\end{array}$} & \multicolumn{2}{|c|}{ January } & \multicolumn{2}{|c|}{ August } & \multirow{2}{*}{$\begin{array}{r}+1- \\
\text { Ft }\end{array}$} \\
\hline & & & & Depth & Elev. & Depth & Elev. & \\
\hline GW-754 & GRIDJ2 & $\mathbf{Y}$ & 907.08 & 3.8 & 924.79 & 13.2 & 915.39 & 9.4 \\
\hline GW -756 & GRIDJ 1 & $\mathbf{Y}$ & 913.94 & 5.8 & 922.12 & 7.2 & 920.72 & 1.4 \\
\hline GW-759 & GRIDG1 & $\mathbf{Y}$ & 966.94 & 17.3 & 976.71 & 19.4 & 974.61 & 2.1 \\
\hline GW-761 & GRIDG2 & $\mathbf{Y}$ & 955.46 & 8.1 & 960.13 & 13.3 & 954.93 & 5.2 \\
\hline GW -765 & GRIDE 1 & $\mathbf{Y}$ & 979.38 & 19.3 & 989.24 & 20.1 & 988.44 & 0.8 \\
\hline GW-767 & GRIDI2 & $\mathbf{Y}$ & 932.82 & 10.5 & 938.04 & 12.6 & 935.94 & 2.1 \\
\hline GW -770 & GRIDG3 & $\mathbf{Y}$ & 928.42 & 13.5 & 931.05 & 14.6 & 929.95 & 1.1 \\
\hline GW-774 & GRIDH2 & $\mathbf{Y}$ & 940.54 & 9.7 & 953.46 & 17.2 & 945.96 & 7.5 \\
\hline
\end{tabular}

Bedrock Interval

\begin{tabular}{|c|c|c|c|c|c|c|c|c|}
\hline $55-2 c$ & Y12 & & 903.65 & 7.8 & 969.05 & 8.6 & 968.25 & 0.8 \\
\hline $56-1 c$ & Y12 & & 896.75 & 6.5 & 962.99 & 6.7 & 962.79 & 0.2 \\
\hline $56-2 c$ & Y 12 & & 888.25 & 9.2 & 955.74 & 9.9 & 955.04 & 0.7 \\
\hline $56-4 C$ & Y12 & & 885.90 & 10.1 & 952.52 & 10.2 & 952.42 & 0.1 \\
\hline $59-1 c$ & Y 12 & & 874.67 & 2.7 & 943.17 & 4.8 & 941.07 & 2.1 \\
\hline GW-109 & s3 & & 881.99 & 3.5 & 993.49 & 4.7 & 992.29 & 1.2 \\
\hline GW-149 & NHP & & 861.51 & 13.7 & 894.02 & 14.1 & 893.62 & 0.4 \\
\hline GW-151 & NHP & $\mathbf{Y}$ & 822.31 & 15.6 & 900.37 & 15.3 & 900.67 & -0.3 \\
\hline$G W-153$ & NHP & & 866.03 & 9.9 & 911.54 & 20.3 & 901.14 & 10.4 \\
\hline$G W-168$ & NHP & $\mathbf{Y}$ & 809.75 & 26.6 & 904.35 & 31.7 & 899.25 & 5.1 \\
\hline$G W-170$ & EXP & $\mathbf{Y}$ & 800.25 & 29.8 & 902.64 & 33.9 & 898.54 & 4.1 \\
\hline GW -172 & EXP & & 803.67 & 15.1 & 909.69 & 20.9 & 903.89 & 5.8 \\
\hline$G W-200$ & RDS & & 915.34 & 15.8 & 953.29 & 18.6 & 950.49 & 2.8 \\
\hline$G W-220$ & NHP & $\mathbf{Y}$ & 874.64 & 20.1 & 895.54 & 16.3 & 899.34 & -3.8 \\
\hline$G W-222$ & NEP & & 887.32 & 8.9 & 902.92 & 10.2 & 901.62 & 1.3 \\
\hline$G W-223$ & NHP & & 824.22 & 8.9 & 902.72 & 10.1 & 901.52 & 1.2 \\
\hline$G W-230$ & EXP & & 546.11 & 12.7 & 908.15 & 16.9 & 903.95 & 4.2 \\
\hline GW -232 & EXP & $\mathbf{Y}$ & 523.17 & 28.7 & 902.52 & 32.3 & 898.92 & 3.6 \\
\hline GW-239 & NHP & $\mathbf{Y}$ & 509.86 & 27.9 & 903.60 & 30.1 & 901.40 & 2.2 \\
\hline$G W-240$ & NHP & $\mathbf{Y}$ & 894.25 & 19.0 & 903.90 & 21.3 & 901.60 & 2.3 \\
\hline GW-251 & s2 & $\mathbf{Y}$ & 958.60 & 11.5 & 992.10 & 22.8 & 980.80 & 11.3 \\
\hline GW-262 & SY & $\mathbf{Y}$ & 983.17 & 17.4 & 1032.27 & 19.2 & 1030.47 & 1.8 \\
\hline$G W-264$ & SY & $Y$ & 990.79 & 27.2 & 1031.10 & 33.8 & 1024.50 & 6.6 \\
\hline$G W-270$ & SY & & 991.60 & 2.1 & 1006.86 & • & • & • \\
\hline$G W-271$ & SY & & 956.39 & 0.9 & 1008.11 & • & - & • \\
\hline GW-275 & SY & & 933.68 & 3.2 & 992.16 & • & • & • \\
\hline$G W-381$ & NHP & $\mathbf{Y}$ & 858.55 & 10.6 & 902.76 & 11.5 & 901.86 & 0.9 \\
\hline$G W-382$ & NHP & $Y$ & 764.16 & 9.3 & 903.87 & 11.8 & 901.37 & 2.5 \\
\hline$G W-385$ & NHP & $\mathbf{Y}$ & 754.67 & 3.9 & 904.97 & 5.0 & 903.87 & 1.1 \\
\hline$G W-603$ & EXP & $\mathbf{Y}$ & 890.11 & 56.3 & 905.00 & 59.6 & 901.72 & 3.3 \\
\hline$G W-604$ & EXP & $Y$ & 853.23 & 55.7 & 905.16 & 59.5 & 901.36 & 3.8 \\
\hline$G W-605$ & EXP & $\mathbf{Y}$ & 882.92 & 10.8 & 908.08 & 11.2 & 907.68 & 0.4 \\
\hline GW-606 & EXP & $\mathbf{Y}$ & 753.63 & 12.8 & 906.59 & 14.4 & 904.99 & 1.6 \\
\hline$G W-733$ & EXP & $Y$ & 707.39 & 54.4 & 904.64 & 58.1 & 900.94 & 3.7 \\
\hline$G W-734$ & EXP & & 907.72 & 35.9 & 904.03 & 39.7 & 900.23 & 3.8 \\
\hline GW-744 & GRIDK 1 & $Y$ & 842.80 & 5.5 & 901.93 & 7.7 & 899.73 & 2.2 \\
\hline
\end{tabular}


APPENDIX $M$

Groundwater Elevations, 1993

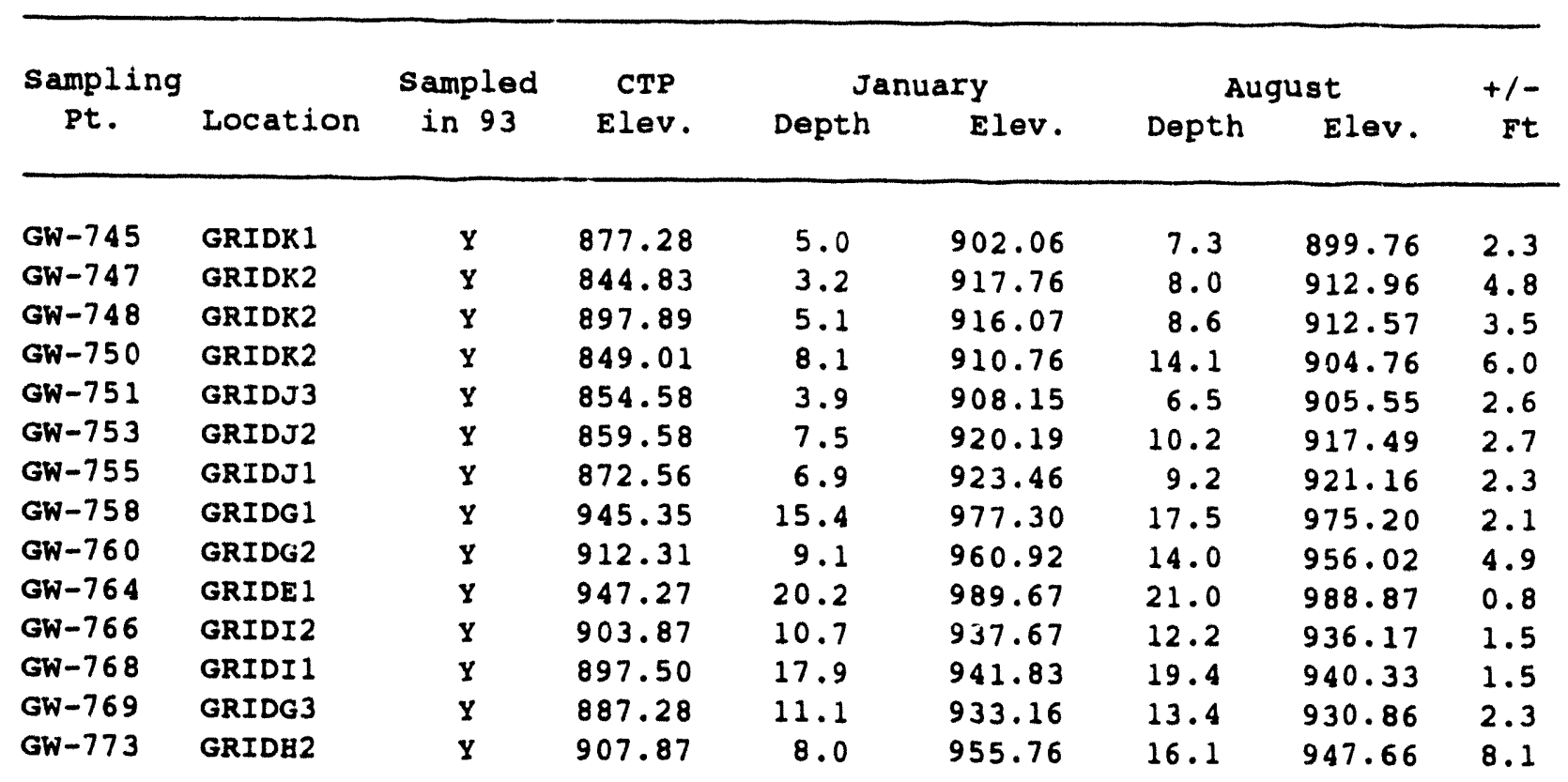




\section{DISTRIBUTION}

DEPARTMENT OE ENERGY

G. W. Bodenstein

W. G. McMillan

P. J. Gross/L. L. Radcliffe

HEALTH, SAFETY, ENVIRONMENT, AND ACCOUNTABILITY ORGANIZATION

D. E. Bohrman

C. C. Hill

W. K. Jago

S. B. Jones

L. W. McMahon

J. E. Powell

E. B. Rundle

L. O. Vaughan

S. W. Wiley

GWPP-File

ENVIRONMENTAL RESTORATION PROGRAM

V. J. Brumback

J. T. Grumski

M. J. Norris

J. A. Vanderlan

C. S. Walker

UNIVERSITY OF TENNESSEE

B. W. McMaster

G. K. Moore

UNIVERSITY OF NEVADA. RENO

L. A. Shevenell

QRISE

S. M. Field
ENVIRONMENTAL SCIENCES DIVISION

R. B. Dreier

T. O. Early

D. D. Huff

C. T. Rightmire

R. R. Turner

D. B. Watson

WASTE MANAGEMENT ORGANIZATION

K. D. Delius

C. W. Hutzler

I. W. Jeter

J. E. Stone

ENGINEERING ORGANIZATION

W. E. Manrod

QUALITY AND TECHNICAL SERVICES

P. Buckley

L. P. Burnett

HSW ENVIRONMENTAL CONSULTANTS

J. R. Walker

H\&R TECHNCIAL ASSOCIATES

P. M. Ezell

SCIENCE APPLICATIONS

INTERNATIONAL CORPORATION

S. Selecman/W. P. Kegley

Y-12 Central Files

A. K. Lee/DOE-OSTI (2) 

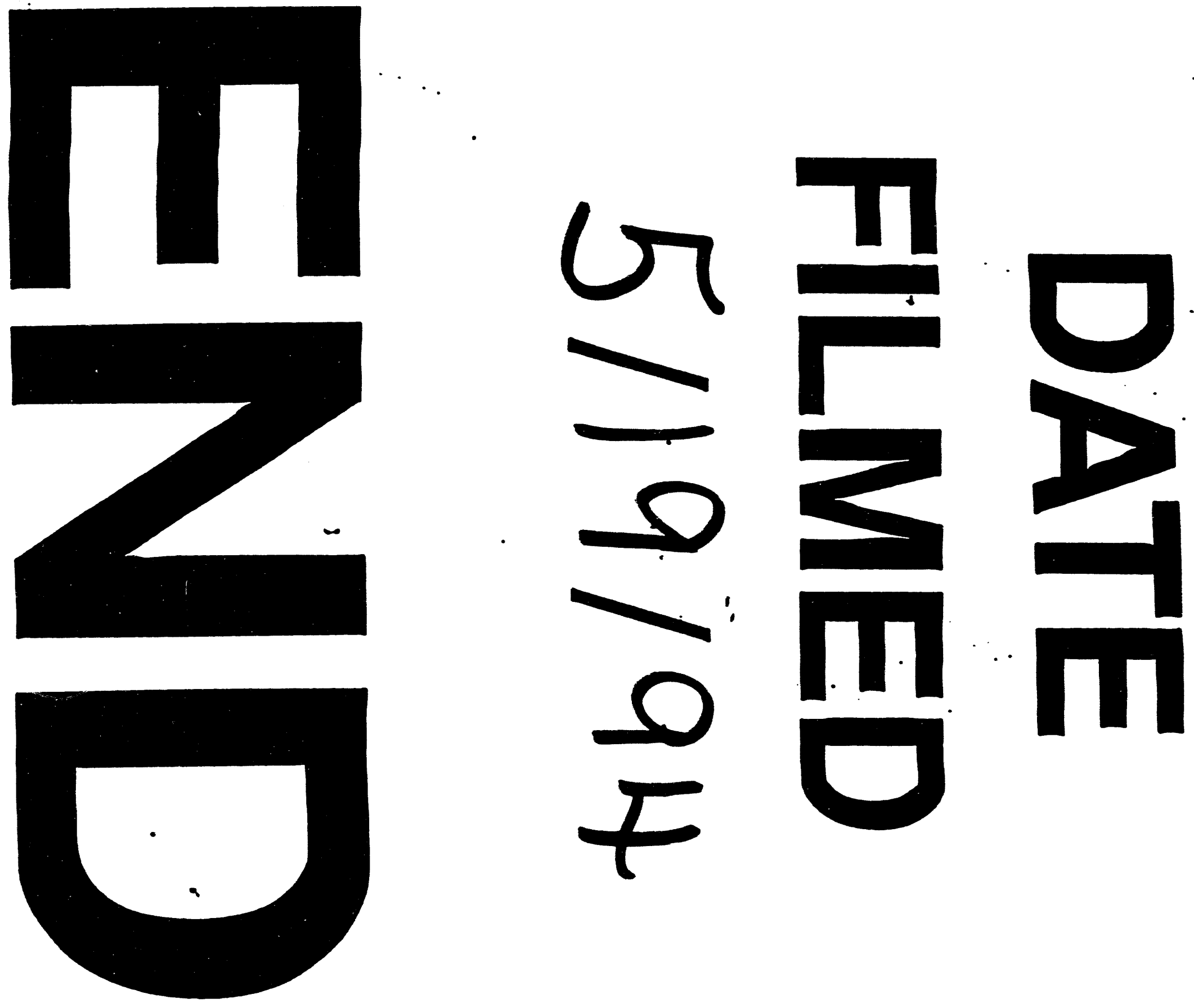
$\mid$ 